LAWRENCE LIVERMORE NATIONAL LABORATORY

High Bandwidth Rotary

Fast Tool Servos and a

Hybrid Rotary/Linear

Electromagnetic Actuator

R. C. Montesanti

September 2005 
This document was prepared as an account of work sponsored by an agency of the United States Government. Neither the United States Government nor the University of California nor any of their employees, makes any warranty, express or implied, or assumes any legal liability or responsibility for the accuracy, completeness, or usefulness of any information, apparatus, product, or process disclosed, or represents that its use would not infringe privately owned rights. Reference herein to any specific commercial product, process, or service by trade name, trademark, manufacturer, or otherwise, does not necessarily constitute or imply its endorsement, recommendation, or favoring by the United States Government or the University of California. The views and opinions of authors expressed herein do not necessarily state or reflect those of the United States Government or the University of California, and shall not be used for advertising or product endorsement purposes.

This work was performed under the auspices of the U.S. Department of Energy by University of California, Lawrence Livermore National Laboratory under Contract W-7405-Eng-48. 


\section{High Bandwidth Rotary Fast Tool Servos and a Hybrid Rotary/Linear Electromagnetic Actuator by}

Richard Clement Montesanti

B.S. Mechanical Engineering Northeastern University

S.M. Mechanical Engineering Massachusetts Institute of Technology (1987)

Submitted to the Department of Mechanical Engineering in partial fulfillment of the requirements for the degree of

Doctor of Philosophy in Mechanical Engineering at the

\section{MASSACHUSETTS INSTITUTE OF TECHNOLOGY}

September 2005

(C) Richard C. Montesanti 2005. All rights reserved.

The author hereby grants to MIT and LLNL permission to reproduce and to distribute publicly paper and electronic copies of this thesis document in whole or in part.

Author

Department of Mechanical Engineering August 5, 2005

Certified by

David L. Trumper Professor of Mechanical Engineering Thesis Supervisor Accepted by

Professor Lallit Anand Chairman, Department Committee on Graduate Students 
This document was prepared as an account of work sponsored by an agency of the United States Government. Neither the United States Government nor the University of California nor any of their employees, makes any warranty, express or implied, or assumes any legal liability or responsibility for the accuracy, completeness, or usefulness of any information, apparatus, product, or process disclosed, or represents that its use would not infringe privately owned rights. Reference herein to any specific commercial product, process, or service by trade name, trademark, manufacturer, or otherwise, does not necessarily constitute or imply its endorsement, recommendation, or favoring by the United States Government or the University of California. The views and opinions of authors expressed herein do not necessarily state or reflect those of the United States Government or the University of California, and shall not be used for advertising or product endorsement purposes.

This work was performed under the auspices of the U.S. Department of Energy by University of California, Lawrence Livermore National Laboratory under Contract W-7405-Eng-48. 


\title{
High Bandwidth Rotary Fast Tool Servos and a Hybrid Rotary/Linear Electromagnetic Actuator
}

\author{
by \\ Richard Clement Montesanti \\ Submitted to the Department of Mechanical Engineering \\ on August 5, 2005, in partial fulfillment of the \\ requirements for the degree of \\ Doctor of Philosophy in Mechanical Engineering
}

\begin{abstract}
This thesis describes the development of two high bandwidth short-stroke rotary fast tool servos and the hybrid rotary/linear electromagnetic actuator developed for one of them. Design insights, trade-off methodologies, and analytical tools are developed for precision mechanical systems, power and signal electronic systems, control systems, normal-stress electromagnetic actuators, and the dynamics of the combined systems.

A fast tool servo (FTS) is a high-speed auxiliary servo axis that is added to a diamond turning machine (ultra-precision lathe) to allow generating free-form nonaxisymmetric or textured surfaces on a workpiece. A rotary fast tool servo produces an in-and-out motion of the tool relative to a workpiece by swinging the tool along an arc having a fixed radius. The rotary fast tool servos developed in this project were designed for diamond turning prescription textured surfaces on small spherical workpieces (diameters in the range of $10 \mathrm{~mm}$ or less), and are suitable for generating free-form non-axisymmetric surfaces on similar-sized workpieces. Straightforward modifications would allow them to be used on larger workpieces. These rotary fast tool servos set new benchmarks for demonstrated closed-loop bandwidth $(2 \mathrm{kHz}$ and $10 \mathrm{kHz}$ ) and tool tip acceleration (400 g).

The first machine, referred to as the $2 \mathrm{kHz}$ rotary fast tool servo, uses a commercially available moving-magnet galvanometer as the actuator (Lorentz force), and provides proof-of-principles for a flexure bearing, small diamond tool and mounting method, circuit topology for a high bandwidth current-mode amplifier, and control system design. The following closed-loop performance is demonstrated for the $2 \mathrm{kHz}$ rotary fast tool servo: $-3 \mathrm{~dB}$ bandwidth of $2 \mathrm{kHz}, 20 \mathrm{~g}$ tool tip acceleration at $2 \mathrm{kHz}$, maximum tool travel of $50 \mu \mathrm{m} \mathrm{PP}$, and tool position noise level of $10 \mathrm{~nm} \mathrm{PP}$. The $2 \mathrm{kHz}$ FTS is integrated with a diamond turning machine and used to produce optical quality textured surfaces on the face and outside diameter of aluminum workpieces while operating at $2 \mathrm{kHz}$. The machining tests validate that a rotary-type fast tool servo can be used to produce optical quality surfaces on a spherical workpiece from its pole to its equator.

The second machine, referred to as the $10 \mathrm{kHz}$ rotary fast tool servo, incorporates
\end{abstract}


the proof-of-principles from the first machine and is the vehicle for developing the hybrid rotary/linear electromagnetic actuator used in it. The actuator is a normalstress variable reluctance machine with a demonstrated order of magnitude increase in the peak torque and in the ratio of peak torque divided by the electrical power at its terminals, when compared to the actuator used in the $2 \mathrm{kHz}$ FTS. By integrating the tool holder directly to the moving mass of the actuator to form a single rigid body, the overall torque-to-inertia ratio for the system and the frequency of the first uncoupled-mass resonance are both increased. The following closed-loop performance is demonstrated for the $10 \mathrm{kHz}$ rotary fast tool servo: $-3 \mathrm{~dB}$ bandwidth of $10 \mathrm{kHz}$, $400 \mathrm{~g}$ tool tip acceleration at $5 \mathrm{kHz}, 870 \mathrm{~g}$ tool tip acceleration at $10 \mathrm{kHz}$ (aided by a stable mechanical resonance), maximum tool travel of $70 \mu \mathrm{m} \mathrm{PP}$, and tool position noise level of 1.4 to $2.5 \mathrm{~nm} \mathrm{rms}$ (depending on the magnitude of the bias flux used).

The hybrid rotary/linear electromagnetic actuator utilizes a constant bias magnetic flux, which linearizes the torque versus drive-current relationship for the actuator and provides up to half of the torque-producing magnetic flux in the rotor/stator air gaps. The actuator is similar to the rotary actuators used to drive and sustain a resonance in a mechanical oscillator in certain electric engraving heads. This research is distinguished from the prior art by the ability to generate closed-loop arbitrary trajectories for the tool tip. Using a separate current-mode amplifier for each stator half allows demonstrating closed-loop control of the rotary and linear degrees of freedom that are inherent in this class of actuators. This research is further distinguished from the prior art by a magnetic circuit that substantially decouples certain magnetic flux paths when a coil is used instead of a permanent magnet to provide the bias magnetic flux. This reduces the complexity of the actuator electrical dynamics from a MIMO system to a SISO system, and allows using loop-shaping techniques with classical control theory to design the control systems.

Torque control for the hybrid rotary/linear actuator in the $10 \mathrm{kHz}$ FTS is independent of force control, but force control requires a torque-generating current to act as an operating point. Alternate magnetic circuit topologies that fully decouple torque and force control are described and compared. Future work that utilizes the linear mode as an active suspension for improving the performance of a predominantly rotary system is considered. Using the experience gained by designing, building, and testing the $10 \mathrm{kHz}$ FTS and hybrid rotary/linear actuator, future work involving alternate concepts for the actuator is suggested for a follow-on rotary fast tool servo, and a high bandwidth steering mirror.

Thesis Supervisor and Committee Chairman:

David L. Trumper, Professor of Mechanical Engineering

Thesis Committee Members:

James L. Kirtley Jr., Professor of Electrical Engineering

Samir A. Nayfeh, Assistant Professor of Mechanical Engineering

James K. Roberge, Professor of Electrical Engineering 


\section{Acknowledgements}

This research most notably benefited from the guidance provided by the members of my thesis committee: MIT Professors David L. Trumper, James L. Kirtley Jr., Samir A. Nayfeh, and James K. Roberge.

Professor Trumper was my research advisor and I am especially grateful for the time that I spent working with him and for his guidance from start to finish during my doctoral program. Professor Trumper created and sustains a stimulating environment in his research group at MIT for gaining a hardware-oriented understanding of precision mechanical hardware, electronics, motion control, system dynamics, and magnetics. When I first arrived in Professor Trumper's group, I had little to no working knowledge in all but the first of those subjects, and I joked with him that some day I would have a sufficient enough background to contribute to a conversation about them. Later, during the conceptual design phase for the $10 \mathrm{kHz}$ fast tool servo and its electromagnetic actuator, we were having fun brain-storming and evaluating a myriad of possibilities and trade-offs in technical areas that had earlier been mysteries to me. I was able to run with him then because he took the time to stroll with me earlier when I was learning to walk. I am fortunate to have had the opportunity to work alongside him while evaluating and troubleshooting electronic and motion control systems, and appreciated his encouragement to push on the envelope of my abilities.

Professor Kirtley taught me a considerable amount about electromagnetic machines and electrical power systems. After completing a course that he teaches on these topics, I continued visiting his office on a regular basis and always felt welcomed. Professor Kirtley helped me develop, evaluate, and sort through many of the design ideas on the hybrid electromagnetic actuator described in this thesis. I am especially grateful for the time he spent helping me extend my understanding of electromagnetic systems. I enjoyed our many conversations, appreciated his encouragement and openmannered candor, and am grateful for his willingness to engage my seemingly endless stream of questions. 
Professor Nayfeh inspired me to be scholarly in my approach to gaining an understanding of a topic. I enjoyed his effervescent enthusiasm for machine design and the many philosophical conversations that we had on that subject and others. Professor Nayfeh always found time for discussion when I approached him with questions, and he helped me expand and deepen my understanding of the mechanics of materials. I am especially grateful for his encouragement to not shy away from using adhesivelybonded joints in the critical mechanical components in the $10 \mathrm{kHz}$ fast tool servo.

Professor Roberge, and one of his teaching assistants from the late 1980's, taught me classical control theory in 2001 through his "Electronic Feedback Systems" video course. There are two remarkable things about this. First, the video tapes where produced fifteen years earlier, while I was finishing my masters degree in mechanical engineering at MIT. Second, the teaching assistant was a graduate student named David Trumper. By a fortunate twist of fate, I had ended up learning classical control theory from the same two people who would have probably taught me the subject if I had immediately continued my studies after my masters program. While I was learning the material via the video tapes, Professor Roberge graciously invited me to drop by his office if I had questions. Later, when I was developing the control systems described in this thesis, I had many conversations with him that provided me with useful insights and deepened my understanding of the subject.

My colleagues in Professor Trumper's Precision Motion Control Laboratory at MIT truly enriched my experience there. It was a joy to be constantly surrounded by their seemingly boundless energy, enthusiastic curiosity, and capable intelligence. They were largely responsible for the day-to-day level of excellence in the Lab, were always willing to engage in discussions around the hardware or at the blackboard, and provided me with help when I needed it. 
Augusto Barton is a fine mechanical design engineer and a gentleman. I appreciated his good nature and ability to maintain a positive attitude in the Lab.

Marten Byl maintained continuity of the Lab, and helped me find my way around when I first arrived. Marty helped me learn to build Simulink ${ }^{\circledR}$ models and implement them using the dSPACE GmbH real-time controller board. He saved me a considerable amount of time by wiring together the electrical components in the diamond turning machine described in this thesis.

Joe Cattell provided me with many fresh perspectives on the topics that we discussed, and helped me understand some of the subtleties associated with Adaptive Feedforward Cancellation compensation.

David Cuff was always willing to let me "think aloud" about things that I was working on, and provided me with useful feedback and insights.

Larry Hawe brought a refreshing outlook on life to the Lab, and always took the time to help me solve computer and software problems that were getting the best of me.

Katie Lilienkamp helped me become more proficient writing MATLAB ${ }^{\circledR}$ scripts. The Simulink ${ }^{\circledR}$-based dynamic signal analyzer that Katie developed allowed me to efficiently gather the experimental frequency response data reported in this thesis.

Xiaodong Lu provided me with an excellent role model of a doctoral student by combining his exemplary work ethic with his inspiring drive and ability to acquire a masterful understanding of concepts and hardware across a broad range of technologies. An excellent teacher, Xiaodong always found time to help me understand topics and ideas that were new to me, and he taught me a considerable amount about electronic circuit design and troubleshooting. I am fortunate that our research shared many of the same technologies and challenges - we often created momentum for each other - and I enjoyed our many philosophical and practical discussions on a wide range of engineering subjects.

Aaron Mazzeo always had a receptive ear when I needed someone to talk to. 
Dave Otten was a constant source of insight when it came to troubleshooting electronic circuits and exploring mechanical design trade-offs. Dave was always a pleasure to be around, and when my thinking on a topic became too focused, I could depend on him to help me step back for a fresh top-level view.

Andrew Stein helped me learn to build Simulink ${ }^{\circledR}$ models and implement them using the dSPACE GmbH real-time controller board, and he helped me become proficient in producing detailed mechanical drawings using Pro/Engineer.

Marty Vona helped me when I started learning to design and build electronic circuits. Ross Hatton, Amar Kendale, Mike Liebman, Vijay Shilpiekandula, and Yi Xie were additional members of the Precision Motion Control Lab that contributed to my memorable experience there.

Gerry Wentworth, Mark Belanger, Dave Dow, and Pat McAtamney in the Laboratory for Manufacturing and Productivity machine shop at MIT where immensely helpful in resurrecting my old machining skills, teaching me new ones, and helping out when the task at hand exceeded my abilities.

David Rodriguera, Maggie Sullivan, Denise Moody, Laura Zaganjori, and Sally Stiffler helped me navigate through MIT's administrative world, made it refreshingly simple to procure items and track expenses, and helped me maintain a balanced perspective on life at MIT.

Leslie Regan remembered my name when I walked into her office as a former MIT student that she had not seen for fourteen years. When I told her that I was considering returning to MIT as a doctoral candidate she asked, "Do you know what you are getting into? Does your wife know what it will be like? Are you really sure you want to do this?" The Mechanical Engineering graduate students at MIT are extremely fortunate to have someone that sincerely cares about their well-being and is proactive in helping them navigate through the system. 
Joan Kravit always greeted me with a friendly "hello", and helped me with administrative issues at MIT.

Vivian Mizuno helped to make Professor Kirtley's lab at MIT a home-away-fromhome for me.

MIT Professor Ain Sonin had an open-door policy, and I enjoyed our many impromptu conversations and his youthful enthusiasm during our discussions on engineering topics. I appreciated his encouragement to think beyond qualitative explanations and use the analytical insights that I was acquiring, and the role model he provided of a gentleman scholar.

Harvard University Professor Paul Horowitz and staff member Tom Hayes taught me a considerable amount about the art of electronics during the semester-long course that I took with them.

Among the many people at MIT that I had the benefit of associating with during my doctoral program, I would like to especially acknowledge the following individuals for sharing their time, knowledge, and friendship: Shorya Awtar, Joaquin Blaya, Blake Gleason, Paulo Goncalves, Dave Micus, Neil Murray, Greg Radighieri, Jonathan Rohrs, Keith Santarelli, Jim Serdy, Paul Slovenski, Kripa Varanasi, Justin Verdirame, Andrew Wilson, and Josh Wilson. 
The research described in this thesis was accomplished during a work assignment with the Lawrence Livermore National Laboratory (LLNL). Funding was provided by LLNL, primarily through its Laboratory Directed Research and Development program (LDRD). The LDRD program is a funding mechanism for encouraging and supporting high technical risk engineering and scientific research that is viewed to have a potential significant impact on the Laboratory's ability to fulfill its primary mission of scientific research in the interest of National Security [109]. LDRD funding is awarded year to year on a Laboratory-wide competitive basis at LLNL for a maximum of three years. This research benefited from three years of LDRD support, and this thesis serves as a final report of the work accomplished.

Among the many people at LLNL that I had the benefit of working with since I joined the Laboratory in 1987, I would like to especially acknowledge the following individuals for their direct and indirect contributions to this research.

First, my sincere gratitude to the members of the management staff at LLNL who supported my participation in this research. Jens Mahler provided me with the initial support from the Engineering Directorate, and made it possible for me to pursue this research and an advanced degree. Hugh Watling was a steadfast advocate of my efforts while at MIT, and provided funding during the last year of this research. Jeff Klingmann was instrumental in getting the LDRD project started, and managed many of the logistics at LLNL while I was resident at MIT, which is on the other side of the country.

Dennis Atkinson provided my earliest examples of how a project engineer takes care of his team and gets tasks accomplished, and taught me that often, "just good enough is perfect" (adequate is optimum).

Doug Baird provided my earliest guidance on designing and conducting experiments involving optical systems, lasers, precision mechanical alignment hardware, and the related measurement equipment. Doug taught me how to maintain a useful record in a lab notebook. 
Charlie Cass was my chief mechanic when I developed a new diamond flycutting machine and vertical-axis positioning system for LLNL. Charlie taught me to consider how the machines that I designed would be fabricated and assembled, what their likely failure modes would be, and to make sure that they could be maintained and serviced. Todd Decker provided me with examples of how a project engineer balances identifying, discussing, and planning what needs to be accomplished, and then gets the job done. Bob Donaldson was my most significant mentor in the field of precision engineering, and I had the good fortune of working with him during repairs, troubleshooting, and upgrades of the Large Optics Diamond Turning Machine (LODTM) at LLNL. Bob instilled in me the engineering rigor and deterministic approach that are needed to understand and design state-of-the-art precision machines, and he taught me how to efficiently gain sufficient analytical insights with simple hand-calculations.

Al Farris provided my earliest examples of how to develop a concept for a new machine into an executable mechanical design.

Layton Hale is a fine precision engineer, and I had the good fortune of many conversations with him on the subject during previous projects at LLNL. Layton's impassioned ability to generate ideas, identify and analyze the core technical issues, and pursue perfection are exemplary.

Stan Locke taught me by example how to elevate the techniques of mechanical design to the art of machine design. Stan's sense for an aesthetic that equates to a sound mechanical design provided me an approach that was worth emulating.

Steve Patterson was one of my mentors in the field of precision engineering. Steve taught me how to reduce an engineering problem to its essential underlying physics and develop a quantitative understanding based on a first-principles analysis.

Sam Thompson was one of my long-time colleagues for troubleshooting, repairing, and designing precision machine tools for LLNL. Sam's passion for exploring machines, asking questions that others often missed, and tenacious pursuit of answers was inspiring. 


\section{Dedication}

To my wife Katrina and son Ian. Together you made our time at MIT a celebration of living life as an adventure, instead of just waiting to arrive at the other end of what was often a difficult journey.

Katrina. I am very fortunate to be travelling along the path of my life with such a sincere, considerate, and vivacious partner. You were often my climbing partner in the mountains before we moved to MIT, and you provided me with an attentive bombproof belay while at MIT. When challenges in my work left me feeling run-out too far beyond what felt safe, knowing that you were there gave me the confidence to keep moving towards my goal. You caught my falls, and helped me regain my footing and continue ascending again. I am truly grateful that we tied-in together.

Ian. You were three months old when we moved into student housing on the MIT campus, and almost five years old when we left. Towards the end of our stay, upon hearing Mom and me discuss moving on, you said, "MIT has everything a person could ever want, why would we ever want to leave?" I am deeply happy that your first years were so incredibly rich and satisfying.

To my Mom, Norma Montesanti. You nurtured my childhood scientific curiosity, encouraged my passion for learning, and taught me to never stop growing.

To my Dad, Clement Montesanti. You kindled my earliest desires to invent, trained me to be a craftsman with machine tools, and instilled in me a strong work ethic.

To my brothers and sisters: Donna, Steven, Terri, and Bob. Your friendship, love, and emotional support have been constants in my world.

To the memory of my grandfather, Donald Wentworth. You passed on to me your "watchmaker's eye", which you received from your father, a clockmaker. You gave me a sense of pride in old-fashioned Yankee craftsmanship, and a desire to live up to that tradition. 


\section{Contents}

1 Introduction $\quad 47$

1.1 Background . . . . . . . . . . . . . . . . . . . 48

1.2 Project Summary . . . . . . . . . . . . . . . . . . . 51

1.2.1 The $2 \mathrm{kHz}$ Rotary Fast Tool Servo . . . . . . . . . . . . 55

1.2.2 The $10 \mathrm{kHz}$ Rotary Fast Tool Servo . . . . . . . . . . . . . . . 60

1.2.3 Hybrid Rotary/Linear Actuator . . . . . . . . . . . . . 62

1.3 Thesis Organization . . . . . . . . . . . . . . . 67

2 Motivation and Context $\quad 69$

2.1 Introduction . . . . . . . . . . . . . . . . . . . . . 69

2.2 Fast Tool Servos . . . . . . . . . . . . . . . . . . 70

2.2.1 Motivation for Fast Tool Servo Development . . . . . . . . 70

2.2.2 Motivation for a Rotary-Type Fast Tool Servo . . . . . . . . . 72

2.2.3 Fast Tool Servos in the Literature . . . . . . . . . . . . . . . . 75

2.3 Electric Engraving Heads . . . . . . . . . . . . . . . . 80

2.3.1 Method of Operation . . . . . . . . . . . 83

2.3.2 Actuators ................... . . 84

2.3.3 Bearing Systems . . . . . . . . . . . . . . . . . . 94

2.3.4 Damping. . . . . . . . . . . . . . . . 97

2.3.5 Control . . . . . . . . . . . . . . . . . . 100

2.3.6 Sensors . . . . . . . . . . . . . . . . . 103

2.3.7 Tool Mounting . . . . . . . . . . . . . . . . . . 108

2.4 Additional Pertinent Prior Art . . . . . . . . . . . . . . . . . . 110 
2.4.1 Radial Plus Tip/Tilt Actuator with Flux-Biasing . . . . . . . 110

2.4.2 Normal-Stress Linear Actuator with Flux-Biasing . . . . . . . 114

2.4.3 Shear-Stress Rotary Actuator with Flux-Biasing . . . . . . . . 115

2.4.4 Crossed-Blade Flexure Suspension . . . . . . . . . . . . . . . 116

2.5 Conclusions . . . . . . . . . . . . . . . . . . . . . 118

3 The 2 kHz Rotary Fast Tool Servo 119

3.1 Introduction . . . . . . . . . . . . . . . . . . . . . . . . . 119

3.2 Design Philosophy . . . . . . . . . . . . . . . . . . . . . . 120

3.3 Machine Topology Concepts . . . . . . . . . . . . . . . . . . . . . . . 122

3.4 Designing for Stiffness . . . . . . . . . . . . . . . . 123

3.5 Manufacturing Tolerances,

Assembly Complexity, and Contingencies . . . . . . . . . . . . . . . 127

3.6 Mechanical Design . . . . . . . . . . . . . . . . . . . . 129

3.6.1 Tool . . . . . . . . . . . . . . . . . . . . . . . 129

3.6 .2 Swing-Arm (Rotor) . . . . . . . . . . . . . 133

3.6.3 Flexure Blades . . . . . . . . . . . . . . . . . . . . 133

3.6.4 Trade-Off Study for Flexure Blades . . . . . . . . . . . . . . . 138

3.6.5 Base and Associated Hardware . . . . . . . . . . . . . . . . . 148

3.6.6 Mechanical Damper . . . . . . . . . . . . . . . . . . . . . . . 148

3.6.7 Actuator . . . . . . . . . . . . . . . . . 149

3.6.8 Sensors . . . . . . . . . . . . . . . . . . . . . . 152

3.7 System Dynamics . . . . . . . . . . . . . . . . . . . . . . . . . . . 154

3.7.1 Modelled Plant Dynamics . . . . . . . . . . . . . 155

3.7.2 Measured Plant Dynamics . . . . . . . . . . . . . . . . . 158

3.7.3 Equivalent Linear Mass

and Passive Dynamic Stiffness Seen at the Tool Tip . . . . . . 161

3.8 Servo Test Bed . . . . . . . . . . . . . . . . . . . . . . . 163

3.9 Electrical Design . . . . . . . . . . . . . . . . . 165

3.9.1 Current-Mode Amplifier . . . . . . . . . . . . . . . . . 165 
3.9.2 Voltage Budget and Power Dissipated in the Amplifier . . . . 166

3.9.3 Electrical Component Layout . . . . . . . . . . . . . . . . . 169

3.9.4 Brief Comments on Troubleshooting Electrical Systems . . . . 171

3.10 Control System . . . . . . . . . . . . . . . . . . . . . . . . 174

3.10.1 Digital Controller Prototyping Environment . . . . . . . . . 175

3.10.2 Double Lead, Lag, and Notch Filter Compensation . . . . . . 178

3.10.3 Double Lead, Lag, and Phase-Stabilizing Pole Compensation . 179

3.10.4 Viscous Damping and Lag Compensation . . . . . . . . . . . . 184

3.11 Results . . . . . . . . . . . . . . . . . . . . . . . . . 194

3.11 .1 Machining Tests . . . . . . . . . . . . . . . . 197

3.11 .2 Demonstrated Peak Torque . . . . . . . . . . . . . . . . . 199

3.12 Conclusions . . . . . . . . . . . . . . . . . . 203

4 The 10 kHz Rotary Fast Tool Servo 205

4.1 Introduction . . . . . . . . . . . . . . . . . . . 205

4.2 Design Philosophy . . . . . . . . . . . . . . . . 206

4.3 Magnetic Circuit . . . . . . . . . . . . . . . . . . . . . 208

4.4 Machine Topology Concepts . . . . . . . . . . . . . . . . . . . . . . . 210

4.5 Mechanical Design . . . . . . . . . . . . . . . . . . . 219

4.5.1 Rotor and Stator Cores . . . . . . . . . . . . . . . . . 221

4.5.2 Rotor ...................... . . 226

4.5.3 Stator . . . . . . . . . . . . . . . . . 228

4.5.4 Mounting the Rotor to the Stator . . . . . . . . . . . . . . 235

4.5.5 Tool Holder . . . . . . . . . . . . . . . . . . . . . . . . . . . . 244

4.5 .6 Flexures . . . . . . . . . . . . . . . . . . . . . 245

4.5.7 Sensors . . . . . . . . . . . . . . . . . . . . 250

4.5.8 Finite Element Analysis of the Rotor . . . . . . . . . . . . . . 251

4.5.9 Mechanical Damping . . . . . . . . . . . . . . . . . . . . . . 259

4.5.10 Adhesive-Bonded Assemblies . . . . . . . . . . . . . . . . 262

4.5.11 Hand-Winding Coils . . . . . . . . . . . . . . . . . . . 267 
4.6 System Dynamics . . . . . . . . . . . . . . . . . . . . . 268

4.6.1 Negative Springs from the Magnetic Fluxes . . . . . . . . . . . 269

4.6.2 Modelled Plant Dynamics . . . . . . . . . . . . . . . . 275

4.6.3 Measured Plant Dynamics . . . . . . . . . . . . . . . . . . . 278

4.6.4 Equivalent Linear Mass and Passive Dynamic Stiffness Seen at the Tool Tip . . . . . . 289

4.7 Electrical Design . . . . . . . . . . . . . . . . . . . . . . . 291

4.7.1 Current-Mode Amplifier . . . . . . . . . . . . . . . . . . . . 293

4.7.2 Voltage Budget and Power Dissipated in the Amplifier . . . . 295

4.7.3 Tuning the Current Loop with a Surrogate Magnetic Circuit . 299

4.7.4 Measured Flux Balance in the Rotor/Stator Air Gaps . . . . . 303

4.7.5 Coupled versus Uncoupled Current Loops . . . . . . . . . . . 304

4.7.6 Implementing Electronic Damping in Analog Circuitry . . . . 307

4.8 Integrated Trade-Off Study . . . . . . . . . . . . . . . . . . . . . 310

4.8.1 Major Trade-off Areas . . . . . . . . . . . . . . . . . . 311

4.8.2 Procedure and Results . . . . . . . . . . . . . . . . . . . . . 312

4.8.3 Departure from an Optimum Design for Survivability . . . . . 322

4.9 Control System . . . . . . . . . . . . . . . . . . . . . . . . 324

4.9 .1 Low-Noise Implementation . . . . . . . . . . . . . . . . 327

4.9.2 High-Acceleration Implementation . . . . . . . . . . . . . . 332

4.9 .3 Sensor Noise . . . . . . . . . . . . . . . . . . . . . . . . . . 342

4.10 Results . . . . . . . . . . . . . . . . . . . . . . 347

4.10.1 Maximum tool tip Acceleration and Stroke . . . . . . . . . . 347

4.10.2 Demonstration of the Linear Mode of the Actuator . . . . . . 349

4.10.3 Temperature Rise in the Rotor and Stator Cores . . . . . . . . 354

4.10.4 Demonstrated Peak Torque and Flux Density . . . . . . . . . 360

4.11 Conclusions . . . . . . . . . . . . . . . . . . . 363 
5.1 Introduction . . . . . . . . . . . . . . . . . . 365

5.2 Electromagnetic Characteristics

of the Actuator . . . . . . . . . . . . . . . . . . 367

5.2.1 Physical Quantities and Units for Magnetic Circuits . . . . . . 368

5.2.2 Magnetic Circuit for the Actuator . . . . . . . . . . . . . . . . 369

5.2.3 Torque is Proportional to the Common Current in the Steering Flux Coils . . . . . . . . 370

5.2.4 Large Rotations of the Rotor . . . . . . . . . . . . . . . 378

5.2.5 Force is Proportional to the Differential Current in the Steering Flux Coils . . . . . . . 384

5.2.6 Back-EMF Produced by the Rotary and Linear Modes . . . . 387

5.2 .7 MMF Drop in the Iron . . . . . . . . . . . . . . . . . . . . 388

5.3 Electromagnetic Design of the Actuator . . . . . . . . . . . . . . 391

5.3.1 Reluctance Model . . . . . . . . . . . . . . . . . . . . . . . 392

5.3 .2 Inductance of the Actuator . . . . . . . . . . . . . . . . . . . 399

5.3.3 Electrical Power Needed to Drive the AC Flux . . . . . . . . . 401

5.3.4 Allowable Coil Current and Sizing the Coil Window Area . . . 403

5.3.5 Voltage versus Current Trade-off for the Coils . . . . . . . . . 406

5.3.6 Litz Wire for the Steering Coils . . . . . . . . . . . . . . . 410

5.4 Candidate Magnetic Circuit Topologies . . . . . . . . . . . . . . . . . 412

5.4 .1 Sandwiched Rotor Design . . . . . . . . . . . . . . . . . . 412

5.4 .2 Pinched Rotor Design . . . . . . . . . . . . . . . 413

5.4.3 Reversed-Roles Sandwiched Rotor Design . . . . . . . . . . . . 414

5.4.4 Comparison of Candidate Magnetic Circuit Topologies .. . . 416

5.5 Soft Magnetic Materials for the Actuator . . . . . . . . . . . . . . 418

5.5.1 Material Selection Strategy _. . . . . . . . . . . . . . . 419

5.5.2 Candidate Materials and Commercially Available Forms . . . 421

5.5.3 Power Loss in Soft Magnetic Materials . . . . . . . . . . . . . 428

5.5.4 Mechanical Design Considerations . . . . . . . . . . . . . 436 
5.5.5 Machining Tape-Wound Cores . . . . . . . . . . . . . . . . . . 437

5.5.6 Comparison of Candidate Soft Magnetic Materials . . . . . . . 438

5.6 Conclusions . . . . . . . . . . . . . . . . . . . . . . . 444

\section{Conclusions, Highlighted Results,}

Contributions, and Future Work 445

6.1 Overall Conclusions . . . . . . . . . . . . . . . . . . . 445

6.2 Highlighted Results . . . . . . . . . . . . . . . . . . . . . 447

6.2.1 The $2 \mathrm{kHz}$ Rotary Fast Tool Servo . . . . . . . . . . . . . . . 447

6.2 .2 The $10 \mathrm{kHz}$ Rotary Fast Tool Servo . . . . . . . . . . . . . . . 448

6.2.3 Hybrid Rotary/Linear Actuator . . . . . . . . . . . . . . . . . 449

6.3 Primary Contributions . . . . . . . . . . . . . . . . . . 452

6.4 Future Work . . . . . . . . . . . . . . . . . . . . . . . . . 453

6.4.1 Adaptive Feedforward Cancellation Compensation

to Reduce Following Error . . . . . . . . . . . . . . . 453

6.4.2 Machine a Workpiece with the $10 \mathrm{kHz}$ FTS . . . . . . . . . . . 454

6.4.3 Constructing Precision Laminated Cores . . . . . . . . . . . . 455

6.4.4 Heat Generation in the Rotor and Stator Cores . . . . . . . . 456

6.4.5 Flexure Bearing Design for

a Non Axially-Over-Constrained Rotor . . . . . . . . . . . . 457

6.4.6 Optimize the Design of the Flexure Blades . . . . . . . . . . . 461

6.4.7 Alternate Bearing Types for Supporting the Rotor . . . . . . . 461

6.4.8 Reduced Flexure Bearing Designs

Using a Magnetic Suspension . . . . . . . . . . . . . . 462

6.4.9 Balanced X-rotor for a One-Axis Steering Mirror . . . . . . . 464

6.4.10 Two-Axis Steering Mirror . . . . . . . . . . . . . . . . . 467

6.4.11 Low-Cost Rotary Actuator . . . . . . . . . . . . . . . . . . . . 468

6.5 Final Comments . . . . . . . . . . . . . . . . . . . . 473

A Selected Control System Design Techniques $\quad 475$

A.1 Loop Shaping with Lead and Lag Compensators . . . . . . . . . . . . 475 
A.2 Fast Inner Loop Acts as a Constant Gain Block . . . . . . . . . . . . 481

A.3 Notch Filter Design . . . . . . . . . . . . . . . . . . . . 482

B MATLAB ${ }^{\circledR}$ Files $\quad 487$

B.1 step_change_oscillator.m . . . . . . . . . . . . . . . . 487

B.2 FTS_modeled_dynamics.m . . . . . . . . . . . . . . . . . . . 491

B.3 push_pull_power_loss_02.m . . . . . . . . . . . . . . . . . 497

B.4 elastic_properties_Ni_Fe_001.m . . . . . . . . . . . . . . . . . 501

B.5 PSD_scope_data.m . . . . . . . . . . . . . . . . 505

B.6 flux_torque_force_01.m . . . . . . . . . . . . . . . 511

C Integrating the $2 \mathrm{kHz}$ FTS with a Diamond Turning Machine $\quad 521$

C.1 Compensating and Tuning the DTM Slides . . . . . . . . . . . . . . 522

C.2 Graphical User Interface and Controller for the DTM . . . . . . . . . 524

C.3 Graphical User Interface and Controller for the $2 \mathrm{kHz}$ FTS . . . . . . 525

C.4 Generating a Sinusoidal Tool Path from

the Workpiece Spindle Encoder Output . . . . . . . . . . . . . . . . . 529

C.5 Second Harmonics in Wavy Surfaces Machined on the Outside Diameter of a Workpiece . . . . . . . . . . . . . 534

C.6 Preferred Sequences of Machining Passes used to Produce the Wavy Surfaces . . . . . . . . . . . . . . . 537 


\section{List of Figures}

1-1 Schedule showing the development of the $2 \mathrm{kHz}$ and $10 \mathrm{kHz}$ rotary fast tool servos. . . . . . . . . . . . . . . . . 50

1-2 The $2 \mathrm{kHz}$ rotary fast tool servo on a diamond turning machine (left), and its rotating element not including the actuator (right). . 53

1-3 The $10 \mathrm{kHz}$ rotary fast tool servo on a diamond turning machine, and the rotor for the fast tool servo with its flexure bearing (inset). 54

1-4 Control system block diagram for the $2 \mathrm{kHz}$ FTS. . . . . . . . . . . 57

1-5 Bode plot of the measured negative loop transmission for the $2 \mathrm{kHz}$ FTS tool position loop. . . . . . . . . . . . . . . 58

1-6 Bode plot of the measured small-signal closed-loop response for the $2 \mathrm{kHz}$ FTS tool position loop. . . . . . . . . . . . . . 58

1-7 Close-up view of the $2 \mathrm{kHz}$ FTS tool tip engaging the face of a workpiece. . . . . . . . . . . . . . . . 59

1-8 Portion of the surface of a wavy part produced with the $2 \mathrm{kHz}$ FTS (left), 3-D surface map of a portion of that surface (right, upper), and profile across the grooves (right, lower). . . . . . . . . 60

1-9 Bode plot of the measured negative loop transmission for the tool position for the low-noise implementation of the $10 \mathrm{kHz}$ FTS. . . . 62

1-10 Bode plot of the measured small-signal closed-loop response for the tool position for the low-noise implementation of the $10 \mathrm{kHz}$ FTS. .

1-11 Model of the magnetic circuit for the hybrid actuator with coils (left), and without coils to show flux lines (right). . . . . . . . 64

1-12 Sketch of the magnetic circuit for the actuator. . . . . . . . . . 65 
2-1 Sketch showing a tool tip following a complex workpiece surface topology (left), an example of a desired complex surface that can be single-point machined with a fast tool servo (center), and an example spherical workpiece that might have that complex surface

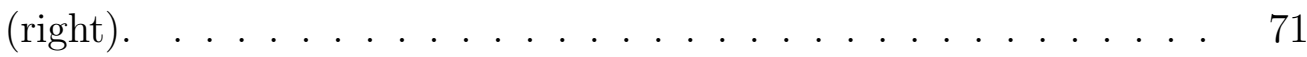

2-2 Basic topologies for Rotary (left) and Linear (right) fast tool servos. 74

2-3 Sketch of a fast tool servo and a two-axis lathe with a B-axis for machining a spherical workpiece. . . . . . . . . . . . . 74

2-4 Comparison of the $2 \mathrm{kHz}$ and $10 \mathrm{kHz}$ rotary fast tool servos to some representative fast tool servos reported in the literature. . . . . . . 76

2-5 Schematic cross-sectional view of the long-stroke rotary fast tool servo and turning machine by Ludwick and Trumper [99]. . . . . . 78

2-6 The $10 \mathrm{kHz}$ and $2 \mathrm{kHz}$ rotary fast tool servos next to the long-stroke rotary FTS by Ludwick and Trumper [99], left to right, respectively. 79

2-7 Control signals used to operate an electric engraver. . . . . . . . . . 84

2-8 A normal-stress variable reluctance actuator with flux biasing used in an electric engraver. . . . . . . . . . . . . . . . . . 87

2-9 A normal-stress variable reluctance actuator used in an electric engraver. . . . . . . . . . . . . . . . . . . 88

2-10 A normal-stress variable reluctance actuator used in an electric engraver. . . . . . . . . . . . . . . . . . . 89

2-11 A normal-stress variable reluctance actuator used in an electric engraver. . . . . . . . . . . . . . . . . . 90

2-12 A voice coil actuator used in an electric engraver. . . . . . . . . . . 91

2-13 A magnetostrictive actuator used in an electric engraver. . . . . . . 92

2-14 A magnetostrictive actuator used in an electric engraver. . . . . . . 93

2-15 A pneumatic actuator used in an engraving head. . . . . . . . . . . 94

2-16 Torsion rods used to support and guide a rotating shaft in an engraving head. . . . . . . . . . . . . . . . . . . 95

2-17 Radial flexures used to resist lateral forces in an engraving head. . 96 
2-18 Coulomb damping used in an engraving head. . . . . . . . . . . . . 98

2-19 Viscous damping used in an engraving head. . . . . . . . . . . . . . 99

2-20 Tuned-mass damping used in an engraving head. . . . . . . . . . . 100

2-21 Detail of cuts for holding ink made in a gravure cylinder by an engraving head. . . . . . . . . . . . . . . . . . . . 101

2-22 Simulation of a mass-spring oscillator without damping acted upon by a sequence of step force inputs. . . . . . . . . . . . . . . . . . . 102

2-23 Sensor for controlling drift and vibration of the tool in an engraving head. ....................... . . 105

2-24 Methods for sensing the displacement of the tool in an engraving head. ........................ 106

2-25 Trapezoidal tool profile and receiving slot for mounting a cutting tool in an engraving head. . . . . . . . . . . . . . . . . . 109

2-26 Schematic view of a radial plus tip/tilt actuator. . . . . . . . . . 111

2-27 Magnetic circuit for a radial plus tip/tilt actuator. . . . . . . . . . 112

2-28 A first alternate magnetic circuit for a radial plus tip/tilt actuator. 113

2-29 A second alternate magnetic circuit for a radial plus tip/tilt actuator. 113

2-30 Normal-stress variable reluctance linear actuator employing fluxbiasing. . . . . . . . . . . . . . . . . . . 115

2-31 Normal-stress variable reluctance linear actuator employing fluxbiasing. . . . . . . . . . . . . . . . . 116

2-32 Shear-stress variable reluctance rotary actuator employing fluxbiasing. . . . . . . . . . . . . . . . . 117

2-33 Crossed-blade flexures for supporting a shaft in a galvanometer. . . 117

3-1 The $2 \mathrm{kHz}$ rotary fast tool servo. . . . . . . . . . . . . . . . 121

3-2 The $2 \mathrm{kHz}$ rotary fast tool servo on a diamond turning machine (left), and its rotating element not including the actuator (right). . 122 
3-3 Concept sketch for a vertical-axis and a horizontal-axis rotary fast tool servo used with a T-base lathe and a rotary table for machining a spherical workpiece. . . . . . . . . . . . . . . . . 124

3-4 Concept sketch for a linear-type fast tool servo used with an R- $\theta-\mathrm{X}$ lathe for machining a spherical workpiece. . . . . . . . . . . . . 124

3-5 Mechanical components and actuator for the $2 \mathrm{kHz}$ FTS. . . . . . . 130

3-6 Diamond tool with clamping hardware sitting on a dime. . . . . . . 132

3-7 The swing-arm (rotor) with its flexure bearing and a diamond tool for the $2 \mathrm{kHz}$ FTS. . . . . . . . . . . . . . . . . . . . . . 134

3-8 Four flexure blades with tensioning devices. . . . . . . . . . . . 138

3-9 Top view of $2 \mathrm{kHz}$ FTS swing-arm mounted to the base with four flexure blades. . . . . . . . . . . . . . . . . . . . . 139

3-10 Geometry of a flexure blade before and after rotation of the swingarm (far left and second from left, respectively). . . . . . . . . . . . 139

3-11 Nomenclature for fluctuating stress (left), and Modified Goodman line (right). . . . . . . . . . . . . . . . . . . . . 142

3-12 Final parametric study for the 0.006 inch thick flexure blades used in the $2 \mathrm{kHz}$ FTS. . . . . . . . . . . . . . . . . . . 146

3-13 Experimental set-up for verifying calculations used to design the flexure blades. . . . . . . . . . . . . . . . . . . . 148

3-14 Mechanical viscous damper used in the $2 \mathrm{kHz}$ rotary fast tool servo. 150

3-15 Close-up view of the $2 \mathrm{kHz}$ FTS tool tip engaging the face of a workpiece. . . . . . . . . . . . . . . . 153

3-16 Scaling the differential measurement from the eddy current sensors to a position measurement for the tool tip. . . . . . . . . . . . . 154

3-17 Simplified model for the mechanical dynamics of the combined motor and swing-arm for the $2 \mathrm{kHz}$ FTS. . . . . . . . . . . . . 155

3-18 Electrical circuit and equivalent block diagram for the electrical dynamics of the motor for the $2 \mathrm{kHz}$ FTS. . . . . . . . . . . . 158 
3-19 Block diagram showing the electrical dynamics of the actuator inside the current loop for the current-mode amplifier for the $2 \mathrm{kHz}$ FTS. 158

3-20 Bode plot of the measured tool position dynamics for the $2 \mathrm{kHz}$ FTS (including a lead compensator). . . . . . . . . . . . . . . 159

3-21 Bode plot of the modelled tool position dynamics for the $2 \mathrm{kHz}$ FTS (including a lead compensator). . . . . . . . . . . . . . 160

3-22 Root locus of the modelled tool position dynamics for the $2 \mathrm{kHz}$ FTS (including a lead compensator) . . . . . . . . . . . . . 160

3-23 Nyquist diagram of the modelled tool position dynamics for the 2 kHz FTS (including a lead compensator). . . . . . . . . . . . . . 161

3-24 Sketches showing the relationship between a rotary inertia and an equivalent linear mass (left three figures), and the Bode plot for the equivalent mass-spring system showing the inverse of the passive dynamic stiffness $k_{P D S}$ (right). . . . . . . . . . . . 163

3-25 Components for the servo test bed. . . . . . . . . . . . . . . . . . 164

3-26 The fully assembled servo test bed. . . . . . . . . . . . . . . . . . . 164

3-27 Schematic diagram of the electrical circuit for the current-mode amplifier used with the $2 \mathrm{kHz}$ FTS. . . . . . . . . . . . . 166

3-28 Schematic diagram of the power amplifier, motor coil resistance and inductance, Back-EMF, and sense resistor for the $2 \mathrm{kHz}$ FTS (left), and phasor diagram showing the relationship between the real and imaginary components of the output voltage of the power amplifier (right)

3-29 Plot showing relationships between the voltage, current, and power in the motor (load), and the supply voltages and dissipated power in the power amplifier for the $2 \mathrm{kHz}$ FTS. . . . . . . . . . . . . 170

3-30 Schematic layout of the electrical components for the $2 \mathrm{kHz}$ FTS. . 171

3-31 The as-built analog compensation circuit and power amplifier for the $2 \mathrm{kHz}$ FTS. . . . . . . . . . . . . . . . . . . 172

3-32 Control system block diagram for the $2 \mathrm{kHz}$ FTS. . . . . . . . . . . 175 
3-33 Captured oscilloscope screen showing DAC glitches on the command voltage $V_{c}$ to the current-mode amplifier (upper trace), and the response of the current compensation as seen at the input voltage $V_{i p}$ to the power operational amplifier (lower trace). . . . . . 177

3-34 Detail of the voltage signals shown in Figure 3-33. . . . . . . . . . . 177

3-35 Block diagram for the digital controller for the tool position for the $2 \mathrm{kHz}$ FTS without viscous damping, using a double lead, lag, and notch filter compensator. . . . . . . . . . . . . . . . . 178

3-36 Bode plot of the measured negative loop transmission for the tool position for the $2 \mathrm{kHz}$ FTS without viscous damping, using a double lead, lag, and notch filter compensation. . . . . . . . . . . 180

3-37 Bode plot of the modelled negative loop transmission for the tool position for the $2 \mathrm{kHz}$ FTS without viscous damping, using a double lead, lag, and notch filter compensation. . . . . . . . . . . . 180

3-38 Root locus of the modelled negative loop transmission for the tool position for the $2 \mathrm{kHz}$ FTS without viscous damping, using a double lead, lag, and notch filter compensation. . . . . . . . . . . . 18

3-39 Root locus of the modelled negative loop transmission for the tool position for the $2 \mathrm{kHz}$ FTS without viscous damping, using a double lead and lag compensation. . . . . . . . . . . . . . . . . 182

3-40 Nyquist diagram of the modelled negative loop transmission for the tool position for the $2 \mathrm{kHz}$ FTS without viscous damping, using a double lead, lag, and notch filter compensation. . . . . . . . . . 183

3-41 Detail of the Nyquist diagram of the modelled negative loop transmission for the tool position for the $2 \mathrm{kHz}$ FTS without viscous damping, using a double lead, lag, and notch filter compensation. . 183

3-42 Block diagram for the digital controller for the tool position for the $2 \mathrm{kHz}$ FTS without viscous damping, using a double lead, lag, and phase-stabilizing pole compensator. . . . . . . . . . . . . . . . 184 
3-43 Bode plot of the measured negative loop transmission for the tool position for the $2 \mathrm{kHz}$ FTS without viscous damping, using a double lead, lag, and phase-stabilizing pole compensation. . . . . . . . 185

3-44 Bode plot of the modelled negative loop transmission for the tool position for the $2 \mathrm{kHz}$ FTS without viscous damping, using a double lead, lag, and phase-stabilizing pole compensation. . . . . . . . . . 185

3-45 Root locus of the modelled negative loop transmission for the tool position for the $2 \mathrm{kHz}$ FTS without viscous damping, using a double lead, lag, and phase-stabilizing pole compensation. . . . . . . . . 186

3-46 Nyquist diagram of the modelled negative loop transmission for the tool position for the $2 \mathrm{kHz}$ FTS without viscous damping, using a double lead, lag, and phase-stabilizing pole compensation. . . . . . 186

3-47 Detail of the Nyquist diagram of the modelled negative loop transmission for the tool position for the $2 \mathrm{kHz}$ FTS without viscous damping, using a double lead, lag, and phase-stabilizing pole compensation. . . . . . . . . . . . . . . . . 187

3-48 Detail of the Nyquist diagram of the modelled negative loop transmission for the tool position for the $2 \mathrm{kHz}$ FTS without viscous damping, using a double lead and lag compensation without a phase-stabilizing pole (or a notch filter). . . . . . . . . . . . . 188

3-49 Block diagram for the digital controller for the tool position for the $2 \mathrm{kHz}$ FTS with viscous damping, using a series lag compensator. . 188

3-50 Bode plot of the measured negative loop transmission for the tool position for the $2 \mathrm{kHz}$ FTS with viscous damping, using a series lag compensator. . . . . . . . . . . . . . . . . 191

3-51 Bode plot of the measured small-signal closed-loop response for the tool position for the $2 \mathrm{kHz}$ FTS with viscous damping, using a series lag compensator. . . . . . . . . . . . . . . . . . . 191

3-52 Measured step response for the tool position for the $2 \mathrm{kHz}$ FTS with viscous damping, using a series lag compensator. 
3-53 Measured following error during machining tests at $2 \mathrm{kHz}$ for the tool position for the $2 \mathrm{kHz}$ FTS with viscous damping, using a series lag compensator. . . . . . . . . . . . . . . . . . . 192

3-54 Bode plot of the modelled negative loop transmission for the tool position for the $2 \mathrm{kHz}$ FTS with viscous damping, using a series lag compensator. . . . . . . . . . . . . . . . . . . . 194

3-55 Bode plot of the modelled negative loop transmission for the tool position for the $2 \mathrm{kHz}$ FTS with viscous damping, using a series lag compensator.

3-56 Root locus of the modelled negative loop transmission for the tool position for the $2 \mathrm{kHz}$ FTS with viscous damping, using a series lag

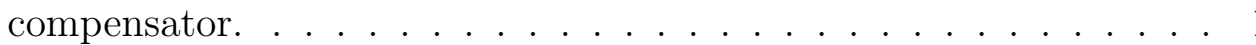

3-57 Nyquist diagram of the modelled negative loop transmission for the tool position for the $2 \mathrm{kHz}$ FTS with viscous damping, using a series lag compensator. . . . . . . . . . . . . . . . 196

3-58 Detail of the Nyquist diagram of the modelled negative loop transmission for the tool position for the $2 \mathrm{kHz}$ FTS with viscous damping, using a series lag compensator. . . . . . . . . . . . . . . . . 196

3-59 Close-up view of the $2 \mathrm{kHz}$ FTS tool tip about to engage the outside diameter of a workpiece.

3-60 Close-up view of the textured surface on the face of a workpiece machined with the $2 \mathrm{kHz}$ FTS.

3-61 Surface measurement of the face of a workpiece machined with the $2 \mathrm{kHz}$ FTS, showing a two-dimensional profile across the grooves. . 199

3-62 Surface measurement of the face of a workpiece machined with the $2 \mathrm{kHz}$ FTS, showing a two-dimensional profile along a groove. . . . 200

3-63 Surface measurement of the outer diameter of a workpiece machined with the $2 \mathrm{kHz}$ FTS, showing a two-dimensional profile across the grooves. 
3-64 Surface measurement of the outer diameter of a workpiece machined with the $2 \mathrm{kHz}$ FTS, showing a two-dimensional profile along a groove. 201

4-1 The $10 \mathrm{kHz}$ rotary fast tool servo. . . . . . . . . . . . . . 206

4-2 The $10 \mathrm{kHz}$ rotary fast tool servo on a diamond turning machine, and the rotor for the fast tool servo with its flexure bearing (inset). 207

4-3 Model of the magnetic circuit for the $10 \mathrm{kHz}$ rotary fast tool servo. 209

4-4 Model showing directions of magnetic fluxes for the $10 \mathrm{kHz}$ rotary fast tool servo. . . . . . . . . . . . . . . . . . 209

4-5 T-rotor layout with nested stator cores. . . . . . . . . . . . . . 212

4-6 Detail of T-rotor layout with front poles arranged to put rear flexure in tension. . . . . . . . . . . . . . . . . . . 212

4-7 X-rotor layout with nested stator cores. . . . . . . . . . . . . 213

4-8 Balanced X-rotor layout with nested stator cores. . . . . . . . . . . 213

4-9 Early concepts for shelled-rotor design. . . . . . . . . . . . . . . . . 214

4-10 Later concept for shelled square-rotor design. . . . . . . . . . . . . 216

4-11 Early concepts for torque-tube design for the rotor. . . . . . . . . . 217

4-12 Mid-evolution concepts for the torque-tube design for the rotor. . . 218

4-13 Final concepts for torque-tube design for the rotor. . . . . . . . . . 219

4-14 Mechanical components for the $10 \mathrm{kHz}$ FTS. . . . . . . . . . . . . . 221

4-15 Model of the $10 \mathrm{kHz}$ FTS with cut-outs, looking up from the bottom-front. . . . . . . . . . . . . . . . 222

4-16 Rotor and stator cores for the $10 \mathrm{kHz}$ rotary fast tool servo. . . . . 224

4-17 Spare $50 \% \mathrm{Ni}-50 \%$ Fe rotor and stator cores for the $10 \mathrm{kHz}$ rotary fast tool servo with several laminations peeled back. . . . . . . . 225

4-18 Wound-tape core blanks for the rotor. . . . . . . . . . . . . . . 225

4-19 Wound-tape core blanks for the stators. . . . . . . . . . . . 226

4-20 The rotor with its tool arm and flexure bearing for the $10 \mathrm{kHz}$ FTS. 229

4-21 Model of the $10 \mathrm{kHz}$ FTS rotor with its tool arm and flexure bearing (left), and a cross-section of the rotor (right). . . . . . . . . . 230 
4-22 The rotor core, ceramic stiffeners, and flexure hubs for the $10 \mathrm{kHz}$ FTS, with epoxy applied to the surfaces to be bonded together. . . . . . 231

4-23 Partial assembly of the rotor core, ceramic stiffeners, and flexure hubs for the $10 \mathrm{kHz}$ FTS. . . . . . . . . . . . . . . . . 231

4-24 Full assembly of the rotor core, ceramic stiffeners, and flexure hubs for the $10 \mathrm{kHz}$ FTS, after cleaning off the excess epoxy adhesive. . 232

4-25 Clamping of the full assembly of the rotor core, ceramic stiffeners, and flexure hubs for the $10 \mathrm{kHz}$ FTS, during cure of the epoxy adhesive. . . . . . . . . . . . . . . . . . 232

4-26 Mounting the flexure blades to the rotor: setting the front/back blades perpendicular to the rotor pole faces. . . . . . . . . . . . . 233

4-27 Mounting the flexure blades to the rotor: setting the right/left blades perpendicular to the sides of the rotor core. . . . . . . . . . 233

4-28 One of the two fully assembled stator halves for the $10 \mathrm{kHz}$ FTS. . 235

4-29 Mechanical model of a stator half for the $10 \mathrm{kHz}$ FTS with a cut-out revealing the major components. . . . . . . . . . . . . 236

4-30 Stator housing prepared with epoxy to receive the stator core. . . . 237

4-31 Bonding fixture used to register the stator pole faces relative to the front face of the stator housing. . . . . . . . . . . . . . . . 237

4-32 Clamping the stator core against the bonding fixture. . . . . . . . . 238

4-33 Steering coils being dry-fitted to the stator core and housing. . . . 238

4-34 Steering coils being bonded to the stator core and housing with a potting compound. . . . . . . . . . . . . . . . 239

4-35 Preparing the stator core and cured potting compound to receive the inner and outer stator core braces. . . . . . . . . . . . . . 239

4-36 A fully assembled stator half before final grinding. . . . . . . . . . 240

4-37 The two stator halves being match-ground with a surface grinder. . 240

4-38 Fitting a terminal piece of back-iron to a fully assembled stator half. 241

4-39 Model of the rotor mounted via the flexure blades to one of the two stator halves. . . . . . . . . . . . . . . . . . . . . 242 
4-40 The two stator halves with the rotor aligned and partially attached to one of the stator halves.

4-41 The two stator halves and the rotor after attaching the stator halves together.

4-42 A back-lit photo of the fully assembled rotor and stator showing the four $50 \mu \mathrm{m}$ rotor/stator air gaps. . . . . . . . . . . . . . . 244

4-43 Tool holder for the $10 \mathrm{kHz}$ FTS, shown with its rotation axis lying in a horizontal plane during static balancing. . . . . . . . . . . . . 245

4-44 Final parametric study for the 0.010 inch thick flexure blades used in the $10 \mathrm{kHz}$ FTS.

4-45 Measuring the axial stiffness of the flexure-suspended rotor (left), and the stiffness at the tool in a direction parallel to the rotor centerline (right). . . . . . . . . . . . . . . . . . 250

4-46 Capacitance sensors and tool holder for the $10 \mathrm{kHz}$ FTS. . . . . . . 251

4-47 Predicted vibration modes of the flexure-suspended rotor using an FEA model. . . . . . . . . . . . . . . . . . . 253

4-48 Predicted vibration modes of the flexure-suspended rotor using an FEA model. . . . . . . . . . . . . . . . . . . . . . 253

4-49 Predicted vibration modes of the flexure-suspended rotor using an FEA model. . . . . . . . . . . . . . . . . . . . . 254

4-50 Predicted vibration modes of the flexure-suspended rotor using an FEA model. . . . . . . . . . . . . . . . . . . . . . . . . 254

4-51 Principle-axes indices for materials with transversely isotropic properties. .......................... 256

4-52 Screen-shot of the Pro/Mechanica FEA model of the flexure-suspended

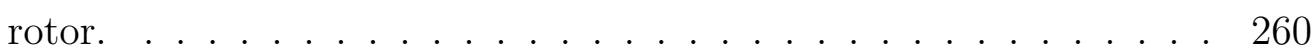

4-53 Screen-shot of the Pro/Mechanica material definition page for the laminated rotor core. . . . . . . . . . . . . . . . . . . 260 
4-54 Adding mechanical damping to the $10 \mathrm{kHz}$ FTS by filling the gap between the tool holder and the centerline capacitance sensor with grease; shown before closing the gap. . . . . . . . . . . . . . . . . 261

4-55 Adding mechanical damping to the $10 \mathrm{kHz}$ FTS by filling the gap between the tool holder and the centerline capacitance sensor with grease; shown after closing the gap. . . . . . . . . . . . . . . 262

4-56 Hand-winding the steering flux coils using a counter-winding technique from the center of the wire bundle out towards the ends. . . . 268

4-57 Sketch of the magnetic circuit showing distribution of steering and bias fluxes when rotor is in its neutral position (rotation and translation)

4-58 Sketch of the magnetic circuit showing large rotation of the rotor and asymmetric distribution of bias flux. . . . . . . . . . . . . 272

4-59 Sketch of the magnetic circuit showing extreme rotation of the rotor and attendant magnetic detent from asymmetric distribution of bias flux.

4-60 Sketch of the magnetic circuit showing small front/back translation of the rotor and asymmetric distribution of steering flux. . . . . . . 273

4-61 Sketch of the magnetic circuit showing extreme front/back translation of the rotor and attendant magnetic detent from asymmetric distribution of steering flux. . . . . . . . . . . . . . . . 274

4-62 Simplified mechanical-magnetic dynamics of the rotational degree of freedom for the rotor. . . . . . . . . . . . . . . 276

4-63 Simplified mechanical-magnetic dynamics of the translational degree of freedom for the rotor. . . . . . . . . . . . . . . . 277

4-64 Electrical circuit and equivalent block diagram for the electrical dynamics of the rotary mode of one stator half in the actuator used in the $10 \mathrm{kHz}$ FTS. . . . . . . . . . . . . . . . . . . . 278

4-65 Block diagram combining the electrical dynamics of one stator half with the electrical dynamics of its current-mode amplifier. . . . . . 279 
4-66 Sketch showing how the relative magnitudes of the spring constants for the torsional springs from the flexures and from the bias flux affects the two open-loop poles for the rotary mode dynamics. . . . 280

4-67 Bode plot of the measured negative loop transmission for the tool position using a bias flux coil current of 0.260 A. . . . . . . . . . 281

4-68 Bode plot of the measured negative loop transmission for the tool position using a bias flux coil current of 0.468 A. . . . . . . . . . . 281

4-69 Bode plot of the measured negative loop transmission for the tool position using a bias flux coil current of 0.524 A. . . . . . . . . . . 282

4-70 Bode plot of the measured negative loop transmission for the tool position using a bias flux coil current of 0.535 A . . . . . . . . . . 282

4-71 Bode plot of the measured negative loop transmission for the tool position using a bias flux coil current of 0.545 A. . . . . . . . . . 283

4-72 Bode plot of the measured negative loop transmission for the tool position using a bias flux coil current of 0.624 A . . . . . . . . . . 283

4-73 Bode plot of the measured plant for the tool position using a moderate current of $0.545 \mathrm{~A}$ in the bias flux coil (low noise implementation), with FEA model predictions mapped to resonant peaks. . . 285

4-74 High frequency detail for a Bode plot of the measured plant for the tool position using a moderate current of $0.545 \mathrm{~A}$ in the bias flux coil (low noise implementation), with FEA model predictions mapped to resonant peaks. . . . . . . . . . . . . . . . 285

4-75 Bode plot of the measured plant for the tool position using a high current of $1.5 \mathrm{~A}$ in the bias flux coil (high acceleration implementation). . . . . . . . . . . . . . . 286

4-76 Measured step response of the rotor with no damping. . . . . . . . 287

4-77 Measured step response of the rotor with electronic damping. . . . 288

4-78 Measured step response of the rotor with mechanical damping. . . . 288

4-79 Measured step response of the rotor with electronic and mechanical damping. . . . . . . . . . . . . . . . . . 289 
4-80 Measured frequency responses of the negative loop transmission of the tool position comparing electronic damping to mechanical-pluselectronic damping. . . . . . . . . . . . . . . . . . . . . . 290

4-81 Power amplifier (back) and current compensation (front) circuit boards for the $10 \mathrm{kHz}$ FTS.

4-82 Copper plate heat sink with the four power operational amplifiers and nine cooling fans for the $10 \mathrm{kHz}$ FTS. . . . . . . . . . . . 293

4-83 Schematic diagram of the electrical circuit for one of the two identical current-mode amplifiers used to drive the series-connected pairs of steering flux coils in the $10 \mathrm{kHz}$ rotary fast tool servo. . . . . . . 295

4-84 Schematic diagram of the power amplifier, a series-connected pair of steering flux coils, the torque-mode Back-EMF, and sense resistor for the $10 \mathrm{kHz}$ FTS (left), and phasor diagram showing the relationship between the real and imaginary components of the output voltage of the power amplifier (right). . . . . . . . . . . 296

4-85 Plot showing relationships between the voltage, current, and power in the torque-mode of the actuator (load), and the supply voltages and dissipated power in the power amplifier. . . . . . . . . . . . 300

4-86 Plot showing relationships between the voltage, current, and power in the torque-mode of the actuator (load), and the supply voltages and dissipated power in the power amplifier. . . . . . . . . . . . . 301

4-87 Surrogate magnetic circuit for tuning the power amplifiers for the $10 \mathrm{kHz}$ FTS. . . . . . . . . . . . . . . . . 302

4-88 Measured 1.0 A step responses for each of the four power amplifier channels when driving the surrogate magnetic circuit. . . . . . . . 303

4-89 Schematic diagram showing the relationship between the bias flux (triple-head arrows), steering fluxes (double-head arrows), and currents needed to produce $\mathrm{a}+\theta$ rotation of the rotor. . . . . . . . . . 304 
4-90 Measured induced voltages at the terminals of the four steering flux coils for a $100 \mathrm{~Hz}$ AC excitation of the bias flux coil, illustrating the proper phasing. . . . . . . . . . . . . . . . . 305

4-91 Overlay of the measured induced voltages at the terminals of the four steering flux coils for a $100 \mathrm{~Hz}$ AC excitation of the bias flux coil, illustrating that the bias flux in the four rotor/stator air gaps is balanced within $7 \% \ldots \ldots \ldots$. . . . . . . . . . . 305

4-92 Simple model (left) and corresponding electrical circuit (right) for the case of driving each of the two steering flux coils on a stator core with its own power amplifier. . . . . . . . . . . . . . . 306

4-93 Block diagram corresponding to the simple model for driving each of the two steering flux coils on a stator core with its own power amplifier. . . . . . . . . . . . . . . . 307

4-94 Simple model (left) and corresponding electrical circuit (right) for the case of driving a series connected pair of steering flux coils on a stator core with one power amplifier. . . . . . . . . . . . . . 307

4-95 Re-wired version of the current compensation circuit board for implementing electronic damping in the $10 \mathrm{kHz}$ FTS. . . . . . . . . . 309

4-96 Circuit diagram for implementing electronic damping in the $10 \mathrm{kHz}$ FTS. ...... 310

4-97 Major design trade-offs involved in determining the dimensions of the rotor and stator assemblies for the $10 \mathrm{kHz}$ FTS. . . . . . . . . . 312

4-98 Sketch showing the relationship between the dimensions of the rotor core, the rotor stiffeners, and the stator core.

4-99 Example of how most of the performance metrics for the rotor assembly were visualized when the stiffener diameter and rotor core height were varied. . . . . . . . . . . . . . . . . . . 320

4-100 The final design space for the $10 \mathrm{kHz}$ FTS trade-off study. . . . . . 321 
4-101 Solid models showing the differences between the baseline rotor assembly design from the trade-off study (left), and the more mechanically robust version used in the $10 \mathrm{kHz}$ FTS (right). . . . . . . 324

4-102 Control system block diagram for the $10 \mathrm{kHz}$ FTS, with electronic damping. . . . . . . . . . . . . . . . . . . . 326

4-103 Block diagram for the digital controller for the tool position for the low-noise implementation of the $10 \mathrm{kHz}$ FTS. . . . . . . . . . . . . 328

4-104 Bode plot of the measured negative loop transmission for the tool position for the low-noise implementation of the $10 \mathrm{kHz}$ FTS. . . . 328

4-105 Bode plot showing high frequency detail of the measured negative loop transmission for the tool position for the low-noise implementation of the $10 \mathrm{kHz}$ FTS. . . . . . . . . . . . . . . . . . 329

4-106 Nyquist diagram of the measured negative loop transmission for the tool position for the low-noise implementation of the $10 \mathrm{kHz}$ FTS. . 330

4-107 Bode plot of the measured small-signal closed-loop response for the tool position for the low-noise implementation of the $10 \mathrm{kHz}$ FTS. . 330

4-108 Bode plot showing high frequency detail of the measured smallsignal closed-loop response for the tool position for the low-noise implementation of the $10 \mathrm{kHz}$ FTS. . . . . . . . . . . . . . . 331

4-109 Measured step response for the tool position for the low-noise implementation of the $10 \mathrm{kHz}$ FTS. . . . . . . . . . . . . . . 332

4-110 Measured following error for the low-noise implementation of the $10 \mathrm{kHz}$ FTS for a $100 \mathrm{~Hz}$ sinusoidal tool tip trajectory. . . . . . . . 333

4-111 Measured following error for the low-noise implementation of the $10 \mathrm{kHz}$ FTS for a $300 \mathrm{~Hz}$ sinusoidal tool tip trajectory. . . . . . . . 333

4-112 Measured following error for the low-noise implementation of the $10 \mathrm{kHz}$ FTS for a $500 \mathrm{~Hz}$ sinusoidal tool tip trajectory. . . . . . . . 334

4-113 Measured following error for the low-noise implementation of the $10 \mathrm{kHz}$ FTS for a $1 \mathrm{kHz}$ sinusoidal tool tip trajectory. . . . . . . . 334 
4-114 Block diagram for the digital controller for the tool position for the high-acceleration implementation of the $10 \mathrm{kHz}$ FTS. . . . . . . . . 335

4-115 Bode plot of the measured negative loop transmission for the tool position for the high-acceleration implementation of the $10 \mathrm{kHz}$ FTS. 336

4-116 Bode plot showing high frequency detail of the measured negative loop transmission for the tool position for the high-acceleration implementation of the $10 \mathrm{kHz}$ FTS. . . . . . . . . . . . . . 336

4-117 Nyquist diagram of the measured negative loop transmission for the tool position for the high-acceleration implementation of the $10 \mathrm{kHz}$ FTS. . . . . . . . . . . . . . . . . . 337

4-118 Bode plot of the measured small-signal closed-loop response for the tool position for the high-acceleration implementation of the $10 \mathrm{kHz}$ FTS. . . . . . . . . . . . . . . . . . . . . . 338

4-119 Bode plot showing high frequency detail of the measured smallsignal closed-loop response for the tool position for the high-acceleration implementation of the $10 \mathrm{kHz}$ FTS. . . . . . . . . . . . . . 338

4-120 Measured step response for the tool position for the high-acceleration implementation of the $10 \mathrm{kHz}$ FTS. . . . . . . . . . . . . . . 339

4-121 Measured following error for the high-acceleration implementation of the $10 \mathrm{kHz}$ FTS for a $100 \mathrm{~Hz}$ sinusoidal tool tip trajectory. . . . 340

4-122 Measured following error for the high-acceleration implementation of the $10 \mathrm{kHz}$ FTS for a $300 \mathrm{~Hz}$ sinusoidal tool tip trajectory. . . . 340

4-123 Measured following error for the high-acceleration implementation of the $10 \mathrm{kHz}$ FTS for a $500 \mathrm{~Hz}$ sinusoidal tool tip trajectory. . . . 341

4-124 Measured following error for the high-acceleration implementation of the $10 \mathrm{kHz}$ FTS for a $1 \mathrm{kHz}$ sinusoidal tool tip trajectory. . . . . 341

4-125 Captured oscilloscope data of the noise measurement for the lownoise implementation of the $10 \mathrm{kHz}$ FTS. . . . . . . . . . . . . 345

4-126 Calculated power spectral density of the noise measurement for the low-noise implementation of the $10 \mathrm{kHz}$ FTS. . . . . . . . . . . . 346 
4-127 Captured oscilloscope data of the noise measurement for the highacceleration implementation of the $10 \mathrm{kHz}$ FTS. . . . . . . . . . . . 346

4-128 Calculated power spectral density of the noise measurement for the high-acceleration implementation of the $10 \mathrm{kHz}$ FTS. . . . . . . . . 347

4-129 Measured closed-loop tool trajectory showing $400 \mathrm{~g}$ tool tip acceleration during operation at $5 \mathrm{kHz}$ with the high-acceleration implementation of the $10 \mathrm{kHz}$ FTS (upper curve). . . . . . . . . . . . 348

4-130 Measured closed-loop tool trajectory showing 870 g tool tip acceleration during operation at $10 \mathrm{kHz}$ with the high-acceleration implementation of the $10 \mathrm{kHz}$ FTS (upper curve). . . . . . . . . . . 349

4-131 Measured closed-loop tool tip trajectory showing full travel of $\pm 34 \mu \mathrm{m}$ during operation at $100 \mathrm{~Hz}$ with the low-noise implementation of the $10 \mathrm{kHz}$ FTS (upper curve). . . . . . . . . . . . . . . 350

4-132 Demonstration of the linear mode of the actuator in the $10 \mathrm{kHz}$ FTS. 352

4-133 Demonstration of the linear mode of the actuator in the $10 \mathrm{kHz}$ FTS. 352

4-134 Demonstration of the linear mode of the actuator in the $10 \mathrm{kHz}$ FTS. 353

4-135 Data showing that centerline motion observed while demonstrating the linear mode is not caused by rotation of the rotor. . . . . . . . 353

4-136 Locations of the thermocouples used to measure the temperature of the rotor core (left) and stator core (right). . . . . . . . . . 355

4-137 Measured temperature of the rotor and stator cores during approximately 3 min of $400 \mathrm{~g}$ operation at $5 \mathrm{kHz}$, and then during a $20 \mathrm{~min}$ cool-down period after turning off the $10 \mathrm{kHz}$ FTS. . . . . . . . . . 356

4-138 Simplified lumped-parameter thermal model used to calculate experimental core power losses from measured thermal time constants. 357

4-139 Detail of the measured temperature of the stator core during approximately 3 min of $400 \mathrm{~g}$ operation at $5 \mathrm{kHz}$. . . . . . . . . . 358

4-140 Detail of the measured temperature of the rotor core during approximately $3 \mathrm{~min}$ of $400 \mathrm{~g}$ operation at $5 \mathrm{kHz}$. . . . . . . . . . . 359 
5-1 Model of the magnetic circuit for the $10 \mathrm{kHz}$ rotary fast tool servo.

5-2 Model showing directions of magnetic fluxes for the $10 \mathrm{kHz}$ rotary

5-3 Sketch of the magnetic circuit showing torque generation for the actuator used in the $10 \mathrm{kHz}$ FTS. . . . . . . . . . . . . . . . 370

5-4 Sketch of an alternate magnetic circuit using a permanent magnet to produce the bias flux.

5-5 Sketch of the magnetic circuit showing torque generation for a large rotation angle of the rotor. . . . . . . . . . . . . . . . . 378

5-6 Sketch of the magnetic circuit showing torque and force generation for the actuator used in the $10 \mathrm{kHz}$ FTS. . . . . . . . . . . . . . . . 384

5-7 Reluctance model of the magnetic circuit for the actuator in the $10 \mathrm{kHz}$ rotary fast tool servo for small rotations of the rotor, showing the flux in the four rotor/stator air gaps due to the bias and steering fluxes. . . . . . . . . . . . . . . . . . . . . . . . 394

5-8 Reluctance model of the magnetic circuit for the actuator in the $10 \mathrm{kHz}$ rotary fast tool servo for small rotations of the rotor, showing the flux in the four rotor/stator air gaps due to the bias flux. . 398

5-9 Reluctance model of the magnetic circuit for the actuator in the $10 \mathrm{kHz}$ rotary fast tool servo for small rotations of the rotor, showing the flux in the four rotor/stator air gaps due to the steering flux.

5-10 Results of a numerical analysis for the sandwiched rotor magnetic circuit. .

5-11 Sketch of the magnetic circuit showing torque and force generation for the pinched rotor magnetic circuit.

5-12 Results of a numerical analysis for the pinched rotor magnetic circuit. 415

5-13 Sketch of the magnetic circuit showing torque and force generation for the reversed-roles sandwiched rotor magnetic circuit. . . . . . . 415 
5-14 Results of a numerical analysis for the reversed-roles sandwiched rotor magnetic circuit. . . . . . . . . . . . . . . . . . 416

5-15 Sketch of the magnetic circuit for the $10 \mathrm{kHz}$ FTS showing the nature of the flux in the rotor core, stator cores, and back-iron, for the designed peak operation.

5-16 Magnetic flux density versus magnetic field intensity for a single, saturating $\mathrm{AC}$ cycle for virgin soft magnetic material with a zero initial condition (01234561); a minor hysteresis loop for a symmetrical AC cycle (fcdaef); and a minor hysteresis loop for a one-sided AC cycle (abcda). . . . . . . . . . . . . . . . 4

6-1 Sketch of the magnetic circuit for the actuator. . . . . . . . . . . . 450

6-2 Experimental results using Adaptive Feedforward Cancellation compensation to reduce the following error for a $20 \mathrm{~Hz}$ air cut with the Rotary fast tool servo by Ludwick and Trumper [99]. . . . . . . . . 454

6-3 The swing-arm (rotor) with its flexure bearing for the $2 \mathrm{kHz}$ FTS. . 458

6-4 The rotor with its flexure bearing for the $10 \mathrm{kHz}$ FTS. . . . . . . . 458

6-5 Model of the rotor in the $10 \mathrm{kHz}$ FTS with the lower flexure blades modified to provide axial compliance at one end of the rotor. . . . . 460

6-6 Model of the rotor in the $10 \mathrm{kHz}$ FTS with the lower flexure blades replaced with rods to provide axial compliance at one end of the rotor.

6-7 Sketch of a first variation of a reduced flexure bearing that uses the linear mode of the actuator as a magnetic suspension to help support the rotor.

6-8 Sketch of a second variation of a reduced flexure bearing that uses the linear mode of the actuator as a magnetic suspension to help support the rotor. 464 
6-9 Sketch of a third variation of a reduced flexure bearing that uses the linear mode of the actuator as a magnetic suspension to help support the rotor. . . . . . . . . . . . . . . 465

6-10 Sketch of the balanced X-rotor layout shown in Figure 4-8 with the stator cores un-nested (left), then with the rotor stretched in one direction and compressed in an orthogonal direction (center). . . . 467

6-11 Sketches showing a variation of the X-rotor design to provide a two-axis pointing mirror. . . . . . . . . . . . . . . . 469

6-12 Sketch showing the magnetic circuit for a proposed low-cost rotary actuator.

A-1 Electrical circuit and equivalent block diagram for the electrical dynamics of the motor for the $2 \mathrm{kHz}$ rotary fast tool servo. . . . . . 476

A-2 Block diagram showing the electrical dynamics of the actuator inside the current loop for the current-mode amplifier for the $2 \mathrm{kHz}$ FTS. 476

A-3 Straight-line approximation of the Bode magnitude plot of the negative loop transmission for the current compensation loop used with the $2 \mathrm{kHz}$ rotary fast tool servo (heavy, solid curve). . . . . . . . . 477

A-4 Mapping of the current compensation block diagram (left) to an op-amp circuit (right). . . . . . . . . . . . . . . . . . . . . 479

A-5 Hand-calculation proof showing that a fast inner loop acts like a constant gain block for frequencies below one tenth of its crossover frequency. . . . . . . . . . . . . . . . . . 483

A-6 Sketch of the Bode magnitude plots of a generic under-damped second-order plant and of the zeros and poles of an ideal notch filter. 484

A-7 Bode magnitude and phase plots for a canonical notch filter. . . . . 485

A-8 Bode magnitude and phase plots for a canonical notch filter. . . . . 486

C-1 Bode plot of the measured negative loop transmission for the compensated Z-axis slide in the diamond turning machine. . . . . . . . 523 
C-2 Graphical user interface developed for the diamond turning machine used to machine workpieces with the $2 \mathrm{kHz}$ FTS. . . . . . . . . . . 525

C-3 Top-level Simulink ${ }^{\circledR}$ model used to control the diamond turning machine. . . . . . . . . . . . . . . . 526

C-4 Second-level Simulink ${ }^{\circledR}$ model used to control the Z-axis slide. . . . 527

C-5 Second-level Simulink ${ }^{\circledR}$ model used to control the X-axis slide. . . 527

C-6 Second-level Simulink ${ }^{\circledR}$ model used to control the spindle. . . . . . 528

C-7 Third-level Simulink ${ }^{\circledR}$ model that generates the set of commanded positions used to produce a trajectory for the Z-axis slide in response to a jog command from the GUI for the diamond turning machine. . . . . . . . . . . . . . . . . 528

C-8 Graphical user interface developed to use the $2 \mathrm{kHz}$ FTS with a diamond turning machine to produce wavy surfaces on a workpiece. 530

C-9 Simulink ${ }^{\circledR}$ model used to control the $2 \mathrm{kHz}$ FTS when used with a diamond turning machine to produce wavy surfaces on a workpiece. 531

C-10 Bode plots of the modelled bandpass filter for the workpiece spindle encoder angle for different attenuation values. . . . . . . . . . . . . 532

C-11 Detail of the Bode plots of the modelled bandpass filter for the workpiece spindle encoder angle for different attenuation values. . . 533

C-12 Schematic representation of the sinusoidal tool path relative to a previously machined "flat" surface on the workpiece. . . . . . . . . 535

C-13 Surface measurement of the outer diameter of a workpiece machined with the $2 \mathrm{kHZ}$ FTS when using an excessively aggressive first depth-of-cut with the sinusoidal tool tip trajectory. . . . . . . 536

C-14 The author carefully jogging the Z-axis slide of the DTM while establishing initial contact between the diamond tool in the $2 \mathrm{kHz}$ FTS and a rotating workpiece. . . . . . . . . . . . . . 539 


\section{List of Tables}

2.1 Key to labels in Figure 2-4 ("Comparison of the $2 \mathrm{kHz}$ and $10 \mathrm{kHz}$ rotary fast tool servos to some representative fast tool servos reported in the literature"). . . . . . . . . . . . . . . 76

4.1 Selected properties of the two-part epoxy structural adhesives that were final candidates for bonding the rotor and stator assemblies. . . 264

4.2 Approximate viscosity (Poise) of some common materials at room temperature $[54] \ldots \ldots \ldots \ldots \ldots \ldots \ldots$

4.3 Noise measurements for the capacitance sensor that measures displacement of the tool in the $10 \mathrm{kHz}$ FTS. . . . . . . . . . . . . . . . . 344

5.1 Comparison of the magnetic circuits shown in Figures 5-6, 5-11, and $5-13$.

5.2 Selected physical and mechanical properties for some of the candidate soft magnetic materials. . . . . . . . . . . . . . . . . . . 426

5.3 Raw material properties and typical values for tape-wound cut core materials. . . . . . . . . . . . . . . . . . . 442

5.4 Magnetic characteristics, acceleration factor, and power loss for tapewound cut core materials with a maximum flux density of $11 \mathrm{kG}$ rms (16 kG peak) at $10 \mathrm{kHz}$ sinusoidal.

5.5 Magnetic characteristics, acceleration factor, and power loss for tapewound cut core materials with a $10 \mathrm{kHz}$ sinusoidal flux density adjusted so that power loss does not exceed that of 0.001 " thick $50 \% \mathrm{Ni}-$

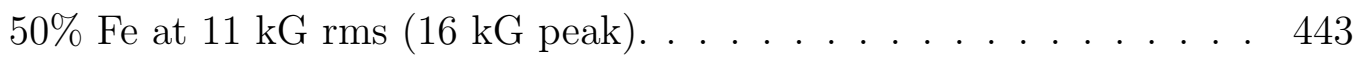


5.6 Magnetic characteristics, acceleration factor, and power loss for tapewound cut core materials with maximum "usable" flux density at $10 \mathrm{kHz}$ sinusoidal. . . . . . . . . . . . . . . . . . . . 443

C.1 Preferred cutting sequence used to produce the initial smooth (nonwavy) surface on a workpiece using the $2 \mathrm{kHz}$ FTS. . . . . . . . . . . 538

C.2 Preferred cutting sequence used to produce the wavy surface on a workpiece using the $2 \mathrm{kHz}$ FTS. . . . . . . . . . . . . . . . . 539 


\section{Chapter 1}

\section{Introduction}

One of the members of my thesis committee, Professor James Kirtley, once lightheartily commented to me: "we are engaged in the useful arts". It is my hope that this thesis adequately reflects the type, breadth, and scope of work that was necessary to develop the machines described by it. Theory and analysis by themselves would not have assured the success of these machines. Good ideas can fall into obscurity if they are not formulated in a manner that allows reasonably executing them into working hardware. Machine design requires a systematic and rigorous approach, but it is more than just a collection of heuristic rules. There is an art to translating a complex set of technical challenges into a seemingly simple design, fabricating and assembling complex hardware, debugging electronic circuits, and seeing, listening to, and feeling what a machine needs when it does not work as intended. Mens et Manus.

In this chapter I develop a background that describes what a fast tool servo is and what it is used for, establish a time-line for the work described in this thesis, and summarize the results of this research. The chapter concludes with brief descriptions for the chapters in this thesis. 


\subsection{Background}

This thesis describes the development of two high bandwidth short-stroke rotary fast tool servos and the hybrid rotary/linear electromagnetic actuator developed for one of them. This research involved the technical disciplines of design, analysis, fabrication, testing, and troubleshooting of precision mechanical systems, power and signal electronic systems, control systems, a normal-stress electromagnetic machine, and the dynamics of the combined systems.

A fast tool servo (FTS) is an auxiliary servo axis that is added to a diamond turning machine to allow generating free-form non-axisymmetric or textured surfaces on a workpiece. ${ }^{1}$ A rotary fast tool servo produces an in-and-out motion of the tool relative to a workpiece by swinging the tool along an arc having a fixed radius. The bandwidth of the fast tool servos described in this thesis is one to two orders of magnitude higher than that of the servo axes in the diamond turning machines that they would typically be used with.

The project began when I joined Professor David Trumper's Precision Motion Control Laboratory [7] at MIT in January 2001 and started learning about control systems and electronics. I had previously worked for fourteen years designing and building precision instruments and machine tools for the Lawrence Livermore National Laboratory (LLNL), cultivating a rich background in the mechanical aspects of precision machine design. In the Fall of 1999 I attended a one day seminar taught by Professor Trumper entitled "Precision Mechatronics". He introduced me to a universe of topics, strategies, and techniques for electronics, controls, and system design that was beyond what my background encompassed. By the end of that day it was clear to me that if I wanted to continue designing precision machines then I would need more than just a one-day tour of this "other" universe. I knew that I had to go back to school. On the one hand the fast tool servos and actuator described in this thesis represent new state-of-the-art machines with significant potential application.

\footnotetext{
${ }^{1} \mathrm{~A}$ diamond turning machine is an ultra-precision lathe that uses a gem-quality diamond with an accurately defined cutting edge for the tool, and is used to single-point machine optical quality surfaces with sub-micron figure accuracy.
} 
Furthermore, they led to ideas that hold promise for the development of other high bandwidth and precision motion control systems. On the other hand these machines served as vehicles for learning about electronics, control systems, electromagnetic machines, system dynamics, and integrated system design.

Figure 1-1 shows a schedule with milestones for this project. The first year was focused on two things. The first was gaining analytical and hands-on experience in control systems and electronics. The second was preparing for the Ph.D. qualifying examinations. I expect that this second item would typically not be worth mentioning, but then my situation was not typical: the undergraduate curriculum and Master of Science work that had prepared me for Ph.D. study had happened 15-22 years earlier! Work on the $2 \mathrm{kHz}$ rotary fast tool servo began in March 2002 with the development of conceptual designs. The detailed design effort began in May 2002, and the machine was fully assembled and running closed-loop nine months later in January 2003. Two significant activities ran in parallel with the development of the $2 \mathrm{kHz}$ FTS and made it possible to realize closed-loop control during the same month that FTS was fully assembled. The first was the development of a servo testbed an experimental set-up that used the same actuator, sensors, and payload inertia that the $2 \mathrm{kHz}$ FTS used - that allowed developing the current-mode amplifier and control system for the $2 \mathrm{kHz}$ FTS. The second was the development of the current-mode amplifier for the $2 \mathrm{kHz}$ FTS. During the summer of $2003 \mathrm{I}$ developed a controller for a diamond turning machine (DTM) and integrated the $2 \mathrm{kHz}$ FTS with that DTM. The first wavy part — a diamond-turned workpiece with 250 cycle/rev sinusoidal perturbations and an optical quality surface finish — was machined with the $2 \mathrm{kHz}$ FTS in September 2003. Conceptual designs for the $10 \mathrm{kHz}$ FTS and its hybrid rotary and linear electromagnetic actuator began in April 2003. The detailed design effort began in April 2004, and the machine was fully assembled nine months later in December 2004. The 10 kHz FTS was running closed-loop in February 2005, eleven months from the start of the detailed design effort. A significant activity that ran in parallel with the development of the $10 \mathrm{kHz}$ FTS was the fabrication, testing, and debugging of its current-mode power amplifier. Extended testing of the 


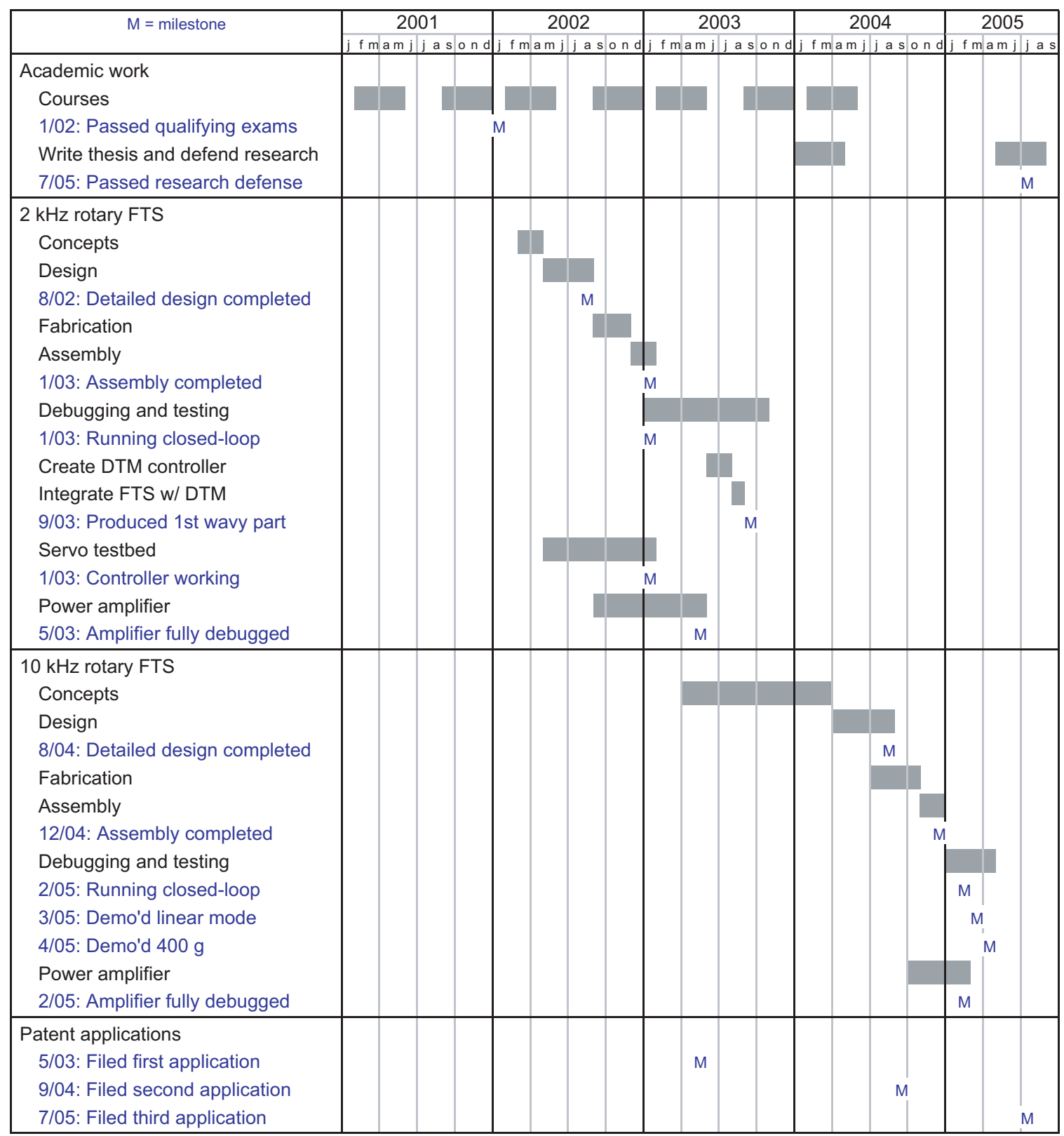

Figure 1-1: Schedule showing the development of the $2 \mathrm{kHz}$ and $10 \mathrm{kHz}$ rotary fast tool servos. Development of the hybrid rotary/linear actuator is embedded in the $10 \mathrm{kHz}$ FTS effort. 
$10 \mathrm{kHz}$ FTS ran until the end of May 2005, at which time we called that work complete and I focused on writing this thesis.

I did not machine a workpiece with the $10 \mathrm{kHz}$ rotary fast tool servo. Instead, I used the time that had been planned for those machining tests to more deeply explore the control issues for the rotary and linear degrees of freedom of our new actuator, and to demonstrate closed-loop control of both of those modes. I have no doubts that the $10 \mathrm{kHz}$ FTS will be able to machine a workpiece as well as or better than what was demonstrated with the $2 \mathrm{kHz}$ FTS. It has the high mechanical stiffness at the tool tip needed for machining a workpiece. Regarding dynamic stiffness from the controller, its position loop compensation has enough authority and robustness to pull the rotor off the open-loop unstable magnetic detent caused by the bias flux, and snap it to a stand still at the neutral position when closing the loop. With the controller turned off, I cannot move the rotor by hand off of that magnetic detent, so I am confident that the $10 \mathrm{kHz}$ FTS can handle the small cutting forces involved in diamond turning.

\section{$1.2 \quad$ Project Summary}

The $2 \mathrm{kHz}$ and $10 \mathrm{kHz}$ rotary fast tool servos described in this thesis were designed for diamond turning precision textured surfaces on small spherical workpieces (diameters in the range of $10 \mathrm{~mm}$ or less), and are suitable for generating free-form non-axisymmetric surfaces on similar-sized workpieces. Straightforward modifications would allow them to be used on larger workpieces, making them applicable to direct machining contact lenses or contact lens molds. These rotary fast tool servos establish new benchmarks for demonstrated closed-loop bandwidth $(2 \mathrm{kHz}$ and $10 \mathrm{kHz}$ ) and tool tip acceleration (400 g) when compared to the fast tool servos that I found in the literature.

A goal for this research was to develop a design for a short-stroke rotary fast tool servo capable of closed-loop operation at frequencies up to $10 \mathrm{kHz}$ and a maximum tool tip acceleration of $1000 \mathrm{~g}$. To control a fast tool servo at $10 \mathrm{kHz}$, the mechanical 
resonant modes need to fall into at least one of the following categories: (1) be well above that frequency; (2) be observable and controllable; or (3) not significantly affect the position of the tool tip. Achieving a $1000 \mathrm{~g}$ tool tip acceleration requires developing a high torque relative to a small moving mass. Unlike a typical servo system where the payload and the moving element of the actuator are often two discrete elements (e.g.: a spindle driven by a motor through a coupling), realizing the proposed high bandwidth and acceleration requires merging those two entities into one moving mass to increase the frequency of resonances and decrease the rotational inertia.

Instead of spending an inordinate amount of time during the early stages of this research on paper studies trying to converge on an optimal integrated design for the payload and actuator, we first developed the $2 \mathrm{kHz}$ rotary fast tool servo shown in Figure 1-2 by designing a novel mechanism that uses a commercially available movingmagnet galvanometer (Lorentz force actuator). The moving mass of the actuator is coupled to the tool arm through a finite stiffness connection, resulting in a first uncoupled mode frequency of $5 \mathrm{kHz}$. Although this approach fits the "typical servo system" description above, it allowed establishing the proof-of-principles needed to develop the $10 \mathrm{kHz}$ rotary fast tool servo shown in Figure 1-3, and provided me the experience with control systems and electronics that I needed.

For the $10 \mathrm{kHz}$ FTS the moving masses of the actuator and the payload are merged into a single moving mass by attaching the tool arm directly to the rotating element of a novel actuator. By getting rid of the uncoupled mode, the first mechanical resonance is a relatively higher frequency non-rigid body mode of the moving mass structure. Placing the position feedback sensors directly behind the tool and careful design of the support structure allows hiding certain flexible modes of the system to further improve high frequency controllability. Additionally, the merged mass approach results in a lower overall moving mass and a higher torque-to-inertia ratio.

During the design phase for the $10 \mathrm{kHz}$ FTS, a 900 g maximum tool tip acceleration design was chosen over a $1000 \mathrm{~g}$ design because it allowed using the same flexure mounting hardware that proved successful with the $2 \mathrm{kHz}$ FTS. This decision allowed 


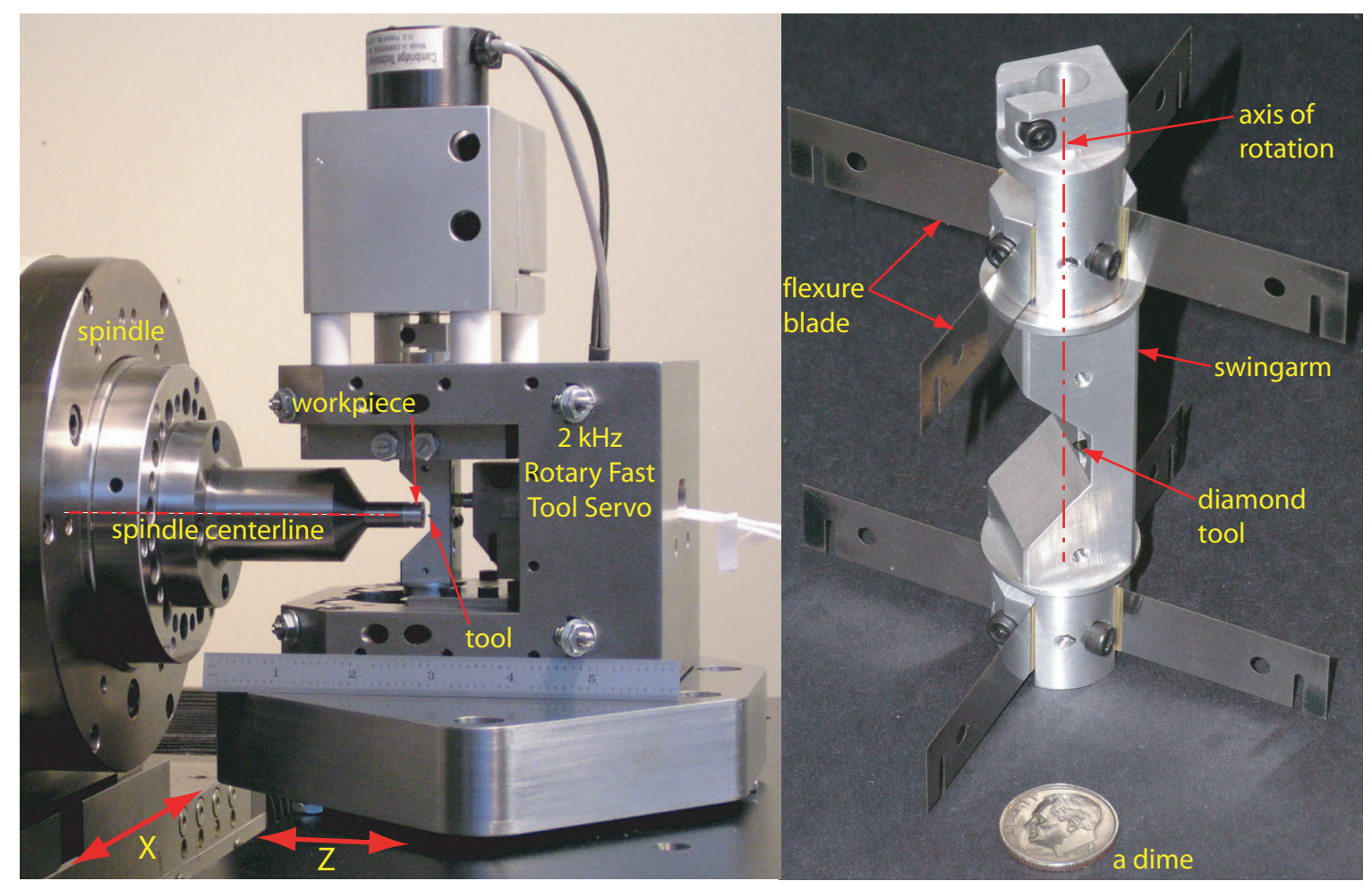

Figure 1-2: The $2 \mathrm{kHz}$ rotary fast tool servo on a diamond turning machine (left), and its rotating element not including the actuator (right).

keeping the flexure bearing off the list of high-risk items for this new machine. A second, more conservative decision was made to depart from the optimized $900 \mathrm{~g}$ design to a $600 \mathrm{~g}$ design by increasing the dimensions for certain components in the rotor to better assure the survival of the system during anticipated tests. Having gained confidence with the successful tests and performance demonstrations for the $10 \mathrm{kHz}$ FTS and its actuator, design parameters can be adjusted in future versions to achieve tool tip accelerations of $1000 \mathrm{~g}$ and beyond.

At the heart of the $10 \mathrm{kHz}$ rotary fast tool servo is a normal-stress variable reluctance actuator that we developed. In addition to producing the torque that is needed for the $10 \mathrm{kHz}$ rotary fast tool servo, the actuator produces a radially-directed force and is therefore referred to as a hybrid rotary/linear actuator. Certain types of electric engraving heads use an actuator that is similar to the hybrid actuator. In the case of the engraving heads, the actuator is used to drive and sustain a resonance in a mechanical oscillator. This is in sharp contrast to the closed-loop arbitrary trajec- 


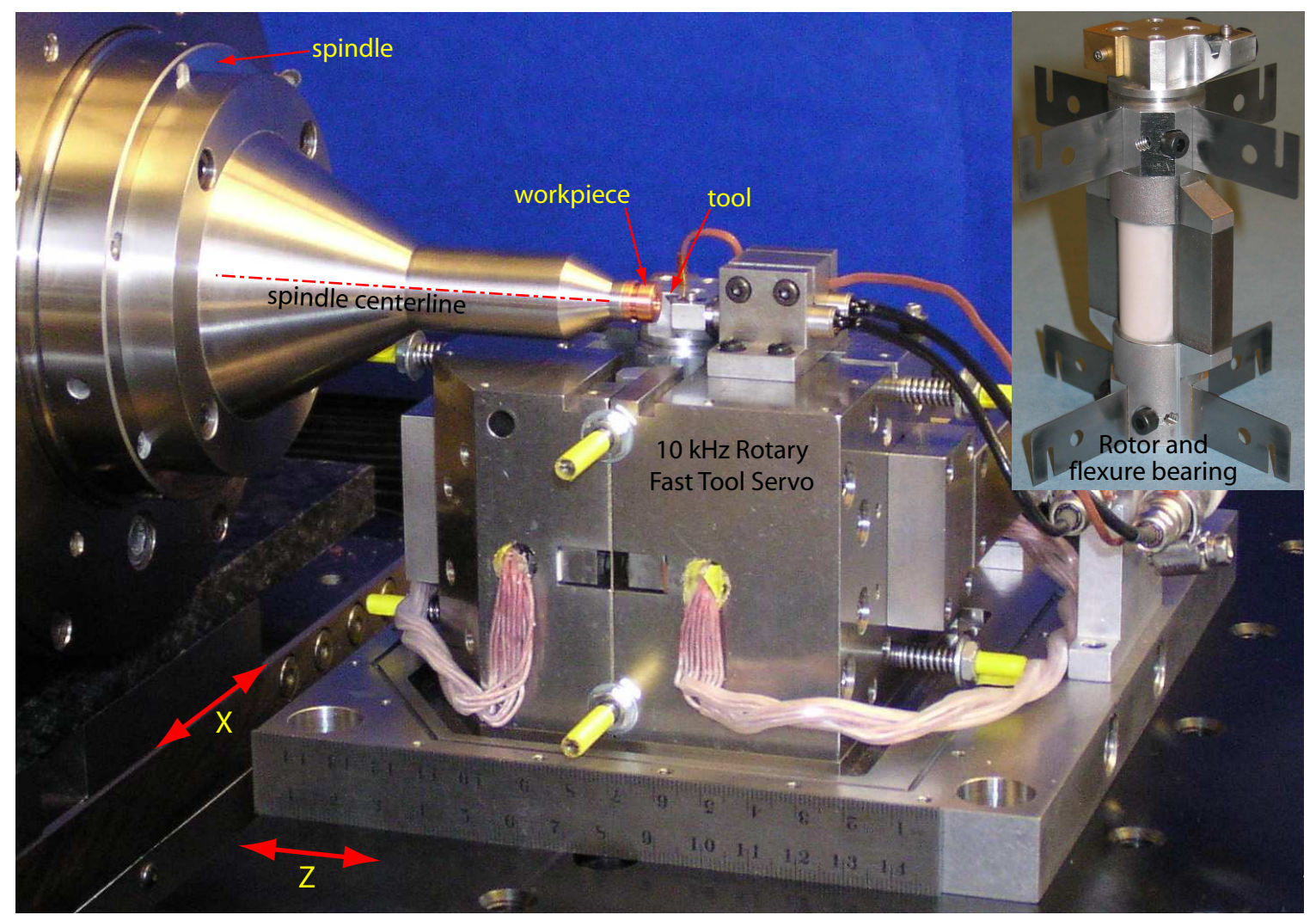

Figure 1-3: The $10 \mathrm{kHz}$ rotary fast tool servo on a diamond turning machine, and the rotor for the fast tool servo with its flexure bearing (inset). 
tories for the tool tip that are demonstrated with the $10 \mathrm{kHz}$ FTS, which represents the first published example that I am aware of demonstrating the use of this class of actuator in a non-resonating application. Using a separate current-mode amplifier for each stator half allows demonstrating closed-loop control of the rotary and linear degrees of freedom that are inherent in this class of actuators. Our work is further distinguished from the prior art by a magnetic circuit that decouples certain magnetic flux paths when a coil is used instead of a permanent magnet to provide the bias magnetic flux. This reduces the complexity of the actuator electrical dynamics from a MIMO system to a SISO system, and allows using loop-shaping techniques with classical control theory to design the control systems.

\subsubsection{The $2 \mathrm{kHz}$ Rotary Fast Tool Servo}

To establish certain proof-of-principles I designed, built, and tested a first new rotary fast tool servo having a continuously variable closed-loop operating frequency up to $2 \mathrm{kHz}$. Referred to as the $2 \mathrm{kHz}$ FTS, it provides the following proof-of-principles for the later $10 \mathrm{kHz}$ rotary fast tool servo: over-constrained flexures for guiding reciprocating rotary motion, small diamond tool and mounting method, circuit topology for a high bandwidth current-mode amplifier, and control system design. Machining tests with the $2 \mathrm{kHz}$ FTS validate that a rotary-type fast tool servo can be used to produce optical quality surfaces on a spherical workpiece from its pole to its equator.

Figure 1-2 shows the $2 \mathrm{kHz}$ rotary fast tool servo mounted on a diamond turning machine and engaging a workpiece. The swing-arm (rotor) for the fast tool servo and its flexure bearing are also shown in Figure 1-2. The swing-arm carries a diamond tool at a swing radius of $5 \mathrm{~mm}$ and has a full-travel rotation angle of $\pm 5 \mathrm{mrad}$. The small rotation angle allows using over-constrained flexures to guide the rotary motion. A total of eight flexures are used, four above the tool and four below it. The inner ends of the flexures are fixed to the swing-arm, and the outer ends are fixed to the base. To minimize the unwanted transverse motion of the tool tip caused by motion along an arc, the tool tip is positioned in a front-back plane that contains the axis of rotation. This transverse motion is only $\pm 63 \mathrm{~nm}$ for the full tool tip travel 
of $\pm 25 \mu \mathrm{m}$, making it negligible for most diamond turning applications.

A flexure bearing utilizing over-constrained flexures was chosen because it satisfies the following requirements: (1) establish a non-wandering center of rotation; (2) add low to negligible rotational inertia to the moving mass; (3) survive oscillatory motion without requiring maintenance; (4) be stiff enough to support the cutting tool to maintain a desired depth of cut when using the FTS to single-point machine optical quality surfaces on a workpiece; and (5) be serviceable if damaged.

The position of the tool is measured by a pair of eddy current sensors operating differentially and looking at the back of the swing-arm on either side of the axis of rotation and near the elevation of the tool. This is best seen in Figure 1-7, which shows the diamond tool engaging a workpiece during a cutting test.

The $2 \mathrm{kHz}$ FTS has a demonstrated closed-loop sinusoidal tool stroke of $50 \mu \mathrm{m} \mathrm{PP}$ at low frequencies and $2.5 \mu \mathrm{m} \mathrm{PP}$ at $2 \mathrm{kHz}(20 \mathrm{~g}$ tool tip acceleration). The demonstrated $20 \mathrm{~g}$ tool tip acceleration at $2 \mathrm{kHz}$ required a peak torque of $0.20 \mathrm{~N} \cdot \mathrm{m}$ from the actuator. That demonstrated peak torque is $71 \%$ of the specified continuous-operation peak torque of $0.28 \mathrm{~N} \cdot \mathrm{m}$ for the actuator. The ratio of the demonstrated peak torque while accelerating the swing arm divided by the corresponding apparent time-average electrical power of $120 \mathrm{VA}$ at the terminals of the actuator is $1.7 \times 10^{-3} \mathrm{~N} \cdot \mathrm{m} / \mathrm{VA}$.

A different measure of performance for the actuator used in the $2 \mathrm{kHz}$ FTS is the ratio of the continuous-operation peak torque divided by the rotational inertia of the rotor without the swing arm. For the demonstrated peak torque of $0.20 \mathrm{~N} \cdot \mathrm{m}$ and the actuator moving mass of $6.4 \mathrm{gm} \cdot \mathrm{cm}^{2}$, that ratio is $3.1 \times 10^{5} \mathrm{~N} \cdot \mathrm{m} /\left(\mathrm{kg} \cdot \mathrm{m}^{2}\right)$. Using the designed peak torque of $0.28 \mathrm{~N} \cdot \mathrm{m}$ increases the ratio to $4.4 \times 10^{5} \mathrm{~N} \cdot \mathrm{m} /\left(\mathrm{kg} \cdot \mathrm{m}^{2}\right)$.

The $2 \mathrm{kHz}$ FTS uses a hybrid analog and digital control system. The block diagram for the control system used with the $2 \mathrm{kHz}$ FTS is shown in Figure 1-4. The faster current loop is implemented with analog electronics to avoid the phase delay inherent with the sampling process of a digital controller, while the slower position loop is implemented with a PC-based digital controller.

Figure 1-5 shows a Bode plot of the measured negative loop transmission for the $2 \mathrm{kHz}$ FTS tool position loop when the integral mechanical viscous damper is used. 


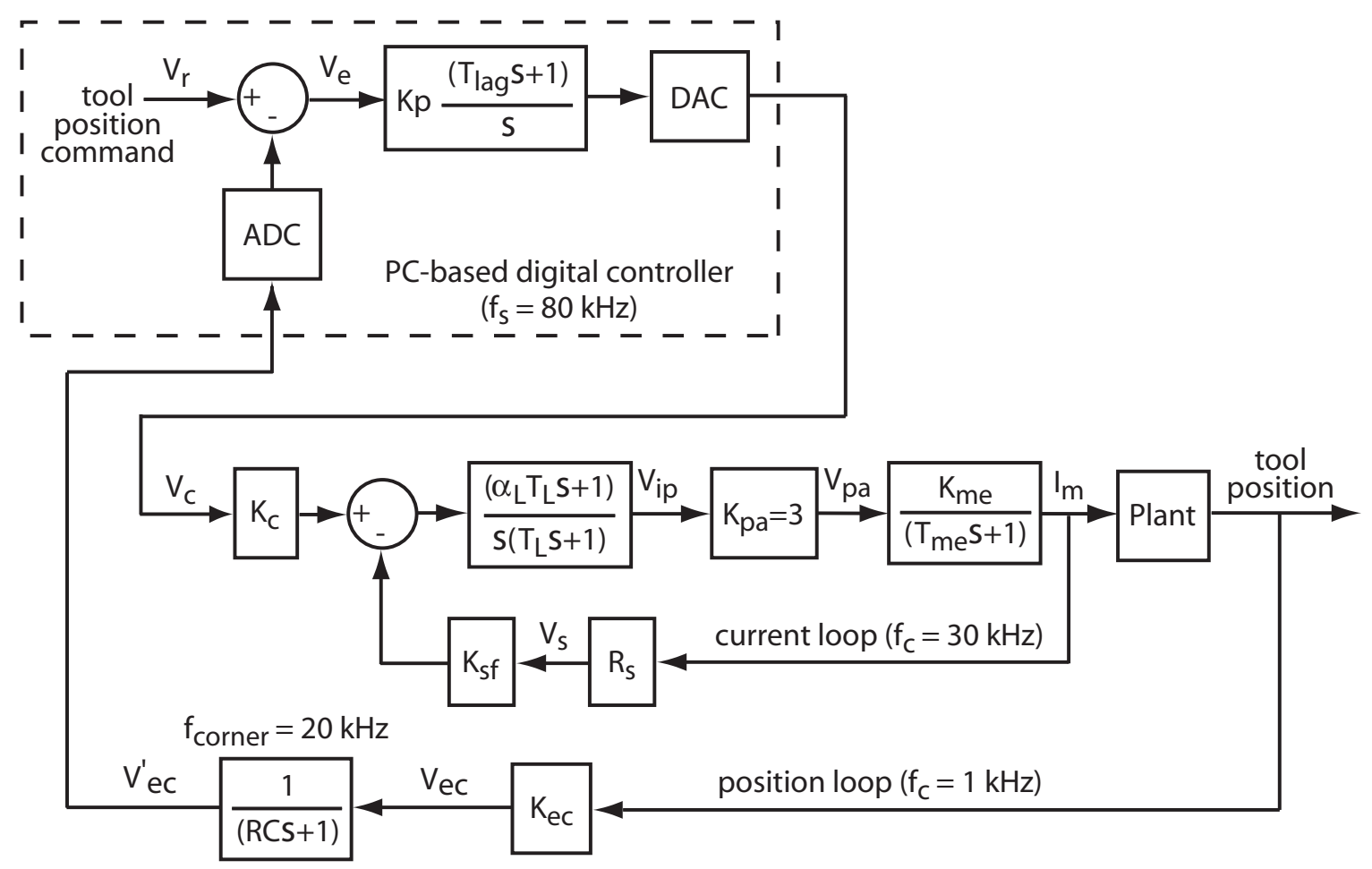

Figure 1-4: Control system block diagram for the $2 \mathrm{kHz}$ FTS.

The crossover frequency for this system is $1 \mathrm{kHz}$ with a phase margin of $41^{\circ}$ and a gain margin of 6 dB. Figure 1-6 shows the corresponding measured small-signal closedloop response. The $-3 \mathrm{~dB}$ closed-loop bandwidth is just above $2 \mathrm{kHz}$. The closed-loop magnitude and phase angle are fairly smooth curves up to $2 \mathrm{kHz}$, suggesting that a possible future addition of a feedforward compensator could be effective up to that frequency. Essentially, the feedforward controller applies a frequency-dependent magnitude and phase adjustment to the commanded reference signal to compensate for the known closed-loop response of the system.

The $2 \mathrm{kHz}$ FTS was integrated with a diamond turning machine and used to produce optical quality textured surfaces on the face and outside diameter of a handful of $9 \mathrm{~mm}$ diameter aluminum workpieces. With the fast tool servo operating at $2 \mathrm{kHz}$ and providing 20 g maximum tool tip acceleration, I machined 250 radial grooves on the face and 250 axial grooves on the outside diameter of the workpiece while it was rotating at 480 RPM. Figure 1-7 shows a close-up view of the tool engaging the face of one of these workpieces. 

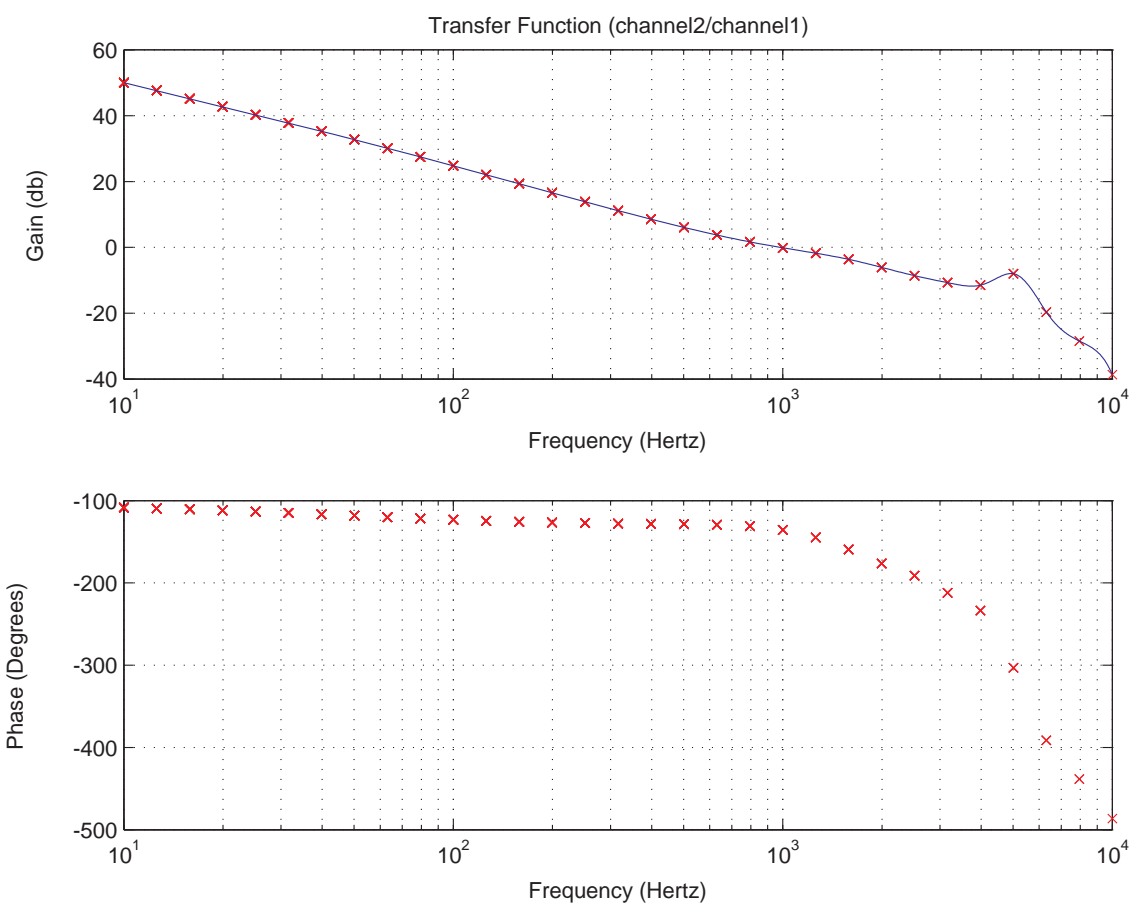

Figure 1-5: Bode plot of the measured negative loop transmission for the $2 \mathrm{kHz}$ FTS tool position loop.
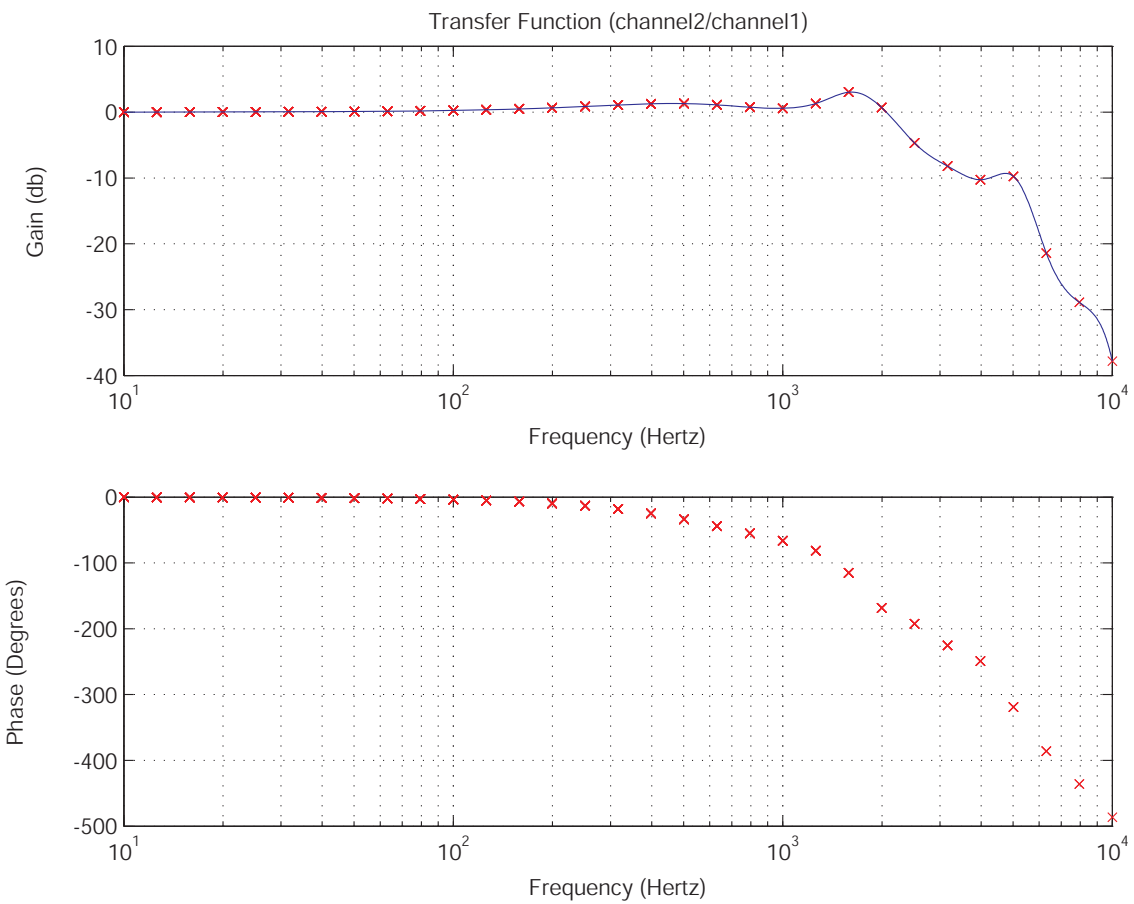

Figure 1-6: Bode plot of the measured small-signal closed-loop response for the $2 \mathrm{kHz}$ FTS tool position loop. 


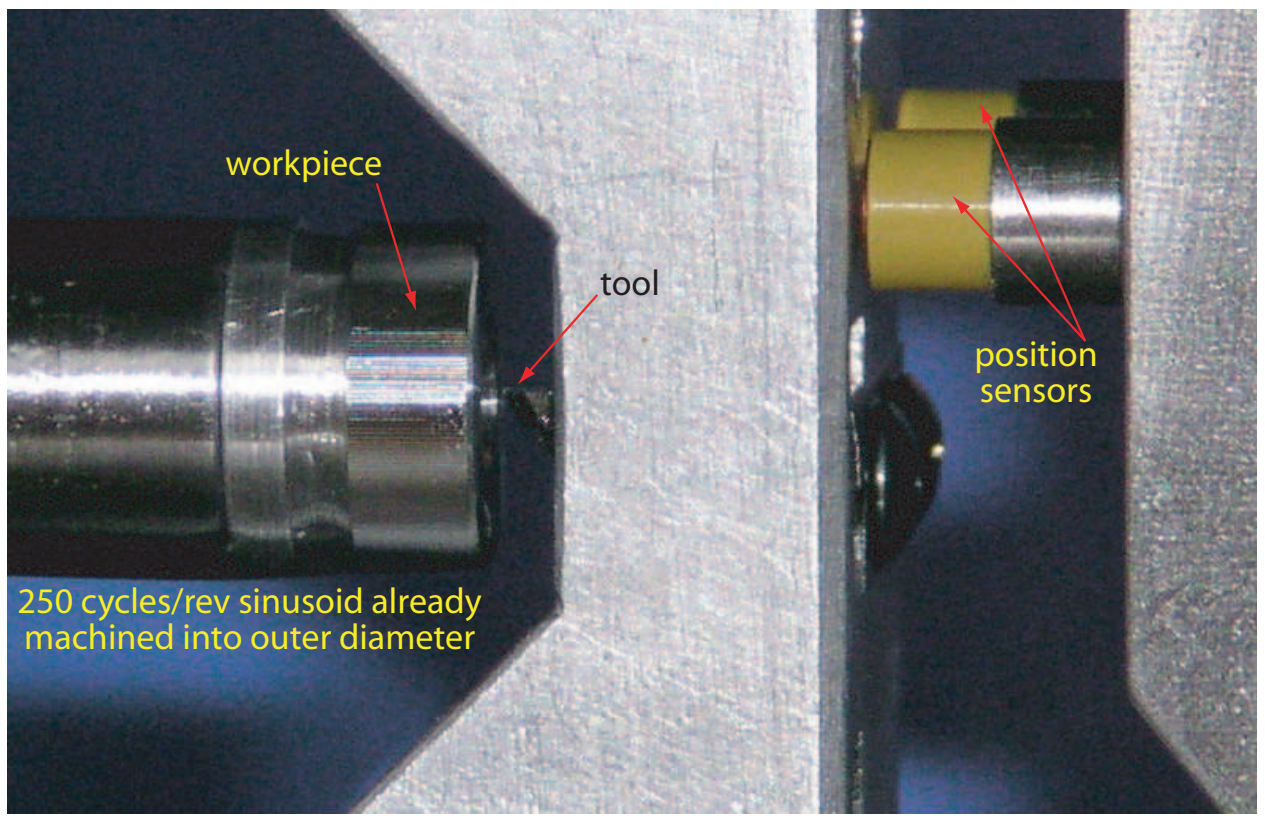

Figure 1-7: Close-up view of the $2 \mathrm{kHz}$ FTS tool tip engaging the face of a workpiece.

Figure 1-8 shows a portion of the face of a finished workpiece, along with optical measurements of that surface. Tracing a profile along the face at any fixed radius, the grooves describe a sinusoidal waveform with $2.2 \mu \mathrm{m}$ PV and a spatial wavelength ranging from $110 \mu \mathrm{m}$ at the outer diameter to $30 \mu \mathrm{m}$ at the inner diameter. The measured roughness along a groove (across the feed marks) is $12 \mathrm{~nm} \mathrm{rms,} \mathrm{which} \mathrm{is}$ very good considering that the diamond turning machine was in a less than optimum operating environment given its close proximity to heavy rotating machinery sharing the same concrete foundation. Similar textured surfaces were machined on the outside diameter of the workpieces, and the measured roughness along a groove in that case is $10 \mathrm{~nm} \mathrm{rms.}$

By machining the face and outside diameters of workpieces and producing optical quality surfaces having approximately the same roughness, I demonstrated that the disturbance to the diamond turning machine from the reaction torque produced by the $2 \mathrm{kHz}$ rotary fast tool servo was fairly constant for the two cases. This result reinforces the motivation for using a rotary-type FTS to machine from the pole to the equator on a spherical workpiece. 

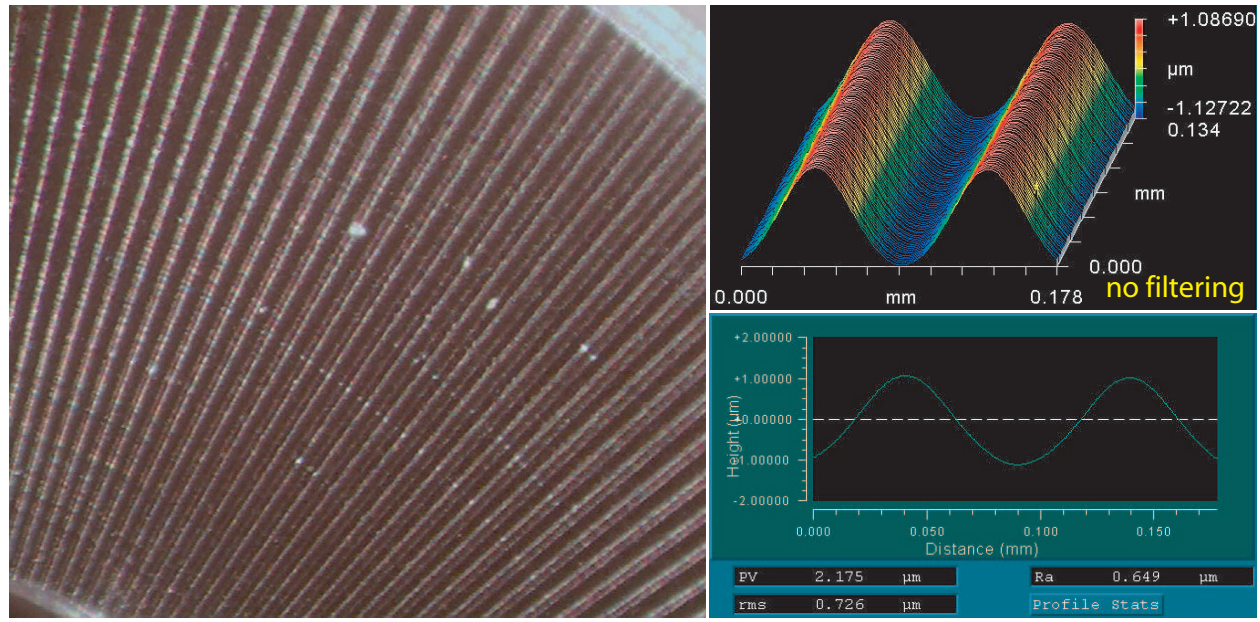

Figure 1-8: Portion of the surface of a wavy part produced with the $2 \mathrm{kHz}$ FTS (left), 3-D surface map of a portion of that surface (right, upper), and profile across the grooves (right, lower).

\subsubsection{The $10 \mathrm{kHz}$ Rotary Fast Tool Servo}

Using knowledge gained from the $2 \mathrm{kHz}$ FTS, I designed, built, and tested a second new rotary FTS having a continuously variable closed-loop operating frequency up to $10 \mathrm{kHz}$. Referred to as the $10 \mathrm{kHz}$ FTS, it incorporates several key concepts developed with the earlier $2 \mathrm{kHz}$ rotary fast tool servo: over-constrained flexures for guiding reciprocating rotary motion, small diamond tool and mounting method, circuit topology for a high bandwidth current-mode amplifier, and control system design.

The most significant advances from the $2 \mathrm{kHz}$ FTS to the $10 \mathrm{kHz}$ FTS are the use of a novel normal-stress variable reluctance actuator and the integration of the payload directly with the moving element of the actuator to form a single rigid body. The first advance provides an order of magnitude increase in actuator force density. The second advance reduces the total inertia of the moving mass and increases the frequency of the first system resonance by getting rid of the uncoupled mode present in the $2 \mathrm{kHz}$ FTS. Combined, these two advances made possible the factor of 20 increase in the demonstrated unaided ${ }^{2}$ tool tip acceleration compared to the $2 \mathrm{kHz}$ FTS. A

\footnotetext{
2 "Unaided" means that the actuator supplies all of the torque needed to accelerate the rotor and is not assisted by the stored energy of a mechanical resonance.
} 
trade-off study was used for balancing the conflicting requirements for the mechanical, magnetic, and electrical design of the actuator.

Figure 1-3 shows the $10 \mathrm{kHz}$ rotary fast tool servo mounted on a diamond turning machine and engaging a workpiece. The rotor for the fast tool servo and its flexure bearing are also shown in Figure 1-3. The rotor consists of the moving element of the actuator and the tool holder that is directly attached to it. The tool holder carries a diamond tool at a swing radius of $12.7 \mathrm{~mm}$ (0.500 in) and has a full-travel rotation angle of $\pm 2.8 \mathrm{mrad}$. The small rotation angle allows using over-constrained flexures to guide the rotary motion. A total of eight flexures are used, four above the rotor core and four below it. The inner ends of the flexures are fixed to the rotor, and the outer ends are fixed to the stator. To minimize the unwanted transverse motion of the tool tip caused by motion along an arc, the tool tip is positioned in a front-back plane that contains the axis of rotation. This transverse motion is only $\pm 48 \mathrm{~nm}$ for the full tool tip travel of $\pm 35 \mu \mathrm{m}$, making it negligible for most diamond turning applications.

The position of the tool is measured by a capacitance sensor looking at the back of the tool arm directly behind the tool. The tool position noise level is 1.4 to $2.5 \mathrm{~nm} \mathrm{rms}$, depending on the magnitude of the bias flux being used. A second capacitance sensor measures translation of the centerline of the tool arm, and is used to control the linear mode of the actuator. The bias magnetic flux in the actuator is easily adjusted by changing the current in the coil that produces it — which allowed establishing a high-acceleration implementation and a low-noise implementation of the $10 \mathrm{kHz}$ FTS.

The $10 \mathrm{kHz}$ rotary fast tool servo has a demonstrated closed-loop sinusoidal tool stroke of $70 \mu \mathrm{m} \mathrm{PP}$ at low frequencies and $8.0 \mu \mathrm{m} \mathrm{PP}$ at $5 \mathrm{kHz}$ (400 g tool tip acceleration). With the aid of a stable mechanical resonance at $10 \mathrm{kHz}$ that stores and recovers energy supplied by the actuator, a closed-loop sinusoidal tool stroke of $4.2 \mu \mathrm{m} \mathrm{PP}$ at $10 \mathrm{kHz}$ is demonstrated with this machine ( $870 \mathrm{~g}$ tool tip acceleration).

The $10 \mathrm{kHz}$ FTS uses a hybrid analog and digital control system similar to the system used with the $2 \mathrm{kHz}$ FTS and shown in Figure 1-4. The faster current loop is implemented with analog electronics to avoid the phase delay inherent with the 

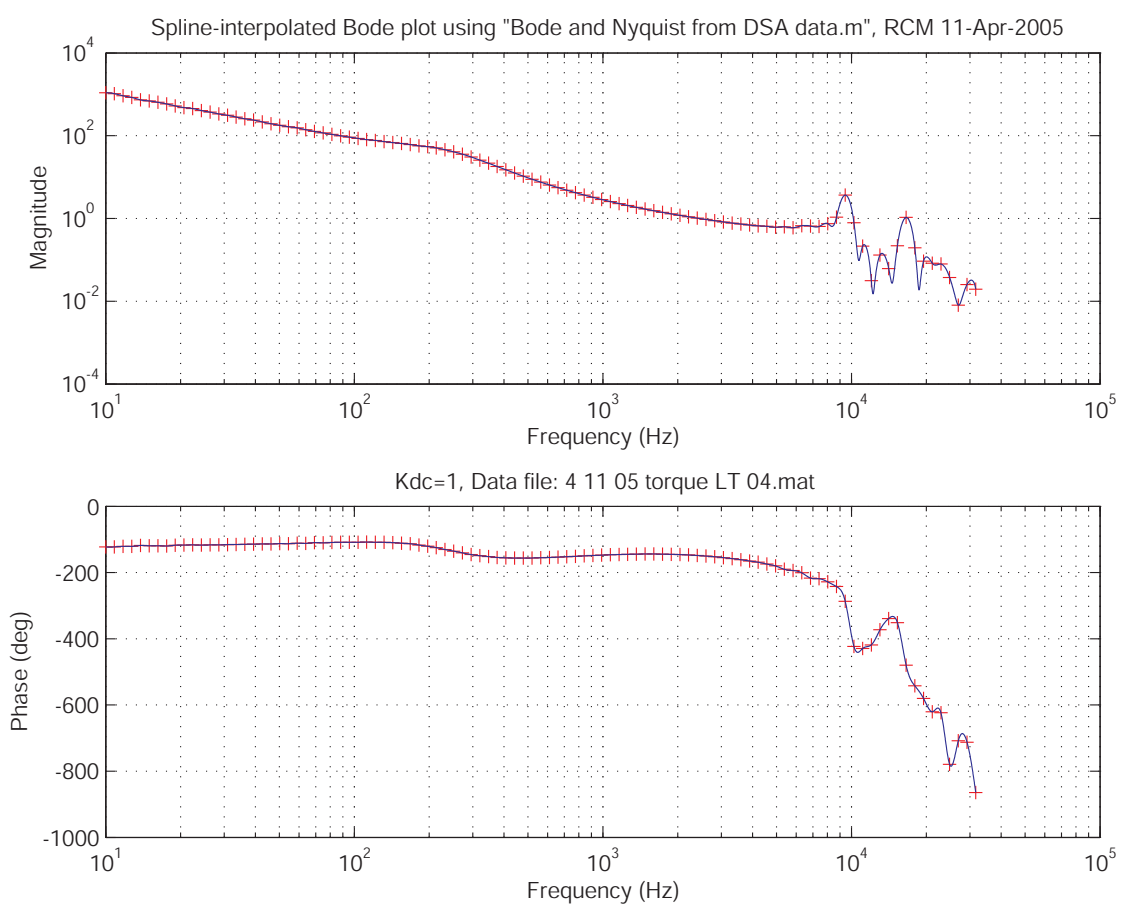

Figure 1-9: Bode plot of the measured negative loop transmission for the tool position for the low-noise implementation of the $10 \mathrm{kHz}$ FTS.

sampling process of a digital controller.

Figure 1-9 shows a Bode plot of the measured negative loop transmission for the tool position for the low-noise implementation of the $10 \mathrm{kHz}$ FTS. The crossover frequency for this system is $2.3 \mathrm{kHz}$ with a phase margin of $32^{\circ}$ and a gain margin of $5 \mathrm{~dB}$. Figure 1-10 shows the corresponding measured small-signal closed-loop response. The $-3 \mathrm{~dB}$ closed-loop bandwidth is $10 \mathrm{kHz}$. The closed-loop magnitude and phase angle are fairly smooth curves up to $5 \mathrm{kHz}$, suggesting that a possible future addition of a feedforward compensator would be effective up to that frequency.

\subsubsection{Hybrid Rotary/Linear Actuator}

Figure 1-11 shows a model of the magnetic circuit for the actuator that we developed for the $10 \mathrm{kHz}$ rotary fast tool servo. The rotor core is a rectangular prism suspended between two opposing C-shaped stator cores with nominal air gaps of $50 \mu \mathrm{m}$ at the four pole faces. The rotor and stator cores are made of laminated soft magnetic material to reduce the hysteretic and eddy current losses associated with the AC 

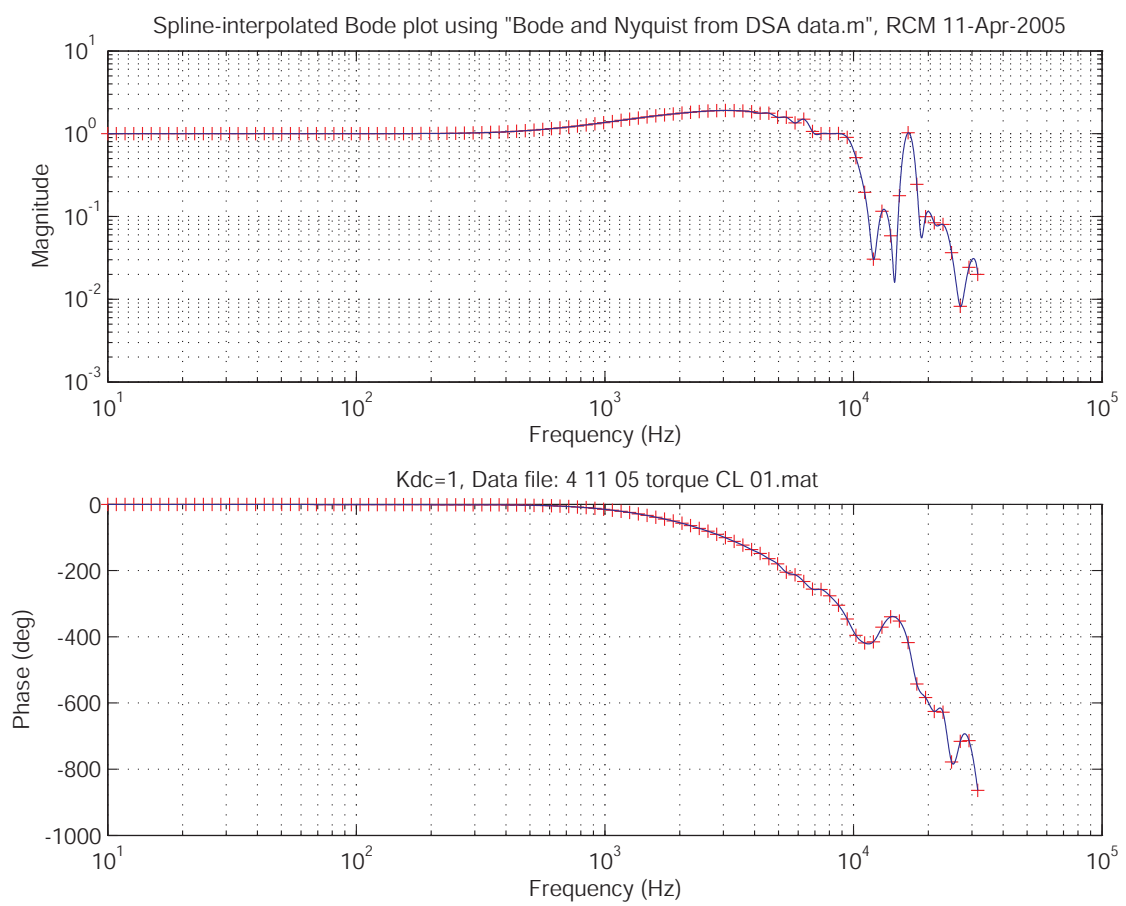

Figure 1-10: Bode plot of the measured small-signal closed-loop response for the tool position for the low-noise implementation of the $10 \mathrm{kHz}$ FTS.

steering flux. A DC coil provides a biasing magnetic flux in the four air gaps. Each stator core with its pair of $\mathrm{AC}$ coils forms a magnetic circuit with the rotor core. Considering one of the stator cores, energizing its AC coils in a manner that produces flux in a common direction creates a steering flux that circulates around that stator core and the rotor core via the two air gaps between the two. The bias and steering fluxes add at one of those gaps and subtract at the other. The same thing occurs between the other stator core and the rotor core. With proper phasing of the AC fluxes in the two stator cores and balanced magnitudes of DC and AC fluxes, the fluxes can be made to add in diagonally opposed air gaps and subtract in the opposite diagonally opposed air gaps, resulting in a net torque on the rotor with nominally no net force on it. The bias flux linearizes the relationship between the torque and steering-coil currents, which makes it easier to accurately control the actuator. Since the bias flux supplies up to half of the torque-producing magnetic flux in the rotor/stator air gaps, it provides a factor of two reduction in the reactive electrical power needed to drive the AC flux during peak operation when compared to a design that does not use a 

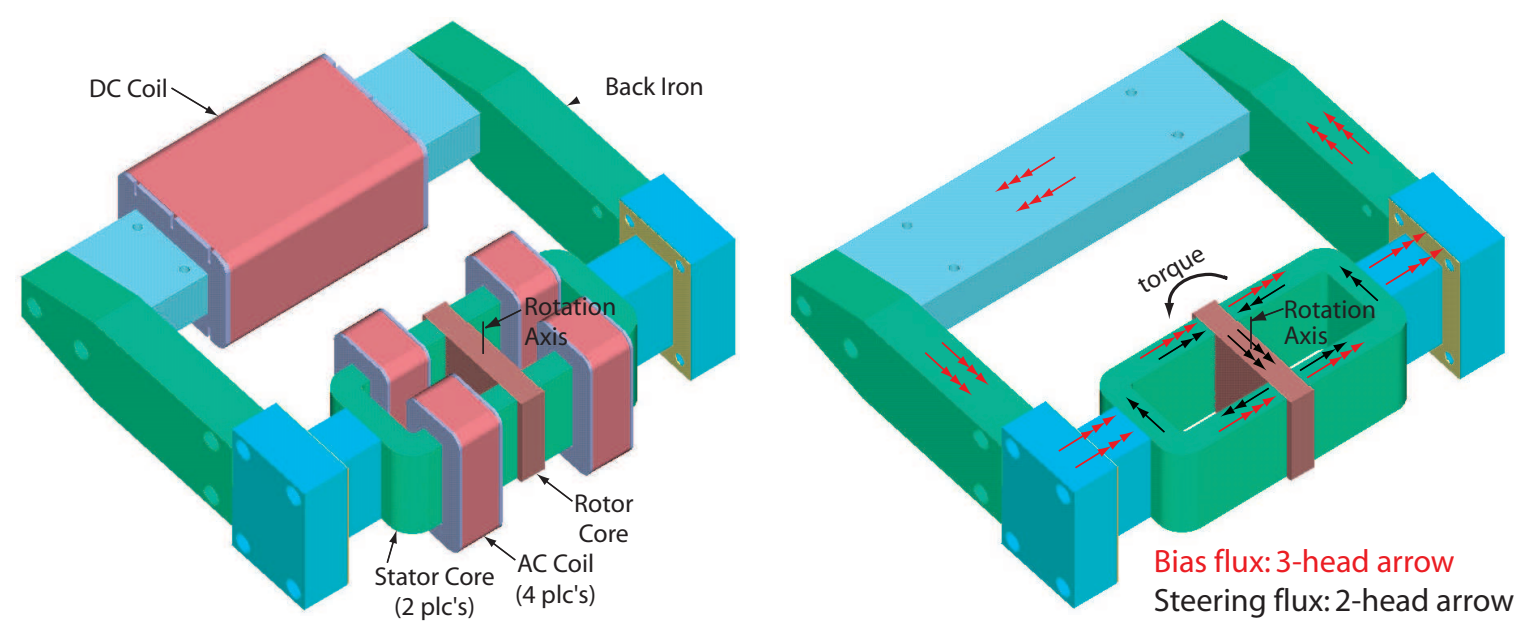

Figure 1-11: Model of the magnetic circuit for the hybrid actuator with coils (left), and without coils to show flux lines (right).

bias flux.

The $400 \mathrm{~g}$ tool tip acceleration at $5 \mathrm{kHz}$ demonstrated with the $10 \mathrm{kHz}$ FTS required a peak torque of $2.5 \mathrm{~N} \cdot \mathrm{m}$ from the actuator. That demonstrated peak torque is a factor of 12 greater than the analogous demonstrated peak torque for the $2 \mathrm{kHz}$ FTS. The demonstrated peak torque for the $10 \mathrm{kHz}$ FTS corresponds to a demonstrated peak magnetic flux density of $1.2 \mathrm{~T}$ in the air gaps between the rotor and stator. The demonstrated peak torque and peak flux density are $68 \%$ and $82 \%$ of our design goals of $3.7 \mathrm{~N} \cdot \mathrm{m}$ and $1.5 \mathrm{~T}$, respectively. The ratio of the demonstrated peak torque while accelerating the tool arm in the $10 \mathrm{kHz}$ FTS divided by the corresponding apparent time-average electrical power of $160 \mathrm{VA}$ at the terminals of the actuator is $16 \times 10^{-3} \mathrm{~N} \cdot \mathrm{m} / \mathrm{VA}$. This ratio is a factor of 9 greater than the same demonstrated ratio of $1.7 \times 10^{-3} \mathrm{~N} \cdot \mathrm{m} / \mathrm{VA}$ for the actuator used in the $2 \mathrm{kHz}$ FTS.

Another way to compare the performance of the two actuators is to consider the ratio of the continuous-operation peak torque divided by the rotational inertia of the rotor without the tool arm. For the $10 \mathrm{kHz}$ FTS, the demonstrated peak torque of $2.5 \mathrm{~N} \cdot \mathrm{m}$ and a rotor inertia of $70 \mathrm{gm} \cdot \mathrm{cm}^{2}$ gives a ratio of $3.6 \times 10^{5} \mathrm{~N} \cdot \mathrm{m} /\left(\mathrm{kg} \cdot \mathrm{m}^{2}\right)$. Using the designed peak torque of $3.7 \mathrm{~N} \cdot \mathrm{m}$ increases the ratio to $5.3 \times 10^{5} \mathrm{~N} \cdot \mathrm{m} /\left(\mathrm{kg} \cdot \mathrm{m}^{2}\right)$. These values are similar to the analogous values for the state-of-the-art galvanometer used for the actuator in the $2 \mathrm{kHz}$ FTS. 


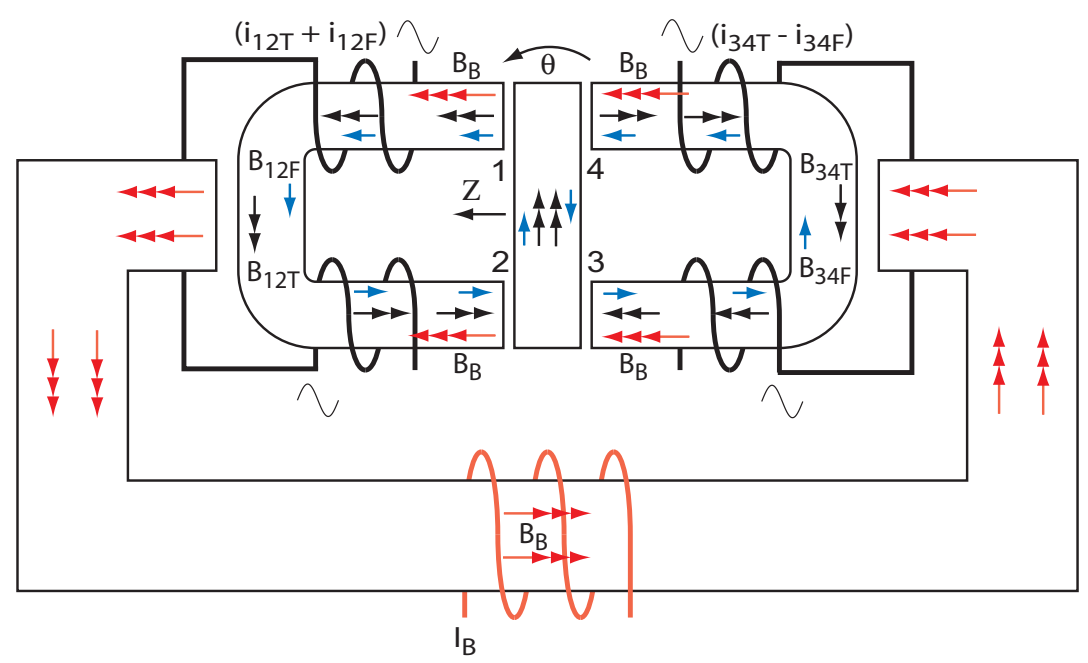

Figure 1-12: Sketch of the magnetic circuit for the actuator.

For a fair comparison of the actuators used in the $10 \mathrm{kHz}$ FTS and in the $2 \mathrm{kHz}$ FTS, one should consider that the actuator in the $10 \mathrm{kHz}$ FTS would need to be cooled if it were to run continuously at the performance levels described in this section. The provisions that were made for cooling the $10 \mathrm{kHz}$ FTS are described at the end of this section.

The bias flux causes the rotary mode of the actuator to be open-loop unstable. Referring to Figure 1-12, if the steering fluxes are set to zero and the rotor is displaced clockwise, then the bias flux will preferentially flow through stator pole 4 , the rotor, and stator pole 2, causing the rotor to turn until it makes contact with stator poles 2 and 4. Adding the torque-generating steering fluxes depicted by the doublehead arrows in Figure 1-12 counteracts this effect. Adding a sensor for measuring the rotation and a control system for the steering fluxes turns this rotary mode into a rotary actuator.

A permanent magnet can be used instead of the current-carrying coil shown in Figure 1-12 to produce the bias flux. I found it advantageous to use a coil because it allowed adjusting the magnitude of bias flux during testing of the actuator. The air gaps between the stator cores and the back-iron (the portion carrying the bias coil) are needed to reduce the magnetic coupling between the two stator cores when a bias coil is used instead of a permanent magnet. This reduces the electrical dynamics 
for the actuator from a multiple-input multiple-output (MIMO) system to a pair of nominally identical single-input single-output (SISO) systems when using separate, independently-controlled power amplifiers for the two stator halves. Independent control of each stator half allows simultaneously controlling the linear and rotary degrees of freedom of the actuator. These two SISO systems are in parallel to each other and conveniently treated as a single SISO system, making it possible to design the controller using classical control system loop-shaping techniques.

In addition to the bias flux and torque generating fluxes shown in Figure 1-11, Figure 1-12 includes the force generating fluxes depicted by the single-head arrows. There are two ways to generate a radial force in the Z-direction on the rotor. The first is as shown in Figure 1-12 for the case when the rotor is centered between the two stator halves and the two torque-producing steering currents $i_{12 T}$ and $i_{34 T}$ are nominally equal. In this case, a net force in the positive Z-direction is produced by increasing the steering coil current for the left stator half to $i_{12}=\left(i_{12 T}+i_{12 F}\right)$, and decreasing the steering coil current for the right stator half to $i_{34}=\left(i_{34 T}-i_{34 F}\right)$. The second way to generate a force is for the case when the rotor is slightly closer to one of the stator halves and there are equal steering coil currents for both stator halves. For example, if the rotor was initially closer to the right stator half, then the force generating fluxes shown in Figure 1-12 would cause the rotor to move to the left. Adding a sensor for measuring the Z-direction displacement and a control system for the steering coil currents turns this linear mode into a linear actuator.

For the magnetic circuit shown in Figure 1-12, torque control is independent of force control, but force control requires a torque-generating current to act as an operating point. I demonstrate closed-loop control of the torque mode and of the force mode for the hybrid actuator used in the $10 \mathrm{kHz}$ rotary fast tool servo, and use the force mode to demonstrate electronic damping. This thesis provides a discussion on alternate magnetic circuits having fully decoupled rotary and linear modes, and suggests future work that utilizes the force mode as an active suspension for improving the performance of a predominantly rotary system.

Heat generation due to magnetic losses in the stator and rotor cores is significant at 
high operating frequencies (around $5 \mathrm{kHz}$ and above). The rotor is thermally isolated from the rest of the $10 \mathrm{kHz}$ FTS by the flexure bearing, so the temperature rise in the rotor core is more of an issue than it is for the stator cores. The $10 \mathrm{kHz}$ FTS has provisions for forced cooling of the rotor and stator with a fluid — most likely a gas because of the small air gaps. Included in the suggested future work are experiments using forced cooling of the $10 \mathrm{kHz}$ FTS, and experiments using a mockup of the magnetic circuit to more deeply investigate the thermal losses in the rotor and stator cores.

\subsection{Thesis Organization}

The chapters in this thesis are organized as follows:

- Chapter 1 establishes the background for this research and provides a summary of the work accomplished.

- Chapter 2 discusses the motivation for this research and explores the pertinent prior art.

- Chapter 3 describes the development and performance of the $2 \mathrm{kHz}$ rotary fast tool servo. Insights are developed for the design of precision mechanical systems and troubleshooting electrical systems.

- Chapter 4 describes the development and performance of the $10 \mathrm{kHz}$ rotary fast tool servo. Insights are developed for the mechanical modelling of laminated cores, design of adhesive-bonded assemblies, dynamics of the combined magnetic and mechanical systems, and an integrated trade-off study.

- Chapter 5 develops the analytical tools and insights used to design the electromagnetic actuator used in the $10 \mathrm{kHz}$ fast tool servo, explores alternate magnetic circuits for the actuator, and reviews candidate soft magnetic materials. 
- Chapter 6 presents the conclusions for this thesis, highlights the results of the work accomplished, lists the primary contributions of this research, and suggests future work.

- Appendix A contains selected control system design techniques that were used in this research.

- Appendix B documents selected MATLAB ${ }^{\circledR}$ scripts that were developed as tools for this research.

- Appendix $\mathrm{C}$ describes integrating and using the $2 \mathrm{kHz}$ rotary fast tool servo with a diamond turning machine to produce test parts. 


\section{Chapter 2}

\section{Motivation and Context}

In this chapter I discuss the motivation for developing the $2 \mathrm{kHz}$ and $10 \mathrm{kHz}$ rotary fast tool servos, and establish a context for this research by examining the prior art. I consider two major bodies of work for the prior art: fast tool servos and electric engraving heads. The chapter concludes by considering additional pertinent prior art.

\subsection{Introduction}

Other than the $2 \mathrm{kHz}$ and $10 \mathrm{kHz}$ rotary fast tool servos described in this thesis, I found only one other example of a rotary-type fast tool servo [99] as compared with many linear-type fast tool servos.

The $2 \mathrm{kHz}$ and $10 \mathrm{kHz}$ rotary fast tool servos establish new benchmarks among all of the types of fast tool servos that I found in the literature for bandwidth, tool stroke, and maximum closed-loop tool tip acceleration. The details on the demonstrated values for those performance criteria are presented in Section 1.2. It is worth noting that the two linear-type fast tool servos developed by Lu and Trumper [97, 96, 98] stand out among all of the others in the literature review, and the more recent of those two devices sets the current benchmark for highest bandwidth. ${ }^{1}$

The hybrid rotary/linear actuator that we developed for the $10 \mathrm{kHz}$ rotary fast

\footnotetext{
${ }^{1}$ Xiaodong Lu and I were Ph.D. candidates working concurrently in Professor David Trumper's Precision Motion Control Lab [7] at MIT.
} 
tool servo is a normal-stress variable reluctance device that is similar to the actuators used in many of the electric engraving heads that I found in the literature. Based on that literature, it is apparent that the actuator in all of the electric engraving heads is used to drive and sustain a resonance in a mechanical oscillator. This is in sharp contrast to the closed-loop arbitrary trajectories for the tool tip that are achieved with the $10 \mathrm{kHz}$ FTS. Furthermore, the literature for electric engravers does not mention that this class of actuators has an inherent linear degree of freedom in addition to the desired rotary degree of freedom.

\section{$2.2 \quad$ Fast Tool Servos}

The fast tool servos found in the literature are all linear types except for one example of a long-stroke, medium bandwidth rotary-type device described by Ludwick and Trumper [99]. The two different topologies, rotary and linear, are described and contrasted in Section 2.2.2. Because the linear types deal with many of the same issues as the rotary fast tool servos described in this thesis - actuation at high frequencies, small displacement sensing, motion guidance, high stiffness, and real-time motion control - a review of them is pertinent and lends perspective to the operating frequencies and tool travels attained by the work described in this thesis.

\subsubsection{Motivation for Fast Tool Servo Development}

The $2 \mathrm{kHz}$ and $10 \mathrm{kHz}$ rotary fast tool servos described in this thesis were designed to specifically accommodate fabricating precision textured surfaces on spherical target components for high energy density physics (HEDP) experiments on the National Ignition Facility laser at the Lawrence Livermore National Laboratory (LLNL) [92]. In addition to that application, these fast tool servos are suitable for generating freeform non-axisymmetric surfaces on workpieces having diameters in the range of $10 \mathrm{~mm}$ or less. As a spin-off from this research, the hybrid rotary/inear actuator developed for the $10 \mathrm{kHz}$ FTS provides a proof-of-principle for a general-purpose two-degreeof-freedom electromagnetic actuator with a broader array of potential applications, 

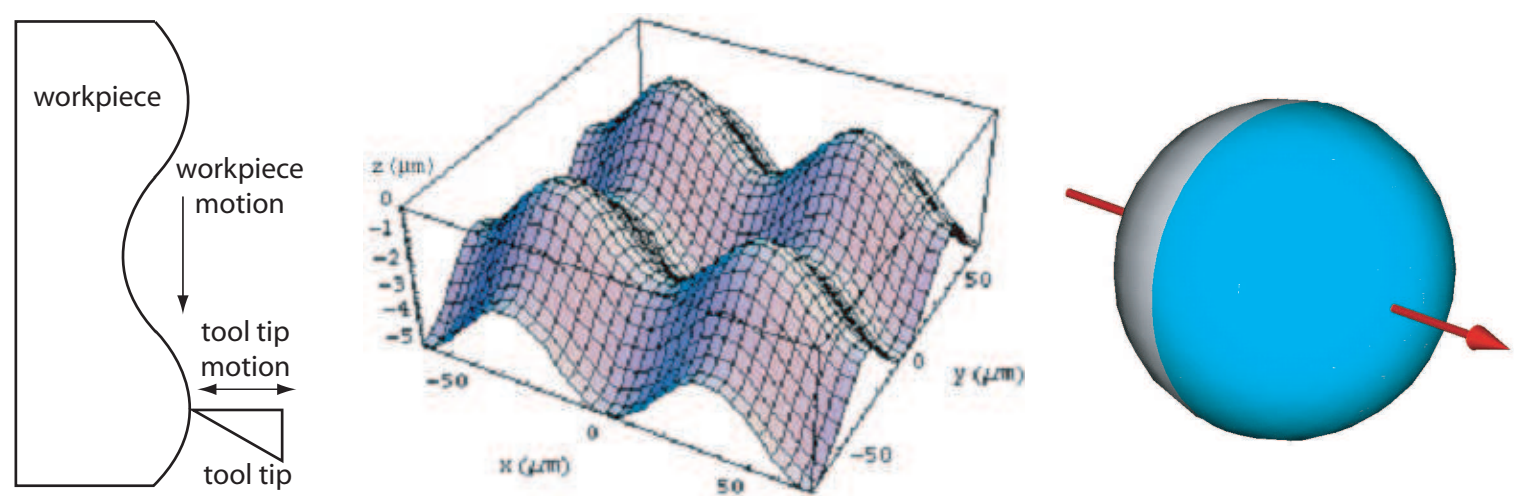

Figure 2-1: Sketch showing a tool tip following a complex workpiece surface topology (left), an example of a desired complex surface that can be single-point machined with a fast tool servo (center), and an example spherical workpiece that might have that complex surface (right).

some of which are mentioned in Section 6.4. Ultra-precision single-point machining (diamond turning) is currently the most accurate and efficient approach for fabricating HEDP target surfaces with relatively long spatial wavelength features and optical quality surface roughness [109]. The rotary fast tool servos described in this thesis will extend this capability to shorter spatial wavelength features, for instance, $50 \mu \mathrm{m}$ long and $5 \mu \mathrm{m} \mathrm{PV}$ at 2 to $10 \mathrm{kHz}$ machining frequencies, while having a low risk for implementation. High bandwidth, defined here as $2 \mathrm{kHz}$ and above, will enable reasonable production rates of the desired target surfaces and better accommodate the minimum allowable surface cutting speed requirements of certain plastic target components. The left-hand sketch in Figure 2-1 illustrates an example of a complex workpiece surface topography that a fast tool servo can follow. The center sketch in Figure 2-1 shows an example desired workpiece surface, which could be on a planar part, wrapped around a cylindrical part, or wrapped around a spherical part like the one shown in the right-hand sketch in that figure.

LLNL is currently in the process of advancing its capability to fabricate the target surfaces needed to provide quantitative corroboration of the physics codes being developed for science-based Stockpile Stewardship. Science-based Stockpile Stewardship allows LLNL to continue fulfilling a primary responsibility to the United States for assessing and certifying the safety and reliability of the Nation's nuclear weapons in 
the absence of nuclear testing, using a combination of computer simulations and nonnuclear weapons tests [2]. Large-scale laser systems such as the 40-beam Omega laser at the University of Rochester and the planned 192-beam National Ignition Facility (NIF) laser at LLNL are essentially tools for delivering sufficient energy accurately in space and time to a fusion-fuel target to initiate, and in the case of the NIF, sustain, fusion of hydrogen atoms into helium along with an attendant release of energy. A significant aspect of stockpile stewardship requires modelling how the degradation of the surfaces of certain components involved in the fusion chain affects the behavior and stability of the fusion process. The $2 \mathrm{kHz}$ and $10 \mathrm{kHz}$ rotary fast tool servos provide LLNL an extended capability for fabricating prescribed complex target surfaces with sufficient accuracy to allow conducting the advanced physics experiments needed to continue refining the physics codes being developed. In addition to stockpile stewardship, advances in those physics codes will move the broader nuclear fusion community closer to an eventual realization of fusion energy as a plausible means for producing consumer energy [93].

Fast tool servos are currently used in the ophthalmic industry to directly machine contact lenses and contact lens molds. Current commercially available fast tool servos are limited to operation at 1 to $2 \mathrm{kHz}$, so a $10 \mathrm{kHz}$ FTS can potentially increase throughput in the ophthalmic industry by a factor of five to ten. ${ }^{2}$ Additionally, the actuator that we developed for the $10 \mathrm{kHz}$ FTS is easily adapted for use in a one or two axis steering mirror for directing light beams, and may also be of interest to those working in the field of electric engraving heads.

\subsubsection{Motivation for a Rotary-Type Fast Tool Servo}

In his Ph.D. thesis, Steve Ludwick [99] provides an excellent overview and comparison of a rotary topology and a linear topology for fast tool servos. He describes the advantages of a rotary topology from the standpoint of maximum achievable tool tip acceleration based on thermal limits of the actuator. In a nutshell, Ludwick [99] de-

\footnotetext{
${ }^{2}$ At the time of this writing, the piezoelectric actuated fast tool servo by Weck (point $\mathrm{H}$ in Figure 2-4) is commercially available in the United States from Precitech, Inc. [124].
} 
scribes that a rotary topology with a tool swing radius that is larger than the radius of the coil in a moving-coil actuator allows magnifying the acceleration produced in the coil. Contrast this with a linear topology, where all elements have the same acceleration. Ludwick further describes how the reaction torque from a rotary-type FTS is generally less of a disturbance to the turning machine than the reaction force from a linear-type FTS. The remainder of this section explores this last topic — disturbance to the turning machine - as it pertains to machining a spherical workpiece with a fast tool servo.

The basic topologies for a rotary fast tool servo and a linear fast tool servo are shown in Figure 2-2. A rotary fast tool servo is preferred in certain diamond-turning applications that are intolerant to the reaction force developed by a linear fast tool servo. For instance, in the case where a goal is to produce a textured surface on a spherical workpiece, a fast tool servo is mounted on a relatively slow rotary axis (B-axis) that allows the tool to engage the workpiece at all points from its pole to its equator, as depicted in Figure 2-3. A rotary-type mechanism oriented with its rotation axis parallel to the $\mathrm{B}$-axis generates a reaction torque on the $\mathrm{B}$-axis. The $\mathrm{B}-$ axis can be allowed to float as a reaction mass or be locked and allowed to transmit the torque to the machine structure. In the first case the B-axis acting as a reaction mass transmits a negligible disturbance to the machine structure. In the latter case, the machine structure experiences a disturbance torque whose value does not depend on the angle of the B-axis. In contrast, a linear fast tool servo will generate a reaction force on the B-axis that passes through it to the machine structure supporting it. This is generally not a problem when the B-axis is positioned so that the reaction force is parallel to the direction of travel of the slide carrying the B-axis. In that case the relatively heavier slide carrying the B-axis can act as a reaction mass to the linear fast tool servo. However, when the B-axis is positioned so that a component of the reaction force is perpendicular to the direction of travel of that slide, that force component is transmitted by the slide to the machine structure as a disturbance. To the extent that the tool/workpiece interaction is affected by disturbances to the machine structure, unless the reaction force is dealt with, a linear fast tool servo 

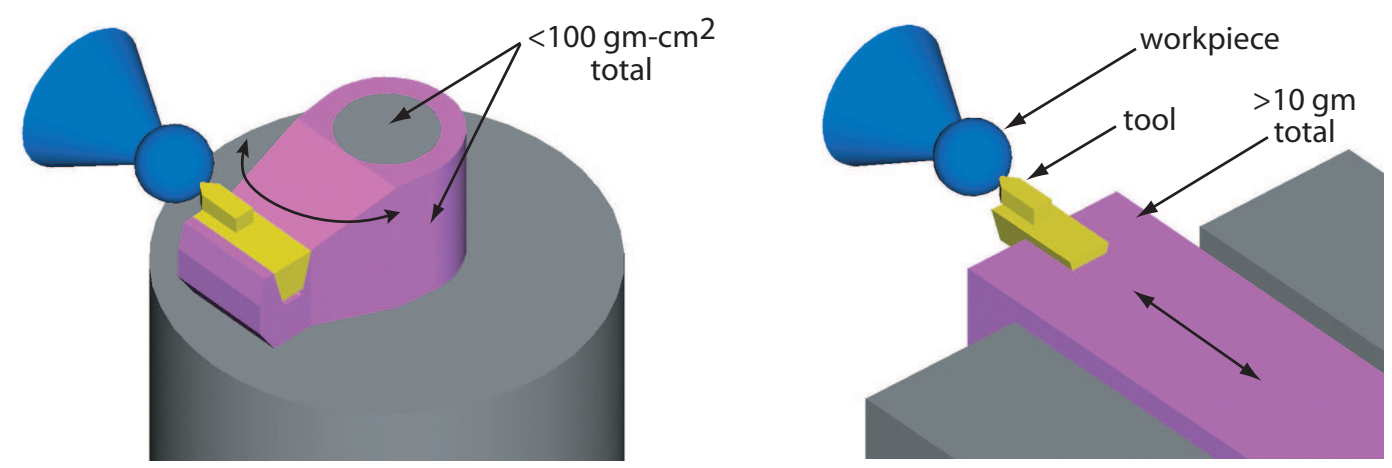

Figure 2-2: Basic topologies for Rotary (left) and Linear (right) fast tool servos.
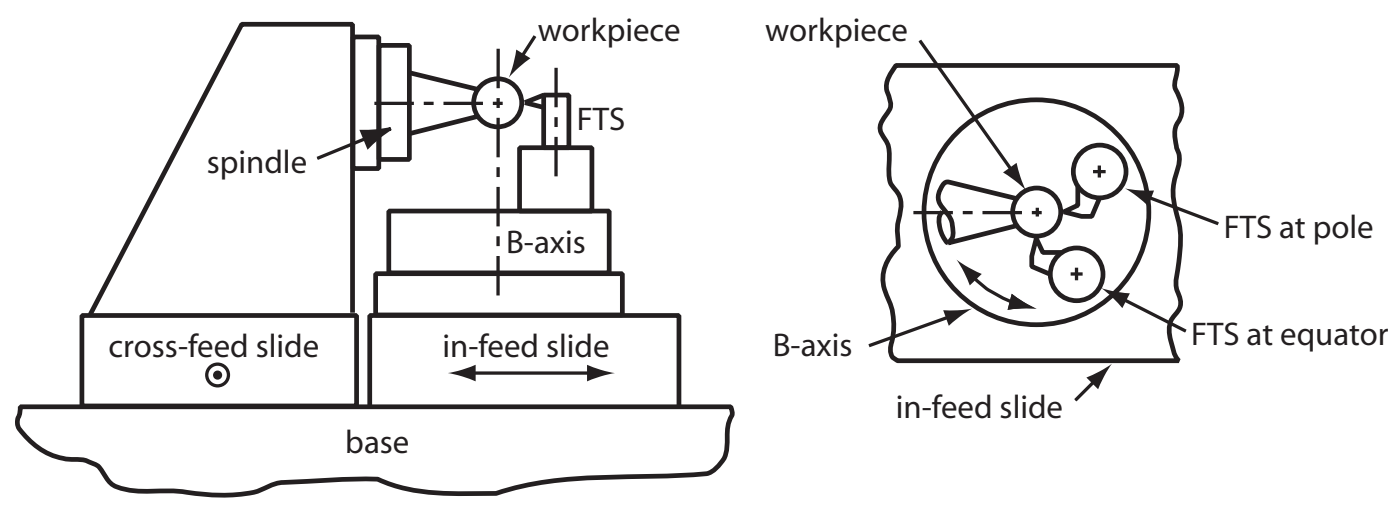

Figure 2-3: Sketch of a fast tool servo and a two-axis lathe with a B-axis for machining a spherical workpiece. Side view (left). Top view showing tool at equator and at pole of workpiece (right).

will thus produce variations in the desired surface texture as a function of latitude between the pole and equator on a spherical workpiece.

The above discussion is quantified by comparing a conservatively high total rotary inertia of $100 \mathrm{gm} \cdot \mathrm{cm}^{2}$ for a rotary FTS with a $5 \mathrm{~mm}$ tool swing radius (twice the inertia of the $2 \mathrm{kHz}$ FTS rotor) and an optimistically low total moving mass of $10 \mathrm{gm}$ for a linear FTS. In the case of a $1000 \mathrm{~g}$ tool tip acceleration, the reaction torque from the rotary FTS and reaction force from the linear FTS are $20 \mathrm{~N} \cdot \mathrm{m}$ and $100 \mathrm{~N}$, respectively. If the FTS is mounted on a B-axis that is allowed to float, a reaction torque causes an angular acceleration of the rotating element of the B-axis creating a tangential displacement between the surface of a spherical workpiece and the tool. Using a conservatively low rotary inertia of $52,000 \mathrm{gm} \cdot \mathrm{cm}^{2}$ for the moving element of the B-axis (e.g. a non-motorized 4 inch diameter air bearing spindle), a $20 \mathrm{~N} \cdot \mathrm{m}$ rotary 
FTS reaction torque produces a negligible $\pm 5 \mathrm{~nm}$ tangential motion between the tool tip and a $5 \mathrm{~mm}$ diameter spherical workpiece. In comparison, without an effective reaction mass between the linear FTS and the B axis, the $100 \mathrm{~N}$ reaction force is transmitted directly through the B-axis as a disturbance to the machine structure, which is surely a large disturbance.

It is worth mentioning that one of the disadvantages that Ludwick [99] discusses regarding tool path generation for a rotary fast tool servo is generally not an issue for the short-stroke rotary fast tool servos described in this thesis. In addition to the in-and-out tool tip travel that a rotary FTS creates there is a parasitic lateral displacement of the tool tip. For large stroke rotary devices this lateral motion needs to be accounted for because it represents a significant displacement of the tool tip across the workpiece. In the case of the short-stroke $2 \mathrm{kHz}$ and $10 \mathrm{kHz}$ rotary fast tool servos the maximum lateral tool tip displacement is $63 \mathrm{~nm}$ and $48 \mathrm{~nm}$, respectively. ${ }^{3}$ This is $2 \%-6 \%$ of the typical $1-3 \mu \mathrm{m}$ spacing of the machining grooves on a diamondturned workpiece, and is negligible in most cases.

\subsubsection{Fast Tool Servos in the Literature}

The use of a fast tool servo with a diamond turning machine is broadly reported in the literature for single-point machining of non-axisymmetric or textured surfaces on a workpiece $[48,49,84,117]$ and for on-the-fly error correction [90, 121]. During the last twenty years researchers have developed many fast tool servos, almost always focused on a specific intended application.

Figure 2-4 provides a comparison of some representative fast tool servos described in the literature, and Table 2.1 is a key to the labels in that figure. In most cases the references report maximum stroke and maximum small signal bandwidth, which are not simultaneously achievable.

\footnotetext{
${ }^{3}$ These values come from simple geometry using the Pythagorean theorem and the following values. $2 \mathrm{kHz}$ FTS: $5 \mathrm{~mm}$ tool swing radius and $25 \mu \mathrm{m}$ half-stroke. $10 \mathrm{kHz}$ FTS: $12.7 \mathrm{~mm}$ tool swing radius and $35 \mu \mathrm{m}$ half-stroke.
} 


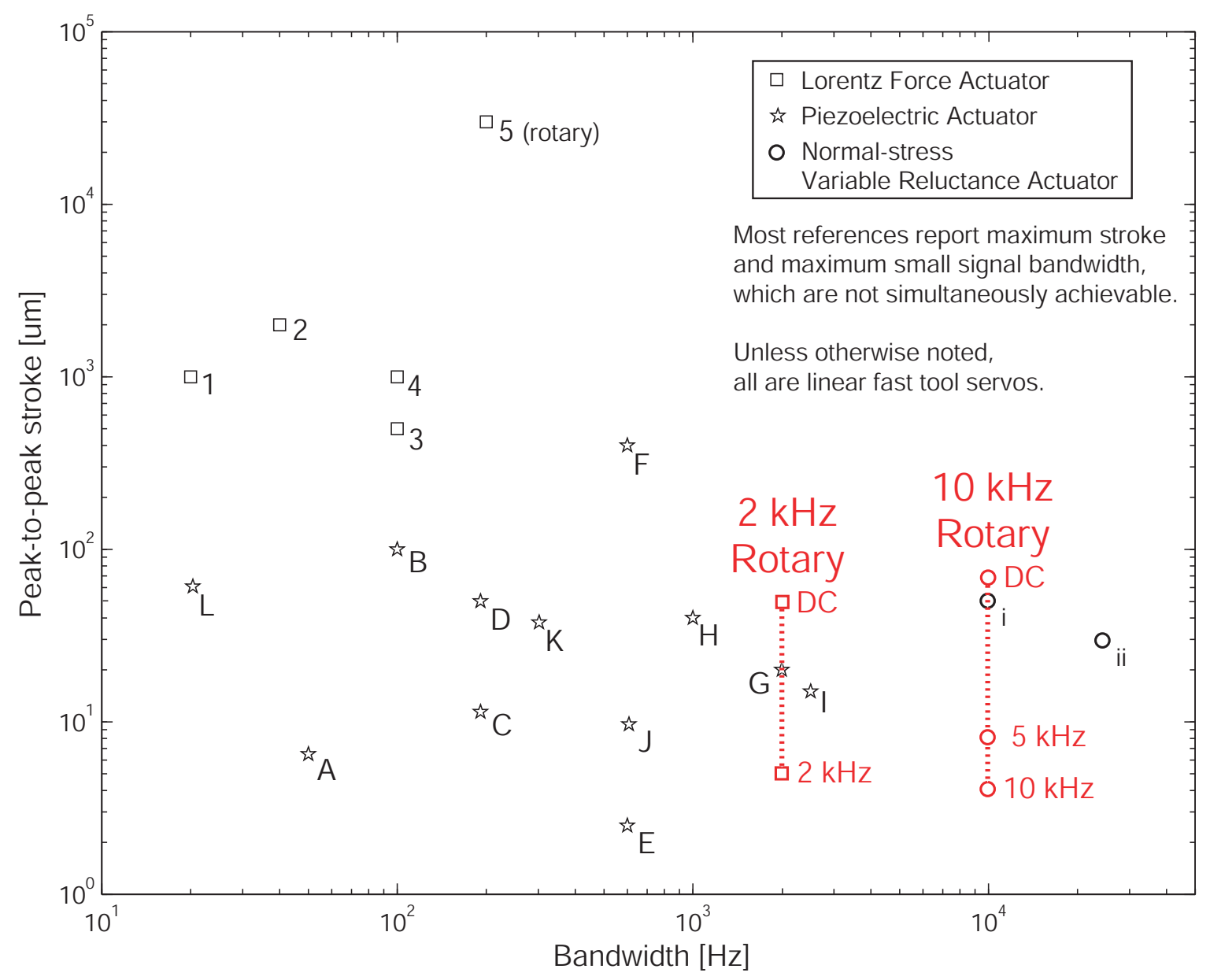

Figure 2-4: Comparison of the $2 \mathrm{kHz}$ and $10 \mathrm{kHz}$ rotary fast tool servos to some representative fast tool servos reported in the literature. Earlier version of this figure courtesy of Lu and Trumper, MIT.

Table 2.1: Key to labels in Figure 2-4.

\begin{tabular}{|l|l|c|l|c|l|}
\hline \multicolumn{2}{|c|}{ Lorentz force actuator } & \multicolumn{4}{|c|}{ Piezoelectric actuator } \\
\hline 1 & Todd \& Cuttino [143] & A & Kuuno [90] & G & Dow \& Miller [49] \\
\hline 2 & Weck [147] & B & Cuttino [43] & H & Weck [147] \\
\hline 3 & Douglass [48] & C & Jared \& Dow [84] & I & Okazaki [117] \\
\hline 4 & Greene \& Shinstock [65] & D & Rasmussen [129, 128] & J & Kaji [87] \\
\hline 5 & Ludwick \& Trumper [99] & E & Patterson \& Magrab [121] & K & Altintas \& Woronko [11] \\
\hline \multicolumn{5}{|c|}{ Normal-stress variable reluctance actuator } \\
\hline & F & Falter \& Youden [55] & L & Zhu et al [153] \\
\hline i & Lu \& Trumper [97, 96] & ii & Lu \& Trumper [98] & \\
\hline
\end{tabular}


For a sinusoidal tool tip trajectory, the peak tool tip acceleration for any simultaneously achieved pair $(\omega, a)$ is equal to $a \omega^{2}$, where $a$ is the tool tip stroke amplitude and $\omega$ is the operating frequency. Therefore, lines of constant peak tool tip acceleration have a slope of -2 on a log-log plot of peak-to-peak stroke versus bandwidth, such as the plot in Figure 2-4. Since so few of the references shown in Figure 2-4 report a simultaneously achieved pair $(\omega, a)$, lines of constant peak tool tip acceleration are not included in the figure because they would be misleading for the data shown.

Referring to Figure 2-4, note that except for the one long stroke medium bandwidth system by Ludwick and Trumper [99], all of the other fast tool servos are linear-type systems. The advantage of a rotary-type fast tool servo over a linear-type for producing a textured surface on a spherical workpiece is discussed in Section 2.2.2. The astute reader, upon checking the bibliography at the end of this thesis, may wonder about the "Rotary Fast Tool Servo" described in [87]. In fact, this reference shows a linear device that moves a cup-type grinding wheel in and out while the whole thing rotates on a spindle.

Still referring to Figure 2-4, all of the fast tool servos besides the two developed by $\mathrm{Lu}$ and Trumper $[97,96]$ use either a Lorentz force actuator or a piezoelectric actuator. Generally speaking, a Lorentz force actuator is well-suited for a longstroke fast tool servo, while a piezoelectric actuator is well-suited for a short-stroke system. Although a piezoelectric actuator is capable of high bandwidths and lever arrangements can be used to extend the effective stroke, it presents unique problems including heat generation due to mechanical and electrical losses in the material, large and expensive high-voltage/high-current power supplies, and additional system dynamics created by inclusion of the actuator in the structural loop of the system. Early in this project we identified a normal-stress variable reluctance actuator as being a preferred choice for a high bandwidth rotary fast tool servo because it is capable of force densities that are an order of magnitude higher than conventional Lorentz force and shear-stress variable reluctance actuators, and can have simpler power supply requirements than a comparably-sized piezoelectric actuator.

Figure 2-5 is a schematic cross-sectional view of the long-stroke rotary fast tool 


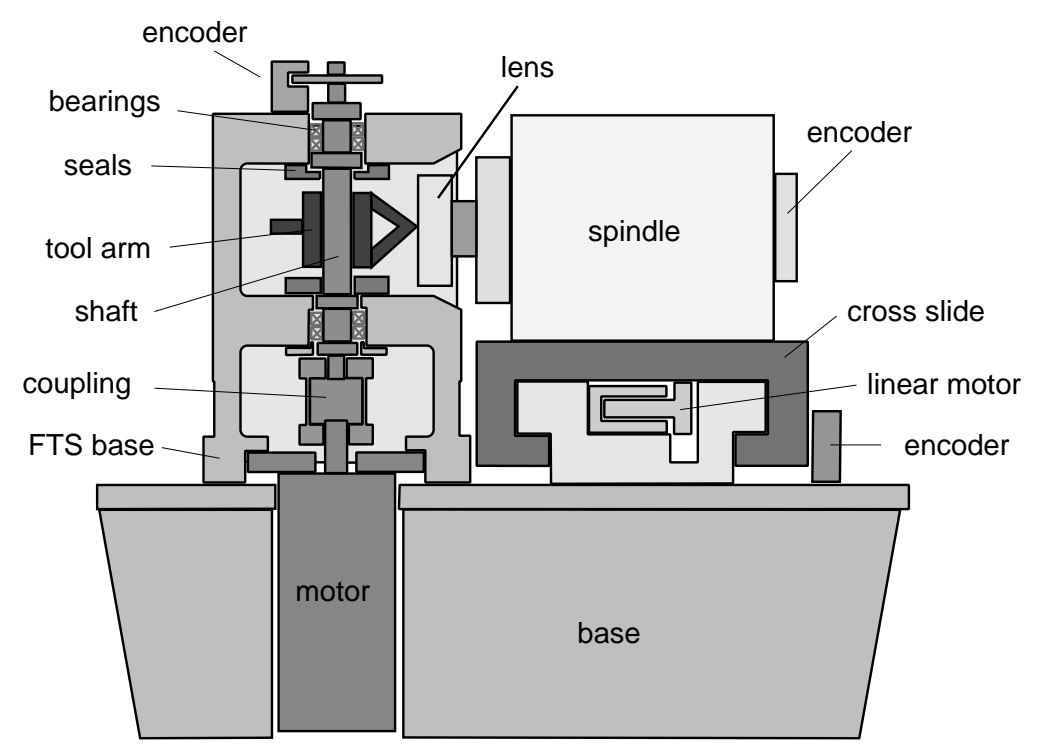

Figure 2-5: Schematic cross-sectional view of the long-stroke rotary fast tool servo and turning machine by Ludwick and Trumper [99]. Figure courtesy of Steve Ludwick.

servo and turning machine by Ludwick and Trumper [99]. The fast tool servo comprises the assembly on the left: motor, FTS base, coupling, shaft, tool arm, seals, bearings, and encoder. It has a $40 \mathrm{~mm}$ tool stoke, a closed-loop bandwidth of $230 \mathrm{~Hz}$, and can produce a $50 \mathrm{~g}$ tool tip acceleration. This FTS and turning machine were specifically designed for single-point machining plastic spectacle lenses having high excursion two cycle per azimuthal revolution surfaces for astigmatism correction.

Figure 2-6 shows the $2 \mathrm{kHz}$ and $10 \mathrm{kHz}$ rotary fast tool servos next to the longstroke rotary FTS by Ludwick and Trumper [99]. Note that the motor for the longstroke FTS is not shown, and an example of one of the spectacle lenses produced with it is sitting on top of the base. Figure 2-6 illustrates two different classes of rotary fast tool servos developed in the Precision Motion Control Laboratory (PMC) [7] at MIT: long-stroke and short-stroke, with three orders of magnitude difference in tool stroke. The earlier long-stroke rotary FTS project provided invaluable corporate knowledge for the PMC lab, and the work described in this thesis benefited immensely from that project. In particular, Ludwick [99] established benchmark work that quantified the advantages of a rotary topology over a linear one for achieving high tool tip accelerations and lower machine base disturbances. 


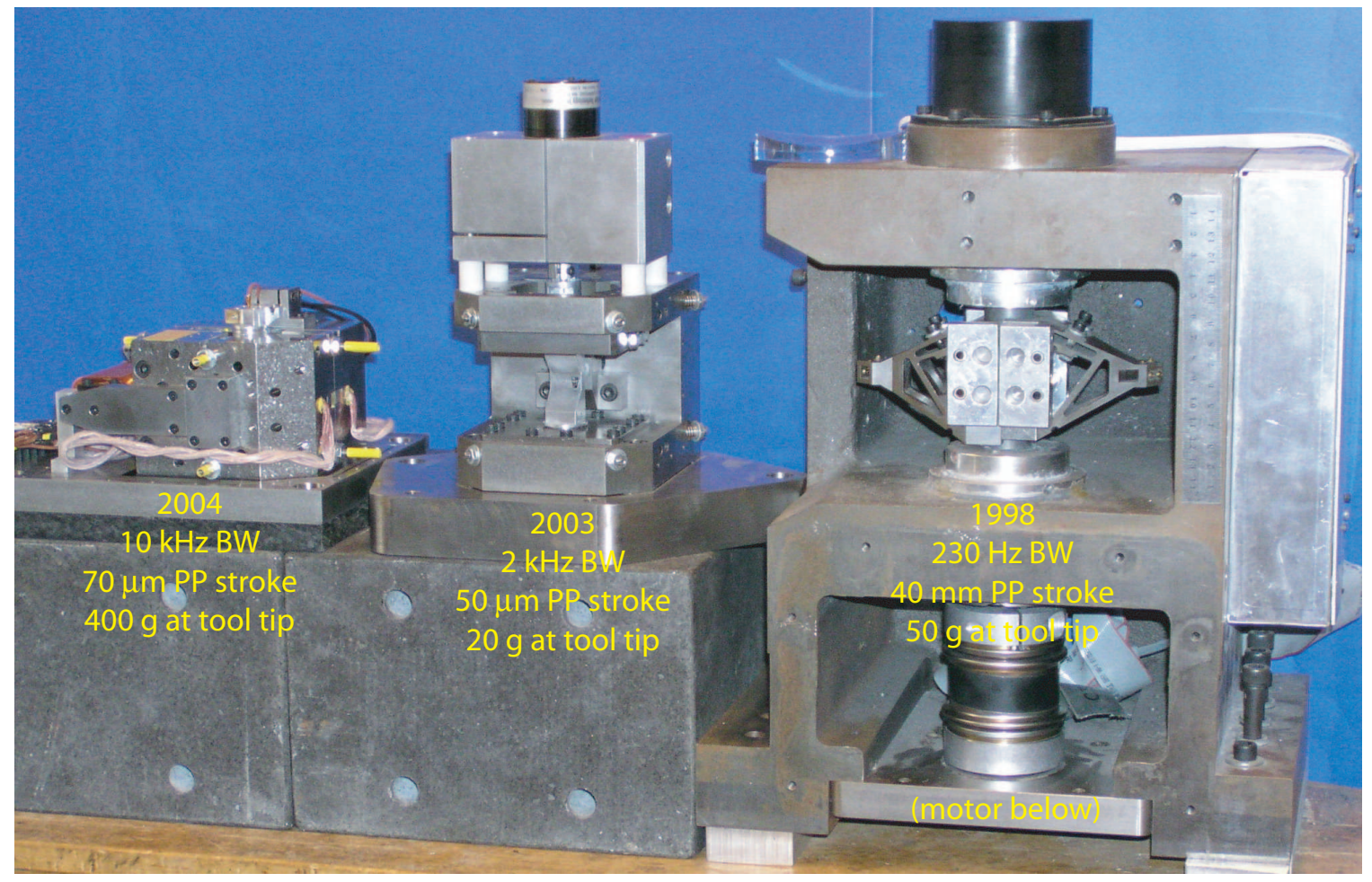

Figure 2-6: The $10 \mathrm{kHz}$ and $2 \mathrm{kHz}$ rotary fast tool servos next to the long-stroke rotary FTS by Ludwick and Trumper [99], left to right, respectively. 


\subsection{Electric Engraving Heads}

Electric engraving heads are used to produce the image carriers used in the intaglio, or gravure printing process. The electric engraving heads are a class of compact, high repetition-rate devices that have much in common with fast tool servos. Whereas rotary fast tool servos are more rare in the literature than the linear types, most electric engravers utilize a rotary architecture. A search of U.S. patents indicates that electric engravers are a fairly mature technology, with prior art from at least as far back as 1937 teaching concepts that are pertinent to the rotary fast tool servos described in this thesis.

A general description of the intaglio printing process and comparisons with other printing processes can be found in the Encyclopædia Britannica [6]. The Gravure Association of America is a good source of information about the gravure industry and the gravure intaglio printing process. The link "What is Gravure?" on their web site provides a thorough introduction to the process:

Gravure is an intaglio printing process. The image carrier has the image cut or etched below the surface of the non-image area. On the gravure image carrier (usually a copper cylinder), all the images are screened, creating thousands of tiny cells. During printing, the image carrier is immersed in fluid ink. As the image carrier rotates, ink fills the tiny cells and covers the surface of the cylinder. The surface of the cylinder is wiped with a doctor blade, leaving the non-image area clean while the ink remains in the recessed cells. Substrate is brought into contact with the image carrier with the help of an impression roll. At the point of contact, ink is drawn out of the cells onto the substrate by capillary action. Gravure is used for publications, catalogs, Sunday newspaper supplements, labels, cartons, packaging, gift-wrap, wall and floor coverings, and a variety of precision coating applications [3].

Most electric engraving heads have an architecture similar to that of a rotary fast tool servo; a cutting tool - typically a diamond — is carried at a fixed radius by 
an arm that is fixed to a rotating element. An actuator acts on the rotating element to cause it to reciprocate, which in turn causes the tool to oscillate along an arc. The reciprocating motion is the result of an intentional mechanical resonance. A bias signal is superimposed on that resonance to cause the tool tip to proportionally engage or disengage with the workpiece. In contrast to the typical fixed-frequency resonance for an engraving head, a distinguishing feature of a rotary fast tool servo is its ability to generate not only a continuous range of tool tip oscillatory frequencies, but most notably, real-time free-form tool tip trajectories. The typical engraving head is capable of significant motion only at a fixed mechanical resonant frequency.

Buechler [27] in U.S. patent 4,357,633 dated 1982 describes an engraving head that uses a normal-stress variable reluctance actuator with flux biasing ${ }^{4}$ having an operating frequency in the range of 3 to $5 \mathrm{kHz}$, a maximum rotary stroke of $4.4 \mathrm{mrad}$ $\left(0.25^{\circ}\right)$ peak-to-peak $(\mathrm{PP})$, and a maximum tool travel of $100 \mu \mathrm{m}$ PP. This particular engraving head is referred to again in later patents by Holowko [76] in 1995, Beckett [20] in 1997, and again by Beckett [21] in 2003. It appears that the 3 to $5 \mathrm{kHz}$ engraving head described by Buechler [27] in 1982 has withstood the test of time. The prior art for this variable reluctance actuator is traced back to a 1937 patent by Losier [95] who teaches flux biasing, and since he makes no specific claims on the actuator in that patent it is presumed prior art as of his 1932 filing date. ${ }^{5}$

Buechler describes an engraving head in U.S. patent 5,491,559 dated 1996 that uses a magnetostrictive actuator intended to operate at "frequencies in excess of $5 \mathrm{KHz}$ or even $10 \mathrm{KHz} . "[30$, column 1 line 55].

Excellent detailed descriptions of complete engraving systems and methods of their use are given by Buechler [28, 30], Beckett [20], and Holowko [76]. Current information on commercially available gravure systems can be found on the web site of the Max Daetwyler Corporation [5], a leading manufacturer of engraving systems in the United States. As of February 2001 Daetwyler had acquired Ohio Electronic

\footnotetext{
${ }^{4}$ Flux biasing: combining a bias magnetic flux and a steering magnetic flux to generate a timevarying force or torque on an armature.

${ }^{5}$ The search path to the Losier U.S. 2,092,765 patent started with "Klischograph", which is a German word for an engraving machine. This led to U.S. patent 5,633,801 by Hada [66], who cited U.S. patent 4,052,739 by Wada [146], who cited Losier.
} 
Engravers, which was the assignee of many of the engraving head patents cited in this thesis. ${ }^{6}$ Another source for current information on commercial engraving systems is the web site of HELL Gravure Systems GmbH, of Germany [4]. Although these web sites lack the level of detail found in the patents, they do provide insight on the level of performance that is currently being marketed.

While searching for literature on electric engraving heads I did not find any published papers describing the devices. All of the detailed information on engraving heads described in this thesis was gleaned from patents; suggesting a highly competitive industry. It is worth noting that the patents represent a snap shot in time and that the technology most likely continued to evolve after the patent application was filed. Although the patents reveal most of the details of the devices, in some cases certain details necessary for a device to operate are omitted. I discovered that many of those details were considered knowledge already in the public domain. Learning this involved a fun piece of research, which went as follows. I called the corporate office of one of the companies that was the assignee for many of the engraving head patents that I was reviewing. I read a list of inventor names to the operator and she said that none of them were on her roster. She did give me the name and number of someone "technical" that might be able to help, but that route did not produce results quick enough so I considered it a dead end and moved on to a different approach. I choose an inventor name, Tony Beckett, from one of the more recent patents and did an on-line phone number search in the state where the assignee is located. The assumption here was that Tony still worked at that company and probably lived near by. I ended up with multiple phone numbers for his name, so I cross-referenced the home addresses with the assignee's location and sorted them according to a "reasonable-commute-towork" criteria. The first number that I called was answered by the inventor's wife. I told her that I was a college student doing research work, and she said that her husband would be delighted to talk to me and gave me a phone number that would reach him. I called Tony, and described my questions regarding what seemed to be

\footnotetext{
${ }^{6}$ On February 15, 2001 the web site of Ohio Electronic Engravers (http://www.ohioEE.com) was redirecting visitors to the Daetwyler web site.
} 
missing elements of the magnetic circuits in some of the patents that I was studying. He told me that one of his co-inventors, John Fraser, could tell me more about the magnetic circuits than he could, and gave me his phone number. I called John, and he filled in the missing details that he considered knowledge in the public domain at the time. John also taught me the word "Klischograph", which allowed me to broaden my literature search on electric engraving heads.

\subsubsection{Method of Operation}

When used to engrave an image carrier, engraving heads are essentially open-loop devices in the sense that the position of the tool tip is not strictly controlled during its entire travel between the end points on its in and out path. The position of the end points on that path relative to the workpiece, and of an intermediate position used to produce "highlight" cells, is the primary variable controlling the volume of the ink well that is produced. Methods of real-time sensing of the cutting tool appear to be concerned with establishing and maintaining these end and intermediate points $[62,41]$.

The 3 to $5 \mathrm{kHz}$ device described by Holowko [76] and the 5 to $10 \mathrm{kHz}$ device described by Buechler [30] provide examples of the typical method of operation of an engraving head $[76,30]$. They both operate by constantly oscillating the tool at some resonant frequency and superimposing on that motion a series of step inputs ("video signal") that cause the tool to engage either fully or partially with the workpiece, or not touch it at all. In the case of the 5 to $10 \mathrm{kHz}$ engraving head described by Buechler [30] the tool oscillates at the third resonant frequency of the actuator (in the range of 10 to $15 \mathrm{kHz}$ ) and a 3 to $5 \mathrm{kHz}$ video signal is superimposed on that motion. Figure 2-7 is from Holowko [77] and shows how the workpiece 10 is typically machined by a tool 20 by superimposing a series of step inputs ( 82 , "video signal") onto the signal that is sustaining the mechanical resonance ( 80 , "AC signal") ${ }^{7} \mathrm{~A}$ more thorough discussion on the control signals shown in Figure 2-7 is presented in Section 2.3.5; as discussed there, the waveforms shown in Figure 2-7 are not quite

\footnotetext{
${ }^{7} \mathrm{~A}$ similar figure is found in the Buechler $[30,31,32]$ patents.
} 


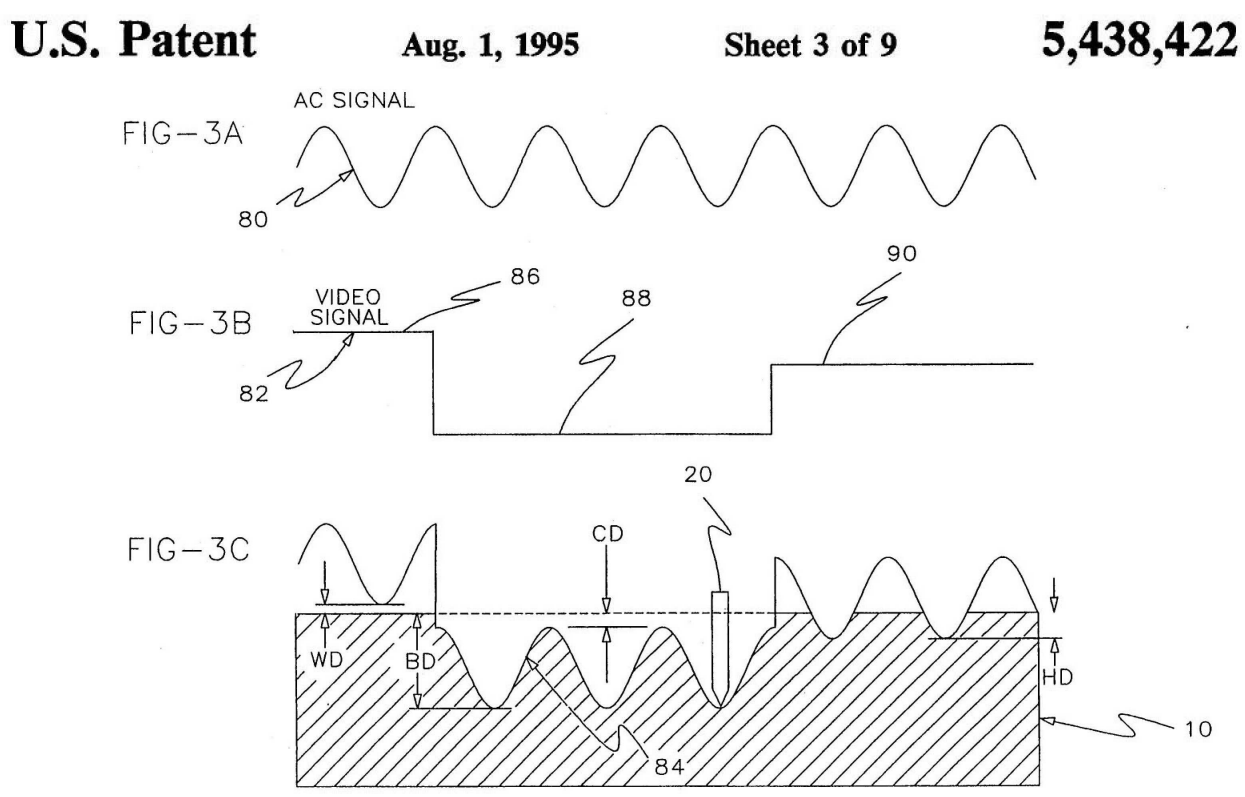

Figure 2-7: Control signals used to operate an electric engraver. From Holowko [77], U.S. Patent 5,438,422.

physically correct.

Although the DC level and $\mathrm{AC}$ amplitude of the tool tip motion is typically closed-loop controlled, because engraving heads typically lack the ability to generate free-form tool path trajectories, they are considered open-loop devices in this thesis. Notwithstanding their open-loop nature, the seventy-plus years of evolution associated with engravers provides a wealth of information about what does and does not work regarding actuators, bearing systems, and damping for devices that produce small rotary motions at operating frequencies in the range of 3 to $10 \mathrm{kHz}$. We now proceed to detailed discussions on the aspects of electric engraving heads that provide pertinent knowledge for the design of our rotary fast tool servos.

\subsubsection{Actuators}

For the literature that I reviewed, the most often cited actuator is a limited-rotation normal-stress variable reluctance device with magnetic flux biasing, similar in nature to the actuator used in the $10 \mathrm{kHz}$ rotary fast tool servo. It is worth noting that this type of actuator is cited in patents from 1937 through 2003, apparently testifying 
to its ongoing success and utility. Other types of actuators found in the literature include Lorentz force, magnetostrictive, and pneumatic. A fairly extensive search of U.S. patents on engraving heads revealed only one mention of using a piezoelectric actuator, but only briefly as if a side issue [136, column 4, line 39]. Of course, this does not rule out the existence of a piezoelectric actuated engraving head, but it does attest to an apparent lack of popularity for the concept.

\section{Normal-Stress Variable Reluctance Rotary Actuators Employing Flux Biasing}

Losier [95] describes a normal-stress variable reluctance actuator with flux biasing in U.S. patent 2,092,765 dated 1937. Figure 2-8 is from this patent. A pair of C-shaped pole pieces 110 and 111 form the stator. An armature 127 forming air gaps (not labelled) with the pole pieces pivots on a rotation axis (not labelled) established by the flexible loops 128 at both ends of the armature. The left-hand flexible loop is hidden behind the screw 115 in "Fig 6". The rotation axis is normal to the view in "Fig 5", at the intersection of the horizontal and vertical mid-planes of the pole pair, and running left to right through the center of the armature in "Fig 6". An ovalshaped coil 125 encircles the armature and fits within the pole pieces. A DC-current electromagnet 109 establishes a constant bias magnetic flux in the pole pieces via the bias flux yoke 108. The DC flux travels vertically through the pole pieces, the armature, and the air gaps between them. An AC current in the coil 125 produces a steering flux that adds with the bias flux in one set of diagonal air gaps, and subtracts from the bias flux in the opposite set of diagonal air gaps, developing a torque on the armature. Rotation of the armature causes vertical motion of the toolholder 130. The tool holder is restrained by the diaphragm flexure 136 to allow only the desired vertical motion. As mentioned earlier, Losier makes no specific claims on the actuator in this patent, so it is presumed to be prior art as of his 1932 filing date.

The magnetic circuit for the actuator taught by Losier in 1937 illustrates the same principles used in many of the engravers that followed it, and is similar to the "sandwiched rotor" design for the hybrid rotary/linear actuator used in the $10 \mathrm{kHz}$ FTS. 
However, Losier [95] and the patents that followed do not mention that there is a linear mode inherent in this class of actuators. Furthermore, they do not teach the use or importance of a high reluctance path for separating the steering fluxes from portions of the bias flux path when a coil is used to provide the bias flux. This last point is important for decoupling the two stator cores, which I found necessary from a practical standpoint for controlling the linear mode of our actuator. A detailed discussion on the high reluctance path for decoupling the steering fluxes is provided in Section 5.3.1.

Baar [15] describes using a pair of C-shaped electromagnets in an actuator for an engraving head in U.S. patent 3,964,382 dated 1976. Figure 2-9 is from this patent. A pair of C-shaped laminated iron cores 1, each with a multi-turn wound coil 5, form the stator. A rotor 7 forming air gaps 3 with the stator is fixed to a shaft 6 which carries a lever 14 holding a tool 15. Not disclosed in this patent is the bias magnetic flux circuit needed to generate torque in the device, which was considered knowledge in the public domain at the time [61]. The magnetic circuit for this actuator is essentially the same as the one taught by Losier [95] in 1937, with the notable difference being the use of two coils, one around each of the stator cores, instead of a single coil around the rotor. Baar does not make any claim in this patent on the actuator.

The poles of the rotor in Figure 2-9 are tapered. This reduces the rotational inertia compared to a design with straight rotor poles, providing a higher torqueto-inertia ratio for a given flux density on the pole faces. The taper also increases the frequencies of the non-rigid body vibration modes of the rotor. Note that the cross-sectional area for the flux path through the rotor via the rotor pole faces is not constant. For the rotor shown in Figure 2-9, the maximum flux density occurs at the base of the triangular rotor poles. The rotors for the patents described next, and shown in Figures 2-10 and 2-11, also have tapered poles; suggesting a de facto consensus on the benefits of adding a taper to the rotor. Note that in the case of the designs shown in Figures 2-9 and 2-11, the rotor/stator air gaps can be adjusted by translating the stator cores towards-and-away from the rotor. For the design shown in Figure 2-10, the tapered poles allow adjusting the air gaps in a similar manner. 
Sept. 14, 1937.

G. E. LOSIER

$2,092,765$
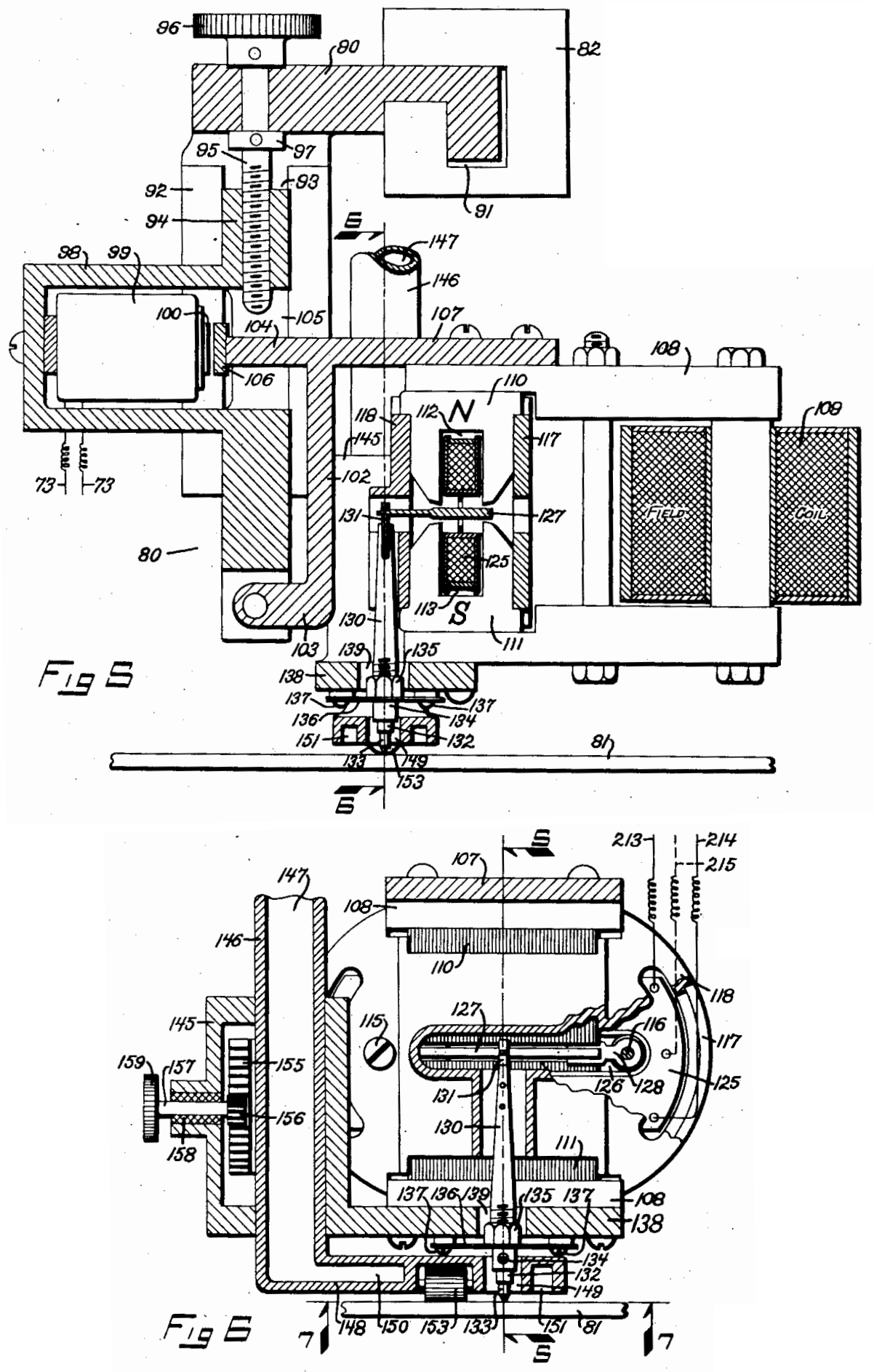

Figure 2-8: A normal-stress variable reluctance actuator with flux biasing used in an electric engraver. From Losier [95], U.S. Patent 2,092,765. 


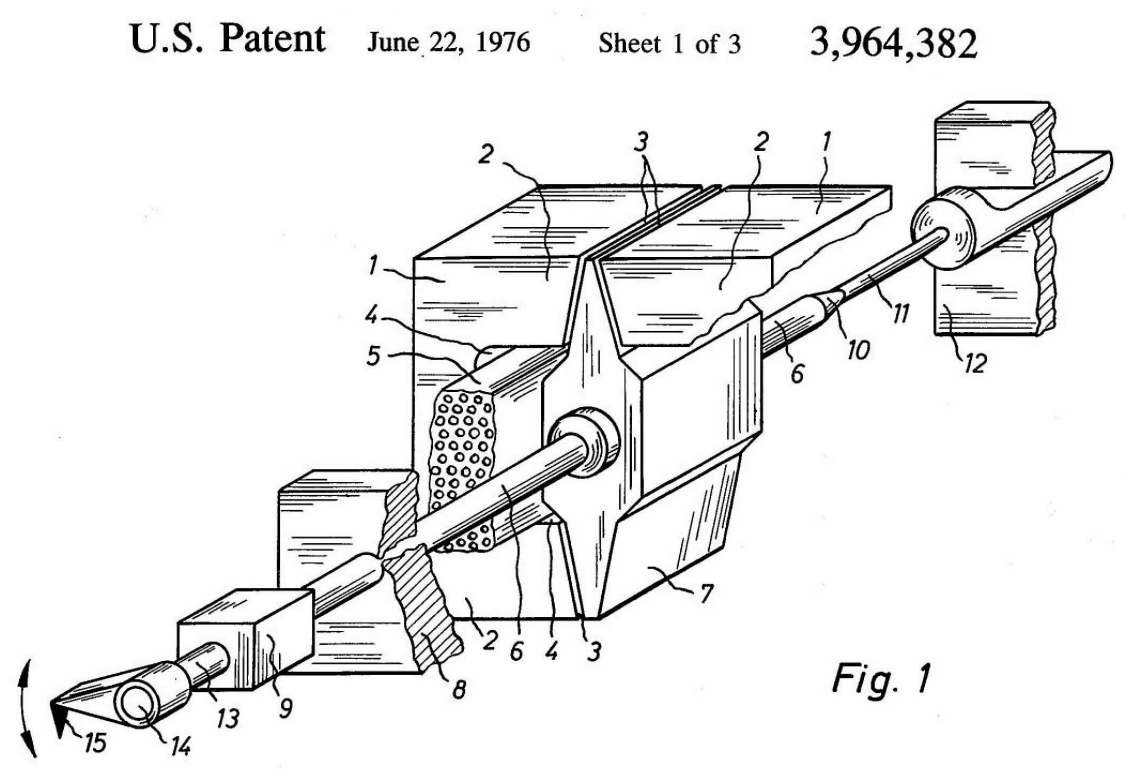

Figure 2-9: A normal-stress variable reluctance actuator used in an electric engraver. From Baar [15], U.S. Patent 3,964,382.

The significance of this is discussed in Section 6.4.9.

Buechler [27] mentions using a pair of C-shaped electromagnets in an actuator in U.S. patent 4,357,633 dated 1982. Figure 2-10 is from this patent. A pair of Cshaped laminated iron cores each having a multi-turn wound coil comprise the two electromagnets 61 that form the stator. A rotor 58 forming air gaps 63 with the stator is fixed to a shaft 30 which carries a lever (not shown) holding a tool (not shown). Not disclosed in this patent is the bias magnetic flux circuit needed to generate torque in the device, which was considered knowledge in the public domain at the time [61]. The element indicated as 26 is described as a "metal housing", and may be involved in the undisclosed DC magnetic circuit. The magnetic circuit for this actuator is similar to the "pinched rotor" design described in this thesis. Buechler does not make any claim in this patent on the actuator.

Hada [66] describes using a pair of C-shaped electromagnets in an actuator for an engraving head in U.S. patent 5,663,801 dated 1997. Figure 2-11 is from this patent. A pair of C-shaped laminated magnetic material cores form a stator 35. A rotor 34 centered in the stator forms four air gaps (not labelled). A permanent magnet 36 


\section{U.S. Patent Nov. 2, $1982 \quad$ Sheet 1 of $3 \quad 4,357,633$}

FIG-3

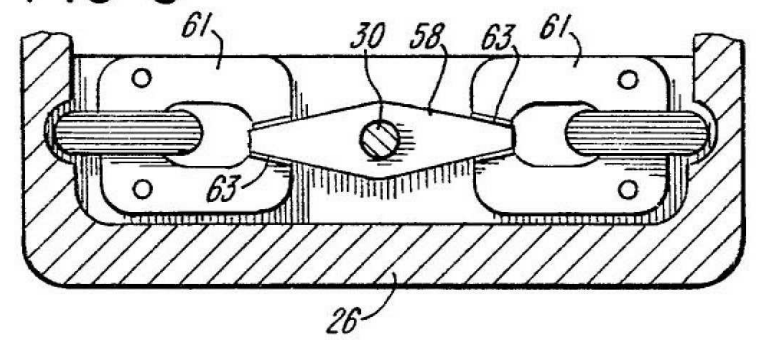

Figure 2-10: A normal-stress variable reluctance actuator used in an electric engraver. From Buechler [27], U.S. Patent 4,357,633.

establishes a constant bias flux through the stator and rotor. An AC current though the coil 37, which is wrapped around the rotor and in the interior region of the stator, causes flux addition in diagonal air gaps and flux subtraction in the opposite diagonal air gaps, developing a torque on the armature. Rotation of the armature causes rotation of the affixed shaft 31 and stylus 30, the later carrying a tool 32 that moves in and out along an arc "B". This is essentially the same actuator taught by Losier [95] in 1937, with the notable difference being the use of a permanent magnet for providing the bias flux. Hada does not make any claim in this patent on the actuator.

\section{Lorentz Force}

Raible [126] describes using a Lorentz force device, specifically a voice coil, as an actuator for an engraving head in U.S. patent 2,925,464 dated 1960. Figure 2-12 is from this patent. A permanent magnet 69 creates a DC flux that passes through the lower yoke 67, the central hollow core 70, and the upper yoke 68 after passing through the radial air gap (not labelled) between 70 and 68. A coil 64 located in the air gap shares a centerline with the non-magnetic rod 71 and is mounted to the non-magnetic plate 72. The plate 72 and the lower end of the rod 71 are mounted to parallel leaf springs 73 that guide the up and down motion of the rod 71 caused by an AC current through the coil. Motion of the rod 71 is transferred via the hook 74 to a lever 75 


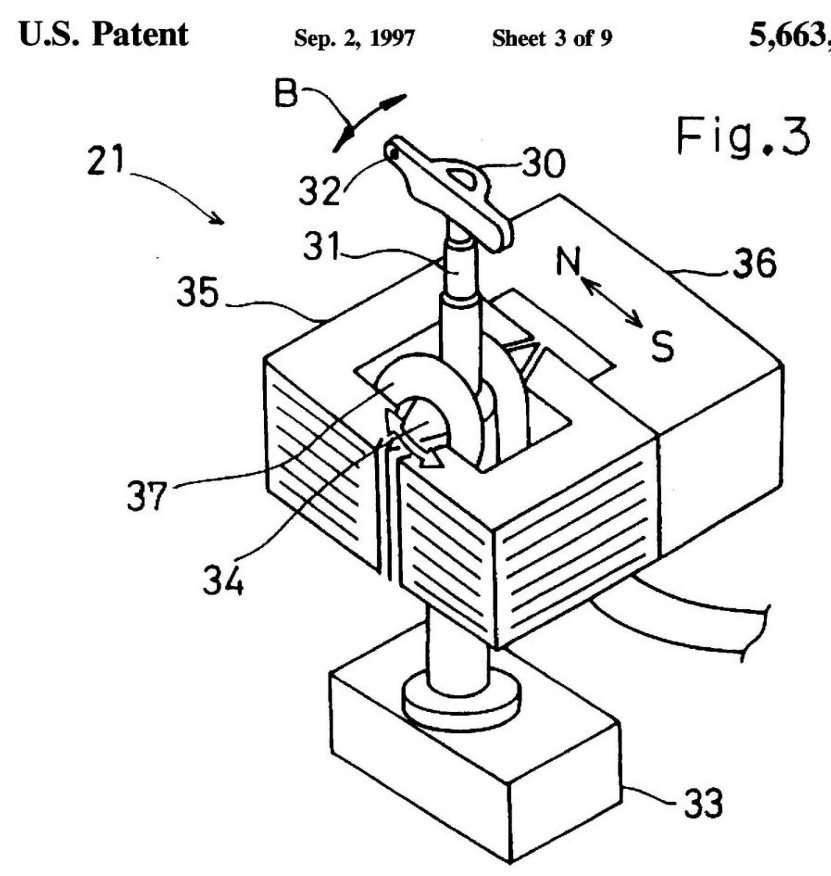

Figure 2-11: A normal-stress variable reluctance actuator used in an electric engraver. From Hada [66], U.S. Patent 5,663,801.

having a leaf spring hinge 76 . The lever acts on a rod 82 that is guided by parallel leaf springs 79 and 80 to cause up and down motion of the tool 81. Raible does not make any specific claim in this patent on the Lorentz force voice coil actuator. In contrast to the reaction torque produced by angular-acceleration only of the moving masses in the rotary actuators described in the previous subsection, the linear-acceleration only of the moving mass in the actuator shown in Figure 2-12 produces a reaction force.

\section{Magnetostrictive}

Buechler [30, 31, 32] describes a magnetostrictive actuator in U.S. patents 5,491,559 dated 1996, 5,671,064 dated 1997, and 5,731,881 dated 1998. The last two patents are continuations on the first one. Figure 2-13 is from the first of these patents. A length change of the magnetostrictive actuator 52 causes an axial displacement of the piston rod 66 which acts on the mechanical amplifier arm 80. The amplifier arm is a simply supported beam that is fixed at one end 80a through flexural elements 88 and 90, and 


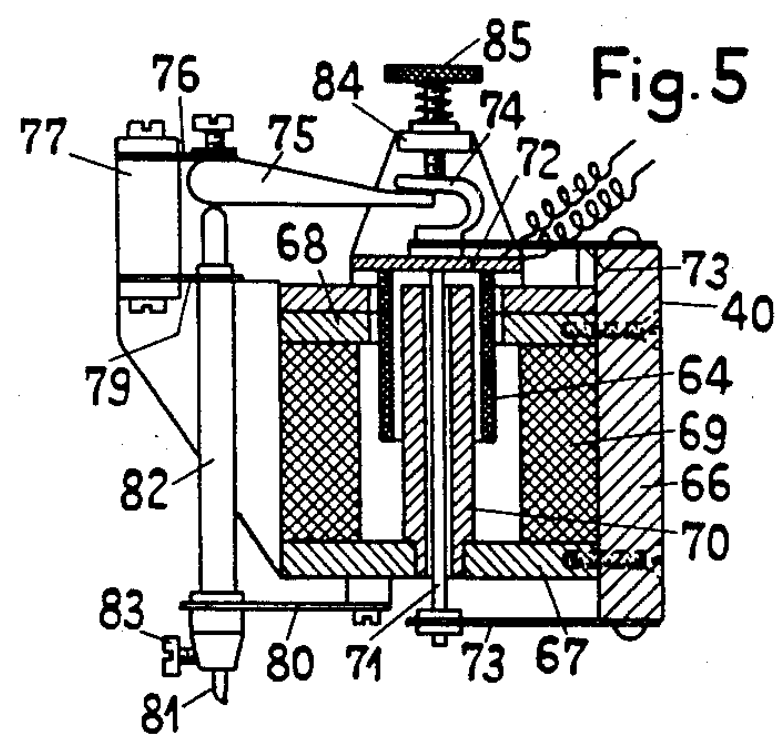

Figure 2-12: A voice coil actuator used in an electric engraver. From Raible [126], U.S. Patent 2,925,464.

carries a stylus arm 92 that holds a tool 95. The flexural elements allow the amplifier arm to pivot at the end 80a, causing motion of the tool along an arc. The actuator produces a linear motion of the piston rod 66 , which is converted into a rotary motion of the amplifier arm and stylus arm. The actuator is axially preloaded by introducing pressurized fluid via a passage 76 into the cylinder 64 to bear down on a piston 68 . Improvements described in the later patents include a simpler mechanical means for preloading the actuator. The system operates at the third harmonic resonance of the actuator, which is in the range of 10 to $15 \mathrm{kHz}$. The control signal to the actuator is a composite of the "AC signal" and "DC video signal", which are described in detail in Section 2.3.5. Acceleration of the linear-moving elements of the actuator produces a force on the amplifier arm that causes it to rotate, and produces a reaction force at the pivot of the amplifier arm. Since the linear-moving elements of the actuator have a finite mass, the force produced by the stationary portion of the actuator is greater than the reaction force on the pivot. Considering the stationary portion of the actuator and the pivot to be integral with the housing, the forces acting on the 


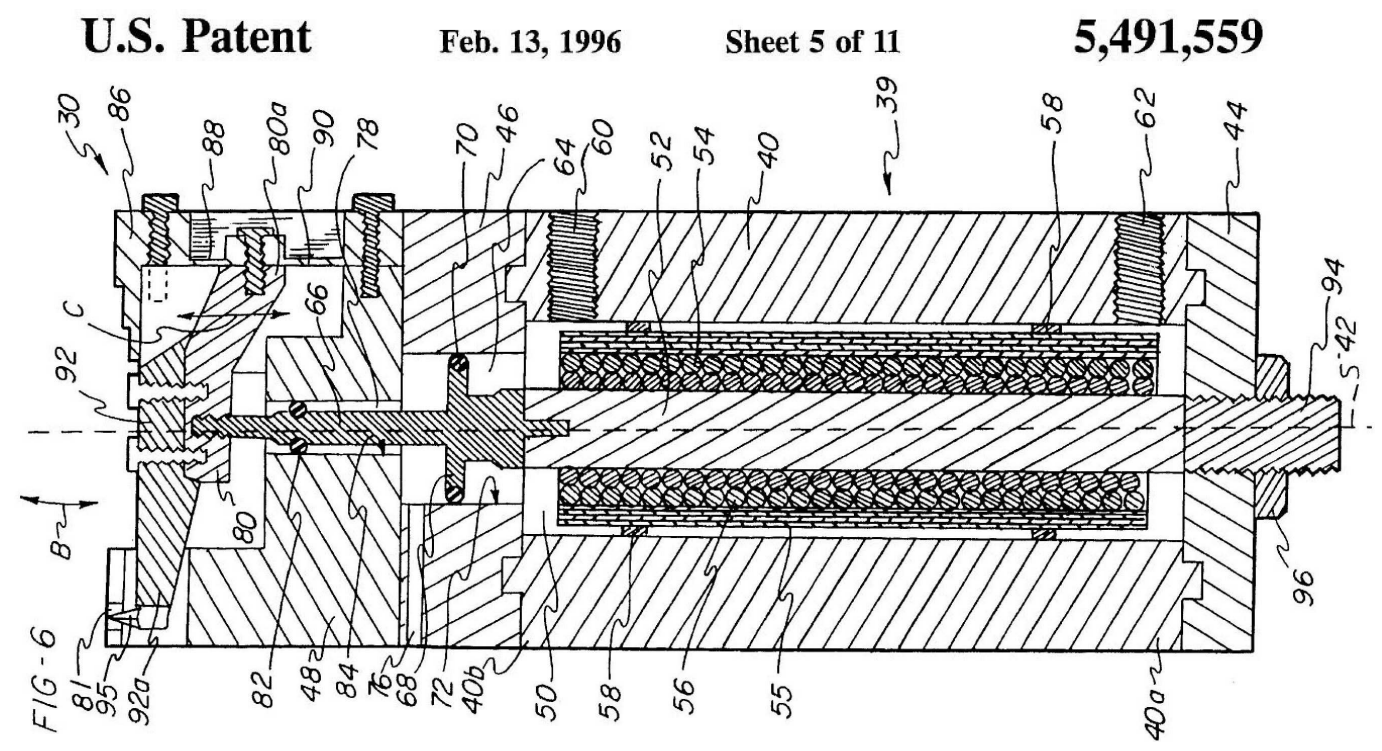

Figure 2-13: A magnetostrictive actuator used in an electric engraver. From Buechler [30], U.S. Patent 5,491,559.

housing from the actuator and amplifier arm create an unbalanced couple that can be resolved into a force and a torque. Therefore, this actuator produces a reaction force and a reaction torque. Another way to think of this is as follows. The reaction force is equal to the product of the linear acceleration of the linear-moving portion of the actuator and its mass, and the reaction torque is equal to the product of the angular acceleration of the rotating portion — the amplifier arm, stylus arm, and tool — and its rotational inertia.

Attendant with length changes of the magnetostrictive material are mechanical strains that generate a substantial amount of thermal energy. Additional significant sources of heating are magnetic hysteresis losses in the actuator 52, Ohmic losses in the coils 54 and 56, and eddy currents in the steel laminate back iron 55. The last of the three Buechler patents covering the magnetostrictive actuator describes a cryogenic cooling mechanism using liquid nitrogen to manage this thermal challenge.

Sermund [136] describes the use of a magnetostrictive actuator in U.S. patent 6,421,576 B1 dated 2002. Figure 2-14 is from this patent. A magnetostrictive actuator 4 is attached to a stationary rear plate 7 via a first force sensor 6 . The opposite end of the actuator carries a movable front plate 8 that holds the tool 2. A second 


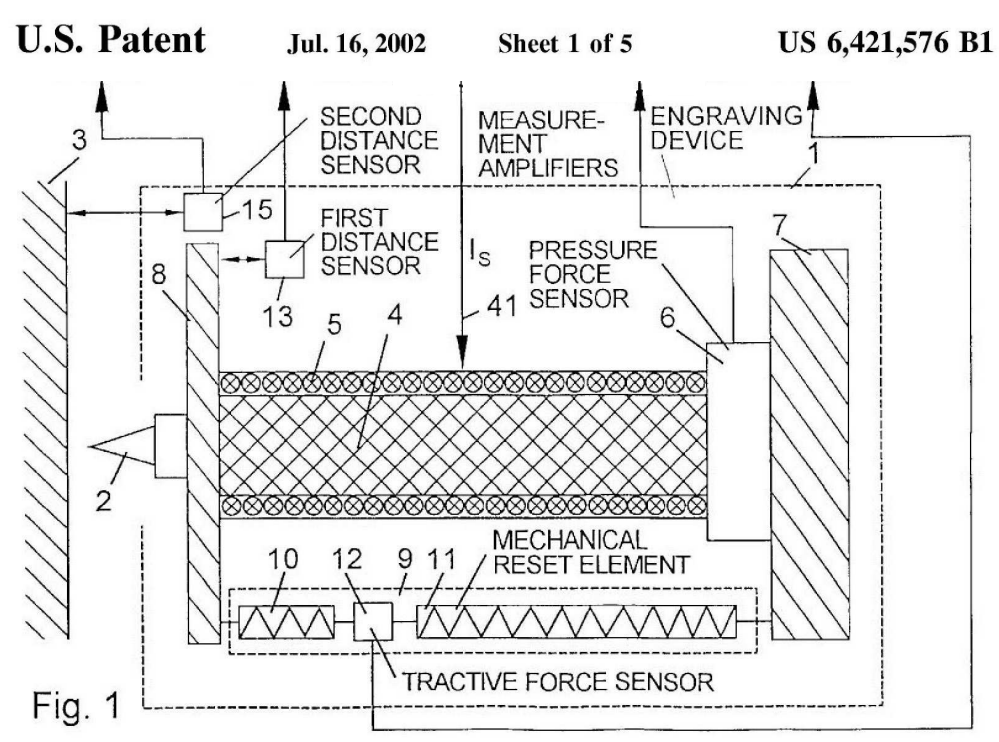

Figure 2-14: A magnetostrictive actuator used in an electric engraver. From Sermund [136], U.S. Patent 6,421,576 B1.

force sensor 12 joins the front plate 8 and rear plate 7 via tension springs 10 and 11 . Length changes in the actuator cause a linear in-and-out motion of the tool. Sermund mentions that the actuator could also be made of a piezoelectric or electrostrictive material. The linear-acceleration only of the moving mass in this actuator produces a reaction force.

\section{Pneumatic}

Ito [83] describes a pneumatic actuator in U.S. patent 6,099,177 dated 2000. Figure 215 is from this patent. A spring 8 pushes against a piston $7 \mathrm{~b}$ and causes the tool tip $7 \mathrm{c}$ to move away from the workpiece "T". This motion of the piston pushes against and compresses an O-ring 6 to block inlet passages 5b. Pressurized air enters the upper chamber $3 \mathrm{~b}$ and increases the pressure above the piston, causing motion of the piston and O-ring in a direction that reduces the compressive deflection in the O-ring and moves the tool towards the workpiece. This motion continues until the O-ring no longer blocks the inlet passages, then air escapes between the O-ring and the piston into the bottom chamber $4 \mathrm{~b}$ and out of the exhaust passages $4 \mathrm{~d}$. The escaping air causes a momentary reduction in air pressure in the upper chamber (because it does 


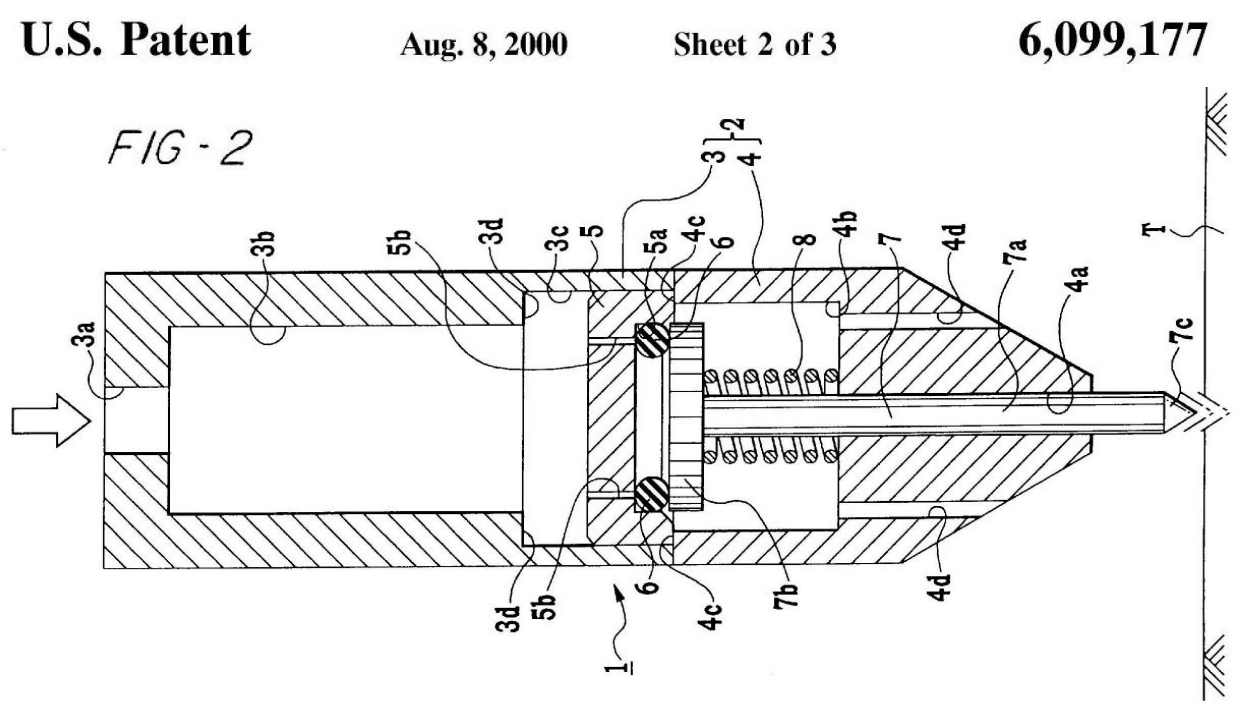

Figure 2-15: A pneumatic actuator used in an engraving head. From Ito [83], U.S. Patent 6,099,177.

not instantaneously refill with air), allowing the spring to push the piston and Oring in a direction that moves the tool away from the workpiece and block the inlet passages. Thus we complete one cycle of the vibration motion of the tool 7 . Note that the system appears to lack a definitive hard-stop to control the displacement of the tool towards the workpiece. The linear-acceleration only of the moving mass in this actuator produces a reaction force.

\subsubsection{Bearing Systems}

Most of the bearing systems found in the literature use one or more torsion rods to provide axial and radial constraints on the rotating element and act as the torsional spring for the system. Radially extending flexures are reported for increasing the lateral stiffness of the rotating element and for acting as a torsional spring, but are not described as a method for providing an axial constraint.

\section{Torsion Rods}

Baar [15] describes using a torsion rod in U.S. patent 3,964,382 dated 1976, which was described earlier and shown in Figure 2-9. The single torsion rod 11 provides an axial 


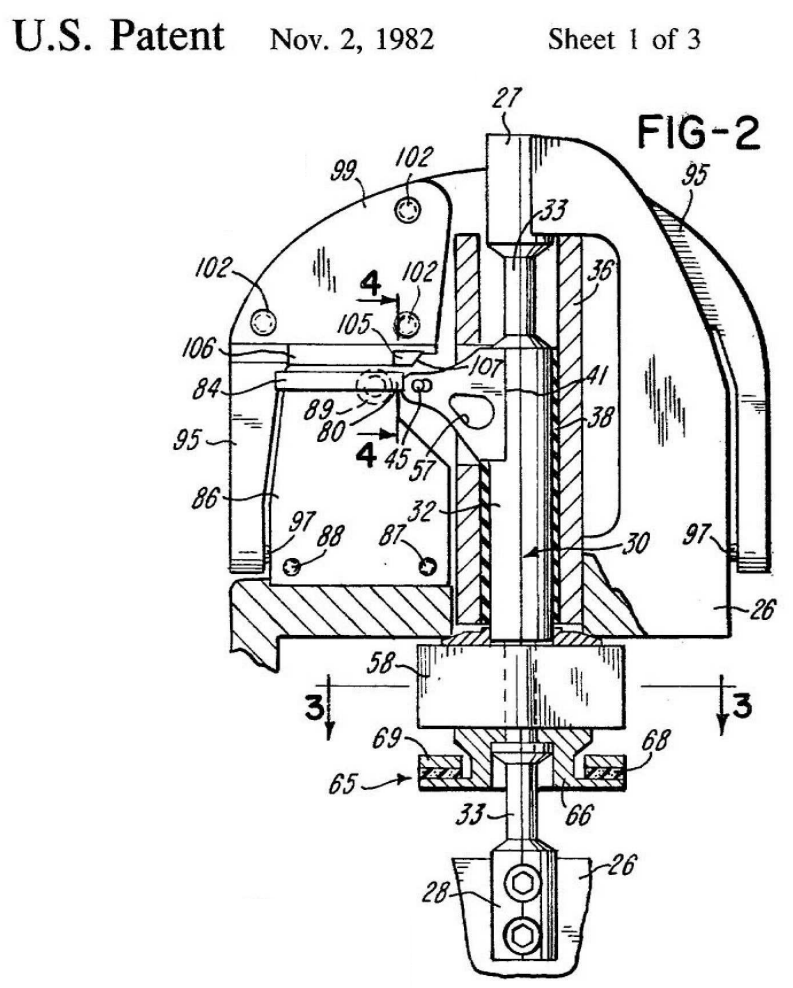

Figure 2-16: Torsion rods used to support and guide a rotating shaft in an engraving head. From Buechler [27], U.S. Patent 4,357,633.

constraint on the shaft 6 and a radial constraint on one end of it, and is the torsional spring for the system. The other end of the shaft is supported by a bearing 8 .

Buechler [27] describes using a pair of torsion rods, one on either side of a rotating element, in U.S. patent 4,357,633 dated 1982. Figure 2-16 is from this patent. The two torsion rods 33 provide an axial constraint on the shaft 30 and radial constraints on both ends of it, and form the torsional spring for the system. Item 58 in this figure is the variable reluctance actuator described earlier. The layer of resilient material 38 reduces lateral movement or vibration of the shaft.

\section{Radial Flexures}

Buechler [27, 29, 28] describes using "support rods" to resist lateral forces on a rotating element in U.S. patent 4,357,633 dated 1982, U.S. patent 4,438,460 dated 1984, and U.S. patent 4,450,486 dated 1984. He makes specific claims to them in the first 


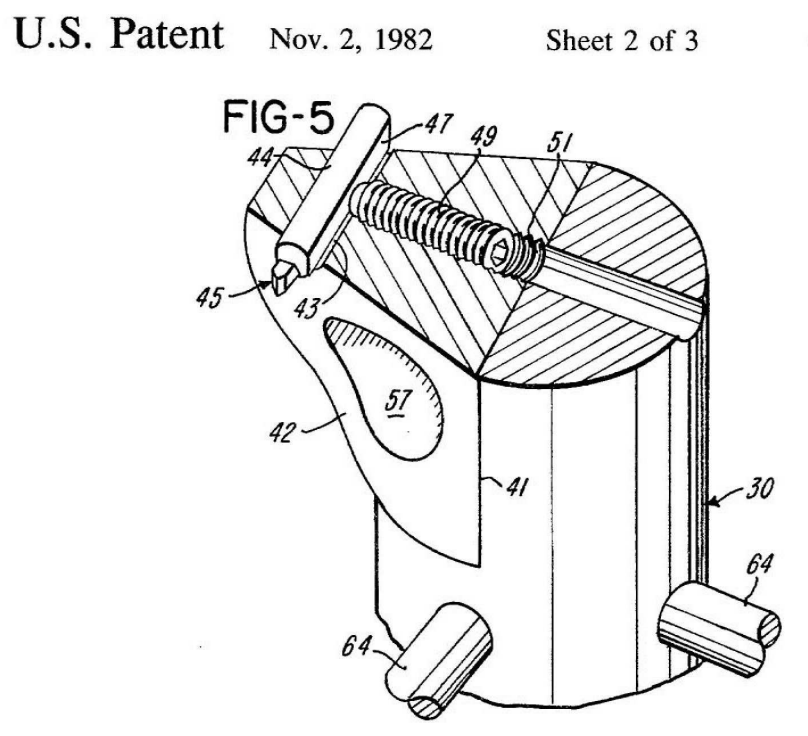

Figure 2-17: Radial flexures used to resist lateral forces in an engraving head. From Buechler [27], U.S. Patent 4,357,633.

of these three patents. Figure 2-17 is from U.S. patent 4,357,633. The radial rods 64 project laterally from the shaft 30 and are fixed at their distal ends (not shown). A lever 42 is rigidly connected to the shaft and carries a tool 45 . Buechler describes the radial rods as providing lateral support of the shaft and possibly acting as torsional springs, but does not mention them being used to provide axial support. Referring to Figures 2-17 and 2-16:

The rods 64 provide the shaft 30 with high lateral stiffness in X-Y [lateral] directions while also providing for high frequency oscillation of the shaft. The support rods 64 substantially eliminate the friction which would be produced by an intermediate support bearing and also minimize hysteresis [that contact with the resilient material damper 38 would cause] ... [27, column 5 line 61$]$.

... if desired, the torsional spring support portions 33 [torsion rods] at opposite ends of the shaft may be replaced by corresponding sets of supports rods 64 [27, column 6 line 7]. 
The support rods are in fact radial flexures, but it is apparent from the circular cross-section shown in the figure that their intended function is to provide a radial constraint on the shaft and to act as a torsional spring for the system. In the form shown they do not provide a significant axial constraint on the shaft. The following excerpts from claims by Buechler [27] further indicate that the support rods were not intended to provide an axial constraint on the rotating shaft:

[From claim 5] ... said shaft supporting means including at least one elongated spring element rigidly connected to said shaft and projecting laterally from said shaft, said spring element being positioned to prevent lateral movement of said shaft in a direction generally parallel to the movement of said stylus tip [lateral direction] ... [27, column 10 line 67]. [From claim 9] Apparatus as defined in claim 5 wherein a plurality of spring elements are connected to said shaft at peripherally spaced locations [27, column 12 line 11].

In contrast, the flat blade style radial flexures taught by this thesis are designed to provide high-stiffness radial and axial constraints on the supported shaft while minimizing the stiffness of the unwanted torsional spring that they add to the system.

\subsubsection{Damping}

Methods for using Coulomb and viscous damping on a rotating element are reported in the literature, as well as the use of tuned-mass damping for suppressing specific torsional resonances.

\section{Coulomb Damping}

Baar [15] describes methods for using Coulomb friction to provide damping to the rotary shaft that carries the tool arm of an engraver in U.S. patent 3,964,382, dated 1976. Figure 2-18 is from this patent. Plastic blocks 17 or 20 are held by a stationary member 19 so that they press against and rub the rotating shaft 6 . In the case of 
U.S. Patent June 22, 1976

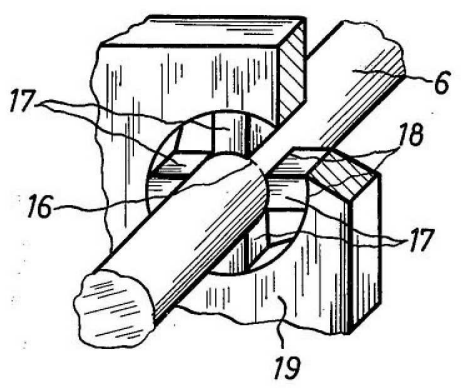

Fig. 2

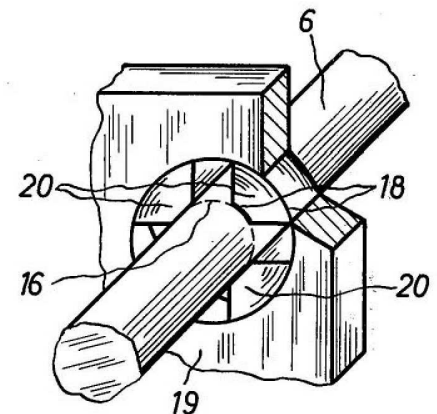

Fig. 3
$3,964,382$

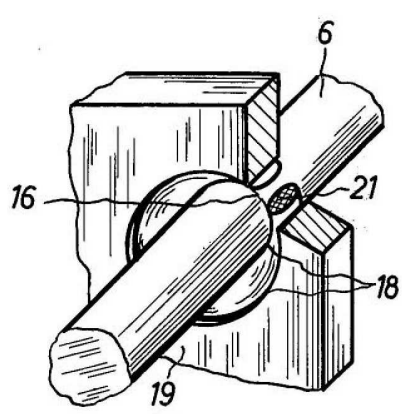

Fig. 4

Figure 2-18: Coulomb damping used in an engraving head. From Baar [15], U.S. Patent $3,964,382$.

"Fig. 4" the plastic blocks are replaced with a plastic ring 21. Inherent with Coulomb friction is a stick-slip behavior that would increase the difficulty of achieving precise motion control over the entire range of travel of a rotor. In the case of an electric engraving head, where controlling the end points and an intermediate point is a goal, stick-slip may not be as much of an issue, but it does diminish the ability to operate in continuous oscillation mode.

\section{Viscous Damping}

Baar [15] describes methods for applying viscous damping to the rotary shaft that carries the tool arm of an engraver in U.S. patent 3,964,382, dated 1976. Figure 2-19 is from this patent. A full disk 22 or partial disk 25 is fixed to the rotating shaft 6 . The outer portion of the full or partial disks are immersed in a viscous fluid, "preferably grease" [15, column 6 line 21], held in the stationary container 23 or 26. Rotation of the disk shears the viscous fluid and dissipates energy into it. This is the same method of viscous damping used in the $2 \mathrm{kHz}$ rotary fast tool servo described by this thesis. All of the claims in this patent by Baar [15] pertain to the Coulomb and viscous damping methods just described. 


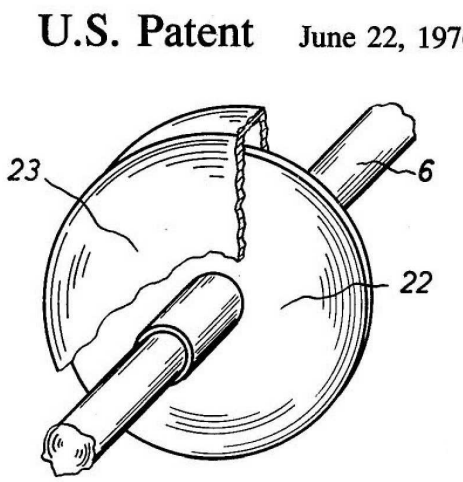

Fig. 5
Sheet 3 of $3 \quad 3,964,382$

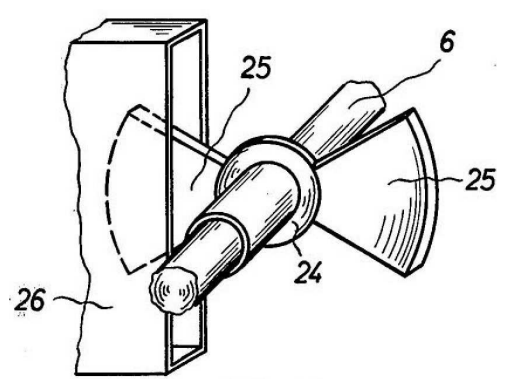

Fig. 6

Figure 2-19: Viscous damping used in an engraving head. From Baar [15], U.S. Patent $3,964,382$.

\section{Tuned-mass Damping}

Buechler [27] describes the use of tuned-mass dampers to damp unwanted rotary oscillations in an engraver in U.S. patent 4,357,633 dated 1982. Figure 2-20 is from this patent. The dampers shown in "FIG-7" and "FIG-8" are pure tuned-mass dampers. The plate 66 is fixed to the rotating shaft 30. In "FIG-7" a set of weights 69 is attached to the plate via a resilient material 68. In "FIG-8" heavy particles 74 such as lead shot are embedded in a resilient material 73. These dampers, especially the one shown in "FIG-7", are analogous to the harmonic dampers used in certain automobile engines for damping an unwanted torsional resonance in the crankshaft. Adjusting the mass of the weights and/or shear stiffness of the resilient material affects the center frequency of the damper. Buechler makes a claim in this patent [27, claim 8] on the damping systems shown in Figure 2-20.

Notice that in the cases of "FIG-9" and "FIG-10" in Figure 2-20 that the tunedmass dampers introduce an additional torsional spring to the system. In FIG-9 one side of the resilient material 73 with heavy particles 74 has a mechanical connection to a stationary plate 76 , causing the resilient material to act as a torsional spring. In FIG-10 one side of the set of weights 69 has a mechanical connection to a stationary plate 76 via a second layer of resilient material 68, again causing the resilient material to act as a torsional spring. 


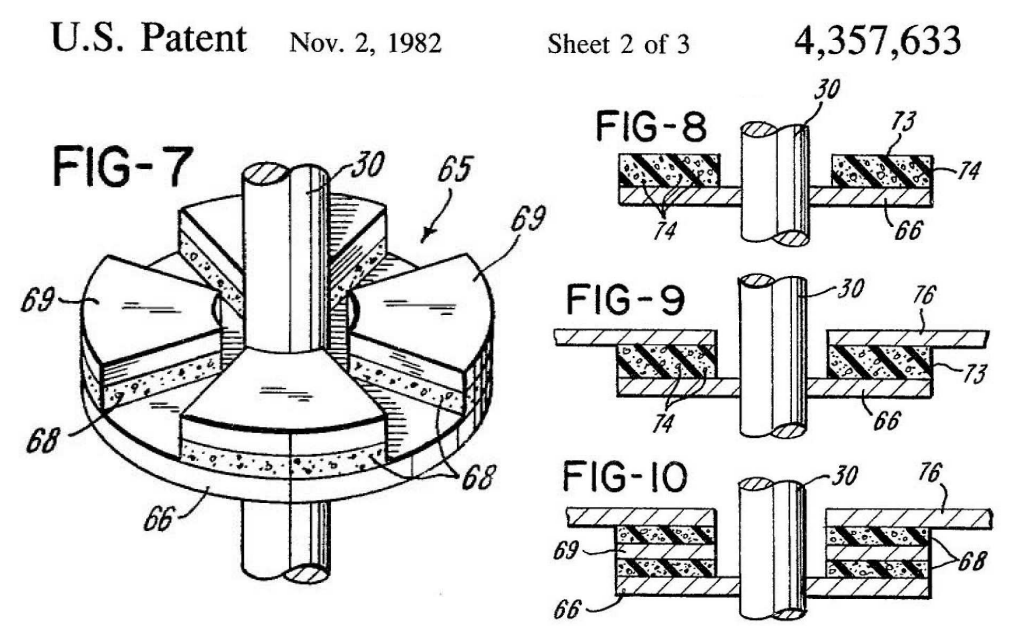

Figure 2-20: Tuned-mass damping used in an engraving head. From Buechler [27], U.S. Patent 4,357,633.

\subsubsection{Control}

When used to engrave an image carrier, engraving heads are essentially open-loop devices in the sense that the position of the tool tip is not strictly controlled during its entire travel between the end points on its in and out path. The control issues for an engraving head are more concerned with controlling the location, depth, and width of the ink wells that are cut into the gravure cylinder at a fixed spacing.

Holowko [77] describes a method for controlling an engraving head in U.S. patent 5,438,422 dated 1995. Figure 2-21 is from this patent. The dotted-line arrows 30 show three engraving tracks along the circumference of a gravure cylinder that has been cut by an engraving head. The black cells 70 hold large amounts of ink,and are connected by channels 72 . White cells (not shown) are areas that have not been cut and will therefore not hold ink after the doctor blade cleans the inked cylinder before printing. The highlight cells 76 hold a small amount of ink. Referring back to Figure 2-7, controlling the depth of the black cells "BD", white cells "WD", and highlight cells "HD" controls the tonal values of the printed image. ${ }^{8}$ In the present case of Holowko [77], the gains for the AC driving signal and the square wave video signal are set to the nominal values needed for achieving the desired cell width and

\footnotetext{
${ }^{8}$ The cell width is related to the cell depth by the geometry of the cutting tip of the tool.
} 


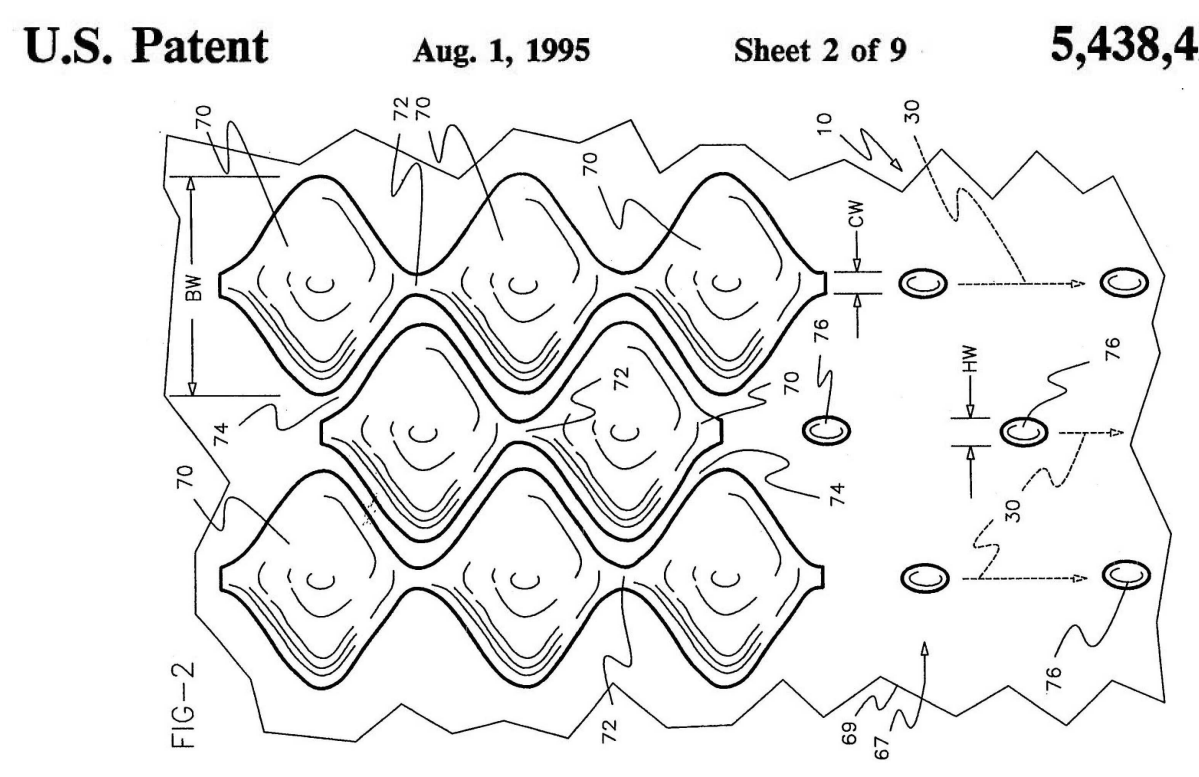

Figure 2-21: Detail of cuts for holding ink made in a gravure cylinder by an engraving head. From Holowko [77], U.S. Patent 5,438,422.

depth during the initial set up of the engraving machine. A video camera provides on-the-fly feedback information on the width of the cells, which is used to adjust the gains that affect them.

It is worth considering how a surprisingly agile displacement response can be achieved with a resonating device. Figure 2-22 is the simulated response of a massspring oscillator without damping to a sequence of step force inputs. ${ }^{9}$ By ignoring damping and the disturbance forces from the tool-workpiece interaction, a simple mass-spring model for the moving element of an engraving head can be used for developing insight into how the depth of cut of the tool can be adjusted within a single oscillation cycle.

The mass is initially at rest with zero displacement and zero input. At the time corresponding to point "A" a positive step input produces the oscillation shown between "A" and "B". At time "B" the mass has zero velocity and the system has potential energy stored in the spring, which exerts a downward force on the mass. The negative step force input at time "B" adds with the spring downward force to cause the larger amplitude half-cycle oscillation between "B" and "C". At time "C"

\footnotetext{
${ }^{9}$ The MATLAB ${ }^{\circledR}$ code used to generate this figure is contained in Appendix B.1.
} 


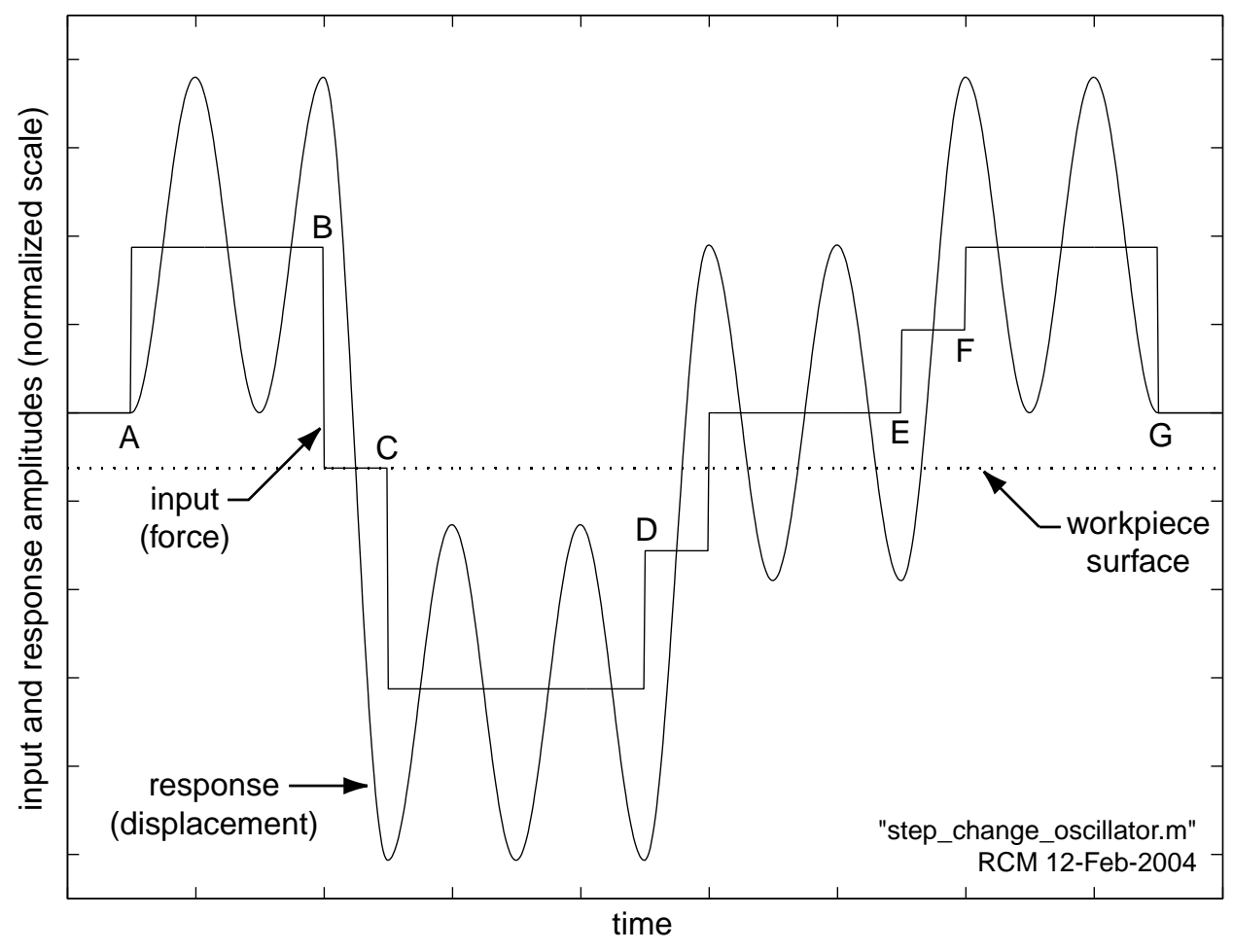

Figure 2-22: Simulation of a mass-spring oscillator without damping acted upon by a sequence of step force inputs.

the mass again has zero velocity and the system has potential energy stored in the spring, which now exerts an upward force on the mass. The negative step force input at time "C" subtracts from the spring upward force, preventing the mass from returning to the height it had at "B", and establishing the original amplitude oscillation shown between "C" and "D". And so it goes, step input forces adding with and subtracting from the spring force on the mass during a zero-velocity initial condition, to produce the trajectory shown. Of course, in the actual system an additional sinusoidal force input would have to be superimposed on the sequence of steps to make up for energy loss and thereby keep the oscillation going.

Notice that the period of oscillation of the mass is preserved from "A" to "G". Looking at the displacement curve relative to the "workpiece surface", moving from left to right note the creation of one "white" cell, three interconnected "black" cells, two "highlight" cells, and one "white cell". Additionally, the input was tailored to return the mass to rest with zero displacement at time " $G$ ". It is interesting to compare 
the input and displacement curves shown between times "A" and "F" in Figure 2-22 to what is typically depicted as the "video signal" and "machined workpiece surface" in the patents reviewed in this thesis, as was shown in Figure 2-7. In particular, referring to Figure 2-7, notice the abrupt change in the tool path attendant with the falling edge of the video signal. The instantaneous displacement implies that the mechanical resonance was overwhelmed by an infinite force, which is not likely. I suspect that the waveforms shown in Figure 2-7 are meant to be illustrative only, and not exact representations of what actually occurs.

Sermund [136] describes methods for controlling the cutting depth of an engraving head in U.S. patent 6,421,576 B1 dated 2002. He makes specific mention of using position and force feedback to reduce drift, control the actuator, and compensate for variations in the hardness of a workpiece. Referring back to Figure 2-14, the force sensors 6 and 12 provide feedback on the actuator force and the cutting force. A first distance sensor 13 and a second distance sensor 15 form a metrology loop between the workpiece 3 and the tool 2 .

Buechler [28] makes specific claims on using band reject filters in a control system to avoid exciting certain mechanical resonances in an electric engraver in U.S. Patent 4,450,486 dated 1984.

Crewe [41] describes using tool position feedback in a closed-loop fashion to control the depth of cut of an engraving head in U.S. Patent 5,818,605 dated 1998. The goal appears to be concerned with efficiently establishing the depths of the black, white, and highlight cells during the set up of the machine on a new workpiece, and maintaining those depths during the engraving process. Crewe describes being able to correct the depth from one cell to the next at cell-to-cell frequencies of $5 \mathrm{kHz}[41$, column 3 line 15].

\subsubsection{Sensors}

For an electric engraving head, real-time sensing of the position of the cutting tool relative to the workpiece appears to be concerned with establishing and maintaining the two end points and at least one intermediate point of the tool along its path 
of travel. Methods for measuring the tool position relative to the engraving head, and in one case, directly relative to the cylinder being engraved, are reported in the literature and discussed next.

Fraser [62] describes using a sensor for controlling the drift and vibration of a tool used in an engraving head in U.S. Patent 5,029,011. Figure 2-23 is from this patent. A tool 62 is mounted to an arm 60 fixed to a shaft 50 that rotates in an oscillatory manner. A light source 72 produces a light beam 74 that reflects off a mirror 76 fixed to the shaft. The reflected light illuminates a position sensor 70 which comprises a first light detector 78 and a second light detector 79. A lens 77 may be used. Rotation of the shaft causes more light to fall on one or the other of the two detectors, and the resulting electronic signal is processed to infer in and out motion of the tool.

Note that the tool is separated from the sensor mirror 76 by the arm 60 and the shaft 50. This may be a preferable arrangement in applications where the region around the tool could be hostile to the sensor, for example, due to cutting fluids or debris generated during use of the device, and when a means for protecting the sensor were not practicable. However, this arrangement does have potential drawbacks. Measurement errors can result from mechanically or thermally induced relative motion between the tool, mirror, light source, detector, and lens. Furthermore, nonrigid-body behavior of one or more of those elements could be another source of error.

As an interesting aside, Fraser [63] also describes a method for mapping the pitch errors of a leadscrew and generating a look-up table for real-time error correction in U.S. patent 5,329,215 dated 1994. Fraser was concerned with the accurate placement of engraving tracks on a gravure cylinder, but his work may be pertinent to other leadscrew-driven devices, like certain diamond turning machines employing that type of drive.

Crewe [41] et al describe a multitude of different sensor types and arrangements for providing feedback information on the position of the tool in an engraving head in U.S. Patent 5,818,605 dated 1998. They cover the use of capacitance, resistance, impedance, optical, piezoelectric, and eddy current sensors, and describe topologies 


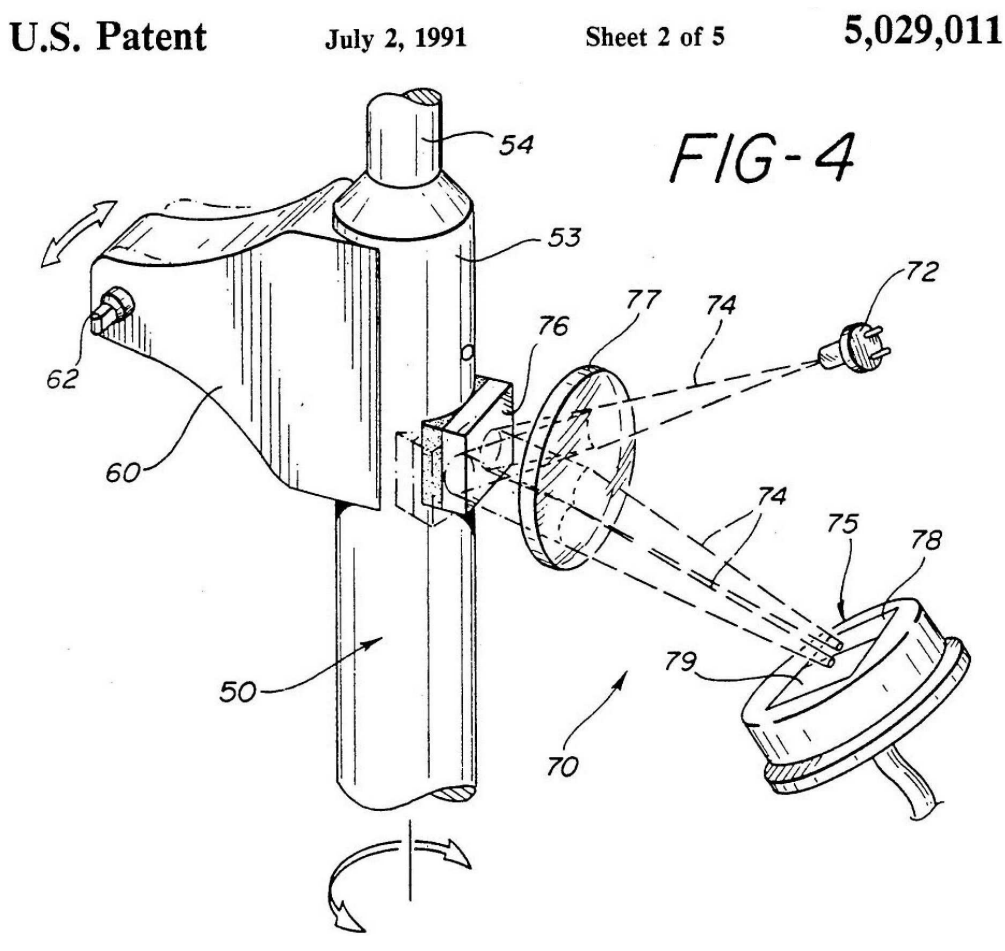

Figure 2-23: Sensor for controlling drift and vibration of the tool in an engraving head. From Fraser [62], U.S. Patent 5,029,011.

for measuring the tool displacement relative to a stationary portion of the engraving head and relative to the workpiece. Figure 2-24 is a compilation of figures from this patent. The rotating arm, referred to as a "stylus" 14, has a pivot axis 16 and carries a diamond tool tip 18 that engages a workpiece 22 with a cutting depth 20. Note that in all of the figures shown in Figure 2-24, except for "FIG. 7", the measurement point on the stylus is on the opposite side of the pivot from the tool. This arrangement is susceptible to the same measurements errors described earlier due to any non rigidbody behavior of the components, which is especially an issue at operating frequencies in the kilohertz range where the flexible modes of small mechanical components often reside.

In "FIG. 1A" a first capacitor plate 26 and a second capacitor plate 28 are fixed to a stationary portion of the engraving head 12. A third capacitor plate 30 is attached to the stylus via an arm 31 and forms a first and second capacitor with the first and second capacitor plates. Differential changes in capacitance caused by rotation of the 
U.S. Patent
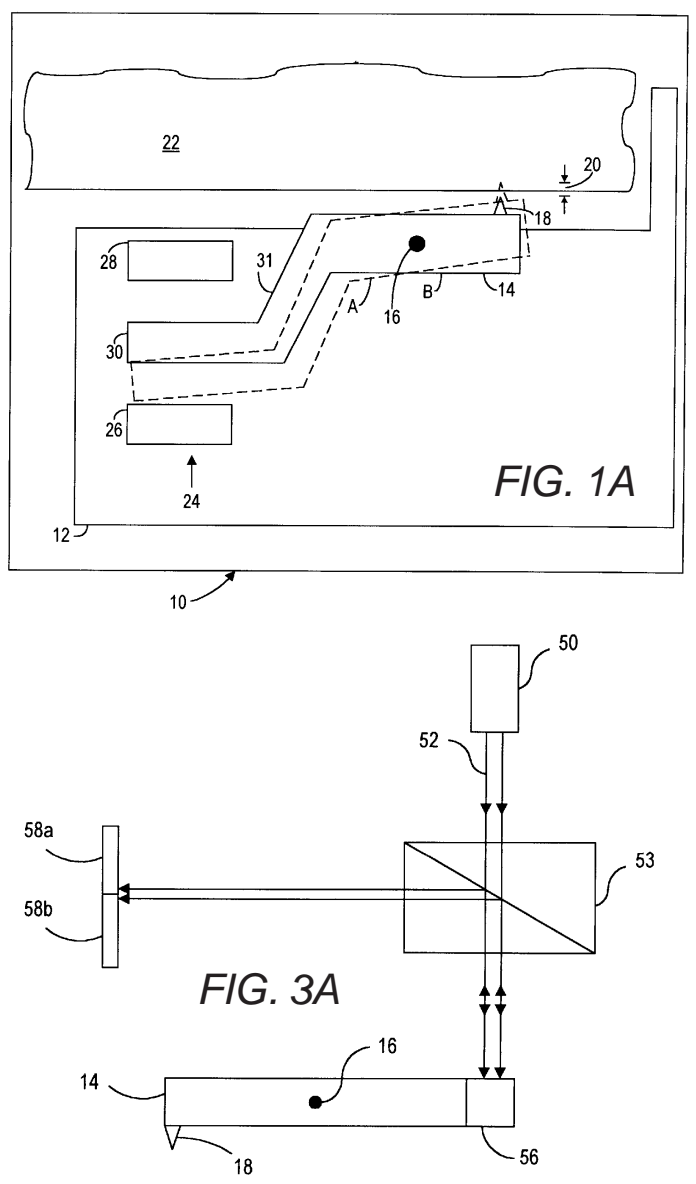

14

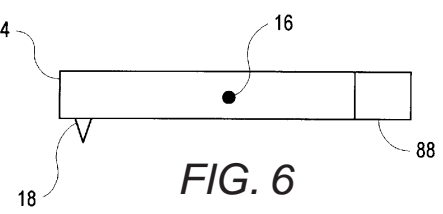

Oct. 6, 1998

$5,818,605$
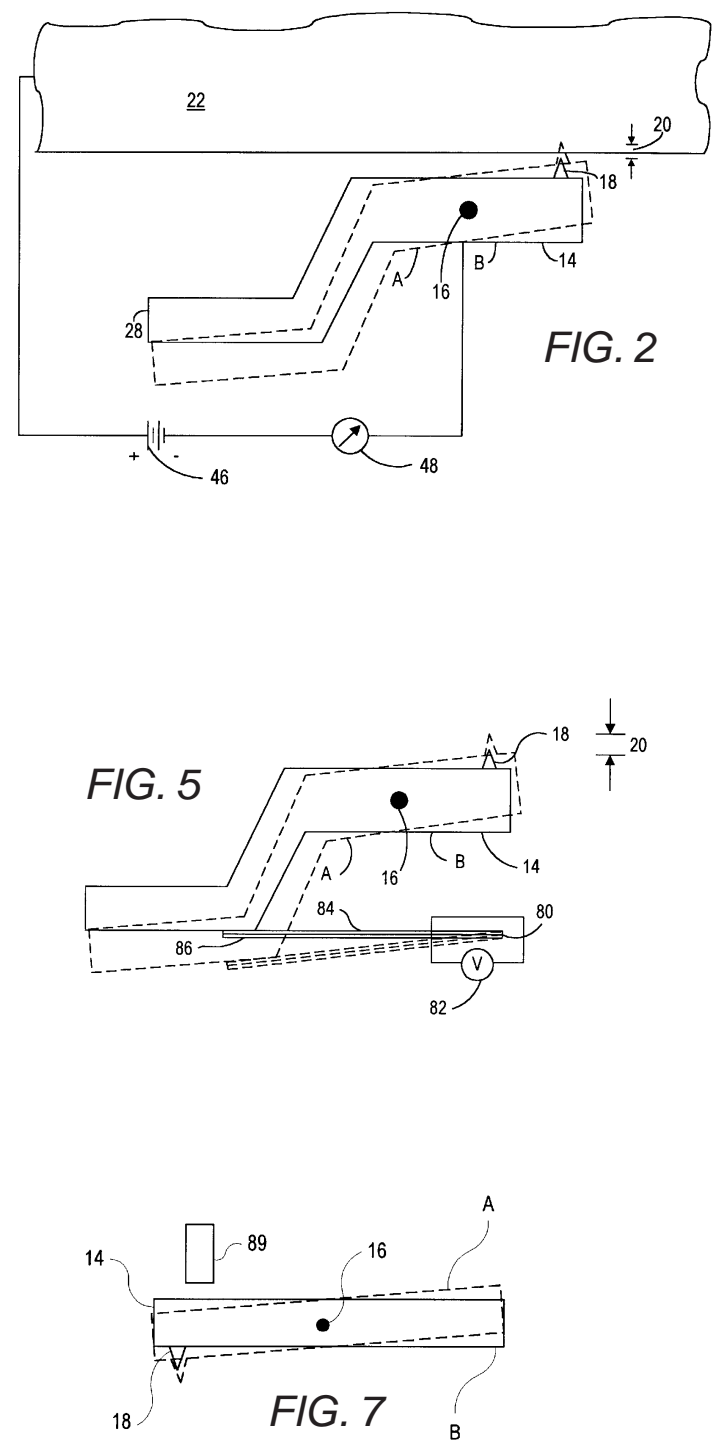

Figure 2-24: Methods for sensing the displacement of the tool in an engraving head. From Crewe [41], U.S. Patent 5,818,605. 
stylus are sensed by an electronic circuit to infer the depth of cut.

In "FIG. 2" an electrical circuit is established between the workpiece 22 and the stylus 14 via the diamond tip 18 embedment in the workpiece. Changes in the depth of cut result in a change in the resistance or impedance of the circuit, which is sensed by an electronic circuit to infer the depth of cut.

In "FIG. 3A" a laser beam 52 from a laser 50 passes through a beam splitter 53 , reflects off of a mirror 56 attached to the stylus 14, and is directed onto a split photo detector $58 \mathrm{a}$ and $58 \mathrm{~b}$ by the beam splitter. Rotation of the stylus causes a change in the amount of light reaching each detector, which is sensed by an electronic circuit to infer the depth of cut. In the text of the patent it is mentioned that the mirror could be mounted on the same end of the stylus as the tool 18. Three additional figures in the patent (not shown here) illustrate other method of optical measurement using a mirror on the stylus. One is a variation on what is shown in "FIG. 3A", while the other two use different optical arrangements with a single photo detector.

In "FIG. 5A" a piezoelectric rod 80 consisting of a first piezoelectric half 84 and a second piezoelectric half 86 are connected to the stylus 14 and a stationary portion (not shown). The two halves 84 and 86 are made of materials having different piezoelectric properties. Rotation of the stylus causes a deflection of the piezoelectric rod, generating voltages in the two halves which is sensed by an electronic circuit to infer the depth of cut.

In "FIG. 6" an eddy current sensor 88 is mounted to the stylus 14 on the opposite side of the pivot 16 from the tool 18. The sensor 88 is in close proximity to the copper plated workpiece (not shown) which is on the same side of the stylus as the tool. Changes in the distance between the sensor and the workpiece affect the impedance of the sensor, which is sensed by an electronic circuit to infer the depth of cut.

In "FIG. 7" an eddy current sensor 89 is mounted to a stationary portion (not shown) of the engraving head and measures the displacement of the stylus 14 on the same side of the pivot 16 as the tool 18. Changes in the distance between the sensor and the stylus affect the impedance of the sensor, which is sensed by an electronic circuit to infer the depth of cut. Note that the sensor arrangement in "FIG. 7" is 
similar to the placement of the capacitance sensor behind the tool in the $10 \mathrm{kHz}$ rotary fast tool servo. "FIG. 7" is also similar to the arrangement of the two eddy current sensors, one on each side of the pivot axis, used in the $2 \mathrm{kHz}$ rotary fast tool servo. In this later case, the use of a differential measurement between the two eddy current sensors allows an option to also measure translation of the pivot.

As mentioned earlier in Section 2.3.5, Sermund [136] shows the use of position and force feedback to reduce drift, control the actuator, and compensate for variations in the hardness of a workpiece in U.S. patent 6,421,576 B1 dated 2002.

\subsubsection{Tool Mounting}

Most of the engraving heads found in the literature have a rotary topology similar to that of a rotary fast tool servo. A tool is mounted at a fixed radius from the axis of rotation of a shaft so that rotation of the shaft causes an in and out motion of the tool along an arc $[15,66,27,29]$. The tools are meant to be replaced during the lifetime of the machine, so a means for doing so while minimizing the set-up time for a new tool is often provided.

Bornhorst [25] describes using a trapezoidal tool profile and receiving slot in U.S. patent 5,475,914 dated 1995. Figure 2-25 is from this patent. Bornhorst describes it as follows using "stylus cartridge" to describe the "diamond stylus" [tool tip] plus the tool "holder" [tool shank] that carries it:

The tool holder 32 is formed with a trapezoidal cross-section which is received within a corresponding elongated trapezoidally-shaped aperture 34 formed in the stylus arm 20 [25, column 5 line 6].

As the screws 40, 42 are tightened down on the tool holder 32, opposing upper and lower tool holder surfaces 50, 52, which are angled toward each other to form the trapezoidal shape, will move into wedging frictional engagement with respective angled upper and lower surfaces 54, 56 of the aperture 34 to firmly lock the cartridge 22 into position and to establish the angular orientation of the stylus 30 relative to the stylus arm 20. 


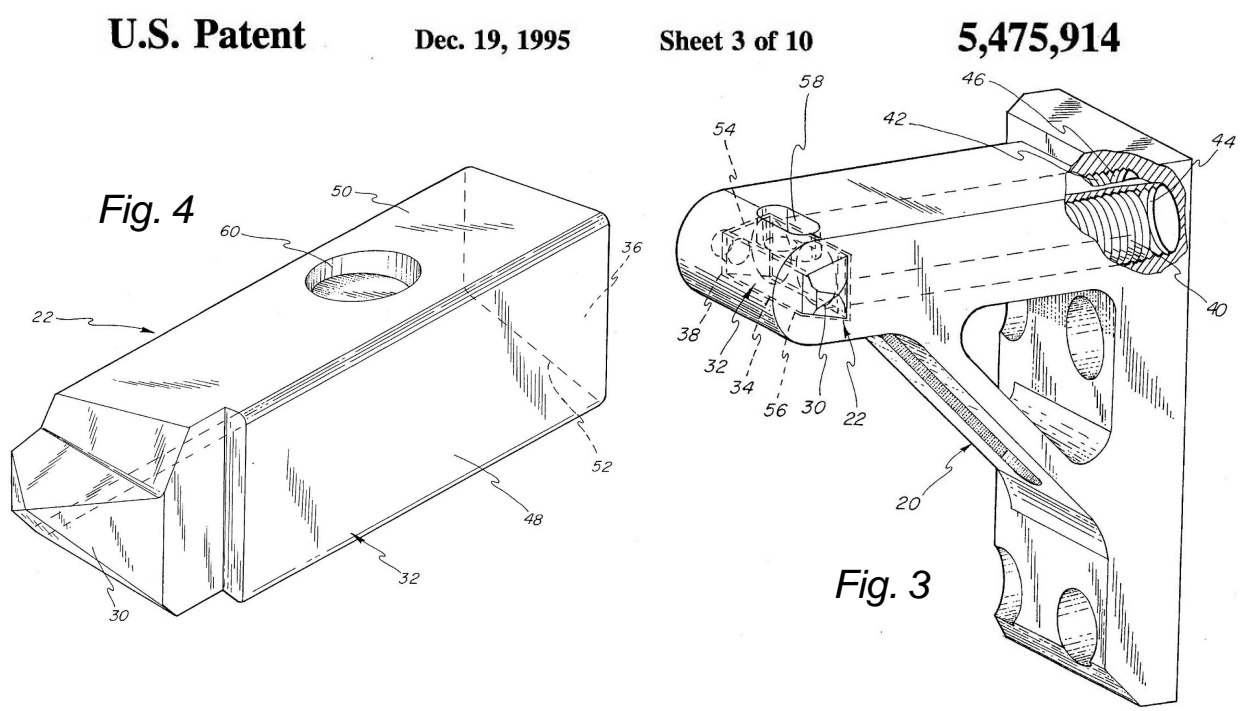

Figure 2-25: Trapezoidal tool profile and receiving slot for mounting a cutting tool in an engraving head. From Bornhorst [25], U.S. Patent 5,475,914.

When it is desired to remove the cartridge 22 from the stylus arm 20, a tool (not shown) may be inserted into an elongated slot 58 formed in the top surface of stylus arm $20 \ldots$ [25, column 5 line 22].

The use of a tapered fit between two objects that employ friction to hold them together is well known. For example, a large array of standard taper fits between two bodies is described in a 1980 third-printing of the twenty-first edition of "Machinery's Handbook" [115]. Notwithstanding the prior art on tapered fits, the method of tool mounting taught by Bornhorst [25] is similar to the tapered fit between the tool and tool holder used in the $2 \mathrm{kHz}$ and $10 \mathrm{kHz}$ rotary fast tool servos. However, this thesis teaches a substantial improvement on a tapered fit by providing recesses in the central portions of the two faces of the tool that contact the receiving slot to establish exactly four deterministic areas of contact between the tool and the slot. ${ }^{10}$ These prescribed areas of contact allow avoiding the possibility of the tool and slot making contact at or near the central portions of the contacting faces, which would reduce the stiffness of the connection between the tool tip and the tool holder and

\footnotetext{
${ }^{10}$ Alternatively, the recesses can be in the central portions of the two faces of the receiving slot that contact the tool.
} 
could lead to a bi-stable orientation of the tool relative to the holder. The elongated slot (58) in Figure 2-25 is intended for facilitating removal of the tool, and is not described to be a means of preventing contact between the tool and the holder near the central portion of the tool on that one side.

\subsection{Additional Pertinent Prior Art}

The selected prior art in this section provides additional examples of magnetic circuits that use flux biasing, and describes a design for a crossed-blade flexure bearing.

\subsubsection{Radial Plus Tip/Tilt Actuator with Flux-Biasing}

Studer [139] describes an assortment of magnetic circuits for realizing a radial plus tip/tilt actuator that use magnetic flux biasing in U.S. patent 4,634,191 dated 1987. This actuator is a normal-stress variable reluctance device. Figures 2-26, 2-27, 2-28, and 2-29 are from this patent. This work by Studer illustrates the possibility of realizing multiple magnetic circuits for producing the same set of desired controllable motions, and how rearranging one circuit into a different topology can ease the burden of realizing independent control of multiple degrees of freedom.

Figure 2-26 shows a schematic view of the actuator. The moving element 12 is supported by radial and axial magnetic fields and does not touch the stator 10 . This actuator controls radial motion in directions perpendicular to the "Z" axis, and rotational motion around the ' $\mathrm{X}$ ' and ' $\mathrm{Y}$ ' axes. The four quadrant segments $22_{1}, 22_{2}$, $22_{3}$, and $22_{4}$ are separated by high reluctance paths to allow realizing independent rotations around the $\mathrm{X}$-axis and $\mathrm{Y}$-axis. Note that the moving element 12 does not contact the stator 10, and is free to rotate about the Z-axis. Figure 2-27 is a crosssection taken along the $\mathrm{X}$-axis and is used to illustrate radial motion along the $\mathrm{X}$-axis and rotation around the Y-axis. A similar cross-section (not shown) taken along the Y-axis would illustrate radial motion along the Y-axis and rotation around the $\mathrm{X}$ axis. Upper and lower permanent magnets 16 and 18 provide bias flux in paths 46 and 48 respectively. Coils $32_{1}, 32_{3}, 34_{1}$, and $34_{3}$ provide steering flux. Rather than 


\section{U.S. Patent Jan. 6, $1987 \quad$ Sheet 1 of $5 \quad 4,634,191$}

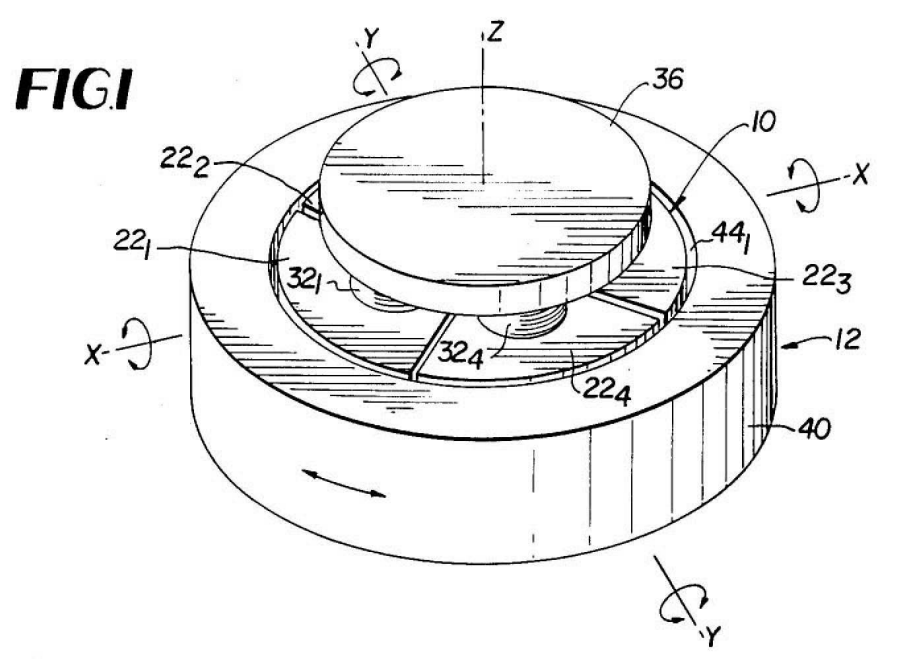

Figure 2-26: Schematic view of a radial plus tip/tilt actuator. From Studer [139], U.S. Patent 4,634,191.

paraphrase, the following excerpt from [139] clearly explains how the device works:

The flux coils $32_{1}-32_{4}$ and $34_{1}-34_{4}$ of FIG. 3 operate to produce both radial forces and torque producing forces depending on the manner in which they are energized. If, for example, the upper and lower flux coils are energized in the same direction to provided magnetic flux which circulates as shown by flux paths 50 and 52 and which returns in the same direction through the central gap $44_{2}$ and the middle disc member 14 as shown, then radial forces are produced. However, if the upper and lower flux coils are energized in opposition to generate magnetic flux which circulates as shown by the outer flux path 54 which traverses the gaps $44_{1}$ and $44_{3}$ while being cancelled through the flux path including the central gap $44_{2}$, then torsional forces are produced [139, column 4 line 58].

Figure 2-28 is a cross-section taken along the $\mathrm{X}$-axis of the actuator illustrating a first alternate magnetic circuit. Upper and lower permanent magnets 16 and 18 provide bias flux in paths 46 and 48 respectively. Radial motion along the $\mathrm{X}$-axis is controlled by coils $41_{1}$ and $41_{3}$ which provide steering flux in paths 57 and $57^{\prime}$. 


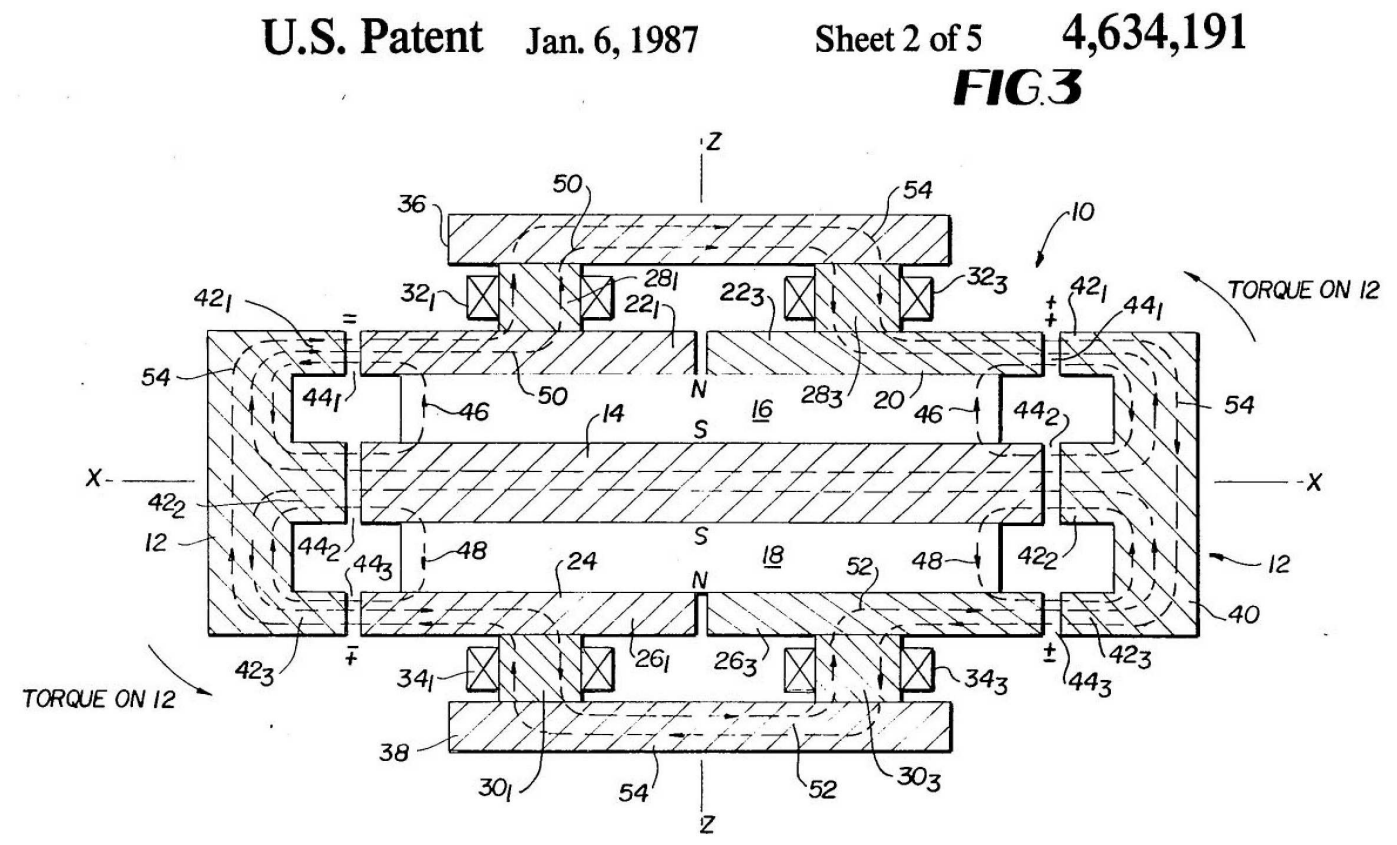

Figure 2-27: Magnetic circuit for a radial plus tip/tilt actuator. From Studer [139], U.S. Patent 4,634,191.

Rotation around the $\mathrm{Y}$-axis is controlled by coils $37_{1}, 37_{3}, 39_{1}$, and $39_{3}$ which provide steering flux in path 55. Note that the use of different coils for producing radial motion and rotational motion reduces the burden of realizing independent control of those motions.

Figure 2-29 is a cross-section taken along the X-axis of the actuator illustrating a second alternate magnetic circuit. The bias magnetic flux 46 is provided by a single permanent magnet 16'. Radial motion along the X-axis is controlled by coils $37_{1}$, $37_{4}, 39_{1}$, and the unlabelled coil $39_{4}$ below $37_{4}$ which provide steering flux in path 57 . Rotation around the $\mathrm{Y}$-axis is controlled by coils $41_{1}, 41_{4}, 43_{1}$, and $43_{4}$ which provide steering flux in paths 55 and 55'. The return pathes connecting the fluxes on the right labelled 55 and 55' with the fluxes on the left labelled 55 and 55' are out of the plane of the page through the annular ring of the moving element 12. As with the first alternate magnetic circuit, using different coils for producing the radial and rotational motions makes it easier to independently control those motions. 


\section{U.S. Patent Jan. 6, $1987 \quad$ Sheet 3 of $5 \quad 4,634,191$}

\section{FIG.4}

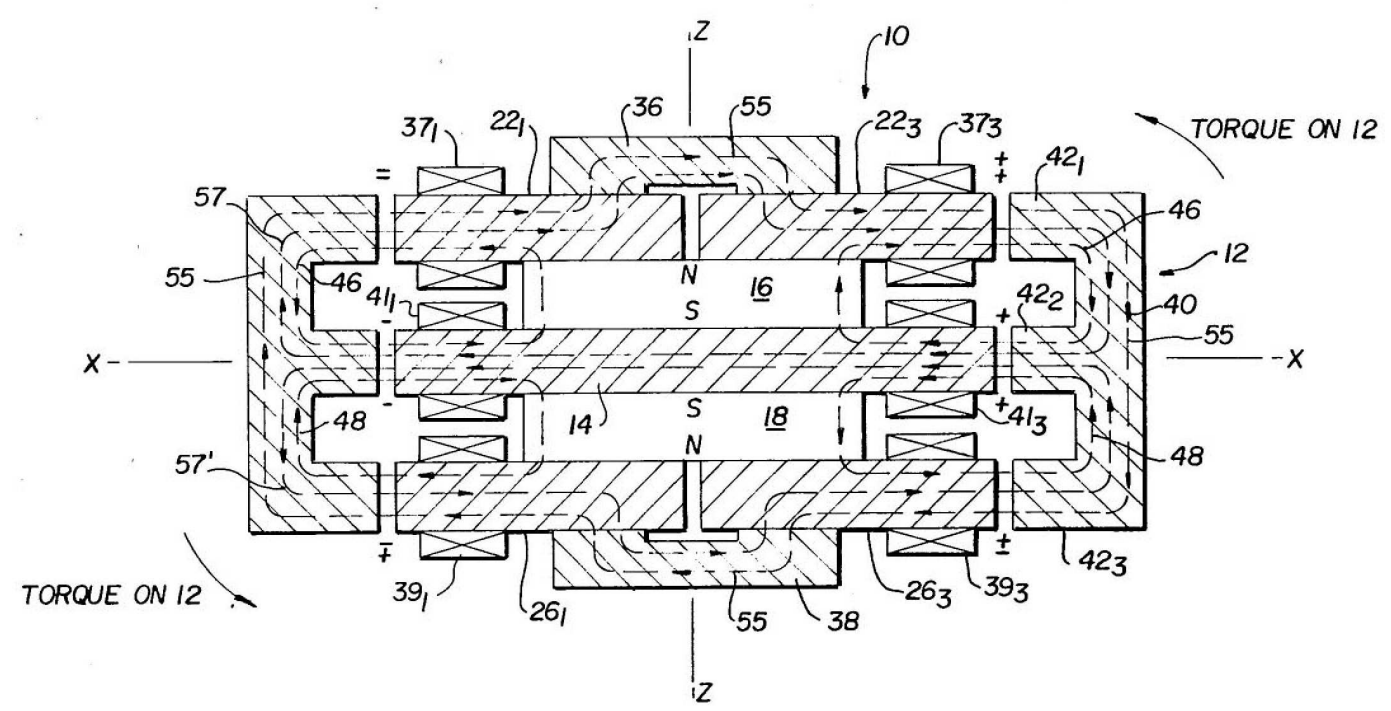

Figure 2-28: A first alternate magnetic circuit for a radial plus tip/tilt actuator. From Studer [139], U.S. Patent 4,634,191.

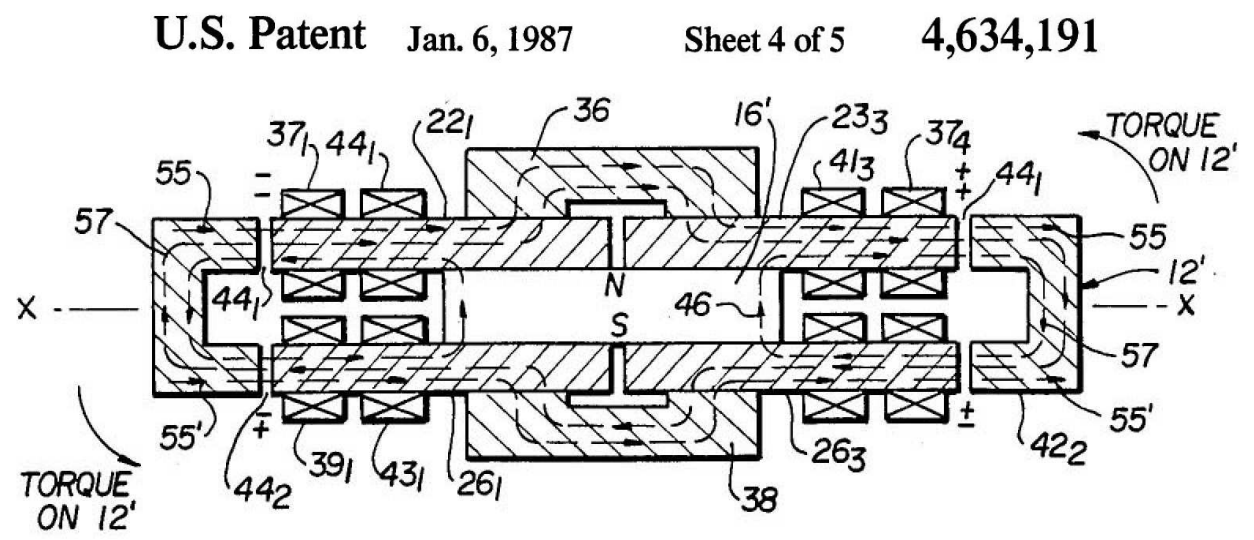

FIG.5

Figure 2-29: A second alternate magnetic circuit for a radial plus tip/tilt actuator. From Studer [139], U.S. Patent 4,634,191. 


\subsubsection{Normal-Stress Linear Actuator with Flux-Biasing}

Berling [22] describes a reciprocating linear motion actuator that uses magnetic flux biasing in U.S. patent 5,719,543 dated 1998. This actuator is a normal-stress variable reluctance device. Figure 2-30 is from this patent. The two permanent magnets 16 and 18 are each sandwiched between pairs of pole pieces 20 and 22, and 24 and 26, respectively. The north poles of the magnets face the bottom of the page in Figure 230. The piston (armature) 80 is held in the neutral position shown in Figure 2-30 by the compression springs 62 and 72, and forms air gaps 82 and 84 with the pole pieces. Coils 102, 104, 112, and 114 surround the pole pieces. To create a net force on the piston to the left, coils 102 and 112 create flux in their pole pieces that points to the right, and coils 104 and 114 create flux in their pole pieces that point to the left. This causes flux addition in the air gaps at pole pieces 20 and 22, and flux subtraction in the air gaps at pole pieces 24 and 26. Reversing the direction of the coil-produced fluxes reverses the direction of the net force on the piston. The magnetically permeable pieces 40 and 42 are separated from the pole pieces by low permeability spacers 44 and 46, and provide a return path for the permanent magnet flux for the side of the piston that has flux subtraction. The compression springs store and supply mechanical energy to create an efficient oscillatory system. It is worth noting that the claims in this patent pertain to a device for creating linear reciprocating motion, and do not mention using closed-loop feedback control to provide an active linear suspension as is taught by this thesis.

Flynn [57] describes a normal-stress variable reluctance linear actuator that uses magnetic flux biasing in U.S. patent 6,342,746. A schematic representation of this actuator is shown in Figure 2-31. A permanent magnet 112 contacts the two pole pieces 114 and 116 and provides a bias flux. The two armature pieces 118 and 120 are connected by a shaft 320 and form air gaps (not labelled) with the pole pieces. The current in the steering coils 122, 124, 126, and 128 is controlled to cause flux addition with the bias flux on one armature, and flux subtraction on the other armature. The flux addition and subtraction for the case shown in Figure 2-31 causes the armatures 


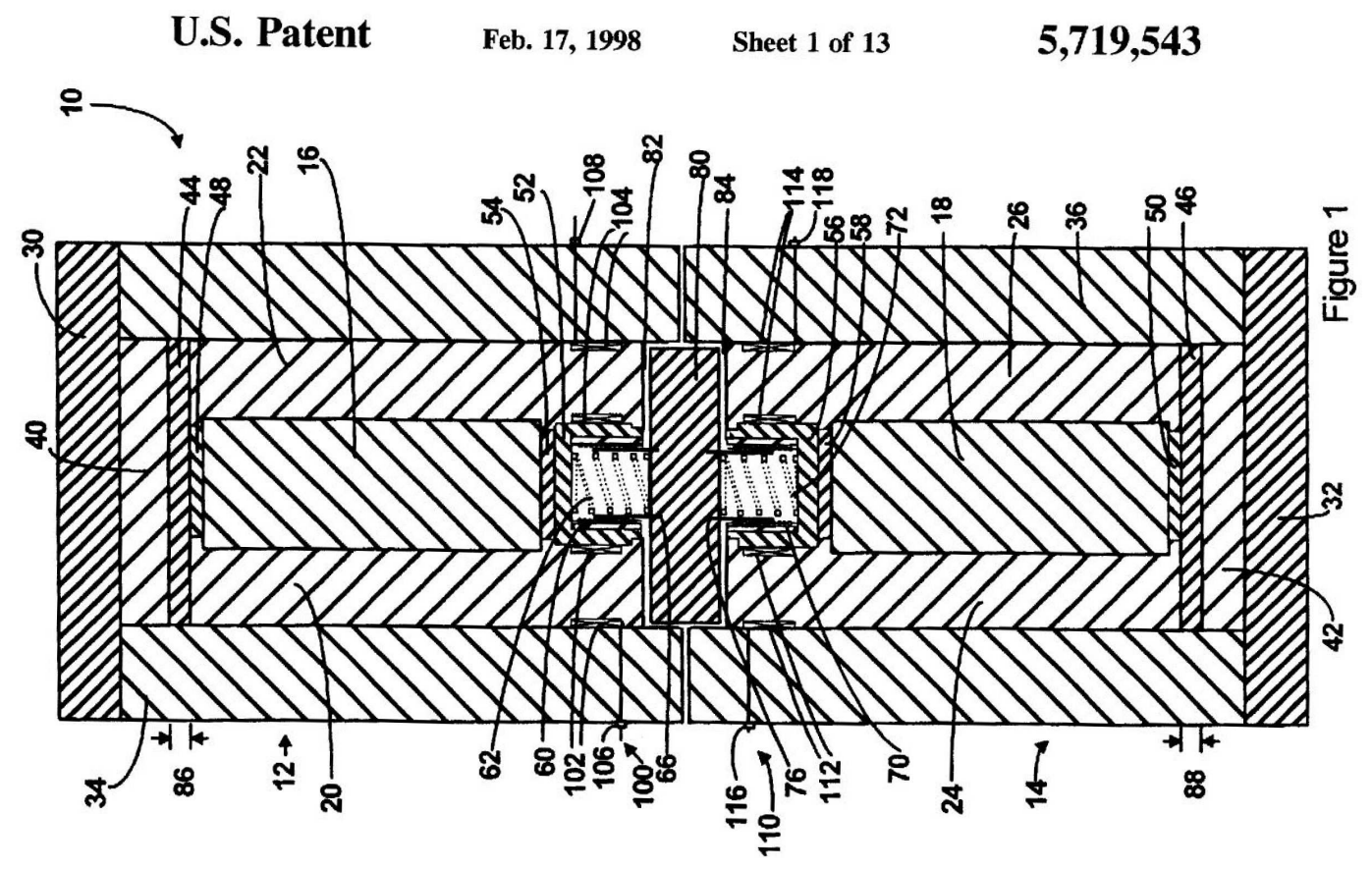

Figure 2-30: Normal-stress variable reluctance linear actuator employing flux-biasing. From Berling [22], U.S. Patent 5,719,543.

to translate in direction depicted by arrow 390. Reversing the direction of the currents in the steering coils cause the armature to translate in direction depicted by arrow 392. All of the claims in this patent [57] are on a rotary device mentioned in it described in the next section - so this linear device is assumed to be prior art to it.

\subsubsection{Shear-Stress Rotary Actuator with Flux-Biasing}

Flynn [57] describes a shear-stress variable reluctance rotary motion actuator that uses magnetic flux biasing in U.S. patent 6,342,746 dated 1998. A schematic representation of this actuator is shown in Figure 2-32. It is included in this prior art review because it provides an example of using permanent magnets to produce a bias flux in a rotary device. Permanent magnets 1130 and 1140 provide bias flux through pole piece stacks 1110 and 1120 via the rotor 1160 and the unlabelled air gaps between the rotor and pole pieces. Coils 1116 and 1126 provide steering flux for the device. When the coil current is phased to produce a steering flux that reinforces the bias flux from permanent magnet 1140 in poles 1154 and 1156, then that steering 


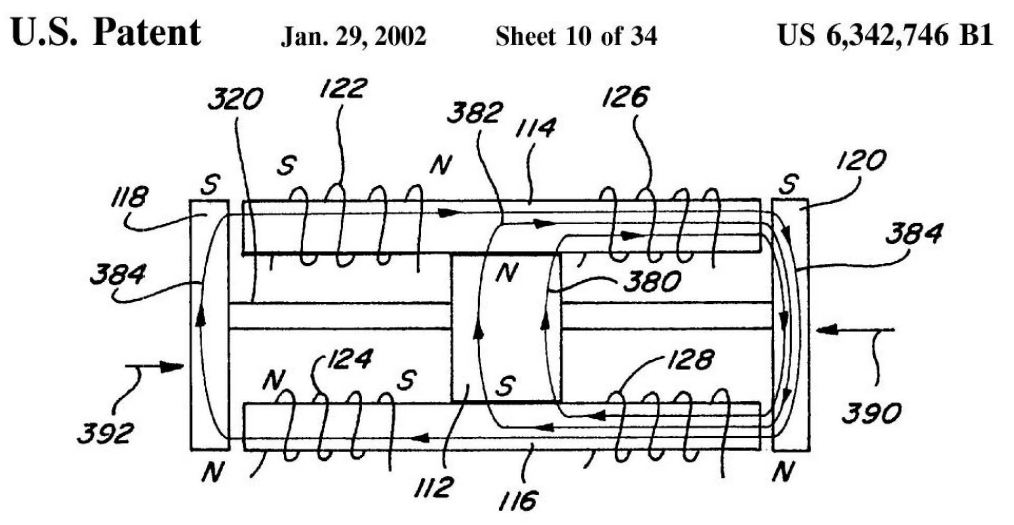

Fig. $16 \mathrm{~A}$

Figure 2-31: Normal-stress variable reluctance linear actuator employing flux-biasing. From Flynn [57], U.S. Patent 6,342,746.

flux opposes the bias flux from permanent magnet 1130 in poles 1152 and 1158. In this case, the rotor turns clockwise (viewed from above) to minimize the reluctance between the rotor protuberances 1166 and poles 1156 and 1154. Reversing the coil current reverses the rotation.

\subsubsection{Crossed-Blade Flexure Suspension}

DeBoalt [45] describes using a pair of crossed-blade flexures for supporting a shaft in a galvanometer in U.S. patent 6,265,794 dated 2001. Figure 2-33 is from this patent. The two flexure blades 34 and 36 provide an axial constraint and a radial constraint on the shaft 30, and form a torsional spring for the system. Each flexure is fixed at one end to the shaft 30 , and at the other end to the base 38 . Note that because one end of each flexure has an unconstrained degree of freedom for motion (the end attached to the shaft), rotation of the shaft will cause the axis of rotation 40 to wander. This is in sharp contrast to the relatively fixed axis of rotation provided by the flexure suspension design used for the $2 \mathrm{kHz}$ FTS and $10 \mathrm{kHz}$ FTS. What makes the DeBoalt [45] design unique is the provision for adjusting the resonant frequency of the shaft by changing the ratio of arm lengths " $A$ " and " $\beta$ " in Figure 2-33. 


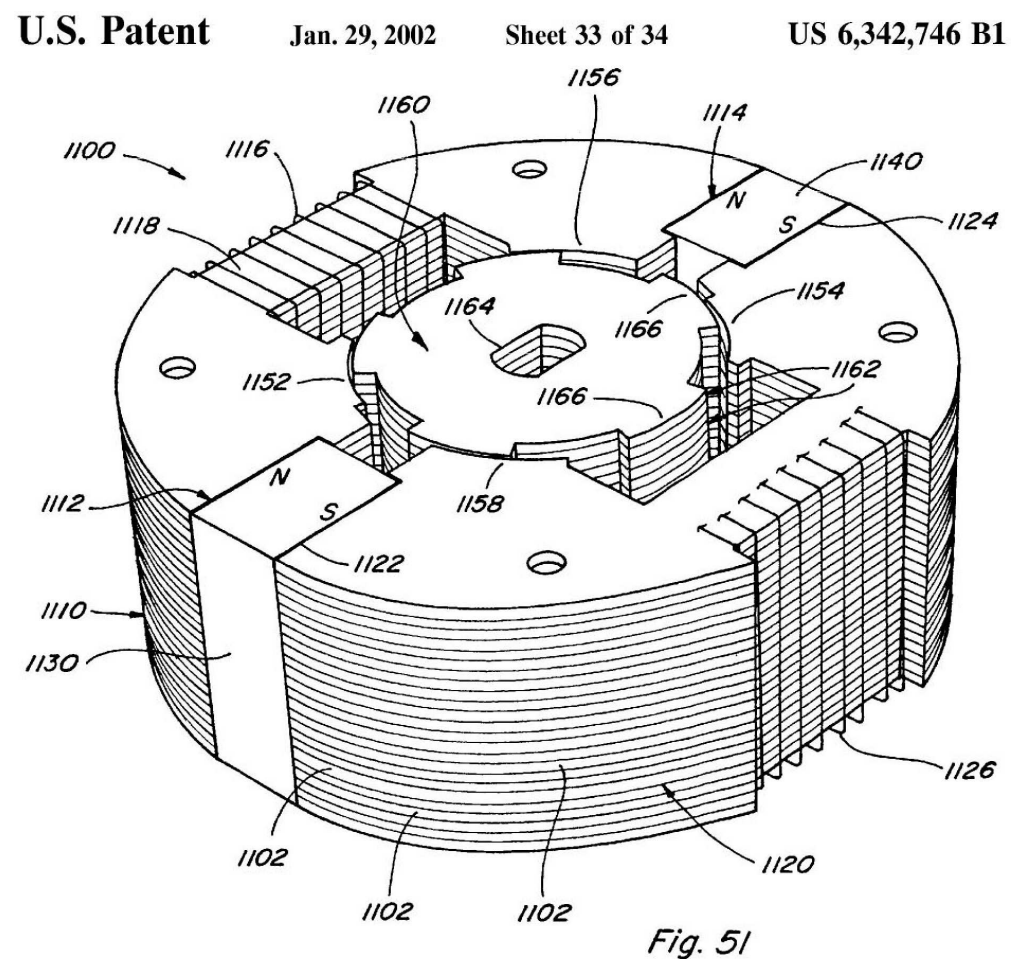

Figure 2-32: Shear-stress variable reluctance rotary actuator employing flux-biasing. From Flynn [57], U.S. Patent 6,342,746.

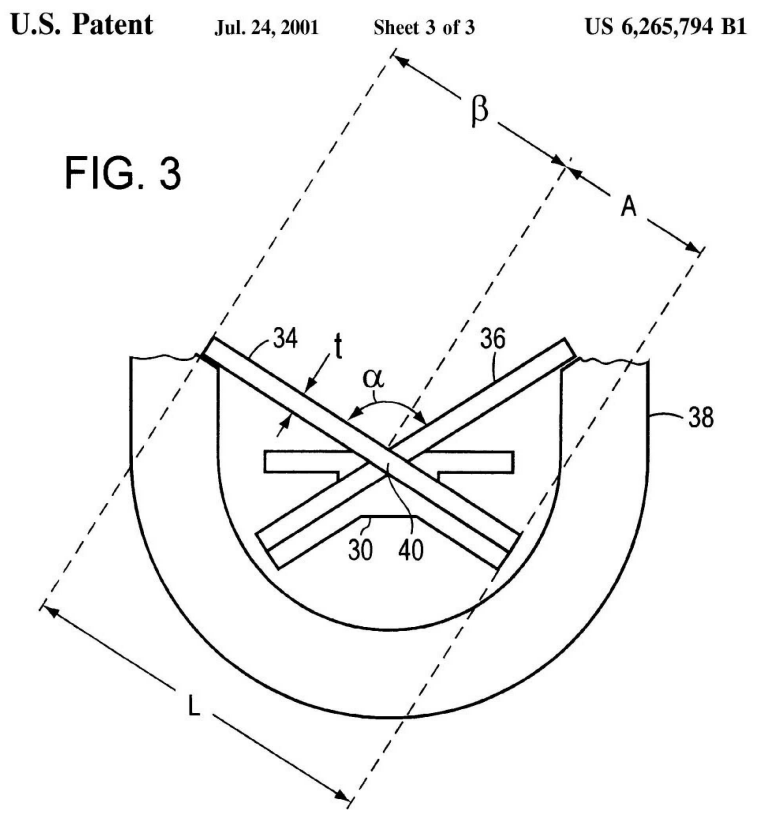

Figure 2-33: Crossed-blade flexures for supporting a shaft in a galvanometer. From DeBolt [45], U.S. Patent 6,265,794. 


\subsection{Conclusions}

A major motivation for our sponsor's interest in this research was to develop new fast tool servos that would allow extending the current capability to diamond turn precision surfaces on target components for high energy density physics experiments in support of the Science-based Stockpile Stewardship program for the United States.

When machining from the pole to the equator on a spherical workpiece, the disturbance to the diamond turning machine from the reaction torque from a rotary-type FTS can be smaller and more uniform than the disturbance caused by the reaction force from a linear-type FTS. A survey of a large assortment of fast tool servos described in the literature indicates that most of them are linear types, and that the $2 \mathrm{kHz}$ and $10 \mathrm{kHz}$ rotary fast tool servos developed in this research establish new benchmarks for bandwidth, tool stroke, and maximum closed-loop tool tip acceleration.

Most of the electric engraving heads that I found in the literature utilize a rotary architecture. The literature on engraving heads is exclusively - as far as I could find - in the form of patents, with prior art from at least as far back as 1937 teaching concepts that are pertinent to this research. Of particular interest are the normalstress variable reluctance rotary actuators employing flux biasing, bearing systems, damping, and tool mounting.

Having established the motivation for this research and a framework of prior art to provide context for the $2 \mathrm{kHz}$ and $10 \mathrm{kHz}$ rotary fast tool servos, we are ready to consider those two new machines and the hybrid rotary/linear actuator. 


\section{Chapter 3}

\section{The $2 \mathrm{kHz}$ Rotary Fast Tool Servo}

In this chapter I describe the development and measured performance of the $2 \mathrm{kHz}$ rotary fast tool servo (2 kHz FTS). I begin by reviewing the philosophical guidelines for the design, and survey the major conceptual designs that were considered. Additional philosophical background in machine design is provided through discussions on designing for stiffness, a trade-off between manufacturing tolerances and assembly complexity, and incorporating contingencies in the design. I then explore the $2 \mathrm{kHz}$ FTS through detailed discussions on the design, analysis, and fabrication associated with the mechanical design, system dynamics, electrical design, and the control system. The chapter concludes by presenting the major test results for the machine.

\subsection{Introduction}

The $2 \mathrm{kHz}$ FTS is shown in Figure 3-1. Figure 3-2 provides context for this machine by showing it mounted on a diamond turning machine and engaging a workpiece. The $2 \mathrm{kHz}$ FTS provides the following proof-of-principles for the later $10 \mathrm{kHz}$ rotary fast tool servo: over-constrained flexures for guiding reciprocating rotary motion, small diamond tool and mounting method, high bandwidth current-mode amplifier, and control system design. It was also a vehicle for me to advance my knowledge of electronics and control systems enough to pursue the more technically challenging $10 \mathrm{kHz}$ FTS. Furthermore, the $2 \mathrm{kHz}$ FTS validates that a rotary-type fast tool servo 
can be used to produce optical quality surfaces on a spherical workpiece from its pole to its equator.

\subsection{Design Philosophy}

The philosophy that guided the design of the $2 \mathrm{kHz}$ rotary fast tool servo included the following points:

- Develop a fast tool servo that can be integrated with an auxiliary rotary axis on a diamond turning machine and used to machine a $10 \mathrm{~mm}$ diameter or smaller spherical or cylindrical workpiece.

- Start with a reasonably smallest commercially available diamond tool, then design the fast tool servo around that tool.

- Design for stiffness at the tool tip. Radial and axial stiffness of the swingarm that carries the tool are provided by the mechanism, torsional stiffness is provided by the controller.

- Design a flexure bearing system that can be easily serviced if one or more of the flexure blades is damaged.

- Place a position sensor as close to the tool as reasonably possible to hide certain flexible modes of the system and improve high frequency controllability.

- Use a commercially available actuator to allow focusing early efforts on development of the mechanism, the current-mode amplifier, and the hybrid analogdigital control system needed for the high bandwidth FTS's described in this thesis.

- Develop a servo test bed that uses the same actuator, sensors, and payload inertia as the $2 \mathrm{kHz}$ FTS to allow developing the current-mode amplifier and control system in parallel with the development of the $2 \mathrm{kHz}$ FTS mechanism. 


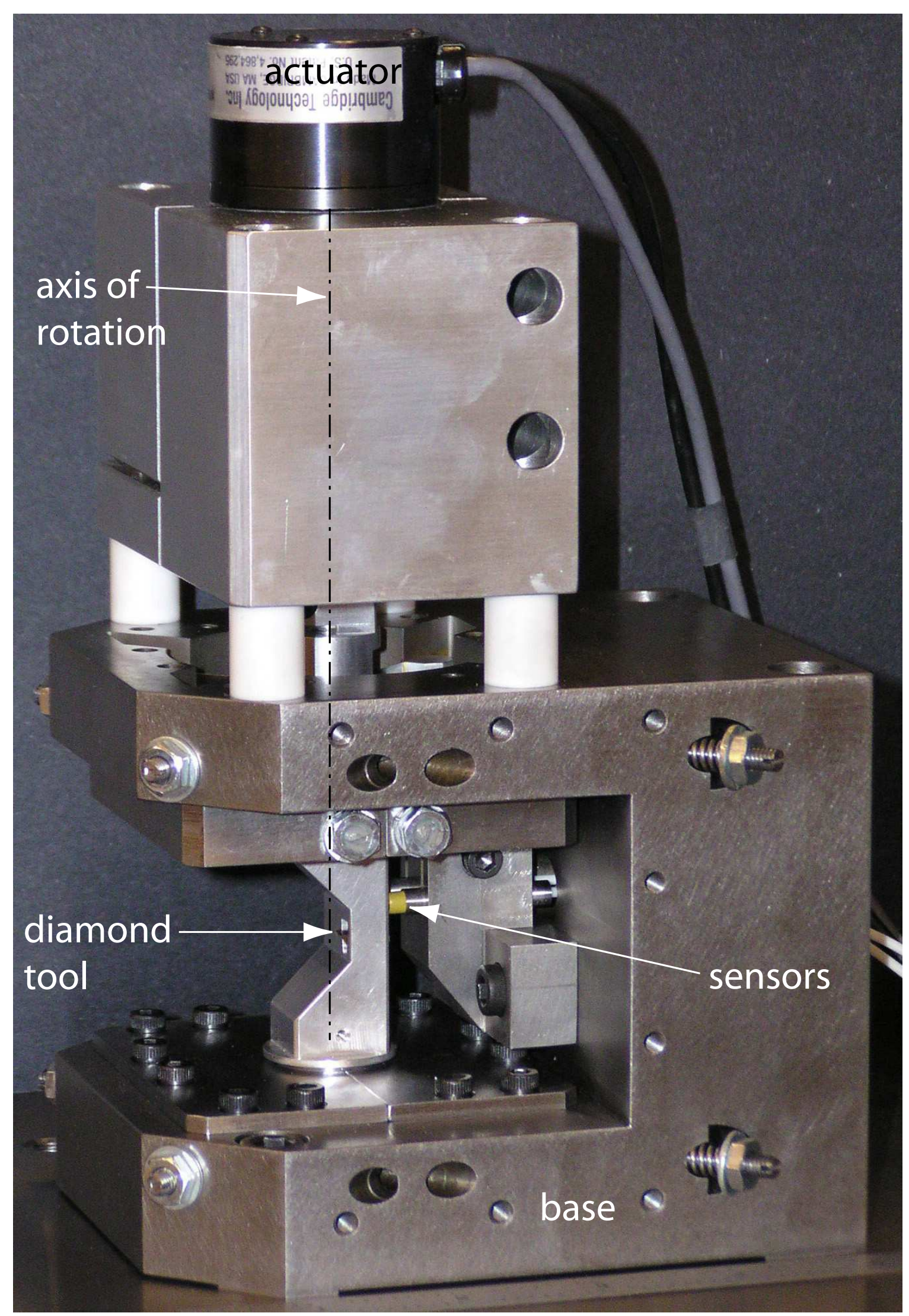

Figure 3-1: The $2 \mathrm{kHz}$ rotary fast tool servo. 


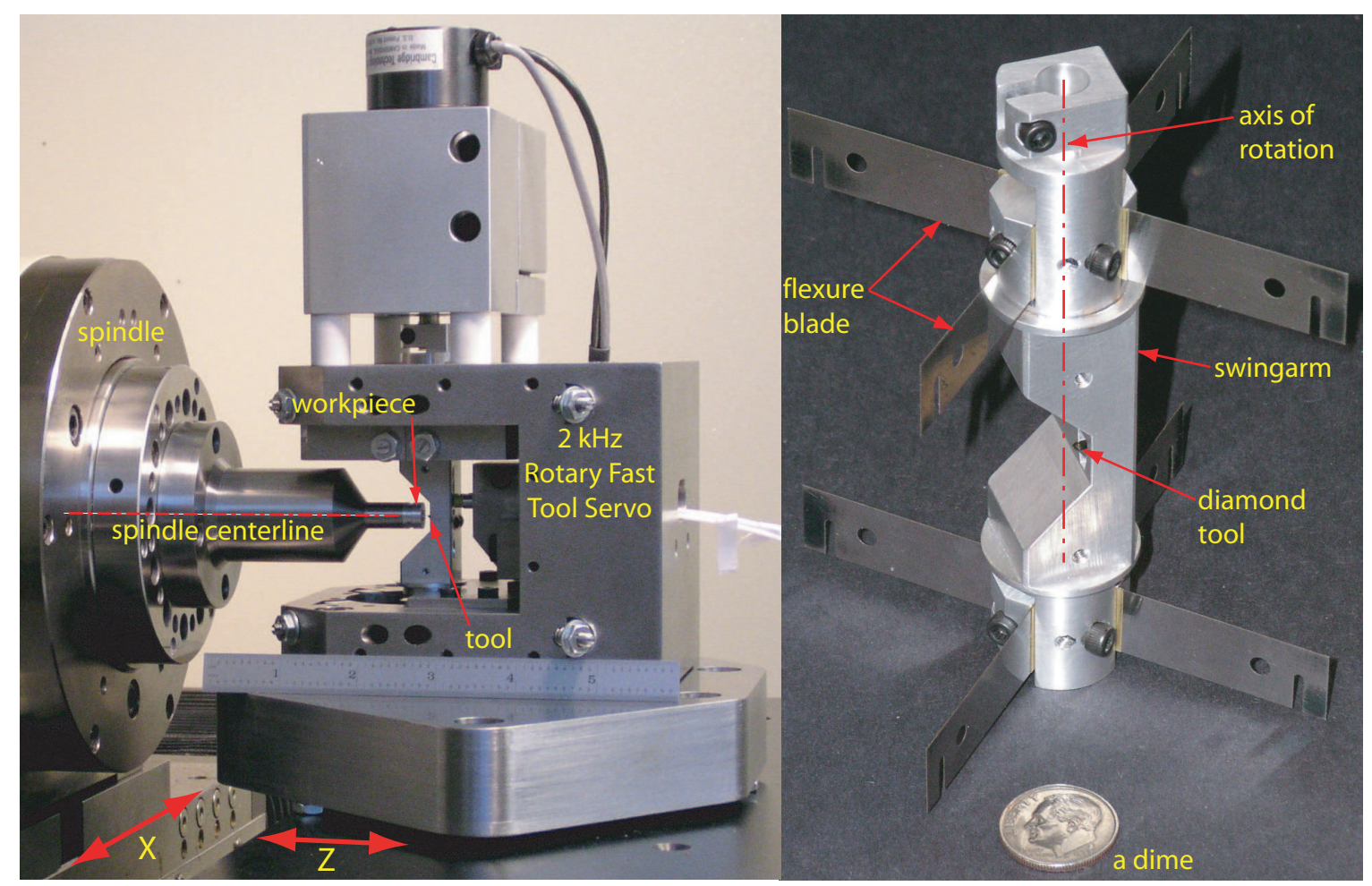

Figure 3-2: The $2 \mathrm{kHz}$ rotary fast tool servo on a diamond turning machine (left), and its rotating element not including the actuator (right).

\subsection{Machine Topology Concepts}

Figure 2-3 in Section 2.2.2 shows how a fast tool servo could be integrated with a diamond turning machine and a rotary table to machine a sphere from its equator to its pole. Early concepts for the $2 \mathrm{kHz}$ FTS considered if its axis of rotation should be oriented in a vertical or horizontal position, and also explored the possibility of using a linear fast tool servo for machining a spherical workpiece. The left-hand sketch in Figure 3-3 depicts a T-base lathe carrying a horizontal-axis workpiece spindle on an $\mathrm{X}$-axis slide-way, and a Z-axis slide-way carrying a B-axis rotary table upon which a rotary fast tool servo would be mounted. The right-hand sketch in Figure 3-3 shows a can-shaped vertical-axis rotary FTS mounted on the B-axis rotary table, and the center sketch shows a horizontal-axis rotary FTS mounted to that rotary table. Note that the reaction torque $\left(\mathrm{T}_{R}\right)$ from the vertical-axis FTS causes a yaw disturbance to the Z-axis slide-way that is independent of the rotation angle for the B-axis rotary table. In the case of the horizontal-axis FTS, the reaction torque causes a pitch or 
roll disturbance to the Z-axis slide-way that depends on the rotation angle of the rotary table. For the T-base lathe shown, a pitch disturbance to the Z-axis slideway is more likely to couple into a vibration mode that causes motion of the tool towards and away from the workpiece, and suggests that a vertical-axis rotary FTS is preferred. Another reason for a vertical-axis rotary FTS is that the B-axis rotary table can be used as a reaction mass to absorb the reaction torque produced by the FTS, as was described in Section 2.2.2. Yet another motivation for a vertical-axis rotary FTS is that for the T-base lathe shown in Figure 3-3, a combination of X, Z, and $\mathrm{B}$ motions allow the tool to reach all points on the workpiece, whereas the same cannot be said for the horizontal-axis rotary FTS (specifically at the center of the workpiece).

Figure 3-4 is a sketch showing a layout using a linear-type fast tool servo on an R- $\theta$-X lathe to machine a sphere from its equator to its pole. The FTS in Figure 3-4 is the small appendage shown at the top-front of the R-axis slide-way. Note that the reaction force from the linear-type FTS causes a disturbance to the lathe that depends on the angle $\theta$, and that the R-axis slide-way can be used as a reaction mass to absorb that reaction force. An alternative to the layout shown in Figure 3-4 is to replace the rotary FTS in Figure 3-3 with a linear FTS and a reaction mass. Since T-base lathes are readily available — we had one for this project — and compensating the reaction force in a linear-type FTS adds additional layers of complexity, we chose to pursue a vertical-axis rotary topology for the $2 \mathrm{kHz}$ FTS and the follow-on $10 \mathrm{kHz}$ rotary fast tool servo.

\subsection{Designing for Stiffness}

Many ultra-precision machine tools have a complex arrangement of multiple slideways that provide multiple degrees of freedom of motion between the tool and the machine base. Formal machine motion error budgets are often used to keep track of how the various error motions from each component contribute to the net error motion of the tool relative to the base and ultimately relative to the workpiece, and 


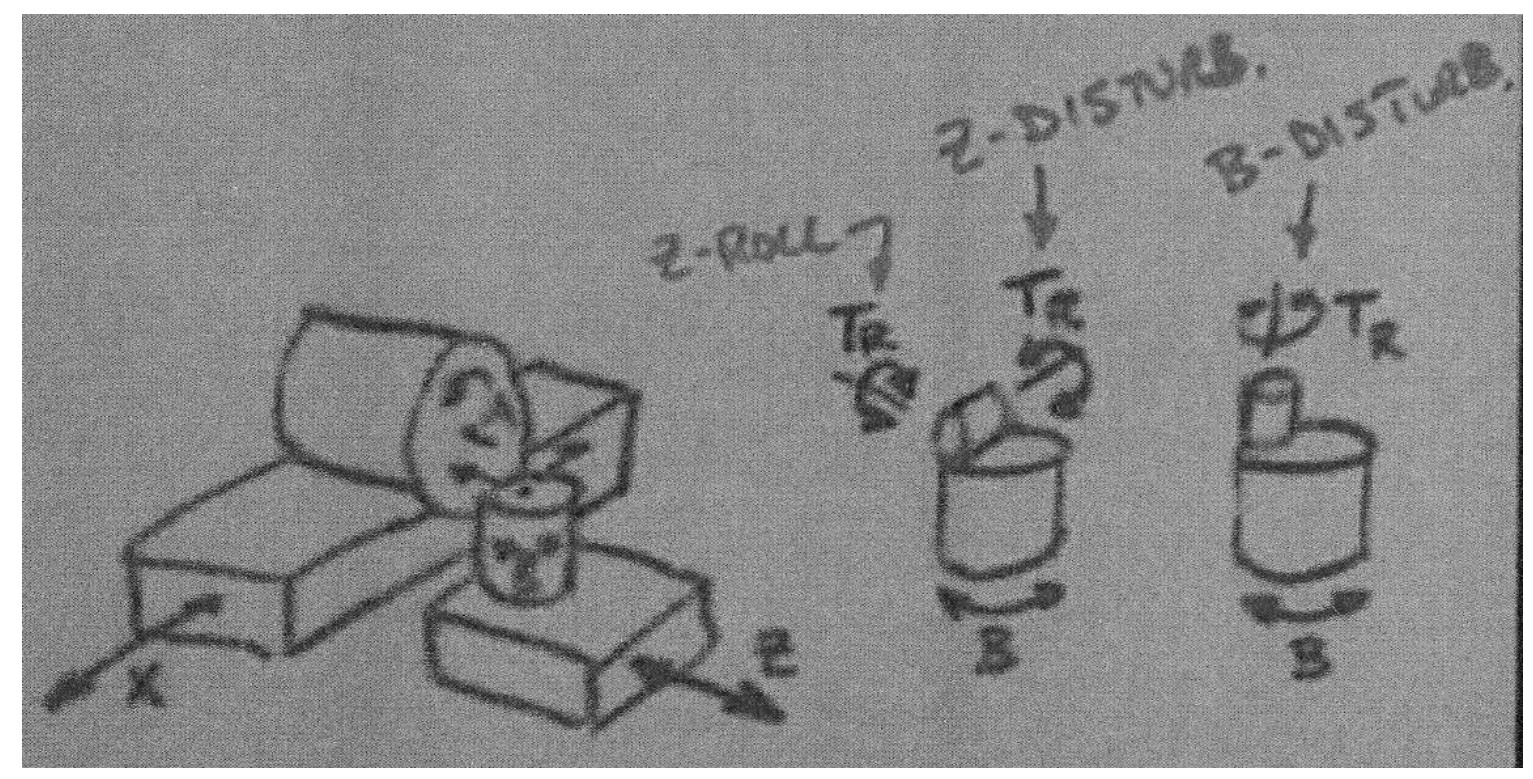

Figure 3-3: Concept sketch for a vertical-axis and a horizontal-axis rotary fast tool servo used with a T-base lathe and a rotary table for machining a spherical workpiece.

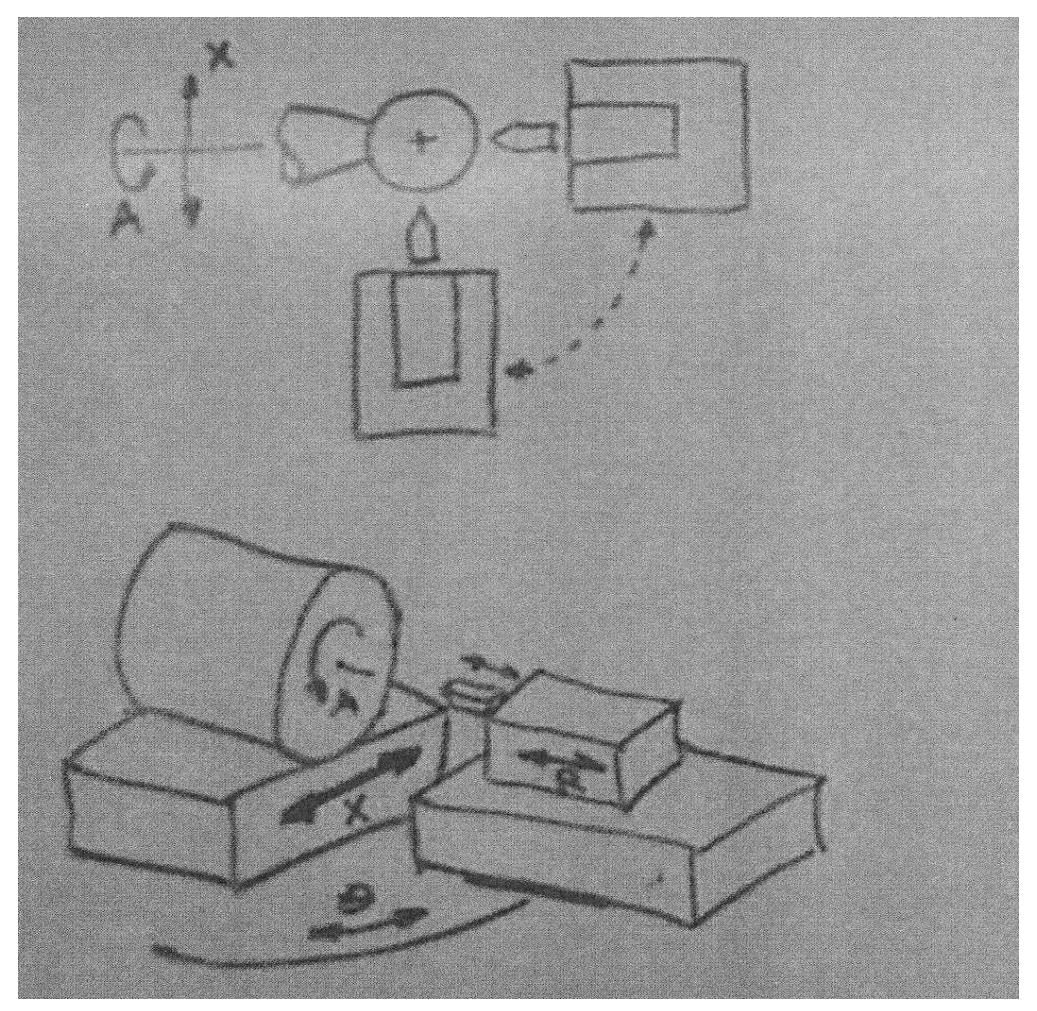

Figure 3-4: Concept sketch for a linear-type fast tool servo used with an R- $\theta-\mathrm{X}$ lathe for machining a spherical workpiece. 
to specify acceptable limits for each contribution to that net error. The $2 \mathrm{kHz}$ FTS and the follow-on $10 \mathrm{kHz}$ rotary fast tool servo do not have formal error budgets for the motion between the tool and the base because there is only one degree of freedom of motion for each of those machines. ${ }^{1}$ Instead, an informal stiffness budget was used to guide the mechanical design for these machines.

The stiffness of the tool tip relative to the base is provided by two things: the mechanical stiffness of the mechanism and the dynamic stiffness provided by the controller. The radial and axial stiffness of the rotor that carries the tool is provided by the flexures, their attachments to the rotor and the base, the rotor itself, and the base. The stiffness of the tool is also involved, as is the connection between the tool and the rotor. The torsional stiffness of the rotor is provided by the closed-loop dynamic stiffness of the actuator. The stiffness budget viewed all of the mechanical components and interfaces from the tool to the base as springs, and set a net stiffness for the assembly of at least $20 \mathrm{~N} / \mu \mathrm{m}$ in the radial and axial directions. As a starting point, all of the components and interfaces were allowed the same stiffness. If the design of a particular component or joint started to be driven too hard by this requirement then it was relaxed for that aspect of the machine and tightened up elsewhere. I mentioned that the stiffness budget was informal: I kept track of it loosely in my notes and firmly in my mind. The point of this project was not to belabor the mechanical design any more than necessary to assure that the mechanism would not be the source of difficulty when attempting to demonstrate the performance goals. Therefore, I reserved analytical rigor and strict bookkeeping for only those aspects of the project that absolutely needed it.

My previous experience designing and building precision machine tools had taught me that a good rule-of-thumb was that the stiffness at the tool tip relative to the machine base needed to be at least $20 \mathrm{~N} / \mu \mathrm{m}$, and that the same stiffness was required for the workpiece relative to the machine base. The following discussion provides a simple quantitative basis for this philosophy.

\footnotetext{
${ }^{1}$ Although the actuator for the $10 \mathrm{kHz}$ FTS has a second, linear degree of freedom, the deflections caused by it are negligible in this work.
} 
I tend to think of precision mechanical hardware in terms of compliance and in U.S. customary units. A $20 \mathrm{~N} / \mu \mathrm{m}$ stiffness is equivalent to a $10 \mu \mathrm{in} / \mathrm{lb}$ compliance. In ultra-precision single-point machining, final depths of cut are often on the order of $20 \mu \mathrm{in}(0.5 \mu \mathrm{m})$ or less. The two major forces that develop between the tool and workpiece are a thrust force that tends to push the two objects away from each other, and a cutting force that is parallel to the major relative velocity vector between the tool and workpiece. The shallow depths of cut typically used develop small thrust and cutting forces, and can be bounded by the following example.

A negative rake angle ${ }^{2}$ tool is used when machining certain crystalline optics, and a layer of cutting fluid at least 0.001 inch $(25 \mu \mathrm{m})$ thick covers the workpiece [108]. While studying the machining process for these crystalline optics in a previous project, we measured thrust and cutting forces that were as high as $1 \mathrm{lb}$, and determined that they were fairly independent of the depths of cut that were used [107]. Considering that the thickness of the oil film was approximately two orders of magnitude greater than the minimum depth of cut, and that the negative rake angle of the tool would tend to make it ride up on the oil, we concluded that the hydrodynamic forces that developed between the tool and oil were the major contributors to the observed thrust and cutting forces.

Using $1 \mathrm{lb}$ as an upper limit for the thrust and cutting forces developed during single-point ultra-precision machining, consider what a thrust force of that magnitude would do to the tool tip and workpiece if they both have a compliance of $10 \mu \mathrm{in} / \mathrm{lb}$. Both will deflect $10 \mu$ in away from each other, producing a net relative deflection of $20 \mu \mathrm{in}$ between the two. If the desired depth of cut was $20 \mu \mathrm{in}$, then the tool and workpiece would have pushed each other completely out of engagement and the tool would be cutting only the cutting fluid that was on the workpiece. The cutting force acts in the non-sensitive direction ${ }^{3}$ and therefore not as much of an issue for

\footnotetext{
${ }^{2}$ The rake angle is the angle between the normal of the workpiece where it contacts the tool and the surface of the tool that is removing material. Example of a positive rake angle tool: the blade of a hand-held snow shovel being pushed along a snow-covered driveway; it peels the snow up from the pavement. Example of a negative rake angle tool: a butter knife spreading butter onto a piece of bread; it smears the butter down onto the bread.

${ }^{3}$ The sensitive direction is normal to the workpiece at the contact point with the tool, and is the direction that establishes the depth of cut. The non-sensitive direction is perpendicular to this
} 
maintaining contact between the tool and workpiece. It is worth mentioning that both the thrust force and cutting force can be associated with the regenerative chatter that occurs in certain single-point machining operations. Designing ultra-precision machine tools with a tool tip stiffness of $20 \mathrm{~N} / \mu \mathrm{m}$ or greater has served me well for avoiding that issue.

\subsection{Manufacturing Tolerances,}

\section{Assembly Complexity, and Contingencies}

When considering the geometric form, fit, and dimensions for every component in a machine, the designer can trade off manufacturing tolerances against the complexity of assembling the machine. All fabricated components have some level of error, so the designer balances the acceptable level of those errors against the degree of adjustability needed to allow assembling the machine and correcting for accumulated errors. For the $2 \mathrm{kHz}$ FTS, and later for the $10 \mathrm{kHz}$ FTS, I tended towards looser tolerances than what a person might expect are needed for the components for a precision machine tool, and accepted the slightly increased complexity of assembling machines that had provisions for adjusting critical alignments between key components. A second benefit of a design that allows adjusting the as-assembled components is that it can allow contingencies for modifying certain characteristics of the machine, such as stiffness, by changing key components.

One of the guidelines that I followed for these machines was that all of the mechanical components would be manufactured to standard machine shop form and dimension tolerances: \pm 0.001 inch to \pm 0.002 inch $( \pm 25 \mu \mathrm{m}$ to $\pm 50 \mu \mathrm{m})$ for most of the tighter tolerances, and \pm 0.0002 inch to \pm 0.0005 inch $( \pm 5 \mu \mathrm{m}$ to $\pm 12 \mu \mathrm{m})$ for the occasional ground surface. The $2 \mathrm{kHz}$ FTS has over 85 mechanical components, and the $10 \mathrm{kHz}$ FTS has over 100 mechanical components. Both are prototype machines, and everyone involved with them is seeing things for the first time; from me as the designer, engineer coordinating fabrication, and technician assembling the sensitive direction. 
machine, to the machinists receiving a print for a new part. Parts do not always go together as intended, even if they meet the manufacturing tolerances that were set for them, and manufactured parts do not always meet their tolerances. Tightening manufacturing tolerances not only drives up the cost for a component, it also increases the time needed to fabricate it and increases the risk that it fails to meet that tolerance. Furthermore, time is lost when a component has to be re-manufactured, or when a new vendor has to be found and qualified. In the end, when building a prototype and trying to adhere to some semblance of a schedule, ${ }^{4}$ you end up having to work with what you get. Instead of designing for an idealized world were everything goes together as planned, I provided strategic fall-back positions so that I could make due with the as-machined components that I received. Being able to successfully assemble a prototype machine that has a few more pieces than might be needed in future versions goes a lot faster than not being able to assemble a prototype that lacked the extra pieces needed for contingencies.

As an example, consider the brass shims that are visible at the joints between the flexure blades and the swing-arm in Figure 3-7. The main motivation for these shims was to provide contingency in case my stiffness calculations for the flexure bearing were incorrect to the extent that the as-built measured stiffness was unacceptable. If this were to happen, I could use thicker flexure blades and thinner shims, and not have to modify the more complicated swing-arm which had a significant lead-time to manufacture. As it turned out, the as-built stiffness was close to my predictions and acceptable. A second motivation for those shims was that they helped avoid tightening the manufacturing tolerances on the cut-outs in the swing-arm for the flexures. Using standard machine shop tolerances meant that those cut-outs might end up over-sized or under-sized relative to the nominal thickness of the flexures. Furthermore, the upper set of cut-outs might not be adequately aligned with the lower set of cut-outs. Rather than tighten the tolerances for these cut-outs, I could selectively adjust the shims to move the effective mating surface for each flexure blade as needed.

\footnotetext{
${ }^{4}$ I say "semblance" because this is research, after all.
} 


\subsection{Mechanical Design}

Referring to Figure 3-1, the swing-arm carries a diamond tool at a swing radius of $5 \mathrm{~mm}$ and has a full-travel rotation angle of $\pm 5 \mathrm{mrad}$. The small rotation angle allows using over-constrained flexures to guide the rotary motion. A total of eight flexures are used, four above the tool and four below it. The inner ends of the flexures are fixed to the swing-arm, and the outer ends are fixed to the base. An advantage of a small swing radius for the tool is that the unwanted transverse motion of the tool tip is only $\pm 63 \mathrm{~nm}$ for the full travel of $\pm 25 \mu \mathrm{m}$, making it negligible for most diamond turning applications. A drawback of using a small swing radius is that the actuator acceleration is de-magnified as reflected into tool tip acceleration. ${ }^{5}$ All of the mechanical components and the actuator for the $2 \mathrm{kHz}$ FTS are shown in Figure 3-5. The are more than 85 individual parts, and the design required 50 sheets of detailed mechanical drawings.

\subsubsection{Tool}

Achieving a high tool tip acceleration with a rotary fast tool servo requires applying a high torque to a small moving mass. Many of the fast tool servos described in the literature use the same heavy-shanked tools intended for the fixed tool posts used in conventional turning operations. In some cases, the mass of some of those tools is close to the total moving mass of the swing-arm plus tool for the $2 \mathrm{kHz}$ FTS. Although the $2 \mathrm{kHz}$ FTS was not intended to establish a new benchmark for tool tip acceleration, one of the proof-of-principles that it provided for the follow-on $10 \mathrm{kHz}$ rotary fast tool servo is the tool and its method of attachment. Knowing that the $10 \mathrm{kHz}$ FTS would require as small a tool as reasonably possible, I began the mechanical design for the $2 \mathrm{kHz}$ FTS with the tool. ${ }^{6}$ A typical diamond tool consists of a tool shank that holds

\footnotetext{
${ }^{5}$ De-magnified relative to the acceleration of the moving element of the actuator. Ludwick [99] describes that using a tool swing radius that is larger than the radius of the coil in a moving-coil actuator allows magnifying the acceleration produced by the coil. As mentioned in Section 2.2.2 this is one of the benefits of a rotary topology over a linear one for a fast tool servo.

6 "Reasonable" meant a tool that could be manufactured by a commercial vendor using existing techniques and equipment, at a price commensurate with its standard tools.
} 


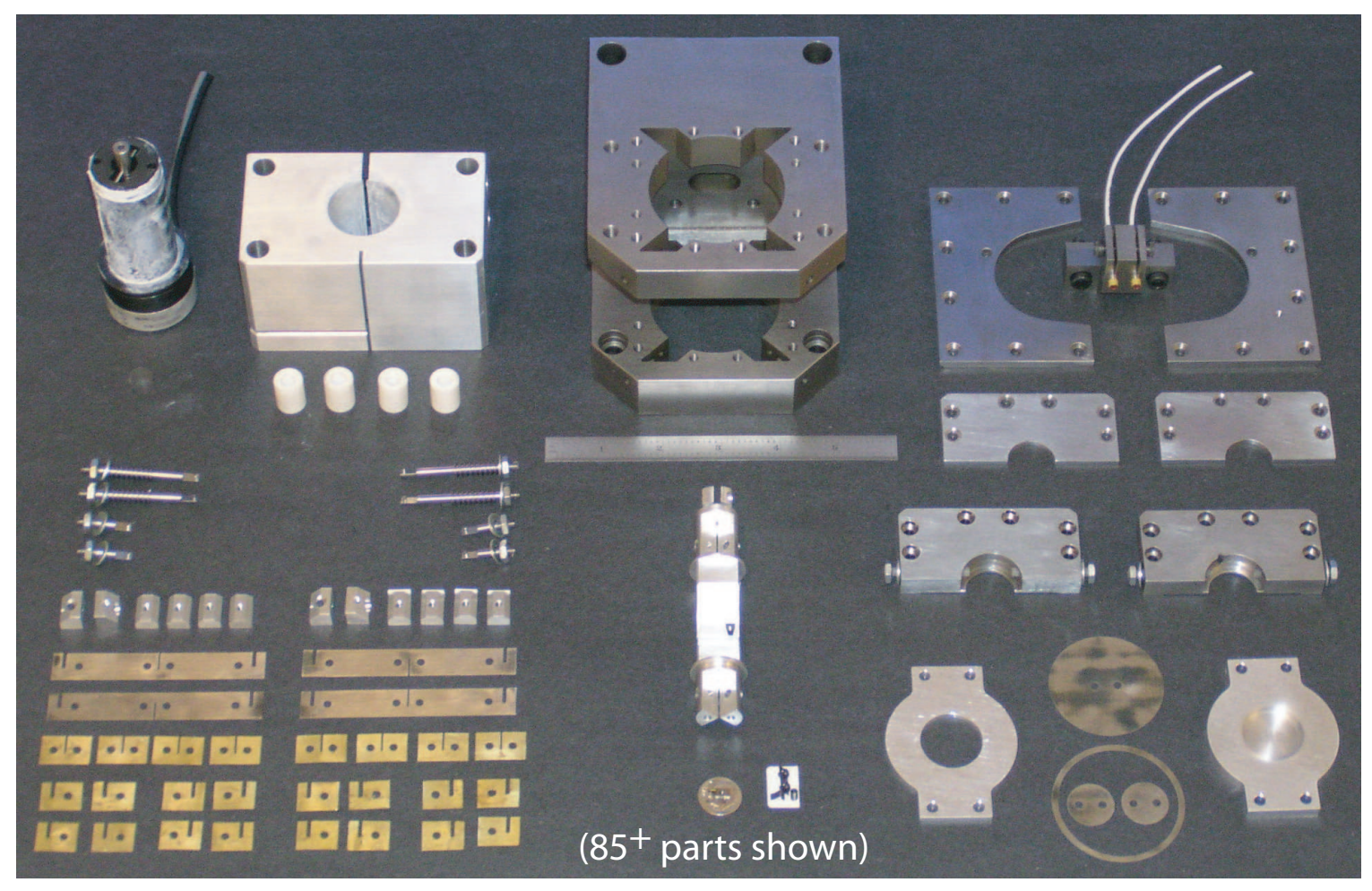

Figure 3-5: Mechanical components and actuator for the $2 \mathrm{kHz}$ FTS.

a gem-quality diamond having an accurately defined cutting edge. The diamond is bonded to the tool shank, and the tool shank provides a larger and tougher mechanical element for mounting the tool to the machine tool that uses it as a cutter. A diamond is used because the cutting edge can be made significantly sharper than the edge of an equivalent metal or ceramic tool. Furthermore, the high thermal conductivity of diamond allows it to dissipate the heat generated at the tool-workpiece interface and prevent the fine cutting edge from rapidly breaking down from thermal effects.

Figure 3-6 shows the diamond tool used in both the $2 \mathrm{kHz}$ FTS and the $10 \mathrm{kHz}$ FTS. The nose of the tool is in the form of an inverted right-angle cone whose base is the top surface of the diamond tip. The cutting edge is defined by the intersection of that cone and the top surface, and has a radius in the plane of the top surface of $25 \mu \mathrm{m}$. This tool was manufactured by Chardon Tool [1], weighs only 0.4 gm (diamond plus steel shank), and turns out to be a fairly standard size and geometry. I had previous experience with Chardon Tool, so I called them and talked with Mike Hunter. After describing what I was attempting to build and what I was looking for in a tool, Mike 
told me that some of the standard tools that they manufactured for electric engraving heads were similar in size to what I needed. ${ }^{7}$ He mentioned that some of the smaller tools used a taper to increase the clamping force between the tool and its holder, and suggested that I consider doing the same. Referring to Figure 3-6, the sides of the tool shank form a $30^{\circ}$ taper angle that is received by a matching tapered slot in the swing-arm. The tool is a blend of the small shank size and style used with certain electric engraving heads, and the diamond tip style typically used for single-point ultra-precision machining.

To insure a high stiffness connection between the tool and the swing-arm, I added a relief to the central portions of the two faces of the tool that contact the receiving slot to establish exactly four deterministic areas of contact between the tool and the slot. These prescribed areas of contact allow avoiding the possibility of the tool and slot making contact at or near the central portions of the contacting faces, which would reduce the stiffness of the connection between the tool tip and the tool holder and could lead to a bi-stable orientation of the tool relative to the holder. Conceptually, the four pads on the tool shank and the two receiving surfaces of the slot are the inverse of a cylinder supported by two V-blocks. Although the cylinder in this case has an infinite radius, the small contact areas provide elastic averaging and an attendant rigid connection.

A leaf spring is used to transmit the hold-down force from a setscrew to the center of the tool shank. The leaf spring isolates the tool from the screw rotation which would have tended to grab and move the tool during tightening. Also, since the axis of the hold-down screw is not perpendicular to the top of the tool shank, the leaf spring isolates the tool from the force component produced by the screw that would have pushed the tool forward during tightening. ${ }^{8}$ Hidden under the leaf spring in Figure 3-6 is a 1-72 thru-hole in the tool shank that allows two alternate ways of seating the tool in a tapered slot: pulling down with a 1-72 screw that comes up from

\footnotetext{
${ }^{7}$ This was a portent of what I would later discover about electric engraving heads during my literature search, which occurred after this conversation with Mike.

${ }^{8}$ The hold-down screw comes in from the back of the swing-arm at an angle of $70^{\circ}$ from the top of the tool shank. This angle allows accessing the hold-down screw without having to disturb the flexure mounting hardware at the top or bottom of the swing-arm.
} 


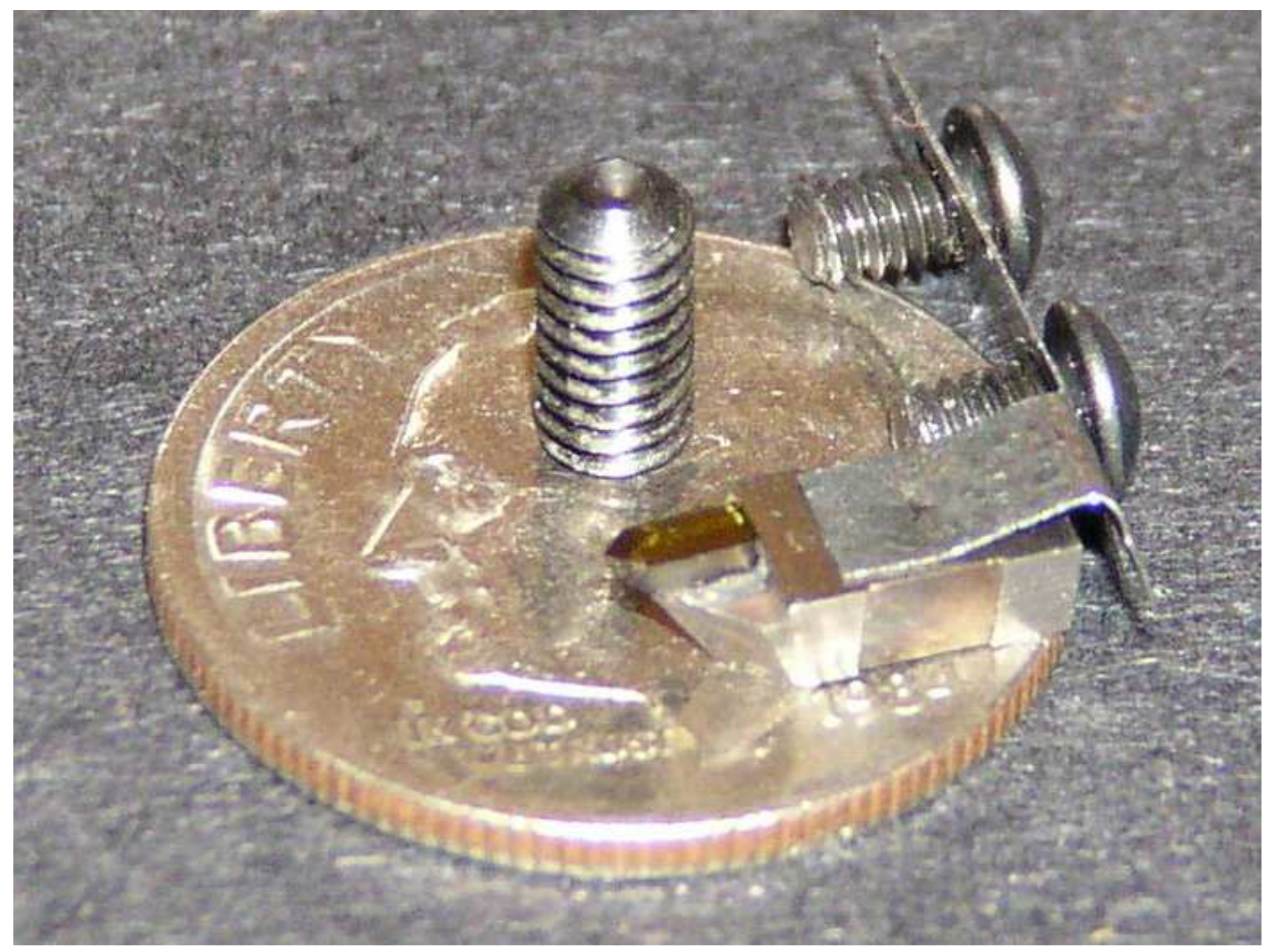

Figure 3-6: Diamond tool with clamping hardware sitting on a dime.

below, or pushing down with an 0-80 screw - whose threads pass through the hole with clearance - from above. The $10 \mathrm{kHz}$ FTS described in Section 4-1 uses this last method to realize a light-weight attachment of the tool to the toolholder.

Certainly a very light-weight approach would be to omit the tool shank and bond the diamond directly to the moving element of the fast tool servo. However, this would have turned the tool into a development project that would have competed with the more important technical challenges for this project. Specifically, special tooling would be required for lapping and polishing the diamond tip. Furthermore, removing and replacing a worn or damaged cutting edge would involve significantly disturbing the FTS mechanism in comparison to using a tool with a shank. Bonding the diamond directly to the moving element of a fast tool servo is still worth considering for future work that attempts to optimize the torque-to-inertia ratio. 


\subsubsection{Swing-Arm (Rotor)}

The rotor for the $2 \mathrm{kHz}$ FTS is referred to as the "swing-arm" because its main function is to hold the tool a fixed distance, the so called swing radius, from the axis of rotation. The swing-arm with its flexure bearing and a diamond tool is shown in Figure 3-7. The tool is mounted in a tapered slot, and is offset $5 \mathrm{~mm}$ from the axis of rotation. To minimize the parasitic lateral motion of the tool tip caused by motion along an arc, the tool tip is positioned in a front-back plane that contains the axis of rotation. The central large cut-out in the swing-arm allows a workpiece to reach the tool tip. The upper and lower portions of the swing-arm that hold the flexure blades are referred to as the flexure hubs. Each flexure hub has two $90^{\circ}$ cut-outs that intersect at the centerline of the swing-arm. These cut-outs have matching clamping blocks that fix the inner ends of the flexure blades to the swing-arm. Just inboard of the flexure hubs is an upper and lower flange that form part of a labyrinth seal with the base that supports the swing-arm. The split-clamp rigid coupling at the top of the swing-arm receives the output shaft of the actuator. A $125 \mu \mathrm{m}$ thick plastic shim lines the inside surface of this coupling, and accommodates residual misalignment between the swing-arm axis of rotation and the actuator axis of rotation after the two are mated. A rigid coupling was feasible because the full range of angular travel for the swing-arm is only $\pm 5 \mathrm{mrad}$. The rotational inertia for the swing-arm, including the tool, the clamping hardware, and fasteners, is $51 \mathrm{gm} \cdot \mathrm{cm}^{2}$.

\subsubsection{Flexure Blades}

The swing-arm is supported by an array of over-constrained flexure blades that extend radially from the swing-arm and are clamped at both ends: at the swing-arm and at the base. This flexure bearing satisfies the following requirements: (1) guide the rotary motion of the swing arm and establish a non-wandering center of rotation; (2) add low to negligible rotational inertia to the moving mass; (3) survive oscillatory motion without requiring maintenance; (4) be stiff enough to support the cutting tool to maintain a desired depth of cut when using the FTS to single-point machine optical 


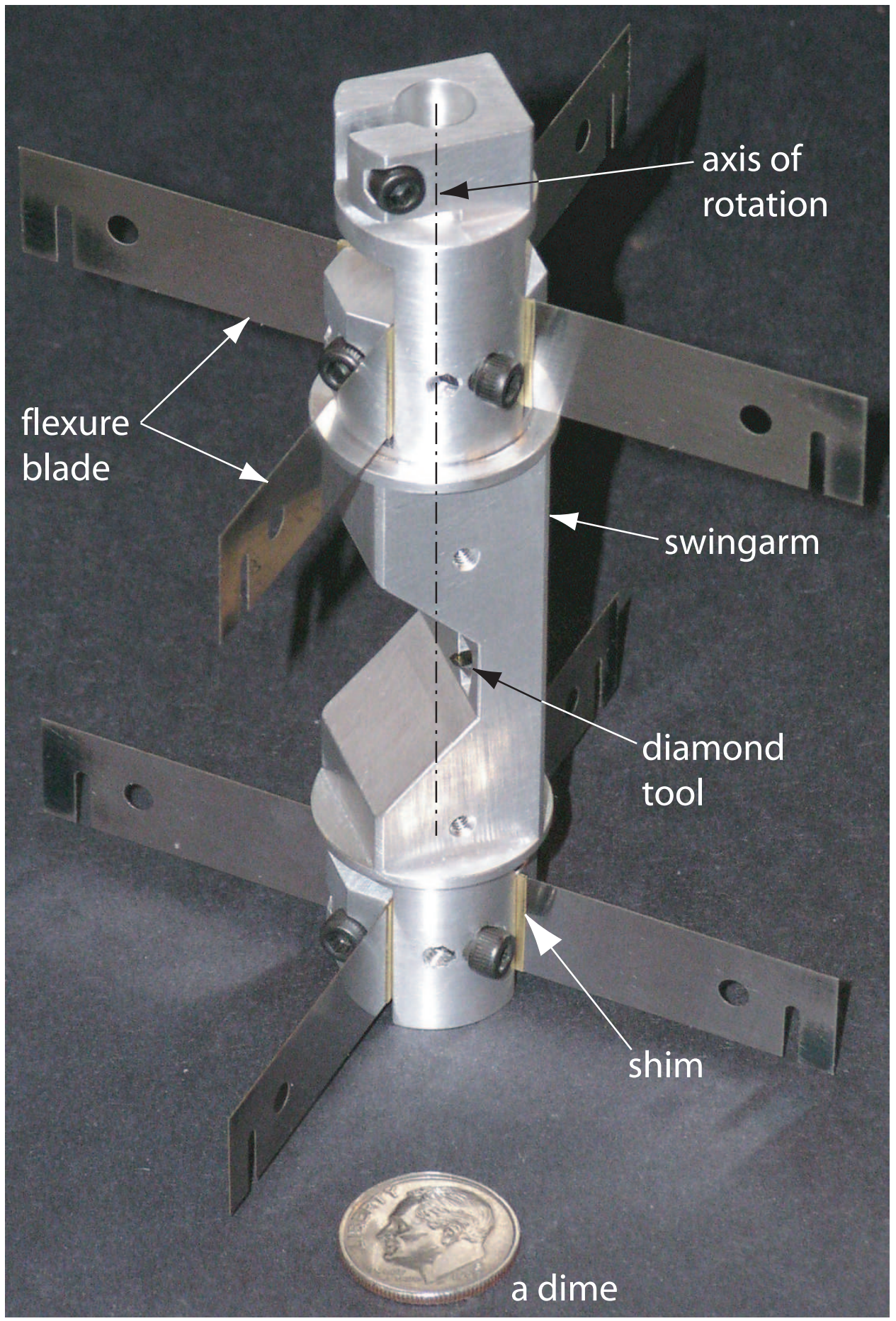

Figure 3-7: The swing-arm (rotor) with its flexure bearing and a diamond tool for the $2 \mathrm{kHz}$ FTS. 
quality surfaces on a workpiece; and (5) be serviceable if damaged. An example of a more typical crossed-blade flexure bearing was discussed in Section 2.4.4 and shown in Figure 2-33. In that case the axis of rotation moves when the shaft angle changes. Smith [138] provides an analysis of a crossed-blade flexure bearing and of a related cartwheel hinge whose axis of rotation also moves when the shaft angle changes, albeit less than it does for the crossed-blade bearing. Of course, the non-wandering axis of rotation associated with over-constrained flexure blades comes with a price: the tensile stresses increase more rapidly with rotation angle than it does with the crossed-blade or cartwheel hinge bearings. However, a significant benefit of the over-constrained flexure blade design is that the flexures can be readily preloaded in tension. The crossed-blade bearing and cartwheel hinge are both cantilever type supports, which makes preloading them in tension difficult unless they are used in opposing pairs, in which case they become over-constrained flexures.

At $2 \mathrm{kHz}$ operation, the flexure blades undergo one million cycles of a fluctuating stress in approximately eight minutes, so fatigue life is a crucial consideration. The flexure blades and their clamping hardware add less rotational inertia to the moving mass than a comparable stiffness ball bearing would, and are much more tolerant to the small amplitude oscillatory motion that would likely cause fretting failure in a rolling element bearing. I chose steel as the material for the flexure blades for two reasons. The first is because steel has an endurance limit; the material can survive an infinite number of cycles if the stresses remain below the endurance limit, which is one-half of the yield strength for steels having a tensile strength up to 200 ksi. For steels with a higher tensile strength, the endurance limit is approximately $100 \mathrm{ksi}$ [137]. The second, and perhaps more compelling practical reason, is that steel was a straightforward material for me to fabricate the flexures from in the machine shop at MIT that I had access to.

Figure 3-8 shows four flexure blades and the pre-tensioning hardware used with them. To ease manufacturing and assembly, pairs of collinear flexures are formed from a single piece. Each flexure blade is a 0.006 inch thick by 0.500 inch wide by 0.750 inch long piece of 1095 blue-tempered spring steel that was polished along its long axis 
to minimize initiation of fatigue cracks, and is preloaded with a $10 \mathrm{lb}$ tensile force before clamping the ends. This pre-tension is intended to assure that each flexure blade remains in tension when the swing-arm is rotated through its working range, helping to maintain a non-wandering axis of rotation. The pre-tensioning devices consist of adjustable front anchor points and spring-loaded rear anchor points. Hooks on the ends of the anchors engage the open slots at the outer ends of the flexure blades. Adjusting the location of the nuts on the two front anchor points changes the position of the intersection of the flexure blades in the plane formed by the four blades. The compression springs in the rear anchor points bear against a stationary base and provide a nearly constant pre-tension force ${ }^{9}$ in the flexure blades for small adjustments of the front anchor points. The holes in the flexure blades are clearance holes for the screws that pass through and provide the clamping force via the clamping blocks used to constrain the inner and outer ends of the flexure blades.

Referring back to Figure 3-7, two sets of four flexures support the rotor. The flexures are first aligned to the rotor and each other, then clamped to the rotor. Figure 3-9 shows a top view of the swing-arm mounted to the base with four flexure blades. Note that one of the two inner clamping blocks and all four of the outer clamping blocks are visible in Figure 3-9. The inner clamping blocks are designed to insure that they contact the flexure blades at the outer diameter of the swing-arm. The included angle of the clamping blocks is toleranced to be equal to or slightly less than $90^{\circ}$, and the included angle of the receiving cut-outs is equal to or slightly more than $90^{\circ}$. The portion of the swing-arm below the flexures is hidden by the split plate that forms a labyrinth seal with the swing-arm.

One thing that I would do differently is to modify the inner and outer clamping blocks to better insure that the clamping pressure is applied to the desired fixedend locations on the flexure blades. The pressure distribution between the clamping blocks and flexure blades shown in Figure 3-9 is most likely higher near the clamping

\footnotetext{
${ }^{9}$ Of course, the change in pre-tension force $\Delta F$ is given by: $\Delta F=k \Delta x$, where $k$ is the spring constant and $\Delta x$ is the change in deflection of the spring. For both the $2 \mathrm{kHz}$ FTS and $10 \mathrm{kHz}$ FTS, $k=46 \mathrm{lb} / \mathrm{in}$. Moving the axis of rotation by $\Delta x=0.001 \mathrm{inch}$ by adjusting the nut on one of the anchor points causes $\Delta F=0.05 \mathrm{lb}(0.2 \mathrm{~N})$, or $0.4 \%$ of the nominal $50 \mathrm{~N}$ pre-tension force.
} 
screw than near the edges. This would result in a longer effective length for the flexure blades and an attendant decrease in mechanical stiffness. In the worst case, insufficient pressure at the edges of a clamping block would allow micro-slipping that could initiate fretting wear in the flexure blade. Relieving the central portion of the clamping blocks, as suggested by the dashed line on the lower-right clamping block in Figure 3-9, would better insure the desired pressure distribution. This observation is made in hindsight after building the $2 \mathrm{kHz}$ FTS and $10 \mathrm{kHz}$ FTS with the flexure clamping hardware shown in Figure 3-9.

It is worth discussing why I decided to use discrete flexure blades with clamping blocks. Although it is possible to use a process such as wire electro-discharge machining (wire EDM) to fabricate the rotor, flexure blades, and base as one monolithic structure, this approach has a significant number of serious drawbacks. The first is a pragmatic one: if one of the flexures were to become damaged the whole assembly would be ruined. The flexure blades used in the $2 \mathrm{kHz}$ FTS and $10 \mathrm{kHz}$ FTS were designed to be removed and replaced in case one or more of them were damaged. The second drawback is that if the thickness of the flexures needed to be changed it would require a difficult modification to the assembly or a completely new one. The third is that producing a pre-tension force in the flexures would be more difficult to accomplish. The fourth is that a wire-EDM surface has a thin, brittle re-cast layer that would have to be removed by polishing so that sites for crack initiations could be mitigated. Whereas polishing a flat strip of metal is easy to do, it would be extremely difficult to polish the flexure blades in a monolithic assembly.

An alternate flexure bearing that might be suitable for the rotary fast tool servos described in this thesis is the cruciform hinge described by Smith [138]. Basically, it is similar in function as the torsion rod labelled 33 in Figure 2-16 of Section 2.3.3, and has a cross-shaped cross-section instead of a circular one. A cruciform hinge provides a narrower axial profile than the over-constrained flexure bearing used with the $2 \mathrm{kHz}$ and $10 \mathrm{kHz}$ rotary fast tool servos, but would result in a taller overall assembly if they are to provide the same low torsional stiffness. This trade-off may be acceptable in an application where tall and thin is preferred to short and wide. 


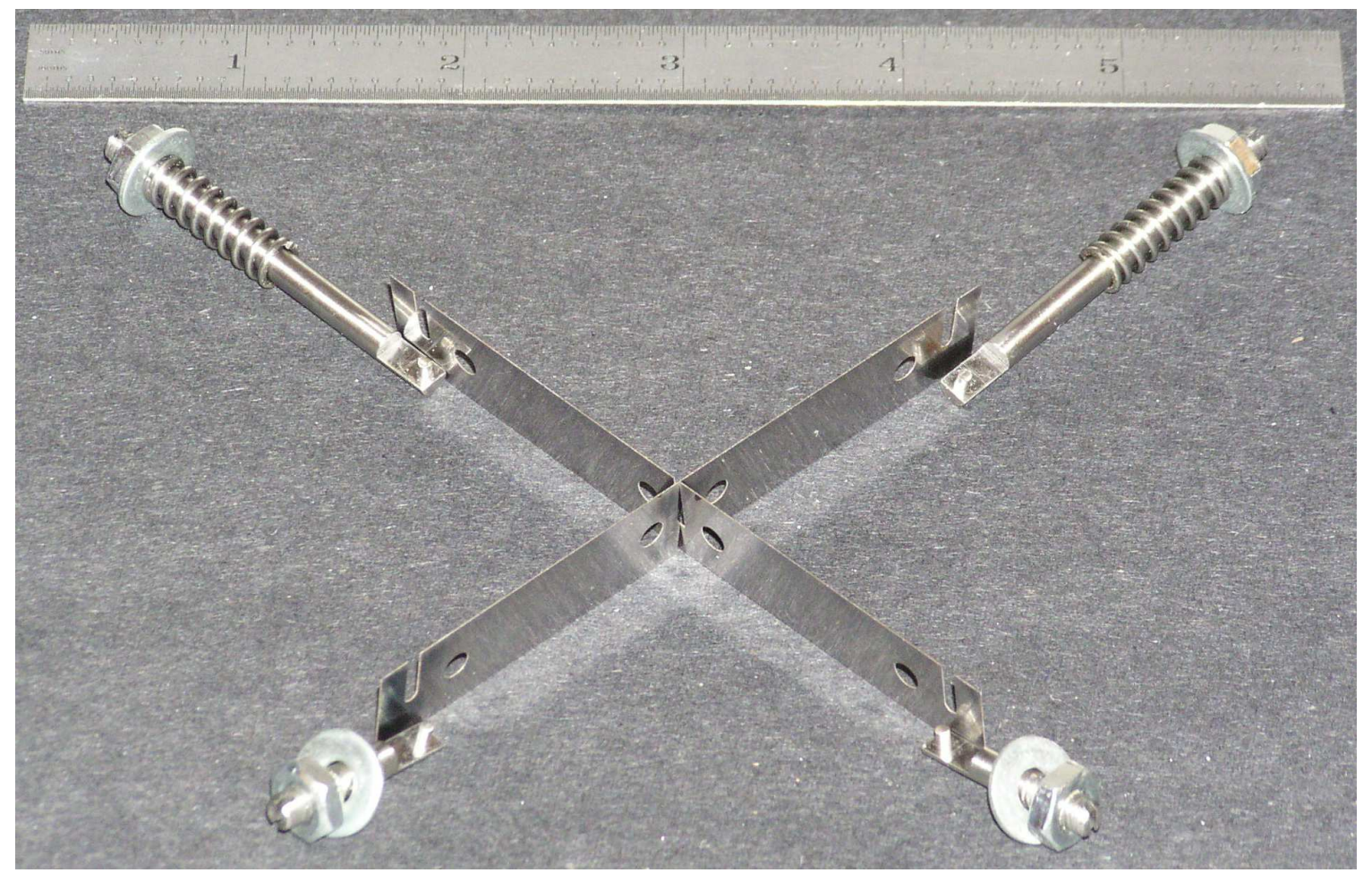

Figure 3-8: Four flexure blades with tensioning devices.

\subsubsection{Trade-Off Study for Flexure Blades}

Figure 3-10 is a sketch showing the geometry of a flexure blade before and after rotation of the swing-arm, and the three cases of simple beam bending that were used with superposition to approximate the deformation of a flexure blade. This approximation allowed me to quickly develop a set of closed-form equations for determining the values for the following design criteria: maximum and minimum stresses in the deformed flexure blade, the clamping force required to restrain the flexure blades, and the torque required to rotate the swing-arm. These closed-form equations, along with other equations for the axial and radial stiffness provided by the flexure suspension, allowed using a spreadsheet-based trade-off study for determining acceptable dimensions for the flexure blades. The goal of the analysis discussed in this section was to quickly gain sufficient guidance for choosing the dimensions of the flexures, then move on with the design of the other aspects of the $2 \mathrm{kHz}$ FTS. Therefore, I treated the equations as providing an order-of-magnitude analysis for the design of the flexure blades, and used a generous factor of safety when using them. 


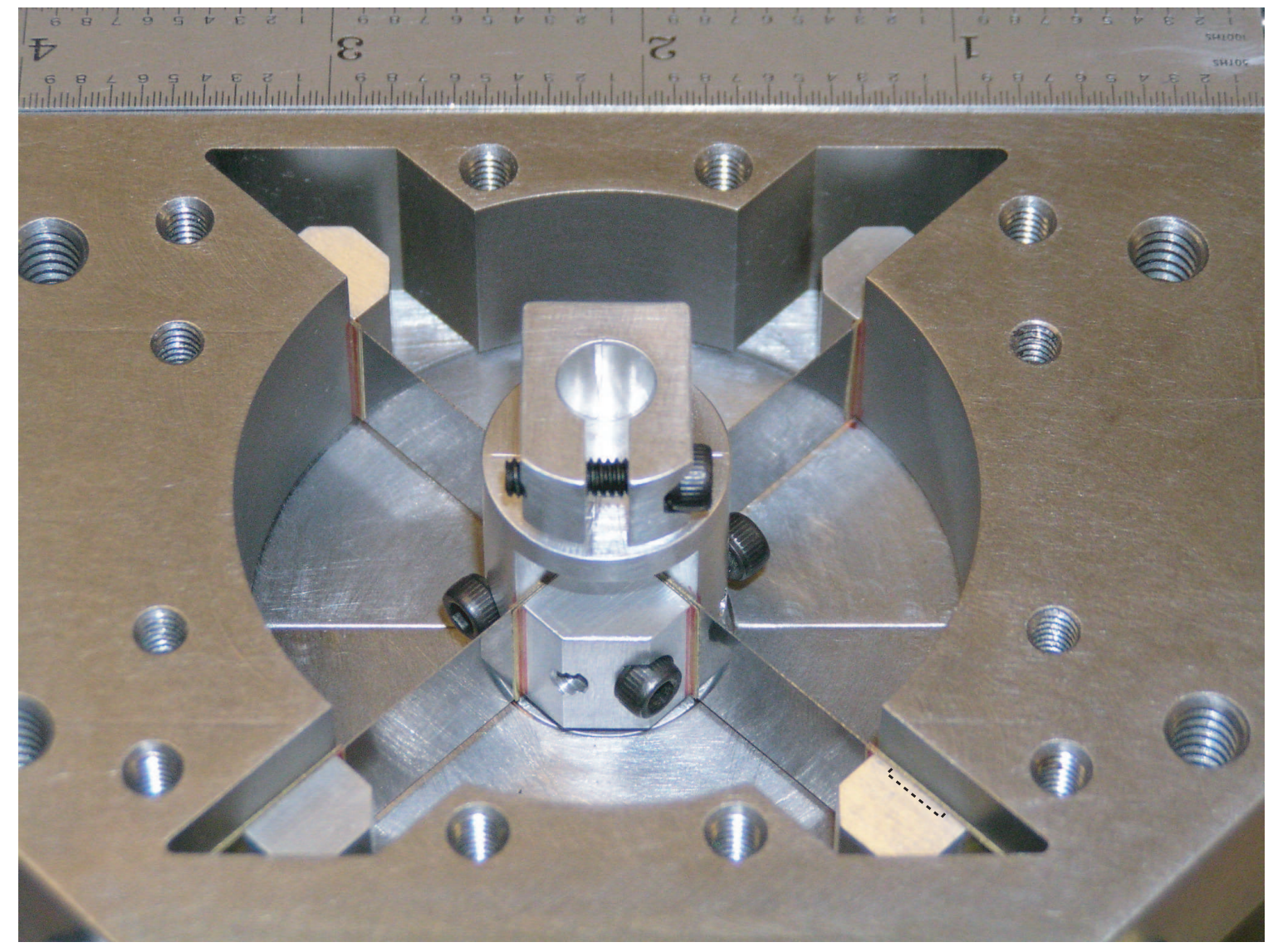

Figure 3-9: Top view of $2 \mathrm{kHz}$ FTS swing-arm mounted to the base with four flexure blades. Dashed line on the lower-right clamping block shows possible modification for better-defined location of the clamping force on the flexure blade.

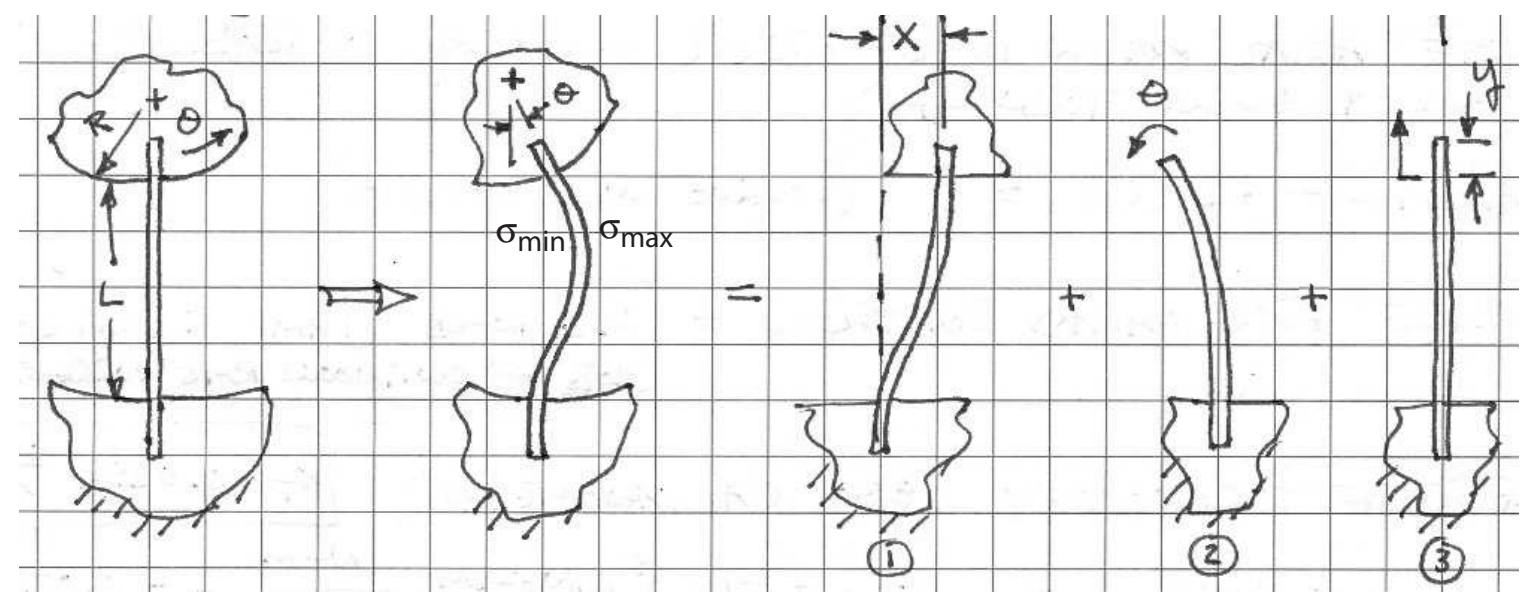

Figure 3-10: Geometry of a flexure blade before and after rotation of the swing-arm (far left and second from left, respectively). The three cases of simple beam bending that were used with superposition to obtain closed-form equations relating the stress and strain of the deformed flexure blade (right three figures). 


\section{Closed-Form Equations for the Flexure Blades}

The thickness, width, and length of each flexure blade are given by $t, b$, and $L$, respectively. $E$ is the modulus of elasticity of the flexure blade, and $\theta$ is the angle of rotation of the rotor. The displacements $x$ and $y$ shown in Figure 3-10 are related to the radius $R$ of the flexure hub by $x=R \sin (\theta)$ and $y=R[1-\cos (\theta)]$, respectively.

Referring to the circled numbers for each particular beam bending case shown in Figure 3-10, the maximum fiber stress ${ }^{10}$ for each case is given by (3.1), (3.2), and (3.3). The tensile preload force $F_{P L}$ on the flexure blade produces the preload stress $\sigma_{P L}$ given by (3.4). A positive-signed stress denotes tension, and a negative-signed stress denotes compression.

$$
\begin{gathered}
\sigma_{1, \text { max }}=\frac{3 t E}{L^{2}} x=\frac{3 t E}{L^{2}} R \sin (\theta) \\
\sigma_{2, \max }=\frac{t E}{2 L} \theta \\
\sigma_{3, \max }=\frac{E}{L} y=\frac{E}{L} R[1-\cos (\theta)] \\
\sigma_{P L}=\frac{F_{P L}}{t b}
\end{gathered}
$$

Referring to Figure 3-10, consider the shape of the flexure blade after rotation of the rotor. Note that the tensile forces on the ends of the flexure due to the axial stretching represented by case 3 are actually not collinear, and produce a moment load on the flexure that increases the maximum bending stress. This analysis neglects the additional stresses from that moment load because it is assumed negligible for the small displacements involved.

The maximum stress $\sigma_{\max }$ and minimum stress $\sigma_{\min }$ occur at the locations indicated in Figure 3-10, and are given by (3.5) and (3.6), respectively.

\footnotetext{
${ }^{10}$ Closed-from equations relating the maximum moment and deflection for cases 1 and 2 can be found in [131]. Case 1 is equivalent to a cantilevered beam having a length that is half the length of the flexure, and a maximum deflection equal to $x / 2$. Case 3 is simple uniaxial strain.
} 


$$
\begin{gathered}
\sigma_{\text {max }}=\sigma_{1, \max }+\sigma_{2, \max }+\sigma_{3, \max }+\sigma_{P L} \\
\sigma_{\text {min }}=\sigma_{3, \max }+\sigma_{P L}-\sigma_{1, \max }-\sigma_{2, \max }
\end{gathered}
$$

The mean stress $\sigma_{\text {mean }}$ and amplitude of the stress fluctuation $\sigma_{a m p l}$ are:

$$
\sigma_{\text {mean }}=\frac{\sigma_{\max }+\sigma_{\min }}{2} \quad ; \quad \sigma_{\text {ampl }}=\frac{\sigma_{\max }-\sigma_{\min }}{2}
$$

Figure 3-11 shows a sketch of a fluctuating stress and the relationship between $\sigma_{\text {max }}, \sigma_{\text {min }}, \sigma_{\text {mean }}$, and $\sigma_{\text {ampl }}$. Also shown in Figure 3-11 is the modified Goodman line ${ }^{11}$, which relates the mean stress $\sigma_{\text {mean }}$ and amplitude of the stress fluctuation $\sigma_{a m p l}$ to the endurance limit $S_{e}$ and ultimate strength $S_{u}$ of the material. Recall that the endurance limit $S_{e}$ is the fatigue strength for the case of complete stress reversal $\left(\sigma_{\text {mean }}=0\right)$. The modified Goodman line is the line joining $S_{e}$ to $S_{u}$ on the tensionside of the vertical axis. For a given mean stress $\sigma_{\text {mean }}$, the modified Goodman line indicates the corresponding fatigue strength $S_{f}$. In essence, the endurance limit $S_{e}$ is derated when the mean stress is tensile. On the compression-side of the vertical axis, the fatigue strength is equal to the endurance limit because a compressive mean stress does not significantly affect the fatigue strength [137, page 202].

The reader may wonder about the following: if compressive stresses are developed in the flexure blade, do we run the risk of losing a net tensile preload force in the blade? First, I assume that at every cross-section the stress distribution varies linearly from one side of the flexure blade to the other. As long as the magnitude of the tensile stress on one side is greater than the magnitude of the compressive stress on the other, the net axial force acting on each cross-section will be a tensile force. Referring to

\footnotetext{
${ }^{11}$ Shigley [137] calls it the "modified Goodman line", but Hamrock et. al. [68] and Machinery's Handbook [116] call it the "Goodman line". The first two references agree on the name for the "modified Goodman diagram", which "bends" the Goodman line to take into account the yield strength of the material, and shows that a compressive mean stress does not reduce the fatigue strength. The third reference does not mention the modified Goodman diagram. I have adopted the terminology used by Shigley [137] because it is the textbook that I used when I first studied this subject. Furthermore, when creating Figure 3-11, I arranged the compression-side of the relationship between the mean stress and the stress amplitude to match the modified Goodman diagram.
} 

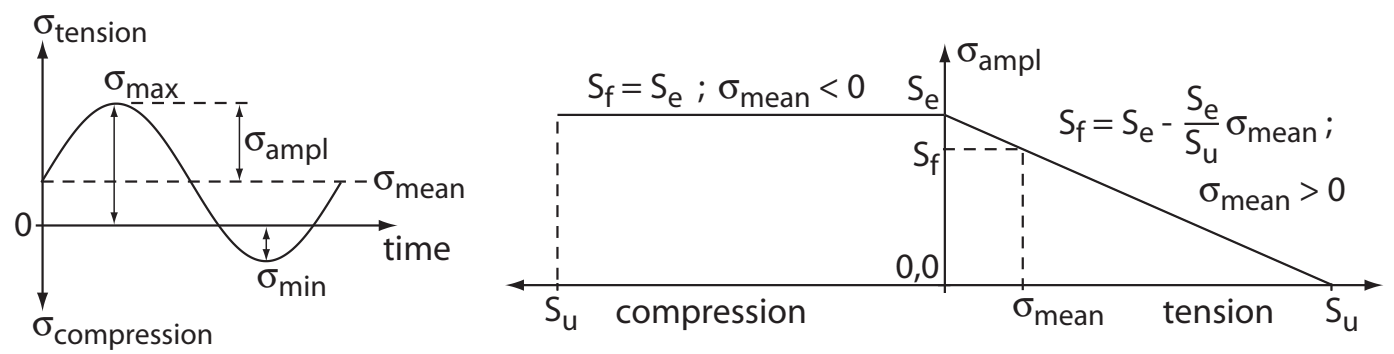

Figure 3-11: Nomenclature for fluctuating stress (left), and Modified Goodman line (right). Adapted from Shigley [137, page 200]

Figure 3-10, note that for case 1 and case 2 that the stresses on opposite sides of the flexure blades have equal magnitudes but opposite signs. The tensile stresses added by case 3 and the preload force insure that we have a net tensile force acting on each cross-section of the flexure blade.

The axial stiffness that the eight flexure blades provide to the swing-arm depends on the in-plane stiffness of each blade, and is given by (3.7), where $G$ is the shear modulus of the material. In this case the flexures are modelled using the short beam assumptions that shear deformation dominates and beam bending is negligible. The minimum radial stiffness that the eight flexure blades provide to the swing-arm occurs in a direction that is parallel to the plane of any of the flexures, and is given by (3.8):

$$
\begin{gathered}
k_{\text {axial }}=\frac{8 t b G}{L} \\
k_{\text {radial }}=\frac{4 t b E}{L}
\end{gathered}
$$

The clamping force required to prevent the ends of the flexure blades from slipping due to the tensile force developed during rotation of the swing-arm is assumed to be dominated by the axial elongation associated with case 3 in Figure 3-10. Using the tensile stress shown in (3.3) for this case, and a coefficient of static friction of $\mu_{s}$, after substitutions the normal-direction clamping force needed to restrain the ends of the flexure blades is given by (3.9): 


$$
N_{\text {clamp }}=\frac{t b}{\mu_{s}} \frac{E}{L} R[1-\cos (\theta)]
$$

The torque required to rotate the swing-arm through an angle $\theta$, starting from its neutral position, is approximated by considering the forces and torques necessary to cause the deflections shown in cases 1, 2, and 3 in Figure 3-10, and the moment arms involved for cases 1 and 2. The total torque required to rotate the swing-arm is given by (3.10), where the terms are due to cases 1,2 , and 3 , respectively:

$$
T_{8 \text { blades }}=8\left[\frac{E b t^{3}}{L^{3}} R^{2} \sin (\theta)+\frac{E b t^{3}}{12 L} \theta+\frac{E b t}{L} R^{2}[1-\cos (\theta)] \sin (\theta)\right]
$$

\section{Spreadsheet-Based Parametric Study}

The closed-form equations developed for the flexures are the engine for a spreadsheetbase parametric study that provided the insight necessary to decide on the dimensions for the flexure blades. Three parameters for the flexure blades were varied: thickness, width, and length. To allow using two-dimensional graphs to present the data for any particular study, width and length were varied while thickness was held constant. A fourth parameter, rotation angle, was held fixed at a value corresponding to the full rotation of the swing-arm from its neutral position to the hard stop. Different studies used different thicknesses. Starting with flexure dimensions that seemed intuitively reasonable, I varied each dimension about that starting point to determine local minima and maxima in the design space.

The set of graphs shown in Figure 3-12 represent the final parametric study for the 0.006 inch thickness value that was chosen. The desired design spaces are indicated by the horizontal lines with vertical arrows at their ends. Because so much depended on the flexure blades surviving prolonged high frequency operation, and it was likely that I would be driving the swing-arm into the hard stops during eventual debugging of the control system, I set a factor of safety of 10 as a minimum requirement for the fatigue life. At $2 \mathrm{kHz}$ operation, $10^{7}$ cycles only takes 83 minutes — roughly the total 
running time to machine one small workpiece with a fast tool servo — so I designed the flexures for an infinite fatigue life.

Each flexure blade in the $2 \mathrm{kHz}$ FTS is a 0.006 inch thick by 0.500 inch wide by 0.750 inch long piece of blue-tempered 1095 spring steel (ASTM A 682), which has an endurance limit of $100 \mathrm{ksi}$ [58]. The radius of the flexure hub is $0.375 \mathrm{inch}$, and the maximum rotation angle of the rotor (to the hard stops) is $\pm 5.0 \mathrm{mrad}$. Using (3.5) and (3.6), the maximum and minimum stresses are:

$$
\begin{gathered}
\sigma_{\max }=1791+597+186+3333=5907 \mathrm{psi}(\text { tension }) \\
\sigma_{\text {min }}=186+3333-1791-597=1131 \text { psi (tension) }
\end{gathered}
$$

The corresponding mean stress and amplitude of the stress fluctuation are:

$$
\sigma_{\text {mean }}=\frac{5907+1131}{2}=3519 \mathrm{psi}(\text { tension }) \quad ; \quad \sigma_{\text {ampl }}=\frac{5907-1131}{2}=2388 \mathrm{psi}
$$

When performing the original analysis for the flexure blades during the design phase for the $2 \mathrm{kHz}$ FTS, I had forgotten the distinction between the case of complete stress reversal and the case of a fluctuating stress, and designed the flexures for complete stress reversal using the maximum stress and the full endurance limit. This led to a factor of safety of 17 for fatigue failure. Later, one of the members of my thesis committee, Professor Samir Nayfeh, pointed out this error, which had led to an overly conservative set of dimensions for the flexure blades. Re-evaluating the flexure blades for the $2 \mathrm{kHz}$ FTS, for the actual case of a fluctuating stress and a derated fatigue strength due to the mean tensile stress, reveals a factor of safety for fatigue of 41. Clearly this is too conservative. For the later $10 \mathrm{kHz}$ FTS, the flexures have a more reasonable - yet still conservative - factor of safety of 11 for the fluctuating stress case.

For the axial stiffness and radial stiffness requirements for the flexure suspension 
I choose $500,000 \mathrm{lb} /$ in $(100 \mathrm{~N} / \mu \mathrm{m})$ and 1,000,000 lb/in $(200 \mathrm{~N} / \mu \mathrm{m})$, respectively. Referring back to the discussion in Section 3.4, these values represented $20 \%$ and $10 \%$ of the stiffness budget for the non-sensitive and sensitive direction at the tool tip, respectively. I accepted a little less stiffness in both cases by choosing a smaller flexure width of 0.500 inch, which kept the clamping force and torque requirements down. Another reason for a smaller width was that it kept the flexure hubs on the swing-arm from getting longer, which would have decreased the torque-to-inertia ratio for this machine. A more thorough trade-off analysis on this last point was performed for the $10 \mathrm{kHz}$ rotary fast tool servo, and is discussed in Section 4.8.

The clamping force needed to prevent the flexure blades from slipping at the inner and outer clamping blocks was set at $1 \mathrm{lb}$. It seemed reasonable to expect that a clamping force of at least 10 to 20 times higher could be produced by the single 5-40 screw that was being used at each clamping block. Regarding the torque required to rotate the swing-arm from its neutral position to one of the hard stops, I set the design point at $5 \%$ of the maximum continuous-operation peak torque that the actuator could produce, so that the majority of the actuator torque would be available to accelerate the swing-arm. In this case, it was $5 \%$ of $0.28 \mathrm{~N} \cdot \mathrm{m}(2.5 \mathrm{lb} \cdot \mathrm{in})$, or $0.12 \mathrm{lb} \cdot \mathrm{in}$.

It is worth mentioning that the desired design spaces on the graphs shown in Figure 3-12 were used as guidelines for choosing the dimensions for the flexure blades, and were not meant to be hard and fast binary decision lines. For instance, note that I traded off stiffness by conservatively picking a flexure blade length that provided a factor of safety for fatigue of at least 15. This flexure suspension was a new design for me, the stiffness chosen was adequate, and I wanted extra assurance that the flexures would not fail during my experiments with the $2 \mathrm{kKz}$ rotary fast tool servo. Ultimately, this flexure suspension proved itself a success, and I relaxed the factor of safety for fatigue back down to 10 for the later $10 \mathrm{kHz}$ rotary fast tool servo. 


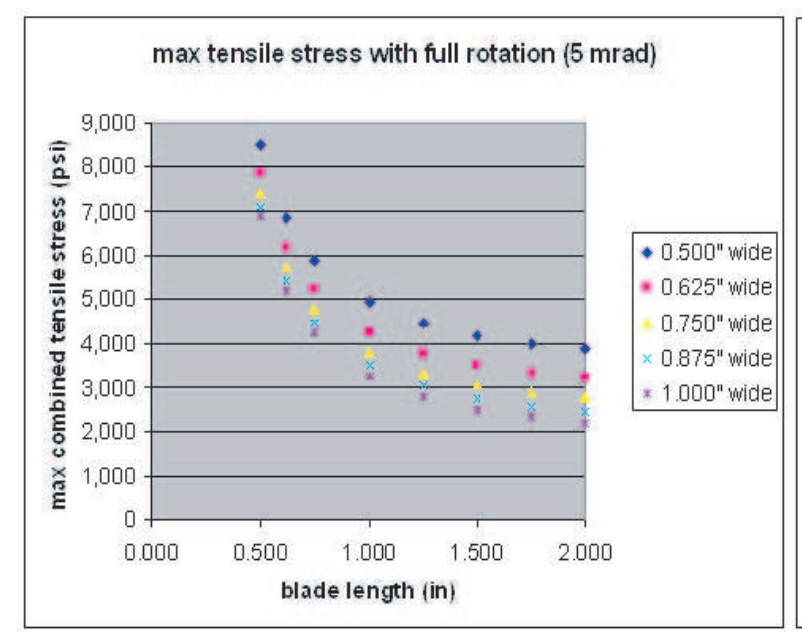

F.S. for endurance limit w/ complete stress reversal
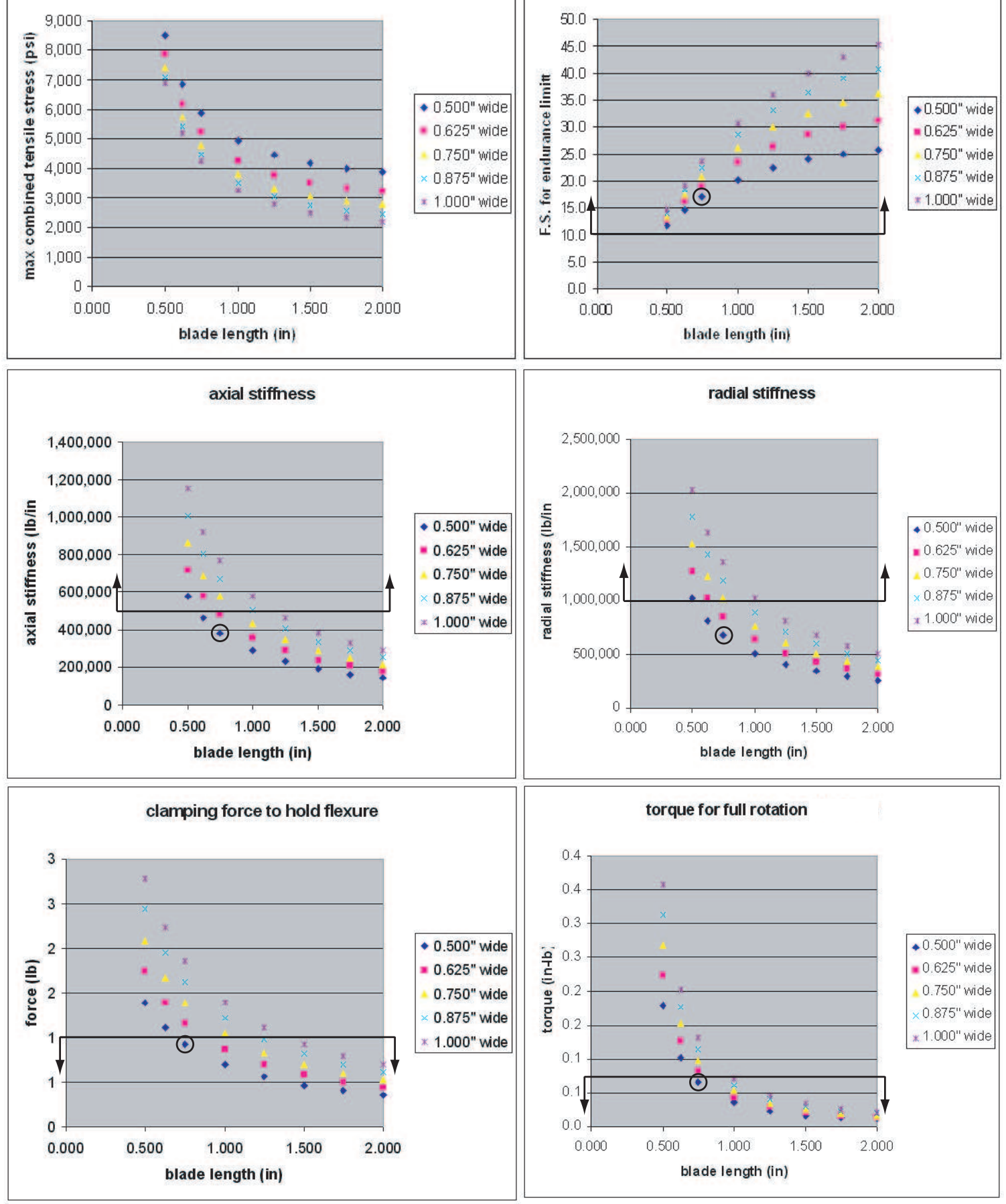

Figure 3-12: Final parametric study for the 0.006 inch thick flexure blades used in the $2 \mathrm{kHz}$ FTS. Full rotation of the swing-arm to a hard stop $(5.0 \mathrm{mrad})$. Factor of safety for fatigue based on the conservative case of complete stress reversal using the maximum stress and the full endurance limit. 


\section{Experimental Verification of Calculations for Flexures}

Because the flexures were so critical to the success of the $2 \mathrm{kHz}$ FTS mechanism, I decided to conduct a quick test to verify the calculations used in my analysis. Figure 3-13 shows the experimental set-up used to do this. Using a single piece to form two flexure blades, I clamped a mock rotor to the center and fixed the outer ends with clamping blocks to simulate the constraint condition for the flexures used in the $2 \mathrm{kHz}$ FTS. Each of these test flexures is a 0.015 inch thick by 0.500 inch wide by 0.750 inch long piece of 1095 blue-tempered spring steel. Using a wrench that was attached to the mock rotor, a 1.0 inch-lb torque produced a rotation of $0.5^{\circ}(\approx 10 \mathrm{mrad})$. Extrapolating the result to eight flexure blades, the experiment predicted that a 4.0 inch-lb torque would be needed to produce a 10 mrad rotation. The predicted torque required for that rotation, based on (3.10), is 6.9 inch-lb, which is $72 \%$ higher than the experimental torque. Considering the crude torque measurement used in this quick experiment, I accepted that the difference between predicted and experimental values was most likely due to a combination of measurement error and less than ideal end constraint conditions for the flexures in the experiment. Since the two values agreed within a factor of two, the experiment provided me enough confidence in the flexure calculations to proceed, especially since I had provided a contingency for increasing the thickness of the flexure blades by as much as a factor of three if I needed to.

\section{Measured Stiffness of the Flexure-Suspended Swing-arm}

Referring to Figure 3-1, the measured open-loop stiffness of the flexure-supported swing-arm at the plane of the tool along the centerline is $19 \mathrm{~N} / \mu \mathrm{m}$ and $13 \mathrm{~N} / \mu \mathrm{m}$ in the axial and radial directions, respectively. In Section 3.4 I mentioned that a goal was a net stiffness for the assembly of $20 \mathrm{~N} / \mu \mathrm{m}$ in the axial and radial directions. The actual radial stiffness is most likely higher than the measured radial stiffness because it was difficult to be sure that the test force and displacement sensor were both acting exactly on the centerline of the rotor, so the radial stiffness measurement 

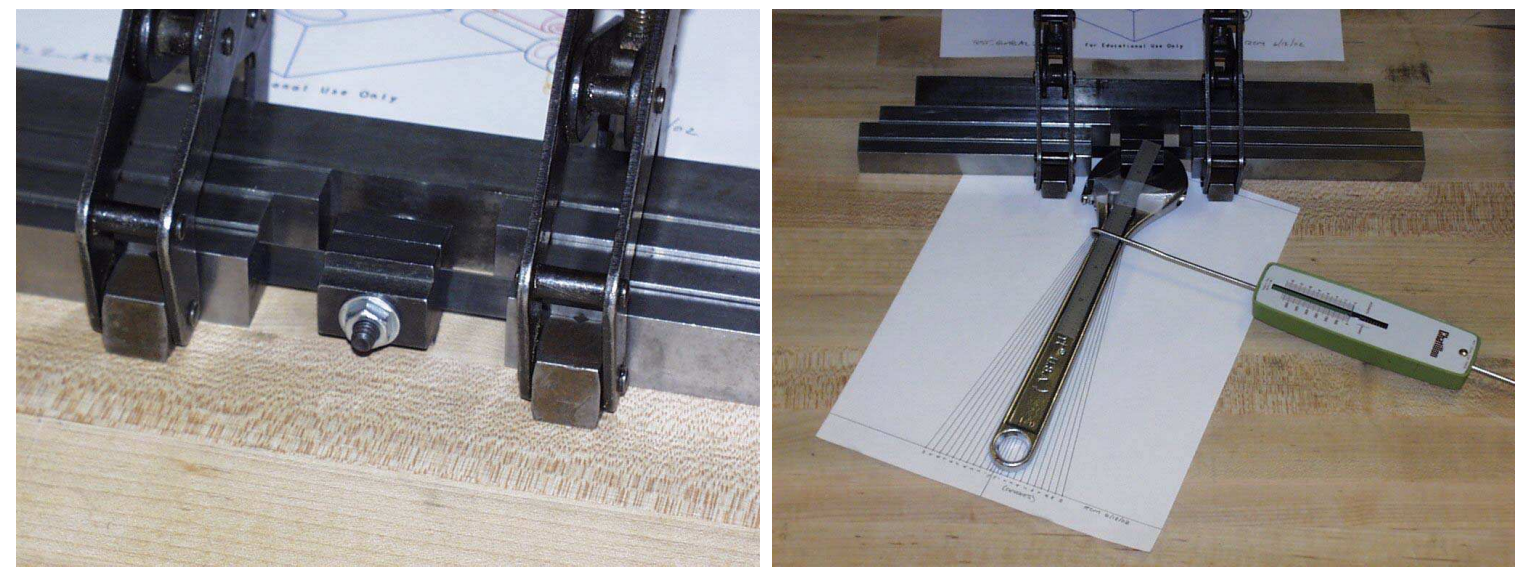

Figure 3-13: Experimental set-up for verifying calculations used to design the flexure blades. Close-up of the test flexure, mock rotor, and outer clamps (left). The addition of a lever arm (the wrench), a scale, a spring-loaded force gauge, and a protractor (right).

includes rotation of the swing-arm.

\subsubsection{Base and Associated Hardware}

The base was machined from a single piece of class 35 grey cast iron. This material was chosen instead of steel because of its lower coefficient of thermal expansion and better internal damping. Referring to Figure 3-1, the lower cover plate that forms a labyrinth seal with the bottom portion of the swing-arm is visible. The upper cover plate is thicker so that it can accept four screws that form the hard-stops for the swing-arm. The ends of two of those screws and their check nuts are visible in Figure 3-1. The four hard-stop screws engage the swing-arm in diagonal pairs to limit rotation and protect the flexure blades.

\subsubsection{Mechanical Damper}

A mechanical viscous damper is attached to the bottom of the swing-arm and is effective at damping the first two modes of torsional vibration for the $2 \mathrm{kHz}$ FTS. The first mode is the swing-arm and actuator moving-mass rotating in phase with each other as a single mass suspended by the torsional spring produced by the flexure suspension. The second mode is those two discrete masses moving out of phase with 
each other via the torsional spring produced by the rigid coupling of the swing-arm and relatively thin output shaft of the actuator. Figure 3-14 shows this rotary viscous damper, which consists of a thin circular plate attached to the bottom of the swingarm. A $0.1 \mathrm{~mm}$ gap between each face of the circular plate and the adjacent stationary surface is filled with DuPont Krytox ${ }^{\circledR}$ GPL-206 grease, with a viscosity of 810 cSt at $20^{\circ} \mathrm{C}$ [52]. This damper reduced the peak amplitudes of the $5 \mathrm{kHz}$ resonance between the swing-arm and the actuator moving-mass by $20 \mathrm{~dB}$, making it possible to use a simple lag compensator for stabilizing the tool position control loop with a crossover frequency of $1 \mathrm{kHz}$. Although it is possible to produce electronic damping via the actuator by differentiating the angular position of the swing-arm, I did not pursue this avenue. This was because the mechanical damper provides a more direct connection to the rotor where it is needed, is not subject to sensor or computation noise, and was included in the original mechanical design of the $2 \mathrm{kHz}$ FTS. Regarding the guidelines that I used to design the damper, I used the maximum diameter disc that would fit into the available space between the outer clamping blocks for the flexures, adjusted the thickness of the damping disc so that its inertia was no more than $10 \%$ of the total inertia of the composite swing-arm, and set the thickness of the gaps to a reasonable value from a fabrication and assembly standpoint. Shims between the swing-arm and damping disc allowed me to accommodate for accumulated fabrication errors in the assembly. Based on the control system tests described in Section 3.10.4, the damper worked successfully.

\subsubsection{Actuator}

The $2 \mathrm{kHz}$ rotary fast tool servo uses a Cambridge Technologies Incorporated (CTI) model 6880 moving-magnet Lorentz force galvanometer for the actuator. It is intended for use with steering mirrors having a rotational inertia in the range of $3-64 \mathrm{gm} \cdot \mathrm{cm}^{2}$, and has a rotation range of $40^{\circ} \mathrm{PP}$. The CTI 6880 is a state-of-the-art galvanometer with a high torque-to-inertia ratio, and represents many years of evolution in a commercially competitive field. It has a specified torque constant of $0.0254 \mathrm{~N} \cdot \mathrm{m} / \mathrm{amp}$ and a maximum continuous current rating of $7.7 \mathrm{~A}$ rms or $11 \mathrm{~A}$ peak. From these values 

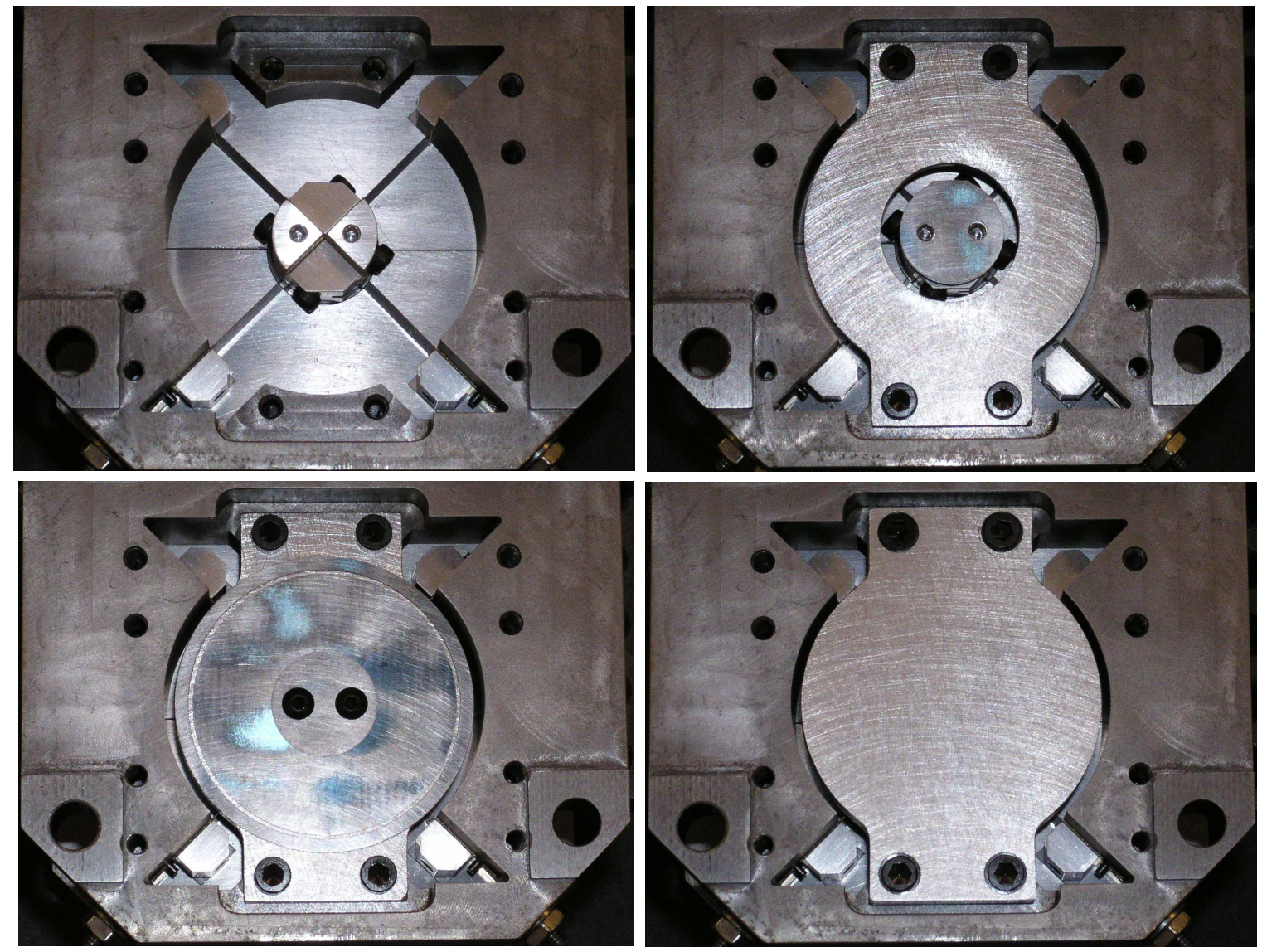

Figure 3-14: Mechanical viscous damper used in the $2 \mathrm{kHz}$ rotary fast tool servo. Bottom of swing-arm and base, and lower set of flexure blades (upper left). Upper stationary plate (covering flexure blades) and spacer on bottom of swing-arm (upper right). Damping disc and outer spacer ring (lower left). Lower stationary plate covering damping disc (lower right). 
the specified continuous-operation peak torque is $0.28 \mathrm{~N} \cdot \mathrm{m}$, which when combined with its moving mass inertia of $6.4 \mathrm{gm} \cdot \mathrm{cm}^{2}$ gives the actuator a rated torque-toinertia ratio of $4.4 \times 10^{5} \mathrm{~N} \cdot \mathrm{m} /\left(\mathrm{kg} \cdot \mathrm{m}^{2}\right)$ [34]. The actuator is shown in the upper left corner of Figure 3-5 next to its aluminum mounting block. The long, narrower diameter portion shown covered with heat-sink compound contains the magnet and coils for the actuator. An advantage of a moving-magnet design is that all of the current-carrying coils are adjacent to the outer housing where heat from electrical losses can be easily removed by conduction through the housing. This design allows using a higher continuous current than a comparably sized moving coil design when internal fluid cooling is not employed. Figure 3-1 shows the actuator in its mounting block on the $2 \mathrm{kHz}$ FTS. Only the back-end electronics package for the actuator is visible above the mounting block.

Dividing the specified continuous-operation peak torque of $0.28 \mathrm{~N} \cdot \mathrm{m}$ by the combined inertia of $57 \mathrm{gm} \cdot \mathrm{cm}^{2}$ for the swing-arm and the moving element of the actuator, the specified peak continuous angular acceleration is $49 \times 10^{3} \mathrm{rad} / \mathrm{sec}^{2}$. Multiplying this value by the tool swing radius of $5 \mathrm{~mm}$ gives a specified peak continuous linear tool tip acceleration of $246 \mathrm{~m} / \mathrm{sec}^{2}$, or $25 \mathrm{~g}$.

The mounting block acts as a simple heat sink for the actuator. The four ceramic spacers between the mounting block and the FTS base help reduce the flow of the heat from the actuator into the rest of the fast tool servo. While using the $2 \mathrm{kHz}$ FTS to diamond turn the workpiece described in Section 3.11.1, the actuator was operated continuously for at least one hour at $71 \%$ of the specified maximum continuous current, or 5.5 A rms. During that time, I measured the following steady-state operating temperatures: the ambient air temperature was $81^{\circ} \mathrm{F}\left(31^{\circ} \mathrm{C}\right)$, the temperature at the top of the mounting block was $132{ }^{\circ} \mathrm{F}\left(55^{\circ} \mathrm{C}\right)$, and the temperature at the top of the FTS base was $91^{\circ} \mathrm{F}\left(33^{\circ} \mathrm{C}\right)$. The actuator mount is designed to be wrapped by an optional fluid-cooled jacket that allows removing the heat generated by the actuator from the work zone on the diamond turning machine that the FTS is used with. 


\subsubsection{Sensors}

The position of the tool is measured by a pair of eddy current sensors operating differentially and looking at the back of the swing-arm on either side of the axis of rotation and near the elevation of the tool. This is best seen in Figure 3-15, which shows the diamond tool engaging a workpiece during a cutting test. The eddy current sensor package is from Kaman Instrumentation [88]: differential amplifier model DIT$5200-15 \mathrm{~N}$ and probe model $15 \mathrm{~N}$. The eddy current sensor amplifier produces a $\pm 10 \mathrm{~V}$ signal for the $\pm 25 \mu \mathrm{m}$ tool travel, and has a specified bandwidth of $0-20 \mathrm{kHz}$ which is adequate for the $2 \mathrm{kHz}$ FTS. The specified root-mean-square electrical noise for this sensor system, presumably over that bandwidth ${ }^{12}$, expressed as an equivalent tool tip displacement, is $0.2 \mathrm{~nm} \mathrm{rms}$. The measured equivalent electrical noise level for this sensor system, after A/D conversion in the digital control system for the $2 \mathrm{kHz}$ FTS, is $4 \mathrm{mV} \mathrm{PP}$, which corresponds to $10 \mathrm{~nm} \mathrm{PP}$ at the tool tip.

Approximately half of the sensor noise is due to the measured 6 bits PP noise in the analog-to-digital converter used in the control system, which corresponds to a tool tip motion of $5 \mathrm{~nm}$ PP. A significant portion of the other half of that noise is due to the inadequate measurement set-up that I used. At the time, I was a novice when it came to measuring micro-volts and simply connected an oscilloscope to the sensor amplifier output with no regard to the noise floor of the oscilloscope, common-mode noise, or the use of a low-pass filter to establish a known cut-off frequency for the measurement. By the time that I built the $10 \mathrm{kHz}$ FTS I had learned about these things, and used a more adequate measurement set-up and rigorous approach (see Section 4.9.3). Unfortunately, I did not remeasure the noise levels for the $2 \mathrm{kHz}$ FTS because I had loaned the power amplifier that I built for it to another student in our lab for his experiments, making it more difficult than it was worth to run the $2 \mathrm{kHz}$ FTS again before finishing this research. Subsequently, the actual noise level for these sensors at the frequencies of interest for the $2 \mathrm{kHz}$ FTS is probably not as bad as the value that I report.

\footnotetext{
${ }^{12}$ The manufacturer's specification sheet does not mention what bandwidth is associated with the noise specification.
} 


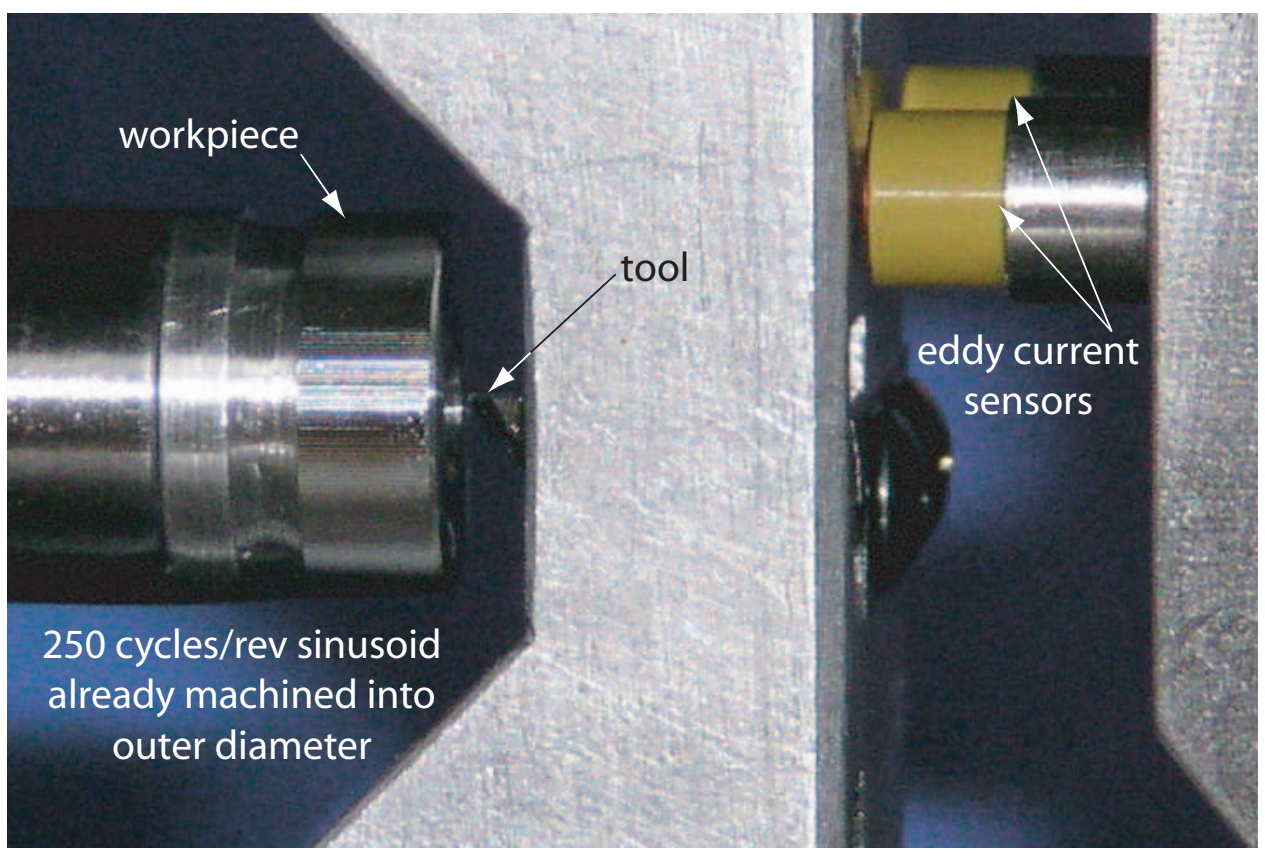

Figure 3-15: Close-up view of the $2 \mathrm{kHz}$ FTS tool tip engaging the face of a workpiece.

The eddy current sensors were chosen over capacitance sensors in this case for two reasons. First, unlike capacitance sensors, eddy current sensors are not affected by cutting fluids that might migrate into the air gaps between the sensors and the swing-arm, so seals for the sensors are not needed. Second, I had used capacitance sensors in previous projects but had not used eddy current sensors before and wanted to gain experience with them. Before finalizing the choice on the type of sensors, I evaluated a borrowed pair of eddy current sensors using the servo test bed described in Section 3.8, and found them to be acceptable for the $2 \mathrm{kHz}$ FTS.

The differential measurement provides information on the rotation angle of the swing-arm. Figure 3-16 shows an LVDT displacement sensor ${ }^{13}$ being used to scale the differential measurement from the eddy current sensors to a position measurement for the tool tip. A benefit of using differential sensors instead of a single sensor is that common-mode electrical noise is rejected. If desired, a measure of the commonmode signal could be used to measure front-to-back translation of the centerline of the swing-arm.

\footnotetext{
${ }^{13}$ LVDT: linear variable differential transformer.
} 


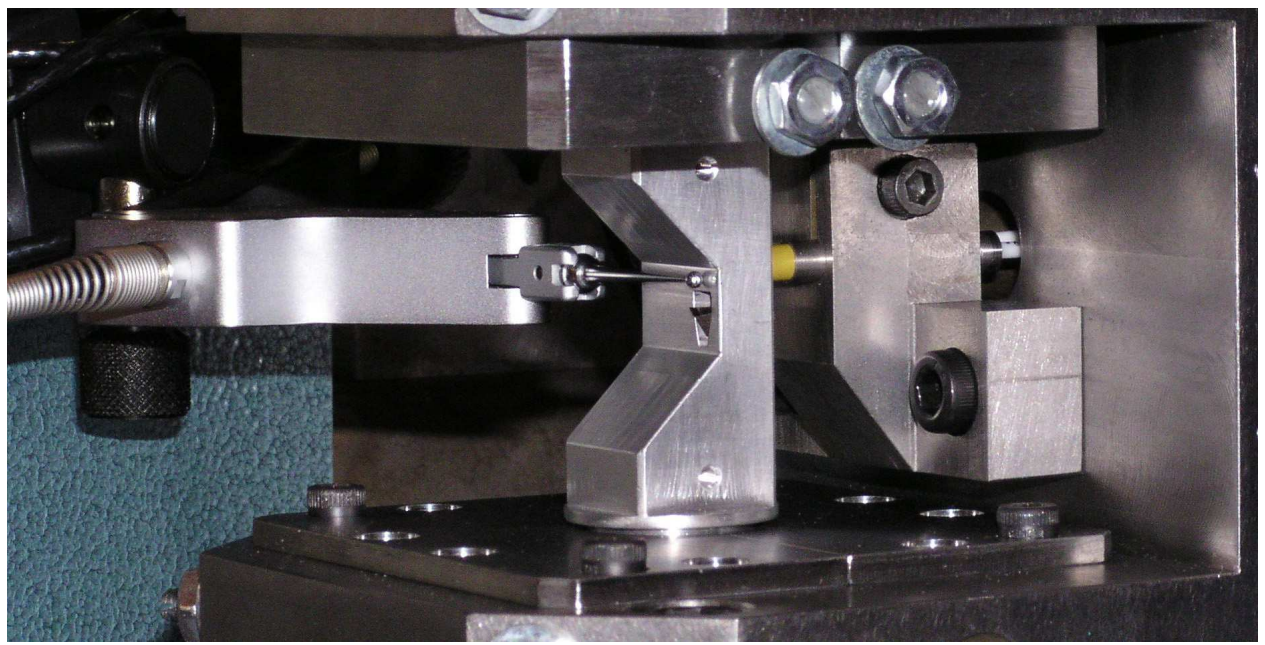

Figure 3-16: Scaling the differential measurement from the eddy current sensors to a position measurement for the tool tip.

\subsection{System Dynamics}

The simplified dynamics models shown in Figures 3-17 and 3-18 provide sufficient insight for designing the compensation for the $2 \mathrm{kHz}$ FTS control system. For this discussion and others in this thesis, the actuator is sometimes referred to as the "motor". The modelled dynamics were used to guide the design of the $2 \mathrm{kHz}$ FTS and predict its performance. Additionally, the modelled dynamics are used in the following manner to develop insight into the measured dynamics for the system. A parametric model describing the plant and compensation dynamics is adjusted until the Bode plot of the modelled negative loop transmission for the tool position matches the Bode plot of the measured negative loop transmission. ${ }^{14}$ From that model, a root locus is generated, upon which the open-loop and closed-loop poles and zeros for the system are identified, and a Nyquist diagram is generated. Regarding the level of rigor employed in matching the parametric model to the measured dynamics, it is

\footnotetext{
${ }^{14}$ For the reader unfamiliar with the term "negative loop transmission", it is a concept that I learned from Professor James Roberge at MIT, and is used in his seminal book "Operational Amplifiers" [132]. Consider a feedback loop such as the one shown in Figure 3-19. Break the loop at a convenient location, insert a test signal at the downstream side of that break, and set all of the other inputs to zero. The negative loop transmission is the gain that the test signal experiences when it is transmitted around the loop to the upstream side of the break. Since negative feedback is almost always used, the test signal experiences a sign inversion when it passes through the summation block, so strictly speaking the gain in the loop is negative. Instead of reporting a negative-valued loop gain, the positive-valued "negative loop transmission" is used.
} 


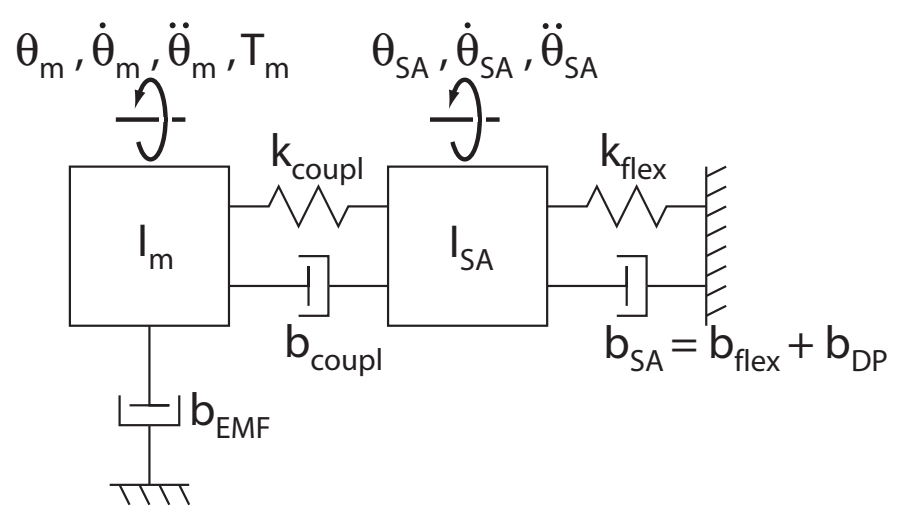

Figure 3-17: Simplified model for the mechanical dynamics of the combined motor and swing-arm for the $2 \mathrm{kHz}$ FTS.

not as high as would be needed if I had to design an inverse-dynamics compensator for the plant, which I did not do for this thesis. The level of rigor was sufficient for providing insight to the measured dynamics and gaining confidence in the robustness of the stability of the system.

\subsubsection{Modelled Plant Dynamics}

\section{Mechanical Dynamics}

Figure 3-17 depicts a model for the mechanical dynamics of the combined motor and swing-arm. The moving element of the motor has rotational inertia $I_{m}$, rotation angle $\theta_{m}$, and is acted on by the torque $T_{m}$ developed by the motor. The swing-arm has rotational inertia $I_{S A}$, rotation angle $\theta_{S A}$, is connected to the moving element of the motor with torsional spring $k_{\text {coupl }}$ and torsional damper $b_{\text {coupl }}$, and is connected to ground with torsional spring $k_{f l e x}$ (the flexure blades) and torsional damper $b_{S A}$. The damper $b_{S A}$ is equal to the sum of the damping in the flexures $b_{\text {flex }}$ (primarily at the end connections) and the damping provided by the viscous damping plate $b_{D P}$. The velocity-dependent back-EMF developed by the motor is treated as a torsional damper $b_{E M F}$ between ground and the moving element of the motor.

Referring to Figure 3-17, equations of motion are derived by writing torque balances for each mass (rotational inertia). For the moving element of the motor, the 
torque balance is given by (3.11):

$$
\sum T=I_{m} \ddot{\theta}_{m}=T_{m}-\left(b_{E M F}+b_{\text {coupl }}\right)\left(\dot{\theta}_{m}-\dot{\theta}_{S A}\right)-k_{\text {coupl }}\left(\theta_{m}-\theta_{S A}\right)
$$

Substituting the Laplace variable $s$ for the time differential operator, and setting $b_{m}=b_{E M F}+b_{\text {coupl }},(3.11)$ is rearranged into (3.12):

$$
\theta_{m}=\frac{\left(b_{m} s+k_{\text {coupl }}\right) \theta_{S A}+T_{m}}{I_{m} s^{2}+b_{m} s+k_{\text {coupl }}}
$$

For the swing-arm, the torque balance is given by (3.13), which leads to (3.14):

$$
\begin{gathered}
\sum T=I_{S A} \ddot{\theta}_{S A}=b_{\text {coupl }}\left(\dot{\theta}_{m}-\dot{\theta}_{S A}\right)+k_{\text {coupl }}\left(\theta_{m}-\theta_{S A}\right)-b_{S A} \dot{\theta}_{S A}-k_{f l e x} \theta_{S A} \\
\theta_{S A}=\frac{b_{\text {coupl }} s+k_{\text {coupl }}}{I_{S A} s^{2}+\left(b_{\text {coupl }}+b_{S A}\right) s+\left(k_{\text {coupl }}+k_{\text {flex }}\right)} \theta_{m}
\end{gathered}
$$

Substituting (3.12) into (3.14) and rearranging gives (3.15):

$$
\theta_{S A}=\left[\begin{array}{c}
\left.\frac{b_{\text {coupl }} s+k_{\text {coupl }}}{\left[I_{S A} s^{2}+\left(b_{\text {coupl }}+b_{S A}\right) s+\left(k_{\text {coupl }}+k_{\text {flex }}\right)\right]\left[I_{m} s^{2}+b_{m} s+k_{\text {coupl }}\right]}\right] \\
-\left[b_{\text {coupl }} b_{m} s^{2}+k_{\text {coupl }}\left(b_{\text {coupl }}+b_{m}\right) s+k_{\text {coupl }}^{2}\right]
\end{array}\right] T_{m}
$$

Examination of (3.15) reveals that the simplified dynamics model can be represented by a generalized transfer function consisting of a first-order zero and two complex second-order poles, as shown in (3.16): 


$$
\theta_{S A}=\frac{K_{d c}\left(T_{z} s+1\right)}{\left[\frac{s^{2}}{\omega_{1}^{2}}+\frac{2 \zeta_{1}}{\omega_{1}} s+1\right]\left[\frac{s^{2}}{\omega_{2}^{2}}+\frac{2 \zeta_{2}}{\omega_{2}} s+1\right]} T_{m}
$$

By adjusting the parameters in (3.16), simple pole-zero models are fitted to the measured negative loop transmission for the position loop for the different compensators used with the $2 \mathrm{kHz}$ FTS.

\section{Electrical Dynamics}

Figure 3-18 relates the electrical circuit for the motor to an equivalent block diagram that depicts the electrical dynamics of the motor. The motor coil has resistance $R_{c}$ and inductance $L_{c}$. The velocity-dependent back-EMF developed by the actuator is represented by the dependent voltage source $K_{m} \dot{\theta}_{m}$. The voltage $V_{s}$ at the top of the sense resistor $R_{s}$ provides a measure of the motor current $I_{m}$ and is used in the feedback controller for the current-mode amplifier. Note that the sense resistor current $I_{s}$ is essentially equal to the motor current $I_{m}$ because the feedback current $I_{f}$ goes to a high impedance input on an operational amplifier, and is therefore essentially zero. The back-EMF is modelled as a disturbance voltage on the output voltage $V_{p a}$ of the power amplifier. This allows not having to explicitly break out a measure of the motor angular velocity $\dot{\theta}_{m}$ when considering the mechanical dynamics for the system. The damping provided by feedback of the motor angular velocity - in the form of the back-EMF - is treated implicitly by the damping term $b_{E M F}$ shown in Figure 3-17.

Figure 3-19 is a block diagram showing the electrical dynamics of the actuator combined with the electrical dynamics of the current-mode amplifier used to drive the actuator. The first two blocks in the forward path of the current loop are the compensation and the power operational amplifier circuit for the amplifier. The gains $K_{c}$ and $K_{s f}$ are chosen to balance the command and feedback signals, respectively, into the current compensation circuit. The back-EMF as a disturbance on $V_{p a}$ is omitted because it is captured in the damping terms for the mechanical dynamics. 

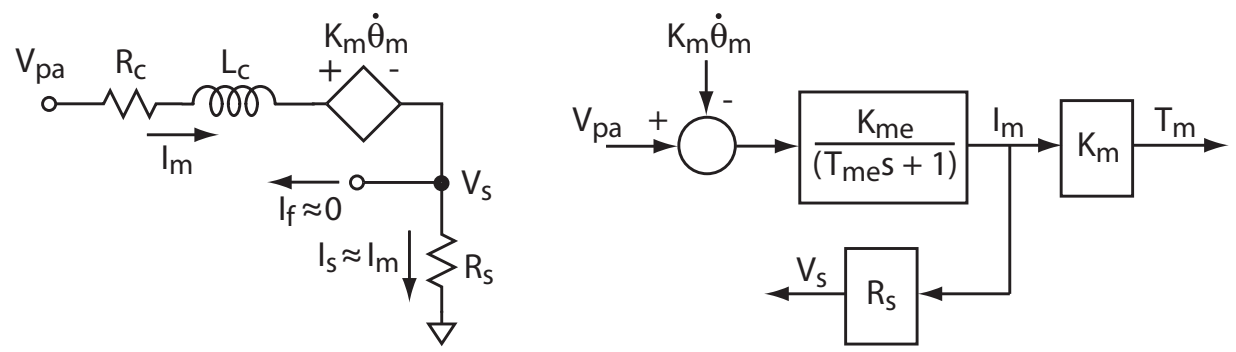

$$
\begin{aligned}
& K_{\text {me }}=\frac{1}{\left(R_{C}+R_{s}\right)} \\
& T_{m e}=\frac{L_{c}}{\left(R_{c}+R_{s}\right)}
\end{aligned}
$$

Figure 3-18: Electrical circuit and equivalent block diagram for the electrical dynamics of the motor for the $2 \mathrm{kHz}$ FTS.

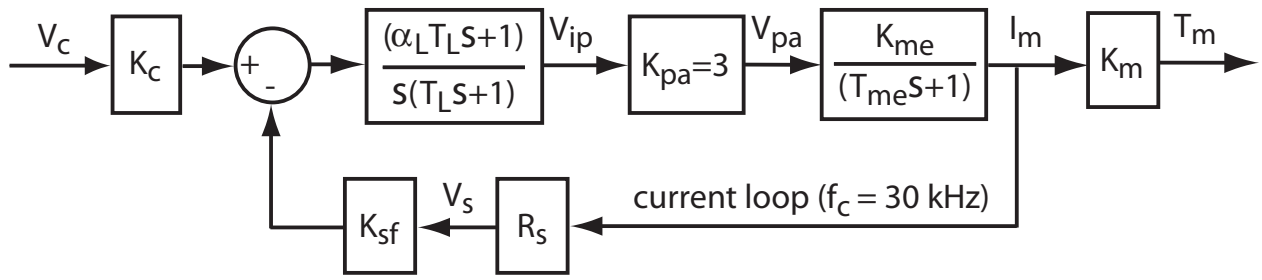

Figure 3-19: Block diagram showing the electrical dynamics of the actuator inside the current loop for the current-mode amplifier for the $2 \mathrm{kHz}$ FTS.

The crossover frequency for the current loop is an order of magnitude higher than the crossover frequency for the tool position (swing-arm angle $\theta_{S A}$ ) loop. Therefore, the current loop can be considered to be a constant gain of $1 /\left(K_{s f} R_{s}\right)$ at the frequencies of interest for the tool position. ${ }^{15}$ Note that the electrical dynamics for the actuator have been placed inside the loop with the current-mode amplifier to facilitate the design of the current compensation.

\subsubsection{Measured Plant Dynamics}

The measured tool position dynamics for the $2 \mathrm{kHz}$ FTS can be seen in Figure 320 , which is a Bode plot of the measured negative loop transmission of the tool position for the $2 \mathrm{kHz}$ FTS before viscous damping was added to the system. The dynamics shown in Figure 3-20 include the lead compensator that was present when the measurement was made. The first coupled torsional mode of the swing-arm and moving element of the motor, suspended by the flexure suspension which acts as a weak torsional spring, produces a $20 \mathrm{~dB}$ peak at $200 \mathrm{~Hz}$. The first uncoupled torsional

\footnotetext{
${ }^{15} \mathrm{~A}$ proof for this claim is provided in Section A-5.
} 

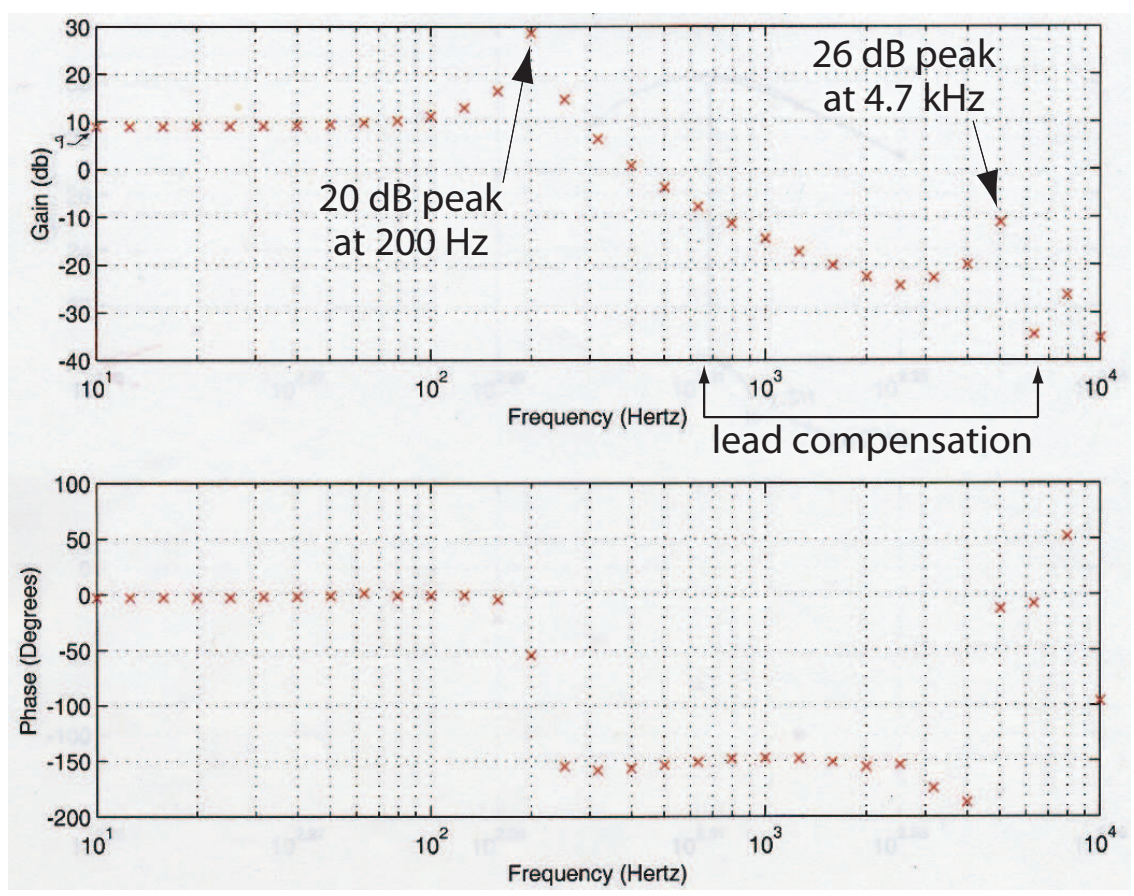

Figure 3-20: Bode plot of the measured tool position dynamics for the $2 \mathrm{kHz}$ FTS (including a lead compensator).

mode of those two masses produces a $26 \mathrm{db}$ peak at $4.7 \mathrm{kHz}$.

The parametric model for the plant dynamics are based on the two pairs of complex second-order poles shown in the generalized transfer function in (3.16). In Section 3.10.4 I show that the first-order zero in (3.16) may be significant for the system with viscous damping. However, for the purposes of this section I neglect that zero because I am concerned with fitting a model to the measured data for the undamped system. Figure 3-21 is based on that fitted model, and shows a Bode plot of the modelled negative loop transmission of the tool position for the $2 \mathrm{kHz}$ FTS, including the lead compensator that was present when the actual dynamics were measured. Figure 3-22 shows the corresponding root locus for that model. The small circles represent the open-loop and closed-loop zeros, the x's represent the open-loop poles, and the solid squares represent the close-loop poles. Figure 3-23 shows the corresponding Nyquist diagram for the model. The small "+" sign represents the -1 point. The Bode plot, Root locus, and Nyquist diagram for the modelled dynamics were all generated using the MATLAB ${ }^{\circledR}$ script in Section B.2. 

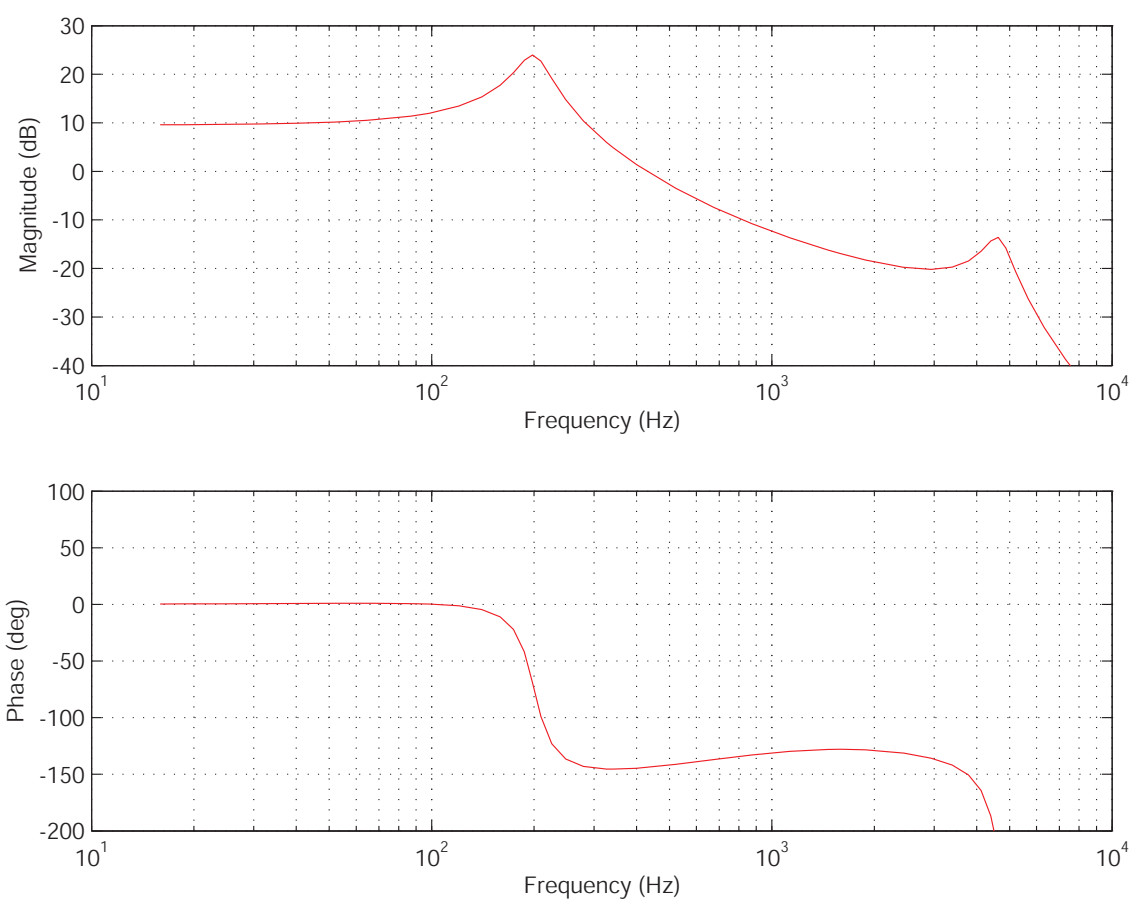

Figure 3-21: Bode plot of the modelled tool position dynamics for the $2 \mathrm{kHz}$ FTS (including a lead compensator).

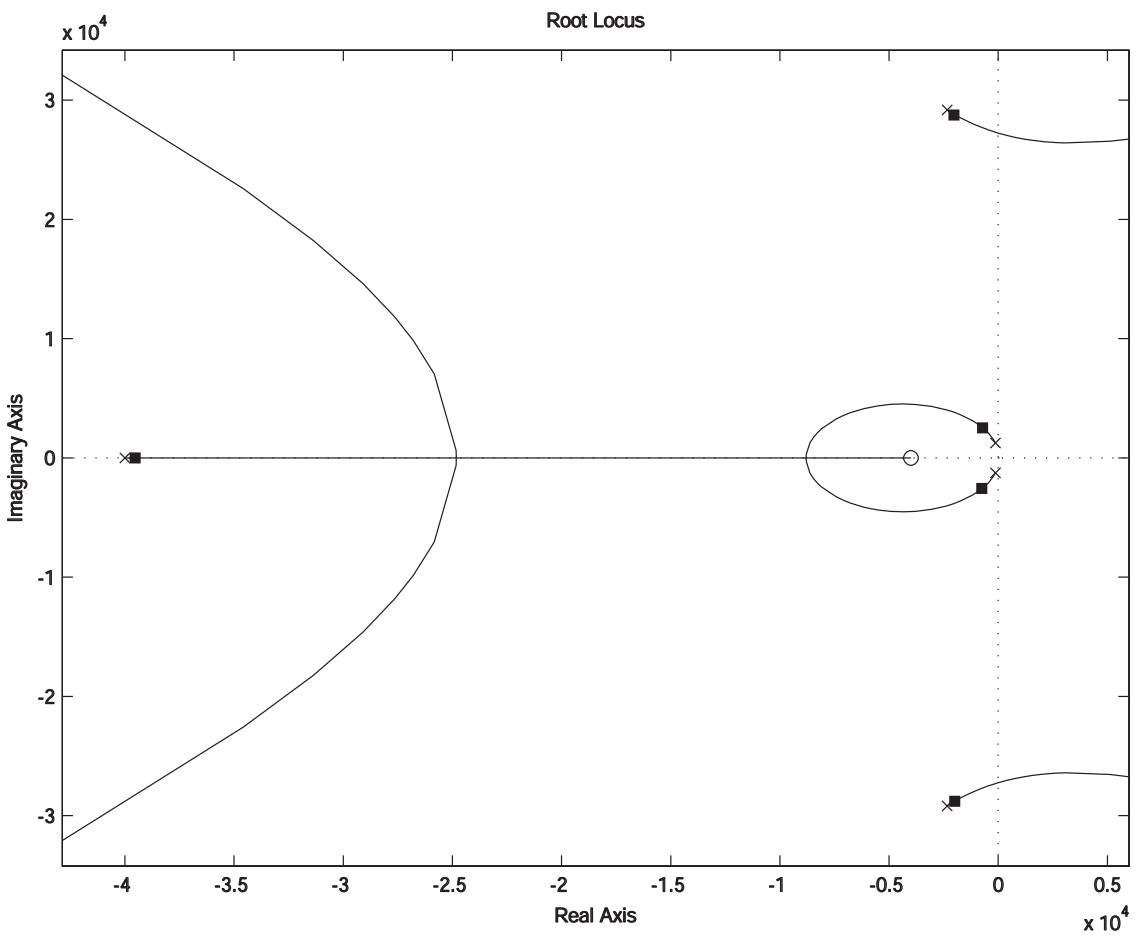

Figure 3-22: Root locus of the modelled tool position dynamics for the $2 \mathrm{kHz}$ FTS (including a lead compensator). Closed-loop poles represented by filled squares. 


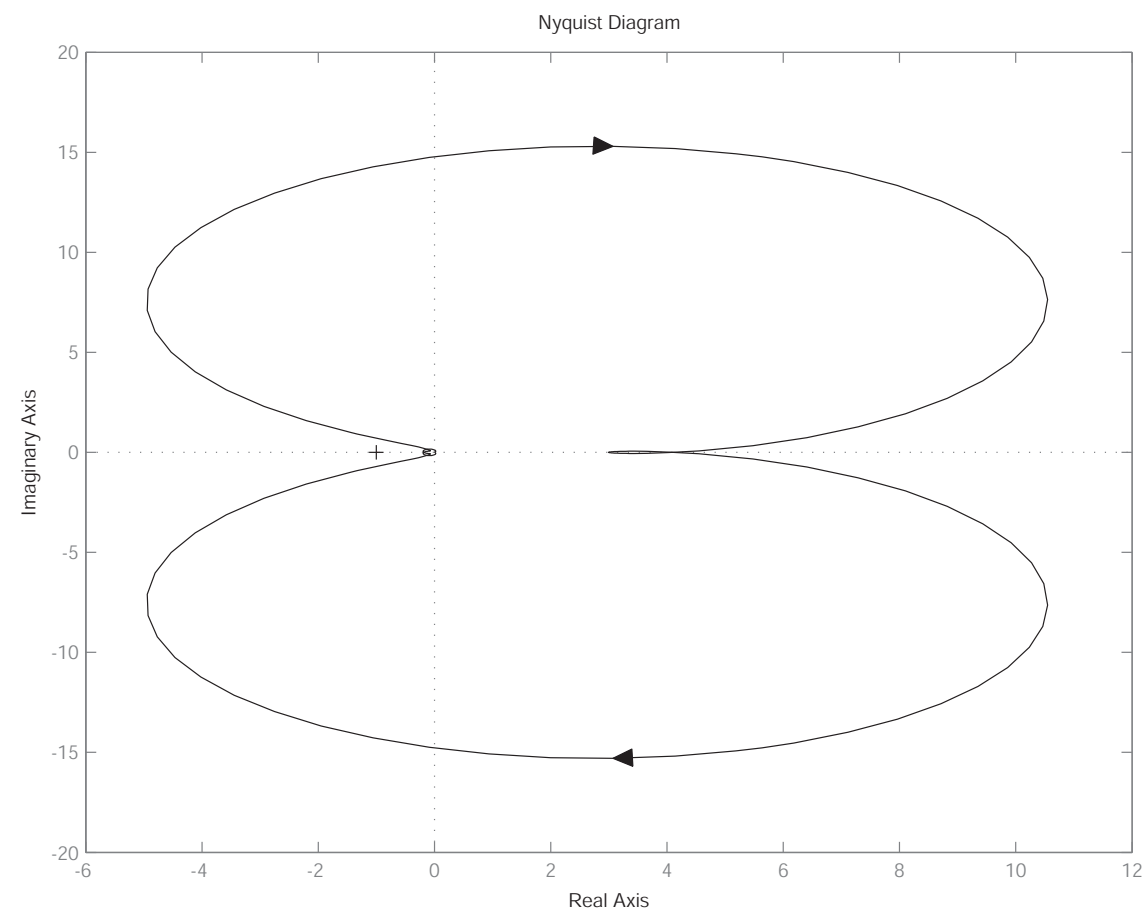

Figure 3-23: Nyquist diagram of the modelled tool position dynamics for the $2 \mathrm{kHz}$ FTS (including a lead compensator).

\subsubsection{Equivalent Linear Mass and Passive Dynamic Stiffness Seen at the Tool Tip}

The rotary inertia of the swing-arm and the tool swing radius can be used to determine an equivalent linear mass for the tool. That equivalent linear mass is then used to determine the passive stiffness (no feedback control) seen at the tool tip. This passive stiffness is due to inertia only, and since it is a function of frequency it is referred to as the passive dynamic stiffness seen at the tool tip. The passive dynamic stiffness provides a metric for describing the tool tip stiffness that a workpiece would feel from a particular fast tool servo without consideration of the control system. Closed-loop control increases the dynamic stiffness seen at the tool tip, except in the vicinity of the crossover frequency, where it may be reduced. For two different fast tool servos to have the same net dynamic stiffness at the tool tip, the one with a lower passive dynamic stiffness requires more controller authority. Since the position loop for the $2 \mathrm{kHz}$ FTS has a crossover frequency of $1 \mathrm{kHz}$, I choose that frequency value as a point of reference for reporting the passive dynamic stiffness seen at the tool tip. 
The sketch on the left in Figure 3-24 shows a rotary inertia $I$ connected to ground via a torsional spring $k_{T}$ and being acted upon by a torque $T$. The second sketch from the left in Figure 3-24 shows an equivalent linear mass $m$, which is modelled as a point mass at the end of a lever arm $R$, being acted on by a force $F=T / R$. The relationship between this mass and the rotary inertia is given by $m=I /\left(R^{2}\right)$. The third sketch from the left in Figure 3-24 shows the equivalent linear mass-spring system, where the linear (translational) spring is denoted by $k_{L}$. The sketch on the right in Figure 3-24 shows the Bode plot for the equivalent mass-spring system. For a linear spring the relationship between force, stiffness, and displacement is given by $F=k x$, respectively. Since $k=F / x$, the Bode plot in Figure 3-24 shows the compliance $\left(1 / k_{P D S}\right)$ of the mass as a function of frequency, or the inverse of the passive dynamic stiffness $k_{P D S}$. At frequencies above the resonance, the stiffness $k_{P D S}$ is dominated by the mass, and increases with frequency. At frequencies below the resonance, the stiffness $k_{P D S}$ is simply the stiffness of the spring $k_{L}$.

Note that if we increase the stiffness of the spring $k_{L}$ by a factor of $N$, for frequencies below the resonance the curve moves down by a factor of $N$, and the frequency of the resonance moves up by the square-root of $N$. Since the high frequency slope of the curve is -2 decade/decade, for frequencies above the resonance the curve does not move. This is what we expect, since the high frequency stiffness is dominated by the mass, which we did not change in this thought exercise. By a similar argument, if we increase the mass, the high frequency stiffness increases and the low frequency stiffness remains unchanged.

An intuitive appreciation for the passive dynamic stiffness $k_{P D S}$ suggested by the Bode plot in Figure 3-24 can be gained by qualitatively considering the system behavior at low and high frequencies. At low frequencies (below the break point) the spring dominates — slowly push on the mass and you feel the stiffness of the spring only. At high frequencies (above the break point) the mass dominates - quickly push on the mass and you feel its inertia. Push even quicker and you need more force to produce the same acceleration - the system gets stiffer as the frequency is increased.

For the $2 \mathrm{kHz}$ FTS, the combined rotational inertia of the swing-arm and moving 

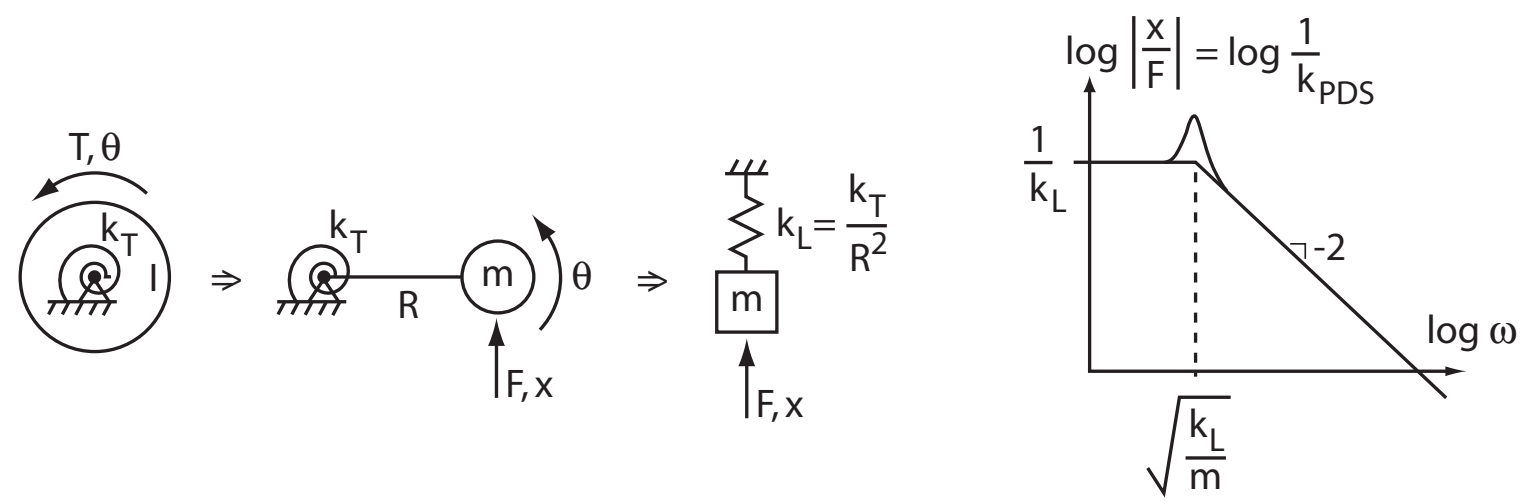

Figure 3-24: Sketches showing the relationship between a rotary inertia and an equivalent linear mass (left three figures), and the Bode plot for the equivalent mass-spring system showing the inverse of the passive dynamic stiffness $k_{P D S}$ (right).

element of the motor is $57 \mathrm{gm} \cdot \mathrm{cm}^{2}$. Combined with a $5 \mathrm{~mm}$ tool swing radius, this results in an effective linear mass of $0.23 \mathrm{~kg}$ as seen at the tool tip. The measured rigid body torsional mode at $200 \mathrm{~Hz}$ suggests a torsional spring constant for the flexure suspension of $k_{T}=9.6 \mathrm{~N} \cdot \mathrm{m} / \mathrm{rad}$, which leads to a linear spring constant of $k_{L}=0.38 \mathrm{~N} / \mu \mathrm{m}$. Using the construction shown in Figure 3-24, the passive dynamic stiffness seen at the tool tip is $9 \mathrm{~N} / \mu \mathrm{m}$ at $1 \mathrm{kHz}$. Note that the flexures provide only $4 \%$ of the passive torsional stiffness at $1 \mathrm{kHz}$.

\subsection{Servo Test Bed}

A servo test bed was developed in parallel with the mechanical design for the $2 \mathrm{kHz}$ FTS. It combined the actuator and sensors for the $2 \mathrm{kHz}$ FTS with a payload having the same rotary inertia as the swing-arm. Figure 3-25 shows the components for the servo test bed and Figure 3-26 shows the fully assembled system. The servo test bed allowed developing the current-mode amplifier and control system for the $2 \mathrm{kHz}$ FTS in parallel with the design, fabrication, and assembly efforts for the mechanical components for the FTS. This allowed me to compress the development schedule shown in Figure 1-1 for the $2 \mathrm{kHz}$ FTS, and made it possible to realize closed-loop control of the FTS during the same month that it was fully assembled. 


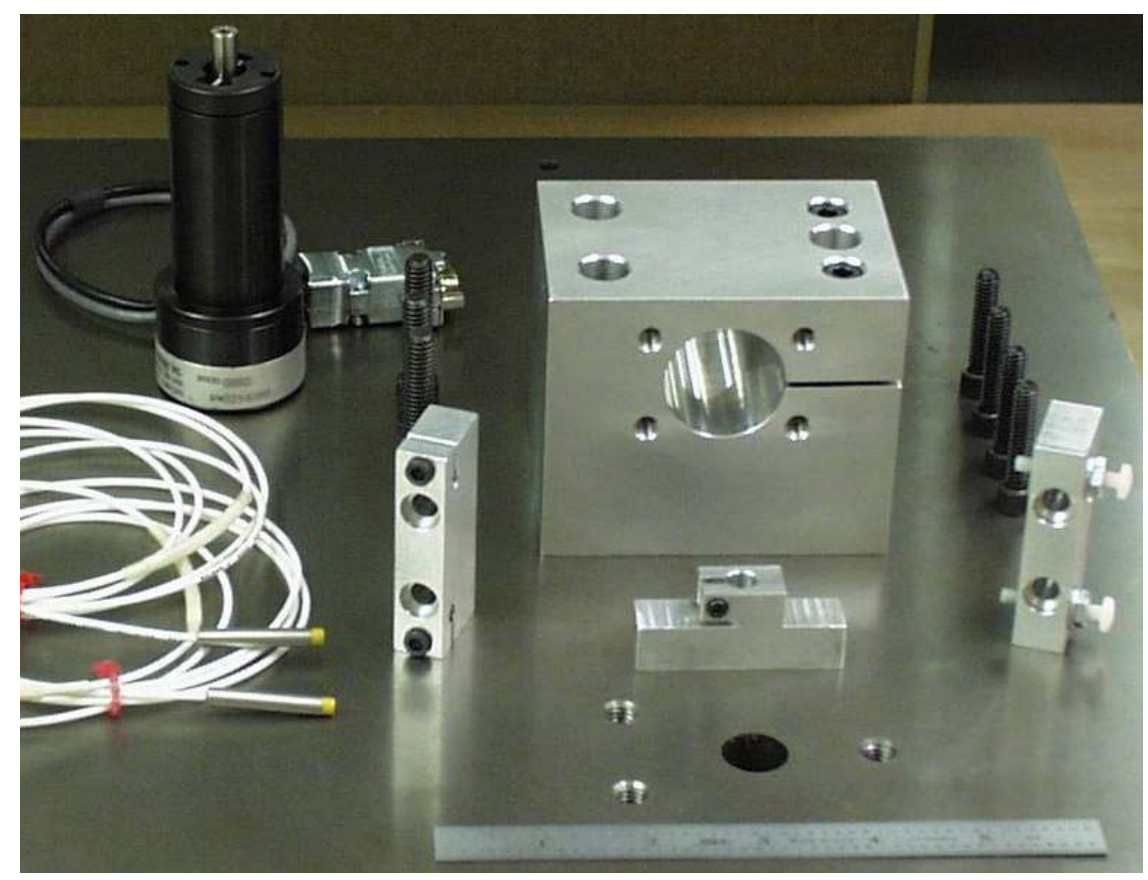

Figure 3-25: Components for the servo test bed.

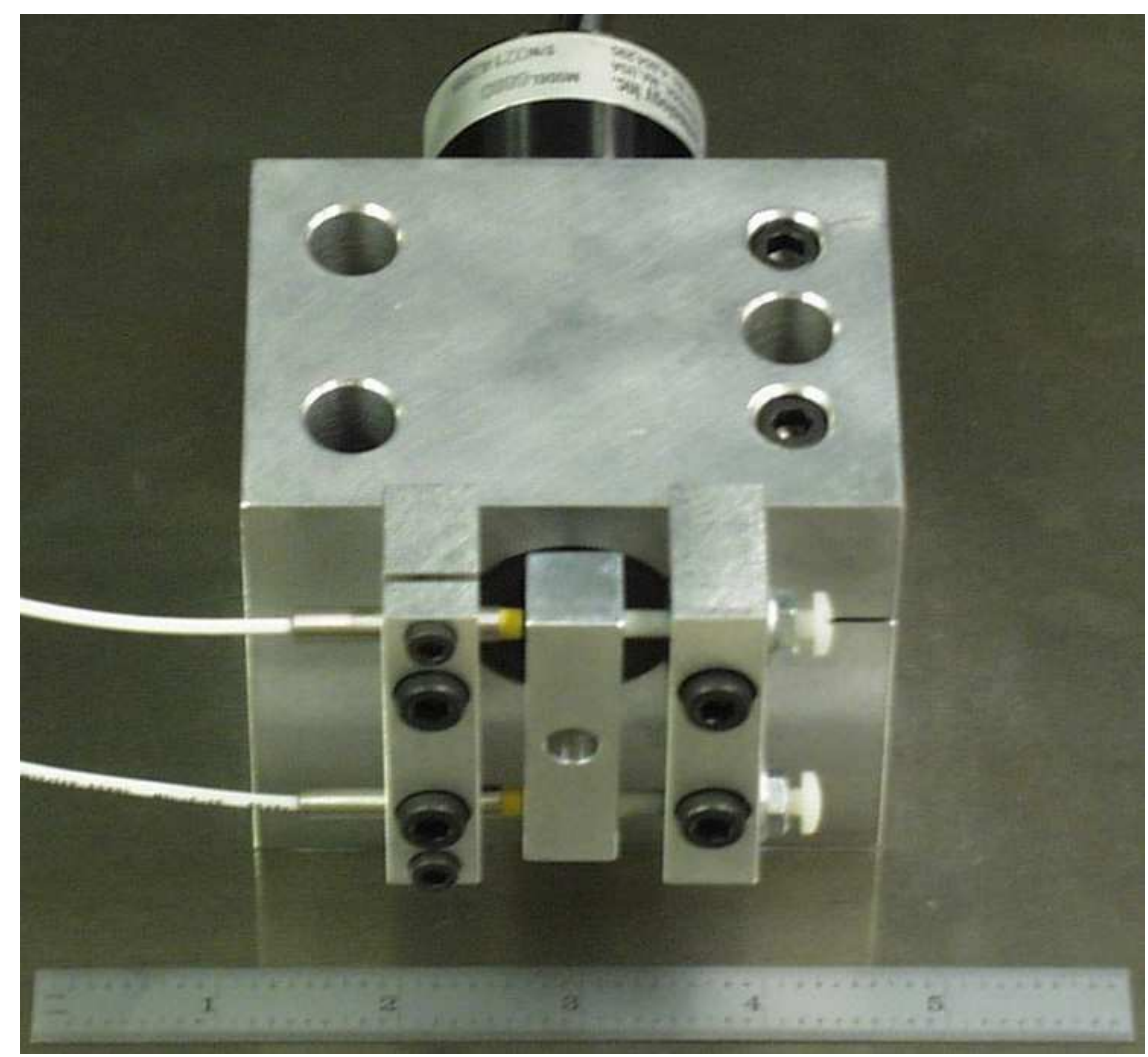

Figure 3-26: The fully assembled servo test bed. 


\subsection{Electrical Design}

I designed and fabricated a current-mode amplifier for the $2 \mathrm{kHz}$ rotary fast tool servo. This type of amplifier is often referred to as a torque-mode amplifier because for many types of actuators torque is proportional to the current flowing through the coil. The actuators used in both the $2 \mathrm{kHz}$ and $10 \mathrm{kHz}$ rotary fast tool servos fall into this category. Figure 3-27 is a schematic diagram of the electrical circuit for the current-mode amplifier, and includes the electrical dynamics of the actuator. A one-for-one mapping can be made between the circuit shown in Figure 3-27 and the block diagram shown in Figure 3-19 (not including the block labelled $\mathrm{K}_{m}$ ).

\subsubsection{Current-Mode Amplifier}

Our design goal of a closed-loop bandwidth of $2 \mathrm{kHz}$ for the tool position suggested designing the current-mode amplifier to have a loop transmission crossover frequency of at least $20 \mathrm{kHz}$, and the achieved $30 \mathrm{kHz}$ crossover satisfies this. The first operational amplifier in the forward path of the loop in Figure 3-27 is the analog controller (current compensation) for the amplifier. Note that the current compensation has a pure integrator (the $30 \mathrm{pF}$ capacitor in the local feedback path) which would cause the output of that operational amplifier to quickly drift to the value of one of its supply voltages if the outer feedback loop was not present. Details on the loop-shaping technique used to design the current compensation, and the mapping between the compensation circuit components in Figure 3-27 and the corresponding blocks in Figure 3-19, are given in Appendix A.1. The last operational amplifier in the forward path of the loop in Figure 3-27 is the power operational amplifier, which is an Apex Microtechnology Corporation PA04 [12] configured for a non-inverting DC gain of 3. The measured gain of the power amplifier is essentially constant up to $1 \mathrm{Mhz}$, with negligible phase up to $100 \mathrm{kHz}$ and only about $-8^{\circ}$ of phase at $200 \mathrm{kHz}$. Therefore the power operational amplifier is treated as a constant gain of 3 for the frequencies of interest for the current-mode amplifier. The remaining three operational amplifiers in Figure 3-27 are differential amplifiers used to isolate the ground noise present in the 


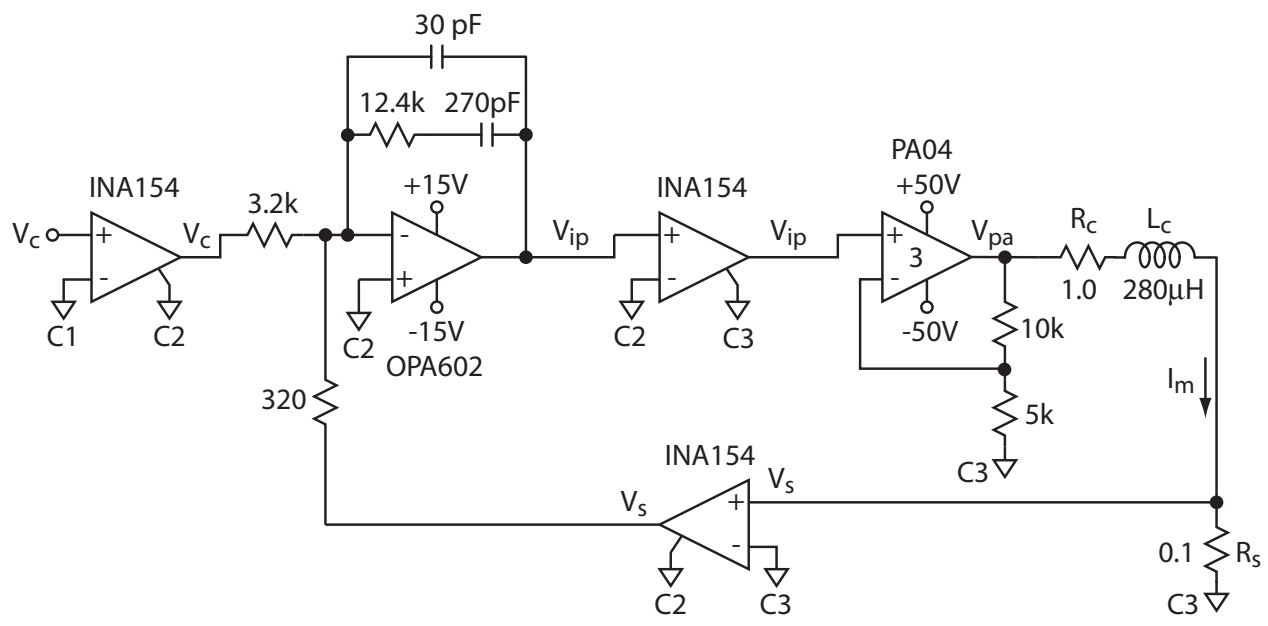

Figure 3-27: Schematic diagram of the electrical circuit for the current-mode amplifier used with the $2 \mathrm{kHz}$ FTS.

three sections of the circuit. "C1" is the signal ground (common) for the PC-based digital control system that provides the command signal $V_{c}$, "C2" is the signal ground for the analog controller (current compensation), and "C3" is the signal ground for the power amplifier and motor (actuator). The feedback voltage $V_{s}$ is a measure of the motor current $I_{m}$ provided by the sense resistor $R_{s}$.

\subsubsection{Voltage Budget and Power Dissipated in the Amplifier}

The power amplifier drives three things in the actuators: (1) the inductive load; (2) the resistive load; and (3) the velocity-dependent back-EMF. For a sinusoidally varying tool tip trajectory of $z_{t}=A \sin (\omega t)$, the peak velocity of $\dot{z}_{t p k}=A \omega$ occurs at $z_{t}=0$, and the peak acceleration of $\ddot{z}_{t p k}=A \omega^{2}$ occurs at the end points of travel of the tool tip, where the actuator needs to produce the peak torque and therefore draws the peak current (torque is proportional to the coil current). Keeping track of positive and negative directions, the maximum tool tip velocity lags the maximum tool tip acceleration by $90^{\circ}$. Since the peak velocity lags the peak current by $90^{\circ}$, the peak back-EMF is in phase with the peak voltage across the inductor. If the actuator was a pure inductive load, then for a sinusoidally varying coil current the peak current would lag the peak voltage by $90^{\circ}$. At the frequencies of interest, I show that the resistive load is appreciable, and therefore the coil current lags the output 

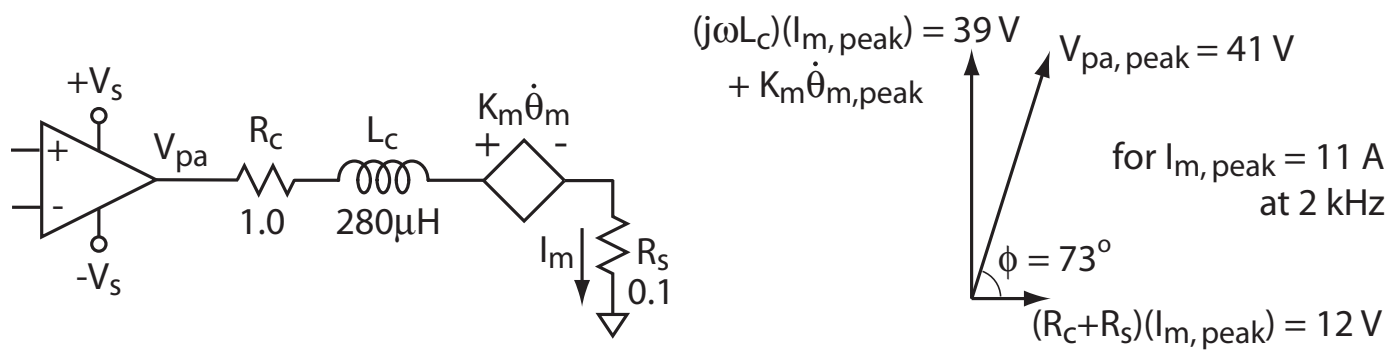

Figure 3-28: Schematic diagram of the power amplifier, motor coil resistance and inductance, Back-EMF, and sense resistor for the $2 \mathrm{kHz}$ FTS (left), and phasor diagram showing the relationship between the real and imaginary components of the output voltage of the power amplifier (right). Phasor diagram is for the maximum specified continuous peak current of $11 \mathrm{~A}$ for the actuator and $2 \mathrm{kHz}$ operation.

voltage of the power amplifier by less than $90^{\circ}$.

The left side of Figure 3-28 shows a schematic diagram of the power amplifier, motor, and sense resistor for the $2 \mathrm{kHz}$ FTS. The relationship between the output voltage of the power amplifier $V_{p a}$, the motor current $I_{m}$, coil resistance $R_{c}$, coil inductance $L_{c}$, sense resistor $R_{s}$, motor constant $K_{m}$, and motor angular velocity $\dot{\theta}_{m}$ is determined by Kirchoff's Voltage Law and given by (3.17):

$$
V_{p a}=\left(R_{c}+R_{s}\right) I_{m}+L_{c} \frac{d I_{m}}{d t}+K_{m} \dot{\theta}_{m}
$$

Using the specified actuator torque constant of $0.0254 \mathrm{~N} \cdot \mathrm{m} / \mathrm{amp}$ and peak continuous current of $11 \mathrm{~A}$, the peak tool tip acceleration at $2 \mathrm{kHz}$ operation is $25 \mathrm{~g}$. This leads to a peak angular velocity of $4.0 \mathrm{rad} / \mathrm{sec}$ for the motor. Combined with the component values shown in Figure 3-28, and the specified actuator torque constant of $0.0254 \mathrm{~N} \cdot \mathrm{m} / \mathrm{amp}$ and peak continuous current rating of $11 \mathrm{~A}$, the terms in (3.17) are given by (3.18), (3.19), and (3.20):

$$
\begin{gathered}
\left(R_{c}+R_{s}\right) I_{m}=(1.0+0.1) 11=12 \mathrm{~V} \\
L_{c} \frac{d I_{m}}{d t}=\left(280 \times 10^{-6}\right)\left(14 \times 10^{4}\right)=39 \mathrm{~V}
\end{gathered}
$$




$$
K_{m} \dot{\theta}_{m}=(0.0254) 4.0=0.10 \mathrm{~V}
$$

Examination of (3.18), (3.19), and (3.20) reveals that the peak back-EMF is negligible compared to the resistive and inductive loads. The right side of Figure 3-28 shows a phasor diagram depicting the relationship between the real and imaginary components of the output voltage of the power amplifier, and indicates that the peak motor current of 11 A lags the peak output voltage of $41 \mathrm{~V}$ from the power amplifier by $73^{\circ}$ when operating at $2 \mathrm{khz}$. The power operational amplifier used with the $2 \mathrm{kHz}$ FTS can swing to within $9 \mathrm{~V}$ of the supply voltages, suggesting minimum supply voltages of $\pm 50 \mathrm{~V}$. It is worth mentioning that the maximum voltage slew rate for this case is $0.5 \mathrm{~V} / \mu \mathrm{sec}$, which is well within the specified $40 \mathrm{~V} / \mu$ sec for the power operational amplifier.

Figure 3-29 shows the relationships between the voltage, current, and power in the motor (load), and the supply voltages and dissipated power in the power amplifier for the $2 \mathrm{kHz}$ FTS. To determine the power dissipation in the output stage of the power operational amplifier we consider the voltage drop across it — voltage of the active supply minus the output voltage of the operational amplifier - and the current flowing through it. The active supply is the one with the same sign as the current being supplied by the operational amplifier. Note that because the motor current is out of phase with the output voltage of the power amplifier that the signs of the two are opposite at certain places in the cycle, which leads to a higher power dissipation in the operational amplifier than would occur for a purely resistive load. The numerical analysis used to generate Figure 3-29 is listed in Appendix B.3, and indicates that the average power dissipated in the power operational amplifier is 284 VA during the peak operating conditions described above. The specified maximum power dissipation for the power operational amplifier used for the $2 \mathrm{kHz}$ FTS is $200 \mathrm{~W}$, or $70 \%$ of $284 \mathrm{VA}$. Since the power dissipated in the operational amplifier scales with the square of the motor current, the allowable maximum continuous peak current for $2 \mathrm{kHz}$ operation with $\pm 50 \mathrm{~V}$ supplies is thus limited to $9.2 \mathrm{~A}$. 
For the work performed for this thesis with the $2 \mathrm{kHz}$ FTS I used $\pm 36 \mathrm{~V}$ power supplies because that is what was available in our lab at the time. Subtracting the $9 \mathrm{~V}$ drop from the supply voltage to the maximum output voltage for the power operational amplifier gives a peak output voltage of $\pm 29 \mathrm{~V}$ with these supplies. Referring back to the phasor diagram in Figure 3-28, the voltages scale linearly with current and therefore the peak available motor current at $2 \mathrm{kHz}$ is $7.8 \mathrm{~A}$. This is consistent with the demonstrated torque of $0.20 \mathrm{~N} \cdot \mathrm{m}$ at $2 \mathrm{kHz}$, which requires a peak current of 7.7 A. Using the same numerical analysis that was used to generate Figure 329, the average power dissipated in the power operational amplifier is 146 VA during the demonstrated peak operating conditions with $\pm 36 \mathrm{~V}$ power supplies, which is well within the $200 \mathrm{~W}$ specifications for the power operational amplifier.

\subsubsection{Electrical Component Layout}

As mentioned earlier in Section 3.9.1, differential amplifiers are used to isolate the ground noise present in the three sections of the circuit. Referring to Figure 3-30, "C1" is the signal ground (common) for the PC-based digital control system, "C2" is the signal ground for the analog controller (current compensation), and "C3" is the signal ground for the power amplifier and motor (actuator). The three signal grounds need to be referenced to a common voltage. The power supplies for the analog compensation, sensor amplifier, and power amplifier are all initially floating relative to the earth safety ground. To avoid ground loop currents between those circuit elements, the signal grounds for the analog compensation, sensor amplifier, and power amplifier are arranged in a parallel connection and tied together at the single point shown schematically above the power amplifier in Figure 3-30. From a ground loop perspective, it is unfortunate that the signal ground "C1" for the PCbased controller is connected locally to the earth safety ground. I could have made the single-point connection with earth ground at the chassis of the PC, but since I was using different computers during the course of this work I decided to associate the earth safety ground with the power amplifier so that I was assured that it was always present. Note that the cooling fan has its own dedicated power supply and 
"push pull power loss 02.m", 2 kHz rotary fast tool servo

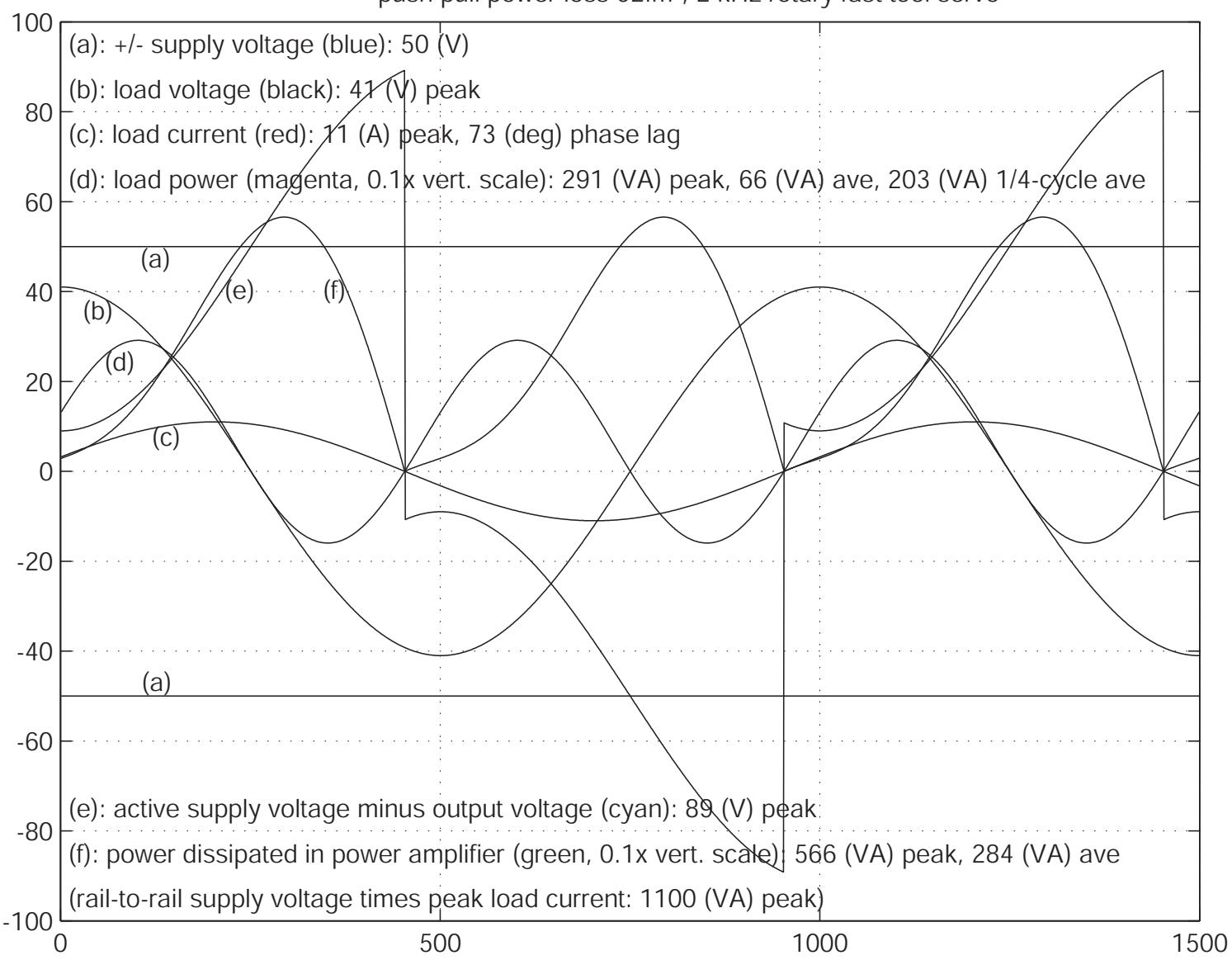

Figure 3-29: Plot showing relationships between the voltage, current, and power in the motor (load), and the supply voltages and dissipated power in the power amplifier for the $2 \mathrm{kHz}$ FTS. 


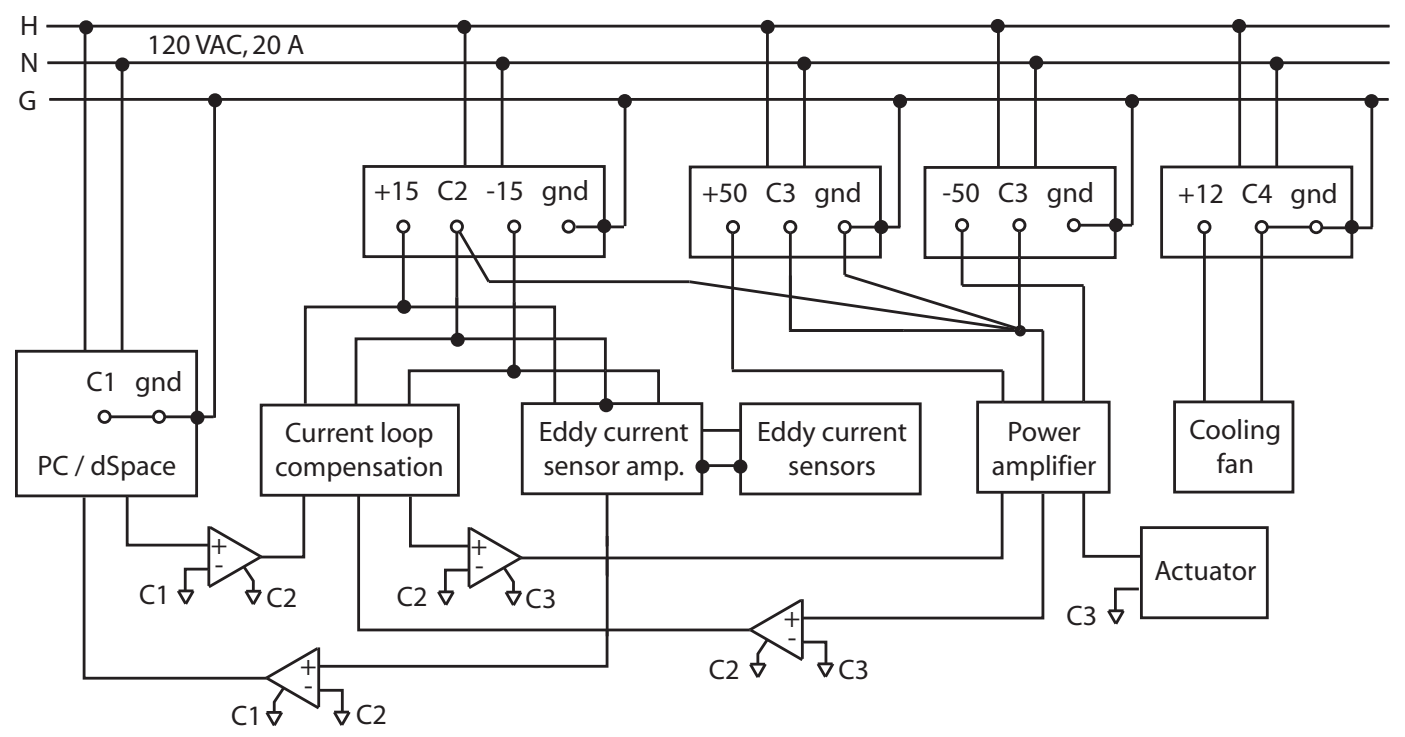

Figure 3-30: Schematic layout of the electrical components for the $2 \mathrm{kHz}$ FTS.

that its common is not connected to the single-point ground connection. It turns out that the cooling fan, which was salvaged from a discarded PC power supply, injects significant noise into its power lines.

Figure 3-31 shows the as-built analog compensation circuit and power amplifier for the $2 \mathrm{kHz}$ FTS. Even though the analog compensation circuit resides on a prototyping board and there is a preponderance of long wires connecting the components, adherence to properly isolating commons and avoiding ground loops allowed the system to perform adequately for this research.

\subsubsection{Brief Comments on Troubleshooting Electrical Systems}

Achieving a $30 \mathrm{kHz}$ crossover frequency with the current-mode amplifier for the $2 \mathrm{kHz}$ FTS was not a trivial task. A significant amount of troubleshooting accompanied the development of the analog compensation circuit and power amplifier. This is one of the areas where the science and art of engineering meet, and in my case the respective venues were the theoretical underpinnings used to predict and explain observed phenomena, and the hard-learned accumulation of hands-on experience in what does and does not work. This topic could easily fill tens of pages, and entire books have been written on the subject. For the purposes of this thesis I merely 


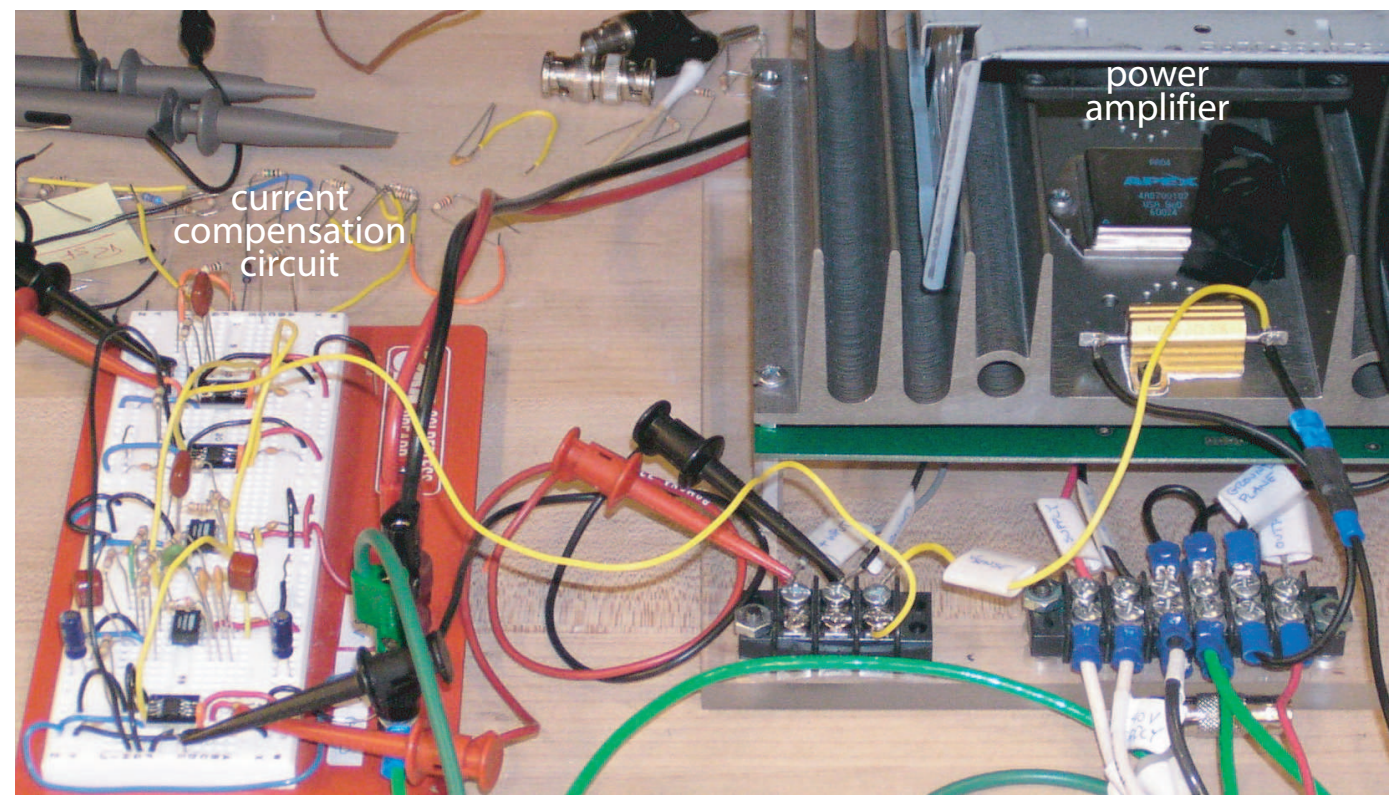

Figure 3-31: The as-built analog compensation circuit and power amplifier for the $2 \mathrm{kHz}$ FTS.

provide a list of the topics that were significant for me, along with the references that I found useful.

- Grounding and ground loops. Ott [118], Barnes [16], and Fowler [59] provide excellent discussions on grounding and ground loops. They describe singlepoint and multi-point grounding, provide motivations for choosing one type over the other according to the nature of the circuits involved, and discuss safety grounding. They also provide clear explanations on what a ground loop is and how it can affect the behavior of a circuit.

- By-pass capacitors. These provide a local energy supply (or sink) for circuit components that would otherwise be adversely affected by the inherent inductance of the circuit leads to the power supplies. Inadequate by-pass capacitance was often the source of unwanted oscillations in some of my operational amplifier circuits. A mix of small-valued low-inductance capacitors and larger capacitors was usually needed. The application notes provided by the manufacturer of the power operational amplifiers [12] used in the $2 \mathrm{kHz}$ and $10 \mathrm{kHz}$ rotary fast tool servos were useful starting points. Ott [118] and Barnes [16] 
discuss lumped-impedance models for a capacitor that provide insight to their actual behavior, and provide data on the characteristics of common capacitors that is useful when deciding which type to select. Additional useful insights regarding by-pass capacitors are provided by Roberge [132] and Horowitz and Hill [78].

- Dealing with electronic noise. Ott [118], Barnes [16], and Mardiguian [103] provide valuable insight to troubleshooting and dealing with electronic noise. I especially appreciated Ott's [118] description of the three items needed for an electronic circuit to be affected by noise: a noise source, a transmission media, and a noise receiver. Looking at a noise problem as a chain consisting of those three links allowed me to be more deterministic in my troubleshooting and noise mitigation efforts.

- Inductive coupling versus capacitive coupling. Fowler [59] describes a useful diagnostic that can help the troubleshooter decide which noise transmission medium between two parallel conductors is more likely: inductive coupling or capacitive coupling. The fix for each type is different, so knowing which one to consider first can speed up a troubleshooting effort. Fowler credits Van Doren [145] for the following idea, in which the diagnostic ratio (dv/dt)/(di/dt) pertains to the source-side of the noise circuit:

For frequencies above $3 \mathrm{kHz}$, a useful diagnostic for determining the mechanism is the ratio of the rate of change in voltage to the rate of change in current (Van Doren, 1991). For the special cases of sinusoidal signals or resistive loads, the ratio is impedance; otherwise, it is a pseudo impedance value. A low value $(<377 \Omega)$ indicates a large change in current and inductive coupling; a high value $(>377 \Omega)$ indicates a large change in voltage and capacitive coupling. At very high frequencies (>20 MHz), a ratio near $377 \Omega$ indicates electromagnetic coupling. 
Diagnostic ratio or pseudo impedance $=(\mathrm{dv} / \mathrm{dt}) /(\mathrm{di} / \mathrm{dt})[59$, page 187].

- Large-amplitude output signals near small-amplitude input signals. One particularly interesting cause of an unwanted oscillation in an operational amplifier was caused by not paying adequate attention to the routing of the wires between and around the analog current compensation circuit and the power amplifier. It turned out that a wire carrying a large-amplitude signal from the output of the power amplifier was too close and in parallel with a wire connected to the non-inverting terminal of the compensation operational amplifier. A recipe for disaster indeed, as this provided an unintended positive feedback network to the current-mode amplifier. This mistake occurred when I was still relatively "young" in my real-world experience with electronics. It drove home the lesson that although a circuit diagram may not show unwanted positive feedback connections, the placement of components and conductors in the actual circuit needs to be scrutinized to make sure that additional connections via un-modelled capacitive or inductive links do not exist.

\subsection{Control System}

Both the $2 \mathrm{kHz}$ and $10 \mathrm{kHz}$ rotary fast tool servos utilize a hybrid analog and digital control system. The block diagram for the control system used with the $2 \mathrm{kHz}$ FTS is shown in Figure 3-32. The inner (current) loop forms the current-mode power amplifier. Feedback of the actuator current is used to compensate for the electrical dynamics of the actuator. This essentially removes the actuator electrical dynamics from the outer (tool position) loop at the operating frequencies of interest. The current loop has a crossover frequency of $30 \mathrm{kHz}$, and is implemented with analog electronics to avoid the phase delay inherent with the sampling process of a digital controller. The tool position loop has a crossover frequency of $1 \mathrm{kHz}$ and is implemented with a digital controller using a sampling rate of $80 \mathrm{kHz}$. During the development of the $2 \mathrm{kHz}$ FTS, three different compensators for the tool position 


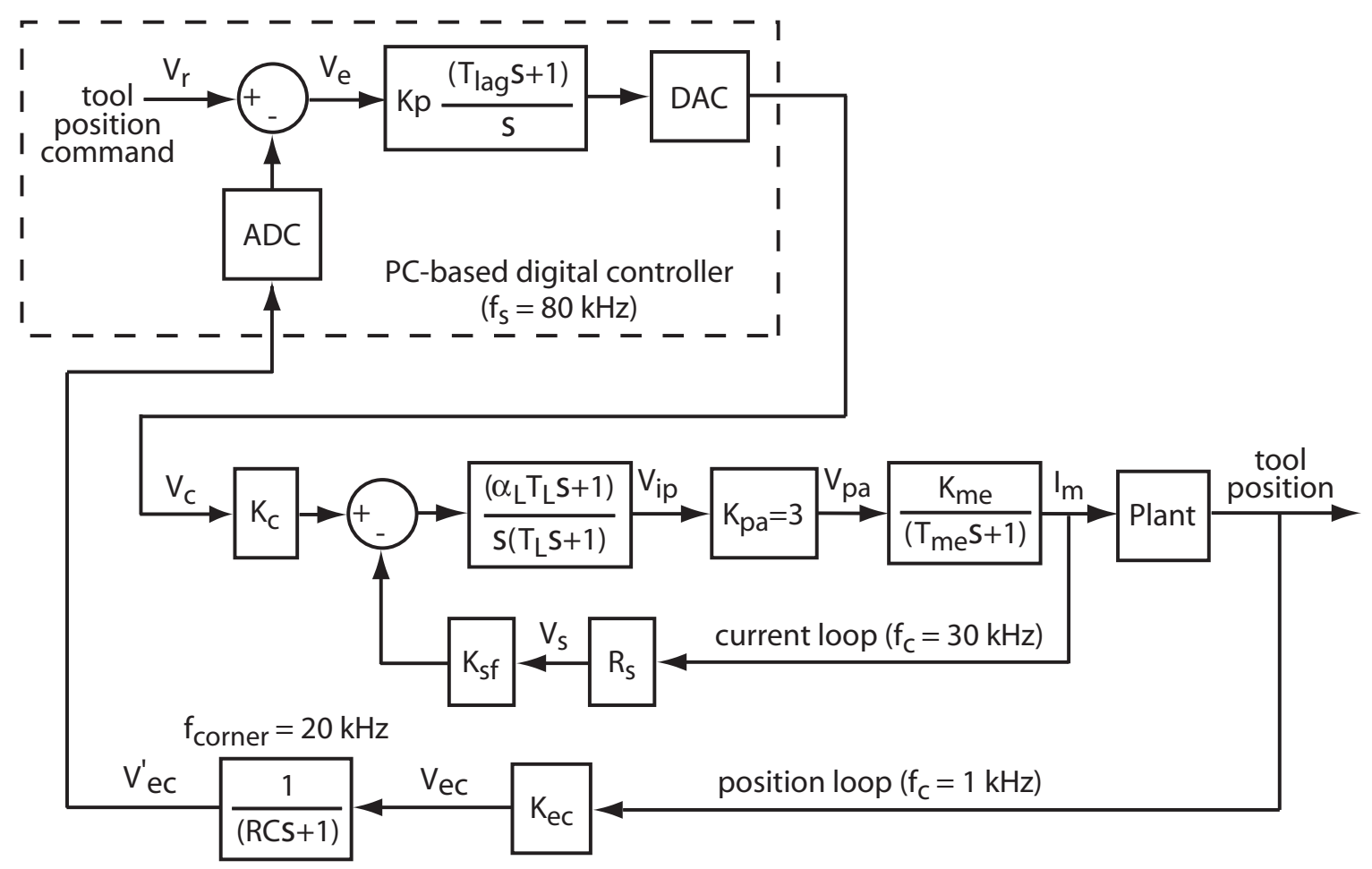

Figure 3-32: Control system block diagram for the $2 \mathrm{kHz}$ FTS.

were used. The first two - lead-lag with a notch filter, and lead-lag with a phasestabilizing pole - were used before viscous damping was added to the system. The third compensator, lag only, was sufficient after viscous damping was added via the damping plate. In all cases, series compensation in the forward path was used for the tool position. The block diagram in Figure 3-32 shows the lag compensation used for the system with viscous damping. Note the use of an anti-aliasing filter preceding the analog-to-digital converter in Figure 3-32. The loop-shaping technique that I used to design the compensators for the $2 \mathrm{kHz}$ and $10 \mathrm{kHz}$ rotary fast tool servos is described in detail in Section A.1.

\subsubsection{Digital Controller Prototyping Environment}

The combination of MATLAB ${ }^{\circledR}$, Simulink ${ }^{\circledR}$, Real-Time Workshop ${ }^{\circledR}$, and a PC-based dSPACE GmbH 1103-board digital controller allowed me to quickly develop, implement, test, and modify prototype controllers for the $2 \mathrm{kHz}$ and $10 \mathrm{kHz}$ rotary fast tool servos. MATLAB ${ }^{\circledR}[104]$ is a computer programming language that works with 
matrix-based data and has an extensive library of functions that are useful for control system design. Simulink ${ }^{\circledR}[104]$ is an extension of MATLAB ${ }^{\circledR}$ and provides a block diagram environment for designing and simulating control systems. Real-Time Workshop ${ }^{\circledR}$ [104] automatically generates a C-code representation of a Simulink ${ }^{\circledR}$ model. The dSPACE GmbH [51] real-time controller board integrates dedicated processors with a variety of input and output interfaces, and uses proprietary software to automatically implement the Real-Time Workshop ${ }^{\circledR}$ C-code. Additional dSPACE GmbH proprietary software allows creating a graphical user interface (GUI) for the control system.

The 16-bit analog-to-digital converters on the dSPACE GmbH board have a measured noise level of 6 bits PP, which corresponds to a tool motion of $5 \mathrm{~nm} \mathrm{PP}$ for the $2 \mathrm{kHz}$ FTS. The digital-to-analog converters on the dSPACE board generate a significant glitch at every transition between the previously held output value to the new one. Measurement of these $180 \mathrm{mV}$ PP "DAC glitches" are shown in the upper trace in the captured oscilloscope screen shown in Figure 3-33, which is a portion of a $1 \mathrm{kHz}$ sinusoidal command voltage $V_{c}$ to the current-mode amplifier. The lower trace in Figure 3-33 is the response of the current compensation as seen at the input voltage $V_{i p}$ to the power operational amplifier. The resulting $80 \mathrm{kHz}$ pulse train is too fast for the position loop to follow because the mechanical system acts as a low-pass filter with a cut-off well below that frequency. However, the current loop with its cross-over frequency of $30 \mathrm{kHz}$ is able to respond to that pulse train, and therefore the power amplifier does unnecessary work trying to follow it. I attempted to attenuate the DAC glitches with an analog RC low-pass filter, but the required cut-off frequency unacceptably reduced the phase margin for the position loop. A detail of one of the DAC glitches and the resulting spike in the input signal to the power operational amplifier is shown in Figure 3-34.

It may be possible that the digital inputs to the DAC's are not being set and allowed to become stable before the gate signal is enabled. Since the DAC's are hard-wired into the dSPACE board, and the glitches were not a show-stopper for this research, I chose to not pursue troubleshooting and improving their performance. 


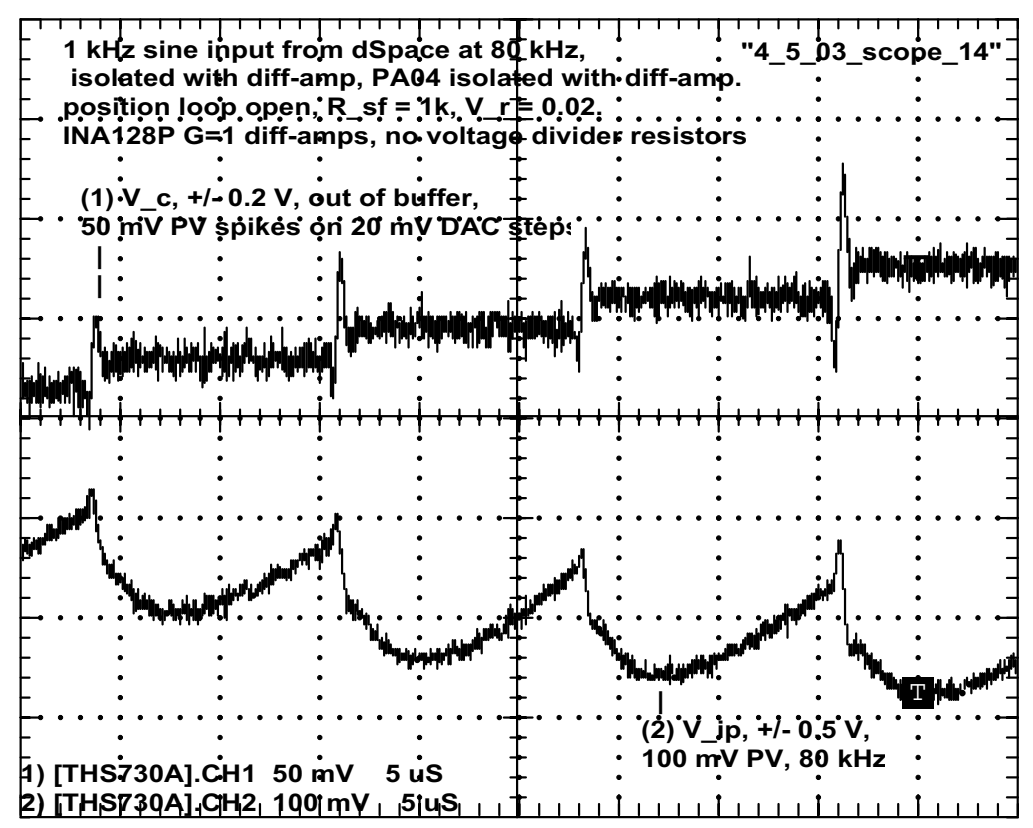

Figure 3-33: Captured oscilloscope screen showing DAC glitches on the command voltage $V_{c}$ to the current-mode amplifier (upper trace), and the response of the current compensation as seen at the input voltage $V_{i p}$ to the power operational amplifier (lower trace). The command signal $V_{c}$ is a $1 \mathrm{kHz}$ sinusoid produced by the digital controller running with an $80 \mathrm{kHz}$ sampling rate.

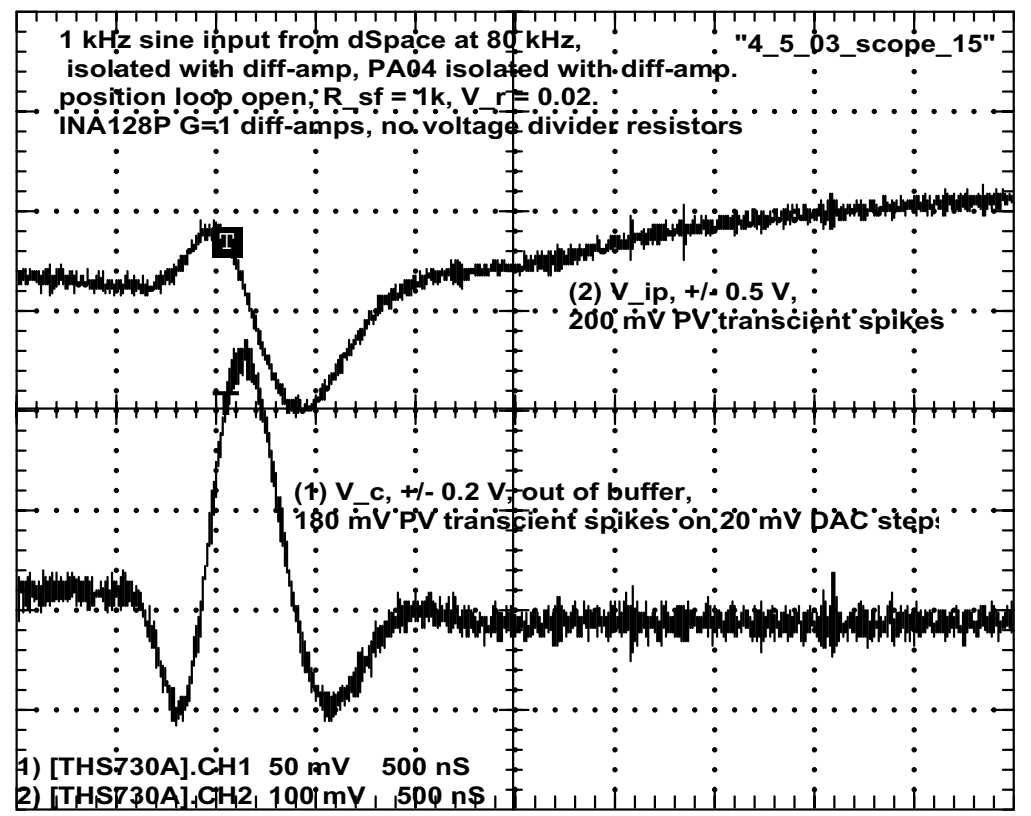

Figure 3-34: Detail of the voltage signals shown in Figure 3-33. Command voltage $V_{c}$ to the current-mode amplifier (lower trace), and the response of the current compensation as seen at the input voltage $V_{i p}$ to the power operational amplifier (upper trace). 


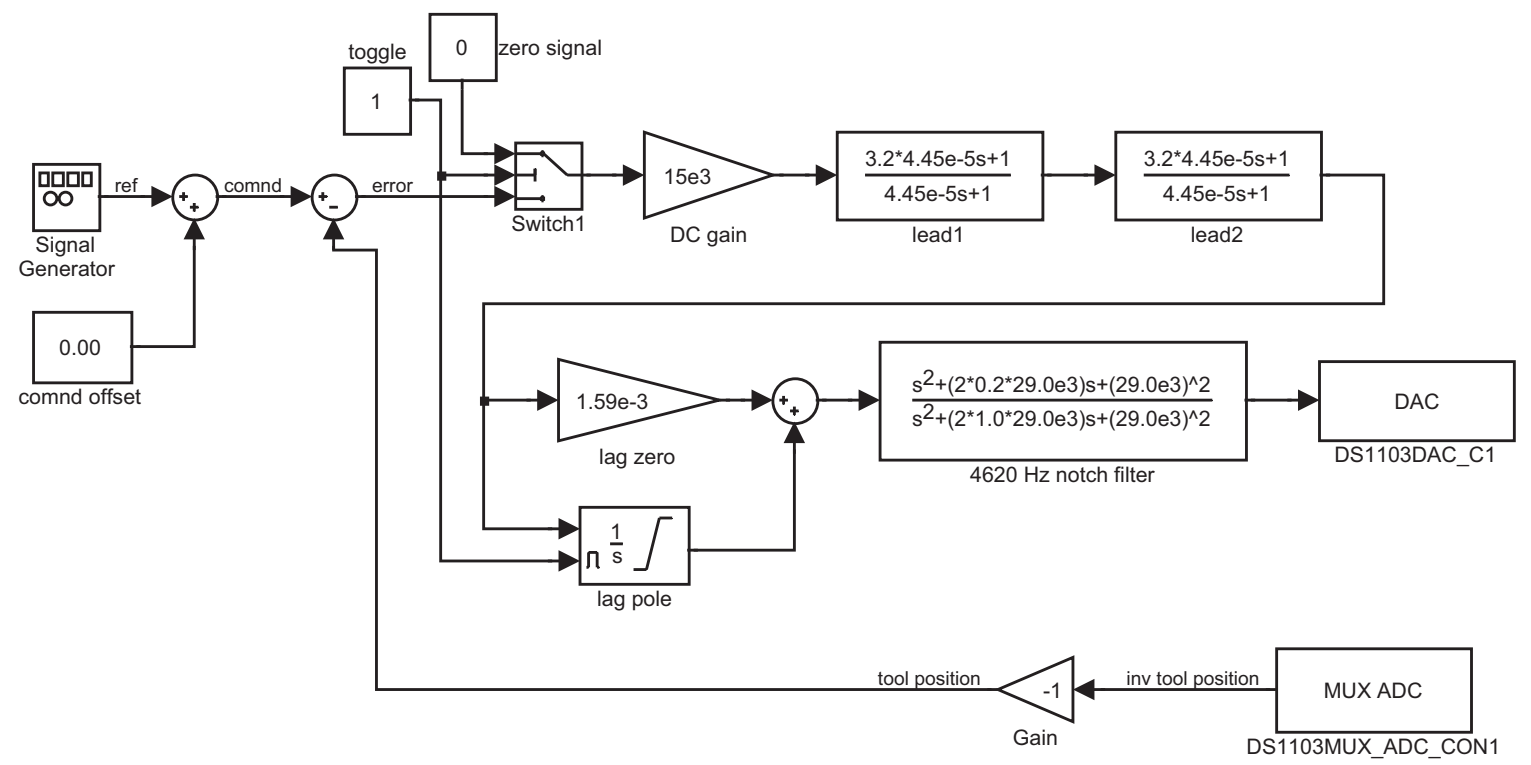

Figure 3-35: Block diagram for the digital controller for the tool position for the $2 \mathrm{kHz}$ FTS without viscous damping, using a double lead, lag, and notch filter compensator.

\subsubsection{Double Lead, Lag, and Notch Filter Compensation}

Figure 3-35 shows the block diagram for the digital controller for the tool position for the $2 \mathrm{kHz}$ FTS without viscous damping, using a series compensator consisting of a double lead, lag, and notch filter compensator. A couple of extra elements are present in this block diagram, which is actually the Simulink ${ }^{\circledR}$ model that was implemented in the real-time controller. The "toggle", "zero signal", and "switch1" blocks allow using the dSPACE GmbH GUI to turn the controller on and off and reset the integrator. The "signal generator" block provides a reference input signal for testing and debugging the controller.

Both of the two lead compensators use $\alpha=3.2$ and a time constant $T_{\text {lead }}=$ $4.45 \times 10^{-5}$ sec. The lag compensator consists of a pure integrator and a zero with a time constant $T_{l a g}=1.59 \times 10^{-3}$ sec. The guidelines described in Section A.3 were used to design the notch filter, which has a center frequency of $4620 \mathrm{~Hz}$ and damping ratios of $\zeta_{z}=0.2$ and $\zeta_{p}=1.0$ for the notch zeros and poles, respectively.

Figure 3-36 shows a Bode plot of the measured negative loop transmission for the tool position for the $2 \mathrm{kHz}$ FTS without viscous damping, using the double lead, lag, 
and notch filter compensator. The crossover frequency for this system is $1 \mathrm{kHz}$ with a phase margin of $13^{\circ}$ and a gain margin of $5 \mathrm{~dB}$.

The parametric model described in Section 3.7 is used to develop insight into the measured dynamics for this system. The model is adjusted until the Bode plot of the modelled negative loop transmission for the tool position sufficiently matches the Bode plot of the measured negative loop transmission, and then a root locus and Nyquist diagram are generated for the model. Figure 3-37 shows a Bode plot of the modelled negative loop transmission for the tool position for the $2 \mathrm{kHz}$ FTS without viscous damping, using the double lead, lag, and notch filter compensation. Figure 338 shows the corresponding root locus for that model. The small circles represent the open-loop and closed-loop zeros, the x's represent the open-loop poles, and the solid squares represent the close-loop poles.

For comparison purposes, Figure 3-39 shows the root locus for the model without the notch filter. Note that a pair of closed-loop poles end up in the right-half plane when the notch filter is omitted. Figure 3-40 shows the Nyquist diagram for the model with the notch filter, and Figure 3-41 is a detail of that Nyquist diagram.

\subsubsection{Double Lead, Lag, and Phase-Stabilizing Pole Com- pensation}

An alternate compensator for the $2 \mathrm{kHz}$ FTS without viscous damping replaces the notch filter with a phase-stabilizing pole. Professor David Trumper of MIT taught me this technique. In essence, for certain systems with a plant resonance that causes the Bode magnitude for the negative loop transmission to be close to unity at the frequency of the resonance, with an attendant phase angle near $-180^{\circ}$, adding a firstorder pole with a corner frequency near the frequency of the resonance pushes down the phase to well below $-180^{\circ}$ while only slightly decreasing the magnitude of the resonance, resulting in a stable loop. For the $2 \mathrm{kHz}$ FTS the extra pole is placed at $5 \mathrm{kHz}$ to phase-stabilize the plant resonance at $4.7 \mathrm{kHz}$. This reduces the magnitude and phase angle of the loop at that resonance by approximately $3 \mathrm{db}$ and $45^{\circ}$, 

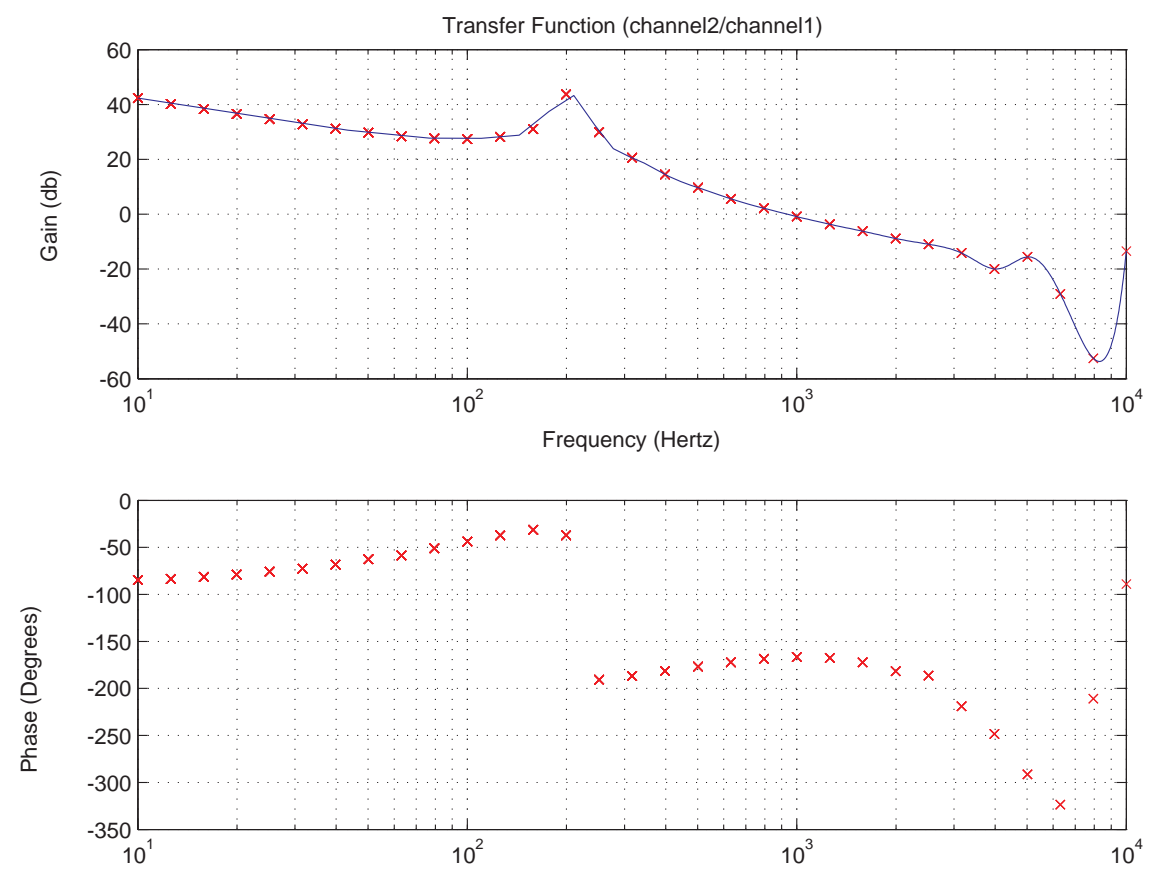

Figure 3-36: Bode plot of the measured negative loop transmission for the tool position for the $2 \mathrm{kHz}$ FTS without viscous damping, using a double lead, lag, and notch filter compensation.
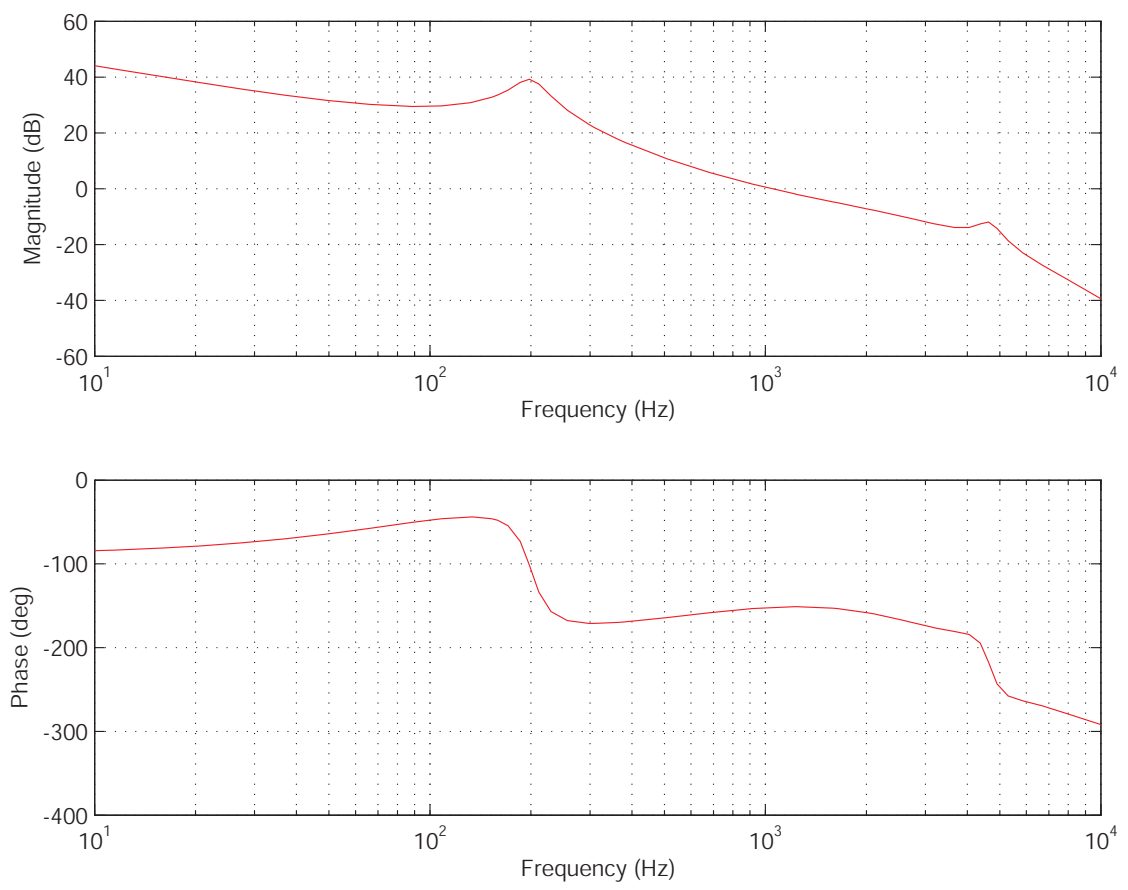

Figure 3-37: Bode plot of the modelled negative loop transmission for the tool position for the $2 \mathrm{kHz}$ FTS without viscous damping, using a double lead, lag, and notch filter compensation. 


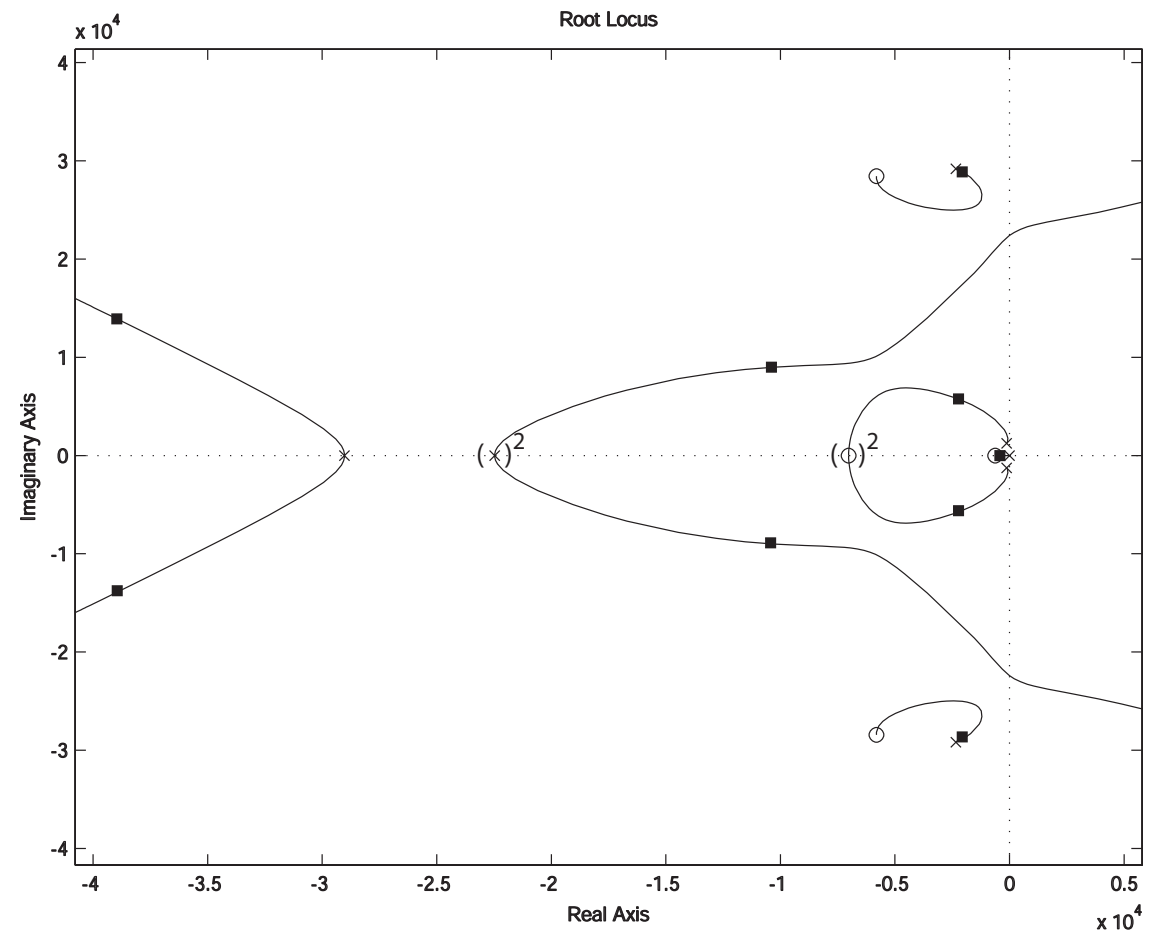

Figure 3-38: Root locus of the modelled negative loop transmission for the tool position for the $2 \mathrm{kHz}$ FTS without viscous damping, using a double lead, lag, and notch filter compensation. Closed-loop poles indicated by solid squares.

respectively, and stabilizes the system. An illustration on how this technique works is provided in this section by comparing the Nyquist diagrams for the negative loop transmission with and without the phase-stabilizing pole.

Figure 3-42 shows the block diagram for the digital controller for the tool position for the $2 \mathrm{kHz}$ FTS without viscous damping, using a series compensator consisting of a double lead, lag, and phase-stabilizing pole. Both of the two lead compensators use $\alpha=3.2$ and a time constant $T_{\text {lead }}=4.45 \times 10^{-5}$ sec. The lag compensator consists of a pure integrator and a zero with a time constant $T_{\text {lag }}=1.59 \times 10^{-3} \mathrm{sec}$. The phase-stabilizing pole has a corner frequency of $5 \mathrm{kHz}$.

Figure 3-43 shows a Bode plot of the measured negative loop transmission for the tool position for the $2 \mathrm{kHz}$ FTS without viscous damping, using the double lead, lag, and phase-stabilizing pole compensator. The crossover frequency for this system is $1 \mathrm{kHz}$ with a phase margin of $23^{\circ}$ and a gain margin of $5 \mathrm{~dB}$.

Figure 3-44 shows a Bode plot of the modelled negative loop transmission for the 


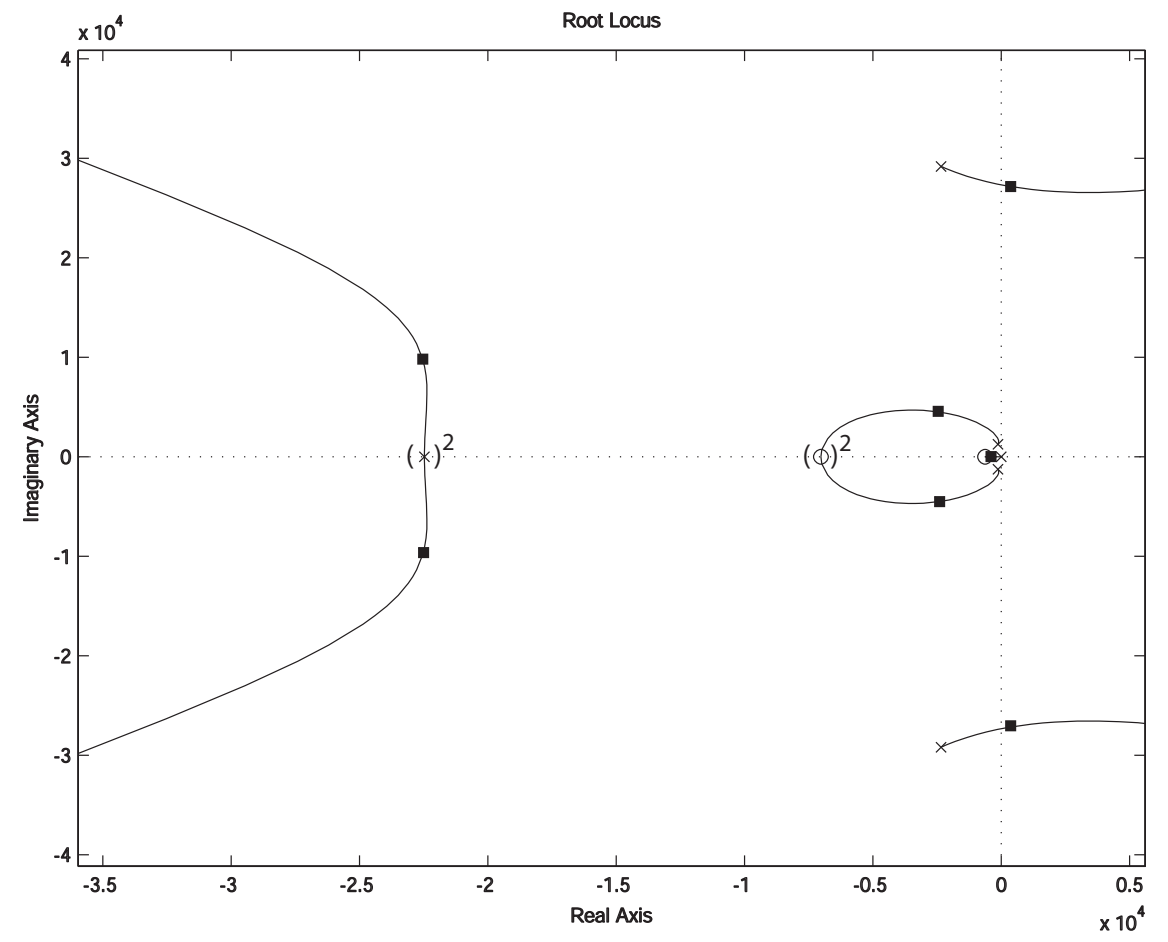

Figure 3-39: Root locus of the modelled negative loop transmission for the tool position for the $2 \mathrm{kHz}$ FTS without viscous damping, using a double lead and lag compensation. The notch filter is omitted for comparison to Figure 3-38. Closed-loop poles indicated by solid squares. 


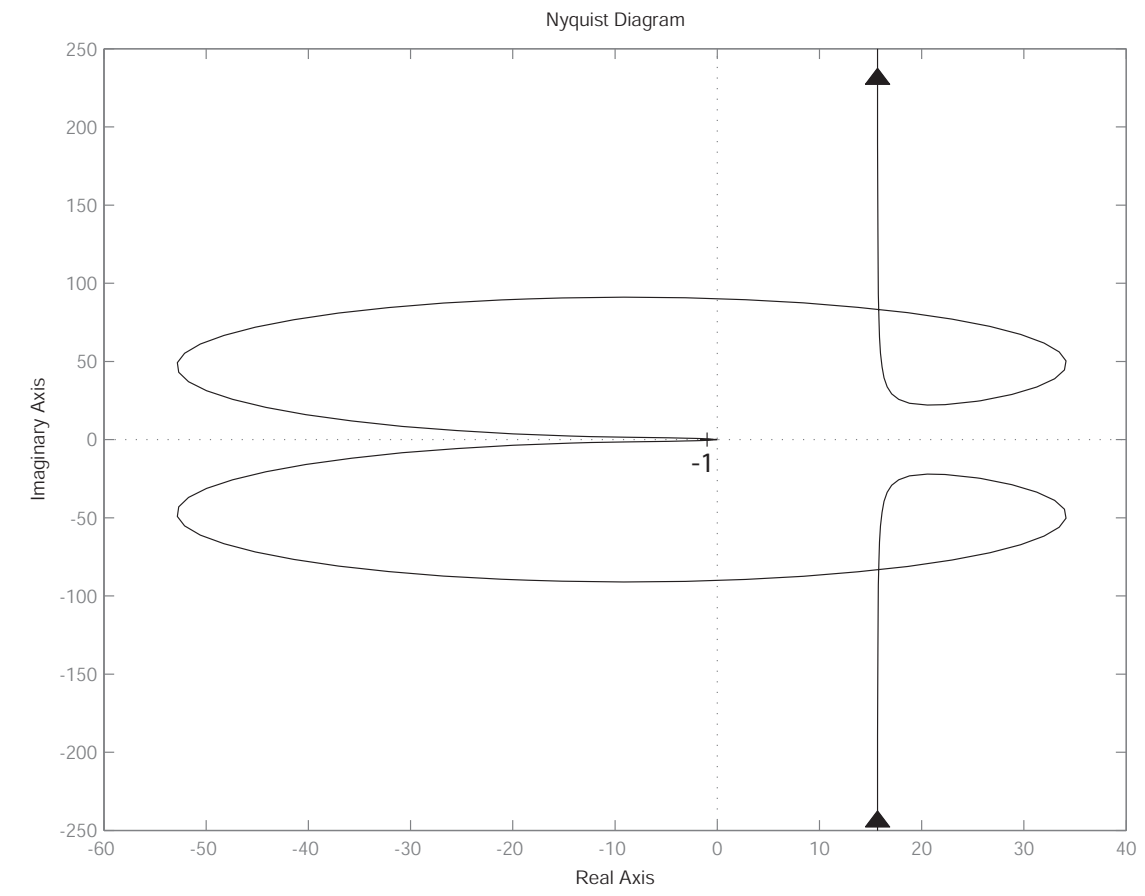

Figure 3-40: Nyquist diagram of the modelled negative loop transmission for the tool position for the $2 \mathrm{kHz}$ FTS without viscous damping, using a double lead, lag, and notch filter compensation.

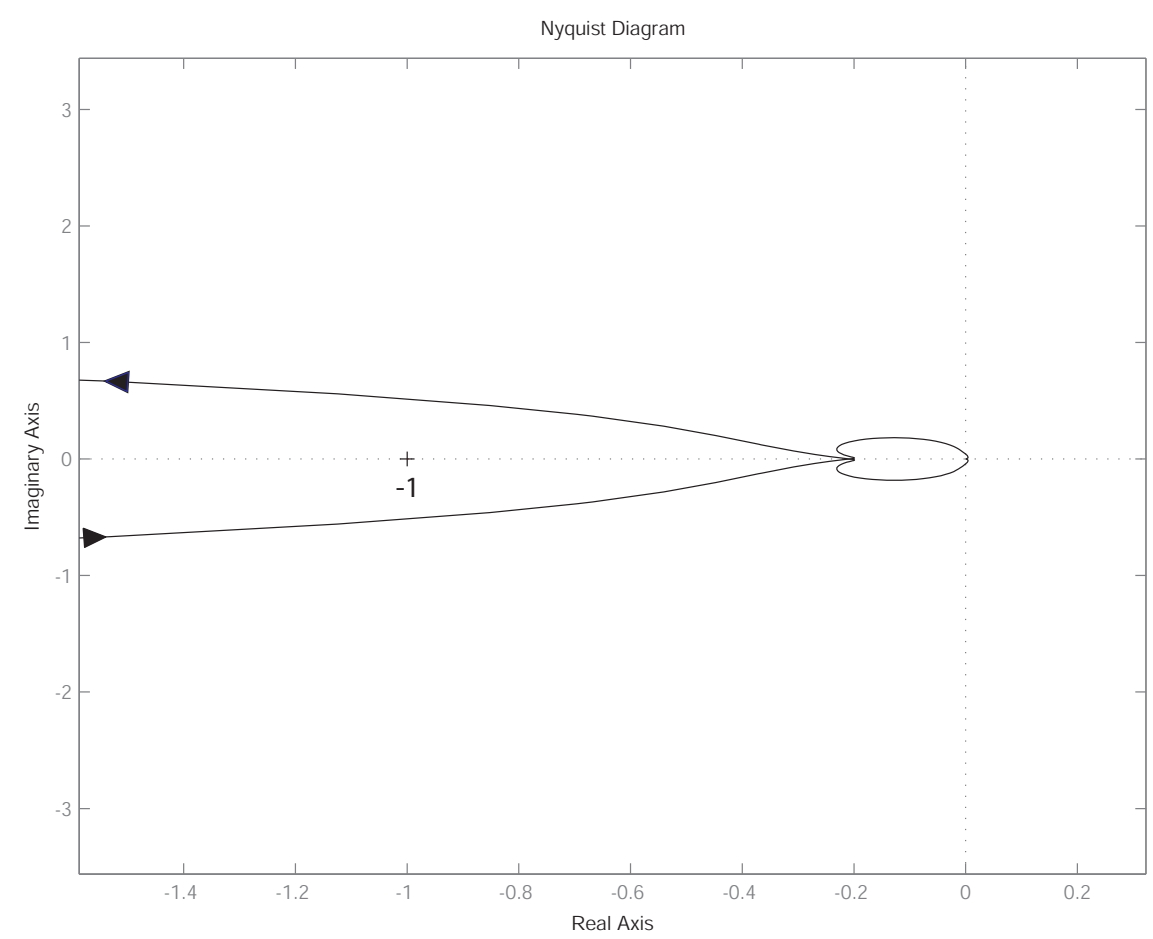

Figure 3-41: Detail of the Nyquist diagram of the modelled negative loop transmission for the tool position for the $2 \mathrm{kHz}$ FTS without viscous damping, using a double lead, lag, and notch filter compensation. 


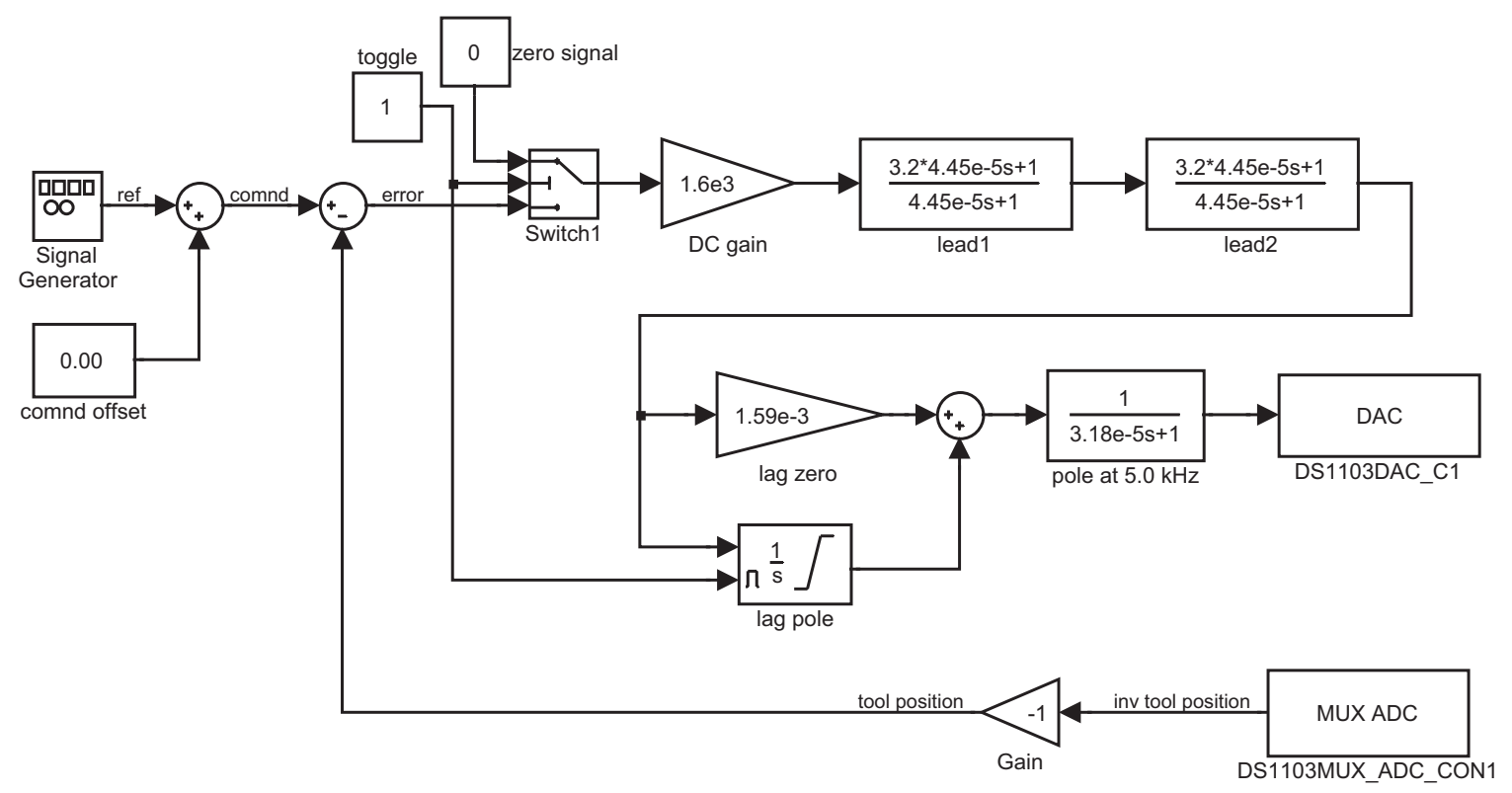

Figure 3-42: Block diagram for the digital controller for the tool position for the $2 \mathrm{kHz}$ FTS without viscous damping, using a double lead, lag, and phase-stabilizing pole compensator.

tool position for the $2 \mathrm{kHz}$ FTS without viscous damping, using the double lead, lag, and a phase-stabilizing pole compensation. Figure 3-45 shows the corresponding root locus for that model. The small circles represent the open-loop and closed-loop zeros, the x's represent the open-loop poles, and the solid squares represent the closed-loop poles. Figure 3-46 shows the corresponding Nyquist diagram for the model, and Figure 3-47 is a detail of that Nyquist diagram.

For comparison purposes, Figure 3-48 shows a detail of the Nyquist diagram for the loop without the phase-stabilizing pole. Notice how the two encirclements of the -1 point in Figure 3-48 are rotated away from the -1 point in Figure 3-47 by the extra negative phase from the phase-stabilizing pole.

\subsubsection{Viscous Damping and Lag Compensation}

Controlling the $2 \mathrm{kHz}$ FTS became a lot easier after I added viscous damping to the system via the damping plate described in Section 3.6.6. I had planned on using a lead and lag compensator, but it turned out that the system was so well damped that a lead compensator was not needed, so I added only the lag compensator to increase 

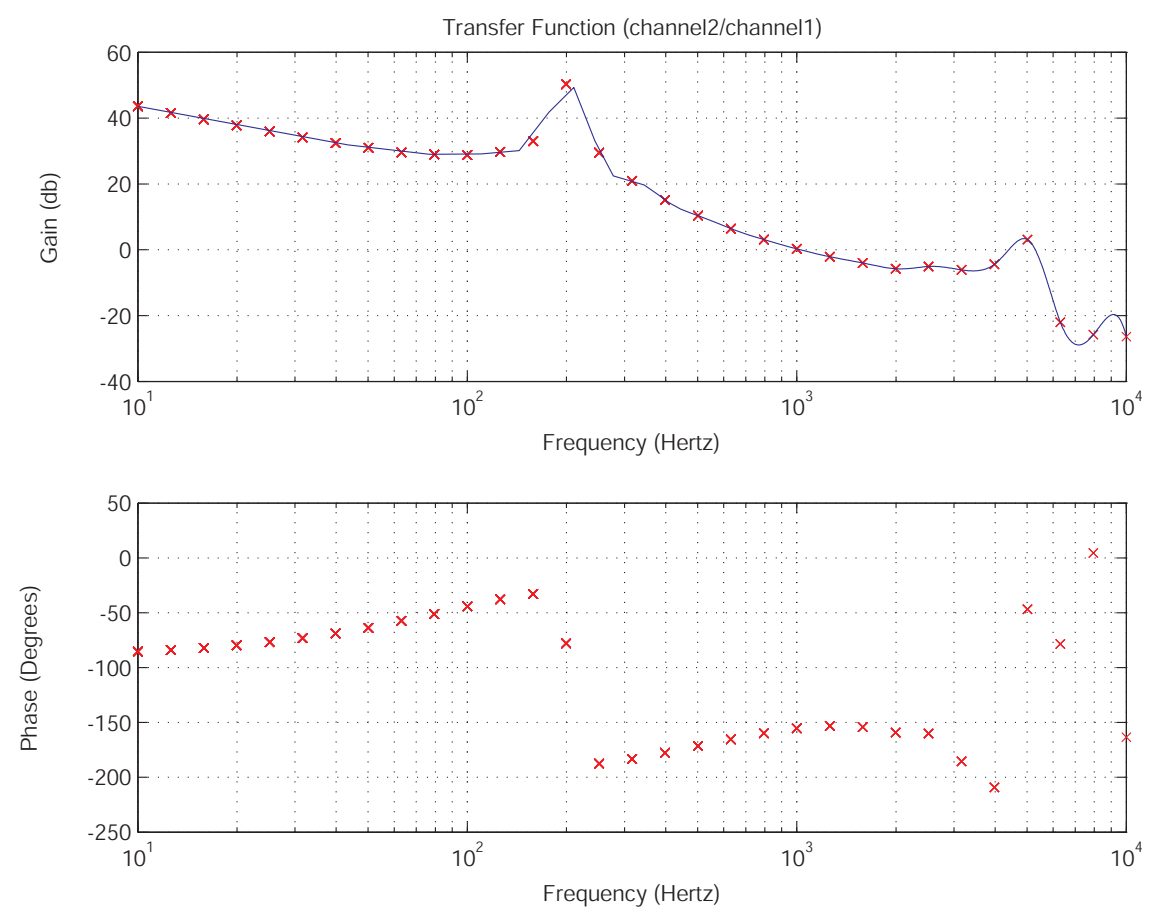

Figure 3-43: Bode plot of the measured negative loop transmission for the tool position for the $2 \mathrm{kHz}$ FTS without viscous damping, using a double lead, lag, and phase-stabilizing pole compensation.
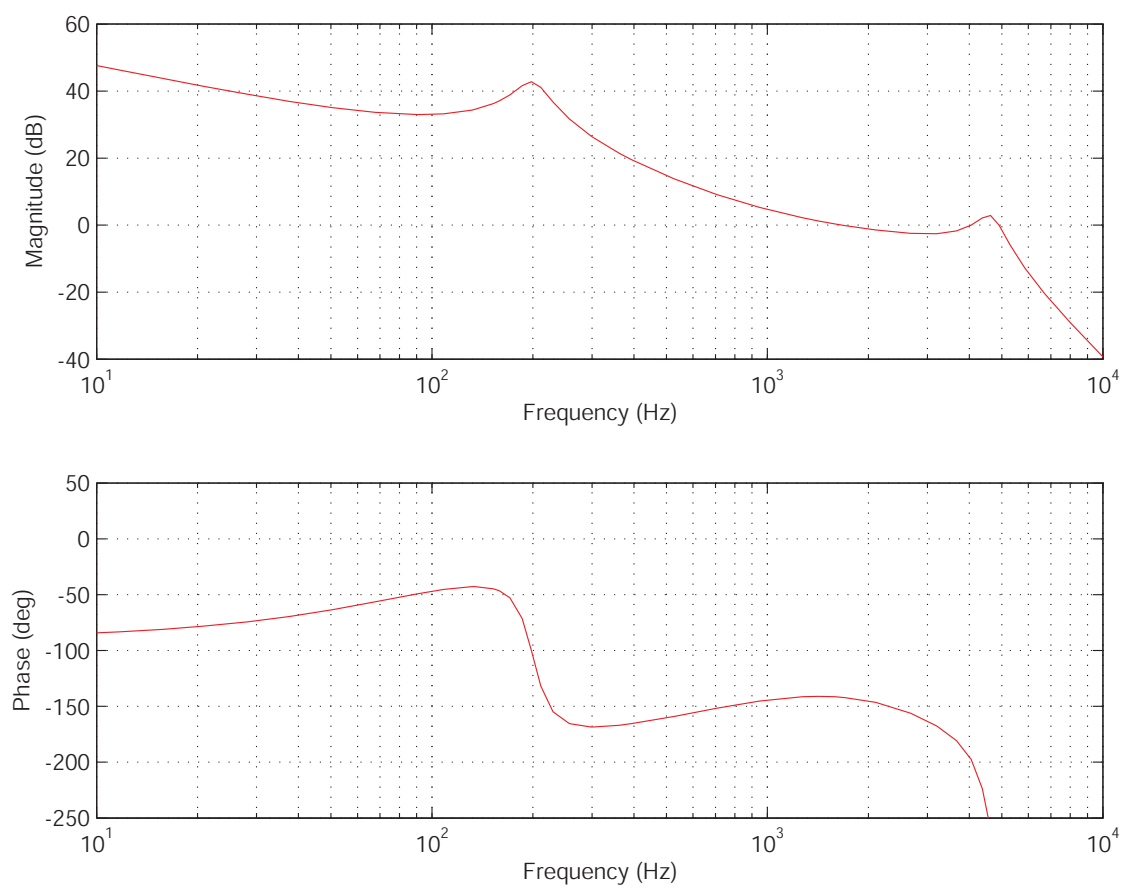

Figure 3-44: Bode plot of the modelled negative loop transmission for the tool position for the $2 \mathrm{kHz}$ FTS without viscous damping, using a double lead, lag, and phasestabilizing pole compensation. 


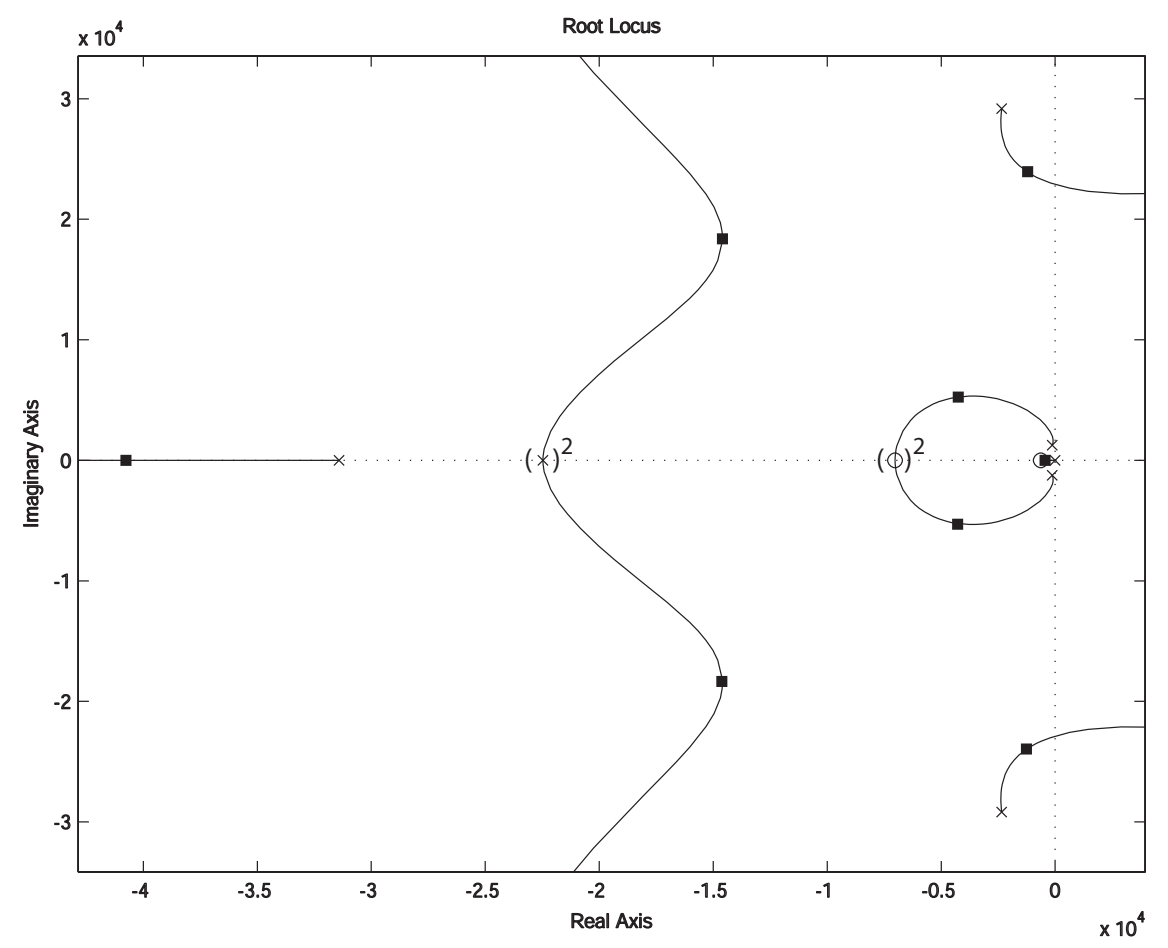

Figure 3-45: Root locus of the modelled negative loop transmission for the tool position for the $2 \mathrm{kHz}$ FTS without viscous damping, using a double lead, lag, and phase-stabilizing pole compensation. Closed-loop poles indicated by solid squares.

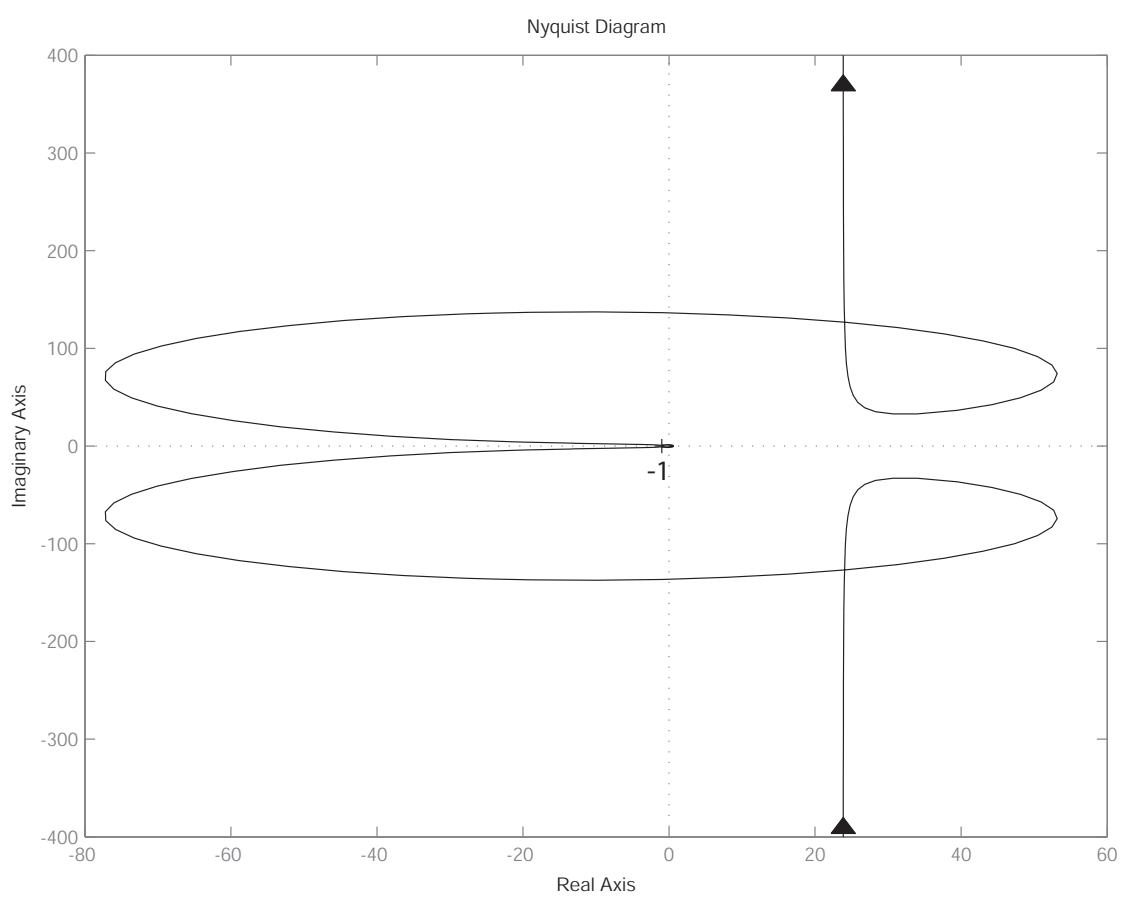

Figure 3-46: Nyquist diagram of the modelled negative loop transmission for the tool position for the $2 \mathrm{kHz}$ FTS without viscous damping, using a double lead, lag, and phase-stabilizing pole compensation. 


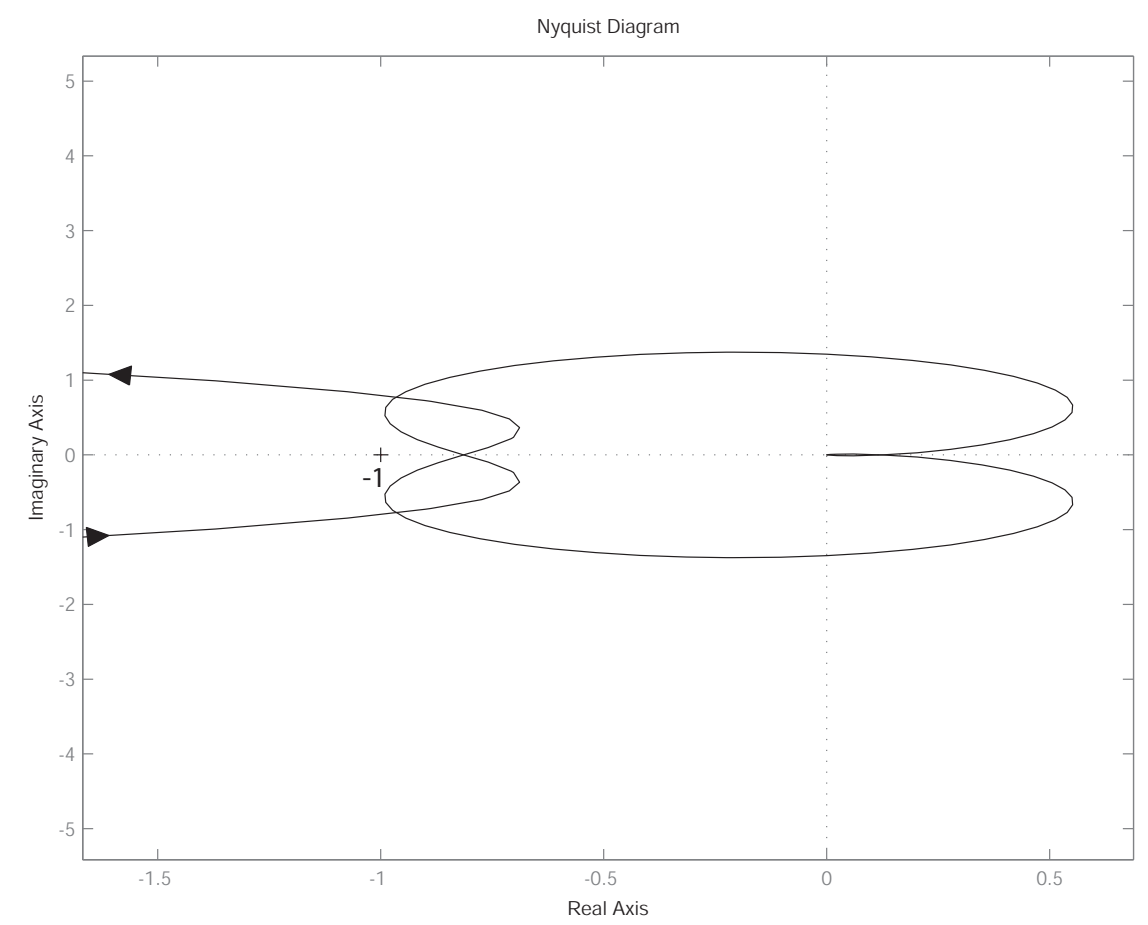

Figure 3-47: Detail of the Nyquist diagram of the modelled negative loop transmission for the tool position for the $2 \mathrm{kHz}$ FTS without viscous damping, using a double lead, lag, and phase-stabilizing pole compensation.

the low to mid frequency gain for the system. The viscous damping was so dominant that I was able to move the lag zero from the initial design point of one decade below crossover all the way up to the crossover frequency of $1 \mathrm{kHz}$ and still achieve a phase margin of $41^{\circ}$.

Figure 3-49 shows the block diagram for the digital controller for the tool position for the $2 \mathrm{kHz}$ FTS with viscous damping, using a series lag compensator. The lag compensator consists of a pure integrator and a zero with a time constant $T_{\text {lag }}=1.59 \times 10^{-4} \mathrm{sec}$.

Figure 3-50 shows a Bode plot of the measured negative loop transmission for the tool position for the $2 \mathrm{kHz}$ FTS with viscous damping, using the series lag compensator. The crossover frequency for this system is $1 \mathrm{kHz}$ with a phase margin of $41^{\circ}$ and a gain margin of $6 \mathrm{~dB}$. Note that for frequencies below the resonant peak at $5 \mathrm{kHz}$, the magnitude plot has a slope of approximately -1 (dec/dec) and the phase remains close to $-100^{\circ}$. It is interesting to note that the Bode magnitude for an ideal 


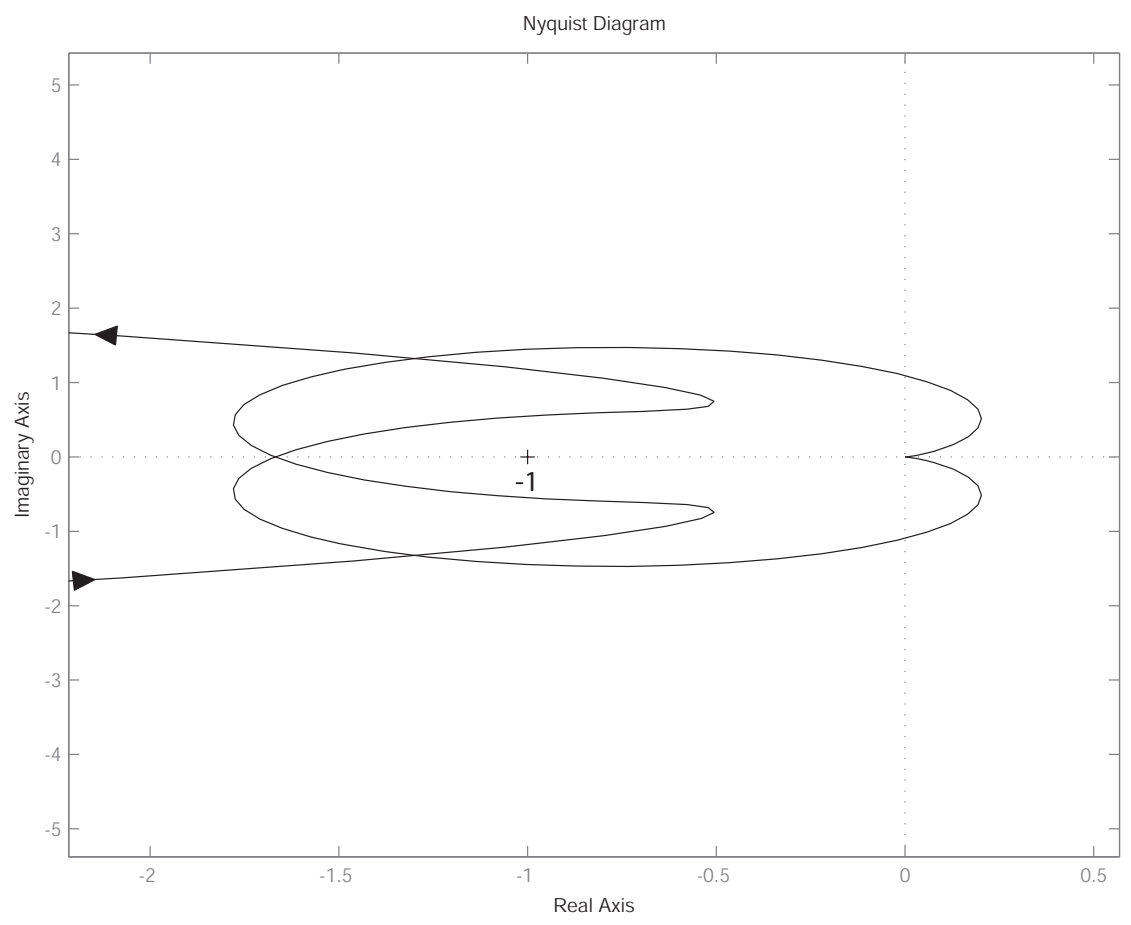

Figure 3-48: Detail of the Nyquist diagram of the modelled negative loop transmission for the tool position for the $2 \mathrm{kHz}$ FTS without viscous damping, using a double lead and lag compensation without a phase-stabilizing pole (or a notch filter). The phasestabilizing pole is omitted for comparison to Figure 3-47.

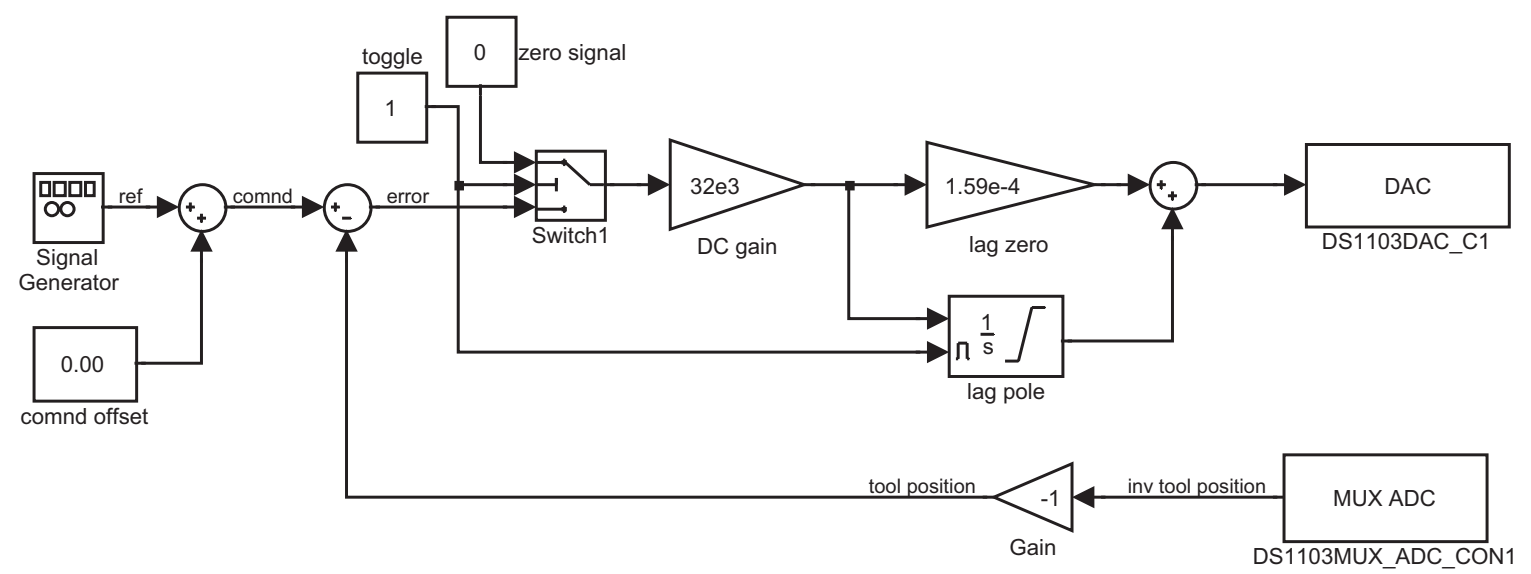

Figure 3-49: Block diagram for the digital controller for the tool position for the $2 \mathrm{kHz}$ FTS with viscous damping, using a series lag compensator. 
inertia-damper system has a slope of -1 (dec/dec) and a phase of $-90^{\circ}$ for frequencies below the corner frequency of the pole produced by the inertia and the damping. This suggests that the dynamics of the $2 \mathrm{kHz}$ FTS with viscous damping is dominated by the rotational inertia and viscous damping.

If we consider a pure inertia-damper system, where $I$ and $b$ are the rotational inertia and damping coefficient, respectively, the transfer function between the applied torque $T_{a}$ and the rotation angle $\theta_{I}$ of the inertia is

$$
\frac{\theta_{I}}{T_{a}}=\frac{(1 / b)}{s[(I / b) s+1]}
$$

The combined rotational inertia of the swing-arm and the rotating element of the motor is $57 \times 10^{-7} \mathrm{~kg} \cdot \mathrm{m}^{2}$. Using that value for the inertia in an ideal inertiadamper system, (3.21) indicates that a corner frequency of $5 \mathrm{kHz}$ for the pole in that system requires a damping coefficient of $0.18 \mathrm{~N} \cdot \mathrm{m} \cdot \mathrm{sec} / \mathrm{rad}$. Converting to the U.S. customary units that my mind and hands are calibrated to, this is equivalent to a damping coefficient of $10 \mathrm{in} \cdot \mathrm{lb} \cdot \mathrm{sec} / \mathrm{rev}$. The swing-arm is essentially a 1 inch diameter shaft. To rotate it with my forefinger and thumb at a speed of $1 \mathrm{rev} / \mathrm{sec}$ I would have to produce a tangential force of $10 \mathrm{lb}$ with each of those fingers if the damping coefficient is $10 \mathrm{in} \cdot \mathrm{lb} \cdot \mathrm{sec} / \mathrm{rev}$. The hard stops for the swing-arm allow it to rotate only \pm 5 mrad. When I rotated the swing-arm by hand, I was moving it back and forth between the hard stops with a round-trip travel time of approximately 1 sec. The small angular displacements involved make it difficult to know with certainty the magnitude of the tangential forces that I was applying. It was less than $1 \mathrm{lb}$, but probably not more than three orders of magnitude less. ${ }^{16}$ Therefore, it is plausible that the viscous damping plate on the bottom of the swing-arm produces a damping coefficient of approximately $10 \mathrm{in} \cdot \mathrm{lb} \cdot \mathrm{sec} / \mathrm{rev}$.

Figure 3-51 shows the measured small-signal closed-loop response for the tool position for the $2 \mathrm{kHz}$ FTS with viscous damping. The -3dB closed-loop bandwidth is just above $2 \mathrm{kHz}$. The closed-loop phase angle changes fairly smoothly up to $2 \mathrm{kHz}$,

\footnotetext{
${ }^{16}$ The difference between a full rotation and a $10 \mathrm{mrad}$ rotation is approximately three orders of magnitude.
} 
suggesting that a feedforward compensator could be effective up to that frequency. However, the magnitude peaking between $1 \mathrm{kHz}$ and $2 \mathrm{kHz}$ will most likely result in reduced accuracy of a feedforward compensator in that frequency range. Ludwick [99] provides an excellent overview of various feedforward controllers that are suitable for a fast tool servo. Essentially, the feedforward controller applies a frequency-dependent magnitude and phase adjustment to the commanded reference signal to compensate for the known closed-loop response of the system.

The step response for a $1 \mu \mathrm{m}$ tool position step is shown in Figure 3-52. This step response has a $170 \mu$ sec rise time (10\% to $90 \%)$ and a $17 \%$ overshoot. Roberge $[132$, page 95] gives the following approximation for the relationship between the frequency and time responses for a system that behaves like a second-order system: $f_{h}=0.35 / t_{r}$, where $f_{h}$ is the half-power $(-3 \mathrm{~dB})$ bandwidth in $(\mathrm{Hz})$ for the closedloop response and $t_{r}$ is the rise time in (sec). According to that relationship, the measured rise time of $170 \mu \mathrm{sec}$ for the $2 \mathrm{kHz}$ FTS is consistent with the measured closed-loop bandwidth of $2 \mathrm{kHz}$. The approximately $1.5 \mathrm{msec}$ long tail in the step response can be attributed to the closed-loop pole-zero doublet at $-1300 \mathrm{sec}^{-1}$ in the root locus of the modelled negative loop transmission shown in Figure 3-56. The $12 \mathrm{~V}$ spike in the feedback voltage from the sense resistor attendant with the start of the $1 \mu \mathrm{m}$ step indicates that the control system is at the threshold of saturation. Assuming that the $12 \mathrm{~V}$ spike is not due to electrical interference, it suggests a $120 \mathrm{~A}$ spike in the motor current $(0.1 \Omega$ sense resistor $)$. If this is the case, then it illustrates the importance of the local by-pass capacitors for the power operational amplifier.

Figure 3-53 shows a measured following error of $-12 \%$ for the tool position for the $2 \mathrm{kHz}$ FTS during machining tests at $2 \mathrm{kHz}$. Note the approximate $180^{\circ}$ phase shift between the commanded and actual tool position, which is consistent with the measured closed-loop transfer function shown in Figure 3-51. The following error can be reduced with the addition of a feedforward compensator. Ludwick [99], Cattell [37], Byl [33], and Lu [98] show that the addition of an Adaptive Feedforward Cancellation compensator can significantly reduce following error, and is discussed in Section 6.4.1.

To fit the parametric model for the $2 \mathrm{kHz}$ FTS without damping to the measured 

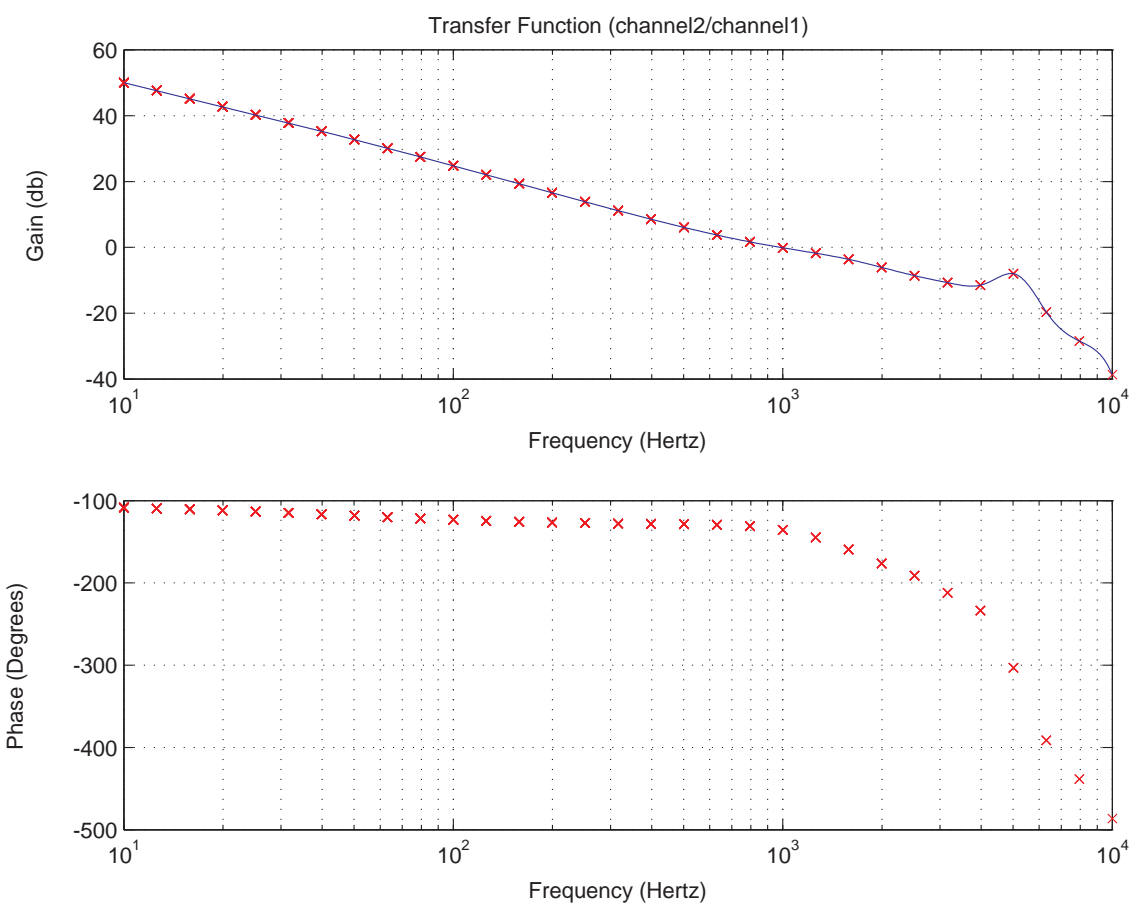

Figure 3-50: Bode plot of the measured negative loop transmission for the tool position for the $2 \mathrm{kHz}$ FTS with viscous damping, using a series lag compensator.
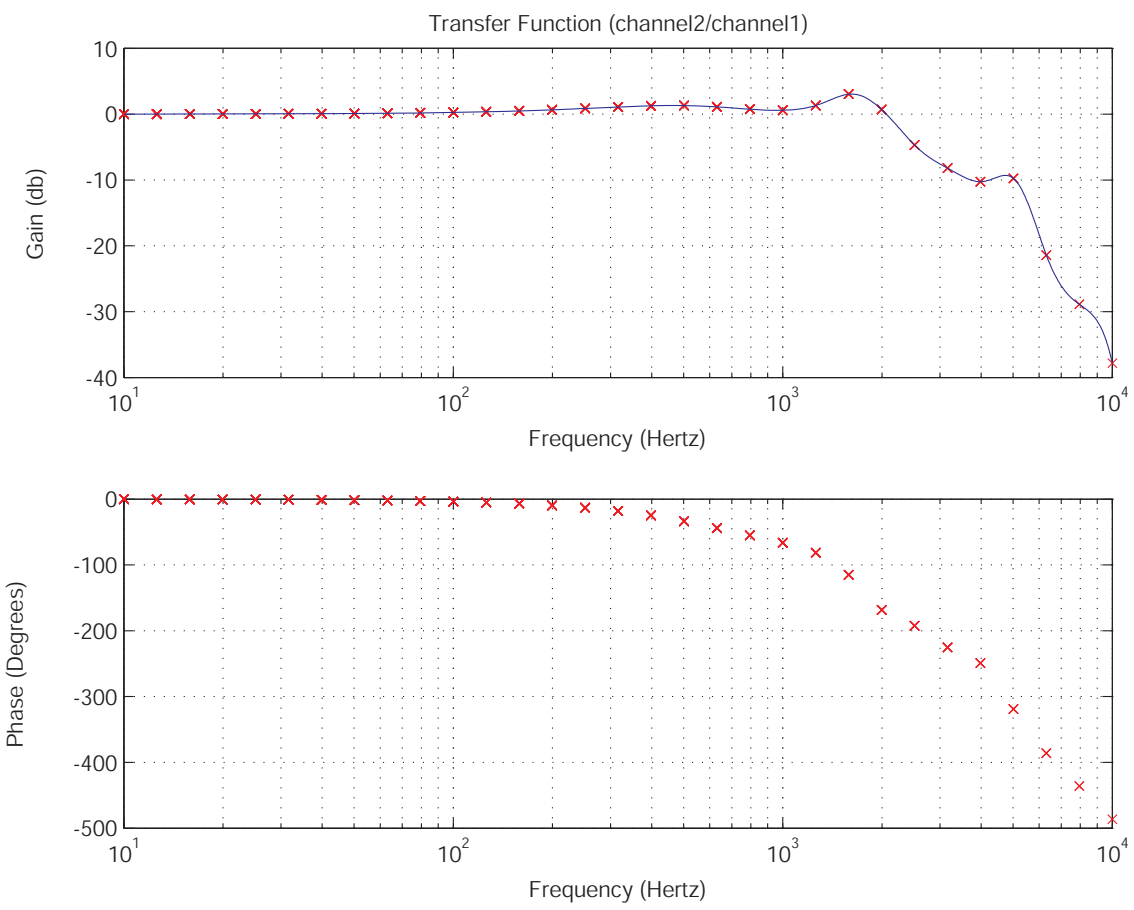

Figure 3-51: Bode plot of the measured small-signal closed-loop response for the tool position for the $2 \mathrm{kHz}$ FTS with viscous damping, using a series lag compensator. 


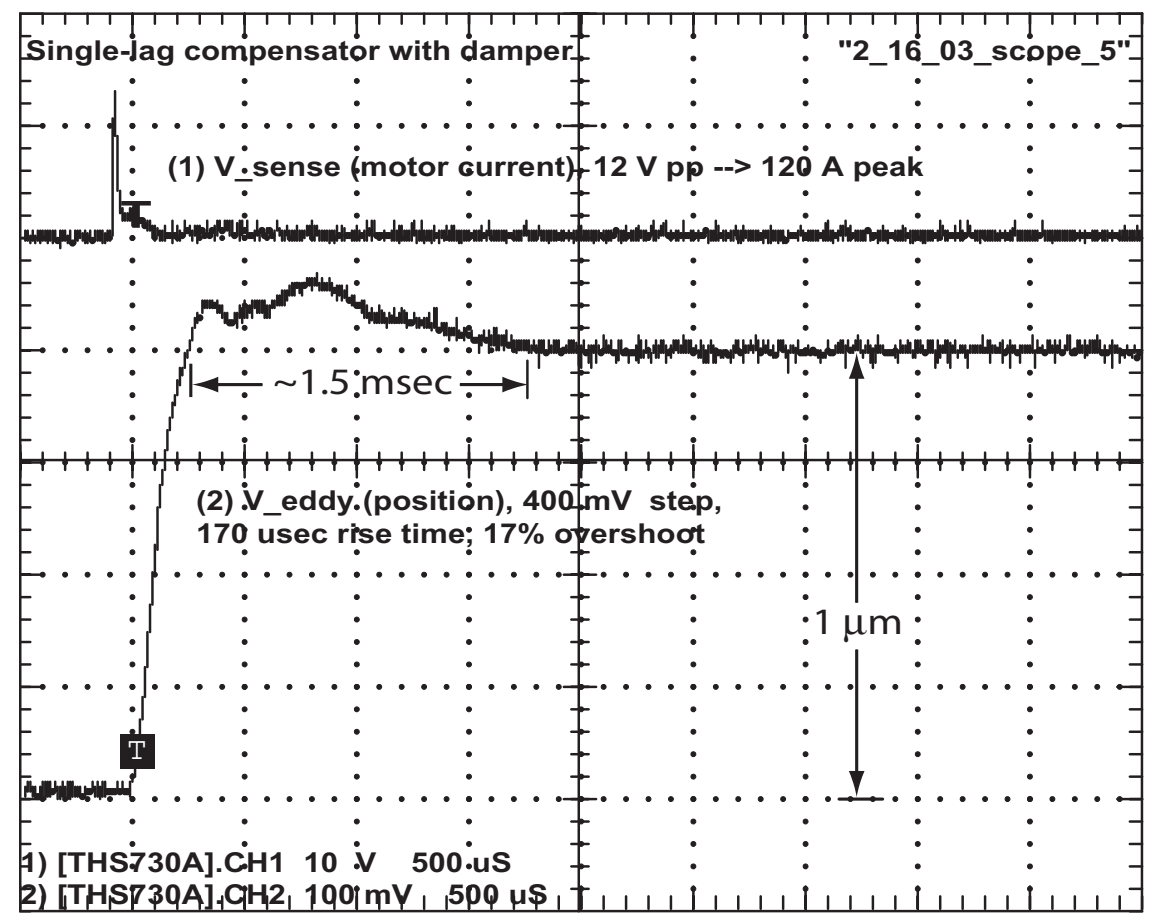

Figure 3-52: Measured step response for the tool position for the $2 \mathrm{kHz}$ FTS with viscous damping, using a series lag compensator.

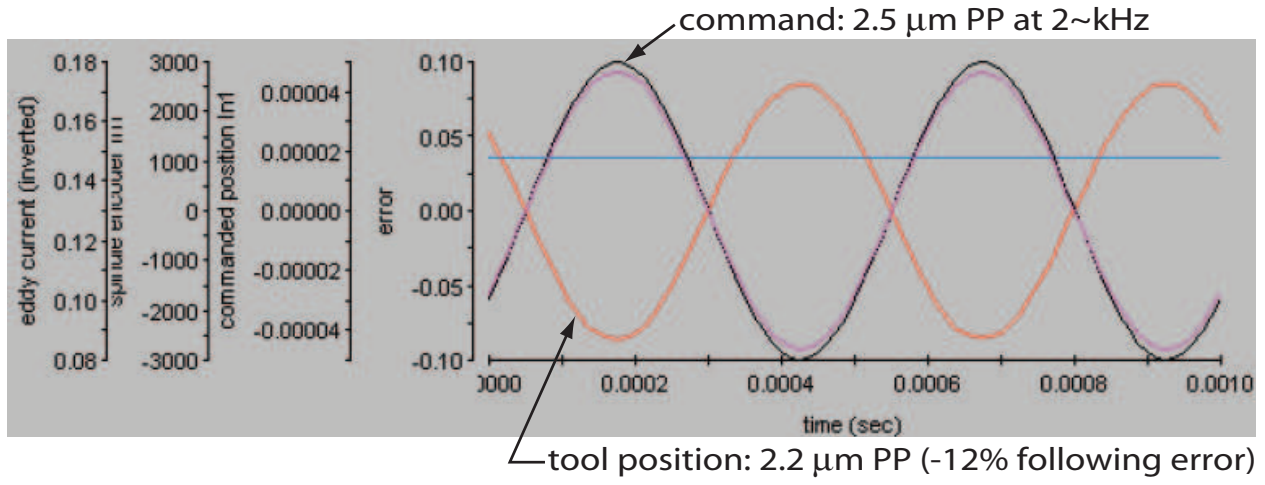

Figure 3-53: Measured following error during machining tests at $2 \mathrm{kHz}$ for the tool position for the $2 \mathrm{kHz}$ FTS with viscous damping, using a series lag compensator. 
negative loop transmission for the system with damping (Figure 3-50) I had to add a zero at $200 \mathrm{~Hz}$. The dynamics model in Section 3.7.1 indicates that the coupling between the motor and the swing-arm creates a zero in the transfer function between the motor torque and swing-arm rotation angle. However, this raises the following issue: if the coupling produces a zero at $200 \mathrm{~Hz}$ in the system with damping, then we would expect that zero to be present in the system without damping because both systems include the same coupling. Without delving into a potentially lengthy analysis on this issue - there are many more significant topics to discuss in this thesis - I qualitatively contribute the difference to the following. The motor torque needed to accelerate the swing-arm has to be transmitted through the series-connected coupling. For equal angular accelerations of the swing-arm, the system with the damping plate attached to the bottom of the swing-arm requires a higher motor torque - to overcome the viscous damping and the added inertia - than the system without it. It is possible that the magnitude of the damping produced by the plastic shim in that coupling increases with torque. If this is the case, then the effects of that damping would be less pronounced in the system without viscous damping.

As a brief aside, I calculate the values of the torsional stiffness and damping that the coupling needs to have to produce a zero at $200 \mathrm{~Hz}$. Referring to Figure 3-17, if the dampers and $k_{\text {flex }}$ are omitted, the undamped natural frequency for the uncoupled mode for the two inertias is given by (3.22):

$$
\omega_{\text {uncoupled }}=\sqrt{\frac{k_{\text {coupl }}\left(I_{m}+I_{S A}\right)}{I_{m} I_{S A}}}
$$

Using (3.22) with the measured frequency of the uncoupled mode of $4.7 \mathrm{kHz}$ and rotational inertias of $6.4 \times 10^{-7} \mathrm{~kg} \cdot \mathrm{m}^{2}$ and $51 \times 10^{-7} \mathrm{~kg} \cdot \mathrm{m}^{2}$ for $I_{m}$ and $I_{S A}$, respectively, the spring constant $k_{\text {coupl }}$ of the coupling is estimated to be $500 \mathrm{~N} \cdot \mathrm{m} / \mathrm{rad}$. Using this spring constant, a zero at $200 \mathrm{~Hz}$ can be accounted for in the full model shown in Figure $3-17$ if the damping coefficient $b_{\text {coupl }}$ is equal to $0.4 \mathrm{~N} \cdot \mathrm{m} \cdot \mathrm{sec} / \mathrm{rad}$ $(20 \mathrm{in} \cdot \mathrm{lb} \cdot \mathrm{sec} / \mathrm{rev})$.

Figure 3-54 shows a Bode plot of the modelled negative loop transmission for 

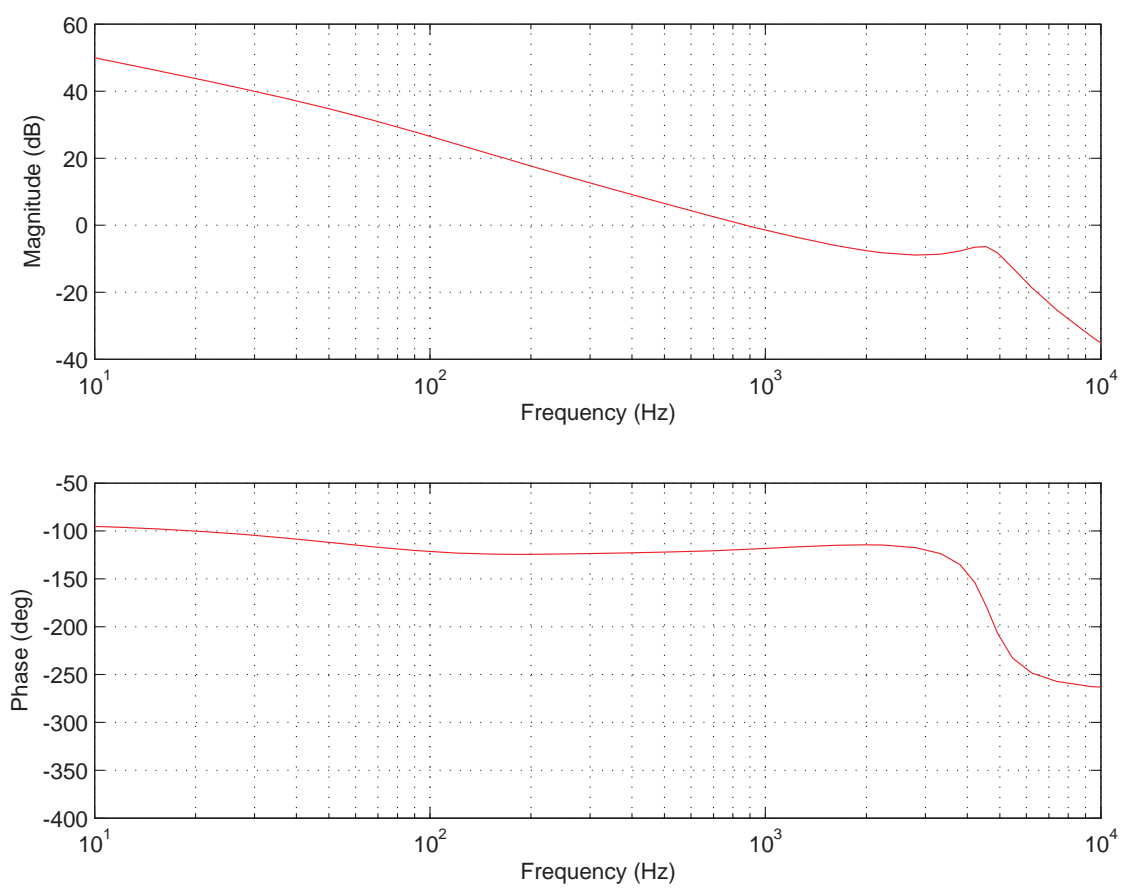

Figure 3-54: Bode plot of the modelled negative loop transmission for the tool position for the $2 \mathrm{kHz}$ FTS with viscous damping, using a series lag compensator.

the tool position for the $2 \mathrm{kHz}$ FTS with viscous damping, using the series lag compensator. Notice that the phase angle stays above $-150^{\circ}$ until the frequency exceeds $1 \mathrm{kHz}$, as it does for the measured system shown in Figure 3-50. For comparison purposes, Figure 3-55 shows the effects of omitting the zero at $200 \mathrm{~Hz}$. Notice that without the zero, the phase angle drops below $-150^{\circ}$ at $100 \mathrm{~Hz}$.

Figure 3-56 shows the root locus for the model. The small circles represent the open-loop and closed-loop zeros, the x's represent the open-loop poles, and the solid squares represent the close-loop poles. Figure 3-57 shows the corresponding Nyquist diagram for the model, and Figure 3-58 is a detail of that Nyquist diagram.

\subsection{Results}

This section presents the significant experimental results for the $2 \mathrm{kHz}$ FTS. The measured frequency and time responses for the $2 \mathrm{kHz}$ FTS are presented earlier in Section 3.10. 

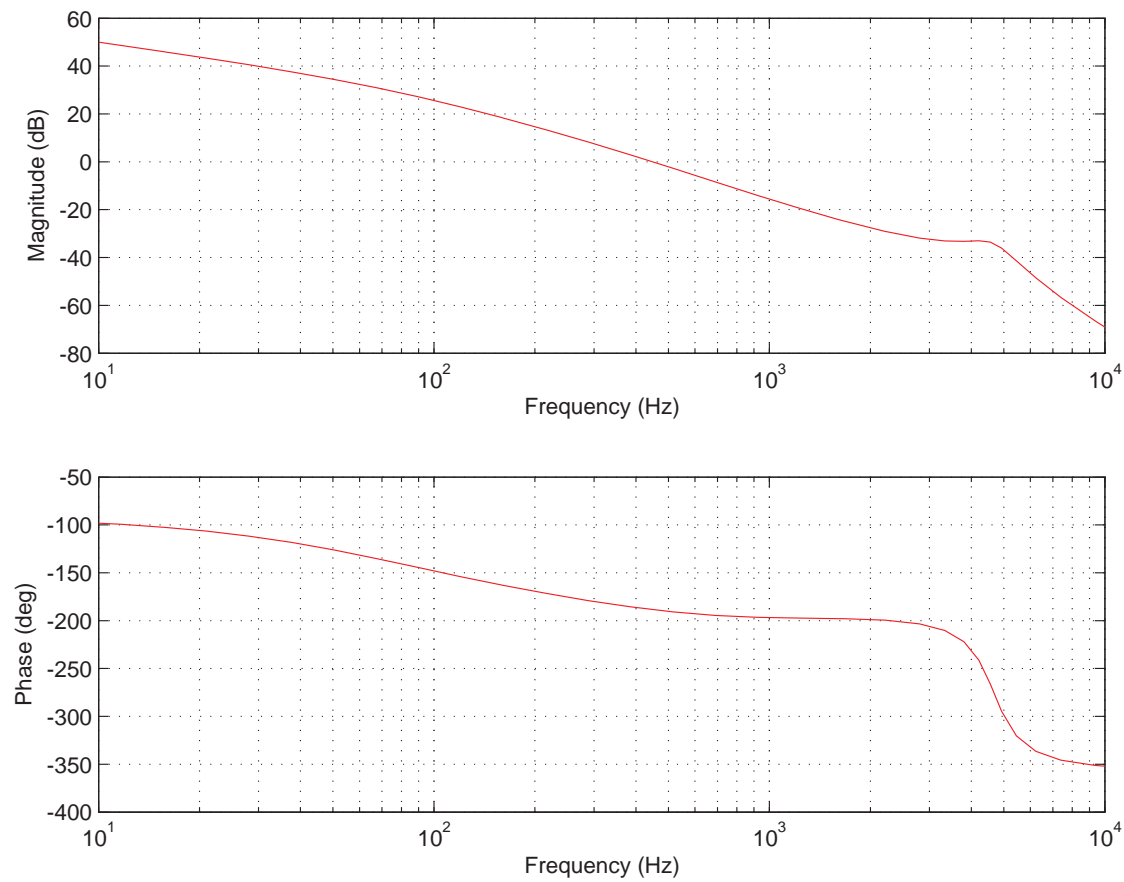

Figure 3-55: Bode plot of the modelled negative loop transmission for the tool position for the $2 \mathrm{kHz}$ FTS with viscous damping, using a series lag compensator. The extra zero at $200 \mathrm{~Hz}$ in the model is omitted for comparison to Figure 3-54.

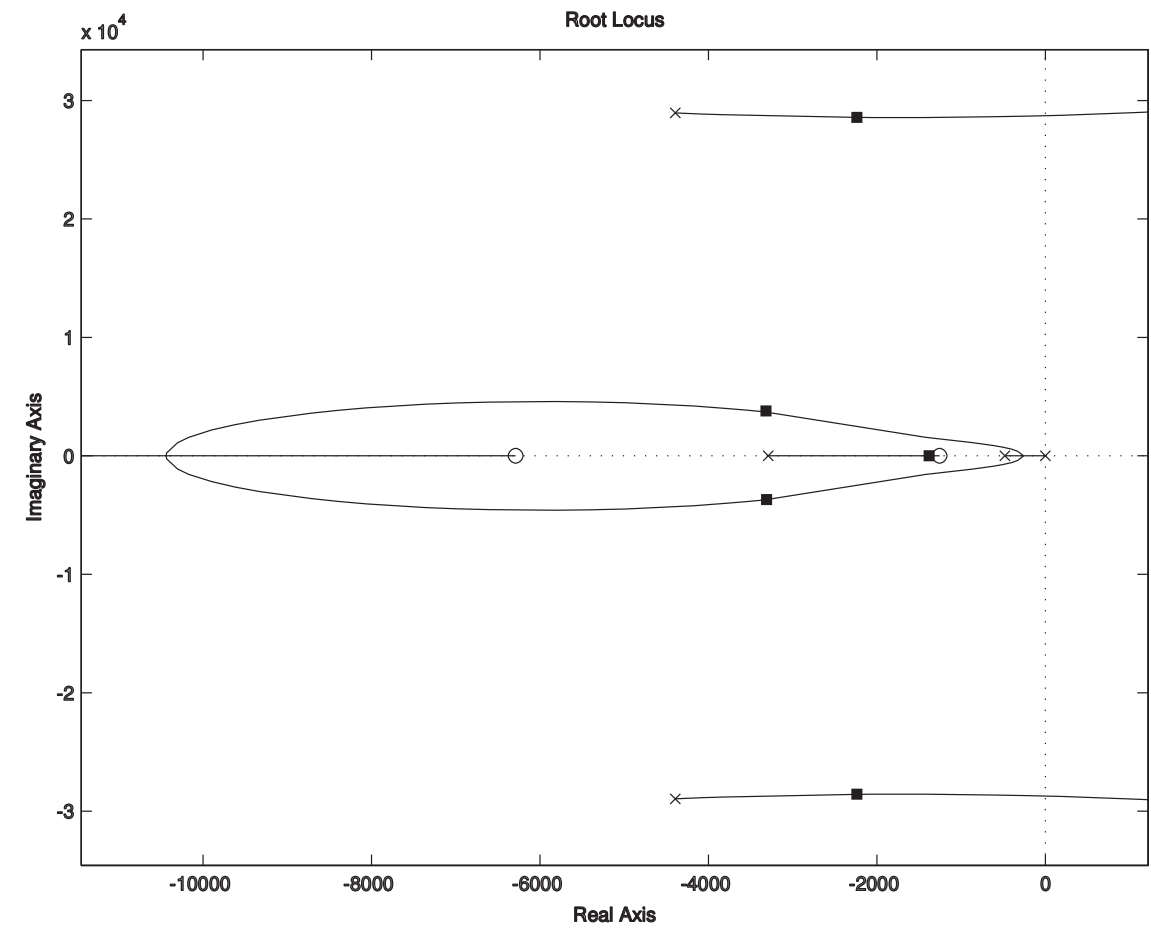

Figure 3-56: Root locus of the modelled negative loop transmission for the tool position for the $2 \mathrm{kHz}$ FTS with viscous damping, using a series lag compensator. Closed-loop poles indicated by solid squares. 


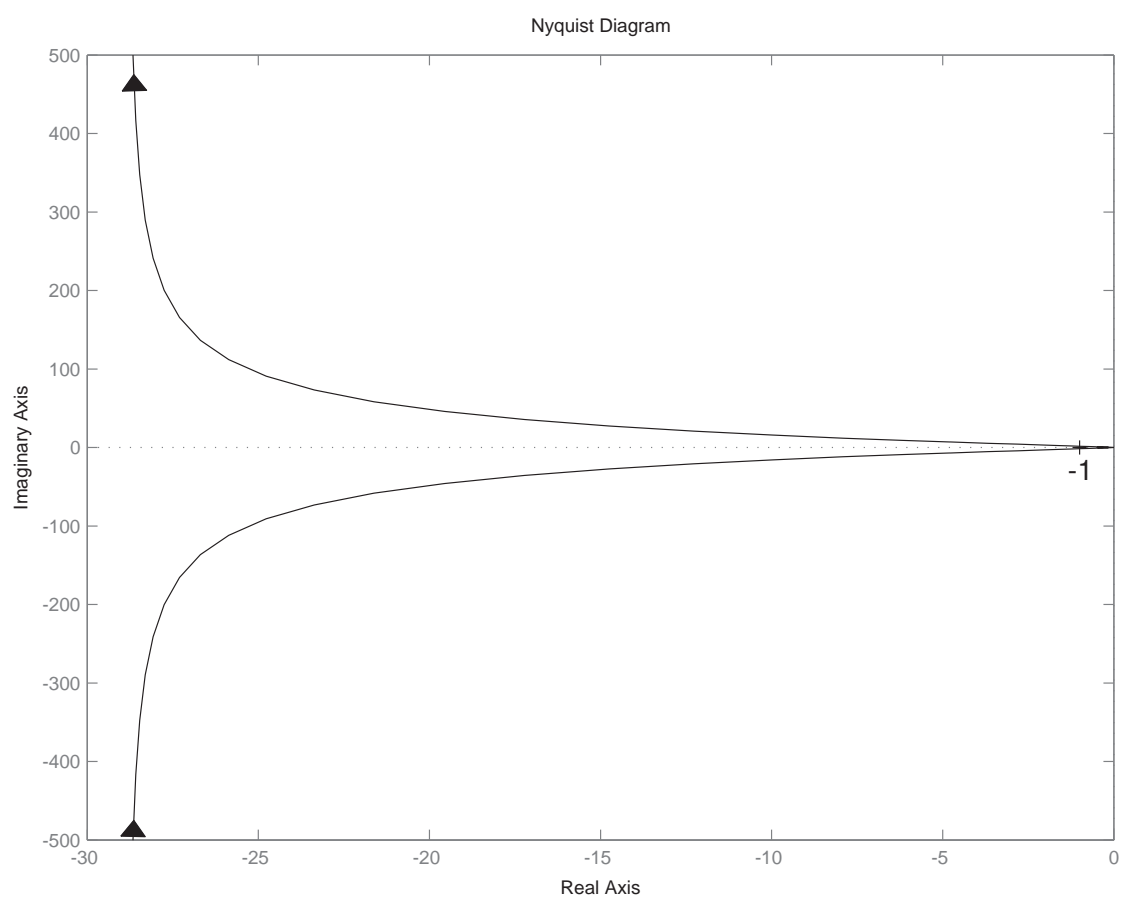

Figure 3-57: Nyquist diagram of the modelled negative loop transmission for the tool position for the $2 \mathrm{kHz}$ FTS with viscous damping, using a series lag compensator.

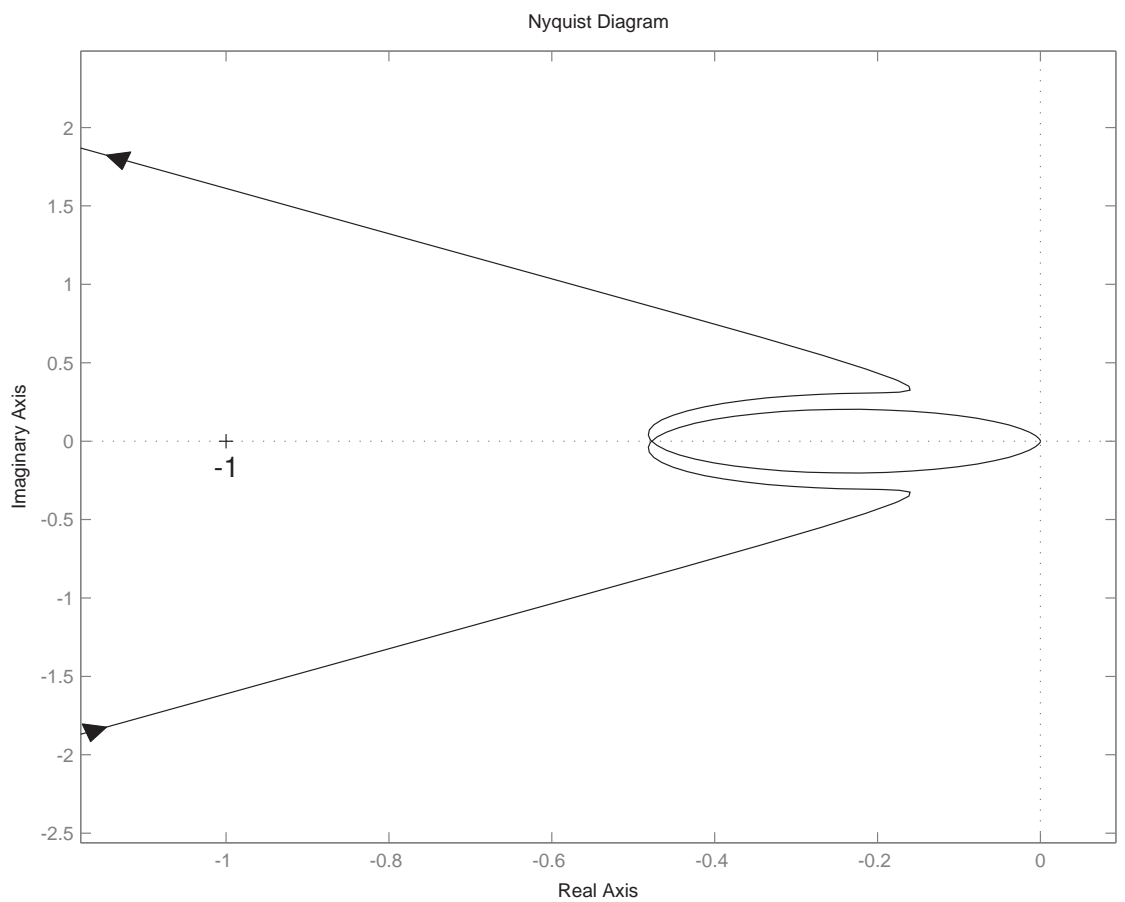

Figure 3-58: Detail of the Nyquist diagram of the modelled negative loop transmission for the tool position for the $2 \mathrm{kHz}$ FTS with viscous damping, using a series lag compensator. 


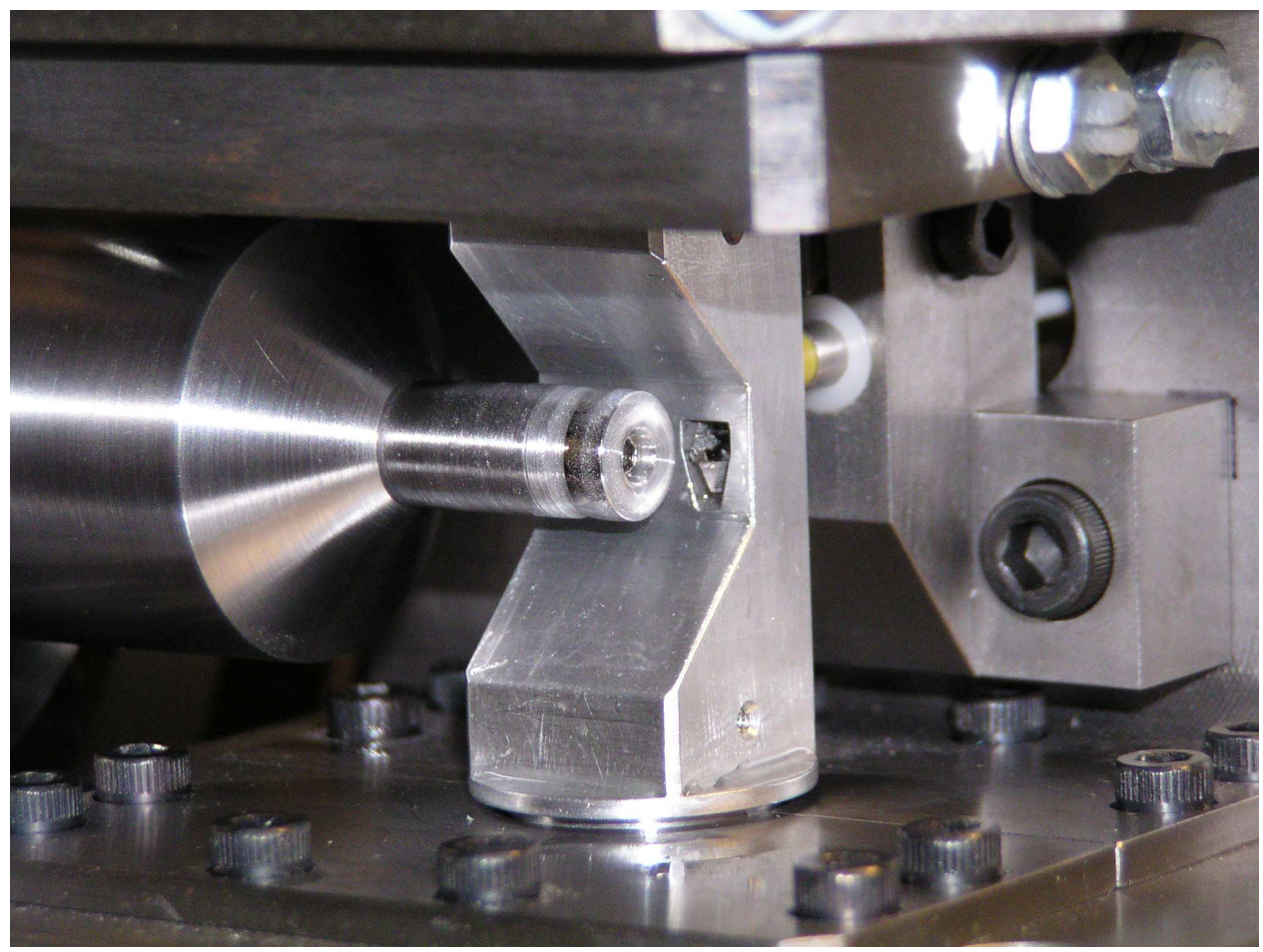

Figure 3-59: Close-up view of the $2 \mathrm{kHz}$ FTS tool tip about to engage the outside diameter of a workpiece.

\subsubsection{Machining Tests}

I integrated the $2 \mathrm{kHz}$ rotary fast tool servo with a Moore Nanotechnology model UPL 350 [110] diamond turning machine (DTM) at MIT, and single-point machined optical quality textured surfaces on the face and outside diameter of a handful of $9 \mathrm{~mm}$ diameter aluminum workpieces. Details on the control system and user interface that I developed for this DTM, and how the $2 \mathrm{kHz}$ FTS was used with it, are provided in Appendix C. Figure 3-2 at the beginning of this chapter shows the $2 \mathrm{kHz}$ FTS mounted on the diamond turning machine and engaging a workpiece. Figure 3-15 in Section 3.6.8 shows a close-up view of the tool engaging the face of a workpiece, while Figure 3-59 shows a close-up of the tool tip about to engage the outside diameter of a workpiece.

I demonstrated $2.2 \mu \mathrm{m}$ PP tool motion at $2 \mathrm{kHz}(20 \mathrm{~g}$ tool tip acceleration) while diamond turning workpieces like the ones shown in Figures 3-15 and 3-59; producing 250 radial grooves on the face and 250 axial grooves on the outside diameter while 


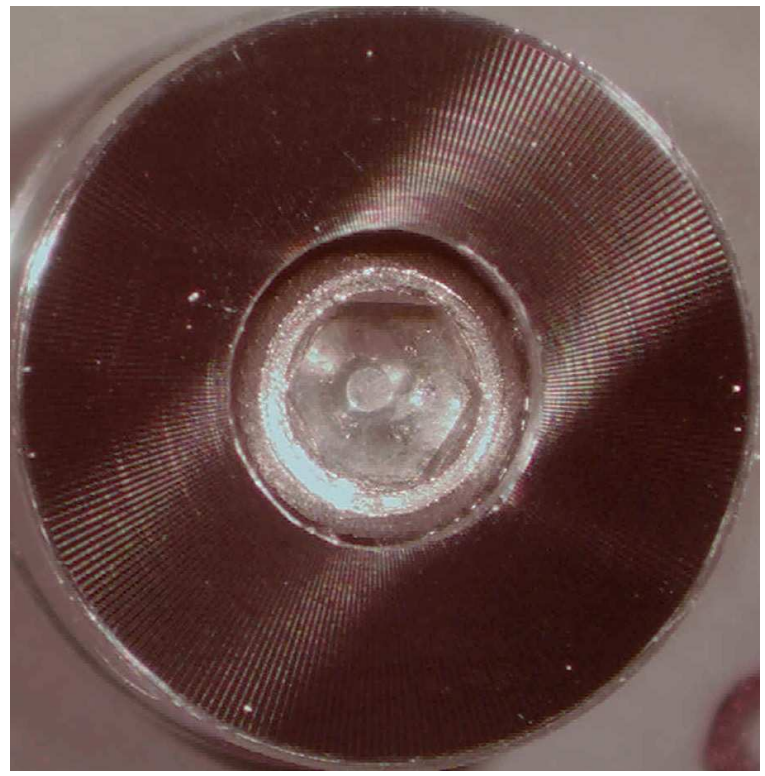

Figure 3-60: Close-up view of the textured surface on the face of a workpiece machined with the $2 \mathrm{kHz}$ FTS.

the workpiece was rotating at 480 RPM. A close-up view of the textured surface on the face of one of the parts is shown in Figure 3-60. Tracing a profile along the face at any fixed radius, the grooves describe a sinusoidal waveform with $2.2 \mu \mathrm{m} \mathrm{PV}$ and a spatial wavelength ranging from $110 \mu \mathrm{m}$ at the outer diameter to $30 \mu \mathrm{m}$ at the inner diameter.

Using a Zygo NewView 5000 white light optical profilometer [154], the measured roughness along a groove (across the feed marks) is $12 \mathrm{~nm} \mathrm{rms,} \mathrm{which} \mathrm{is} \mathrm{very} \mathrm{good}$ considering that the diamond turning machine was in a less than optimum operating environment given its close proximity to heavy rotating machinery sharing the same concrete foundation. Figure 3-61 shows the surface measurement of the face of a workpiece with a two-dimensional profile across the grooves. Figure 3-62 shows that same surface measurement with a two-dimensional profile along the grooves indicating a roughness of $12 \mathrm{~nm} \mathrm{rms}$ for a $130 \mu \mathrm{m}$ scan. Figure 3-63 shows the surface measurement of the outer diameter of a workpiece with a two-dimensional profile across the grooves. Figure 3-64 shows that same surface measurement with a two-dimensional profile along the grooves indicating a roughness of $10 \mathrm{~nm} \mathrm{rms}$ for a $170 \mu \mathrm{m}$ scan. By machining the face and outside diameters of workpieces and producing optical 


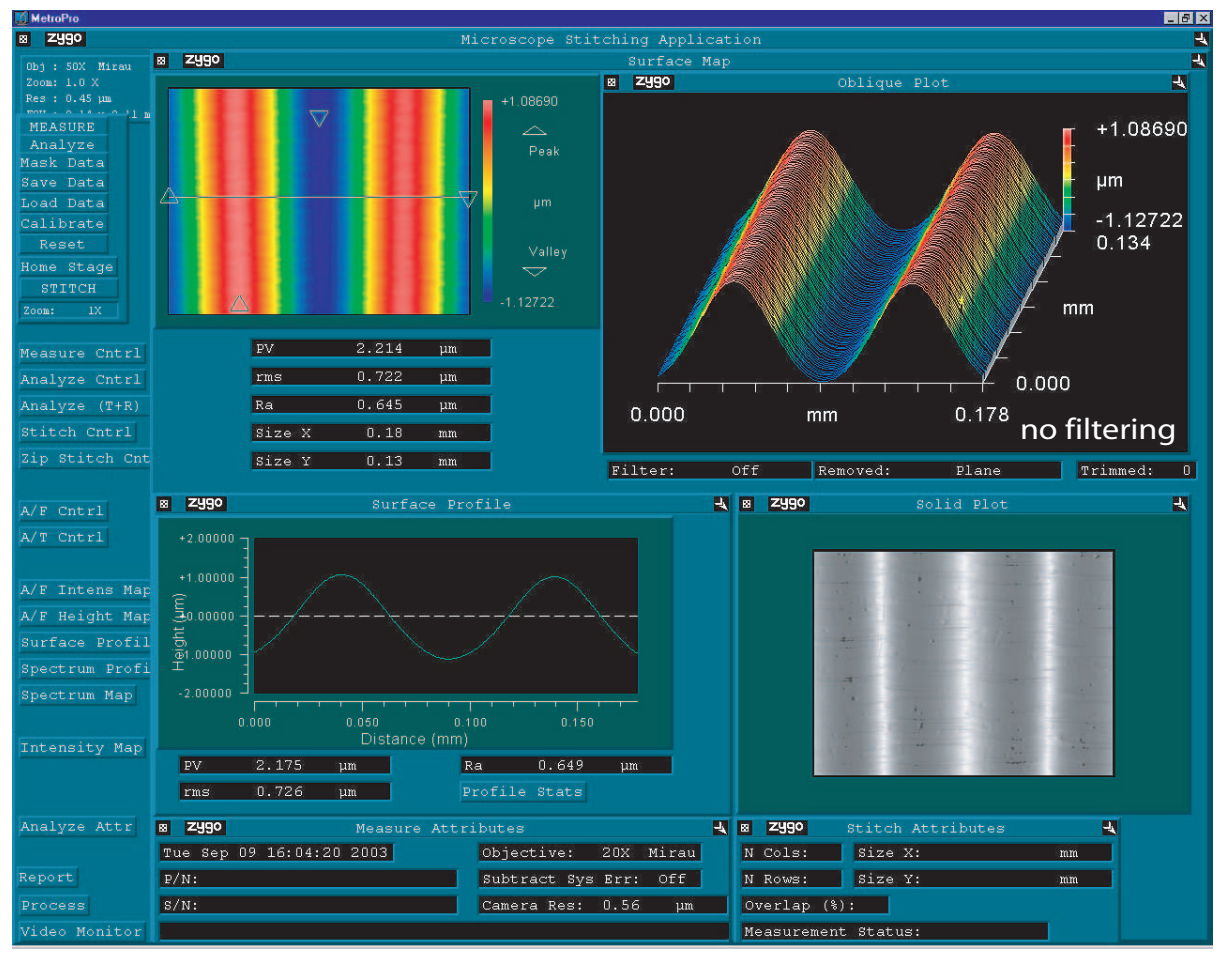

Figure 3-61: Surface measurement of the face of a workpiece machined with the $2 \mathrm{kHz}$ FTS, showing a two-dimensional profile across the grooves.

quality surfaces having approximately the same roughness, I demonstrated that the disturbance to the diamond turning machine from the reaction torque produced by the $2 \mathrm{kHz}$ FTS was fairly constant for the two cases. This result reinforces the motivation for using a rotary-type FTS to machine from the pole to the equator on a spherical workpiece, which was described in Section 2.2.2.

\subsubsection{Demonstrated Peak Torque}

In addition to discussing the demonstrated peak torque from the actuator, this section develops two performance metrics that allow comparing the actuators used in the $2 \mathrm{kHz}$ FTS to the actuator that we developed for the $10 \mathrm{kHz}$ FTS.

Section 3.11.1 mentioned that a peak tool tip acceleration of $20 \mathrm{~g}$ was demonstrated with the $2 \mathrm{kHz}$ FTS while following a $2 \mathrm{kHz}$ sinusoidal tool path trajectory. Running through the math using the swing radius for the tool of $5 \mathrm{~mm}$ and a total rotational inertia of $57 \mathrm{gm} \cdot \mathrm{cm}^{2}$, the $20 \mathrm{~g}$ tool tip acceleration corresponds to a 


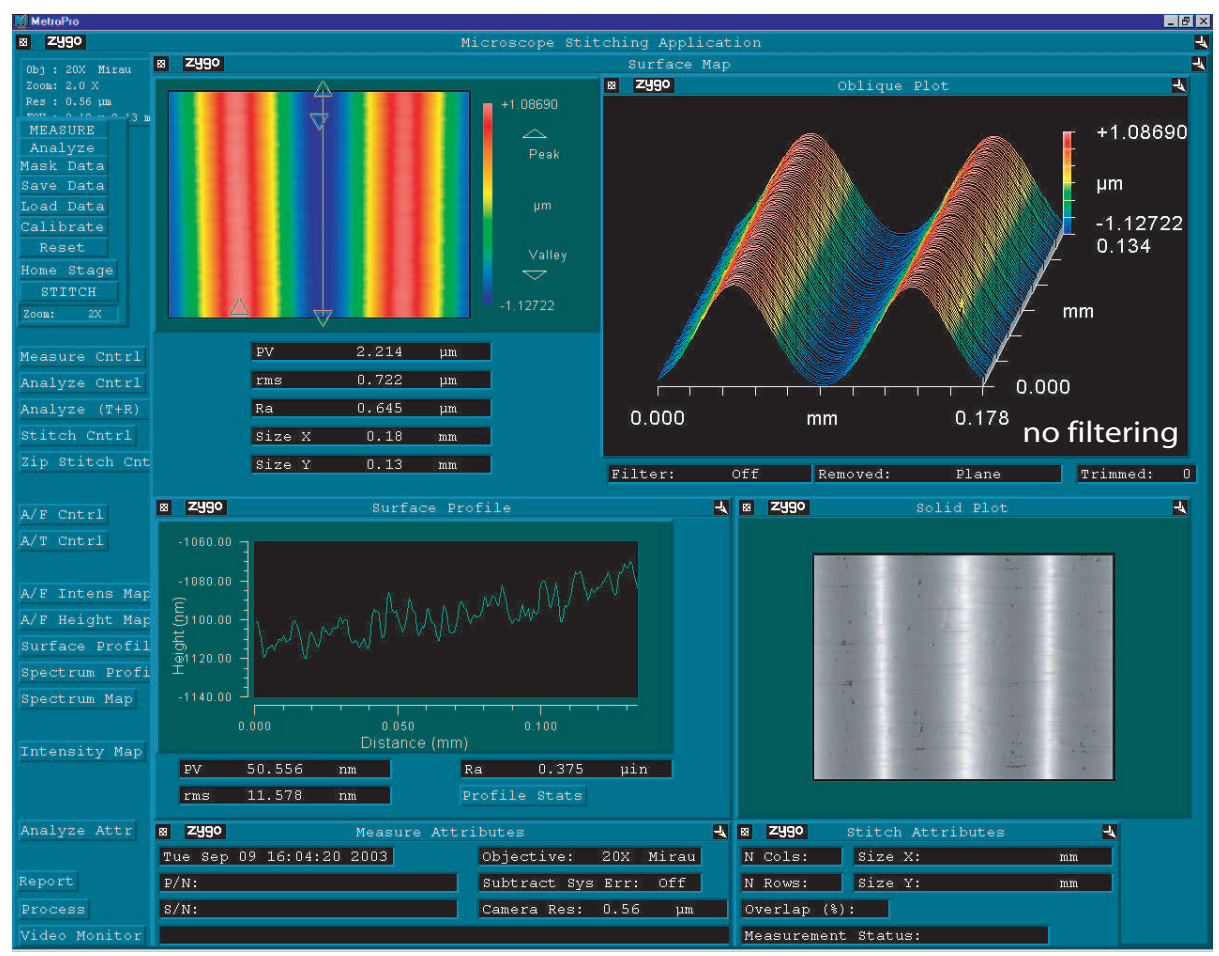

Figure 3-62: Surface measurement of the face of a workpiece machined with the $2 \mathrm{kHz}$ FTS, showing a two-dimensional profile along a groove.

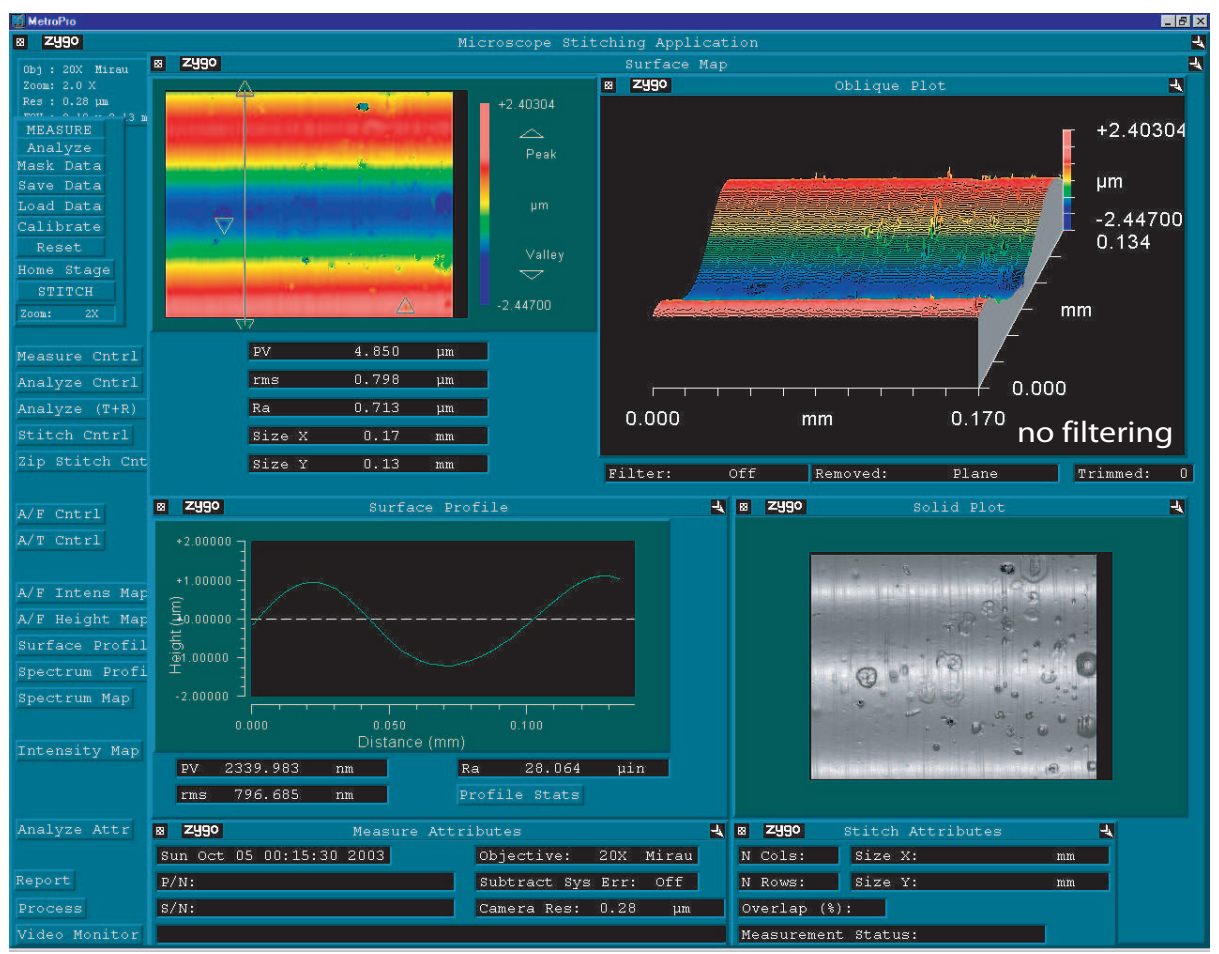

Figure 3-63: Surface measurement of the outer diameter of a workpiece machined with the $2 \mathrm{kHz}$ FTS, showing a two-dimensional profile across the grooves. 


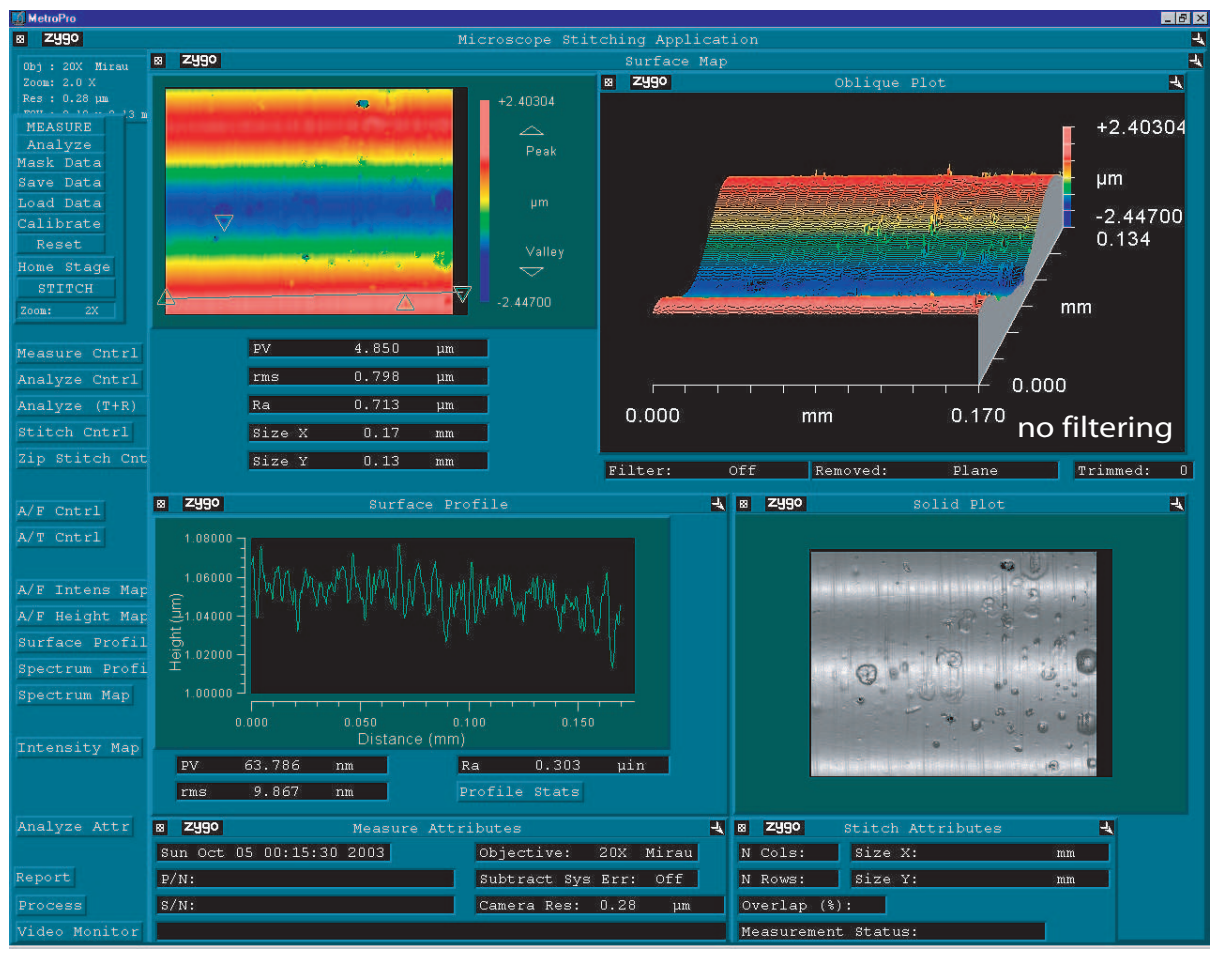

Figure 3-64: Surface measurement of the outer diameter of a workpiece machined with the $2 \mathrm{kHz}$ FTS, showing a two-dimensional profile along a groove.

demonstrated peak torque of $0.20 \mathrm{~N} \cdot \mathrm{m}$. The demonstrated peak torque is $71 \%$ of the specified peak torque of $0.28 \mathrm{~N} \cdot \mathrm{m}$, which is based on a peak current of $11 \mathrm{~A}$ corresponding to the specified maximum continuous current of $7.7 \mathrm{~A} \mathrm{rms}$ for the actuator.

One measure of performance for the actuator used in the $2 \mathrm{kHz}$ FTS is the ratio of the continuous-operation peak torque divided by the unburdened ${ }^{17}$ rotational inertia of the moving mass in the actuator. For the demonstrated peak torque of $0.20 \mathrm{~N} \cdot \mathrm{m}$ and the actuator moving mass of $6.4 \mathrm{gm} \cdot \mathrm{cm}^{2}$, that ratio is $3.1 \times 10^{5} \mathrm{~N} \cdot \mathrm{m} /\left(\mathrm{kg} \cdot \mathrm{m}^{2}\right)$. Using the designed peak torque of $0.28 \mathrm{~N} \cdot \mathrm{m}$ increases the ratio to $4.4 \times 10^{5} \mathrm{~N} \cdot \mathrm{m} /\left(\mathrm{kg} \cdot \mathrm{m}^{2}\right)$. Adding the burden of the $51 \mathrm{gm} \cdot \mathrm{cm}^{2}$ rotational inertia for the swing-arm — which is within the manufacturer's specified range of 3-64 $\mathrm{gm} \cdot \mathrm{cm}^{2}$ for the payload — reduces these ratios by a factor of 0.11 .

Another measure of performance is the ratio of the demonstrated peak torque

\footnotetext{
17 "Unburdened" means that the inertia of the payload — the swing arm in this case — is not included.
} 
while accelerating the swing arm divided by the apparent time-average electrical power at the terminals of the actuator. The demonstrated peak torque of $0.20 \mathrm{~N} \cdot \mathrm{m}$ at $2 \mathrm{kHz}$ required a total peak current of $7.9 \mathrm{~A}$. The corresponding voltage drops across the resistance and the inductance in the actuator are given by (3.18) and (3.19):

$$
\begin{gathered}
\left(R_{c}+R_{s}\right) I_{m}=(1.0+0.1) 7.9=8.7 \mathrm{~V} \\
L_{c} \frac{d I_{m}}{d t}=\left(280 \times 10^{-6}\right)[7.9(2 \pi 2000)]=28 \mathrm{~V}
\end{gathered}
$$

Section 3.9.2 shows that the current in the resistance - and hence the voltage across the resistance - lags the voltage across the inductance by $73^{\circ}$. The timeaverage complex power $\underline{S}$ is the real power $P$ plus the reactive power $Q$, and is related to the complex voltage $\underline{V}$ and complex conjugate $\underline{I}^{*}$ of the current by the following relationship given by Kirtley [89], where the underscore indicates a vector quantity:

$$
\underline{S}=P+j Q=\frac{1}{2} \underline{V} \underline{I}^{*}
$$

Substituting in values for the actuator, we obtain:

$$
\underline{S}=P+j Q=\frac{1}{2} \underline{V} \underline{I}^{*}=\frac{1}{2}(8.7+j 28) 7.9=34 \mathrm{~W}+j 110 \mathrm{VAR}
$$

In this case the real power dissipated in the resistance is significant compared to the reactive power in the inductance. The magnitude of the time-average complex power $|\underline{S}|$ is the apparent power, and is $120 \mathrm{VA}$ for the actuator while demonstrating $0.20 \mathrm{~N} \cdot \mathrm{m}$ at $5 \mathrm{kHz}$. This gives a ratio of the demonstrated peak torque while accelerating the swing arm divided by the apparent time-average electrical power at the terminals of the actuator of $1.7 \times 10^{-3} \mathrm{~N} \cdot \mathrm{m} / \mathrm{VA}$. 


\subsection{Conclusions}

The main purpose of developing the $2 \mathrm{kHz}$ rotary fast tool servo was to establish the proofs-of-principles needed to pursue the more technically challenging $10 \mathrm{kHz}$ FTS. In addition to meeting that goal, by producing optical quality wavy surfaces with approximately the same surface roughness on the face and outside diameter of workpieces I demonstrated that a rotary-type fast tool servo is well suited for diamond turning spherical workpieces from the pole to the equator. Using a commercially available moving-magnet Lorentz force galvanometer for the actuator, the $2 \mathrm{kHz}$ FTS produced a peak closed-loop tool tip acceleration of $20 \mathrm{~g}$, and establishes a new benchmark for a rotary fast tool servo with a demonstrated closed-loop bandwidth of $2 \mathrm{kHz}$.

The following proofs-of-principles were established for developing the follow-on $10 \mathrm{kHz}$ FTS:

- Designed and tested a serviceable over-constrained flexure bearing for guiding reciprocating rotary motion, and showed that it can survive high cycle numbers and provide adequate stiffness for diamond turning.

- Established a mechanical design that integrates the flexure suspension with a small commercially available diamond tool.

- Established a circuit topology for a high bandwidth current-mode amplifier.

- Showed that loop-shaping techniques with Classical Control theory were adequate for designing the control systems.

With these proof-of-principles in hand, we now proceed to the development of the $10 \mathrm{kHz}$ rotary fast tool servo, which adds a new layer of complexity by integrating the tool holder with the moving mass of a novel electromagnetic actuator. 


\section{Chapter 4}

\section{The $10 \mathrm{kHz}$ Rotary Fast Tool Servo}

In this chapter I describe the development and measured performance of the $10 \mathrm{kHz}$ rotary fast tool servo (10 kHz FTS). I begin by reviewing the philosophical guidelines for the design, introduce the magnetic circuit for the actuator to establish context for later discussions, and survey the major conceptual designs that were considered. I then explore the $10 \mathrm{kHz}$ FTS through detailed discussions on the design, analysis, and fabrication associated with the mechanical design, system dynamics, electrical design, integrated trade-off study, and the control system. The chapter concludes by presenting the major test results for the machine.

\subsection{Introduction}

The $10 \mathrm{kHz}$ rotary fast tool servo is shown in Figure 4-1. Figure 4-2 provides context for this machine by showing it mounted on a diamond turning machine and engaging a workpiece. This machine incorporates several key concepts developed with the earlier $2 \mathrm{kHz}$ rotary fast tool servo - the flexure bearing for guiding reciprocating rotary motion, small diamond tool and mounting method, circuit topology for the current-mode amplifier, and control system design — and adds electromagnetic machine design for the novel actuator and an integrated trade-off study for balancing the mechanical, magnetic, and electrical design for that actuator. In addition to establishing new benchmarks for high bandwidth and peak tool tip acceleration for a 


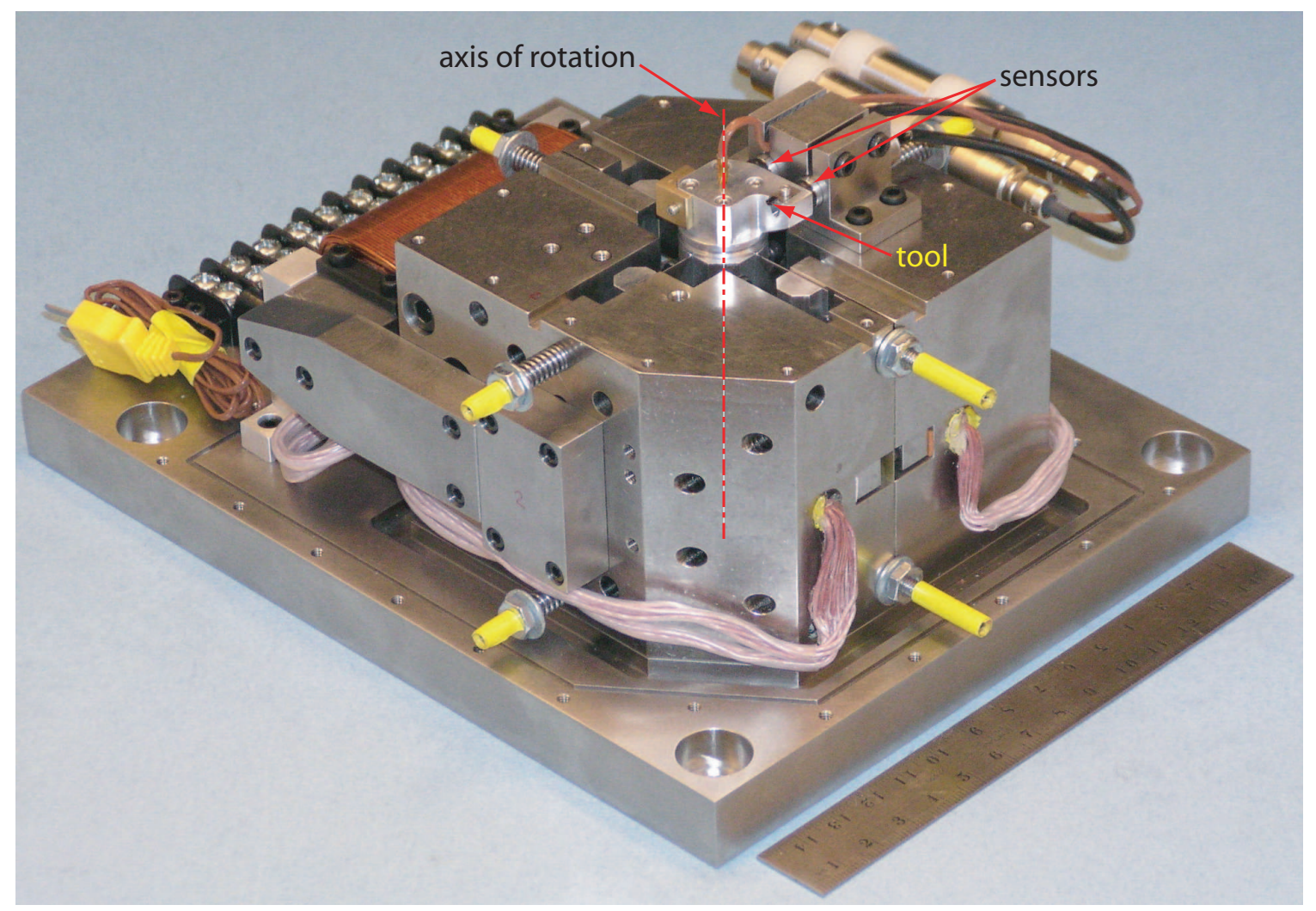

Figure 4-1: The $10 \mathrm{kHz}$ rotary fast tool servo.

rotary fast tool servo, the $10 \mathrm{kHz}$ FTS serves as a proof-of-concept for an actuator that has potential application beyond this research.

\subsection{Design Philosophy}

The philosophy that guided the design of the $10 \mathrm{kHz}$ rotary fast tool servo included the following points:

- Develop a fast tool servo that can be integrated with an auxiliary rotary axis on a diamond turning machine and used to machine a $10 \mathrm{~mm}$ diameter or smaller spherical or cylindrical workpiece.

- Use the same small commercially available diamond tool and mounting method developed with the $2 \mathrm{kHz}$ FTS.

- Design for stiffness at the tool tip. Radial and axial stiffness of the rotor that 


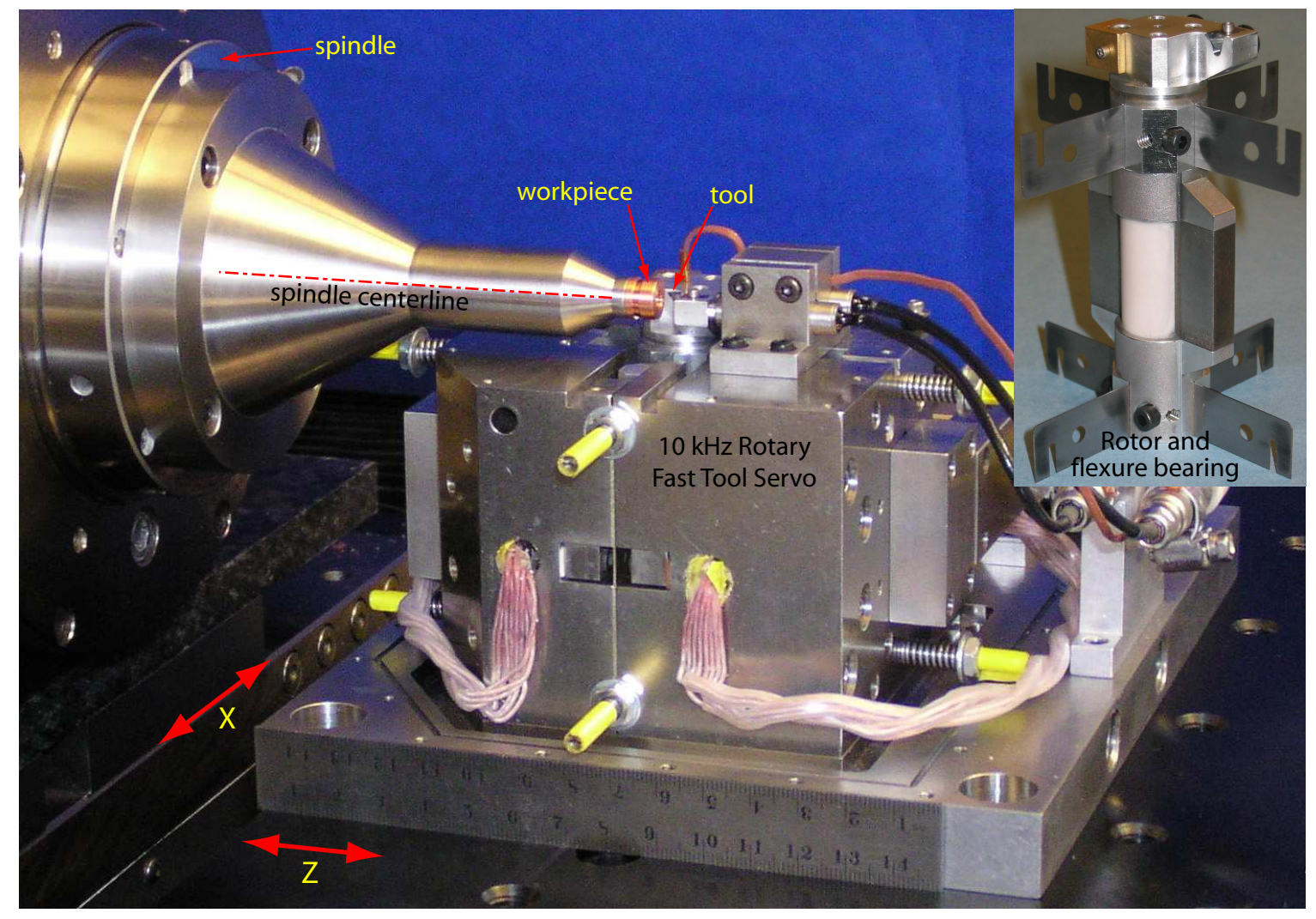

Figure 4-2: The $10 \mathrm{kHz}$ rotary fast tool servo on a diamond turning machine, and the rotor for the fast tool servo with its flexure bearing (inset).

carries the tool are provided by the mechanism, torsional stiffness is provided by the controller.

- Merge the moving masses of the tool holder and the actuator into a single mass. This results in a reduced total moving mass and avoids the first uncoupled rigidbody mode that the $2 \mathrm{kHz}$ FTS has.

- Use the same flexure bearing design that proved successful for the $2 \mathrm{kHz}$ FTS.

- Starting with the soft magnetic core for the rotor, design a custom, high torquedensity actuator that allows direct attachment of a tool holder.

- Split the stator into two halves in a manner that allows controlling all 18 degrees of freedom between the rotor and the two stator cores during assembly of the actuator. The assembled actuator needs to have four uniform, small air gaps, and all eight of the flexure blades suspending the rotor need to be preloaded 
in tension. These challenges can easily be underappreciated when realizing the simplicity of the mechanical design.

- Use a coil instead of permanent magnets to supply bias flux for the actuator. Being able to adjust the bias flux proved invaluable when identifying the dynamics of the as-built system, and allowed establishing a low noise implementation and a high acceleration implementation for the $10 \mathrm{kHz}$ FTS.

- Place a position sensor directly behind the tool to hide certain flexible modes of the system and improve high frequency controllability.

- Add a second position sensor directly on the centerline of the rotor to allow controlling the linear (radial) mode of the actuator.

- Include provisions for active cooling of the actuator and adding covers and seals to protect it. Internal passages for forced fluid cooling are provided to better allow using the $10 \mathrm{kHz}$ FTS in an ultra-precision machining environment where temperature control is important. The design includes the covers and seals needed to prevent contamination of the actuator and sensors from machining debris.

\subsection{Magnetic Circuit}

A brief discussion on the magnetic circuit for the actuator that we developed for the $10 \mathrm{kHz}$ FTS is presented in this section to provide context for many of the other topics that are discussed in this chapter. Since the design, analysis, and fabrication of the actuator represents a significant fraction of the effort involved in developing the $10 \mathrm{kHz}$ FTS, all of Chapter 5 is devoted to it.

In addition to producing the torque that is needed to accelerate the rotor of the $10 \mathrm{kHz}$ FTS, the actuator produces a radial force and is therefore referred to as a hybrid rotary/linear actuator. The actuator consists of a rotor and a stator consisting of two halves. Figures 4-3 and 4-4 show models of the magnetic circuit 


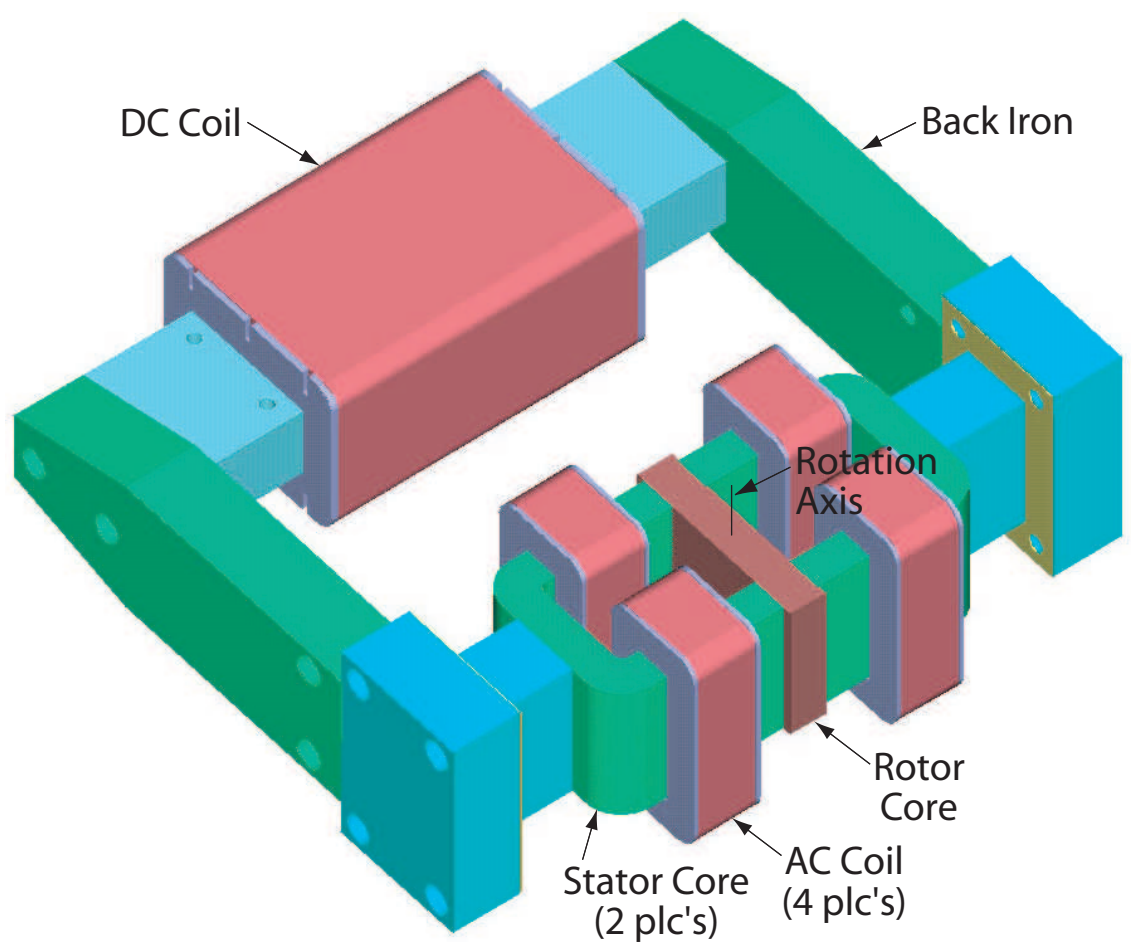

Figure 4-3: Model of the magnetic circuit for the $10 \mathrm{kHz}$ rotary fast tool servo.

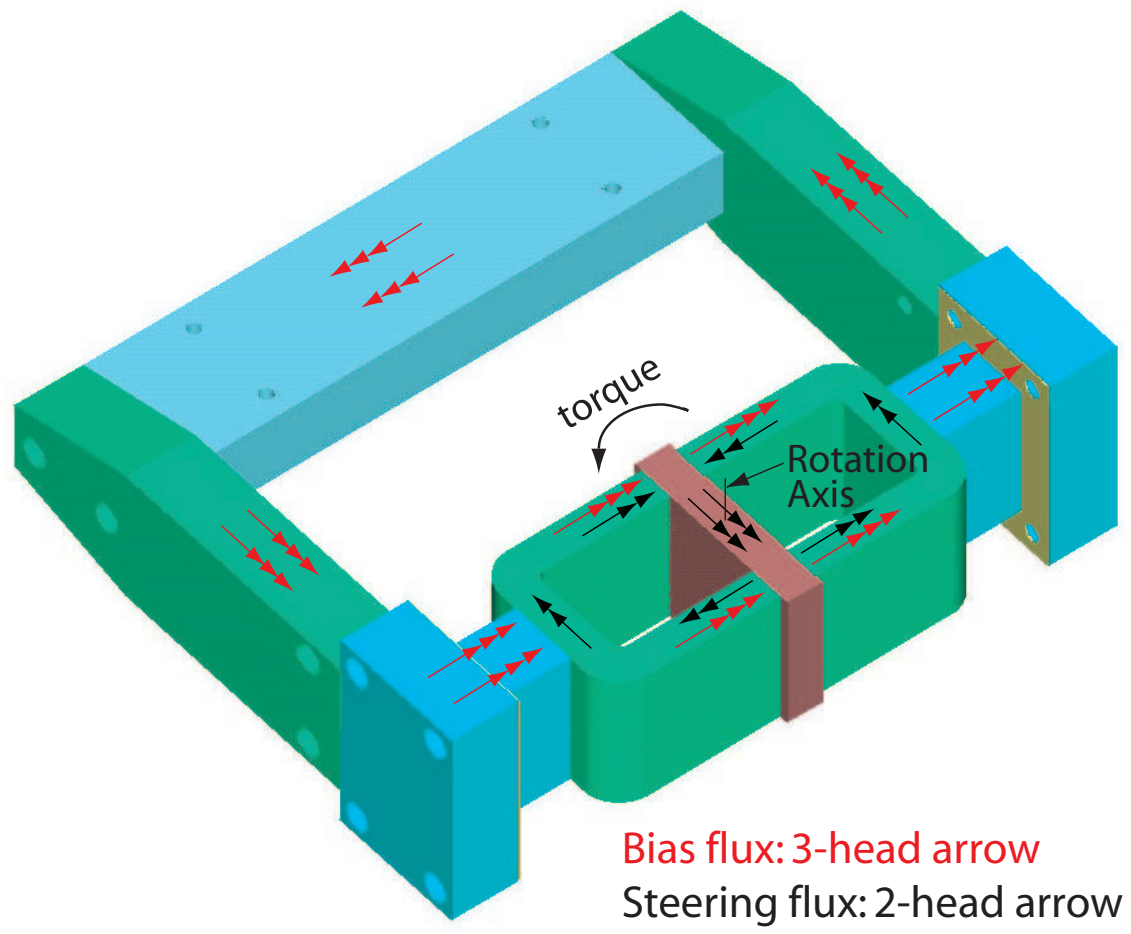

Figure 4-4: Model showing directions of magnetic fluxes for the $10 \mathrm{kHz}$ rotary fast tool servo. 
for the actuator. The DC coil provides a bias flux (triple-head arrows) and the AC coils provide a steering flux (double-head arrows). With appropriate phasing, the bias and steering fluxes add in one pair of diagonally opposed rotor/stator air gaps and subtracts in the other pair, producing a net torque on the rotor. A net radial force is produced on the rotor when the steering fluxes in the two stator cores have different magnitudes.

The bias flux linearizes the relationship between the torque and steering-coil currents, which makes it easier to accurately control the actuator. Since the bias flux supplies up to half of the torque-producing magnetic flux in the rotor/stator air gaps, it provides a factor of two reduction in the reactive electrical power needed to drive the AC flux during peak operation when compared to a design that does not use a bias flux.

Reduced to its most essential elements, the $10 \mathrm{kHz}$ FTS consists of a rotor core that carries a cutting tool and is supported by a bearing system that provides a single degree of freedom for rotation. The two stator cores provide controllable magnetic forces on the rotor core to produce a desired angular acceleration of the core, and to produce a desired net radial force on the core. Sensors provide position feedback to control those magnetic forces.

\subsection{Machine Topology Concepts}

Before selecting the magnetic circuit shown in Figures 4-3 and 4-4 for the $10 \mathrm{kHz}$ FTS, we considered other possible magnetic circuits, the most significant of which are presented in this section. The magnetic circuits described in this section illustrate an evolution that occurred during the design phase for the $10 \mathrm{kHz}$ FTS.

Figure 4-5 is a sketch of a magnetic circuit referred to as the T-rotor layout. The T-shaped rotor is suspended from three flexure blades that attach to two stator cores. The two stator cores are nested, one inside the other, so that the front face of the rotor is completely open. This increases the size of the work zone in front of the rotor so that the cutting tool can engage a large workpiece, or alternatively, so that a steering 
mirror can be integrated with the front face of the rotor for use in an optical system. Permanent magnets (P.M.) are shown as the source of the bias flux in Figure 4-5. Note that the magnetic forces between the outer stator poles and the rotor produce a compressive axial load on the flexure blades, which is undesirable. Figure 4-6 shows a sketch of an alternate arrangement of the outer stator poles and the rotor that leads to a tensile axial load on the flexure blades. Note also that in both cases the magnetic forces on the rotor from the inner stator poles result in a net lateral force on the rotor. The flexure blades for this layout establish an axis of rotation for the rotor that is not coincident with the center of mass of the rotor. Therefore, angular acceleration of the rotor will be produce an undesirable net acceleration of the center of mass of the rotor.

Figure 4-7 is a sketch of a magnetic circuit referred to as the X-rotor layout. It is similar to the T-rotor layout with the outer stator poles arranged to put the rear flexure in tension, which occurs if the flux density between the outer poles and rotor is greater than the flux density between the inner stator poles and rotor. In Section 5.2.3 I show that the magnetic force on the rotor at each air gap depends on the square of the magnetic flux density. Therefore, if the cross-sectional area of the outer poles is less than that of the inner poles, the bias flux produced by the permanent magnets (P.M.) will have a higher density in the outer pole air gaps, resulting in a net force that puts all three flexure blades into tension. Note that the forces produced by the steering fluxes to develop the desired torque also produce a net lateral force on the rotor.

Figure 4-8 is a sketch of a magnetic circuit referred to as the Balanced X-rotor layout. Like the T-rotor and X-rotor designs before it, the rotor is suspended from the nested stator cores by three flexure blades. Note that the magnetic forces on the rotor do not create a net force on it, and that the center of rotation established by the flexure blades is coincident with the center of mass of the rotor. Although Figure 4-8 shows three flexure blades connected directly to the rotor core, the X-rotor layout can accommodate flexure hubs above and below the core in the same fashion as the $10 \mathrm{kHz}$ FTS, or it can use one of the other types of bearings described in Section 6.4.7. 


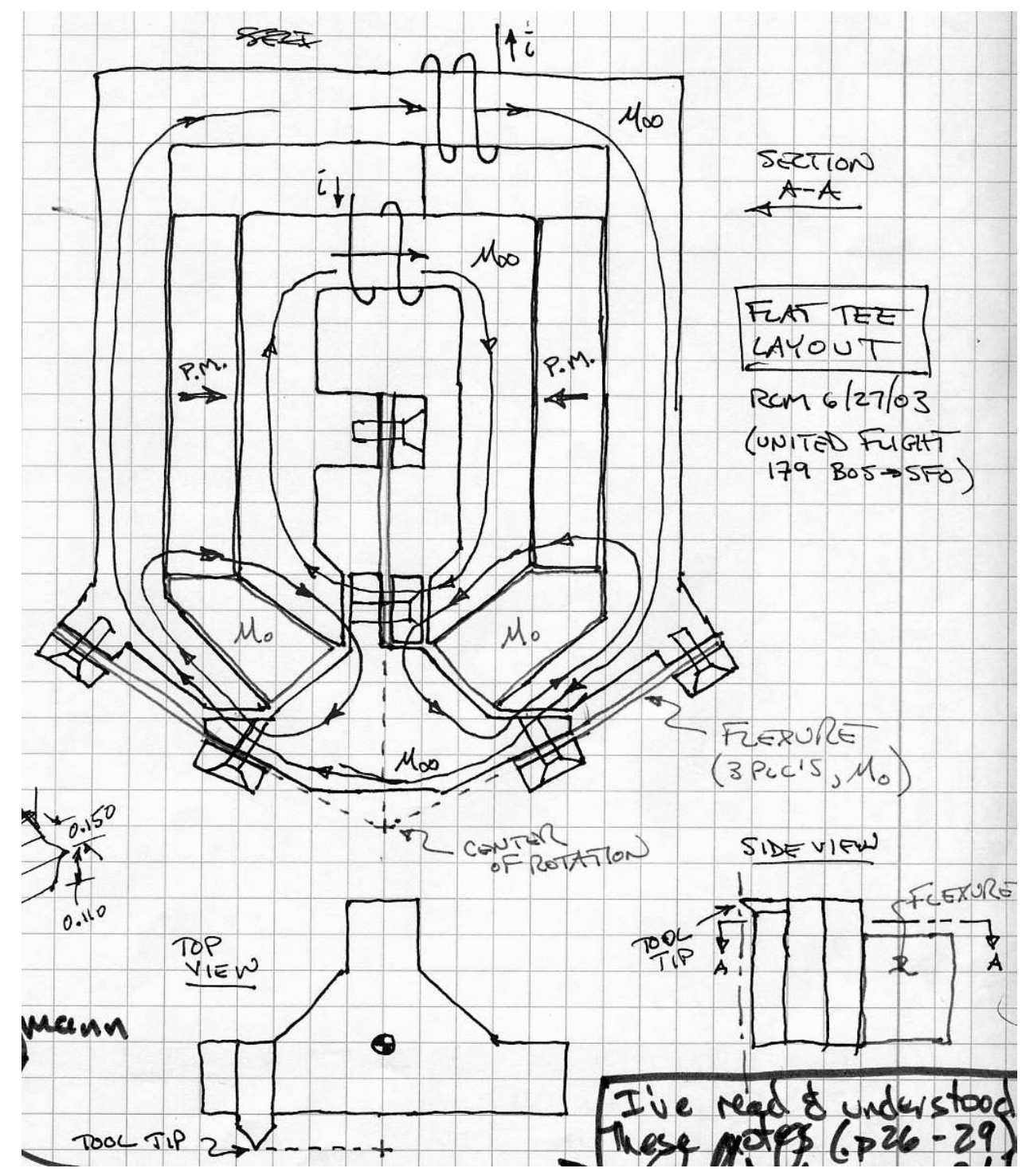

Figure 4-5: T-rotor layout with nested stator cores.

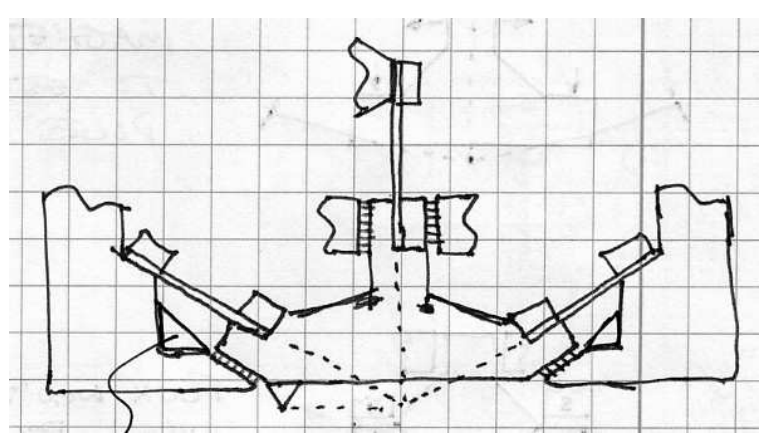

Figure 4-6: Detail of T-rotor layout with front poles arranged to put rear flexure in tension. 


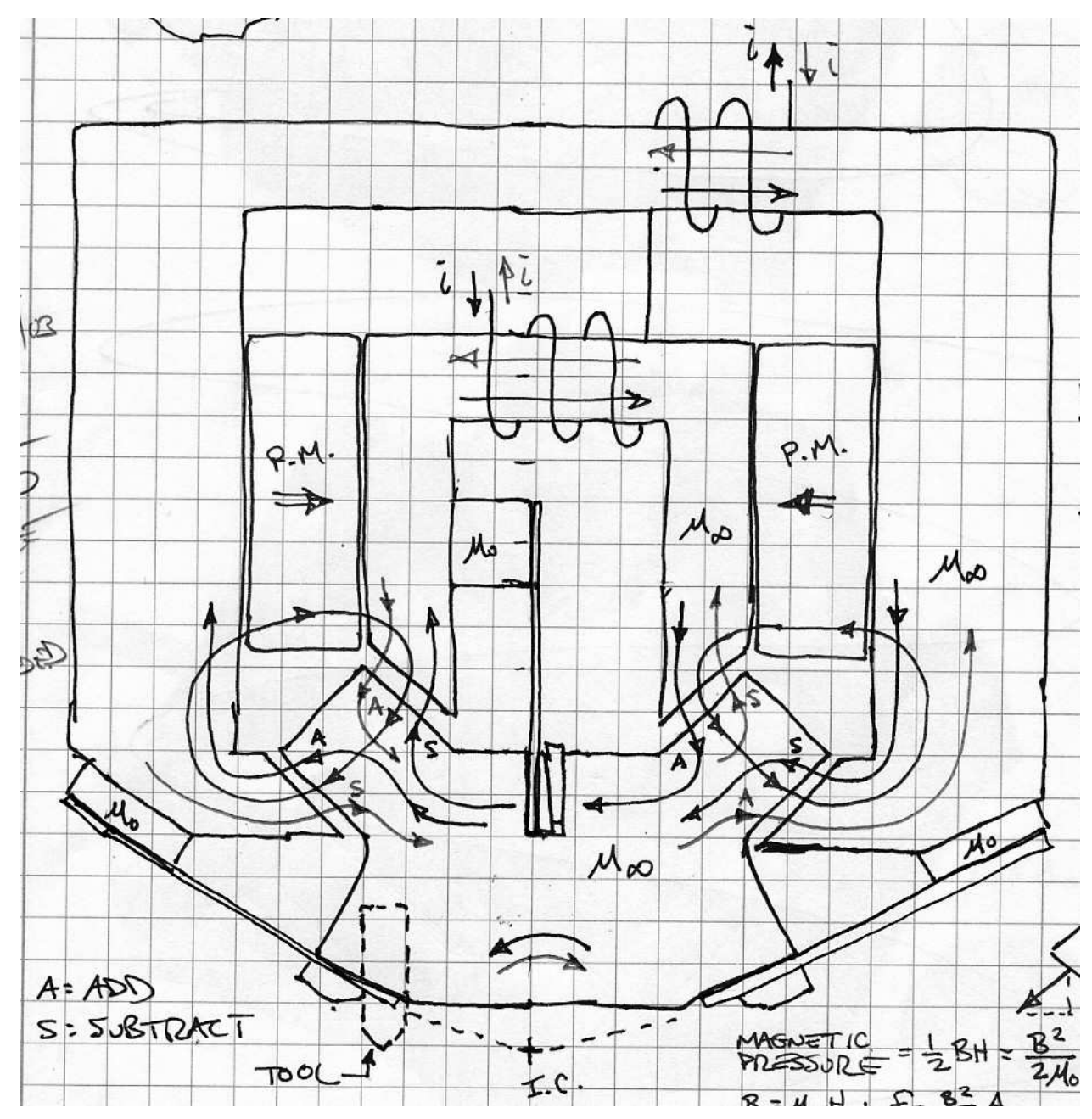

Figure 4-7: X-rotor layout with nested stator cores.

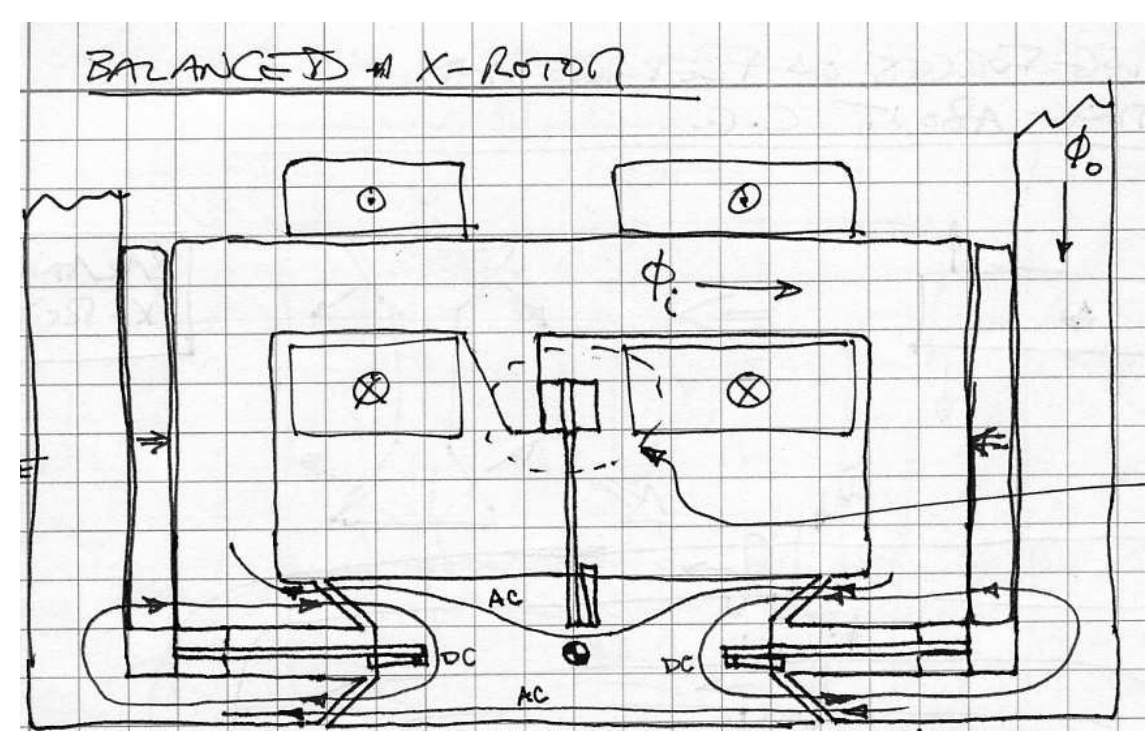

Figure 4-8: Balanced X-rotor layout with nested stator cores. 

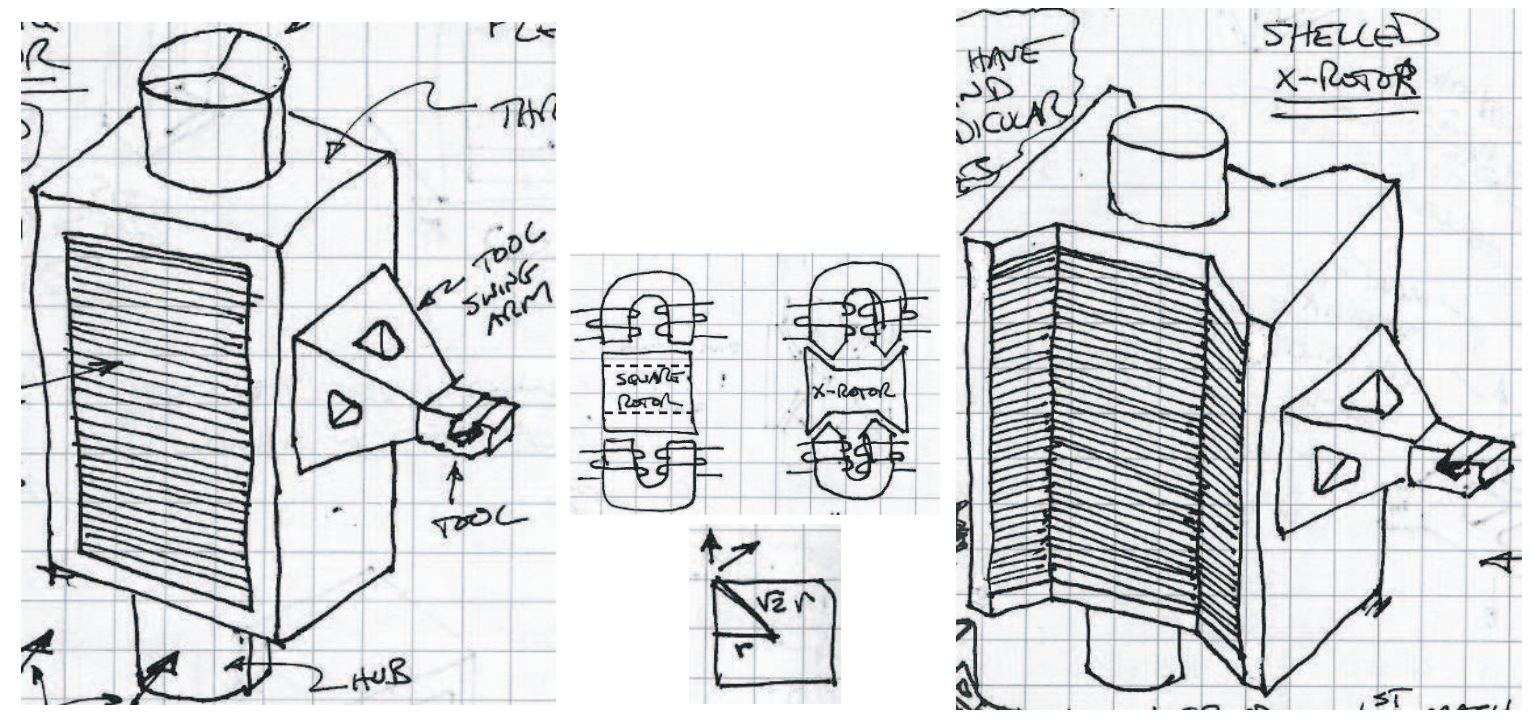

Figure 4-9: Early concepts for shelled-rotor design. Shelled square-rotor (left), shelled $\mathrm{X}$-rotor (right), and sketch comparing the torque-producing lever arms for the squarerotor and X-rotor (center).

At this point during the evolution of the magnetic circuit we began to consider how to provide mechanical support to the relatively fragile laminated rotor core. Figure 4-9 shows sketches of "shelled rotors", designs that support the rotor core by encasing them in a shell of mechanically stronger material. Note the integral hubs for mounting the flexure blades at the top and bottom of each shelled rotor, and the integral tool arm. The torque-producing lever arm for the X-rotor is $41 \%$ longer than that of the square-rotor, as is shown in Figure 4-9. However, a fair comparison requires removing material from the square-rotor to produce the inner rectangle represented by the dashed lines in the figure, so that both rotors have the same minimum cross-sectional area for the magnetic flux. The X-rotor can now be seen as a rectangular rotor with additional mass - the four wedge-shaped pieces added to the outside corners. From the standpoint of optimizing the torque-to-inertia ratio for the rotor, since the rotational inertia increases with the square of the distance between each piece of additional mass and the center of rotation, the advantage of a longer torque-producing lever arm is offset by the increase in rotational inertia.

Figure 4-10 shows a more evolved version of the shelled square-rotor, with the addition of front and back shear panels on the large faces of the rotor core, and 
better use of material for providing a stiff connection of the tool to the shell. Note the indication of "grease" between the ends of the flexure hubs (top and bottom) for providing mechanical damping.

It is worth comparing the magnetic circuits for the concepts discussed so far. In Section 5.4 I describe generic magnetic circuits referred to as the "pinched rotor" design and the "sandwiched rotor" design. An important conclusion in that section is that the torque and force modes are not fully decoupled for the sandwiched rotor, but they are fully decoupled for the pinched rotor. The T-rotor and X-rotor layouts have the same magnetic circuit as the "pinched rotor" design, while the arrangement of the stators and rotor for the shelled rotor concepts creates the same magnetic circuit as the "sandwiched rotor" design. Although the balanced X-rotor layout shown in Figure 4-8 provides advantages over the magnetic circuit that we selected for the $10 \mathrm{kHz}$ FTS, most notably fully decoupled torque and force modes, we decided against it for our first prototype because of the additional fabrication complexity that the extra angles in the cores present. Now that the $10 \mathrm{kHz}$ FTS has been built and used to produce successful test results, if I were to design a follow-on machine I would consider pursuing the version of the balanced X-rotor magnetic circuit described in Section 6.4.9, or the significantly different system described in Section 6.4.11.

Figures 4-11 and 4-12 show sketches of early concepts for what we called the "torque-tube" design for the rotor. Essentially, the rotor core derives bending and torsional stiffness from a tube-like structure that encapsulates the stack of laminations. An important detail to note is that since the rotor core conducts AC flux, the loop around the core formed by the torque tube and the flexure hubs needs to be constructed so that it does not form an electrically conductive closed path. If it did, then the AC flux linked by that loop would generate an AC current in the loop that would lead to appreciable electrical heating. In Figure 4-11 that loop is broken by separating the two halves of a metallic torque tube with an electrically-insulating spacer. In Figure 4-12 the torque tube is made from an electrically non-conducting ceramic material. Note the mention of "glue" in Figure 4-12, as I begin to embrace the idea that the rotor needs to be an adhesive-bonded assembly. 


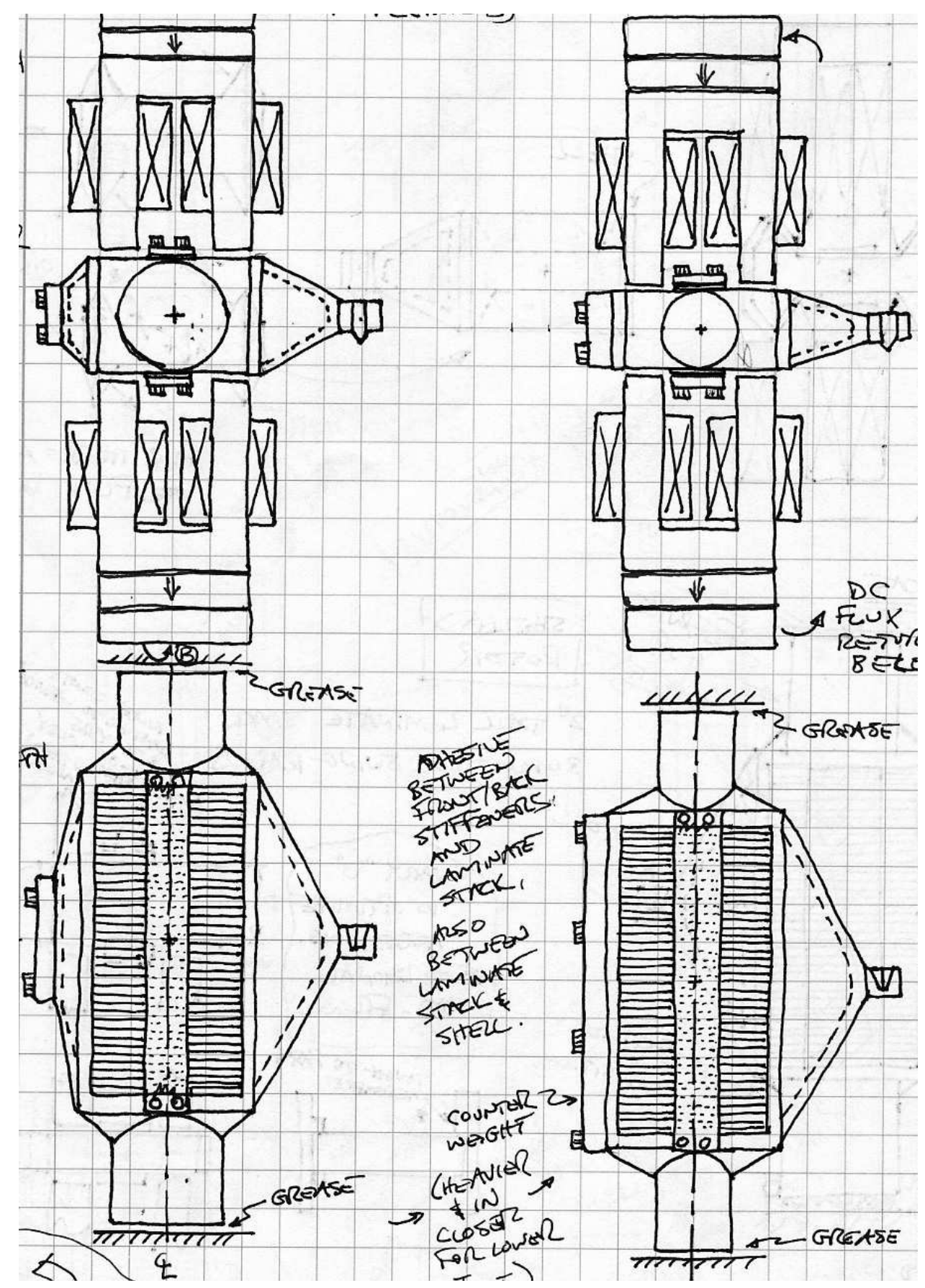

Figure 4-10: Later concept for shelled square-rotor design. 


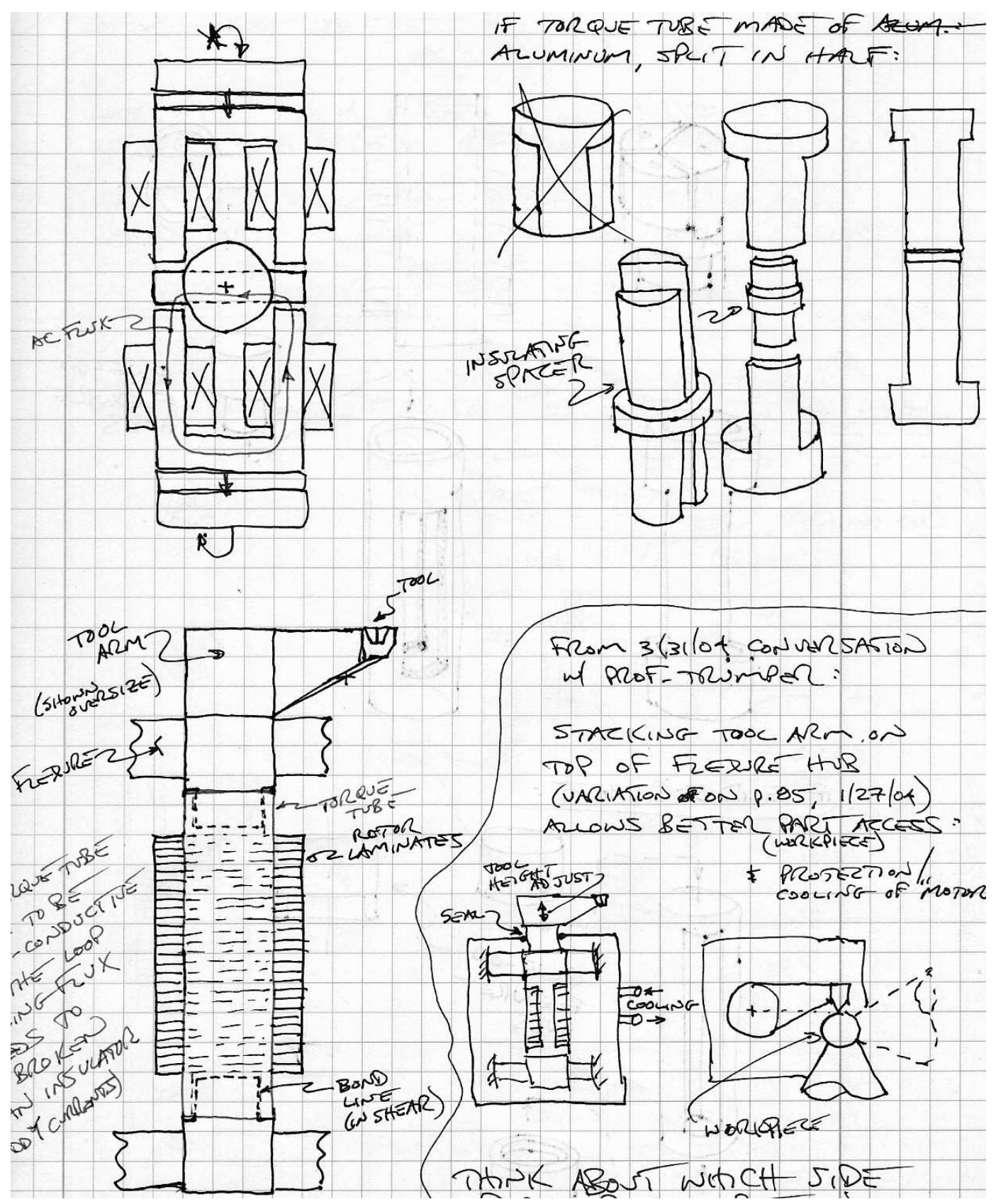

Figure 4-11: Early concepts for torque-tube design for the rotor. 


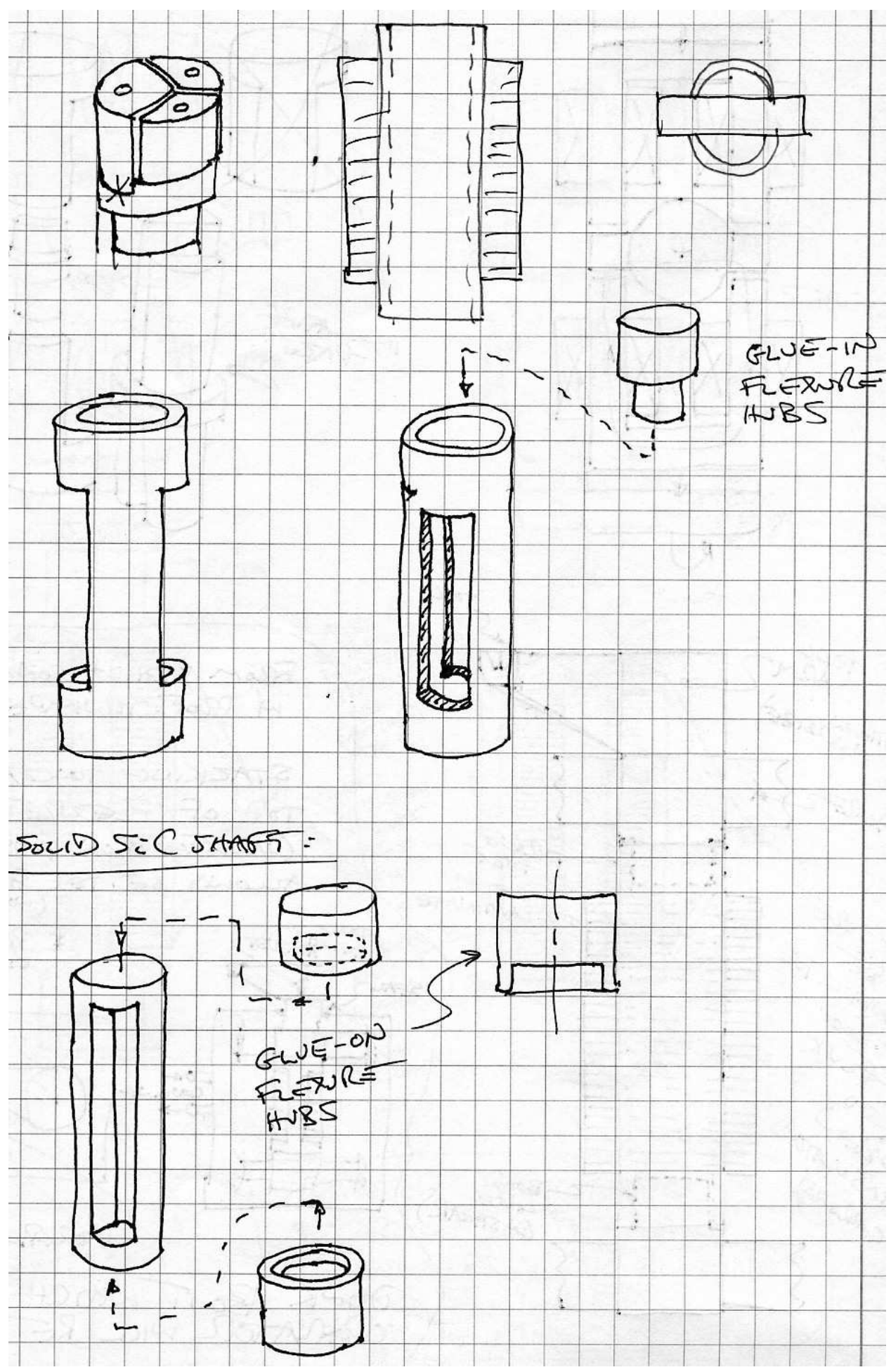

Figure 4-12: Mid-evolution concepts for the torque-tube design for the rotor. 


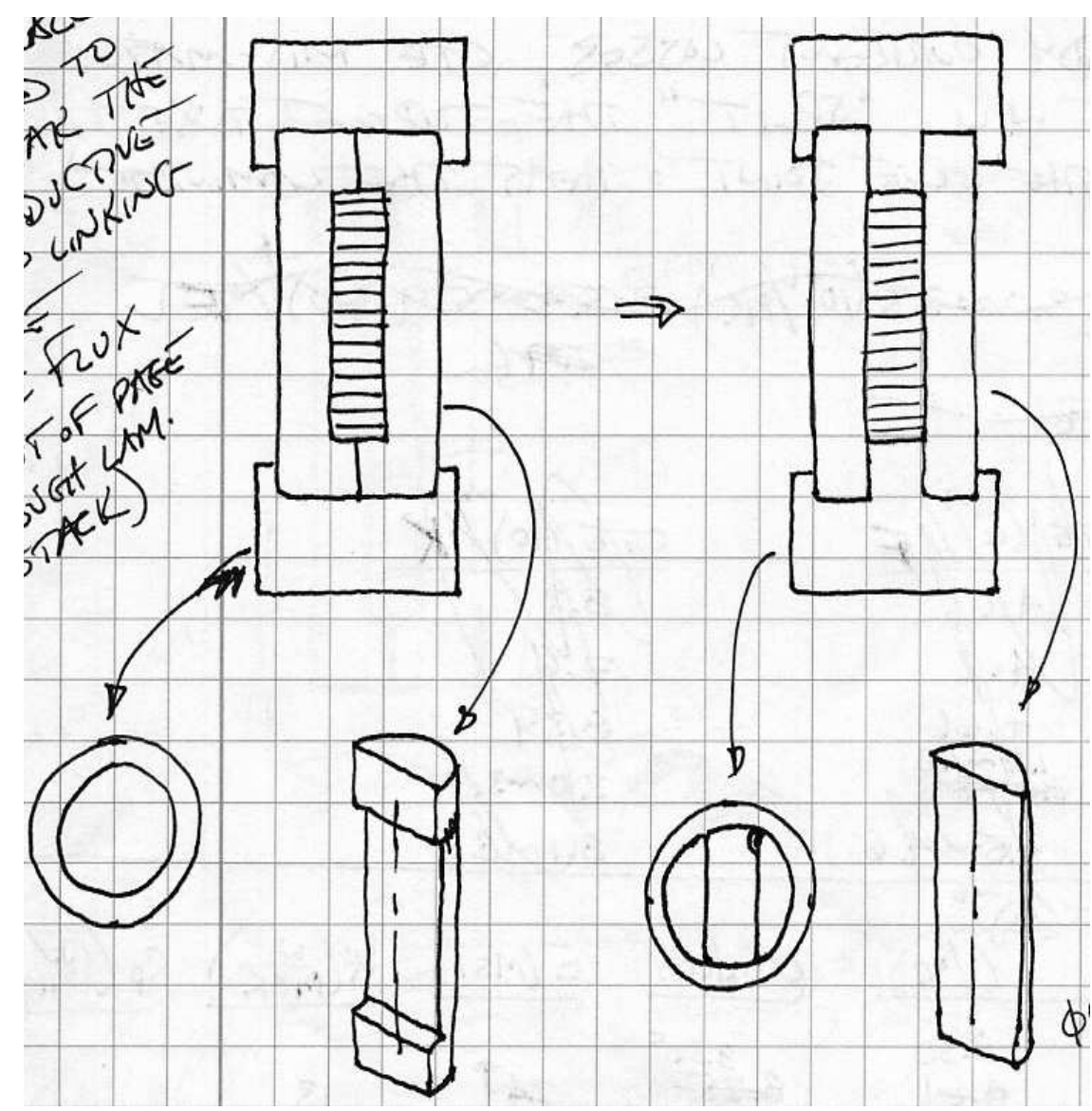

Figure 4-13: Final concepts for torque-tube design for the rotor. The concept for the rotor that I built is on the right.

Figure 4-13 shows the final step in the evolution of the torque-tube design. We are now fully depending on the mechanical integrity of an adhesive-bonded assembly: the rotor core is captured front-to-back between two semicircular ceramic stiffeners, and captured top-to-bottom between two metal flexure hubs.

\subsection{Mechanical Design}

The heart of the mechanism is the mechanical design for the actuator. We start with the rotor core, add enough support structure to protect the fragile laminations and allow attaching the flexure bearing, then directly attach the tool to realize a single rigid body. The rotor is sandwiched between two stator halves in a manner that allows aligning and then fixing the eighteen degrees of freedom for those three bodies when 
establishing the four $50 \mu \mathrm{m}$ rotor/stator air gaps while preserving a tensile preload force in each of the eight flexure blades that support the rotor and establish the axis of rotation.

Referring to Figure 4-1, the tool holder carries a diamond tool at a swing radius of $12.7 \mathrm{~mm}$ (0.500 inch) and has a full-travel rotation angle of $\pm 2.8 \mathrm{mrad}$. The small rotation angle allows using over-constrained flexures to guide the rotary motion. A total of eight flexures are used, four above the rotor magnetic core and four below it. The inner ends of the flexures are fixed to the rotor, and the outer ends are fixed to the stator. The unwanted transverse motion of the tool tip due to motion along an arc is only $\pm 48 \mathrm{~nm}$ for the full travel of $\pm 35 \mu \mathrm{m}$, making it negligible for most diamond turning applications.

Still referring to Figure 4-1, a first capacitance gauge measures the displacement of the tool arm at a location just behind the tool. A second capacitance gauge measures the tool arm displacement at the same elevation but along the rotation axis. The two displacement sensors provide sufficient information for controlling the tool tip position and, in case of an imbalance in the force couple on the rotor produced by the magnetic flux on the pole faces, compensating for axial motion of the rotor. The FTS is designed for a maximum stroke of $70 \mu \mathrm{m}$ peak-to-peak (PP) at low frequencies and was initially designed for a $5 \mu \mathrm{m} \mathrm{PP}$ stroke at $10 \mathrm{kHz}(1000 \mathrm{~g})$. The peak tool tip acceleration of $1000 \mathrm{~g}$ was reduced to $600 \mathrm{~g}$ in a trade-off between having a more mechanically robust system versus the more light-weighted rotor needed for $1000 \mathrm{~g}$. I felt that it was more important to have a robust proof-of-principle machine having a higher likelihood of surviving the anticipated tests, especially since design parameters can be adjusted in future versions to achieve $1000 \mathrm{~g}$ and beyond.

All of the mechanical components and the actuator for the $10 \mathrm{kHz}$ FTS are shown in Figure 4-14. The are more than 100 individual parts, and the design required 55 sheets of detailed mechanical drawings. Figure $4-15$ is a model of the $10 \mathrm{kHz}$ FTS with cut-outs, and provides a sense of the complexity of the arrangement of these components. It took approximately one man-month ${ }^{1}$ of effort to assemble the entire

\footnotetext{
${ }^{1}$ It should be noted that in this case, the "graduate student" man-month is longer than the
} 


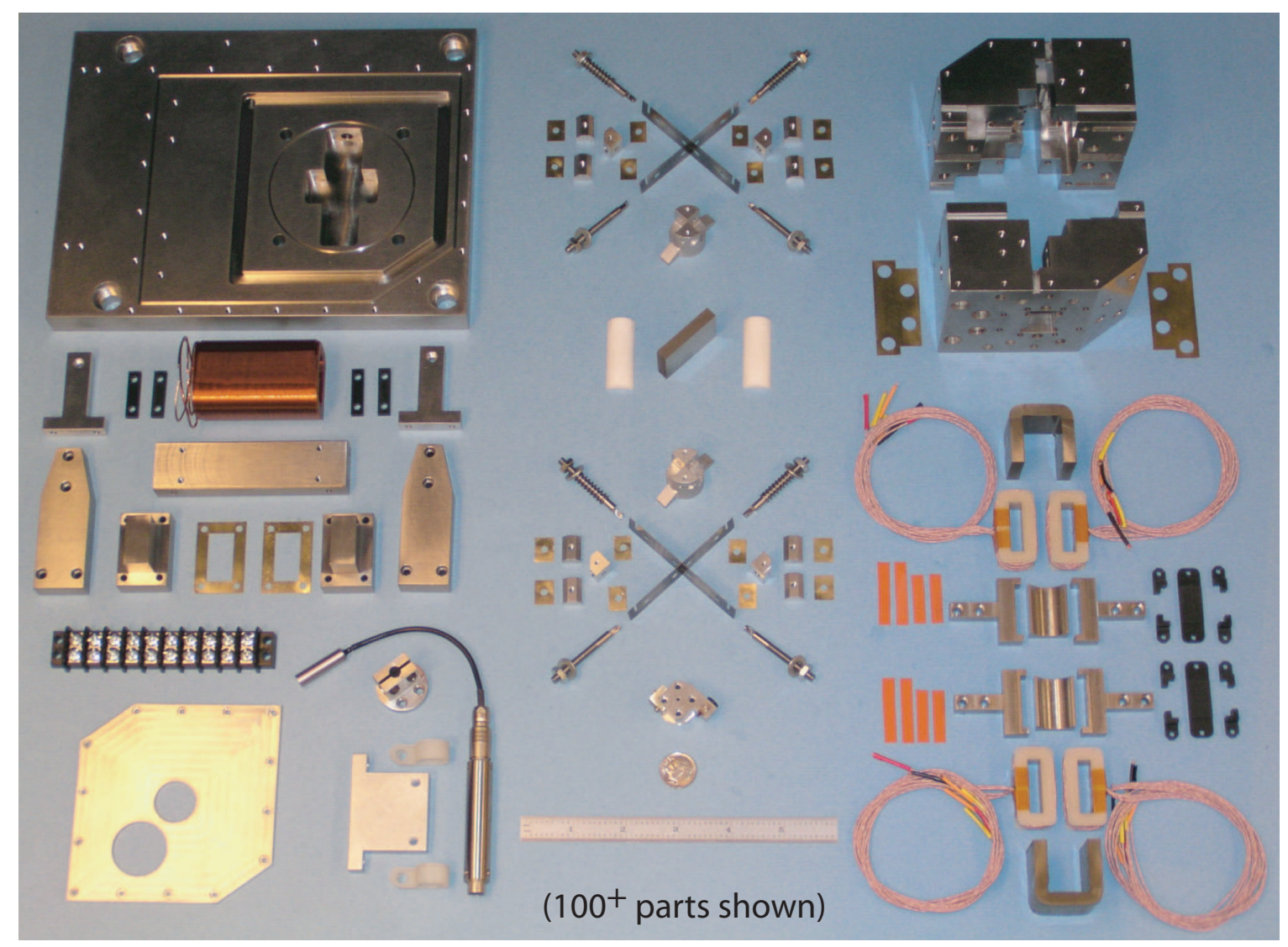

Figure 4-14: Mechanical components for the $10 \mathrm{kHz}$ FTS.

$10 \mathrm{kHz}$ FTS from the components shown in Figure 4-14. The discussions in the following subsections should provide an appreciation for why it took this long — and hopefully by illustrating the challenges of assembling this prototype - convey the sense that one month was actually a short period of time.

\subsubsection{Rotor and Stator Cores}

Figure 4-16 shows the rotor and stator cores for the $10 \mathrm{kHz}$ rotary fast tool servo. The cores are made from $25 \mu \mathrm{m}$ thick laminations of soft magnetic material: nanocrystalline iron-alloy for the rotor core, and $50 \% \mathrm{Ni}-50 \%$ Fe for the stator cores. A detailed discussion on the choice of these materials is presented in Section 5.5.1. Figure 417 illustrates the orientation of the laminations in the cores. The rotor core in the

typical man-month, which is based on a 40 hour work week. A multiplier in the range of 1.5 to 2.0 is probably accurate. 


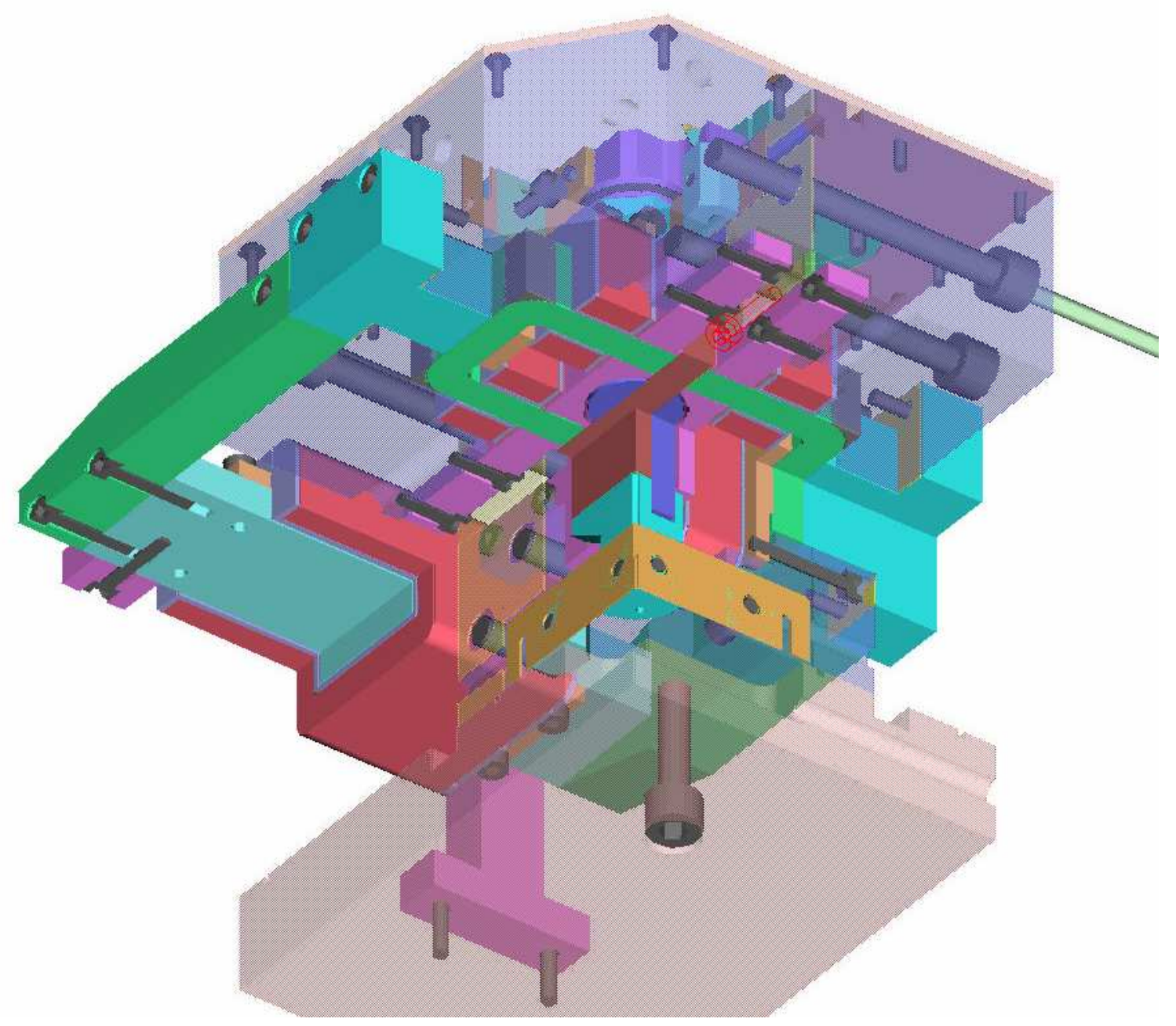

Figure 4-15: Model of the $10 \mathrm{kHz}$ FTS with cut-outs, looking up from the bottomfront. 
machine that I built is made from nanocrystalline iron-alloy laminations that are brittle but are bonded together well. The stator cores are made from $50 \% \mathrm{Ni}-50 \% \mathrm{Fe}$ laminations that are ductile but can be easily peeled apart with your fingernail.

The rotor and stator cores were cut from wound-tape core blanks purchased from Magnetic Metals [100]. Figure 4-18 shows core blanks for the rotor cores, while Figure 4-19 shows core blanks for the stator cores. Each rotor core blank can provide four rotor cores, and each stator core blank can provide two stator cores. The quality of the $50 \% \mathrm{Ni}-50 \%$ Fe core blanks was better than that of the nanocrystalline ironalloy core blanks. Most of the nanocrystalline iron-alloy core blanks had significant voids between adjacent layers of tape, making them partially or wholly unusable. I had ordered roughly six times more core blank material than was needed to produce the rotor and stator cores for the $10 \mathrm{kHz}$ FTS, and this conservatism paid off because I ended up with approximately only three times more satisfactory core blanks for each material than was initially needed by the machine shop that produced the rotor and stator cores.

The rotor and stator cores were cut from their blanks using a wire electro-discharge machining (wire EDM) process. Wire EDM was chosen because it appeared to be a plausible method for machining the laminated materials while not causing appreciable delamination. Section 5.5.5 discusses the various machining processes that I considered. The two major issues that were addressed during wire EDM were insuring that sufficient electrical continuity between each lamination and the wire EDM setup was established and maintained, and that the flush coolant used in the wire EDM process did not cause delamination at the work zone. After a short proof-of-principle experiment with a wire EDM machine shop near MIT [42], a process was established that produced the rotor and stator cores for the $10 \mathrm{kHz}$ FTS. The process was more successful with the $50 \% \mathrm{Ni}-50 \%$ Fe cores than with the nanocrystalline iron-alloy cores. The most notable difference was that the nanocrystalline iron-alloy cores ended up with areas of localized damage that looked as if the material had overheated and exploded, suggesting poor electrical continuity between one or more laminations in the damage zone and the wire EDM setup. The poor electrical continuity could have 


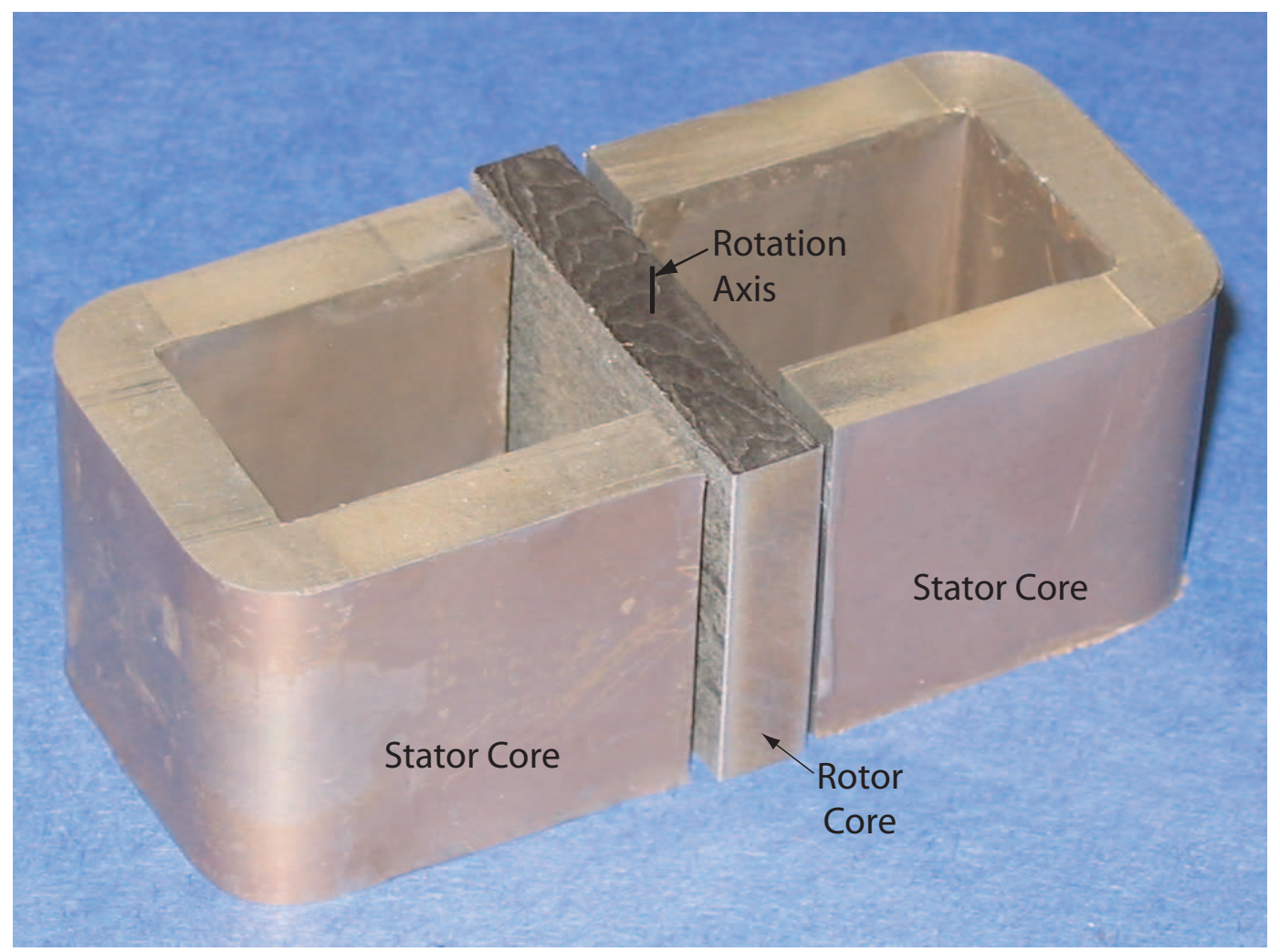

Figure 4-16: Rotor and stator cores for the $10 \mathrm{kHz}$ rotary fast tool servo. Length of air gaps shown larger for clarity.

been due to fractures or voids in the core blank material, or due to inadequate fixturing of the workpiece in the wire EDM setup. The lack of this type of localized damage in the $50 \% \mathrm{Ni}-50 \%$ Fe cores suggests that the wire EDM setup was probably adequate and that the damage in the as-machined nanocrystalline iron-alloy cores is most likely due to discontinuities in the blank material.

In addition to wire electro-discharge machining, the cores were machined using a surface grinder. Grinding across the ends of the laminations, predominantly in a direction parallel to the laminations, was used to produce flat surfaces and to establish perpendicularity and parallelism between surfaces. In the case where the grinding direction was not parallel to the laminations, clamping the laminations prevented excessive fanning out of the laminations. 


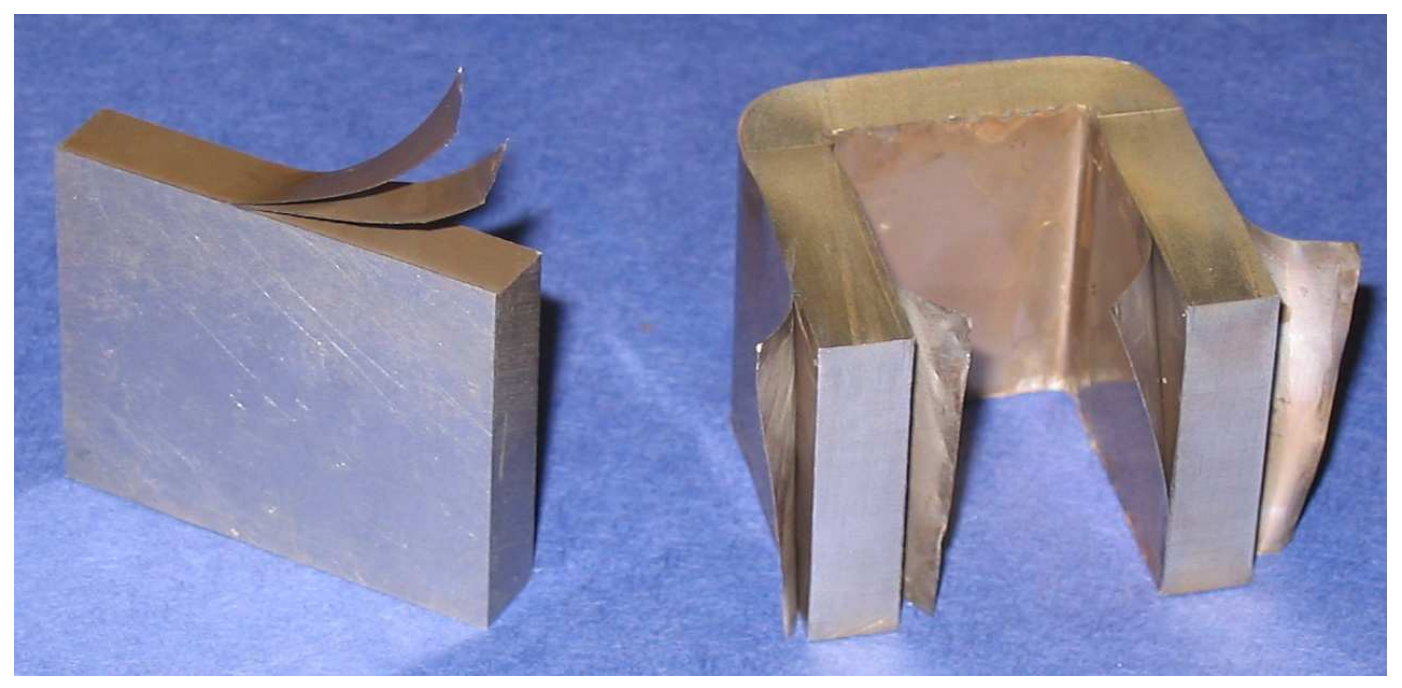

Figure 4-17: Spare 50\% Ni-50\% Fe rotor and stator cores for the $10 \mathrm{kHz}$ rotary fast tool servo with several laminations peeled back. Note that for the machine that I built, the rotor core is made from nanocrystalline iron-alloy.
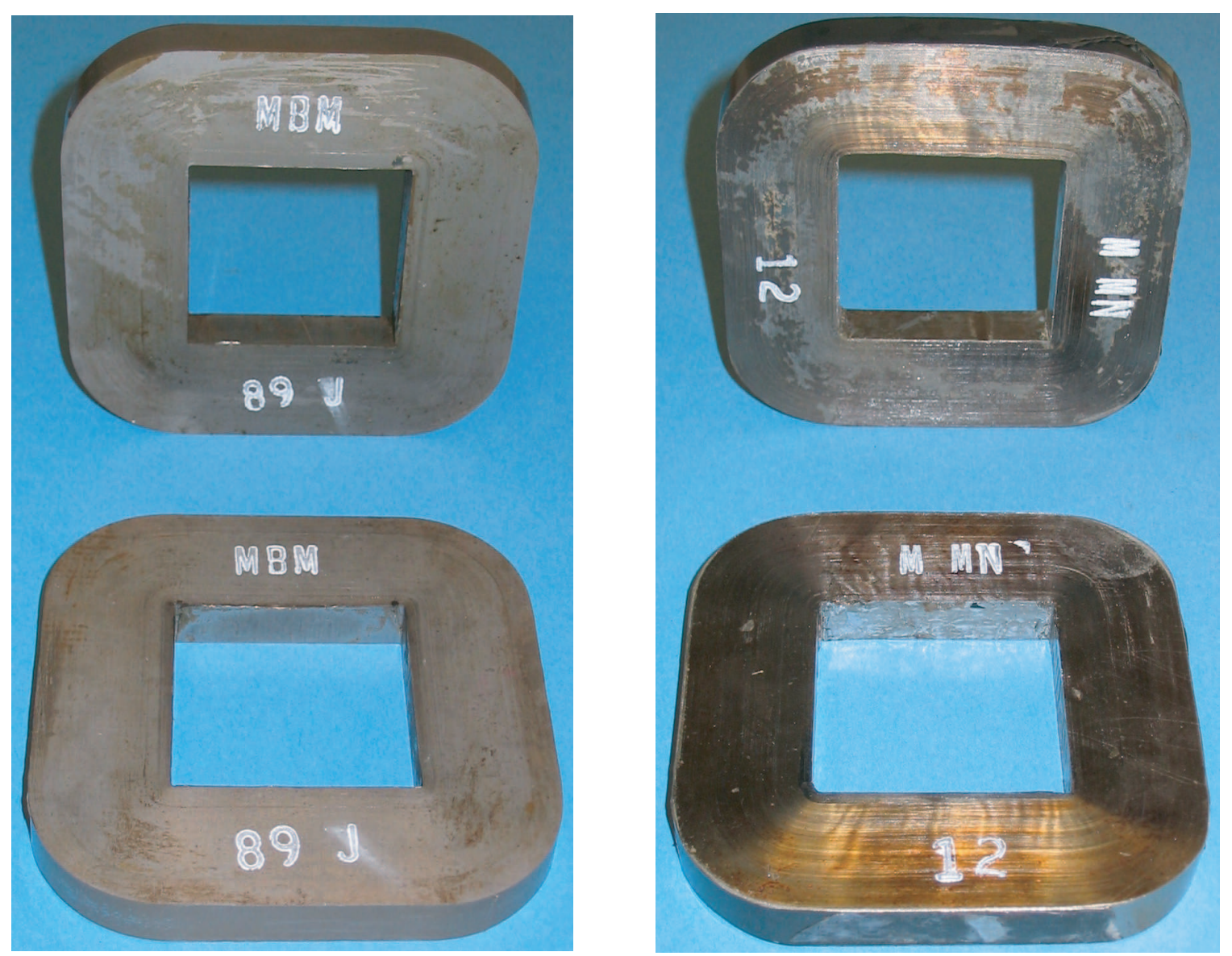

Figure 4-18: Wound-tape core blanks for the rotor. Conventional Ni-Fe (left), and nanocrystalline amorphous $\mathrm{Ni}-\mathrm{Fe}$ (right). Each core blank can provide four rotor cores. 

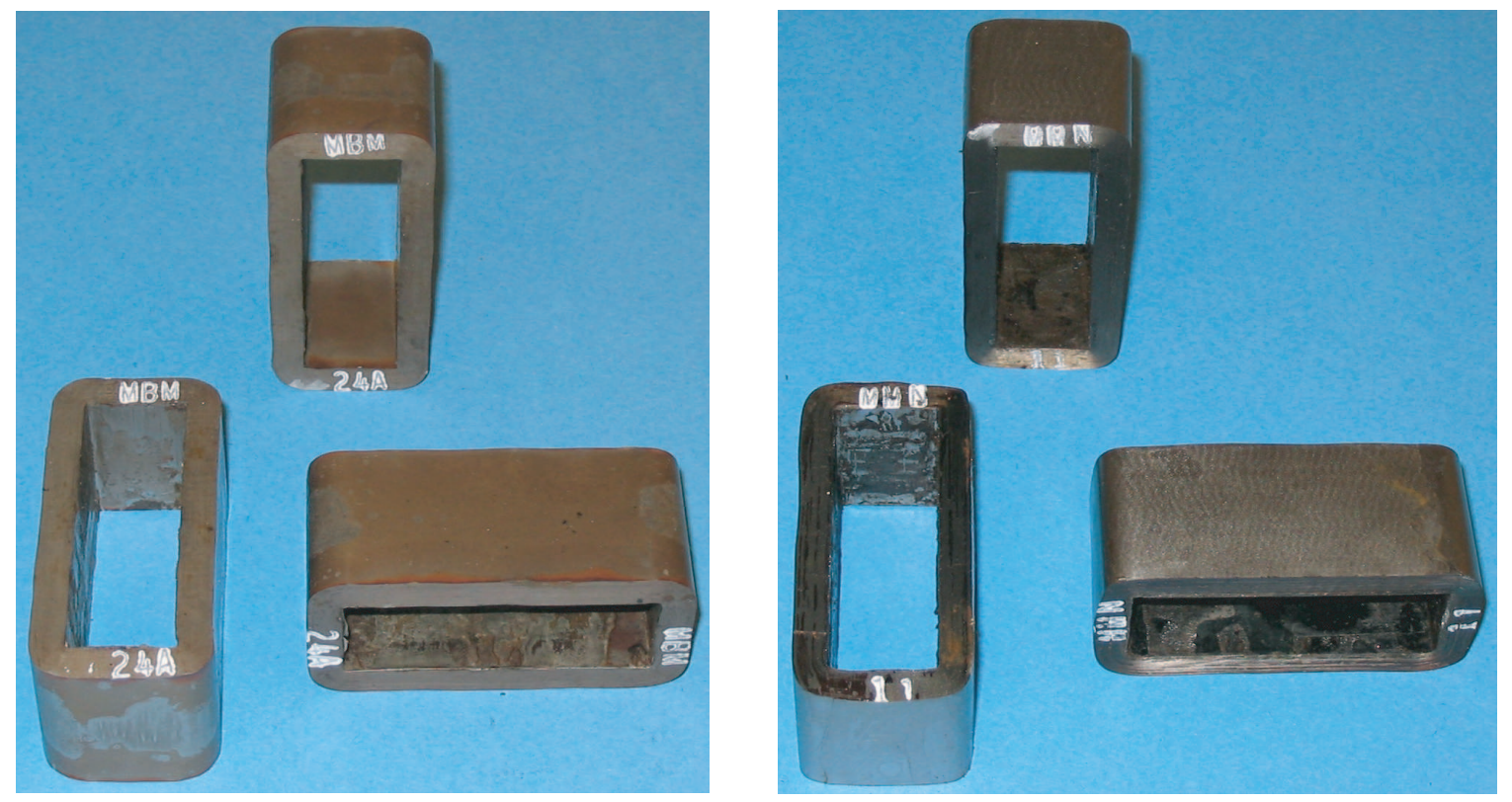

Figure 4-19: Wound-tape core blanks for the stators. Conventional Ni-Fe (left), and nanocrystalline amorphous Ni-Fe (right). Each core blank can provide two stator cores.

\subsubsection{Rotor}

The rotor with its tool arm and flexure bearing are shown in Figure 4-20. Figure 421 shows the corresponding mechanical model. The rotor core lamination layers are perpendicular to the axis of rotation. The rotor core is supported by stiffeners on its front and back, and by the flexure hubs at its top and bottom. The $10 \mathrm{kHz}$ FTS uses the same flexure design that proved successful with the earlier $2 \mathrm{kHz}$ FTS. The flexures are constrained at their inner ends by the flexure hubs and at the outer ends by the stator housing, and establish the axis of rotation for the rotor. Note that the two stiffeners and the two flexure hubs encircle the rotor core. Making the stiffeners from a ceramic material provides high stiffness and avoids producing a conducting loop with the aluminum flexure hubs that would produce eddy current power loss by linking the AC magnetic flux in the rotor core. The stiffeners are made of aluminum oxide, which has a coefficient of thermal expansion close to that of the rotor core.

The rotor core, stiffeners, and flexure hubs are joined together with an epoxy adhesive. All of the adhesive joints have a nominal bond-line thickness of $50 \mu \mathrm{m}$ except for the joints between the ends of the ceramic stiffeners and the flexure hubs, 
which have a nominal bond-line thickness of $500 \mu \mathrm{m}$. Since the epoxy undergoes an approximate $5 \%$ volumetric shrinkage upon curing, the greater shrinkage in the axial direction of the epoxy between the stiffeners and the flexure hubs, relative to the axial direction shrinkage between the rotor core and the flexure hubs, causes the stiffeners to be preloaded in tension and the rotor core to be preloaded in compression while the epoxy is curing. For an order-or-magnitude analysis I assumed that the epoxy undergoes tensile creep for stresses that are greater than $10 \%$ of its rated tensile lap shear strength, and then can sustain a tensile stress equal to that $10 \%$ value without significant subsequent creep. For the epoxy that I used to assembly the rotor, that $10 \%$ value has a lower limit of approximately $300 \mathrm{psi}$, which would produce a $23 \mathrm{lb}$ tensile force in each of the two stiffeners. Using those forces for an order-ofmagnitude analysis, the resulting compressive load on the lamination stack is $46 \mathrm{lb}$, or 130 psi, which produces a negligible axial strain of $0.01 \%$ in the lamination stack. The compressive preload on the rotor core is important because tensile stresses on the core in the axial direction, and especially cleavage stresses, could lead to delamination of the core. A discussion on the design of the adhesive bonded assemblies in the $10 \mathrm{kHz}$ FTS, with details on the adhesives used, is provided in Section 4.5.10.

One of my colleagues in the Precision Motion Control Lab [7] at MIT, Xiaodong $\mathrm{Lu}$, pointed out an interesting "self-protecting" feature of the rotor. During use, magnetic losses in the rotor core will make it the predominant heat source for the rotor. Since the CTE of the stiffeners and rotor core are approximately equal, the rotor core will tend to grow more than the stiffeners, and put the lamination stack into compression.

The dimensions of the rotor core are determined by a trade-off analysis driven by closed-form equations relating those dimensions and the matching dimensions of the stiffeners, flexure hubs, and stator cores to the power losses in the rotor and stator cores, reactive electrical power required to drive the AC flux in the air gaps, current density and cooling requirements for the AC coils, rigid body and non-rigid body first modes of the rotor, and tool acceleration produced by a 1.5 Tesla peak flux density. Finite element analysis models were used to determine the frequency of the first non- 
rigid body modes for a selection of rotor geometries to provide a bounding analysis in the design space, and agree with the closed-form equations. Also folded into the tradeoff is the attractiveness of using the same design for the flexure mounting hardware that proved successful in the earlier $2 \mathrm{kHz}$ FTS. A detailed discussion on the trade-off analysis is provided in Section 4.8 .

Selected steps from the process of bonding the rotor core, ceramic stiffeners, and flexure hubs are shown in Figures 4-22 through 4-25. Note the use of the thread-like $50 \mu \mathrm{m}$ shims used to control the bond-line thickness for most of the joints. Bond-line shims in the radial direction between the flexure hubs and the stiffeners are built into the flexure hubs. Figures 4-26 and 4-27 show the flexure blades being mounted to the fully assembled rotor.

\subsubsection{Stator}

The stator consists of two identical halves that contain the stator core and steering flux coils, and together form the base structure for the $10 \mathrm{kHz}$ FTS. One of the two stator halves is shown in Figure 4-28. Figure 4-29 shows the corresponding mechanical model with a cut-out revealing the major components. Referring to Figure 4-29, the stator core is the central C-shaped structure (green). The laminations near the stator pole faces are supported by the stator core braces (pink). The center brace is the component with the cylindrical cut-out in it, and the two outer braces are the Tshaped components. Note that each outer brace has an extension at its top and bottom that partially wraps around the stator cores. Those extensions form part of the mechanical hard stops between the rotor and stator by engaging the wing-like extensions on the flexure hubs. The two steering flux coils (red) lie just behind the stator pole braces. The component behind the stator core is a terminal piece of the back-iron that carries bias flux to the stator core. The stator core housing is the large component (cyan) that all of the aforementioned components are mounted to. Referring to Figure 4-15, note the arrangement of the rotor and back-iron relative to the two stator halves.

The stator housing and stator core braces are made from non-magnetic 303 stain- 


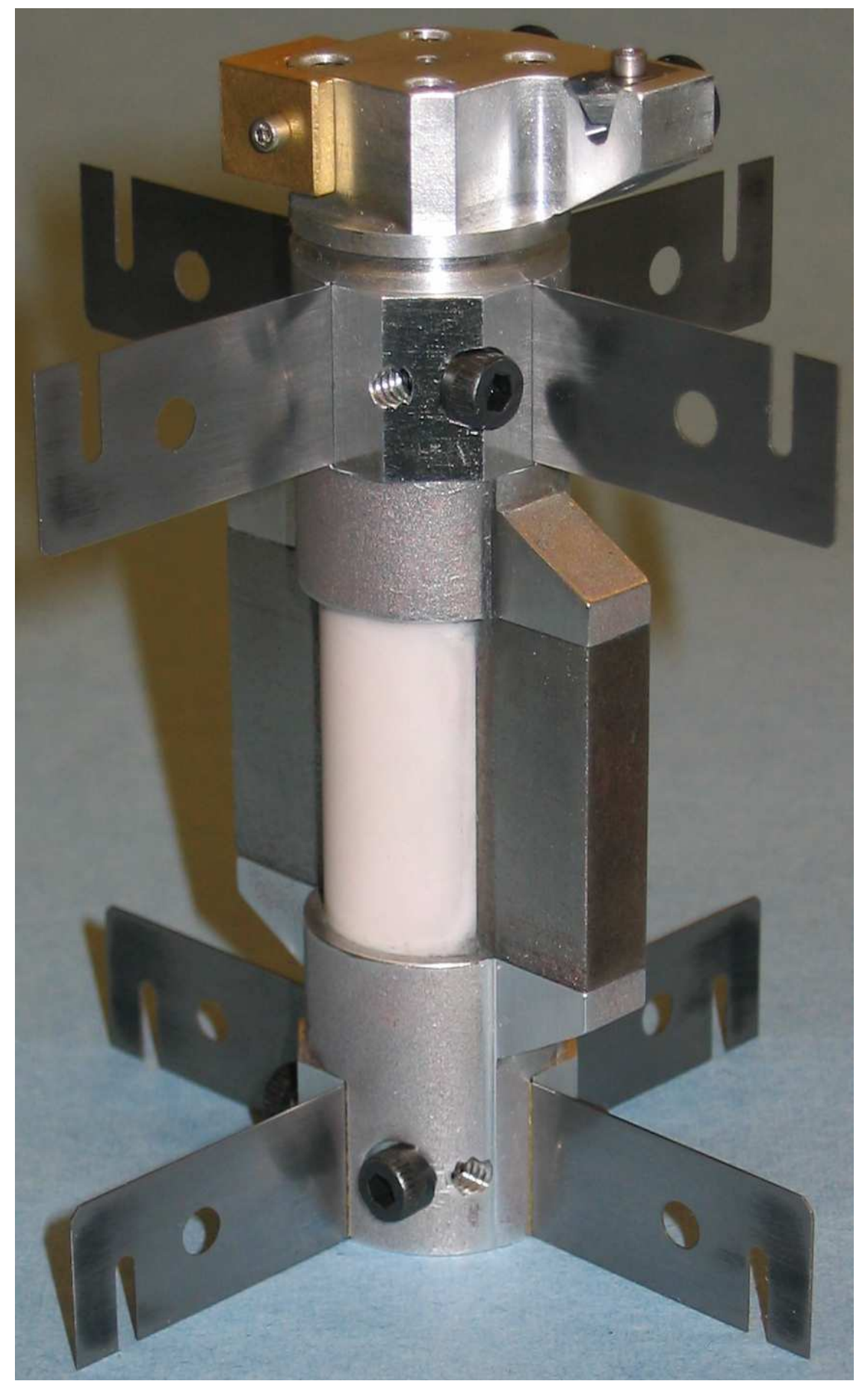

Figure 4-20: The rotor with its tool arm and flexure bearing for the $10 \mathrm{kHz}$ FTS. 

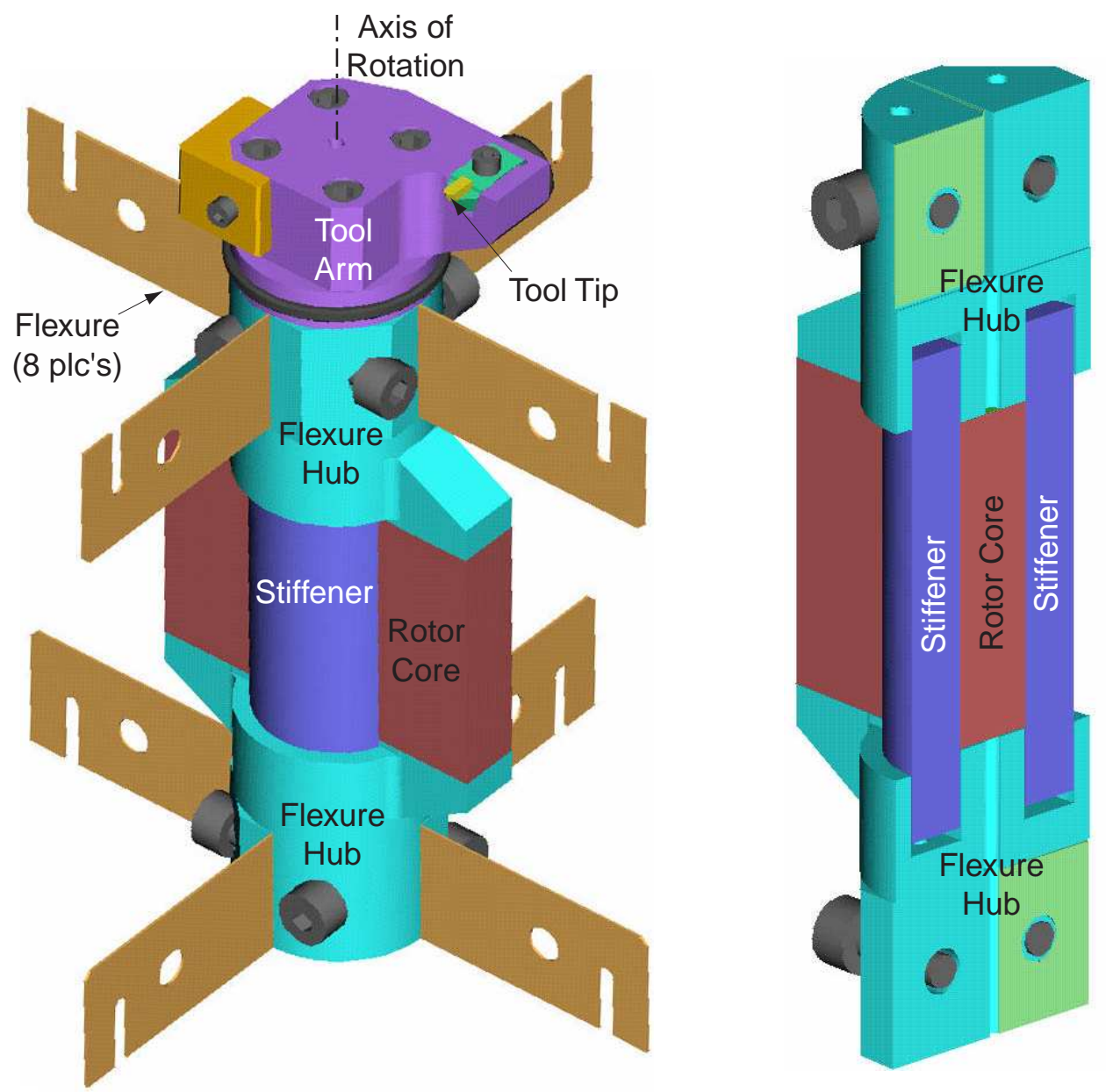

Figure 4-21: Model of the $10 \mathrm{kHz}$ FTS rotor with its tool arm and flexure bearing (left), and a cross-section of the rotor (right). 


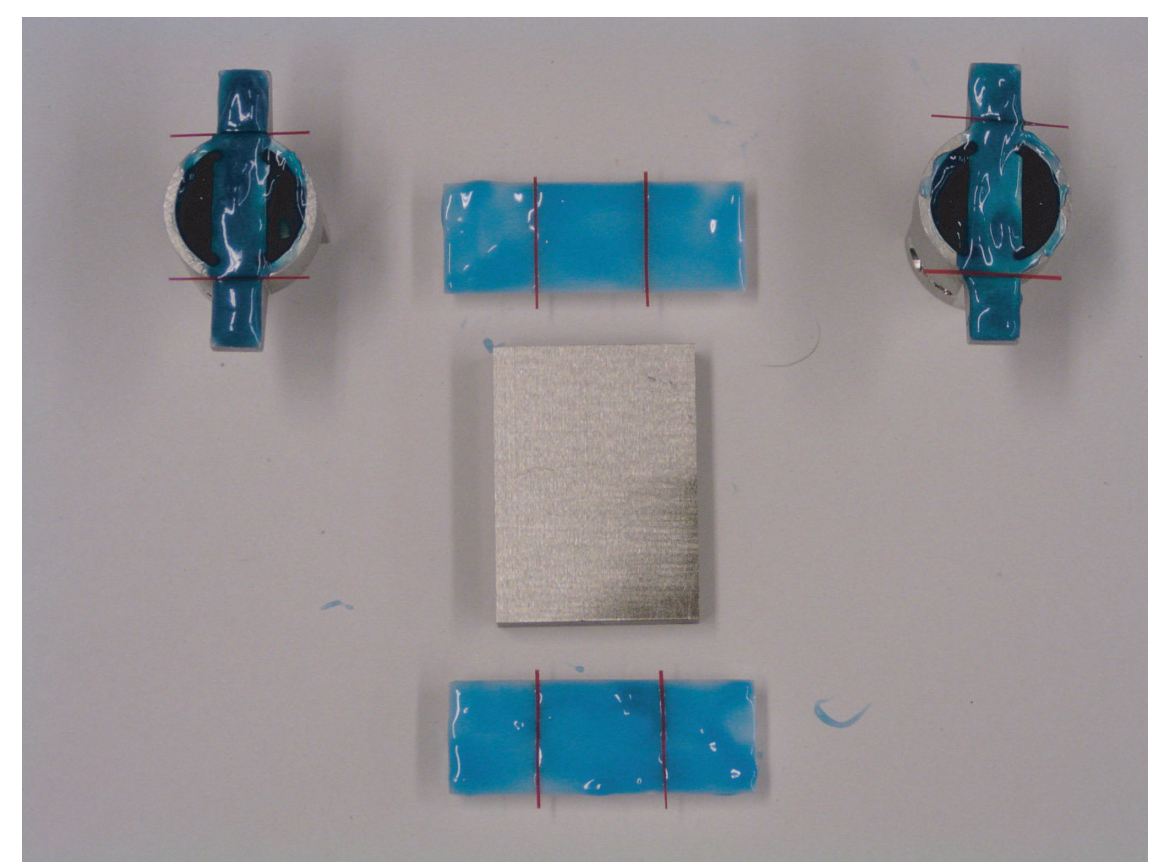

Figure 4-22: The rotor core, ceramic stiffeners, and flexure hubs for the $10 \mathrm{kHz}$ FTS, with epoxy applied to the surfaces to be bonded together.

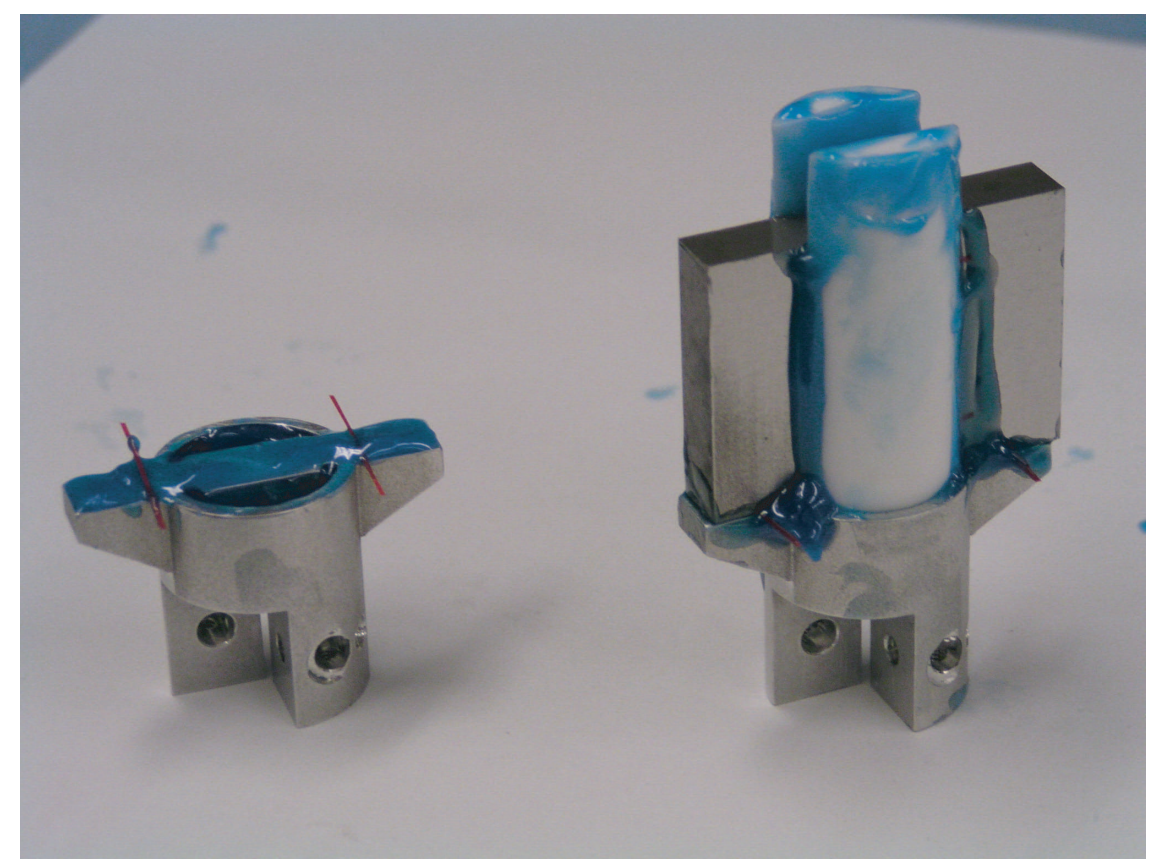

Figure 4-23: Partial assembly of the rotor core, ceramic stiffeners, and flexure hubs for the $10 \mathrm{kHz}$ FTS. 


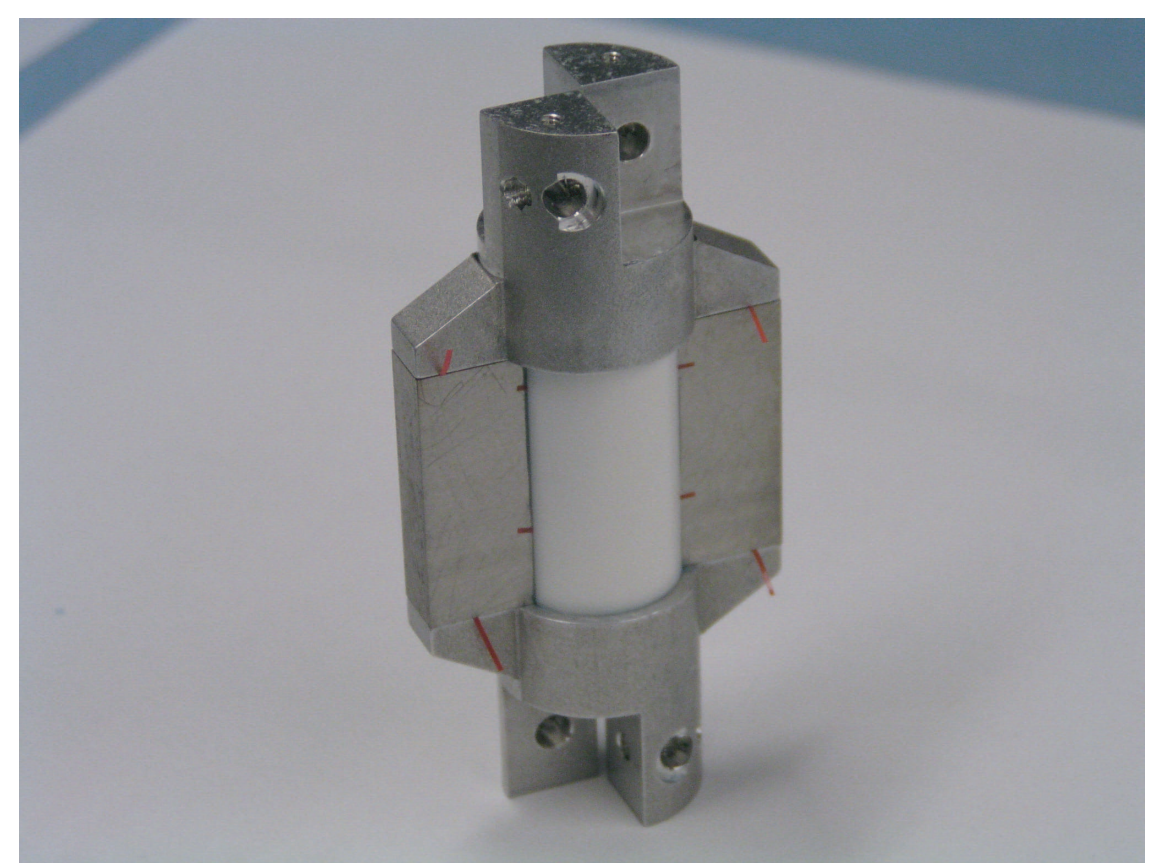

Figure 4-24: Full assembly of the rotor core, ceramic stiffeners, and flexure hubs for the $10 \mathrm{kHz}$ FTS, after cleaning off the excess epoxy adhesive.

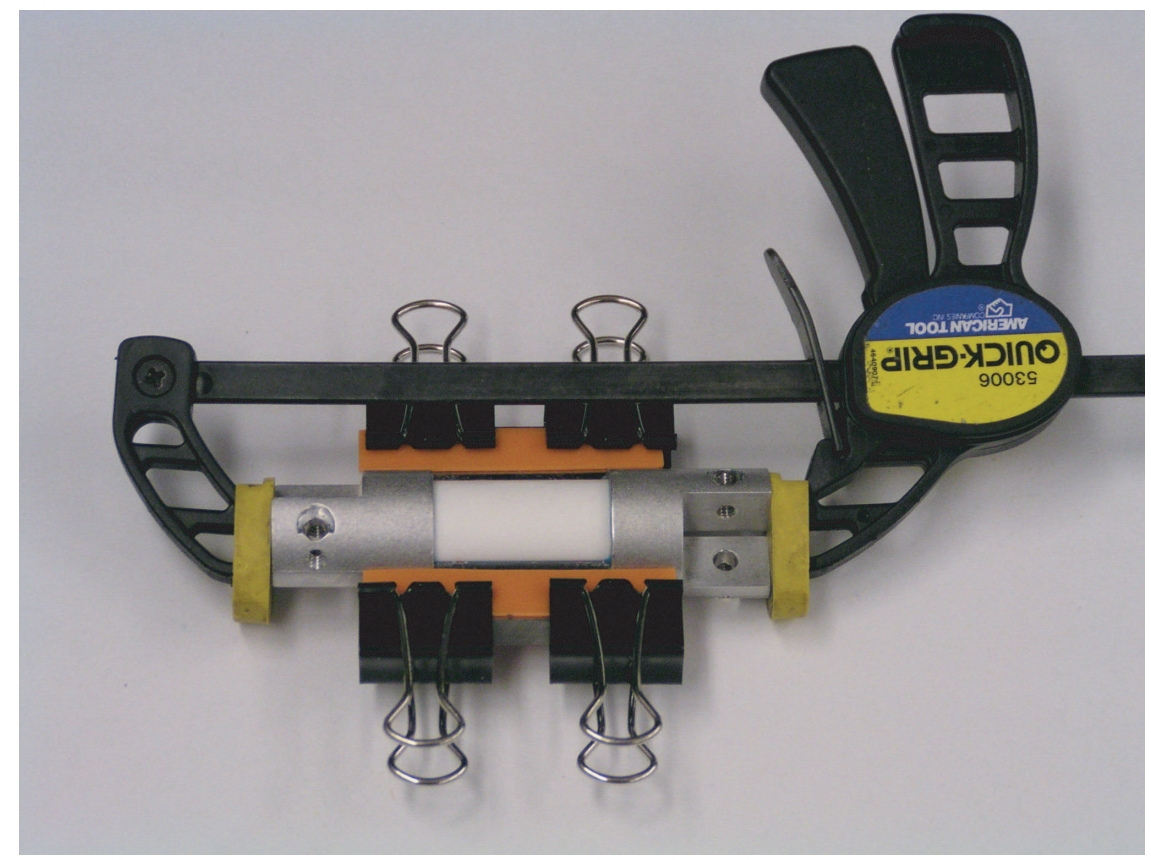

Figure 4-25: Clamping of the full assembly of the rotor core, ceramic stiffeners, and flexure hubs for the $10 \mathrm{kHz}$ FTS, during cure of the epoxy adhesive. 


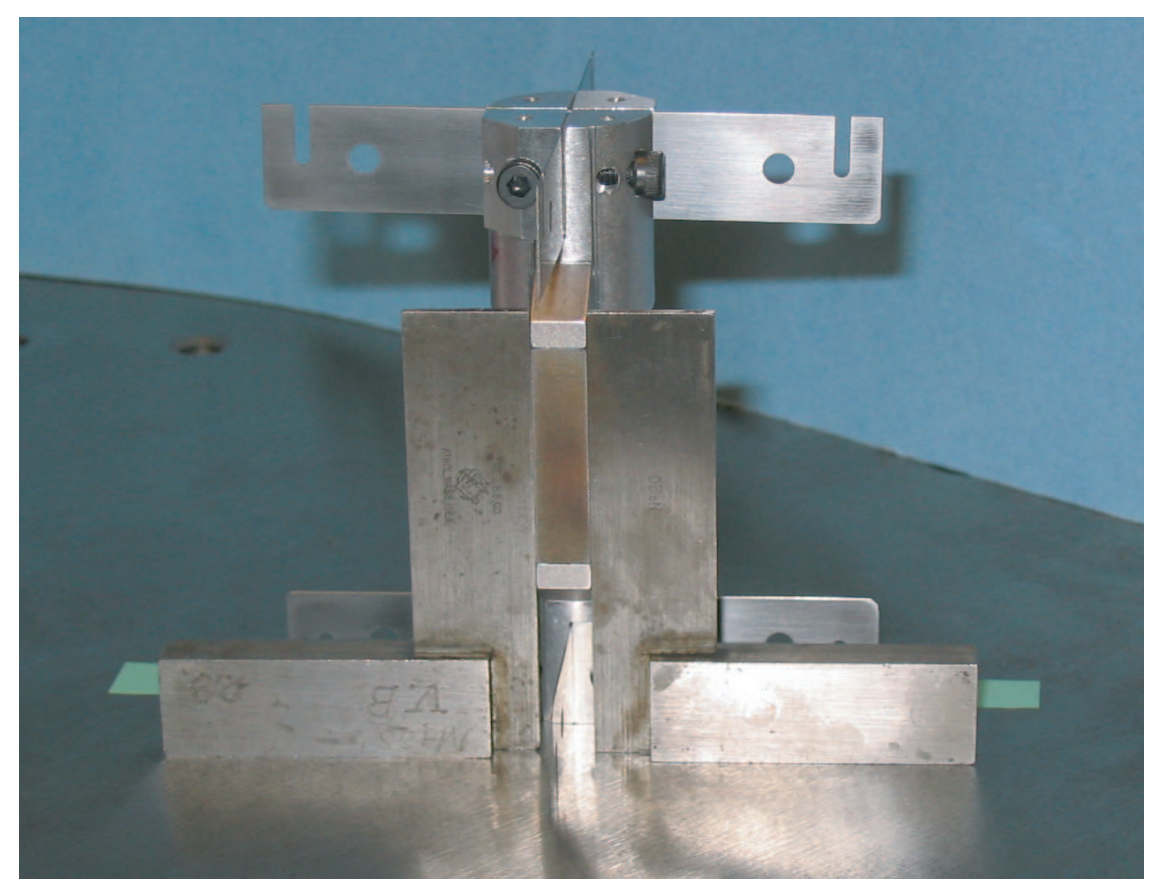

Figure 4-26: Mounting the flexure blades to the rotor: setting the front/back blades perpendicular to the rotor pole faces.

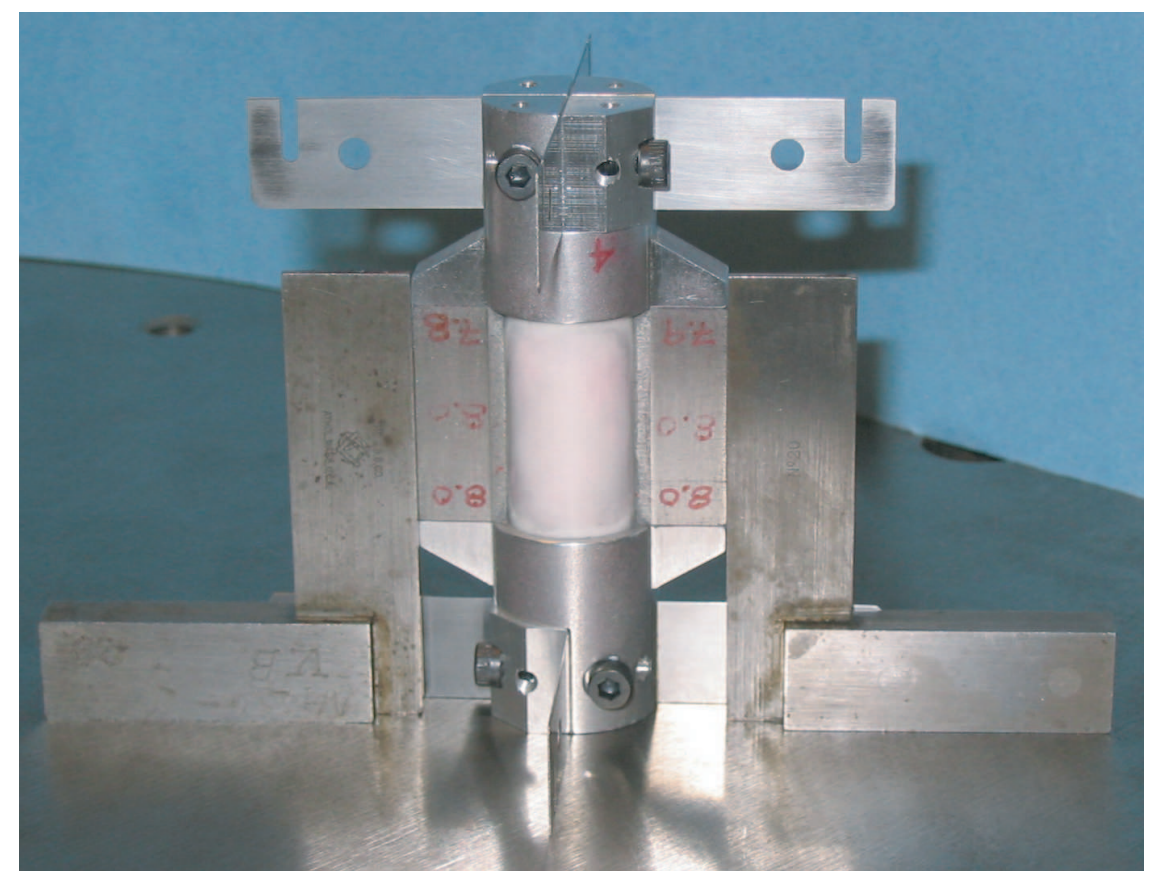

Figure 4-27: Mounting the flexure blades to the rotor: setting the right/left blades perpendicular to the sides of the rotor core. 
less steel with $\mu_{r}=1.02$ [58]. It is worth noting that work-hardening this type of steel appears to slightly increase its magnetic permeability, which should be considered during machining. I chose stainless steel because it allows grinding the critical surfaces to obtain the final flatness, geometry, and alignment of the parts.

The components in each stator half are predominantly joined together with an epoxy adhesive and an epoxy potting compound. Details on the epoxies used are provided in Section 4.5.10. Selected steps from the process of assembling a stator half are shown in Figures 4-30 through 4-38.

In Figure 4-30 the stator housing has been prepared to receive the stator core. A puddle of uncured epoxy lies at the back of the housing, and the outer stator core braces (labelled "1L" and "1R") are temporarily mounted to act as fixtures to locate the stator core.

In Figure 4-31 a fixture consisting of a non-magnetic plate and three permanent magnets is used to register the stator pole faces relative to the front face of the stator housing while the epoxy cures. Figure 4-32 shows the addition of a clamp used to firmly hold the stator pole faces against the fixture during curing of the epoxy. The bottom half of the clamp presses against a rubber plug that passes through the back of the stator housing and contacts the back of the stator core.

Figure 4-33 shows the two steering coils being dry-fitted to the stator core and housing. Figure 4-34 shows the steering coils being bonded to the stator core and housing using a pool of clear potting compound.

In Figure 4-35 the stator core and the cured potting compound have been prepared to receive the inner and outer stator core braces. Uncured epoxy has been applied to the sides of the stator poles and on top of the potting compound. Shims (orange and white) are used to establish the height of the braces relative to the front face of the stator housing.

Figure 4-36 shows the fully assembled stator half before final grinding. Figure 437 shows the two stator halves being match-ground with a surface grinder. This operation established the final flatness of: the front faces of the stator housings, the stator pole faces, and the stator pole braces. It also established the offset distance 


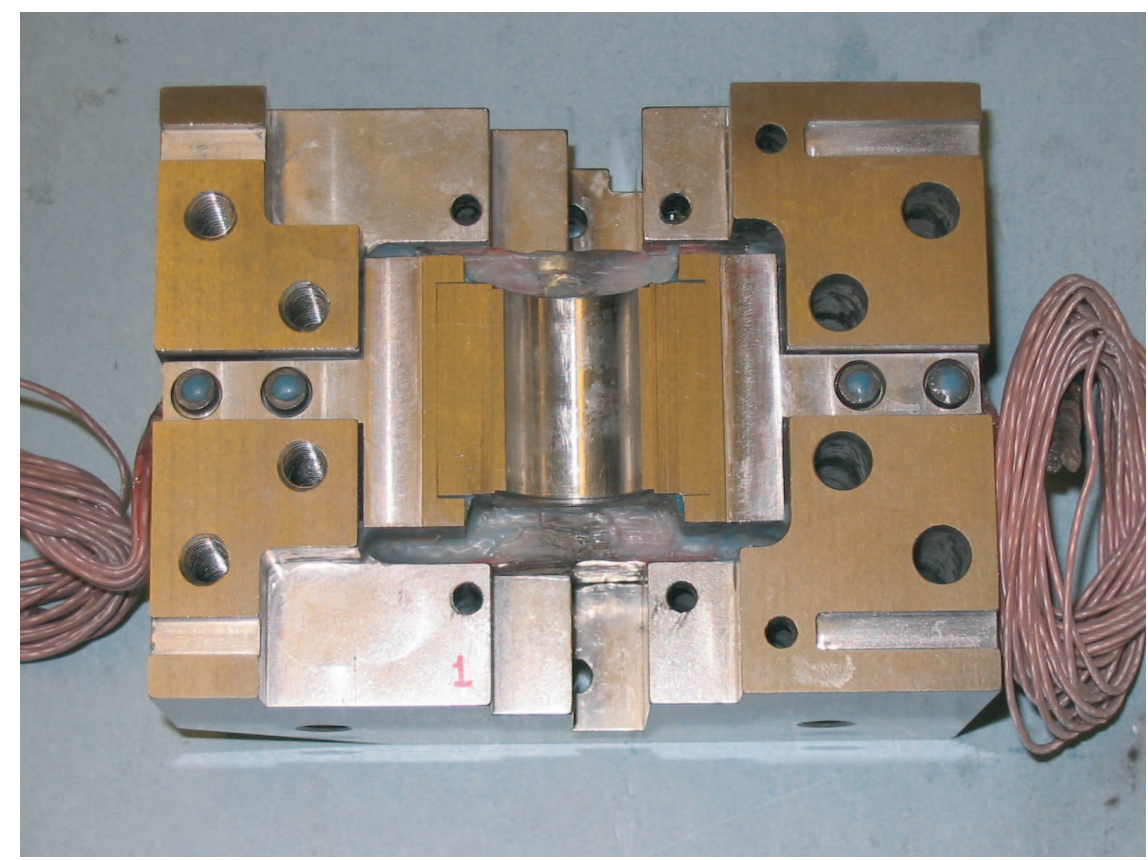

Figure 4-28: One of the two fully assembled stator halves for the $10 \mathrm{kHz}$ FTS.

between the stator pole faces and the front faces of the stator housings, which was a key step in establishing the four $50 \mu \mathrm{m}$ rotor/stator air gaps and the mounting surfaces for the outer ends of the flexure blades.

In Figure 4-38 the terminal piece of the back-iron is being fitted to the fully assembled stator half. The back of the stator core is visible through the central hole in the stator housing. Note the rectangular shim (gold) with the rectangular cut-out that is used to establish the back-iron air gap length.

\subsubsection{Mounting the Rotor to the Stator}

Figure 4-39 shows a model of the rotor mounted to one of the stator housings via the outer ends of four of the eight flexure blades. During an earlier fabrication step the flexure mating surfaces on the front of the stator housings and the stator pole faces were ground flat and parallel to each other. During assembly of the rotor to the stator, shims are used between the ends of the flexure blades and the stator housings to accommodate any stack-up of manufacturing and assembly errors. The other stator half is added to what is shown in Figure 4-39 to complete the magnetic circuit and 


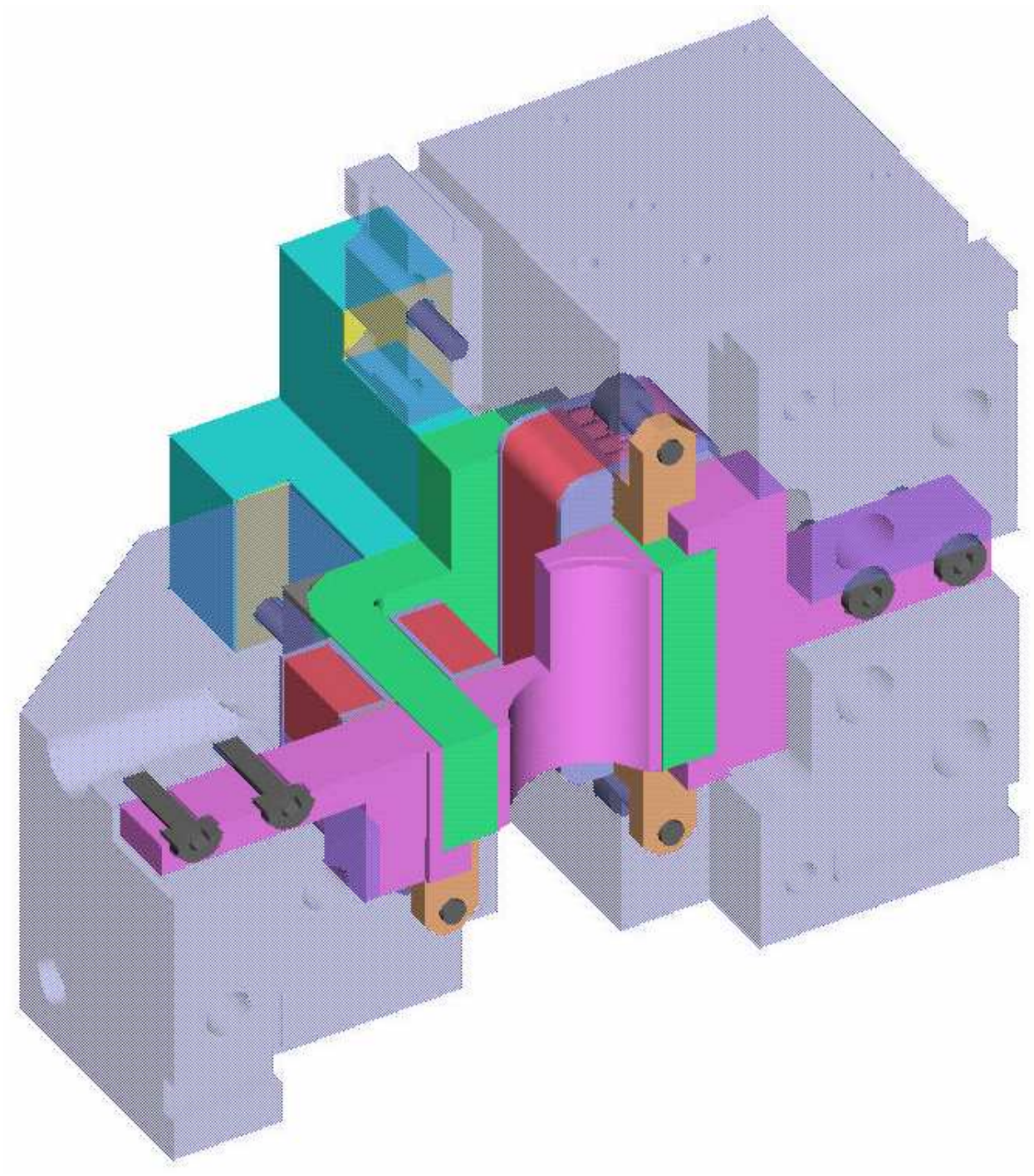

Figure 4-29: Mechanical model of a stator half for the $10 \mathrm{kHz}$ FTS with a cut-out revealing the major components. 


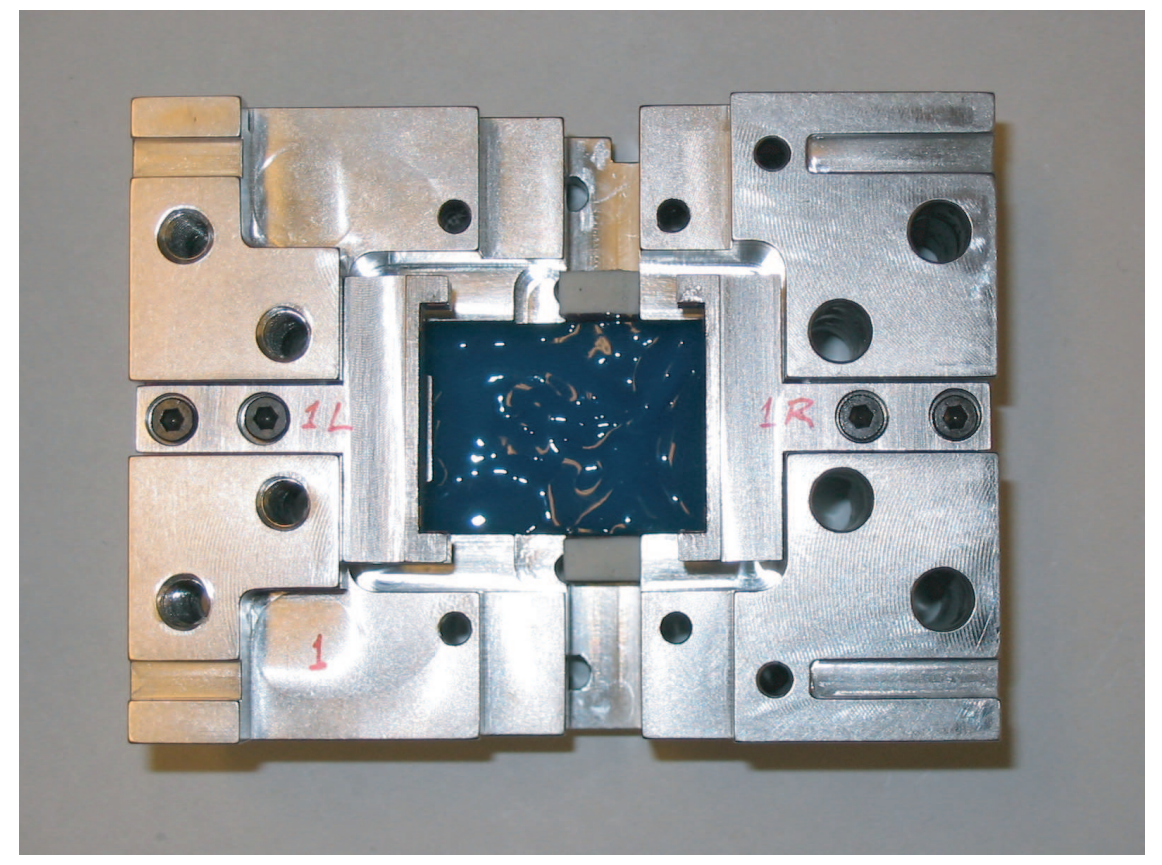

Figure 4-30: Stator housing prepared with epoxy to receive the stator core.

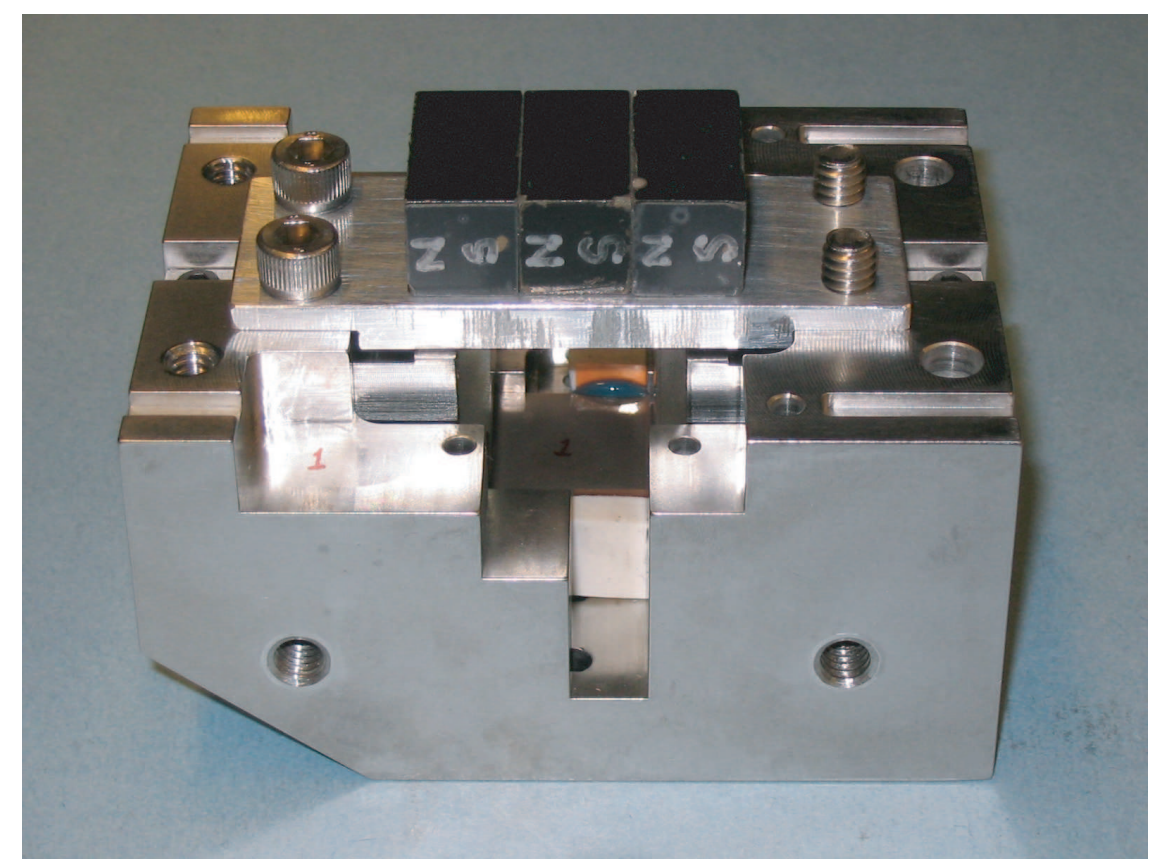

Figure 4-31: Bonding fixture used to register the stator pole faces relative to the front face of the stator housing. 


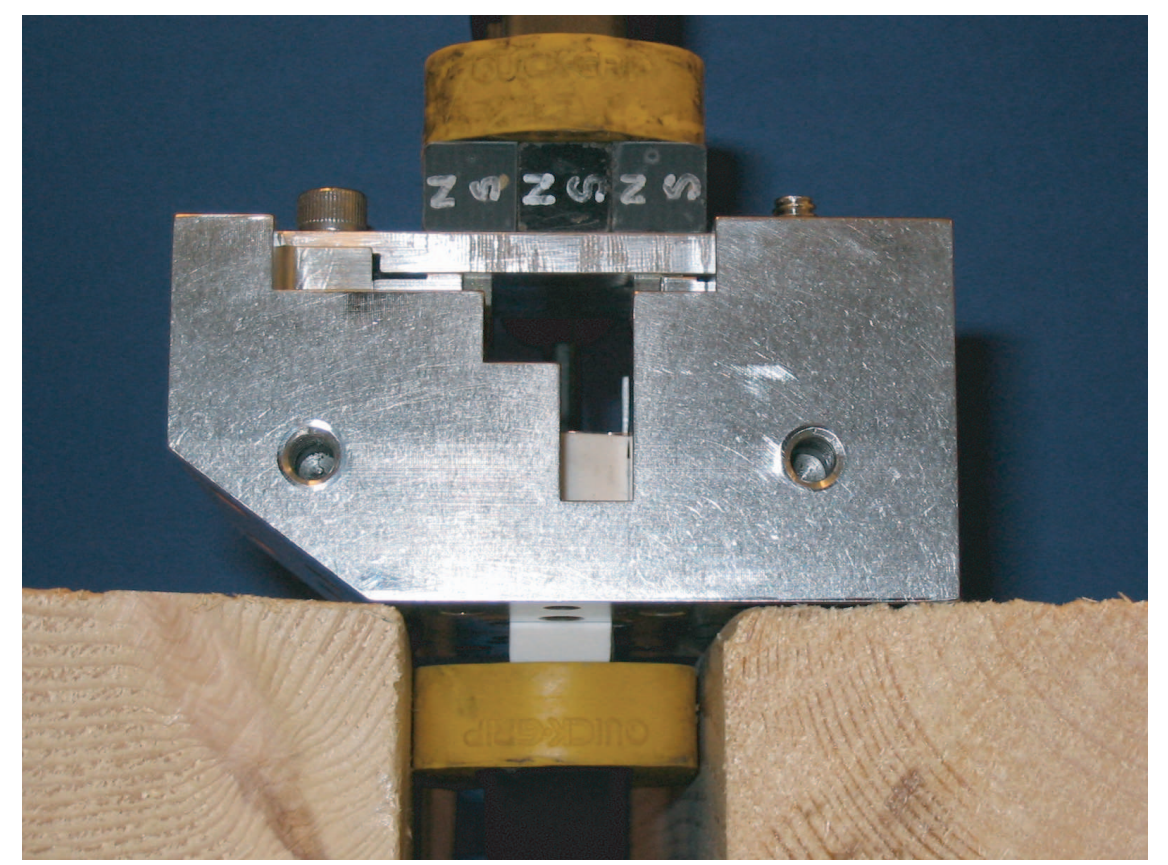

Figure 4-32: Clamping the stator core against the bonding fixture.

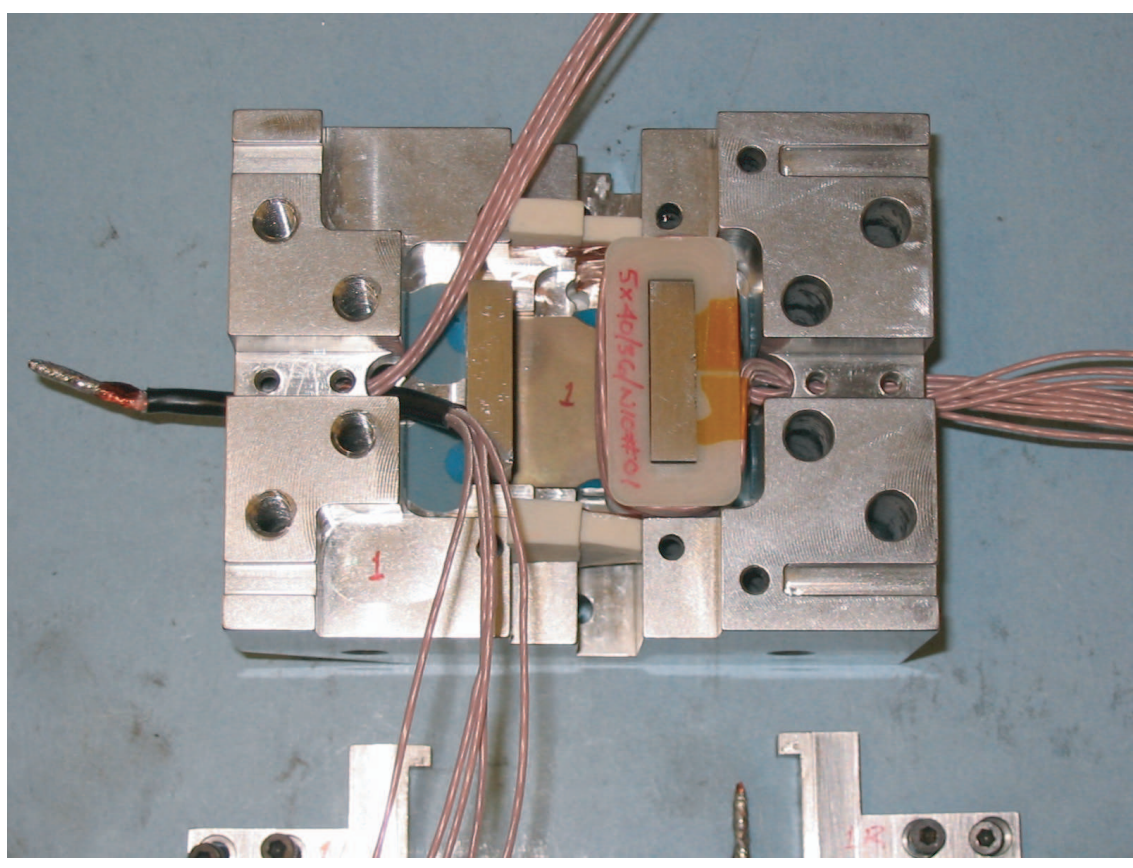

Figure 4-33: Steering coils being dry-fitted to the stator core and housing. 


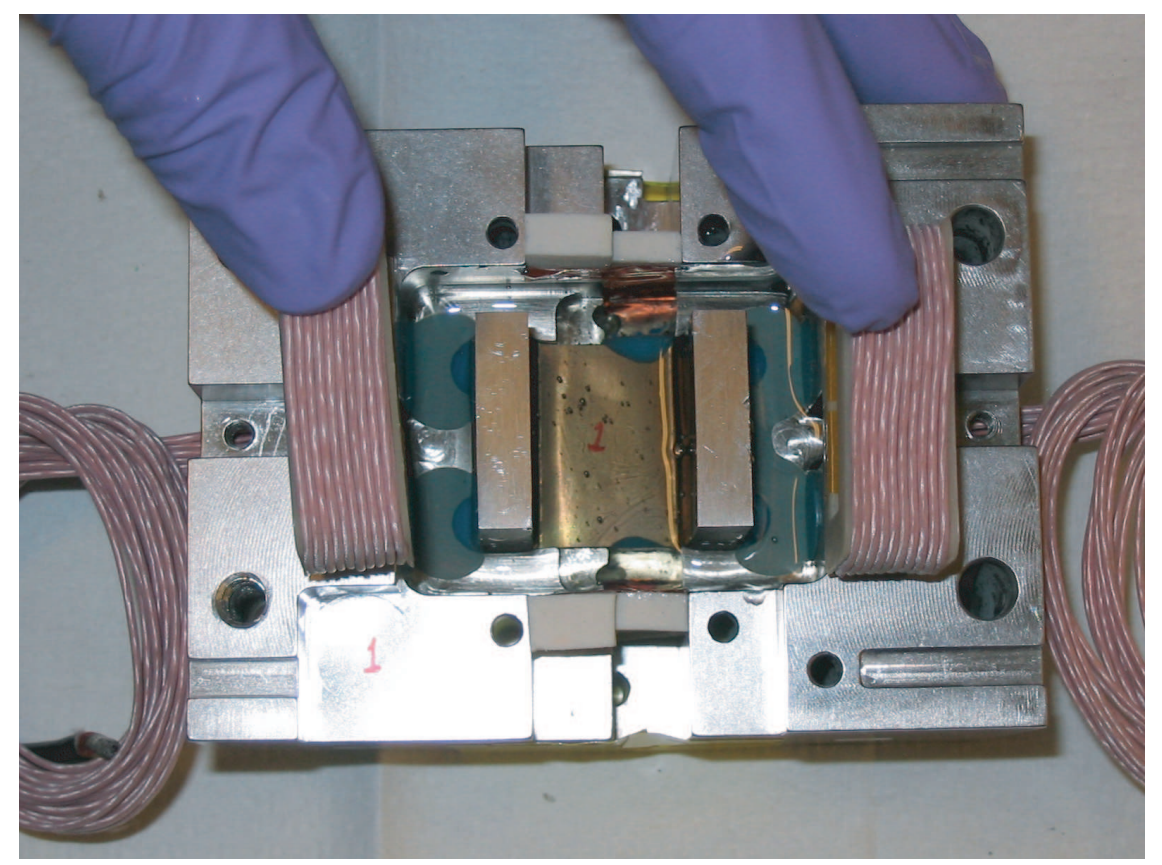

Figure 4-34: Steering coils being bonded to the stator core and housing with a potting compound.

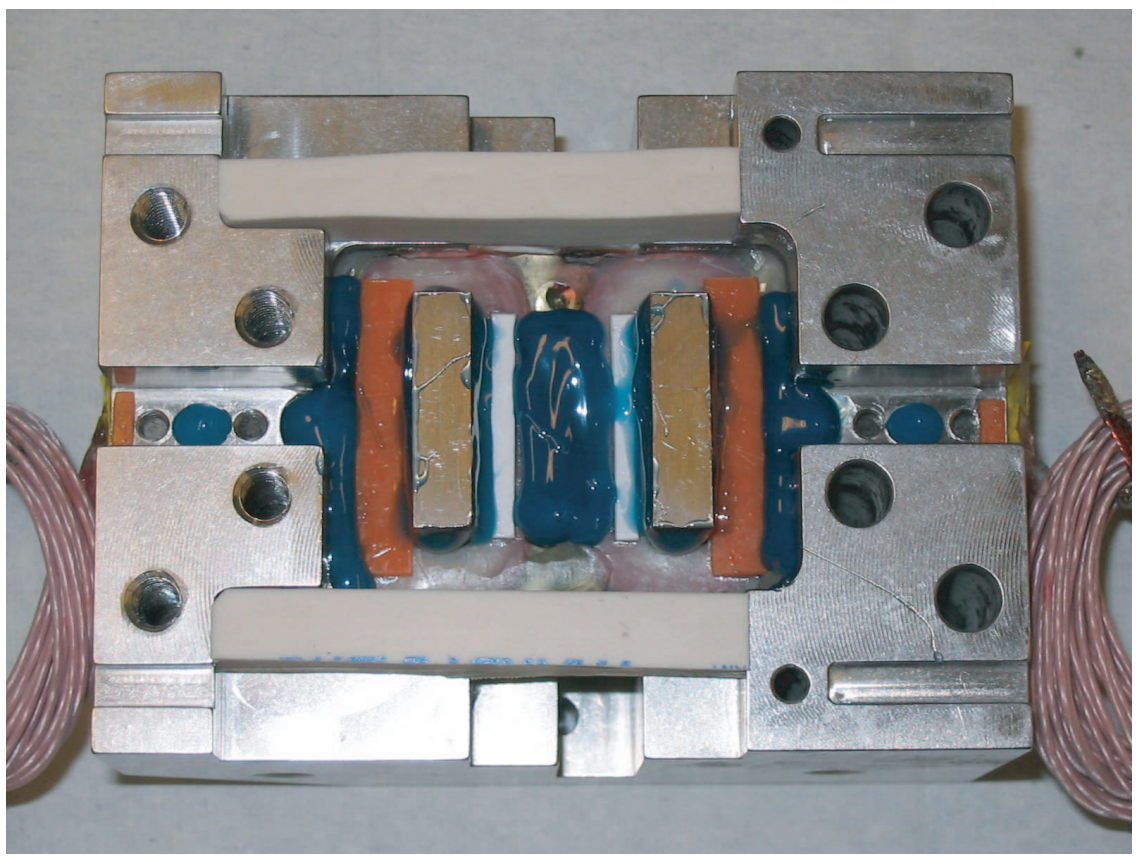

Figure 4-35: Preparing the stator core and cured potting compound to receive the inner and outer stator core braces. 


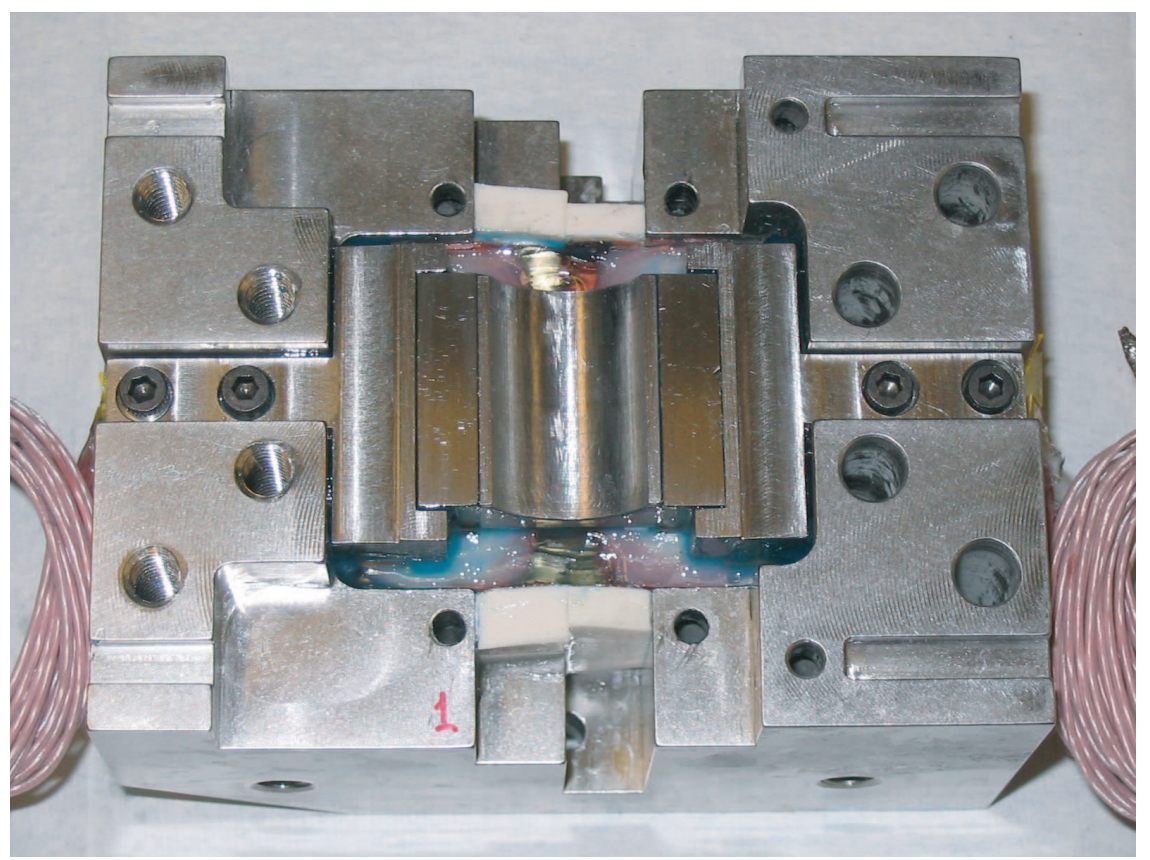

Figure 4-36: A fully assembled stator half before final grinding.

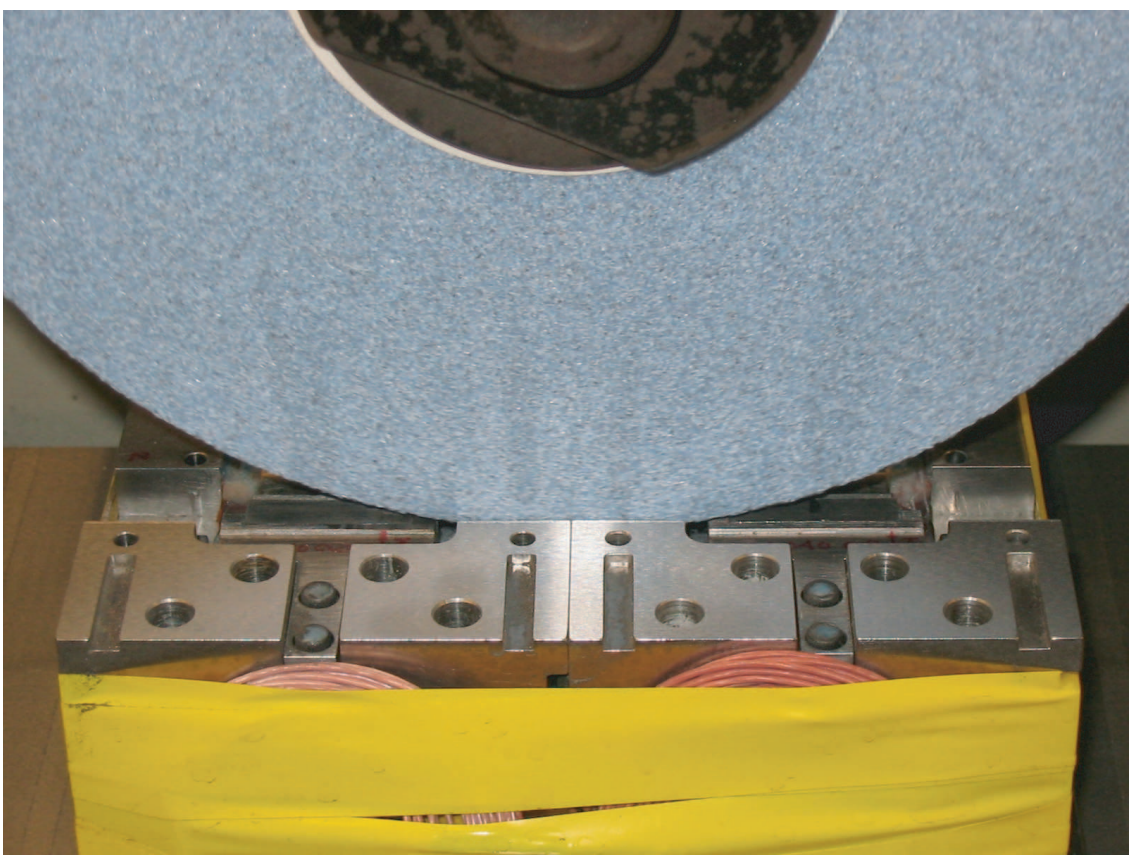

Figure 4-37: The two stator halves being match-ground with a surface grinder. 


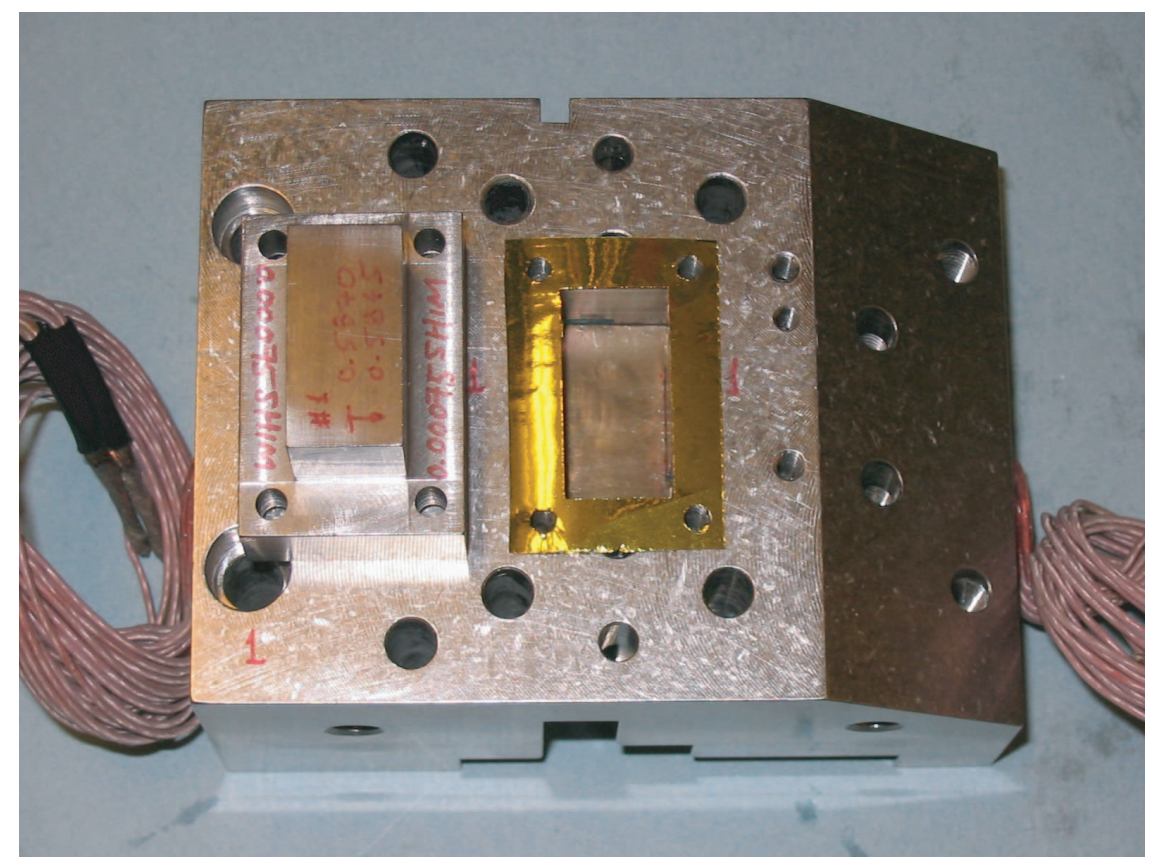

Figure 4-38: Fitting a terminal piece of back-iron to a fully assembled stator half.

provide a stiff support structure for the rotor; essentially encasing the rotor in a solid block of metal with openings provided for the stator cores, steering flux coils, flexures, and the flow of a cooling fluid around the rotor.

Figure 4-40 shows the two stator halves with the rotor aligned and partially attached to one of them. Note the two $50 \mu \mathrm{m}$ plastic shims (red) between the rotor pole faces and the stator poles faces in the left-hand stator half. A second set of these shims are placed between the other two rotor pole faces and the stator pole faces in the right-hand stator half when the two stator halves are brought together. The brass shims on the right side of the front face of each stator housing establish the nominal spacing between the two stator halves.

Figure 4-41 shows the two stator halves and the rotor after the stator halves are aligned and attached to each other. Feeler gauges were used to measure the thickness required for each shim that was placed between the ends of the flexure blades and the stator housings. Before clamping the flexure blades to the stator housings, the pre-tensioning devices visible in Figure 4-41 were adjusted to provide a nominal $50 \mathrm{~N}$ tensile preload on the flexures. These pre-tensioning devices follow the same design 


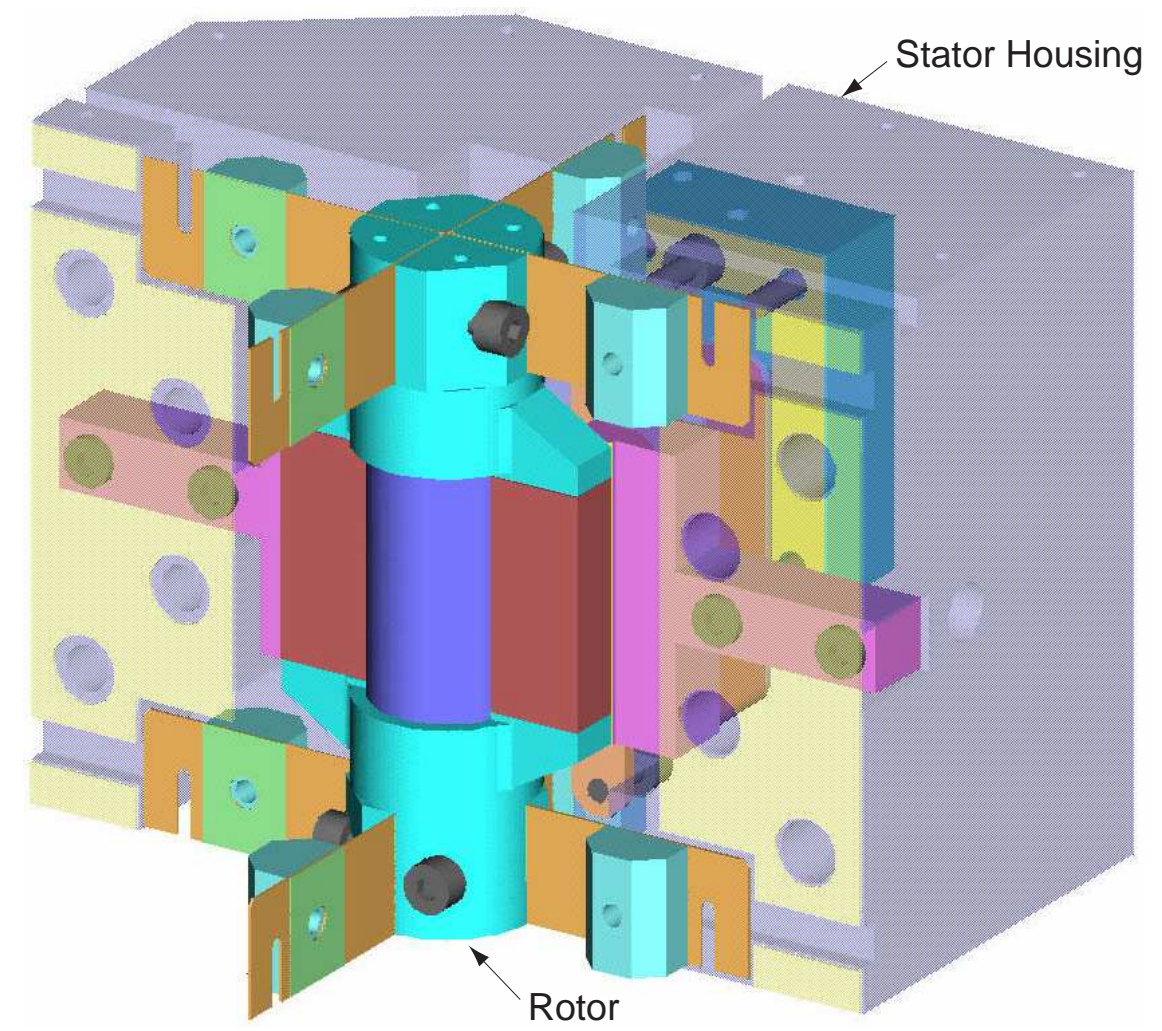

Figure 4-39: Model of the rotor mounted via the flexure blades to one of the two stator halves.

that was used for the $2 \mathrm{kHz}$ FTS, which is described in Section 3.6.3.

Figure 4-42 is a back-lit photo of the fully assembled rotor and stator, after removal of the $50 \mu \mathrm{m}$ plastic shims that were in the four rotor/stator air gaps. The air gaps in Figure 4-42 appear longer than $50 \mu \mathrm{m}$ because the camera was focused on the top of the stator housing and also because of internal reflections of light on the pole faces. Measurements of the four air gap lengths with feeler gauges indicated that they were all in the range of 45 to $50 \mu \mathrm{m}$, which is within $10 \%$ of the nominal $50 \mu \mathrm{m}$ length. Electrical measurements discussed in Section 4.7.4 indicate that the bias flux in the four air gaps is matched to within $7 \%$ when the rotor is in its neutral position, suggesting that the lengths of the air gaps are matched to within $7 \%$. 


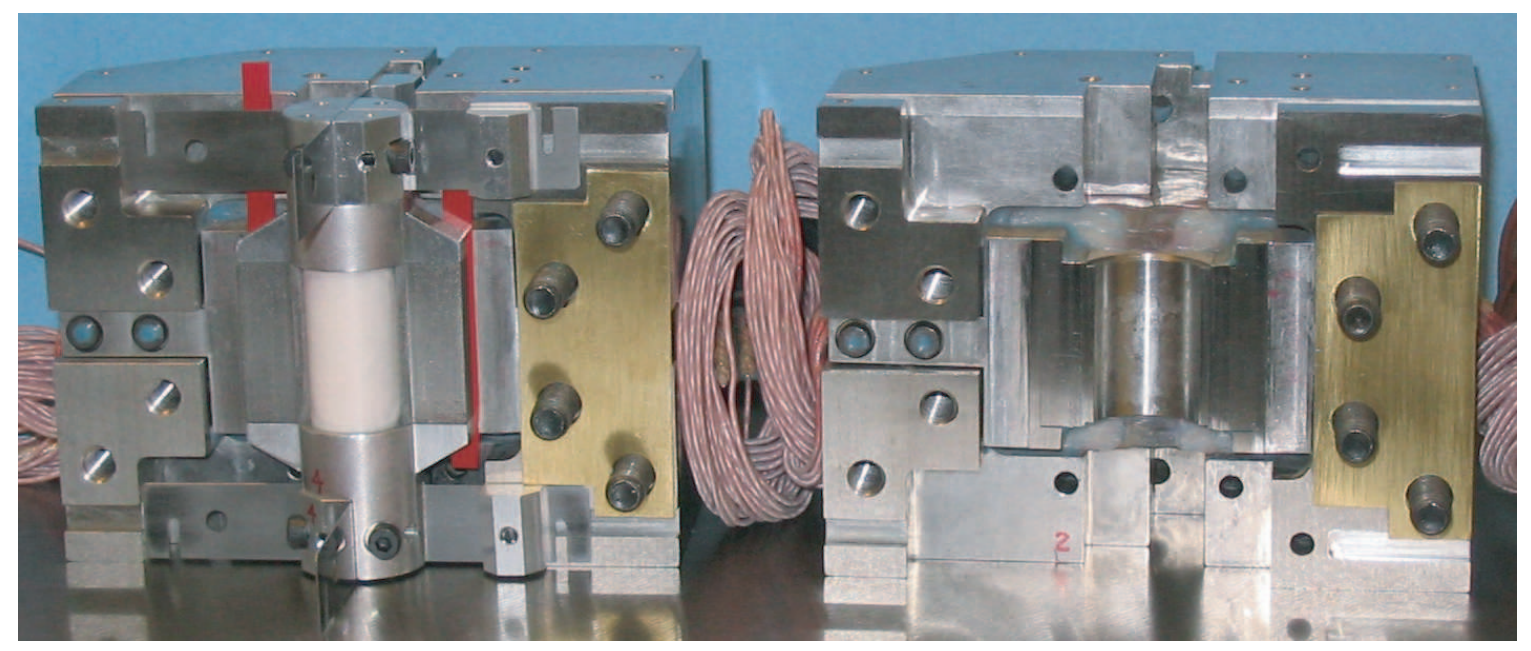

Figure 4-40: The two stator halves with the rotor aligned and partially attached to one of the stator halves.

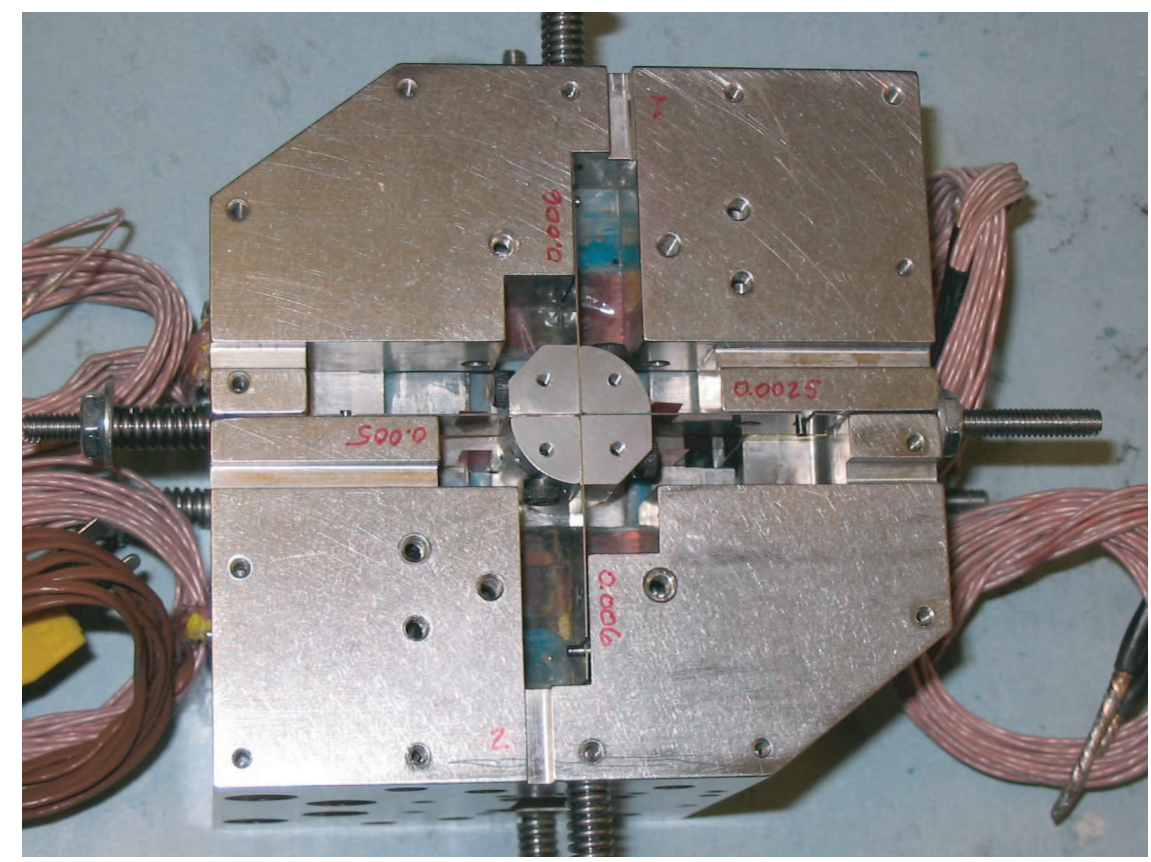

Figure 4-41: The two stator halves and the rotor after attaching the stator halves together. 


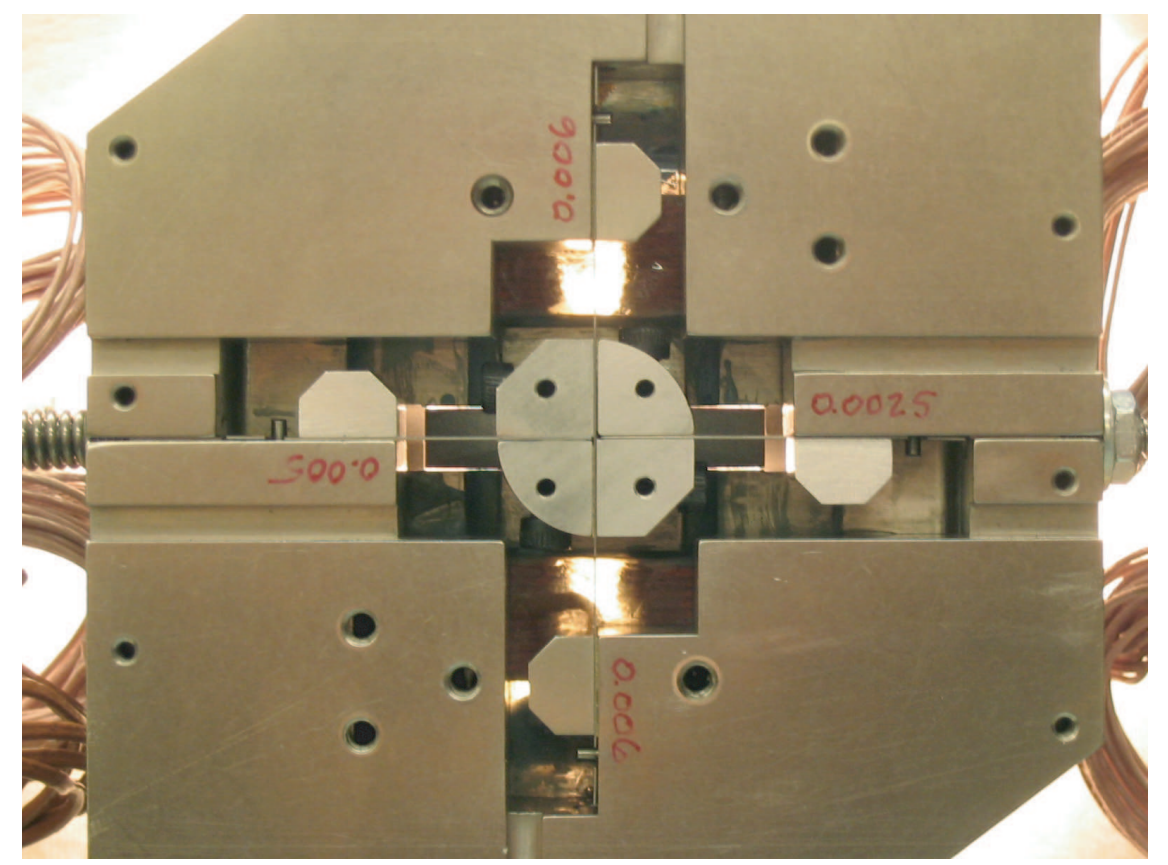

Figure 4-42: A back-lit photo of the fully assembled rotor and stator showing the four $50 \mu \mathrm{m}$ rotor/stator air gaps.

\subsubsection{Tool Holder}

The $10 \mathrm{kHz}$ FTS uses the same tapered-shank tool with four-point contact that was developed for the $2 \mathrm{kHz}$ FTS, and is discussed in detail in Section 3.6.1. Figure 4-43 shows the tool holder for the $10 \mathrm{kHz}$ FTS. Note the use of a single screw to clamp the tool shank into the receiving slot, which is a simpler and lower mass method than was used with the $2 \mathrm{kHz}$ FTS. The brass counterweight on the left side was designed to provide two-plane balance of the tool holder. ${ }^{2}$ The two O-rings surround the target surfaces for the capacitance sensors that provide displacement feedback for the $10 \mathrm{kHz}$ FTS. The O-rings seal against the face of the capacitance sensors, and prevent debris from entering into the sensing area.

\footnotetext{
${ }^{2}$ Two-plane balance: the center of gravity lies on the centerline of the tool holder, and the centers of gravity for any pair of lumped masses that are on opposite sides of the centerline both lay in a plane that is normal to the centerline. If this last condition is not met, rotation will produce a torque around an axis that is perpendicular to the centerline and rotates with the tool holder.
} 


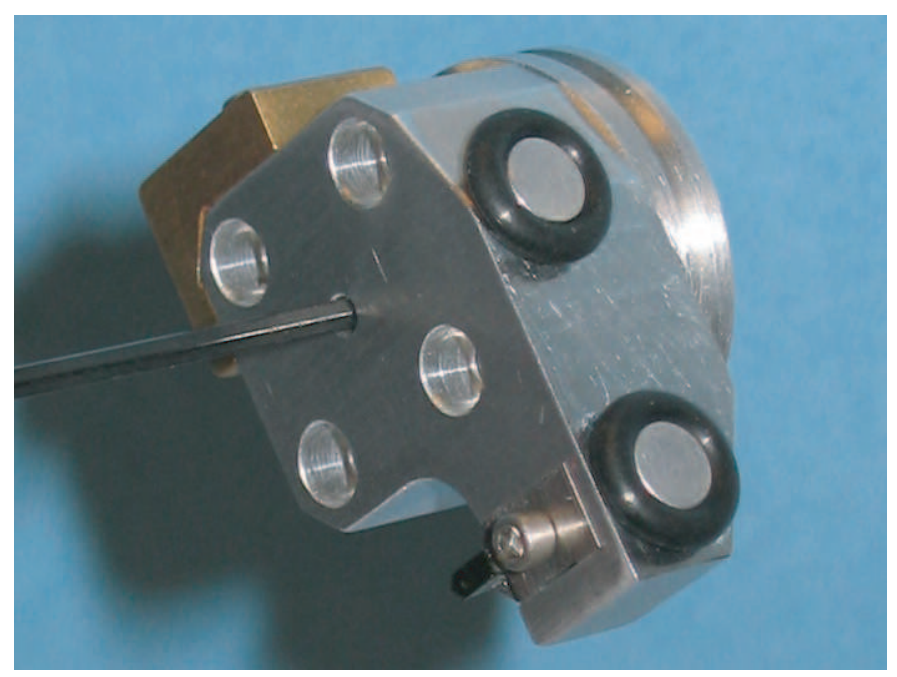

Figure 4-43: Tool holder for the $10 \mathrm{kHz}$ FTS, shown with its rotation axis lying in a horizontal plane during static balancing.

\subsubsection{Flexures}

The rotor is supported by an array of over-constrained flexure blades that extend radially from the rotor and are clamped at both ends: at the rotor and at the stator housing. This flexure bearing follows the same design that was proven successful with the $2 \mathrm{kHz}$ FTS. Section 3.6.3 provides a detailed discussion on the motivation for using a flexure bearing and the analysis that was used to design it. Each flexure blade is a 0.010 inch thick by 0.500 inch wide by 0.375 inch long piece of 1095 bluetempered spring steel that was polished along its long axis to minimize initiation of fatigue cracks, and is preloaded with a $10 \mathrm{lb}$ tensile force before clamping the ends.

Referring to Figure 4-20, note that all of the flexure blades are identical and have a high in-plane stiffness compared to their low out-of-plane bending stiffness. This creates a radial and axial constraint at both ends of the rotor, which is therefore over-constrained in the axial direction. ${ }^{3}$ In the case of the $2 \mathrm{kHz}$ FTS this is not a problem because the rotor does not have a heat generating element in it. However, with the $10 \mathrm{kHz}$ FTS magnetic losses in the rotor core during high frequency operation

\footnotetext{
${ }^{3}$ An "over-constrained rotor" should not be confused with the intentional "over-constrained flexures" used in this design. The flexures are over-constrained in the sense that rotation of the rotor causes an axial strain in the flexure blades. That axial strain is taken into account in the stress analysis for the flexures.
} 
generate heat that will be a problem if the rotor axial thermal growth is not managed. A first approach to addressing this issue is to monitor the temperatures of the rotor and stator and control them by limiting the duration of high frequency operation, or by using a cooling fluid. A second approach is to modify the lower set of flexure blades to make them compliant in the axial direction. The second approach may ultimately be preferable, however it creates additional vibration modes for the rotor and requires modifying the design for the flexure mounting hardware used in the $2 \mathrm{kHz}$ FTS. Thus we stayed with the first approach of an axially over-constrained rotor. This choice allowed focusing this research on testing the new normal-stress variable reluctance actuator with a proven bearing system, albeit with known limitations that can be addressed in future versions. The second approach was carried as a contingency in case the axially over-constrained rotor presented problems during testing of the $10 \mathrm{kHz}$ FTS, and is described in Section 6.4.5.

\section{Design Calculations for the Flexure Blades}

At $10 \mathrm{kHz}$ operation, the flexure blades undergo one million cycles of a fluctuating stress in approximately two minutes, so fatigue life is a crucial consideration. The design of the flexure blades was guided by the same closed-form equations and spreadsheet parametric study that I developed for the $2 \mathrm{kHz}$ FTS and describe in Section 3.6.4.

The set of graphs shown in Figure 4-44 represent the final parametric study for the 0.010 inch thickness value that was chosen. The desired design spaces are indicated by the horizontal lines with vertical arrows at their ends. Because success of the $10 \mathrm{kHz}$ FTS depended on the flexure blades surviving prolonged high frequency operation, and it was likely that I would be driving the rotor into the hard stops during eventual debugging of the control system, I set a factor of safety of 10 as a minimum requirement for the fatigue life. At $10 \mathrm{kHz}$ operation, $10^{7}$ cycles occurs in 17 minutes, so I designed the flexures for an infinite fatigue life.

Each flexure blade in the $10 \mathrm{kHz}$ FTS is a 0.010 inch thick by 0.500 inch wide by 0.375 inch long piece of blue-tempered 1095 spring steel (ASTM A 682), which has 
an endurance limit of $100 \mathrm{ksi}$ [58]. The radius of the flexure hub is $0.438 \mathrm{inch}$, and the maximum rotation angle of the rotor (to the hard stops) is $\pm 2.9 \mathrm{mrad}$. Using (3.5) and (3.6), the maximum and minimum stresses are:

$$
\begin{gathered}
\sigma_{\max }=8120+1160+147+2000=11427 \text { psi }(\text { tension }) \\
\sigma_{\min }=147+2000-8120-1160=-7133 \text { psi (compression) }
\end{gathered}
$$

The corresponding mean stress and amplitude of the stress fluctuation are:

$$
\begin{gathered}
\sigma_{\text {mean }}=\frac{11427+(-7133)}{2}=2147 \text { psi (tension) } \\
\sigma_{\text {ampl }}=\frac{11427-(-7133)}{2}=9280 \mathrm{psi}
\end{gathered}
$$

Using the modified Goodman line in Figure 3-11, the fatigue strength corresponding to the mean stress of 2147 psi is $99 \mathrm{ksi}$. This leads to a factor of safety for fatigue of 11 for the fluctuating stress of 9280 psi.

For the axial stiffness and radial stiffness requirements for the flexure suspension I choose $500,000 \mathrm{lb} /$ in $(100 \mathrm{~N} / \mu \mathrm{m})$ and 1,000,000 lb/in $(200 \mathrm{~N} / \mu \mathrm{m})$, respectively. Referring back to the discussion in Section 3.4, these values represented $20 \%$ and $10 \%$ of the stiffness budget for the non-sensitive and sensitive direction at the tool tip, respectively. Note that although I chose a flexure width of 0.500 inch, smaller widths would satisfy my design criteria. The trade-off here was to use the detailed design for the flexure mounting hardware that was developed with the $2 \mathrm{kHz}$ FTS, at the cost of having a higher rotary inertia due to the taller flexure hubs needed for 0.500 inch wide blades.

The desired design spaces on the graphs shown in Figure 4-44 were used as guidelines for choosing the dimensions for the flexure blades, and were not meant to be hard and fast binary decision lines. For instance, note that the clamping force to hold the end of a flexure blade was set at $1 \mathrm{lb}$. It seemed reasonable to accept the 
clamping force of $1.2 \mathrm{lb}$ needed for the 0.500 inch blade, considering that the single 5-40 screw used at each clamping block would easily produce at least a 10 to 20 times higher clamping force. Regarding the torque required to rotate the swing-arm from its neutral position to one of the hard stops, I set the design point at $5 \%$ of the maximum continuous-operation peak torque that the actuator could produce, so that the majority of the actuator torque would be available to accelerate the swing-arm. In this case, it was $5 \%$ of our design goal of $3.7 \mathrm{~N} \cdot \mathrm{m}(33 \mathrm{lb} \cdot \mathrm{in})$, or $0.2 \mathrm{~N} \cdot \mathrm{m}(1.7 \mathrm{lb} \cdot \mathrm{in})$.

\section{Measured Stiffness of Flexure-Suspended Rotor}

After mounting the rotor to the stator, I measured the mechanical stiffness of the rotor in the axial direction using a hand-held force gauge and an LVDT displacement sensor. Figure 4-45 shows the set-up for measuring the axial stiffness of the rotor along its centerline, and the stiffness at the tool in a direction parallel to the rotor centerline. The measured values are $45 \mathrm{~N} / \mu \mathrm{m}$ and $29 \mathrm{~N} / \mu \mathrm{m}$, respectively. The flexure calculations summarized in Figure 4-44 predict that the axial stiffness of the rotor would be $230 \mathrm{~N} / \mu \mathrm{m}(1.3 \mathrm{lb} / \mu \mathrm{in})$ if the flexure blades were the only compliant components in the mechanical loop connecting the top of the rotor to the bottom of the base of the $10 \mathrm{kHz}$ FTS. The measured axial stiffness of the rotor is one fifth of that value, which is not surprising considering all of the shear joints between the flexure blades and the rotor and stator housings. Those shear joints were taken into consideration a priori when I set the design specification shown in Figure 4-44 for the axial stiffness provided by the flexure blades. The measured axial stiffness of $29 \mathrm{~N} / \mu \mathrm{m}$ at the tool tip meets the $20 \mathrm{~N} / \mu \mathrm{m}$ stiffness (10 $\mu \mathrm{in} / \mathrm{lb}$ compliance) goal described in Section 3.4.

I attempted to measure the radial stiffness of the flexure-suspended rotor along its centerline, but the immediate lack of a good anti-rotation device made the measurement unreliable. Rather than continue pursuing this measurement, I chose to proceed to integrating the $10 \mathrm{kHz}$ FTS with its electronics and control system. 

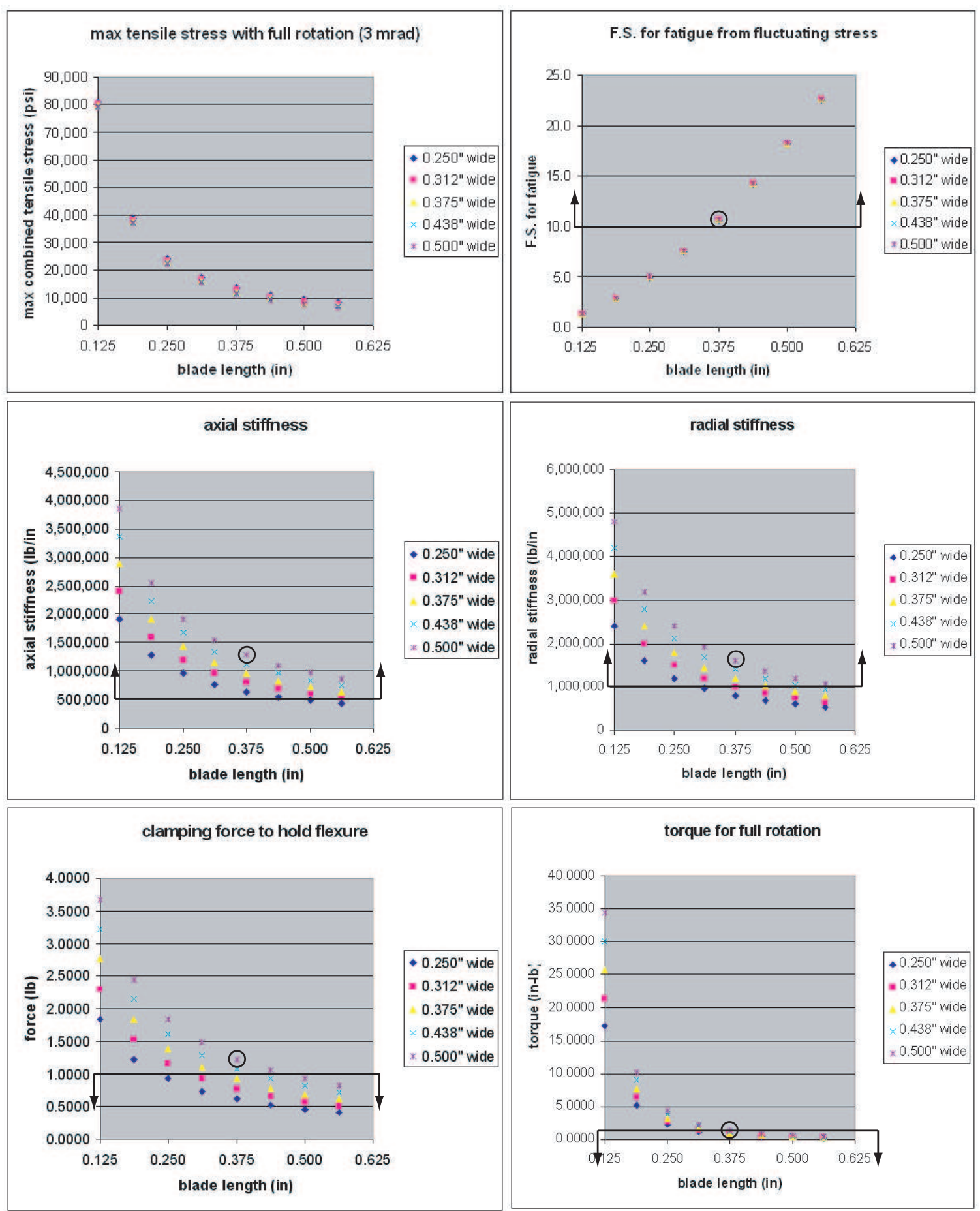

Figure 4-44: Final parametric study for the 0.010 inch thick flexure blades used in the $10 \mathrm{kHz}$ FTS. Full rotation of the rotor to a hard stop (2.9 mrad). Factor of safety for fatigue based on the actual case of a fluctuating stress and a derated endurance limit due to the mean tensile stress. 

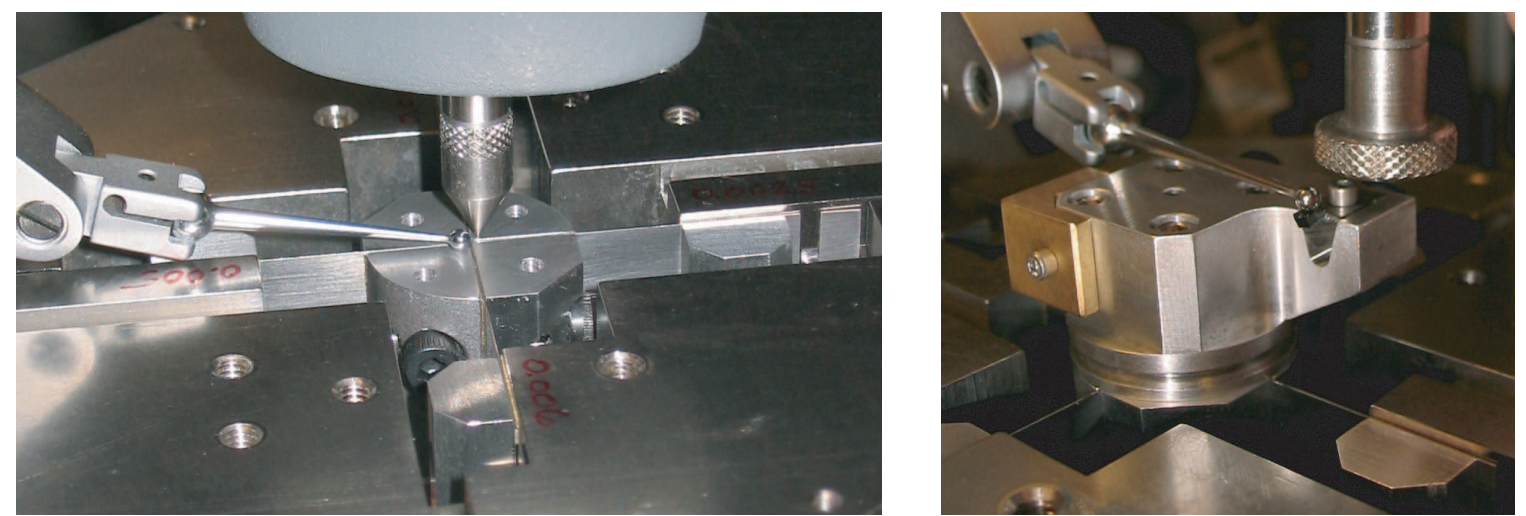

Figure 4-45: Measuring the axial stiffness of the flexure-suspended rotor (left), and the stiffness at the tool in a direction parallel to the rotor centerline (right).

\subsubsection{Sensors}

The position of the tool tip is measured by a capacitance sensor looking at the back of the tool arm directly behind the tool. A second capacitance sensor measures translation of the centerline of the tool arm. Figure 4-46 shows the two capacitance sensors in their mount and the tool holder. The capacitance sensors are from ADE Technologies [9]: amplifier model 5810 and probe model 5552-01. The sensor amplifier produces a $\pm 10 \mathrm{~V}$ signal for the $\pm 35 \mu \mathrm{m}$ tool travel $(3.5 \mathrm{~nm} / \mathrm{mV})$, and has a specified bandwidth of $0-100 \mathrm{kHz}$ which is adequate for the $10 \mathrm{kHz}$ rotary fast tool servo. Setting the space between the sensor and tool arm to approximately $55 \mu \mathrm{m}$ produces a null voltage. The factory measured root-mean-square electrical noise for these sensors over that bandwidth is $0.70 \mathrm{mV}$ rms, which is equivalent to a tool tip displacement of $2.5 \mathrm{~nm} \mathrm{rms}$. Using a $30 \mathrm{kHz}$ low-pass cut-off filter on the sensor signal, the measured locked-rotor sensor noise on the $10 \mathrm{kHz}$ FTS is $0.110 \mathrm{mV} \mathrm{rms,} \mathrm{which} \mathrm{is} \mathrm{equivalent}$ to a tool tip displacement of $0.39 \mathrm{~nm} \mathrm{rms}$. This noise measurement is discussed in Section 4.9.3, along with closed-loop tool tip displacement noise measurements.

The earlier $2 \mathrm{kHz}$ FTS uses eddy current gauges that performed well overall. However, they have a specified bandwidth of $20 \mathrm{kHz}$, which is too low for accurately measuring tool holder displacements occurring at $10 \mathrm{kHz}$. 


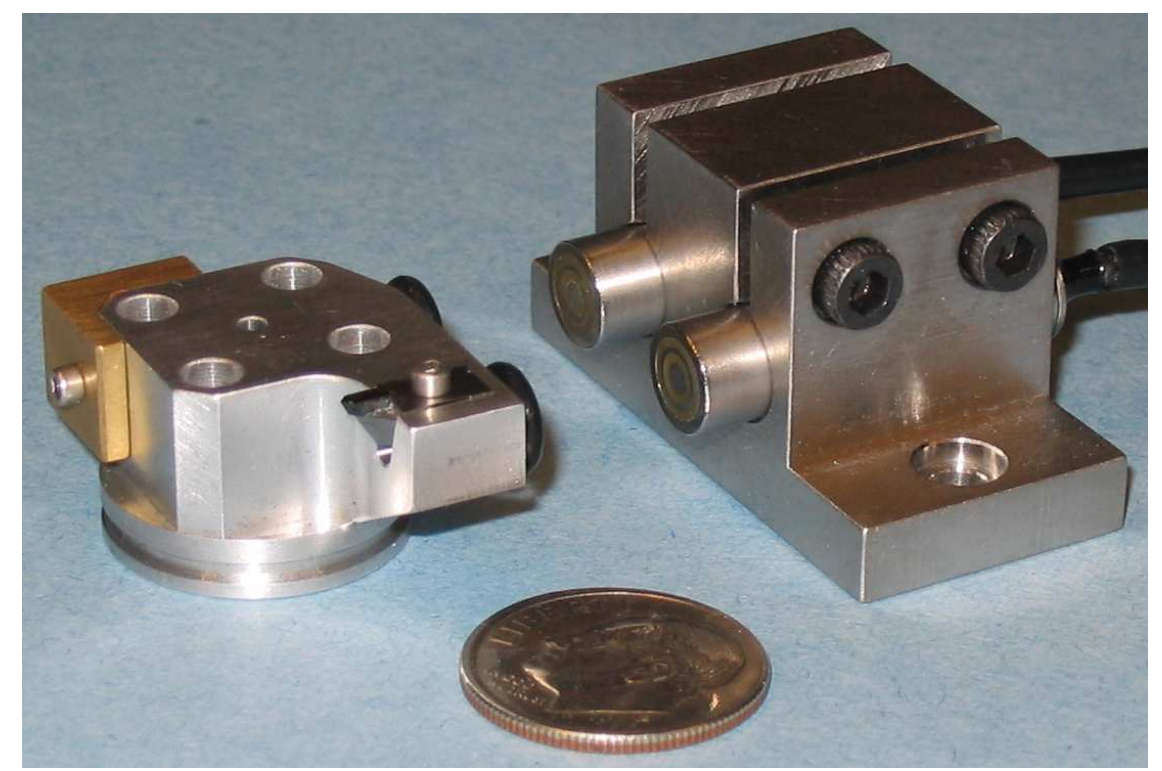

Figure 4-46: Capacitance sensors and tool holder for the $10 \mathrm{kHz}$ FTS. Distance between the sensors and tool holder is greatly exaggerated for illustrative purposes. The dime in the foreground provides a sense of scale.

\subsubsection{Finite Element Analysis of the Rotor}

A finite element analysis (FEA) model was developed and used to predict the frequency and shape of the vibration modes for the flexure-suspended rotor. I used Pro/Engineer [120] to build the solid models for the $10 \mathrm{kHz}$ FTS (and for the $2 \mathrm{kHz}$ FTS), so used the integrated Pro/Mechanica [120] to build the FEA model and perform the analysis. In this section I summarize the results of the FEA model analysis, and offer some insights on how I modelled the laminated rotor core.

The FEA model does not include the $50 \mu \mathrm{m}$ thick layers of epoxy that bond the rotor components together; attempts to include those layers resulted in the analysis failing to reach convergence. Therefore, the FEA model uses a more rigid connection between those components than the real hardware has, which leads to predicted vibration frequencies that are higher than the actual values for the as-built hardware. This last point was confirmed by measurements of certain vibration modes, which are discussed in Section 4.6.3. 


\section{Results of the FEA Model Analysis}

Figures 4-47 through 4-50 show the results of the FEA model analysis of the flexuresuspended rotor. The predicted frequency for each mode is shown in the figures. The labels "sensitive" and "non-sensitive" in Figures 4-47 through 4-50 refer to whether or not the tool position sensor observes the motion as a first-order effect. The hybrid rotary/linear actuator and the two displacement sensors for the $10 \mathrm{kHz}$ FTS can be used to control the rigid body rotation and the front/back translation of the rotor.

The two lateral translation modes shown in Figure 4-48 are referred to as "rigid" modes for two reasons. First, the frequencies for the two modes are the same, which is consistent with the rotor translating as a rigid body that is attached to springs having the same stiffness in both directions. Second, the bending stiffness of the rotor is significantly different for the two directions shown, which would result in different frequencies for the two modes if they were predominantly bending modes of the rotor. The bending of the rotor shown in Figure 4-48 is an exaggeration of the actual displacements of the rotor.

The two non-rigid body torsional modes shown in Figure 4-50 are both stable because the magnitude of the mechanical torsional stiffness of the rotor is greater than the magnitude of the negative torsional spring produced by the magnetic flux in the rotor/stator air gaps. The concept of a negative spring produced by the magnetic flux is discussed in Section 4.6.1.

\section{Modelling the Laminated Rotor Core}

The rotor core consists of $25 \mu \mathrm{m}$ thick iron-alloy laminations bonded together with epoxy using a bond-line thickness of approximately $6-8 \mu \mathrm{m}$. Since the epoxy makes up an appreciable fraction of the stack height of the core, and the stiffness of the epoxy is an order of magnitude less than that of the iron, the elastic properties of the laminated stack are significantly affected by the epoxy. To insure that the FEA model of the rotor would provide a reasonably accurate prediction of the vibration modes and frequencies, I developed a set of compliance coefficients to describe the 

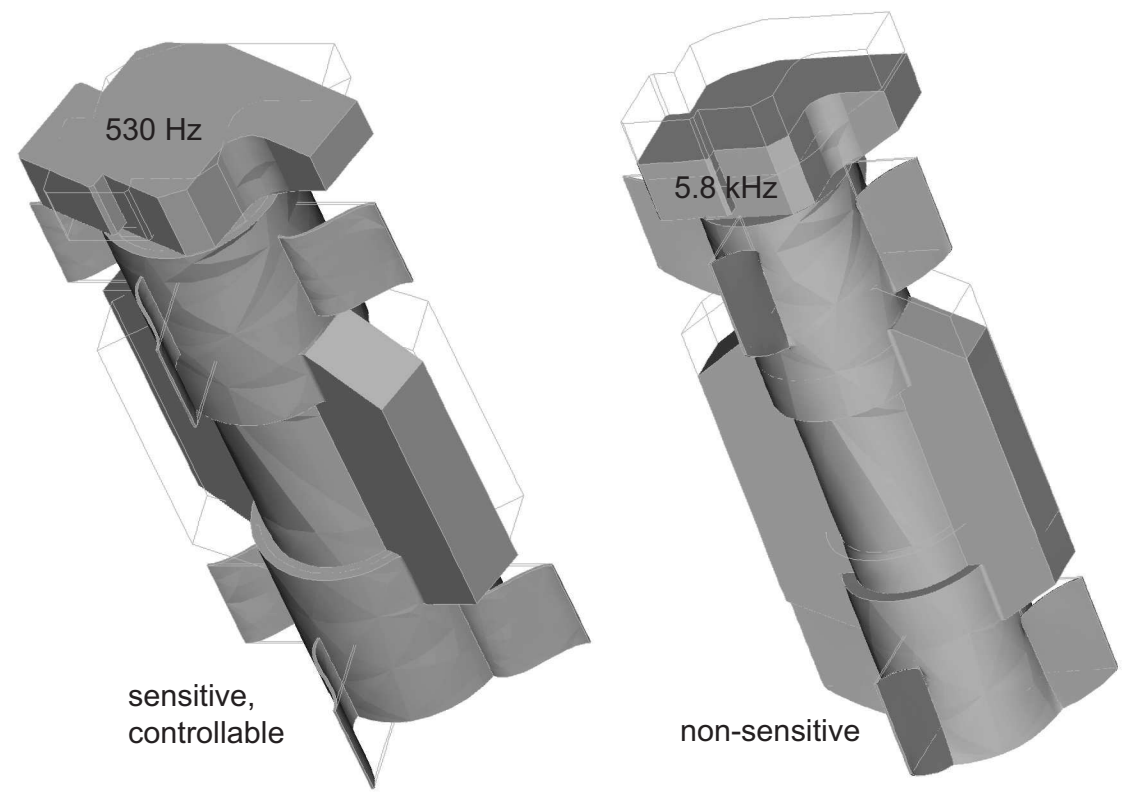

Figure 4-47: Predicted vibration modes of the flexure-suspended rotor using an FEA model. Rigid body modes: rotation (left) and axial translation (right).
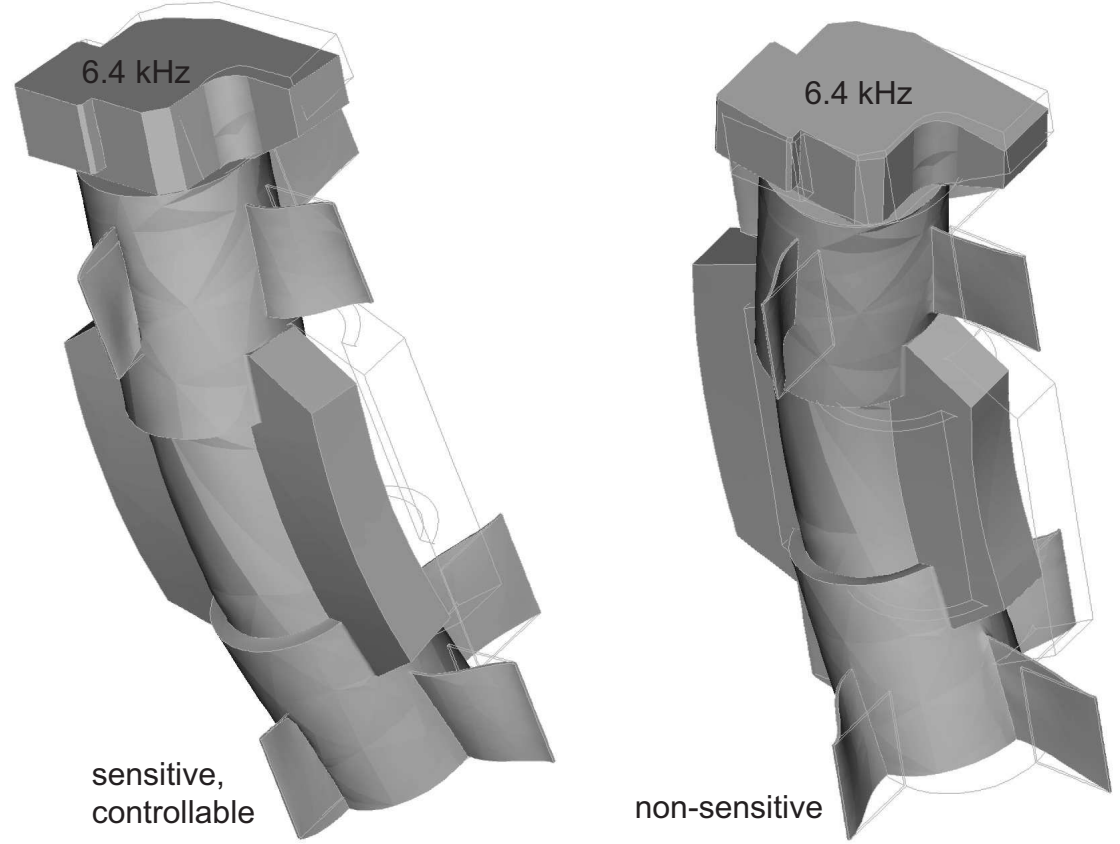

Figure 4-48: Predicted vibration modes of the flexure-suspended rotor using an FEA model. "Rigid" body modes: lateral translations. 

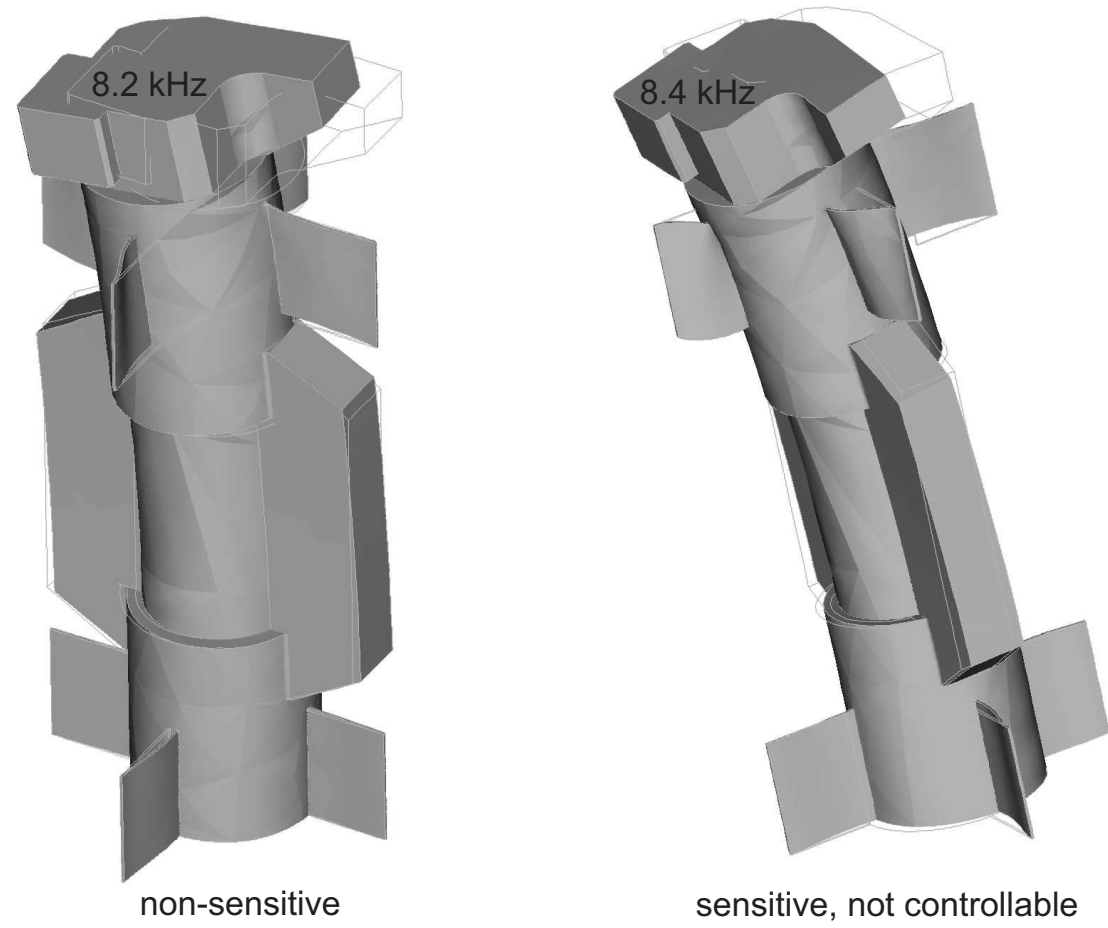

sensitive, not controllable

Figure 4-49: Predicted vibration modes of the flexure-suspended rotor using an FEA model. Tipping modes: rotation plus lateral translation.

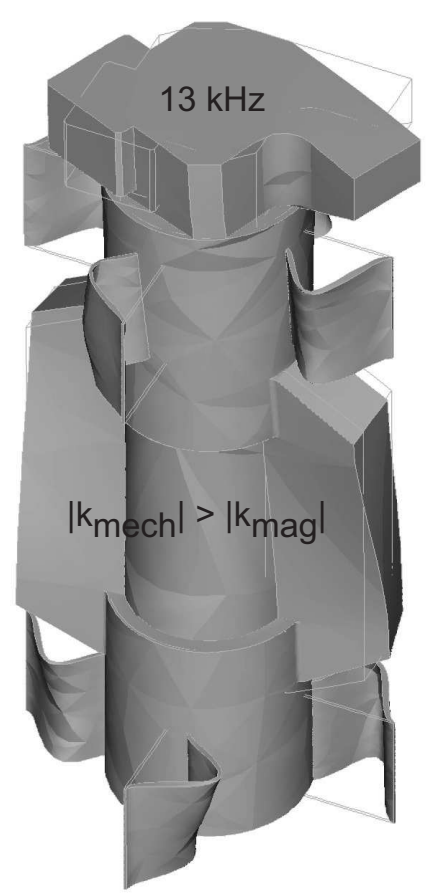

sensitive, not controllable, stable

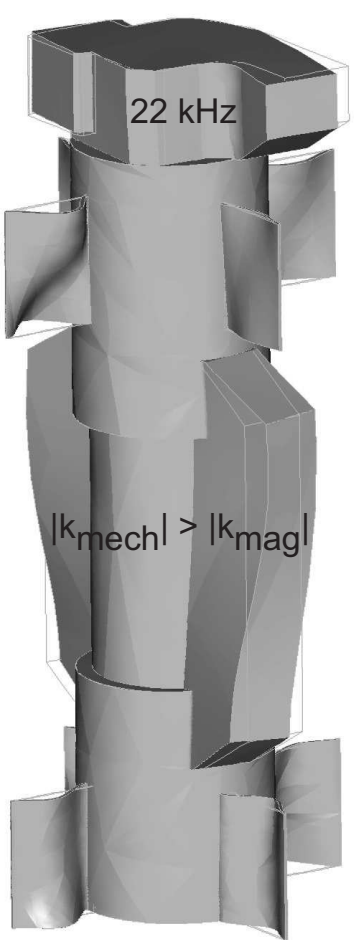

sensitive, not controllable, stable

Figure 4-50: Predicted vibration modes of the flexure-suspended rotor using an FEA model. Non-rigid body torsional modes. 
anisotropic elastic properties for the laminated core.

It had been over twenty years since my one undergraduate course in mechanics of materials, so I needed to do some research and studying to gain sufficient proficiency for developing a set of useful elastic constants to model the laminated rotor core. Professor Samir Nayfeh at MIT suggested that I consider the laminated core as a composite material, and motivated me to dig into the literature on it. I found the books on composite materials and mechanics of materials by the following authors to be useful: Daniel and Ishai [44], Jones [86], Tsai [144], Seed [135], Dowling [50], Crandall [40], and Popov [123]. The book by Daniel and Ishai was my favorite for composite materials. It walked me through the derivation of the compliance matrix for an orthotropic material and for a transversely isotropic material. The book by Popov was my favorite for re-learning the basic principles of stress and strain in a solid.

Daniel and Ishai define an orthotropic material as having "three mutually perpendicular planes of material symmetry" [44, page 41], and then define a transversely isotropic material as follows:

An orthotropic material is called transversely isotropic when one of its principal planes is a plane of isotropy, i.e., at every point there is a plane on which the mechanical properties are the same in all directions. Many unidirectional composites with fibers packed in a hexagonal array, or close to it, can be considered transversely isotropic, with the 2-3 plane (normal to the fibers) as the plane of isotropy [44, page 42].

Figure 4-51 contains a sketch of a unidirectional fiber-reinforced composite with the principle-axes indices that Daniel and Ishai refer to. I considered the laminated rotor core to be a transversely isotropic material having jump discontinuities between the iron and epoxy layers. Figure 4-51 shows a sketch of the laminated rotor core oriented so that its 2-3 planes are isotropic.

The elastic properties of an isotropic material can be fully described by two independent material constants: Young's modulus $E$ and Poisson's ratio $\nu$. Nine inde- 

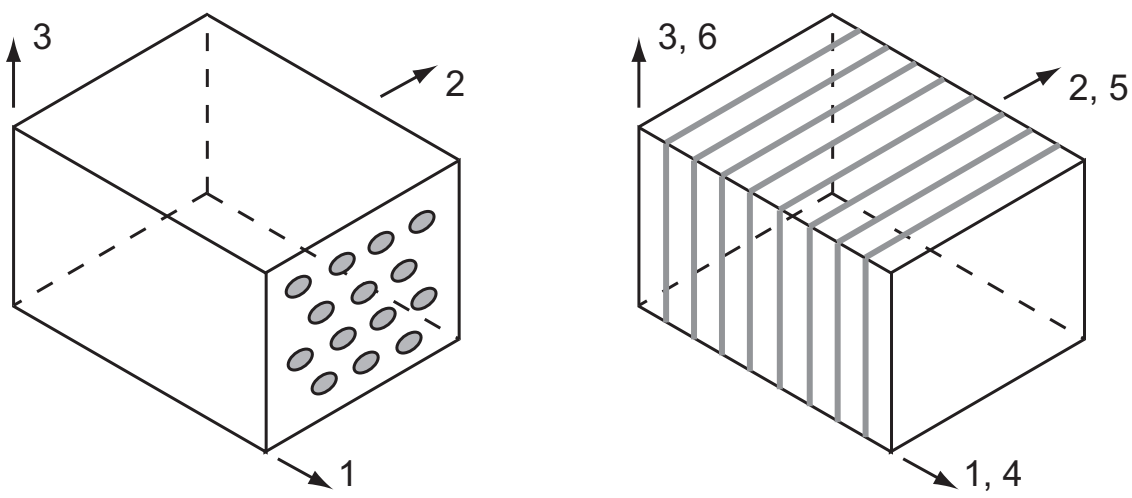

Figure 4-51: Principle-axes indices for materials with transversely isotropic properties. Nomenclature used by Daniel and Ishai [44] for a unidirectional fiber-reinforced composite (left). Nomenclature that I used for the laminated rotor core (right). For both cases, the 2-3 plane is the plane of transverse isotropy, with jump discontinuities for the laminated core.

pendent constants are needed to describe the elastic properties of a three-dimensional orthotropic material, and the symmetry of a transversely isotropic material reduces that number to five independent constants.

Referring to Figure 4-51, the 1, 2, and 3 directions indicate the principle axes for the laminated rotor core. The three principle-axes applied axial stresses are $\sigma_{1}, \sigma_{2}$, and $\sigma_{3}$, and the three induced axial strains are $\epsilon_{1}, \epsilon_{2}$, and $\epsilon_{3}$, respectively. The 4,5 , and 6 directions indicate the normal directions for shear in the 23, 13, and 12 planes, respectively. The three principle-axes applied shear stresses are $\tau_{4}, \tau_{5}$, and $\tau_{6}$, and the three induced shear strains are $\gamma_{4}, \gamma_{5}$, and $\gamma_{6}$, respectively. To handle the jump discontinuities in material properties between the iron and epoxy layers, I considered how each material contributes to the net induced strains of the composite in response to an applied stress, and formulated a net-effective Young's modulus and Poisson's ratio to relate the stress to the net strains.

The elastic constants for the laminated rotor core are related to the Young's modulus $E$, Poisson's ratio $\nu$, and layer thickness for the iron and epoxy by (4.1) through (4.7). The subscripts " 1 " and "2" correspond to the iron and epoxy, respectively. The subscripts "N" and "P" refer to the normal and parallel directions of the laminations, respectively. The stacking factor $S F$ for the laminated rotor core depends on the layer thicknesses $l$ for the iron and epoxy. A modified Young's modulus $E_{2}^{*}$ for 
the epoxy takes into account the increased stiffness for the epoxy layer in the normal direction of the laminations due to a uniaxial strain assumption. ${ }^{4}$

For an applied axial stress in the normal direction of the laminations $\sigma_{1}$, the iron and epoxy layers act like series-connected springs, leading to the net Young's modulus $E_{N}$ for the normal direction given by (4.1).

$$
E_{N}=\frac{E_{1} E_{2}^{*}}{E_{2}^{*}(S F)+E_{1}(1-S F)}
$$

For an applied axial stress in the parallel directions of the laminations $\left(\sigma_{2}\right.$ and $\left.\sigma_{3}\right)$, the iron and epoxy layers act like parallel-connected springs, leading to the net Young's modulus $E_{P}$ for the parallel direction given by (4.2). $E_{P}$ is dominated by $E_{1}$ because the iron is an order of magnitude stiffer than the epoxy and roughly three quarters of the stack height is iron.

$$
E_{P}=(S F) E_{1}+(1-S F) E_{2}
$$

In response to an applied axial stress in the parallel direction of the laminations ( $\sigma_{2}$ or $\sigma_{3}$ ), the induced normal-direction lateral strains $\epsilon_{N}$ in the iron and epoxy layers are both significant. The axial strain in the applied stress direction $\epsilon_{P}$ is related to the applied stress by: $\sigma_{P}=E_{P} \epsilon_{P}$. This leads to the net Poisson's ratio $\nu_{P N}$ given by (4.3).

$$
\nu_{P N}=(S F) \nu_{1}+(1-S F) \nu_{2}
$$

Using (4.1), (4.2), and (4.3), the net Poisson's ratio $\nu_{N P}$ given by (4.4).

$$
\nu_{N P}=\nu_{P N}\left(\frac{E_{N}}{E_{P}}\right)
$$

\footnotetext{
${ }^{4}$ Professor Samir Nayfeh at MIT provides a good tutorial on uniaxial strain in his course notes for a class at MIT [112], and Popov discusses it in [123]. Essentially, the thin epoxy layer (relative to its lateral dimensions) is effectively constrained in its lateral directions by the stiffer iron layers that capture it. For a uniaxial strain condition, the epoxy layer does not undergo the free lateral strain associated with a uniaxial stress condition, and therefore acts stiffer in the normal direction of the layer.
} 
In response to an applied axial stress in the parallel direction of the laminations $\left(\sigma_{2}\right.$ or $\left.\sigma_{3}\right)$, the induced parallel-direction lateral strain $\epsilon_{P}$ in the composite is dominated by the iron. This leads to the net Poisson's ratio $\nu_{P P}$ given by (4.5).

$$
\begin{gathered}
\nu_{P P}=\nu_{1} \\
S F=\left(\frac{l_{1}}{l_{1}+l_{2}}\right) \\
E_{2}^{*}=E_{2}\left(\frac{1-\nu_{2}}{1-\nu_{2}-2 \nu_{2}^{2}}\right)
\end{gathered}
$$

The compliance matrix for the transversely-isotropic description for the laminated rotor core is given by (4.8).

$$
\left[\begin{array}{c}
\epsilon_{1} \\
\epsilon_{2} \\
\epsilon_{3} \\
\gamma_{4} \\
\gamma_{5} \\
\gamma_{6}
\end{array}\right]=\left[\begin{array}{cccccc}
\frac{1}{E_{N}} & \frac{-\nu_{N P}}{E_{N}} & \frac{-\nu_{N P}}{E_{N}} & 0 & 0 & 0 \\
\frac{-\nu_{N P}}{E_{N}} & \frac{1}{E_{P}} & \frac{-\nu_{P P}}{E_{P}} & 0 & 0 & 0 \\
\frac{-\nu_{N P}}{E_{N}} & \frac{-\nu_{P P}}{E_{P}} & \frac{1}{E_{P}} & 0 & 0 & 0 \\
0 & 0 & 0 & \frac{2\left(1+\nu_{P P}\right)}{E_{P}} & 0 & 0 \\
0 & 0 & 0 & 0 & \left(\frac{1+\nu_{P N}}{E_{P}}+\frac{1+\nu_{N P}}{E_{N}}\right) & 0\left(\frac{1+\nu_{P N}}{E_{P}}+\frac{1+\nu_{N P}}{E_{N}}\right)
\end{array}\right]\left[\begin{array}{c}
\sigma_{1} \\
\sigma_{2} \\
\sigma_{3} \\
\tau_{4} \\
\tau_{5} \\
\tau_{6}
\end{array}\right]
$$

A comment is necessary on the indices notation that I used for Poisson's ratio, $\nu$, because I found two opposite sets of notation in the literature. Letting: $i$ be the direction of the applied normal stress, and $j$ be the direction of the induced lateral strain, Daniel and Ishai [44] and Jones [86] use $\nu_{i j}$, while Tsai [144] uses $\nu_{j i}$. I use the same Poisson's ratio notation $\nu_{i j}$ as Daniel and Ishai. The practical significance of this is that after developing the matrix of elastic constants for the laminated rotor core, I discovered that the Pro/Mechanica [120] FEA package that I used follows the Tsai notation. This was easily handled by carefully mapping my elastic coefficients to the coefficients used in the FEA package.

It is worth noting two peculiarities of Pro/Mechanica that involve the elastic 
constants for an anisotropic material. The first is that for an orthotropic material, Pro/Mechanica interchanges rows four and six of the compliance matrix shown in (4.8). Perhaps they do this because the shear stress in my row six is what would remain if a problem was reduced to a plane-strain problem in the 12-plane. The second peculiarity is that for a transversely isotropic material, Pro/Mechanica requires that the Young's modulus in the first direction $E_{1}$ be greater than that of the second $E_{2}$ and third $E_{3}$. Since this is not the case for the laminated rotor core, I had to use the orthotropic material option in Pro/Mechanica, which does allow $E_{1}$ to be less than $E_{2}$ and $E_{3}$.

Figures 4-52 and 4-53 are screen-shots of the Pro/Mechanica FEA model of the flexure-suspended rotor and the corresponding material definition page for the laminated rotor core, respectively. Appendix B.4 contains the Matlab ${ }^{\circledR}$ script that I used to generate the elastic constants in Figure 4-53 using (4.8) and the isotropic material properties for the iron-alloy and the epoxy used in the rotor core. The analysis assumed that the rotor core would be made from $50 \%$ Ni-50\% Fe. For the purposes of the FEA model predictions described in this section, the elastic properties of the $\mathrm{Ni}-$ Fe are not significantly different from those of the nanocrystalline iron-alloy material that was used in the as-built rotor core.

\subsubsection{Mechanical Damping}

Section 4.5.8 describes a "tipping" mode of the rotor that is observable by both of the capacitance sensors, but it is not controllable. To deal with this unwanted motion, we added mechanical damping by filling the gap between the tool holder and the centerline capacitance sensor with grease, as shown in Figures 4-54 and 4-55. The grease is DuPont Krytox ${ }^{\circledR}$ GPL-207, and has a viscosity of 1600 cSt at $20^{\circ} \mathrm{C}[52]$. This damping reduced the Bode magnitude of the uncontrollable tipping mode and the front/back translation mode by at least $25 \mathrm{~dB}$. Quantitative details on the effects of the damping on those modes are presented in Section 4.6.3.

The grease increases the dielectric constant of the space between the capacitance sensor and its target by approximately a factor of two compared to air. This in- 


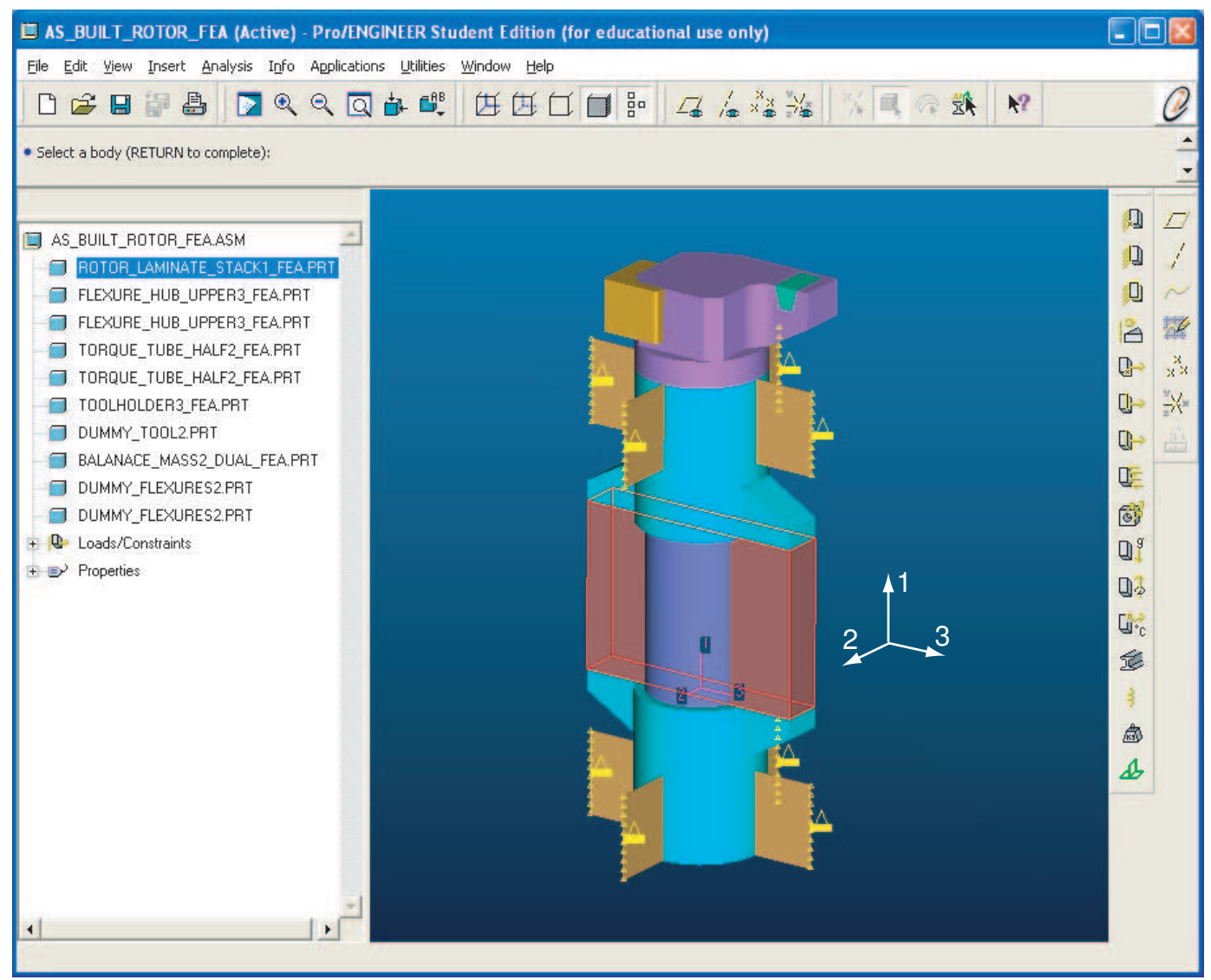

Figure 4-52: Screen-shot of the Pro/Mechanica FEA model of the flexure-suspended rotor.

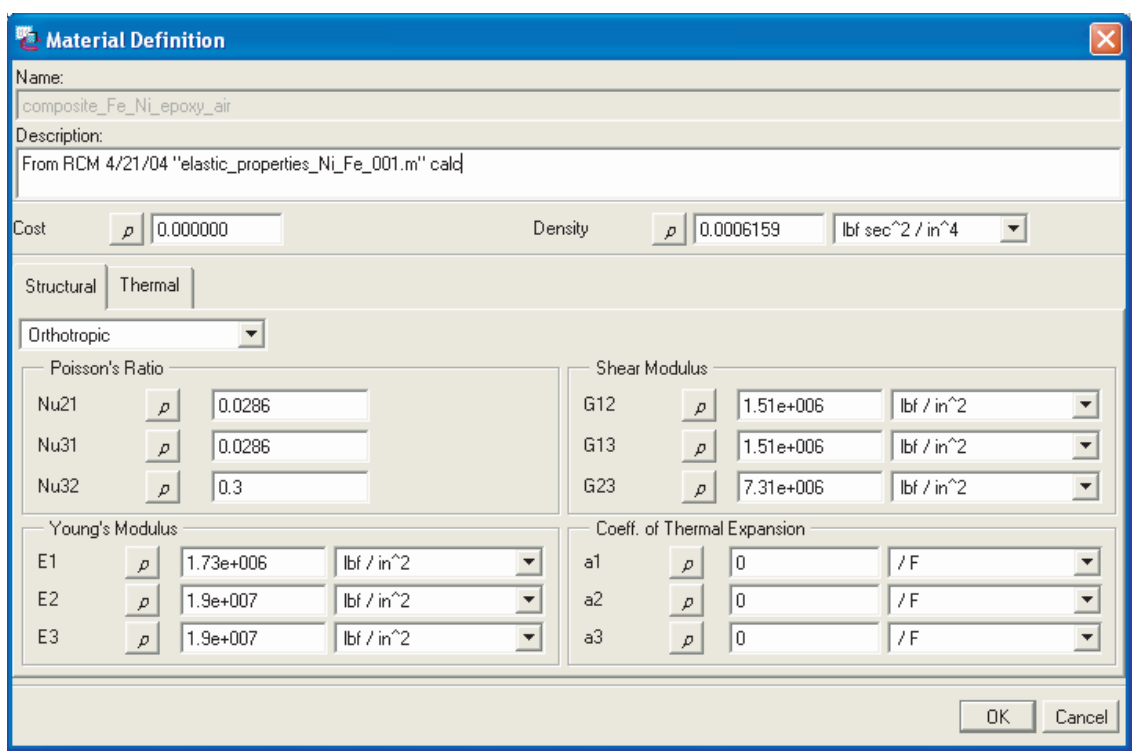

Figure 4-53: Screen-shot of the Pro/Mechanica material definition page for the laminated rotor core. 


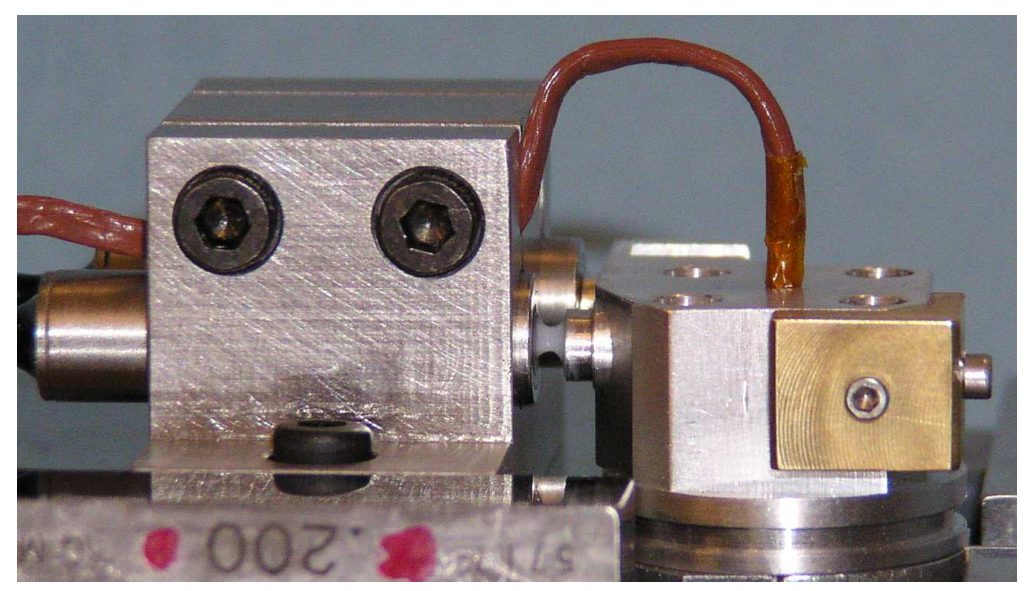

Figure 4-54: Adding mechanical damping to the $10 \mathrm{kHz}$ FTS by filling the gap between the tool holder and the centerline capacitance sensor with grease; shown before closing the gap.

creases the scale factor for the centerline capacitance sensor from $3.5 \mu \mathrm{m} / \mathrm{V}$ for air to $6.0 \mu \mathrm{m} / \mathrm{V}$ for the grease. I determined the new scale factor using the following procedure: (1) Before adding the grease, measure the radial stiffness of the flexure-mounted rotor using a hand-held force gauge and the output of the capacitance sensor. (2) Add the grease and close the sensor/target gap to approximately $25 \mu \mathrm{m}$. This results in an electrical offset seen at the sensor amplifier of approximately $5 \mathrm{~V}$ which is acceptable because the rotor centerline radial displacements during operation are small and the sensor stays within the $\pm 10 \mathrm{~V}$ range. (3) Re-measure the radial stiffness using the same force as in step (1), and calculate the sensor scale factor to match the stiffness measured in step (1).

Fortunately, the grease is not a viscous fluid. If it were, it would have eventually poured out of the gap between the capacitance gauge and the tool holder. After six months, the dab of grease still looked the same as it does in Figure 4-55.

An alternate method of adding mechanical damping would be to introduce a viscous fluid into the rotor/stator air gaps to produce squeeze-film damping. Strict attention to the viscosity of the fluid is needed because the large surface areas involved - relative to the short gap lengths - could easily lead to excessively high damping forces. 


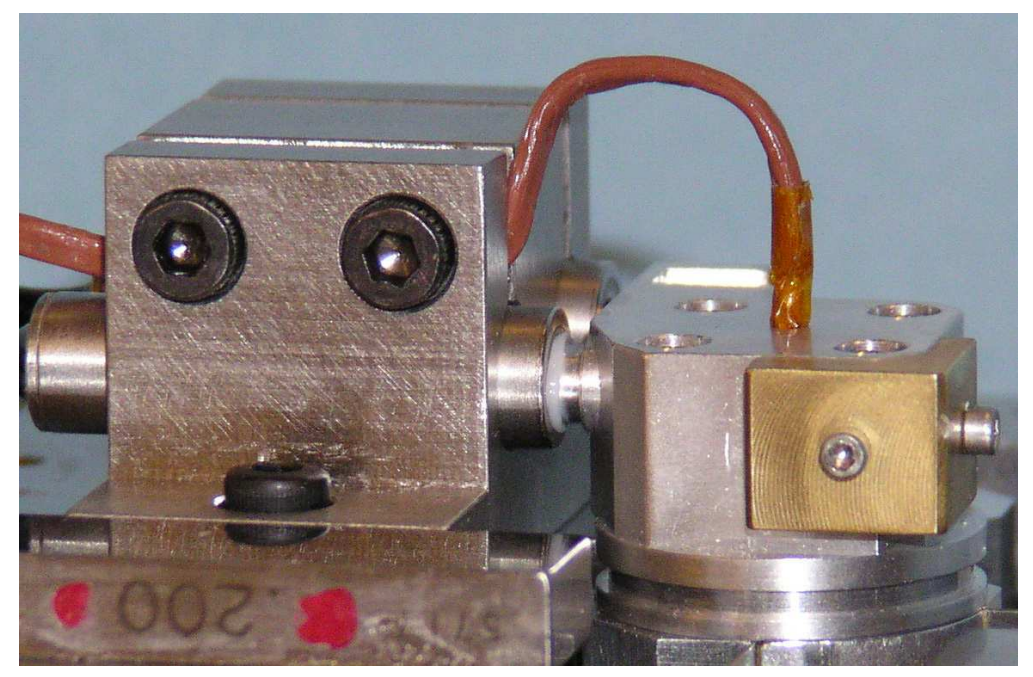

Figure 4-55: Adding mechanical damping to the $10 \mathrm{kHz}$ FTS by filling the gap between the tool holder and the centerline capacitance sensor with grease; shown after closing the gap.

\subsubsection{Adhesive-Bonded Assemblies}

The mechanical integrity of the rotor and stator assemblies depends on the adhesivebonded joints used in their design. I did a significant amount of work learning about adhesives, adhesive-joint design, and surface preparations, but limit the discussion in this thesis to just a few of the more significant issues and provide references for the reader seeking further insight.

By far the single most useful reference that I found was "Handbook of Adhesives and Sealants" by Petrie [122]. It contains everything that I really needed to know to design and manufacture strong and reliable adhesive-bonded assemblies. The handbook includes detailed discussions on theories of adhesion, joint design, surface preparation, and descriptions of more different types of adhesives than I was previously aware of. The scope, depth, and writing style provided me with the next best thing to having an adhesives expert in the room.

\section{Selection Criteria for the Adhesives}

The following criteria guided my decision to down-select to the four aerospace-grade two-part epoxies listed in Table 4.1, and finally select Loctite Hysol ${ }^{\circledR}$ EA 9360 for all 
of the adhesive bonds except for potting the steering coils. The potting compound that I used is 3M Scotch-Weld ${ }^{\mathrm{TM}}$ DP270 Clear [8].

- Tensile lap shear strength. To the extent possible, I designed the adhesivebonded joints so that the predominant loading on the adhesive would be a shear stress. Since it was likely that the rotor and stator would be subjected to heating from magnetic losses, I considered the adhesive shear strength at room temperature and at $180^{\circ} \mathrm{F}$.

- Peel strength. Adhesive-bonded joints are usually weakest when they are subjected to a peeling stress. Although the adhesive joints for the $10 \mathrm{kHz}$ FTS were designed to not be subjected to peeling or cleavage stresses, possible misalignments during assembly could result in those types of stresses being developed. ${ }^{5}$

- Ability to bond to metal and ceramic substrates. The materials involved are iron-alloy laminated soft magnetic cores (held together with their own adhesive), aluminum, stainless steel, and aluminum oxide.

- Cure time. I knew that after applying the adhesive, I would need to time to handle, assemble, and align the components being bonded together. I set thirty minutes as the minimum pot life that I was willing to accept.

- Room temperature cure. The components being assembled have different coefficients of thermal expansion (CTE), so I avoided adhesives that require an elevated temperature cure.

- Viscosity. I wanted a thixotropic ${ }^{6}$ paste that would not pour out of the joints during the assembly and curing steps. Also, I did not want too viscous of an

\footnotetext{
${ }^{5}$ For the reader not familiar with peeling and cleavage stresses, consider the following illustration. Peeling stress: starting with a piece of adhesive tape that has been placed glue-side down on a surface, grab a corner of the tape and lift it so that the tape bends at the zone of separation with the surface. The adhesive is put into tension, with an amplifying prying effect at the separation zone. Cleavage stress: starting with a rigid block that has been glued to a rigid surface, lift one corner or edge of the block. The adhesive is put into tension, but without the same amplifying effect at the separation zone as with peeling.

${ }^{6}$ Toothpaste is an example of a thixotropic fluid. In the absence of an external shear stress it does not flow. Upon application of an external shear stress that decreases in time, it flows at a constant strain rate [149].
} 
Table 4.1: Selected properties of the two-part epoxy structural adhesives that were final candidates for bonding the rotor and stator assemblies. EA 9360 was used for all of the adhesive bonds except for potting the steering coils. Data from Loctite Aerospace [94]. The potting compound is 3M Scotch-Weld ${ }^{T M}$ DP270 Clear [8] (pot life and cure time of 70 minutes and 48 hours, respectively).

\begin{tabular}{|c|c|c|c|c|c|c|c|}
\hline \multirow{2}{*}{$\begin{array}{c}\text { Loctite } \\
\text { Hysol }^{\circledR} \mathrm{p} / \mathrm{n}\end{array}$} & \multirow{2}{*}{$\begin{array}{l}\text { viscosity } \\
\text { (Poise) }\end{array}$} & \multirow{2}{*}{$\begin{array}{l}\text { viscosity } \\
\text { similar to: }\end{array}$} & \multirow{2}{*}{$\begin{array}{l}\text { pot life } \\
\text { (minutes) }\end{array}$} & \multirow{2}{*}{$\begin{array}{c}\text { cure time } \\
\text { at } 77^{\circ} \mathrm{F} \\
\text { (days) }\end{array}$} & \multirow{2}{*}{$\begin{array}{c}\text { peel } \\
\text { strength } \\
(\text { pli })\end{array}$} & \multicolumn{2}{|c|}{$\begin{array}{l}\text { tensile lap shear } \\
\text { strength (psi) }\end{array}$} \\
\hline & & & & & & $77^{\circ} \mathrm{F}$ & $180^{\circ} \mathrm{F}$ \\
\hline EA 9360 & 1200 & lard & 50 & $5-7$ & 50 & 5000 & 3000 \\
\hline EA 9392 & $\sim 5000$ & $>$ peanut butter & 75 & $5-7$ & 20 & 4300 & 2500 \\
\hline EA 9394 & 1600 & peanut butter & 90 & $3-5$ & 5 & 4200 & 3000 \\
\hline EA 9396 & 35 & honey-molasses & $75-90$ & $3-5$ & 25 & 3500 & 3200 \\
\hline
\end{tabular}

adhesive because I needed the adhesive to flow through multiple $50 \mu \mathrm{m}$ gaps during hand-assembly of the parts, and wanted to be sure of complete wetting of the surfaces that were being bonded. The adhesive manufacturers that I considered report viscosity in Poise, a unit that I did not have a working sense of. Table 4.2 provides the approximate viscosity of some common materials, which helped me envision how the different candidate adhesives would flow.

- Color. For the structural adhesives used to bond together the rotor and most of the stator components, color was not important. For the potting compound used to bond the steering coils to the stator core and stator housing, I wanted a clear adhesive so that I could see the coils after it cured.

\section{Theories of Adhesion and Strength of the Bonded-Joint}

Petrie describes adsorption and mechanical interlocking as being the more highly regarded theories of adhesion [122, page 64]. Adsorption is the result of molecular contact between the adhesive and the adherent, and involves secondary or Van Der Waals forces [122, page 59]. Mechanical interlocking is the result of the adhesive penetrating cavities in the surface of the adherent, displacing trapped air, hardening, and locking on [122, page 62]. In both cases, the adherent needs a higher surface energy than the liquid adhesive so that the adhesive can wet it. 
Table 4.2: Approximate viscosity (Poise) of some common materials at room temperature [54].

\begin{tabular}{|l|r|}
\hline Water & 0.01 \\
\hline Blood & 0.10 \\
\hline Ethylene Glycol & 0.15 \\
\hline Motor Oil (SAE 10) & 0.50 \\
\hline Corn Oil & 0.65 \\
\hline Maple Syrup & 1.5 \\
\hline Motor Oil (SAE 40) & 2.5 \\
\hline Motor Oil (SAE 60) & 10 \\
\hline Honey & 20 \\
\hline Molasses & 50 \\
\hline Chocolate Syrup & 100 \\
\hline Ketchup & 500 \\
\hline Lard & 1000 \\
\hline Peanut Butter & 1500 \\
\hline
\end{tabular}

The strength of an adhesively-bonded joint depends on the strength of the adherents, the strength of the bond between the adhesive and the adherent, and the cohesive strength of the adhesive. If the surfaces of the adherents have boundary layers, then they add additional interfaces to the joint structure. Petrie suggests viewing the joint as a structure consisting of these components and interfaces to gain insight into where the weakest link in the chain might lie [122, page 200].

\section{Surface Preparation}

Surface preparation of the adherents is crucial for obtaining a high-strength adhesivebonded joint. Preparation of the adherent involves at least two important steps: (1) removing contaminants that may decrease its surface energy, and (2) removing strongly attached weak boundary layers that may be on the surface [122, page 197]. Contamination of the surface with oil is an example of the first item. A weak oxide layer is an example of the second item. An optional third step is to alter the surface chemistry of the adherent to improve adhesion.

Adhesive-bonded joints are used extensively in the aerospace industry, where high 
strength and reliability are crucial, which led to the development of rigorous surface preparation standards $[82,81,80]$. Additional guidelines on surface preparation is provided by Petrie [122], Wegman [148], and Loctite Aerospace [94]. I did not have immediate access to all of the equipment and processes described in those standards and guidelines, and some of the steps — such as vapor degreasing and producing freshly anodized or acid etched surfaces - would have been overkill for this research. Therefore, I used the information to develop the following procedure for preparing the adherents to be bonded together for the $10 \mathrm{kHz}$ FTS, and tested it with some quick experiments using materials that I had on hand in our lab at MIT before using it on the $10 \mathrm{kHz}$ FTS:

1. Degrease the adherents by ultrasonic cleaning in a $10 \%$ (by volume) solution of Branson OC optical cleaning detergent [26] in water for five minutes.

2. Rinse with clean tap-water rinse. Air dry. Test for water-break ${ }^{7}$ with clean tap-water.

3. Using a piece of steel wool or fine sandpaper, mechanically abrade the surfaces to be bonded to remove any strongly attached weak boundary layers or contaminants.

4. Remove residues from the previous step by ultrasonic cleaning in a $10 \%$ (by volume) solution of Branson OC optical cleaning detergent [26] in water for five minutes.

5. Rinse with clean tap-water rinse. Air dry. Test for water-break with clean tap-water.

\section{Controlling Bond-Line Thickness}

Petrie [122, page 438] recommends a bond-line thickness in the range of $0.002-$ 0.006 inch, and mentions that below that range the strength usually decreases because

\footnotetext{
${ }^{7} \mathrm{~A}$ positive water-break test is when the water sheets the surface of the adherent. If the water forms beads on any part of the surface, then the adhesive will most likely not wet those parts.
} 
of gap tolerance and starvation of adhesive in the joint. A bond-line thickness above that range can lead to an unnecessary decrease in the mechanical stiffness of the assembly. For the rotor assembly, all of the bond-line thicknesses are 0.002 inch $(50 \mu \mathrm{m})$, except at the ends of the stiffeners where a much thicker bond-line was needed (refer to Section 4.5.2). The 0.002 inch bond-line thicknesses were controlled by a combination of thread-like plastic shims and built-in shims. This allowed me to firmly clamp together the components with out the risk of over-squeezing the joint and starving it of adhesive.

An alternate method for controlling the bond-line thickness is to add glass or polymeric micro-balloons to the epoxy. The diameter of the micro-balloons establishes the bond-line thickness. This method may be preferable for a high-production run of adhesively-bonded assemblies. However, in the case of the $10 \mathrm{kHz}$ FTS, the microballoon method would have been more trouble than it was worth, considering that the shimming method allowed me to use the epoxy as-is and was not difficult to do.

For the stator assembly, all of the bond-lines have an as-designed thickness of 0.002 inch, but many of the as-built bond-lines have a thickness greater than 0.006 inch due to the out-of-tolerance conditions of the stator cores. I was not too worried about this because of the nested nature of the adhesively-bonded components in the stator halves.

\subsubsection{Hand-Winding Coils}

In this section I briefly review the technique that I used to hand-wind the steering flux coils onto the bobbins. Although experienced coil winders may already know this method, I mention it for the benefit of someone new to the task, as I was.

If I had simply wrapped wire around the bobbin, starting from one end of the wire and working towards the other end, the starting end of the wire would have to pass around all of the winding layers in order to get out of the bobbin. This would have crowded all of the turns around the bobbin and made it difficult to fill the coil window area. Professor David Trumper at MIT suggested the following simple technique, which worked nicely. 

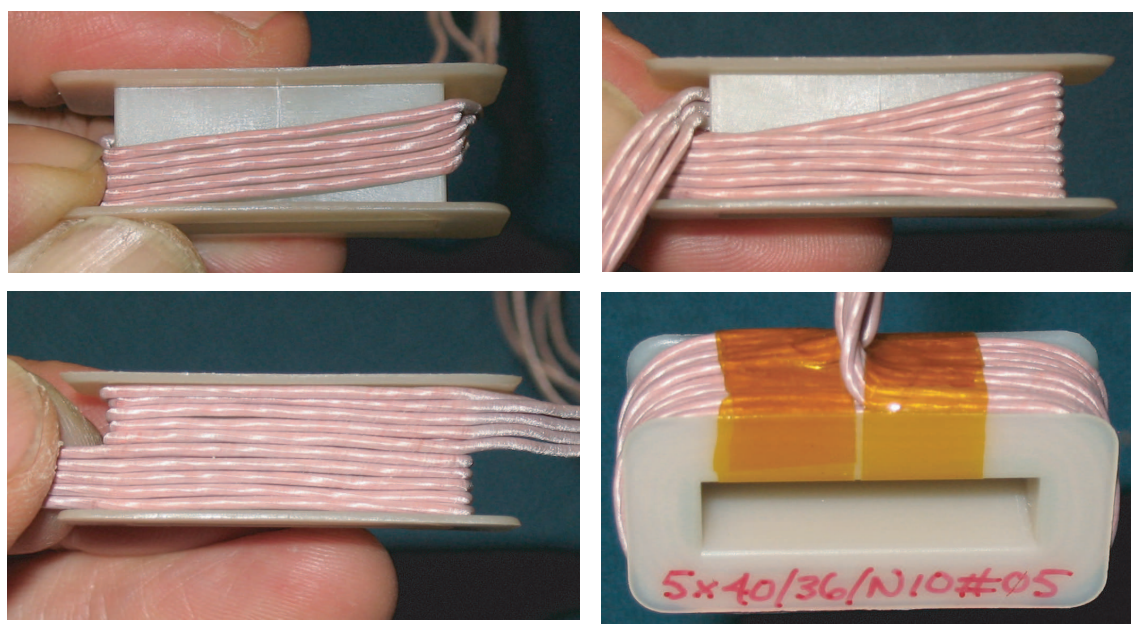

Figure 4-56: Hand-winding the steering flux coils using a counter-winding technique from the center of the wire bundle out towards the ends.

Referring to Figure 4-56, lay the center of the wire bundle diagonally across the bobbin. Wind the two ends of the wire bundle in opposite directions around the bobbin, filling each layer as you proceed. Both ends of the wire bundle will finish on the outside surface of the winding. Note that this method results in a diagonal bump in the finished winding. For the $10 \mathrm{kHz}$ FTS this was preferable to having lost coil window area.

\subsection{System Dynamics}

The dynamics for the $10 \mathrm{kHz}$ FTS include the presence of negative springs from the magnetic flux. Depending on the magnitude of the spring constants of these negative springs relative to the mechanical springs in the system, the rotor is either openloop stable or unstable. In addition to discussing the negative springs, this section describes simplified mechanical models for the rotary and linear modes that include those negative springs, the electrical dynamics for the $10 \mathrm{kHz}$ FTS, and considers the effect of changing the magnitude of the negative spring for the rotary mode. The measured dynamics for a low noise implementation and for a high acceleration implementation of the $10 \mathrm{kHz}$ FTS are discussed, along with the effects of adding electronic and mechanical damping to the system. Chapter 5 provides a detailed 
background for quantifying the characteristics of the magnetic circuits described in this section.

\subsubsection{Negative Springs from the Magnetic Fluxes}

The following thought-exercise and discussion is intended to provide an intuitive appreciation for the negative spring constant from the magnetic flux and the challenges for the control system in dealing with it. Imagine holding a magnet close to a magnetically permeable target and trying to maintain a constant-length gap. For a fairly large gap, if the magnet strength and area are not too great and your finger strength alone is sufficient, then the magnitude of the static stiffness provided by your fingers will dominate and you will be able to maintain an equilibrium. If you attempt to maintain a smaller gap, then you will need to develop a dynamic stiffness in the following manner. If your sensitivity to displacement, reaction time, and hand strength are sufficient, then you can provide adequate closed-loop control on the position of the magnet and prevent it from either being pulled fully out of engagement with the target or slammed into it. These are the challenges for the sensors, sampling rate, and actuator, respectively, for the $10 \mathrm{kHz}$ rotary fast tool servo. If, during your experiment with the magnet you were able to accurately sense the rate of change of the gap length, then you could anticipate whether or not a large displacement was about to occur and preemptively provide a higher balancing force in an attempt to catch the displacement before it ran away. This is what the lead compensator does for the control system used with the $10 \mathrm{kHz}$ rotary fast tool servo. The example just described is for a linear system, but it also applies to a rotary system. For instance, consider a see-saw arrangement consisting of a pair of magnets mounted on a beam, one on each side of the pivot, and the whole thing mounted on a magnetically permeable table. If both magnets are the same size, strength, distance from the pivot, and distance from the target, then the net torque acting on the beam will be balanced. A small perturbation to the angle of the beam will bring one of the magnets closer to the target and move the other magnet further from it. This causes a torque imbalance on the beam that unless countered, causes the beam to rotate it until the nearer magnet 
makes contact with the target.

Referring to the actuator for the $10 \mathrm{kHz}$ FTS and Figure 4-57, assume that all four air gaps have the same cross-sectional area, bias flux, and steering flux (steering coil currents $i_{12 T}$ and $i_{34 T}$ are equal). If the lengths of the air gaps and moment arms are equal, then a force and torque balance on the rotor exists and the rotor will be in an equilibrium position.

\section{Negative Torsional Spring from the Bias Flux}

Consider the case when the steering fluxes $\left(B_{12 T}\right.$ and $\left.B_{34 T}\right)$ in Figure 4-57 are zero. The bias flux density $\left(B_{B}\right)$ produces a normal-direction tensile stress between each of the four stator pole faces and the rotor. The tensile stress at each pole face is proportional to the square of the flux density in its air gap. Rotating the rotor as shown in Figure 4-58 causes an asymmetric distribution of the bias flux in each stator half and develops a torque on the rotor. Continued rotation of the rotor increases the asymmetry of the bias flux distribution and increases that torque. The torque increases in the same direction as the rotation of the rotor. Compare this to a mechanical torsional spring: the torque produced by a mechanical spring increases in the opposite direction of the displacement of the spring. Using the customary description for a linear mechanical spring of: Torque $=($ spring constant $) \times($ angular displacement $)$, the effective torsional spring from the bias flux has a negative spring constant. In Section 5.2.4 I show that for rotation angles small enough to allow using the approximation $\sin \theta \approx \theta$, the torque produced by the negative spring from the bias flux is a linear function of the rotation angle of the rotor from its neutral position, and the spring constant has a square-law dependence on the magnitude of the bias flux.

The flexure suspension for the rotor provides the system with a mechanical torsional spring. The over-constrained nature of the flexures cause the spring constant to increase as the rotor is rotated away from its neutral position. For small rotations about a given operating point, a linear approximation to the spring constant is valid. Furthermore, treating this mechanical spring as having a linear spring constant provides a conservative analysis when considering the rotational stability of the 


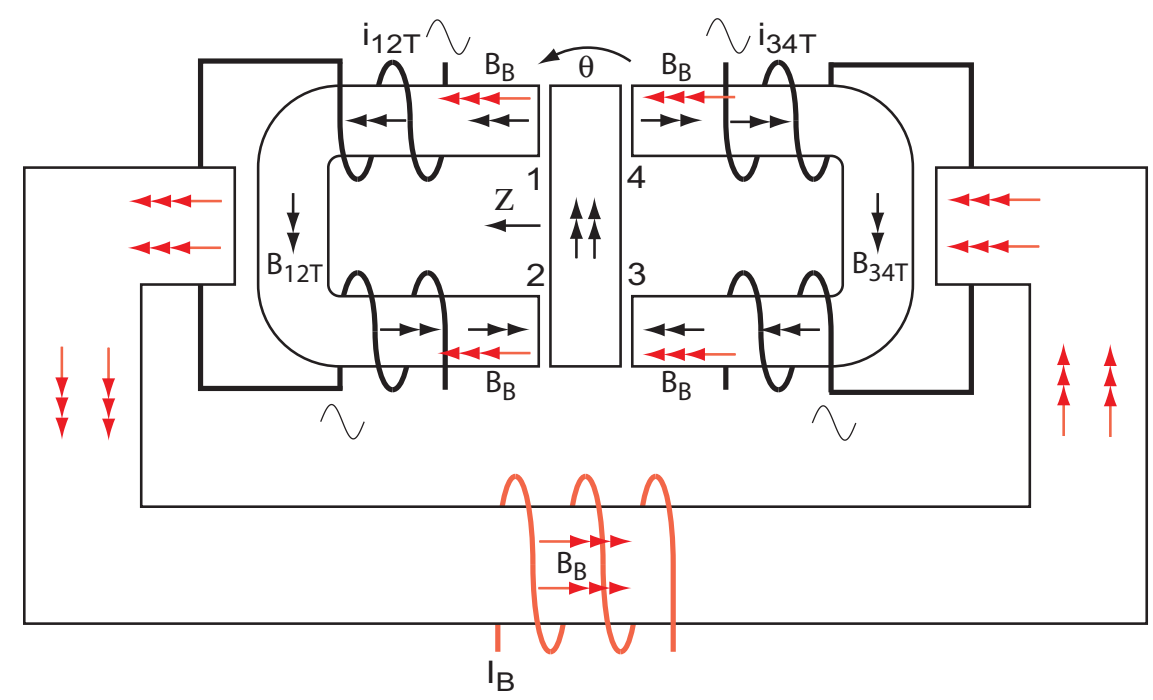

Figure 4-57: Sketch of the magnetic circuit showing distribution of steering and bias fluxes when rotor is in its neutral position (rotation and translation).

rotor. If the magnitude of the spring constant of the negative torsional spring from the bias flux is greater than that of the positive torsional spring from the flexures, then an open-loop rotation perturbation to the rotor will cause it to accelerate until it contacts the stator cores as shown in Figure 4-59.

\section{Negative Linear Spring from the Steering Fluxes}

Referring to Figure 4-57, consider the case when the bias flux $\left(B_{B}\right)$ is zero. The steering fluxes $\left(B_{12 T}\right.$ and $\left.B_{34 T}\right)$ produce a normal-direction tensile stress between each of the four stator pole faces and the rotor. The tensile stress at each pole face is proportional to the square of the flux density in its air gap. If the steering coil currents $\left(i_{12 T}\right.$ and $\left.i_{34 T}\right)$ are held constant, front/back translation of the rotor as shown in Figure 460 causes an increase in the steering flux density in the right-hand stator core and a decrease in the steering flux density in the left-hand stator core, developing a force on the rotor. Continued translation of the rotor increases the steering flux imbalance and increases that force. The force increases in the same direction as the translation of the rotor. This leads to a negative spring constant for the effective linear spring from the steering fluxes, which is treated as having a linear (force/displacement) relationship for small displacements about a given operating point. 


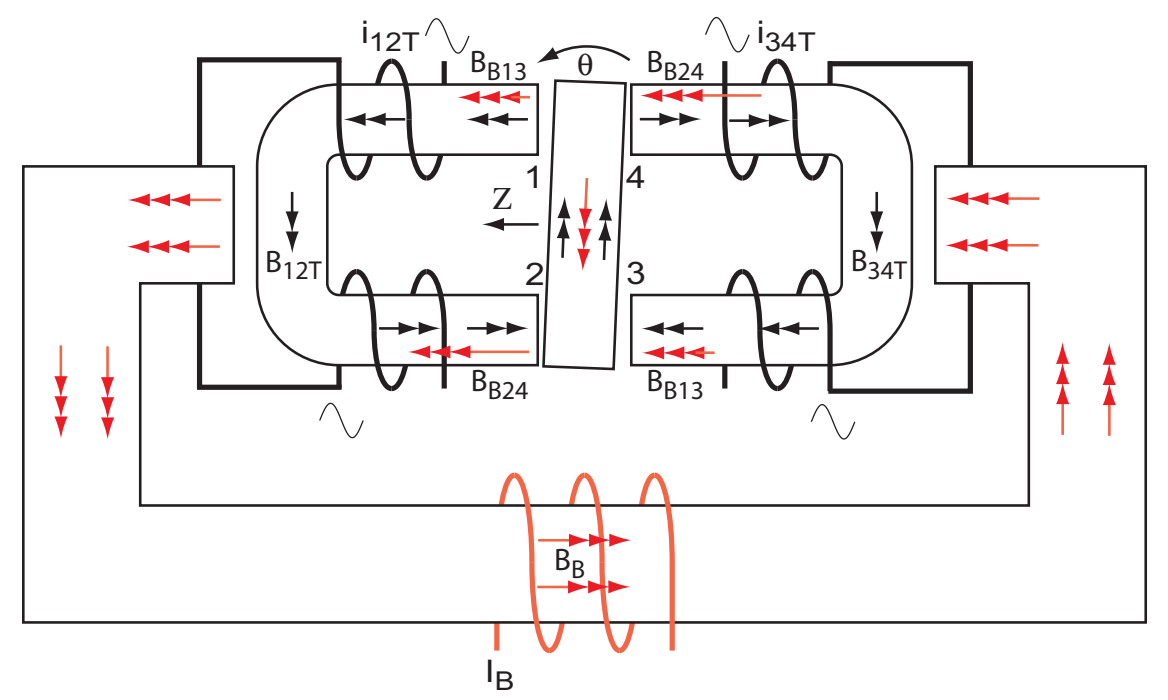

Figure 4-58: Sketch of the magnetic circuit showing large rotation of the rotor and asymmetric distribution of bias flux.

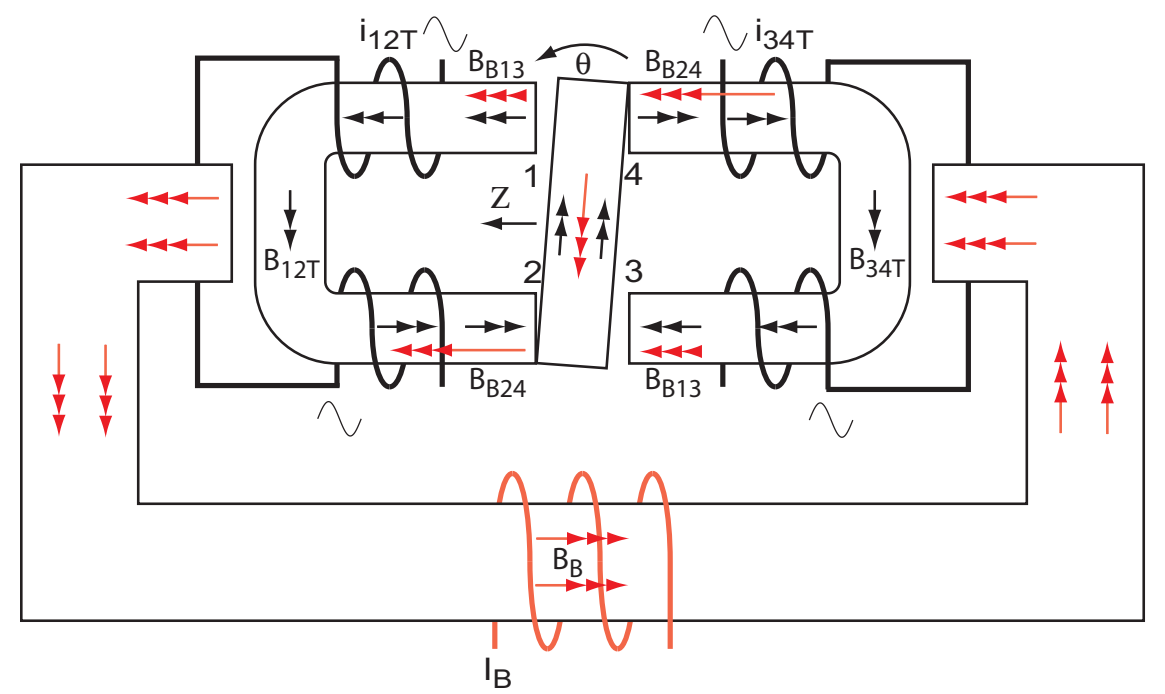

Figure 4-59: Sketch of the magnetic circuit showing extreme rotation of the rotor and attendant magnetic detent from asymmetric distribution of bias flux. Assumes that negative spring from bias flux is stronger than positive spring from torsional stiffness of flexures, which is the case for the $10 \mathrm{kHz}$ FTS. 


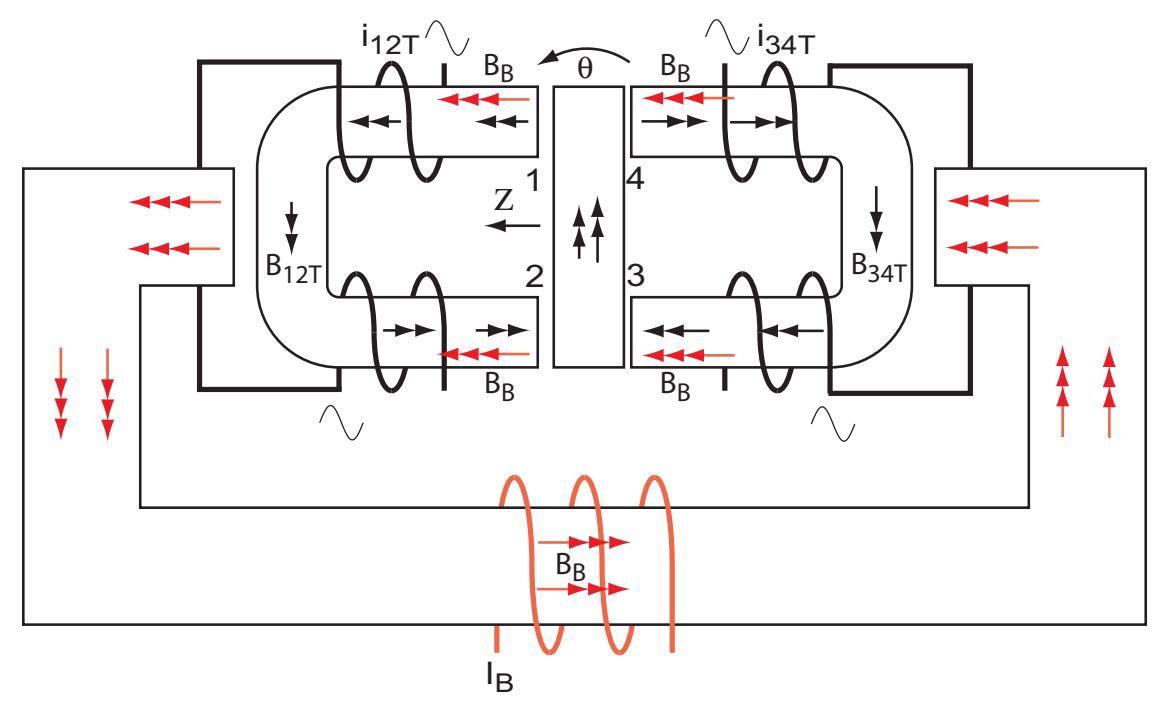

Figure 4-60: Sketch of the magnetic circuit showing small front/back translation of the rotor and asymmetric distribution of steering flux. Steering coil currents held constant with respect to Figure 4-57.

The flexure suspension for the rotor provides the system with a mechanical linear spring. If the magnitude of the spring constant of the negative linear spring from the steering fluxes is greater than that of the positive linear spring from the flexures, then an open-loop translation perturbation to the rotor will cause the rotor to accelerate until it makes contact with the stator as shown in Figure 4-61.

Referring to Figure 4-58, note that rotating the rotor does not significantly affect the reluctance for the steering flux paths, and the asymmetrical bias flux attendant with that rotation does not produce a net force on the rotor. Therefore, to first order the bias flux does not affect the stability of the translation mode of the rotor. Referring to Figure 4-60, note that having different magnitude steering fluxes on the right and left sides of the rotor does not produce a net torque on the rotor. Therefore, to first order the steering fluxes do not affect the stability of the rotation mode of the rotor.

\section{Summary for the Negative Springs from the Magnetic Fluxes}

If the magnitude of the spring constants (torsional and linear) from the flexures is greater than those from the magnetic flux at the operating point for the rotor, then 


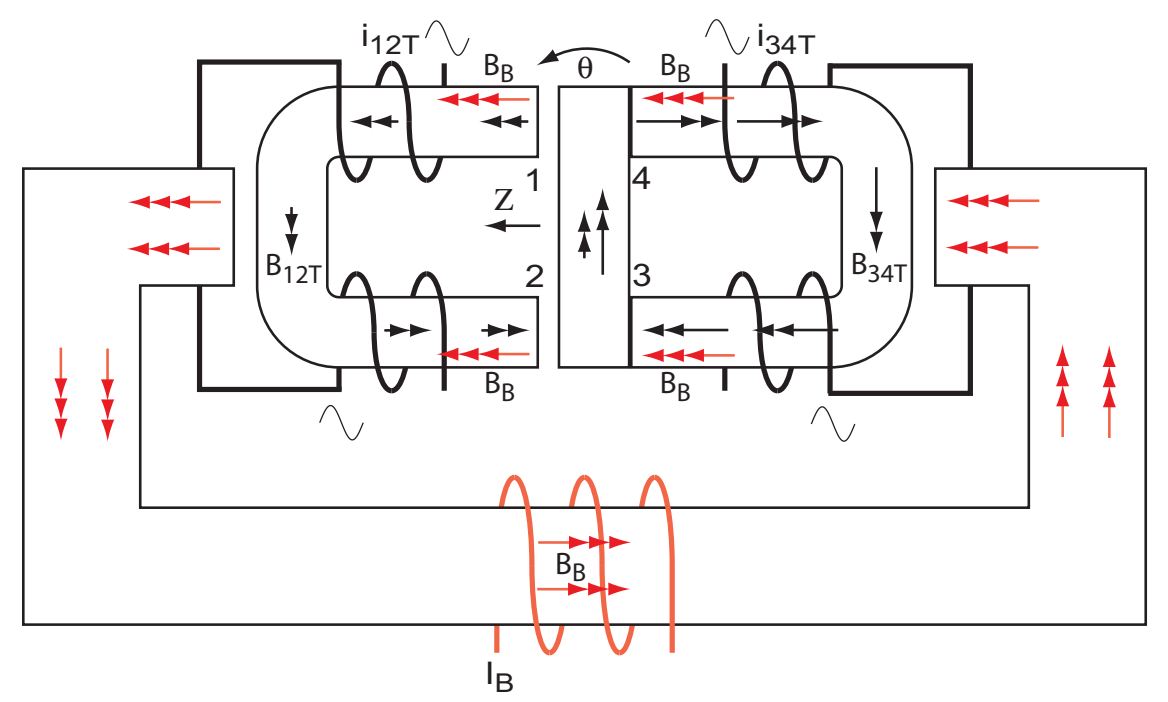

Figure 4-61: Sketch of the magnetic circuit showing extreme front/back translation of the rotor and attendant magnetic detent from asymmetric distribution of steering flux. Steering coil currents held constant with respect to Figure 4-57. Assumes that negative spring from steering flux is stronger than positive spring from radial stiffness of flexures, which is not the case for the $10 \mathrm{kHz}$ FTS.

for a small rotation or translation perturbation the flexures will provide sufficient increases in torque or force to allow them to compensate for the increases in torque or force from the magnetic fluxes, and the system will be stable. On the other hand, if the magnitude of either of the spring constants from the magnetic fluxes is greater than its corresponding mechanical spring from the flexures, then a small perturbation will cause the system to run away in rotation or translation, displacing the rotor to a mechanical hard stop. By design, the magnitude of the spring constant of the positive linear spring from the radial stiffness of the flexures is always greater than the magnitude of the spring constant of the negative linear spring from the steering fluxes. In essence, the rotor is always against the linear direction hard stops: the front/back flexure blades. Since the flexure suspension is designed to provide a torsionally compliant support to the rotor, the magnitude of the spring constant of the negative torsional spring from the bias magnetic flux can easily dominate over it, which leads to the two magnetic detents for the rotor: open-loop full rotation to one or the other set of hard stops, as depicted in Figure 4-59. Examining an earlier assumption, the nominal lengths of the four air gaps, their cross-sectional area, and 
the bias magnetic flux are not all balanced. This causes a net force and torque to be exerted on the flexure suspension, causing a rotation of the rotor to one of the two magnetic detents when the bias flux has at least a moderate value.

\subsubsection{Modelled Plant Dynamics}

Simplified lumped-parameter mechanical-magnetic models for the rotary and linear modes of the flexure-suspended rotor provide sufficient insight for designing the compensation for the $10 \mathrm{kHz}$ rotary fast tool servo control system. As with the $2 \mathrm{kHz}$ FTS, the electrical dynamics of the actuator are combined with the dynamics of the currentmode amplifier.

\section{Rotary Mode Mechanical-Magnetic Dynamics}

Figure 4-62 shows a model of the simplified mechanical-magnetic dynamics of the rotational degree of freedom for the rotor. The rotor has rotational inertia $I_{R}$, rotation angle $\theta$, and is acted on by the magnetic torque $T_{M}$ produced by the actuator. The rotor is connected to ground by two torsional springs, one from the flexure blades $k_{F R}$ and one from the bias magnetic flux $k_{M R}$, and by a torsional damper $b_{R}$. The mechanical portion of the torsional damper is primarily due to energy losses at the end connections of the flexures. The velocity-dependent back-EMF developed by the rotary mode of the actuator is treated as a torsional damper and makes up the electrical portion of $b_{R}$.

The rotary mode dynamics model in Figure 4-62 can be represented by the simple second-order system shown in (4.9):

$$
\theta=\frac{K_{R}}{\left[\frac{s^{2}}{\omega_{R}^{2}}+\frac{2 \zeta_{R}}{\omega_{R}} s+1\right]} T_{M}
$$

Depending on the relative magnitudes of $k_{F R}$ and $k_{M R}$, the two poles in (4.9) are either complex and in the left-half s-plane, both real and at the origin, or both real 


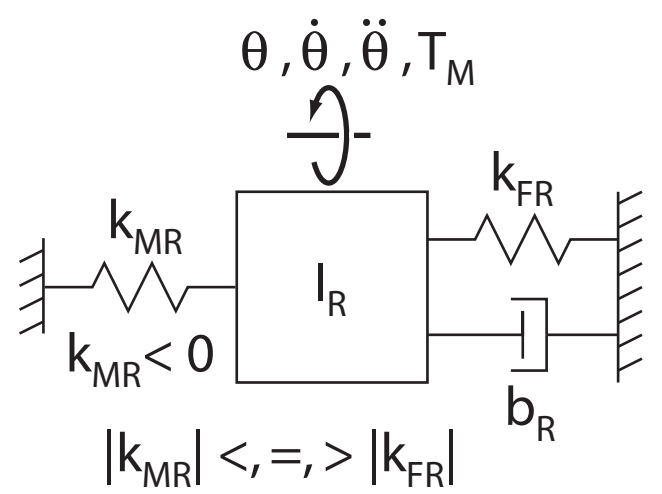

Figure 4-62: Simplified mechanical-magnetic dynamics of the rotational degree of freedom for the rotor.

and mirror images on either side of the imaginary axis. The location of the poles for the rotary mode dynamics, as a function of the bias flux, is explored in Section 4.6.3.

\section{Linear Mode Mechanical-Magnetic Dynamics}

Figure 4-63 shows a model of the simplified mechanical-magnetic dynamics of the translational degree of freedom for the rotor. The rotor has linear inertia $m_{R}$, linear position $z$, and is acted on by the magnetic force $F_{M}$ produced by the actuator. The rotor is connected to ground by two linear springs, one from the flexure blades $k_{F L}$ and one from the steering magnetic fluxes $k_{M L}$, and a linear damper $b_{L}$. The mechanical portion of the linear damper is primarily due to energy losses at the end connections of the flexures. The velocity-dependent back-EMF developed by the linear mode of the actuator is treated as a linear damper and makes up the electrical portion of $b_{L}$.

The linear mode dynamics model in Figure 4-63 can be represented by the simple second-order system shown in (4.10):

$$
z=\frac{K_{L}}{\left[\frac{s^{2}}{\omega_{L}^{2}}+\frac{2 \zeta_{L}}{\omega_{L}} s+1\right]} F_{M}
$$

Since the magnitude of $k_{F L}$ is always greater than that of $k_{M L}$ for the $10 \mathrm{kHz}$ FTS, the two poles in (4.10) are complex and in the left-half s-plane. 


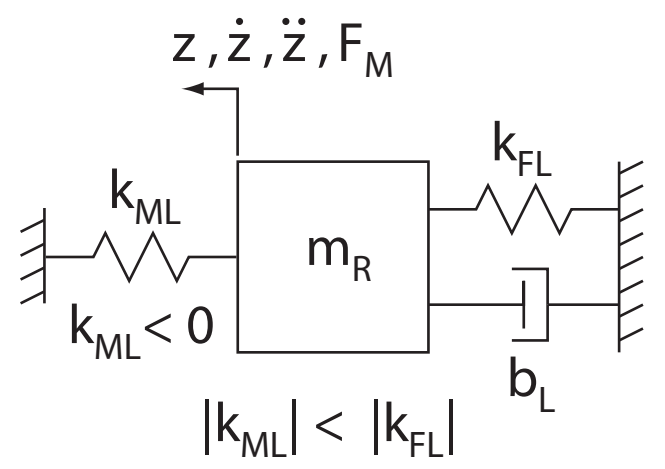

Figure 4-63: Simplified mechanical-magnetic dynamics of the translational degree of freedom for the rotor.

\section{Electrical Dynamics}

The main role of the actuator used in the $10 \mathrm{kHz}$ FTS is to produce a torque, so the current-mode amplifier is tuned to the electrical dynamics for the rotary mode of the hybrid rotary/linear actuator. The electrical dynamics for the linear mode are essentially the same as those for the rotary mode except for a different back-EMF constant and a slight difference in the inductance term that is discussed in Section 5.3.2. Since the back-EMF is captured in the damping terms for the mechanical dynamics, and the difference between the inductance for each mode is negligible for this thesis, the electrical dynamics for the linear mode are treated as being the same as those for the rotary mode.

Each stator half in the actuator is driven by its own current-mode amplifier. Figure 4-64 relates the electrical circuit for the rotary mode for one stator half to an equivalent block diagram that depicts the electrical dynamics. Each steering coil pair has resistance $R_{s c p}$ and inductance $L_{s c p}$. The resistance $R_{s c p}$ includes the resistance of the cables between the power amplifier and the coils. The velocity-dependent backEMF developed by the actuator is represented by the dependent voltage source $K_{T} \dot{\theta}$. Since each stator half provides one half of the motor torque constant $K_{T}$, the backEMF for each stator half is $\left(K_{T} / 2\right) \dot{\theta}$. The voltage $V_{s}$ at the top of the sense resistor $R_{s}$ provides a measure of the torque-producing current $I_{T}$ and is used in the feedback controller for the current-mode amplifier. Note that the sense resistor current $I_{s}$ is 

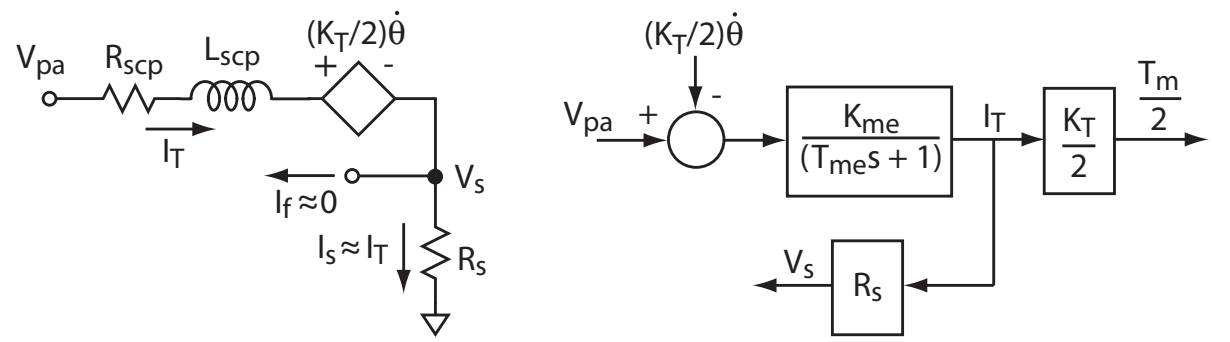

$$
\begin{aligned}
& K_{m e}=\frac{1}{\left(R_{s c p}+R_{s}\right)} \\
& T_{m e}=\frac{L_{s c p}}{\left(R_{s c p}+R_{s}\right)}
\end{aligned}
$$

Figure 4-64: Electrical circuit and equivalent block diagram for the electrical dynamics of the rotary mode of one stator half in the actuator used in the $10 \mathrm{kHz}$ FTS.

essentially equal to the torque-producing current $I_{T}$ because the feedback current $I_{f}$ goes to a high impedance input on an operational amplifier, and is essentially zero. The back-EMF is modelled as a disturbance voltage on the output voltage $V_{p a}$ of the power amplifier. This allows not having to explicitly break out a measure of the rotor angular velocity $\dot{\theta}$ when considering the mechanical dynamics for the system. The damping provided by feedback of the rotor angular velocity — in the form of the back-EMF - is treated implicitly by the damping term $b_{R}$ shown in Figure 4-62.

Figure 4-65 is a block diagram showing the electrical dynamics of one stator half combined with the electrical dynamics of the current-mode amplifier used to drive that stator half. The first two blocks in the forward path of the current loop are the compensation and the power operational amplifier circuit for the amplifier. Details on that compensation and the performance of the current loop are discussed in Section 4.7.1. The gains $K_{c}$ and $K_{s f}$ are chosen to balance the command and feedback signals, respectively, into the current compensation circuit. The back-EMF as a disturbance on $V_{p a}$ is omitted because it is captured in the damping terms for the mechanical dynamics. Since the rotary and linear modes have essentially the same inductance, the output of the block diagram is a general current $I_{s c p}$ for the series-connected pair of steering coils that is applicable to both modes of the actuator.

\subsubsection{Measured Plant Dynamics}

The tool tip position for the $10 \mathrm{kHz}$ FTS is primarily controlled by the rotary mode of the actuator. In this section I explore moving the open-loop poles for that mode by 


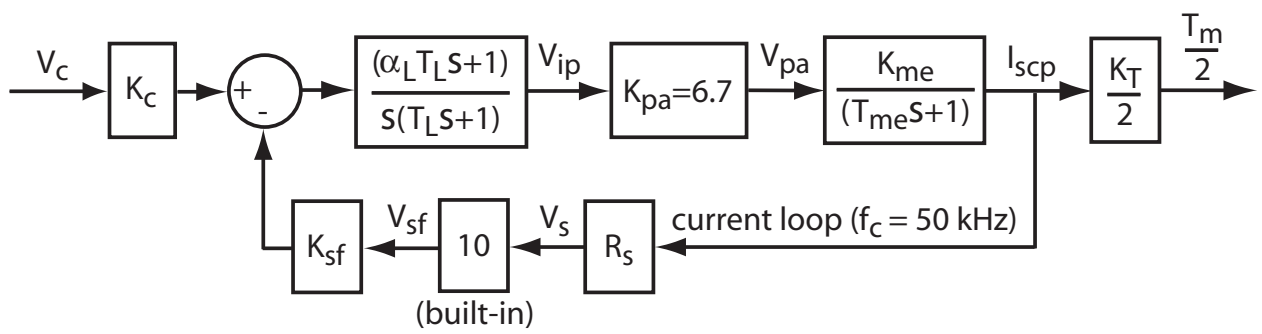

Figure 4-65: Block diagram combining the electrical dynamics of one stator half with the electrical dynamics of its current-mode amplifier.

adjusting the bias flux, compare the measured frequency response for the plant to the predictions from the FEA model, and discuss the effects of electronic and mechanical damping on the radial motion of the rotor.

\section{Moving the Open-Loop Rotary Mode Poles}

One of the benefits of using a coil to provide the bias flux instead of a permanent magnet is that it allows adjusting the magnitude of the bias magnetic flux. This degree of freedom was valuable during early tests when identifying the dynamics of the $10 \mathrm{kHz}$ FTS, and reduced the risk of damaging the machine while developing the control system. Starting with a small bias flux, I was able to measure the smallsignal plant dynamics for the case where the magnitude of the spring constant of the negative torsional spring from the bias flux was less than that of the positive torsional spring from the flexure suspension. Using the measured plant dynamics instead of the modelled plant dynamics, I designed a controller that allowed me to maintain a stable system while incrementally increasing the bias flux until the bias flux torsional spring was stronger than the flexure suspension torsional spring. Figure 4-66 is a sketch showing how the relative magnitudes of these two torsional springs affects the location of the two open-loop poles for the rotary mode dynamics.

Figures 4-67 through 4-72 are Bode plots of the measured negative loop transmission for the tool position using different magnitudes of bias flux. The negative loop transmission for these Bode plots includes a lead compensator centered on $1 \mathrm{kHz}$ (zero at $320 \mathrm{~Hz}$ and pole at $3.2 \mathrm{kHz}$ ) and the mechanical damping described in Section 4.5.9. Note how the natural frequency of the system changes as the spring constant for the 


$$
\begin{aligned}
& k_{\text {flex }}>0 \\
& k_{\text {mag }}<0 \\
& \omega_{n}=\sqrt{\frac{k_{\text {flex }}+k_{\text {mag }}}{I_{R}}}
\end{aligned}
$$
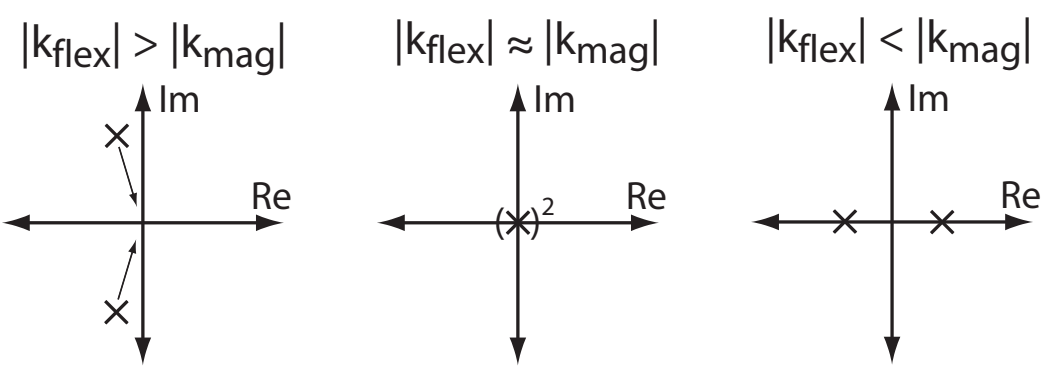

Figure 4-66: Sketch showing how the relative magnitudes of the spring constants for the torsional springs from the flexures and from the bias flux affects the two open-loop poles for the rotary mode dynamics.

torsional spring from the bias flux is varied. The DC gain is not the same in each plot because of one of the following two reasons. First, the motor constant for the rotary mode of the actuator changes linearly with the bias current and it was not important during these tests to exactly adjust the proportional gain for each case. Second, as the magnitudes of the two spring constants approach each other, the system behavior approaches that of a free mass, which has a theoretically infinite DC gain. The inset unscaled pole-maps are for illustrative purposes only: to suggest the approximate relationship of the two open-loop poles for the rotary mode.

In Figures 4-67 and 4-68 the bias flux is set low enough that the torsional spring from the flexure suspension dominates and the system looks like a simple mass-springdamper system. The magnitude plot has prominent peaking and the low-frequency phase angle is near the expected value of $0^{\circ}$.

In Figures 4-69 and 4-70 the torsional spring from the bias flux is becoming significant compared to the torsional spring from the flexure suspension. Note how the peak of the magnitude plots begin to flatten, especially in Figure 4-70, as the system begins to act like a free mass.

In Figures 4-71 and 4-72 the torsional spring from the bias flux dominates and the two poles lay on the real axis. The system now looks like two over-damped first-order poles, and the low-frequency phase angle approaches its DC value of $-180^{\circ}$. 


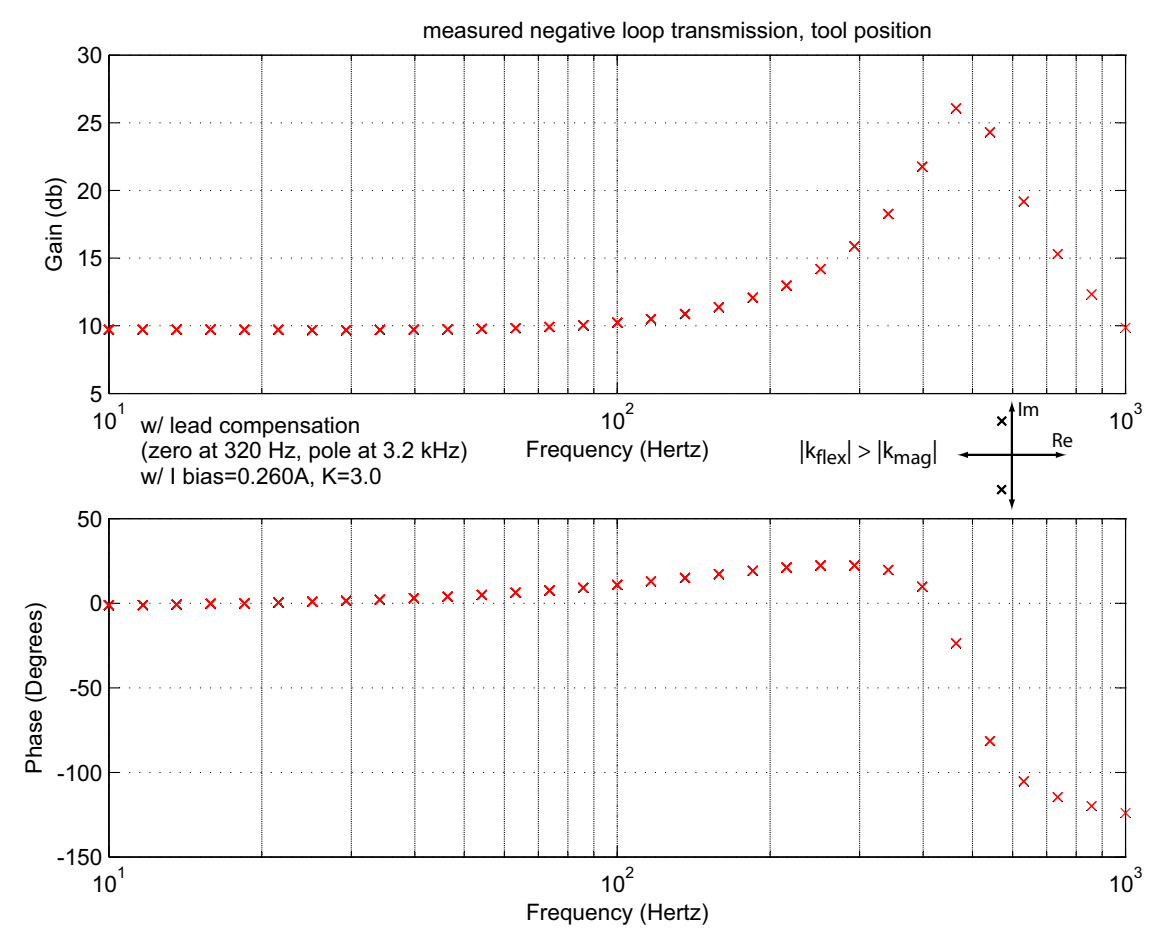

Figure 4-67: Bode plot of the measured negative loop transmission for the tool position using a bias flux coil current of 0.260 A. Torsional spring from the flexure suspension dominates.

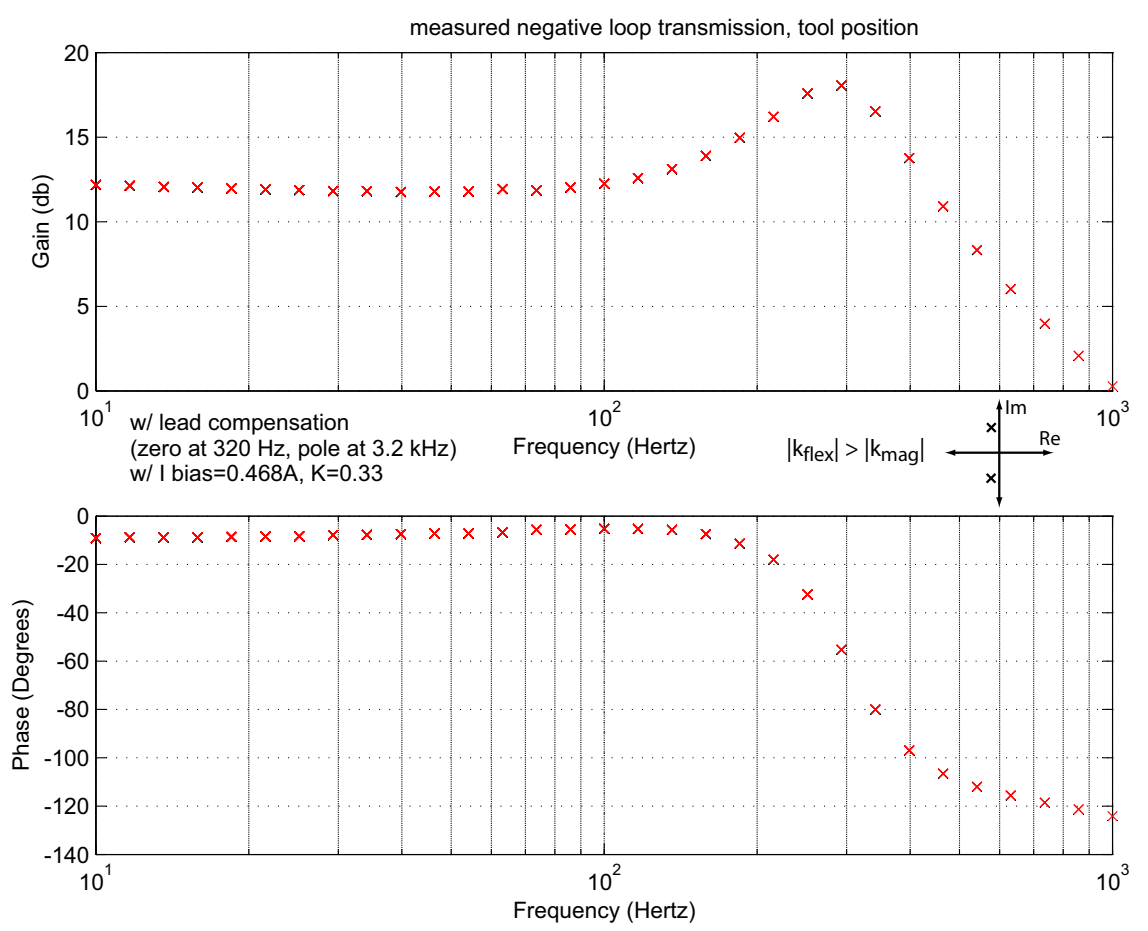

Figure 4-68: Bode plot of the measured negative loop transmission for the tool position using a bias flux coil current of 0.468 A. Torsional spring from the flexure suspension dominates. 


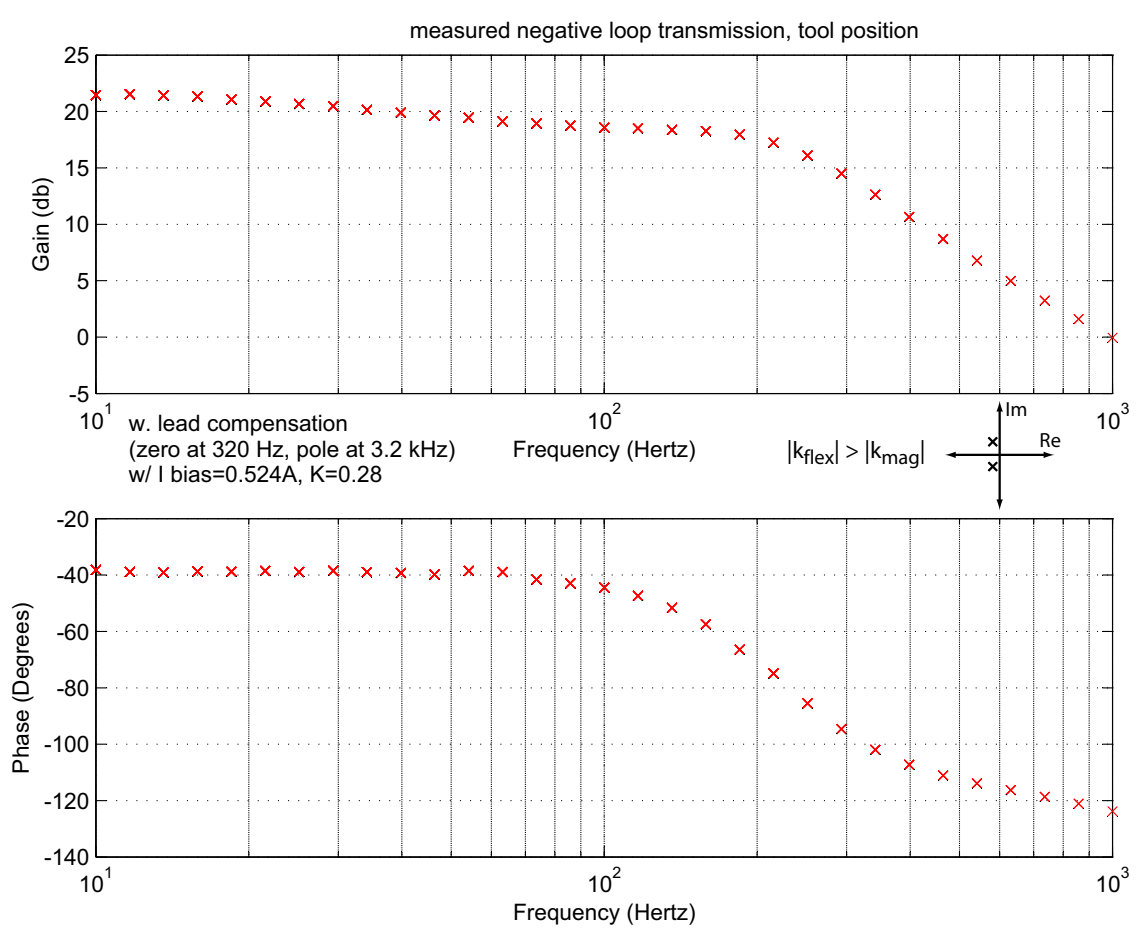

Figure 4-69: Bode plot of the measured negative loop transmission for the tool position using a bias flux coil current of $0.524 \mathrm{~A}$. Torsional springs from the bias flux and flexure suspension are comparable.

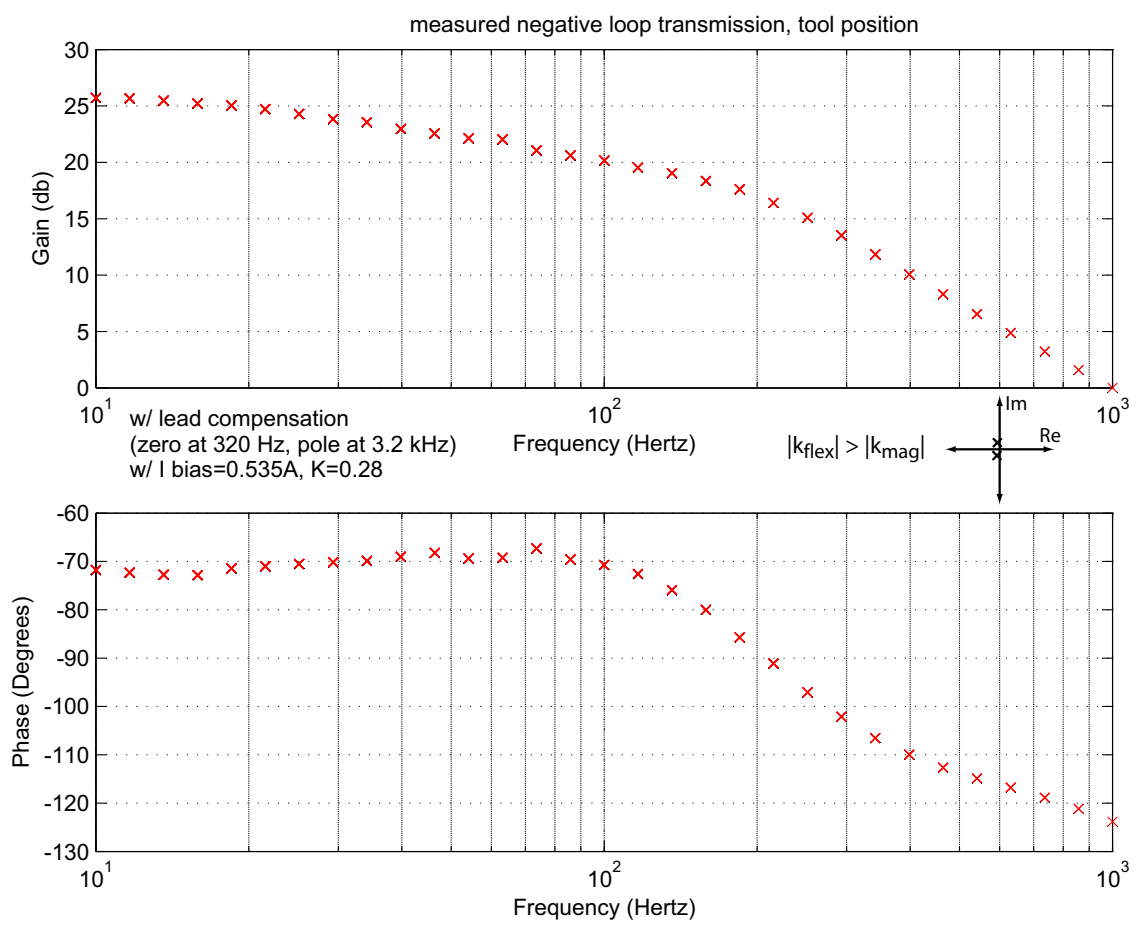

Figure 4-70: Bode plot of the measured negative loop transmission for the tool position using a bias flux coil current of $0.535 \mathrm{~A}$. Torsional springs from the bias flux and flexure suspension are comparable. 


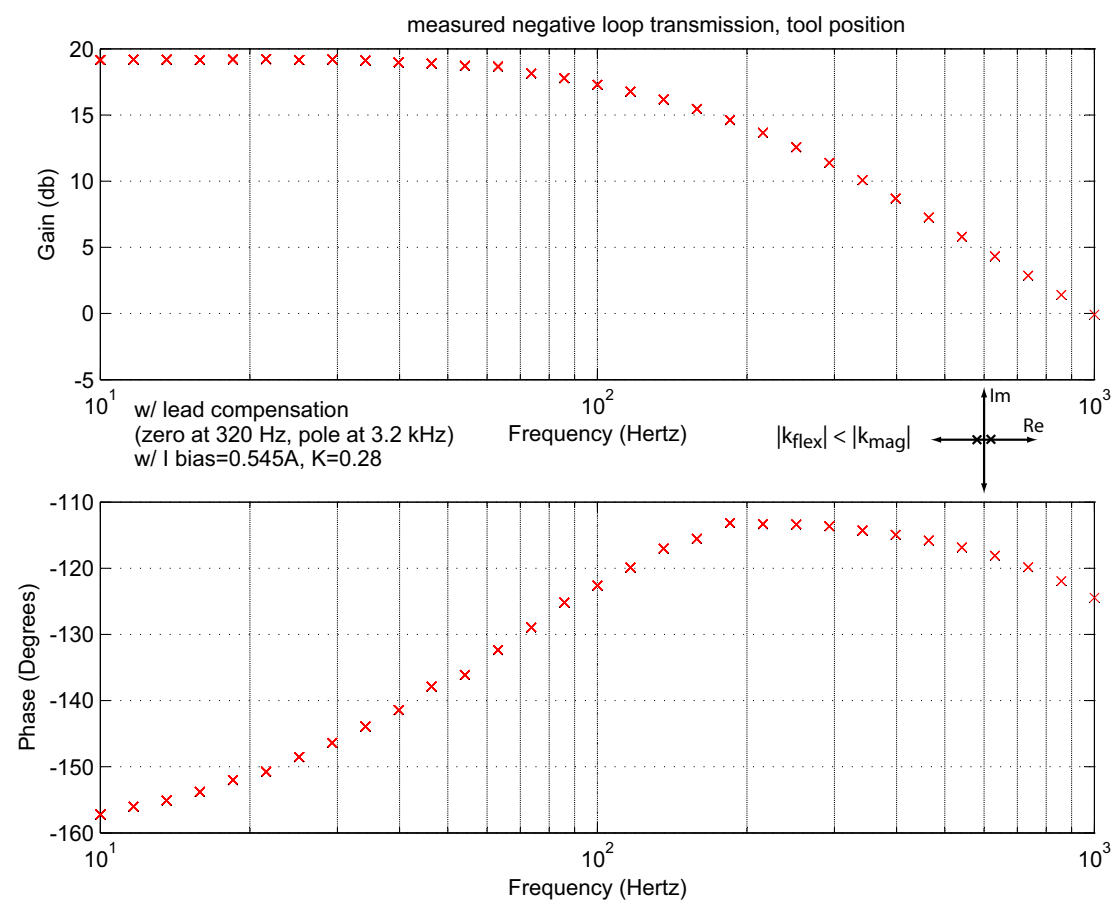

Figure 4-71: Bode plot of the measured negative loop transmission for the tool position using a bias flux coil current of $0.545 \mathrm{~A}$. Torsional spring from the bias flux dominates. The low-noise implementation of the $10 \mathrm{kHz}$ FTS uses a bias coil current of $0.545 \mathrm{~A}$.

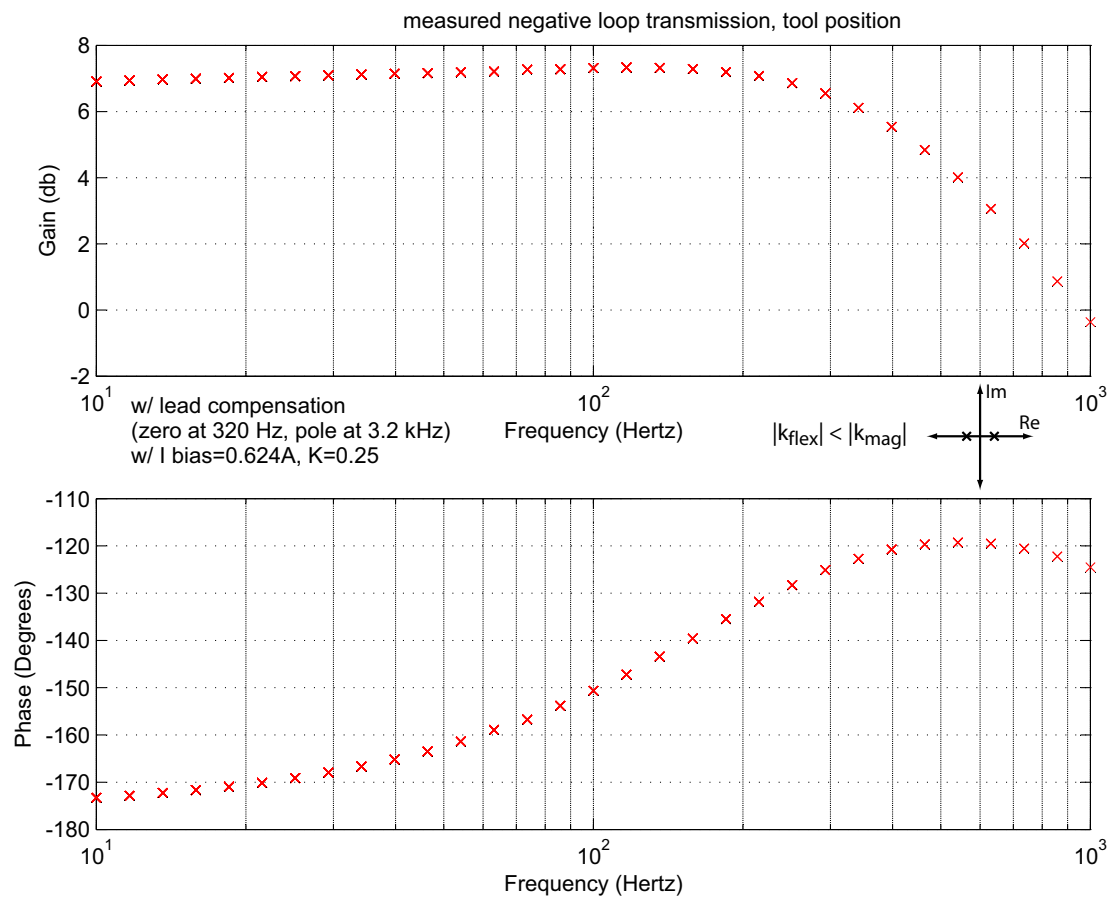

Figure 4-72: Bode plot of the measured negative loop transmission for the tool position using a bias flux coil current of 0.624 A. Torsional spring from the bias flux dominates. 


\section{Measured Flexible Modes of Rotor}

Figure 4-73 is a Bode plot of the measured plant for the tool position using a moderate current of $0.545 \mathrm{~A}$ in the bias flux coil, which sets the torsional spring from the flux slightly less dominant than that from the flexure suspension. The rigid-body torsional mode looks like a simple mass-spring-damper system with its low-frequency phase angle near $0^{\circ} .8$ This is referred to as the "low-noise" implementation because it results in lower position noise at the tool tip than does a more aggressive highacceleration implementation.

The rigid body and first non-rigid body torsional modes for the rotor predicted by the FEA model in Section 4.5.8 are mapped to their respective resonant peaks in Figure 4-73. Details on the higher frequencies are provided by Figure 4-74, which includes the second non-rigid torsional mode of the rotor. The measured frequencies for the rotor modes are lower than the predicted frequencies - most likely because the FEA model uses idealized fixed-ends for the flexures and does not include the compliance due to the epoxy in the bonded joints.

Figure 4-75 is a Bode plot of the measured plant for the tool position using a high current of $1.5 \mathrm{~A}$ in the bias flux coil, which makes the torsional spring from the flux considerably more dominant than that from the flexure suspension. The rigid-body torsional mode looks like two over-damped first-order poles with its lowfrequency phase angle near $-180^{\circ}$, which is consistent with a right-hand plane openloop pole. This is referred to as the "high-acceleration" implementation because it allows a higher peak flux density in the rotor/stator air gaps than does the low-noise implementation. Notice that the high frequency resonant peaks did not move when the current in the bias flux coil was increased from $0.545 \mathrm{~A}$.

\footnotetext{
${ }^{8}$ This result appears to be in contradiction with the measurement results shown in Figure 4-71, where a current of $0.545 \mathrm{~A}$ in the bias flux coil made the torsional spring from the bias flux slightly more dominant than that from the flexure suspension. Comparing the systems shown in Figures 471 and 4-70, a $2 \%$ change in current in the bias flux coil caused a shift in which torsional spring dominates. The dividing line between which spring dominates is likely finer, and evidently not time invariant.
} 

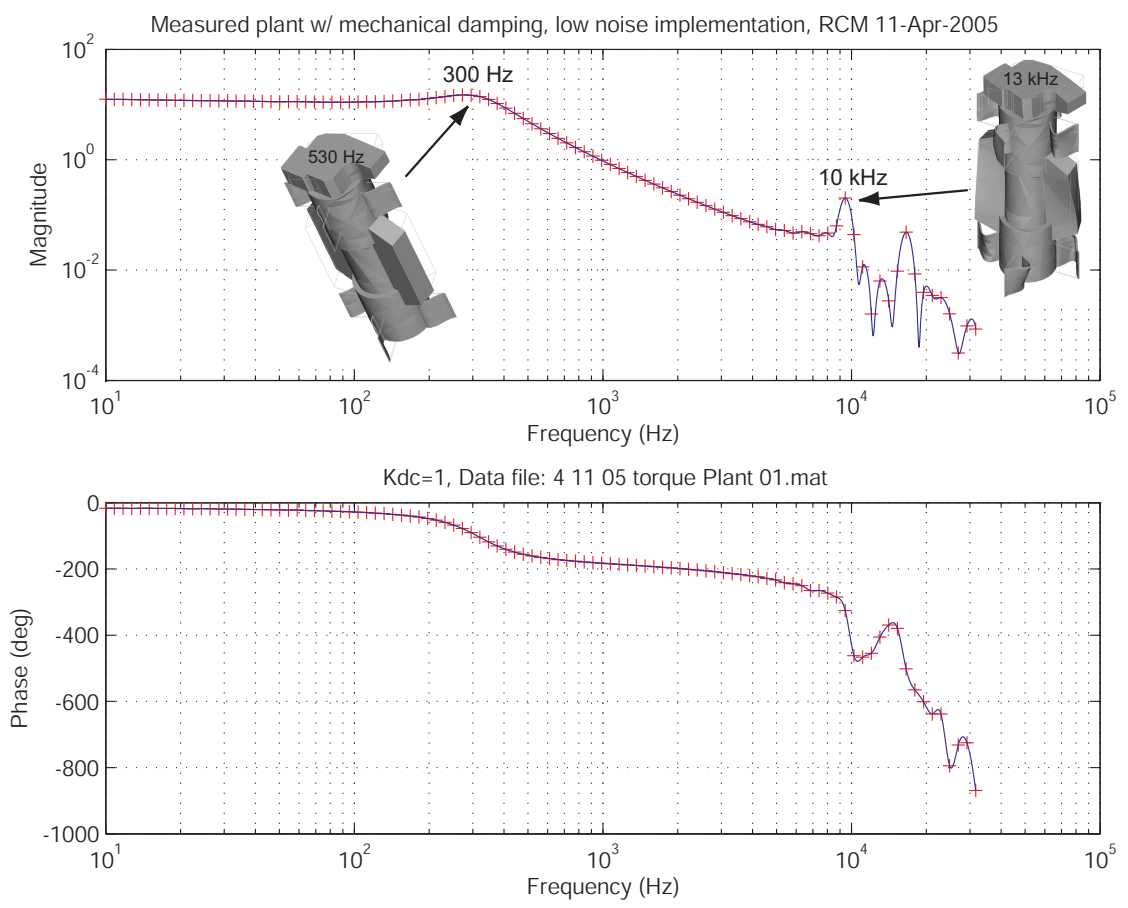

Figure 4-73: Bode plot of the measured plant for the tool position using a moderate current of $0.545 \mathrm{~A}$ in the bias flux coil (low noise implementation), with FEA model predictions mapped to resonant peaks.
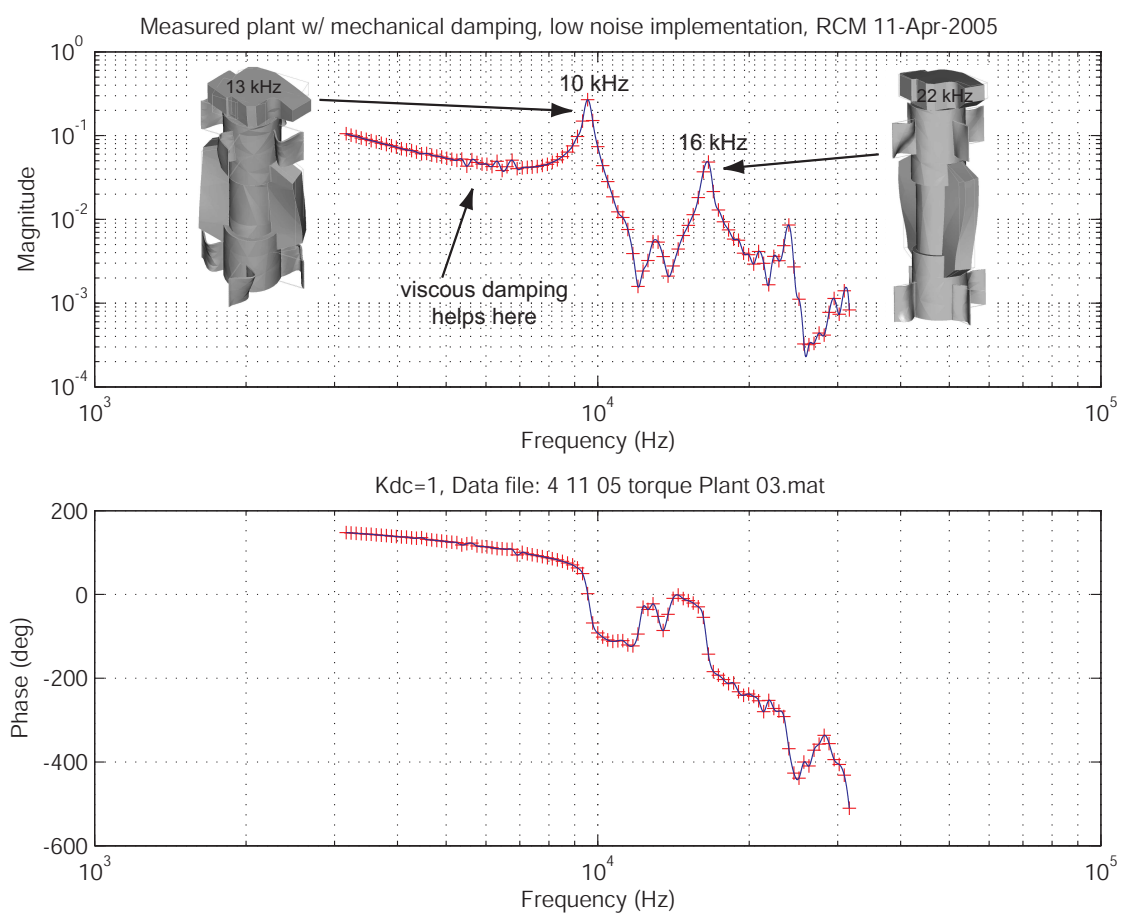

Figure 4-74: High frequency detail for a Bode plot of the measured plant for the tool position using a moderate current of $0.545 \mathrm{~A}$ in the bias flux coil (low noise implementation), with FEA model predictions mapped to resonant peaks. 

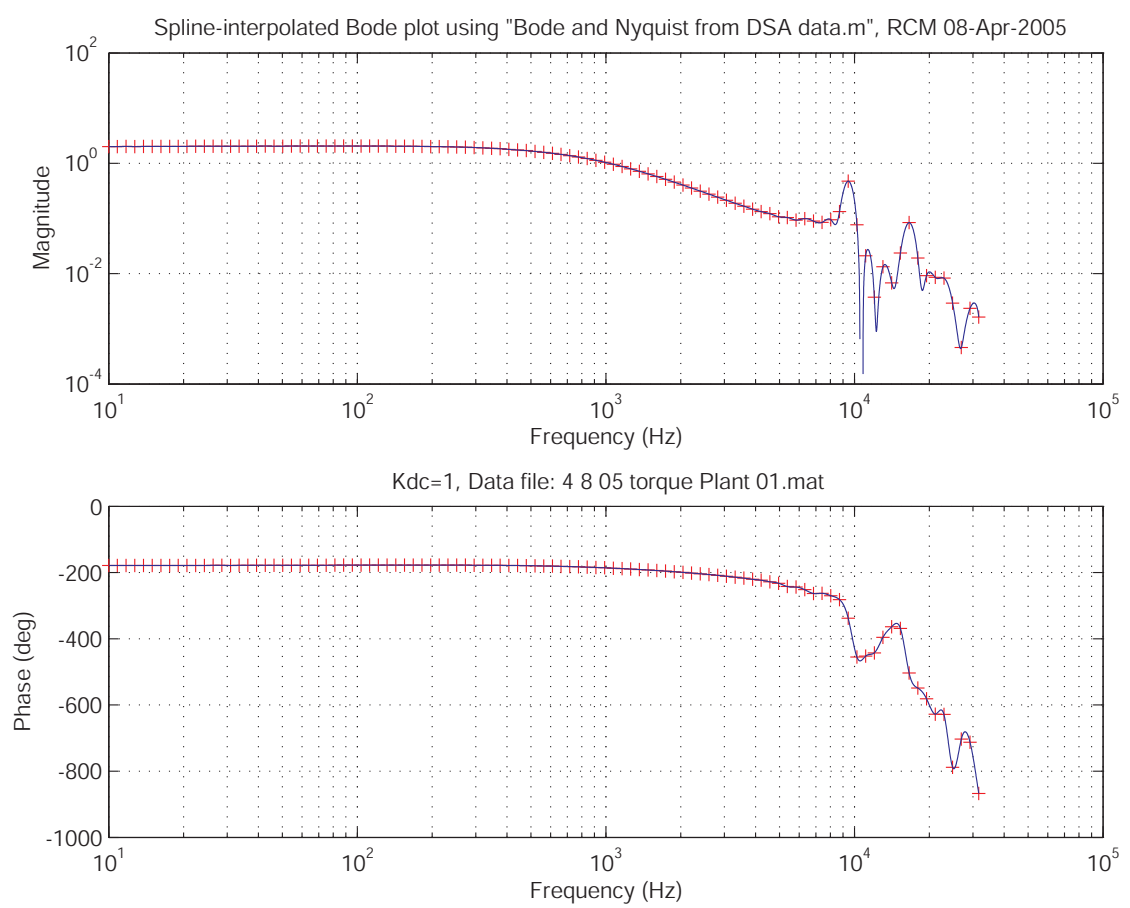

Figure 4-75: Bode plot of the measured plant for the tool position using a high current of $1.5 \mathrm{~A}$ in the bias flux coil (high acceleration implementation).

\section{Effects of Added Damping}

Both electronic and mechanical damping were used to reduce unwanted motion of the rotor due to the front/back translation and tipping vibration modes described in Section 4.5.8. The linear mode of the actuator was used to provide electronic damping to the translation mode only; the actuator does not have a tipping mode degree of freedom. The addition of grease between the tool holder and the centerline capacitance gauge described in Section 4.5.9 provides mechanical damping to both the translation and tipping modes. By knowing which modes each type of damping affects, the measured frequency for each mode is deduced from the observed ring frequency.

The affect of damping was measured by plucking the top edge of the tool holder with my thumbnail, just above the measurement target for the centerline capacitance sensor in the z-direction, and capturing the displacement measured by that sensor with an oscilloscope. In the end, the mechanical damping was superior to the electronic damping, and sufficient by itself to allow robust controller systems for the 


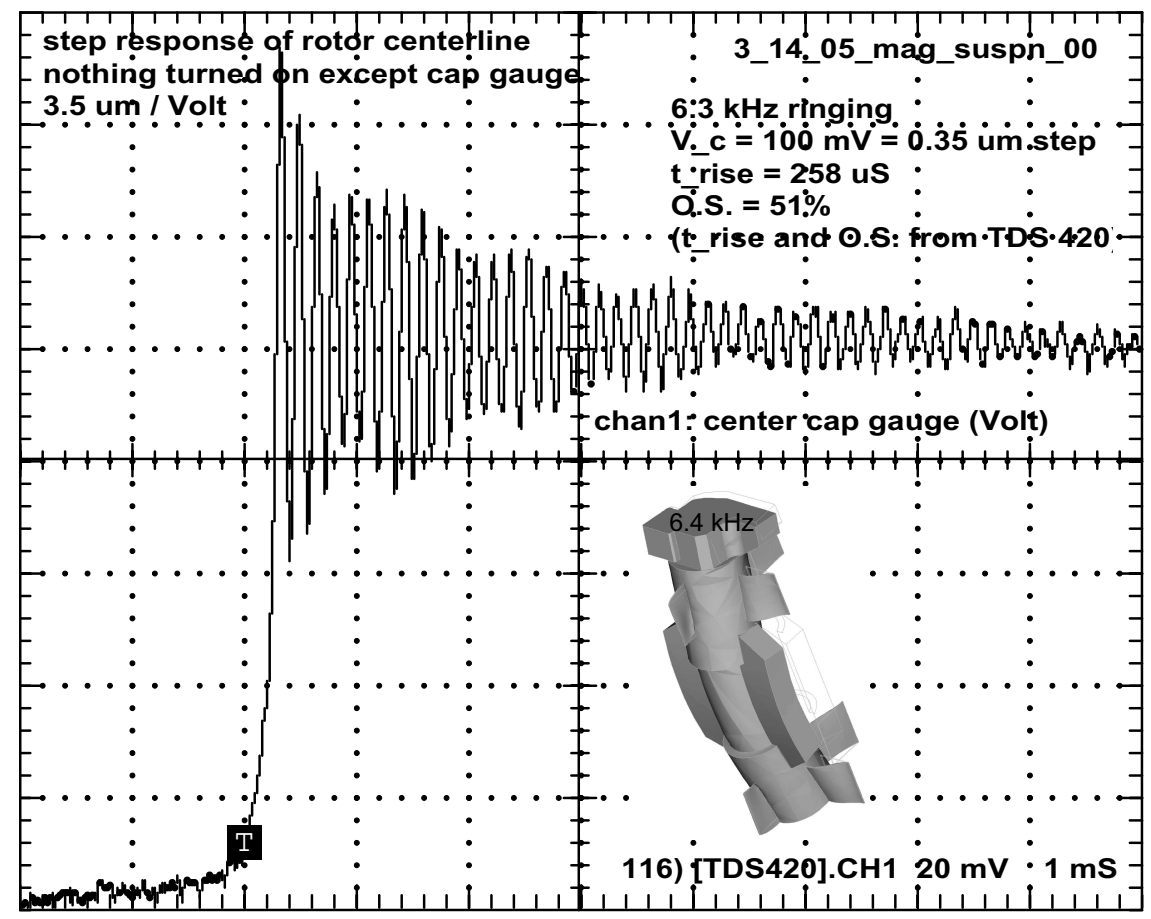

Figure 4-76: Measured step response of the rotor with no damping. Input is an external mechanical step in force on the tool holder at the centerline in the $\mathrm{z}$-direction.

low-noise and high-acceleration implementations of the $10 \mathrm{kHz}$ FTS.

Figure 4-76 is the measured step response of the rotor with no damping. Using subsequent measurements with damping, the measured ring frequency of $6.3 \mathrm{kHz}$ in Figure 4-76 is associated with the front/back translation mode of the flexuresuspended rotor.

Figure 4-77 is the measured step response of the rotor with electronic damping. The measured ring frequency of $5.5 \mathrm{kHz}$ is associated with the front/back tipping mode of the flexure-suspended rotor because the actuator cannot control that mode. Additional measurements discussed later in this section support this conclusion.

Figure 4-78 is the measured step response of the rotor with mechanical damping. Note the significant reduction in ringing compared to the electronic damping shown in Figure 4-77.

Figure 4-79 is the measured step response of the rotor with mechanical-pluselectronic damping. Note that there is no significant advantage to including the electronic damping with the mechanical damping. In fact, the electronic damping 


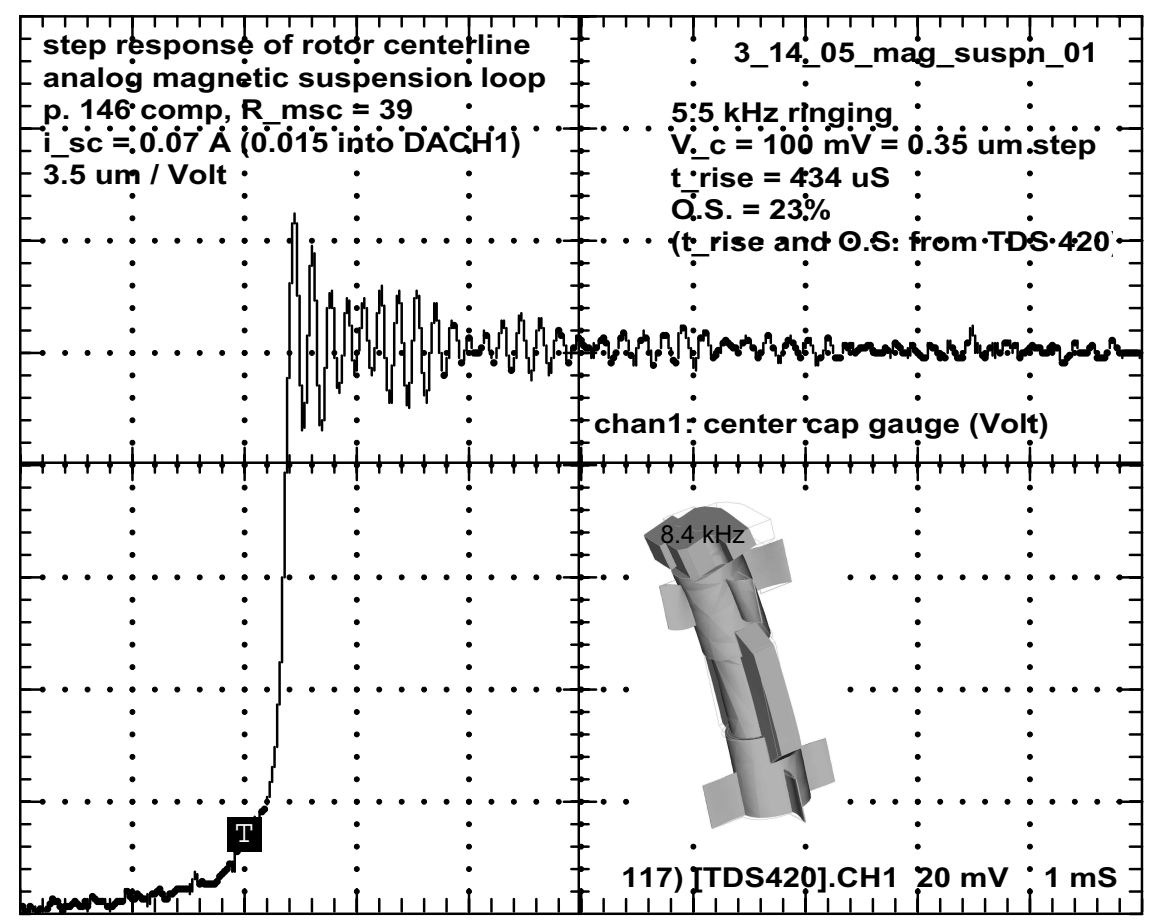

Figure 4-77: Measured step response of the rotor with electronic damping. Input is an external mechanical step in force on the tool holder at the centerline in the z-direction.

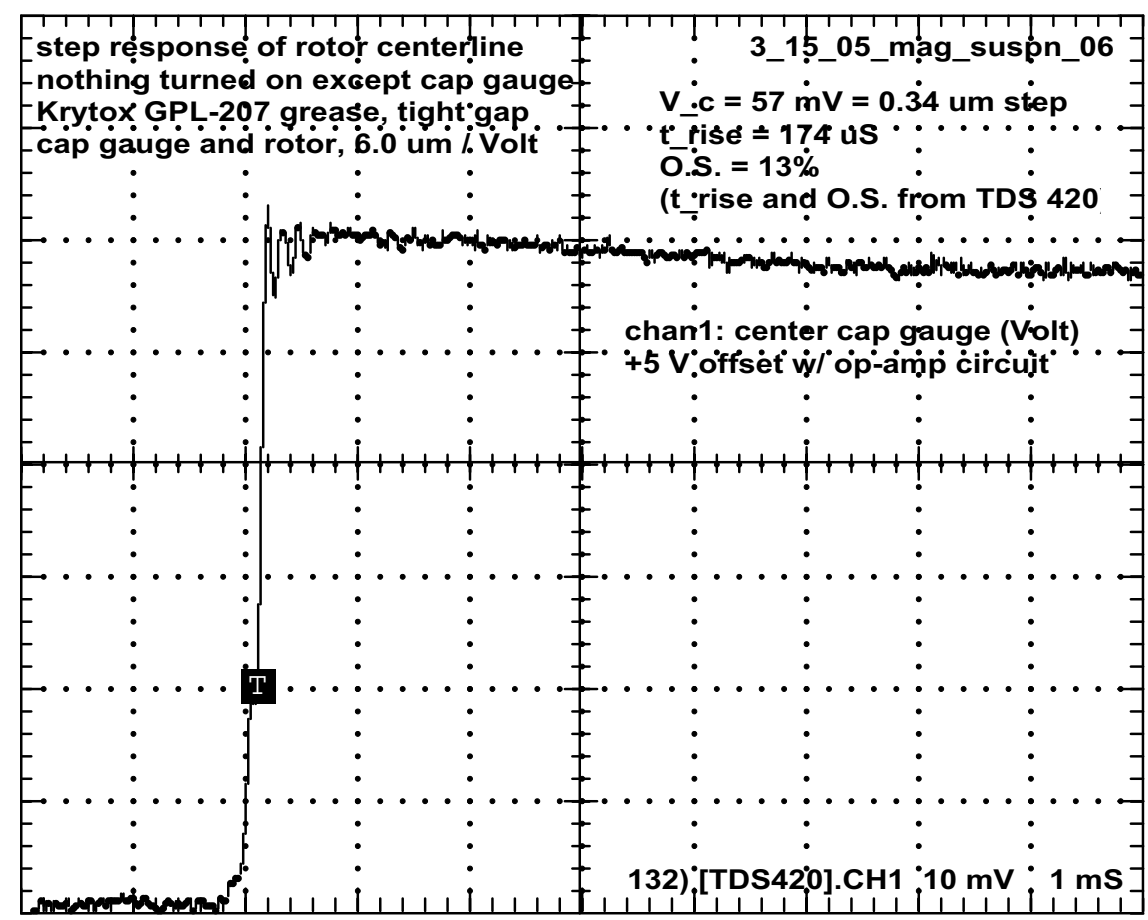

Figure 4-78: Measured step response of the rotor with mechanical damping. Input is an external mechanical step in force on the tool holder at the centerline in the z-direction. 


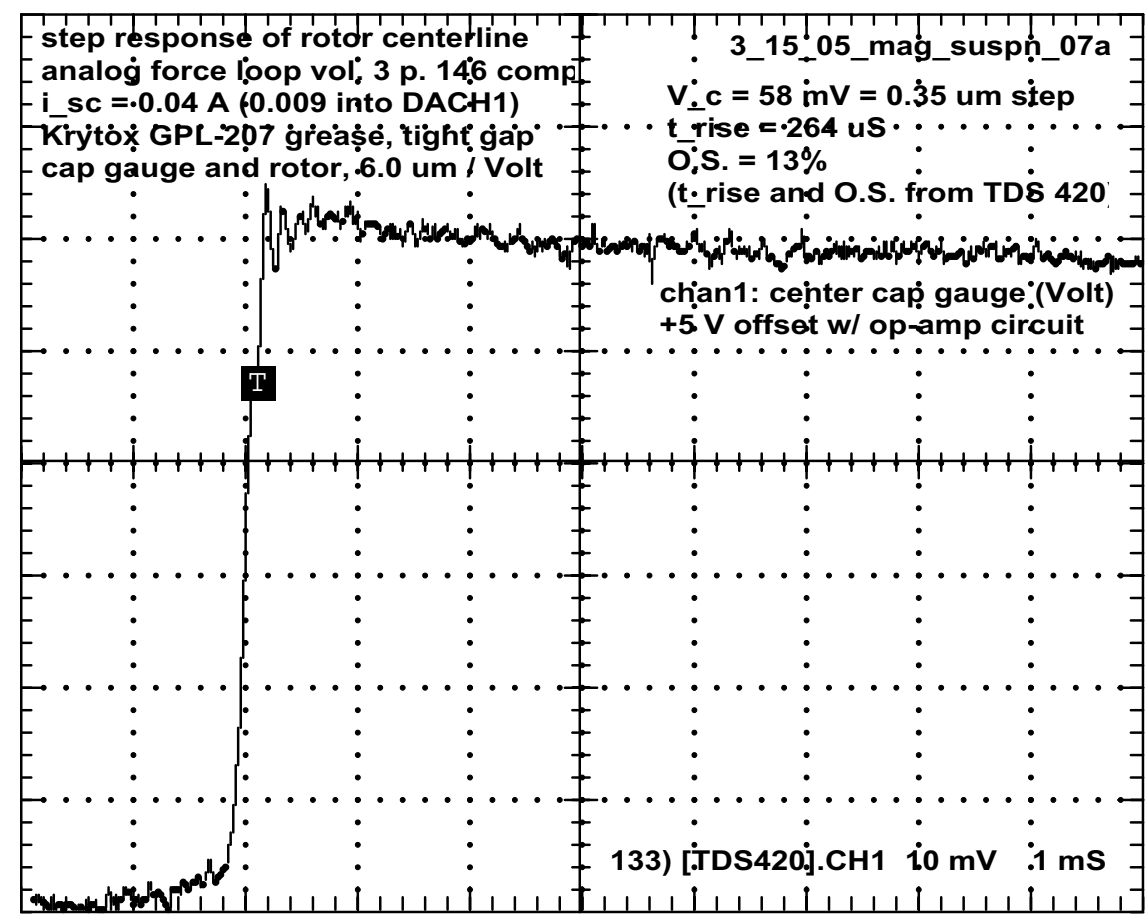

Figure 4-79: Measured step response of the rotor with electronic and mechanical damping. Input is an external mechanical step in force on the tool holder at the centerline in the z-direction.

appears to add noise to the system.

Figure 4-80 shows measured frequency responses of the negative loop transmission of the tool position comparing the affect of electronic damping to the affect of mechanical-plus-electronic damping on the front/back vibration modes. The mechanical damping reduces the resonant peaks by at least $25 \mathrm{~dB}$ beyond what electronic damping alone does. Note that the electronic damping suppresses the resonant peak at $6.3 \mathrm{kHz}$ more than the one at $5.5 \mathrm{kHz}$. This is consistent with the front/back translation capability of the linear mode of the actuator and the rotor modes assigned to the step responses shown in Figures 4-76 and 4-77.

\subsubsection{Equivalent Linear Mass}

\section{and Passive Dynamic Stiffness Seen at the Tool Tip}

The concept of the equivalent linear mass for the rotor and how it is used to determine the passive dynamic stiffness seen at the tool tip is developed in Section 3.7.3. The 

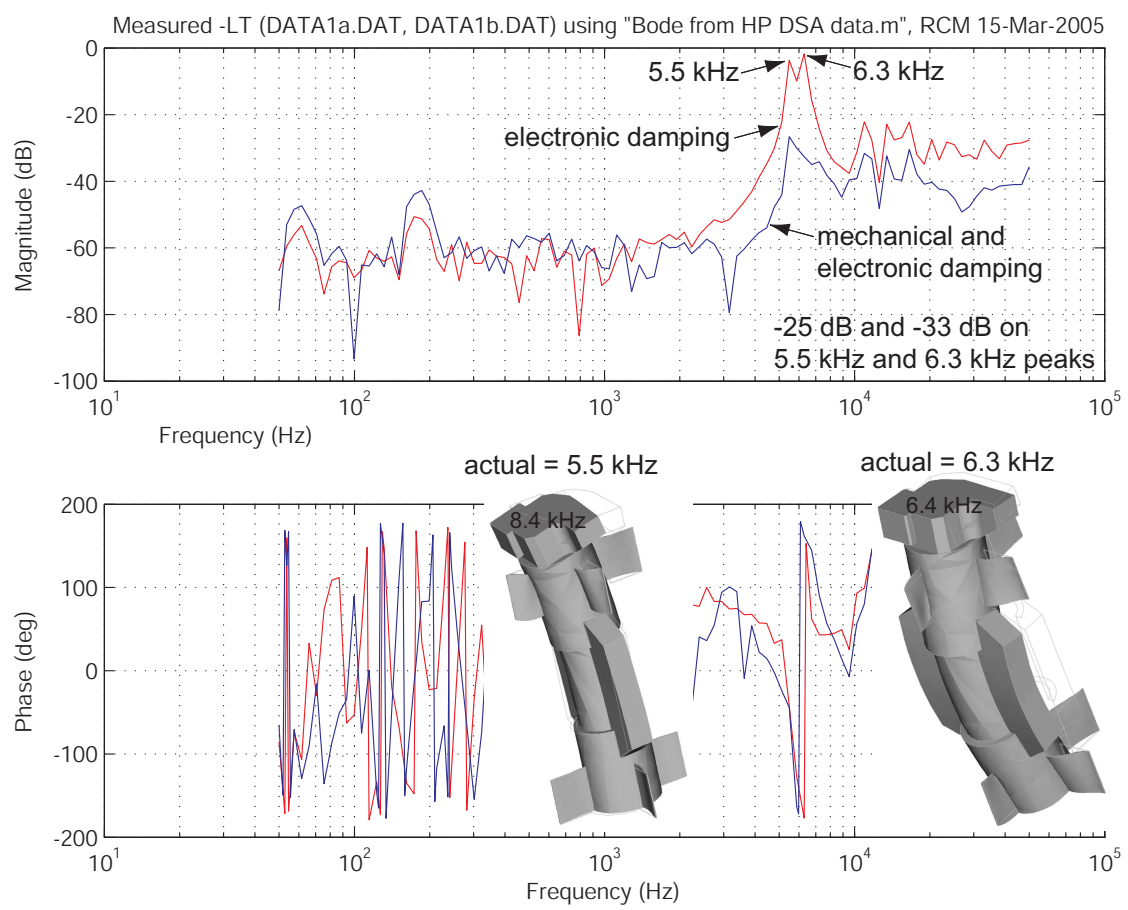

Figure 4-80: Measured frequency responses of the negative loop transmission of the tool position comparing electronic damping to mechanical-plus-electronic damping.

passive dynamic stiffness provides a metric for describing the tool tip stiffness that a workpiece would feel from a particular fast tool servo without consideration of the control system.

For the $10 \mathrm{kHz}$ FTS, the $12.7 \mathrm{~mm}$ tool swing radius combined with a moving mass rotational inertia of $81 \mathrm{gm} \cdot \mathrm{cm}^{2}$ results in an effective linear mass of $0.050 \mathrm{~kg}$ as seen at the tool tip. The measured rigid body torsional mode at $300 \mathrm{~Hz}$ for the low-noise implementation $\left(I_{B}=0.545 \mathrm{~A}\right)$ suggests a torsional spring constant of $k_{T}=90 \mathrm{~N} \cdot \mathrm{m} / \mathrm{rad}$ for the flexure suspension, which leads to a linear spring constant for the equivalent mass of $k_{L}=0.18 \mathrm{~N} / \mu \mathrm{m}$. Using the construction shown in Figure 3-24, the passive dynamic stiffness seen at the tool tip is $2 \mathrm{~N} / \mu \mathrm{m}$ at $1 \mathrm{kHz}$. Note that the flexures provide only $9 \%$ of the passive torsional stiffness at $1 \mathrm{kHz}$.

Closed-loop control increases the dynamic stiffness seen at the tool tip. For two different fast tool servos to have the same net dynamic stiffness at the tool tip, the one with a lower passive dynamic stiffness requires more controller authority. This is the case of the $10 \mathrm{kHz}$ FTS when compared to the $2 \mathrm{kHz}$ FTS, since the later has a 
tool tip passive dynamic stiffness of $9 \mathrm{~N} / \mu \mathrm{m}$ at $1 \mathrm{kHz}$.

\subsection{Electrical Design}

The current-mode amplifier that I built for the $10 \mathrm{kHz}$ FTS uses the same basic circuit topology and current loop compensation that was used with the earlier $2 \mathrm{kHz}$ FTS and is described in Section 3.9.1. Special credit is due to one of my colleagues in the Precision Motion Control Lab at MIT [7], Xiaodong Lu, who produced the fully engineered design for the power amplifier and current compensation circuits used with the $10 \mathrm{kHz}$ FTS. Xiaodong was developing a new high bandwidth linear fast tool servo at the same time that I was developing the $10 \mathrm{kHz}$ rotary fast tool servo, and we had similar requirements for our current-mode power amplifiers. Rather than duplicate efforts, this project provided funding for Xiaodong's work on the circuit design, circuit board layout, and thermal management design for the amplifier. Xiaodong provides details on that system in his Doctoral thesis [98], so I provide only a top-level description in this thesis.

Figure 4-81 shows the power amplifier and current compensation circuit boards for the $10 \mathrm{kHz}$ FTS. Each circuit board contains four channels, and the current compensation board normally resides nested on top of the amplifier board. The power amplifier board is mounted to a large copper plate which acts as a heat sink. Figure 4-82 shows the back side of the copper plate with the four power operational amplifiers and nine high-performance CPU cooling fans used to remove heat from the system. The quiescent current for the entire system while operating the power operational amplifiers in boost mode is $+0.35 \mathrm{~A}$ and $-0.32 \mathrm{~A}$ when using either $\pm 60 \mathrm{~V}$ or $\pm 80 \mathrm{~V}$ power supplies.

Initially, each steering flux coil was driven by its own amplifier. Since the pairs of coils on each stator core are magnetically coupled, each stator half was a multiple input multiple output (MIMO) system. I was able to design adequate compensation to close the four current loops for the four amplifiers, but they were prone to ringing during testing. Ultimately, I connected the two coils on each stator core in series, 


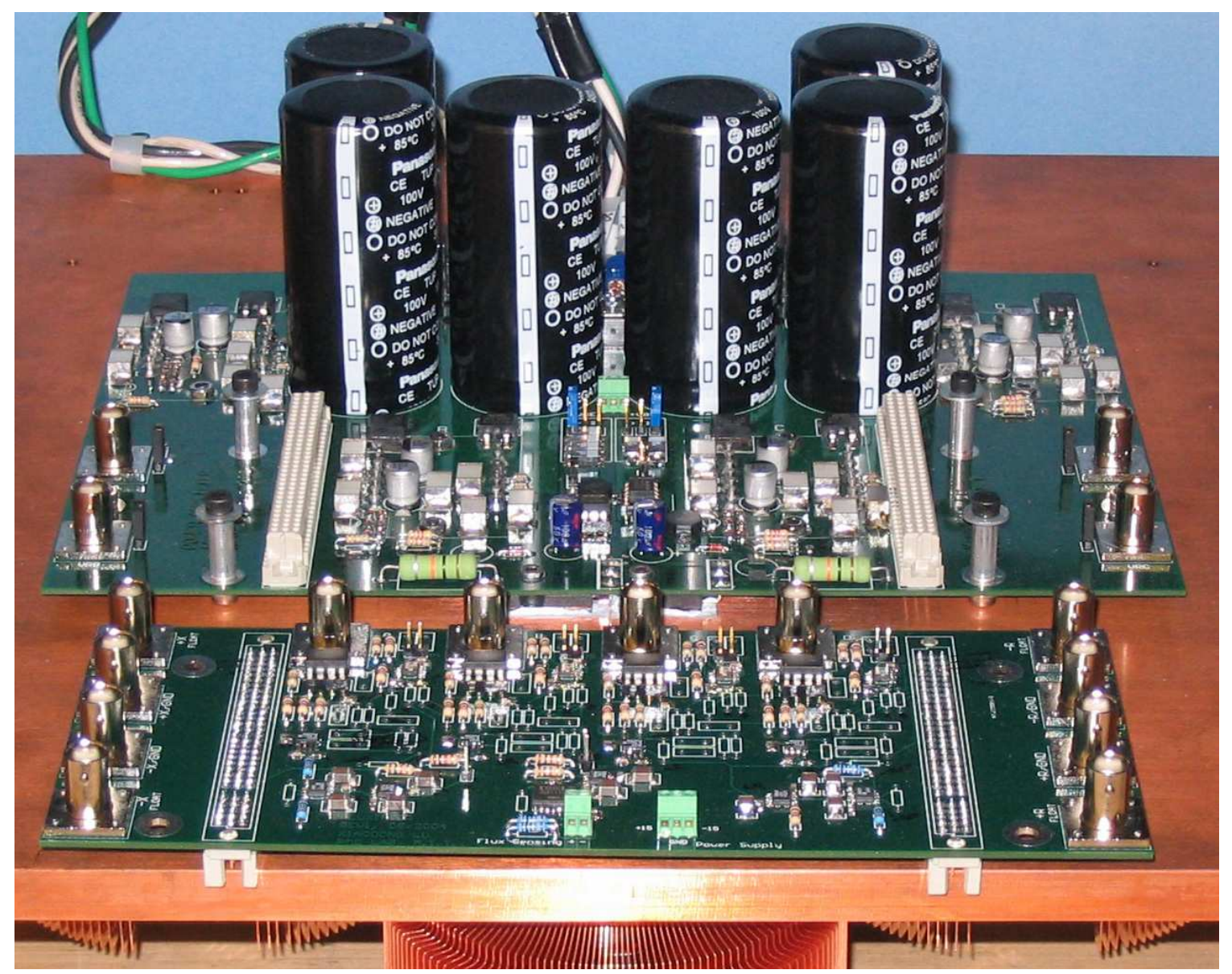

Figure 4-81: Power amplifier (back) and current compensation (front) circuit boards for the $10 \mathrm{kHz}$ FTS.

and drove each pair with its own amplifier. This reduced the complexity of the system dynamics for each stator half to that of a single input single output (SISO) system, and allowed using SISO design techniques to develop a robust control system. Section 5.3.1 discusses how the two stator cores are magnetically decoupled by the air gaps between the back-iron and the stator cores, and by the low permeability of the back-iron compared to the rotor and stator cores. This allows independently driving each stator half with its own power amplifier, which allows controlling the rotary and linear degrees of freedom of the actuator. 


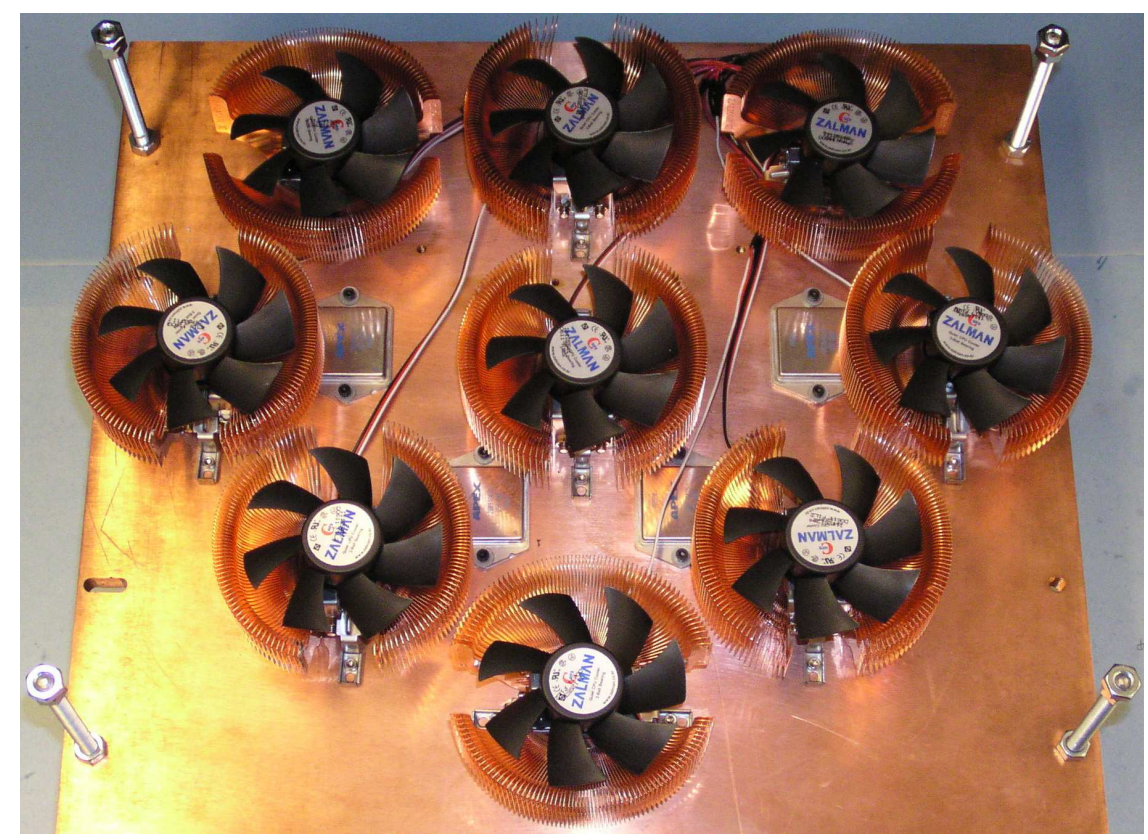

Figure 4-82: Copper plate heat sink with the four power operational amplifiers and nine cooling fans for the $10 \mathrm{kHz}$ FTS.

\subsubsection{Current-Mode Amplifier}

Figure 4-83 is a schematic diagram of the electrical circuit for one of the two identical current-mode amplifiers used with the $10 \mathrm{kHz}$ FTS, and includes the electrical dynamics of one stator half. A one-for-one mapping can be made between the circuit shown in Figure 4-83 and the block diagram shown in Figure 4-65.

Our design goal of a closed-loop bandwidth of $10 \mathrm{kHz}$ for the tool position suggests designing the current-mode amplifiers to have negligible dynamics at and below that frequency. Initial debugging and tuning of the amplifiers with a surrogate singlecoil magnetic circuit for the load established a closed-loop half-power bandwidth of $160 \mathrm{kHz}$ and a phase margin of approximately $36^{\circ}$. Later, when driving pairs of seriesconnected steering flux coils with a single amplifier, the bandwidth was reduced to approximately $80 \mathrm{kHz}$. This is consistent with a designed current loop crossover frequency of $50 \mathrm{kHz}$, which was sufficient for moving forward with testing of the $10 \mathrm{kHz}$ FTS. ${ }^{9}$

\footnotetext{
${ }^{9}$ The initial $160 \mathrm{kHz}$ closed-loop bandwidth and $36^{\circ}$ phase margin are based on the measured step responses of the amplifier channels, which are discussed in Section 4.7.3. The $80 \mathrm{kHz}$ closedloop bandwidth for the loop containing pairs of coils was not measured directly because I needed
} 
The first operational amplifier in the forward path of the loop in Figure 4-83 is the analog controller (current compensation) for the amplifier. The current compensation consists of a pure integrator (the $100 \mathrm{pF}$ capacitor in the local feedback path) and a lead transfer function centered on $100 \mathrm{kHz}$ with an $\alpha=11$. The outer feedback loop — which uses a measurement of the current in the actuator - prevents the integrator from causing the output of the compensation operational amplifier to drift to the value of one of its supply voltages. Details on the loop-shaping technique used to design the current compensation are given in Section A.1. The last operational amplifier in the forward path of the loop is the power operational amplifier, which is an Apex Microtechnology Corporation PA52A [12] configured for a non-inverting DC gain of 6.7. The dynamics of the power operational amplifier are negligible for the frequencies of interest for the current-mode amplifier, so it is treated as a constant gain of 6.7. The resistor network connected to the non-inverting input of the power operational amplifier matches the input impedance of the resistor network connected to the inverting input, and sets the input to ground in case the input connection to the current compensation circuit is broken while power is being supplied to the amplifier. The first operational amplifier in the feedback loop is configured as a differential amplifier for measuring the voltage drop across the sense resistor $R_{s}$. Note that the power ground (PGND) and analog ground (AGND) are tied together at the foot of the sense resistor. The second operational amplifier in the feedback loop provides a summing point for an optional additional input for the current loop. The resistance value $R_{s c p}$ for the series-connected pair of steering flux coils includes the DC resistance of the cables from the power amplifier to the coils.

to proceed with other tests with the $10 \mathrm{kHz}$ FTS. Instead, the new crossover frequency is inferred from the earlier value as follows. Switching from a single coil to a pair of magnetically coupled coils increases the inductance by a factor of four, which lowers the crossover frequency by a factor of four. The value of the resistor bringing the actuator current feedback into the summing point of the compensation operational amplifier was decreased by a factor of two to increase the loop gain by a factor of two. This resulted in a stable and robust current loop, so I did not continue refining it. The net result of these changes is a factor of two reduction in the designed crossover frequency, and hence the estimated factor of two reduction in closed-loop bandwidth from $160 \mathrm{kHz}$ to $80 \mathrm{kHz}$. 


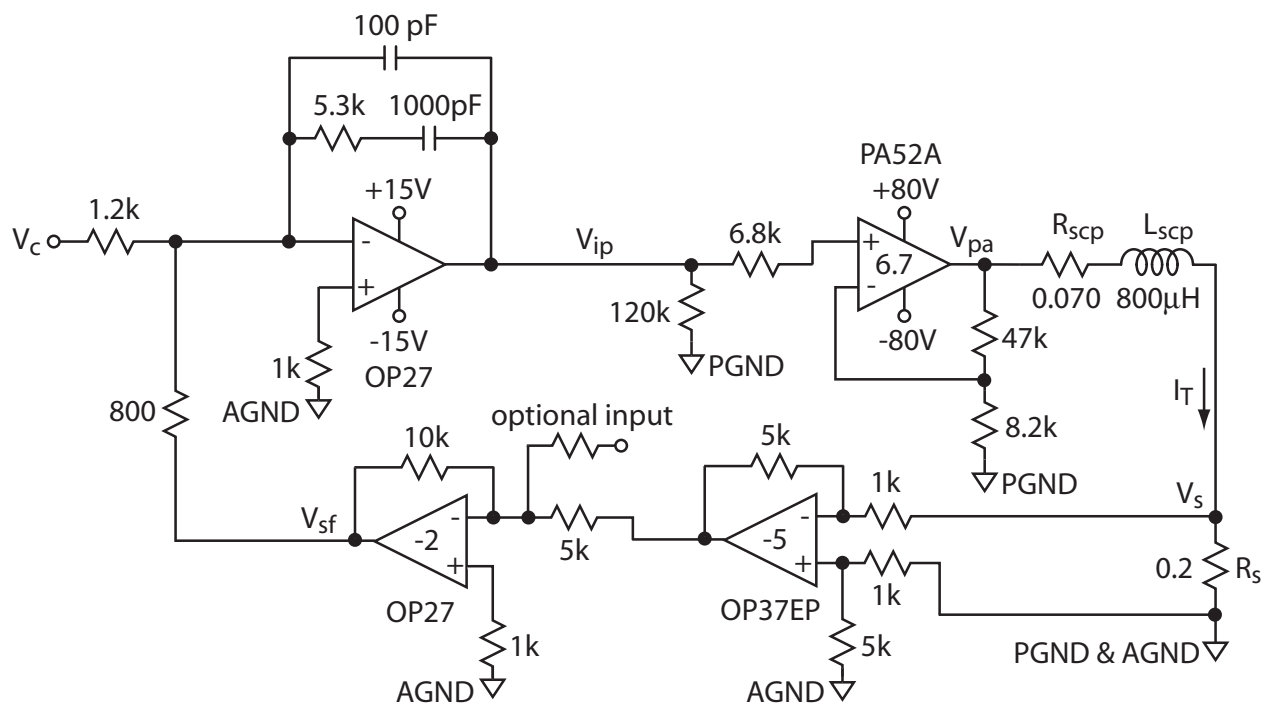

Figure 4-83: Schematic diagram of the electrical circuit for one of the two identical current-mode amplifiers used to drive the series-connected pairs of steering flux coils in the $10 \mathrm{kHz}$ rotary fast tool servo.

\subsubsection{Voltage Budget and Power Dissipated in the Amplifier}

In Section 5.3.5 I describe the consequences of designing the current-mode amplifiers with the assumption that each steering flux coil would be driven by its own dedicated amplifier, but ultimately using one amplifier to drive each series-connected pair of coils. In summary, each of the as-built amplifiers had sufficient power for driving the series-connected pairs of steering flux coils at frequencies up to $5 \mathrm{kHz}$, and I had the good fortune that a stable mechanical resonance at $10 \mathrm{kHz}$ provided the additional power needed for operating at frequencies between $5 \mathrm{kHz}$ and $10 \mathrm{kHz}$. Due to the mutual inductance between the two coils in each of these pairs, the total inductance of the series-connected pair is four times greater than that of a single coil by itself.

The calculations presented in this section are based on driving each series-connected pair of steering flux coils with one amplifier to produce the required $0.75 \mathrm{~T}$ peak steering flux density needed to generate a peak tool tip acceleration of $600 \mathrm{~g}$ at a maximum operating frequency of $5 \mathrm{kHz}$. This is consistent with the electrical dynamics model in Section 4.6.2. The amplifiers were designed to drive the torque mode of the actuator - since that is the main role of the hybrid rotary/linear actuator that we developed - so the analysis in this section considers the torque mode. 


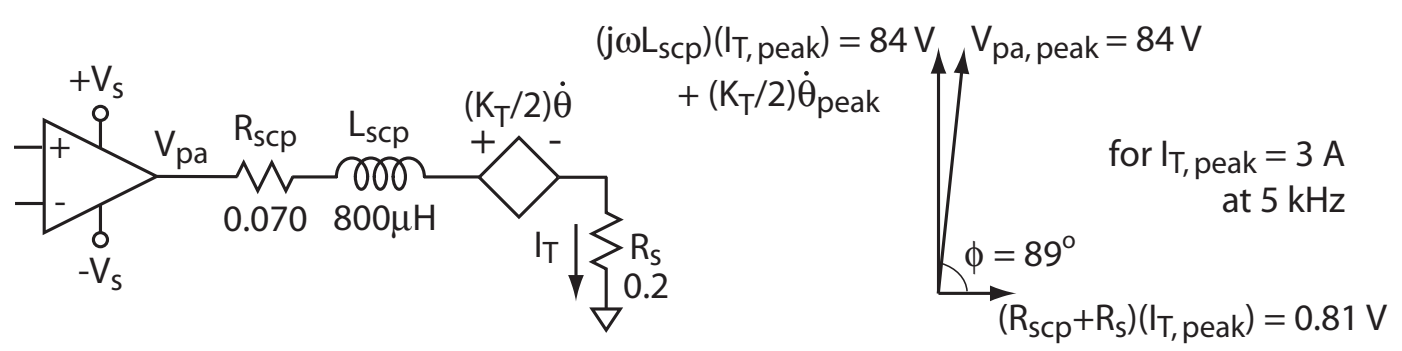

Figure 4-84: Schematic diagram of the power amplifier, a series-connected pair of steering flux coils, the torque-mode Back-EMF, and sense resistor for the $10 \mathrm{kHz}$ FTS (left), and phasor diagram showing the relationship between the real and imaginary components of the output voltage of the power amplifier (right). Phasor diagram is for the maximum specified continuous peak current of $3 \mathrm{~A}$ for the actuator and $5 \mathrm{kHz}$ operation.

Section 5.3.1 shows that the steering coil pair needs to produce a magnetomotive force of $60 \mathrm{amp}$ turns to produce a steering flux density of $0.75 \mathrm{~T}$. A peak current of 3.0 $\mathrm{A}$ is needed to produce that magnetomotive force in the 20 -turns series-connected pair of steering flux coils, and Section 5.3.5 shows that a peak voltage of $75 \mathrm{~V}$ is needed to drive that current at $5 \mathrm{kHz}$.

The power amplifiers drive three things in the actuator: (1) the inductive load; (2) the resistive load; and (3) the velocity-dependent back-EMF. For a sinusoidally varying tool tip trajectory of $z_{t}=A \sin (\omega t)$, the peak velocity of $\dot{z}_{t p k}=A \omega$ occurs at $z_{t}=0$, and the peak acceleration of $\ddot{z}_{t p k}=A \omega^{2}$ occurs at the end points of travel of the tool tip, where the actuator needs to produce the peak torque and therefore draws the peak current (torque is proportional to the coil current). Keeping track of positive and negative directions, the maximum tool tip velocity lags the maximum tool tip acceleration by $90^{\circ}$. Since the peak velocity lags the peak current by $90^{\circ}$, the peak back-EMF is in phase with the peak voltage across the inductor. At the frequencies of interest, I show that the resistive load for the actuator is negligible compared to the inductive load and back-EMF, and therefore the coil current lags the output voltage of the power amplifier by nearly $90^{\circ}$.

The left side of Figure 4-84 shows a schematic diagram of the power amplifier, a series-connected pair of steering flux coils, the torque-mode Back-EMF, and sense resistor for the $10 \mathrm{kHz}$ FTS. The resistance value $R_{s c p}$ for the pair of coils includes the 
DC resistance of the cables from the power amplifier to the coils. ${ }^{10}$ The relationship between the output voltage of the power amplifier $V_{p a}$, the torque-producing current $I_{T}$, resistance of the coil-pair $R_{s c p}$, inductance of the coil-pair $L_{s c p}$, sense resistor $R_{s}$, motor constant $K_{T}$, and motor angular velocity $\dot{\theta}$ is determined by Kirchoff's Voltage Law and given by (4.11). Since each pair of steering flux coils provides one half of the motor torque constant $K_{T}$, the back-EMF for each pair of coils is $\left(K_{T} / 2\right) \dot{\theta}$.

$$
V_{p a}=\left(R_{s c p}+R_{s}\right) I_{T}+L_{s c p} \frac{d I_{T}}{d t}+\left(K_{T} / 2\right) \dot{\theta}
$$

The desired peak tool tip acceleration of $600 \mathrm{~g}$ at an operating frequency of $5 \mathrm{kHz}$ leads to a peak angular velocity of $15 \mathrm{rad} / \mathrm{sec}$ for the rotor. Combined with the component values shown in Figure 4-84, an actuator torque constant of $1.2 \mathrm{~N} \cdot \mathrm{m} / \mathrm{amp}$ for a bias flux density of $0.75 \mathrm{~T}$ (see Section 5.2.3), and a peak continuous current of $3 \mathrm{~A}$, the terms in (4.11) are given by (4.12), (4.13), and (4.14):

$$
\begin{gathered}
\left(R_{s c p}+R_{s}\right) I_{T}=[(0.024+0.046)+0.2] 3=0.81 \mathrm{~V} \\
L_{s c p} \frac{d I_{T}}{d t}=\left(800 \times 10^{-6}\right)\left(9.4 \times 10^{4}\right)=75 \mathrm{~V} \\
\left(K_{T} / 2\right) \dot{\theta}=(1.2 / 2) 15=9 \mathrm{~V}
\end{gathered}
$$

Examination of (4.12), (4.13), and (4.14) reveals that the resistive load is negligible compared to the inductive load and peak back-EMF. The right side of Figure 4-84 shows a phasor diagram depicting the relationship between the real and imaginary components of the output voltage of the power amplifier, and indicates that the peak motor current of $3 \mathrm{~A}$ lags the peak output voltage of $84 \mathrm{~V}$ from the power amplifier by $89^{\circ}$ when operating at $5 \mathrm{kHz}$. For currents up to $10 \mathrm{~A}$, the power operational

\footnotetext{
${ }^{10}$ Litz wire of an appropriate gauge was used to construct the cables that connect the power amplifiers to the coils, so that the increase in resistance attendant with $\mathrm{AC}$ operation would not be significant. Section 5.3.6 provides a discussion on the underlying mechanism for increased AC resistance and Litz wire.
} 
amplifiers used with the $10 \mathrm{kHz}$ FTS can normally swing to within $6 \mathrm{~V}$ of the supply voltages, and to within $1 \mathrm{~V}$ when used in boost mode. This suggests using power supply voltages of at least $\pm 90 \mathrm{~V}$. The maximum voltage slew rate for this case is $2.6 \mathrm{~V} / \mu$ sec, which is well within the specified $50 \mathrm{~V} / \mu$ sec for the power operational amplifiers.

It is interesting to consider how the voltage budget depends on the operating frequency. If the operating frequency is increased from $5 \mathrm{kHz}$ to $10 \mathrm{kHz}$ while maintaining the desired peak tool tip acceleration of $600 \mathrm{~g}$ and a peak continuous current of $3 \mathrm{~A}$, the peak back-EMF voltage decreases from $9 \mathrm{~V}$ to $4.4 \mathrm{~V}$ due to the lower peak velocity attendant with the shorter tool sweep. Meanwhile, the voltage across the inductance increases from $75 \mathrm{~V}$ to $150 \mathrm{~V}$ due to the higher peak (dI/dt) attendant with the higher operating frequency. The net result is a higher combined voltage, $155 \mathrm{~V}$ versus $84 \mathrm{~V}$, for $600 \mathrm{~g}$ at $10 \mathrm{kHz}$.

Figures 4-85 and 4-86 show the relationships between the voltage, current, and power in the torque mode of the actuator (load), and the supply voltages and dissipated power in the power amplifier. Figure 4-85 is based on my original analysis on driving a single, lone steering flux coil with one power amplifier at $10 \mathrm{kHz}$. That analysis erroneously omitted the mutual inductance of the other coil on the shared stator core and neglecting the back-EMF, and indicated a required peak current and voltage of $3.0 \mathrm{~A}$ and $75 \mathrm{~V}$, respectively. These are the same peak values needed to drive only the inductance of the series-connected pair of coils to produce a peak steering flux density of $0.75 \mathrm{~T}$ at $5 \mathrm{kHz} .^{11}$ Therefore, the original analysis for the power dissipated in a power operational amplifier is valid for the case of driving the inductance-only of the series-connected pair at $5 \mathrm{kHz}$. If we increase the power supply voltages to accommodate the back-EMF, we obtain the results shown in Figure 4-86.

To determine the power dissipation in the output stage of the power operational amplifier we consider the voltage drop across it - voltage of the active supply minus the output voltage of the operational amplifier — and the current flowing through it.

\footnotetext{
${ }^{11}(5.97)$ shows that the peak voltage is proportional to the total number of turns times the operating frequency.
} 
The active supply is the one with the same sign as the current being supplied by the operational amplifier. Note that because the actuator current is out of phase with the output voltage of the power amplifier that the signs of the two are opposite at certain places in the cycle, which leads to a higher power dissipation in the operational amplifier than would occur for a purely resistive load. The numerical analysis used to generate Figures 4-85 and 4-86 is similar to the analysis used for the $2 \mathrm{kHz}$ FTS and listed in Appendix B.3. In the case of the $10 \mathrm{kHz}$ FTS, the analysis treats the load as being purely inductive and indicates that the average power dissipated in the power operational amplifier is 172 VA when driving a series-connected pair of coils with one amplifier at $5 \mathrm{kHz}$ to produce a $600 \mathrm{~g}$ tool tip acceleration (Figure 4-86). This is well within the internal power dissipation rating of $250 \mathrm{~W}$ at $75^{\circ} \mathrm{C}$ for the device, and according to the safe operating area specification, allows a case temperature of approximately $90^{\circ} \mathrm{C}$.

A comparison of Figures 4-85 and 4-86 reveals an evolution in my understanding of the relationship between the electrical power in the load and the magnetic power in the air gaps. The earlier Figure 4-85 reports the peak and average real electrical power. In the case of a purely inductive load, the average real electrical power is zero, which does not tell us much about the average magnetic power. The later Figure 486 reports the more meaningful apparent time-average electrical power in the load, which has a direct mapping to the time-average magnetic power in the air gaps. ${ }^{12}$

\subsubsection{Tuning the Current Loop with a Surrogate Magnetic Circuit}

The current compensation circuits were tuned using the loop shaping technique described in Appendix A.1. A surrogate magnetic circuit consisting of one steering flux coil on a stator core, attached to a rotor core with a $50 \mu \mathrm{m}$ plastic shim, was used as the load. This allowed me to complete fabrication and testing of the power amplifier in a parallel effort with the fabrication of the mechanical and magnetic aspects of the

\footnotetext{
${ }^{12}$ The relationship between the apparent time-average electrical power needed to drive the AC flux in the air gaps and the time-average magnetic power in the air gaps is developed in Section 5.3.3.
} 
"push pull power loss 01.m", $10 \mathrm{kHz}$ rotary fast tool servo

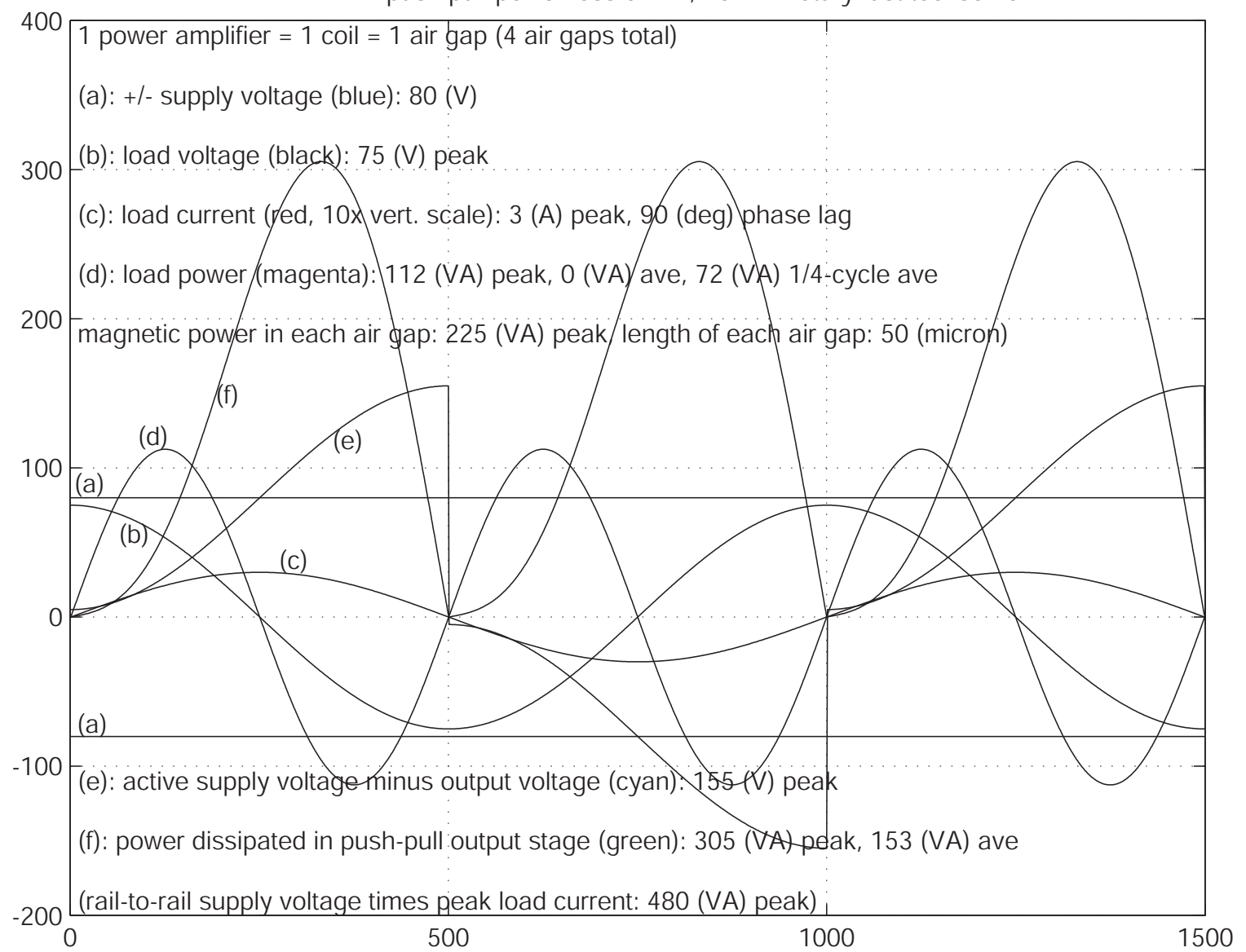

Figure 4-85: Plot showing relationships between the voltage, current, and power in the torque-mode of the actuator (load), and the supply voltages and dissipated power in the power amplifier. Based on the original analysis on driving a single, lone steering flux coil with one power amplifier at $10 \mathrm{kHz}$, and neglecting the back-EMF. Applies to producing a peak steering flux density of $0.75 \mathrm{~T}$ when driving the inductance-only of a series-connected pair of steering flux coils at $5 \mathrm{kHz}$. 
"push pull power loss $015 \mathrm{kHz} 600 \mathrm{~g}$ ", $10 \mathrm{kHz}$ rotary fast tool servo

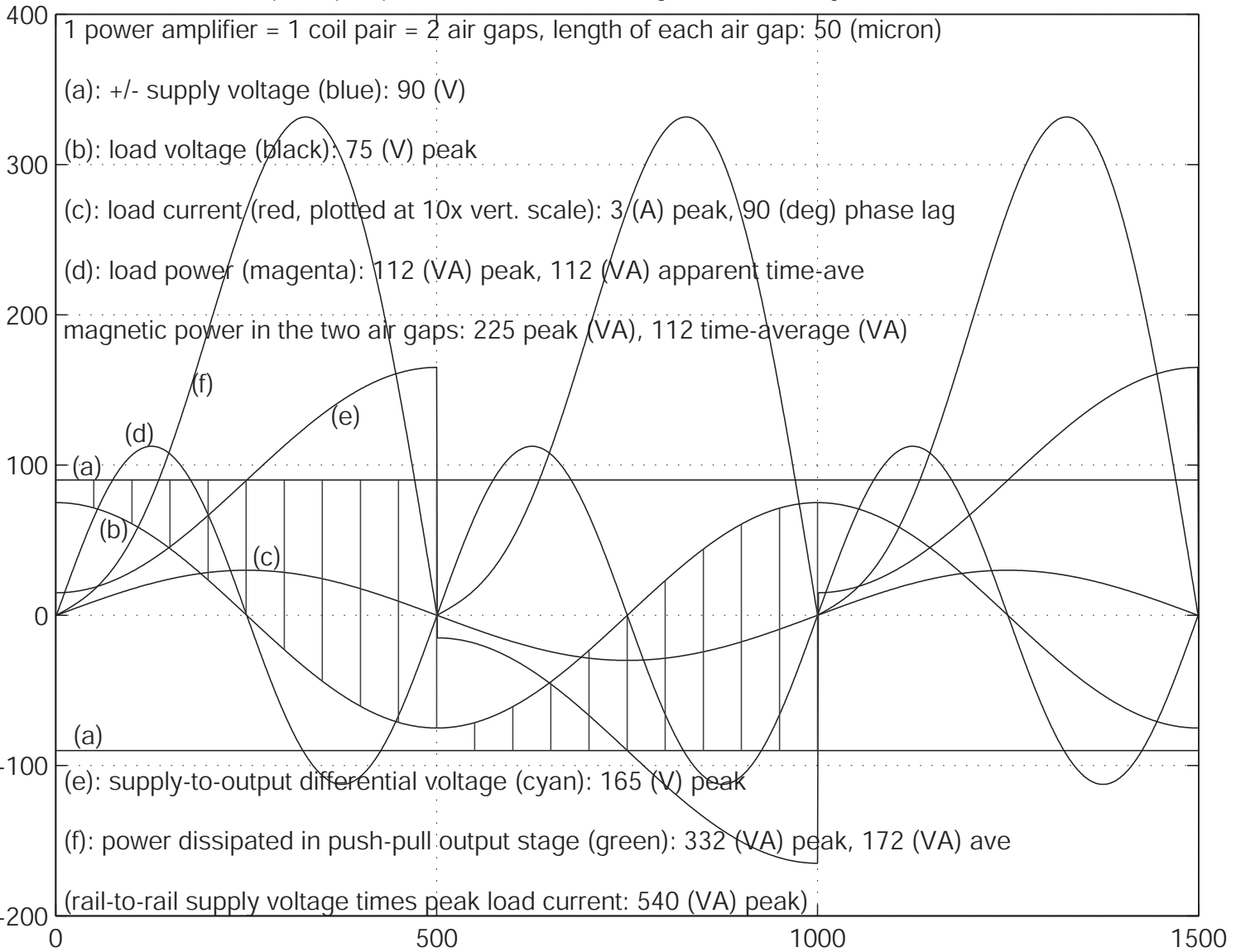

Figure 4-86: Plot showing relationships between the voltage, current, and power in the torque-mode of the actuator (load), and the supply voltages and dissipated power in the power amplifier. Based on driving a pair of series-connected steering flux coils with one power amplifier at $5 \mathrm{kHz}$ to produce a $600 \mathrm{~g}$ tool tip acceleration. Includes higher supply voltages needed to accommodate the back-EMF. 


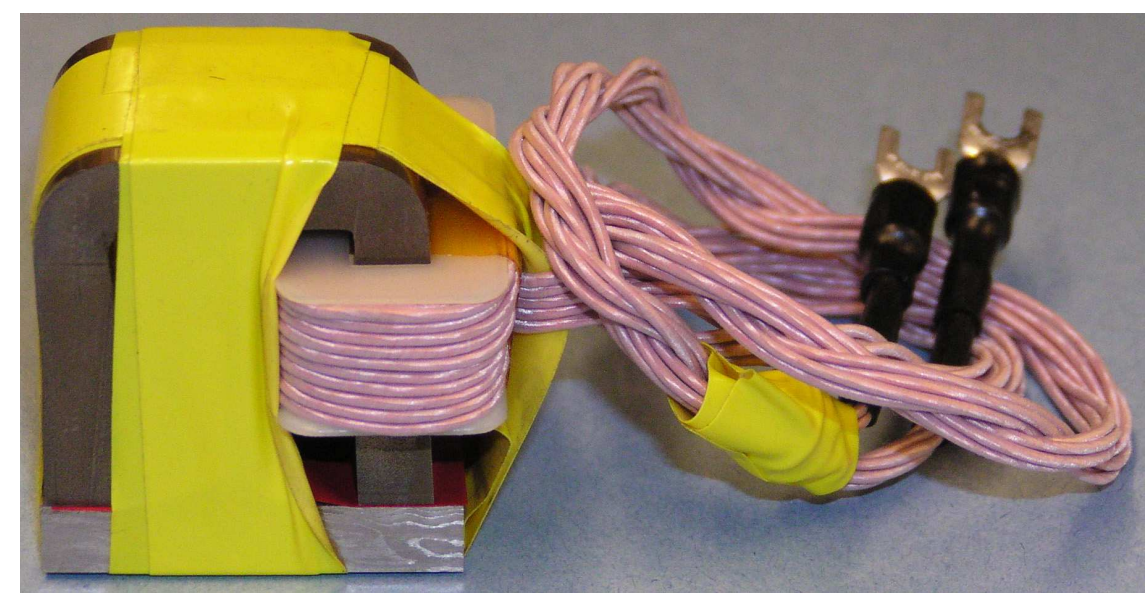

Figure 4-87: Surrogate magnetic circuit for tuning the power amplifiers for the $10 \mathrm{kHz}$ FTS. Consists of one steering flux coil on a stator core, a rotor core, and a $50 \mu \mathrm{m}$ thick plastic shim in each rotor/stator air gap.

10 khz FTS. Figure 4-87 shows the surrogate magnetic circuit.

Figure 4-88 shows the measured 1.0 A step responses for each of the four power amplifier channels when driving the surrogate magnetic circuit (single coil). The rise times of 2.1-2.2 $\mu \mathrm{sec}$ suggest a minimum closed-loop half-power bandwidth of $160 \mathrm{kHz}$ for each power amplifier channel, and the 28-30\% overshoot suggests a damping ratio and phase margin of approximately 0.36 and $36^{\circ}$, respectively. These conclusions are based on the following characteristics for a second-order system, which the power amplifier behaves like at the frequencies of interest for the $10 \mathrm{kHz}$ FTS. For systems that behave like second-order systems at frequencies up to and near their crossover frequency, Roberge [132, page 97] provides the following useful relationship between rise time $t_{r}$ (sec) and closed-loop half-power bandwidth $f_{h}(\mathrm{~Hz}): f_{h} \approx 0.35 / t_{r}$. The peak overshoot $M_{p}$ in the step response of a second order system is related to the damping ratio by the following relationship $[132,114,60]: M_{p}=\exp \left[(-\pi \zeta) /\left(\sqrt{1-\zeta^{2}}\right)\right]$. Franklin, Powell, and Emami-Naeini [60, page 406] provide the following approximation between the phase margin $P M$ in degrees and damping ratio $\zeta$ for a second-order system: $\zeta \approx P M / 100 ; P M<70^{\circ}$. Therefore, if we treat the power amplifier as a second-order system, then the measured overshoot of $30 \%$ leads to a damping ratio of 0.36 , which suggests a phase margin of approximately $36^{\circ}$. Using an argument based on the magnitude and angle of the loop transmission and the closed-loop transfer 


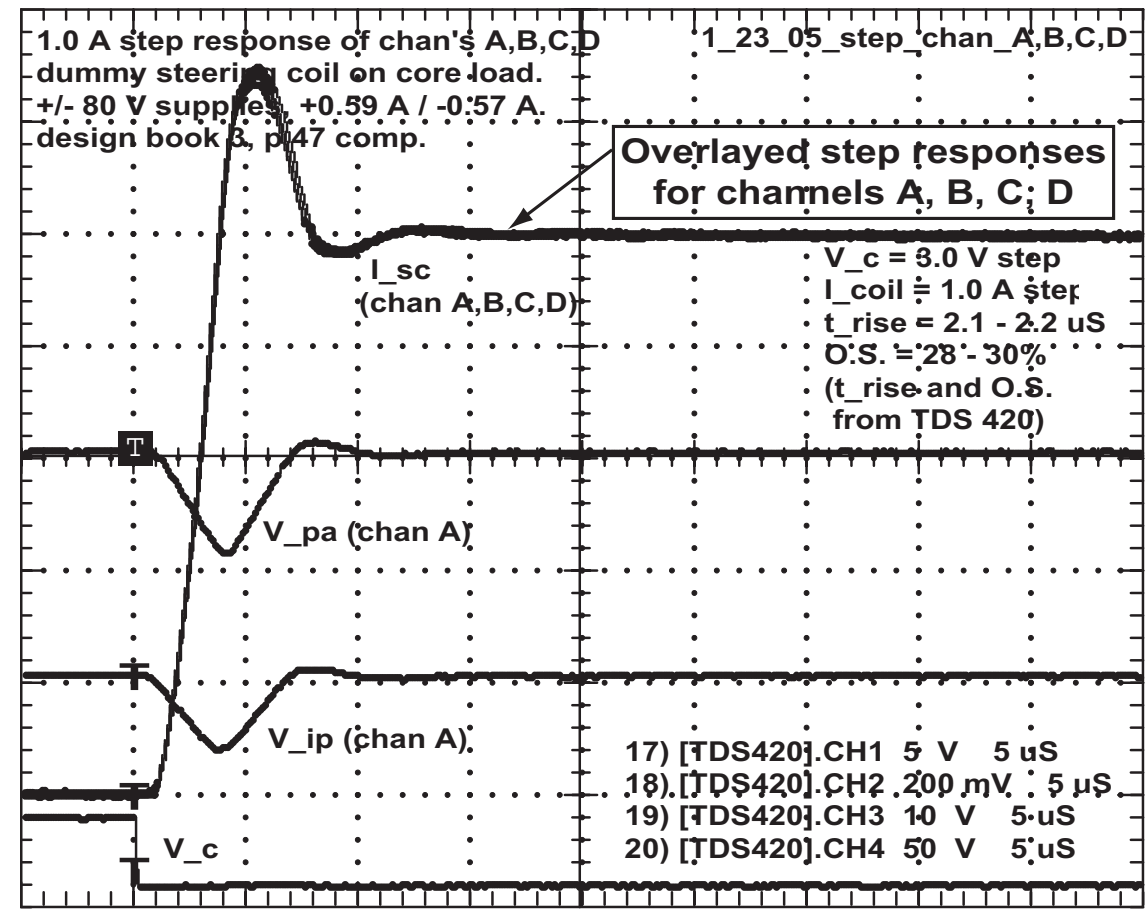

Figure 4-88: Measured 1.0 A step responses for each of the four power amplifier channels when driving the surrogate magnetic circuit.

function in the vicinity of the crossover frequency, Franklin, Powell, and EmamiNaeini [60, page 416] provide approximated curves relating closed-loop bandwidth to phase margin, showing that for $22^{\circ} \leq P M \leq 45$ the bandwidth is approximately equal to 1.8 times the crossover frequency. Using that relationship, a bandwidth of $80 \mathrm{kHz}$ and phase margin of $36^{\circ}$ corresponds to a crossover frequency of $44 \mathrm{kHz}$, which is close to the designed value of $50 \mathrm{kHz}$.

\subsubsection{Measured Flux Balance in the Rotor/Stator Air Gaps}

After assembling the $10 \mathrm{kHz}$ FTS, I used the following method to insure the proper phasing of the four steering flux coils and to measure the flux balance in the four rotor/stator air gaps. With the rotor in its neutral position, I excited the bias flux coil with a $100 \mathrm{~Hz}$ signal and measured the induced voltage at the open terminals of each of the steering flux coils. Essentially, the magnetic circuit is a transformer with one pair of input terminals and four pairs of output terminals. Figure 4-89 is a schematic diagram showing how the bias flux (triple-head arrows) flows through the 


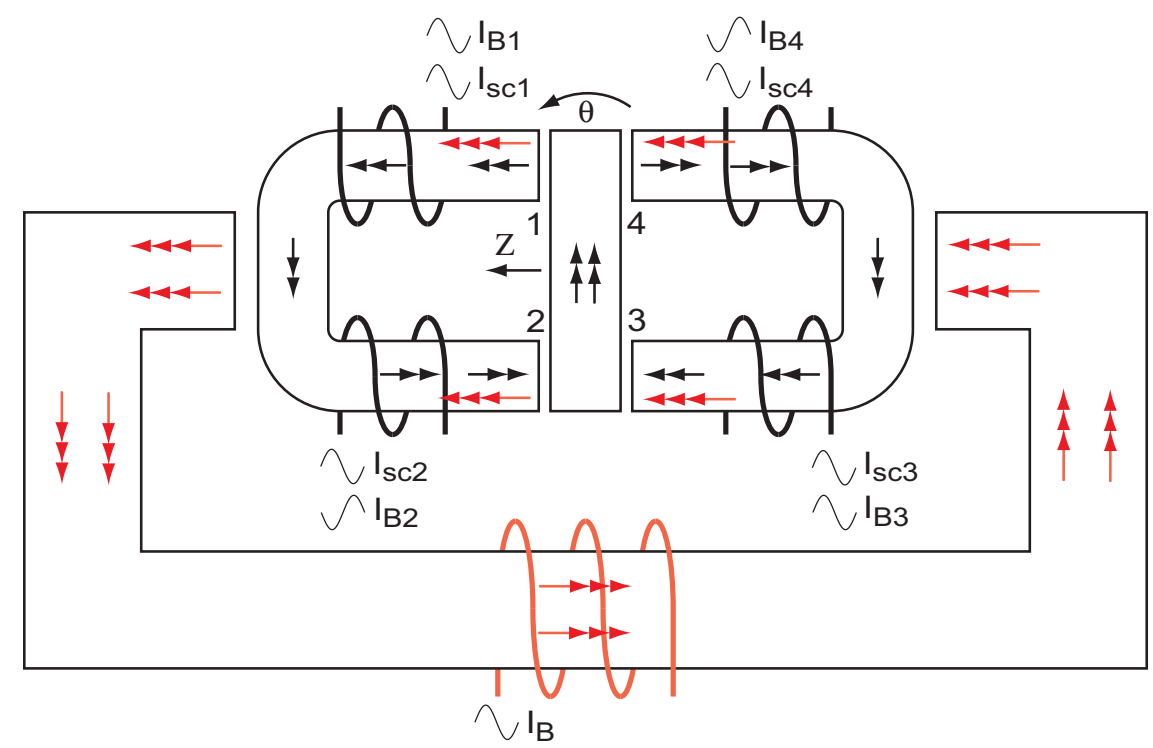

Figure 4-89: Schematic diagram showing the relationship between the bias flux (triplehead arrows), steering fluxes (double-head arrows), and currents needed to produce a $+\theta$ rotation of the rotor. Exciting the bias flux coil with a sinusoidal voltage induces the voltages shown at the terminals of the steering flux coils.

magnetic circuit, and the directions for the four steering fluxes (double-head arrows) needed to produce a $+\theta$ rotation of the rotor. The electrical phasing of the bias flux coil current $I_{B}$ and the steering flux coil currents $i_{s c}$ is shown at the terminals of each of the steering coils. By comparing the induced voltages at the terminals of the steering coils to the desired phasing, I was able to determine the correct positive and negative assignments for all of the coil leads.

Figure 4-90 shows the measured induced voltages at the terminals of the four steering flux coils and illustrates the proper phasing of those coils. The excitation voltage (not shown) leads the voltages in steering coils 1 and 3 by approximately $60^{\circ}$. Figure 4-91 shows the same voltages as in Figure 4-90, but with the signals overlayed to illustrate that the bias flux is balanced in the four rotor/stator air gaps within $7 \%$.

\subsubsection{Coupled versus Uncoupled Current Loops}

In Section 4.7 I mentioned that I initially drove each steering flux coil with its own amplifier. The magnetic coupling between coils on the same stator core results in a MIMO system for each stator half. The alternative was to connect the two coils on 


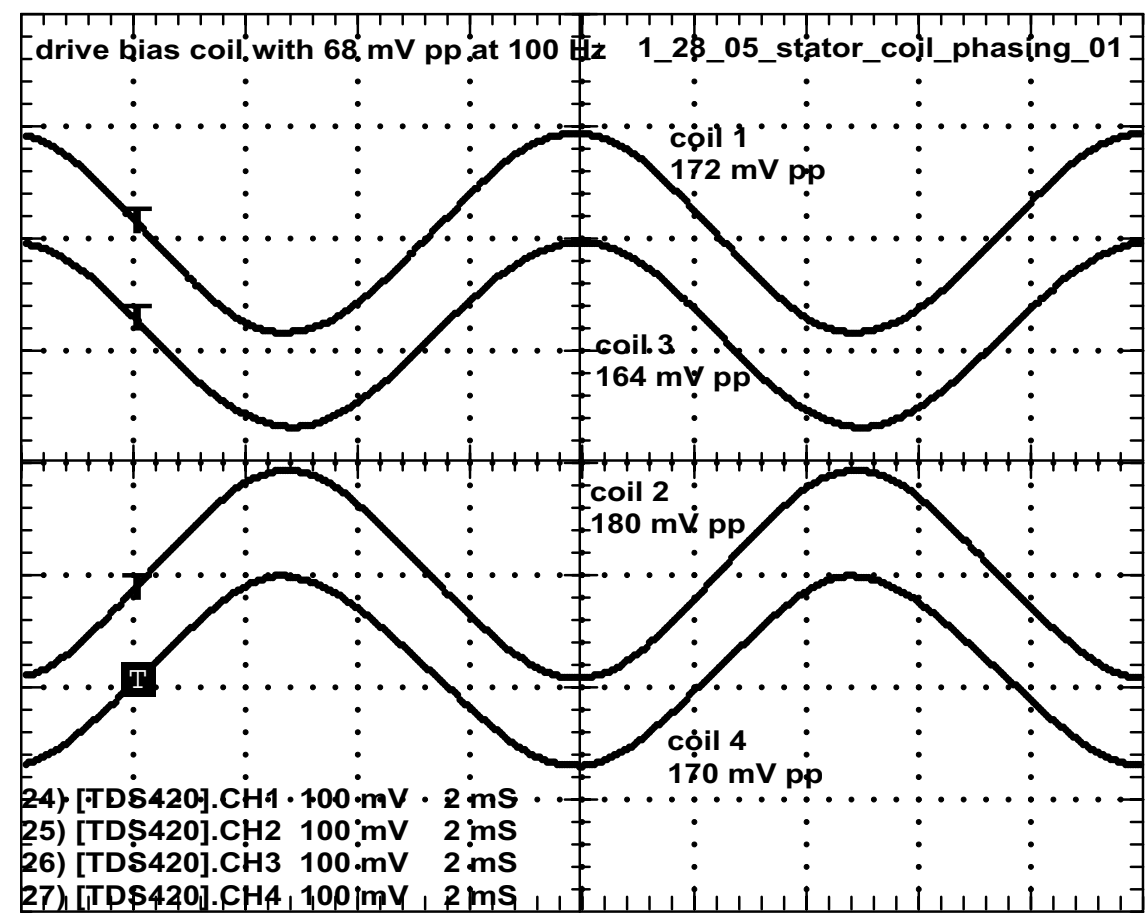

Figure 4-90: Measured induced voltages at the terminals of the four steering flux coils for a $100 \mathrm{~Hz} \mathrm{AC}$ excitation of the bias flux coil, illustrating the proper phasing. DC offsets added to provide visual separation.

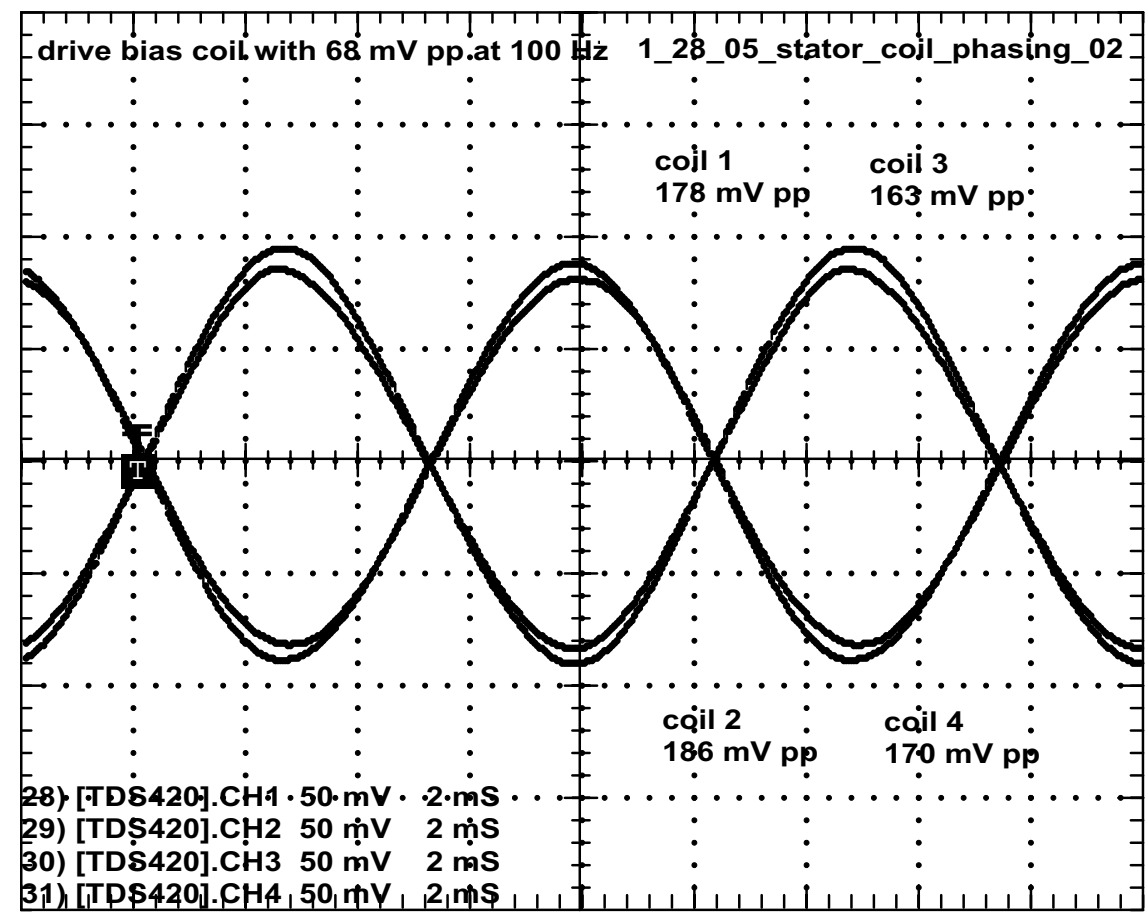

Figure 4-91: Overlay of the measured induced voltages at the terminals of the four steering flux coils for a $100 \mathrm{~Hz}$ AC excitation of the bias flux coil, illustrating that the bias flux in the four rotor/stator air gaps is balanced within $7 \%$. 

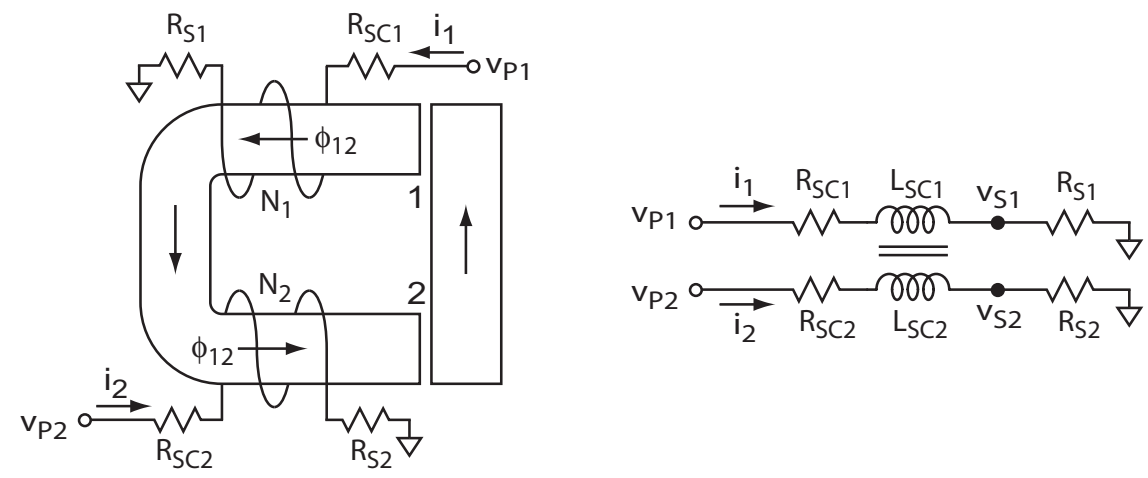

Figure 4-92: Simple model (left) and corresponding electrical circuit (right) for the case of driving each of the two steering flux coils on a stator core with its own power amplifier.

each stator core in series, and drive the resulting SISO system with a one amplifier. I do not discuss the details of the MIMO system compensation in this thesis. Instead, this section illustrates how the coils were coupled and describes the attendant system dynamics.

Figure 4-92 provides a simple model and corresponding electrical circuit for the case of driving each of the two steering flux coils on a stator core with its own power amplifier. The relationship between the output voltages of the power amplifiers $\left(V_{p 1}\right.$ and $\left.V_{p 2}\right)$, coil resistances $\left(R_{s c 1}\right.$ and $\left.R_{s c 2}\right)$ and inductances $\left(L_{s c 1}\right.$ and $\left.L_{s c 2}\right)$, sense resistors $\left(R_{s 1}\right.$ and $\left.R_{s 2}\right)$, and the coil currents $\left(i_{1}\right.$ and $\left.i_{2}\right)$ is given by (4.15):

$$
\left[\begin{array}{c}
V_{p 1} \\
V_{p 2}
\end{array}\right]=\left[\begin{array}{cc}
\left(R_{s c 1}+R_{s 1}+L_{s c 1} s\right) & L_{s c 2} s \\
L_{s c 1} s & \left(R_{s c 2}+R_{s 2}+L_{s c 2} s\right)
\end{array}\right]\left[\begin{array}{l}
i_{1} \\
i_{2}
\end{array}\right]
$$

The determinant of the system matrix in (4.15) is non-zero, so it can be inverted to find the coil currents in terms of the output voltages of the power amplifiers. For simplicity, the resistances and inductances for the two coil circuits are set equal to each other, which is the nominal case for the $10 \mathrm{kHz}$ FTS. This leads to (4.16) and the corresponding MIMO system block diagram in Figure 4-93.

$$
\left[\begin{array}{l}
i_{1} \\
i_{2}
\end{array}\right]=\operatorname{DET}(A)\left[\begin{array}{cc}
\left(R_{s c}+R_{s}+L_{s c} s\right) & -L_{s c} s \\
-L_{s c} s & \left(R_{s c}+R_{s}+L_{s c} s\right)
\end{array}\right]\left[\begin{array}{c}
V_{p 1} \\
V_{p 2}
\end{array}\right]
$$




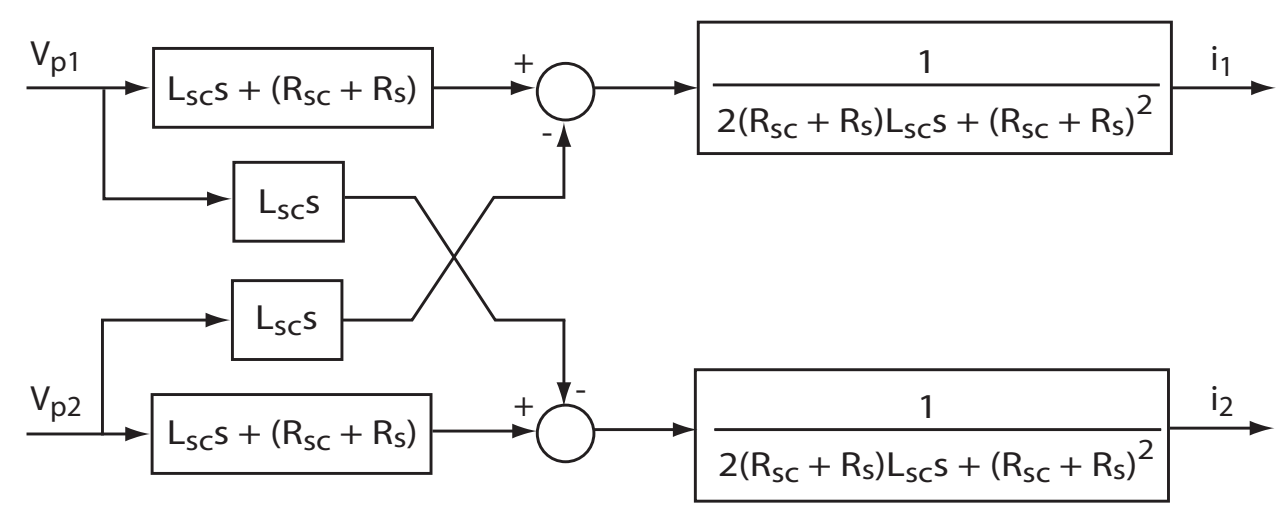

Figure 4-93: Block diagram corresponding to the simple model for driving each of the two steering flux coils on a stator core with its own power amplifier.
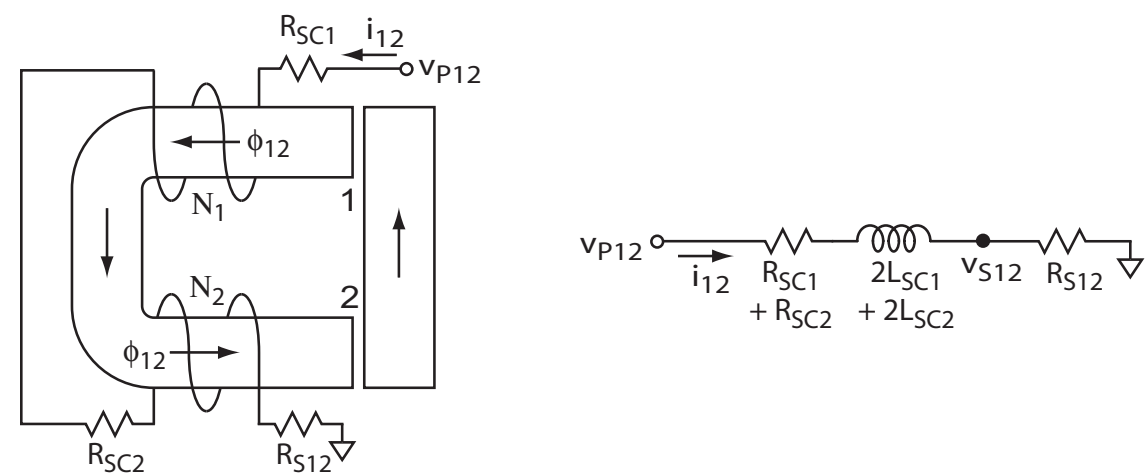

Figure 4-94: Simple model (left) and corresponding electrical circuit (right) for the case of driving a series connected pair of steering flux coils on a stator core with one power amplifier.

Where $\operatorname{DET}(A)$ in $(4.16)$ is:

$$
\operatorname{DET}(A)=\frac{1}{\left[\left(R_{s c}+R_{s}\right)^{2}+2\left(R_{s c}+R_{s}\right) L_{s c} s\right]}
$$

For comparison purposes, Figure 4-92 provides a simple model and corresponding electrical circuit for the case of driving a series connected pair of steering flux coils on a stator core with one power amplifier. Note that this is a SISO system, and represents the final electrical configuration for each stator half in the $10 \mathrm{kHz}$ FTS.

\subsubsection{Implementing Electronic Damping in Analog Circuitry}

Section 4.6.3 mentions that the linear mode of the actuator is used to produce electronic damping of the front/back vibration mode of the flexure-suspended rotor. This 
was achieved by a compensator that differentiates the output from the centerline capacitance sensor and applies an appropriate proportional gain to that synthesized velocity signal. To avoid amplifying high frequency noise in the position feedback signal, a pair of first-order poles is added to the compensator to roll off its high frequency gain. These poles are collocated at $48 \mathrm{kHz}$, which represents a trade-off between limiting the gain on high frequency noise and providing damping at frequencies up to $10 \mathrm{kHz}$. Since the digital control system uses a sampling rate of $75 \mathrm{kHz}$, the electronic damping is implemented in analog electronic circuitry.

The current-mode amplifier for the $10 \mathrm{kHz}$ FTS has provision for adding optional inputs into the current compensation loop (shown in Figure 4-83). Figure 4-95 shows how I re-wired one of the optional inputs to bring in the position feedback signal from the centerline capacitance sensor and feed it to only one of the unused ${ }^{13}$ current compensation channels in the amplifier. The optional input had to be used because the circuit board hard-wires the tool position capacitance sensor to the nominal input for all of the channels. I re-configured the formerly unused channel to realize the differentiator and two poles, and re-configured a second optional input to realize the inverter used to create the differential current command needed by the linear mode of the actuator.

Horowitz and Hill [78, page 225] describe the circuit topology that I used to create the differentiator and two first-order poles with an operational amplifier. Figure 4-96 is the circuit diagram for the electronic damping. $V_{c l}$ is the input voltage from the centerline capacitance sensor. The differential amplifier and unity-gain buffer were already hard-wired into the circuit board. The damping compensation (differentiator and two roll-off poles) is implemented in the operational amplifier circuit to the right of the buffer. The peak gain in that operational amplifier circuit is $1 / 2$, and occurs at the frequency of the two poles $(48 \mathrm{kHz})$. The opposite-signed outputs of the circuit go to the summing points of the current loop compensation operational amplifiers for the two pairs of steering flux coils, creating the differential current command for

\footnotetext{
${ }^{13}$ Now that I was driving the steering flux coils in pairs, two out of the four amplifier channels were not being used.
} 


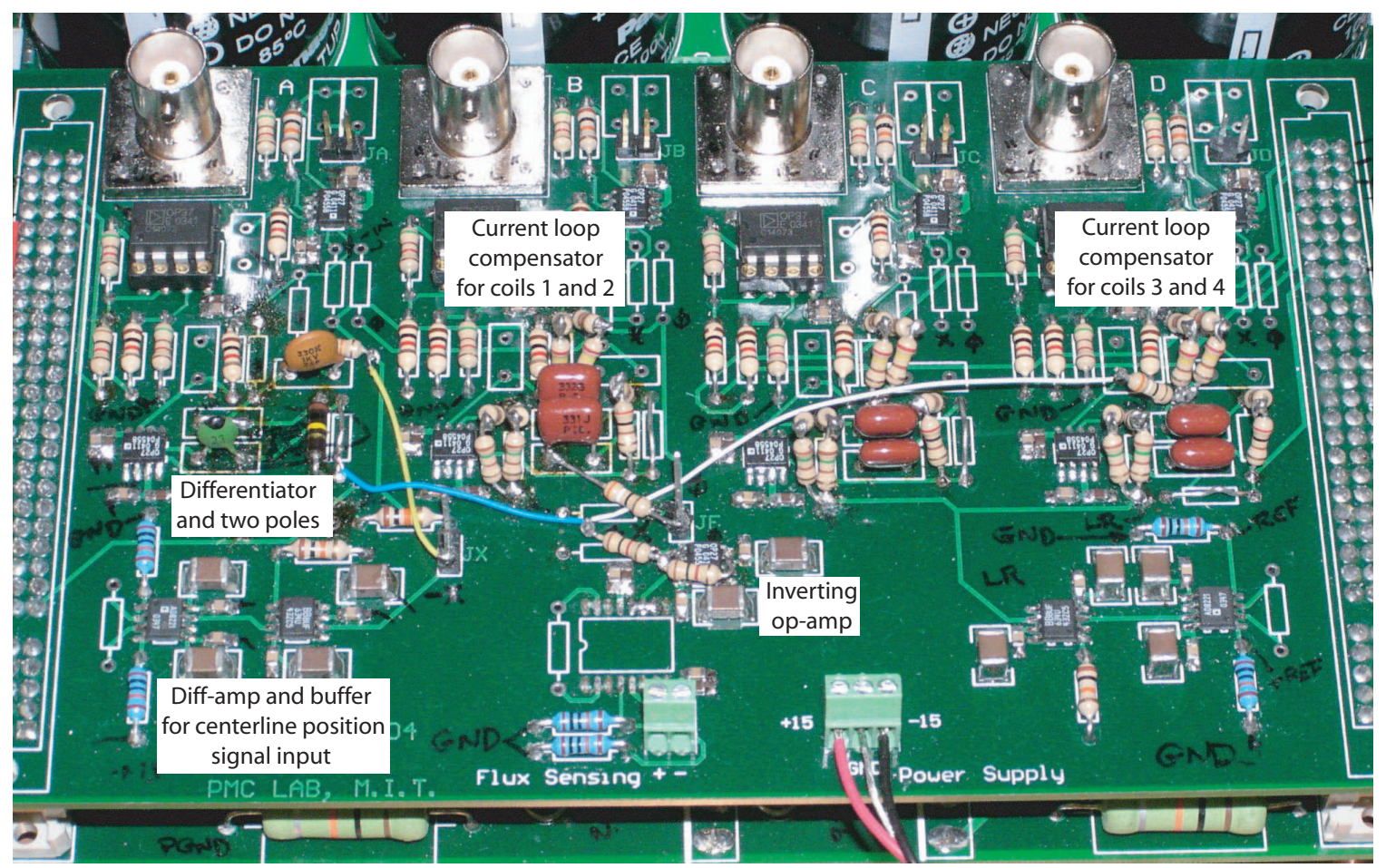

Figure 4-95: Re-wired version of the current compensation circuit board for implementing electronic damping in the $10 \mathrm{kHz}$ FTS.

the linear mode of the actuator. The rotor was rotationally locked and a torqueproducing current of $0.07 \mathrm{~A}$ was provided as an operating point for the linear mode. Additional details on how the rotor was locked are given in Section 4.10.2.

The values of the resistors at the output end of the circuit diagram in Figure 4-96 were determined experimentally. Starting with $10 \mathrm{k} \Omega$, which corresponds to a conservatively low DC gain for the electronic damping circuit, I incrementally decreased the value of the resistors (increased the DC gain) until I observed damping in the system. I then pushed the gain up until the linear mode started ringing at $39 \mathrm{kHz}$, which corresponded to the crossover frequency for the now unstable loop. I then backed off on the gain by a factor of four to achieve a stable system and my desired crossover frequency of $10 \mathrm{kHz}$. This gives a loop gain of 1.7 at the $6 \mathrm{kHz}$ frequency of the front/back translation mode of the rotor, which is sufficient for demonstrating damping of that mode. Since I was going to eventually use and depend on mechanical damping, I did not attempt to modify the compensation for the electronic damping so that I could increase the gain. 


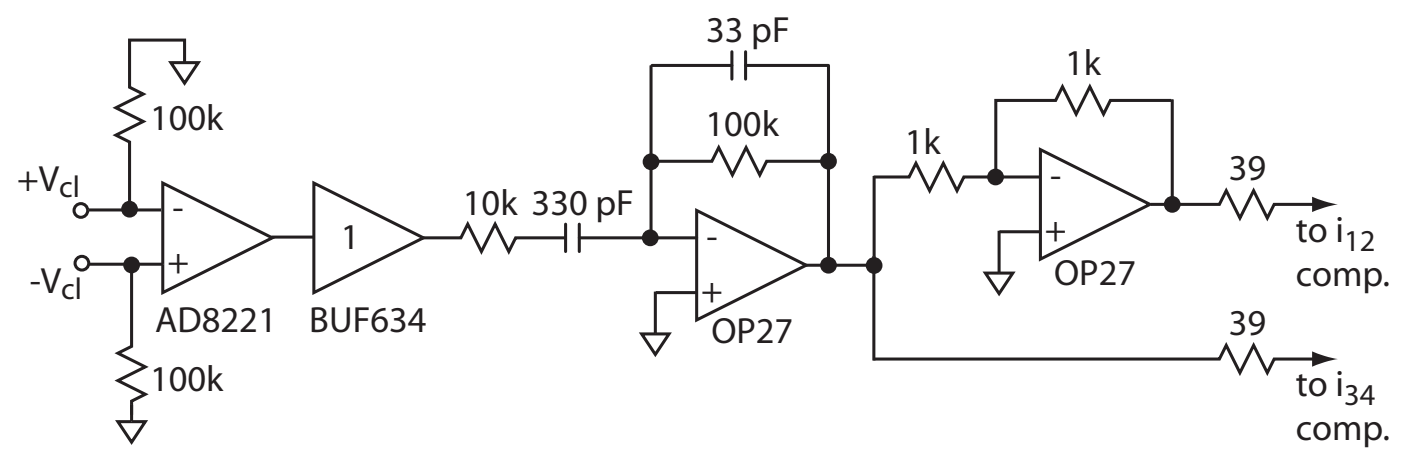

Figure 4-96: Circuit diagram for implementing electronic damping in the $10 \mathrm{kHz}$ FTS.

\subsection{Integrated Trade-Off Study}

To avoid being arbitrary in the decision-making process during the design of the highly integrated actuator and payload for the $10 \mathrm{kHz}$ FTS, I developed a spreadsheet-based trade-off study to allow balancing the requirements of the mechanical, electrical, and magnetic systems. These requirements largely conflict with each other and correspond to a multidimensional design space within which a set of acceptable solutions exist. The rotor and stator layout for the $10 \mathrm{kHz}$ FTS lends itself to a set of closed-form parametric equations that represent the design space and are driven by the dimensions of the rotor core. These equations are the engine for the trade-off study, which produced a set of acceptable choices for the dimensions of the rotor and stator cores for achieving a $1000 \mathrm{~g}$ tool tip acceleration. The final decision was influenced by the earlier success with the flexure suspension used in the $2 \mathrm{kHz}$ FTS. All things being fairly equal with the final candidates, I chose a set of rotor dimensions that allowed using the same detailed design for the flexure suspension hardware, and settled for a baseline tool tip acceleration of $900 \mathrm{~g}$. Ultimately, I departed from the baseline rotor design derived from the trade-off study by increasing the dimensions for certain components in the rotor. This provided a more mechanically robust $600 \mathrm{~g}$ version of the prototype rotor assembly, which increased my confidence that it would survive the anticipated tests for the $10 \mathrm{kHz}$ FTS. It was tempting to go overboard in the trade-off study and represent the design space with a set of three-dimensional plots that would provide "slices" from the higher-dimensional space. However, in the spirit 
of spending only enough time on this topic as needed to provide adequate guidance to move forward with this research, I did not, and instead visualized the design space based on a matrix representation of the data.

\subsubsection{Major Trade-off Areas}

Figure 4-97 illustrates the major design trade-offs involved in determining the dimensions of the rotor and stator assemblies for the $10 \mathrm{kHz}$ FTS. The following list describes these trade-offs.

- Rotor. For a given flux density in the rotor/stator air gaps, a long and slender rotor has a higher torque-to-inertia ratio than a short and thick rotor. However, the flexible modes of the short and thick rotor occur at a higher frequency than do those of the long and slender rotor. The frequency of the flexible modes of the rotor establish limits on the practical maximum operating frequency of the $10 \mathrm{kHz}$ FTS. For a given tool arm swing radius, the added inertia of the tool arm represents a smaller fractional increase in the total inertia for a short and thick rotor. Using the total inertia, the torque-to-inertia ratio sets the maximum achievable tool tip acceleration for a given swing radius of the tool.

- Air gaps. Large rotor/stator air gaps would provide relaxed mechanical tolerances on fabricating and assembling the actuator. The electrical power needed to drive magnetic flux in the air gaps is proportional to the volume of the air gaps. Therefore, small rotor/stator air gaps ease the requirements on the power amplifier for the $10 \mathrm{kHz}$ FTS.

- Flexure length. For a given maximum stress level in the flexure blades, long flexures allow a larger rotation of the rotor than do short flexures. However, the axial and radial stiffness of the flexure suspension decreases when the length of the flexures is increased.

- Core material. A soft magnetic material having a high maximum allowable flux density and low losses will allow producing high magnetic forces on the pole 


\begin{tabular}{|c|c|c|}
\hline long \& slender & ROTOR & short \& thick \\
\hline high torque/inertia ratio & & $\begin{array}{l}\text { high frequency } \\
\text { flexible modes }\end{array}$ \\
\hline
\end{tabular}

\begin{tabular}{lrr|}
\hline large & AIR GAPS & small \\
\hline $\begin{array}{l}\text { relaxed mechanical } \\
\text { tolerances }\end{array}$ & low reactive power \\
to drive flux
\end{tabular}

\begin{tabular}{|c|c|c|}
\hline long & FLEXURE LENGTH & short \\
\hline
\end{tabular}

\begin{tabular}{|lcr|}
\hline high saturation, low loss & CORE MATERIAL & low saturation, high loss \\
\hline high force, low heat & (actually, 4-way) & low force, high heat
\end{tabular}

\begin{tabular}{|lll}
\hline passive cooling COIL CURRENT DENSITY active cooling & C
\end{tabular}

Figure 4-97: Major design trade-offs involved in determining the dimensions of the rotor and stator assemblies for the $10 \mathrm{kHz}$ FTS.

faces with low thermal losses in the cores. The opposite results in low magnetic forces and high power losses. The candidate materials for the rotor and stator represent a four-way trade-off of these attributes because the material with the lowest loss did not have the highest allowable flux density.

- Coil current density. If the window area of the steering flux coils is large enough, then the current density in the coils is low enough to depend on passive cooling of the coils. For a given stator pole face area, the window area depends on the length of the poles that is available for the coil. Therefore, increasing the coil window area requires an increase in the volume of the stator core, and corresponding increase in the stator core losses.

\subsubsection{Procedure and Results}

This section describes how the dimensions of the rotor and stator assemblies affect the performance metrics that were considered during the trade-off study. Systematically 
varying the dimensions allowed surveying the design space in a manner that allowed identifying final candidates for the design. This section concludes with a discussion on how the final decision was made.

\section{Relationship Between the Dimensions of the Rotor and Stator Assemblies}

Figure 4-98 is a sketch showing the relationship between the dimensions of the rotor core, the rotor core stiffeners, and the stator core. The stator and rotor cores each have a constant thickness and have the same height. Setting the thickness of the rotor core equal to the width of the stator pole face provides a nominally constant flux density in the magnetic circuit. From the standpoint of operating the magnetic circuit at the maximum allowable flux density, setting the rotor core thickness greater than this value would add unnecessary mass. The width of the rotor core is set equal to the diameter of the stiffener plus two times the width of the stator pole face. This sets the width of the space between the stator poles to be equal to the diameter of the stiffener, and provides a small clearance between the stiffener and the stator core. ${ }^{14}$ The length of the stator poles was set equal to the length of the steering flux coil window plus an additional 0.125 inch to provide clearance between the coils and the stiffeners.

For a set diameter for the stiffener, changing the thickness of the rotor core causes an equal change in the thickness of the stator core and a corresponding change in the overall size of the stator core. Increasing the height of the rotor core causes an equal increase in the height of the stator cores. The trade-off study proceeded as follows:

1. Set a fixed diameter for the stiffener.

2. Set a fixed height for the rotor and stator cores.

3. Evaluate the performance metrics for different reasonable thickness values for the cores.

\footnotetext{
${ }^{14}$ An oversight during the trade-off study performed for the $10 \mathrm{kHz}$ FTS was the need for a more generous clearance between the stator core and the stiffeners than was provided by the difference between the diameter and the chord length of the stiffener. I increased that clearance during the detailed design of the hardware, but did not re-run the trade-off study because I assumed that it would not have altered the final choice of dimensions for the rotor and stator assemblies.
} 


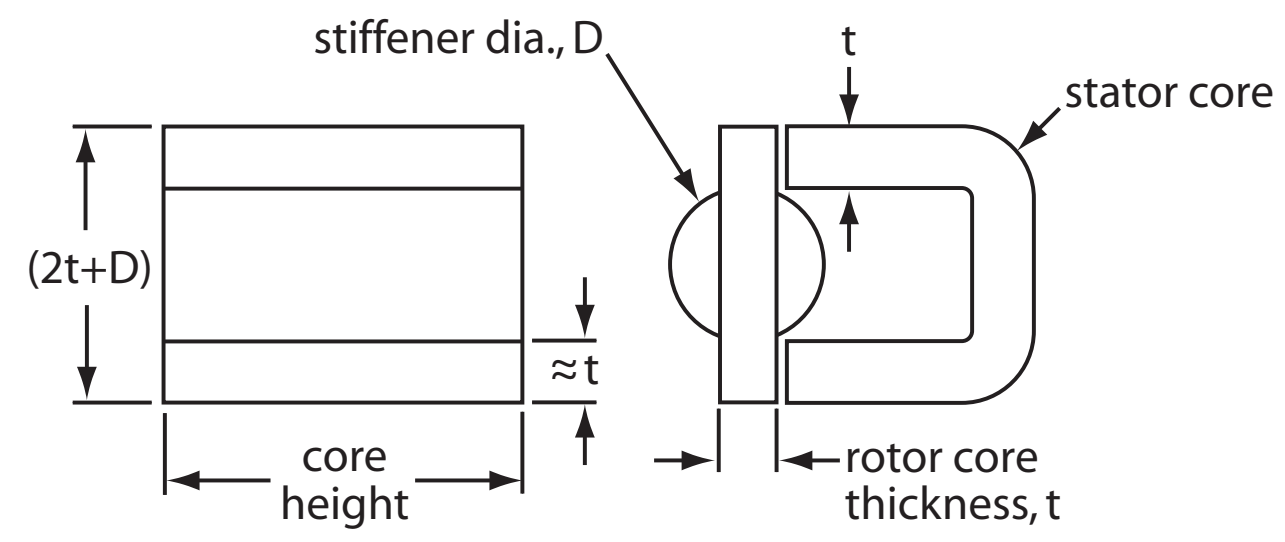

Figure 4-98: Sketch showing the relationship between the dimensions of the rotor core, the rotor stiffeners, and the stator core. Front view of the rotor, lying on its side (left). Top view of the rotor and one stator core (right).

4. Loop back to step (2) and change the height. Repeat this loop enough times to allow considering a reasonable range of height values.

5. Loop back to step (1) and change the diameter of the stiffener. Repeat this loop enough times to allow considering a reasonable range of stiffener diameter values.

\section{Performance Metrics Considered}

The following list describes the performance metrics that were considered during the trade-off study, and how they are affected by the dimensions of the rotor core, stiffeners, and stator core.

- Core losses. The power loss in a core varies linearly with the volume of the core. The trade-off study used 0.001 inch thick laminations of $50 \% \mathrm{Ni}-50 \% \mathrm{Fe}$ as the baseline material for both cores. Figure 5-15 shows that for the designed peak operation, the amplitude of the AC flux density is $1.5 \mathrm{~T}$ in the rotor core and $0.75 \mathrm{~T}$ in the stator core. I used the manufacturer's reported core power losses [101] corresponding to those flux densities for an operating frequency of $10 \mathrm{kHz}$ FTS. The volume and therefore power loss for the rotor and stator cores is driven by the diameter of the stiffener, height of the cores, thickness of the 
cores, and length of the coil window (which turns out to be a function of the stiffener diameter).

- Power to drive the AC flux in the rotor/stator air gaps. Section 5.3.3 develops the following relationship between the apparent time-average electrical power $P_{a, \text { elec }}$ into the series-connected pair of coils on a stator core and the timeaverage magnetic power $P_{a, \text { mag }}$ produced in the two rotor/stator air gaps for that core:

$$
\frac{1}{2} V_{p k} i_{p k}=P_{a, e l e c}=P_{a, m a g}=\frac{1}{2} H_{p k} B_{p k} \omega \forall_{g a p s}=\frac{B_{p k}^{2}}{2 \mu_{0}} \omega\left(A_{p} 2 g_{N}\right)
$$

This relationship assumes that the load is purely inductive, which is a valid assumption for the $10 \mathrm{kHz}$ FTS, and that the magnetic flux density is linearly dependent on the magnetic field intensity for the frequencies of interest (no losses or hysteresis). $V_{p k}$ is the peak load voltage, $i_{p k}$ is the peak load current, $H_{p k}$ is the peak magnetic field intensity, $B_{p k}$ is the peak magnetic flux density, $\omega$ is the operating frequency, and $\forall_{\text {gaps }}$ is the total volume of the air gaps in the magnetic circuit. Based on the design goals of producing a peak AC magnetic flux density of $0.75 \mathrm{~T}$ - the other half of the $1.5 \mathrm{~T}$ peak flux density is provided by the DC bias flux - and an air gap length of $50 \mu \mathrm{m}$, the total apparent timeaverage electrical power needed to drive the $\mathrm{AC}$ flux in all four air gaps is calculated based on the height and thickness of the rotor core (area of the pole faces) for the maximum operating frequency of $10 \mathrm{kHz}$.

- Current density and electrical loss. Each of the two stator poles has a steering flux coil surrounding it. If the coils fill the open space between the stator poles, then the area of that open space is equal to twice the window area for each coil. For the purpose of the trade-off study, each coil is assumed to have a ratio of 2:1 for its length divided by its build-up dimension. ${ }^{15}$ This sets the

\footnotetext{
${ }^{15}$ Section 5.3.4 provides a discussion on window area and build-up. The 2:1 ratio for the coil was set as the minimum allowable from the standpoint of keeping the winding layers close to the core to provide adequate coupling between the core and the magnetic field from the coil.
} 
window area for a pair of coils to be equal to the square of the diameter of the stiffener. Section 5.3 shows that the pair of coils needs to produce an MMF of $60 \mathrm{amp}$-turns to provide a flux density of $0.75 \mathrm{~T}$ in the $50 \mu \mathrm{m}$ long air gaps. The trade-off study used an approximate value for the packing factor of $80 \%$, which in hindsight is a little optimistic. We can now determine the current density in the coils as a function of the diameter of the stiffener. Assuming square end turns for the coils, we can also determine the corresponding electrical loss in each pair of coils.

- Vibration modes: translation modes. The axial and radial direction rigid body translation modes of the flexure-suspended rotor are calculated based on the total mass of the rotor and the stiffness provided by the flexure suspension. The rotor mass includes the rotor core, the two stiffeners, the two flexure hubs, and the tool arm. The dimensions and hence the mass of these components are tied to the dimensions of the rotor core and the stiffeners. The axial and radial stiffness of the flexure suspension is based on eight pre-tensioned 0.010 inch thick by 0.500 inch high by 0.250 inch long steel blades using the analysis described in Section 4.5.6. The tool swing radius was fixed at 0.500 inch. As the stiffener and rotor core dimensions are varied, the total mass and frequency of the corresponding translation modes are calculated.

- Vibration modes: analytical first bending modes with tool arm. Closedform equations were developed for calculating the frequency of the first bending modes of the rotor with the tool arm attached to it. The analysis considers bending in the "front" plane of the rotor (parallel to the pole faces), and bending in the "right" plane (perpendicular to the pole faces). The rotor is modelled as a composite continuous beam consisting of the rotor core and the two stiffeners, with the tool arm as an extended mass attached to one end of the beam. The flexure hubs are at the support points provided by the flexure suspension, so do not translate during the rotor bending modes. The rotational energy of the flexure hubs is neglected because it is assumed to be negligible com- 
pared to the energy stored and recovered by bending of the rotor and motion of the tool arm. Dunkerley's method $[18,127]$ is used to estimate the lowest frequency bending mode of a continuous beam with an attached discrete mass. This method approximates that frequency using the following relationship:

$$
\frac{1}{f_{1}^{2}} \approx \frac{1}{f_{A}^{2}}+\frac{1}{f_{B}^{2}}
$$

Where $f_{1}$ is the frequency of the fundamental mode of the composite beam, $f_{A}$ is the frequency of the fundamental bending mode of the beam acting alone, and $f_{B}$ is the vibration mode of the mass on the equivalent linear spring representation of the support that the beam provides it. Beards [18, page 162] shows through an example that the Dunkerley method provides good agreement with a more rigorous solution derived using Raleigh's energy method, which matches the kinetic and strain energy of the system. I chose the Dunkerley method because it allowed creating a straightforward parametric model for use in the trade-off study.

The beam is modelled as being simply supported with a uniformly distributed mass per unit length. The simple supports allow finite rotation of the flexure hubs and lead to lower (conservative) frequency modes than expected from the pseudo-fixed constraints provided by the flexures. To allow using readily available equations for calculating the bending stiffness and fundamental frequency of the beam [131, page 96, 576], an equivalent single-material beam is used to model the composite beam. Roark and Young describe an equivalent-width technique that allows doing this:

Beams that are constructed of more than one material can be treated by using an equivalent width technique if the maximum stresses in each of the several materials remain within the proportional limit. An equivalent cross section is developed in which the width of each component parallel to the principal axis of bending is increased in the 
same proportion that the modulus of elasticity of that component makes with the modulus of the assumed material of the equivalent beam [131, page 112].

Before using the equivalent-width technique I had to simplify the geometry of the rotor. The chordal sections of the stiffeners are transformed into rectangular sections having the same mass and bending stiffness. The distance between the neutral axes of the rotor core and the neutral axes of the actual stiffeners is preserved when "attaching" the equivalent rectangular stiffeners to the rotor core. As the stiffener and rotor core dimensions are varied, the frequency of the first bending mode of the rotor with the tool arm attached to it is calculated for bending in the front plane and in the right plane.

- Vibration modes: Finite element analysis of selected cases. Using the finite element analysis model discussed in Section 4.5.8, I determined the frequency of the first non-rigid body mode for the rotor with the tool arm attached to it for selected cases of rotor and stiffener dimensions. At least one FEA model was evaluated for each of the stiffener diameters that were considered in the trade-off study. In most cases, multiple rotor core heights and/or thicknesses were considered. In almost all cases, the first non-rigid body mode is a torsional mode. The exceptions are the bending modes for the rotors having a long and slender geometry, in particular when the stiffener diameter was 0.250 inch.

- Tool acceleration. As the dimensions of the stiffeners and rotor core are varied, the rotational inertia of the rotor with the tool arm attached to it, and the lever arms from the axis of rotation to the pole faces is calculated. Using a maximum flux density of $1.5 \mathrm{~T}$ and the relationship between flux density and torque that is developed in Section 5.2.3, the expected tool tip acceleration is calculated for a fixed tool swing radius of 0.500 inch. 


\section{Final Candidates and Final Decision}

Figure 4-99 shows an example of how most of the performance metrics for the rotor assembly were visualized as the stiffener diameter and rotor core height were varied. The data points in the graphs are driven by parametric closed-form equations, and do not include the FEA data. The thickness dimension of the rotor core was held constant for the cases shown in Figure 4-99. The horizontal lines with an attached arrow represent design goals that guided selection of the final candidates. A set of graphs similar to those shown in Figure 4-99 were produced for each thickness value considered for the rotor core during the trade-off study.

Figure 4-100 shows, in matrix format, the final design space that was considered in the trade-off study for the $10 \mathrm{kHz}$ FTS. Note that the rows are arranged in major and minor groupings. The major grouping has a fixed diameter for the stiffeners. Within each major grouping are minor groupings that have fixed heights for the rotor core. The thickness of the rotor core is varied within each minor grouping. The ranges for the dimensions that are considered in Figure 4-100 were picked based on the following: start with a range of component sizes that seemed reasonable from a fabrication standpoint, run the trade-off study using this preliminary range of dimensions, and then expand or contract regions of that preliminary design space based on the results.

The following variables were held constant throughout the final trade-off study: $50 \mu \mathrm{m}$ long rotor/stator air gaps, $1.5 \mathrm{~T}$ peak flux density, $10 \mathrm{kHz}$ operating frequency, a $14 \mathrm{gm}$ tool holder having a 0.500 inch tool swing radius and an $11 \mathrm{gm} \cdot \mathrm{cm}^{2}$ rotational inertia, and flexure hubs to accommodate 0.5 inch wide flexure blades.

Close scrutiny of Figure 4-100 will reveal that at the time of the trade-off study I was keeping track of the peak electrical power needed to drive AC magnetic flux in the air gaps, which happens to be equal to the apparent time-average electrical power for a purely inductive load. ${ }^{16}$

The maximum tool tip acceleration for each minor grouping is highlighted. The baseline design is the highlighted row, "0.625, 0.188, 0.250, 1.000, ...". The baseline

\footnotetext{
${ }^{16}$ For a purely inductive load, the current lags the voltage by $90^{\circ}$. Therefore: $P_{\text {peak }}=\left[V_{\text {peak }} \cos \left(45^{\circ}\right)\right]\left[I_{\text {peak }} \sin \left(45^{\circ}\right)\right]=(1 / 2) V_{\text {peak }} I_{\text {peak }}=P_{\text {ave }}$.
} 

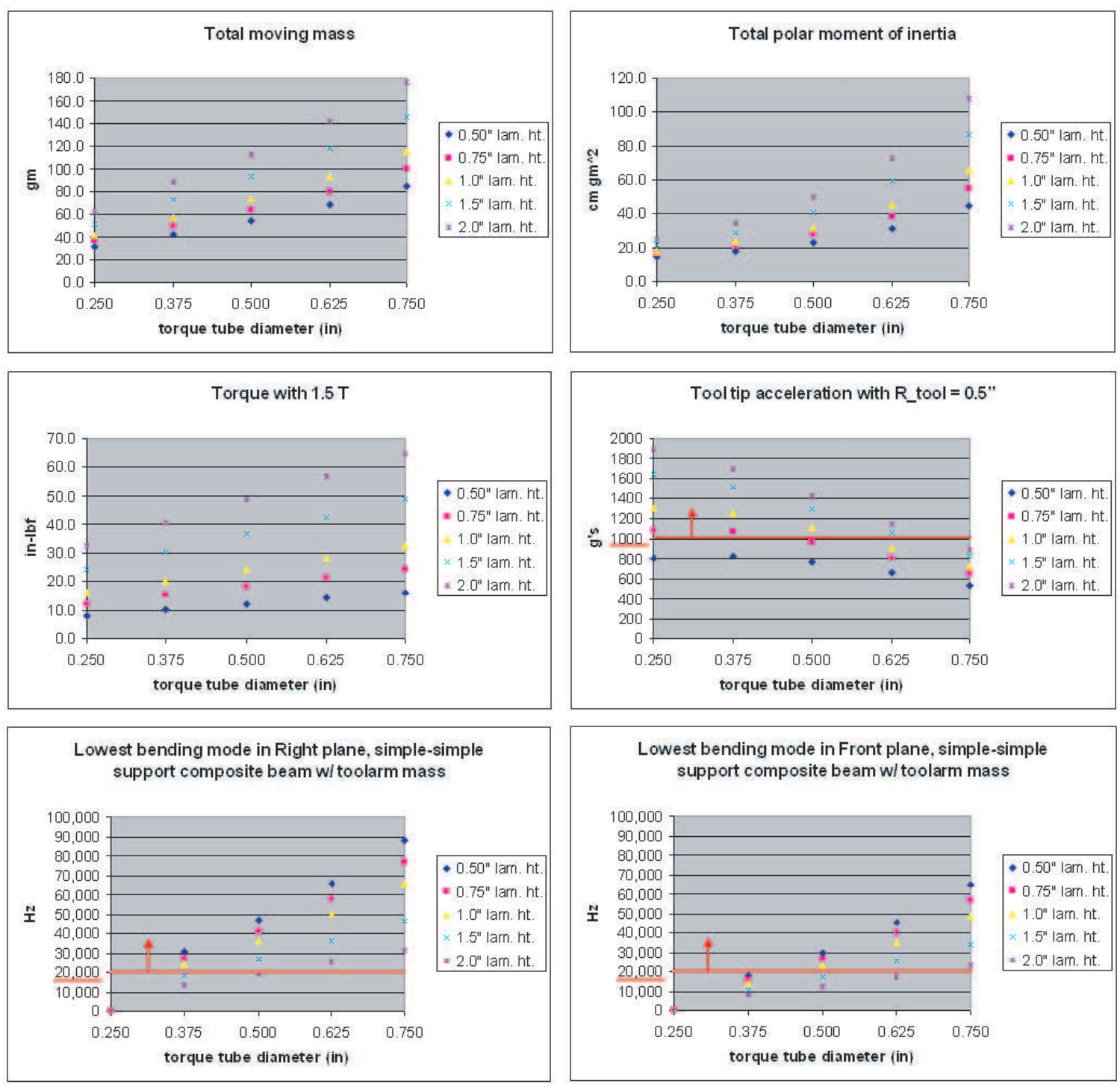

Figure 4-99: Example of how most of the performance metrics for the rotor assembly were visualized when the stiffener diameter and rotor core height were varied. 


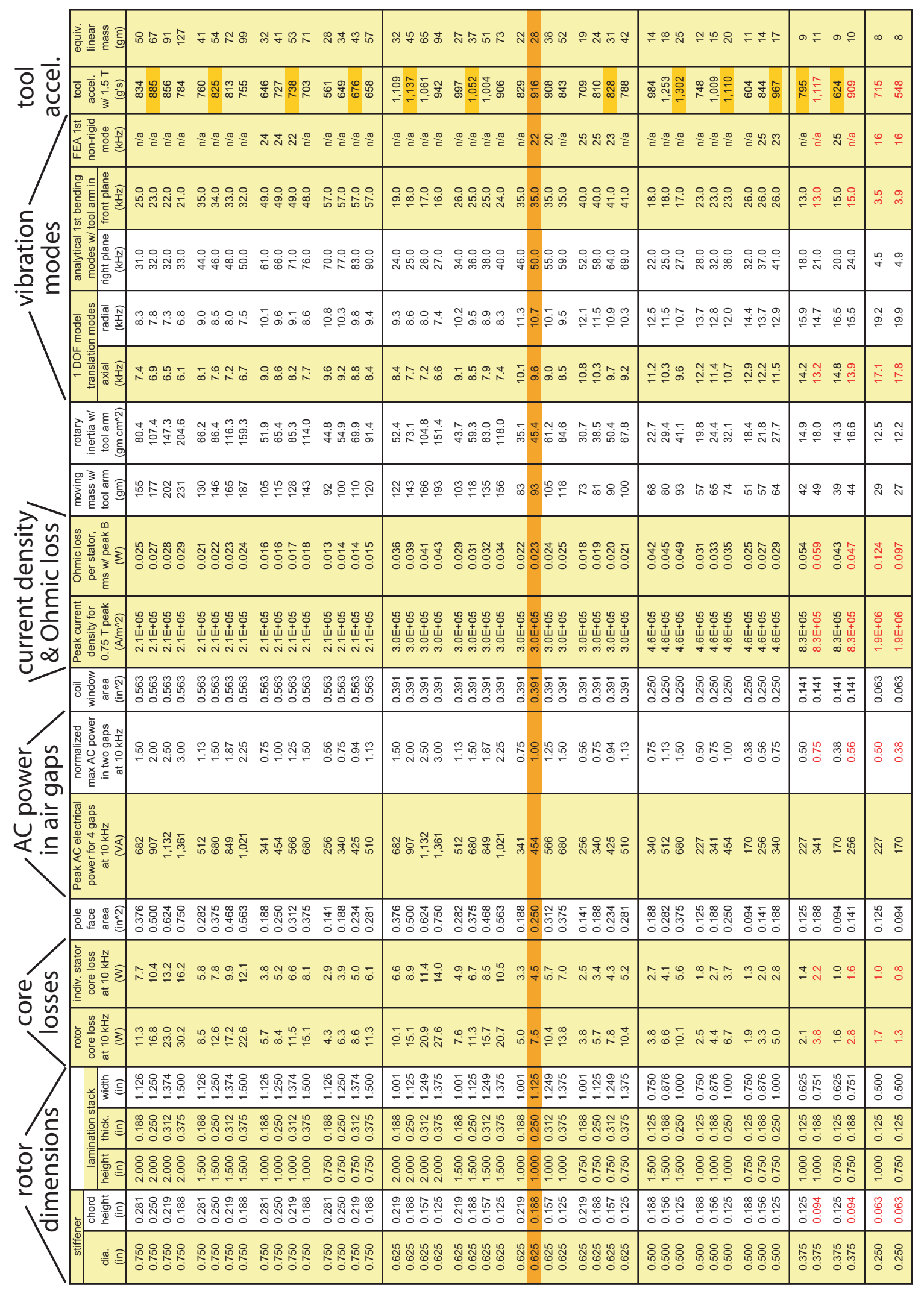

Figure 4-100: The final design space for the $10 \mathrm{kHz}$ FTS trade-off study. The baseline design is the highlighted row, "0.625, 0.188, 0.250, 1.000, ...". 
rotor uses 0.625 inch diameter stiffeners with a 0.250 inch thick by 1.000 inch high rotor core. It provides the best combination of high acceleration, reasonable core losses and $\mathrm{AC}$ electrical power requirement, and adequately high frequency for the first vibration modes, while allowing the flexure mounting hardware design from the $2 \mathrm{kHz}$ FTS to be used. The higher tool tip acceleration for a taller rotor core with the 0.625 inch diameter stiffener was attractive, but I chose to avoid the attendant lower frequency bending modes and higher required AC electrical power. Reducing the stiffener diameter for the baseline to 0.500 inch was an attractive alternate choice for the baseline because it increases the tool tip acceleration by $21 \%$. However, I did not because it decreases the frequency of the bending modes and the smaller flexure hubs would require modifying the proven design for the flexure mounting hardware.

Using the baseline design, I investigated increasing the swing radius for the tool from 0.500 inch to 0.750 inch. The larger tool holder was designed to provide the same support-stiffness for the tool, which increased the total mass and rotational inertia for the rotor assembly by $5 \mathrm{gm}$ and $10 \mathrm{gm} \cdot \mathrm{cm}^{2}$, respectively. The longer swing arm increased the baseline tool tip acceleration from $920 \mathrm{~g}$ to $1120 \mathrm{~g}$, however the first non-rigid body mode of the tool arm decreased from $29 \mathrm{kHz}$ to $18 \mathrm{kHz}$, which I considered unacceptable.

\subsubsection{Departure from an Optimum Design for Survivability}

Designing and fabricating the rotor assembly for the $10 \mathrm{kHz}$ FTS involved a significant set of new issues and unknowns for me. It was my first time designing an electromagnetic actuator and working with laminated soft magnetic materials. It was also the first time that I was depending on adhesive-bonded joints in the critical components of a machine. Since it was more important to this research that the mechanical structure did not fail during assembly and use, we were willing to compromise on the peak tool tip acceleration in return for a more mechanically robust system. I departed from the baseline rotor design derived from the trade-off study by increasing the dimensions for certain components in the rotor. This provided a more mechanically robust $600 \mathrm{~g}$ version of the prototype rotor assembly, which increased 
my confidence that it would survive the anticipated tests for the $10 \mathrm{kHz}$ FTS. Having gained confidence with the successful tests and performance demonstrations for the $10 \mathrm{kHz}$ FTS and its actuator, design parameters can be adjusted in future versions to achieve tool tip accelerations of $1000 \mathrm{~g}$ and beyond.

Figure 4-101 shows a solid model of the rotor assembly based on the baseline choice from the trade-off study, and a model of the more mechanically robust version used in the $10 \mathrm{kHz}$ FTS. The incremental steps leading to the ultimate differences between the two, and the corresponding affect on the peak tool tip acceleration, are described in the following list.

- The baseline peak tool tip acceleration from the trade-off study is $920 \mathrm{~g}$.

- Adding the flexure hub "wings" and the fasteners that were not included in the trade-off study reduced the peak tool tip acceleration to $840 \mathrm{~g}$.

- The height of both flexure hubs was increased by 0.125 inch to increase the width of the adhesive bond-line between the stiffeners and flexure hubs from 0.125 inch to 0.250 inch. This provided a stronger adhesive-bonded joint, and decreased the peak tool tip acceleration to $800 \mathrm{~g}$.

- The width of the rotor and stator cores was increased from 1.125 inch to 1.250 inch to provide a larger clearance between the stator cores and the rotor stiffeners. This reduced the peak tool tip acceleration to $690 \mathrm{~g}$.

- The width of the rotor core was increased from 1.250 inch to 1.375 inch to provide nominally non-flux-carrying outer portions of the rotor core for the "hard stops" between the rotor and stator. The rotational inertia of the rotor plus the tool holder is now $81 \mathrm{gm} \cdot \mathrm{cm}^{2}$, which reduces the peak tool tip acceleration for a 1.5 T flux density to $590 \mathrm{~g}$ (round off to $600 \mathrm{~g}$ ). I wanted to avoid work hardening the nominal flux-carrying portions of the rotor (and stator) cores, which would reduce the magnetic permeability of the material [17]. It is worth noting that if the flexure hub wings were the only portions of the rotor that contacted the hard stops, potentially catastrophic stress levels in the joints between the 


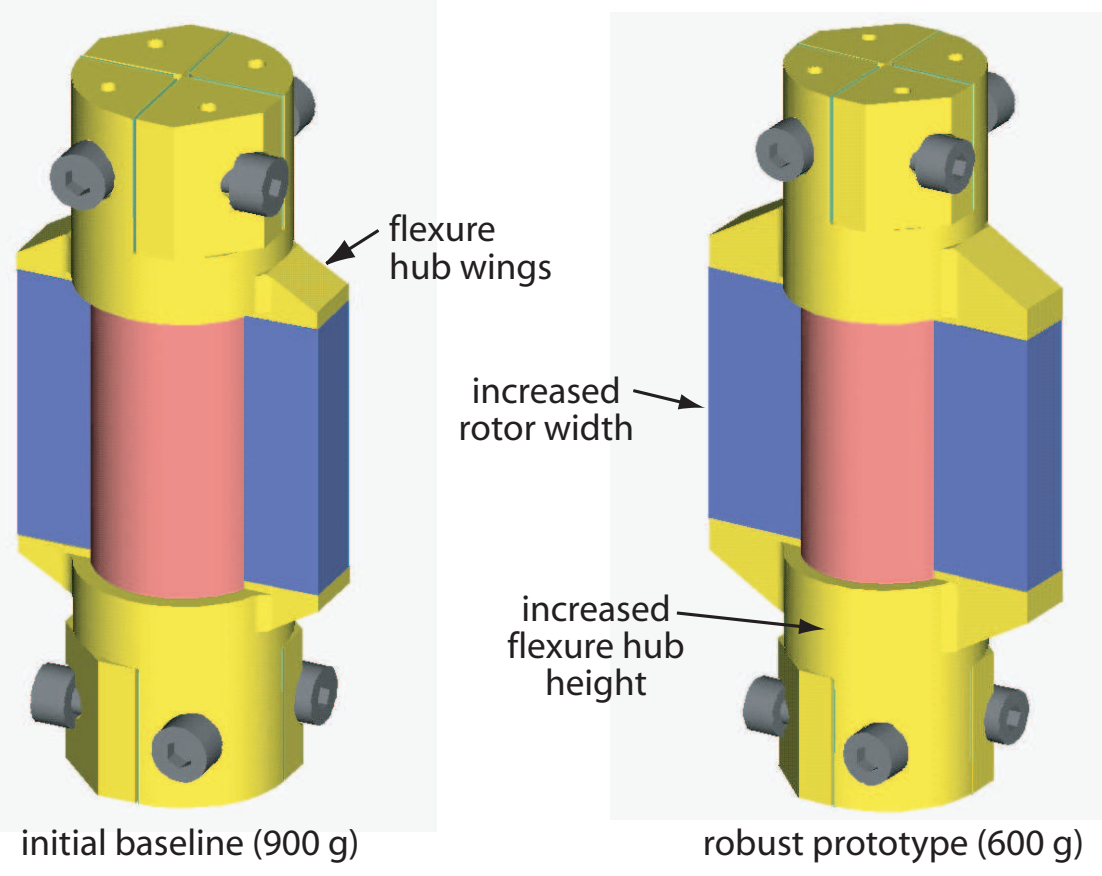

Figure 4-101: Solid models showing the differences between the baseline rotor assembly design from the trade-off study (left), and the more mechanically robust version used in the $10 \mathrm{kHz}$ FTS (right).

rotor core and the flexure hubs would be developed when the magnetic forces acting on the rotor core were driving the rotor into the hard stops.

\subsection{Control System}

This section describes the control system and compensation used to establish a lownoise implementation and a high-acceleration implementation of the $10 \mathrm{kHz}$ FTS. The measured frequency and time responses of the tool position for both implementations is presented and explored. The section concludes with a discussion on the measured noise levels in the signal from the capacitance sensor that provides feedback on the tool tip displacement.

As with the earlier $2 \mathrm{kHz}$ FTS, the $10 \mathrm{kHz}$ FTS utilizes a hybrid analog and digital control system. Feedback of the actuator current is used to compensate for the electrical dynamics of the actuator, essentially removing those dynamics from the tool position loop at the operating frequencies of interest. The block diagram for the 
control system is shown in Figure 4-102. The current loops - one for each stator half - have a crossover frequency of approximately $50 \mathrm{kHz}$, and are implemented with analog electronics to avoid the phase delay inherent with the sampling process of the digital controller. Electronic damping with the linear mode of the actuator is implemented with analog electronics for the same reason. The rotary mode of the actuator is used to control the tool tip position. The tool position loop has a crossover frequency of $2.3 \mathrm{kHz}$ and is implemented with a digital controller using a sampling rate of $75 \mathrm{kHz}$. The digital controller is the same dSPACE GmbH 1103-board [51] PC-based prototyping environment that was used for the $2 \mathrm{kHz}$ FTS and is described in Section 3.10.1.

The torque constant for the actuator in the $10 \mathrm{kHz}$ FTS has a linear dependence on the bias flux coil current. I tuned the position loop compensation for two different bias coil currents to establish the low-noise and the high-acceleration implementations mentioned in Section 4.6. The performance of the current loops is presented in Section 4.7, so is not repeated here. Likewise, details on the analog controller for the electronic damping and its performance are discussed in Sections 4.7.6 and 4.6.3, respectively. The loop-shaping technique that I used to design all of the compensators for the $10 \mathrm{kHz}$ FTS is described in Section A.1.

The "plant" in Figure 4-102 consists of the two second-order mechanical systems for the rotary and linear modes of the rotor that are described in Section 4.6.2. The constant gains $K_{e c t}$ and $K_{e c c}$ are the scaling factors for the capacitance sensors behind the tool and at the centerline of the rotor, respectively. Note that the signal from the tool capacitance sensor passes through an anti-aliasing filter before entering the digital controller. The two "trim" gains in the digital controller allow balancing the nominal torque-only currents to the two pairs of coils. The trim gains are set by commanding a sinusoidal tool motion (rotor rotation) and adjusting the trim values to minimize the front/back translation of the rotor observed by the centerline capacitance sensor. 


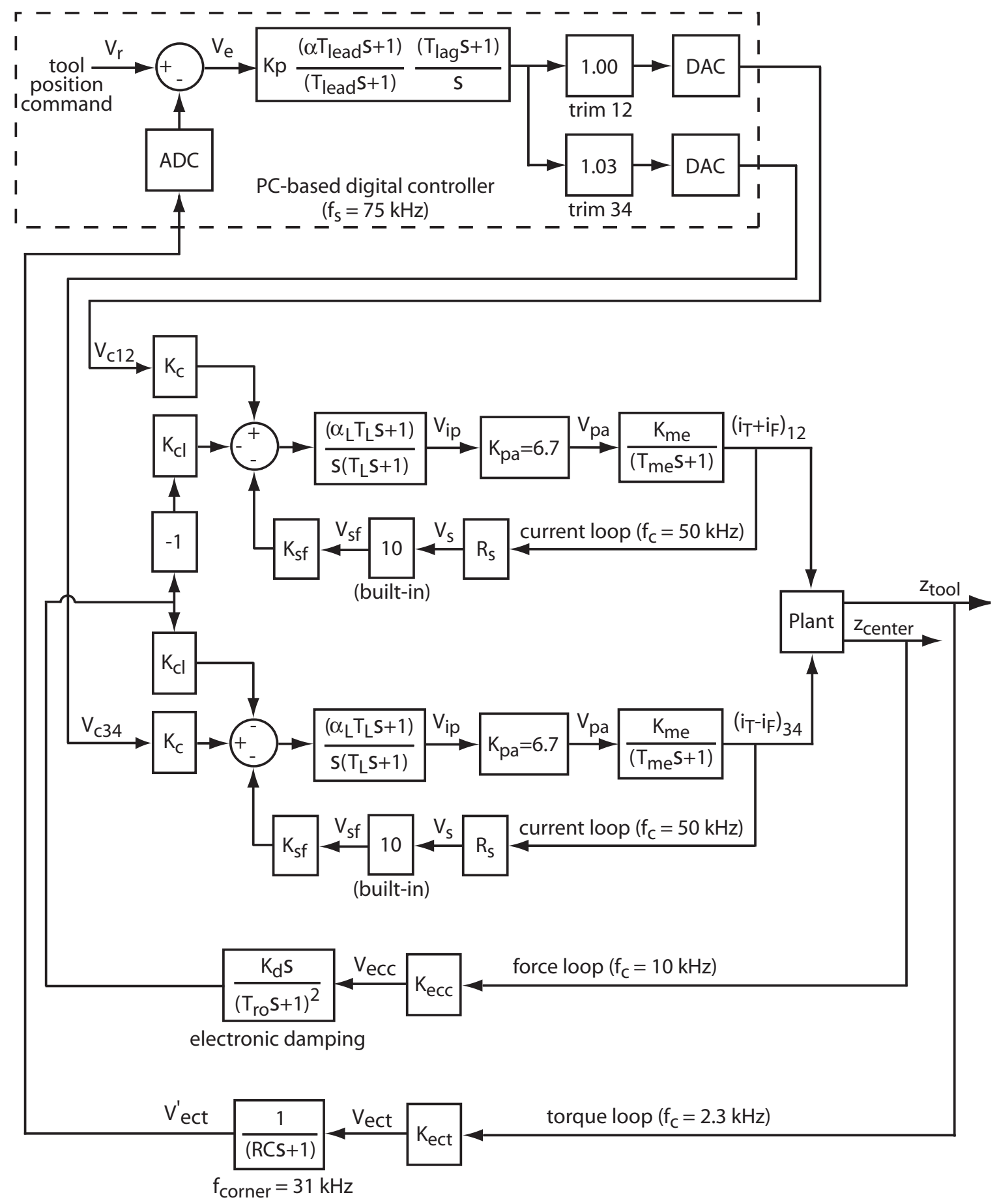

Figure 4-102: Control system block diagram for the $10 \mathrm{kHz}$ FTS, with electronic damping. 


\subsubsection{Low-Noise Implementation}

The current in the bias flux coil is set to $0.545 \mathrm{~A}$ for the low-noise implementation of the $10 \mathrm{kHz}$ FTS. This causes the torsional spring from the flexure suspension to dominate over the torsional spring from the magnetic flux, resulting in no open-loop poles in the right-half plane. The low-noise implementation includes the mechanical damping described in Section 4.5.9. Developing compensation in this case was straightforward because the system behaves as a simple mass-spring-damper.

Figure 4-103 shows the block diagram for the digital controller for the tool position for the low-noise implementation of the $10 \mathrm{kHz}$ FTS, illustrating the lead and lag series compensation that was used. The lead compensator uses an $\alpha=10$ and a time constant of $T_{\text {lead }}=2.19 \times 10^{-5} \mathrm{sec}$ (geometric mean frequency of $2.3 \mathrm{kHz}$ ). The lag compensator consists of a pure integrator and a zero with a time constant of $T_{\text {lag }}=7.96 \times 10^{-4} \mathrm{sec}(200 \mathrm{~Hz})$. A couple of extra elements are present in this block diagram, which is actually the Simulink ${ }^{\circledR}$ model that was implemented in the real-time controller. The "toggle", "zero signal", and "switch1" blocks allow using the dSPACE GmbH GUI to turn the controller on and off and reset the integrator. The "comnd offset" block allows centering the electrical and mechanical rotation angles of the rotor. The "signal generator" block provides a reference input signal for testing and debugging the controller.

Figure 4-104 shows a Bode plot of the measured negative loop transmission for the tool position for the low-noise implementation. The crossover frequency for this system is $2.3 \mathrm{kHz}$ with a phase margin of $32^{\circ}$ and a gain margin of $5 \mathrm{~dB}$. The peaks at $10 \mathrm{kHz}$ and $13 \mathrm{kHz}$ correspond to stable torsional modes of the rotor, which are discussed in Section 4.6.3. Figure 4-105 shows a detail of the measured negative loop transmission, illustrating the damped front/back mechanical modes of the rotor in the frequency range of 5 to $7 \mathrm{kHz}$.

Figure 4-106 shows the Nyquist diagram for the measured negative loop transmission for the low-noise implementation. Note that there are no encirclements of the -1 point, which is consistent with a stable system having no open-loop poles in the 


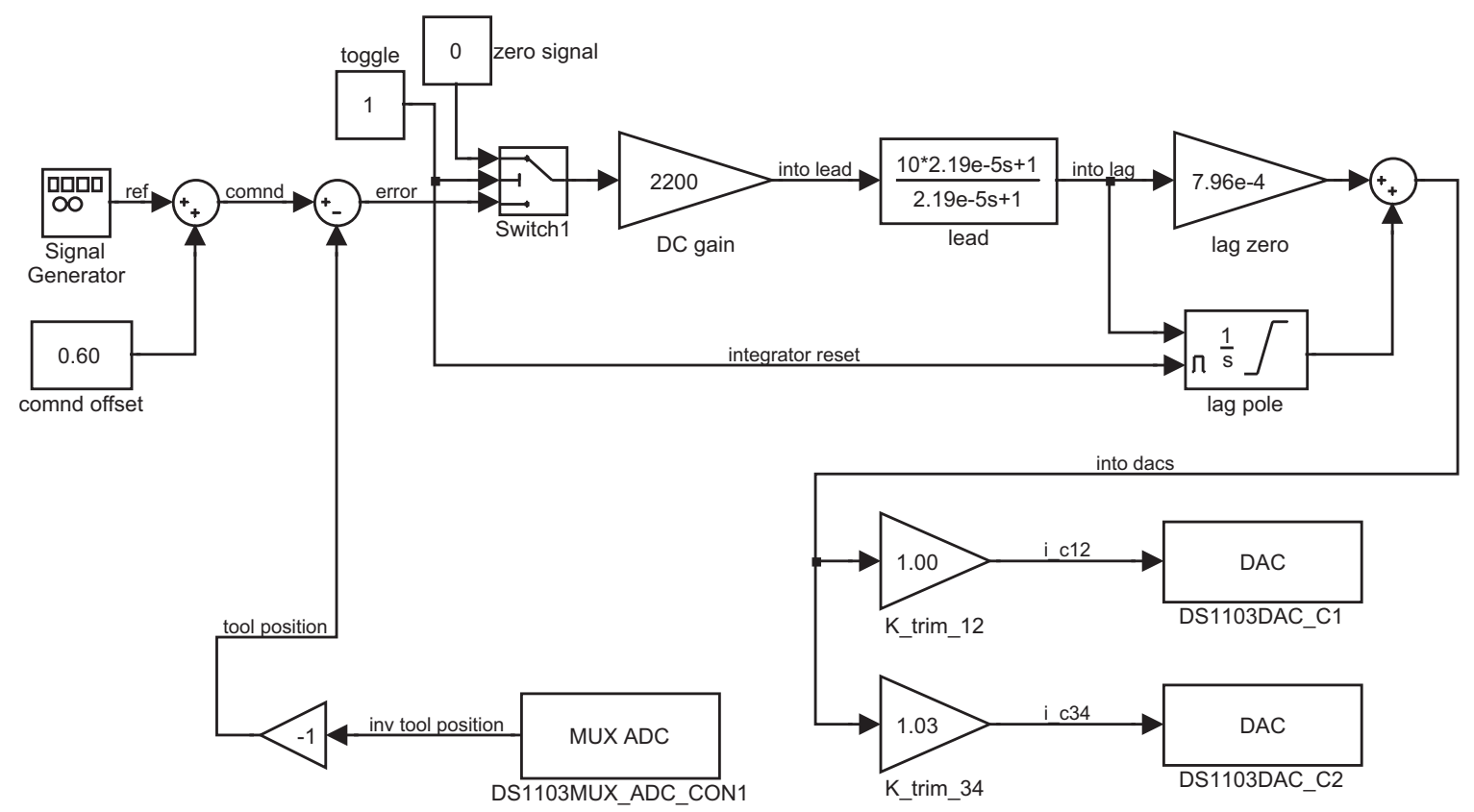

Figure 4-103: Block diagram for the digital controller for the tool position for the low-noise implementation of the $10 \mathrm{kHz}$ FTS.
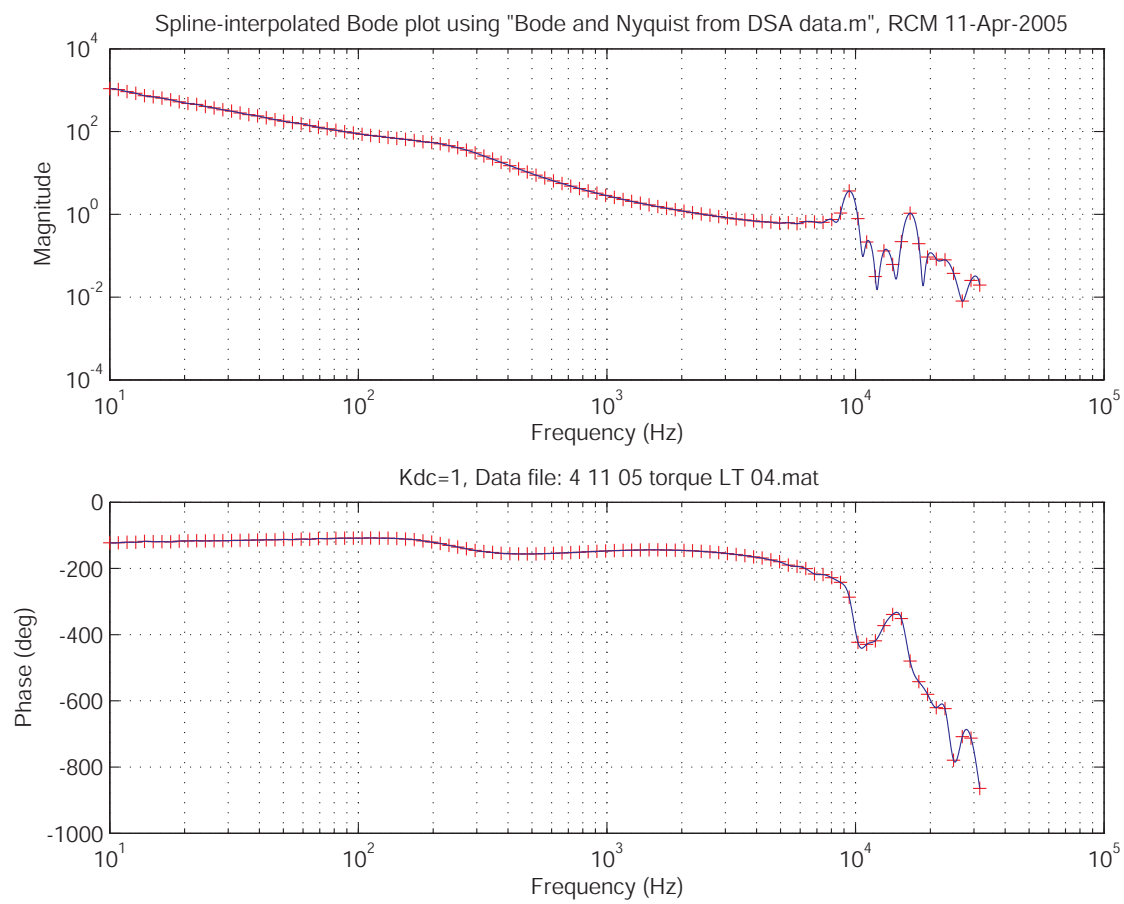

Figure 4-104: Bode plot of the measured negative loop transmission for the tool position for the low-noise implementation of the $10 \mathrm{kHz}$ FTS. 

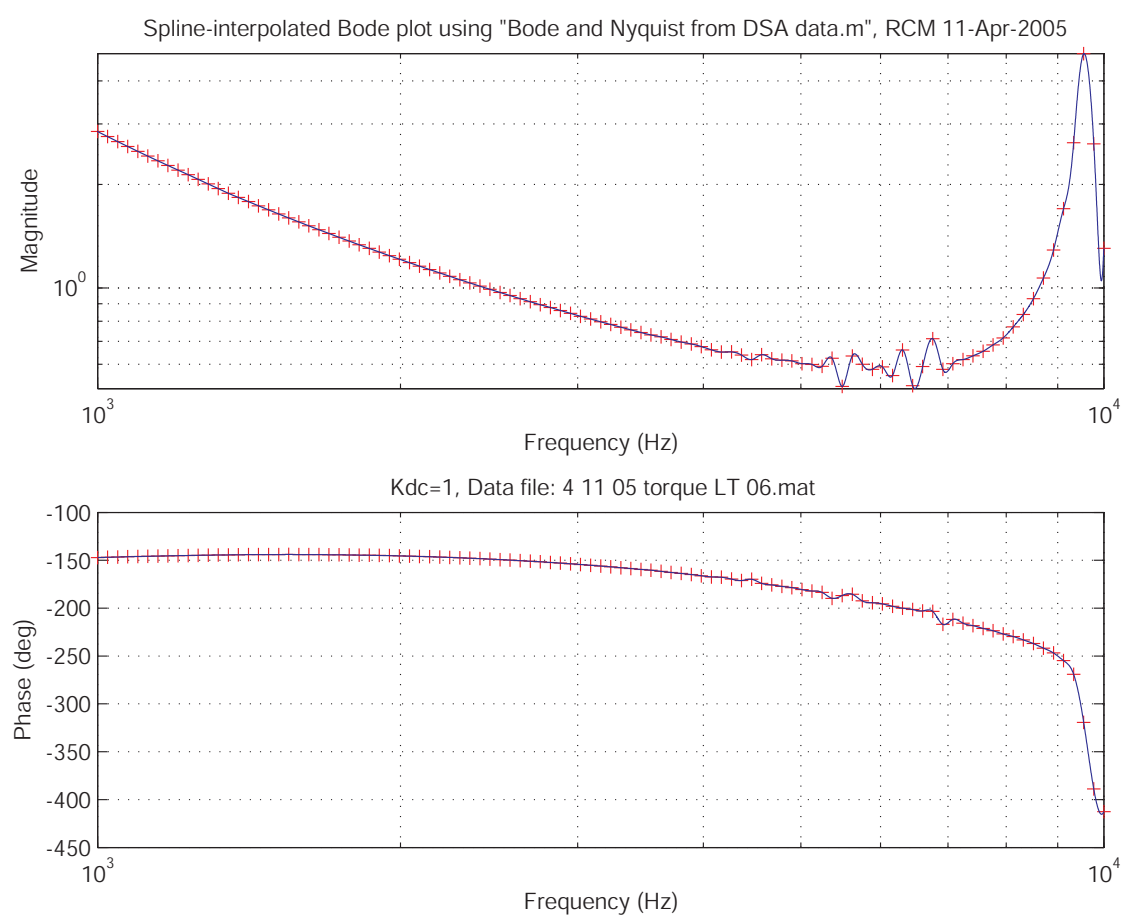

Figure 4-105: Bode plot showing high frequency detail of the measured negative loop transmission for the tool position for the low-noise implementation of the $10 \mathrm{kHz}$ FTS.

right-half plane.

Figure 4-107 shows the measured small-signal closed-loop response for the lownoise implementation. The $-3 \mathrm{~dB}$ closed-loop half-power bandwidth is at $10 \mathrm{kHz}$. Figure 4-105 shows a detail of the measured small-signal closed-loop response. The phase angle changes fairly smoothly up to $5 \mathrm{kHz}$, suggesting that a possible future addition of a feedforward compensator could be effective up to that frequency. However, the ripple in the magnitude plot between $4 \mathrm{kHz}$ and $5 \mathrm{kHz}$ could result in reduced accuracy of a feedforward compensator in that frequency range. Ludwick [99] provides an excellent overview of various feedforward controllers that are suitable for a fast tool servo. Essentially, the feedforward controller applies a frequency-dependent magnitude and phase adjustment to the commanded reference signal to compensate for the known closed-loop response of the system.

The measured step response for the low-noise implementation for a $1 \mu \mathrm{m}$ tool position step is shown in Figure 4-109. This step response has a $32 \mu$ sec rise time (10\% to $90 \%$ ) and a $90 \%$ overshoot. The rise time is consistent with the measured 


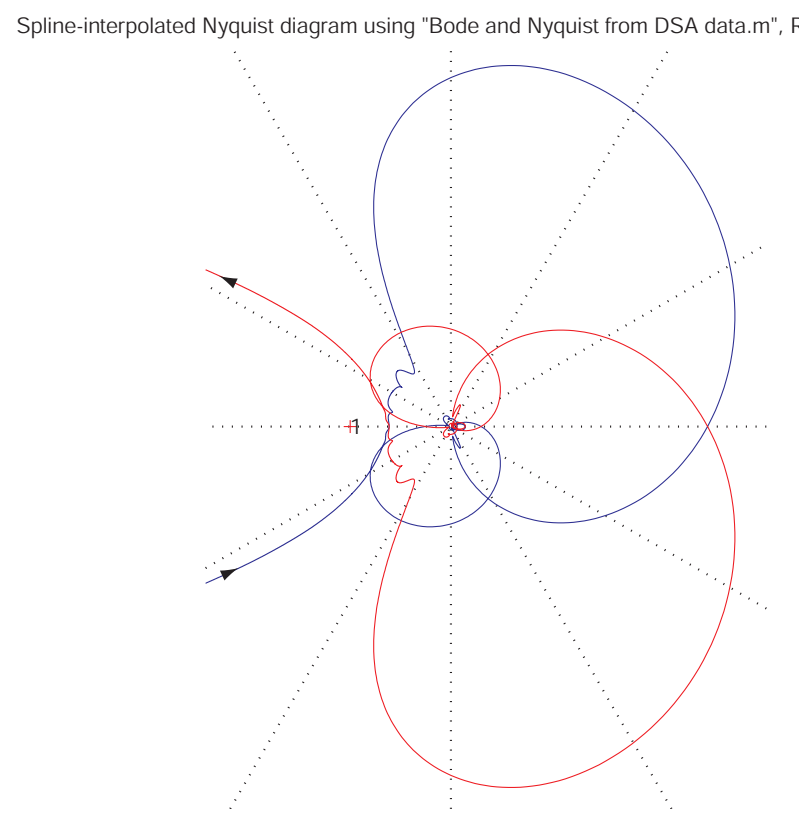

Kdc=1, Data file: 41105 torque LT 04.mat (in on blue, out on red)

Figure 4-106: Nyquist diagram of the measured negative loop transmission for the tool position for the low-noise implementation of the $10 \mathrm{kHz}$ FTS.
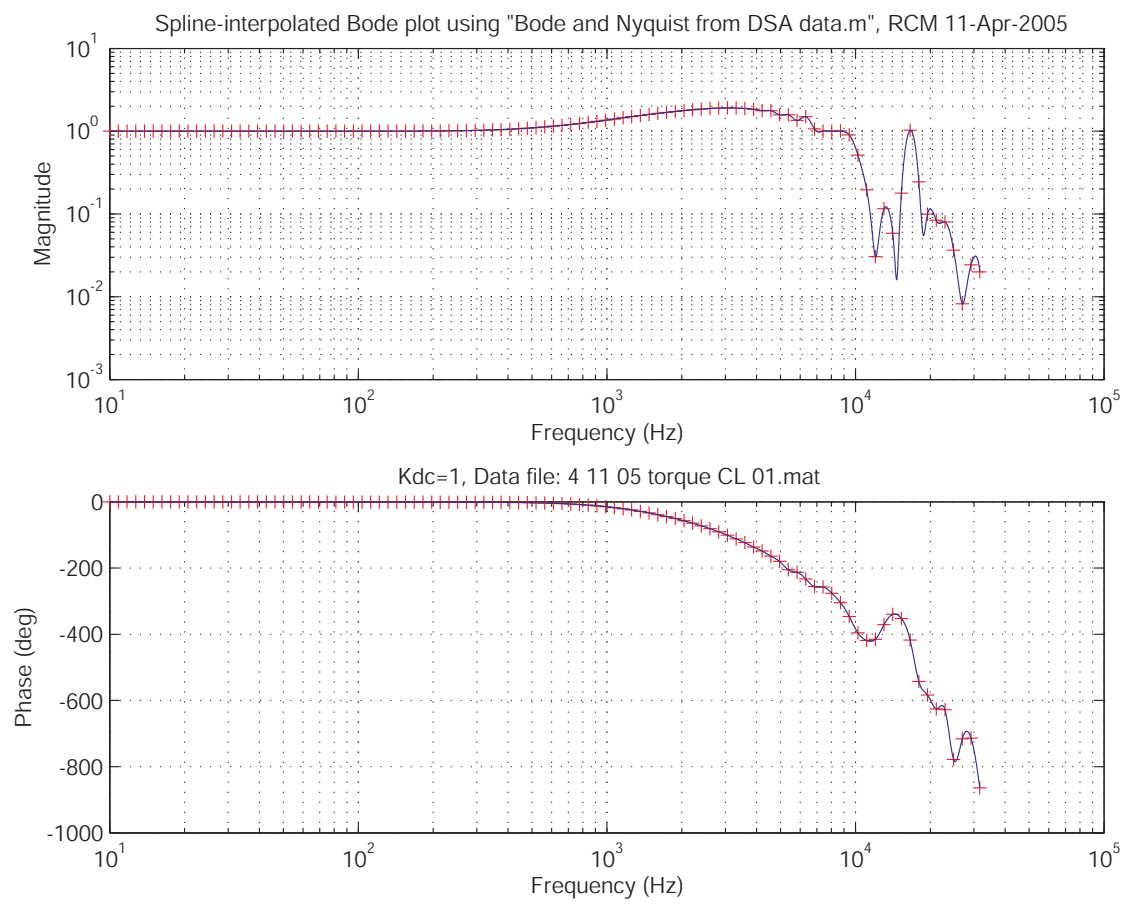

Figure 4-107: Bode plot of the measured small-signal closed-loop response for the tool position for the low-noise implementation of the $10 \mathrm{kHz}$ FTS. 

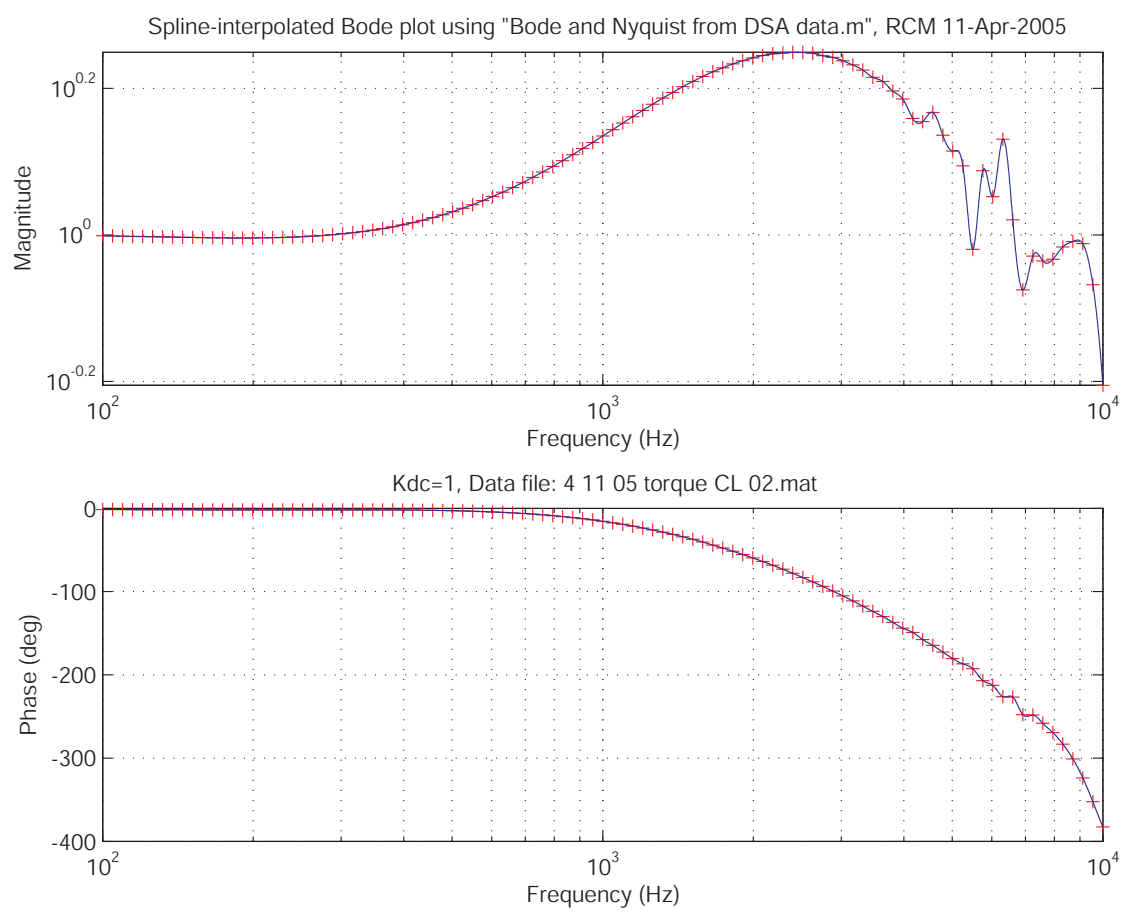

Figure 4-108: Bode plot showing high frequency detail of the measured small-signal closed-loop response for the tool position for the low-noise implementation of the $10 \mathrm{kHz}$ FTS.

closed-loop bandwidth of $10 \mathrm{kHz}$. The overshoot is greater than what would be expected from a second-order system with a phase margin of $32^{\circ}$. Using the relationships in Section 4.7.3 between the frequency response and time response of a second-order system, if we treat the low-noise implementation as a second-order system, then the measured phase margin of $32^{\circ}$ suggests a damping ratio of 0.32 , which leads to an overshoot of $35 \%$. The large measured overshoot is most likely due to the stable mechanical resonance at $10 \mathrm{kHz}$ and the lead compensation, but is not an issue because the fast tool servo is intended for producing sine wave tool tip trajectories.

Figures 4-110, 4-111, 4-112, and 4-113 show the measured following errors for commanded sinusoidal tool tip trajectories for the low-noise implementation. The following errors of $3 \%, 3 \%, 11 \%$, and $50 \%$ at $100 \mathrm{~Hz}, 300 \mathrm{~Hz}, 500 \mathrm{~Hz}$, and $1 \mathrm{kHz}$, respectively, are consistent with the measured closed-loop response. The relatively large following errors - for precision machining uses of the $10 \mathrm{kHz}$ FTS — can be reduced with the addition of a feedforward compensator. Ludwick [99], Cattell [37], Byl [33], and Lu [98] show that the addition of an Adaptive Feedforward Cancellation 


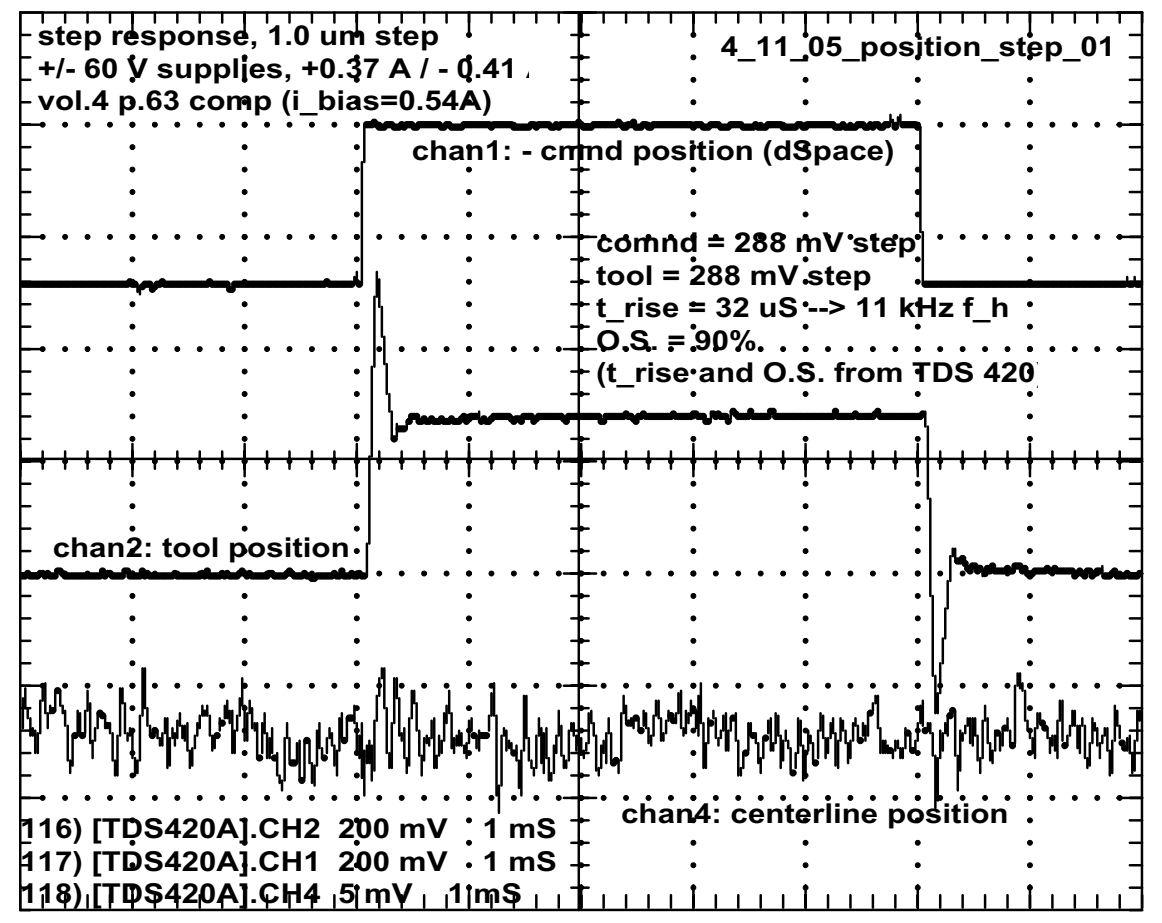

Figure 4-109: Measured step response for the tool position for the low-noise implementation of the $10 \mathrm{kHz}$ FTS.

compensator can significantly reduce following error, and is discussed in Section 6.4.1.

\subsubsection{High-Acceleration Implementation}

The current in the bias flux coil is set to $1.5 \mathrm{~A}$ for the high-acceleration implementation of the $10 \mathrm{kHz}$ FTS. This causes the torsional spring from the magnetic flux to dominate over the torsional spring from the flexure suspension, resulting in an openloop pole in the right-half plane. The high-acceleration implementation includes the mechanical damping described in Section 4.5.9. Developing the compensation in this case proceeded according to the following method. First, obtain a stable system using a low proportional gain with a lead compensator centered on a modest crossover frequency. Next, incrementally increase the gain and move the center frequency of the lead compensator to achieve the desired crossover frequency. Finally, add a lag compensator to increase the low-frequency gain of the negative loop transmission.

Figure 4-114 shows the block diagram for the digital controller for the tool position for the high-acceleration implementation of the $10 \mathrm{kHz}$ FTS. The lead compensator 


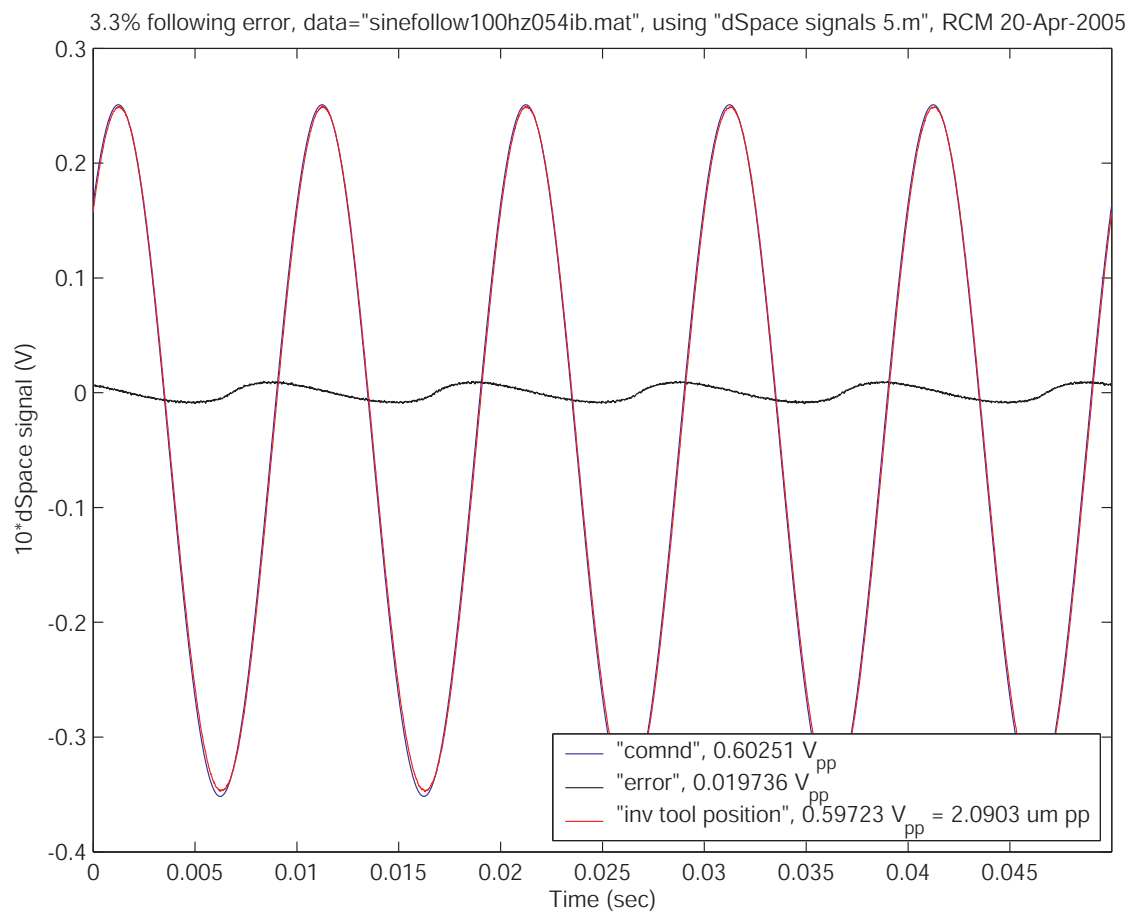

Figure 4-110: Measured following error for the low-noise implementation of the $10 \mathrm{kHz}$ FTS for a $100 \mathrm{~Hz}$ sinusoidal tool tip trajectory.

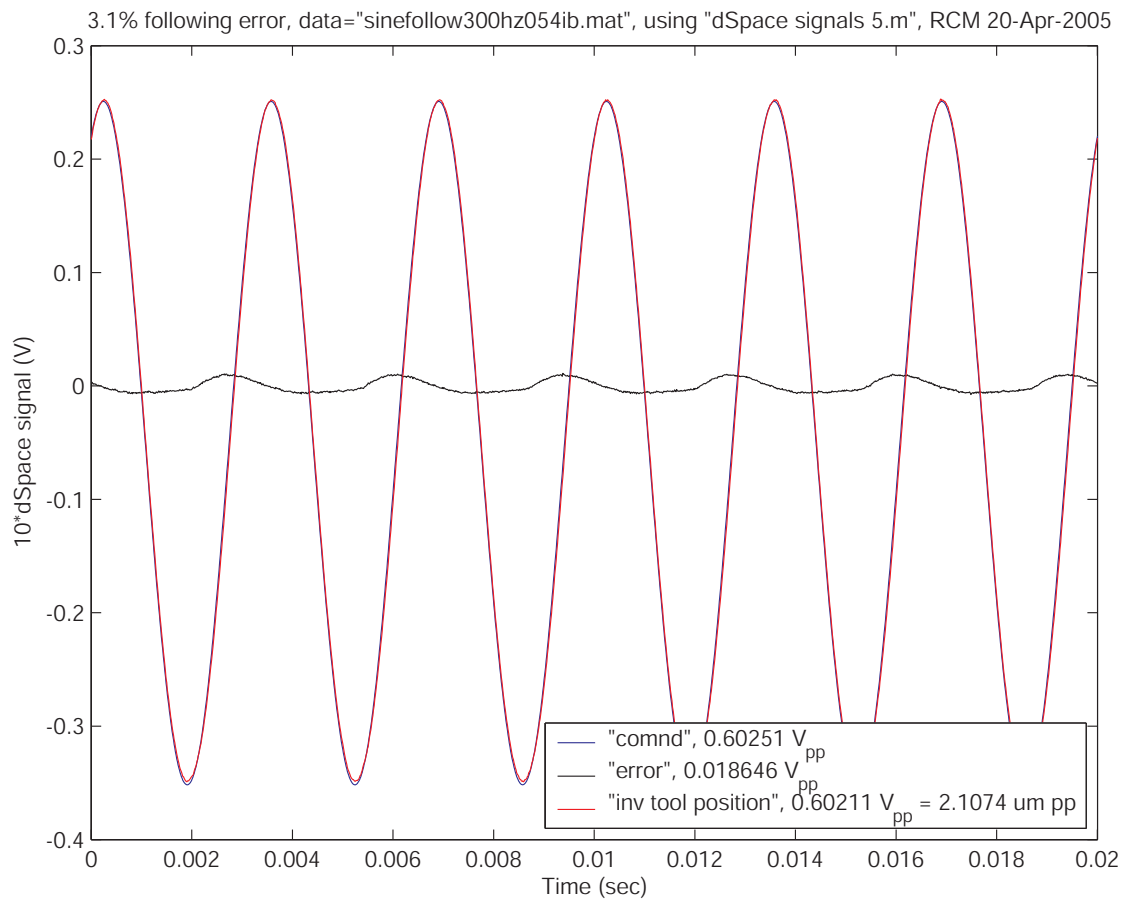

Figure 4-111: Measured following error for the low-noise implementation of the $10 \mathrm{kHz}$ FTS for a $300 \mathrm{~Hz}$ sinusoidal tool tip trajectory. 


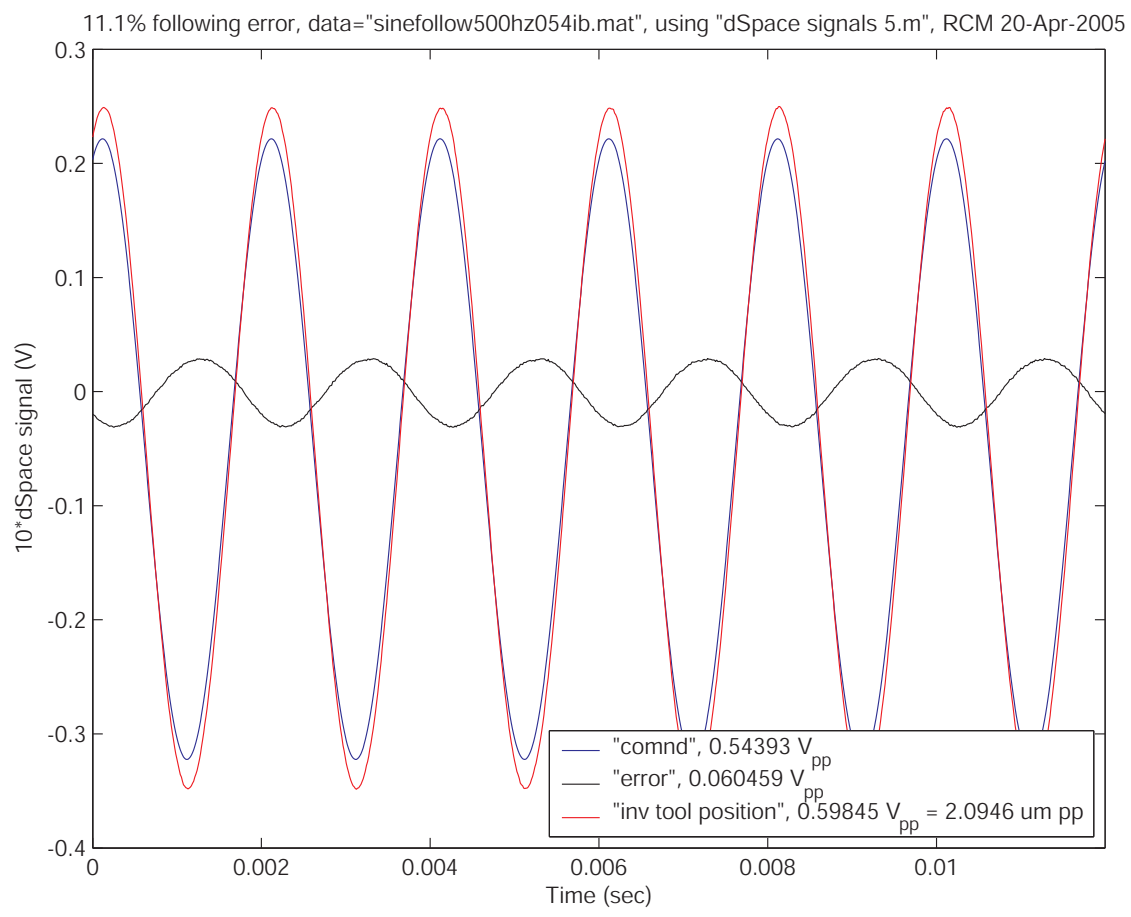

Figure 4-112: Measured following error for the low-noise implementation of the $10 \mathrm{kHz}$ FTS for a $500 \mathrm{~Hz}$ sinusoidal tool tip trajectory.

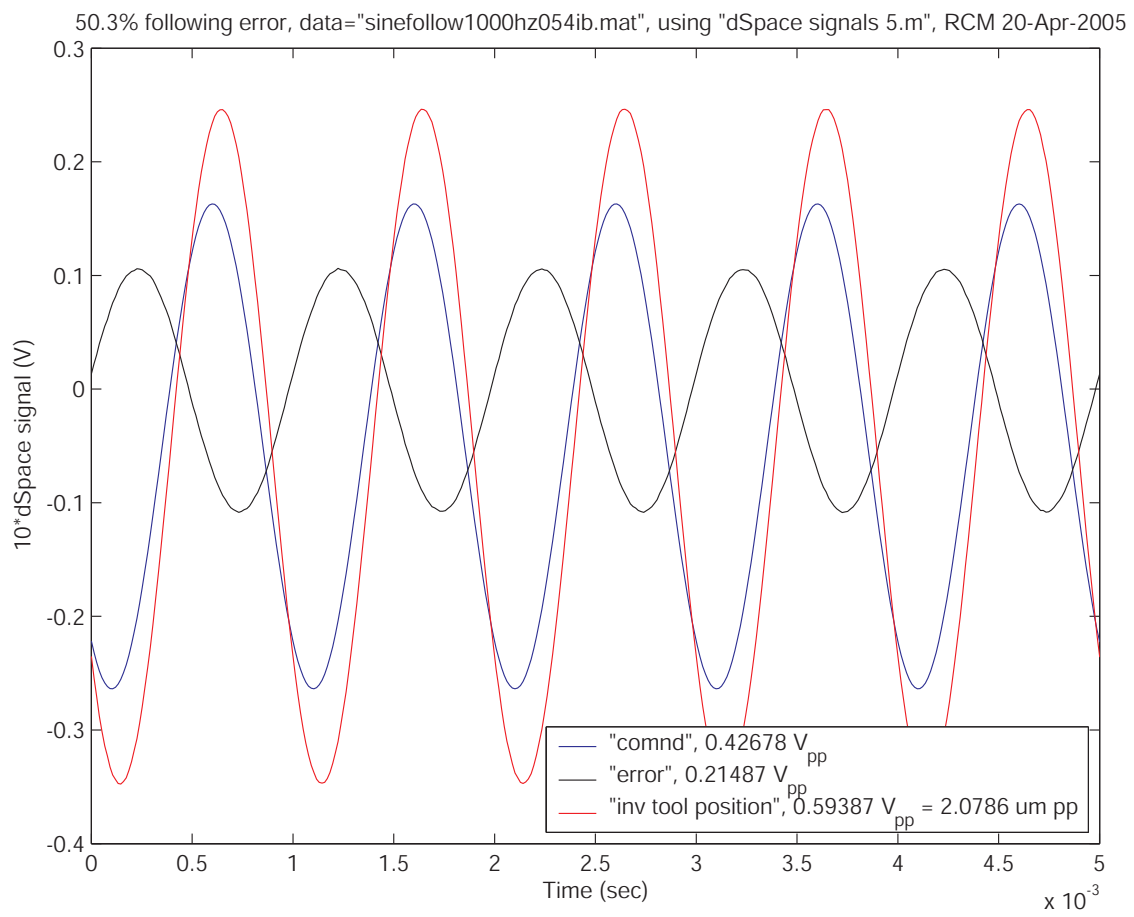

Figure 4-113: Measured following error for the low-noise implementation of the $10 \mathrm{kHz}$ FTS for a $1 \mathrm{kHz}$ sinusoidal tool tip trajectory. 


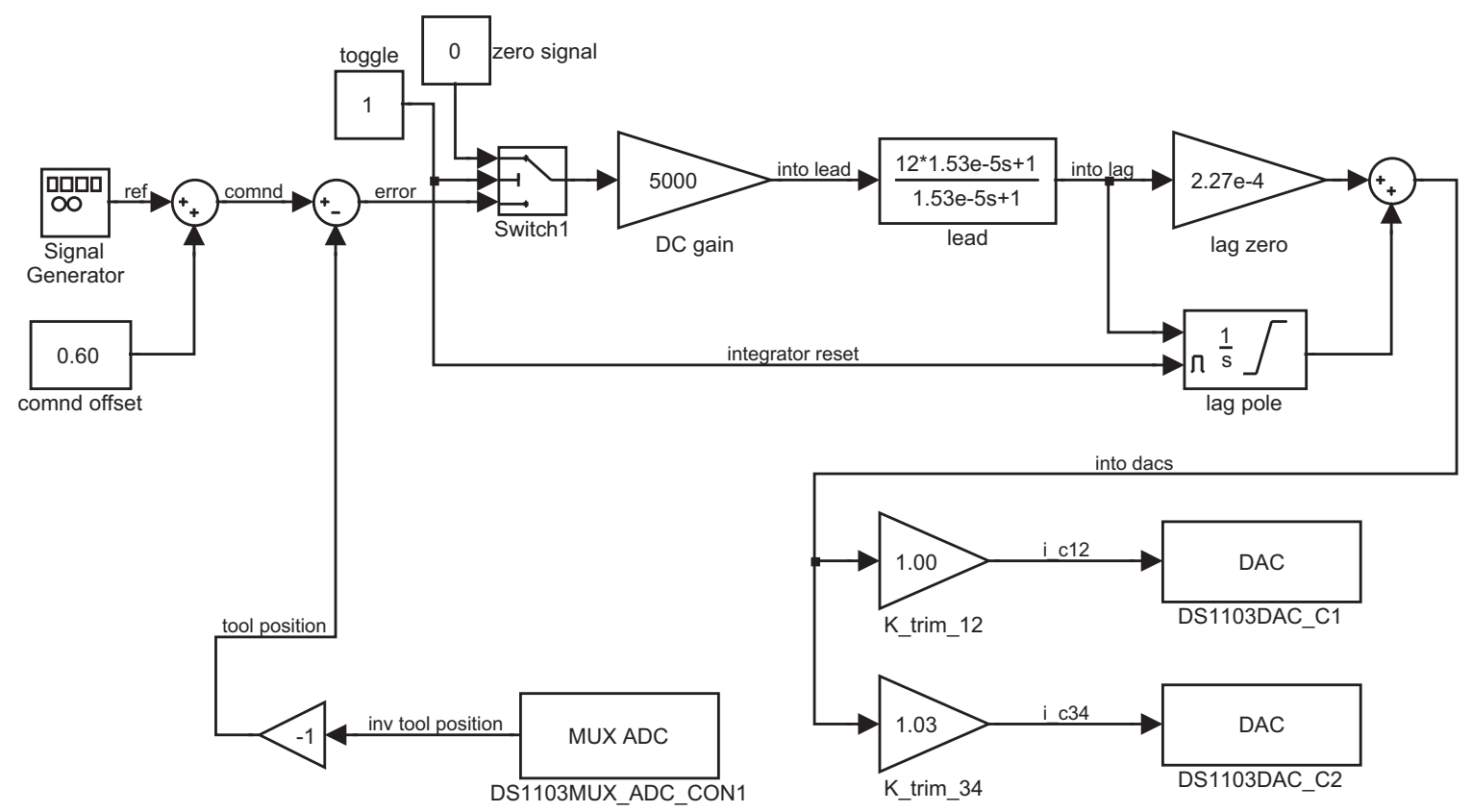

Figure 4-114: Block diagram for the digital controller for the tool position for the high-acceleration implementation of the $10 \mathrm{kHz}$ FTS.

uses an $\alpha=12$ and a time constant of $T_{\text {lead }}=1.53 \times 10^{-5} \mathrm{sec}$ (geometric mean frequency of $3.0 \mathrm{kHz}$ ). The lag compensator consists of a pure integrator and a zero with a time constant of $T_{\text {lag }}=2.27 \times 10^{-4} \mathrm{sec}(700 \mathrm{~Hz})$.

Figure 4-115 shows a Bode plot of the measured negative loop transmission for the tool position for the high-acceleration implementation. The crossover frequency for this system is $2.3 \mathrm{kHz}$ with a phase margin of $22^{\circ}$ and a gain margin of $3 \mathrm{~dB}$. The peaks at $10 \mathrm{kHz}$ and $13 \mathrm{kHz}$ correspond to stable torsional modes of the rotor, which are discussed in Section 4.6.3. Figure 4-116 shows a detail of the measured negative loop transmission, illustrating the damped front/back mechanical modes of the rotor in the frequency range of 5 to7 $\mathrm{kHz}$.

Figure 4-117 shows the Nyquist diagram for the measured negative loop transmission for the high-acceleration implementation. Note the single counterclockwise encirclement of the -1 point, which is consistent with a stable system having one open-loop pole in the right-half plane.

Figure 4-118 shows the measured small-signal closed-loop response for the highacceleration implementation. The $-3 \mathrm{~dB}$ closed-loop half-power bandwidth is at $10 \mathrm{kHz}$. 

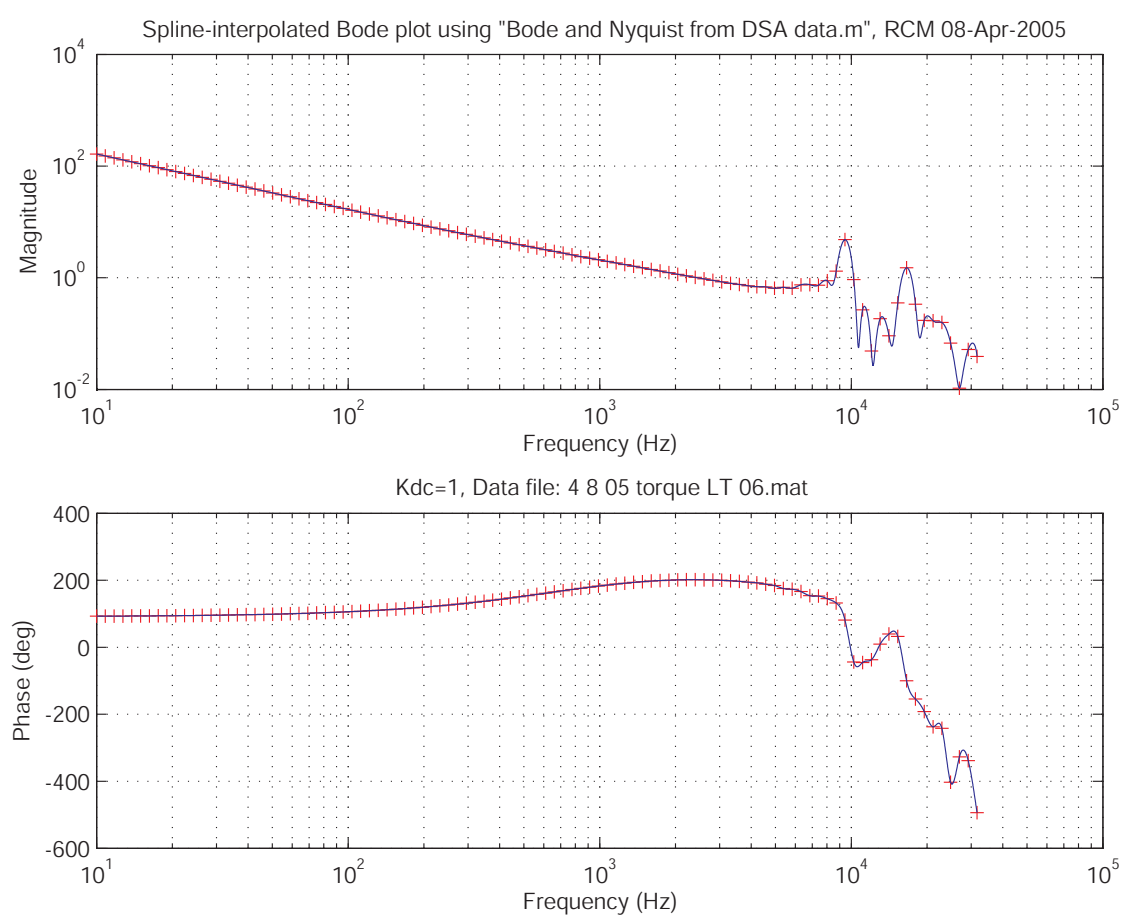

Figure 4-115: Bode plot of the measured negative loop transmission for the tool position for the high-acceleration implementation of the $10 \mathrm{kHz}$ FTS.
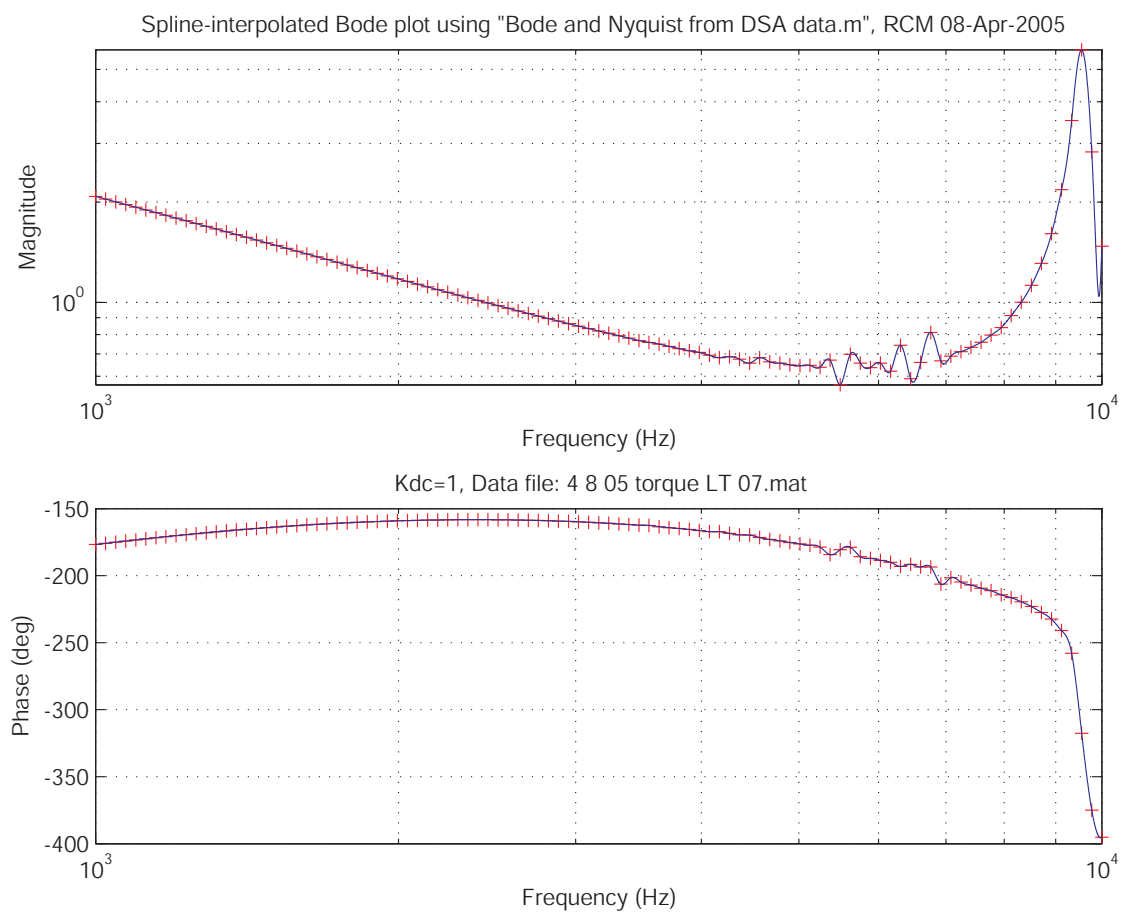

Figure 4-116: Bode plot showing high frequency detail of the measured negative loop transmission for the tool position for the high-acceleration implementation of the $10 \mathrm{kHz}$ FTS. 


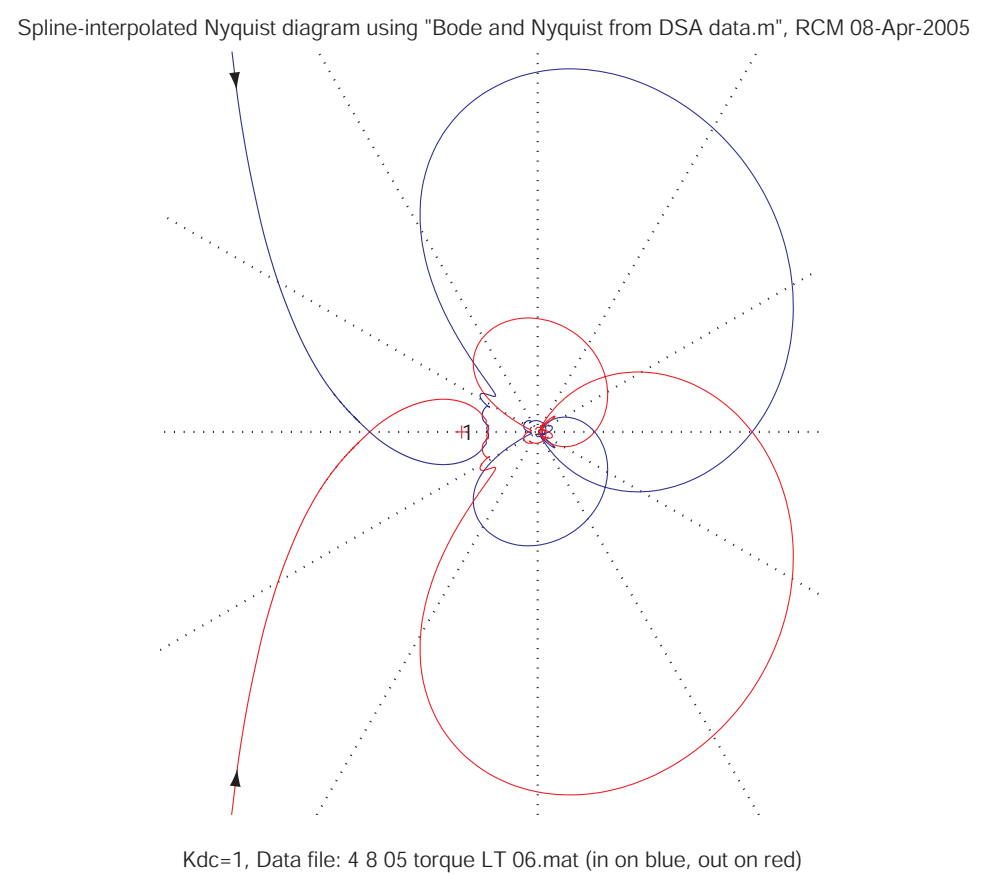

Figure 4-117: Nyquist diagram of the measured negative loop transmission for the tool position for the high-acceleration implementation of the $10 \mathrm{kHz}$ FTS.

Figure 4-119 shows a detail of the measured small-signal closed-loop response. As with the low-noise implementation, the fairly smooth phase angle up to $5 \mathrm{kHz}$, and ripple in the magnitude plot between $4 \mathrm{kHz}$ and $5 \mathrm{kHz}$, suggest that a possible future addition of a feedforward compensator could be effective for frequencies up to 4 to $5 \mathrm{kHz}$.

The measured step response for the high-acceleration implementation for a $1 \mu \mathrm{m}$ tool position step is shown in Figure 4-120. This step response has a $30 \mu$ sec rise time $(10 \%$ to $90 \%)$ and a $150 \%$ overshoot. The rise time is fairly consistent with the measured closed-loop bandwidth of $10 \mathrm{kHz}$. As with the low-noise implementation, the overshoot is greater than what would be expected from a second-order system with a phase margin of $22^{\circ}$. Following the argument given in the discussion on the step response of the low-noise implementation, if we treat the high-acceleration implementation as a second-order system, then the measured phase margin of $22^{\circ}$ suggests a damping ratio of 0.22 , which leads to an overshoot of $49 \%$. The large measured overshoot is most likely due to the stable mechanical resonance at $10 \mathrm{kHz}$ and the lead compensation, but is not an issue because the fast tool servo is intended 

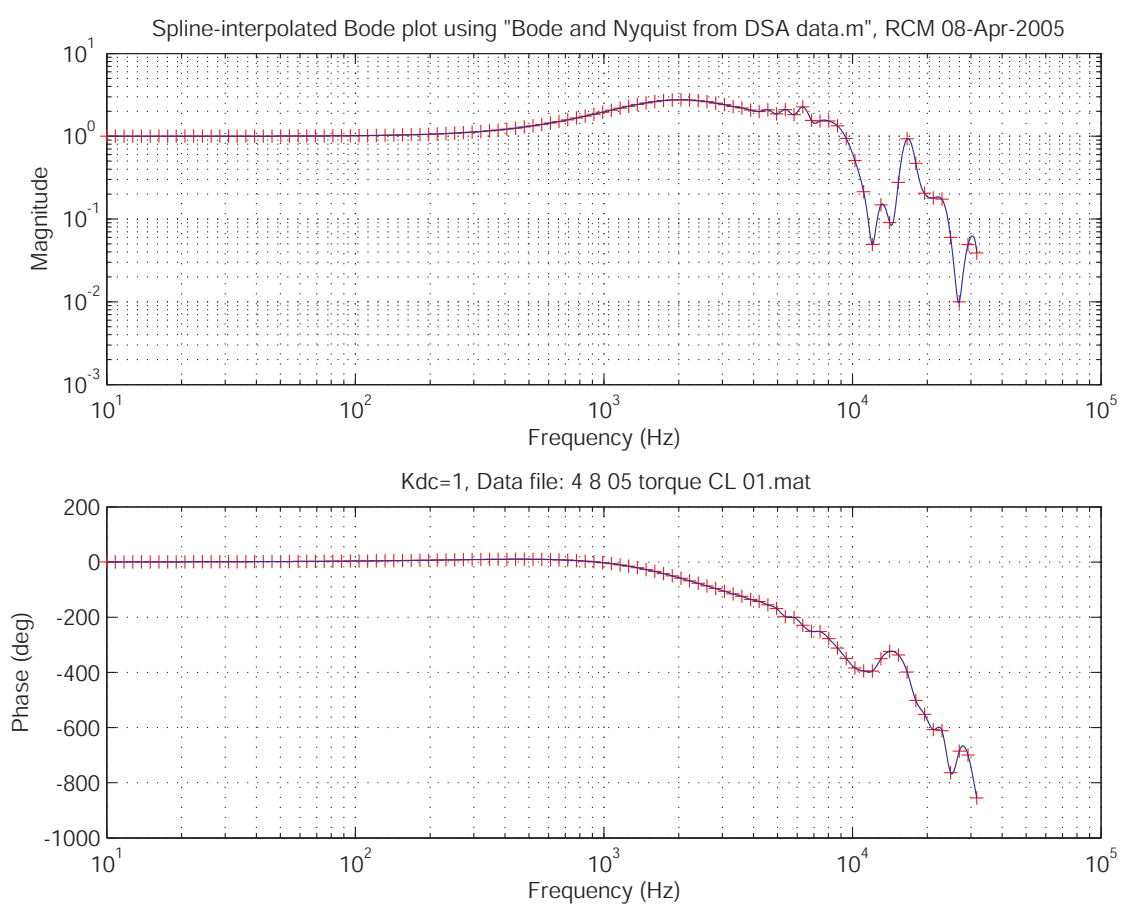

Figure 4-118: Bode plot of the measured small-signal closed-loop response for the tool position for the high-acceleration implementation of the $10 \mathrm{kHz}$ FTS.
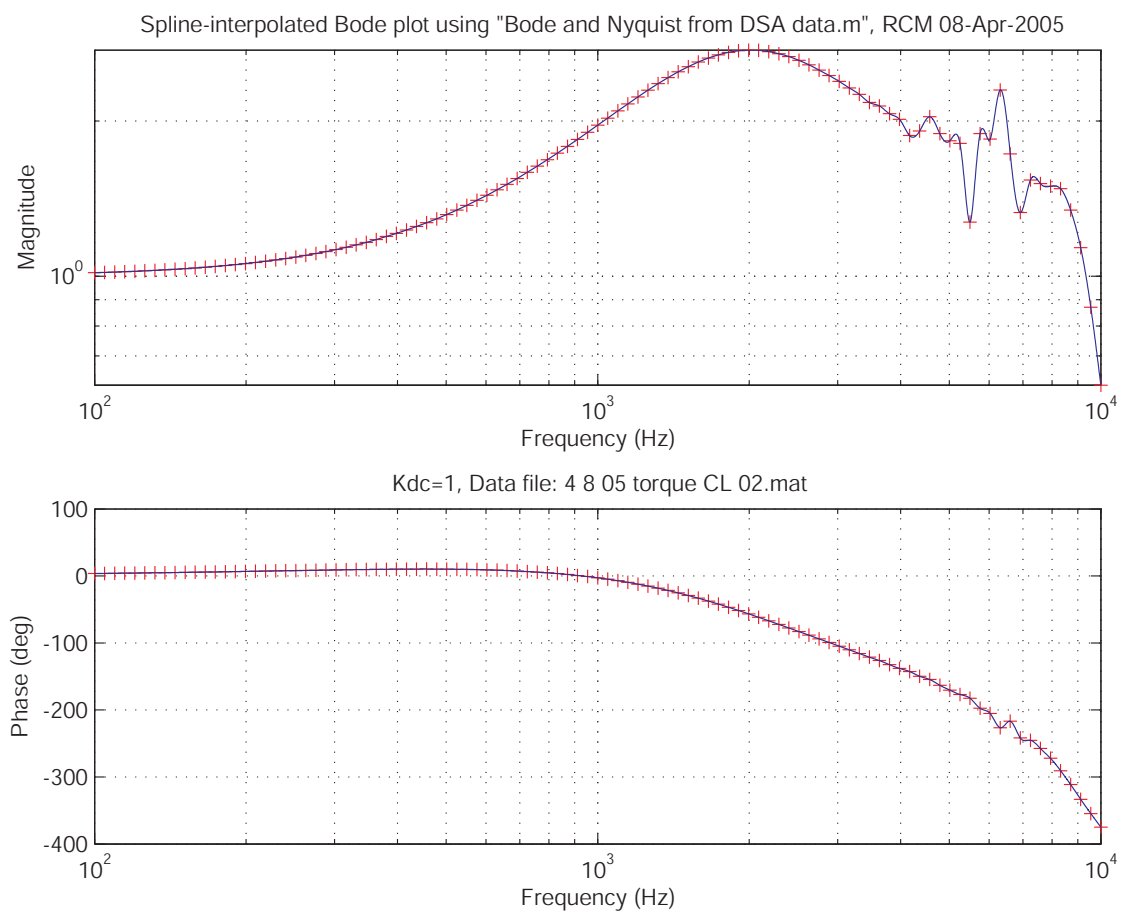

Figure 4-119: Bode plot showing high frequency detail of the measured small-signal closed-loop response for the tool position for the high-acceleration implementation of the $10 \mathrm{kHz}$ FTS. 


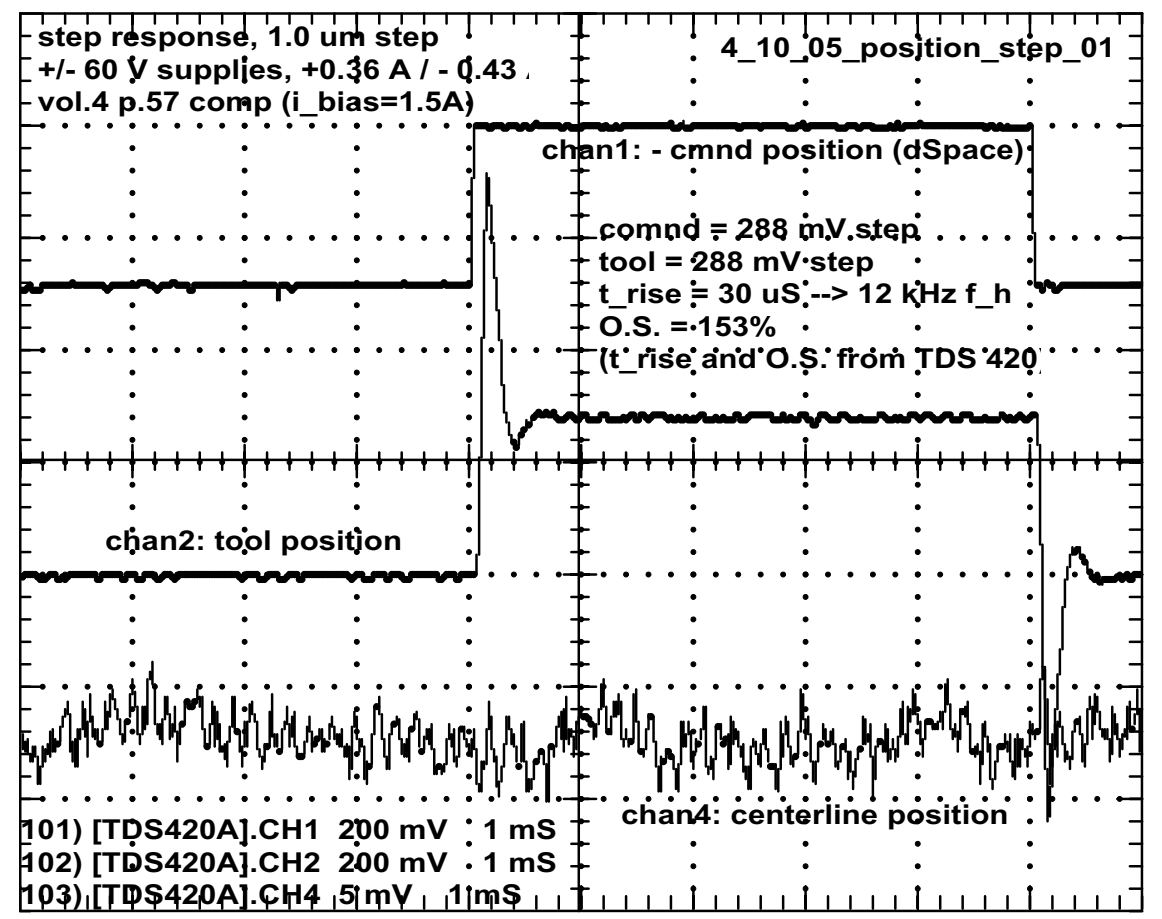

Figure 4-120: Measured step response for the tool position for the high-acceleration implementation of the $10 \mathrm{kHz}$ FTS.

for producing sine wave tool tip trajectories.

Figures 4-121, 4-122, 4-123, and 4-124 show the measured following errors for commanded sinusoidal tool tip trajectories for the high-acceleration implementation. The following errors of 7\%, 20\%,37\%, and $94 \%$ at $100 \mathrm{~Hz}, 300 \mathrm{~Hz}, 500 \mathrm{~Hz}$, and $1 \mathrm{kHz}$, respectively, are consistent with the measured closed-loop response. As is the case for the low-noise implementation, the following errors can be significantly reduced with the addition of a feedforward compensator, and especially with the addition of an Adaptive Feedforward Cancellation compensator. 


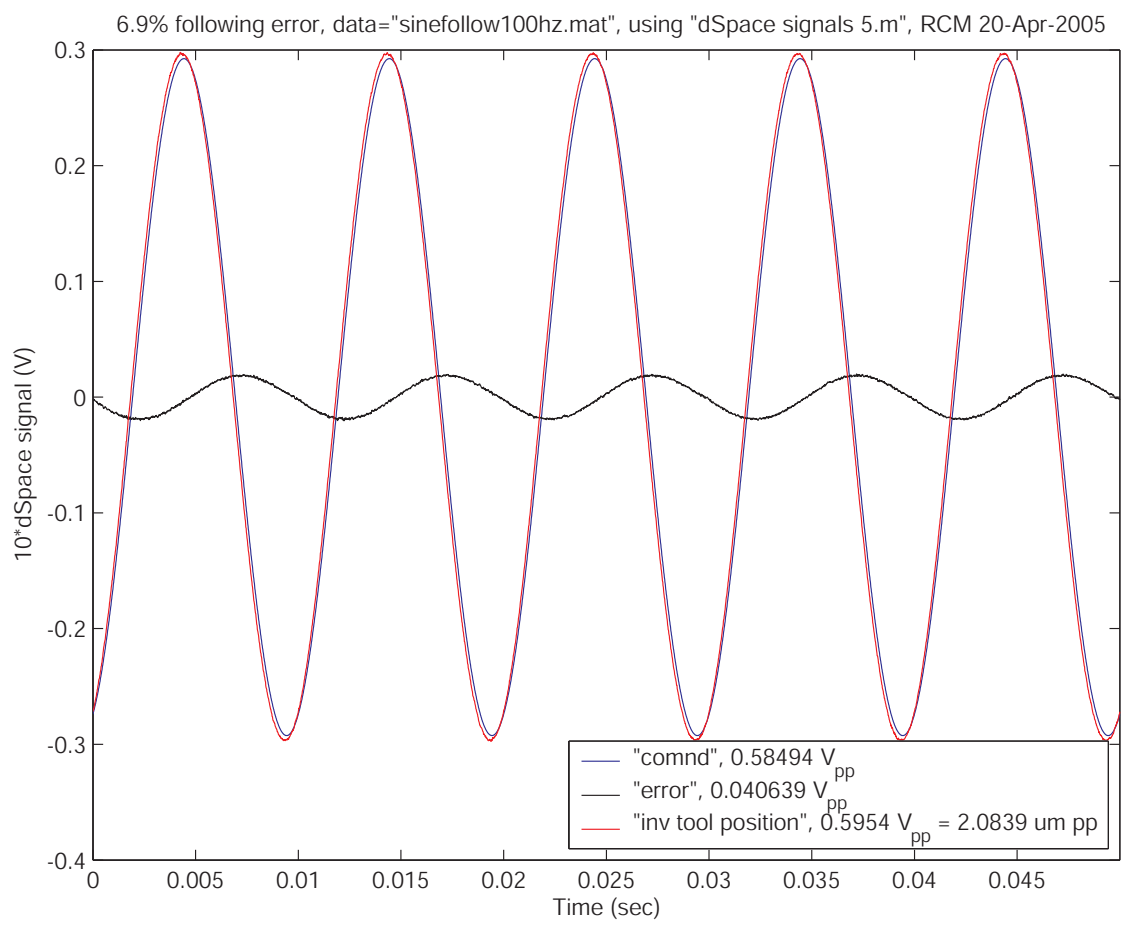

Figure 4-121: Measured following error for the high-acceleration implementation of the $10 \mathrm{kHz}$ FTS for a $100 \mathrm{~Hz}$ sinusoidal tool tip trajectory.

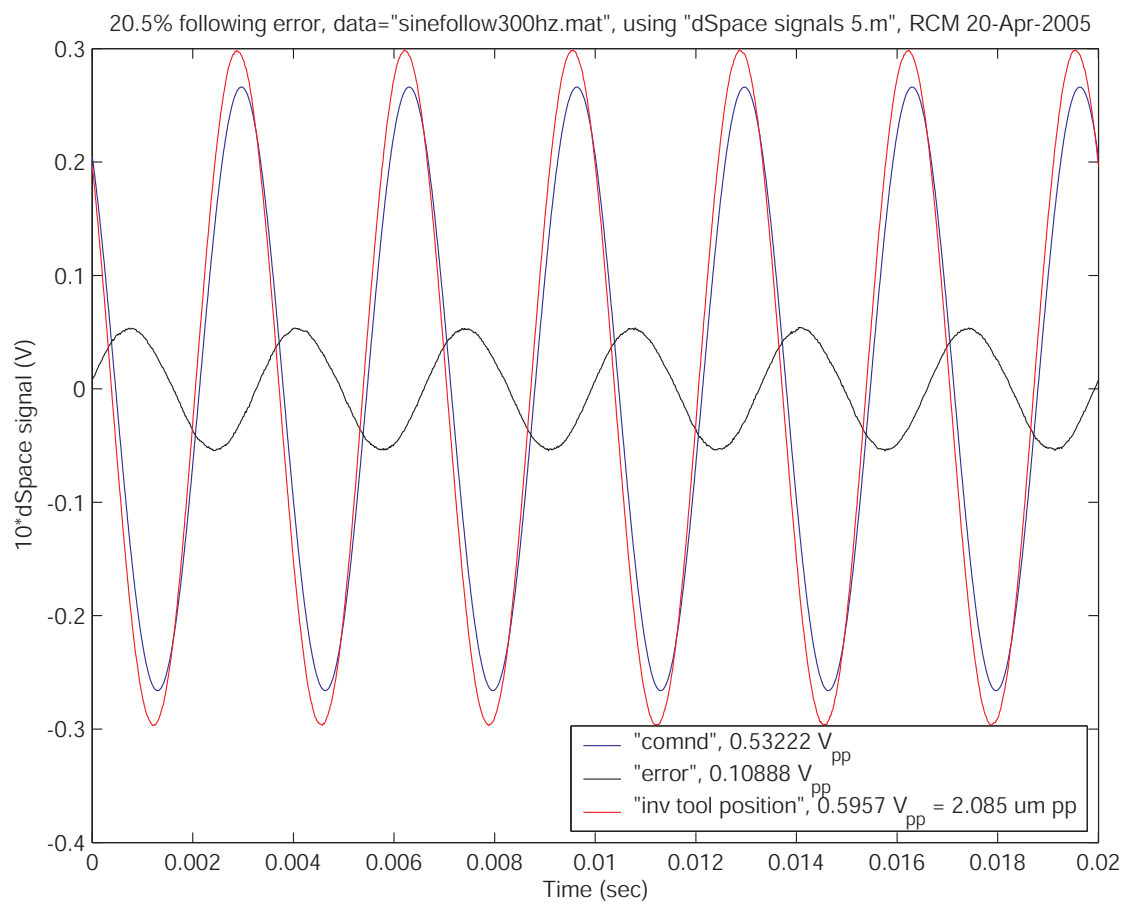

Figure 4-122: Measured following error for the high-acceleration implementation of the $10 \mathrm{kHz}$ FTS for a $300 \mathrm{~Hz}$ sinusoidal tool tip trajectory. 


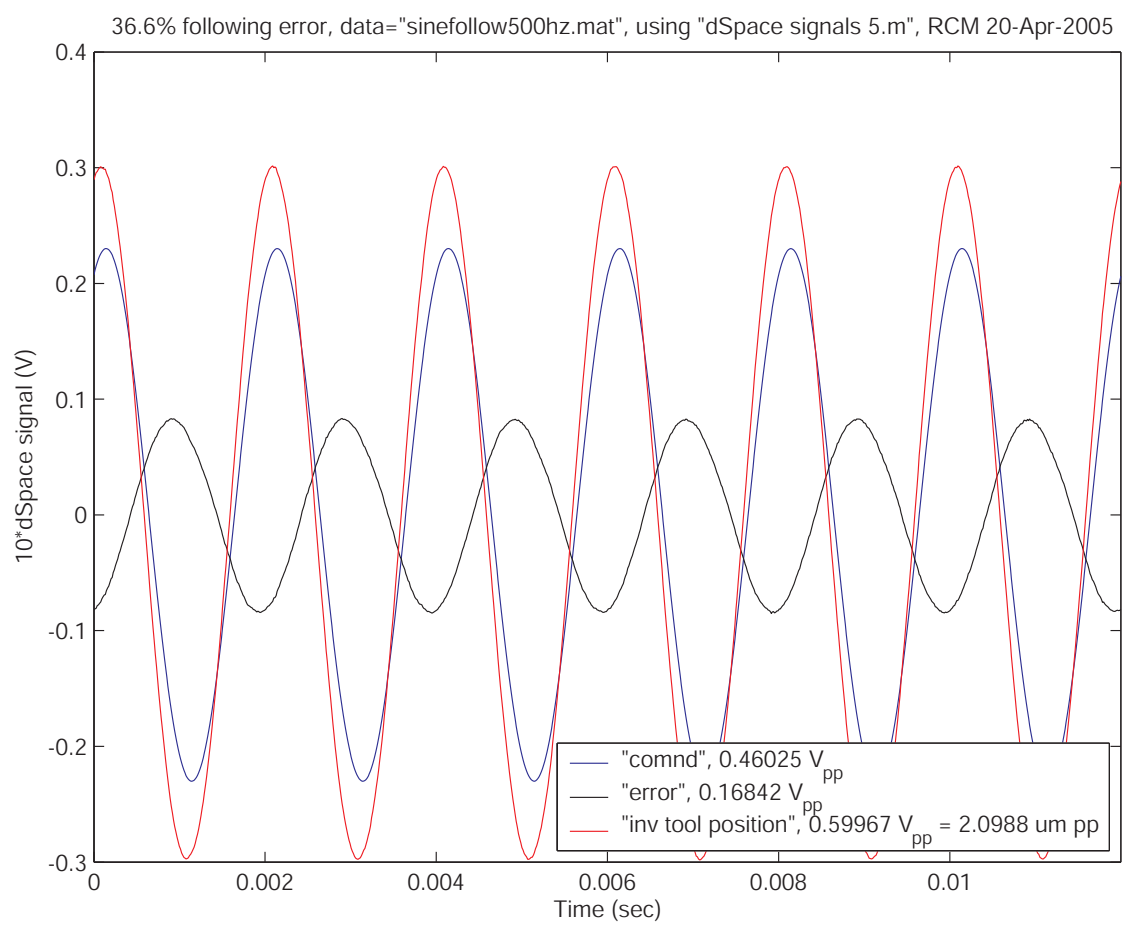

Figure 4-123: Measured following error for the high-acceleration implementation of the $10 \mathrm{kHz}$ FTS for a $500 \mathrm{~Hz}$ sinusoidal tool tip trajectory.

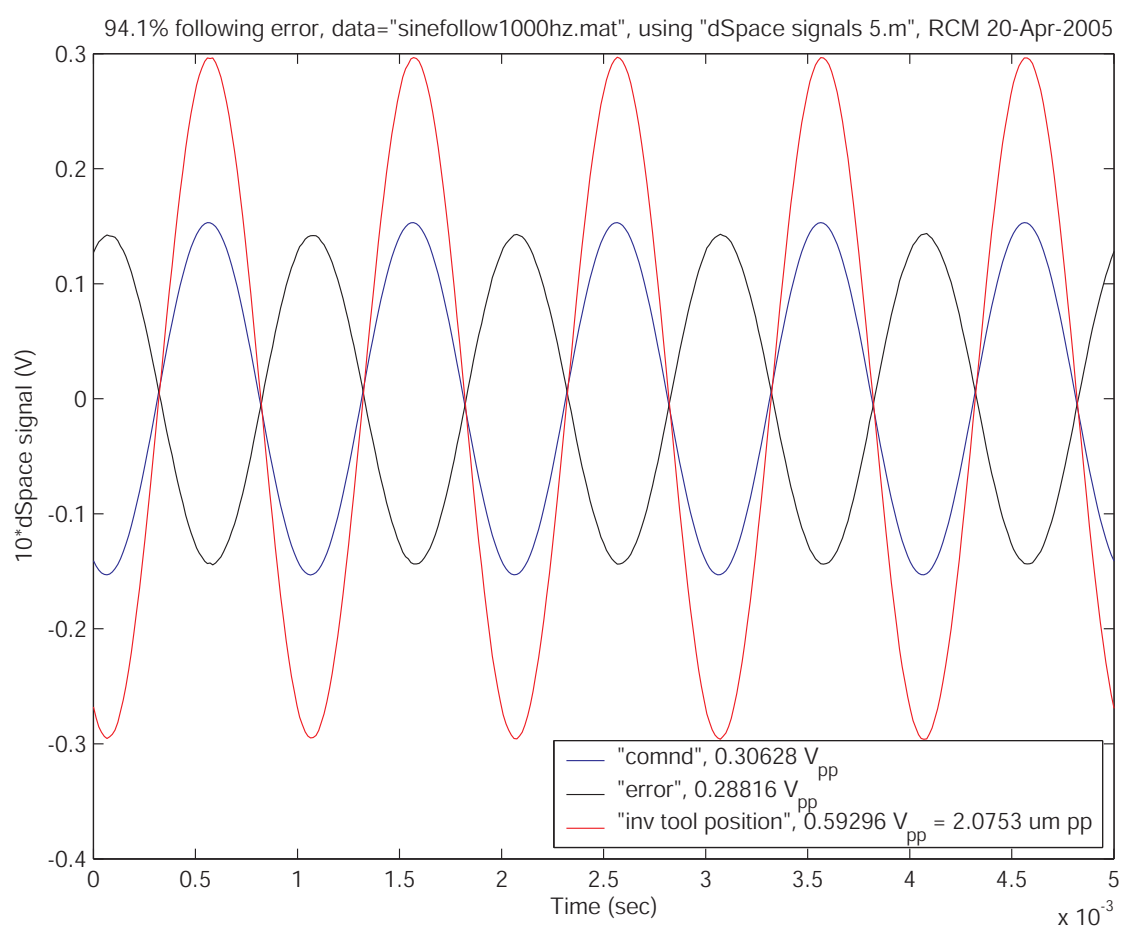

Figure 4-124: Measured following error for the high-acceleration implementation of the $10 \mathrm{kHz}$ FTS for a $1 \mathrm{kHz}$ sinusoidal tool tip trajectory. 


\subsubsection{Sensor Noise}

This section discusses the measurements that I made of the noise levels in the signal from the capacitance sensor that measures the tool tip displacement for the $10 \mathrm{kHz}$ FTS. By incrementally including elements of the control system — turning on the power amplifiers and the fans, attaching the sensor amplifier output to the digital controller, adding the anti-aliasing filter, and closing the position loop - I was able to gain a sense of the relative effect that these elements have on the sensor noise. Ultimately, the measured noise voltages in the signal from the sensor amplifier are converted into an equivalent motion as seen at the tool tip. With the position loop closed, the measured equivalent noise motion at the tool tip is $1.4 \mathrm{~nm} \mathrm{rms}$ and $2.5 \mathrm{~nm} \mathrm{rms}$ for the low-noise $\left(\mathrm{I}_{B}=0.545 \mathrm{~A}\right)$ and high-acceleration $\left(\mathrm{I}_{B}=1.5 \mathrm{~A}\right)$ implementations of the $10 \mathrm{kHz}$ FTS, respectively.

The noise measurement set-up consists of a pair of 10x scope probes, a Tektronix AM502 differential amplifier [141] with a 1000x gain and a low-pass filter with a corner frequency of $30 \mathrm{kHz}$, and a Tektronix TDS 420A digital oscilloscope running at $250 \mathrm{kHz}$ for a 60 msec capture of 15,000 data points.

The differential amplifier and its low-pass filter provides the following benefits. It floats the sensor signal relative to the oscilloscope and rejects common-mode noise in the measurement signal. Since the power of the signal is proportional to the square of the voltage, the $100 \mathrm{x}$ effective ${ }^{17}$ voltage gain increases the power of the signal by a factor of 10,000 relative to the power in the noise floor of the oscilloscope. The low-pass filter provides an anti-aliasing function and removes high frequency noise that the mechanical system of the $10 \mathrm{kHz}$ FTS would not be able to follow, thereby providing a more meaningful noise measurement. The differential input avoids creating a ground loop between the sensor amplifier, differential amplifier, and oscilloscope - the ground clips on the scope leads were not connected to the sensor amplifier.

The measured sensor noise levels are shown in Table 4.3. The raw oscilloscope

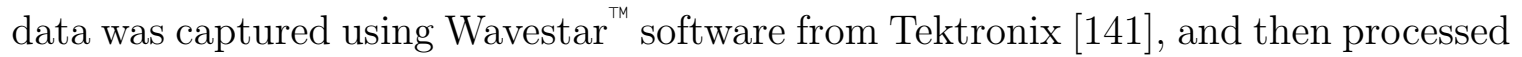

\footnotetext{
${ }^{17}$ The combination of the 10x probes and the 1000x gain in the differential amplifier produces a net gain of 100x.
} 
using the Matlab ${ }^{\circledR}$ script listed in Appendix B.5. The following is a summary evaluation of the measured sensor noise:

- The capacitance sensor amplifier does not appear to pick up significant noise from nearby power supplies or the array of nine cooling fans used in the currentmode amplifier.

- Connecting the sensor amplifier output to the dSPACE A/D converter produces a significant increase in the noise level. This is most likely due to the fact that the shell of the $\mathrm{A} / \mathrm{D}$ converter is at the same noisy ground potential as the personal computer $(\mathrm{PC})$ that the digital controller resides in. Furthermore, that ground connection creates a ground loop between the sensor amplifier and the PC.

- With the sensor amplifier output connected to the A/D converter, the antialiasing filter between the sensor amplifier and the A/D converter does not make a significant difference in the noise level. This further implicates the electrical connection between the digital controller and the sensor amplifier as a significant contributor to an elevated noise floor for the system.

- Closing the position feedback loop increases the sensor noise. A significant contributor to that increase is the measured 6 bits PP noise in the A/D converter, which corresponds to a tool tip motion of $7 \mathrm{~nm}$ PP.

- The need for using an anti-aliasing filter between the sensor amplifier and the digital controller can be seen by comparing the closed-loop measurements with and without it.

- Increasing the bias flux coil current makes the closed-loop $10 \mathrm{kHz}$ FTS more aggressive and increases the noise level seen at the tool tip. The baseline low-noise implementation noise level is $1.4 \mathrm{~nm} \mathrm{rms}$, while the baseline high-acceleration implementation noise level is $2.5 \mathrm{~nm} \mathrm{rms}$. 
- Increasing the power supply voltages for the high-acceleration implementation from $\pm 60 \mathrm{~V}$ to $\pm 80 \mathrm{~V}$ does not significantly increase the noise level.

Table 4.3: Noise measurements for the capacitance sensor that measures displacement of the tool in the $10 \mathrm{kHz}$ FTS. Values are RMS deviation from the average value of 15,000 data points captured by an oscilloscope at $250 \mathrm{kHz}$ (60 msec capture). Signal from 10x scope probes is conditioned before entering the oscilloscope by a Tektronix AM502 differential amplifier with a 1000x gain and a low-pass filter with a corner frequency of $30 \mathrm{kHz}$. Corner frequency of the single-pole anti-aliasing filter is $31 \mathrm{kHz}$. Low-noise and high-acceleration implementations of the $10 \mathrm{kHz}$ FTS correspond to $\mathrm{I}_{B}=0.545 \mathrm{~A}$ and $\mathrm{I}_{B}=1.5 \mathrm{~A}$, respectively.

\begin{tabular}{|l|c|c|}
\hline & $\begin{array}{c}\text { Output of } \\
\text { sensor } \\
\text { amplifier } \\
\mu \mathrm{V}\end{array}$ & $\begin{array}{c}\text { Equivalent } \\
\text { tool tip } \\
\text { motion } \\
\mathrm{nm}\end{array}$ \\
\hline $\begin{array}{l}\text { Noise floor of set-up: } \\
\text { scope probes, differential amplifier, } 30 \mathrm{kHz} \text { LPF, and scope }\end{array}$ & 85 & 0.30 \\
\hline $\begin{array}{l}\text { Capacitance sensor amplifier output } \\
\text { with nothing else connected or turned on }\end{array}$ & 105 & 0.37 \\
\hline $\begin{array}{l}\text { Capacitance sensor amplifier output } \\
\text { with nothing else connected, power supplies and fans on }\end{array}$ & 111 & 0.39 \\
\hline $\begin{array}{l}\text { Sensor amplifier output connected to dSPACE ADC } \\
\text { with anti-aliasing filter }\end{array}$ & 472 & 1.7 \\
\hline $\begin{array}{l}\text { Sensor amplifier output connected to dSPACE ADC } \\
\text { no anti-aliasing filter }\end{array}$ & 462 & 1.6 \\
\hline $\begin{array}{l}\text { Loop closed, } \mathrm{I}_{B}=1.5 \mathrm{~A}, \pm 60 \mathrm{~V} \text { supplies } \\
\text { with anti-aliasing filter }\end{array}$ & 686 & 2.4 \\
\hline $\begin{array}{l}\text { Loop closed, } \mathrm{I}_{B}=1.5 \mathrm{~A}, \pm 60 \mathrm{~V} \text { supplies } \\
\text { no anti-aliasing filter }\end{array}$ & 7134 & 4.0 \\
\hline $\begin{array}{l}\text { Loop closed, } \mathrm{I}_{B}=1.5 \mathrm{~A}, \pm 80 \mathrm{~V} \text { supplies } \\
\text { with anti-aliasing filter }\end{array}$ & 599 & 2.5 \\
\hline $\begin{array}{l}\text { Loop closed, } \mathrm{I}_{B}=0.545 \mathrm{~A}, \pm 60 \mathrm{~V} \text { supplies } \\
\text { with anti-aliasing filter }\end{array}$ & 2.0 \\
\hline $\begin{array}{l}\text { Loop closed, } \mathrm{I}_{B}=0.545 \mathrm{~A}, \pm 60 \mathrm{~V} \text { supplies } \\
\text { no anti-aliasing filter }\end{array}$ & 2.4 \\
\hline
\end{tabular}

Figures 4-125 through 4-128 show the captured raw oscilloscope data and calculated power spectral density (PSD) for the low-noise and the high-acceleration implementations of the $10 \mathrm{kHz}$ FTS. Note the factor of ten difference in vertical scales for the two PSD plots, and the appearance of dominant power at approximately $4.6 \mathrm{kHz}$ 


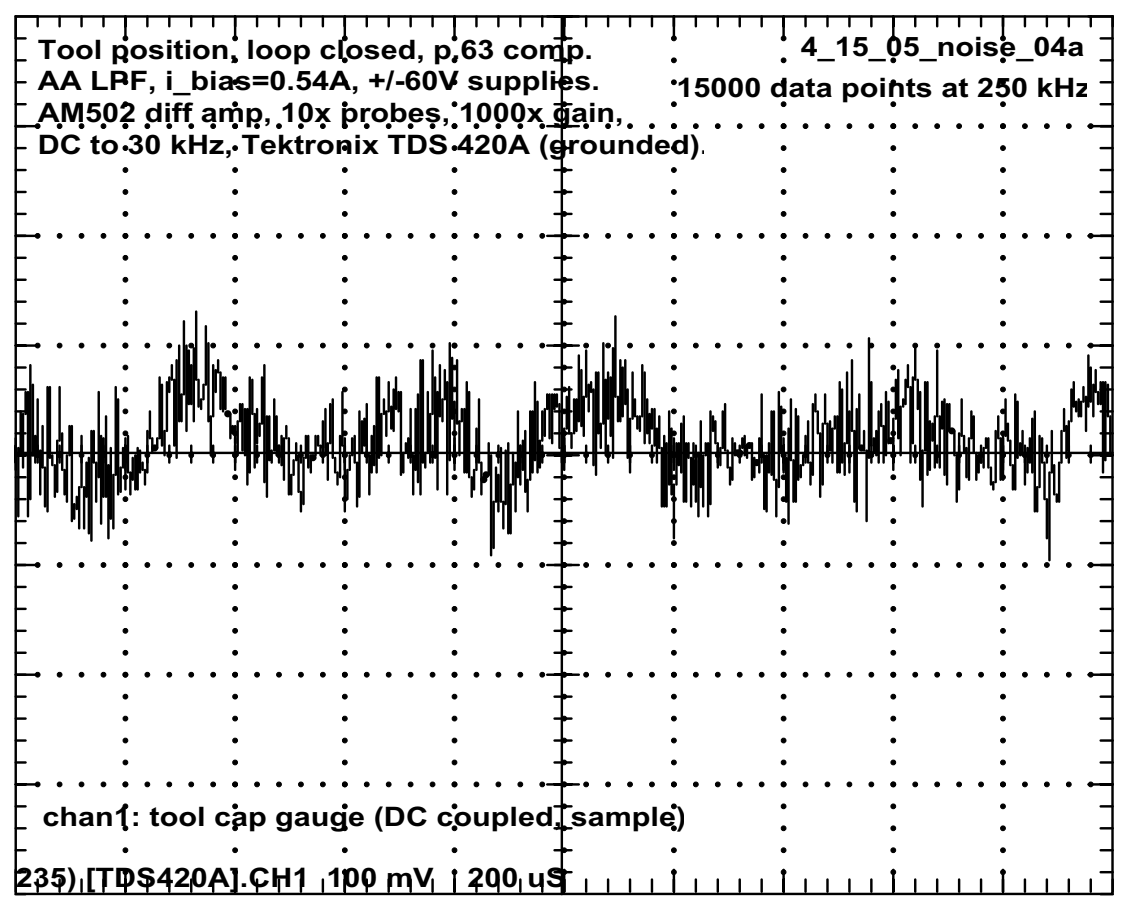

Figure 4-125: Captured oscilloscope data of the noise measurement for the low-noise implementation of the $10 \mathrm{kHz}$ FTS.

in the high-acceleration implementation. I did not have time to investigate the source of the spike at $4.6 \mathrm{kHz}$, and limit my discussion on it in this thesis to the observation that for both implementations, near that frequency there is a small bump in the magnitude of the negative loop transmission with a corresponding phase angle of approximately $-175^{\circ}$ (see Figures 4-105 and 4-116). 

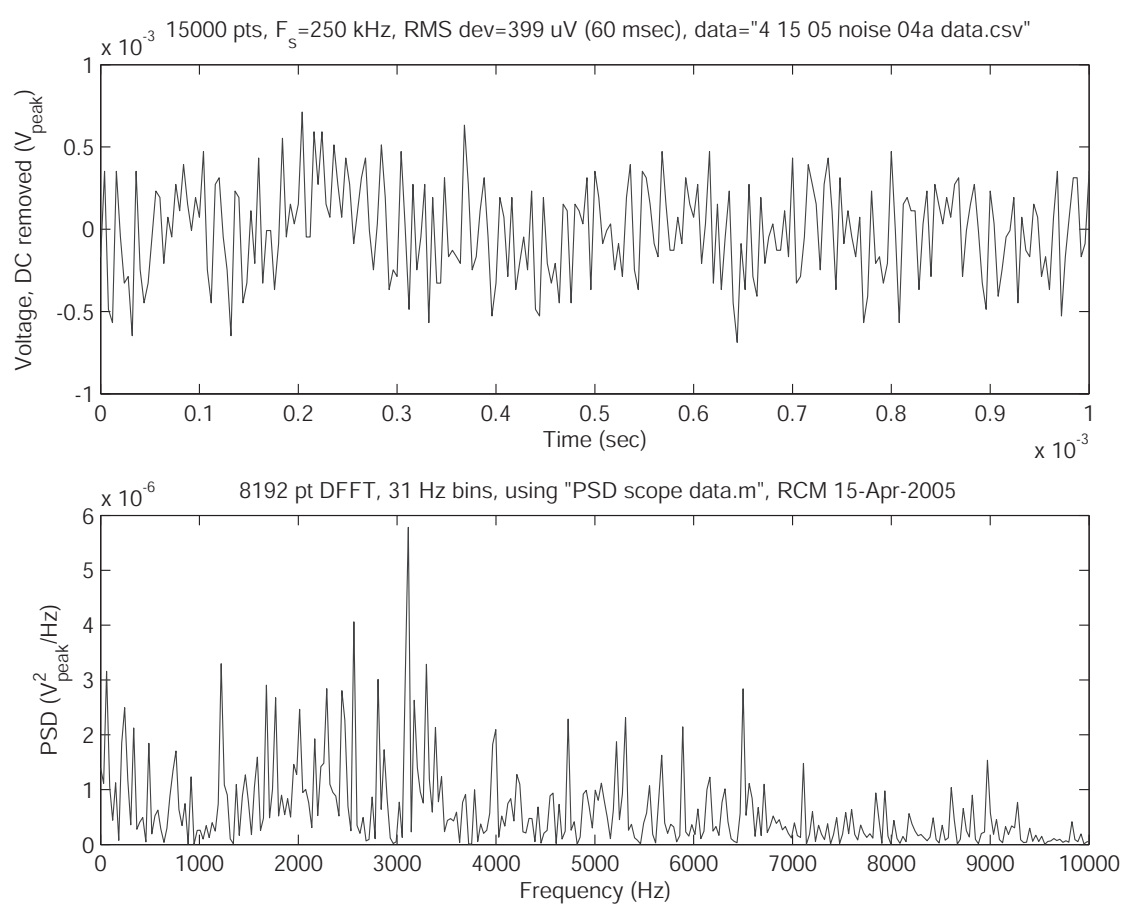

Figure 4-126: Calculated power spectral density of the noise measurement for the low-noise implementation of the $10 \mathrm{kHz}$ FTS.

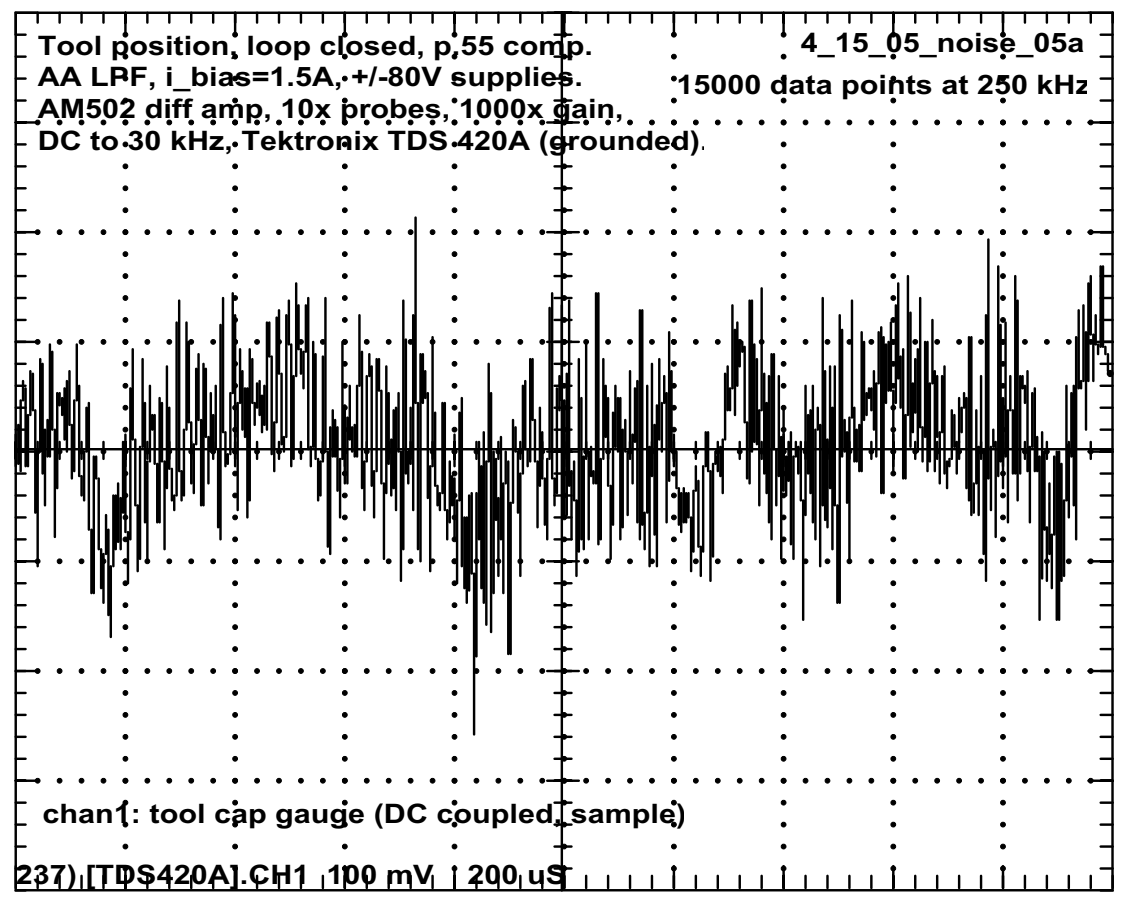

Figure 4-127: Captured oscilloscope data of the noise measurement for the highacceleration implementation of the $10 \mathrm{kHz}$ FTS. 

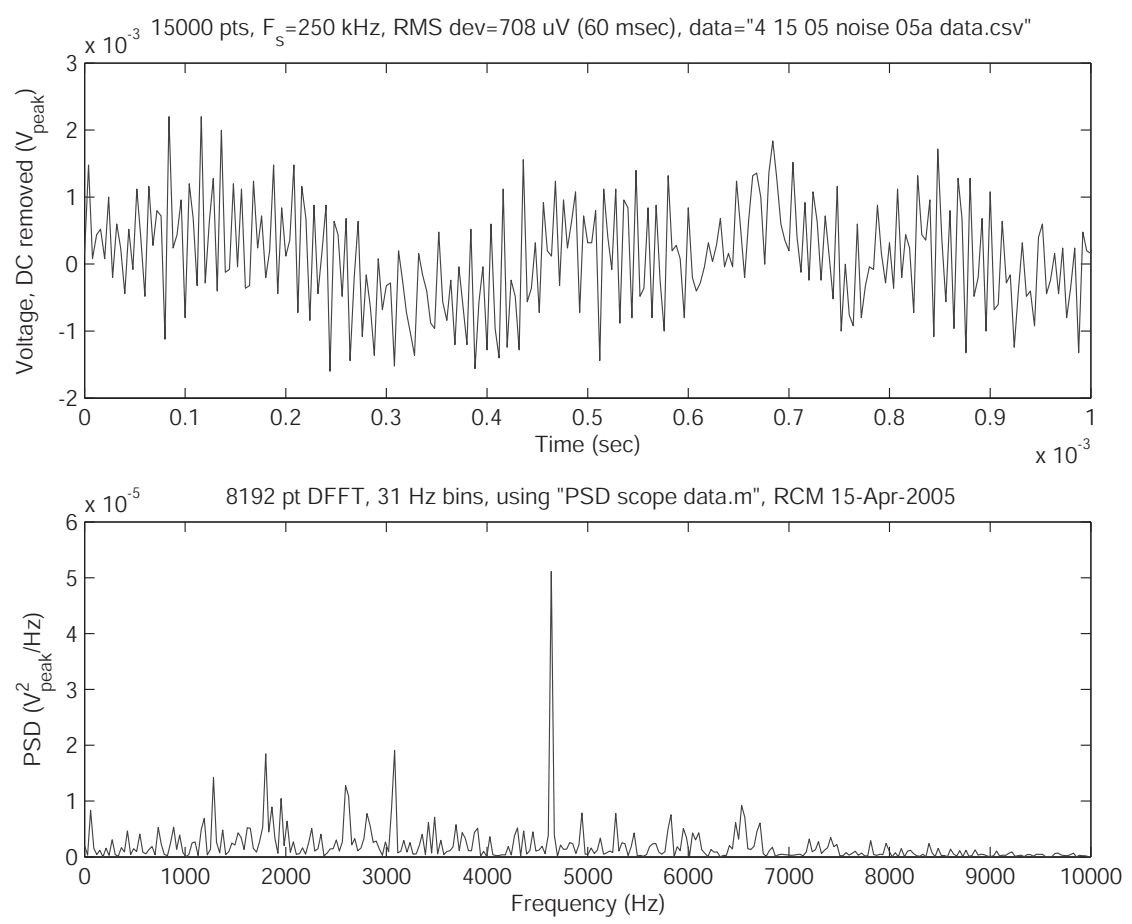

Figure 4-128: Calculated power spectral density of the noise measurement for the high-acceleration implementation of the $10 \mathrm{kHz}$ FTS.

\subsection{Results}

This section summarizes the results of the key tests for the $10 \mathrm{kHz}$ FTS. Those tests include high-acceleration tool tip trajectories, demonstrating control of the linear degree of freedom of the actuator, and heating of the rotor and stator cores during high frequency operation. The demonstrated peak torque and flux density are also discussed.

\subsubsection{Maximum tool tip Acceleration and Stroke}

A major design goal for the $10 \mathrm{kHz}$ FTS was to build a machine that could produce a closed-loop tool tip acceleration of $600 \mathrm{~g}$ when commanding the tool to follow a sinusoidal trajectory. Using the high-acceleration implementation, I demonstrated a sinusoidal tool tip trajectory of $\pm 4.0 \mu \mathrm{m}$ at $5 \mathrm{kHz}$, which corresponds to a $400 \mathrm{~g}$ maximum tool tip acceleration. Figure 4-129 shows the measured tool tip trajectory during the test. Section 4.10.4 describes how this peak tool tip acceleration corre- 


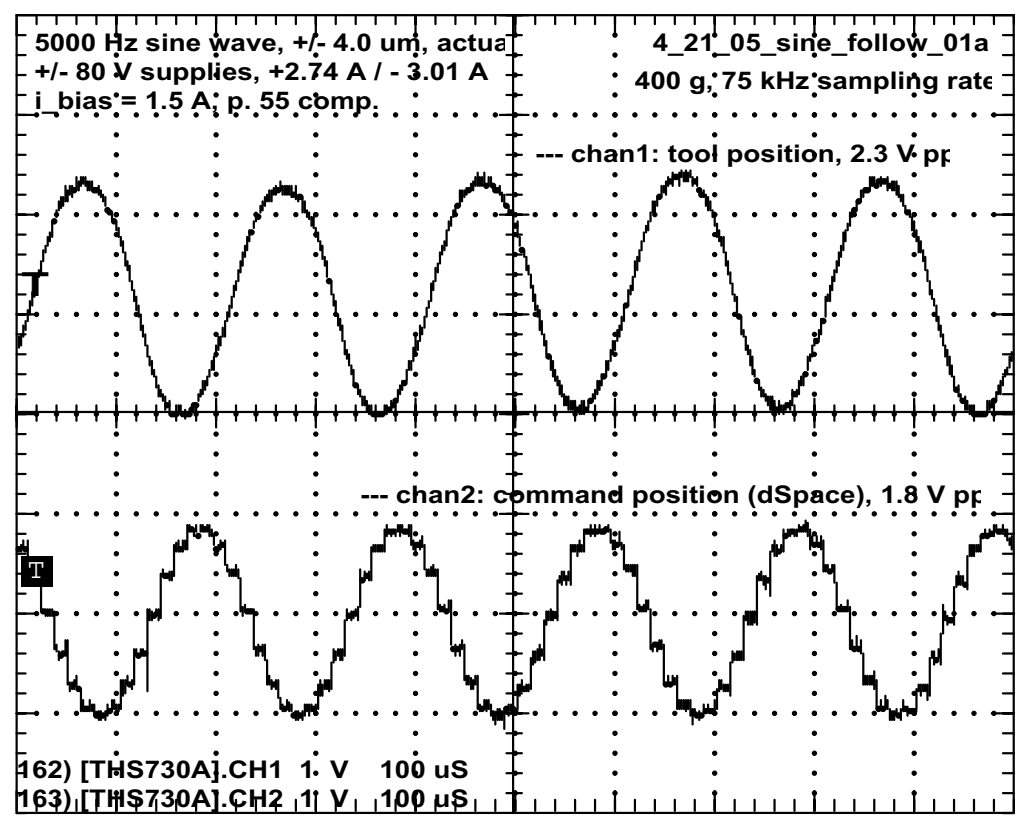

Figure 4-129: Measured closed-loop tool trajectory showing $400 \mathrm{~g}$ tool tip acceleration during operation at $5 \mathrm{kHz}$ with the high-acceleration implementation of the $10 \mathrm{kHz}$ FTS (upper curve). The lower curve is the commanded tool tip position from the digital controller, and the sampling rate of $75 \mathrm{kHz}$ is apparent in the step-like output caused by the zero-order hold of the D/A converter.

sponds to a demonstrated peak flux density of $1.2 \mathrm{~T}$ in the rotor/stator air gaps. It is likely that a higher peak acceleration could have been achieved if the bias flux coil current was increased, but I was running out of time for this research so did not re-tune the control system for a higher bias flux.

Another major design goal for the $10 \mathrm{kHz}$ FTS was to achieve a high tool tip acceleration during closed-loop operation at $10 \mathrm{kHz}$. Using the high-acceleration implementation, I demonstrated a sinusoidal tool tip trajectory of $\pm 2.17 \mu \mathrm{m}$ at $10 \mathrm{kHz}$, which corresponds to a 870 g maximum tool tip acceleration. Figure 4-130 shows the measured tool tip trajectory during the test. In this case the actuator is being aided by the stable first non-rigid body torsional mode of the rotor, which is discussed in Section 4.6.3. That torsional mode stores and releases energy that was originally supplied to the rotor by the actuator, and thereby assists the actuator in building up to and sustaining the commanded sinusoidal tool tip trajectory. It is interesting to note that this behavior could be acceptable in an application where the commanded 


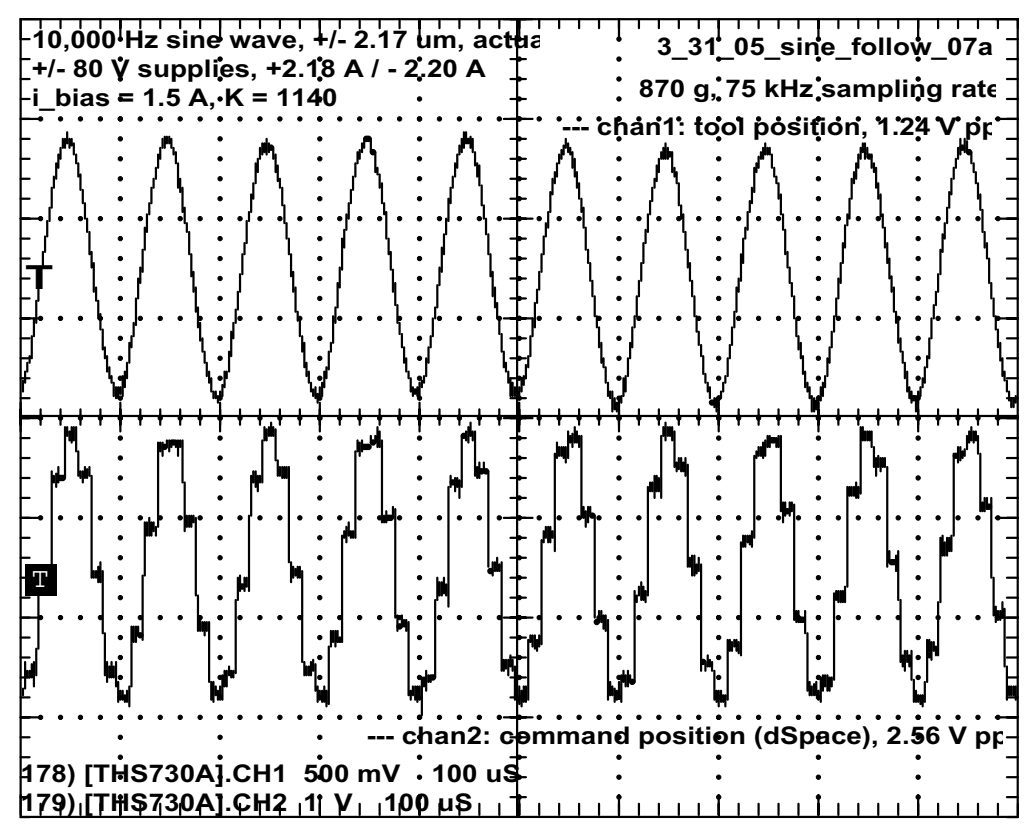

Figure 4-130: Measured closed-loop tool trajectory showing $870 \mathrm{~g}$ tool tip acceleration during operation at $10 \mathrm{kHz}$ with the high-acceleration implementation of the $10 \mathrm{kHz}$ FTS (upper curve). Energy is being stored and released by a stable mechanical resonance at $10 \mathrm{kHz}$. The lower curve is the commanded tool tip position from the digital controller, and the sampling rate of $75 \mathrm{kHz}$ is apparent in the step-like output caused by the zero-order hold of the D/A converter.

frequency and amplitude of a sinusoidal tool path did not have abrupt changes.

The full stroke of the $10 \mathrm{kHz}$ FTS was tested by commanding a $100 \mathrm{~Hz}$ sinusoidal tool tip trajectory, and increasing the amplitude until a flattening in the peaks was observed (and heard!) due to the rotor making contact with the hard stops. Since this test causes some saturation of the power amplifiers when the rotor reaches the hard stops and is still being commanded to go further, I performed it with the less aggressive low-noise implementation of the $10 \mathrm{kHz}$ FTS. Figure 4-131 shows the measured tool tip trajectory during the test.

\subsubsection{Demonstration of the Linear Mode of the Actuator}

Section 4.6.3 describes using the linear mode of the actuator to produce electronic damping of the front/back radial motion of the rotor. In this section I describe a set of experiments that explicitly demonstrate control of the linear mode of the 


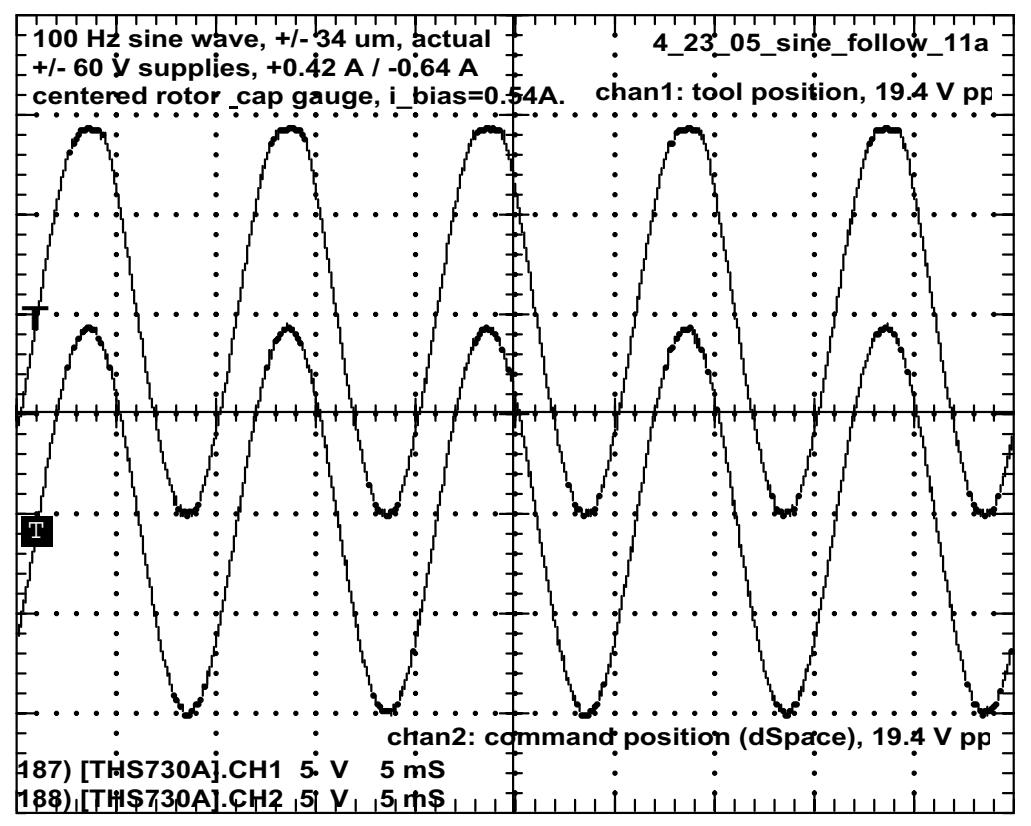

Figure 4-131: Measured closed-loop tool tip trajectory showing full travel of $\pm 34 \mu \mathrm{m}$ during operation at $100 \mathrm{~Hz}$ with the low-noise implementation of the $10 \mathrm{kHz}$ FTS (upper curve). The lower curve is the commanded tool tip position from the digital controller.

actuator. A $100 \mathrm{~Hz}$ sinusoidal force-producing current was applied to the actuator, and the resulting radial displacement of the rotor was observed by measuring the output voltage of the centerline capacitance sensor. The peak magnitude of the radial force developed by the actuator was limited to $9 \mathrm{~N}$ to avoid damaging the flexure suspension, and was sufficient for producing an observable radial displacement of the rotor.

The linear mode of actuator used in the $10 \mathrm{kHz}$ FTS requires a torque-producing current to act as an operating point. For demonstrating electronic damping, the rotor was rotationally locked by filling the rotor/stator air gaps with plastic shims, and a torque-producing current of $1.0 \mathrm{~A}$ was provided as an operating point for the linear mode. This provides a motor constant of $32 \mathrm{~N} / \mathrm{amp}$ for the force mode of the actuator (see (5.61) in Section 5.2.5). Using amplitudes of $0.1 \mathrm{~A}, 0.2 \mathrm{~A}$, and $0.3 \mathrm{~A}$ for the force-producing current produced calculated peak radial forces of $3 \mathrm{~N}, 6 \mathrm{~N}$, and $9 \mathrm{~N}$, and resulted in measured peak-to-peak radial motions of $6 \mathrm{~nm}, 9 \mathrm{~nm}$, and $12 \mathrm{~nm}$, respectively. Dividing the peak force of $9 \mathrm{~N}$ by the corresponding measured 
displacement amplitude of $6 \mathrm{~nm}(12 \mathrm{~nm} / 2)$ suggests a radial stiffness of $1500 \mathrm{~N} / \mu \mathrm{m}$. This is five times higher than the predicted radial stiffness of $280 \mathrm{~N} / \mu \mathrm{m}(1.6 \mathrm{lb} / \mu \mathrm{in})$ for the flexure-suspended rotor (see Figure 4-44). The higher measured stiffness in this case is most likely due to the presence of the shims in the rotor/stator air gaps.

Figures 4-132 through 4-134 show the measured centerline and tool tip displacements for the three tests, along with the differential currents used to drive the linear mode of the actuator. The astute reader will recognize that if the centerline capacitance sensor is not perfectly aligned with the axis of rotation of the rotor, then the motion observed by that sensor could be due to rotation-only of the rotor. Figure 4135 is included to show that the displacements observed by the centerline capacitance sensor in Figures 4-132 through 4-134 are not due to rotation-only of the rotor. Referring to Figure 4-135, a torque command to the actuator produced a measured tool tip motion of $8.4 \mu \mathrm{m}$ pp and a measured radial motion of the rotor of $48 \mathrm{~nm} \mathrm{pp}$. This indicates that the centerline capacitance sensor does have a slight offset from the rotation axis. By comparing the measured centerline motions shown in Figures 4-134 and 4-135, the ratio of $12 \mathrm{~nm}$ pp divided by $48 \mathrm{~nm}$ pp indicates that the observed tool tip motion in Figure 4-134 would have to be $2.1 \mu \mathrm{m}$ pp if the observed centerline motion in Figure 4-134 was due to rotation-only of the rotor, which it is not (by a factor of 13).

In concluding this section, I consider the issue of having to provide the linear mode of the actuator with a torque-producing current to act as an operating point. During the envisioned use of the $10 \mathrm{kHz}$ FTS as a machine tool, an O-ring seal between the capacitance sensors and the tool would be used to prevent debris from entering into the measurement gaps. To insure the integrity of the O-ring seal with the sensor behind the tool, a non-zero torque would need to be maintained on the rotor. Therefore it is possible - although maybe not practical - to consider using the current required to maintain that O-ring seal as the operating point needed by the linear mode, and add an automatic gain control element to the controller for the linear mode to compensate for changes in the motor force constant due to commanded changes in the torque-producing current. 


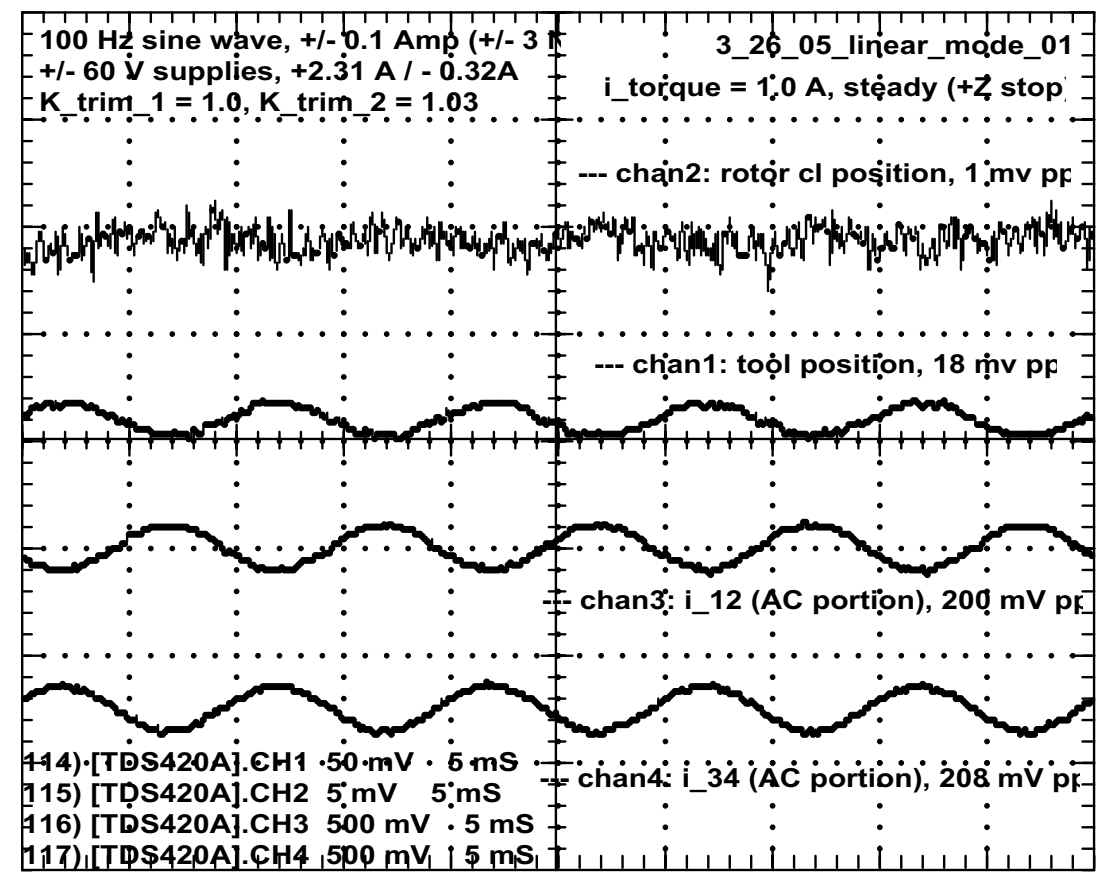

Figure 4-132: Demonstration of the linear mode of the actuator in the $10 \mathrm{kHz}$ FTS. Measured rotor radial motion is $6 \mathrm{~nm}$ pp (upper curve). Corresponding measured tool tip motion is $63 \mathrm{~nm}$ pp (second curve from top). Measured differential currents of \pm 0.1 A used to drive the two pairs of steering flux coils (bottom two curves).

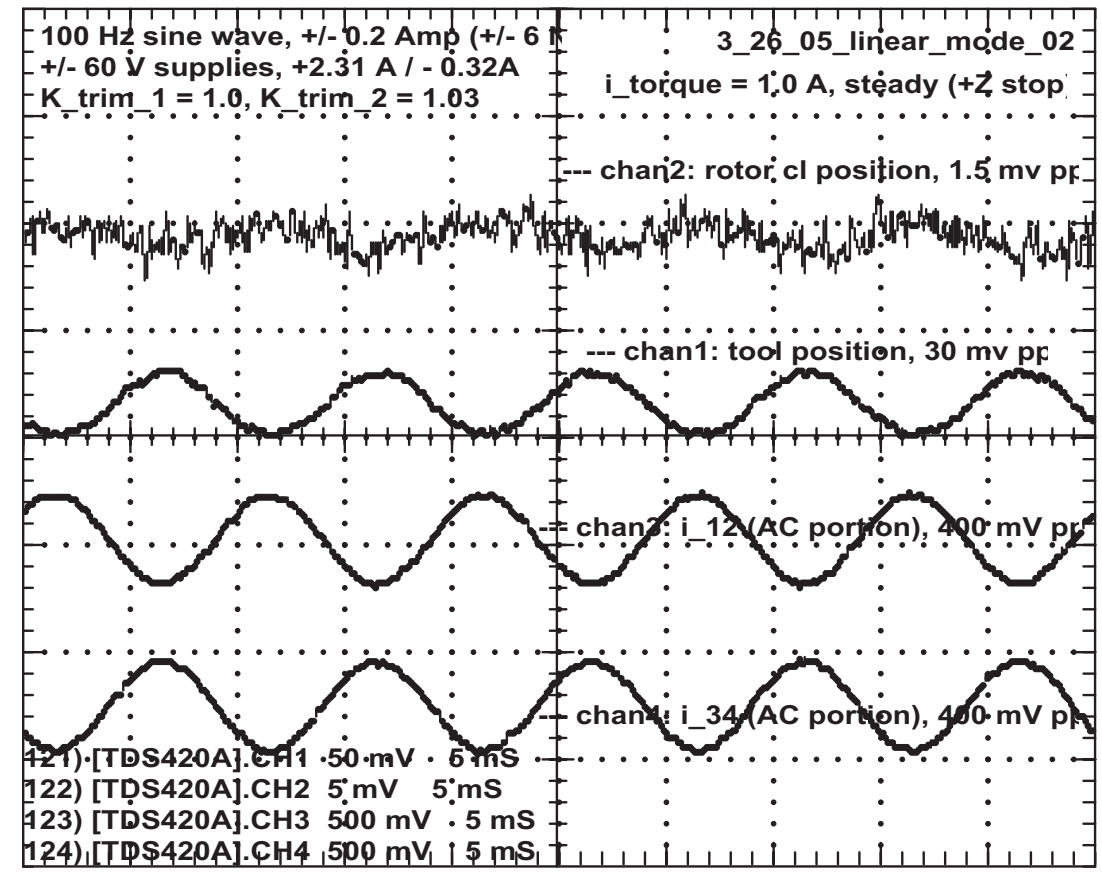

Figure 4-133: Demonstration of the linear mode of the actuator in the $10 \mathrm{kHz}$ FTS. Measured rotor radial motion is $9 \mathrm{~nm}$ pp (upper curve). Corresponding measured tool tip motion is $100 \mathrm{~nm}$ pp (second curve from top). Measured differential currents of $\pm 0.2 \mathrm{~A}$ used to drive the two pairs of steering flux coils (bottom two curves). 


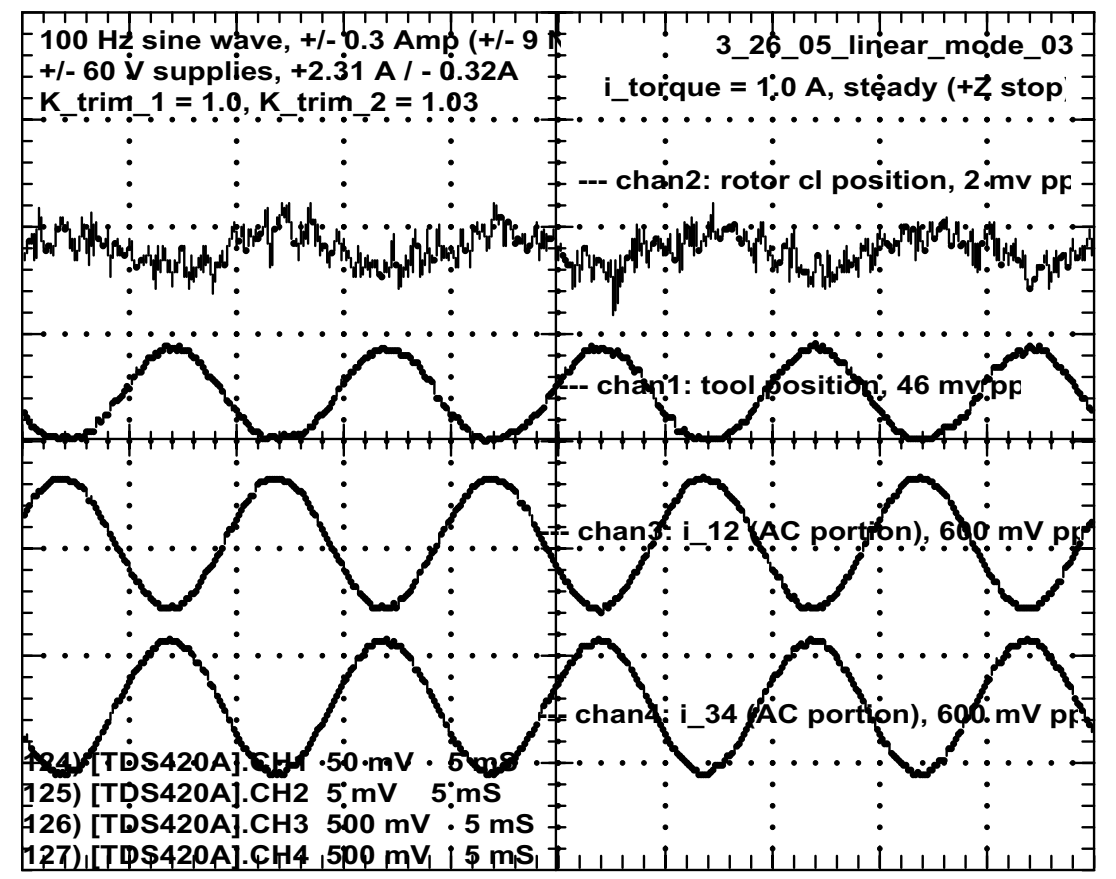

Figure 4-134: Demonstration of the linear mode of the actuator in the $10 \mathrm{kHz}$ FTS. Measured rotor radial motion is $12 \mathrm{~nm}$ pp (upper curve). Corresponding measured tool tip motion is $160 \mathrm{~nm}$ pp (second curve from top). Measured differential currents of $\pm 0.3 \mathrm{~A}$ used to drive the two pairs of steering flux coils (bottom two curves).

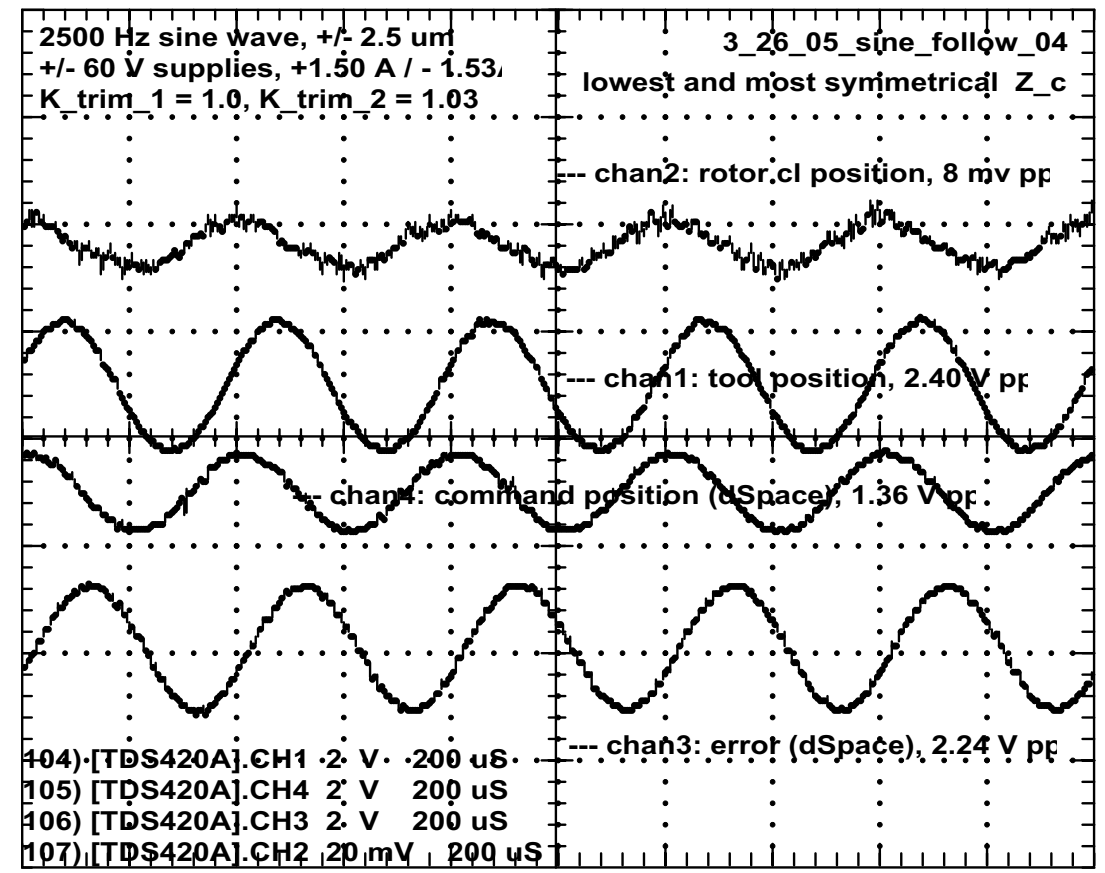

Figure 4-135: Data showing that centerline motion observed while demonstrating the linear mode is not caused by rotation of the rotor. Measured tool tip motion of $8.4 \mu \mathrm{m}$ pp (second curve from top) during commanded rotation of the rotor, and corresponding measured centerline motion of $48 \mathrm{~nm}$ pp (upper curve). 


\subsubsection{Temperature Rise in the Rotor and Stator Cores}

Heat generation due to magnetic losses in the rotor and stator cores is significant at high operating frequencies (around $5 \mathrm{kHz}$ and above). With respect to conductive heat transfer, the rotor is thermally isolated from the rest of the $10 \mathrm{kHz}$ FTS by the flexure bearing, so the temperature rise in the rotor core is more of an issue than it is for the stator cores. Referring to Figure 4-15, the $10 \mathrm{kHz}$ FTS has provisions for forced cooling of the rotor and stator with a fluid - most likely a gas because of the small air gaps. Included in the suggested future work in Section 6.4 are experiments using forced cooling with the $10 \mathrm{kHz}$ FTS and experiments using a mockup of the magnetic circuit to more deeply explore the thermal losses in the rotor and stator cores. In this section I present measurements of the rotor and stator core temperatures during $5 \mathrm{kHz}$ operation, use a lumped-parameter thermal model to estimate the actual rotor and stator core losses, and compare those estimated losses to the predicted core losses.

Figure 4-136 shows the locations of the thermocouples used to measure the temperature of the rotor and stator cores. The thermocouple for the rotor core passes through a hole in the tool arm that is centered on the axis of rotation, and touches the top of the upper flexure hub. This was as close as I could practically get to the rotor core for the $10 \mathrm{kHz}$ FTS, and was acceptable for this research because the majority of the heat generated by the rotor core passes directly into the aluminum flexure hubs. Therefore, the temperature measured by that thermocouple should track the actual temperature of the rotor core, with a time lag. The thermocouple for the stator core is touching the inside back surface of the core, and is held in place by the potting compound used to retain the coils.

Figure 4-137 shows the measured temperature of the rotor and stator cores during approximately 3 minutes of $400 \mathrm{~g}$ operation at $5 \mathrm{kHz}$, and then during a 20 minute cool-down period after turning off the $10 \mathrm{kHz}$ FTS. I ran the fast tool servo until the temperature difference between the rotor and stator cores approached the $10^{\circ} \mathrm{F}$ limit that I had set based on what I considered to be a safe stress level in the flexure blades due to differential thermal growth of the rotor and stator. An alternate flexure- 

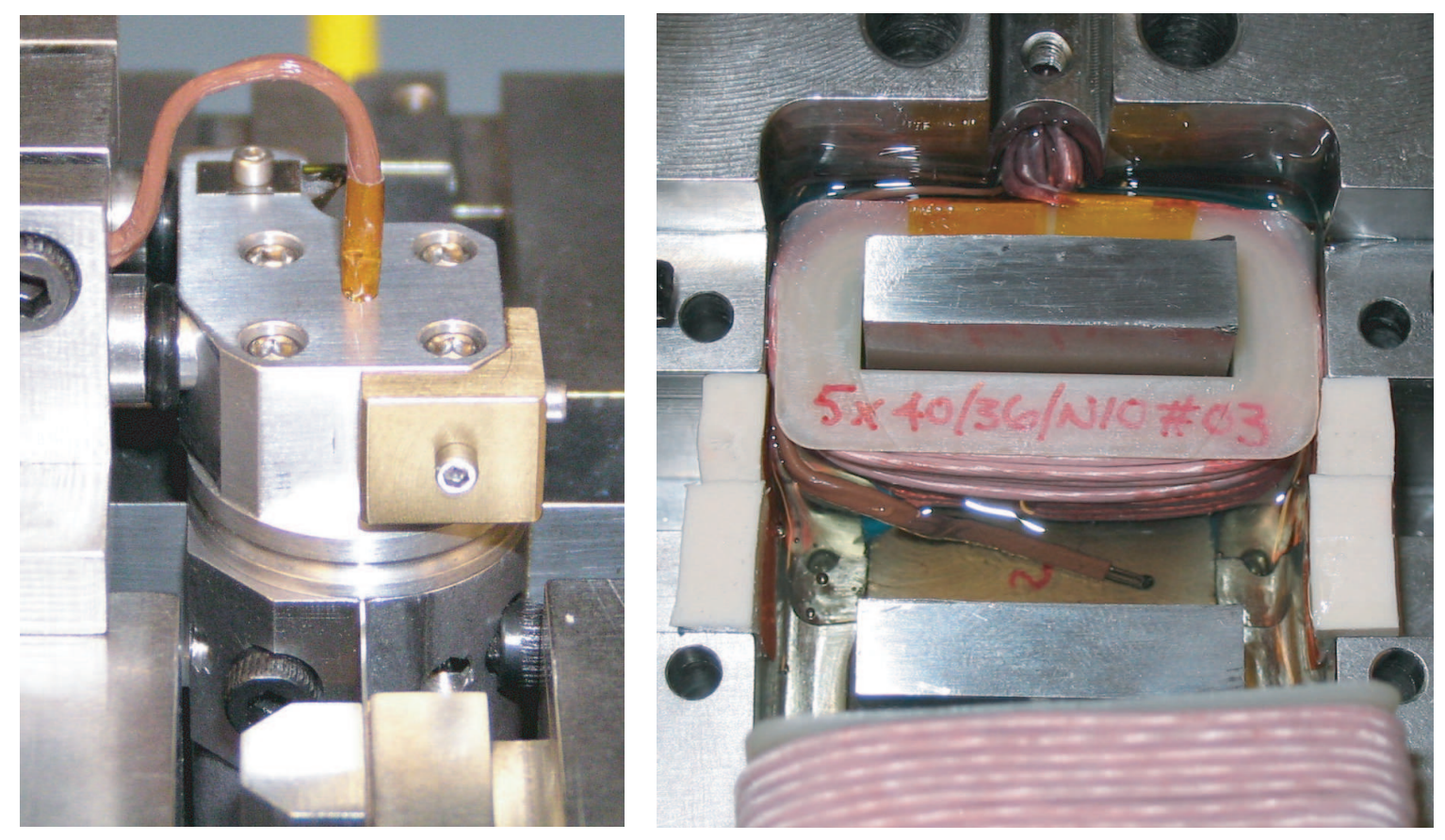

Figure 4-136: Locations of the thermocouples used to measure the temperature of the rotor core (left) and stator core (right).

suspension that reduces the axial components of those stresses is presented in the Section 6.4.5. First-order exponential curves were hand-fitted to the rising and falling temperature profiles to allow correlating the data to first-order thermal models. The measured temperature of the rotor core lags that of the stator core, as expected. Note that the cooling data for the stator core is well represented by different exponential curves for the first and last portions of the cooling cycle. The first of those two curves indicates that the stator core final temperature would have been higher than it actually was. The additional cooling corresponding to the second of those two curves is most likely due to thermal paths not accounted for by the simple first-order model.

Figure 4-138 shows the simplified first-order lumped-parameter thermal model that I use to calculate the actual heat generation in the cores from the measured temperature data. $\dot{Q}_{g e n}$ is the heat generated by the core, $\mathrm{C}_{\text {core }}$ is the thermal capacitance of the core, $\mathrm{R}_{\text {disp }}$ is the thermal resistance between the core and its surroundings, $\mathrm{T}_{\text {core }}$ is the temperature of the core, and $\mathrm{T}_{a m b}$ is the temperature of the surroundings.

Assuming that the rotor and stator cores are initially at the same temperature 


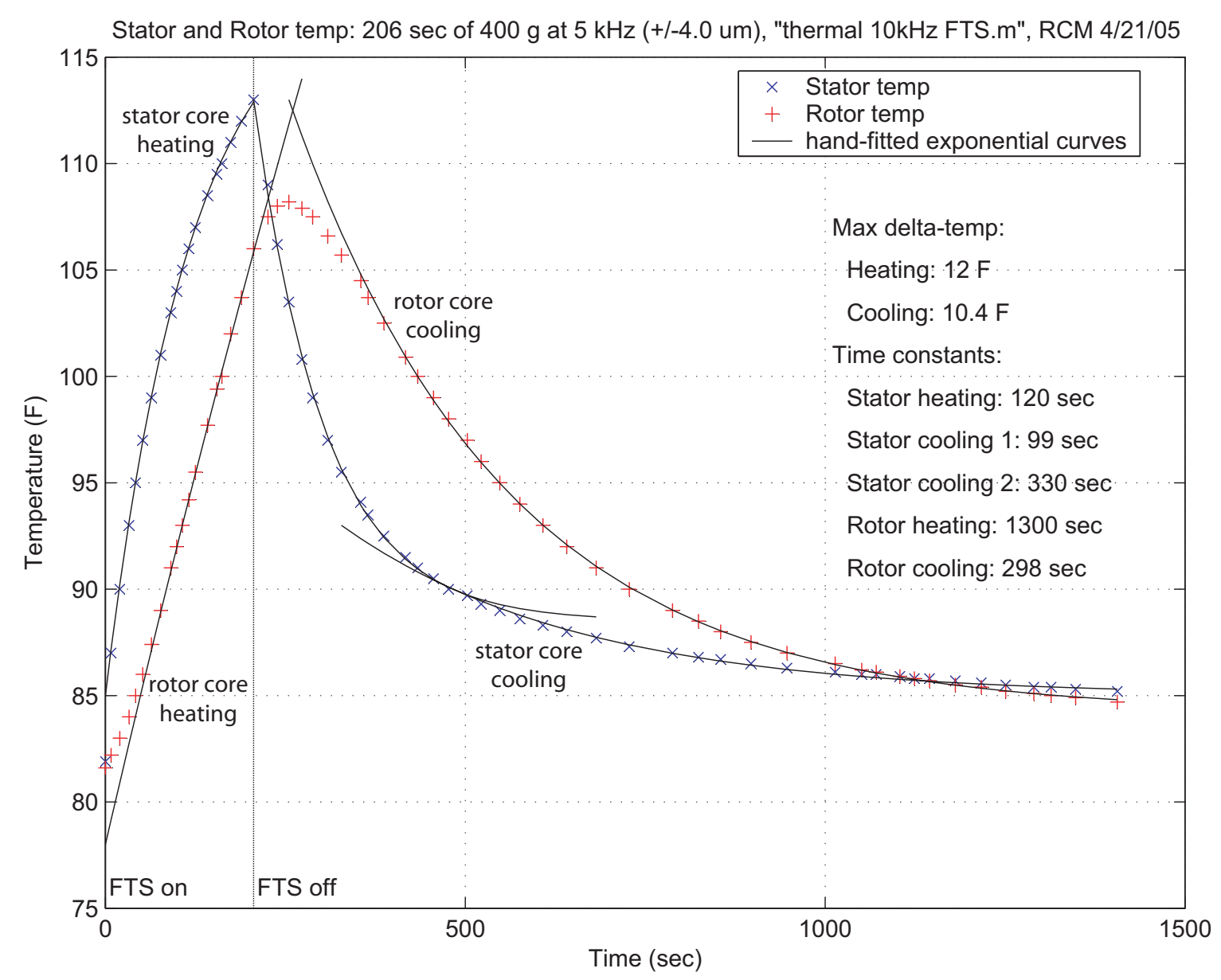

Figure 4-137: Measured temperature of the rotor and stator cores during approximately $3 \mathrm{~min}$ of $400 \mathrm{~g}$ operation at $5 \mathrm{kHz}$, and then during a $20 \mathrm{~min}$ cool-down period after turning off the $10 \mathrm{kHz}$ FTS. The solid lines are exponential curves that were hand-fitted to the rising and falling temperature data points. Time constants for the fitted curves are listed on the right-hand side. 


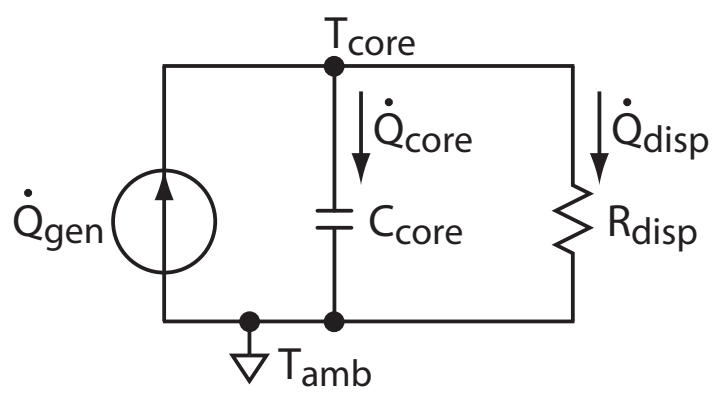

Figure 4-138: Simplified lumped-parameter thermal model used to calculate experimental core power losses from measured thermal time constants.

as their surroundings, at $t=0$, since there is no temperature drop across the thermal resistance, all of the heat generated by a core initially flows into its own thermal capacitance. The heat flow $\dot{Q}_{\text {core }}$ into the thermal capacitance of a core is given by (4.17):

$$
\dot{Q}_{\text {core }}=C_{\text {core }} \frac{d T_{\text {core }}}{d t}
$$

At $\mathrm{t}=0,\left(d T_{\text {core }} / d t\right)$ is equal to the ratio of the difference between the final and initial temperatures divided by the time constant for the system, all of which we obtain from the exponential curves fitted to the measured temperature during heating.

Figure 4-139 provides detail on the heating data and fitted exponential curve for the stator core. Using the fitted curve, $\left(d T_{\text {core }} / d t\right)$ at $\mathrm{t}=0$ is equal to $19^{\circ} \mathrm{C}\left(34^{\circ} \mathrm{F}\right)$ divided by $120 \mathrm{sec}$, or $0.16\left({ }^{\circ} \mathrm{C} / \mathrm{sec}\right)$. The thermal capacitance of the stator core is $42 \mathrm{~J} /{ }^{\circ} \mathrm{K}$. Using (4.17), the measured heat generation by the stator core is $6.7 \mathrm{~W}$. The predicted heat generation based on the core loss data from the manufacturer of the $50 \% \mathrm{Ni}-50 \%$ Fe stator core material for a $0.75 \mathrm{~T}$ peak flux density at $5 \mathrm{kHz}$ operation is $1.8 \mathrm{~W}$, which is a factor of 3.7 times lower than the measured stator core loss of 6.7 W. As an aside, the calculated ohmic heating in the two steering flux coils due to the $\pm 1.8 \mathrm{~A}$ of current that was flowing through them during the experiment is a negligible $0.04 \mathrm{~W}$.

Figure 4-140 provides detail on the heating data and fitted exponential curve for the rotor core. The initial and final data points are ignored during the data fit because they represent a transient behavior that the first-order model does not 


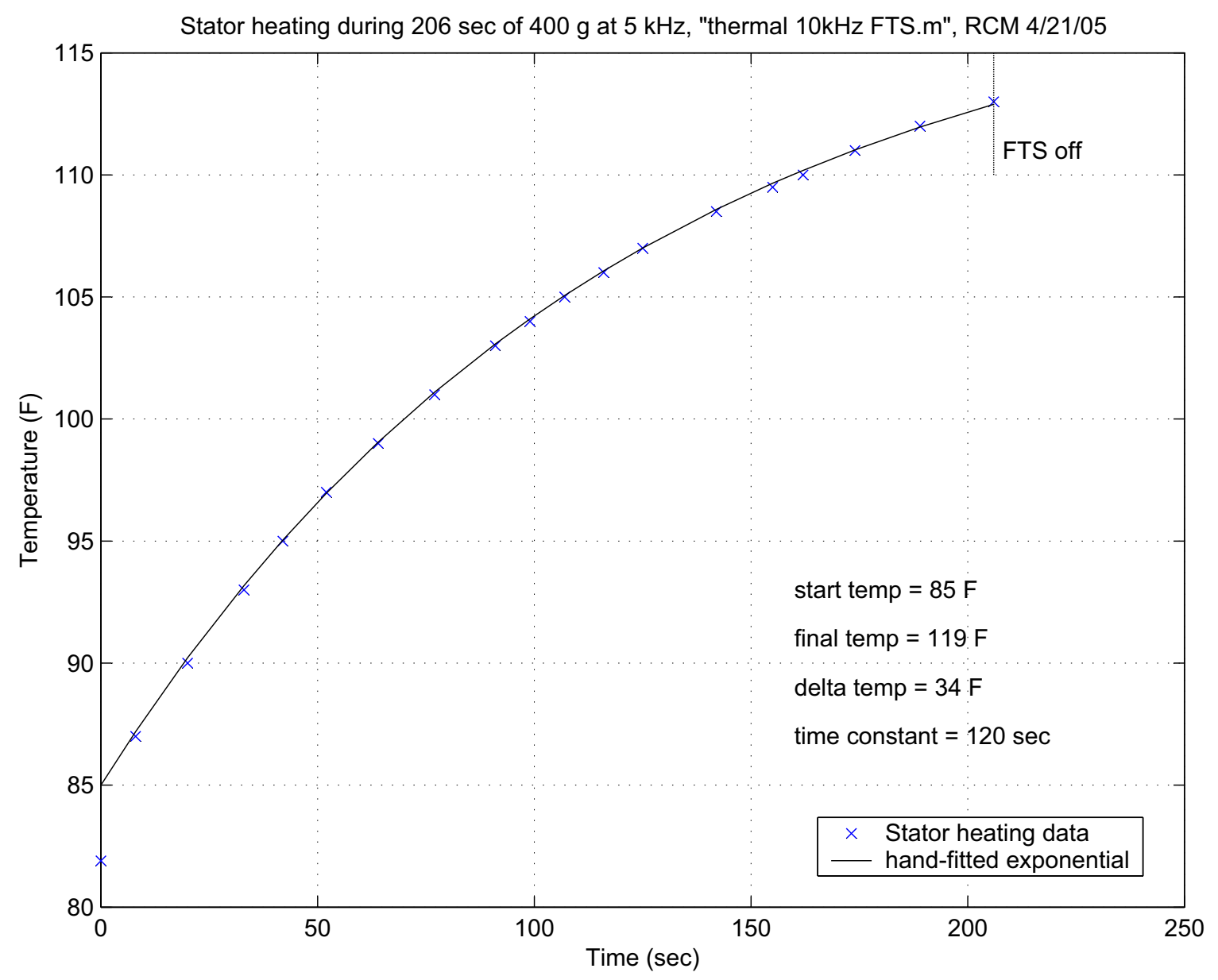

Figure 4-139: Detail of the measured temperature of the stator core during approximately $3 \mathrm{~min}$ of $400 \mathrm{~g}$ operation at $5 \mathrm{kHz}$. The solid line is an exponential curve that was hand-fitted to the data points. The initial and final temperatures and time constants listed on the right-hand side are based on the fitted curve. 


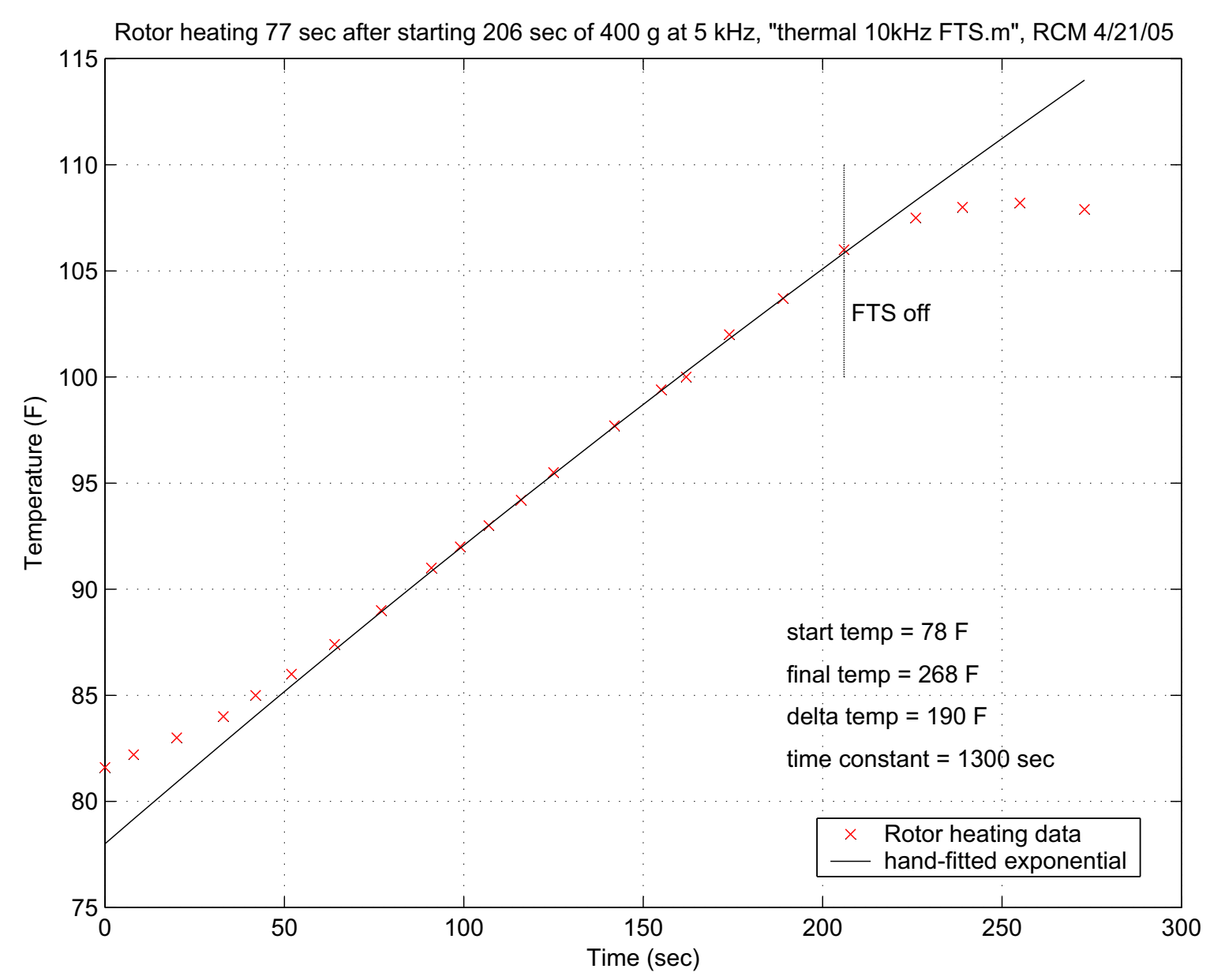

Figure 4-140: Detail of the measured temperature of the rotor core during approximately $3 \mathrm{~min}$ of $400 \mathrm{~g}$ operation at $5 \mathrm{kHz}$. The solid line is an exponential curve that was hand-fitted to the data points. The initial and final data points are ignored during the data fit because they represent a transient behavior that the first-order model does not account for. The initial and final temperatures and time constants listed on the right-hand side are based on the fitted curve.

account for. Using the fitted curve, $\left(d T_{\text {core }} / d t\right)$ at $\mathrm{t}=0$ is equal to $106^{\circ} \mathrm{C}\left(190^{\circ} \mathrm{F}\right)$ divided by $1300 \mathrm{sec}$, or $0.081^{\circ} \mathrm{C} / \mathrm{sec}$. The thermal capacitance of the rotor core is $19 \mathrm{~J} /{ }^{\circ} \mathrm{K}$. Using (4.17), the measured heat generation by the rotor core is $1.5 \mathrm{~W}$. The predicted heat generation based on the core loss data from the manufacturer of the nanocrystalline iron-alloy rotor core material for a $0.75 \mathrm{~T}$ peak flux density at $5 \mathrm{kHz}$ operation is $0.08 \mathrm{~W}$, which is a factor of 19 times lower than the measured rotor core loss of $1.5 \mathrm{~W}$.

Why the discrepancy between the predicted and measured core losses for the rotor 
and stator cores, and why is the difference so large for the rotor core? Section 5.5.6 discusses how the published core loss data indicates that the dominant loss mechanism is eddy current loss and not hysteresis loss. One of the things that we did not successfully achieve when manufacturing the rotor and stator cores was to insure that the laminations were not shorted together at the machined surfaces. I tried using an acid etch to remove the smeared-together layer that was present at the machined surfaces, but it did not work. In the case of the nanocrystalline iron-alloy rotor core, the as-machined core did not meet the flatness requirements and needed a considerable amount of careful hand-sanding to make it flat enough to use. It is likely that this significantly increased the depth of the region where the laminations are shorted together, and exacerbated the eddy current losses for the rotor core. Furthermore, we observed damage in the as-machined nanocrystalline iron-alloy cores that suggested the presence of internal shorting between multiple lamination layers. It is not clear if that damage existing before the wire electro-discharge-machining (EDM) process, or was caused by that process. Internal shorting between the lamination layers would cause higher core losses than predicted by the manufacturer's core loss data.

\subsubsection{Demonstrated Peak Torque and Flux Density}

Section 4.10.1 mentioned that I demonstrated a sinusoidal tool tip trajectory of $\pm 4.0 \mu \mathrm{m}$ at $5 \mathrm{kHz}$, which corresponds to a $400 \mathrm{~g}$ maximum tool tip acceleration. Chasing through the math using the swing radius for the tool of $12.7 \mathrm{~mm}$ and a total rotational inertia of $81 \mathrm{gm} \cdot \mathrm{cm}^{2}$, the $400 \mathrm{~g}$ tool tip acceleration corresponds to a demonstrated peak torque of $2.5 \mathrm{~N} \cdot \mathrm{m}$. The demonstrated peak torque is $68 \%$ of the designed peak torque of $3.7 \mathrm{~N} \cdot \mathrm{m}$, which is based on (5.16) and the assumption that the steering and bias fluxes have the same designed peak magnitudes of $0.75 \mathrm{~T}$ in the rotor/stator air gaps.

During the peak acceleration tests, the current-mode amplifiers were capable of providing a higher voltage and current to the steering flux coils than what was needed to produce the $400 \mathrm{~g}$ tool tip acceleration. Therefore, the demonstrated peak acceleration was limited by the magnitude of the bias magnetic flux density. Due to 
geometric form errors in the as-fabricated stator cores, and imperfect closure of the joints between the components in the back-iron, the effective length of the as-built air gaps between the stator cores and the back-iron is ten times longer than what was planned when choosing the number of turns for the bias coil. Section 5.3.1 describes how the length of those as-built air gaps is calculated. Consequentially, I had to use a higher bias coil current than planned when conducting the high acceleration tests for the actuator, but not high enough to produce the desired $0.75 \mathrm{~T}$ bias flux density. The simple remedy for future work is to use a bias coil with a higher number of turns, or a properly sized permanent magnet for the bias source.

The actuator produces its maximum torque when the magnitude of the steering flux density is equal to the magnitude of the bias flux density: to first-order the steering and bias fluxes add to produce twice the magnitude of each flux density in one pair of diagonally opposed rotor/stator air gaps, and they completely extinguish each other in the other pair of rotor/stator air gaps. Under these conditions, using (5.16) and a torque of $2.5 \mathrm{~N} \cdot \mathrm{m}$, we find that the magnitude of the steering and bias flux densities is $0.6 \mathrm{~T}$ during the demonstrated peak tool tip acceleration of $400 \mathrm{~g}$ at $5 \mathrm{kHz}$. This corresponds to a demonstrated peak flux density of $1.2 \mathrm{~T}$ in the rotor/stator air gaps, which is $82 \%$ of the designed $1.5 \mathrm{~T}$ peak flux density.

One measure of performance for the actuator used in the $10 \mathrm{kHz}$ FTS is the ratio of the continuous-operation peak torque divided by the unburdened ${ }^{18}$ rotational inertia of the rotor. For the demonstrated peak torque of $2.5 \mathrm{~N} \cdot \mathrm{m}$ and a rotor inertia of $70 \mathrm{gm} \cdot \mathrm{cm}^{2}$, that ratio is $3.6 \times 10^{5} \mathrm{~N} \cdot \mathrm{m} /\left(\mathrm{kg} \cdot \mathrm{m}^{2}\right)$. Using the designed peak torque of $3.7 \mathrm{~N} \cdot \mathrm{m}$ increases the ratio to $5.3 \times 10^{5} \mathrm{~N} \cdot \mathrm{m} /\left(\mathrm{kg} \cdot \mathrm{m}^{2}\right)$. Adding the burden of the $11 \mathrm{gm} \cdot \mathrm{cm}^{2}$ rotational inertia for the tool arm reduces these ratios by a factor of 0.86. It is interesting to note that the demonstrated and designed unburdened torque-to-inertia ratios for the $10 \mathrm{kHz}$ FTS straddle the specified unburdened value of $4.4 \times 10^{5} \mathrm{~N} \cdot \mathrm{m} /\left(\mathrm{kg} \cdot \mathrm{m}^{2}\right)$ presented in Section 3.11 .2 for the state-of-the-art galvanometer used in the $2 \mathrm{kHz}$ FTS.

\footnotetext{
18 "Unburdened" means that the inertia of the payload — the tool arm in this case — is not included.
} 
Another measure of performance is the ratio of the demonstrated peak torque while accelerating the tool arm divided by the apparent time-average electrical power at the terminals of the actuator. The demonstrated peak torque of $2.5 \mathrm{~N} \cdot \mathrm{m}$ at $5 \mathrm{kHz}$ required a total peak current of $2.5 \mathrm{~A}$ in each stator half. ${ }^{19}$ The corresponding voltage drops across the total resistance and total inductance in each stator half are given by (4.12) and (4.13) after substituting in values for a pair of series connected steering flux coils:

$$
\begin{gathered}
V_{\text {resistance }}=\left(R_{\text {scp }}+R_{s}\right) I_{T}=[(0.024+0.046)+0.2](2.5)=0.68 \mathrm{~V} \\
V_{\text {inductance }}=L_{\text {scp }} \frac{d I_{T}}{d t}=\left(800 \times 10^{-6}\right)[2.5(2 \pi 5000)]=63 \mathrm{~V}
\end{gathered}
$$

Section 4.7.2 shows that the current in the resistance - and hence the voltage across the resistance - lags the voltage across the inductance by $89^{\circ}$. Using (3.23) from Section 3.11.2 to relate the time-average complex power $\underline{S}$ to the real power $P$ power and the reactive power $Q$, and substituting in values for one stator half, we obtain:

$$
\underline{S}=P+j Q=\frac{1}{2} \underline{V} \underline{I}^{*}=\frac{1}{2}(0.68+j 63) 2.5=0.85 \mathrm{~W}+j 79 \mathrm{VAR}
$$

In this case the power is essentially all reactive. The magnitude of the time-average complex power $|\underline{S}|$ is the apparent power, and is 79 VA for each stator half. Therefore, the total apparent power at the terminals of the actuator while demonstrating $2.5 \mathrm{~N} \cdot \mathrm{m}$ at $5 \mathrm{kHz}$ is $160 \mathrm{VA}$. This gives a ratio of the demonstrated peak torque while accelerating the tool arm divided by the apparent time-average electrical power at the terminals of the actuator of $16 \times 10^{-3} \mathrm{~N} \cdot \mathrm{m} / \mathrm{VA}$. This ratio is a factor of 9 greater than the same demonstrated ratio of $1.7 \times 10^{-3} \mathrm{~N} \cdot \mathrm{m} / \mathrm{VA}$ for the actuator used in the $2 \mathrm{kHz}$ FTS.

It should be noted that for a fair comparison of the actuators used in the $10 \mathrm{kHz}$ FTS

\footnotetext{
${ }^{19}$ The peak current of $2.5 \mathrm{~A}$ is calculated by dividing the peak torque of $2.5 \mathrm{~N} \cdot \mathrm{m}$ by the motor constant of $0.99 \mathrm{~N} \cdot \mathrm{m} / \mathrm{amp}$ for the rotary mode of the actuator (high-acceleration implementation).
} 
and in the $2 \mathrm{kHz}$ FTS, one should consider that the actuator in the $10 \mathrm{kHz}$ FTS would need to be cooled if it were to run continuously at the performance levels discussed in this section.

\subsection{Conclusions}

Using the proof of principles established with the earlier $2 \mathrm{kHz}$ FTS, the $10 \mathrm{kHz}$ rotary fast tool servo establishes a new benchmark for a rotary fast tool servo with a demonstrated closed-loop bandwidth of $10 \mathrm{kHz}$ and a peak closed-loop tool tip acceleration of $400 \mathrm{~g}$ provided entirely by the actuator while operating at a frequency of $5 \mathrm{kHz}$. This corresponds to a peak flux density of $1.2 \mathrm{~T}$ in the rotor/stator air gaps, which is $80 \%$ of our design goal of $1.5 \mathrm{~T}$ for a $600 \mathrm{~g}$ tool tip acceleration. A stable mechanical resonance at $10 \mathrm{kHz}$ allowed demonstrating a closed-loop tool tip acceleration of $870 \mathrm{~g}$ while operating at that frequency.

The following additional items were demonstrated with the $10 \mathrm{kHz}$ FTS:

- Developed a mechanical design that allowed controlling 18 degrees of freedom between the rotor core and the two stator cores to establish four $50 \mu \mathrm{m}$ rotor/stator air gaps and balance the bias flux in the stator poles within $7 \%$.

- Demonstrated that when the bias flux is provided by a coil, air gaps between the stator cores and the bias flux circuit back-iron can provide sufficient enough magnetic decoupling to allow independently controlling the two stator halves with a controller that was designed using SISO system techniques.

- Demonstrated arbitrary point-to-point closed-loop control of the tool tip using a class of actuator that is typically used to drive and sustain a resonance in a mechanical oscillator.

- Demonstrated closed-loop control of the rotary and linear modes of the actuator.

- Heat generation due to magnetic losses in the rotor and stator cores is significant at high operating frequencies (around $5 \mathrm{kHz}$ and above), and requires active 
cooling or additional work to reduce the losses.

- The demonstrated ratio of peak torque to electrical power at the terminals of the hybrid actuator is $16 \times 10^{-} 3 \mathrm{~N} \cdot \mathrm{m} / \mathrm{VA}$, which is a factor of 9 greater than the same demonstrated ratio for the actuator used in the $2 \mathrm{kHz}$ FTS.

The $10 \mathrm{kHz}$ FTS serves as a proof-of-concept for a hybrid rotary/linear electromagnetic actuator that has a broader array of potential applications beyond fast tool servos. In the next chapter we explore the actuator in detail, and develop a set of insights and tools that can be used to design other magnetic circuits and normal-stress electromagnetic actuators. 


\section{Chapter 5}

\section{Hybrid Rotary/Linear Electromagnetic Actuator}

In this chapter I develop the analytical tools and insights used to design the actuator in the $10 \mathrm{kHz}$ rotary fast tool servo, explore alternate magnetic circuits for the actuator, and review the candidate soft magnetic materials that were considered. Using firstprinciples relationships for the physics of electromagnetic systems, sets of equations are developed that allow predicting the electromagnetic characteristics of the actuator and aid the decision-making process during the detailed design of the actuator. The other half of the picture - designing the mechanical aspects of the actuator - is described in the trade-off study in Section 4.8. A general discussion on soft magnetic materials is included to establish an understanding of the underlying issues associated with them.

\subsection{Introduction}

The actuator that I built for the $10 \mathrm{kHz}$ FTS is a normal-stress variable reluctance machine that utilizes a constant bias magnetic flux. The bias flux provides two significant benefits: (1) it linearizes the torque versus drive-current relationship for the actuator, and (2) it provides up to half of the torque-producing magnetic flux in the rotor/stator air gaps. The first benefit makes it easier to accurately control 
the actuator. The second benefit provides a factor of two reduction in the electrical power needed to drive the peak AC flux when compared to a design that does not use a bias flux.

In addition to producing the torque that is needed to accelerate the rotor of the $10 \mathrm{kHz}$ FTS, the actuator produces a radial force and is therefore referred to as a hybrid rotary/linear actuator. The actuator consists of a rotor and a stator consisting of two halves. Figures 5-1 and 5-2 show models of the magnetic circuit for the actuator. The DC coil provides a bias flux (triple-head arrows) and the AC coils provide a steering flux (double-head arrows). With appropriate phasing, the bias and steering fluxes add in one pair of diagonally opposed rotor/stator air gaps and subtracts in the other pair, producing a net torque on the rotor. A net force is produced on the rotor in the front/back direction ${ }^{1}$ when the steering fluxes in the two stator cores have different magnitudes.

In Section 2.3 I show that certain types of electric engraving heads use a rotary actuator that is similar to our hybrid actuator. In the case of the engraving heads, the actuator is used to produce and sustain a resonating mechanical oscillation. In our case, the hybrid actuator is used to demonstrate closed-loop arbitrary trajectories for the tool tip of the $10 \mathrm{kHz}$ FTS. Using a separate current-mode amplifier for each stator half allows demonstrating closed-loop control of the rotary and linear degrees of freedom that are inherent in this class of actuators. Our work is further distinguished from the prior art by a magnetic circuit that decouples certain magnetic flux paths when a coil is used instead of a permanent magnet to provide the bias magnetic flux. This reduces the complexity of the actuator electrical dynamics from a MIMO system to a SISO system, and allows using loop-shaping techniques with Classical Control theory to design the control systems.

A review of candidate soft magnetic materials indicates that designing for a peak flux density of $1.5 \mathrm{~T}$ was reasonable, so we set that as a goal. For the actuator in the $10 \mathrm{kHz}$ FTS, a peak flux density of $1.5 \mathrm{~T}$ in one pair of diagonally opposed

\footnotetext{
${ }^{1}$ The front/back direction for the rotor is parallel to the surface normals of the pole faces when the rotor is in its neutral (no rotation) position.
} 


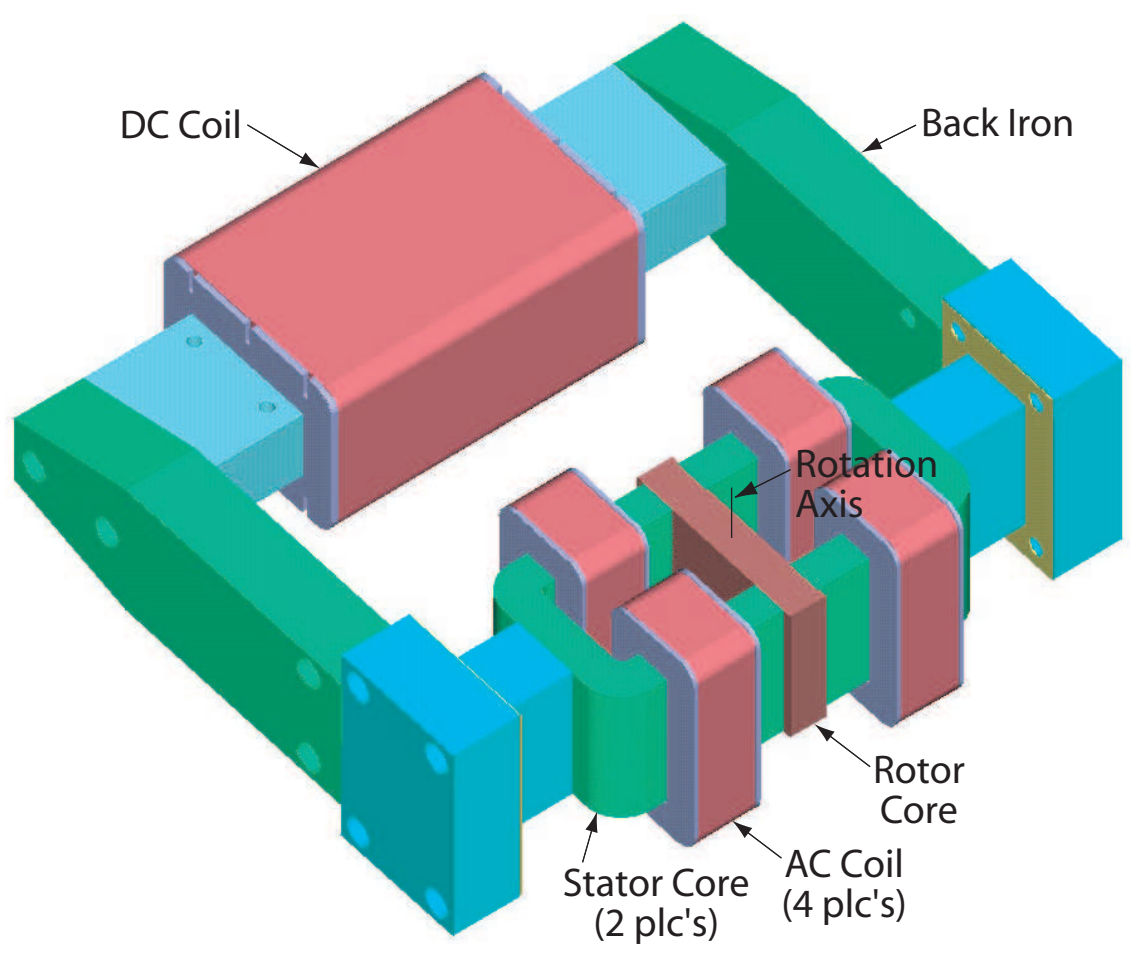

Figure 5-1: Model of the magnetic circuit for the $10 \mathrm{kHz}$ rotary fast tool servo.

rotor/stator air gaps, and flux extinction in the other pair, produces a peak torque of $3.7 \mathrm{~N} \cdot \mathrm{m}$ on the rotor. The corresponding normal force on the pole faces with the $1.5 \mathrm{~T}$ flux density acting on them is $144 \mathrm{~N}$ (32 lb).

For the actuator in the $10 \mathrm{kHz}$ FTS, the torque mode is decoupled from the force mode, but the force mode requires a torque-producing current to act as an operating point and is therefore coupled to the torque mode. This chapter contains a discussion on two alternate magnetic circuits for the actuator that have fully decoupled torque and force modes.

\subsection{Electromagnetic Characteristics of the Actuator}

In this section I establish the physical quantities and units that are used to describe and analyze the magnetic circuits, and discuss the magnetic circuit for the actuator used in the $10 \mathrm{kHz}$ FTS. The majority of this section develops equations that are used 


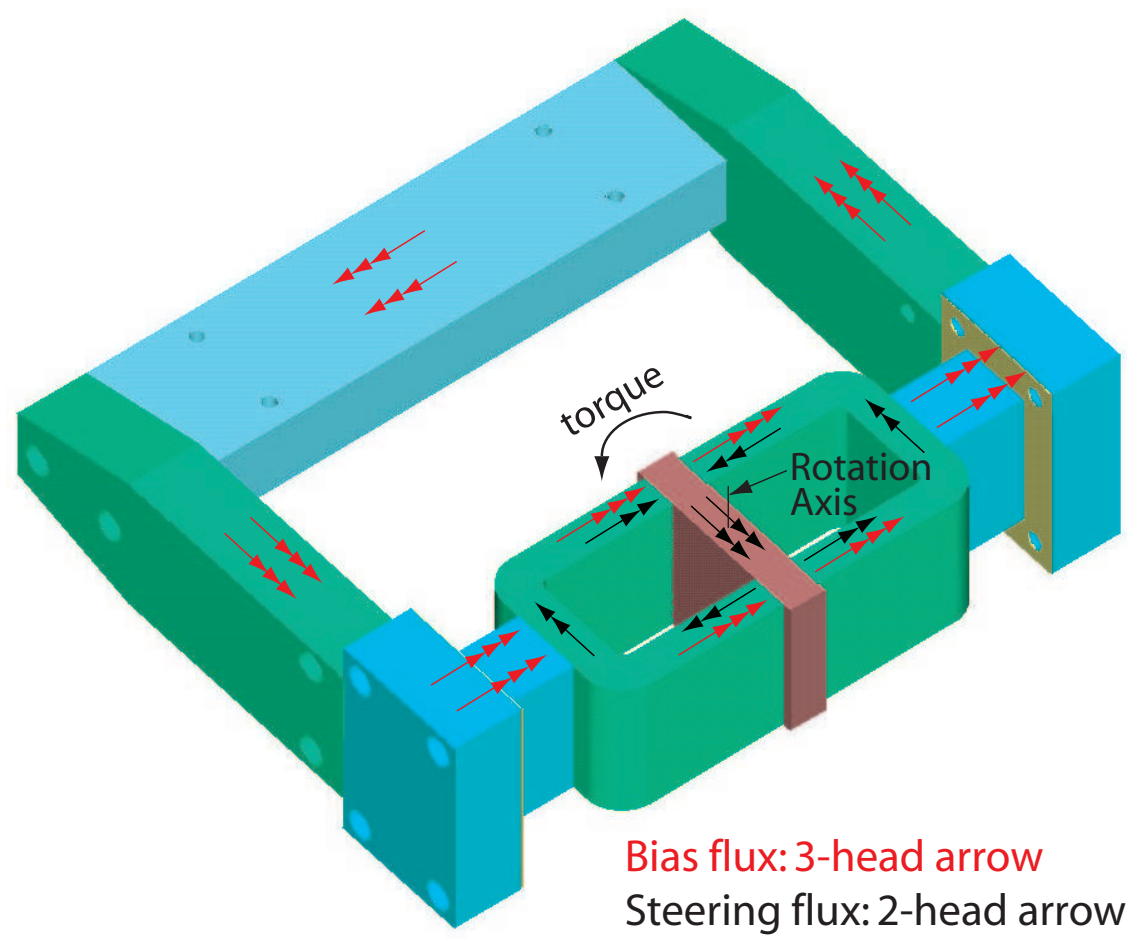

Figure 5-2: Model showing directions of magnetic fluxes for the $10 \mathrm{kHz}$ rotary fast tool servo.

to specify the electromagnetic characteristics for that actuator; specifically the motor constants for the rotary and linear degrees of freedom, and the back-EMF associated with both modes. The general approach can be used to design other normal-stress electromagnetic actuators having a different magnetic circuit topology.

\subsubsection{Physical Quantities and Units for Magnetic Circuits}

The SI system provides a consistent set of units for working with magnetic circuits, and is used throughout this thesis when dealing with electromagnetic topics.

- Magnetic flux density:

$[\mathrm{B}]=$ Weber $/$ meter $^{2}=$ Tesla $=$ volt $\cdot$ sec $/$ meter $^{2}$

- Magnetic field intensity:

$[\mathrm{H}]=\operatorname{amp} /$ meter 
- Magnetic permeability:

$[\mu]=$ Weber $/($ amp $\cdot$ meter $)=$ Tesla $\cdot$ meter $/$ amp $=$ Henry $/$ meter

- Magnetic permeability of free space:

$\mu_{0}=4 \pi \times 10^{-7}$ Weber $/($ amp $\cdot$ meter $)$

- Magnetomotive force:

$[\mathscr{F}]=$ amp·turns

- Magnetic flux:

$[\phi]=$ Weber

- Reluctance:

$[\mathscr{R}]=$ amp.turns/Weber

Note that the product of magnetic flux density $B$ and magnetic field intensity $H$ has the units of energy per unit volume: $[\mathrm{B}][\mathrm{H}]=\left(\right.$ volt.sec.amp $\left./ \mathrm{meter}^{3}\right)$. If we multiply that product by the operating frequency $\omega$ (radians/sec), then we obtain the magnetic power per unit volume.

\subsubsection{Magnetic Circuit for the Actuator}

Figure 5-3 is a top view sketch of the magnetic circuit for the actuator used in the $10 \mathrm{kHz}$ FTS, and shows the bias flux $B_{B}$ (triple-head arrows) and the torqueproducing steering fluxes $B_{12 T}$ and $B_{34 T}$ (double-head arrows). The central rectangular shape is the rotor core, the two C-shapes are the stator cores, and the large structure that appears to be pinching the pair of stator cores is the bias flux back-iron.

During standard operation of the actuator, the bias flux is constant and the steering fluxes are nominally sinusoidal. Section 5.5 presents a more detailed discussion on the nature of the flux in each region of the magnetic circuit. Figure 5-4 shows an alternate version of the magnetic circuit that uses a permanent magnet instead of a coil to produce the bias flux. Note that the permanent magnet creates a high 


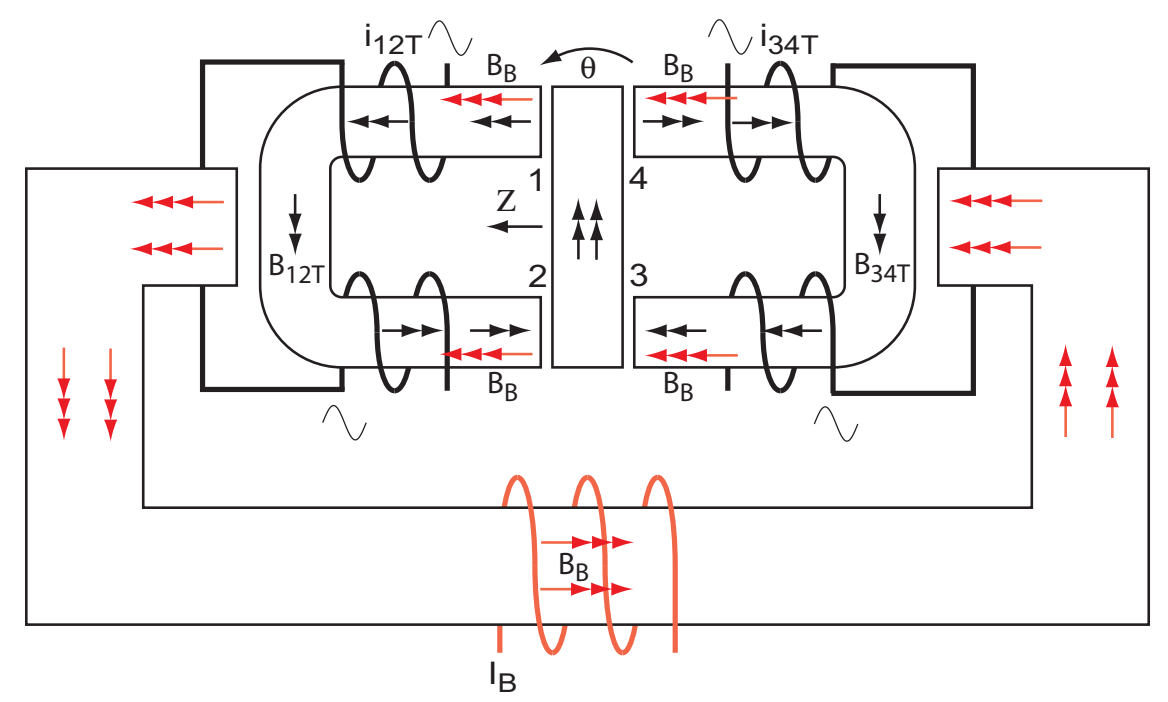

Figure 5-3: Sketch of the magnetic circuit showing torque generation for the actuator used in the $10 \mathrm{kHz}$ FTS.

reluctance path between the two stator cores. In Section 5.3.1 I discuss the need for that high reluctance path. I found that using a coil for the bias flux was advantageous for the prototype actuator used in the $10 \mathrm{kHz}$ FTS because it allowed me to easily adjust the bias flux during debugging and tuning of the control system.

\subsubsection{Torque is Proportional to the Common Current in the Steering Flux Coils}

Starting from first principles, this section shows that for small rotations of the rotor, the torque developed on the rotor is proportional to the common current flowing in the steering flux coils. The small-rotations assumption simplifies the problem and allows developing a convenient equation for the motor constant for the rotary mode of the actuator that is useful for design purposes. That motor constant has a linear dependence on the bias coil current, and an inverse quadratic dependence on the nominal air gaps between the rotor and stator poles. The problem is simplified further by initially neglecting the magnetomotive force (MMF) required to drive flux through the iron, then adding a correction factor for the MMF drop in the back-iron. In Section 5.2.4 I explore the effects of large rotations of the rotor, and show that the small-rotation motor constant is still valid if we add a negative torsional spring 


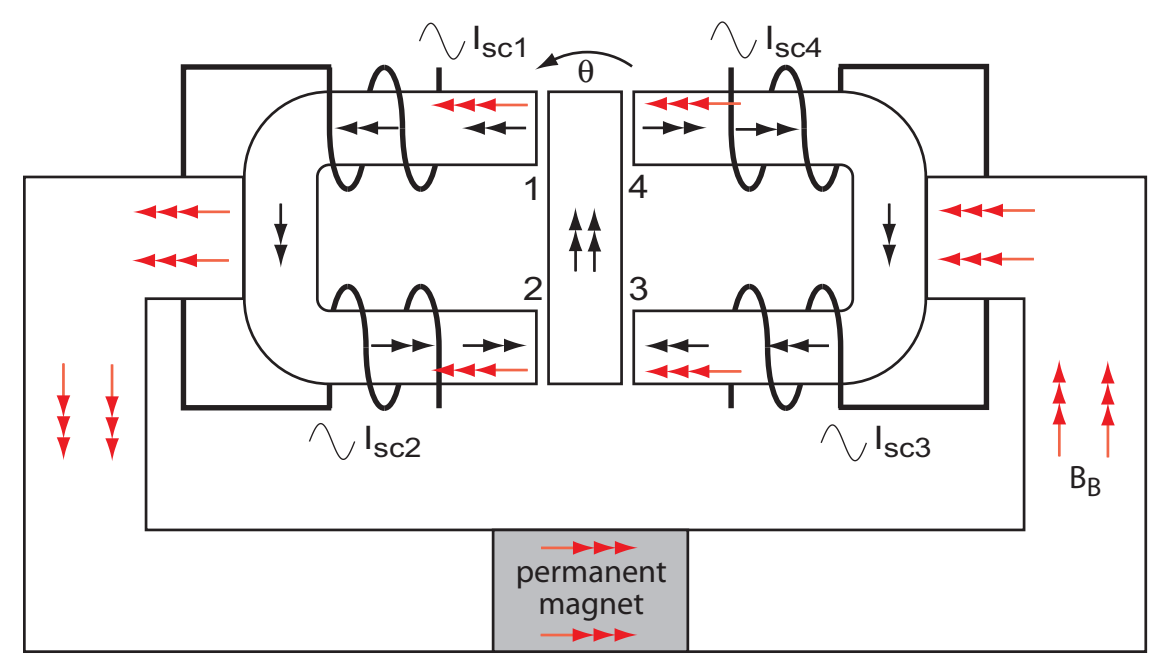

Figure 5-4: Sketch of an alternate magnetic circuit using a permanent magnet to produce the bias flux.

to the system.

Referring to Figure 5-3, for the actuator in the $10 \mathrm{kHz}$ FTS the following conditions and assumptions are established for the analysis in this section:

1. The bias flux splits evenly between poles 3 and 4 , and again at poles 1 and 2 . This is the case when the rotor is in its neutral position (zero rotation angle), and is assumed valid for small rotations of the rotor. This assumption is removed later for the large-rotation analysis in Section 5.2.4.

2. The coils on pole 1 and pole 2 are connected in series and therefore the steering currents $I_{S C 1}$ and $I_{S C 2}$ are equal and are referred to as the torque-producing current $i_{12 T}$ in coils 1 and 2. $N_{12}$ is equal to the sum of the turns in coils 1 and 2. Together, these two coils produce a torque-producing steering flux $B_{12 T}$.

3. The coils on pole 3 and pole 4 are connected in series and therefore the steering currents $I_{S C 3}$ and $I_{S C 4}$ are equal and are referred to as the torque-producing current $i_{34 T}$ in coils 3 and 4. $N_{34}$ is equal to the sum of the turns in coils 3 and 4 . Together, these two coils produce a torque-producing steering flux $B_{34 T}$.

4. The cross-sectional area $A_{p}$ of each of the four poles is equal. 
5. The air gaps between the rotor and the stator poles all have the same nominal length $g_{N}$ when the rotor is in its neutral position.

6. Rotation of the rotor causes the air gaps to assume a trapezoidal shape (when viewed from above, as in Figure 5-3). This analysis treats the air gaps as having a rectangular shape, with the length of the air gaps equal to the average length of the actual gap.

7. The bias coil has $N_{B}$ turns and carries current $I_{B}$ to produce a bias flux $B_{B}$.

8. The cross-sectional area $A_{B I}$ of the back-iron path (which carries the bias flux coil) is twice the cross-sectional area of the poles $A_{p}$. This provides a constant cross-section magnetic path for the bias flux when the rotor is in its neutral position.

9. The leakage of the steering flux into the back-iron is neglected. A discussion on the conditions needed to make this assumption valid is presented in Section 5.3.1.

10. Fringing magnetic fields at the air gaps are neglected.

11. To simplify this analysis, the magnetomotive force (MMF) required to drive flux through the iron is initially neglected. A discussion on the significance of the MMF drop in the iron is presented in Section 5.2.7, where it will be shown that the MMF required to drive bias flux through the back-iron is of the same order of magnitude as the MMF needed to drive the bias flux through the air gaps. A correction factor from Section 5.2.7 is included in the final result in this section to account for the MMF drop in the back-iron. Section 5.2.7 also shows that the MMF required to drive steering flux through the stator and rotor cores is only $10 \%$ of the MMF needed to drive the steering flux through the air gaps, so the reluctance of those cores is negligible for design purposes.

Starting with Ampere's law and an assumption of lumped electromagnetic quantities, we relate the integral of the magnetic field intensity $H$ along a path on a closed 
loop to the magnetomotive force $\mathscr{F}=N i$ in that loop:

$$
\mathscr{F}=N i=\oint H \cdot d l
$$

The magnetic field intensity $H$ and magnetic flux density $B$ in air are related by the magnetic permeability of free space $\mu_{0}$ via the linear constitutive equation:

$$
B=\mu_{0} H \quad \Rightarrow \quad H=\frac{B}{\mu_{0}}
$$

Neglecting fringing fields and the MMF drop in the iron, we use (5.1) and (5.2) to relate the magnetomotive force $(N i)$ and total length of the air gaps $L_{a i r}$ in a closed loop magnetic path having a constant cross-section to the magnetic flux density $B$ in the air gaps:

$$
N i=\frac{B}{\mu_{0}} L_{a i r} \Rightarrow B=\frac{\mu_{0} N i}{L_{a i r}}
$$

Using an argument based on tubes of magnetic flux, Hammond [67, page 146] deduces a useful relationship between the normal-direction magnetic pressure $f$ acting on a body and the normal-direction magnetic field intensity $H$ and magnetic flux density $B$ :

$$
f=\frac{1}{2} B H
$$

Substituting (5.2) into (5.4) gives:

$$
f=\frac{B^{2}}{2 \mu_{0}}
$$

Referring to Figure 5-3, the magnetic force acting on the rotor at each of the air gaps is approximated by assuming a uniform magnetic field in each gap. Using (5.5) and the cross-sectional area $A_{p}$ of the poles, the magnetic force $F$ acting on the rotor at each pole is: 


$$
F=f A_{p}=\frac{B^{2} A_{p}}{2 \mu_{0}}
$$

Letting $R$ be the distance from the center of rotation of the rotor to the center of each pole face in Figure 5-3, the total torque on the rotor about the $\theta$-axis from the magnetic pressure is:

$$
T_{\theta}=\frac{R A_{p}}{2 \mu_{0}}\left(B_{1}^{2}-B_{2}^{2}+B_{3}^{2}-B_{4}^{2}\right)
$$

At each air gap between the rotor and the stator poles, positive magnetic flux density is defined as pointing in the same direction as the surface-normal of the rotor at that air gap. With this definition, the net magnetic flux density acting on the rotor at each air gap is:

$$
\begin{gathered}
B_{1}=B_{B}+B_{12 T} \\
B_{2}=B_{B}-B_{12 T} \\
B_{3}=-B_{B}-B_{34 T} \\
B_{4}=-B_{B}+B_{34 T}
\end{gathered}
$$

Note that I have assumed in this case that the bias flux $B_{B}$ in each stator pole is constant, independent of rotation of the rotor. Squaring each equation (5.8), (5.9), (5.10), and (5.11), we obtain the square of the net magnetic flux density acting on the rotor at each air gap:

$$
\begin{aligned}
& B_{1}{ }^{2}=B_{B}{ }^{2}+B_{12 T}{ }^{2}+2 B_{B} B_{12 T} \\
& B_{2}{ }^{2}=B_{B}{ }^{2}+B_{12 T}{ }^{2}-2 B_{B} B_{12 T}
\end{aligned}
$$




$$
\begin{aligned}
& B_{3}{ }^{2}=B_{B}{ }^{2}+B_{34 T}{ }^{2}+2 B_{B} B_{34 T} \\
& B_{4}{ }^{2}=B_{B}{ }^{2}+B_{34 T}{ }^{2}-2 B_{B} B_{34 T}
\end{aligned}
$$

Substituting (5.12), (5.13), (5.14), and (5.15) into (5.7), note that the squared terms cancel. The total torque on the rotor from the magnetic flux produced by the bias coil and steering coils is thus:

$$
T_{\theta}=\frac{R A_{p}}{2 \mu_{0}}\left(4 B_{B}\right)\left(B_{12 T}+B_{34 T}\right)
$$

As a reference for discussions throughout this thesis regarding the as-designed peak torque for the actuator used in the $10 \mathrm{kHz}$ FTS, we evaluate (5.16) using consistent units and the designed bias flux density and peak steering flux density of $0.75 \mathrm{~T}$ :

$$
T_{\theta}=\frac{(0.0127)\left(1.61 \times 10^{-4}\right)}{2\left(4 \pi \times 10^{-7}\right)}[(4)(0.75)](0.75+0.75)=3.7 \mathrm{~N} \cdot \mathrm{m} ; \text { As-designed peak }
$$

Using (5.3), the magnetic flux densities due to the bias coil and the steering coils are:

$$
\begin{aligned}
& B_{B}=\frac{\mu_{0} N_{B} I_{B, a i r}}{2\left(g_{B I}+g_{N}\right)} \\
& B_{12 T}=\frac{\mu_{0} N_{12} i_{12 T}}{2 g_{N}} \\
& B_{34 T}=\frac{\mu_{0} N_{34} i_{34 T}}{2 g_{N}}
\end{aligned}
$$

Here $g_{N}$ is the nominal length of the rotor/stator air gaps when the rotor is in its neutral position, and $g_{B I}$ is the length of each intentional air gap between the back of the stator cores and the bias flux back-iron.

The bias current $I_{B, \text { air }}$ appearing in (5.17) is the portion of the total bias current 
that is driving the bias flux in the air gaps. Substituting (5.17), (5.18), and (5.19) into (5.16) produces the following linear relationship between the total torque on the rotor and the currents in the bias and steering coils:

$$
T_{\theta}=\frac{\mu_{0} R A_{p}}{2 g_{N}\left(g_{N}+g_{B I}\right)}\left(N_{B} I_{B, a i r}\right)\left(N_{12} i_{12 T}+N_{34} i_{34 T}\right)
$$

Nominally, the total number of turns on each stator core is equal, and so is the torque producing current flowing through both pairs of steering coils. Letting $N_{S C P}$ be the total number of turns for each steering coil pair, and $i_{T}$ be the torque-producing current flowing through the steering coils:

$$
\begin{gathered}
N_{12}=N_{34}=N_{S C P} \\
i_{12}=i_{34}=i_{T}
\end{gathered}
$$

Substituting (5.21) and (5.22) into (5.20) produces:

$$
T_{\theta}=\left[\frac{\mu_{0} R A_{p}}{g_{N}\left(g_{N}+g_{B I}\right)}\left(N_{B} I_{B, a i r}\right) N_{S C P}\right]\left(i_{T}\right)
$$

The analysis in Section 5.2.7 shows that the moderate value of magnetic permeability for the back-iron and the long magnetic circuit paths result in an appreciable MMF drop in the back-iron, while the high value of permeability for the rotor and stator cores results in a negligible MMF drop in those cores. Section 5.2.7 develops a concept referred to as an equivalent extra length of air $l_{e q, b i}$ for the back-iron in the magnetic path whose iron now has infinite magnetic permeability. Using (5.66) in Section 5.2.7, we can account for the MMF drop in the back-iron by including a term for $l_{e q, b i}$ in (5.23), producing the desired result for this section: 


$$
T_{\theta}=\left[\frac{\mu_{0} R A_{p}}{g_{N}\left(g_{N}+g_{B I}+\frac{l_{b i}}{2 \mu_{r, b i}}\right)}\left(N_{B} I_{B}\right) N_{S C P}\right]\left(i_{T}\right)
$$

The motor constant $K_{T}$ for the rotary degree of freedom for the actuator is the term in square brackets in (5.24), and $I_{B}$ is the total bias current needed to drive flux in the bias flux path. Note that this motor constant has a linear dependence on the bias coil current, and an inverse quadratic dependence on the nominal air gaps between the rotor and stator poles. The bias current $I_{B}$ appearing in (5.24) is the total bias current needed to drive flux through the air gaps and the back-iron. $l_{b i}$ is the length of the iron in the bias flux path and $\mu_{r, b i}$ is the relative permeability for that iron.

Using (5.24) with the as-built values in consistent units for the actuator used in the $10 \mathrm{kHz}$ FTS, the motor constant for the rotary degree of freedom is:

$$
\begin{array}{r}
K_{T}=\frac{\left(4 \pi \times 10^{-7}\right)(0.0127)\left(1.61 \times 10^{-4}\right)}{\left(50 \times 10^{-6}\right)\left(\left(50 \times 10^{-6}\right)+\left(520 \times 10^{-6}\right)+\frac{0.3}{2(500)}\right)}(560) I_{B}(20) \\
=0.66 I_{B} \mathrm{~N} \cdot \mathrm{m} / \mathrm{amp} ;\left[I_{B}\right]=\mathrm{amp}
\end{array}
$$

Extensive testing of the $10 \mathrm{kHz}$ FTS used two different values for the bias flux coil current: $I_{B}=0.545 \mathrm{~A}$ and $I_{B}=1.5 \mathrm{~A}$. The corresponding motor constants are $K_{T}=0.36 \mathrm{~N} \cdot \mathrm{m} / \mathrm{amp}$ and $K_{T}=0.99 \mathrm{~N} \cdot \mathrm{m} / \mathrm{amp}$, respectively.

Section 5.3.1 describes how the effective as-built length of the air gaps between the back of the stator cores and the back-iron is ten times larger than the as-designed length, and that a bias coil current of $1.9 \mathrm{~A}$ is needed to produce the desired bias flux density of $0.75 \mathrm{~T}$. Using (5.24) with a bias coil current of $1.9 \mathrm{~A}$ and the as-built values for the actuator, the motor constant for the rotary mode is $1.2 \mathrm{~N} \cdot \mathrm{m} / \mathrm{amp}$ when 


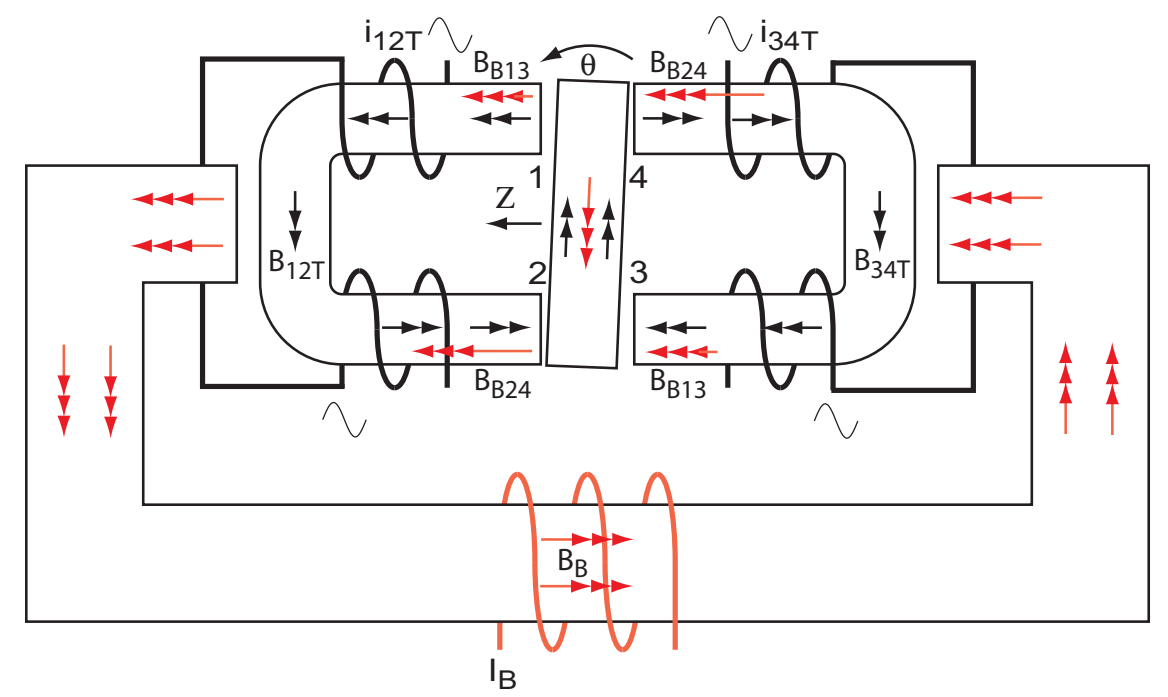

Figure 5-5: Sketch of the magnetic circuit showing torque generation for a large rotation angle of the rotor.

the bias flux density is $0.75 \mathrm{~T}$.

\subsubsection{Large Rotations of the Rotor}

Following the same analytical approach, conditions, and assumptions (not including the first one) that were used in Section 5.2.3, this section explores large rotations of the rotor, and shows that the small-rotation motor constant in (5.24) is valid for large rotations if we add the negative torsional spring to the system caused by the asymmetric distribution of bias flux attendant with a large rotation. I develop the relationship between the total torque on the rotor and the rotation angle of the rotor from its neutral position, and show that for angles small enough to allow using the approximation $\sin \theta \approx \theta$, the torque produced by the negative spring is a linear function of the rotation angle.

Referring to Figure 5-5, notice that the large clockwise rotation of the rotor causes the bias flux to preferentially flow through stator poles 2 and 4 , and thus we need to introduce independent bias flux variables at each pole face. By symmetry, we have $B_{B 13}$ as the bias flux at poles 1 and 3 , and $B_{B 24}$ at poles 2 and 4 . Without closed-loop control, this flux imbalance will cause the rotor to continue rotating clockwise until a hard stop is reached. This open-loop unstable nature of the rotor is discussed in 
Section 4.6.1. The net magnetic flux density acting on the rotor at each air gap is:

$$
\begin{gathered}
B_{1}=B_{B 13}+B_{12 T} \\
B_{2}=B_{B 24}-B_{12 T} \\
B_{3}=-B_{B 13}-B_{34 T} \\
B_{4}=-B_{B 24}+B_{34 T}
\end{gathered}
$$

Squaring each equation (5.25), (5.26), (5.27), and (5.28), we obtain the square of the net magnetic flux density acting on the rotor at each air gap:

$$
\begin{aligned}
& B_{1}{ }^{2}=B_{B 13}{ }^{2}+B_{12 T}{ }^{2}+2 B_{B 13} B_{12 T} \\
& B_{2}{ }^{2}=B_{B 24}{ }^{2}+B_{12 T}{ }^{2}-2 B_{B 24} B_{12 T} \\
& B_{3}{ }^{2}=B_{B 13}{ }^{2}+B_{34 T}{ }^{2}+2 B_{B 13} B_{34 T} \\
& B_{4}{ }^{2}=B_{B 24}{ }^{2}+B_{34 T}{ }^{2}-2 B_{B 24} B_{34 T}
\end{aligned}
$$

Substituting (5.29), (5.30), (5.31), and (5.32) into (5.7), the large-rotation total torque $T_{\theta, l}$ on the rotor from the magnetic flux produced by the bias coil and steering coils is:

$$
T_{\theta, l}=\frac{R A_{p}}{2 \mu_{0}}\left[2\left(B_{B 13}^{2}-B_{B 24}^{2}\right)+2\left(B_{12 T}+B_{34 T}\right)\left(B_{B 13}+B_{B 24}\right)\right]
$$

As a check, substituting $B_{B 13}=B_{B 24}=B_{B}$ into (5.33) produces the same result 
as was shown for small rotations in (5.16). Considering the stator core on the right in Figure 5-5, the sum of the fluxes in the two stator poles is equal to the flux in the back-iron:

$$
\phi_{B}=\phi_{B 13}+\phi_{B 24}
$$

Since the cross-sectional area of the back-iron $A_{B I}$ is twice the cross-sectional area $A_{P}$ of the stator poles:

$$
B_{B}\left(2 A_{p}\right)=B_{B 13} A_{p}+B_{B 24} A_{p} \quad \Rightarrow \quad B_{B 13}+B_{B 24}=2 B_{B}
$$

Substituting (5.35) into (5.33), and separating terms:

$$
T_{\theta, l}=\frac{R A_{p}}{2 \mu_{0}}\left[2\left(B_{B 13}^{2}-B_{B 24}^{2}\right)\right]+\frac{R A_{p}}{2 \mu_{0}}\left(4 B_{B}\right)\left(B_{12 T}+B_{34 T}\right)
$$

The second term on the right side of the equality in (5.36) is the torque on the rotor for small rotations, as shown in (5.16). The first term on the right side of the equality in (5.36) is the torque on the rotor from the asymmetric distribution of the bias flux, which reduces the total torque on the rotor (note the signs for the terms $B_{B 13}$ and $B_{B 24}$, and recall that $B_{B 24}>B_{B 13}$ in this analysis). This is consistent with the description in Section 4.6.1 that the asymmetric distribution of bias flux acts like a negative torsional spring. Therefore, the first term on the right side of the equality in (5.36) is referred to as the torque from the negative spring, $T_{\theta, n s}$. With this definition, we can use the small-rotation motor constant in (5.24) for large rotations if we add the negative torsional spring to the system. Using (5.36), we express the large-rotation total torque $T_{\theta, l}$ on the rotor as the sum of the torque for small rotations $T_{\theta}$ plus the torque from the negative spring, $T_{\theta, n s}$ :

$$
T_{\theta, l}=T_{\theta, n s}+T_{\theta}
$$

The torque for small rotations $T_{\theta}$ is given by (5.16), or alternatively by (5.24), and the torque from the negative spring, $T_{\theta, n s}$ is: 


$$
T_{\theta, n s}=\frac{R A_{p}}{2 \mu_{0}}\left[2\left(B_{B 13}^{2}-B_{B 24}^{2}\right)\right]
$$

The torque from the negative spring $T_{\theta, n s}$ is a function of the rotation angle of the rotor from its neutral position. For rotation angles small enough to allow using the approximation $\sin \theta \approx \theta$, to first-order the torque produced by the negative spring from the bias flux is a linear function of the rotation angle of the rotor from its neutral position. Before proving this assertion and developing a relationship between the torque from the negative spring and the rotor angle, I consider the following two limiting cases. When the rotor is in its neutral position, $B_{B 13}=B_{B 24}=B_{B}$, and the torque from the negative spring is $T_{\theta, n s}=0$, as expected. If the rotor is fully rotated clockwise so that the rotor contacts stator poles 2 and 4 , the torque from the negative spring, $T_{\theta, n s}$ is:

$$
\begin{aligned}
& B_{B 24} \rightarrow 2 B_{B} \\
& B_{B 13} \rightarrow 0
\end{aligned} \quad \Rightarrow \quad T_{\theta, n s} \rightarrow \frac{-R A_{p}}{2 \mu_{0}}\left(8 B_{B}^{2}\right)
$$

The magnitude of the limiting torque from the negative spring in (5.39) is the maximum torque that the actuator can produce, which can be seen by setting $B_{12 T}=$ $B_{34 T}=B_{B}$ in (5.16). It is worth noting that higher steering fluxes, $B_{12 T}=B_{34 T}>$ $B_{B}$, do not increase the torque beyond that value. For a full clockwise rotation of the rotor, because of the rotor angle, the air gaps at 2 and 4 do not completely close and some bias flux still flows through air gaps 1 and 3. Therefore, the torque from the negative spring does not reach the limiting value in (5.39) and the actuator can produce sufficient torque to pull the rotor off of initial contact with stator poles 2 and 4 . I demonstrate this last point with the $10 \mathrm{kHz}$ FTS while closing the loop for the control system.

Using the reluctance model shown in Figure 5-8 for the bias flux circuit, I now develop a relationship between the torque from the negative spring and the rotor angle. From Section 5.3.1, when the rotor is in its neutral position, the reluctance of each rotor/stator air gap is: 


$$
\mathscr{R}_{j}=\frac{g_{N}}{\mu_{0} A_{p}} ; \mathrm{j}=1,2,3,4 \text { (pole face number) }
$$

For rotation angles small enough to allow using the approximation $\sin \theta \approx \theta$, a positive (counterclockwise) rotation of the rotor changes the air gaps by $(R \theta)$, changing the reluctance of the rotor/stator air gaps to:

$$
\mathscr{R}_{1}=\mathscr{R}_{3}=\frac{g_{N}-R \theta}{\mu_{0} A_{p}} \quad ; \quad \mathscr{R}_{2}=\mathscr{R}_{4}=\frac{g_{N}+R \theta}{\mu_{0} A_{p}}
$$

Referring to the reluctance model in Figure 5-8, the magnetic-circuit equivalent to the current divider gives us the following bias fluxes in the stator poles:

$$
\phi_{B 1}=\phi_{B 3}=\phi_{B 13}=\frac{\mathscr{R}_{4}}{\mathscr{R}_{3}+\mathscr{R}_{4}} \phi_{B} \quad ; \quad \phi_{B 2}=\phi_{B 4}=\phi_{B 24}=\frac{\mathscr{R}_{3}}{\mathscr{R}_{3}+\mathscr{R}_{4}} \phi_{B}
$$

Substituting $\phi_{j}=B_{B j} A_{p}$ and $\phi_{B}=B_{B}\left(2 A_{p}\right)$ into (5.42), and cancelling terms:

$$
B_{B 1}=B_{B 3}=B_{B 13}=\frac{g_{N}+R \theta}{g_{N}} B_{B} \quad ; \quad B_{B 2}=B_{B 4}=B_{B 24}=\frac{g_{N}-R \theta}{g_{N}} B_{B}
$$

We perform the following checks, and obtain the expected results:

- Substitute $(R \theta)=0$ into (5.43) for the neutral position of the rotor, and obtain $B_{B 1}=B_{B 2}=B_{B 3}=B_{B 4}=B_{B}$.

- For a full clockwise rotation of the rotor to the stops, $(R \theta)=-g_{N}$, and (5.43) gives us: $B_{B 13}=0$ and $B_{B 24}=2 B_{B}$.

- For a full counterclockwise rotation of the rotor to the stops, $(R \theta)=g_{N}$, and (5.43) gives us: $B_{B 13}=2 B_{B}$ and $B_{B 24}=0$.

Substituting (5.43) into (5.38) gives the torque from the negative spring in terms of the bias flux density and the rotor angle: 


$$
T_{\theta, n s}=\frac{R A_{p}}{\mu_{0}}\left[\left(\frac{g_{N}+R \theta}{g_{N}} B_{B}\right)^{2}-\left(\frac{g_{N}-R \theta}{g_{N}} B_{B}\right)^{2}\right]=\left[\frac{4 R^{2} B_{B}^{2} A_{p}}{\mu_{0} g_{N}}\right] \theta
$$

Examination of (5.44) reveals that for rotation angles small enough to allow using the approximation $\sin \theta \approx \theta$, the torque produced by the negative spring from the bias flux is a linear function of the rotation angle of the rotor from its neutral position. At first glance, this may appear to be a surprising result because the change in the rotor/stator air gaps is a linear function of the rotor angle, and we expect the magnetic pressure on the rotor pole faces to vary with the square of the gap length. However, the symmetry of the geometry causes the square-terms of the rotation angle to cancel, leaving only the linear term of the rotation angle.

The final step is to substitute (5.44) and (5.24) into (5.37) to obtain the final form of the large-rotation total torque on the rotor:

$$
T_{\theta, l}=\left[\frac{4 R^{2} B_{B}^{2} A_{p}}{\mu_{0} g_{N}}\right] \theta+\left[\frac{\mu_{0} R A_{p}}{g_{N}\left(g_{N}+g_{B I}+\frac{l_{b i}}{2 \mu_{r, b i}}\right)}\left(N_{B} I_{B}\right) N_{S C P}\right]\left(i_{T}\right)
$$

The first term on the righthand-side of (5.45) is the torque on the rotor from the negative spring, and the quantity in square brackets in the first term is the spring constant. Note that the spring constant has a square-law dependence on the magnitude of the bias flux. The second term on the righthand-side of (5.45) is the small-rotation torque developed by the actuator, and is the same as derived earlier and given in (5.24). The quantity in square brackets in the second term is the motor constant for the rotary degree of freedom for the actuator. 


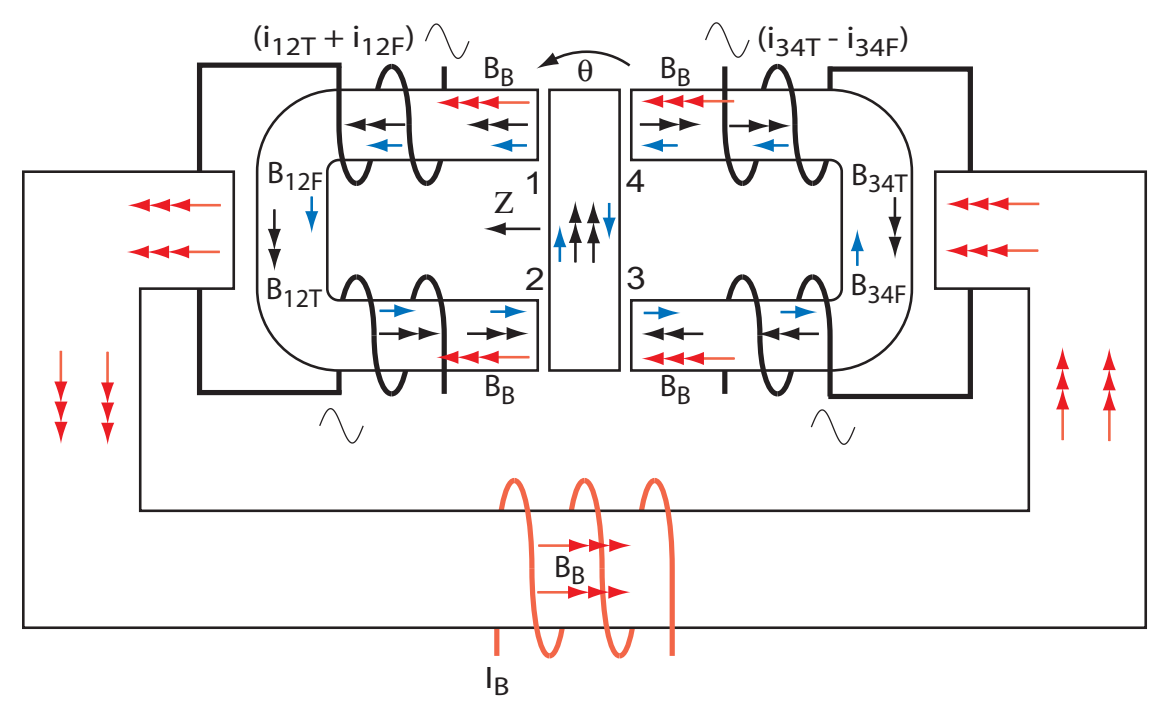

Figure 5-6: Sketch of the magnetic circuit showing torque and force generation for the actuator used in the $10 \mathrm{kHz}$ FTS.

\subsubsection{Force is Proportional to}

\section{the Differential Current in the Steering Flux Coils}

Following the same analytical approach, conditions, and assumptions that were used in Section 5.2.3, this section shows that the force developed on the rotor is proportional to the differential current flowing in the steering flux coils. I develop an equation for the motor constant for the linear degree of freedom for the actuator. That motor constant has a linear dependence on the torque-producing current in the steering coils and an inverse square dependence on the nominal air gaps between the rotor and stator poles. The torque-producing current in the steering coils acts as an operating point for the differential current in the steering coils. In Section 5.4 I discuss alternate magnetic circuit topologies that do not require that operating point and have fully decoupled torque and force characteristics.

Figure 5-6 is a sketch of the magnetic circuit for the actuator used in the $10 \mathrm{kHz}$ FTS, and shows the bias flux $B_{B}$ (triple-head arrows), the torque-producing steering fluxes $B_{12 T}$ and $B_{34 T}$ (double-head arrows), and the force-producing steering fluxes $B_{12 F}$ and $B_{34 f}$ (single-head arrows). The force acting on the rotor at each of the air gaps is approximated by assuming a uniform magnetic field in each gap. The magnetic force $F$ on the rotor at each pole is given by (5.6), and the net magnetic force on the rotor 
in the Z-direction is:

$$
F_{Z}=\frac{A_{p}}{2 \mu_{0}}\left(B_{1}^{2}+B_{2}^{2}-B_{3}^{2}-B_{4}^{2}\right)
$$

As before, positive magnetic flux density is defined as pointing in the same direction as the surface-normal of the rotor at that air gap. With this definition, the net magnetic flux density acting on the rotor at each air gap is:

$$
\begin{gathered}
B_{1}=B_{B}+B_{12 T}+B_{12 F} \\
B_{2}=B_{B}-B_{12 T}-B_{12 F} \\
B_{3}=-B_{B}-B_{34 T}+B_{34 F} \\
B_{4}=-B_{B}+B_{34 T}-B_{34 F}
\end{gathered}
$$

Squaring each equation (5.47), (5.48), (5.49), and (5.50), we obtain the square of the net magnetic flux density acting on the rotor at each air gap:

$$
\begin{aligned}
& B_{1}{ }^{2}=B_{B}{ }^{2}+B_{12 T}{ }^{2}+B_{12 F}{ }^{2}+2 B_{B} B_{12 T}+2 B_{B} B_{12 F}+2 B_{12 T} B_{12 F} \\
& B_{2}{ }^{2}=B_{B}{ }^{2}+B_{12 T}{ }^{2}+B_{12 F}{ }^{2}-2 B_{B} B_{12 T}-2 B_{B} B_{12 F}+2 B_{12 T} B_{12 F} \\
& B_{3}{ }^{2}=B_{B}{ }^{2}+B_{34 T}{ }^{2}+B_{34 F}{ }^{2}+2 B_{B} B_{34 T}-2 B_{B} B_{34 F}-2 B_{34 T} B_{34 F}
\end{aligned}
$$




$$
B_{4}^{2}=B_{B}^{2}+B_{34 T}^{2}+B_{34 F^{2}}-2 B_{B} B_{34 T}+2 B_{B} B_{34 F}-2 B_{34 T} B_{34 F}
$$

Substituting (5.51), (5.52), (5.53), and (5.54) into (5.46), note that the bias flux terms cancel. The total force on the rotor from the magnetic flux produced by the steering coils is:

$$
F_{Z}=\frac{A_{p}}{2 \mu_{0}}\left[2\left(B_{12 T}{ }^{2}-B_{34 T}{ }^{2}\right)+2\left(B_{12 F}{ }^{2}-B_{34 F^{2}}{ }^{2}\right)+4\left(B_{12 T} B_{12 F}+B_{34 T} B_{34 F}\right)\right]
$$

Nominally, the total number of turns on each stator core is equal, and so is the torque producing currents $i_{12 T}$ and $i_{34 T}$ flowing through the pairs of steering coils. Furthermore, the magnitude of the force-producing currents $i_{12 F}$ and $i_{34 F}$ flowing through the pairs of steering coils is equal. $N_{S C P}$ in $(5.21)$ is the total number of turns for each steering coil pair. Letting $i_{T}$ and $i_{F}$ be the torque-producing and force-producing currents flowing through the steering coils, respectively:

$$
\begin{aligned}
& i_{12 T}=i_{34 T}=i_{T} \\
& i_{12 F}=i_{34 F}=i_{F}
\end{aligned}
$$

Combining (5.21), (5.56), and (5.57) with (5.3):

$$
\begin{aligned}
& B_{12 T}=B_{34 T}=B_{T}=\frac{\mu_{0} N_{S C P} i_{T}}{2 g_{N}} \\
& B_{12 F}=B_{34 F}=B_{F}=\frac{\mu_{0} N_{S C P} i_{F}}{2 g_{N}}
\end{aligned}
$$

Progressing in steps, substituting the penultimate terms in (5.58) and (5.59) into (5.55): 


$$
F_{Z}=\frac{A_{p}}{2 \mu_{0}}\left[4\left(B_{T} B_{F}+B_{T} B_{F}\right)\right]=\frac{A_{p}}{2 \mu_{0}}\left(8 B_{T} B_{F}\right)
$$

Substituting the final terms in (5.58) and (5.59) into (5.60) produces the desired result for this section:

$$
F_{Z}=\left[\frac{\mu_{0} A_{p} N_{S C P}^{2}}{g_{N}^{2}}\left(i_{T}\right)\right]\left(i_{F}\right)
$$

The motor constant $K_{F}$ for the linear degree of freedom for the actuator is the term in square brackets in (5.61). Note that this motor constant has a linear dependence on the torque-producing current in the steering coils, and an inverse square dependence on the nominal air gaps between the rotor and stator poles. Also note that the linear degree of freedom for the actuator does not require the bias flux.

Using (5.61) with the as-built values in consistent units for the actuator used in the $10 \mathrm{kHz}$ FTS, the motor constant for the linear degree of freedom is:

$$
K_{F}=\frac{\left(4 \pi \times 10^{-7}\right)\left(1.61 \times 10^{-4}\right)(20)^{2}}{\left(50 \times 10^{-6}\right)^{2}} i_{T}=32 i_{T} \mathrm{~N} / \operatorname{amp} ;\left[i_{T}\right]=\operatorname{amp}
$$

\subsubsection{Back-EMF Produced by the Rotary and Linear Modes}

The rotary and linear modes of the actuator can be thought of as electrical-mechanical transformers. The intended function is to apply an appropriate voltage to the terminals of the steering coils to produce rotary or linear motions of the rotor. Associated with those motions is a speed-dependent generator action that induces a voltage at the terminals of the steering coils that tends to reduce the magnitude of the applied voltage. The induced voltage is referred to as a back electromotive-force (EMF). Analytical expressions relating the rotary and linear motions of the rotor to their respective back-EMF can be derived using Faraday's law to relate the time-rate of change in magnetic flux linked by the steering coils to the motions of the rotor. In this section I do not pursue those equations. Instead, I provide a qualitative explanation on the underlying sources of the back-EMF for each mode of the actuator, and use 
the reciprocity principle for an electrical-mechanical transformer to deduce that the back-EMF constants for the rotary and linear modes are equal to the motor constants for each mode.

\section{Rotary Mode Back-EMF}

Referring to Figures 4-57 and 4-58, note that rotation of the rotor causes a change in the distribution of the bias flux in each stator core pole. Therefore, related to the angular velocity of the rotor is a time-rate of change in flux linked by each steering coil that induces an open-circuit voltage in the coil. By Lenz's law, the induced voltage is in a direction that would cause the coil to produce a magnetic field that opposes the change in flux caused by rotation of the rotor.

\section{Linear Mode Back-EMF}

Referring to Figures 4-57 and 4-60, note that the front/back translation of the rotor causes a change in the magnitude of the steering flux in each stator core. Therefore, related to the linear velocity of the rotor is a time-rate of change in flux linked by each steering coil. Again by Lenz's law, the induced voltage is in a direction that would cause the coil to produce a magnetic field that opposes the change in flux caused by translation of the rotor.

\subsubsection{MMF Drop in the Iron}

In this section I take into account the magnetomotive force required to drive flux through the iron in the magnetic circuits. For a constant cross-section closed-magnetic path consisting of iron and air, we use Ampere's law to relate the magnetomotive force $\mathscr{F}=N i$ driving flux in that loop to the magnetic field intensity $H$ in each material and the total length $l$ of each material in the path. The subscripts $f e$ and air are used in this section to denote iron and air, respectively:

$$
\mathscr{F}=N i=\oint H \cdot d l=H_{f e} l_{f e}+H_{a i r} l_{\text {air }}
$$


The magnetic permeability of a material $\mu$ can be expressed as the product of its relative permeability $\mu_{r}$ and the magnetic permeability of free space $\mu_{0}$ :

$$
\mu=\mu_{r} \mu_{0}
$$

The magnetic field intensity $H$ and magnetic flux density $B$ in a material are related by the magnetic permeability of that material $\mu$ via the linear constitutive equation:

$$
B=\mu H \quad \Rightarrow \quad H=\frac{B}{\mu}=\frac{B}{\mu_{r} \mu_{0}}
$$

Denoting the length and relative permeability of the back-iron as $l_{f e}$ and $\mu_{r, f e}$, respectively, and substituting (5.64) into (5.62):

$$
N i=\frac{B}{\left(\mu_{r, f e}\right)\left(\mu_{0}\right)} l_{f e}+\frac{B}{\mu_{0}} l_{a i r}=\frac{B}{\mu_{0}}\left[\left(\frac{l_{f e}}{\mu_{r, f e}}\right)+l_{\text {air }}\right]
$$

The term $\left(l_{f e} / \mu_{r f e}\right)$ in $(5.65)$ can be considered as an equivalent extra length of air $l_{e q, f e}$ for the iron in the magnetic path whose iron now has infinite magnetic permeability:

$$
l_{e q, f e}=\left(\frac{l_{f e}}{\mu_{r, f e}}\right)
$$

The term in square brackets in (5.65) is the net effective length of air $l_{\text {eff,air }}$ in the magnetic path whose iron now has infinite magnetic permeability:

$$
l_{e f f, a i r}=\left[\left(\frac{l_{f e}}{\mu_{r, f e}}\right)+l_{a i r}\right]
$$

The ratio of $l_{e q, f e}$ divided by $l_{e f f, a i r}$ provides a measure of the significance of the MMF needed to drive flux through the iron relative to the MMF needed to drive flux through the entire magnetic path.

The magnetic permeability $\mu$ of a material is a function of the magnetic flux density $B$ in the material. For design purposes, I consider a maximum flux density of $1.5 \mathrm{~T}$ to obtain the following lower-bound values for the relative permeability for 
the iron components used in the magnetic circuit for the $10 \mathrm{kHz}$ FTS: $\mu_{r}=10,000$ for the rotor and stator cores, and $\mu_{r}=500$ for the back-iron (see Section 5.5).

\section{MMF Drop in the Rotor and Stator Cores}

Referring to Figure 5-3, there are two steering flux paths in the magnetic circuit. One is through the left stator core and the rotor, and the other is through the right stator core and the rotor. For reasons that will be shown using the reluctance models in Section 5.3.1, the steering flux does not substantially flow from one stator core to the other through the back-iron or through the rotor core. The cross-sectional area is substantially uniform along each steering flux path, and is independent of the rotor rotation angle. The length of the nominal air gaps between the rotor and stator cores is $50 \mu \mathrm{m}$, which leads to a total actual length of air in each steering flux path of $l_{\text {air }, s f}=100 \mu \mathrm{m}$.

The total length of iron $l_{f e, b i}$ for each steering flux path is approximately $0.1 \mathrm{~m}$, with a relative magnetic permeability $\mu_{r, s f}=10,000$. Using (5.66), the equivalent extra length of air from the iron in each steering flux path is $l_{e q, s f}=10 \mu \mathrm{m}$, leading to a net effective length of air in each steering flux path of $l_{e f f, a i r}=110 \mu \mathrm{m}$. The ratio of $l_{e q, b i}$ divided by $l_{e f f, a i r}$ indicates that only $9 \%$ of the MMF drop in each steering flux path occurs in the cores, which is negligible for design purposes.

\section{MMF Drop in the Back-Iron}

Referring to Figure 5-3, the bias flux path consists of the stainless steel back-iron 410, 416, or 430F - and the stator cores and rotor core. Since the cross-sectional area of the back-iron is twice that of the stator poles, the cross-sectional area is substantially uniform along the entire bias flux path when the rotor is in its neutral position. During the design phase for the hybrid actuator the lengths of the air gaps between the rotor and stator cores, and between the stator cores and the back-iron, were set at $50 \mu \mathrm{m}$. This leads to a total actual length of air in the as-designed bias flux path of $l_{\text {air }, b f}=200 \mu \mathrm{m}$.

The total length of iron $l_{f e, b f}$ for the bias flux path is approximately $0.4 \mathrm{~m}$, and 
approximately $3 / 4$ of that length is the stainless steel back-iron. Since the magnetic permeability of the rotor and stator cores is approximately twenty times greater than that of the back-iron, the MMF needed to drive the bias flux through the cores can be neglected for design purposes. Therefore, the length of the back-iron $l_{f e, b i}$ is approximately $0.3 \mathrm{~m}$, with a relative magnetic permeability $\mu_{r, b f}=500$. Using (5.66), the equivalent extra length of air from the iron in the bias flux path is $l_{e q, b f}=600 \mu \mathrm{m}$, leading to a net effective length of air in the as-designed bias flux path of $l_{\text {eff,air }}=800 \mu \mathrm{m}$. The ratio of $l_{e q, b f}$ divided by the as-designed $l_{\text {eff,air }}$ indicates that $75 \%$ of the MMF drop in the as-designed bias flux path occurs in the back-iron. Therefore the bias coil current $I_{B, \text { air }}$ needed to drive bias flux in the air gaps only is $25 \%$ of the total current needed for the as-designed bias flux path.

Section 5.3.1 shows that the effective length of the as-built air gaps between the stator cores and the back-iron are considerably larger than the design value; $520 \mu \mathrm{m}$ versus $50 \mu \mathrm{m}$ for each air gap. The equivalent extra length of air from the back-iron is still $l_{e q, b f}=600 \mu \mathrm{m}$, but now the net effective length of air in the as-built bias flux

path is $l_{e f f, a i r}=1740 \mu \mathrm{m}$. The ratio of $l_{e q, b f}$ divided by the as-built $l_{e f f, a i r}$ indicates that $34 \%$ of the MMF drop in the as-built bias flux path occurs in the back-iron. Therefore the bias coil current $I_{B \text {,air }}$ needed to drive bias flux in the air gaps only is $66 \%$ of the total current needed for the as-built bias flux path.

\subsection{Electromagnetic Design of the Actuator}

In this section I develop a set of design equations that are used to establish the electromagnetic specifications for the actuator used in the $10 \mathrm{kHz}$ FTS. The general approach can be used to derive specifications for other normal-stress electromagnetic actuators having a different magnetic circuit topology. For our actuator, designing for a peak flux density of $1.5 \mathrm{~T}$ means that the bias flux and peak steering flux both need to be 0.75 T. Starting with a reluctance model for the magnetic circuit, I determine the magnetomotive force that each coil needs to produce to achieve the desired flux density in the rotor/stator air gaps. I then determine the inductance seen 
at the terminals of the actuator. Establishing allowable currents and the required coil window area based on thermal arguments allows sizing the coils. This section concludes by considering the trade-off of voltage versus current when choosing the number of turns in the steering flux coils, and includes a discussion on Litz wire.

\subsubsection{Reluctance Model}

The reluctance model for the actuator provides a convenient analytical framework to quantify the magnetic fluxes flowing through the magnetic circuit and determine the magnetomotive forces needed to drive the bias flux and steering flux. It also illustrates how the air gaps between the back-iron and the stator cores, and the low permeability of the back-iron compared to the rotor and stator cores, act to magnetically decouple the two stator cores. Using separate power amplifiers for each stator half allows simultaneously controlling the rotary degree of freedom and the linear degree of freedom of the actuator. By magnetically decoupling the two stator cores, we reduce the complexity of the actuator electrical dynamics from a MIMO system to a SISO system, which allows using loop-shaping techniques with classical control theory to design the control systems. Since our primary goal for the hybrid actuator was to produce a high torque for the $10 \mathrm{kHz}$ FTS, this section considers only the toque mode for the actuator.

For the reader who is not already familiar with the concept of reluctance models for analyzing a magnetic circuit, Hubert [79] and Fitzgerald, Kingsley, and Umans [56] provide excellent descriptions and worked-out examples. The magnetomotive force $(\mathrm{MMF}) \mathscr{F}$, flux $\phi$, and reluctance $\mathscr{R}$ are used in a lumped-parameter model of a magnetic circuit as analogues to voltage, current, and resistance, respectively. This allows using well-established electrical circuit analysis techniques for quantifying the MMF needed and for understanding the flow of magnetic flux in the circuit.

For a constant cross-section, homogeneous-material portion of a closed-loop magnetic path, the MMF $\mathscr{F}_{1}$, flux $\phi_{1}$, and reluctance $\mathscr{R}_{1}$ are related by a magnetic circuit equivalent to Ohm's law: 


$$
\mathscr{F}_{1}=\mathscr{R}_{1} \phi_{1} \quad \Rightarrow \quad \mathscr{R}_{1}=\frac{\mathscr{F}_{1}}{\phi_{1}}
$$

We use Ampere's law to relate the integral of the magnetic field intensity $H_{1}$ in that portion of the magnetic path, which has length $L_{1}$, to the magnetomotive force $\mathscr{F}_{1}$ required to drive flux through it:

$$
\mathscr{F}=\oint H \cdot d l \quad \Rightarrow \quad \mathscr{F}_{1}=H_{1} L_{1}
$$

Assuming a constant cross-sectional area $A_{1}$ for that portion of the magnetic path, we relate the magnetic flux $\phi_{1}$ to the magnetic flux density $B_{1}$ :

$$
\phi_{1}=B_{1} A_{1}
$$

Substituting (5.69) and (5.70) into (5.68), and using (5.64), we obtain the following expression for the reluctance $\mathscr{R}_{1}$ of a single-material portion of a magnetic path having length $L_{1}$, constant cross-sectional area $A_{1}$, and magnetic permeability $\mu_{1}$ :

$$
\mathscr{R}_{1}=\frac{H_{1} L_{1}}{B_{1} A_{1}}=\frac{H_{1} L_{1}}{\mu_{1} H_{1} A_{1}}=\frac{L_{1}}{\mu_{1} A_{1}}
$$

Now we are ready to create the lumped-parameter reluctance model of the magnetic circuit for the actuator used in the $10 \mathrm{kHz}$ FTS. In Section 5.2.7 I show that the MMF needed to drive flux through the back-iron was appreciable, and that the MMF needed to drive flux through the rotor and stator cores was negligible (for design purposes).

Figure 5-7 shows the reluctance model for the magnetic circuit shown in Figure 53 for small rotations of the rotor. Each of the steering coils acts as an AC source of MMF: $\left(N_{j} i_{j T}\right) ; j=1,2,3,4$. The subscript $T$ refers to a torque-producing quantity. The bias coil acts as a DC source of MMF: $\left(N_{B} I_{B}\right)$. The reluctance of the air gaps between the rotor and stator poles is denoted by $\mathscr{R}_{j} ; j=1,2,3,4$. The reluctance of the air gaps between the stator cores and the back-iron is denoted by $\mathscr{R}_{\text {bimn }} ; m n=$ 12,34 , and the reluctance of the back-iron is denoted by $\mathscr{R}_{b i}$. Using (5.71) and the 


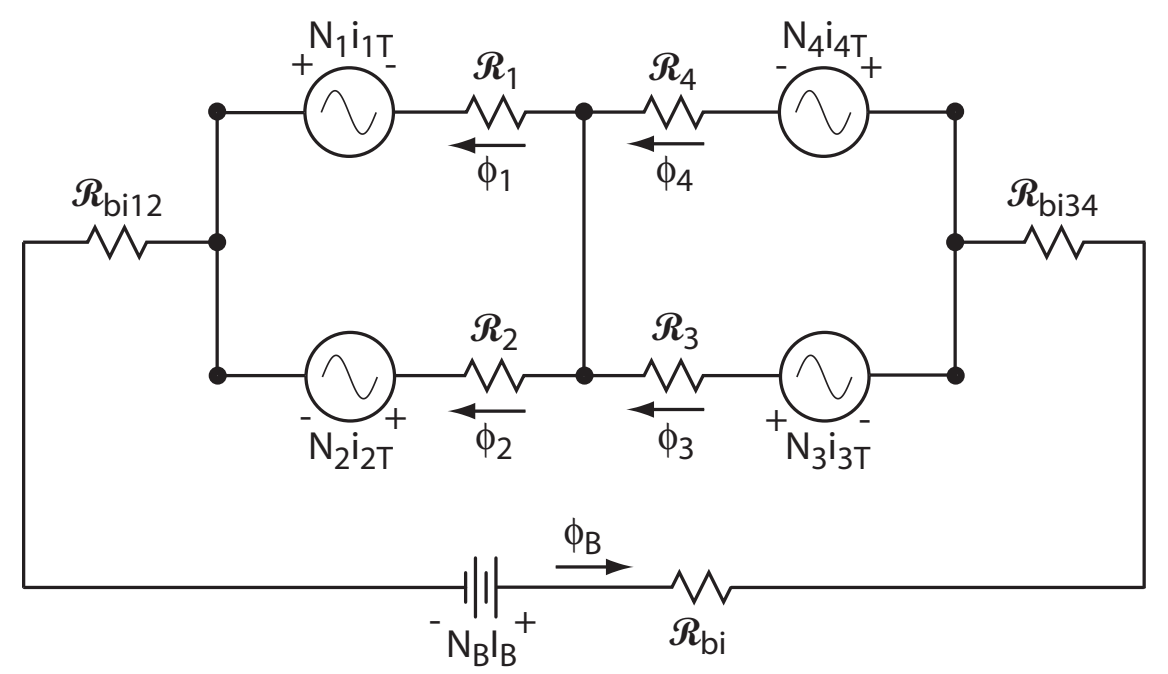

Figure 5-7: Reluctance model of the magnetic circuit for the actuator in the $10 \mathrm{kHz}$ rotary fast tool servo for small rotations of the rotor, showing the flux in the four rotor/stator air gaps due to the bias and steering fluxes.

geometry for the magnetic circuit shown in Figure 5-3:

$$
\mathscr{R}_{j}=\frac{g_{N}}{\mu_{0} A_{p}} \quad ; \quad \mathscr{R}_{b i m n}=\frac{g_{B I}}{\mu_{0}\left(2 A_{p}\right)} \quad ; \quad \mathscr{R}_{b i}=\frac{l_{f e}}{\mu_{r, f e}\left(2 A_{p}\right)}
$$

Where $g_{N}$ is the nominal length of the rotor/stator air gaps, $g_{B I}$ is the length of the air gap between the back of a stator core and the back-iron, $A_{p}$ is the crosssectional area of the stator poles, $l_{f e}$ is the length of the bias flux path through the back-iron, and $\mu_{r, f e}$ is the relative permeability of the back-iron. If a more detailed analysis of the magnetic circuit were needed, the reluctance model can be refined to account for the MMF needed to drive flux through the rotor and stator cores by including additional $\mathscr{R}$ elements for those portions of the magnetic paths.

Using (5.72) and the SI units described in Section 5.2.1, we obtain the following reluctance values for the actuator used in the $10 \mathrm{kHz}$ FTS. The reluctance $\mathscr{R}_{j}$ of the rotor/stator air gaps when the rotor is in its neutral position (centered rotation angle, and centered translation) is: 


$$
\mathscr{R}_{j}=\frac{50 \times 10^{-6}}{\left(4 \pi \times 10^{-7}\right)\left(1.61 \times 10^{-4}\right)}=2.5 \times 10^{5} \text { amp } \cdot \text { turns } / \mathrm{Wb} ;
$$

The reluctance $\mathscr{R}_{b i m n}$ of the air gap between the back of a stator core and the bias flux back-iron is:

$$
\begin{gathered}
\mathscr{R}_{\text {bimn }}=\frac{50 \times 10^{-6}}{\left(4 \pi \times 10^{-7}\right)\left[(2)\left(1.61 \times 10^{-4}\right)\right]}=1.2 \times 10^{5} \text { amp.turns/Wb; as-designed } \\
\mathscr{R}_{\text {bimn }}=\frac{520 \times 10^{-6}}{\left(4 \pi \times 10^{-7}\right)\left[(2)\left(1.61 \times 10^{-4}\right)\right]}=13 \times 10^{5} \text { amp.turns } / \mathrm{Wb} ; \text { as-built }
\end{gathered}
$$

Note that due to geometric form errors in the as-fabricated stator cores, and imperfect closure of the joints between the components in the back-iron, the effective length of the as-built air gaps between the stator cores and the back-iron is ten times larger than the as-designed value. The as-built length of those air gaps is calculated in the next subsection, which discusses the MMF needed to produce the bias flux.

The reluctance $\mathscr{R}_{b i}$ of the bias flux path in the back-iron is:

$$
\mathscr{R}_{b i}=\frac{0.3}{(500)\left(4 \pi \times 10^{-7}\right)\left[(2)\left(1.61 \times 10^{-4}\right)\right]}=15 \times 10^{5} \text { amp.turns } / \mathrm{Wb}
$$

The net flux in the rotor-stator air gaps $\left(\phi_{i} ; i=1,2,3,4\right)$ is found by utilizing the principle of superposition for the linear magnetic circuit shown in Figure 5-7. Figure 5-8 shows the flux in the rotor-stator air gaps $\left(\phi_{j B} ; j=1,2,3,4\right)$ due to the MMF $\left(N_{B} I_{B}\right)$ produced by the bias coil. Figure 5-9 shows the flux in the rotor-stator 
air gaps $\left(\phi_{j T} ; j=1,2,3,4\right)$ due to the $\operatorname{MMF}\left(N_{j} i_{j T} ; j=1,2,3,4\right)$ produced by the steering coils. Our design goal for the $10 \mathrm{kHz}$ FTS was to produce a maximum flux density of $1.5 \mathrm{~T}$ in the rotor-stator air gaps, which requires a maximum flux density of $0.75 \mathrm{~T}$ from both the bias flux and the steering flux.

\section{MMF Needed for 0.75 T Bias Flux}

The magnetic circuit shown in Figure 5-8 is equivalent to a single reluctance $\mathscr{R}_{\text {bias }}$ driven by the MMF $\left(N_{B} I_{B}\right)$ produced by the bias coil. For small rotations of the rotor about its neutral position, $\mathscr{R}_{1}=\mathscr{R}_{2}=\mathscr{R}_{3}=\mathscr{R}_{4}$, and there is nominally no bias flux flowing through the long axis of the rotor. Therefore:

$$
\mathscr{R}_{\text {bias }}=\mathscr{R}_{j}+2 \mathscr{R}_{\text {bimn }}+\mathscr{R}_{\text {bi }}
$$

Setting the cross-sectional area of the back-iron to twice the cross-section area $A_{p}$ of the stator poles, we use (5.68) to determine the MMF $\mathscr{F}_{b}$ that the bias coil needs to produce:

$$
\begin{gathered}
\mathscr{F}_{b}=N_{B} I_{B}=\mathscr{R}_{\text {bias }} B_{B}\left(2 A_{p}\right) \\
N_{B} I_{B}=\left[(2.5+2(1.2)+15) \times 10^{5}\right](0.75)\left[(2)\left(1.61 \times 10^{-4}\right)\right] \\
=480 \text { amp.turns ; as-designed }
\end{gathered}
$$

Section 4.10.4 describes that when demonstrating a peak tool tip acceleration of $400 \mathrm{~g}$ at $5 \mathrm{kHz}$, the actuator produced a peak torque of $2.5 \mathrm{~N} \cdot \mathrm{m}$, which corresponds to a bias flux density of $0.6 \mathrm{~T}$ and a peak steering flux density of $0.6 \mathrm{~T}$. Using (5.72), (5.73), and (5.74) with the operating conditions during the $400 \mathrm{~g}$ demonstration and the already-known as-built values for the actuator, we solve for the length of the as-built air gaps between the back of the stator cores and the back-iron: 


$$
\begin{aligned}
& 560(1.5)=840 \text { amp.turns } \\
= & {\left[\left(2.5+2\left(\frac{g_{B I}}{\left(4 \pi \times 10^{-7}\right)(2)\left(1.61 \times 10^{-4}\right)}\right)+15\right) \times 10^{5}\right](0.60)\left[2\left(1.61 \times 10^{-4}\right)\right] } \\
& \Rightarrow g_{B I}=520 \mu \mathrm{m} ; \text { as-built }
\end{aligned}
$$

To produce the desired $0.75 \mathrm{~T}$ bias flux density with the as-built air gaps between the back of the stator cores and the back-iron, the bias coil needs to produce an MMF of:

$$
\begin{array}{r}
N_{B} I_{B}=\left[(2.5+2(13)+15) \times 10^{5}\right](0.75)\left[(2)\left(1.61 \times 10^{-4}\right)\right] \\
=1040 \text { amp.turns ; as-built }
\end{array}
$$

The as-built bias flux coil has 560 turns. Therefore, a current of $1.9 \mathrm{~A}$ is needed to produce the desired $0.75 \mathrm{~T}$ bias flux density. It is interesting to note that during the early portion of the design phase I sized the bias coil based on a conservative approximation of treating all of the iron in the $0.4 \mathrm{~m}$ bias flux path as having the moderate permeability of $\mu_{r}=500$ for stainless steel. This led to a specification for the bias coil of $N_{B} I_{B}=600$ amp-turns, which is not as far off from the as-built requirement of $1040 \mathrm{amp}$ turns as is the as-designed requirement of $480 \mathrm{amp}$ turns. The ratios are $58 \%$ versus $46 \%$, respectively.

\section{MMF Needed for $0.75 \mathrm{~T}$ Steering Flux}

Referring to the magnetic circuit shown in Figure 5-9, consider the steering flux $\phi_{12 T}$ circulating in the stator core on the left. Since the reluctance of the rotor core is negligible (for design purposes), the steering flux $\phi_{12 T}$ does not cross over the rotor to the stator core on the right. The same argument is true for the steering flux $\phi_{34 T}$ circulating in the stator core on the right. 


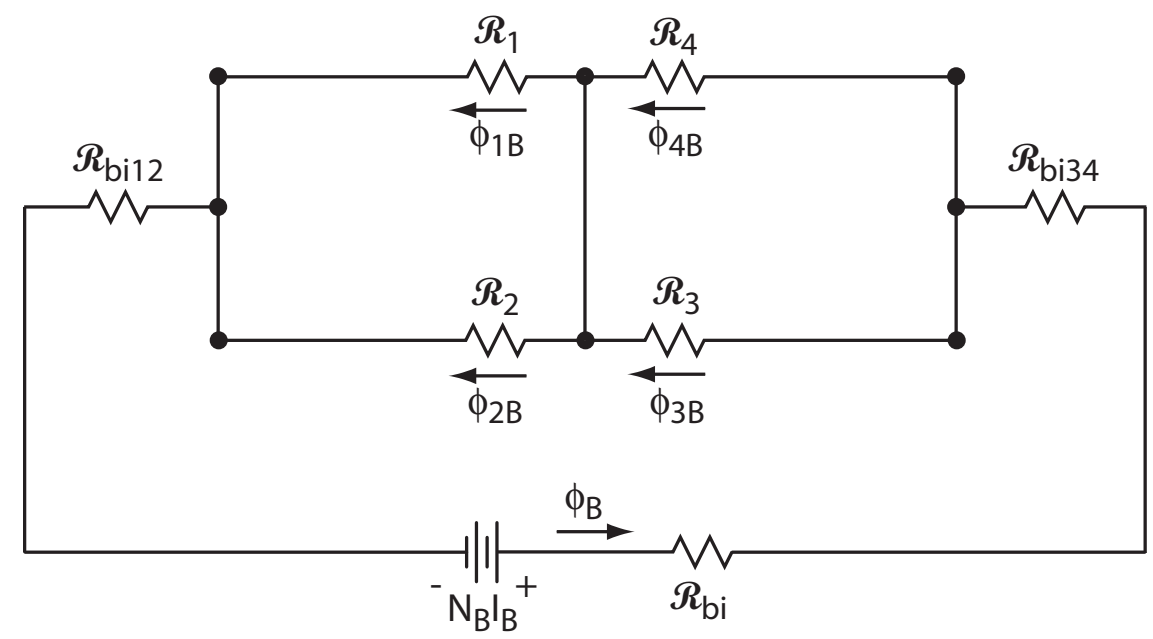

Figure 5-8: Reluctance model of the magnetic circuit for actuator in the the $10 \mathrm{kHz}$ rotary fast tool servo for small rotations of the rotor, showing the flux in the four rotor/stator air gaps due to the bias flux.

Consider the steering flux produced by the coil $\left(N_{1} i_{1}\right)$. The portion that contributes to the steering flux $\phi_{12 T}$ that circulates around the loop formed by the stator core on the left and the rotor core sees a total reluctance of $\left(2 \mathscr{R}_{j}\right)$. The portion $\phi_{b i T}$ that circulates through the back-iron, the stator core on the right, and the rotor core sees a total reluctance of $\left[2 \mathscr{R}_{b i m n}+\mathscr{R}_{b i}+(1.5) \mathscr{R}_{j}\right]$. The ratio of the second total reluctance divided by the first one is 4.2 for the as-designed bias flux path, and 9.0 for the as-built path. Therefore, for design purposes the steering coil flux $\phi_{b i T}$ in the back-iron is neglected. Experimental results with the $10 \mathrm{kHz}$ FTS indicate a lack of significant inductive coupling between the coil pairs on the two stator cores, supporting the validity of this assumption.

Based on the forgoing argument and for small rotations of the rotor, I set the following conditions on the steering coils and use (5.68) to determine the MMF $\mathscr{F}_{s c}$ that each steering coil needs to produce:

$$
\begin{gathered}
N_{j}=N_{s c} \quad ; \quad i_{j T}=i_{s c} \quad ; \quad j=1,2,3,4 \\
\mathscr{F}_{s c}=N_{s c} i_{s c}=\mathscr{R}_{j} B_{m n T} A_{p} \quad ; \quad m n=12,34
\end{gathered}
$$




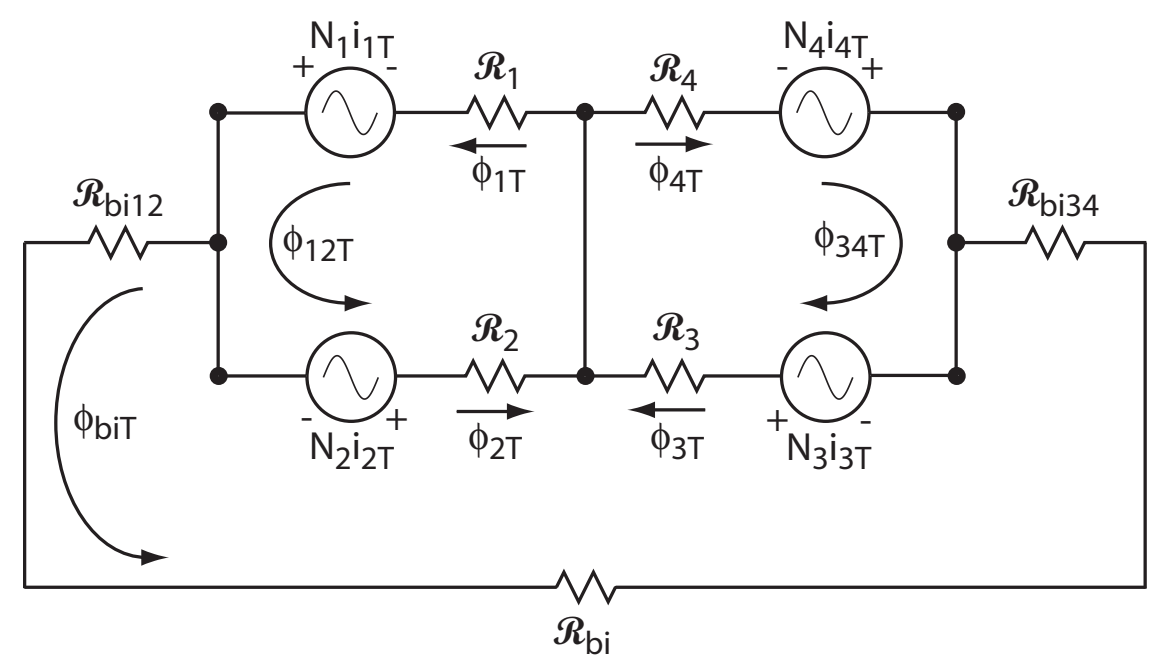

Figure 5-9: Reluctance model of the magnetic circuit for the actuator in the $10 \mathrm{kHz}$ rotary fast tool servo for small rotations of the rotor, showing the flux in the four rotor/stator air gaps due to the steering flux.

$$
N_{s c} i_{s c}=\left[2.5 \times 10^{5}\right](0.75)\left[1.61 \times 10^{-4}\right]=30 \text { amp.turns }
$$

\subsubsection{Inductance of the Actuator}

Referring to the magnetic circuit in Figure 5-3, we consider the steering flux path through the left-hand stator core and the rotor core to determine the inductance at the terminals of the series-connected pair of steering flux coils on that stator core. Due to the symmetry of the actuator, the inductance for the coil pair on the right-hand stator core is the same as it is for the left-hand pair.

Starting with Faraday's law, we relate the time-differential of the total flux linked $\lambda$ by an N-turn coil to the voltage $V$ produced at the terminals of the coil:

$$
V=-\frac{d}{d t}(N \phi)=-\frac{d}{d t}(\lambda)
$$

Where:

$$
\lambda=N \phi
$$


Referring to Figure 5-3, the steering flux $\phi_{12 T}$ in the left-hand stator core is the product of the flux density $B_{12 T}$ and the cross-sectional area of the core $A_{p}$ :

$$
\phi_{12 T}=B_{12 T} A_{p}
$$

Each coil has $N_{s c}$ turns, and since they are connected in series the total number of turns linking the flux $B_{12 T}$ is $N_{s c p}=2 N_{s c}$. Substituting (5.78) into (5.77) gives the flux linked $\lambda_{s c p}$ by the series-connected pair of coils:

$$
\lambda_{s c p}=N_{s c p} B_{12 T} A_{p}
$$

Using (5.18) to express the magnetic flux density $B_{12 T}$ in terms of the total number of turns $N_{s c p}$ linking the flux and the current $i_{s c p}$ in the series-connected coils:

$$
B_{12 T}=\frac{\mu_{0} N_{s c p} i_{s c p}}{2 g_{N}}
$$

Substituting (5.80) into (5.79):

$$
\lambda_{s c p}=N_{s c p}\left[\frac{\mu_{0} N_{s c p} i_{s c p}}{2 g_{N}}\right] A_{p}=\left[\frac{\mu_{0} A_{p} N_{s c p}^{2}}{2 g_{N}}\right] i_{s c p}
$$

Substituting (5.81) into (5.76) and denoting the terminal voltage for the seriesconnected coils as $V_{\text {scp }}$ :

$$
V_{s c p}=-\frac{d \lambda}{d t}=-\left[\frac{\mu_{0} A_{p} N_{s c p}^{2}}{2 g_{N}}\right] \frac{d}{d t}\left(i_{s c p}\right)
$$

For a rotation of the rotor of $\theta$, the total length of air $\left(2 g_{N}\right)$ in this steering flux path does not change. However, for a front/back translation of the rotor of $Z$ the total length of air in the flux path does change, leading to a second term in (5.82) to account for the linear velocity of the rotor. Since the main role of the actuator used in the $10 \mathrm{kHz}$ FTS is to produce a torque, the linear velocity term for $(5.82)$ is not pursued in this thesis.

The term in the square brackets in (5.82) is the inductance $L_{s c p}$ at the terminals of the series-connected pair of steering flux coils: 


$$
L_{s c p}=\left[\frac{\mu_{0} A_{p} N_{s c p}^{2}}{2 g_{N}}\right]
$$

Substituting in values for the actuator used in the $10 \mathrm{kHz}$ FTS, (5.83) produces:

$$
L_{s c p}=\frac{\left(4 \pi \times 10^{-7}\right)\left(1.61 \times 10^{-4}\right)(20)^{2}}{(2)\left(50 \times 10^{-6}\right)}=800 \mu \mathrm{H}
$$

If there was only one steering coil with $\left(N_{s c}=N_{s c p} / 2\right)$ turns on the stator core, the inductance $L_{s c}$ seen at the terminals of that single coil would be:

$$
L_{s c}=\left[\frac{\mu_{0} A_{p} N_{s c}^{2}}{2 g_{N}}\right]=\left[\frac{\mu_{0} A_{p}\left(N_{s c p} / 2\right)^{2}}{2 g_{N}}\right]=\frac{1}{4} L_{s c p}=200 \mu \mathrm{H}
$$

So, what is going on here? Basic circuit theory tells us that two inductors connected in series are equivalent to one inductor having an inductance that is equal to the sum of the two. However, that assumes that there is no mutual inductance between them: the flux linked by one is not linked by the other. In our case however, the two inductors (coils) link the same flux and there is mutual inductance: a time-varying current flowing in one coil produces flux that creates a time-varying current in the other coil. Each coil has a mutual inductance with the other coil, and because the two coils are identical, the mutual inductance for each one is equal to its self-inductance. Hence the factor of four difference between the inductance seen at the terminals of the series-connected pair and the inductance for a single coil by itself. I point this out because during the early design phase I did not take into account that mutual inductance, and sized the power amplifiers to drive each steering coil individually. The consequence of this error is discussed in Section 5.3.5.

\subsubsection{Electrical Power Needed to Drive the AC Flux}

The relationships in the previous section are used to relate the apparent time-average electrical power needed to drive the AC flux in the rotor/stator air gaps to the timeaverage magnetic power in the air gaps. For this derivation I assume that the pair of coils on the stator core act as a purely inductive load with no losses, and that 
the magnetic flux density is linearly dependent on the magnetic field intensity for the frequencies of interest (no hysteresis). For this ideal transformer assumption, we expect that the time-average power in the two different energy domains will be equal, which is what the analysis in this section reveals.

For a purely inductive electrical load with a sinusoidal current of $i=i_{p k} \sin (\omega t)$, the peak current $i_{p k}$ lags the peak voltage $V_{p k}$ by $90^{\circ}$, and the electrical power is purely reactive. Using a result developed in Section 3.11.2, we relate the apparent time-average electrical power $P_{a, e l e c}$ into the load to the magnitude of the time-average complex power $|\underline{S}|$, where the underscore indicates a vector quantity:

$$
P_{a, e l e c}=|\underline{S}|=\frac{1}{2}|\underline{V}|\left|\underline{I}^{*}\right|=\frac{1}{2} V_{p k} i_{p k}
$$

Using (5.82), which relates the voltage to the product of the inductance and the time-rate of change of the current, we obtain the following relationship for the peak voltage:

$$
V_{p k}=\left[\frac{\mu_{0} A_{p} N_{s c p}^{2}}{2 g_{N}}\right]\left(i_{p k} \omega\right)
$$

Substituting (5.87) into (5.86) gives:

$$
P_{a, \text { elec }}=\frac{\mu_{0} A_{p} \omega\left(N_{s c p} i_{p k}\right)^{2}}{4 g_{N}}
$$

Using the conclusion from Section 5.2.1 and (5.2), the instantaneous magnetic power $P_{m a g}$ in the air gaps is related to the magnetic field intensity $H$, the magnetic flux density $B$, the operating frequency $\omega$, and the total volume $\forall_{\text {gaps }}$ of the air gaps in the magnetic circuit by:

$$
P_{\text {mag }}=H B \omega \forall_{\text {gaps }}=\frac{B^{2}}{\mu_{0}} \omega\left(A_{p} 2 g_{N}\right)
$$

Note that $H$ and $B$ are in phase with each other, so with the assumption that they are both sinusoidal, their product looks like a scaled version of $(\sin \theta)^{2}$, which has an average value over one cycle of [23, page 312]: 


$$
\frac{1}{2 \pi} \int_{0}^{2 \pi}(\sin \theta)^{2} d \theta=\left.\frac{1}{2 \pi}\left[\frac{\theta}{2}-\frac{1}{4} \sin (2 \theta)\right]\right|_{0} ^{2 \pi}=\frac{1}{2 \pi}\left(\frac{2 \pi}{2}-0\right)=\frac{1}{2}
$$

Therefore, using (5.89) and (5.90), the time-average magnetic power $P_{a, m a g}$ in the air gaps is:

$$
P_{a, m a g}=\frac{1}{2} H_{p k} B_{p k} \omega \forall_{g a p s}=\frac{B_{p k}^{2}}{2 \mu_{0}} \omega\left(A_{p} 2 g_{N}\right)
$$

Substituting (5.80) into (5.91) gives:

$$
P_{a, m a g}=\frac{1}{2 \mu_{0}}\left(\frac{\mu_{0} N_{s c p} i_{p k}}{2 g_{N}}\right)^{2} \omega\left(A_{p} 2 g_{N}\right)=\frac{\mu_{0} A_{p} \omega\left(N_{s c p} i_{p k}\right)^{2}}{4 g_{N}}
$$

Comparison of (5.88) and (5.92) provides the final result for this section - a relationship between the apparent time-average electrical power into the series-connected pair of coils on a stator core and the time-average magnetic power produced in the two rotor/stator air gaps for that core:

$$
\frac{1}{2} V_{p k} i_{p k}=P_{a, e l e c}=P_{a, m a g}=\frac{1}{2} H_{p k} B_{p k} \omega \forall_{g a p s}=\frac{B_{p k}^{2}}{2 \mu_{0}} \omega\left(A_{p} 2 g_{N}\right)
$$

\subsubsection{Allowable Coil Current and Sizing the Coil Window Area}

For a passively cooled coil in air, Cooner Wire [39] recommends a ratio of wire area divided by current of at least 1000 circ-mils/amp for a passively air-cooled copper wire, and suggests that 800 circ-mils/amp is permissible if moderate heating is acceptable. These values correspond to $2 \times 10^{6} \mathrm{amp} / \mathrm{m}^{2}$ and $2.5 \times 10^{6} \mathrm{amp} / \mathrm{m}^{2}$, respectively. McLyman [105] suggests the same 1000 circ-mils/amp as a rule-of-thumb. Professor David Trumper at MIT described the following order-of-magnitude guidelines for allowable current density in copper: $1 \times 10^{6} \mathrm{amp} / \mathrm{m}^{2}$ can be sustained with passive cooling, and $1 \times 10^{7} \mathrm{amp} / \mathrm{m}^{2}$ requires active cooling. These guidelines are consistent 
with the 1000 circ-mils/amp rule.

For the reader who is not familiar with circ-mils - which is a common abbreviation for circular-mils - it is a useful concept when filling a coil window area with wire. Furthermore, many handbooks use circ-mils for reporting the cross-sectional area for different gauges of wire. In essence, for sizing purposes the round cross-section of the wire is treated as taking up the same space as it would if it were a square crosssection, where the sides of the square are equal to the diameter of the wire. Now the coil window area can be conceptually filled with squares as if the wire were being laid in a row and column fashion. This provides a conservative packing factor for the wire when compared to a hexagonal-close-packed winding. ${ }^{2}$ The wire area in circ-mils can be converted to the actual cross-sectional area of the wire in $\left(\right.$ mils $\left.^{2}\right)$ by multiplying by $(\pi / 4)$; where $($ mils $)=0.001$ inch.

\section{Coil Window Area for the Bias Coil}

As mentioned in Section 5.3.1, during the early design phase I sized the bias coil to provide an MMF of 600 amp-turns. Recall that the bias coil carries a DC current. If the coil consisted on a single turn of copper, the current would be $600 \mathrm{~A}$. Using 1000 circ-mils $/ \mathrm{amp}=2 \times 10^{6} \mathrm{amp} / \mathrm{m}^{2}$ as an acceptable thermal limit for a passively air-cooled coil, the cross-sectional area of the copper needs to be 600,000 circ-mils, or $3.0 \times 10^{-4} \mathrm{~m}^{2}$ :

$$
\begin{array}{r}
(600,000 \text { circ-mils }) \frac{\pi}{4}\left(\frac{\text { mil }^{2}}{\text { circ-mils }}\right)\left(\frac{\text { in }}{1000 \text { mil }}\right)^{2}=0.47 \mathrm{in}^{2}=3.0 \times 10^{-4} \mathrm{~m}^{2} \\
100 \% \text { packing factor }
\end{array}
$$

Since the entire window area is not filled with copper - some area is lost to the empty space between windings and to insulation - a packing factor needs to be included. Note that the ratio, $(\pi / 4=0.79)$ in the conversion from (circ-mils) to actual

\footnotetext{
${ }^{2}$ In practice, it can be more difficult to maintain a hexagonal-close-packed winding if many turns are involved, so designing for a row and column packing is appropriate unless significant extra effort for winding the coil is specified.
} 
area in $\left(\mathrm{mil}^{2}\right)$ provides a reasonable — although slightly optimistic — approximation for a packing factor. Therefore, a quick design value for the required window area can be obtained by dropping the factor of $(\pi / 4)$ in the calculation above:

$$
(600,000 \text { circ-mils })\left(\frac{\text { in }}{1000 \text { mil }}\right)^{2} \Rightarrow 0.60 \mathrm{in}^{2} ; \pi / 4=79 \% \text { packing factor }
$$

The window area of 0.60 inch $^{2}$ roughly corresponds to a more rigorously derived window area of 0.625 inch $^{2}$ that was based on an analysis that assumed a hexagonalclose-packed winding and considered area lost to the enamel insulation on the wire. It is worth noting that 0.625 inch $^{2}$ corresponds to a packing factor of $75 \%$.

\section{Coil Window Area for Each Steering Coil}

In Section 5.3.1 I determined that each steering coil needs to produce a peak MMF of 30 amp.turns. The steering coils carry an AC current, so heating should be based on the rms current associated with the peak current. However, due to the non-linear relationship between flux density $B$ and the magnetic field intensity $H$ in the cores, a non-sinusoidal current is needed to produce a sinusoidal flux. Hubert [79, page 110] shows that for a transformer driven by a sinusoidal voltage source, this non-linear relationship causes odd-multiple harmonics to be present in the nominally sinusoidal current waveform, resulting in peaking in the current waveform. ${ }^{3}$ Therefore, as a conservative measure, I sized the steering flux coil windows as if the 30 amp-turns were a DC current. Following the same procedure used above for the bias coil, and using 1000 circ-mils/amp $=2 \times 10^{6} \mathrm{amp} / \mathrm{m}^{2}$ as an acceptable thermal limit for a passively air-cooled coil, the cross-sectional area of the copper needs to be 30,000 circmils:

\footnotetext{
${ }^{3}$ As an aside, this leads to one possible explanation for why odd-multiple harmonics of $60 \mathrm{~Hz}$ show up as noise on low-power circuits that are inductively coupling with nearby $60 \mathrm{~Hz}$ transformers. This is especially true if the $60 \mathrm{~Hz}$ transformer involved is being operated near magnetic saturation.
} 
$\left(30,000\right.$ circ-mils) $\frac{\pi}{4}\left(\frac{\text { mil }^{2}}{\text { circ-mils }^{2}}\right)\left(\frac{\text { in }}{1000 \text { mil }}\right)^{2}=0.024 \mathrm{in}^{2} ; 100 \%$ packing factor

$(30,000$ circ-mils $)\left(\frac{\text { in }}{1000 \text { mil }}\right)^{2} \Rightarrow 0.030 \mathrm{in}^{2} ; \pi / 4=79 \%$ packing factor

The steering flux coils for the $10 \mathrm{kHz}$ FTS are buried in potting compound inside of the stator housing, so are not exposed to the ambient air like the bias flux coil is. Therefore, I used the guideline of 1000 circ-mils/amp for passively air-cooled copper wire as a starting point. I found a standard-sized bobbin that would closely fit on the stator pole, and provided a coil window area of $0.087 \mathrm{inch}^{2}$. This provides a factor of three larger window area than the above calculation indicates is necessary based on 1000 circ-mils/amp. It allowed increasing the amount of copper in the window, and provided me with some margin on how tightly I had to wind the coils. Regarding how to dissipate the heat produced in the steering coil if it had turned out to be an issue - and it did not - the mechanical design of the stator provides an option for forced liquid cooling if necessary.

\subsubsection{Voltage versus Current Trade-off for the Coils}

In Section 5.3.1 I determined the magnetomotive force in Ampere-turns that the bias and steering coils need to supply. As the designer, we need to choose the number of coil turns and corresponding peak current that we want to use. To avoid being arbitrary in this decision, in this section I consider the trade-off between the peak voltage at the terminals of a coil and the peak current in the coil based on the number of turns. The steering coils carry an AC current, so their inductance comes into play and I choose the number of turns corresponding to the peak voltage that I want to drive with the power amplifiers. The trade-off for the bias coil is simpler because it carries only a DC current, so voltage and current are related by the coil resistance. 
When trading off voltage and current we consider how many coil turns will be needed to produce the required MMF. A lower current requires more turns, which increases the difficulty of maintaining a tight wire packing. For the $10 \mathrm{kHz}$ FTS, the bias coil was machine-wound with epoxy-bonded wire and no bobbin, and the steering coils were hand-wound with served Litz wire on a bobbin.

\section{Peak Voltage and Current for the Steering Coils}

In this analysis I assume that the resistance of the coil is negligible compared to the impedance due to the inductance of the coil at the frequencies of interest for the $10 \mathrm{kHz}$ FTS. In Section 4.7.2 I show this to be a valid assumption.

For a series-connected pair of coils on a stator core, I assume that the current $i_{s c p}$ in the coils is sinusoidal with a peak value of $i_{p k}:{ }^{4}$

$$
i_{s c p}=i_{p k} \sin (\omega t) \Rightarrow \frac{d}{d t}\left(i_{s c p}\right)=i_{p k} \omega \cos (\omega t)
$$

The magnetic flux density in the core is sinusoidal and in phase with the current. Using (5.80) and denoting the peak flux density as $B_{p k}$ :

$$
i_{s c p}=\frac{\left[B_{p k} \sin (\omega t)\right] 2 g_{N}}{\mu_{0} N_{s c p}} \Rightarrow i_{p k}=\frac{B_{p k} 2 g_{N}}{\mu_{0} N_{s c p}}
$$

Treating the coil pair as a purely inductive load, the corresponding voltage $v_{s c p}$ at the terminals of the series-connected pair of coils is:

$$
v_{s c p}=L_{s c p} i_{p k} \omega \cos (\omega t)
$$

Substituting (5.83) and (5.95) into (5.96), the peak voltage $v_{p k}$ is:

$$
\begin{array}{r}
v_{s c p}=\left[\frac{\mu_{0} A_{p} N_{s c p}^{2}}{2 g_{N}}\right]\left[\frac{B_{p k} 2 g_{N}}{\mu_{0} N_{s c p}}\right] \omega \cos (\omega t)=A_{p} N_{s c p} B_{p k} \omega \cos (\omega t) \\
\Rightarrow \quad v_{p k}=A_{p} N_{s c p} B_{p k} \omega
\end{array}
$$

\footnotetext{
${ }^{4}$ Even though we know from Section 5.3.4 that there are odd-multiple harmonics present.
} 
Given a design goal for the peak flux density, depending on which other variables we know - air gap length, cross-sectional area of the poles, maximum operating frequency, peak current, and peak voltage — we can use either (5.95) or (5.97) to determine the number of turns for the coil pair. For the $10 \mathrm{kHz}$ FTS, I set a maximum voltage for the power amplifier as a design criteria, and used (5.97) to determine the number of turns for the series-connected pair of coils:

$$
N_{s c p}=\frac{v_{p k}}{A_{p} B_{p k} \omega}
$$

For the $10 \mathrm{kHz}$ FTS I chose $v_{p k}=75 \mathrm{~V}$ so that I could use power supplies that I already had and end up with a peak current in the range of 5 to $10 \mathrm{~A}$, which allowed using the same circuit topology for the current-mode amplifier that was successful with the $2 \mathrm{kHz}$ FTS. One design goal was to produce a $0.75 \mathrm{~T}$ peak flux density in the rotor/stator air gaps at $10 \mathrm{kHz}$. Using (5.98) and these two conditions, we obtain the total number of turns of the series-connected coil pair for the actuator used in the $10 \mathrm{kHz}$ FTS:

$$
N_{s c p}=\frac{75}{\left(1.61 \times 10^{-4}\right)(0.75)[(2 \pi) 10,000]}=10 \text { turns }
$$

We now use (5.95) to calculate the peak current $i_{p k}$ in the coil pair:

$$
i_{p k}=\frac{(0.75)(2)\left(50 \times 10^{-6}\right)}{\left(4 \pi \times 10^{-7}\right)(10)}=6.0 \mathrm{amp}
$$

Note that this result is consistent with the $30 \mathrm{amp} \cdot$ turns required from each one of the coils:

$$
N_{s c} i_{p k}=\left(N_{s c p} / 2\right) i_{p k}=(5 \text { turns })(6.0 \mathrm{Amp})=30 \text { amp } \cdot \text { turns }
$$

At this point the story gets more interesting for the $10 \mathrm{kHz}$ FTS. During the early design phase when I was specifying the electronic components, I had not yet fully appreciated the significance of the mutual inductance between the two coils on each stator core, and erroneously neglected it. I sized the power amplifiers assuming 
that each coil would be driven by its own independent current-mode amplifier, and wound each coil with 10 turns. When I tried to close the loops for the two pairs of amplifiers - each amplifier in a pair was driving one of the two coils on the same stator core - I quickly learned about the mutual inductance and realized that each steering flux circuit was a MIMO system. The expedient solution was to use a series connection for the coils on each stator core, and drive each steering flux circuit as a SISO system, which worked fine. The net result of this error is that the actual total number of turns for the series-connected coils on each stator core is 20 , not 10, and (5.97) tells us that we would need $V_{p k}=150 \mathrm{~V}$ to produce $0.75 \mathrm{~T}$ at $10 \mathrm{kHz}$. The stroke of good luck here is that the $10 \mathrm{kHz}$ FTS has a stable mechanical resonance at $10 \mathrm{kHz}$, so the peak current specified in this section was not needed. The measured negative loop transmission for the $10 \mathrm{kHz}$ FTS indicates that the actuator provides all of the torque to accelerate the rotor for operating frequencies up to approximately $5 \mathrm{kHz}$ (See Figure 4-105). Above that frequency, the actuator gets help from the stable mechanical resonance. If we plug $75 \mathrm{~V}$ and $5 \mathrm{kHz}$ into (5.98), we find that the total number of turns for the series-connected pair of coils is 20 , which is consistent with what I built. In retrospect, to produce $30 \mathrm{amp} \cdot$ turns at $10 \mathrm{kHz}$ with each of the as-built steering coils, the analysis of this section indicates that the power amplifiers need to be arranged in pairs forming bridge circuits that can supply twice the voltage of what I built for this research.

\section{Peak Voltage and Current for the Bias Coil}

The bias coil carries only a DC current, so the voltage and current are related by the resistance of the coil. In Section 5.3.4 I show that a window area of 0.625 inch $^{2}$ corresponds to a $75 \%$ packing factor and 1000 circ-mils/amp for a bias coil MMF of 600 amp-turns. That window area corresponds to a 2.5 inch long coil with a build-up of 0.25 inch. Note that I chose the window area to be wide and short to keep the individual wire turns closer to the back-iron and minimize leakage inductance [105]. Glover [64] provides data on the number of turns-per-inch that can be wound for various sizes of enamel-coated wire, and the resistance-per-length for those sizes. 
Using that data I created a spreadsheet that filled the window area with various sizes of wire, and calculated the total number of turns, resistance, current, and voltage for each case. After trading-off various wire sizes with the difficulty of winding the coil - smaller wire gauges require more turns and increases the difficulty of maintaining a tight wire packing - I specified \#20 AWG copper wire. This led to the following bias coil design specifications for producing 610 amp.turns: 580 turns, 0.49 Ohm total resistance, $1.05 \mathrm{~A}$, and $0.52 \mathrm{~V}$. The coil was machine-wound with epoxy-bonded wire. The dimensions of the coil were exact, but it ended up having only 560 turns, which is $97 \%$ of the specified number of turns and was acceptable.

\subsubsection{Litz Wire for the Steering Coils}

Litz $^{5}$ wire is used to construct the steering flux coils for the $10 \mathrm{kHz}$ FTS because they carry an alternating current at frequencies up to $10 \mathrm{kHz}$. Sullivan [140] provides an excellent discussion on the construction of Litz wire, and the roles of skin effect and proximity effect losses associated with high frequency alternating currents. The skin effect losses are due to the following. Associated with the time-rate of change of the intended current in a conductor is a time-varying magnetic field which induces an eddy current (in accordance with Faraday's law) that opposes the intended current at the center of the conductor and reinforces it towards the outside surface of the conductor. The net result is that at sufficiently high frequencies the intended current flows near the outside surface of the conductor, effectively reducing the cross-sectional area of the conductor and increasing its resistance. This effect can be reduced by decreasing the cross-sectional area of the conductor, and for a given frequency a strand diameter can be chosen so that its entire cross-section carries the intended current. Sullivan describes the proximity effect losses to be due to "the tendency for current to flow in other undesirable patterns - loops or concentrated distributions - due to the presence of magnetic fields generated by nearby conductors" [140]. Litz wire is constructed by twisting or weaving small diameter strands into bundles, and then twisting of weaving the bundles into a cable. Sullivan [140] further describes skin

\footnotetext{
5 "Litz" is from the German word for woven wire: "litzendraht".
} 
effect and proximity effect losses at the individual strand level, and at the bundle level. Professor David Trumper at MIT provided me with the following intuitive way of thinking about the woven cable. Since alternating currents are forced to the outside of the conductor, weave the individual strands and bundles so that each one occasionally reaches the outside surface of the cable so that it can pick up current before returning back towards the center of the cable. If all of the strands "come up for current" often enough, then they all will be carrying the alternating current. To the extent that certain strands do not carry any of the alternating current, the $\mathrm{AC}$ resistance of the cable will be higher than the DC resistance.

For $10 \mathrm{kHz}$ alternating currents, [74], [39] and [113] recommend that the individual strands be \#33 AWG or smaller. Recall from Section 5.3.5 that during the early design phase I had specified that each steering coil needs 10 turns of wire, which meant that a peak current of $3.0 \mathrm{~A}$ is needed to produce the $30 \mathrm{amp} \cdot \mathrm{turns}$ specified in Section 5.3.1. In accordance with the above discussion on skin and proximity effects at the strand and bundle levels, I specified a Litz cable construction that allowed me to fill the window coil area with 10 turns. However, it proved extremely difficult to hand-wind the relatively large cable onto the small, rectangular bobbin. The bend radii of the cable at the corners of the bobbin became progressively larger as I wound the coil, resulting in a loosely packed bobbin that would not fit into the stator for the $10 \mathrm{kHz}$ FTS. I exercised my fall-back plan: use four parallel bundles of 60 strands of twisted \#36 AWG copper wire that was readily available from MWS Wire Industries [111]. The resulting cable provides 6000 circ-mils of copper, resulting in a thermally conservative 2000 circ-mils/amp or $1.5 \times 10^{6} \mathrm{amp} / \mathrm{m}^{2}$. Twisting the four bundles would reduce bundle-level losses, but then the coil would have been too large to fit into the stator. I could have experimented with trying to twist only three bundles, which would have provided an acceptable 1500 circ-mils/amp, but the four parallel bundles were adequate and I needed to proceed to other aspects of building the $10 \mathrm{kHz}$ FTS. 


\subsection{Candidate Magnetic Circuit Topologies}

In this section I compare the magnetic circuit for the hybrid rotary/linear actuator used in the $10 \mathrm{kHz}$ FTS to two other candidate magnetic circuits. Whereas the force mode is coupled to the torque mode for the actuator that I built, the other two magnetic circuits result in fully decoupled torque and force modes. A comparison of the properties of the three circuit topologies is presented, along with a discussion on why we chose the magnetic circuit that we did for the $10 \mathrm{kHz}$ FTS.

In Sections 5.2.3, 5.2.4, and 5.2.5 I developed closed-form equations relating the torque and force on the rotor caused by the addition and subtraction of the bias and steering fluxes in the rotor/stator air gaps. The analytical methods used in those sections can be used to derive the same relationships for the other magnetic circuit topologies described in this section. For the purposes of this thesis, I use the numerical analysis shown in Appendix B.6 to explore and compare the torque and force modes for the magnetic circuit topologies discussed in this section.

It is worth noting that the values of some of the parameters that I used in the numerical model, such as the number of coil turns and coil currents, are not the same as the actual values for the actuator in the $10 \mathrm{kHz}$ FTS. I used convenient "round numbers" in the numerical model to explore the differences between the candidate magnetic circuit topologies, and did that work before establishing the final operating parameters for the actuator. Therefore, the numerical results for torque and force from the model in Appendix B.6 do not match the actual experimental values, but they do show the general trends.

\subsubsection{Sandwiched Rotor Design}

The magnetic circuit showing how torque and force are generated by the actuator used in the $10 \mathrm{kHz}$ FTS is shown in Figure 5-6. I refer to this circuit topology as the sandwiched rotor design because the rotor core appears to be sandwiched between the two stator cores. The upper plot in Figure 5-10 shows that the torque caused by the torque-producing current $i_{\text {torque }}$ is independent of the value of the force-producing 


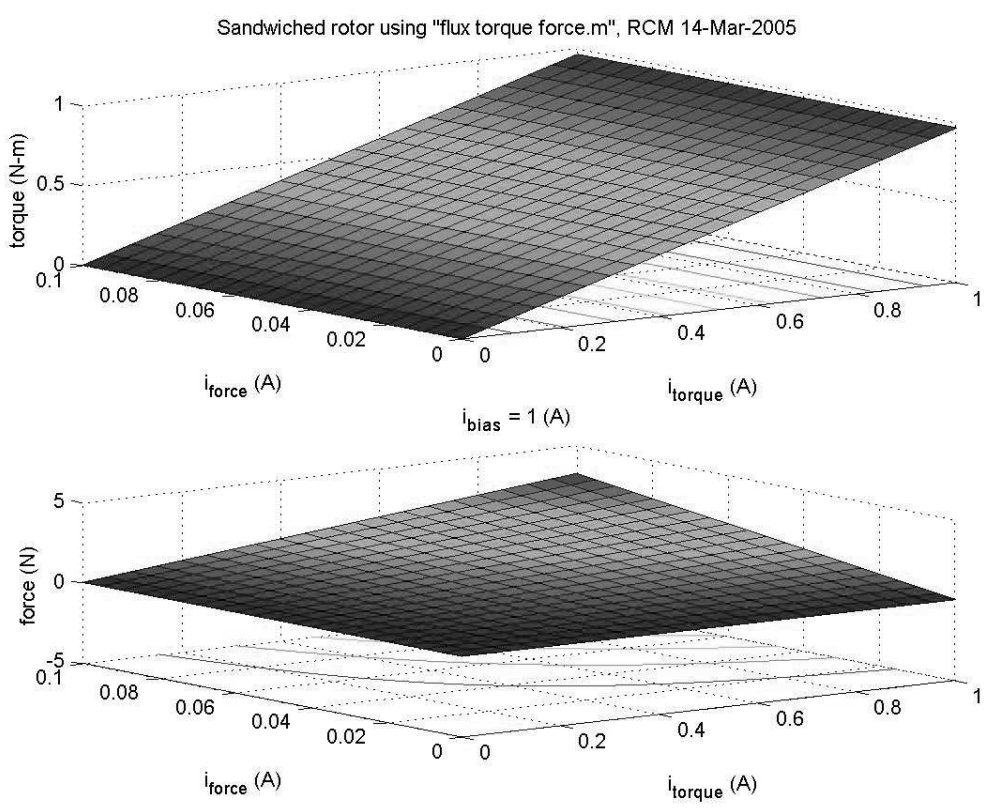

Figure 5-10: Results of a numerical analysis for the sandwiched rotor magnetic circuit. Torque as a function of torque-producing current $i_{\text {torque }}$ (upper plot). Force as a function of force-producing current $i_{\text {force }}$ (lower plot).

current $i_{\text {force }}$. The lower plot in Figure 5-10 shows that the force caused by the forceproducing current $i_{\text {force }}$ is dependent on the value of the torque-producing current $i_{\text {torque }}$. This is consistent with the results shown in Sections 5.2.3 and 5.2.5, and visually illustrates that a non-zero value of $i_{\text {torque }}$ is needed to act as an operating for the force-producing current $i_{\text {force }}$.

\subsubsection{Pinched Rotor Design}

Figure 5-11 is a sketch of the first alternate magnetic circuit for the hybrid rotary/linear actuator, and shows the bias flux $B_{B}$ (triple-head arrows), the torqueproducing steering fluxes $B_{12 T}$ and $B_{34 T}$ (double-head arrows), and the force-producing steering fluxes $B_{12 F}$ and $B_{34 F}$ (single-head arrows). I refer to this circuit topology as the pinched rotor design because the two stator cores appear to be pinching the rotor core. The upper plot in Figure 5-12 shows that the torque caused by the torque-producing current $i_{\text {torque }}$ is independent of the value of the force-producing current $i_{\text {force }}$. The lower plot in Figure 5-12 shows that the force caused by the force- 


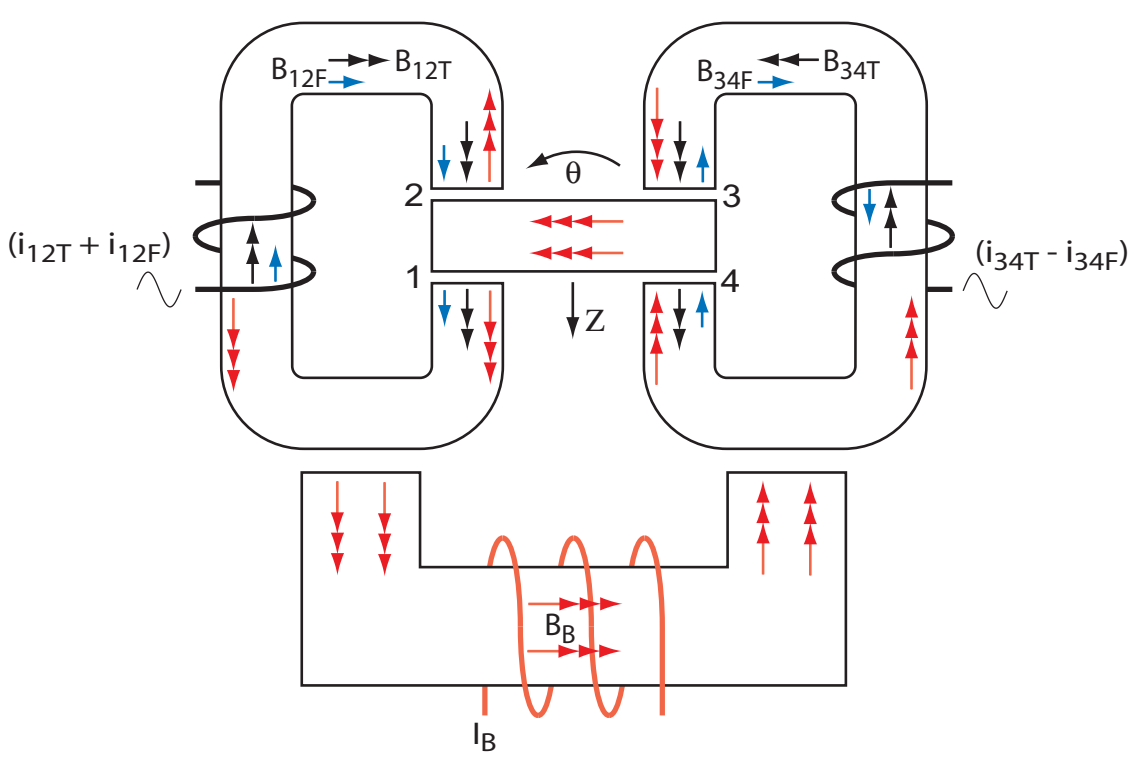

Figure 5-11: Sketch of the magnetic circuit showing torque and force generation for the pinched rotor magnetic circuit.

producing current $i_{\text {force }}$ is independent of the value of the torque-producing current $i_{\text {torque }}$.

\subsubsection{Reversed-Roles Sandwiched Rotor Design}

Figure 5-13 is a sketch of the second alternate magnetic circuit for the hybrid rotary/linear actuator, and shows the bias flux $B_{B}$ (triple-head arrows), the torqueproducing steering fluxes $B_{12 T}$ and $B_{34 T}$ (double-head arrows), and the force-producing steering fluxes $B_{12 F}$ and $B_{34 F}$ (single-head arrows). I refer to this circuit topology as the reversed-roles sandwiched rotor design because it has the same appearance as the sandwiched rotor design, but the roles of the bias flux and torque flux coils have been reversed. Note that the force-producing flux is superimposed on the bias flux. The upper plot in Figure 5-14 shows that the torque caused by the torque-producing current $i_{\text {torque }}$ is independent of the value of the force-producing current $i_{\text {force }}$. The lower plot in Figure 5-14 shows that the force caused by the force-producing current $i_{\text {force }}$ is independent of the value of the torque-producing current $i_{\text {torque }}$. 

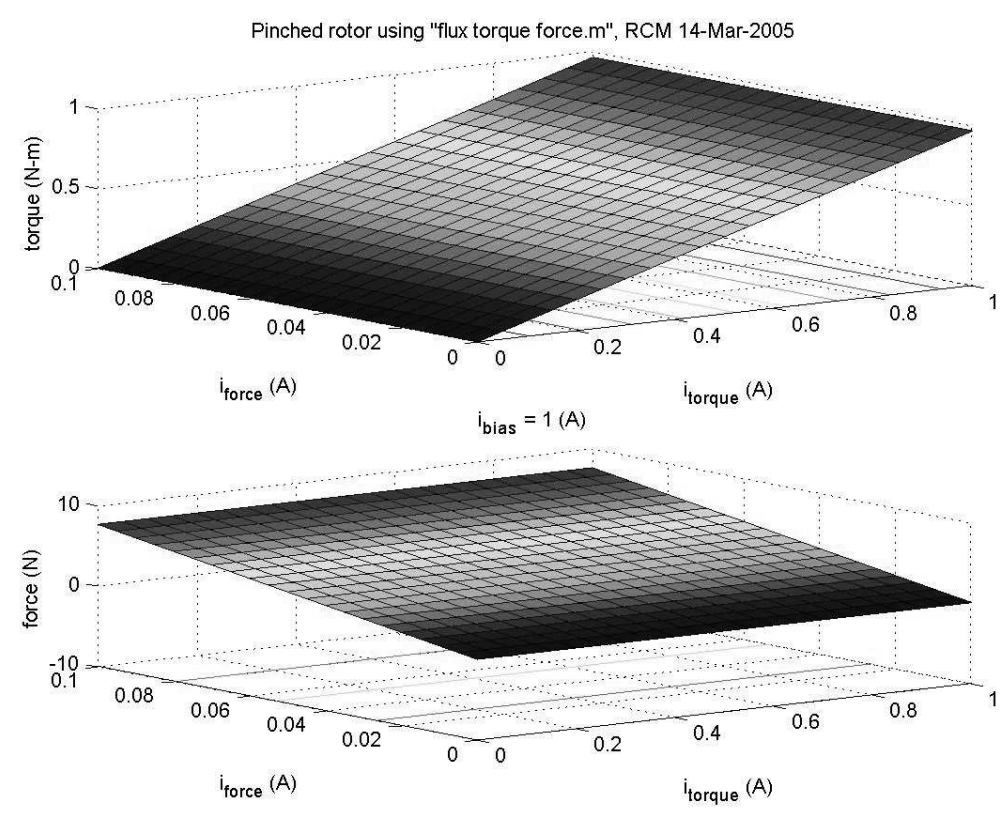

Figure 5-12: Results of a numerical analysis for the pinched rotor magnetic circuit. Torque as a function of torque-producing current $i_{\text {torque }}$ (upper plot). Force as a function of force-producing current $i_{\text {force }}$ (lower plot).

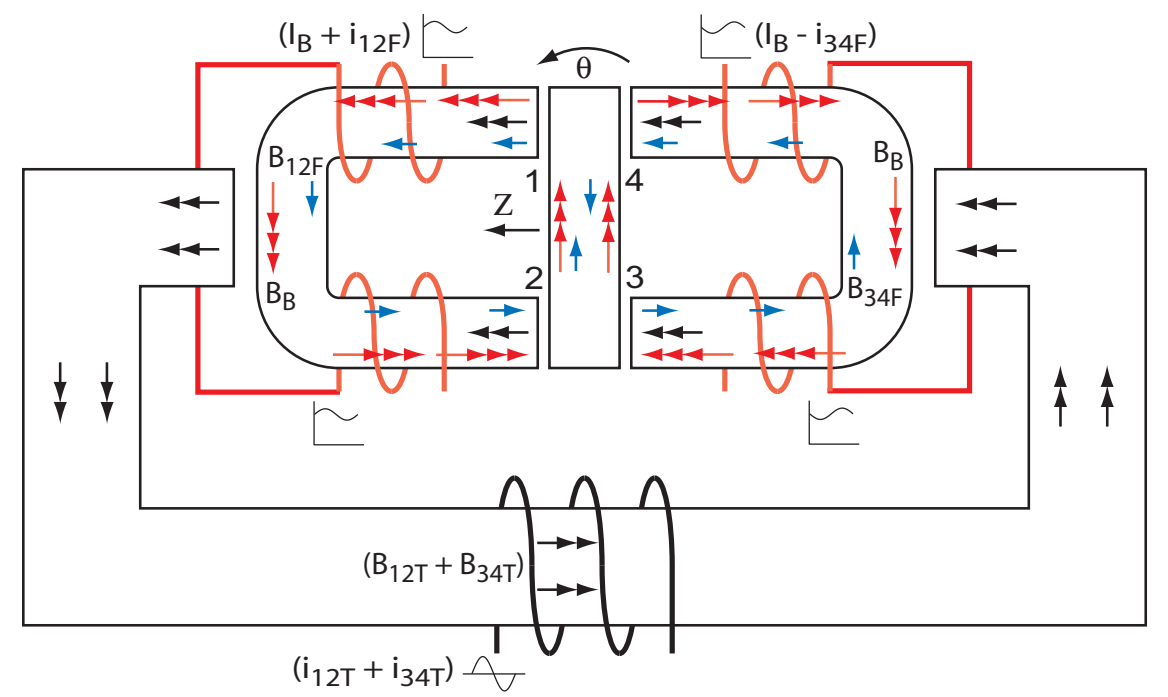

Figure 5-13: Sketch of the magnetic circuit showing torque and force generation for the reversed-roles sandwiched rotor magnetic circuit. 

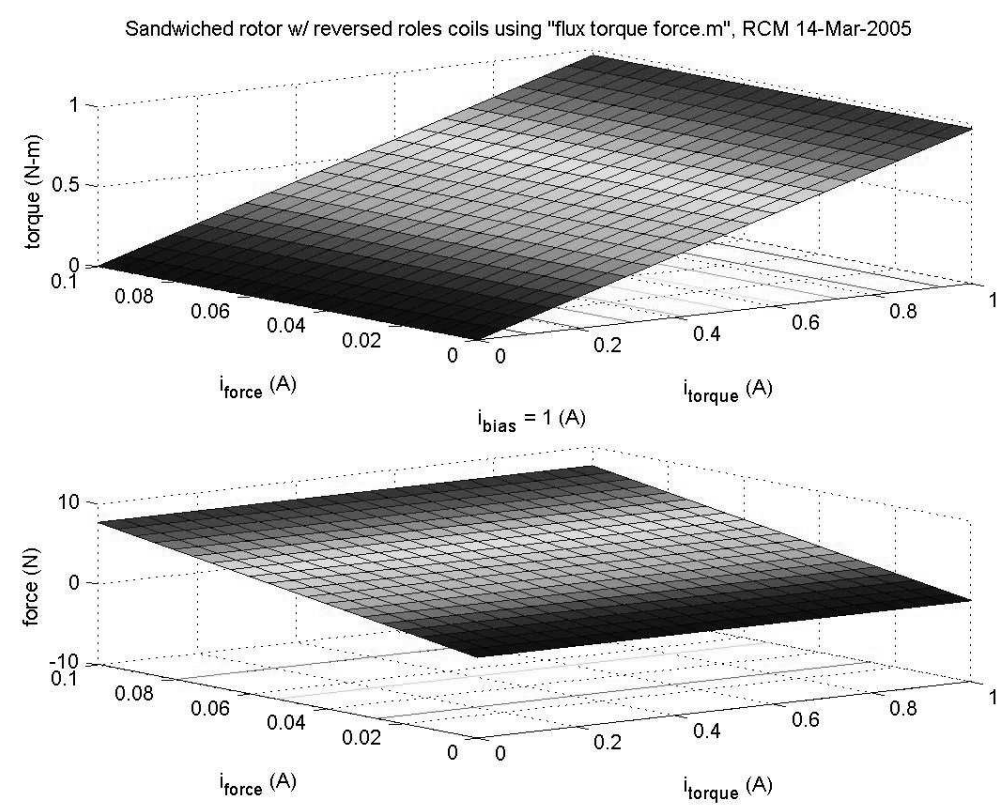

Figure 5-14: Results of a numerical analysis for the reversed-roles sandwiched rotor magnetic circuit. Torque as a function of torque-producing current $i_{\text {torque }}$ (upper plot). Force as a function of force-producing current $i_{\text {force }}$ (lower plot).

\subsubsection{Comparison of Candidate Magnetic Circuit Topologies}

Table 5.1 provides a comparison of key characteristics of the three different magnetic circuit topologies described in this section. Note that the order of the items in Table 5.1 does not imply relative importance, and that I did not include any weighting factors in the table.

Based on a quick glance of Table 5.1, it may appear that the sandwiched rotor design that we chose for the $10 \mathrm{kHz}$ FTS is not as desirable as the pinched rotor or the reversed-roles sandwiched rotor. However, during the conceptual design phase for the $10 \mathrm{kHz}$ FTS we identified that being able to adjust the length of the air gaps between the rotor and stator poles was extremely important for our the first prototype. In the case of the sandwiched rotor designs, if the rotor/stator air gaps were not the correct length after grinding the stator pole faces, they could be adjusted by changing the thickness of the shims that are designed into the assembly. This is certainly not possible with the pinched rotor design shown in Figure 5-11. Our primary goal for the actuator used in the $10 \mathrm{kHz}$ FTS was to provide a high torque and to demonstrate 
Table 5.1: Comparison of the magnetic circuits shown in Figures 5-6, 5-11, and 5-13.

\begin{tabular}{|l|c|c|c|}
\hline Item & $\begin{array}{c}\text { Sandwiched } \\
\text { Rotor }\end{array}$ & $\begin{array}{c}\text { Pinched } \\
\text { Rotor }\end{array}$ & $\begin{array}{c}\text { Reversed } \\
\text { Roles } \\
\text { Sandwiched } \\
\text { Rotor }\end{array}$ \\
\hline Torque is a linear function of current & Yes & Yes & Yes \\
\hline Relative torque for a given current & $1 \mathrm{x}$ & $1 \mathrm{x}$ & $1 \mathrm{x}$ \\
\hline Torque is not affected by force command & Yes & Yes & Yes \\
\hline Force is a linear function of current & Yes & Yes & Yes \\
\hline Relative force for a given current & $1 / 2 \mathrm{x}(\mathrm{max})$ & $1 \mathrm{x}$ & $1 \mathrm{x}$ \\
\hline Force is not affected by torque command & No & Yes & Yes \\
\hline Force does not require an operating point torque & No & Yes & Yes \\
\hline Force constant is independent of torque command & No & Yes & Yes \\
\hline One steering coil is okay on each stator piece & Yes & Yes & Yes \\
\hline Torque does not depend on leakage flux paths & Yes & Yes & Yes \\
\hline Force does not depend on leakage flux paths & Yes & Yes & Yes \\
\hline Rotor heat generation from AC flux & Higher & Lower & Lower \\
\hline Stator heat generation from AC flux & $1 \mathrm{x}$ & $1 \mathrm{x}$ & $1 \mathrm{x}$ \\
\hline Back-iron heat generation from AC flux & None & None & Significant \\
\hline Able to adjust air gaps after grinding pole faces & Yes & No & Yes \\
\hline Large face of rotor available for mounting a mirror & No & Yes & No \\
\hline
\end{tabular}

control of the linear mode that is inherent in this class of actuators. Therefore it was acceptable to proceed with a magnetic circuit that has a lower rating than the others with respect to the force mode, but provides more flexibility during fabrication and assembling. Regarding the reversed-roles sandwiched rotor design, although it provides fully decoupled torque and force modes, and allows adjusting the rotor/stator air gaps, it requires using laminated high-permeability iron in the back-iron magnetic path which would add an additional layer of design and fabrication complexity to building the actuator for the prototype $10 \mathrm{kHz}$ FTS, as well as having significantly higher inductance in the steering coil flux path.

In Section 4.4 I describe a balanced X-rotor layout that we considered when developing different possible magnetic circuit topologies for the $10 \mathrm{kHz}$ FTS. Close examination of the balanced X-rotor design shown in Figure 4-8 reveals that it has the same magnetic circuit as the pinched rotor design, and that the rotor/stator air gaps can be adjusted by translating each stator core towards and away from the rotor centerline. We decided against an X-rotor layout for the $10 \mathrm{kHz}$ FTS because of 
the additional fabrication complexity that the extra angles in the cores present. I revisit the balanced X-rotor in the discussion on future work in Section 6.4.9, and contemplate how nesting the stator cores makes the large face of the rotor available for directly mounting a mirror; creating an integrated high bandwidth actuator and steering mirror for use in an optical system.

\subsection{Soft Magnetic Materials for the Actuator}

This section provides a discussion on the issues that I considered when selecting the soft magnetic materials for the actuator used in the $10 \mathrm{kHz}$ FTS. After describing the overall selection strategy, I present a summary of the candidate materials and commercially available forms that were considered, review the sources of power loss in soft magnetic materials, discuss mechanical design concerns, and compare the magnetic properties of the candidate materials.

The primary considerations during the selection of the soft magnetic materials for the actuator are the ability to carry the peak flux, thermal losses, commercial availability, ability to fabricate into the desired forms with the required tolerances, and being able to integrate the material into a design that is robust enough to survive the anticipated mechanical loads during use.

The magnetic circuit for the hybrid rotary/linear actuator contains soft magnetic material that carries DC flux, AC flux, and mixed AC and DC flux. During steadystate operation the DC magnetic flux does not cause heating of the cores. The AC magnetic flux produces hysteresis and eddy current losses in the core material that result in heat generation. A primary requirement when choosing the core material is to be able to operate at a high enough flux density to achieve the desired torque for the actuator while not generating an inordinate amount of thermal energy loss. When designing the actuator I followed a practical method described by Kirtley in [19]: use empirical loss data from the manufacturer of the material rather than attempt to accurately calculate the combined hysteresis and eddy current losses based on theory. Additional concerns for the rotor and stator core material are stiffness (modulus of 


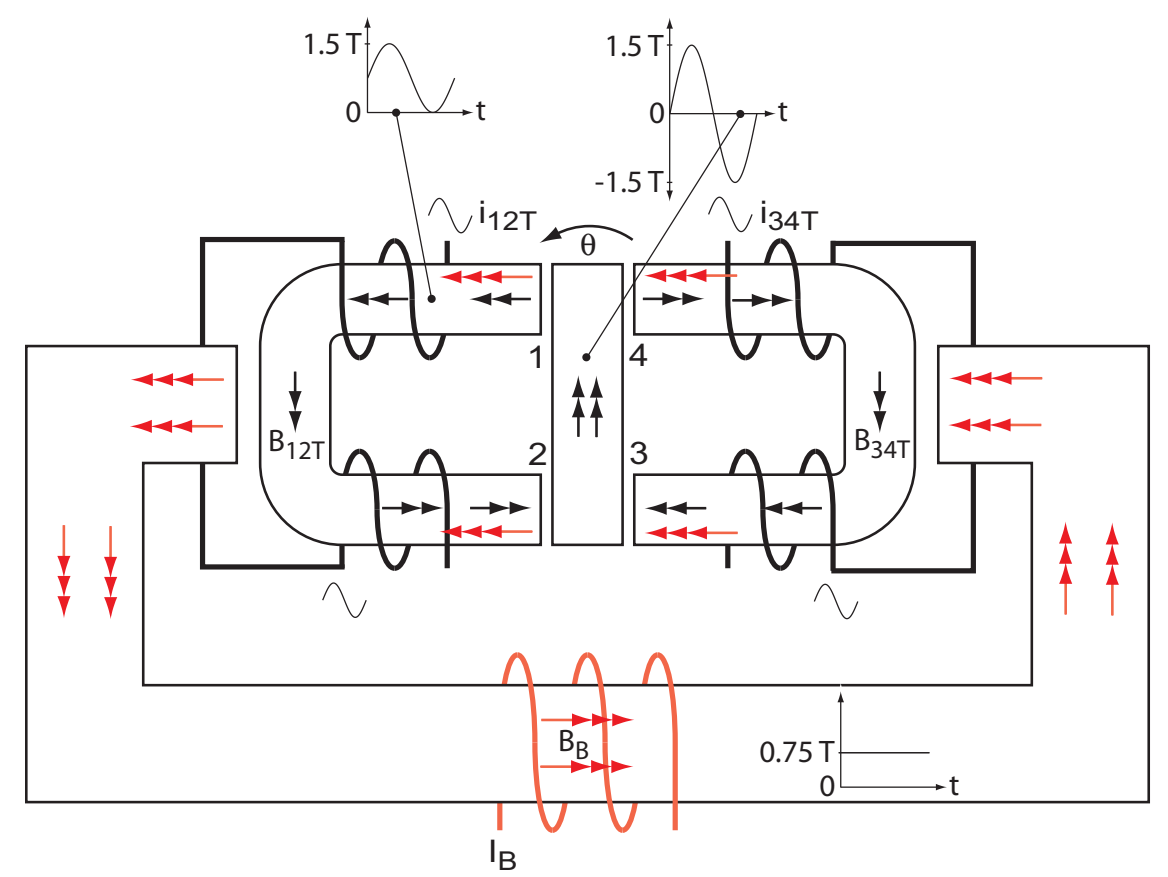

Figure 5-15: Sketch of the magnetic circuit for the $10 \mathrm{kHz}$ FTS showing the nature of the flux in the rotor core, stator cores, and back-iron, for the designed peak operation.

elasticity), strength, and coefficient of thermal expansion (CTE).

Figure 5-15 shows the nature of the magnetic flux in the rotor core, stator cores, and back-iron for the actuator used in the $10 \mathrm{kHz}$ FTS, for the designed peak operation. To produce a $1.5 \mathrm{~T}$ peak flux density in the rotor/stator air gaps we use a DC bias flux of $0.75 \mathrm{~T}$ and an $\mathrm{AC}$ steering flux with a peak of $0.75 \mathrm{~T}$. This results in a predominantly $\mathrm{AC}$ flux in the rotor core with a peak amplitude of $1.5 \mathrm{~T}(1.1 \mathrm{~T} \mathrm{rms})$, and a predominantly mixed AC and DC flux in the stator cores with a peak amplitude of $0.75 \mathrm{~T}(0.53 \mathrm{~T} \mathrm{rms})$ superimposed on constant bias flux of $0.75 \mathrm{~T}$.

\subsubsection{Material Selection Strategy}

I compiled and evaluated manufacturer's published data on power loss measurements and magnetic properties for a selection of soft magnetic materials that are commercially available as tape-wound cores. I developed metrics to compare the achievable rotor acceleration and core power loss for each material based on the allowable flux density, material density, and power loss at a maximum flux density amplitude of 
$1.6 \mathrm{~T}$ at $10 \mathrm{kHz}(1.1 \mathrm{~T} \mathrm{rms}) .{ }^{6}$ These metrics - a normalized acceleration factor and the core power loss - were used to down-select to three candidate materials in 0.001" thick laminations: (1) $50 \% \mathrm{Ni}-50 \% \mathrm{Fe}$, (2) amorphous iron-alloy, and (3) nanocrystalline iron-alloy. The $50 \% \mathrm{Ni}-50 \% \mathrm{Fe}$ was found to provide the best combination of high rotor acceleration and low power loss for the conventional iron-alloy laminate materials. This conclusion agrees with the experimental work by Williams and Trumper to identify the best choice of a conventional iron-alloy laminate material for developing high forces with low heat loss for a particular magnetic bearing actuator [151], although they were concerned with a lower frequency. The amorphous iron-alloy, with a lower density and the same peak flux capability as the Ni-Fe, provides a $14 \%$ higher rotor acceleration but has a $50 \%$ increase in core loss for a peak amplitude of $0.75 \mathrm{~T}$ at $10 \mathrm{kHz}$. The nanocrystalline iron-alloy, with a lower density and lower peak flux capability than the $\mathrm{Ni}-\mathrm{Fe}$, provides a $7 \%$ lower rotor acceleration with an $80 \%$ decrease in core loss for a peak amplitude of $0.75 \mathrm{~T}$ at $10 \mathrm{kHz}$.

For the $10 \mathrm{kHz}$ FTS, the rotor core is made from a nanocrystalline iron-alloy with a reported relative permeability in the range of 50,000-80,000 for a flux density of 2-11 kG rms at $10 \mathrm{kHz}$ [101]. The stator cores are made from $50 \% \mathrm{Ni}-50 \% \mathrm{Fe}$ with a reported relative permeability in the range of 10,000-20,000 for a flux density of 2-11 kG rms at $10 \mathrm{kHz}$ [101]. The nanocrystalline iron-alloy material was selected for the rotor core because its lower reported core loss would better allow conducting tests of the $10 \mathrm{kHz}$ FTS without using a cooling fluid to remove heat from the rotor core, which has a poor thermally conductive path to the stator housing via the flexure blades. Also, the laminations in nanocrystalline iron-alloy cores are bonded together better than the laminations in the $50 \% \mathrm{Ni}-50 \% \mathrm{Fe}$, which reduces the risk of pulling apart the rotor core when forces are applied to it via the flexures hubs. The $50 \% \mathrm{Ni}-$ $50 \%$ Fe material was picked for the stator cores because they have a good thermally conductive path to the stator housing - ameliorating the higher power loss — and the as-fabricated nanocrystalline iron-alloy stator cores had significant damage (see

\footnotetext{
${ }^{6} \mathrm{~A}$ peak amplitude of $1.6 \mathrm{~T}$ was used instead of $1.5 \mathrm{~T}$ because the manufacturer's data that I used reports flux in terms of rms-values using whole-numbers in units of kilo-Gauss (kG). $10 \mathrm{kG}=$ $1 \mathrm{~T}$.
} 
discussions in Sections 4.5.1 and 5.5.5).

\subsubsection{Candidate Materials and Commercially Available Forms}

This section discusses the commercially available soft magnetic materials that I considered when designing the $10 \mathrm{khz}$ FTS. We chose to use standard, commercially available tape-wound cores for building the stator and rotor cores. This avoiding having to bond together stacks of thin laminations ourselves, and nominally saved time while developing the $10 \mathrm{kHz}$ FTS. I say "nominally" because I ended up having problems with the tape-wound cores, which are discussed in Section 4.5.1. Iron powder polymer composites and ferrites were considered for the rotor and stator cores, but were ruled out because of low permeability and higher power loss, and low saturation flux density, respectively. Stainless steel was used for the back-iron material, which carries a DC magnetic flux.

\section{Tape-wound Cores}

Tape-wound cores are normally used to build low-loss electrical transformers and inductors. The cores are manufactured by winding a thin tape-form of a soft magnetic material around a mandrel and using an epoxy adhesive to bond the layers together. ${ }^{7}$ The resulting core is typically cut in half to produce two C-shaped halves. The cut faces are polished to produce flat surfaces for subsequent rejoining, and then chemically etched to remove any soft magnetic material that may have smeared across lamination layers and caused them to make electrical contact with each other. The cut core allows dropping a pre-wound coil onto each leg of the "C" before joining the two halves, which is generally easier to do than winding the coil onto a closed O-shaped core. Referring to Figures 4-17 and 4-19, each stator core in the actuator for the $10 \mathrm{kHz}$ FTS corresponds to one of the C-shaped cut-cores.

\footnotetext{
${ }^{7}$ The details of how the layers are bonded together are usually considered proprietary information by the material suppliers. Petrie, in his excellent reference "Handbook of Adhesives and Sealants", mentions that a solvent-based solid thermosetting epoxy adhesive "may be used to coat and then bond laminations of electrical steel for motor applications. The adhesive is applied and then dried through solvent evaporation. Once the parts are ready to be bonded, they are stacked under pressure. Heat is then applied to achieve flow and final chemical crosslinking [122, page 313]."
} 
Referring to Figures 4-17 and 4-18, the rotor core in the actuator is a simple stack of laminations that is produced by cutting a section from a large tape-wound core.

The three candidate materials for the actuator $-50 \% \mathrm{Ni}-50 \% \mathrm{Fe}$, amorphous iron-alloy, and nanocrystalline iron-alloy — are available in 0.001" thick laminate tape-wound cores from the Magnetic Metals Corporation [101]. Table 5.2 provides a comparison of some of the physical and mechanical properties of these materials. The $50 \% \mathrm{Ni}-50 \%$ Fe base material is Alloy $49^{\circledR}$ produced by Carpenter Technology [35]. The other two materials are discussed in more detail in the next section.

\section{Amorphous and Nanocrystalline Fe Alloys}

The amorphous iron-alloy and nanocrystalline iron-alloy tape-wound cores that I considered using in the $10 \mathrm{kHz}$ FTS were standard commercially available items from the Magnetic Metals Corporation [100], which uses the names Namglass ${ }^{\circledR} 1$ and Namglass ${ }^{\circledR}$ 4, respectively. Both materials use a base ribbon material supplied by other vendors. The base material for Namglass 1 is Metglas ${ }^{\circledR} 2605$ SA1 Alloy [106] (originally from Allied Signal/Honeywell), and for Namglass 4 it is Finemet ${ }^{\circledR}$ FT-3 from Hitachi Metals America [73]. In both cases, Magnetic Metals adds a proprietary insulation coating called Namlite ${ }^{\circledR}$ to the material to reduce eddy current losses in the wound cores "by $30 \%$ or more at higher frequencies, i.e. $5 \mathrm{kHz}$ or higher, compared to otherwise equivalent uncoated iron based amorphous alloys [101, 47]."

The amorphous iron-alloy and nanocrystalline iron-alloy are both produced by a chill-block melt spinning process. A stream of the molten metal alloy is impinged upon a cold substrate to cause the rapid cooling needed to bypass crystallization (long-range atomic order) and create a ribbon of material with an amorphous structure (random arrangement of atoms) [46]. Cooling rates as high as $1 \times 10^{6}{ }^{\circ} \mathrm{C} / \mathrm{sec}$ are reported in the literature [73]. The nanocrystalline iron-alloy material undergoes a subsequent annealing process that produces $10 \mathrm{~nm}$ grains surrounded by a $10 \%$ to $15 \%$ amorphous phase $[13,102]$. Both materials are relatively brittle when compared to the traditional soft magnetic materials, such as the $50 \% \mathrm{Ni}-50 \% \mathrm{Fe}$.

For the pure amorphous material, DeCristofaro [46] states: "In crystalline metals 
such as grain-oriented silicon steel, structural features of comparable size to domain walls, such as dislocations and grain boundaries, can impede domain-wall motion. Amorphous metals magnetize more easily than crystalline metals because they lack such features. . . . The eddy-current component of magnetic losses is also minimized in amorphous metals. The atomic disorder and high solute content (metalloid and nonmetal components) of amorphous metals limit the mean-free path of electrons, resulting in electrical resistivity two to three times those of crystalline alloys." Comparison of the resistivity values shown in Table 5.3 for the candidate materials verifies this last point.

In the case of the nanocrystalline material, the manufacturer's literature is not specific about the mechanism for explaining why the nano-scale grains result in high permeability and low loss. In a paper published by Hitachi Metals, Ltd. in 1988, Yoshizawa [152] states: "The mechanism whereby FINEMET shows excellent soft magnetic properties is not fully understood. However, it is suggested that this is due to the decrease of local magnetic anisotropy by reducing the grain size and lower magnetostriction than Fe-based amorphous alloys." Based on the discussion on total power losses later in Section 5.5.3, the improved magnetic properties of the nanocrystalline material may also be due to the smaller micro eddy currents and lower domain wall velocities associated with a very fine grain structure.

\section{Iron Powder Polymer Composites}

Powdered iron-alloys having attractive soft magnetic properties can be compacted into net-shape components. Hanejko and Rutz [70], [134], and [71] describe powdered iron-alloys that are commercially available from the Hoeganaes Corporation [75]. An extrapolation of data that I found in the literature indicates that a compacted iron powder polymer composite intended for AC flux applications has a core loss that is a factor of 2.5 higher than that of 0.001 " laminations of $50 \% \mathrm{Ni}-50 \%$ Fe for a flux density of $5 \mathrm{kG} \mathrm{rms} \mathrm{at} 10 \mathrm{kHz}$. Details on that extrapolation are provided later in this section. More significantly for the $10 \mathrm{kHz}$ FTS, the non-magnetic material between the iron particles causes the permeability of the powdered iron composite 
to be at least ${ }^{8}$ a factor of 50 lower than that of the $50 \% \mathrm{Ni}-50 \% \mathrm{Fe}$. To be fair, it is worth noting that iron powder polymer composites are useful for producing complicated three-dimensional shapes with modest core losses, especially if the extra Ampere-turns of MMF needed to drive the flux is not an issue.

For DC applications an iron-based powder is compacted into the desired shape and then typically sintered to increase the part strength. For AC applications the iron powder is coated with a polymer that bonds the particles together while providing electrical insulation between them. The non-magnetic material in the spaces between the iron particles act as distributed air gaps in the core, significantly decreasing the net magnetic permeability of the material. AC powdered metal components are not sintered after compaction because it would produce physical contact between the iron particles that would ruin the bulk resistivity of the material. The compacted materials are brittle (no elongation during tensile testing), have transverse rupture strengths in the range of 12,000 psi to 35,000 psi depending on whether or not a thermal curing step occurs after compaction, and have a modulus of elasticity of approximately $0.5 \times 10^{6}$ psi $[70,134,69]$. Note that the modulus of elasticity is in the range of typical values for engineering plastics, suggesting that the stiffness of the compacted iron powder polymer is controlled by the plastic matrix.

The compacted iron powder polymer composite that I considered using for the rotor and stator cores is $\mathrm{TC} 80^{\circledR}$, which is commercially available from the Hoeganaes Corporation [75]. Table 5.2 provides a comparison of some of the physical and mechanical properties of $\mathrm{TC} 80^{\circledR}$ to those of the other materials that I considered. The TC80 ${ }^{\circledR}$ powder is intended for high frequency applications, and has an oxide coating in addition to the polymer coating to increase electrical resistivity and therefore decrease eddy current losses. The amount of polymer in the TC $80{ }^{\circledR}$ material is $0.75 \%$ by weight, or approximately $5 \%$ by volume [69]. The composite density for TC $80^{\circledR}$ is $7.2 \mathrm{~g} / \mathrm{cc}$, it has a maximum relative permeability of 210, and a saturation flux density in the range of $18 \mathrm{kG}$ peak [134]. The flux density values reported in [70, 134, 71] are

\footnotetext{
${ }^{8}$ I say "at least" a factor of 50 lower because the permeability data that I found reports a maximum DC relative permeability of 210 . I expect the permeability to be lower at the frequencies of interest for the $10 \mathrm{kHz}$ FTS.
} 
for the composite material [69].

The following extrapolation of available data indicates that the $\mathrm{TC} 80^{\circledR}$ powder has the same core loss as 0.001 " thick laminations of $3 \% \mathrm{Si}-97 \%$ Fe for a flux density of $5 \mathrm{kG}$ rms at $10 \mathrm{kHz}$. Rutz [134] provides data showing that the core loss of TC80 ${ }^{\circledR}$ at $5 \mathrm{kG}$ rms and $10 \mathrm{kHz}$ was a factor of nine less than the loss in 0.007 " thick laminations of non-oriented $3 \% \mathrm{Si}-97 \%$ Fe. Using the core loss data on tape laminates from [101], I found that 0.001 " thick laminations of oriented $3 \% \mathrm{Si}-97 \%$ Fe have the same loss as TC80 ${ }^{\circledR}$ at that flux density and frequency. It appears reasonable to extrapolate that the core loss for both of those materials is comparable at $11 \mathrm{kG} \mathrm{rms} \mathrm{at} 10 \mathrm{kHz}$, when considering: (1) that at higher frequencies the eddy current losses dominate and the skin depth for the laminate materials being considered are all at least equal to the half-thickness, and (2) the permeability for the $\mathrm{Si}-\mathrm{Fe}$ at $11 \mathrm{kG}$ rms is higher than at $5 \mathrm{kG}$ rms. At $11 \mathrm{kG} \mathrm{rms}$ and $10 \mathrm{kHz}$, the core loss for 0.001 " thick laminations of $3 \% \mathrm{Si}-97 \% \mathrm{Fe}$ is 2.5 times higher than it is for the same thickness of $50 \% \mathrm{Ni}-50 \% \mathrm{Fe}$ (see Table 5.4).

Compared to 0.001 " laminations of $50 \% \mathrm{Ni}-50 \% \mathrm{Fe}$, the estimated 2.5 times higher

core loss for the $\mathrm{TC} 80^{\circledR}$ and its factor of 50 times lower relative permeability — which would require a significantly higher MMF to drive flux in the core - motivated me to not use iron-powder polymer composites for the stator and rotor cores. Future work involving a complicated rotor or stator shape should include investigating whether or not the potential benefits of net-shaped powder metallurgy components would outweigh the drawbacks of higher core loss and required MMF.

\section{Ferrites}

Ferrites are ceramic oxides having a resistivity that is roughly a million times higher than that of the magnetic alloys already discussed, making their eddy current losses insignificant in comparison. I considered using ferrites for the rotor and stator cores, but a low saturation flux density in the range of $0.3-0.5 \mathrm{~T}$ [38] renders them unsuitable for use in a high acceleration actuator, where a high force-to-inertia ratio is required. 
Table 5.2: Selected physical and mechanical properties for some of the candidate soft magnetic materials.

\begin{tabular}{|c|c|c|c|c|}
\hline & $\begin{array}{c}50 \% \mathrm{Ni}-50 \% \mathrm{Fe} \\
\text { (a) }\end{array}$ & $\begin{array}{c}\operatorname{Namglass}^{\circledR}{ }^{\circledR} \\
\text { (b) }\end{array}$ & $\begin{array}{l}\operatorname{Namglass}^{\circledR}{ }^{(} 4 \\
\text { (c) }\end{array}$ & $\begin{array}{l}\mathrm{TC}^{(\mathrm{R})} \\
\quad(\mathrm{d})\end{array}$ \\
\hline Relative permeability & $\begin{array}{c}10,000 \\
\text { to } 20,000(\mathrm{i})\end{array}$ & $\begin{array}{c}9,000 \\
\text { to } 10,000(\mathrm{i})\end{array}$ & $\begin{array}{c}50,000 \\
\text { to } 80,000(\mathrm{i})\end{array}$ & $210(\mathrm{j})$ \\
\hline $\begin{array}{l}\text { Modulus of elasticity } \\
\text { (psi) }\end{array}$ & $24 \times 10^{6}(\mathrm{e})$ & $14.5 \times 10^{6}$ & $17.3 \times 10^{6}$ & $0.5 \times 10^{6}$ \\
\hline $\begin{array}{c}\text { Tensile strength } \\
(\mathrm{psi})\end{array}$ & $130 \times 10^{3}(\mathrm{e})$ & $\begin{array}{l}145 \times 10^{3} \\
\text { to } 290 \times 10^{3}\end{array}$ & $308 \times 10^{3}$ & $\mathrm{n} / \mathrm{a}$ \\
\hline $\begin{array}{l}\text { Transverse rupture strength } \\
\qquad(\mathrm{psi})\end{array}$ & $\mathrm{n} / \mathrm{a}$ & $\mathrm{n} / \mathrm{a}$ & $\mathrm{n} / \mathrm{a}$ & $\begin{array}{l}12 \times 10^{3} \\
\text { to } 35 \times 10^{3}(\mathrm{f})\end{array}$ \\
\hline $\begin{array}{c}\mathrm{CTE} \\
\left(\times 10^{-} 6 /{ }^{\circ} \mathrm{F}\right)\end{array}$ & 4.61 & 4.2 & 4.66 & (h) \\
\hline $\begin{array}{l}\text { Thermal conductivity } \\
\left(\mathrm{W} / \mathrm{m} /{ }^{\circ} \mathrm{K}\right)\end{array}$ & 13.01 & $(\mathrm{~g})$ & 8.15 & $(\mathrm{~h})$ \\
\hline \multicolumn{5}{|c|}{ (a) Alloy $49^{\circledR}$ cold-rolled strip base material from Carpenter Technology [35]. } \\
\hline \multicolumn{5}{|c|}{$\begin{array}{l}\text { (b) Metglas }{ }^{\circledR} \text { 2605SA1 amorphous iron-alloy base material } \\
\text { from Allied Signal/Honeywell [106]. }\end{array}$} \\
\hline \multicolumn{5}{|c|}{$\begin{array}{l}\text { (c) Finemet }{ }^{\circledR} \text { FT-3 nanocrystalline iron-alloy base material } \\
\text { from Hitachi Metals America }[73,72,13] \text {. }\end{array}$} \\
\hline \multicolumn{5}{|c|}{ (d) Iron powder polymer composite from Hoeganaes Corp [70]. } \\
\hline \multicolumn{5}{|c|}{ (e) Cold rolled strip. } \\
\hline \multicolumn{5}{|c|}{ (f) Higher value if thermally cured after compaction. } \\
\hline \multicolumn{5}{|c|}{$\begin{array}{l}\text { (g) Not reported in sources cited. Since "glass-like", } \\
\text { expect it to be lower than that of } 50 \% \mathrm{Ni}-50 \% \mathrm{Fe} \text {. }\end{array}$} \\
\hline \multicolumn{5}{|c|}{$\begin{array}{l}\text { (h) Not reported in sources cited. Since plastic matrix, expect it to be } \\
\text { less favorable than } 50 \% \mathrm{Ni}-50 \% \text { Fe for a precision machine application. }\end{array}$} \\
\hline \multicolumn{5}{|c|}{ (i) For $2-11 \mathrm{kG}$ rms at $10 \mathrm{kHz}[100]$. } \\
\hline \multicolumn{5}{|c|}{ (j) Maximum DC permeability [134]. } \\
\hline
\end{tabular}




\section{Stainless Steel for the Back-Iron}

The back-iron material carries a DC flux equal to one half of the peak flux in the rotor/stator air gaps. During steady-state operation the DC flux does not generate hysteresis or eddy current losses, so a solid iron-based material is used. The DC flux and lower peak flux level in the back-iron allows considering steels that have attractive mechanical and fabrication properties. The lower permeability of structural steels relative to the soft magnetic materials used for the rotor and stator cores - requires considering the drop in magnetomotive force in the back-iron when sizing the coil used to produce the DC bias flux. That MMF drop is explored in Section 5.2.7.

I specified the use of 410,416, or 430F stainless steel for the DC-flux portions of the magnetic circuit because they were all acceptable from a magnetic standpoint, and I did not want to over-constrain the machine shops that fabricated the components. The relative permeability for these ferromagnetic stainless steels is reported by the American Society for Metals [58] to be in the range 600-1000; I picked 500 as a conservative value. I chose 416 and $430 \mathrm{~F}$ because of their high machinability ratings of $110 \%$ and $91 \%$, respectively, and included 410 because some machine shops keep this material in their stock room and consider it fine to work with even though its machinability rating is 54\%. The machinability ratings are from [36] and [58], and are relative to a $100 \%$ machinability rating for 1212 carbon steel. As a comparison, properly annealed high-purity cast magnetic ingot iron has a relative permeability in the range 1500-3500 [85]. I choose stainless steel over cast iron because the differences in permeability were not an issue for the $10 \mathrm{kHz}$ FTS; there was ample room to accommodate the larger coil and power supply needed to produce a $0.75 \mathrm{~T}$ bias flux in the stainless steel. If the size and weight of that coil and its power supply where an issue - for example, if the actuator was going to be onboard an air- or space-borne mission - then the choice of material for the back-iron would have to be revisited. As an aside, weight and size issues would most likely motivate the use of a permanent magnet instead of a coil for producing the bias flux. Ultimately, for the $10 \mathrm{kHz}$ FTS I chose stainless steel for the following pragmatic reason. Prototype machines tend to 
have an initial flurry of activity, then sit idle and unused for a long time. If continued work with the $10 \mathrm{kHz}$ FTS did not occur after completing this research, I did not want the machine to be a rusted paperweight.

\subsubsection{Power Loss in Soft Magnetic Materials}

Magnetic hysteresis and eddy currents are the two loss mechanisms usually considered for a soft magnetic material subjected to an alternating (AC) magnetic field. Rather than attempt to formulate an accurate prediction of total power loss based on theory, the discussions in this section are used to establish a scientific framework for understanding soft magnetic materials and to motivate the use of empirical loss data to design the actuator.

Introductory descriptions of the behavior of magnetic materials and ways to quantify hysteresis and eddy current losses can be found in [19], [56], [67], [79], [150], and [105]. A deeper treatment of these loss mechanisms and additional ones, and discussions on the concept of magnetic domains and their behavior as one traces out the B-H curve are presented by "The Vacuumschmelze Handbook" [24], Chen [38], and Jiles [85]. The Vacuumschmelze Handbook and work by Chen are contemporaries of each other, both being originally published in 1977. The handbook presents an excellent discussion accessible to an engineer not already familiar with the topic, while the later is a monograph providing both a clear discussion of fundamental principles and greater theoretical detail. The book by Jiles is a more recent (1998) text book on the subject of magnetic materials. A practical discussion on basic magnetics that complements the manufacturer's data on the tape laminates used to build the actuator for the $10 \mathrm{kHz}$ FTS is given by the Arnold Engineering Company [14]. The following is an amalgamated distillation of what I learned from these sources.

\section{Background on Soft Magnetic Materials}

The ferromagnetic materials used to guide magnetic flux have magnetic domains that will align with the direction of an applied magnetic field. In metallic magnetic 
materials each crystal in the grain structure has multiple magnetic domains in it. Qualitatively speaking, each magnetic domain can be thought of as a source of magnetic flux, like a tiny bar magnet. ${ }^{9}$ When a magnetic domain aligns with an applied magnetic field the total magnetic flux in the field will increase. Generally speaking, a "soft" magnetic material requires less energy to reorient the magnetic domains than a "hard" one does, and so the flux-guiding portions of a magnetic circuit use soft magnetic materials to minimize the energy loss when it is desired to change the flux density in the circuit. In the case of iron-based alloys, soft and hard correspond to the mechanical hardness of the material.

When a magnetic field is applied to a material that initially has randomly oriented magnetic domains, energy goes into the material to change the size and orientation of the magnetic domains to align them with the applied field. This is accomplished first by domain growth through domain wall movement, and second by domain rotation. A certain amount of the energy put into the material is non-recoverable and is dissipated as heat, resulting in the hysteretic behavior of the material when the applied magnetic field is reversed. Figure 5-16 shows a sketch of hysteresis curves that is typical of the "round loop" soft magnetic materials considered for the actuator cores. ${ }^{10}$ The horizontal and vertical scales of the hysteresis curves shown in Figure 5-16 were chosen for illustrative purposes, as were there shapes and sizes of the hysteresis loops.

If a material initially has randomly oriented magnetic domains then the net magnetic flux per unit area, or flux density $B$, is zero. As the intensity of an applied magnetic field is increased from zero, domains that were already aligned with the applied field grow by moving their domain walls, and cause an attendant increase in the magnetic flux density through a surface that is perpendicular to the direction of the applied field. At some point, while increasing the applied magnetic field intensity $H$, domain walls will encounter obstacles in the form of impurities or imperfections in the grain structure of the material. The domain walls minimize their free energy by

\footnotetext{
${ }^{9}$ Chen in [38, page 66] provides an excellent description of the magnetic domain theory proposed by Pierre Weiss in 1929, and an ample theoretical discussion supporting it. Jiles [85] devotes three chapters to discussing magnetic domains, domain walls, and domain processes.

${ }^{10}$ The three typical shapes for hysteresis loops are round loop ("Form R"), rectangular loop ("Form Z"), and flat loop ("Form F") [24].
} 
"sticking" to these obstacles. If the applied field was reduced before the domain walls reached these obstacles then the domains would return to their original size without energy loss (no hysteresis). This region of operation corresponds to the initial, low slope portion of the B-H curve (near point " 0 " in Figure 5-16). On the other hand, once a domain wall attaches to an impurity or imperfection a finite amount of nonrecoverable energy is required to free it. In the case of an increasing applied magnetic field, motion of the domain wall pauses until sufficient energy is built up to allow the domain wall to free itself from the obstacle in what is called a "Barkhausen jump", which is the source of the hysteretic energy loss [38, 24]. Since Barkhausen jumps depend on the presence of impurities and imperfections in the grain structure, hysteresis is affected by heat treatment or cold-working of the material. The steep portions of the B-H curve correspond to the domain growth associated with Barkhausen jumps. As the applied magnetic field continues to be increased, the domain walls eventually stop moving and further alignment of the domains is accomplished by rotation of the electron spin axes that are not already aligned with the applied field. The transition from Barkhausen jumps to rotation of the spin axes corresponds to the knee of the B-H curve, and rotation of the spin axes is a reversible process [38]. As the magnetic field intensity continues to be increased fewer non-oriented magnetic domains are available to contribute flux, so the rate of flux density increase falls off, and the material is said to be entering magnetic saturation. Eventually, all of the available magnetic domains become oriented with the applied magnetic field, at which point the material is fully saturated. The absolute permeability $\mu$ of the material is the ratio of the flux density $B$ divided by the magnetic field intensity $H$, and for soft magnetic materials its value depends on the magnitude of the magnetic field intensity. Thus, the absolute permeability provides a measure of how much the flux density increases for a unit increase in the magnetic field intensity at a given operating point on the B-H curve. ${ }^{11}$ The references sometimes refer to the induced magnetic flux density $B$ as the "induction".

\footnotetext{
${ }^{11}$ The relative permeability $\mu_{r}$ is customarily used for soft magnetic materials, and is the ratio of the absolute permeability $\mu$ divided by the permeability of free space $\mu_{0}$.
} 


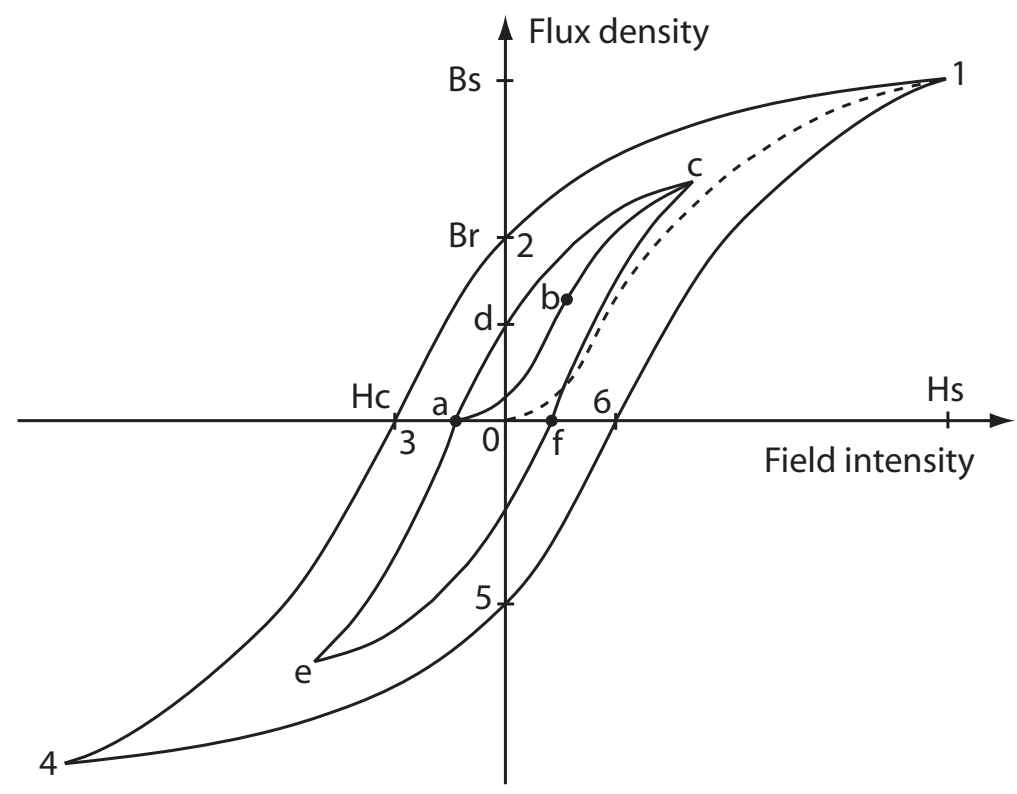

Figure 5-16: Magnetic flux density versus magnetic field intensity for a single, saturating AC cycle for virgin soft magnetic material with a zero initial condition (01234561); a minor hysteresis loop for a symmetrical AC cycle (fcdaef); and a minor hysteresis loop for a one-sided AC cycle (abcda).

\section{Hysteresis Loss}

Figure 5-16 illustrates how the magnetic flux density in a soft magnetic material responds to a single, saturating $\mathrm{AC}$ cycle of a magnetic field, starting with virgin material and a zero initial condition. At point " 0 " the magnetic domains are randomly oriented and the material has a permeability close to but greater than the permeability of free space $\mu_{0}$. The low slope of the B-H curve at point " 0 " indicates that the initial incremental increase in applied magnetic field does not cause as much domain growth as subsequent increases will. As the curve proceeds along the dashed line from point " 0 " to point " 1 " there is a steep portion corresponding to the maximum permeability for the material, followed by a reduction in the slope and hence permeability as the material enters saturation. Eventually, the material becomes saturated at point "1", and starts to act more like free space as the magnetic field intensity is increased further, and hence the permeability at point "1" is approaching the permeability of free space $\left(\mu_{0}\right)$. When the magnetic field intensity is decreased from its saturation value $\mathrm{H}_{s}$ the flux density decreases from its saturation value $\mathrm{B}_{s}$ and the curve proceeds 
from point " 1 " to point "2". At point "2" the curve meets the B-axis at the point $\mathrm{H}=0, \mathrm{~B}=\mathrm{B}_{r}$. At this $\mathrm{B}$-axis crossing some of the magnetic domains in the material are still aligned to the past direction of the applied magnetic field, and so continue to produce a remanent flux density $\mathrm{B}_{r}$ in the absence of an applied magnetic field. To unalign these magnetic domains the magnetic field intensity has to be reversed to a field strength corresponding to the coercivity $\mathrm{H}_{c}$ of the material at point " 3 ". In effect, the material had become partially magnetized in going from point "0" to "1" to " 2 ", and has to be demagnetized between points "2" and " 3 " before the flux density can change directions. The cycle then continues from point " 3 " to " 4,5 , and 6 ", and then back to "1" to trace out the major hysteresis loop.

During the process of aligning the magnetic domains energy is dissipated as heat in the material. It can be shown $[19,56,67]$ that the energy loss per cycle due to hysteresis is equal to the area of the hysteresis loop on a B-H curve. Power loss due to hysteresis is found by multiplying the loop area by the operating frequency and volume of the material, assuming a consistent set of units. ${ }^{12}$ Roughly speaking, the coercivity $\mathrm{H}_{c}$ of a material provides a sense of the width of its hysteresis loop and therefore a relative measure of its hysteresis loss compared to other soft magnetic materials having similarly shaped hysteresis curves. Equation 5.99 provides a simplified expression for hysteresis power loss to illustrate the linear dependency on loop area and frequency. If the SI system is used with frequency expressed in $(\mathrm{rad} / \mathrm{sec})$, then $\forall_{H}$ is the volume of the core in $\mathrm{m}^{3}$.

$$
P_{H}=\forall_{H}(\text { loop area) (frequency) }
$$

The nature of the magnetic flux in the magnetic circuit for the hybrid rotary/linear actuator depends on the location in the circuit. Figure 5-15 provides a schematic representation of the magnetic flux in the actuator. The back-iron carries a DC flux, the rotor core carries a predominantly symmetrical AC flux, and the stator cores

\footnotetext{
${ }^{12}$ The SI system provides a consistent set of units: $[\mathrm{B}]=$ Tesla $=$ volt $\cdot \mathrm{sec} /$ meter $^{2},[\mathrm{H}]=\mathrm{amp} /$ meter. Therefore: $[\mathrm{B}][\mathrm{H}]=$ volt.amp.sec $/ \mathrm{meter}^{3}$, which is energy per unit volume. Expressing frequency in $(\mathrm{rad} / \mathrm{sec})$ provides volumetric power loss in $\mathrm{W} / \mathrm{m}^{3}$.
} 
carry a unipolar (one-sided) AC flux.

Referring to Figure 5-16, the minor hysteresis loop (fcdaef) represents the nonsaturating symmetrical AC flux in the rotor core, and the minor hysteresis loop (abcda) represents the one-sided AC flux in the stator core. Point "b" corresponds to the flux density produced by the DC bias flux, and point "c" corresponds to the maximum flux density achieved in the rotor/stator air gaps of the actuator (ignoring sign). Note for both cases that when at point "c", merely reducing the field intensity to zero (point "d") is not sufficient to "extinguish" the flux in the air gap. The field intensity needs to be reversed to the value corresponding to point "a" to achieve zero flux in the air gap. For design purposes, since the material manufacturer's core loss data is based on a symmetrical AC flux, I assume that the losses associated with the one-sided AC flux in the stator cores is approximately the same as the losses for a symmetrical AC flux with the same peak-to-peak flux density.

\section{Eddy Current Loss}

According to Faraday's law, a time-varying magnetic field will produce an electric field. If this occurs in a conductor then the electric field will create an electric current which, according to Ampere's law, produces its own magnetic field. Lenz's law states that the direction of the current produced is such that the magnetic field produced by that current tends to cancel the change in the applied magnetic field. Accordingly, the AC magnetic flux in an electrically conducting magnetic material gives rise to electric currents that flow in circles ("eddies") whose axes of rotation are everywhere aligned with the applied magnetic field. The eddy current produces an ohmic power loss in the material $P_{E}$. It can be shown that for a fully penetrating applied magnetic field that the eddy current loss is proportional to the squares of the frequency, flux density, and thickness of the flux carrying material, and inversely proportional to the resistivity of the material $[19,38]$. Therefore, for a given time-varying applied magnetic field, the magnitude of the eddy current can be reduced by decreasing the cross-sectional area of the material carrying the magnetic flux and by increasing its resistivity. This leads to the ubiquitous use in AC magnetic circuits of thin laminates 
with a high electrical resistivity relative to pure iron. Equation 5.100 provides a simplified expression for the eddy current power loss to illustrate this relationship, where $K_{E}$ is a constant of proportionality.

$$
P_{E}=K_{E} \frac{(\text { frequency })^{2}(\text { flux density })^{2}(\text { thickness })^{2}}{(\text { resistivity })}
$$

The magnetic field produced by the eddy current has a cancelling effect on the applied AC magnetic field and inhibits its penetration into the full thickness of the material intended for carrying magnetic flux. This cancelling effect is zero at the surface of the material and increases with depth into the material, giving rise to the concept of a "skin depth" for the material, which is the depth at which the applied magnetic field is reduced to $1 / e$ times its value at the surface [85]. Roughly speaking, limiting the thickness of the material to $1 / 3$ of the skin depth (or less) assures a thorough penetration of the applied magnetic field and efficient use of the magnetic flux carrying material. Professor Kirtley at MIT pointed out to me that the skin depth is also the same as the thickness of a conductor carrying a uniform current with the same voltage gradient (loss). The skin depth $\delta$ is given by Equation 5.101 [19], where $\omega$ is the frequency of the $\mathrm{AC}$ magnetic field, $\mu$ is the magnetic permeability of the material, and $\rho$ is the electrical resistivity of the material.

$$
\delta=\sqrt{\frac{2 \rho}{\omega \mu}}
$$

Agarwal [10] describes a method of calculating eddy current losses in saturating iron. Although this more accurately represents the case for the actuator in the $10 \mathrm{kHz}$ FTS than full penetration does, that refinement is not pursued here. The full penetration discussion above serves the purpose of illustrating the square law dependency of eddy current power loss on frequency, flux density, and thickness, and its linear relationship to the electric resistivity of the material, setting the stage for considering the total power loss. 


\section{Total Power Loss in Soft Magnetic Materials}

A comparison of Equations 5.99 and 5.100 reveals the dominance of hysteresis loss at low frequencies. The square law proportionality of eddy current loss to frequency results in its dominance above a certain frequency. In practice however, adding the theoretical hysteresis and "classical" eddy current losses does not account for the entire energy loss observed in a thin soft magnetic material subjected to an AC flux.

Chen [38, page 147] describes an "anomalous energy loss" or "eddy-current anomaly" that becomes prominent as the sheet thickness becomes smaller than approximately five widths of the magnetic domains. He describes that the there are "micro" eddy currents in each magnetic domain, and that the microcurrents of different domains are not aligned to each other, so that the classical treatment of the "macro" eddy current (described earlier) underestimates the total ohmic power loss. Chen illustrates how the anomalous energy loss dominates over the hysteresis and classical eddy current losses as the peak flux density or frequency is increased, and points out that a finer domain structure reduces the role of the anomalous energy loss relative to the classical eddy current loss: "If the domain structure is very fine, meaning the number of domain walls is enormously large, the velocity of an individual wall is expected to be small, as would be the induced microcurrents. The magnetization then should be quite homogeneous, and the classical eddy-current loss caused by the macrocurrents in the second term should dominate [38, page 150]." This is consistent with my hypothesis earlier in Section 5.5.2 regarding the low energy loss in nanocrystalline iron-alloy material. Furthermore, Overshott and Foot [119] describe experimental results that correlate a decrease in hysteresis and anomalous eddy current losses with a decrease in domain wall spacing.

Boll, on the other hand, attributes the eddy current anomaly to electron "spin relaxation" and "spin precession" [24, page 40, 74]. Energy is required to overcome the angular momentum of the electron spin and work against the precession of the spin axis to reorient the axis with the applied field. Boll goes further and describes a "gyromagnetic limiting frequency" as an upper limit on the frequency of an AC 
magnetic flux that a material will allow, attributes this limit to the spin precession, and shows that it is in the megahertz range for metal and ferrite soft magnetic materials [24, page 75]. Chen also discusses damping due to "field-induced precessions of the spin axes" [38, page 161].

\subsubsection{Mechanical Design Considerations}

The permeability of metallic soft magnetic materials is strongly influenced by the grain structure of the material [38, 24], and plastic deformation of the material certainly affects the grain structure. A specific concern for the $10 \mathrm{kHz}$ FTS is whether or not the pole faces of the rotor and the stator can be allowed to make energetic mechanical contact with each other, as would be the case if that contact is the defacto "hard stops" for the actuator. Baudouin and Houbaert [17] conducted recent experiments that quantify the effects of uniaxial deformation on the magnetic properties of electrical (soft magnetic) steel. Test pieces of $0.65 \mathrm{~mm}$ (0.025 inch) thick $3 \%$ Silicon non-oriented steel were mechanically deformed in a controlled manner, then their magnetic properties including permeability were measured. Using a flux density of $1 \mathrm{~T}$ at $50 \mathrm{~Hz}$ they found that the maximum permeability was reduced by an asymptotically approached value of $65 \%$ for mechanical strains in the range of 6 to $15 \%$. Rupture of the specimens occurred at tensile strains greater than $22 \%$, so an upper limit of a $70 \%$ reduction in permeability due to work hardening seems to be a reasonable value for assessing an order-of-magnitude effect.

A reduction in the permeability of the rotor or stator cores would require additional Ampere-turns of MMF to produce the desired air gap flux. From the standpoint of the prototype $10 \mathrm{kHz}$ FTS, a more serious concern regarding repeated energetic contact between the rotor and stator cores is an increase in core loss due to electrical shorting of the laminations. This is a main motivation for increasing the width of the rotor core in the $10 \mathrm{kHz}$ FTS so that it extends laterally past the stator pole faces. The extended portions of the rotor core form hard stops with the non-magnetic braces that surround the stator poles. The area of mechanical contact on the rotor core is outside of the intended AC flux paths, so to first order the additional core loses 
from repeated mechanical contact are lower than they would be if contact occurred at regions in the AC flux paths. Regarding a decrease in permeability caused by mechanical strain, during the design of the $10 \mathrm{kHz}$ FTS careful attention was paid to avoid creating stress concentrations in the $\mathrm{AC}$ flux carrying components due to mechanical connections and attachments.

\subsubsection{Machining Tape-Wound Cores}

Tape-wound cores are usually intended for use in transformers and inductors. A closed-loop shape is built up by winding the tape onto a mandrel, and an epoxy adhesive is used to bond the layers together. The resulting core is typically cut into two C-shaped halves with an abrasive cut-off saw, then the cut surfaces are polished flat and chemically etched to remove material that might be electrically shorting the laminations together. The rotor and stator cores for the $10 \mathrm{kHz}$ FTS required tighter dimensional and geometric form tolerances than those associated with standard cut tape-wound cores. Therefore I obtained oversized, un-cut tape-wound cores as a starting point for producing the laminated cores for the $10 \mathrm{kHz}$ FTS. I chose wire electro-discharge machining (wire-EDM) as the process for cutting the tape-wound cores because it allows maintaining the requisite dimensional tolerances, and it seemed plausible that it would allow machining the cores without tearing the relatively weakly bonded lamination layers from each other.

I searched for literature that discussed machining tape-wound cores but did not find any. The closest pertinent paper that I found is by Emura [53], which discussed the influence of various cutting processes on the magnetic properties of single laminations of electrical steel. He found that cutting affects the permeability at $1.5 \mathrm{~T}$ more than it affects power loss. Unfortunately, wire-EDM was not among the processes that he considered - punching, guillotine cutting, laser cutting, and photo-corrosion.

Based on power loss predictions, I down-selected to two candidate materials for the rotor and stator cores: $50 \% \mathrm{Ni}-50 \% \mathrm{Fe}$ and nanocrystalline iron-alloy. The $50 \% \mathrm{Ni}-$ $50 \%$ Fe tape-wound cores can easily be peeled apart with a knife or fingernail, but the base material is fairly ductile. The nanocrystalline iron-alloy tape-wound cores are 
much tougher to peel apart, and the base material is significantly glass-like and brittle. I conducted quick experiments to confirm that both materials could be easily handsanding, both parallel and perpendicular to the lamination layers. This indicating that grinding would be a viable process if appropriate attention was paid to backing up the material to prevent roll-over of the edges of the laminations.

I established a small development contract with a local machine shop [42] that specialized in wire-EDM, and supplied them with two different types of tape-wound cores made from $0.001 "$ thick material $-50 \% \mathrm{Ni}-50 \% \mathrm{Fe}$ and nanocrystalline ironalloy — that I obtained from the Magnetic Metals Corporation [100]. The amorphous iron-alloy appeared to be physically similar to the nanocrystalline iron-alloy, so I assume that the machinability of the two is similar. The most significant challenge was establishing electrical continuity with every lamination layer in the core so that the wire-EDM process would work. We found that the nanocrystalline iron-alloy cores were more prone to localized burning and blow-out of the material, suggesting poor electrical continuity between one or more laminations in the damage zone and the wire EDM setup. The poor electrical continuity could have been due to fractures or voids in the core blank material, or due to inadequate fixturing of the workpiece in the wire EDM setup. The lack of this type of localized damage in the $50 \% \mathrm{Ni}-50 \% \mathrm{Fe}$ cores suggests that the wire EDM setup was probably adequate and that the damage in the as-machined nanocrystalline iron-alloy cores is most likely due to discontinuities in the blank material. Grinding was successful with both materials. In all cases, the as-machined surfaces have shorted lamination layers. I conducted a series of simple experiments to explore removing the shorted material using an acid-based etchant, but they were not successful. Section 6.4 includes suggested future work to address the shorted laminations caused by machining.

\subsubsection{Comparison of Candidate Soft Magnetic Materials}

Data published by the Magnetic Metals Corporation [101] for the standard, commercially available tape-wound cores that I considered for the rotor and stator cores are compiled in this section. That data includes empirical loss data based on power 
measurements for samples subjected to the flux densities and frequencies of interest for the $10 \mathrm{kHz}$ FTS. For the purposes of comparison, I consider a peak flux density of 1.6 Tesla (11 kG rms) and a sinusoidal operating frequency of $10 \mathrm{kHz}$. Some of the materials have useable peak flux densities below $1.6 \mathrm{~T}$, which I note as appropriate.

The manufacturer's core power loss data is presented in [101] as a series of fairly straight lines on a log-log plot of power loss (ordinate) versus flux density (abscissa). The slopes of the lines are in the range of 1.7 to 2.0, indicating that the core losses roughly vary with the square of the flux density, and are therefore dominated by eddy current losses.

In this section I develop and use normalized acceleration factors and normalized core power losses to compare the relative merits of the candidate materials. I chose 0.001 " thick laminations of $50 \% \mathrm{Ni}-50 \%$ Fe with a peak flux density of $1.6 \mathrm{~T}$ at $10 \mathrm{kHz}$ as a baseline for these comparisons because it is the best choice among the conventional soft magnetic metals. Since achieving a high tool tip acceleration is a prime goal for the $10 \mathrm{kHz}$ FTS, producing high forces on the pole faces of the rotor is necessary but not sufficient. Therefore, in addition to considering the maximum usable flux density and power loss for the materials, the mass density of the rotor core material is considered.

\section{Flux Density in the Core and Material Working Density}

Tape-wound cut cores are primarily used as transformer cores, so producing an acceleration of the core material is not a typical concern for the designer using them. There are certain unstated assumptions in the Magnetic Metals Corporation data [101] that may not be immediately apparent to a reader not already familiar with transformer design, and they are important for designing the actuator. I gathered the following four points through conversations with one of their engineers [47].

1. Flux density values are rms.

2. There are spaces between the laminations in a stack that do not carry magnetic flux. "Stacking factor" $S F$ is used to indicate the height fraction of a stack of 
laminations that is actually the desired soft magnetic material.

3. Flux density data is based on the "net" cross-sectional area of a core, not the "gross" or physical cross-sectional area of the stack of laminations. The difference between net and gross area is best explained with an example. Assume a stack of laminations with a physical cross-sectional area of $\left(1^{\prime \prime} \times 1^{\prime \prime}\right)$ and a stacking factor of 0.75 . The gross area is equal to the physical area of 1 inch $^{2}$, which is also referred to as the composite area since it includes the area between the soft magnetic material. The net area is equal to the cross-sectional area of the intended magnetic material, which is $0.75 \mathrm{inch}^{2}$ in this case. This distinction is important to keep in mind when sizing the stator and rotor cores.

4. "Material working density" is a phrase coined in this thesis to take into account the mass of the non-flux carrying material in the spaces between the laminations, which adds deadweight to the rotor. The spaces between the laminations is partially filled with epoxy when the laminations are bonded together, and a $50 \%$ fill-factor is a reasonable estimate. The insulation on the laminations is an oxide coating with a thickness measured in Angstroms, so its weight can be neglected. Since we want to accelerate the rotor core, the mass of the epoxy — which represents a burden to the flux-carrying material — is considered. Using an estimated specific gravity of 1.0 for the epoxy, the material working density is obtained by adding $((1-S F) / S F)(0.5)(1 \mathrm{~g} / \mathrm{cc})$ to the density of the flux-carrying material.

\section{Normalized Acceleration Factor}

Normalized acceleration factors are used to take into account the different working densities and allowable peak flux densities for the candidate materials, and are based on the following argument. The angular acceleration of the rotor core is equal to the torque applied to it divided by its rotational inertia. The applied torque is proportional to the magnetic forces on the rotor core, which in turn are proportional to the square of the flux density in the rotor/stator air gaps. The rotary inertia is 
proportional to the material working density of the rotor. Therefore, the acceleration factor for a material is defined as the square of the flux density being considered divided by the material working density. The normalized acceleration factors are found by dividing all of the acceleration factors by the acceleration factor for the baseline 0.001 " thick laminations of $50 \% \mathrm{Ni}-50 \%$ Fe with a peak flux density of $1.6 \mathrm{~T}$ at $10 \mathrm{kHz}$.

\section{Comments on Tables 5.3 through 5.6}

A key point to observe in Table 5.3 is the lower maximum "usable" flux densities for $\mathrm{Ni}-\mathrm{Fe}-\mathrm{Mo}$ and nanocrystalline iron-alloy relative to the others. This results in lower normalized acceleration factors for these two low-loss materials - especially for the Ni-Fe-Mo - when compared to the others. Also note that the minimum thickness available for the $\mathrm{Co}-\mathrm{Fe}-\mathrm{V}$ is $0.002 "$.

In Table 5.4 the maximum flux density is set at $11 \mathrm{kG}$ rms $(1.6 \mathrm{~T} \mathrm{pk})$ to correspond with the value used in the trade-off study for the actuator in the $10 \mathrm{kHz}$ FTS (see Section 4.8). Notice that all of the materials provide essentially the same normalized acceleration factor, except for the Ni-Fe-Mo due to its lower maximum flux density. The other notable differences in Table 5.4 are the power losses, which can be as much as 2.8 times the loss for the baseline 0.001 " thick laminations of $50 \% \mathrm{Ni}-50 \% \mathrm{Fe}$.

In Table 5.5 the flux density for each material was reduced so that they all have the same power loss as the baseline 0.001 " thick laminations of $50 \% \mathrm{Ni}-50 \% \mathrm{Fe}$. Note that the power losses for Ni-Fe-Mo and nanocrystalline iron-alloy were already lower than that of the baseline, so the flux density for those materials was not lowered. The baseline material stands out for acceleration among the other conventional metal laminates, but the amorphous and nanocrystalline Fe alloys make a respectable showing with $80 \%$ and $93 \%$ normalized acceleration factors, respectively.

In Table 5.6 each material is operated at its maximum flux density. This comparison is done more for completeness than anything else. Note that Co-Fe-V could provide 1.8 times the acceleration of the baseline 0.001 " Ni-Fe if a factor of 4.7 increase in core power loss was acceptable, which it is not. 
Table 5.3: Raw material properties and typical values for tape-wound cut core materials. Based on data from Magnetic Metals Corporation [101, 47].

\begin{tabular}{|c|c|c|c|c|c|c|c|}
\hline & & & & & coercive & rms flus & density $^{\mathrm{c}}$ \\
\hline material & $\begin{array}{c}\text { density }^{\mathrm{a}} \\
(\mathrm{g} / \mathrm{cc})\end{array}$ & $\begin{array}{l}\text { resistivity } \\
(\mu \Omega-\mathrm{cm})\end{array}$ & $\begin{array}{l}\text { thick. } \\
\text { (in) }\end{array}$ & $\begin{array}{l}\text { ave. } \\
\mathrm{SF}^{\mathrm{b}}\end{array}$ & $\begin{array}{l}\text { force } \\
(\mathrm{Oe})\end{array}$ & $\begin{array}{l}\text { usable }^{\mathrm{d}} \\
(\mathrm{kG})\end{array}$ & $\begin{array}{c}\text { optimal }^{\text {e }} \\
(\mathrm{kG})\end{array}$ \\
\hline $3 \% \mathrm{Si}-97 \% \mathrm{Fe}$ & 7.67 & 47 & 0.001 & 0.79 & 0.6 & 14 & $11-13$ \\
\hline $3 \% \mathrm{Si}-97 \% \mathrm{Fe}$ & 7.67 & 47 & 0.002 & 0.87 & 0.5 & 16 & 11-13 \\
\hline $50 \% \mathrm{Ni}-50 \% \mathrm{Fe}$ & 8.25 & 45 & 0.001 & 0.79 & 0.1 & 15 & 11 \\
\hline $50 \% \mathrm{Ni}-50 \% \mathrm{Fe}$ & 8.25 & 45 & 0.002 & 0.87 & 0.1 & 15 & 11 \\
\hline $79 \% \mathrm{Ni}-15 \% \mathrm{Fe}-5 \% \mathrm{Mo}$ & 8.77 & 60 & 0.001 & 0.79 & 0.028 & 8 & 4 \\
\hline $49 \% \mathrm{Co}-49 \% \mathrm{Fe}-2 \% \mathrm{~V}$ & 8.15 & 26 & 0.002 & 0.87 & 0.18 & 20 & $11-16$ \\
\hline $\begin{array}{c}\text { Namglass }{ }^{\circledR} 1 \\
\text { amorphous iron-alloy }\end{array}$ & 7.19 & 130 & 0.001 & 0.75 & 0.2 & 15 & $4-12$ \\
\hline $\begin{array}{c}\text { Namglass }^{\circledR} 4 \\
\text { nanocrystalline iron-alloy }\end{array}$ & 7.3 & 120 & 0.001 & 0.75 & 0.008 & 10 & $1-9$ \\
\hline (a) Density of the flux-car & ying mate & & & & & & \\
\hline (b) "SF" $\equiv$ stacking facto & the heigl & fraction th & t carrie & flux i & a stack & laminat & \\
\hline (c) Flux density is based & the net & re area $(\mathrm{ph}$ & sical st & ck heis & ht times $S$ & F) $[47]$. & \\
\hline (d) "Usable" flux density & terminol & by used by & 01] to & dicate & a maximu & & \\
\hline
\end{tabular}

Table 5.4: Magnetic characteristics, acceleration factor, and power loss for tapewound cut core materials with a maximum flux density of $11 \mathrm{kG}$ rms (16 kG peak) at $10 \mathrm{kHz}$ sinusoidal. Based on data from Magnetic Metals Corporation [101, 47].

\begin{tabular}{|c|c|c|c|c|c|c|c|}
\hline material & $\begin{array}{l}\text { thick. } \\
\text { (in) }\end{array}$ & $\begin{array}{l}\text { working } \\
\text { density } \\
(\mathrm{g} / \mathrm{cc})\end{array}$ & $\begin{array}{l}\text { rms flux } \\
\text { density } \\
(\mathrm{kG})\end{array}$ & $\begin{array}{l}\text { relative } \\
\text { perm. }\end{array}$ & $\begin{array}{l}\text { skin } \\
\text { depth } \\
\text { (in) }\end{array}$ & $\begin{array}{l}\text { normal. } \\
\text { accel. } \\
\text { factor }\end{array}$ & $\begin{array}{c}\text { power } \\
\text { loss } \\
(\mathrm{W} / \mathrm{lb})\end{array}$ \\
\hline $3 \% \mathrm{Si}-97 \% \mathrm{Fe}$ & 0.001 & 7.80 & 11 & 6,000 & 0.0018 & 1.07 & 230 \\
\hline $3 \% \mathrm{Si}-97 \% \mathrm{Fe}$ & 0.002 & 7.74 & 11 & 5,000 & 0.0019 & 1.08 & 300 \\
\hline $50 \% \mathrm{Ni}-50 \% \mathrm{Fe}$ & 0.001 & 8.38 & 11 & 20,000 & 0.0009 & 1.00 & 90 \\
\hline $50 \% \mathrm{Ni}-50 \% \mathrm{Fe}$ & 0.002 & 8.32 & 11 & 11,000 & 0.0013 & 1.01 & 200 \\
\hline $79 \% \mathrm{Ni}-15 \% \mathrm{Fe}-5 \% \mathrm{Mo}$ & 0.001 & 8.90 & 8 & 2,500 & 0.0031 & 0.50 & 30 \\
\hline $49 \% \mathrm{Co}-49 \% \mathrm{Fe}-2 \% \mathrm{~V}$ & 0.002 & 8.22 & 11 & 6,000 & 0.0013 & 1.02 & 250 \\
\hline $\begin{array}{c}\text { Namglass }{ }^{\circledR} 1 \\
\text { amorphous iron-alloy }\end{array}$ & 0.001 & 7.36 & 11 & 10,000 & 0.0023 & 1.14 & 110 \\
\hline $\begin{array}{c}\text { Namglass }^{\circledR} 4 \\
\text { nanocrystalline iron-alloy }\end{array}$ & 0.001 & 7.47 & 10 & 60,000 & 0.0009 & 0.93 & 12 \\
\hline
\end{tabular}


Table 5.5: Magnetic characteristics, acceleration factor, and power loss for tapewound cut core materials with a $10 \mathrm{kHz}$ sinusoidal flux density adjusted so that power loss does not exceed that of 0.001 " thick $50 \%$ Ni-50\% Fe at $11 \mathrm{kG}$ rms (16 kG peak). Based on data from Magnetic Metals Corporation [101, 47].

\begin{tabular}{|c|c|c|c|c|c|c|c|}
\hline material & $\begin{array}{c}\text { thick. } \\
\text { (in) }\end{array}$ & $\begin{array}{c}\text { working } \\
\text { density } \\
\text { (g/cc) }\end{array}$ & $\begin{array}{c}\text { rms flux } \\
\text { density } \\
(\mathrm{kG})\end{array}$ & $\begin{array}{c}\text { relative } \\
\text { perm. }\end{array}$ & $\begin{array}{c}\text { skin } \\
\text { depth } \\
\text { (in) }\end{array}$ & $\begin{array}{c}\text { normal. } \\
\text { accel. } \\
\text { factor }\end{array}$ & $\begin{array}{c}\text { power } \\
\text { loss } \\
(\mathrm{W} / \mathrm{lb})\end{array}$ \\
\hline $3 \% \mathrm{Si}-97 \% \mathrm{Fe}$ & 0.001 & 7.80 & 6.3 & 4,400 & 0.0020 & 0.35 & 90 \\
\hline $3 \% \mathrm{Si}-97 \% \mathrm{Fe}$ & 0.002 & 7.74 & 5.8 & 3,800 & 0.0022 & 0.30 & 90 \\
\hline $50 \% \mathrm{Ni}-50 \% \mathrm{Fe}$ & 0.001 & 8.38 & 11 & 20,000 & 0.0009 & 1.00 & 90 \\
\hline $50 \% \mathrm{Ni}-50 \% \mathrm{Fe}$ & 0.002 & 8.32 & 8.5 & 10,000 & 0.0013 & 0.60 & 90 \\
\hline $79 \% \mathrm{Ni}-15 \% \mathrm{Fe}-5 \% \mathrm{Mo}$ & 0.001 & 8.90 & 8 & 2,500 & 0.0031 & 0.50 & 30 \\
\hline $49 \% \mathrm{Co}-49 \% \mathrm{Fe}-2 \% \mathrm{~V}$ & 0.002 & 8.22 & 5.7 & 4,300 & 0.0015 & 0.27 & 90 \\
\hline $\begin{array}{c}\text { Namglass } \\
{ }^{\circledR}\end{array}$ & 0.001 & 7.36 & 9.2 & 10,000 & 0.0023 & 0.80 & 90 \\
\hline $\begin{array}{c}\text { amorphous iron-alloy } \\
\text { nanocrystalline iron-alloy }\end{array}$ & 0.001 & 7.47 & 10 & 60,000 & 0.0009 & 0.93 & 12 \\
\hline
\end{tabular}

Table 5.6: Magnetic characteristics, acceleration factor, and power loss for tapewound cut core materials with maximum "usable" flux density at $10 \mathrm{kHz}$ sinusoidal. Based on data from Magnetic Metals Corporation [101, 47].

\begin{tabular}{|c|c|c|c|c|c|c|c|}
\hline material & $\begin{array}{l}\text { thick. } \\
\text { (in) }\end{array}$ & $\begin{array}{l}\text { working } \\
\text { density } \\
\text { (g/cc) }\end{array}$ & $\begin{array}{l}\text { rms flux } \\
\text { density } \\
(\mathrm{kG})\end{array}$ & $\begin{array}{c}\text { relative } \\
\text { perm. }\end{array}$ & $\begin{array}{l}\text { skin } \\
\text { depth } \\
\text { (in) }\end{array}$ & $\begin{array}{c}\text { normal. } \\
\text { accel. } \\
\text { factor }\end{array}$ & $\begin{array}{c}\text { power } \\
\text { loss } \\
\text { (W/lb) }\end{array}$ \\
\hline $3 \% \mathrm{Si}-97 \% \mathrm{Fe}$ & 0.001 & 7.80 & 14 & 5,300 & 0.0019 & 1.74 & 500 \\
\hline $3 \% \mathrm{Si}-97 \% \mathrm{Fe}$ & 0.002 & 7.74 & 16 & 3,000 & 0.0025 & 2.29 & 800 \\
\hline $50 \% \mathrm{Ni}-50 \% \mathrm{Fe}$ & 0.001 & 8.38 & 15 & 4,000 & 0.0021 & 1.86 & 150 \\
\hline $50 \% \mathrm{Ni}-50 \% \mathrm{Fe}$ & 0.002 & 8.32 & 15 & 3,000 & 0.0024 & 1.87 & 300 \\
\hline $79 \% \mathrm{Ni}-15 \% \mathrm{Fe}-5 \% \mathrm{Mo}$ & 0.001 & 8.90 & 8 & 2,500 & 0.0031 & 0.50 & 30 \\
\hline $49 \% \mathrm{Co}-49 \% \mathrm{Fe}-2 \% \mathrm{~V}$ & 0.002 & 8.22 & 20 & 3,100 & 0.0018 & 3.37 & 700 \\
\hline $\begin{array}{c}\left.\text { Namglass }^{(\mathbb{}}\right) \\
\text { amorphous iron-alloy }\end{array}$ & 0.001 & 7.36 & 15 & 7,000 & 0.0027 & 2.12 & 250 \\
\hline $\begin{array}{c}\text { Namglass }^{(\mathrm{R})} 4 \\
\text { nanocrystalline iron-alloy }\end{array}$ & 0.001 & 7.47 & 10 & 60,000 & 0.0009 & 0.93 & 12 \\
\hline
\end{tabular}




\subsection{Conclusions}

The concepts and topics discussed in this chapter - the predicted performance characteristics of the actuator based on first-principles analyzes, the detailed electromagnetic design of the actuator, and a discussion on soft magnetic materials — provide the insight and analyzes that were needed to design and build the actuator in the $10 \mathrm{kHz}$ FTS. The successful performance of that actuator - due in large part to the guidance provided by this chapter - is discussed in Chapter 4, so is not repeated here. Hopefully, this chapter will be of use to other researchers that are not already intimately familiar with the topics that are discussed in it, and provide a sufficiently comprehensive set of ideas to allow understanding and developing a normal-stress electromagnetic actuator. 


\section{Chapter 6}

\section{Conclusions, Highlighted Results, Contributions, and Future Work}

In this chapter I draw the major conclusions for this thesis, highlight the results of the work accomplished, list the primary contributions of this research, and explore suggested future work.

\subsection{Overall Conclusions}

The main results of this research are the development of two new high bandwidth short-stroke rotary fast tool servos and a hybrid rotary/linear electromagnetic actuator. The rotary fast tool servos were designed for diamond turning prescription textured surfaces on small spherical workpieces (diameters in the range of $10 \mathrm{~mm}$ or less), and are suitable for generating free-form non-axisymmetric surfaces on similarsized workpieces. Straightforward modifications would allow them to be used on larger workpieces. These rotary fast tool servos establish new benchmarks for demonstrated closed-loop bandwidth $(2 \mathrm{kHz}$ and $10 \mathrm{kHz})$ and tool tip acceleration (400 g) when compared to the fast tool servos that I found in the literature. Both machines benefit from earlier work by Ludwick [99] who establishes the advantages of a rotary-type fast tool servo over a linear-type from the standpoints of maximum achievable tool tip acceleration based on thermal limits of the actuator and a reduction in the disturbance 
to the diamond turning machine caused by the fast tool servo.

The flexure bearing and control system for the $2 \mathrm{kHz}$ FTS proved themselves robust enough to tolerate the external disturbances that occur when using that FTS to machine a workpiece. The $10 \mathrm{kHz}$ FTS uses a similar flexure bearing, and tests of its control system indicate that its robustness matches or exceeds that of the $2 \mathrm{kHz}$ FTS. The closed-loop magnitude and phase angle for the $2 \mathrm{kHz}$ FTS and the $10 \mathrm{kHz}$ FTS are fairly smooth curves up to $2 \mathrm{kHz}$ and $5 \mathrm{kHz}$, respectively. This suggests that a possible future addition of a feedforward compensator could be effective up to that frequency. Essentially, the feedforward controller applies a frequency-dependent magnitude and phase adjustment to the commanded reference signal to compensate for the known closed-loop response of the system. This research did not include trying to reduce the observed following errors for commanded sinusoidal tool trajectories. Ludwick [99], Cattell [37], Byl [33], and Lu [98] already established that the addition of an Adaptive Feedforward Cancellation compensator can significantly reduce those following errors, so I devoted my time during this research to demonstrating principles in new areas.

The literature for electric engraving heads, predominantly in the form of patents dating back to 1937, teaches concepts that are pertinent to the rotary fast tool servos described in this thesis. In particular, many of the electric engravers use an actuator that is similar to our hybrid rotary/linear electromagnetic actuator, but they use it to drive and sustain a resonance in a mechanical oscillator. The closed-loop arbitrary trajectories for the tool tip that I demonstrate with the $10 \mathrm{kHz}$ FTS represents the first published example that I am aware of demonstrating the use of this class of actuator in a non-resonating application. Our work is further distinguished from the prior art by a magnetic circuit that substantially decouples certain magnetic flux paths when a coil is used instead of a permanent magnet to provide the bias magnetic flux. This reduces the complexity of the actuator electrical dynamics from a MIMO system to a SISO system, and allows using loop-shaping techniques with classical control theory to design the control systems.

The two high bandwidth short-stroke rotary fast tool servos developed in this re- 
search hold promise for extending the existing capability at the Lawrence Livermore National Laboratory (LLNL) [91] for diamond turning prescription textured surfaces on small spherical workpieces. In addition to that application, these fast tool servos are suitable as-is for generating free-form non-axisymmetric surfaces on workpieces having diameters in the range of $10 \mathrm{~mm}$ or less. Straightforward modifications would allow them to be used on larger workpieces, making them applicable to direct machining contact lenses or contact lens molds.

The hybrid rotary/linear actuator developed for the $10 \mathrm{kHz}$ FTS provides a proof-of-principle for a general-purpose two degree-of-freedom electric machine with a broader array of potential applications, some of which are mentioned in Section 6.4. The electro-magnetic-mechanical design of its rotor, stator, and flexure bearing system could provide useful ideas to researchers designing new electric engraving heads, or other high bandwidth oscillatory systems, such as a steering mirror for an optical system. Furthermore, this thesis teaches how to achieve closed-loop arbitrary trajectories for the tool tip over its entire stroke when using a class of actuators that are typically used to drive a mechanical resonator. This last point could open new doors for improving control of the tool tip in an electric engraving head.

\subsection{Highlighted Results}

This section contains highlights of the results reported in the earlier chapters of this thesis for the $2 \mathrm{kHz}$ FTS, $10 \mathrm{kHz}$ FTS, and hybrid rotary/linear actuator.

\subsubsection{The $2 \mathrm{kHz}$ Rotary Fast Tool Servo}

The $2 \mathrm{kHz}$ rotary fast tool servo is shown in Figures 3-1 and 3-2. It uses a commercially available moving-magnet galvanometer as the actuator (Lorentz force) and provides proof-of-principles for a flexure bearing, small diamond tool and mounting method, circuit topology for a high bandwidth current-mode amplifier, and control system design. The following closed-loop performance is demonstrated for the $2 \mathrm{kHz}$ rotary fast tool servo: $-3 \mathrm{~dB}$ bandwidth of $2 \mathrm{kHz}, 20 \mathrm{~g}$ tool tip acceleration at $2 \mathrm{kHz}$, 
maximum tool travel of $50 \mu \mathrm{m} \mathrm{PP}$, and tool position noise level of $10 \mathrm{~nm}$ PP.

The demonstrated $20 \mathrm{~g}$ tool tip acceleration at $2 \mathrm{kHz}$ required a peak torque of $0.20 \mathrm{~N} \cdot \mathrm{m}$ from the actuator. That demonstrated peak torque is $71 \%$ of the specified continuous-operation peak torque of $0.28 \mathrm{~N} \cdot \mathrm{m}$ for the actuator. The ratio of the demonstrated peak torque while accelerating the swing arm divided by the corresponding apparent time-average electrical power of $120 \mathrm{VA}$ at the terminals of the actuator is $1.7 \times 10^{-3} \mathrm{~N} \cdot \mathrm{m} / \mathrm{VA}$.

A different measure of performance for the actuator used in the $2 \mathrm{kHz}$ FTS is the ratio of the continuous-operation peak torque divided by the rotational inertia of the rotor without the swing arm. For the demonstrated peak torque of $0.20 \mathrm{~N} \cdot \mathrm{m}$ and the actuator moving mass of $6.4 \mathrm{gm} \cdot \mathrm{cm}^{2}$, that ratio is $3.1 \times 10^{5} \mathrm{~N} \cdot \mathrm{m} /\left(\mathrm{kg} \cdot \mathrm{m}^{2}\right)$. Using the designed peak torque of $0.28 \mathrm{~N} \cdot \mathrm{m}$ increases the ratio to $4.4 \times 10^{5} \mathrm{~N} \cdot \mathrm{m} /\left(\mathrm{kg} \cdot \mathrm{m}^{2}\right)$.

The $2 \mathrm{kHz}$ FTS was integrated with a diamond turning machine and used to produce optical quality textured surfaces having approximately the same surface roughness on the face and outside diameter of aluminum workpieces while operating at $2 \mathrm{kHz}$. These machining tests demonstrate that the disturbance to the diamond turning machine from the reaction torque produced by the $2 \mathrm{kHz}$ rotary fast tool servo was fairly constant for the two cases, and supports the motivation for using a rotary-type FTS to machine from the pole to the equator on a spherical workpiece. Furthermore, prolonged periods of operation at $2 \mathrm{kHz}$ while machining workpieces indicate that the $2 \mathrm{kHz}$ FTS is suitable as-is for integration with a diamond turning machine to produce parts on a regular basis.

\subsubsection{The $10 \mathrm{kHz}$ Rotary Fast Tool Servo}

The $10 \mathrm{kHz}$ rotary fast tool servo is shown in Figures 4-1 and 4-2. It incorporates the proof-of-principles from the $2 \mathrm{kHz}$ FTS and is the vehicle for developing the hybrid rotary/linear electromagnetic actuator used in it. To reduce the number of high risk items and better assure the survival of the system during anticipated tests, we chose to pursue a $600 \mathrm{~g}$ version of the design instead of a riskier light-weighted design meeting the initial goal of $1000 \mathrm{~g}$. Having gained confidence with the successful testing and 
performance of the $10 \mathrm{kHz}$ FTS and its actuator, design parameters can be adjusted in future versions to achieve tool tip accelerations of $1000 \mathrm{~g}$ and beyond.

The actuator for the $10 \mathrm{kHz}$ FTS is a novel normal-stress variable reluctance machine with a demonstrated order of magnitude increase in the peak torque and ratio of peak torque to electrical power at the terminals of the actuator, when compared to the actuator used in the $2 \mathrm{kHz}$ FTS. By integrating the tool holder directly to the moving mass of the actuator to form a single rigid body we increase the overall torqueto-inertia ratio for the system and increase the frequency of the first uncoupled-mass resonance. Combined, these two advances made possible the factor of 20 increase in the demonstrated unaided ${ }^{1}$ tool tip acceleration compared to the $2 \mathrm{kHz}$ FTS.

The following closed-loop performance is demonstrated for the $10 \mathrm{kHz}$ rotary fast tool servo: $-3 \mathrm{~dB}$ bandwidth of $10 \mathrm{kHz}, 400 \mathrm{~g}$ tool tip acceleration at $5 \mathrm{kHz}, 870 \mathrm{~g}$ tool tip acceleration at $10 \mathrm{kHz}$ (utilizing a stable mechanical resonance), maximum tool travel of $70 \mu \mathrm{m}$ PP, and tool position noise level of 1.4 to $2.5 \mathrm{~nm}$ rms (depending on the magnitude of the bias flux used).

Many of the most significant results for the $10 \mathrm{kHz}$ FTS have to do with its actuator, which is discussed next.

\subsubsection{Hybrid Rotary/Linear Actuator}

Figures 5-1, 5-2, and 5-6 show models and a sketch of the magnetic circuit for the actuator that we developed for the $10 \mathrm{kHz}$ rotary fast tool servo. The actuator utilizes a constant bias magnetic flux, which linearizes the torque versus drive-current relationship for the actuator and provides up to half of the torque-producing magnetic flux in the rotor/stator air gaps. This makes it easier to accurately control the actuator, and reduces the electrical power needed to drive the peak AC flux by a factor of two when compared to a design that does not use a bias flux. Using a coil instead of a permanent magnet to supply the bias flux for the actuator allowed establishing a low noise implementation and a high acceleration implementation for

\footnotetext{
1 "Unaided" means that the actuator supplies all of the torque needed to accelerate the rotor and is not assisted by the stored energy of a mechanical resonance.
} 


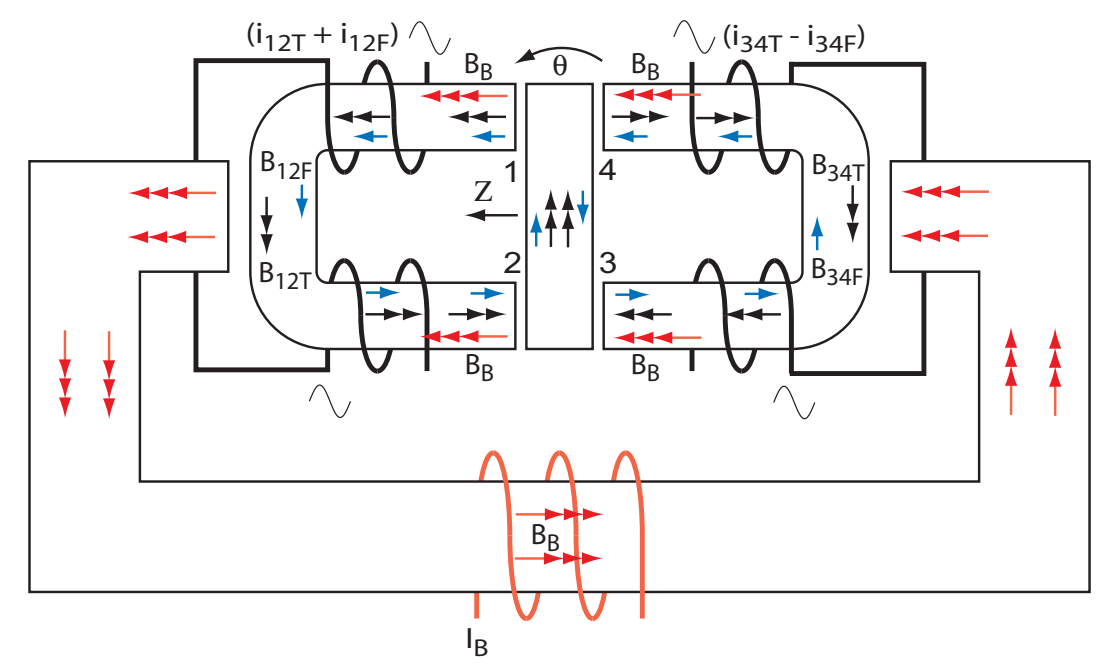

Figure 6-1: Sketch of the magnetic circuit for the actuator.

the $10 \mathrm{kHz}$ FTS.

The air gaps between the back-iron and the stator cores provide adequate enough magnetic decoupling of the two stator cores to allow independently controlling the magnetic flux in each stator half. Decoupling the two stator halves allows independently driving each half with its own current-mode amplifier and demonstrating closed-loop control of the rotary and linear degrees of freedom that are inherent in this class of actuators. The linear degree of freedom is used to demonstrate electronic damping of front/back vibration modes of the rotor. By extension, this suggests that the linear degree of freedom can be controlled as an active suspension for improving the performance of a predominantly rotary system. For the hybrid actuator that I built, torque control is independent of force control, but force control requires a torque-generating current to act as an operating point. In this thesis I describe alternate magnetic circuits having fully decoupled rotary and linear modes.

The mechanical design for the actuator allows controlling all 18 degrees of freedom between the rotor and the two stator cores during assembly of the actuator. Inductance measurements indicate that the bias magnetic flux in the four stator poles is balanced within $7 \%$. Therefore, the four $50 \mu \mathrm{m}$ air gaps between the rotor and stator are matched within approximately $7 \%$, or $3 \mu \mathrm{m}$.

The $400 \mathrm{~g}$ tool tip acceleration at $5 \mathrm{kHz}$ demonstrated with the $10 \mathrm{kHz}$ FTS re- 
quired a peak torque of $2.5 \mathrm{~N} \cdot \mathrm{m}$ from the actuator. That demonstrated peak torque is a factor of 12 greater than the analogous demonstrated peak torque for the $2 \mathrm{kHz}$ FTS. The demonstrated peak torque for the $10 \mathrm{kHz}$ FTS corresponds to a demonstrated peak magnetic flux density of $1.2 \mathrm{~T}$ in the rotor/stator air gaps. The demonstrated peak torque and peak flux density are $68 \%$ and $82 \%$ of our design goals of $3.7 \mathrm{~N} \cdot \mathrm{m}$ and $1.5 \mathrm{~T}$, respectively. The ratio of the demonstrated peak torque while accelerating the tool arm in the $10 \mathrm{kHz}$ FTS divided by the corresponding apparent time-average electrical power of $160 \mathrm{VA}$ at the terminals of the actuator is $16 \times 10^{-3} \mathrm{~N} \cdot \mathrm{m} / \mathrm{VA}$. This ratio is a factor of 9 greater than the same demonstrated ratio of $1.7 \times 10^{-3} \mathrm{~N} \cdot \mathrm{m} / \mathrm{VA}$ for the actuator used in the $2 \mathrm{kHz}$ FTS.

Another way to compare the performance of the two actuators is to consider the ratio of the continuous-operation peak torque divided by the rotational inertia of the rotor without the tool arm. For the $10 \mathrm{kHz}$ FTS, the demonstrated peak torque of $2.5 \mathrm{~N} \cdot \mathrm{m}$ and a rotor inertia of $70 \mathrm{gm} \cdot \mathrm{cm}^{2}$ gives a ratio of $3.6 \times 10^{5} \mathrm{~N} \cdot \mathrm{m} /\left(\mathrm{kg} \cdot \mathrm{m}^{2}\right)$. Using the designed peak torque of $3.7 \mathrm{~N} \cdot \mathrm{m}$ increases the ratio to $5.3 \times 10^{5} \mathrm{~N} \cdot \mathrm{m} /\left(\mathrm{kg} \cdot \mathrm{m}^{2}\right)$. These values are similar to the analogous values for the state-of-the-art galvanometer used in the $2 \mathrm{kHz}$ FTS.

For a fair comparison of the actuators used in the $10 \mathrm{kHz}$ FTS and in the $2 \mathrm{kHz}$ FTS, one should consider that the actuator in the $10 \mathrm{kHz}$ FTS would need to be cooled if it were to run continuously at the performance levels described in this section. The mechanical design for the $10 \mathrm{kHz}$ FTS includes provisions for active cooling of the actuator by flowing a fluid through internal passages.

The demonstrated peak magnetic flux, and therefore peak demonstrated tool tip acceleration, was limited by the magnitude of the bias magnetic flux density. Due to geometric form errors in the as-fabricated stator cores, and imperfect closure of the joints between the components in the back-iron, the effective length of the as-built air gaps between the stator cores and the back-iron is ten times longer than what was planned when choosing the number of turns for the bias coil. Consequentially, I had to use a higher bias coil current than planned when conducting the high acceleration tests for the actuator. This caused higher Ohmic heating in the passively cooled coil 
than planned, resulting in a steady-state coil temperature of $107^{\circ} \mathrm{F}$. Although that temperature is not excessive from a safety standpoint, it is generally considered unacceptable for a component that is to be integrated with a diamond turning machine. The simple remedy for future work is to use a bias coil with a higher number of turns, or a properly sized permanent magnet for the bias source. Heat generation due to magnetic losses in the stator and rotor cores is significant at the higher operating frequencies (around $5 \mathrm{kHz}$ and above), and deserves additional work.

\subsection{Primary Contributions}

The primary contributions of this thesis are:

1. Designs for two new rotary fast tool servos and experimental demonstration of their performance capabilities.

2. Insights, trade-off methodologies, and analytical tools for designing and building precision mechanical systems, power and signal electronic systems, control systems, normal-stress electromagnetic actuators, and the dynamics of the combined systems.

3. Design method and demonstration for an over-constrained flexure bearing for guiding small-amplitude rotary motion.

4. A technical and historical review of electric engraving heads, and their connection to high bandwidth rotary fast tool servos and a new hybrid rotary/linear actuator.

5. Magnetic circuit topologies, theory, and design relationships for a normal-stress hybrid rotary/linear electromagnetic actuator.

6. Design trade-off method for a highly integrated actuator and payload involving the mechanical, electrical, and magnetic systems.

7. Three patent applications. 


\subsection{Future Work}

During the development of the $2 \mathrm{kHz}$ FTS and the $10 \mathrm{kHz}$ FTS a myriad of technical challenges arose that could not all be pursued in this research. One of the "tricks" to successfully building a new machine is to limit the number of small research projects contained in the overall project so that you can actually deliver something that works and meets the major goals. Distinguishing between efforts that are merely interesting and those that are necessary is one way to accomplish this. Also, during the course of executing this research, new areas of concern were discovered that either required immediate attention in order to proceed, or could be put off for potential future work. This section discusses work that would have been interesting but was not necessary for demonstrating the goals of this research, mentions some new areas of concern that I discovered and set aside, and suggests future projects that use this research as a staring point.

\subsubsection{Adaptive Feedforward Cancellation Compensation to Reduce Following Error}

Ludwick [99], Cattell [37], Byl [33], and Lu [98] establish that the addition of an Adaptive Feedforward Cancellation (AFC) compensator can significantly reduce following errors for commanded sinusoidal tool trajectories, and reject disturbances at frequencies that are harmonics of the workpiece spindle rotation frequency. Essentially, the AFC compensator adds resonators at the frequencies where greater control authority is desired. These resonators theoretically give the magnitude of the negative loop transmission infinite gain at those frequencies, and therefore the steady-state error at those frequencies approaches zero. Careful design of the AFC compensator allows preserving the phase margin for the loop. Furthermore, the AFC compensator can be designed so that the frequencies of the resonators track variations in the spindle rotation speed. Figure 6-2 shows experimental results from Ludwick [99] illustrating how an AFC compensator reduced the following error by three orders of magnitude. Future work with the $2 \mathrm{kHz}$ FTS and the $10 \mathrm{kHz}$ FTS should include adding AFC 
Commanded

Position (cm)

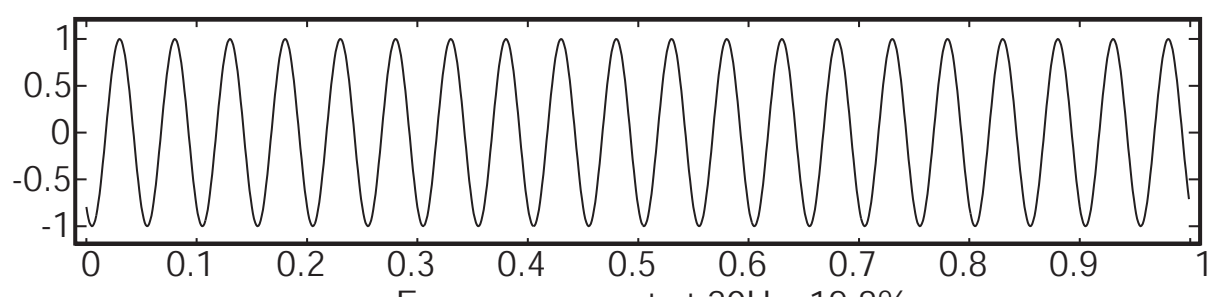

Error component at 20Hz: $19.8 \%$
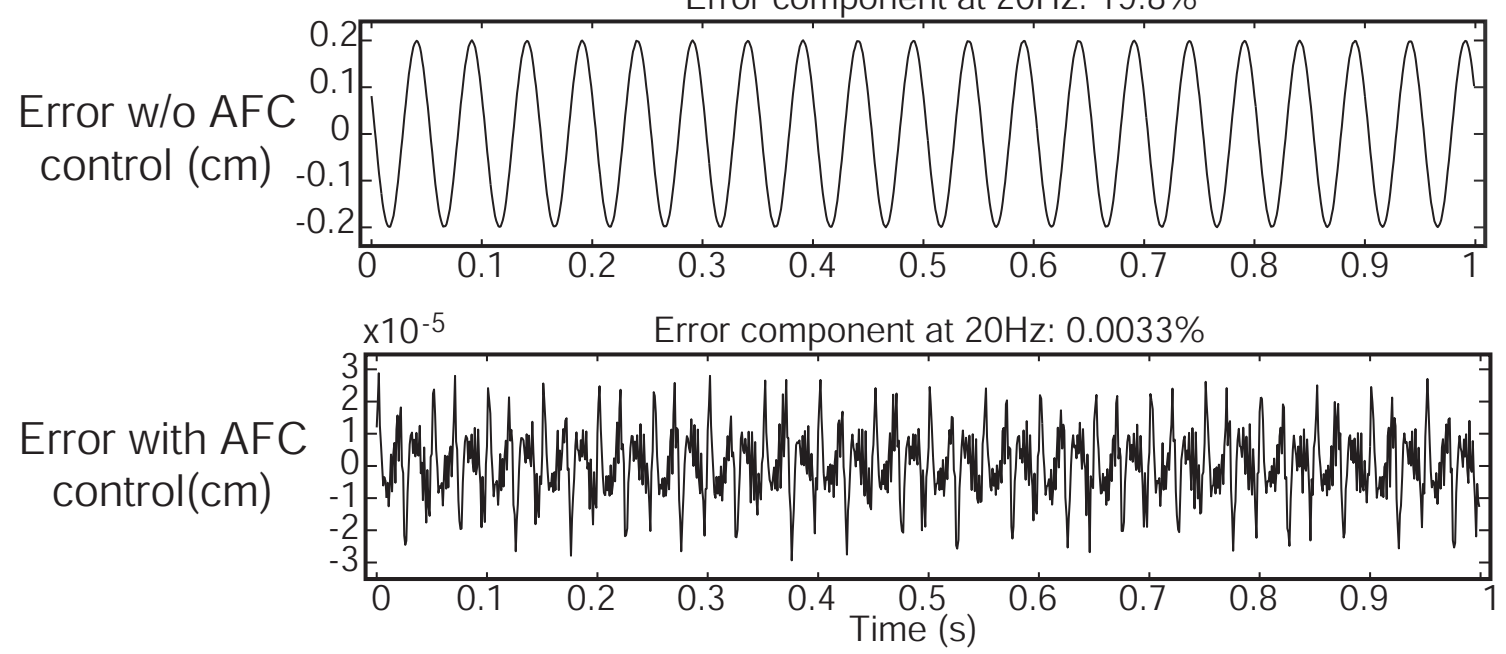

Figure 6-2: Experimental results using Adaptive Feedforward Cancellation compensation to reduce the following error for a $20 \mathrm{~Hz}$ air cut with the Rotary fast tool servo by Ludwick and Trumper [99]. Figure courtesy of Byl, Ludwick, and Trumper (MIT, 2004).

compensation.

\subsubsection{Machine a Workpiece with the $10 \mathrm{kHz}$ FTS}

I did not machine a workpiece with the $10 \mathrm{kHz}$ rotary fast tool servo. Instead, I used the time that had been planned for those machining tests to more deeply explore the control issues for the rotary and linear degrees of freedom of our new actuator, and to demonstrate closed-loop control of both of those modes. I have no doubts that the $10 \mathrm{kHz}$ FTS will be able to machine a workpiece as well as or better than what was demonstrated with the $2 \mathrm{kHz}$ FTS. The $10 \mathrm{kHz}$ FTS has the high mechanical stiffness at the tool tip needed for machining a workpiece. Regarding dynamic stiffness from the controller, its position loop compensation has enough authority and robustness to pull the rotor off the open-loop unstable magnetic detent caused by the bias flux, and snap it to a stand still at the neutral position when closing the loop. With the 
controller turned off, I can not move the rotor by hand off of that magnetic detent, so I am confident that the $10 \mathrm{kHz}$ FTS can handle the small cutting forces involved in diamond turning.

Future work should include using the $10 \mathrm{kHz}$ FTS to machine a workpiece, preferably while testing the addition of an Adaptive Feedforward Cancellation compensator to demonstrate reduced following error.

\subsubsection{Constructing Precision Laminated Cores}

The tape-wound cores used to built the rotor and stator cores for the $10 \mathrm{kHz}$ FTS were not as good as expected, and machining them into the precision forms that were needed proved to be difficult. A worthwhile future research effort would be to develop a method for fabricating precision-shaped laminated cores from the raw softmagnetic tape material. The knowledge and experience gained while fabricating and bonding the rotor and stator assemblies for the $10 \mathrm{kHz}$ FTS should provide sufficient guidance and confidence for pursuing this suggested future work. As a starting point, Petrie suggests that solvent-based adhesives can be used to bond soft magnetic steel laminations to each other:

Solvent-based adhesives use organic solvent to reduce viscosity for easier application. The solvent is simply a carrier for the "solids" part of the adhesive. . . . Solvent adhesives are applied by spraying, dipping, or brushing, but the solvent must be driven off before the joint is assembled. . . Solvent-based adhesives may be chemically crosslinked, usually through the application of a curing agent (either incorporated directly into single part systems or added as a second part to the base resin) and heat. The application of a solid thermosetting epoxy via solution coating is an example. Such an adhesive may be used to coat and then bond laminations of electrical steel for motor applications. The adhesive is applied and then dried through solvent evaporation. Once the parts are ready to be bonded, they are stacked under pressure. Heat is then applied 
to achieve flow and final chemical crosslinking [122, page 312].

This future work should include establishing a method to remove the layer of shorted laminations from the machined surfaces of the cores, which are a suspected major source of eddy current losses. Furthermore, the observed apparent internal shorting damage between lamination layers in the as-machined nanocrystalline ironalloy cores should be investigated. It is not clear if that damage existing before the wire electro-discharge-machining (EDM) process that was used to machine the cores, or was caused by that process. Section 5.5.5 contains a discussion on this issue, and concludes that the damage is most likely due to discontinuities in the blank material. The future work proposed in this section is closely related to the suggested future work in Section 6.4.4, which should accompany it.

\subsubsection{Heat Generation in the Rotor and Stator Cores}

Section 4.10.3 shows that the temperature rise in the rotor and stator cores during operation at $5 \mathrm{kHz}$ was an issue, and attributed the power loss in the cores to excessive eddy current losses. This problem needs to be addressed if high-frequency operation is desired from an electromagnetically driven actuator similar in construction to the one used in the $10 \mathrm{kHz}$ FTS, especially if passive cooling is desired.

There are at least two possible avenues to pursue in this area. The first is to perform experiments using forced cooling of the $10 \mathrm{kHz}$ FTS. The second is to construct a mockup of the magnetic circuit used in the $10 \mathrm{kHz}$ FTS - with adequate instrumentation for monitoring temperatures — and accurately quantify the thermal losses in the rotor and stator cores for various operating frequencies and peak flux densities. Since the second avenue does not depend on the $10 \mathrm{kHz}$ FTS and readily allows experimenting with different materials, fabrication techniques, and samples, it is discussed further in this section.

Looking at the schematic diagram in Figure 5-6 of the magnetic circuit for the $10 \mathrm{kHz}$ FTS, one can envision that it would be a straightforward task to build a thermal testbed for it by fixing the components of the magnetic circuit to a non- 
magnetic holder, such as a board. Thermocouples could be readily attached to the desired locations, and components could be interchanged to allow comparing cores that are fabricated using different materials or techniques. The thermal testbed could also provide useful data on whether or not attempts to reduce eddy current heating in the cores is being affected by the effort in that area, as suggested in Section 6.4.3.

\subsubsection{Flexure Bearing Design for a Non Axially-Over-Constrained Rotor}

The swing-arm (rotor) with its flexure bearing for the $2 \mathrm{kHz}$ rotary fast tool servo is shown in Figure 6-3. Note that the upper flexure blades and lower flexure blades are identical and have a high in-plane stiffness compared to their low out-of-plane bending stiffness. This creates a radial and axial constraint at both ends of the rotor, which is therefore over-constrained in the axial direction. If the axial length of the rotor changes relative to the axial spacing of the portions of the base that fix the outer ends of the flexures, then an unwanted stress will develop in the flexures. Thermal growth of the rotor that is not matched by thermal growth of the base is one mechanism that can cause such a difference in axial length. In the case of the $2 \mathrm{kHz} F T S$, the predominant heat generating component is the actuator, which is isolated from the swing arm and base by thermal insulators, so the concern of unmatched thermal growth of the swing arm and base is mitigated in this case.

The rotor with its flexure bearing for the $10 \mathrm{kHz}$ rotary fast tool servo is shown in Figure 6-4. Note that the flexure suspension over-constrains the rotor in the axial direction in the same manner described above for the $2 \mathrm{kHz}$ FTS. Thermal growth in this case may be more significant than for the $2 \mathrm{kHz}$ FTS because magnetic losses in the rotor core generate heat in the rotor and therefore cause a change in the axial length of the rotor during operation. Similar magnetic losses in the stator cores generate heat in the stator housings that causes a change in the axial spacing of the outer ends of the flexures during operation. Stresses in the flexures due to the differential axial growth can be kept within a tolerable level by: (1) prescribing strict operat- 


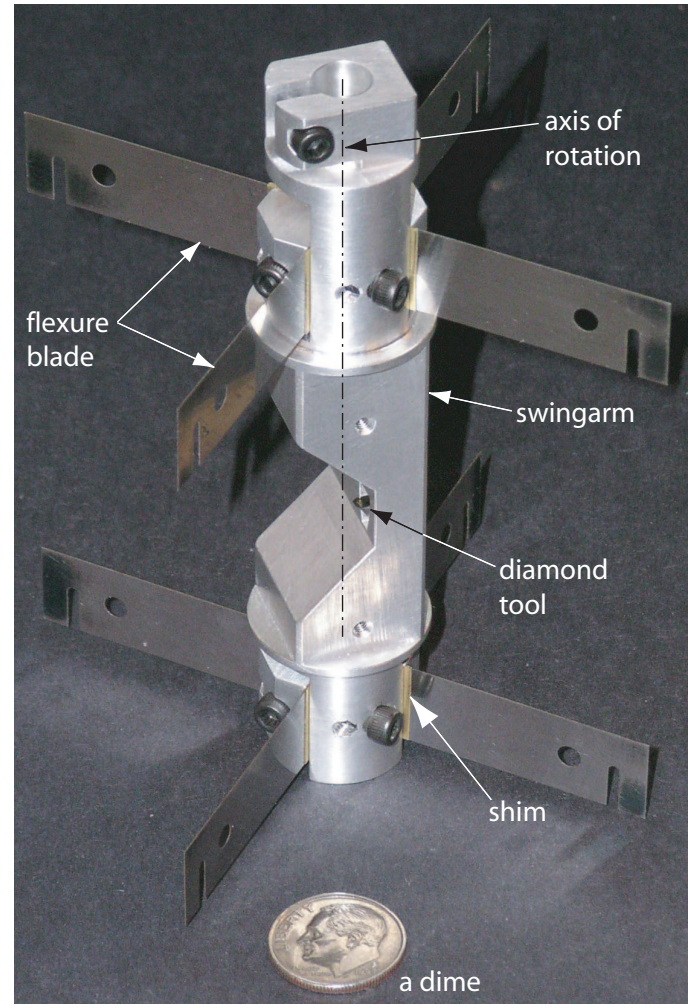

Figure 6-3: The swing-arm (rotor) with its flexure bearing for the $2 \mathrm{kHz}$ FTS.

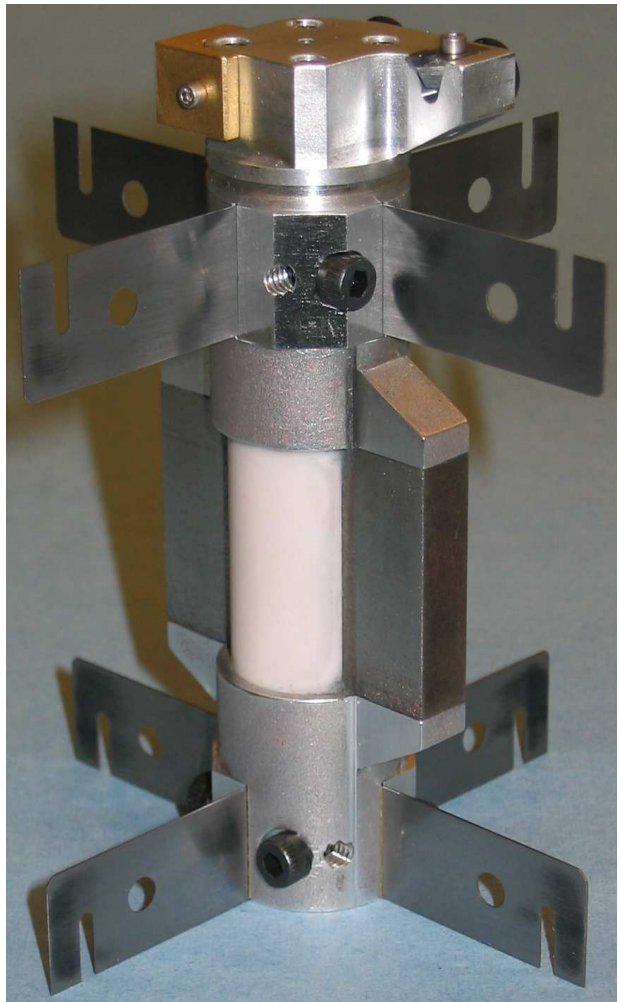

Figure 6-4: The rotor with its flexure bearing for the $10 \mathrm{kHz}$ FTS.

ing conditions so that the differential thermal growth between the rotor and stator housings is kept within an acceptable level; (2) the use of a cooling fluid to maintain an acceptable differential temperature between the rotor and stator housings; or (3) modify the in-plane stiffness of either the lower or upper flexures so that the rotor is less over-constrained in the axial direction. Solution (1) was used during testing of the $10 \mathrm{kHz}$ FTS: I monitored the temperature of the rotor and stator cores during operation and turned off the FTS when heating became excessive. Solution (2) is readily implemented with the as-built $10 \mathrm{kHz}$ FTS which has provisions for forced cooling of the rotor and stator with a fluid - most likely a gas because of the small air gaps.

Referring to Figures 6-3 and 6-4, note that in both cases the similarity between the upper and lower flexures allows using the same mounting hardware for both sets of flexures, which saved design and fabrication time during this research. Also note that in both cases, the stiffness of the radial and torsional constraints provided to 
the rotor by the lower flexures is the same as the stiffness of the radial and torsional constraints provided by the upper flexures. If this were not the case, especially for the $10 \mathrm{kHz}$ FTS, additional vibration modes of the rotor could have become an issue. For future designs of a flexure-suspended rotor, a different trade-off might be made between the advantages of having identical mounting hardware and the same radial and torsional constraint stiffness at both ends of the rotor, against the disadvantages of having an over-constrained rotor. Solution (3) can be realized by the alternate flexure bearing designs shown in Figures 6-5 and 6-6.

Referring to Figure 6-5, the lower flexures have radial slots cut into them to create ligaments that reduce the axial stiffness that those flexures provide to the rotor, compared to the higher axial stiffness that the upper flexures provider. In this case, the rotor is essentially axially fixed by the upper flexures and has a relaxed axial constraint from the lower flexures. The size of the slots and number of ligaments can be varied to adjust the stiffness characteristics of the lower flexures. The upper and lower flexures both provide radial constraints to the rotor, and together establish the axis of rotation. A differential axial length change of the rotor relative to the stator housings is accommodated by the reduced axial-direction stiffness provided by the lower flexures, and the stresses in all of the flexures are lower than in the case with identical upper and lower flexures. The similarity in the flat-blade construction of the upper and lower flexures allows using the same mounting hardware for both. Note that as depicted in Figure 6-5, the stiffness of the radial and torsional constraints provided to the rotor by the lower flexures is not the same as the stiffness of the radial and torsional constraints provided by the upper flexures, which can lead to additional vibration modes of the rotor compared to the case of identical upper and lower flexures. Increasing the overall height of the lower flexure blades would allow setting the total cross-sectional area of the ligaments to be equal to that of the upper flexures. In that case, the stiffness of the radial and torsional constraints provided to the rotor would nominally be the same for the upper and lower sets of flexures.

Referring to Figure 6-6, the lower flexures have an elliptical cross-section to reduce the axial stiffness that those flexures provide to the rotor compared to the higher axial 


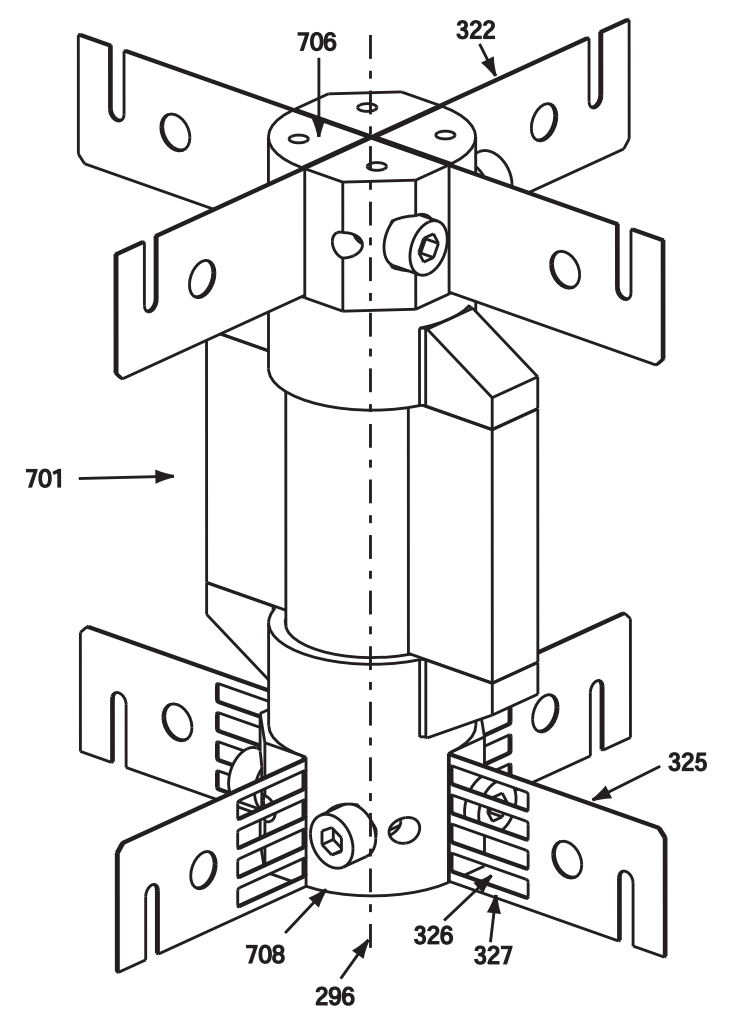

Figure 6-5: Model of the rotor in the $10 \mathrm{kHz}$ FTS with the lower flexure blades modified to provide axial compliance at one end of the rotor.

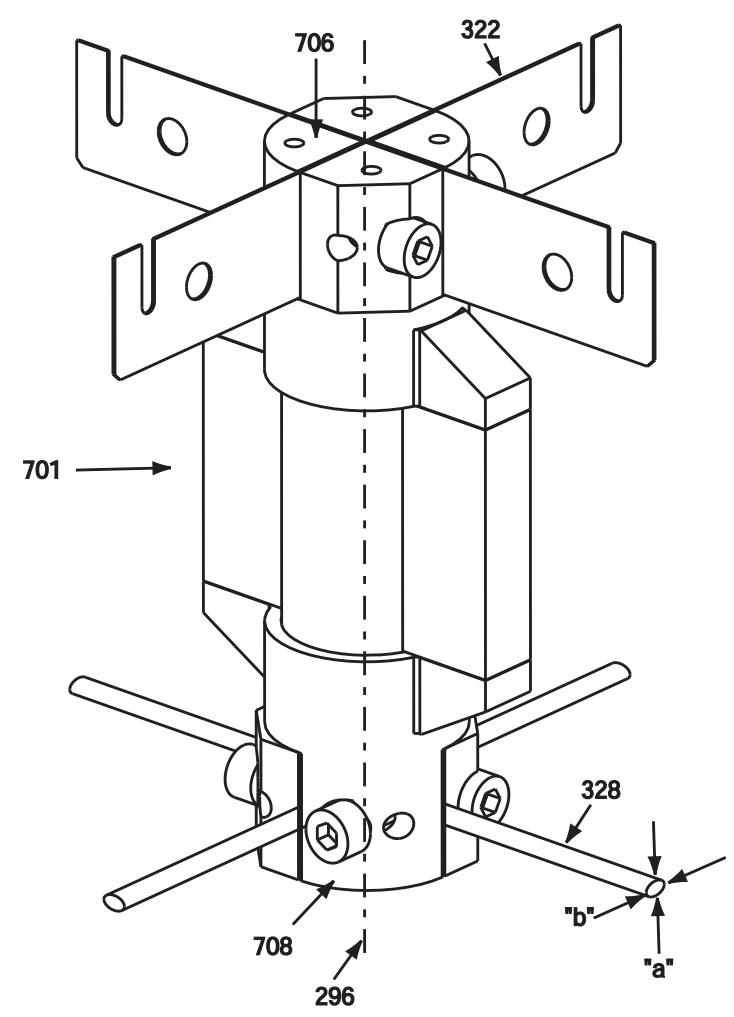

Figure 6-6: Model of the rotor in the $10 \mathrm{kHz}$ FTS with the lower flexure blades replaced with rods to provide axial compliance at one end of the rotor.

stiffness provided by the upper flexures. In this case the lower flexures are the same in form and function as the support rods used in certain electric engraving heads (see Section 2.3.3). The rotor is essentially axially fixed by the upper flexures, has a relaxed axial constraint from the lower flexures, and the axis of rotation is established by the two sets of flexures. A differential axial length change of the rotor relative to the stator housings is accommodated by the reduced axial-direction stiffness of the lower flexures, and the stresses in all of the flexures are lower than in the case with identical upper and lower flexures. The cross-section dimensions "a" and "b" of the lower flexures can be chosen so that the stiffness of the radial and/or torsional constraints provided to the rotor by the lower flexures is the same as the stiffness of the radial and torsional constraints provided by the upper flexures. This would avoid the additional vibration modes of the rotor attendant with the design shown in Figure 6-5. Alternatively, the lower flexures can have a rectangular cross-section 
to achieve that same purpose. In either case for the design shown in Figure 6-6, the different shapes of the upper flexures and lower flexures require different mounting hardware for fixing them to the rotor and to the stator.

\subsubsection{Optimize the Design of the Flexure Blades}

In Section 4.5.6, which discusses the flexure blades for the $10 \mathrm{kHz}$ FTS, I mention that I accepted a larger rotary inertia for the flexure hubs than was necessary. I chose to use 0.500 in wide flexure blades so that I could utilize the same detailed design for the flexure mounting hardware that was developed for the $2 \mathrm{kHz}$ FTS. The analysis in Section 4.5.6 indicates that smaller width blades would have been sufficient, which would reduce the height of the flexure hubs and decrease the rotary inertia of the rotor. Future work that attempts to increase the torque-to-inertia ratio for a rotor that uses a flexure bearing like the one described in this thesis should explore this point, and perhaps refine the order-of-magnitude analysis that was used (Section 3.6.4)

In Section 3.6.3 I mention that the inner and outer clamping blocks should be modified to better insure that the clamping pressure is applied to the desired fixed-end locations on the flexure blades. Relieving the central portion of the clamping blocks, as suggested in Figure 3-9, would better insure the desired pressure distribution.

\subsubsection{Alternate Bearing Types for Supporting the Rotor}

To minimize the number of different research areas during this research, the $2 \mathrm{kHz}$ FTS and $10 \mathrm{kHz}$ FTS both used the same flexure bearing design. The fast tool servos and actuator described in this thesis are prototypes that serve as proofs of principle, and will hopefully serve as starting points for new designs. Depending on the particular application or economics associated with producing multiple units, it may be preferable to use a different type of bearing to support the rotor. Referring to Figures 6-3 and 6-4, the geometry of the flexure hubs suggest that the following types of bearings could be used to support the rotor: 
- Gas-film radial and thrust bearings. For example, externally pressurized air bearings.

- Fluid-film radial and thrust bearings. For example, externally pressurized oil bearings.

- Rubber-sheet radial bearing, with thrust support from an axial torsion arm. The reader is referred to the original work by Rivin [130] on the design of rubber-sheet bearings for limited travel applications.

These alternate bearings could work with features similar to the hubs, or they could interact directly with a suitably designed rotor core. In the case where the actuator provides rotations about two axes, such as the tip and tilt arrangement described in Section 6.4.10, the alternate bearing could be a spherical version of one of the types listed above.

\subsubsection{Reduced Flexure Bearing Designs Using a Magnetic Suspension}

I demonstrated closed-loop control of the torque mode and of the force mode for the hybrid rotary/linear actuator used in the $10 \mathrm{kHz}$ FTS, and used the force mode to demonstrate electronic damping. In Section 5.4 I discussed alternate magnetic circuits having fully decoupled rotary and linear modes. In this section I explore some of the possibilities of utilizing the linear degree of freedom as an active suspension for improving the performance of a predominantly rotary system.

Figure 6-7 is a sketch showing the top view of the rotor for the $10 \mathrm{kHz}$ FTS. In this case, the front and back flexures have been replaced with springs. As drawn, the flexures do not constrain translation of the rotor in the front/back direction, so the rotation axis established by the flexures is free to translate in that direction. The linear mode of the hybrid actuator can be used as an active suspension to control translation of the rotor in the direction not constrained by the flexures. The springs 


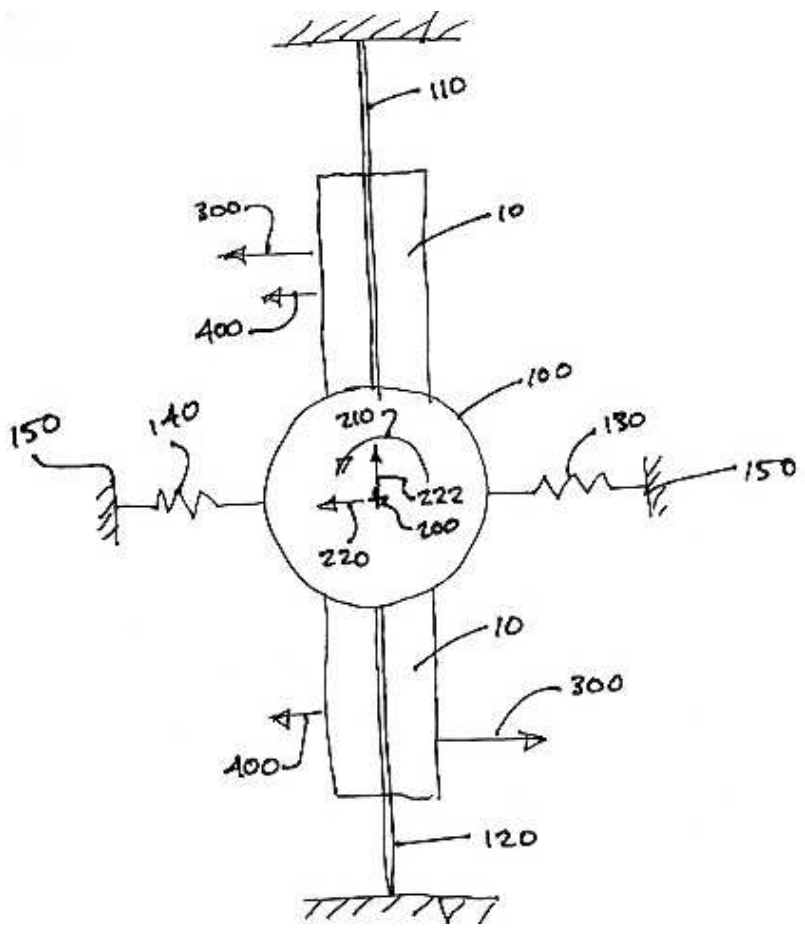

Figure 6-7: Sketch of a first variation of a reduced flexure bearing that uses the linear mode of the actuator as a magnetic suspension to help support the rotor.

shown in the figure are optional: including them could make it easier to develop the control system for the magnetic suspension.

Figure 6-8 shows a variation of the rotor assembly shown in Figure 6-7. In Figure 6-8 the flexure blades are oriented to substantially constrain the three translation degrees of freedom of the rotor. Bending of the flexure blades attendant with rotation of the rotor will cause the axis of rotation to wander in the plane of the figure. The linear mode of the hybrid actuator can be used as an active suspension to offset translation of that axis in the front/back direction, which may be adequate for an application that is insensitive to translation of the axis in the orthogonal direction.

Figure 6-9 shows a variation of the rotor assembly shown in Figure 6-8. The flexure blades still substantially constrain the three translation degrees of freedom of the rotor and lead to an axis of rotation that wanders in the plane of the figure. Again, the linear mode of the actuator can be used to compensate for translation of that axis in the front/back direction, which may be adequate for an application that is insensitive to translation of the axis in the perpendicular direction. 


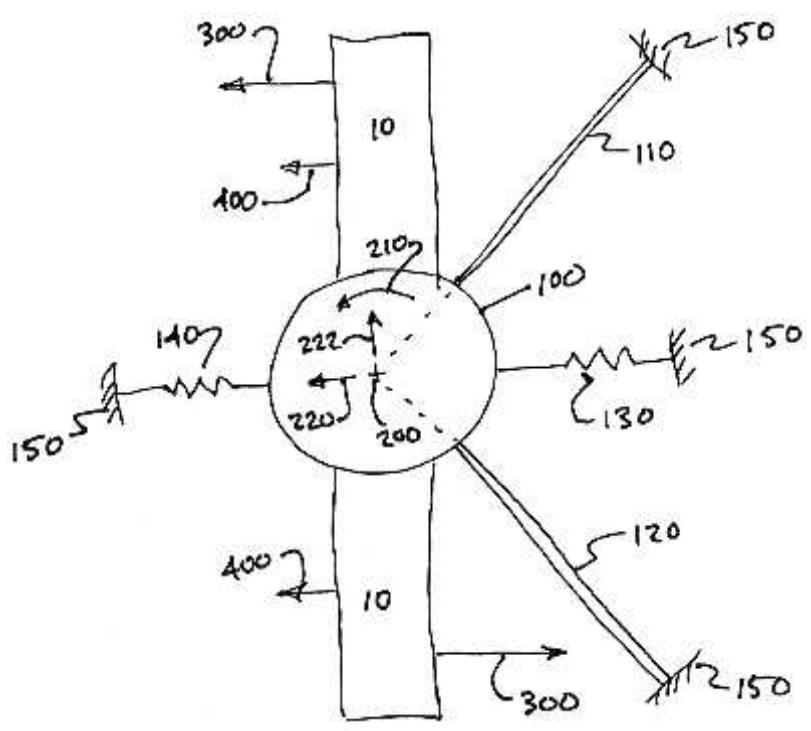

Figure 6-8: Sketch of a second variation of a reduced flexure bearing that uses the linear mode of the actuator as a magnetic suspension to help support the rotor.

Taken together, Figures 6-7, 6-8, and 6-9 represent three possible arrangements of using two flexure blades, optional helper springs, and the linear mode of the actuator to provide a bearing for a rotor. A continuum exists for the possible angles between the flexure blades and the rotor. Furthermore, it is possible to use a set of upper and lower flexures as with the 10 kHZ FTS, or to use only one set of flexures: upper, lower, or somewhere in between.

\subsubsection{Balanced X-rotor for a One-Axis Steering Mirror}

In Section $4.4 \mathrm{I}$ mentioned that if I were to design a follow-on machine to the $10 \mathrm{kHz}$ FTS I would consider pursuing a version of the balanced X-rotor magnetic circuit shown in Figure 4-8. We decided against the balanced X-rotor for the first prototype - the $10 \mathrm{kHz}$ FTS - because of the additional fabrication complexity that the extra angles in the cores present. With the knowledge gained from building the $10 \mathrm{kHz}$ FTS and its successful testing, future work can more assuredly pursue a version of the balanced X-rotor magnetic circuit. The following list enumerates the reasons for this choice, and suggests that the hybrid actuator developed for the $10 \mathrm{kHz}$ FTS is the prototype for an integrated high bandwidth actuator and steering 


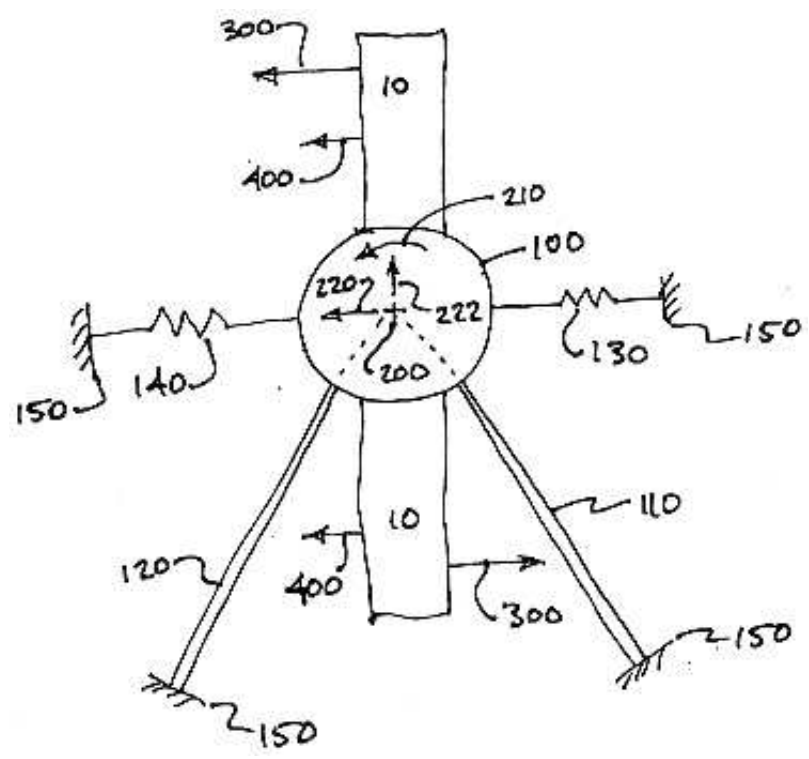

Figure 6-9: Sketch of a third variation of a reduced flexure bearing that uses the linear mode of the actuator as a magnetic suspension to help support the rotor.

mirror for use in an optical system.

1. The magnetic circuit for the balanced X-rotor has the same topology as the "pinched rotor" design described in Section 5.4.2, and therefore has fully decoupled torque and force modes.

2. Unlike the pinched rotor design shown in Figure 5-11, the rotor/stator air gaps in the balanced X-rotor design can be adjusted after final grinding of the pole faces. This is because translation of each stator core, towards and away from the rotor centerline, produces an equal change in the lengths of the air gaps due to the angled pole faces. This removes the biggest show-stopper listed for the pinched rotor design in Table 5.1, making it the clear choice for a preferred magnetic circuit.

3. As shown in Figure 4-8, the stators for the balanced X-rotor can be nested, one inside the other, so that the front face of the rotor is completely open. This increases the size of the work zone in front of the rotor. If the resulting actuator is used in a fast tool servo, then the cutting tool can engage a large workpiece. Alternatively, if the actuator is used to rotate a steering mirror in an optical 
system, the mirror can be made integral with the exposed front face of the rotor. If there is no clear advantage to nesting the stator cores for a particular application for the actuator, then they do not have to be.

4. The magnetic forces on the balanced X-rotor are nominally balanced and do not create a net force on it. Furthermore, the center of rotation established by the flexure-suspension bearing is coincident with the center of mass of the rotor. Therefore, the magnetic forces and angular acceleration of the rotor nominally do not produce forces on the bearing.

5. Figure 4-8 shows three flexure blades connected directly to the rotor core for the balanced X-rotor. Alternatively, the rotor can accommodate flexure hubs above and below the core in the same fashion as the $10 \mathrm{kHz}$ FTS, or it can use one of the other types of bearings described in Section 6.4.7.

6. The bias flux for the balanced X-rotor can be provided by a coil as with the $10 \mathrm{kHz}$ FTS, or by permanent magnets as shown in Figure 4-8.

It is worth noting that the tapered-rotor actuator by Buechler [27] described in Section 2.3 and shown in Figure 2-10 has most of the qualities listed above for the balanced X-rotor. Most notably, it is a pinched rotor design with fully decoupled torque and force modes, and the rotor/stator air gaps can be adjusted by translating the stator cores. The notable difference is that the actuator in Figure 2-10 does not provide a completely open front face for the rotor when the stator cores are nested.

The similarities between the tapered-rotor actuator and the balanced X-rotor actuator can be seen in Figure 6-10, which modifies the aspect ratio of the balanced X-rotor shown in Figure 4-8 by stretching the rotor in one direction and compressing it in an orthogonal direction, resulting in a rotor/stator layout that is similar to the one by Buechler [27] shown in Figure 2-10 but with inverted angles at the rotor/stator air gaps. The metamorphosed X-rotor can be considered as an inverted-tapered rotor, with the four wedge-shaped pieces adding mass to the outside corners. Note that the lever arms from the pole faces to the axis of rotation are greater for the invertedtapered rotor. Since the rotational inertia increases with the square of the distance 

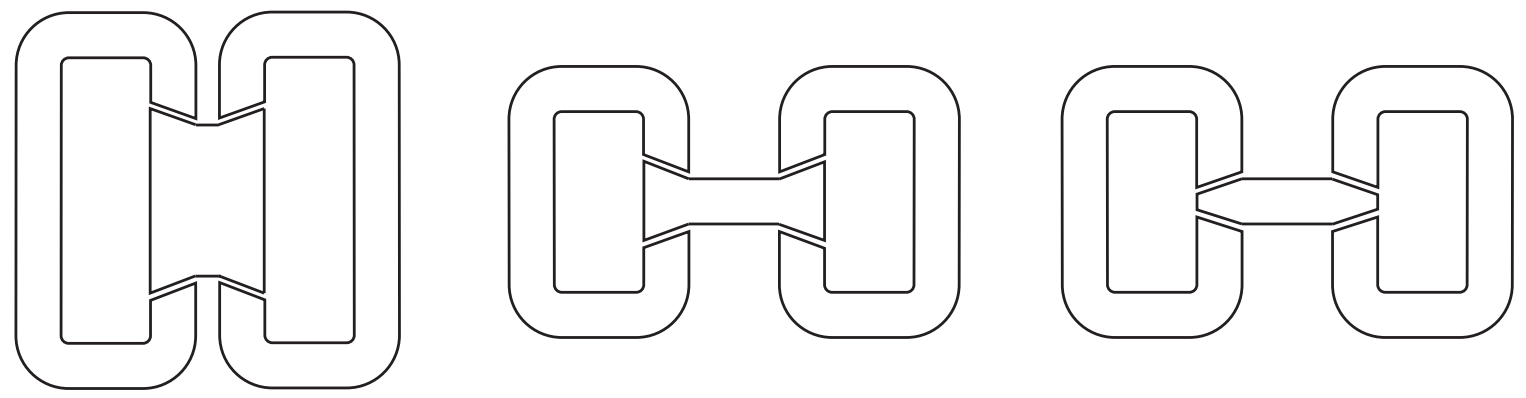

Figure 6-10: Sketch of the balanced X-rotor layout shown in Figure 4-8 with the stator cores un-nested (left), then with the rotor stretched in one direction and compressed in an orthogonal direction (center). Sketch of rotor and stator from Buechler [27] (right).

between each piece of additional mass and the center of rotation, in this case the increase in the torque-producing lever arm is outpaced by the increase in inertia. The net result is that the metamorphosed X-rotor has a lower torque-to-inertia ratio than a similar-sized tapered rotor. Considering a tapered rotor to be a version of an X-rotor, deciding between the two requires considering the trade-off between the required torque-to-inertia ratio versus the need to provide a completely open front face for the rotor.

\subsubsection{Two-Axis Steering Mirror}

Figure 6-11 shows sketches depicting how a variation of the hybrid rotary/linear actuator that we developed can be used to actuate a two-axis pointing mirror. This particular variation starts with the Balanced X-rotor magnetic circuit layout that is shown in Section 4.4. Note that one of the stator halves is folded back and nested inside of the other stator half to form a first stator pair. A second stator pair is arranged orthogonally to the first stator pair in a manner allowing both pairs to engage the same rotor. The rear view of the rotor shows at least four flexure blades and one torque rod for collectively supporting rotor. The flexure blades are arranged in a radial fashion in pairs around the rotor, and all nominally point at the center of gravity of the rotor. The torque rod extends from the rotor along a line passing through the center of gravity of the rotor, and is substantial enough to constrain translation of the rotor in a direction parallel to the long axis of the torque rod. 
Together, the four flexures and torque rod constrain all motions of the rotor except for rotations around two orthogonal axes that lie in a plane nominally parallel to the front surface of the rotor and passing through the center of gravity of the rotor. The cross-sectional view in Figure 6-11 cuts through the center of the torque rod and one pair of flexures, and shows the inner and outer stators of one of the stator pairs. The cross-section through the other pair of flexures and stators looks substantially the same. Coordinated actuation of each of the two axes produces coordinated two-axis rotation of the rotor, which can have an integral mirrored surface or an integral mount for holding a mirror. Note that the torque rod acts as the third flexure for each of the flexure pairs, and is thin in two directions to allow bending around two axes.

\subsubsection{Low-Cost Rotary Actuator}

Figure 6-12 shows a sketch of the magnetic circuit for a simple, potentially low-cost, normal-stress variable reluctance rotary actuator that uses a rubber sheet permanent magnet for producing a bias flux and for forming a bearing between the rotor and the stator. A rubber sheet permanent magnet is a sheet of elastic material that has permanent magnet properties. For example, a rubber sheet permanent magnet can be a $0.5 \mathrm{~mm}$ thick layer of rubber impregnated with particles of ceramic permanent magnet that are oriented in a common direction. The rubber sheet permanent magnet is captured between the central poles of the stator and the rotor, where the curved mating surfaces have have a common center to form an axis of rotation for the rotor that is coincident with the center of gravity of the rotor. The concept shown in Figure 6-12 was motivated by my discussions with MIT Professor David Trumper on the use of rubber sheet bearings in limited-motion systems, and with MIT Professor James Kirtley who suggested considering a design that uses a rubber sheet permanent magnet to act as the bearing and provide the bias flux. Note the large potential work zone at the front face of the rotor. If the actuator is used in a fast tool servo, then the cutting tool can be arranged to engage a large workpiece. Alternatively, if the actuator is used to rotate a steering mirror in an optical system, that mirror can be made integral with the exposed front face of the rotor or mounted directly to it. 


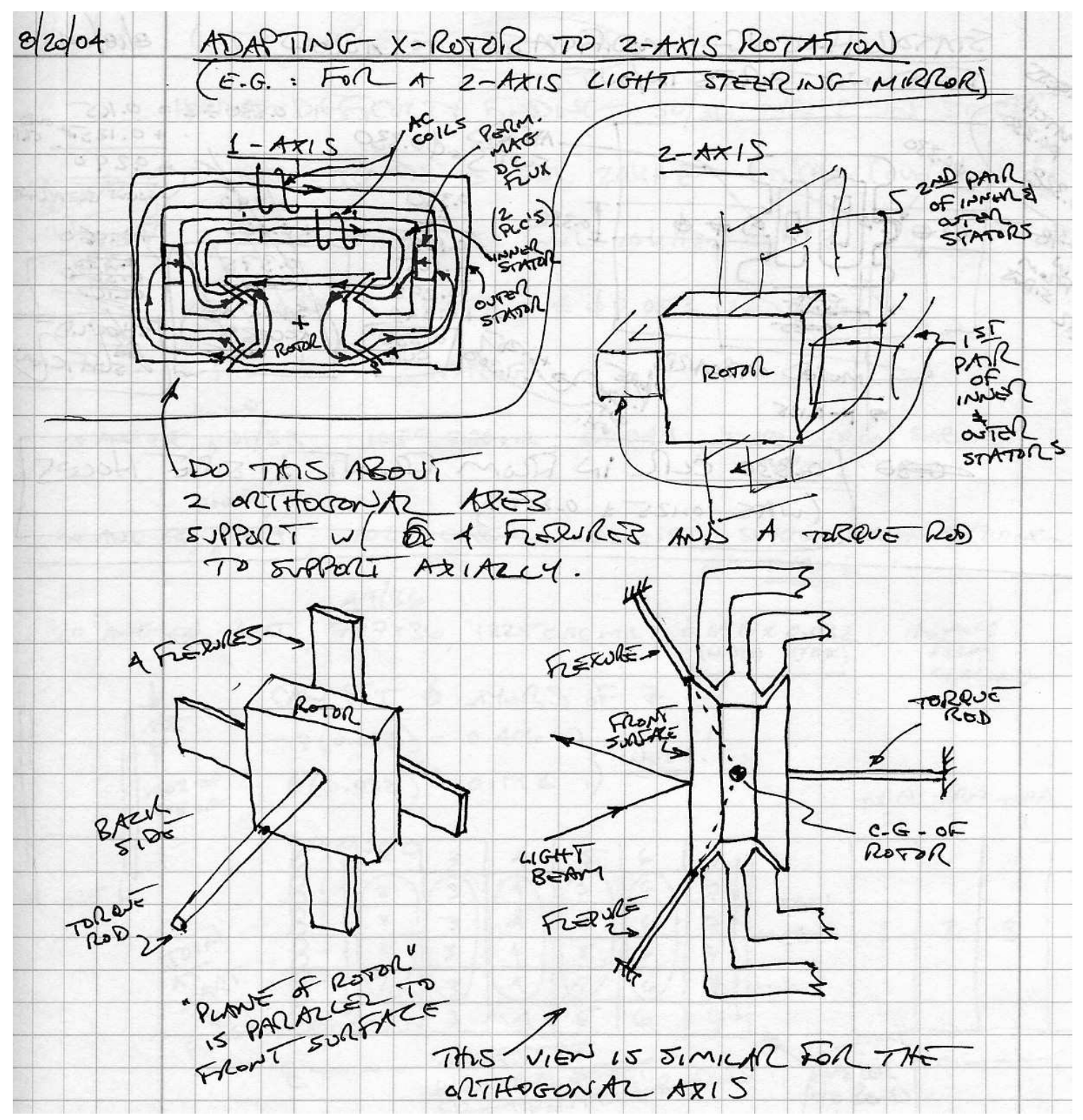

Figure 6-11: Sketches showing a variation of the X-rotor design to provide a twoaxis pointing mirror. Balanced X-rotor magnetic circuit with first stator pair (upper, left). A second stator pair is arranged orthogonally to the first stator pair (upper, right). Rear view of the rotor showing at least four flexure blades and one torque rod for supporting the rotor (lower, left). Cross-sectional view through the center of the torque rod and one of the pairs of flexures (lower, right). 


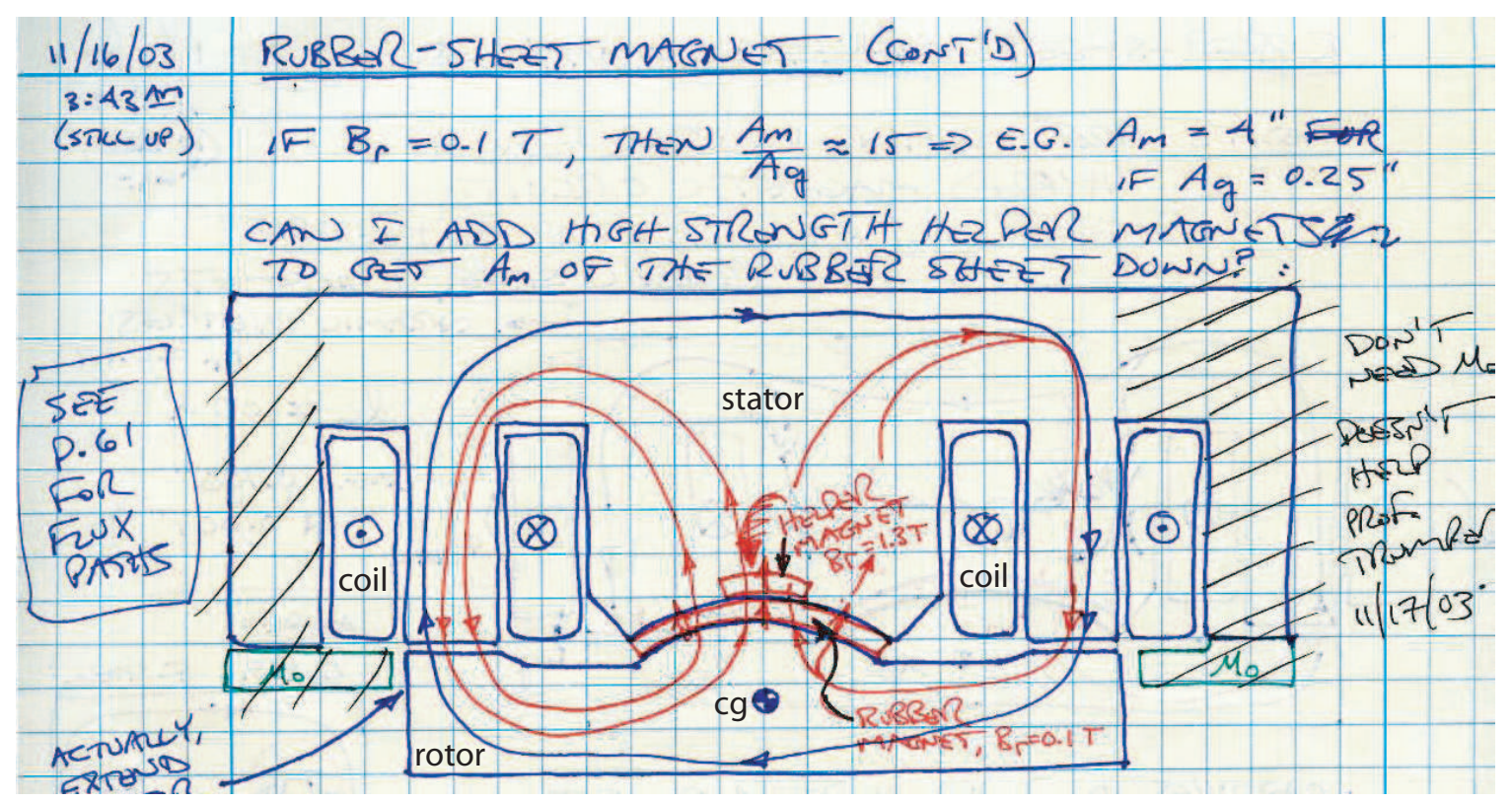

Figure 6-12: Sketch showing the magnetic circuit for a proposed low-cost rotary actuator. A rubber sheet permanent magnet provides the bearing between the rotor and stator, and provides the bias flux. The steering flux is provided by the pair of coils.

The use of one or more rubber sheets to form a bearing is taught by Rivin [130], who describes constructing a mechanical bearing using alternating layers of rubber and metal. The particular composition and thickness of the layers is chosen to achieve the desired mechanical characteristics for the bearing of low shear stiffness in a direction parallel to the layers, and high stiffness in a direction perpendicular to the plane of the layers. A significant attribute of a rubber sheet bearing is that it can tolerate the net compressive force produced by the magnetic attraction between the rotor and stator in the actuator shown in Figure 6-12; that actuator would be difficult to realize using flexure blades because of their limited tolerance to a compressive force.

Referring to Figure 6-12, the bias flux starts at the rubber sheet permanent magnet, enters the central pole of the magnetically permeable stator core, splits and circulates through the stator core to the left and right stator poles, crosses the air gaps between the stator core and the left and right rotor poles, travels through the magnetically permeable rotor core to the rotor central pole, and returns to the rubber sheet permanent magnet. To develop a torque on the rotor, the coils are used to produce a steering flux that travels along a path consisting of the back of the stator core, 
the left and right stator poles, the air gaps between the stator core and the left and right rotor poles, and the rotor core. Note that the rubber sheet permanent magnet extends across the entire width of the central pole of the stator and rotor cores, and is significantly thicker than the nominal length of the left and right rotor/stator air gaps. Therefore, the rubber sheet permanent magnet provides a high reluctance path that substantially prevents the steering flux from flowing across the space between the central poles of the stator and rotor cores, separating the steering flux path from the bias flux path. For the magnetic flux directions depicted in Figure 6-12, the bias flux and steering flux add in the right-hand air gap and subtract in the left-hand air gap, producing a net torque on the rotor that causes it to rotate in a counterclockwise direction. Reversing the direction of the current in the coils reverses the direction of the steering flux, which reverses the direction of the torque acting on the rotor. Note that due to the square-law relationship between magnetic flux and normal-direction force, the flux addition and subtraction in the outer air gaps changes the magnitude of the net magnetic force acting on the rotor and causes a lateral shift of that force.

Rivin [130] describes using multiple sheets of rubber separated by sheets of metal to achieve a desired stiffness ratio - shear-direction stiffness divided by normaldirection stiffness - for the bearing. Therefore, a first natural progression for the actuator depicted in Figure 6-12 is to replace the rubber sheet permanent magnet with a composite made up of alternating layers of rubber sheet permanent magnet and high magnetic permeability metal. A second natural progression is to consider a composite consisting of separate layers of rubber, high magnetic permeability metal, and permanent magnet. By moving the permanent magnet material out of the rubber sheet, the mechanical and magnetic properties of the bearing and the bias flux source can be more readily tailored to meet the performance goals for the actuator. For instance, this would allow: avoiding the possible break down of the rubber in a rubber sheet permanent magnet due to the motion of the particles of ceramic permanent magnet in the rubber during repeated cycles of shear deformation; adjusting the thickness and type of elastomer used in a rubber sheet based on the stiffness requirements for the bearing, independent of the bias flux requirement; and using a 
stronger permanent magnet to produce a desired bias flux. In practice, it may be impractical to fabricate and fit a curved permanent magnet to the curved surfaces of the composite rubber sheet permanent magnet. In that case, a flat permanent magnet extending across the entire width of the central stator pole would be preferred.

The addition of the "helper magnet" shown in Figure 6-12 was motivated by the recognition that standard rubber sheet permanent magnets have a low remanent flux $\left(\mathrm{B}_{r} \approx 0.1 \mathrm{~T}\right)$, which drives up the required cross-sectional area of the central stator and rotor poles. For example, if a total flux of $1.5 \mathrm{~T}$ is desired in the outer rotor/stator air gaps, with $0.75 \mathrm{~T}$ provided by a rubber sheet permanent magnet having $\mathrm{B}_{r}=0.1 \mathrm{~T}$, and $0.75 \mathrm{~T}$ provided by the steering flux coils, then the crosssectional area of the central pole needs to be 7.5 times larger than the combined crosssectional area of the outer air gaps. It is interesting to note that when I drew the sketch shown in Figure 6-12 I had not yet fully internalized a thorough understanding of the behavior of permanent magnets. As drawn in Figure 6-12, the flux from one face of the helper magnet will predominantly circulate around the magnet — through the high permeability stator core material surrounding it — to its other face, instead of circulating through the intended bias flux path. Later sketches reflect a growing appreciation for this issue - which is obviously solved by increasing the width of the helper magnet so that it extends across the entire width of the central stator pole but I did not amend any of the sketches because I had considered the ideas captured enough to stop thinking about the actuator depicted in Figure 6-12, and returned to considering the actuator that we were developing for the $10 \mathrm{kHz}$ FTS.

The actuator depicted in Figure 6-12 can readily be extended to a two-axis rotary system, like the steering mirror described in Section 6.4.10. In this case, the sketch in Figure 6-12 can be considered as one of two orthogonal cross-sections through a two-axis rotary actuator. The curved mating surfaces of the central poles of the stator and the rotor would be sections of spheres that have common centers forming two orthogonal axes of rotation for the rotor. If the rubber sheet permanent magnet bearing were replaced with a gas-film or fluid-film bearing and a permanent magnet, then the bias flux would provide a compressive preload for the bearing. 


\subsection{Final Comments}

When I embarked on this research, the successful testing results that were eventually demonstrated with the $2 \mathrm{kHz}$ and $10 \mathrm{kHz}$ rotary fast tool servos, and the hybrid rotary/linear electromagnetic actuator, were not assured. The diamond turning experiments with the $2 \mathrm{kHz}$ FTS could have been plagued with tool chatter due to an inadequately stiff flexure bearing for the rotor or insufficient disturbance rejection by the control system. The rotor in the $10 \mathrm{kHz}$ FTS could have stayed "stuck" to the hard stops or oscillated like an old-fashioned door buzzer in the rotary or linear mode, despite the controller efforts on the steering flux in the actuator. The over-constrained flexure blades in either machine could have performed poorly because of inadequate alignment during assembly, failed due to fatigue or thermally-induced stresses, or been accidently broken by crashing the tool tip or rotor into an unforgiving object. None of these things happened. I mention them because a casual observer could take for granted that the machines performed as intended, and under-appreciate the design approach and execution that led to the success of this research. If you have read this far, then you are certainly not a casual observer.

I can now breath a long-awaited sigh of relief, and smile with the satisfaction of knowing that this research developed and demonstrated new state-of-the-art machines and proof-of-principles with significant potential application. Writing this thesis allowed me to compile many of the significant design insights, trade-off methodologies, and analytical tools that I developed and used during this research, and make a broader benefit of my work available to the technical community interested in the areas of precision mechanical systems, power and signal electronic systems, control systems, normal-stress electromagnetic actuators, and the dynamics of the combined systems. 


\section{Appendix A}

\section{Selected Control System Design Techniques}

This appendix contains selected techniques that I used to design the analog and digital controllers for both the $2 \mathrm{kHz}$ and $10 \mathrm{kHz}$ rotary fast tool servos.

\section{A.1 Loop Shaping with Lead and Lag Compen- sators}

The current compensation circuit described for the $2 \mathrm{kHz}$ rotary fast tool servo in 3.9.1 and shown in Figure 3-27 provides a case study for describing the loop shaping technique that I favored when designing the series lead and lag compensators for the analog and digital controllers for both the $2 \mathrm{kHz}$ and $10 \mathrm{kHz}$ rotary fast tool servos. For convenience, Figure 3-18 and Figure 3-19 from Section 3.7.1 are repeated in this appendix as Figure A-1 and Figure A-2, respectively.

As was mentioned in Section 3.9.1, the constant gain block $\mathrm{K}_{p a}=3$ in Figure A-2 represents the power operational amplifier, whose dynamics are negligible for the frequencies of interest for the current-mode amplifier. The motor electrical dynamics are in the block after the power operational amplifier. In the case of the $2 \mathrm{kHz}$ FTS, the coil inductance $L_{c}$ and resistance $R_{c}$ combine with the current sense 

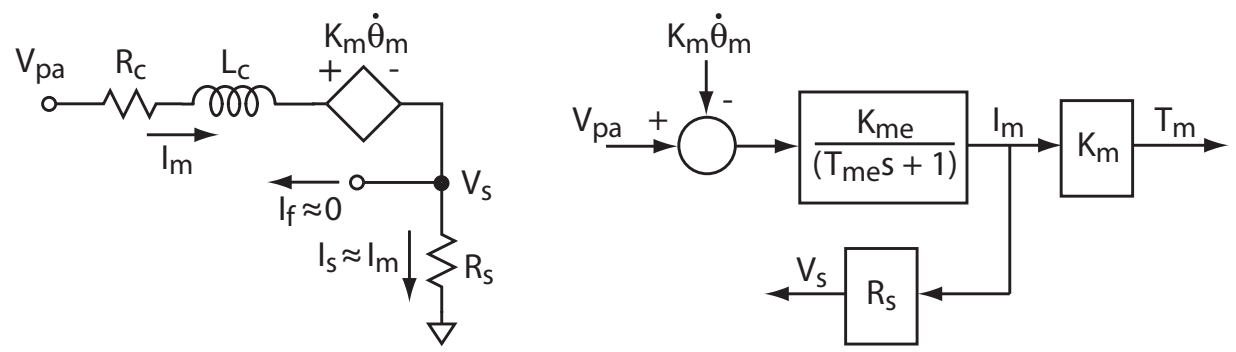

$$
\begin{aligned}
& K_{\text {me }}=\frac{1}{\left(R_{C}+R_{s}\right)} \\
& T_{\text {me }}=\frac{L_{c}}{\left(R_{c}+R_{s}\right)}
\end{aligned}
$$

Figure A-1: Electrical circuit and equivalent block diagram for the electrical dynamics of the motor for the $2 \mathrm{kHz}$ rotary fast tool servo.

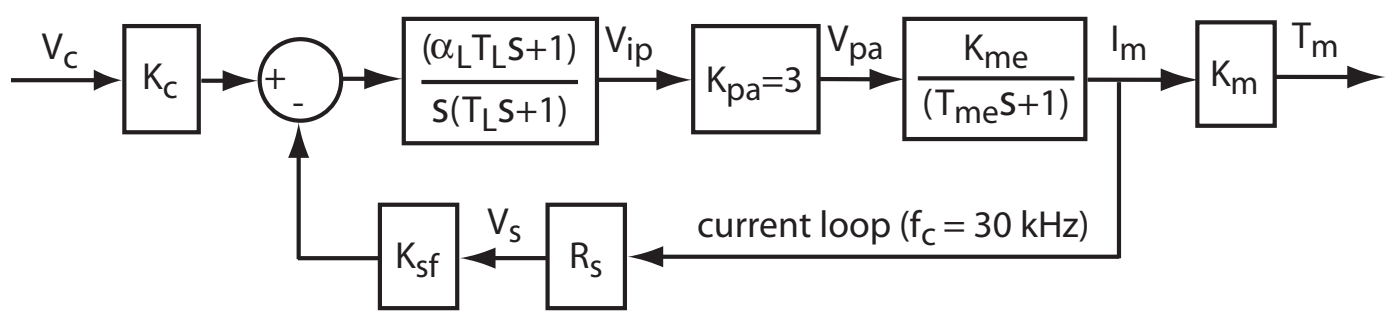

Figure A-2: Block diagram showing the electrical dynamics of the actuator inside the current loop for the current-mode amplifier for the $2 \mathrm{kHz}$ FTS.

resistor $\left(R_{s}\right)$ to create a time constant of $T_{m e}=2.55 \times 10^{-4}$ sec and a DC gain of $\mathrm{K}_{m e}=0.91 \mathrm{amp} /$ volt. The compensation dynamics for the current loop are in the block preceding the power amplifier and consist of a lead transfer function cascaded with a pure integrator. The constant gain term of the compensator was put on the input side of the summing junction in the form of the gains $\mathrm{K}_{c}$ and $\mathrm{K}_{s f}$ to make the assignment of resistor values for the compensation op-amp circuit more straightforward.

The solid upper curve in Figure A-3 is a straight-line approximation of the Bode magnitude plot of the negative loop transmission for the current compensation loop used with the $2 \mathrm{kHz}$ rotary fast tool servo. Starting at the low frequency end, the pure integrator provides the current loop with a high gain for rejecting low frequency disturbances. As we move to the right towards higher frequencies along the magnitude curve we encounter the pole from the motor electrical dynamics $\left(1 / \mathrm{T}_{m e}\right)$, which causes the slope to decrease from -1 (dec/dec) to -2 (dec/dec). Before reaching the desired crossover frequency $\left(\omega_{c}\right)$ we encounter the lead zero $\left(1 / \alpha \mathrm{T}_{L}\right)$, which brings the slope back up to -1 (dec/dec) so that we can pass through crossover with a positive phase 
margin. After crossover we reach the lead pole $\left(1 / \mathrm{T}_{L}\right)$, which rolls off the gain at -2 (dec/dec) to avoid high frequency noise. More will be said about high frequency noise later in this section. Regarding the phase angle, which is not shown in the figure, in the case of our current compensation circuit the calculated angle stays above $-180^{\circ}$ for frequencies below and around crossover. If there was a larger spread between the motor electrical pole and the lead zero, then greater attention would need to be paid to the possibility of the phase angle falling to $-180^{\circ}$ or lower before crossover, which could lead to conditional stability of the loop. The measured closed-loop performance of the current loop for the $2 \mathrm{kHz}$ FTS has a $-3 \mathrm{~dB}$ point at $30 \mathrm{kHz}$ and negligible phase up to $4 \mathrm{kHz}$.

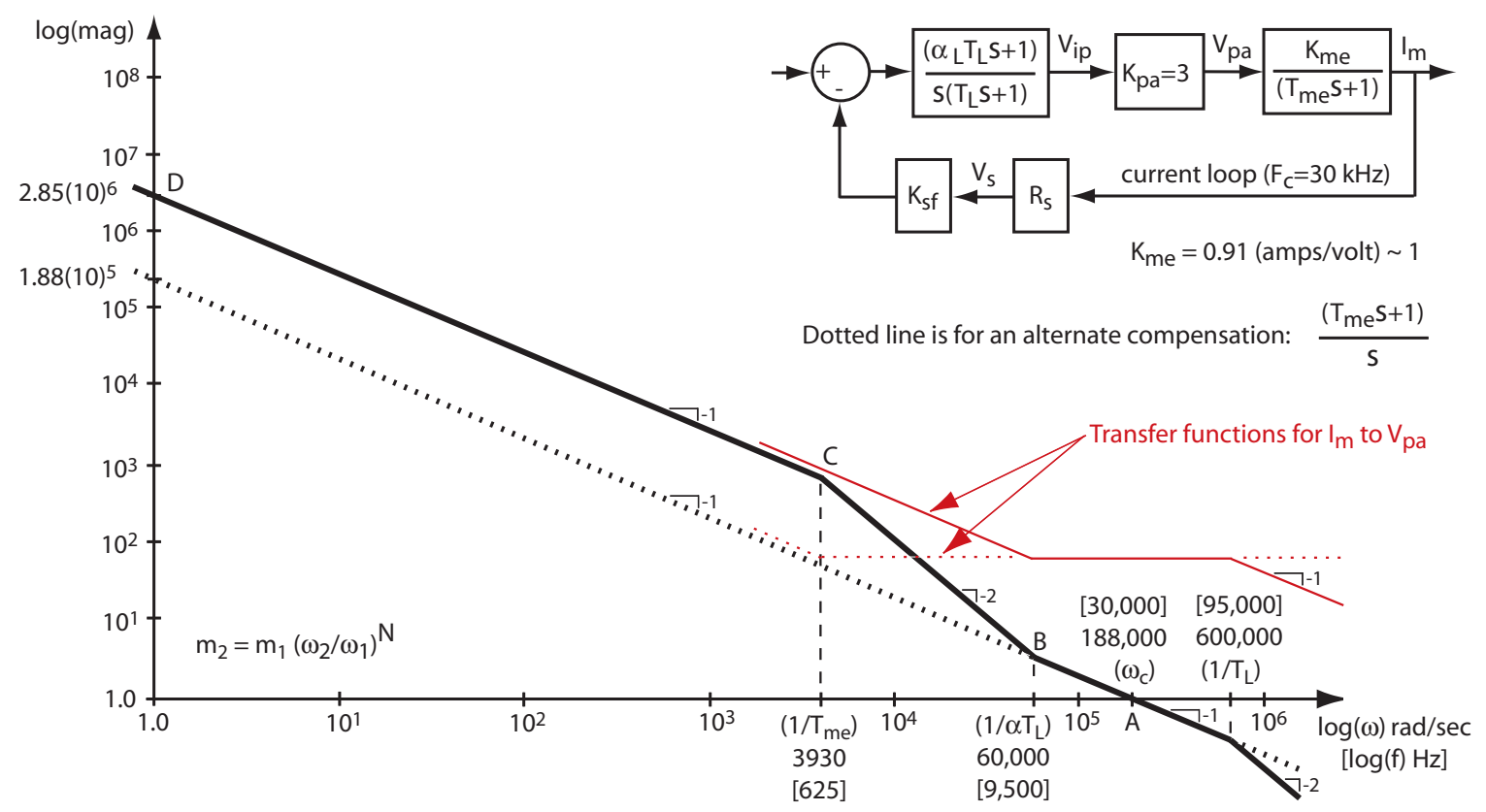

Figure A-3: Straight-line approximation of the Bode magnitude plot of the negative loop transmission for the current compensation loop used with the $2 \mathrm{kHz}$ rotary fast tool servo (heavy, solid curve). Block diagram for the current compensation loop for the $2 \mathrm{kHz}$ FTS (upper right inset).

Designing the current control loop compensation is accomplished by first assigning values to the lead transfer function, and then moving backwards along the magnitude plot from the crossover point to determine the product of the constant gain terms in the loop transmission. Choosing $\alpha=10$ for the lead transfer function provides a 
good balance between a maximum phase advance of $55^{\circ}$ and limiting the increase in gain on the higher frequency signals in the compensation network to a factor of 10 . The time constant $\left(\mathrm{T}_{L}\right)$ is picked by setting the geometric mean of the lead zero and pole equal to the crossover frequency so that the maximum phase advance occurs at crossover.

The current compensation circuit for the $2 \mathrm{kHz}$ rotary fast tool servo does not include the classic lag compensator: a low frequency pole followed by a higher frequency zero. The pole in a lag compensator increases the low frequency magnitude of the negative loop transmission, which equates to a higher controller authority for rejecting low frequency disturbances. It would be desirable to maintain that higher controller authority all the way up to the crossover frequency for the loop, but at some point before reaching crossover the effects of the lag pole need to be "turned off" by the lag zero in order to have an acceptable phase margin for stability. Roberge [132] suggests that a good starting point for placing the lag zero is one decade below crossover, so that the effect of the negative phase from the lag pole on the phase margin is limited to $6^{\circ}$.

If conditional stability is not an issue, placing the lag pole at zero - making it a pure integrator - provides the loop transmission with theoretically infinite gain at DC operation. For the reader who is unfamiliar with the concept of conditional stability, a thorough discussion is presented by Roberge in [132]. Essentially, it is possible for a feedback loop to be stable even thought the phase angle of the negative loop transmission passes down through $-180^{\circ}$ and then back up through it before reaching crossover, as long as the magnitudes associated with those crossings are not unity. The loop is considered conditionally stable because events that change the magnitude of the negative loop transmission but not its phase - saturation of a power amplifier is an example - can cause the system to enter into an unwanted stable resonance. For the reader that has access to the Barker Engineering Library at MIT, Professor James Roberge of MIT provides an excellent explanation on conditional stability in video tape number 17 of his recorded MIT lecture series entitled "Electronic Feedback Systems" [133]. 
To determine the product of the constant gain terms in the loop transmission we start at crossover and use the similar triangles relationship for the log-log plot shown in the lower-left corner of Figure A-3 to move backwards along the magnitude plot until reaching the gain at a frequency of $1 \mathrm{rad} / \mathrm{sec}$. Since all of the transfer functions in the current loop shown in Figure A-2 (and repeated in the inset in Figure A3) and are expressed in Bode format, it is straightforward to calculate an accurate approximation of the magnitude at point " $\mathrm{D}$ ", which corresponds to a frequency of $1 \mathrm{rad} / \mathrm{sec}$. The exact magnitude of the loop transmission for any frequency $\omega$ is found by substituting $s=j \omega$ into the product of the transfer functions in the loop. If the inverse of the time constants for all of the first-order singularities in the loop are much greater than $1 \mathrm{rad} / \mathrm{sec}$, then the contributions from those singularities to the loop magnitude at $1 \mathrm{rad} / \mathrm{sec}$ is negligible and the product of the constant gain terms in the loop transmission provides an accurate approximation of the magnitude at point "D". For control system design purposes, a factor of 10 suffices for "much greater than". If the condition above for the time constants is not met, then point "D" can be moved to a lower frequency that does allow satisfying that condition. As an interesting aside, the product of the constant gain terms found using this graphical method agreed within $1 \%$ of the value found using a more rigorous approach with a MATLAB ${ }^{\circledR}$ script.
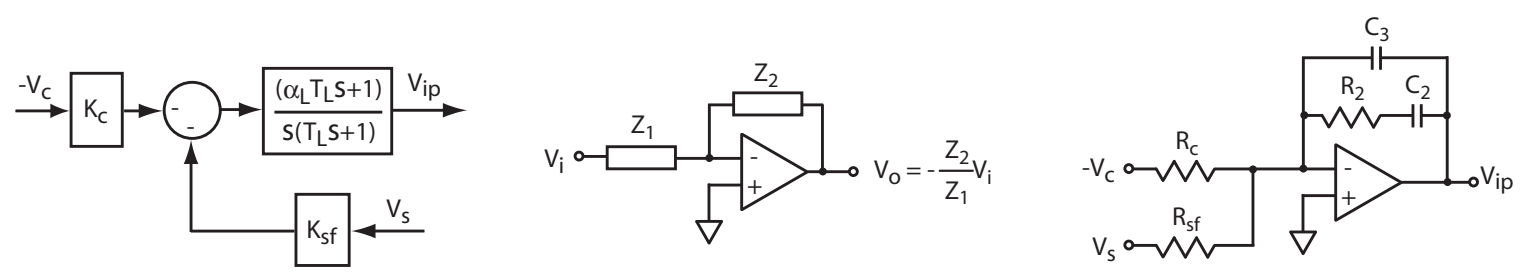

Figure A-4: Mapping of the current compensation block diagram (left) to an op-amp circuit (right). The circuit with the generic complex impedances (center) provides the mapping.

Figure A-4 shows the mapping from the current compensation block diagram to an op-amp circuit. One way to understand this mapping is to consider qualitatively what we want each element in the compensation transfer function to do. At low frequencies we want the compensation to act like an integrator. At mid frequencies, just before 
and around crossover, we want the zero to compensate for the integrator and make the gain constant. At high frequencies (above crossover) we want the pole to roll off the gain. The middle circuit with the generic complex impedances in Figure A-4 shows the ideal op-amp relationship between the input and output, which is a valid assumption because of the high speed op-amp that I used. ${ }^{1}$ Referring to the actual op-amp circuit on the right in Figure A-4, for the moment ignore the capacitor $\mathrm{C}_{3}$. At low frequencies the capacitor $\mathrm{C}_{2}$ lets very little current through the feedback path, so its impedance dominates over that of the resistor $R_{2}$ and we have an integrator. At mid frequencies the impedance of the capacitor $\mathrm{C}_{2}$ becomes negligible compared to that of $\mathrm{R}_{2}$, so the gain approaches a constant value of $\left(-\mathrm{R}_{2} / \mathrm{R}_{c}\right)$ or $\left(-\mathrm{R}_{2} / \mathrm{R}_{s f}\right)$, depending on which input is being considered. Now consider the capacitor $\mathrm{C}_{3}$, whose value is chosen so that its impedance is high at low and mid frequencies, and low at high frequencies. At low and mid frequencies $\mathrm{C}_{3}$ looks like an open circuit and acts as if it were not there, and at high frequencies $\mathrm{C}_{3}$ looks like a short and the gain of the circuit approaches zero. The inverting terminal of an op-amp configured as shown in Figure A-4 acts as a summing point for the individual input signals acting through their respective impedance $\left(Z_{1}\right)$, so superposition applies. To obtain the passive component values, the ratio of complex impedances $\left(Z_{2} / Z_{1}\right)$ is formed for each input in terms of the component variables, and then terms are matched with the coefficients for the corresponding transfer function in the block diagram. The values of the gains $\mathrm{K}_{s f}$ and $\mathrm{K}_{c}$ are set so that a \pm 10 amps swing in the motor current $\left(\mathrm{I}_{m}\right)$ causes the same voltage swing at the inverting terminal as does a \pm 10 volt swing in the command voltage $\left(\mathrm{V}_{c}\right)$.

The lower, dotted line with a constant slope of -1 (dec/dec) in Figure A-3 represents an alternate current compensation network that might be attractive to use. Its zero is placed to cancel the stable pole from the motor electrical dynamics, and the free integrator provides high gain at low frequencies. The circuit topology for this compensation is the same as the one shown in Figure A-4 after omitting the capaci-

\footnotetext{
${ }^{1}$ The current compensation circuit for the $2 \mathrm{kHz}$ FTS uses a Burr-Brown OPA602 operational amplifier [142], which is unity-gain stable and has a $3.5 \mathrm{MHz}$ gain-bandwidth product.
} 
tor $\mathrm{C}_{3}$. Finding the product of the constant gain terms in the loop transmission and assigning values to the analog component are accomplished by the same methods described above. The alternate compensation provides a constant phase angle of $-90^{\circ}$ at all frequencies, and therefore a conservative phase margin of $90^{\circ}$. Note that the gain at frequencies below the motor pole is approximately a factor of ten less than that of the more aggressive compensation that I used (described earlier). Therefore, the more aggressive compensation is better at rejecting low to mid frequency disturbances in the current control loop. The difference in gain between the two compensators is in the op-amp circuit, and a factor of ten increase requires paying closer attention to the signal levels in the current loop. Also, note that the alternate compensation needs an additional high frequency pole above the crossover frequency to roll off its gain to avoid high frequency noise. This is done by including the capacitor $\mathrm{C}_{3}$ shown in Figure A-4.

With regard to high frequency noise and the need to roll off the gain of the compensator after crossover, considering the internal signal $\mathrm{V}_{i p}$ of the current loop in Figure A-3 provides the necessary insight. The transfer functions from $I_{m}$ to $V_{p a}$ for the baseline and alternate compensation are shown in Figure A-3. Note that in both cases, since the pole from the motor electrical dynamics is outside of the compensation, the gain from $I_{m}$ to $V_{p a}$ is not reduced by the motor pole $\left(1 / \mathrm{T}_{m e}\right)$. If the high frequency pole where missing from the compensator, then once we reach the frequency of the compensator's zero the gain of the op-amp circuit would stay at a constant (high) value for all higher frequencies, making the circuit sensitive to any high frequency noise. This is unnecessary, and easily avoided by including the high frequency pole in the compensation.

\section{A.2 Fast Inner Loop Acts as a Constant Gain Block}

Figure A-5 shows a block diagram of a generic fast inner loop inside a generic slower outer loop. The hand-calculations in Figure A-5 go through the proof that for frequencies below one tenth the crossover frequency for the inner loop, for design purposes 
the inner loop can be treated as a constant gain block equal to the inverse of its feedback loop gain.

\section{A.3 Notch Filter Design}

The compensator for the tool position for the $2 \mathrm{kHz}$ rotary fast tool servo without viscous damping that was presented in Section 3.10.2 includes a notch filter. The topic of designing notch filters does not appear in most of the controls text books that I reviewed. Franklin, Powell, and Emani-Naeini [60] present a good explanation that provided me insight for designing a notch filter. A key concept for me was a description by Professor David Trumper of MIT that a notch filter can be viewed as a pair of complex zeros and a pair of complex poles. In this section I provide highlights on some of the issues that I considered when designing a notch filter.

Viewing the notch filter as a pair of complex zeros and a pair of complex poles, if these zeros and poles have the same natural frequency then the notch filter has unity gain at frequencies below and above that natural frequency. This allows adjusting the behavior of the notch filter without greatly upsetting the negative loop transmission gain at other frequencies. The depth and width of the notch, and its affect on the phase margin for the negative loop transmission, is adjusted by changing the damping ratios for the complex zeros and complex poles. The canonical form for this type of notch filter is:

$$
(T F)_{n o t c h}=\frac{s^{2}+2 \zeta_{z} \omega_{z} s+\omega_{z}^{2}}{s^{2}+2 \zeta_{p} \omega_{p} s+\omega_{p}^{2}}
$$

Consider what a notch filter does. It's zeros compensate for the pair of stable plant poles that are producing the resonance that we wish to suppress. The notch poles cancel the gain of the notch zeros at frequencies above the natural frequency of the notch zeros. Without the notch poles, the gain of the notch zeros would run away at higher frequencies. Figure A-6 is a sketch of the Bode magnitude plots of a generic under-damped second-order plant and of the zeros and poles of an ideal notch filter. In this ideal case the peak from the notch zeros exactly cancels the peak from the 
PROOF OF RULE-OF-THUMB FOR

$$
\frac{\operatorname{RCM} 3 / 22 / 04}{6 / 25}
$$

inNer coop $\geq 10 x$ FAster titan ouTER coop
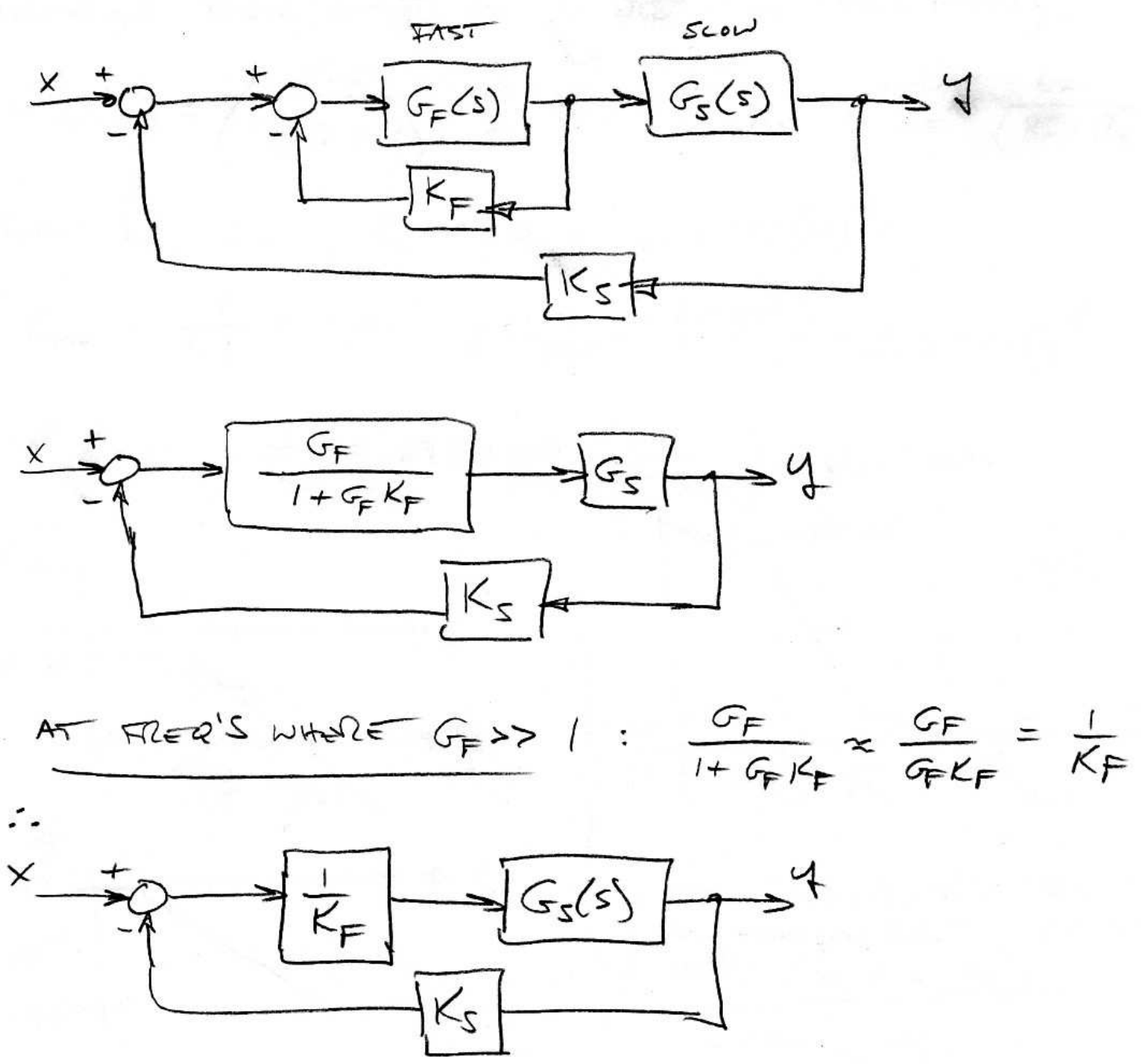

inNER coop looks like A PURE GAN oF $\left(\frac{1}{K_{F}}\right)$ AT GREQ'S WHORE GP $(S) \gg 1$

Figure A-5: Hand-calculation proof showing that a fast inner loop acts like a constant gain block for frequencies below one tenth of its crossover frequency.

483 


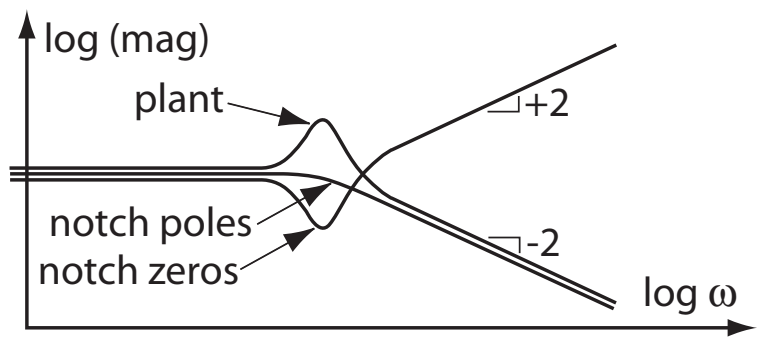

Figure A-6: Sketch of the Bode magnitude plots of a generic under-damped secondorder plant and of the zeros and poles of an ideal notch filter. Curves shown slightly separated vertically for clarity.

plant resonance, and the notch poles cancel the high frequency gain from the notch zeros. Note that the notch zeros need to be under-damped to provide a peak, and that the notch poles have little or no peaking.

An important thing to consider when designing a notch filter is the nature of the plant resonance that we want to reduce. That resonance can most likely be excited by a broad band of frequencies centered about some center frequency, and that center frequency is probably not stationary. Therefore, a sharp notch will most likely be too narrow to be effective, and it will most likely miss the resonance at some point in time.

When designing a notch filter for a plant resonance above the crossover frequency for the negative loop transmission, a trade-off is needed between the amount of attenuation that the notch zeros provides and the effect of the notch poles on the phase margin for the loop. Figure A-7 and Figure A-8 show the Bode magnitude and phase plots for the canonical notch filter in (A.1) with the natural frequency for the zeros and poles both set to $4620 \mathrm{~Hz}$. In Figure A-7 the damping ratio for the notch zeros is varied, and the damping ratio for the notch poles is fixed. Decreasing the damping ratio of the notch zeros deepens the notch but it also decreases the phase angle for frequencies below the notch center frequency. In Figure A-8 the damping ratio for the notch zeros is fixed, and the damping ratio for the notch poles is varied. Increasing the damping ratio of the notch poles deepens the notch by decreasing the peaking from those poles, but it also decreases the negative phase from those poles at frequencies below the notch natural frequency. In both cases, a deeper notch reduces the 

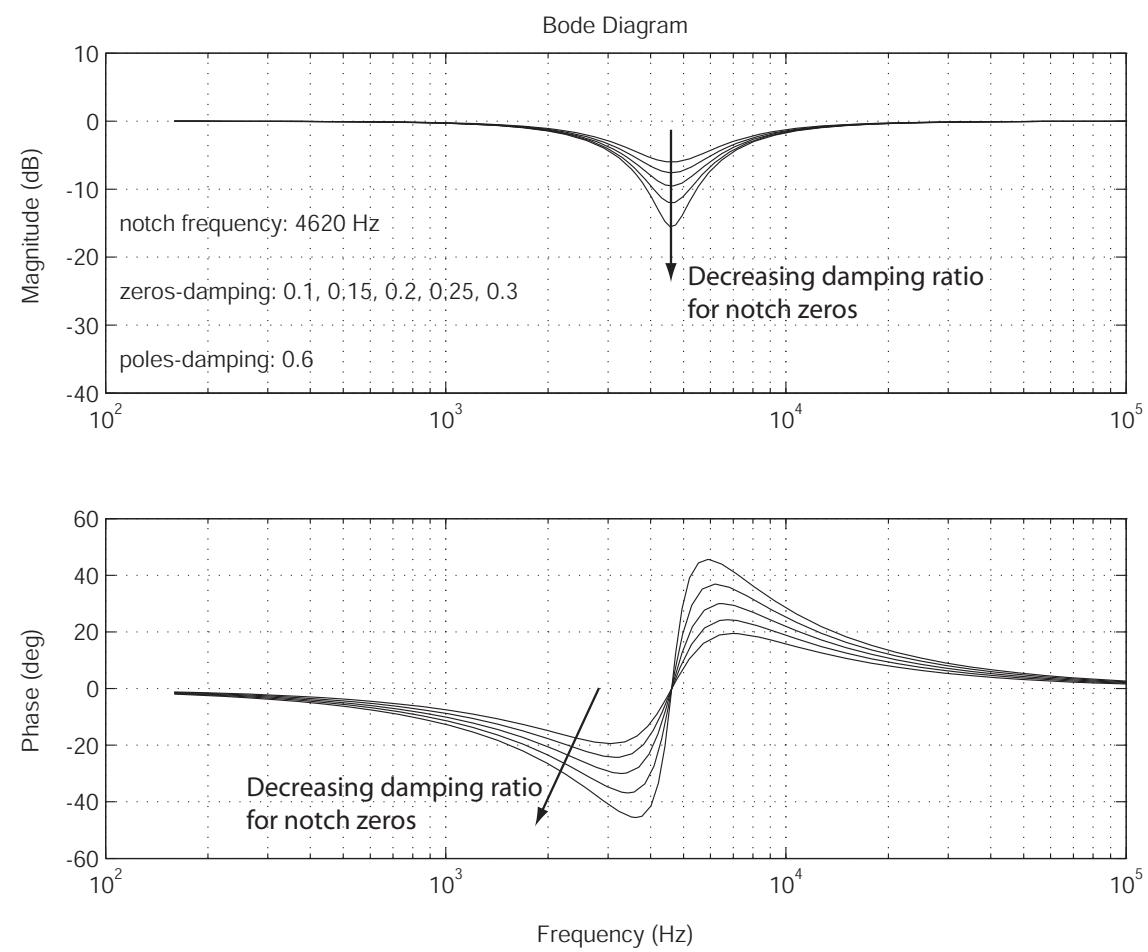

Figure A-7: Bode magnitude and phase plots for a canonical notch filter. The natural frequencies of the notch zeros and poles are equal, the damping ratio for the notch zeros is varied, and the damping ratio for the notch poles is fixed.

phase angle of the negative loop transmission at frequencies below the notch natural frequency, but the effect is less severe when the damping ratio of the notch zeros is used to adjust the depth of the notch.

Regarding where to place the notch filter in the control system - in the forward path or in the feedback path - for the $2 \mathrm{kHz}$ rotary fast tool servo without viscous damping I found that placing the notch filter in the forward path provided better results than placing it in the feedback path. The following qualitative explanation supports that observation. If the frequency of the reference (command) signal is the same as the plant resonance, then a notch filter in the forward path will attenuate that egregious reference signal before it reaches the plant. If the notch filter is in the feedback path, then that egregious command signal passes through the forward path without attenuation and excites the plant resonance, and the feedback sensor is somewhat "blinded" to that resonance by the attenuation in the feedback path. In the case of an external disturbance to the plant at the same frequency as the resonance, 

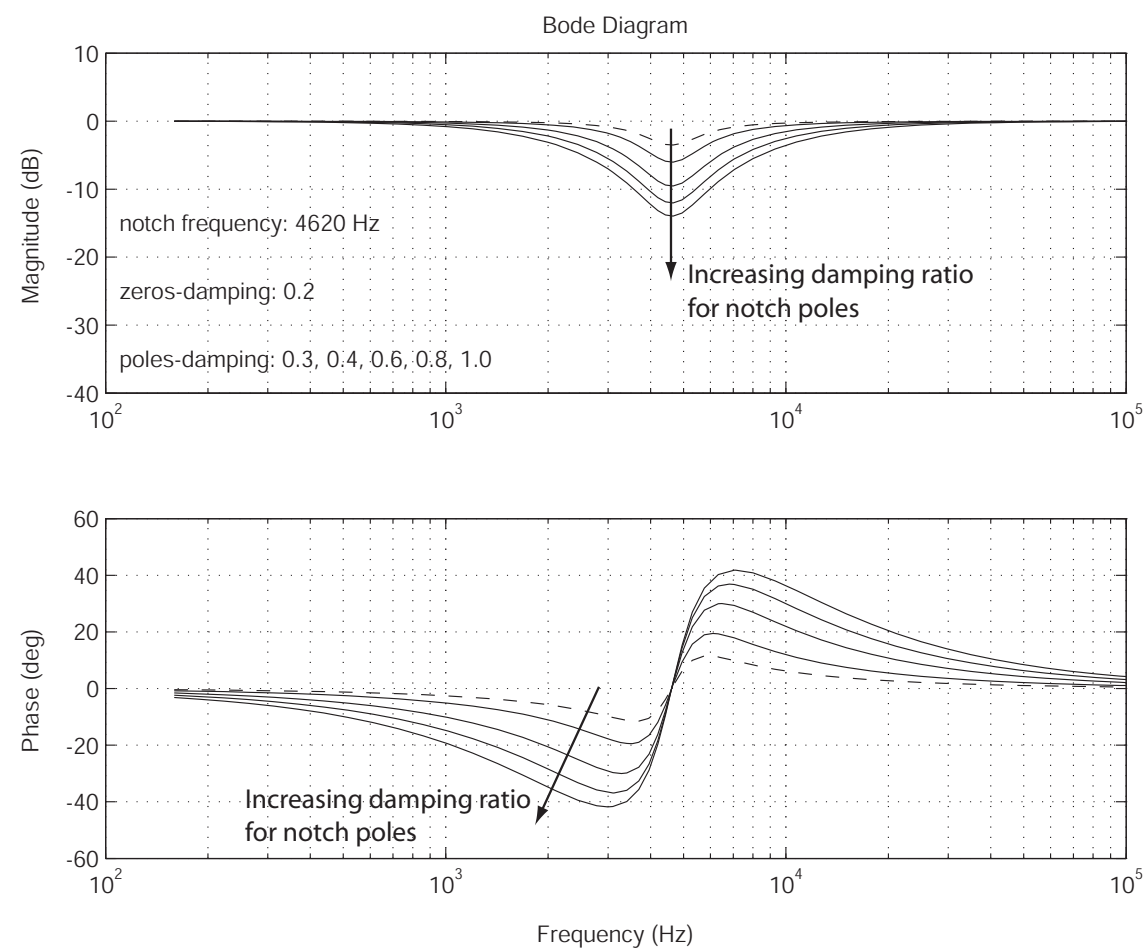

Figure A-8: Bode magnitude and phase plots for a canonical notch filter. The natural frequencies of the notch zeros and poles are equal, the damping ratio for the notch zeros is fixed, and the damping ratio for the notch poles is varied.

in the absence of a command signal the loop acts the same for the notch filter in the forward path and in the feedback path. Therefore, if the possibility exists that the command signal might excite the plant resonance, it is better to place the notch filter in the forward path. 


\section{Appendix B}

\section{MATLAB $^{\circledR}$ Files}

This appendix contains selected MATLAB ${ }^{\circledR}$ scripts that I developed as tools for designing and testing the $2 \mathrm{kHz}$ and $10 \mathrm{kHz}$ rotary fast tool servos and the hybrid rotary/linear actuator.

I had to insert additional line breaks to prevent the text from running off of the pages of this thesis, and added an ellipsis (...) to indicate those breaks. The reader is warned that although MATLAB ${ }^{\circledR}$ uses the ellipsis to indicate that the command line continues on the next line, execution of a script could get fouled if MATLAB ${ }^{\circledR}$ does not like the placements used in this thesis.

\section{B.1 step_change_oscillator.m}

\% filename: "step_change_oscillator.m", Rick Montesanti 2/12/04

$\%$ Simulation of a mass-spring oscillator without damping acted upon $\%$ by a sequence of step force inputs. A simplified model for making $\%$ step changes in the depth of cut of an electric engraver by $\%$ superimposing step inputs and a sinusoidal oscillation.

$\%$

clear all;

hold off 
$\%$

$\%$ system model

$m=1$

$\mathrm{k}=(2 * \mathrm{pi})^{\wedge} 2$

$\mathrm{f}=\operatorname{sqrt}(\mathrm{k} / \mathrm{m}) /(2 * \mathrm{pi})$

num $=[1 / \mathrm{m}]$;

$\operatorname{den}=\left[\begin{array}{lll}1 & 0 & \mathrm{k} / \mathrm{m}\end{array}\right]$;

sys $=\mathrm{tf}($ num, $\mathrm{den})$

$\%$

$\%$ input vector

$u 1=\operatorname{zeros}(50,1)$;

u2 $=0.75 *$ ones $(150,1)$;

$\mathrm{u} 3=-0.25 *$ ones $(50,1)$;

$\mathrm{u} 4=-1.25 *$ ones $(200,1)$;

$\mathrm{u} 5=-0.625 *$ ones $(50,1)$;

$u 6=\operatorname{zeros}(150,1)$;

$u 7=0.375 *$ ones $(50,1)$;

u $8=0.75 *$ ones $(150,1)$;

u9=zeros $(50,1)$;

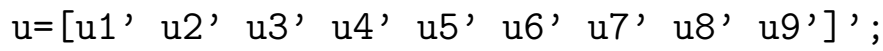

numstep=numel $(u)$

tstep $=0.01$

t=0:tstep: ((numstep-1)*tstep) ;

$\%$

$\%$ simulate response

$\operatorname{lsim}($ sys, $u, t)$

$\%$

$\%$ plot results

$\operatorname{axis}([0,($ numstep*tstep) $,-0.055,0.045]$ )

xlabel ('time') 
ylabel('input and response amplitudes (not to scale)')

hold on

$\operatorname{plot}(t,(u * 0.025))$

title(['"step change oscillator.m", RCM ', date])

$\operatorname{plot}(t,(-(0.025 * 0.25) *$ ones (numstep)) , 'k: ')

$\%$

\%end script 


\section{B.2 FTS_modeled_dynamics.m}

\% filename: "FTS_modeled_dynamics.m", Rick Montesanti 7/1/05

$\%$ Script for producing simple modeled dynamics for $2 \mathrm{kHz}$ FTS.

\% -loop transmission, root locus (find C.L. poles with

$\%$ "gain=1.0" points),

$\%$ and Nyquist.

$\%$ Uncomment single $\%$ lines to make that model active.

$\%$

clear all

$\%$

$s=\operatorname{tf}(' s ')$;

$\%$

\% \% \% \% \% \% \% \% \% \% \% \% \% \% \% \% \% \% \% \% \% \% \% \% \% \% \% \% \% \% \% \% \% \% \% \% \% \% \% \% \% \% \% \% \% \% \% \% \% \% \% \% \% \% \% \% \% \% \% \%

$\% 2 \mathrm{kHz}$ FTS, no damping, lead only, MIT vol. 4 p. $63 \%$ \% \% \% \% \% \% \% \% \% \% \% \% \% \% \% \% \% \% \% \% \% \% \% \% \% \% \% \% \% \% \% \% \% \% \% \% \% \% \% \% \% \% \% \% \% \% \% \% \% \%

\% see "1_7_03_baseline_1" loop transmission

$\%$ plant

$\mathrm{K}=3 ; \%$ adjust to fit model to measured LT

w1 $=200 * 2 *$ pi ;

zeta $1=0.1 ; \%$ adjust to fit model to measured LT

w2 $=4660 * 2 * \mathrm{pi}$;

zeta $2=0.08 ; \%$ adjust to fit model to measured LT

$\mathrm{Gp}=1 /\left(\left(\left(\mathrm{s}^{\wedge} 2 / \mathrm{w} 1 \wedge 2\right)+(2 * z e t a 1 / \mathrm{w} 1) * \mathrm{~s}+1\right) *\left(\left(\mathrm{~s}^{\wedge} 2 / \mathrm{w} 2 \wedge 2\right)+\ldots\right.\right.$

$(2 *$ zeta2/w2)*s +1$))$;

$\%$ lead compensation, SINGLE

alpha_lead=10;

tau_lead=2.5e-5;

Glead $=(($ alpha_lead $*$ tau_lead $* s+1) /($ tau_lead $* s+1))$; 
$\mathrm{Gc}=\mathrm{K} * \mathrm{Glead}$;

$\%$ dSpace gain

$\% \mathrm{~K}=5$

$\%$ measured loop transmission

$\% \mathrm{FC}=400 \mathrm{~Hz}$

$\% \mathrm{PM}=$ unstable

$\%$ GM=unstable

$\%$

\% \% \% \% \% \% \% \% \% \% \% \% \% \% \% \% \% \% \% \% \% \% \% \% \% \% \% \% \% \% \% \% \% \% \% \% \% \% \% \% \% \% \% \% \% \% \% \% \% \% \% \% \% \% \% \% \% \% \% \% \% \% \% \%

$\% \% 2 \mathrm{kHz}$ FTS, no damping, double-notch fiter, MIT vol.4 p.90\% \% \% \% \% \% \% \% \% \% \% \% \% \% \% \% \% \% \% \% \% \% \% \% \% \% \% \% \% \% \% \% \% \% \% \% \% \% \% \% \% \% \% \% \% \% \% \% \% \% \% \% \% \% \% \% \% \% \% \% \% \% \% \% \% \%

$\%$ see "1_28_03_lt_1" loop transmission

$\%$ plant

$\% \mathrm{~K}=10000 ; \%$ adjust to fit model to measured LT

$\% \mathrm{w} 1=200 * 2 * \mathrm{pi}$;

$\%$ zeta $1=0.1 ; \%$ adjust to fit model to measured LT

$\% \mathrm{w} 2=4660 * 2 * \mathrm{pi}$;

$\%$ zeta $2=0.08 ; \%$ adjust to fit model to measured LT

$\% \mathrm{Gp}=1 /\left(\left(\left(\mathrm{s}^{\wedge} 2 / \mathrm{w} 1 \wedge 2\right)+(2 * z e t a 1 / \mathrm{w} 1) * \mathrm{~s}+1\right) *\left(\left(\mathrm{~s}^{\wedge} 2 / \mathrm{w} 2 \wedge 2\right)+\ldots\right.\right.$

$\%(2 * z e t a 2 / \mathrm{w} 2) * \mathrm{~s}+1))$;

$\%$ lead compensation, DOUBLE

$\%$ alpha_lead=3.2;

$\%$ tau_lead $=4.45 e-5$;

$\%$ Glead $=(($ alpha_lead $*$ tau_lead $* s+1) /($ tau_lead $* s+1)) * \ldots$

$\%(($ alpha_lead $*$ tau_lead $* s+1) /($ tau_lead $* s+1))$;

$\%$ lag compensation (zero and integrator)

$\%$ tau_lag=1.59e-3;

$\%$ Glag $=($ tau_lag*s +1$) / \mathrm{s}$;

$\%$ notch filter

$\% \mathrm{wz}=4620 * 2 * \mathrm{pi}$; 
$\%$ zetaz=0.2;

$\%$ wp=wZ;

$\%$ zetap $=1.0$;

$\%$ Gnotch $=\left(\mathrm{s}^{\wedge} 2+2 * z e t a z * w z * s+\mathrm{wz}^{\wedge} 2\right) /\left(\mathrm{s}^{\wedge} 2+2 *\right.$ zetap*wp*s $\left.+\mathrm{wp}^{\wedge} 2\right)$;

$\%$

$\% \mathrm{Gc}=\mathrm{K} * \mathrm{Glead} * \mathrm{Glag} *$ Gnotch $;$

$\%$ dSpace gain

$\% \mathrm{~K}=15000$

$\%$ measured loop transmission

$\%$ FC=1000 Hz

$\% \quad \mathrm{PM}=13$ degrees

$\%$ GM $=\sim 5 \mathrm{~dB}$

$\%$

\% \% \% \% \% \% \% \% \% \% \% \% \% \% \% \% \% \% \% \% \% \% \% \% \% \% \% \% \% \% \% \% \% \% \% \% \% \% \% \% \% \% \% \% \% \% \% \% \% \% \% \% \%

$\% 2 \mathrm{kHz}$ FTS, no damping, extra pole, MIT vol. 4 p. $103 \%$

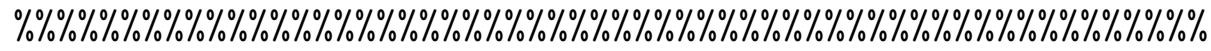

$\%$ see "2_2_03_1t_1" loop transmission

$\%$ plant

$\% \mathrm{~K}=15000 ; \%$ adjust to fit model to measured LT

$\% \mathrm{w} 1=200 * 2 * \mathrm{pi}$;

$\%$ zeta $1=0.1 ; \%$ adjust to fit model to measured LT

$\% \mathrm{w} 2=4660 * 2 * \mathrm{pi}$;

$\%$ zeta $2=0.08 ; \%$ adjust to fit model to measured LT

$\% \mathrm{Gp}=1 /\left(\left(\left(\mathrm{s}^{\wedge} 2 / \mathrm{w} 1{ }^{\wedge} 2\right)+(2 * \mathrm{zeta} 1 / \mathrm{w} 1) * \mathrm{~s}+1\right) *\left(\left(\mathrm{~s}^{\wedge} 2 / \mathrm{w} 2^{\wedge} 2\right)+\ldots\right.\right.$

$\%(2 *$ zeta $2 /$ w2 $) * \mathrm{~s}+1))$;

$\%$ lead compensation, DOUBLE

$\%$ alpha_lead=3.2;

$\%$ tau_lead $=4.45 e-5$;

$\%$ Glead $=(($ alpha_lead $*$ tau_lead $* s+1) /($ tau_lead $* s+1)) * \ldots$

$\%(($ alpha_lead $*$ tau_lead $* s+1) /($ tau_lead $* s+1))$; 
$\%$ lag compensation (zero and integrator)

$\%$ tau_lag=1.59e-3;

$\% \mathrm{Glag}=($ tau_lag*s+1) $/ \mathrm{s}$;

$\%$ extra pole

$\%$ tau_ep $=1 /(5000 * 2 * \mathrm{pi})$;

$\%$ Gextrapole=1/(tau_ep*s+1) ;

$\% \%$

$\% \mathrm{Gc}=\mathrm{K} * \mathrm{Glead} * \mathrm{Glag} * \mathrm{Gextrapole}$;

$\%$ dSpace gain

$\% \quad \mathrm{~K}=1600$

$\%$ measured loop transmission

$\% \quad F C=1000 \mathrm{~Hz}$

$\% \quad \mathrm{PM}=23$ degrees

$\% \quad \mathrm{GM}=\sim 5 \mathrm{~dB}$

$\%$

$\% \% \% \% \% \% \% \% \% \% \% \% \% \% \% \% \% \% \% \% \% \% \% \% \% \% \% \% \% \% \% \% \% \% \% \% \% \% \% \% \%$

$\% \% 2 \mathrm{kHz}$ FTS, damping, MIT vol.4 p.151\% $\% \% \% \% \% \% \% \% \% \% \% \% \% \% \% \% \% \% \% \% \% \% \% \% \% \% \% \% \% \% \% \% \% \% \% \% \% \% \% \% \% \%$

$\%$ see "3_14_03_1t_3" loop transmission

$\%$ plant

$\% \mathrm{~K}=20000 ; \%$ adjust to fit model to measured LT

$\% \mathrm{w} 1=200 * 2 * \mathrm{pi}$;

$\%$ zeta $1=1.5 ; \%$ adjust to fit model to measured LT

$\% \mathrm{w} 2=4660 * 2 * \mathrm{pi}$;

$\%$ zeta $2=0.15 ; \%$ adjust to fit model to measured LT

$\% \mathrm{Gp}=1 /\left(\left(\left(\mathrm{s}^{\wedge} 2 / \mathrm{w} 1^{\wedge} 2\right)+(2 * z e t a 1 / \mathrm{w} 1) * \mathrm{~s}+1\right) *\left(\left(\mathrm{~s}^{\wedge} 2 / \mathrm{w} 2^{\wedge} 2\right)+\ldots\right.\right.$ $\%(2 *$ zeta $2 /$ w2 $) * s+1))$;

$\%$ lag compensation (zero and integrator)

$\%$ tau_lag $=1.59 e-4$

$\% \mathrm{Glag}=($ tau_lag*s+1) $/ \mathrm{s}$; 
$\%$ extra zero, TO GET THE MODEL TO FIT THE DATA

$\%$ note that damping in the coupling adds a zero, but it's high freq.

$\%$ tau_ez=1/(200*2*pi);

$\%$ Gextrazero $=($ tau_ez*s+1) ;

$\%$

$\% \mathrm{Gc}=\mathrm{K} * \mathrm{Glag} *$ Gextrazero ;

$\%$ dSpace gain

$\% \quad \mathrm{~K}=32000$

$\%$ measured loop transmission

$\%$ dSpace gain

$\% \quad F C=1000 \mathrm{~Hz}$

$\%$ PM=41 degrees

$\% \quad \mathrm{GM}=6 \mathrm{~dB}$

$\%$

$\% \% \% \% \% \% \% \% \% \% \% \% \% \% \% \% \% \% \% \% \%$

$\%$ calculate results $\%$

$\% \% \% \% \% \% \% \% \% \% \% \% \% \% \% \% \% \% \% \% \% \% \%$

$\%$

$\%$ negative loop transmission

[mag, phase, freq] =bode (Gp*Gc) ;

freq $=f r e q /(2 * p i)$;

index $=$ size ( freq);

index $(:, 2)=[]$;

for $i=1:$ index

$\operatorname{magdb}(i)=20 * \log 10(\operatorname{mag}(:,:, i)) ;$

phase1(i) $=\operatorname{phase}(:,:, i)$;

end

$\%$ Bode plot

subplot $(2,1,1)$

semilogx (freq, magdb, 'r') 


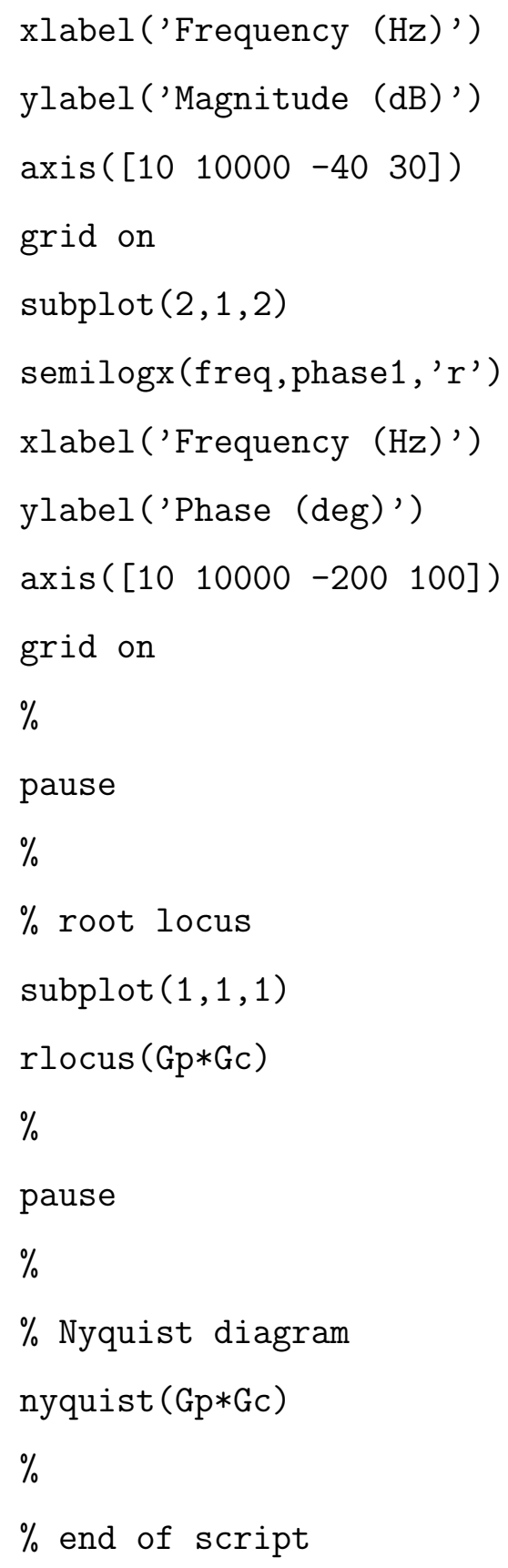




\section{B.3 push_pull_power_loss_02.m}

\% filename: "push_pull_power_loss_02.m", Rick Montesanti 11/15/04

$\%$ Power dissipation in a power amplifier push-pull output stage

$\%$ when driving an arbitrary load (can set phase lag of current).

\% Based on "push_pull_power_loss_01.m", RCM 7/24/04

$\%$

$\%$ units: volt, amp, s

$\%$

clear all

$\%$

$\%$ magnitude of plus and minus supply voltages

$\mathrm{Vs}=50$;

$\%$ peak load voltage

$\mathrm{Vl} p=41$

$\%$ peak load current

Ilp $=11$;

$\%$ phase lag of load current behind load voltage

phi $=(\mathrm{pi} / 2) *(73 / 90)$;

$\%$ number of data points per cycle

$\mathrm{N}=1000$;

$\%$ radian frequency

omega $=(2 * \mathrm{pi}) /(\mathrm{N}-1)$;

$\%$ generate waveforms for 1.5 cycles

for $\mathrm{n}=1: 1.5 * \mathrm{~N}$

$\%$ waveforms

Vsupply (n)=Vs ;

$\mathrm{VI}(\mathrm{n})=\mathrm{Vl}$ * $* \cos ($ omega $*(\mathrm{n}-1))$;

Il $(\mathrm{n})=\mathrm{Il} \mathrm{p} * \cos (\operatorname{omega} *(\mathrm{n}-1)-\mathrm{phi})$;

$\%$ load power 
$\mathrm{Pl}(\mathrm{n})=\mathrm{Vl}(\mathrm{n}) * \mathrm{Il}(\mathrm{n})$;

$\%$ voltage drop across push-pull stage

if $\mathrm{Il}(\mathrm{n})>0$

$\mathrm{Vpp}(\mathrm{n})=\mathrm{Vs}-\mathrm{Vl}(\mathrm{n})$;

elseif $I l(n)<0$

$\operatorname{Vpp}(\mathrm{n})=-\mathrm{Vs}-\mathrm{VI}(\mathrm{n})$;

else

$\operatorname{Vpp}(\mathrm{n})=\mathrm{Vs}-\mathrm{VIp} ;$

end

$\%$ power dissipation in push-pull stage

$\operatorname{Ppp}(\mathrm{n})=\operatorname{abs}(\operatorname{Vpp}(\mathrm{n}) * \mathrm{II}(\mathrm{n}))$;

end

$\%$ average sinewave power for load and push-pull

$\%$ stage over one cycle of the load voltage

sum_Pl=0;

sum_Ppp $=0$;

for $n=1: N$

sum_Pl=sum_Pl+Pl(n);

sum_Ppp=sum_Ppp+Ppp (n);

end

ave_Pl= $(1 / \mathrm{N}) *$ sum_Pl ;

ave_Ppp $=(1 / \mathrm{N}) *$ sum_Ppp;

$\%$ average sinewave power for load over a $1 / 4$ cycle

$\%$ of load voltage (ave power for an inductor $=0$ )

sum_Pl=0;

for $n=1:(N / 4)$

sum_Pl=sum_Pl+Pl(n);

end

ave_quarter_cycle_Pl=(4/N)*sum_Pl ;

$\%$ peak values 


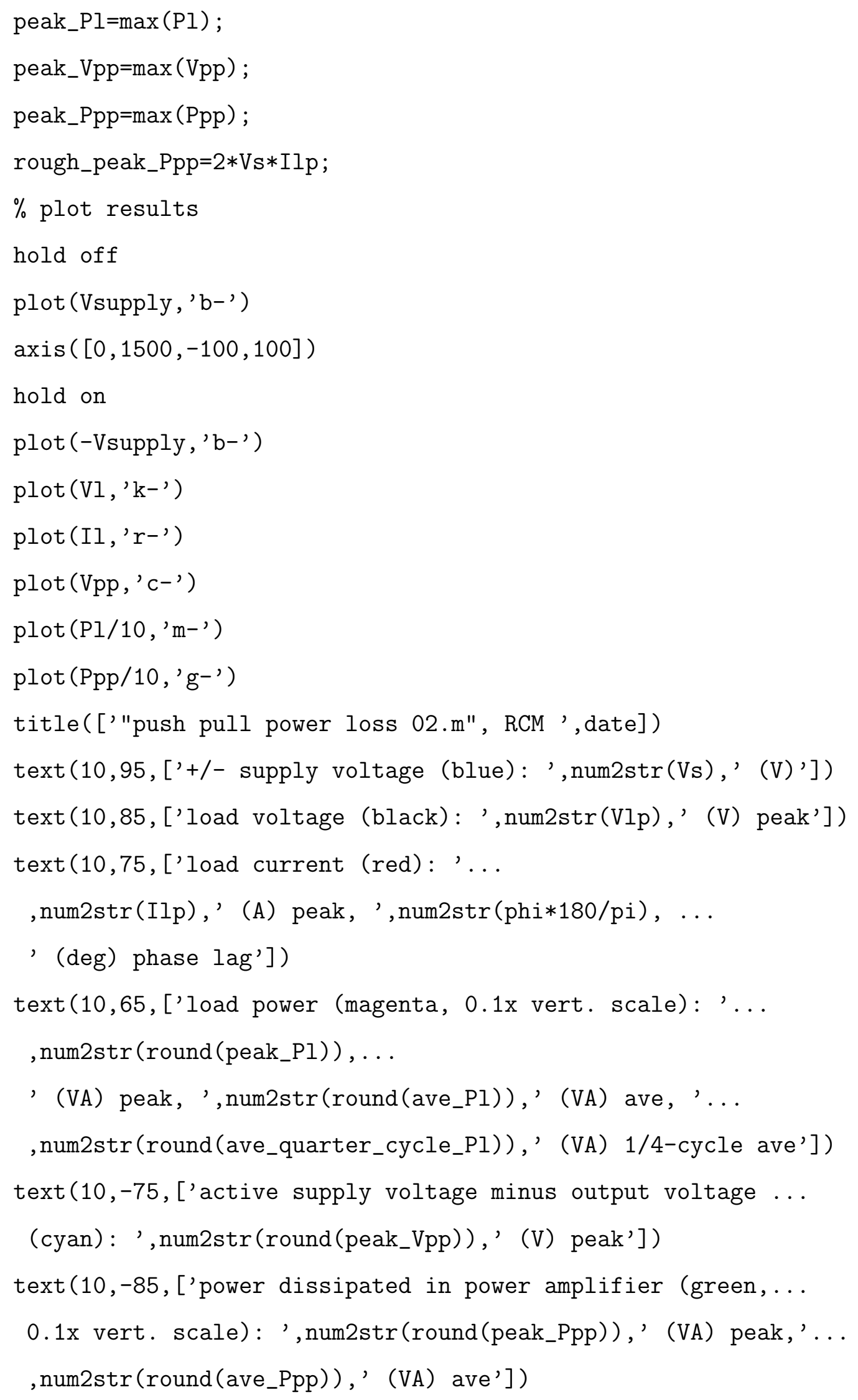


text (10,-95, ['(rail-to-rail supply voltage times peak load... current: ', num2str (round (rough_peak_Ppp)),' (VA) peak)'])

grid on

$\%$

$\%$ end of script 


\section{B.4 elastic_properties_Ni_Fe_001.m}

$\%$ Determines material elastic constants for the rotor $\%$ lamination stack.

$\%$ Specifically, 0.001" 50\% Ni-50\% Fe with SF=0.79.

$\%$ Treats the lamination stack of iron and epoxy as transversely

$\%$ isotropic.

$\%$ Based on RCM 4/12/04 and RCM 4/7/04 calculations.

$\%$ Rick Montesanti 4/21/04

$\%$ Updated 4/13/04 version to include increased Young's modulus

$\%$ in normal direction due to uniaxial strain condition of $\%$ constrained epoxy layer.

\% filename: "elastic_properties_Ni_Fe_001.m"

$\%$

clear all

$\%$

date

$\%$

units $=\operatorname{sprintf}\left({ }^{\prime} \% \mathrm{~s}^{\prime}\right.$, , $^{\prime}$ in $\left., 1 \mathrm{bf}, \mathrm{sec}^{\prime}\right)$

$\%$

$\%$ isotropic material properties

$\% \quad 50 \% \mathrm{Ni}-50 \% \mathrm{Fe}$

$\mathrm{E} 1=24 \mathrm{e} 6 \% \mathrm{psi}$

nu1 $=0.3$

rho1=0.0007635 \% lbf-sec^2/in^4

$\%$

\% epoxy (using properties from Pro/Mechanica: "epoxy, unfilled

$\%$ casing resin Cook, adv. mechanics)

E_epoxy $=0.4351 \mathrm{e} 6 \% \mathrm{psi}$

nu_epoxy $=0.37$ 


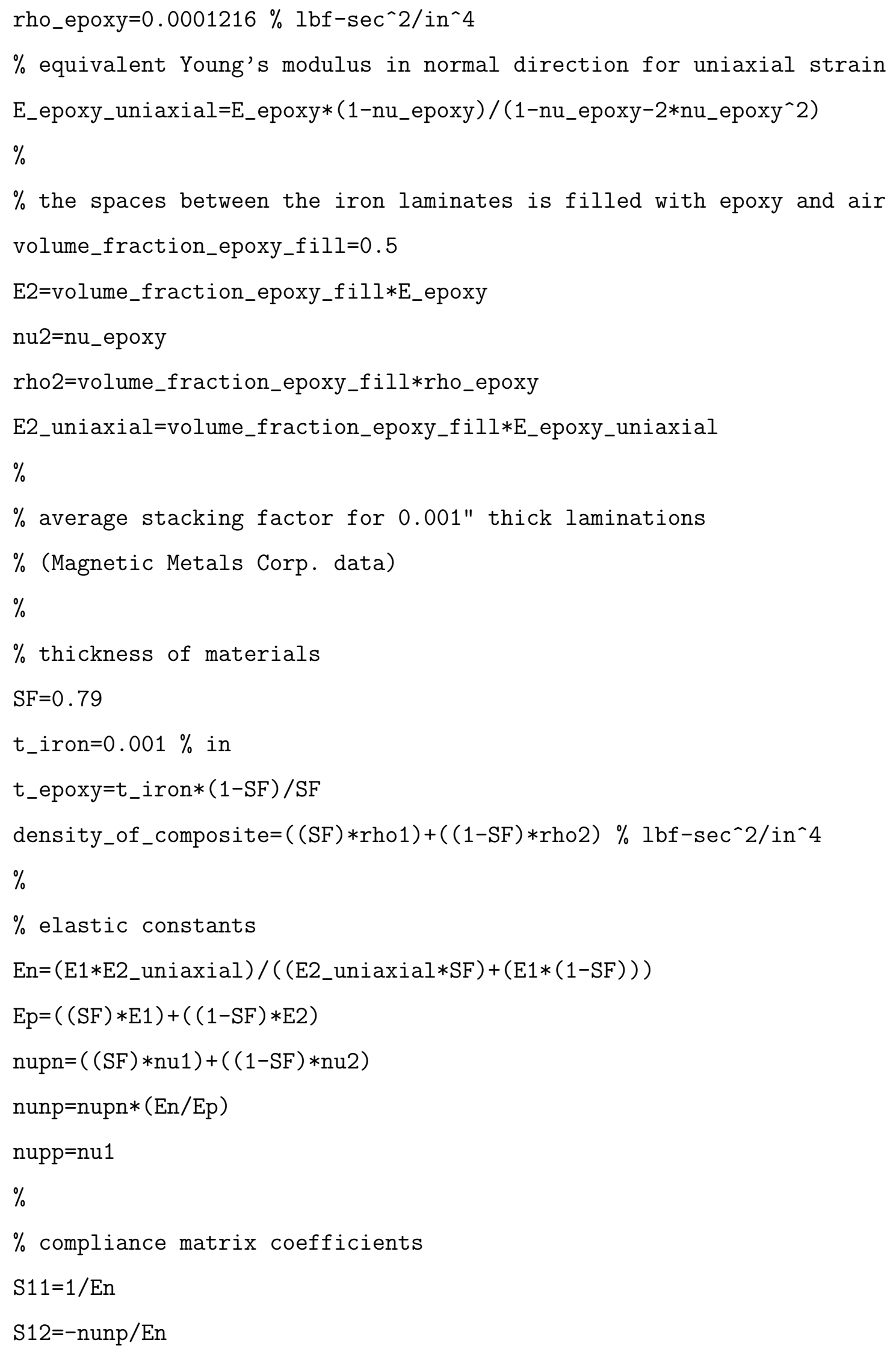




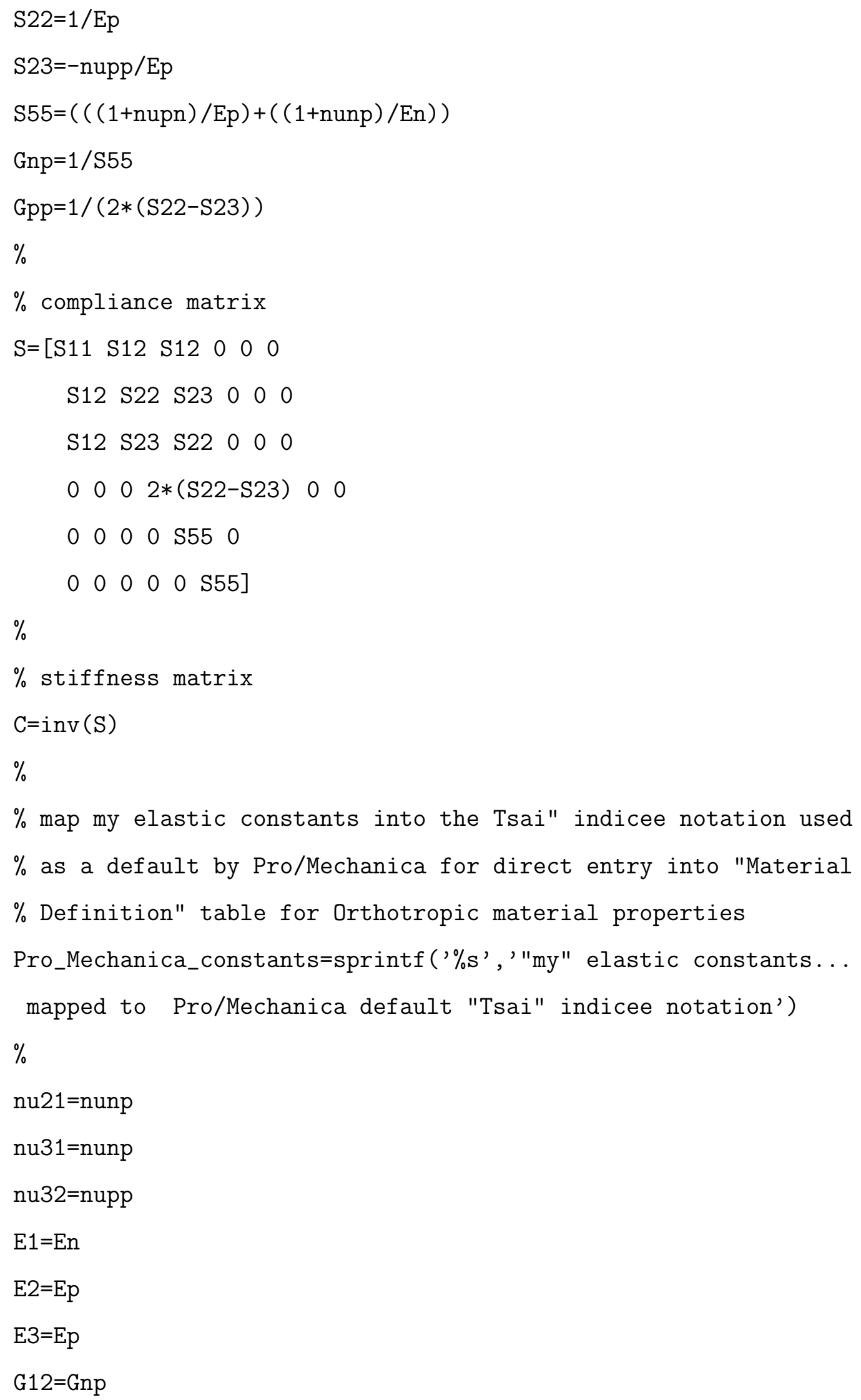


G13=Gnp

G23=Gpp

rho=density_of_composite

$\%$

$\%$ end of script 


\section{B.5 PSD_scope_data.m}

\% filename: "PSD_scope_data.m", Rick Montesanti 4/7/05

$\%$ Script for loading previously saved data from a scope trace,

$\%$ computing the FFT and PSD, and plotting the data and PSD.

$\%$ Baseline is for a 5000 point data file.

\% Data was saved in a Comma Separated Value (.csv) file

$\%$ (in working directory) with the following form:

$\%$ | S | Volts | S | Volts | S | Volts | ...

$\%$

$\%$ NOTE: Need to delete first line in Tektronix .csv data files so

$\%$ that Matlab does not choke on "S" and "Volts" column headings.

$\%$

clear all; \% clear all variables

$\%$

$\%$ load scope data

$\% \quad * * * * * * * * * * * * * * * * * * * * * * * * * * * * * * * * * * * * * * * * * * * * * * * * * * * * * * * * * * * * * * * * *$

$\% * * *$ BE SURE CORRECT FILE NAME AND GAIN APPEAR IN NEXT 3 LINES ***

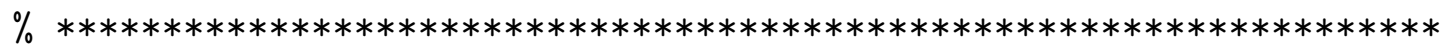

data='4_15_05_noise_05a_data.csv'

data_file_name='4 1505 noise 05a data.csv' \% replace

$\%$ underscores with spaces

diff_amp_gain=100; \% gain from differential amplifier

$\%$ (set to 1.0 if no diff amp)

raw_data=csvread (data);

$\% * * * * * * * * * * * * * * * * * * * * * * * * * * * * * * * * * * * * * * * * * * * * * * * * * * * * * * * * * * * * *$

$\%$

$\mathrm{x}_{\text {ddata }}=\mathrm{raw} \_$data $(:, 1)$;

y_data=raw_data $(:, 2) /$ diff_amp_gain; \% remove gain from

$\%$ differential amplifier 
y_data=y_data-mean(y_data); \% remove DC offset from y_data

size_x $=$ size (x_data);

size_y=size(y_data);

num_y_data $=$ size $_{-} y(1)$

$\%$ determine total time and time spacing of data

total_time=x_data (num_y_data) $-x_{\text {_data }}(1)$

$T_{-} s=x_{-} \operatorname{data}(2)-x_{-} \operatorname{data}(1)$

$F_{-} s=\operatorname{round}\left(1 / T_{-} s\right)$

$\%$

\% test script using Matlab help-file example (w/ improvements):

$\% 50 \mathrm{~Hz}$ and $120 \mathrm{~Hz}$ signal with noise, sampled at $1 \mathrm{kHz}$

$\%$ ts $=0.001$

$\%$ fs $=$ round $(1 /$ ts $)$

$\% t=0:$ ts:0.6;

$\% \mathrm{x}=\sin (2 * \mathrm{pi} * 50 * \mathrm{t})+\sin (2 * \mathrm{p} i * 120 * \mathrm{t}) ;$

$\% y=x+2 * \operatorname{randn}(\operatorname{size}(t))$;

$\%$ subplot $(2,1,1)$

$\% \operatorname{plot}(1000 * t(1: 50), \mathrm{y}(1: 50))$

\%title('Signal Corrupted with Zero-Mean Random Noise')

$\% x l a b e l$ ('time (milliseconds)')

$\% Y=f f t(y, 512) ;$

$\%$ Pyy $=$ Y. $* \operatorname{conj}(\mathrm{Y}) / 512$;

$\% \mathrm{f}=\mathrm{fs} *(0: 256) / 512$;

$\%$ subplot $(2,1,2)$

$\% \operatorname{plot}(f$, Pyy $(1: 257))$

$\%$ title('Frequency content of y')

$\% x l$ abel ('frequency (Hz)')

$\%$ pause

$\%$

$\%$ compute power spectral density of $\mathrm{y}_{\text {_data }}$ using a $2^{\wedge} \mathrm{N}$ point $\mathrm{DFFT}$ 
$\%$ (baseline is $2^{\wedge} 11=2048$, increase if freq bins are too coarse) num_points_fft=2^13

$\%$ check to make sure record length is long enough

$\%$ (possible computation errors if padded with zeros)

if num_points_fft > num_y_data

disp('WARNING: FFT is padded with zeros: possible errors.')

disp('Decrease \# of FFT points, or increase record length.')

end

fft_y_data=fft(y_data,num_points_fft);

size_fft=size(fft_y_data);

psd_y_data=fft_y_data.*conj(fft_y_data)/num_points_fft;

size_psd=size (psd_y_data);

freqs $=F \_s *(0:($ num_points_fft/2))/num_points_fft;

$\%$

$\%$ compute the rms (standard) deviation for the ADC signal

$\%$ root of the mean of the squares of the deviations from the average) rms_deviation_y_data=std(y_data);

$\%$

$\%$ plot data

$\%$ first plot

subplot $(2,1,1)$

plot (x_data,y_data)

$\% \operatorname{axis}([0$ total_time $-1 \quad 1]$ )

\%axis 'auto y'

title([num2str(num_y_data), ' pts, F_s=', . .

num2str(round $\left.\left(1 \mathrm{e}-3 * F_{-} s\right)\right), ' \mathrm{kHz}$, RMS dev=', .. .

num2str (round (1e6*rms_deviation_y_data)), uV (', . .

num2str(round(1e3*total_time)),' msec), data=" ', . .

data_file_name, '"'])

xlabel ('Time (sec)') 
ylabel ('Voltage, DC removed (V_p_e_a_k)')

$\%$ graph the first the first half of the PSD points (it's symmetrical) subplot $(2,1,2)$

plot (freqs,psd_y_data $(1:($ num_points_fft/2+1)))

$\operatorname{axis}\left(\left[\begin{array}{llll}0 & 1000 & 0 & 100\end{array}\right]\right)$

axis 'auto $\mathrm{y}^{\prime}$

title([num2str(num_points_fft),' pt DFFT, ',...

num2str (round (F_s/num_points_fft)) , . .

' $\mathrm{Hz}$ bins, using "PSD scope data.m", RCM ', date])

xlabel ('Frequency (Hz)')

ylabel ('PSD (V^2_p_e_a_k/Hz)')

$\%$

pause

$\%$

$\%$ second plot

subplot $(2,1,1)$

plot (x_data,y_data)

$\operatorname{axis}\left(\left[\begin{array}{llll}0 & 0.001 & -1 & 1\end{array}\right]\right)$

axis 'auto $\mathrm{y}^{\prime}$

title([num2str(num_y_data), ' pts, $F_{-} s=', \ldots$

num2str(round $\left.\left(1 \mathrm{e}-3 * F_{-} s\right)\right), ' \mathrm{kHz}$, RMS dev=', ...

num2str(round (1e6*rms_deviation_y_data)), 'uV (', . .

num2str(round(1e3*total_time)),' msec), data=" '...

,data_file_name,'"')])

xlabel ('Time (sec)')

ylabel ('Voltage, DC removed (V_p_e_a_k)')

$\%$ graph the first the first half of the PSD points (it's symmetrical)

subplot $(2,1,2)$

plot (freqs,psd_y_data $(1:($ num_points_fft $/ 2+1)))$

$\operatorname{axis}\left(\left[\begin{array}{lllll}0 & 10000 & 0 & 100\end{array}\right)\right.$ 


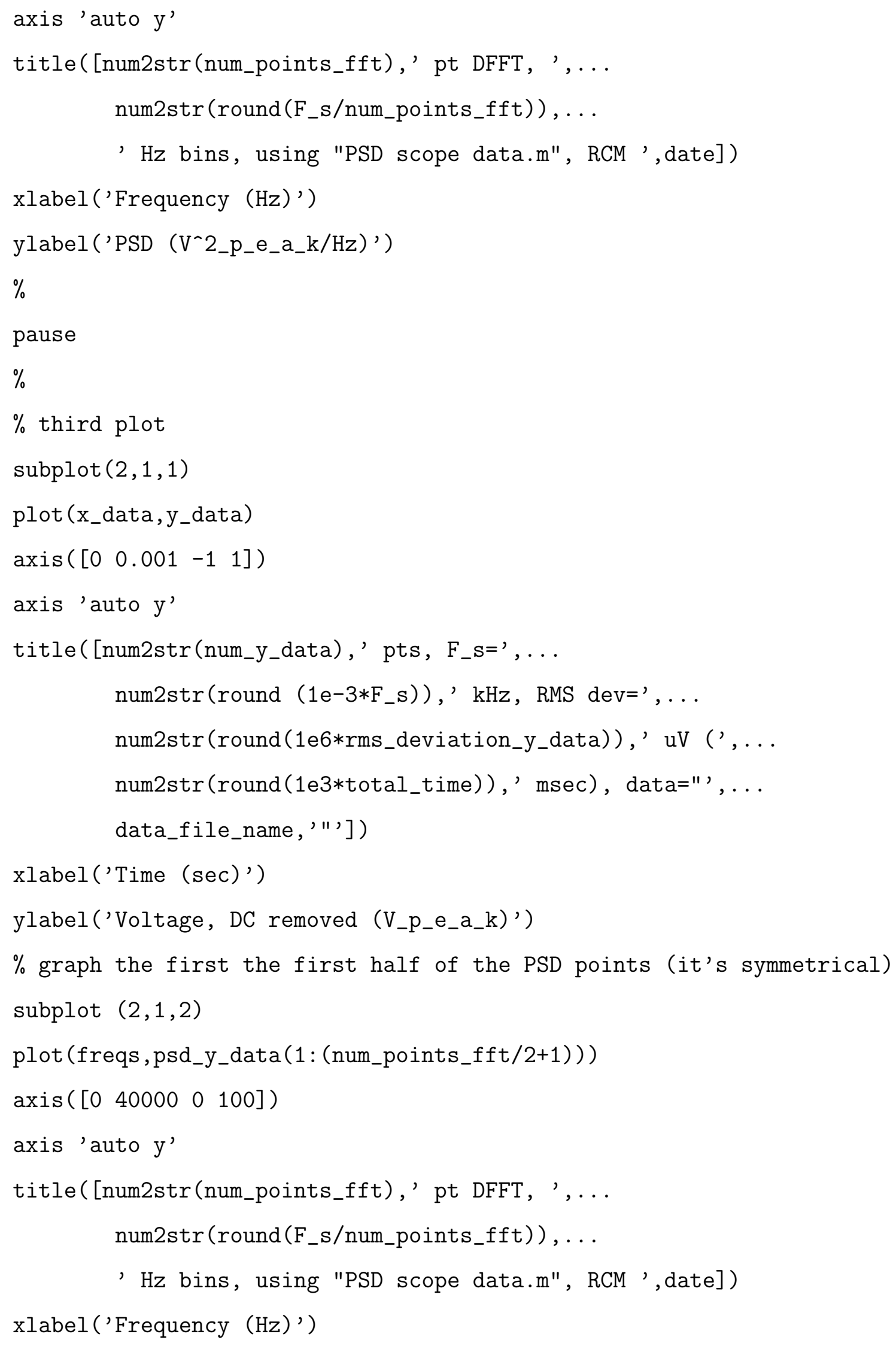




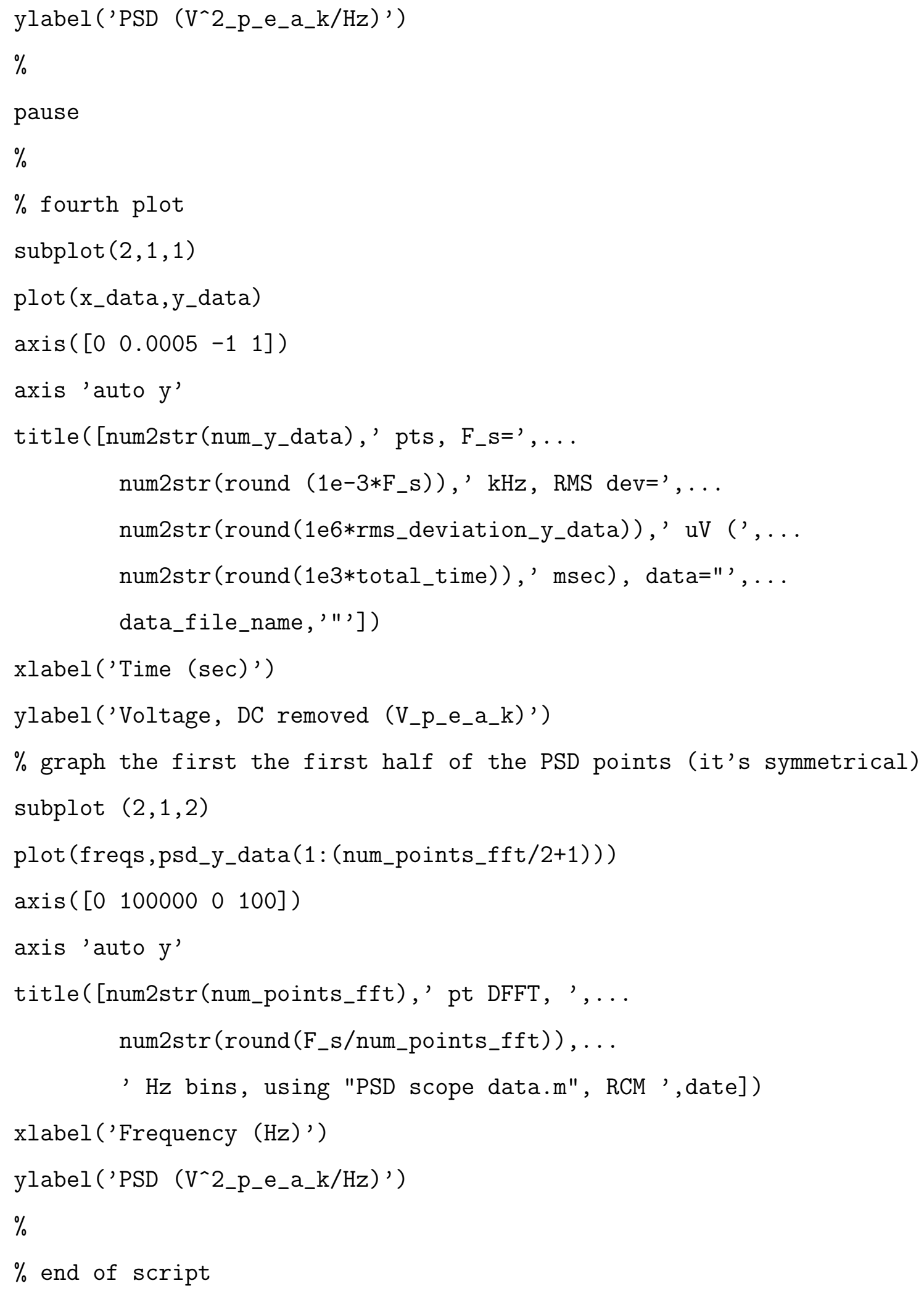




\section{B.6 flux_torque_force_01.m}

\% filename: "flux_torque_force_01.m", Rick Montesanti 3/12/05

\% corrected on 8/22/05: fixed erroneous factor of 2 on bias flux.

\% Based on RCM 3/12/05 "sandwiched versus pinched rotor" calc

\% units: m, kg, sec, rad, Tesla, Ampere, Henry, Volt, Ohm, Farad

$\%$

$\%$ sandwiched rotor pinched rotor

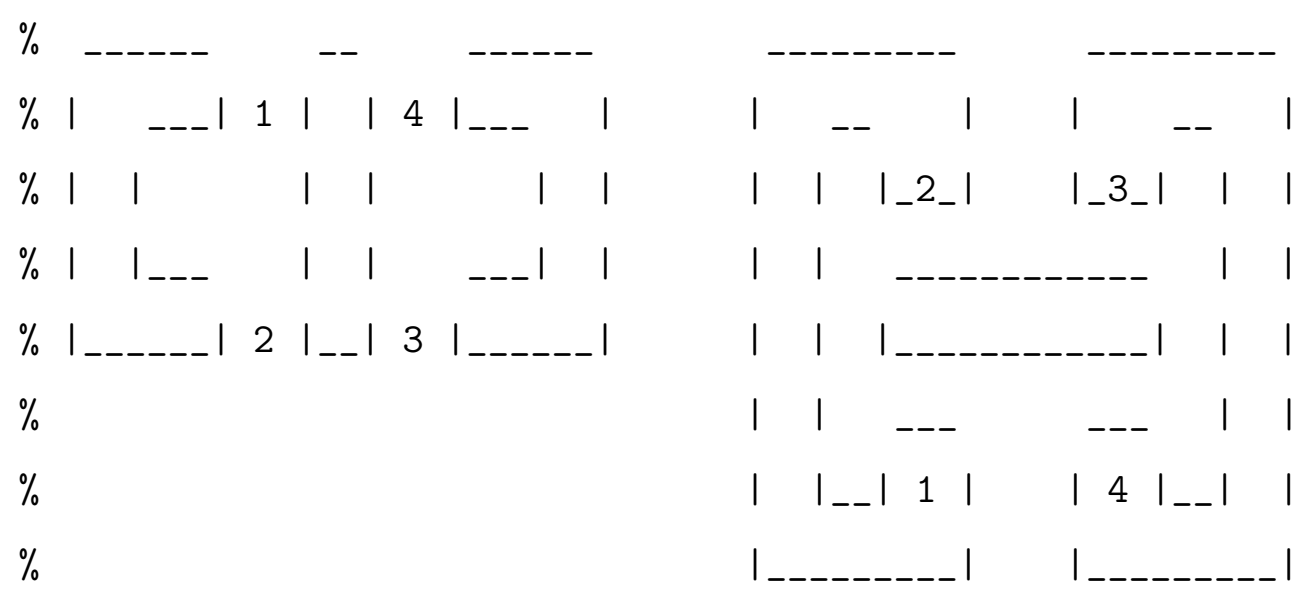

clear all

$\%$

$\%$ equations for calculating fluxes from

$\%$ air gap lengths, turns, and currents

$\%$ permiablity of air (Henry/m)

u_0 $=4 *$ pi $* 1 e^{-7}$;

$\%$ nominal air gap length for each rotor pole

g_n $=50 e-6$

$\%$ air gap length between each stator core and back iron

$g_{-} b=250 e-6$

$\%$ number of total turns on stator core \#12

N_12 $=20$;

$\%$ number of total turns on stator core \#34

N_34 $=20$; 
$\%$ number of turns on bias core

N_b $=560$;

$\%$ current in bias coil

$i_{-} b=1.0$

$\%$ number of increments for steering coil current (plus initial)

$\mathrm{n}=21$

$\%$ midpoint

$\mathrm{n} \_\operatorname{mid}=\mathrm{round}(\mathrm{n} / 2)$

$\%$ currents in steering coils for producing torque

i_t $=$ linspace $(0,1.0, \mathrm{n})$;

$\%$ currents in steering coils for producing force

$i_{-} f=l$ inspace $(0,0.1, n)$;

$\%$ current in steering coil \#12 for producing torque

i_12t=i_t;

$\%$ current in steering coil \#12 for producing force

i_12f $=i_{-} f$;

$\%$ current in steering coil \#34 for producing torque

i_34t $=i_{-} t$;

$\%$ current in steering coil \#34 for producing force

i_34f $=$ i_f

$\%$ flux density due to coil \#12 current for torque

B_12t $=\left(u_{-} 0 * N_{-} 12 * i_{-} 12 t\right) /\left(2 * g \_n\right)$;

$\%$ flux density due to coil \#12 current for force

B_12f $=\left(u_{-} \_* N_{-} 12 * i_{-} 12 f\right) /\left(2 * g \_n\right)$;

$\%$ flux density due to coil \#34 current for torque

B_34t $=\left(u_{-}\right.$0 $\left.* N_{-} 34 * i \_34 t\right) /\left(2 * g \_n\right)$;

$\%$ flux density due to coil \#34 current for force

B_34f $=\left(u_{-} 0 * N_{-} 34 * i_{-} 34 f\right) /\left(2 * g \_n\right)$;

$\%$ flux density due to bias coil

B_b $=\left(u_{-} 0 * N_{-} b * i_{-} b\right) /\left(2 *\left(g_{-} b+g \_n\right)\right)$; 
$\%$ net flux at pole faces

$\%$ sandwiched rotor

$B_{-} 1 s=B_{-} b+B_{-} 12 t+B_{-} 12 f ;$

B_2s = B_b - B_12t - B_12f;

B_3s $=-B_{-} b-B_{-} 34 t+B_{-} 34 f$;

$B_{-} 4 s=-B_{-} b+B_{-} 34 t-B_{-} 34 f$;

$\%$ pinched rotor

$B_{-} 1 p=B_{-} b+B_{-} 12 t+B_{-} 12 f ;$

B_2p $=$ B_b - B_12t - B_12f;

B_3p $=-$ B_f $_{-}-$B_34t + B_34f

$\mathrm{B}_{-} 4 \mathrm{p}=-\mathrm{B}_{-} \mathrm{b}+\mathrm{B}_{-} 34 t-\mathrm{B}_{-} 34 \mathrm{f}$;

$\%$ sandwiched rotor with reversed roles of bias and torque coils

B_1sr = B_b + B_12t + B_12f ;

B_2sr $=-B_{-} b+$ B_12t - B_12f ;

B_3sr = -B_b - B_34t + B_34f ;

B_4sr $=$ B_b - B_34t - B_34f;

$\%$

$\%$ dimensions for calculating forces and torques

$\%$ area of stator pole faces

$A_{-} p=(0.25 * 1.00) /\left(39.37^{\wedge} 2\right)$;

$\%$ cross-sectional area of back iron at stator core

$A_{-} b=2 * A_{-} p ; \%$ (not used in calc; reminder of an assumption in eqn's)

$\%$ distance from center plane to center of stator pole faces

$\mathrm{R}=((0.25+0.75) / 2) /(39.37)$;

$\%$

$\%$ torques and forces acting on rotor; indexing i_t and i_f at

$\%$ the same time

for $i=1: n$

$\%$ sandwiched rotor 


$$
\begin{aligned}
& \mathrm{T}_{-} \mathrm{s}(\mathrm{i})=\left(\left(\mathrm{R} * \mathrm{~A}_{-} \mathrm{p}\right) /\left(2 * \mathrm{u}_{-} 0\right)\right) *\left(\mathrm{~B}_{-} 1 \mathrm{~s}(\mathrm{i})^{\wedge} 2-\mathrm{B}_{-} 2 \mathrm{~s}(\mathrm{i})^{\wedge} 2 \ldots\right. \\
& + \text { B_3s(i)^2 - B_4s(i)^2); } \\
& \mathrm{F}_{-} \mathrm{s}(\mathrm{i})=\left(\mathrm{A}_{-} \mathrm{p} /\left(2 * \mathrm{u}_{-} 0\right)\right) *\left(\mathrm{~B}_{-} 1 \mathrm{~s}(\mathrm{i})^{\wedge} 2+\mathrm{B}_{-} 2 \mathrm{~s}(\mathrm{i})^{\wedge} 2 \ldots\right.
\end{aligned}
$$

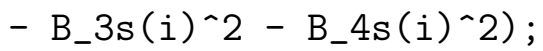

$$
\begin{aligned}
& \left.+B_{-} 3 p(i)^{\wedge} 2-B_{-} 4 p(i)^{\wedge} 2\right) \text {; } \\
& F_{-} p(i)=\left(A_{-} p /\left(2 * u_{-} 0\right)\right) *\left(B_{-} 1 p(i) \wedge 2-B_{-} 2 p(i) \wedge 2 \ldots\right. \\
& - \text { B_3p(i)^2 + B_4p(i)^2); }
\end{aligned}
$$

$\%$ sandwiched rotor with reversed roles of bias and torque coils

$$
\mathrm{T}_{-} \mathrm{sr}(\mathrm{i})=\left(\left(\mathrm{R} * \mathrm{~A}_{-} \mathrm{p}\right) /\left(2 * \mathrm{u}_{-} 0\right)\right) *\left(\mathrm{~B}_{-} 1 \mathrm{sr}(\mathrm{i})^{\wedge} 2-\mathrm{B}_{-} 2 \mathrm{sr}(\mathrm{i})^{\wedge} 2 \ldots\right.
$$$$
\left.+B_{-} 3 \operatorname{sr}(i) \frown 2-B_{-} 4 \operatorname{sr}(i) \frown 2\right) \text {; }
$$$$
\mathrm{F}_{-} \mathrm{sr}(\mathrm{i})=\left(\mathrm{A}_{-} \mathrm{p} /\left(2 * \mathrm{u}_{-} 0\right)\right) *\left(\mathrm{~B}_{-} 1 \mathrm{sr}(\mathrm{i})^{\wedge} 2+\mathrm{B}_{-} 2 \mathrm{sr}(\mathrm{i})^{\wedge} 2 \ldots\right.
$$$$
\text { - B_3sr(i)^2 - B_4sr(i)^2); }
$$

end

$\%$

$\%$ generate data for 3D mesh plot

[i_t_mesh,i_f_mesh] = meshgrid(i_t,i_f);

$\%$ current in steering coil \#12 for producing torque

i_12t_mesh=i_t_mesh;

$\%$ current in steering coil \#12 for producing force

i_12f_mesh=i_f_mesh;

$\%$ current in steering coil \#34 for producing torque

i_34t_mesh=i_t_mesh;

$\%$ current in steering coil \#34 for producing force

i_34f_mesh=i_f_mesh;

$\%$ flux density due to coil \#12 current for torque

B_12t_mesh $=\left(u_{-} 0 * N_{-} 12 * i \_12 t \_m e s h\right) /\left(2 * g \_n\right)$;

$\%$ flux density due to coil \#12 current for force

B_12f_mesh $=\left(u_{-} 0 * N_{-} 12 * i_{-} 12 f \_m e s h\right) /\left(2 * g \_n\right)$; 
$\%$ flux density due to coil \#34 current for torque

B_34t_mesh $=\left(u_{-} 0 * N_{-} 34 * i \_34 t \_m e s h\right) /\left(2 * g \_n\right)$;

$\%$ flux density due to coil \#34 current for force

B_34f_mesh $=\left(u_{-} 0 * N_{-} 34 * i_{-} 34 f \_m e s h\right) /\left(2 * g \_n\right)$;

$\%$

$\%$ net flux at pole faces

$\%$ sandwiched rotor

B_1s_mesh $=$ B_b + B_12t_mesh + B_12f_mesh;

B_2s_mesh $=$ B_b - B_12t_mesh - B_12f_mesh;

B_3s_mesh $=-$ B_b - B_34t_mesh + B_34f_mesh;

B_4s_mesh $=-$ B_b + B_34t_mesh - B_34f_mesh;

$\%$ pinched rotor

B_1p_mesh $=$ B_b + B_12t_mesh + B_12f_mesh;

B_2p_mesh $=$ B_b - B_12t_mesh - B_12f_mesh;

B_3p_mesh $=-$ B_b - B_34t_mesh + B_34f_mesh;

B_4p_mesh $=-$ B_b + B_34t_mesh - B_34f_mesh;

$\%$ sandwiched rotor with reversed roles of bias and torque coils

B_1sr_mesh $=$ B_b + B_12t_mesh + B_12f_mesh;

B_2sr_mesh $=-B_{-} b+$ B_12t_mesh - B_12f_mesh;

B_3sr_mesh $=-$ B_b - B_34t_mesh + B_34f_mesh;

B_4sr_mesh = B_b - B_34t_mesh - B_34f_mesh;

$\%$

$\%$ torques and forces acting on rotor

$\%$ sandwiched rotor

T_s_mesh $=\left(\left(\mathrm{R} * \mathrm{~A} \_\mathrm{p}\right) /\left(2 * \mathrm{u}_{-} 0\right)\right) *\left(\mathrm{~B}_{-} 1 \mathrm{~s} \_\right.$mesh. ${ }^{\wedge} 2-\mathrm{B}_{-} 2 \mathrm{~s} \_m e s h . ` 2 \ldots$

+ B_3s_mesh. $22-$ B_4s_mesh. $\left.{ }^{2} 2\right)$;

F_s_mesh $=\left(A_{-} p /\left(2 * u_{-} 0\right)\right) *\left(B_{-}\right.$1s_mesh. ${ }^{\wedge} 2+$ B_2s_mesh. ${ }^{\wedge} 2 \ldots$

- B_3s_mesh.^2 - B_4s_mesh. ${ }^{\wedge} 2$ );

$\%$ pinched rotor

T_p_mesh $=\left(\left(\mathrm{R} * \mathrm{~A}_{-} \mathrm{p}\right) /\left(2 * \mathrm{u}_{-} 0\right)\right) *\left(\mathrm{~B}_{-} 1 \mathrm{p} \_\right.$mesh. ${ }^{\wedge} 2-\mathrm{B}_{-} 2 \mathrm{p} \_m e s h .{ }^{\wedge} 2 \ldots$ 
+ B_3p_mesh.^2 - B_4p_mesh.`2);

F_p_mesh $=\left(A_{-}\right.$p $\left./\left(2 * u_{-} 0\right)\right) *\left(B_{-} 1\right.$ p_mesh. ${ }^{\wedge} 2-$ B_2p_mesh. ${ }^{\wedge} 2 \ldots$

- B_3p_mesh.`2 + B_4p_mesh.`2);

$\%$ sandwiched rotor with reversed roles of bias and torque coils

T_sr_mesh $=\left(\left(\mathrm{R} * \mathrm{~A}_{-} \mathrm{p}\right) /\left(2 * \mathrm{u}_{-} 0\right)\right) *\left(\mathrm{~B}_{-} 1 \mathrm{sr} \_\mathrm{mesh} .{ }^{\circ} 2-\mathrm{B}_{-} 2 \mathrm{sr} \_\mathrm{mesh} .{ }^{\circ} 2 \ldots\right.$

+ B_3sr_mesh.^2 - B_4sr_mesh.`2);

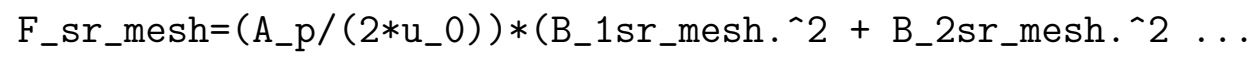

- B_3sr_mesh.`2 - B_4sr_mesh.`2);

$\%$

$\%$ plot results

$\%$ sandwiched rotor

$\%$ 3D plots

$\%$ default three-dimensional view: view $(a z, e l), a z=-37.5$, el $=30$ subplot $(2,1,1)$

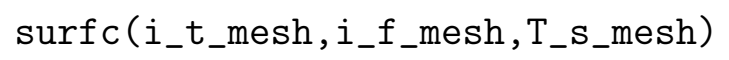

\%contour3 (i_t_mesh, i_f_mesh, T_s_mesh)

\%surface(i_t_mesh,i_f_mesh_mesh, 'EdgeColor', [ 8.8 .8 .8] , ...

'FaceColor', 'none')

view $(-37.5,30)$

title(['Sandwiched rotor using "flux torque force.m", RCM ', date])

xlabel ('i_t_o_r_q_u_e (A)')

ylabel ('i_f_o_r_c_e (A)')

zlabel ('torque $(\mathrm{N}-\mathrm{m})$ ')

subplot $(2,1,2)$

$\operatorname{surfc}$ (i_t_mesh,i_f_mesh,F_s_mesh)

\%contour3 (i_t_mesh,i_f_mesh,F_s_mesh)

\%surface(i_t_mesh,i_f_mesh_mesh, 'EdgeColor', [ $\left[\begin{array}{lll}.8 & .8 & .8\end{array}\right], \ldots$

'FaceColor' , 'none')

$\%$ hidden off

view $(-37.5,30)$ 


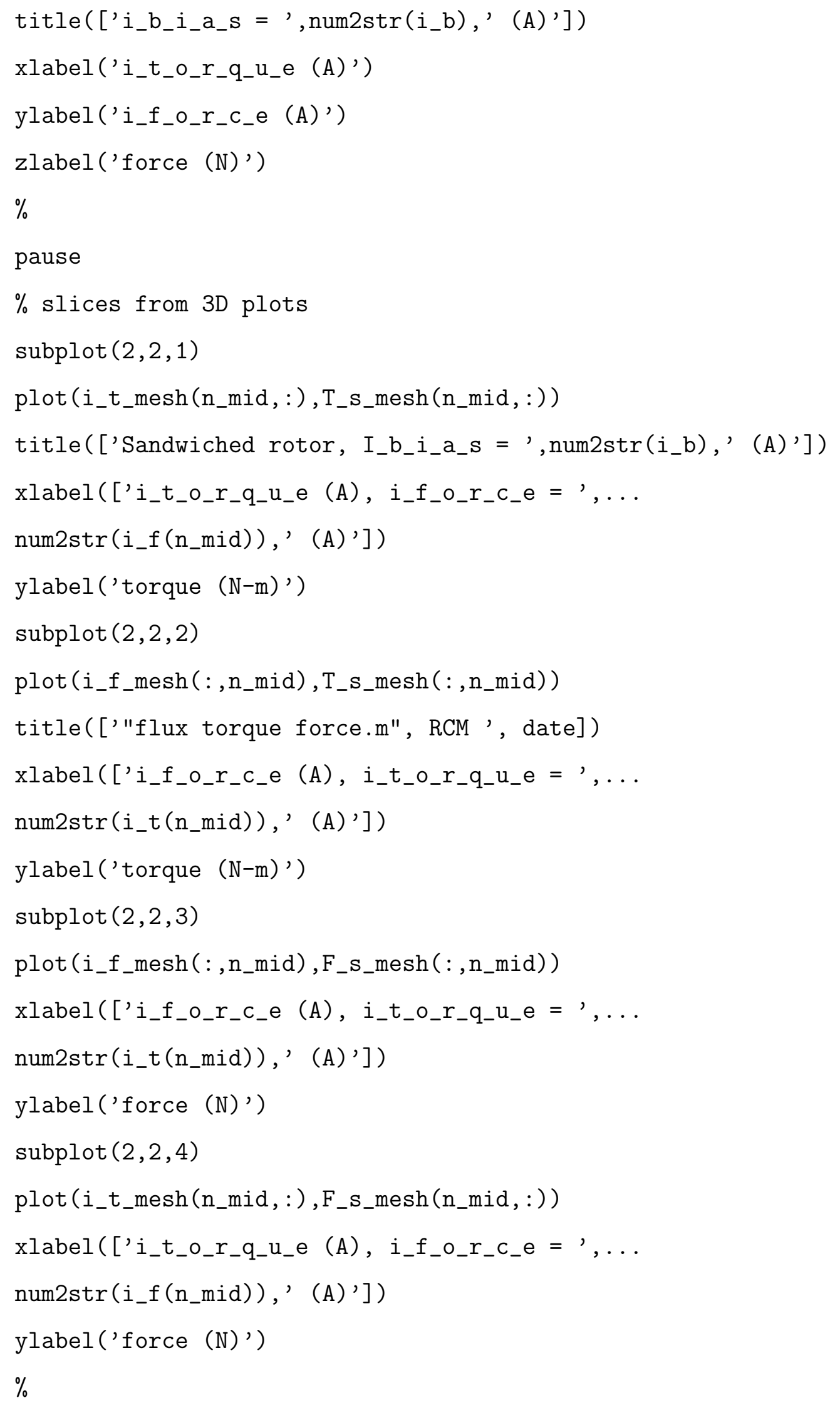




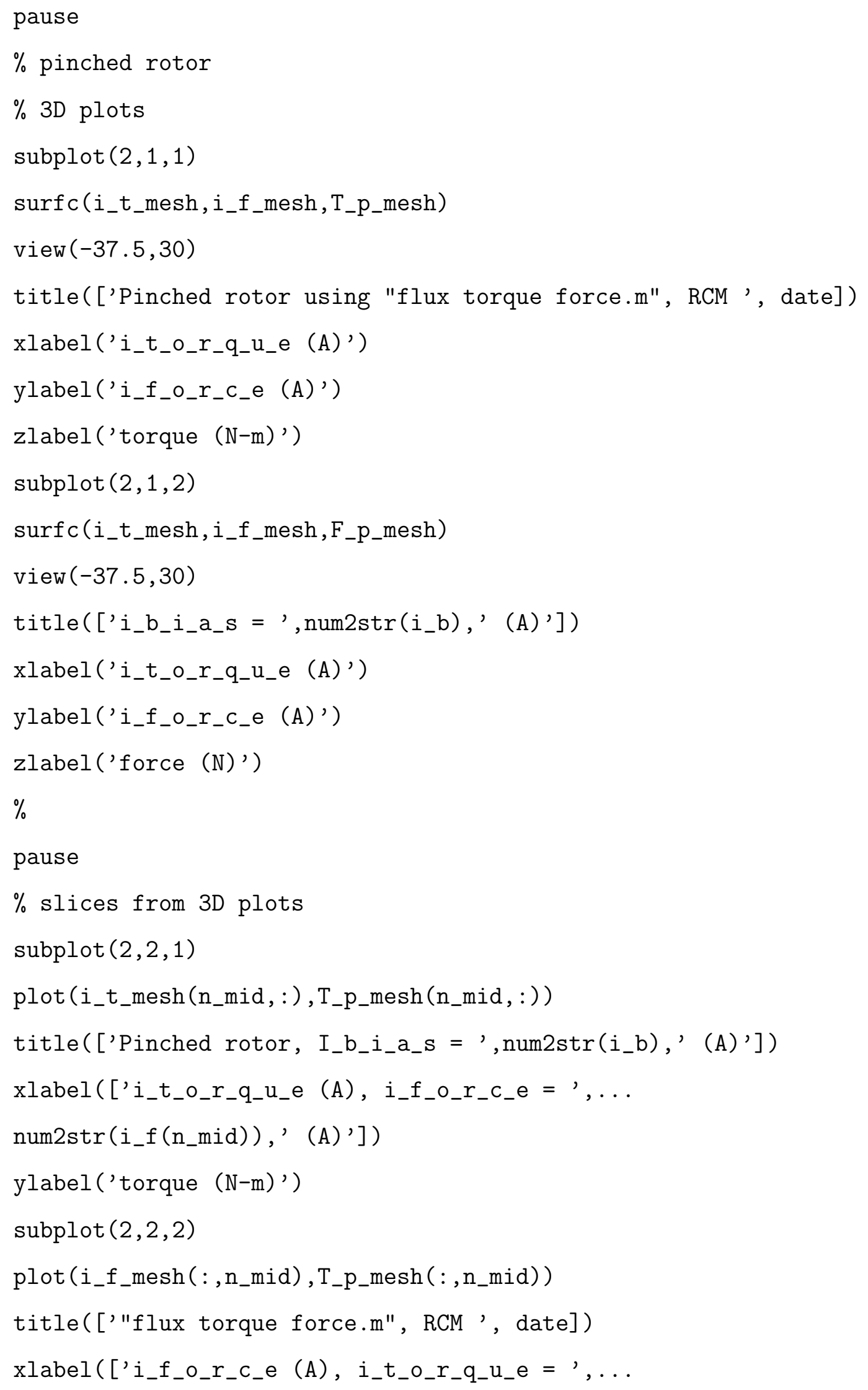




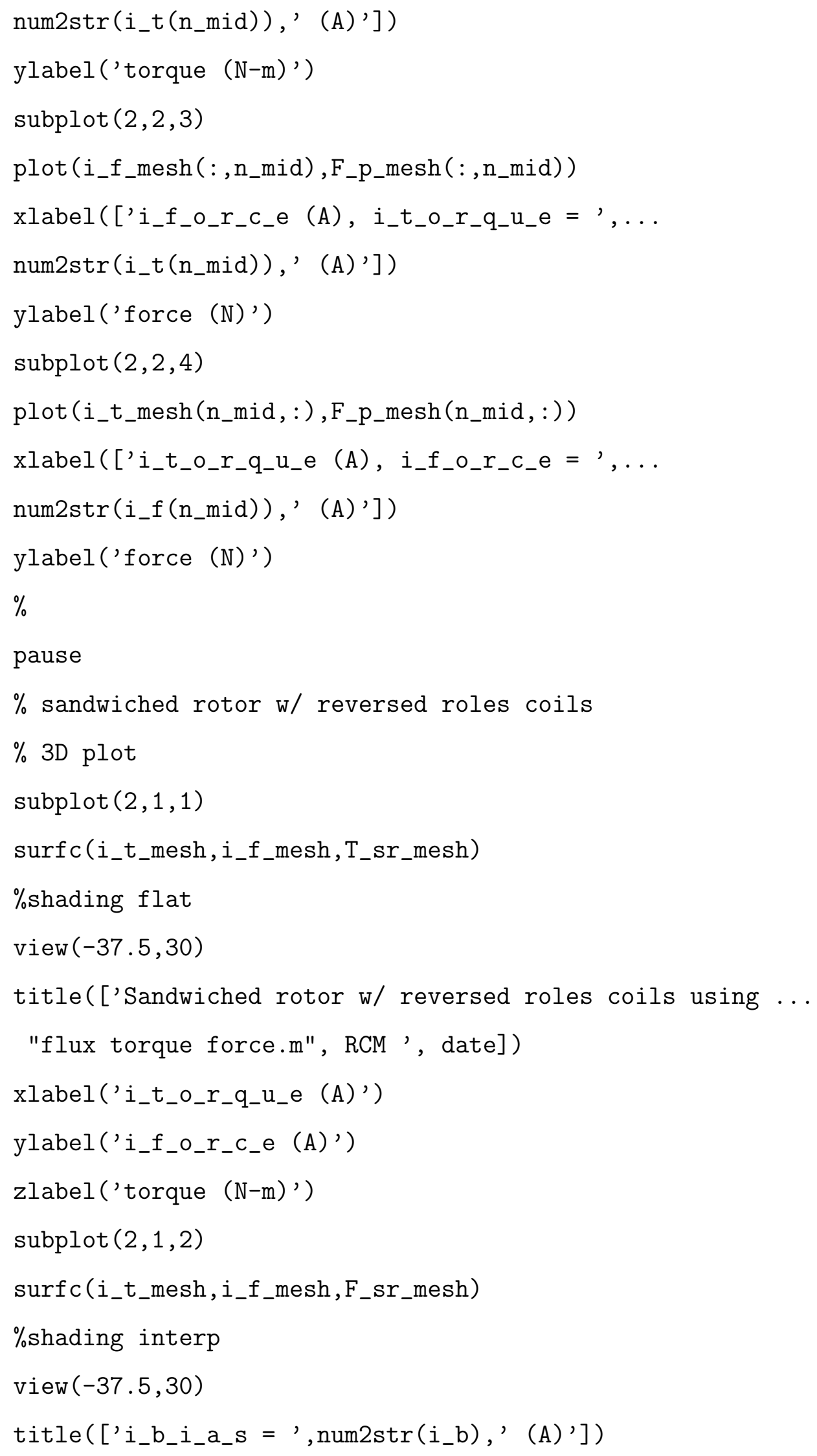




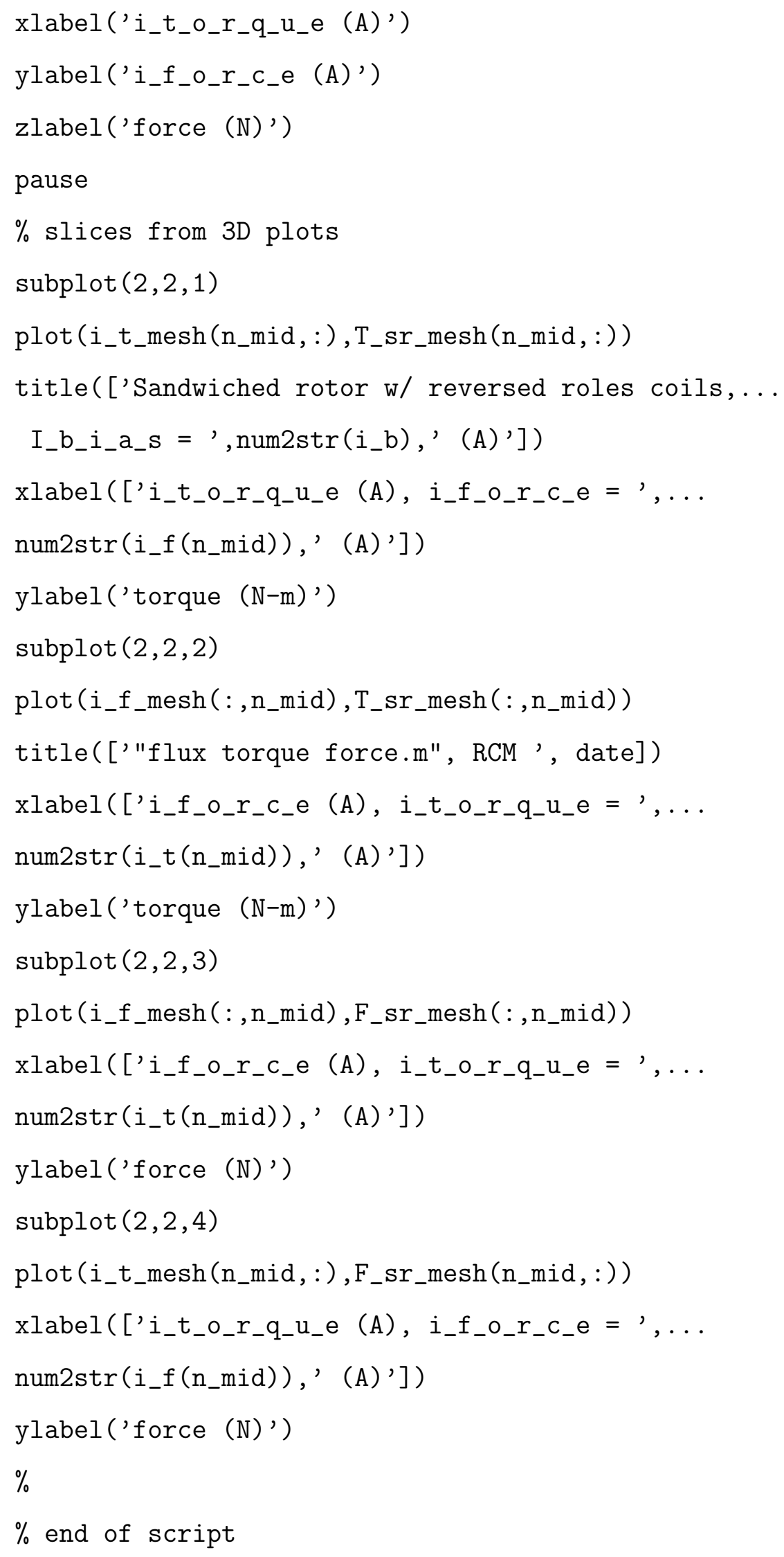




\section{Appendix $\mathrm{C}$}

\section{Integrating the $2 \mathrm{kHz}$ FTS with a Diamond Turning Machine}

This appendix describes how I integrated and used the $2 \mathrm{kHz}$ rotary fast tool servo with a diamond turning machine (DTM) at MIT to conduct the machining tests described in Section 3.11.1.

Figure 3-2 shows the $2 \mathrm{kHz}$ FTS mounted on the DTM and engaging a workpiece, Figure 3-15 shows a close-up view of the tool engaging the face of a workpiece, and Figure 3-59 shows a close-up of the tool tip about to engage the outside diameter of a workpiece. The workpieces have 250 radial grooves on the face and 250 axial grooves on the outside diameter. In both cases the grooves have a sinusoidal profile of $2.2 \mu \mathrm{m}$ PP, and were machined while the workpiece was rotating at 480 RPM (2000 grooves per second).

The diamond turning machine is a Moore Nanotechnology model UPL 350 [110], which consists of two slide-ways arranged in a Tee configuration. The slides are supported and guided by hydrostatic oil bearings and driven by integral linear motors. The workpiece spindle is a Professional Instruments motorized air bearing spindle model 4B [125], and is mounted on the cross-slide (X-axis). This DTM did not have a controller, so my first task was to identify the pin-outs of the input and output connections for the sensors and motor drives, develop closed-loop controllers 
for the linear motors, and create a Graphical User Interface (GUI) that would allow me to coordinate motion of the slides and spindle so that I could use the DTM to machine workpieces. I used the same digital controller prototyping environment that is described in Section 3.10.1: MATLAB $^{\circledR}$ [104] and Simulink ${ }^{\circledR}$ [104] software with the dSPACE GmbH [51] real-time controller board.

To test the controller that I developed for the DTM, I used a fixed tool in a static holder mounted to the Z-axis to diamond turn the face and outside diameter of one of the $9 \mathrm{~mm}$ diameter aluminum workpieces that I would later machine groves into with the $2 \mathrm{kHz}$ FTS. I then replaced the tool holder with the $2 \mathrm{kHz}$ FTS, commanded the tool tip to hold a constant position, and repeated the earlier test to convince myself that the mechanical hardware and control system for the $2 \mathrm{kHz}$ FTS were robust enough to withstand the cutting forces involved in diamond turning. The surface finish of the workpieces machined by the two set-ups was comparable, so I proceeded to using the $2 \mathrm{kHz}$ FTS to produce wavy surfaces on a workpiece.

Two separate control systems are used to generate a wavy surface on the workpiece: one for the DTM and one for the fast tool servo. The DTM is commanded as if it were using a tool held in a static holder. The sinusoidal tool tip trajectory for the fast tool servo is generated by using the workpiece spindle encoder signal to generate the requisite sine wave. The large motions of the DTM generate the base smooth surfaces on the workpiece, and the small motions of the FTS generate the wavy surfaces that are superimposed on those base surfaces.

\section{C.1 Compensating and Tuning the DTM Slides}

Figure C-1 shows the negative loop transmission for the compensated Z-axis slide. The slide has a mechanical resonance between 1 and $2 \mathrm{kHz}$. Instead of using a notch filter, which would be sensitive to changes in the plant - for instance, changing the mass of the slide when switching from the static tool holder to the fast tool servo I used a phase-stabilizing pole located at $300 \mathrm{~Hz}$ (see Section 3.10.3 for a discussion on this technique). Using a lead-lag compensator with the phase-stabilizing pole, 

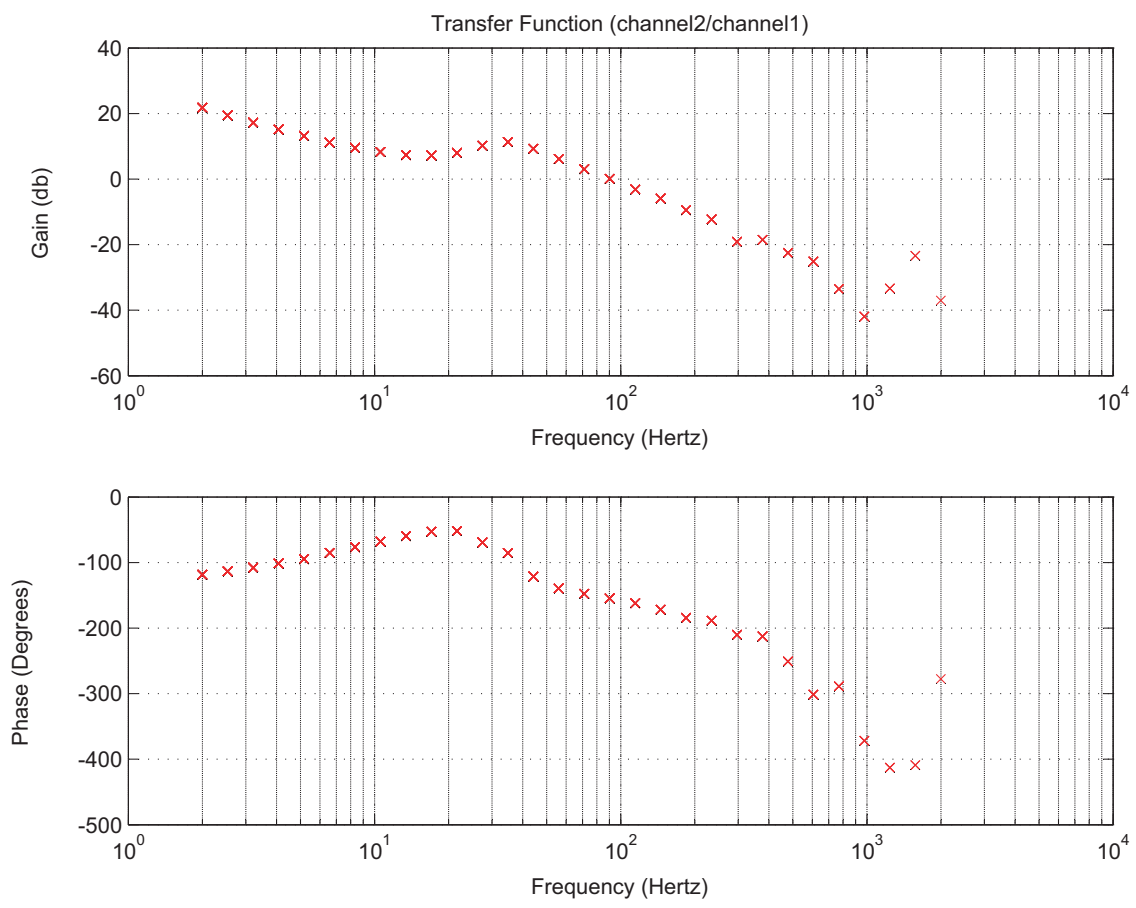

Figure C-1: Bode plot of the measured negative loop transmission for the compensated Z-axis slide in the diamond turning machine.

I established a crossover frequency of $90 \mathrm{~Hz}$ with a phase margin of $30^{\circ}$ for the Zaxis slide. The same technique - a lead-lag compensator with a phase-stabilizing pole - was used for compensating the $\mathrm{X}$-axis slide, which carries the relatively heavy workpiece spindle. In the case of the $\mathrm{X}$-axis slide, a crossover frequency of $75 \mathrm{~Hz}$ and phase margin of $30^{\circ}$ were established.

The resonance at approximately $35 \mathrm{~Hz}$ in Figure C-1 is probably due to the cables between the slide and the machine base, which act as a damped spring for small motions of the slide. Chasing through the numbers, the slide mass of $70 \mathrm{kG}$ and a resonant frequency of $35 \mathrm{~Hz}$ indicate a spring rate of $17 \times 10^{3} \mathrm{lb} /$ in, or a compliance of $60 \mu \mathrm{in} / \mathrm{lb}$. Using a hand-held force gauge, I pushed the open-loop slide with as small a force and displacement as practical for a quick test, and monitored the displacement using the Z-axis encoder signal. The force and displacement of $0.5 \mathrm{lb}$ and $8 \mu \mathrm{in}$ respectively indicate a compliance of $16 \mu \mathrm{in} / \mathrm{lb}$, which is not too far off from the expected value of $60 \mu \mathrm{in} / \mathrm{lb}$ considering the crudeness of the test and the fact that I was near the resolution limits of the measurement equipment. 


\section{C.2 Graphical User Interface and Controller for the DTM}

Figure C-2 shows the graphical user interface (GUI) that I developed for the diamond turning machine using the dSPACE GmbH [51] software. The GUI is connected to the real-time implementation of the Simulink ${ }^{\circledR}$ [104] models that I developed for the following tasks: (1) controlling the X-axis slide, the Z-axis slide, and the workpiece spindle; (2) generating commanded position trajectories for the slides; and (3) monitoring hardware and safety limits for the machine. The GUI provides the following minimum set of features needed for safely applying power to the machine, establishing initial contact between the tool and workpiece, and executing a manual machining sequence on the workpiece:

1. Selectively enable the power amplifiers for the DTM axes (spindle, X-slide, and Z-slide).

2. Set the spindle rotation speed and maximum torque.

3. Reset the encoders and commanded positions for the $\mathrm{X}$ and $\mathrm{Z}$ slides. This allows setting the "home" position for the machine at the current position of the $\mathrm{X}$ and $\mathrm{Z}$ slides.

4. Close the loops on the X and Z slide control systems (labelled "uncage" the controller).

5. Set the speed for commanded moves of the $\mathrm{X}$ and $\mathrm{Z}$ slides.

6. Set the jog increment for moving the $\mathrm{X}$ and $\mathrm{Z}$ slides.

7. Jog the $\mathrm{X}$ or $\mathrm{Z}$ slides in their positive or negative directions.

8. Stop the $\mathrm{X}$ or $\mathrm{Z}$ slide movement that is currently being executed.

9. Monitor the hardware and safety limits for the machine. 


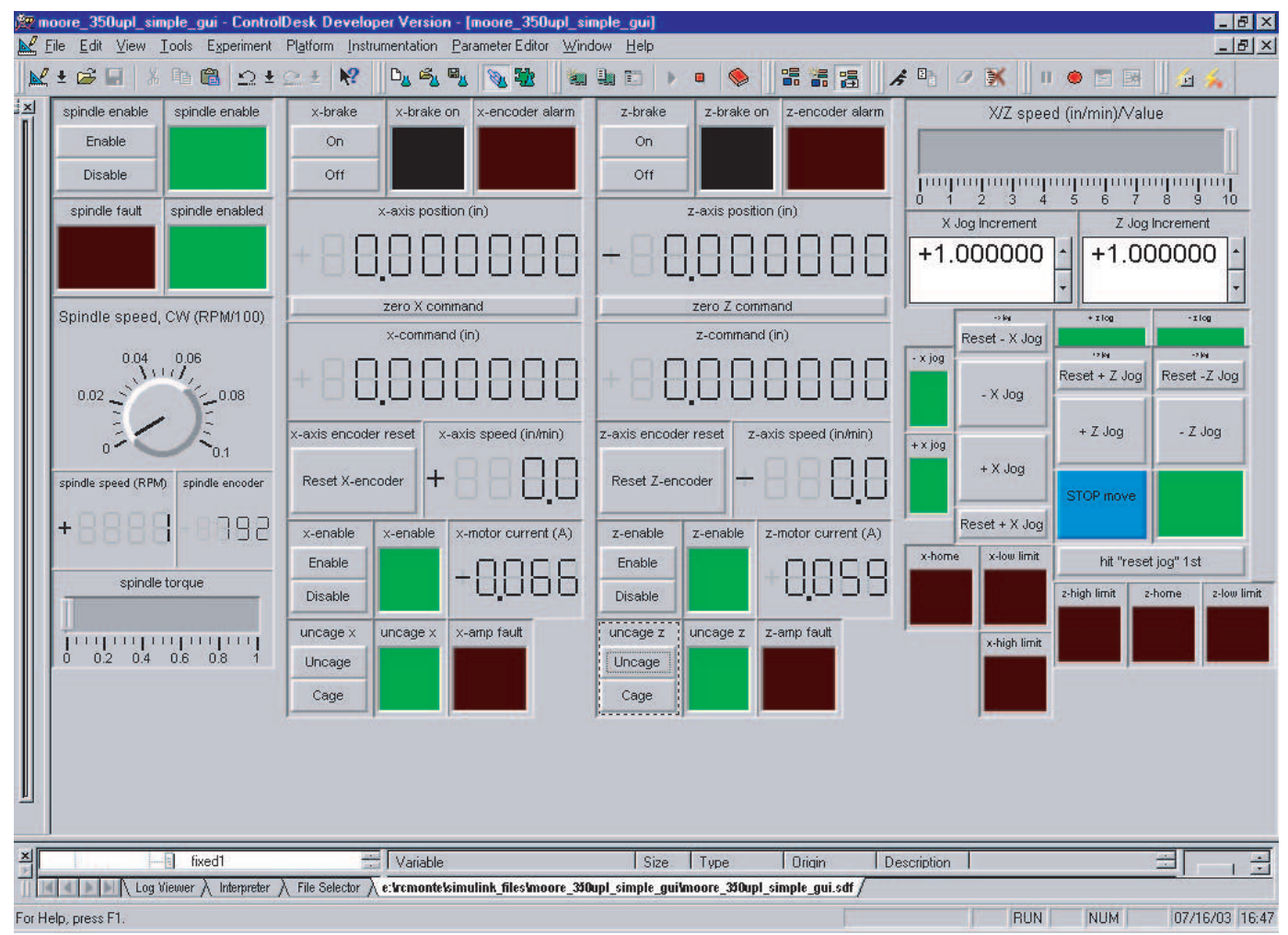

Figure C-2: Graphical user interface developed for the diamond turning machine used to machine workpieces with the $2 \mathrm{kHz}$ FTS.

10. For all of the DTM axes, hard-wired emergency-stop buttons (not shown) disconnect the power amplifiers from their energy sources.

Figures C-3 through C-7 show the Simulink ${ }^{\circledR}$ models that are connected to the GUI for the diamond turning machine.

\section{C.3 Graphical User Interface and Controller for the $2 \mathrm{kHz}$ FTS}

Figure C-8 shows the graphical user interface that I developed to allow using the $2 \mathrm{kHz}$ FTS with the diamond turning machine to produce wavy surfaces on a workpiece. This GUI is written with the dSPACE GmbH [51] software, and is connected to the real-time implementation of the Simulink ${ }^{\circledR}$ [104] model shown in Figure C-9, which controls the fast tool servo and generates the sinusoidal tool tip trajectory from 

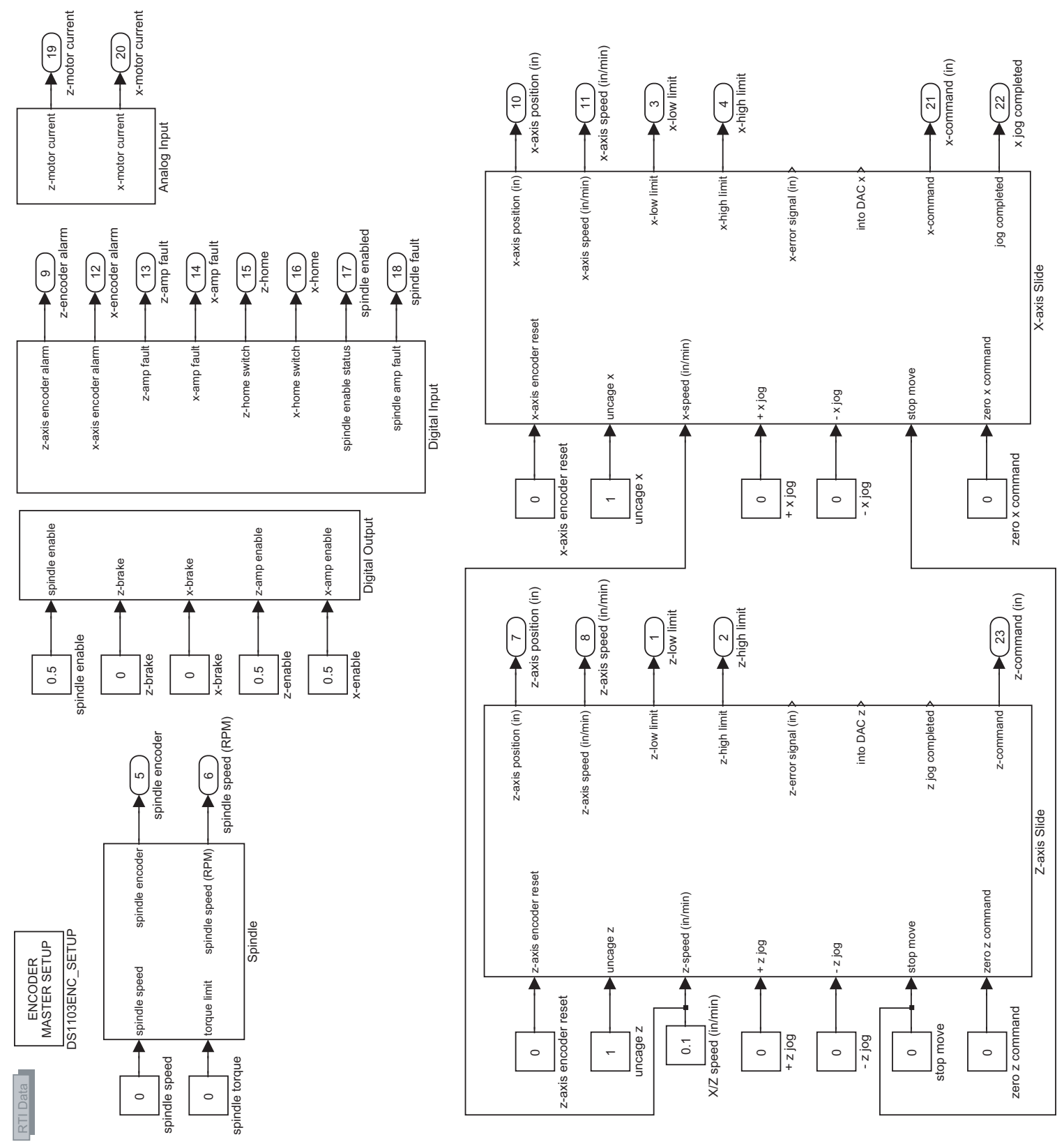

Figure C-3: Top-level Simulink ${ }^{\circledR}$ model used to control the diamond turning machine. 

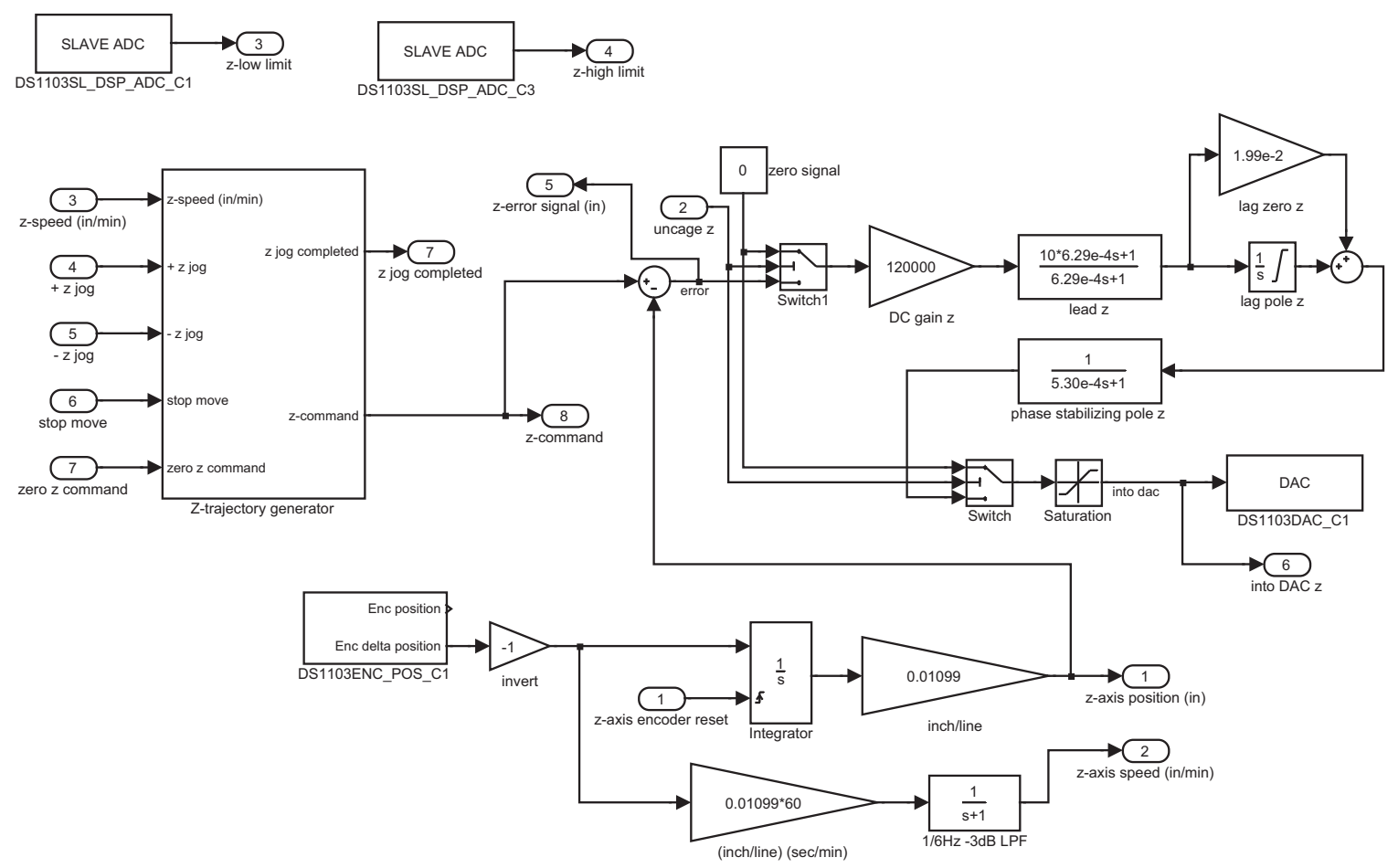

Figure C-4: Second-level Simulink ${ }^{\circledR}$ model used to control the Z-axis slide. This model is an expansion of the block labelled "Z-axis Slide" in Figure C-3.

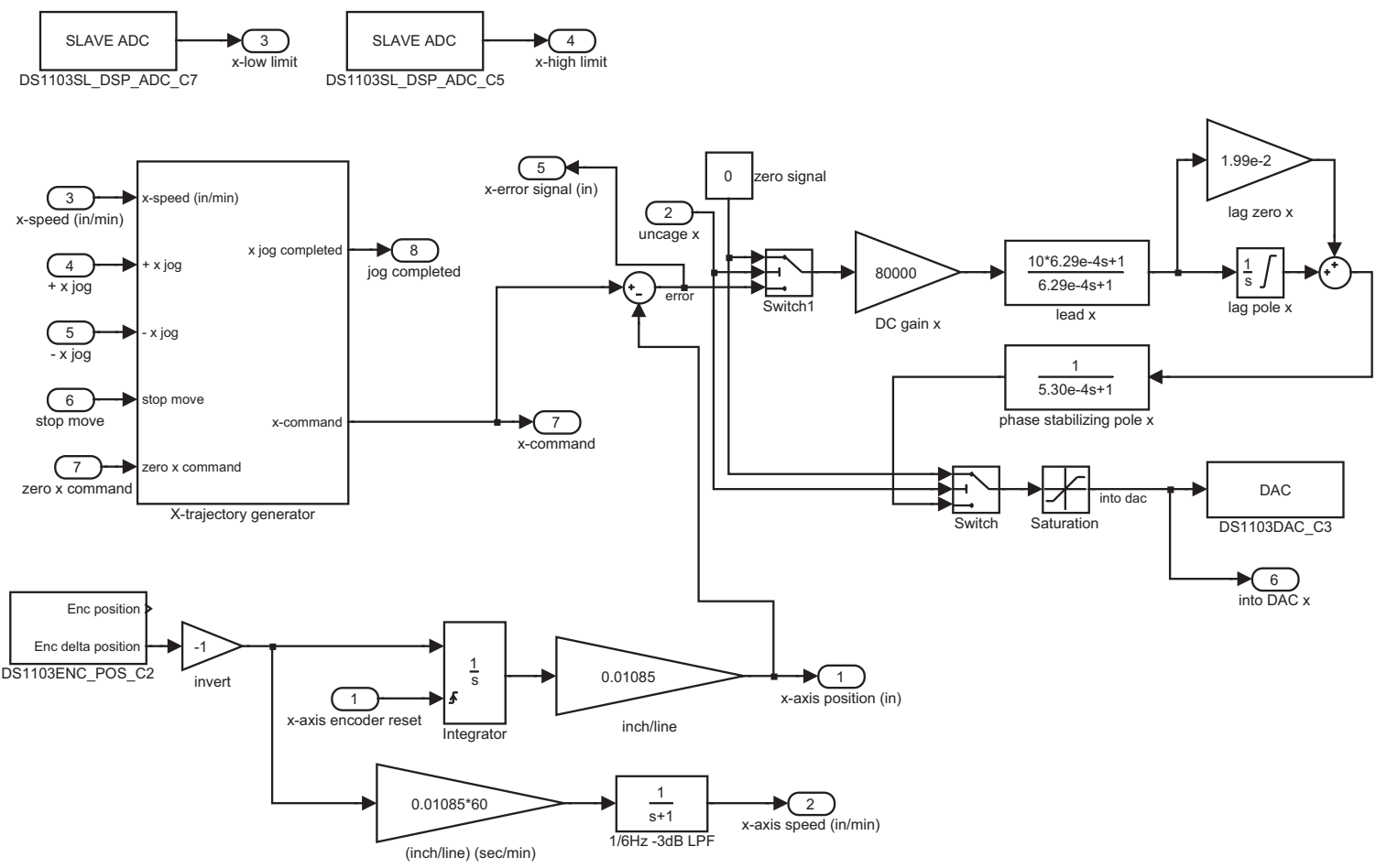

Figure C-5: Second-level Simulink ${ }^{\circledR}$ model used to control the X-axis slide. This model is an expansion of the block labelled "X-axis Slide" in Figure C-3. 

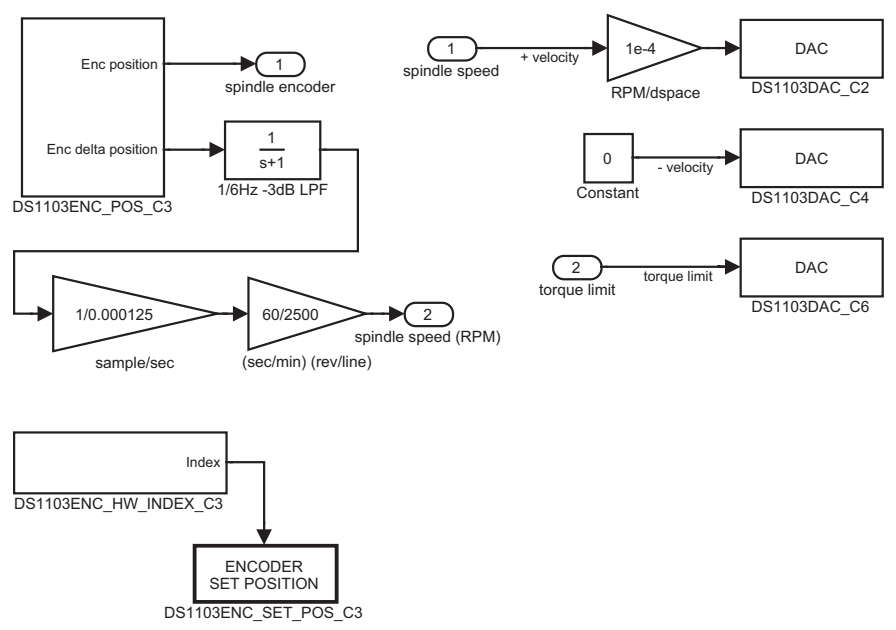

Figure C-6: Second-level Simulink ${ }^{\circledR}$ model used to control the spindle. This model is an expansion of the block labelled "Spindle" in Figure C-3.

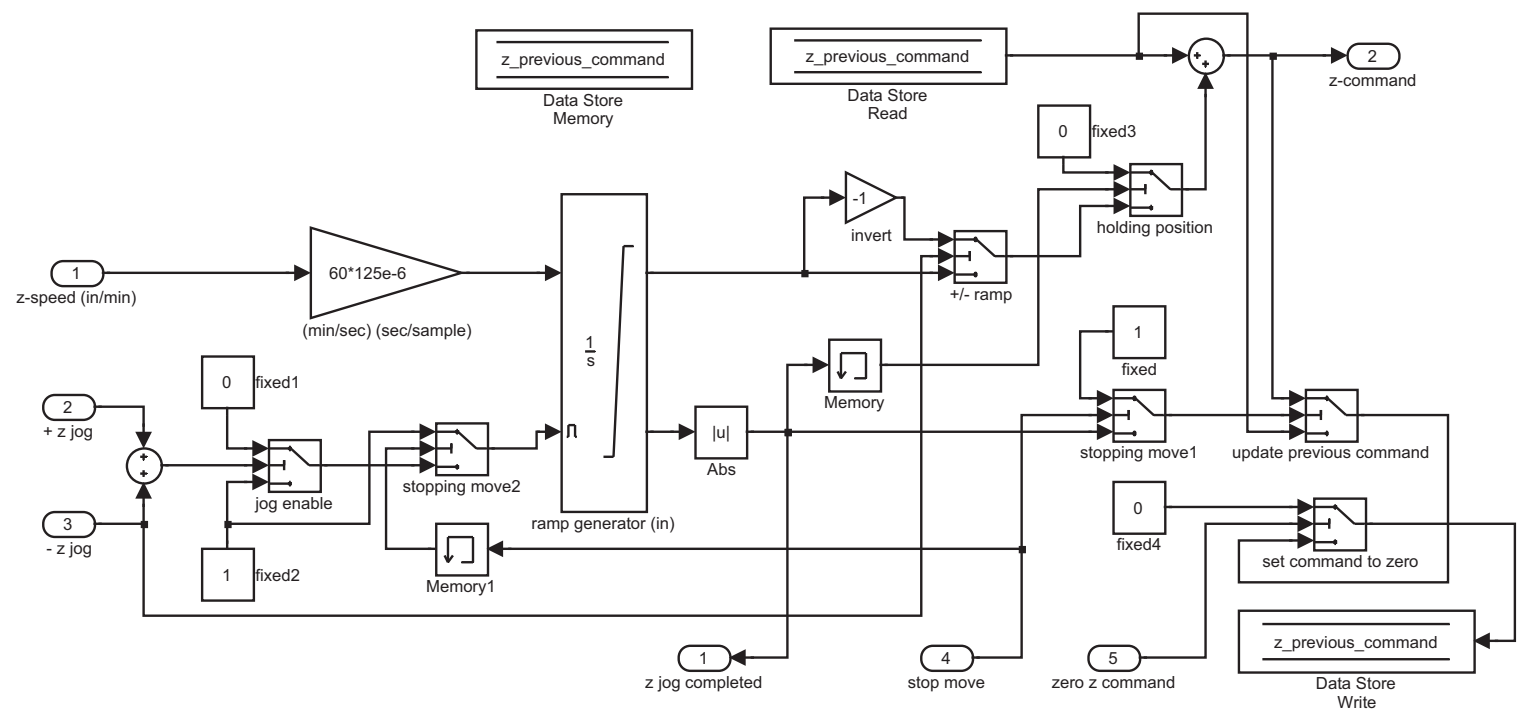

Figure C-7: Third-level Simulink ${ }^{\circledR}$ model that generates the set of commanded positions used to produce a trajectory for the Z-axis slide in response to a jog command from the GUI for the diamond turning machine. This model is an expansion of the block labelled "Z-trajectory generator" in Figure C-4. The model for generating the commanded position trajectory for the $\mathrm{X}$-axis slide is identical to the one shown in the figure, with the exception of the labels. 
a measurement of the workpiece spindle angle. The GUI for the $2 \mathrm{kHz}$ FTS allows controlling and/or monitoring the following items:

1. Close the loop for the fast tool servo control system.

2. Adjust the DC tool position offset for the fast tool servo.

3. Monitor the workpiece spindle encoder output value.

4. Adjust the amplitude and frequency of a test sine wave used to generate a tool tip trajectory that is independent of the workpiece spindle encoder angle.

5. Adjust the amplitude of the sine wave generated from the workpiece spindle encoder angle. The frequency of that sine wave is hard-coded into the Simulink ${ }^{\circledR}$ model for simplicity.

6. Allow choosing the source for the generated sine wave used to generate the tool tip trajectory.

7. Add or remove the filter used to smooth the sine wave generated from the workpiece spindle encoder angle.

8. Monitor the signal levels in the control system for the $2 \mathrm{kHz}$ FTS.

\section{C.4 Generating a Sinusoidal Tool Path from the Workpiece Spindle Encoder Output}

The sinusoidal tool tip trajectory for the fast tool servo is produced by generating a sine wave based on the angular position of the workpiece spindle. The spindle angle is measured by an encoder that is integrated with the spindle motor and used to provide feedback to the velocity control system that is built into the spindle motor amplifier. At 2500 lines/rev, the resolution of the spindle encoder is too coarse for producing the desired 250 smooth sinusoidal grooves per revolution of the workpiece (10 data points 


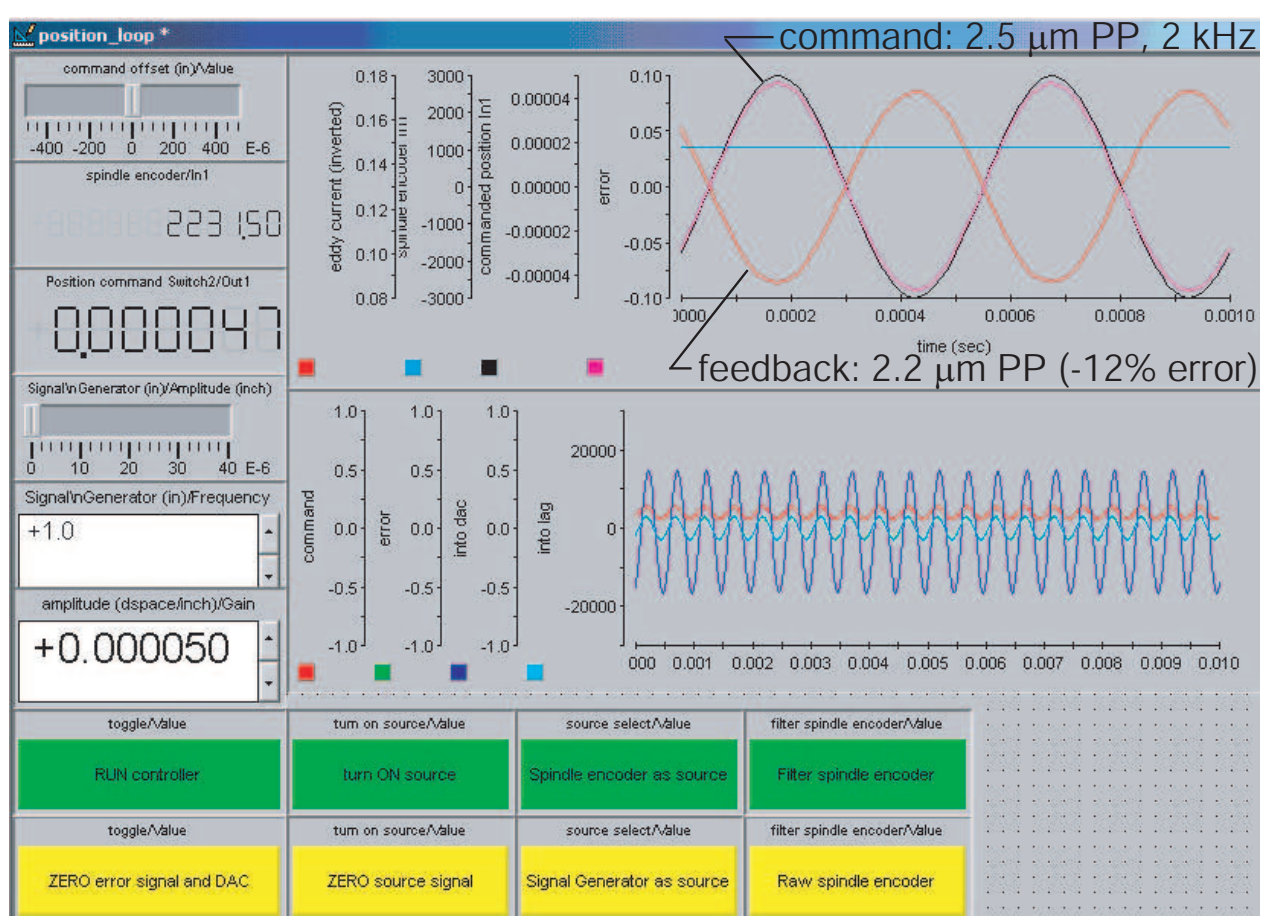

Figure C-8: Graphical user interface developed to use the $2 \mathrm{kHz}$ FTS with a diamond turning machine to produce wavy surfaces on a workpiece.

per cycle). That resolution is improved by the dSPACE encoder input electronics, which divides each encoder line into quarter lines (40 data points per cycle). However, time and amplitude jitter associated with the sampling process of the digital control system produces unwanted noise in the sine wave that is generated for commanding the tool tip trajectory for the fast tool servo. Using the design technique described in Section A.3, I designed a bandpass filter to smooth the sine wave that is generated from the sampled encoder signal. Using the encoder index pulse to reset the encoder signal every revolution insures that the dSPACE encoder register does not overflow while machining a workpiece: the register range of $\pm 2^{21}$ is exceeded within two minutes for 2500 line/rev at 480 RPM.

Figure C-10 shows Bode plots of the modelled bandpass filter for the workpiece spindle encoder angle for different attenuation values. Note that increasing the attenuation of the out-of-band frequencies causes a larger shift in phase when passing from one side of the pass frequency to the other. Figure C-11 shows a detail of the Bode plots of the modelled bandpass filter, and a schematic representation of how 


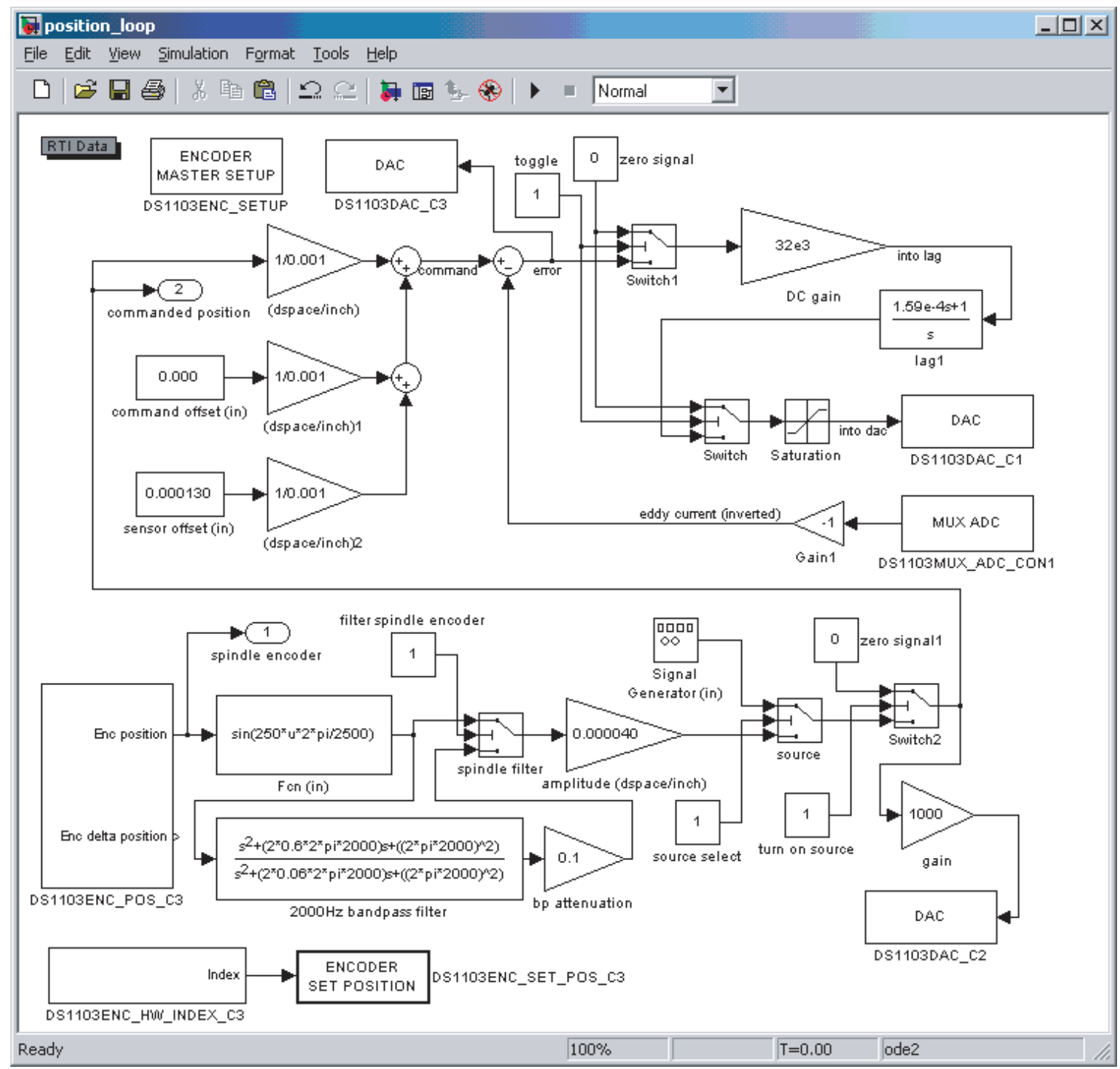

Figure C-9: Simulink ${ }^{\circledR}$ model used to control the $2 \mathrm{kHz}$ FTS when used with a diamond turning machine to produce wavy surfaces on a workpiece. 


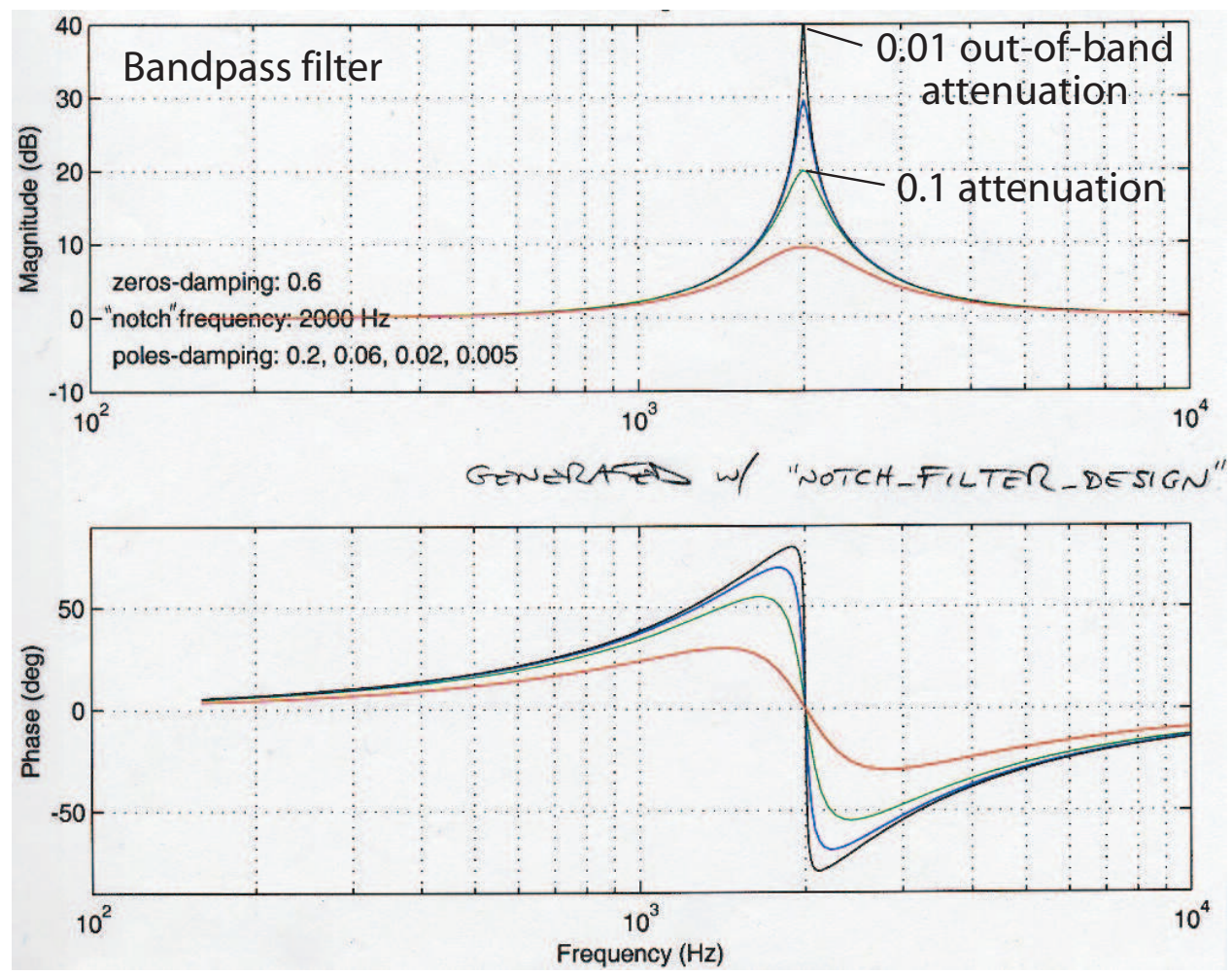

Figure C-10: Bode plots of the modelled bandpass filter for the workpiece spindle encoder angle for different attenuation values.

the expected $\pm 0.1 \%$ speed variations for the workpiece spindle could cause a phase shift between the sine waves produced in successive revolutions of the workpiece, which degrades the smoothness of the machined surface. I set the notch filter outof-band attenuation to 0.1 as a compromise between smoothing the noise in the sine wave generated from the sampled workpiece spindle encoder signal, and increasing the roughness of the machined surface due to phase shifts caused by the notch filter and spindle speed variations.

It is interesting to note that the high bandwidth of the $2 \mathrm{kHz}$ FTS allows it to reproduce enough of the power in the sampled encoder signal noise that I could easily hear it. Using the GUI for the FTS to toggle the spindle encoder filter in and out of the control system, I could hear the sound of the noise fall and rise, respectively. I could also hear a difference in the noise level when I changed the value of attenuation for the out-of-band frequencies. 


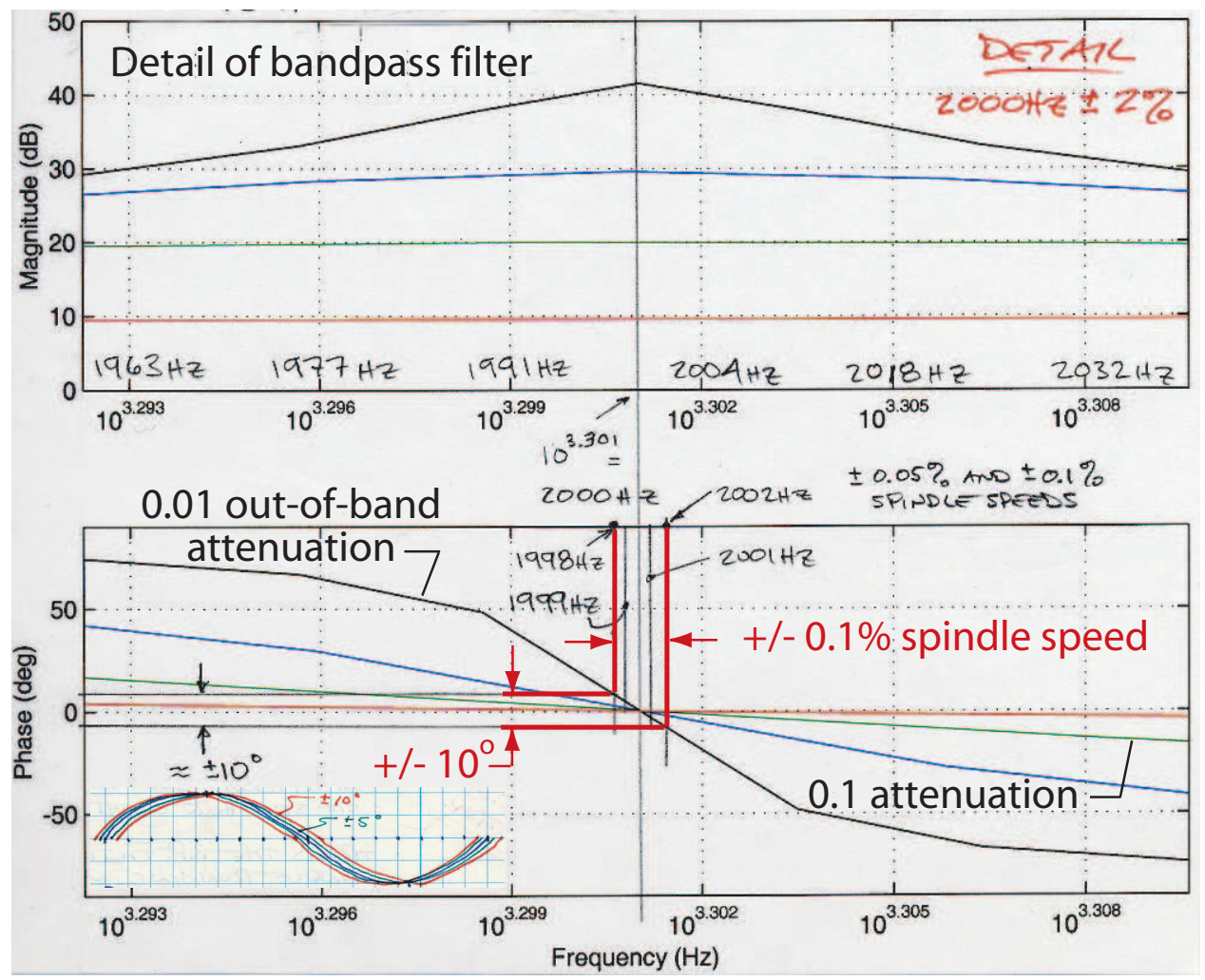

Figure C-11: Detail of the Bode plots of the modelled bandpass filter for the workpiece spindle encoder angle for different attenuation values. Schematic representation of how the expected $\pm 0.1 \%$ spindle speed variations could cause a phase shift between the sine waves produced in successive revolutions of the workpiece (inset). 


\section{C.5 Second Harmonics in Wavy Surfaces Machined on the Outside Diameter of a Workpiece}

While developing a preferred taper-down sequence for the depth-of-cut for successive machining passes on a workpiece when creating a wavy surface, I observed an interesting phenomena that occurs when machining the outside diameter of the part if the first depth-of-cut is too aggressive. Essentially, the tool forces associated with the interrupted cutting during the first machining pass with a sinusoidal tool tip trajectory act as a forcing function on the workpiece and its attachment to the spindle. It will be shown that this forcing function contains a significant second harmonic of the frequency of the desired sine wave. Keeping the forces of the interrupted cut low enough allows each successive machining pass to remove the second harmonics that were machined into the surface during the previous machining pass, and allows converging to the desired final wavy surface.

To machine a wavy surface on a workpiece, the fast tool servo is first commanded to hold a fixed tool position while successive machining passes are made to produce an initial smooth surface on the workpiece and establish a known relationship between the FTS and the workpiece. The FTS is then commanded to produce a sinusoidal tool tip trajectory that is synchronized with the workpiece spindle rotation angle, and a series of successive machining passes are made to produce the desired wavy surface. Figure C-12 shows a schematic representation of this first sinusoidal tool path relative to the previously machined "flat" surface (flat, relative to the in-andout trajectory of the tool tip). Note that the first machining pass with a sinusoidal in-and-out trajectory of the tool tip results in an interrupted cut as the tool engages and disengages the workpiece. Consider the force that develops between the tool and workpiece in the normal direction of the workpiece surface (the thrust force). When the tool is not engaged in the workpiece the thrust force is zero. At the instant that the tool engages the workpiece there is an impact force due to the finite normal approach velocity between the tool and workpiece. This causes an elastic deflection in both the tool and in the workpiece. Assuming that the workpiece and tool were 


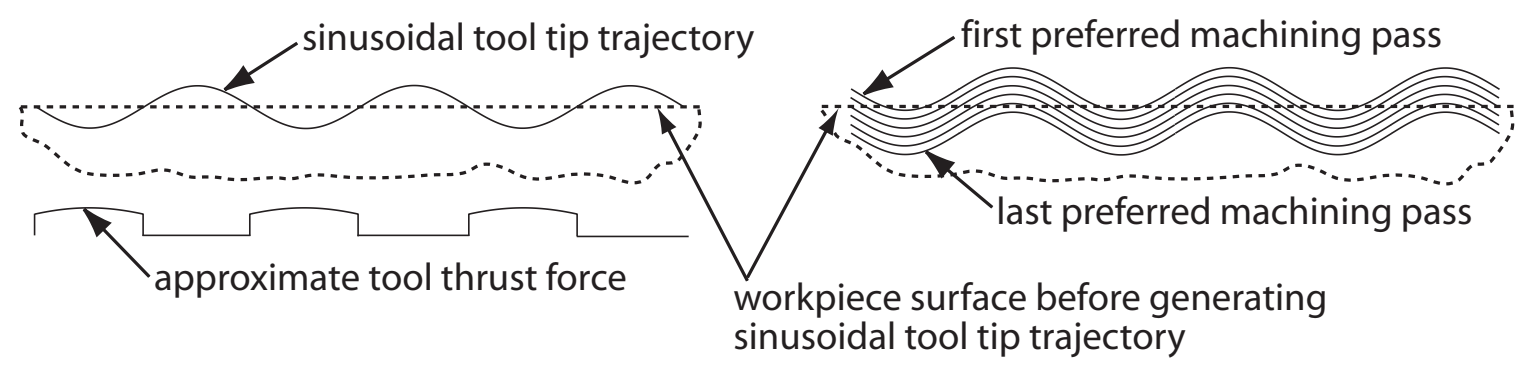

Figure C-12: Schematic representation of the sinusoidal tool path relative to a previously machined "flat" surface on the workpiece. An excessively aggressive first sinusoidal tool path (left). A preferred sequence of machining passes used to avoid generating second harmonics in the desired wavy surface on the finished workpiece (right).

not bounced out of engagement by that impact, the thrust force generally increases as the depth of penetration increases, then decreases as the depth of penetration decreases. At the instant when the tool disengages from the workpiece the thrust force quickly reduces to zero (pseudo-impact force). The general result is a thrust force profile that has a boxcar appearance as depicted in Figure C-12. The Fourier transform of that force profile has a fundamental component at the same frequency as the desired wavy surface, and a significant component at twice the frequency of the desired wavy surface. Since the workpiece and its attachment to the spindle essentially form a series of mechanical springs (with some damping), the interrupted cutting causes second harmonic motion of the workpiece relative to the tool tip. The tool tip experiences the same second harmonic of the thrust force, and resists it only to the extent of its passive and dynamic stiffness at that frequency.

Figure C-13 shows the surface measurement of the outer diameter of a workpiece machined with the $2 \mathrm{kHZ}$ FTS when using an excessively aggressive first depth-of-cut with the sinusoidal tool tip trajectory. Note the presence of the second harmonic in the two-dimensional profile across the grooves. While machining the surface shown in Figure C-13, the tool position feedback signal did not have a noticeable second harmonic component in it. Also, a similar second harmonic did not appear when machining the face of a workpiece with the same cutting sequence that was used to produce the surface shown in Figure C-13. These two facts suggest that the second harmonic motion responsible for the surface shown in Figure C-13 occurred on the 


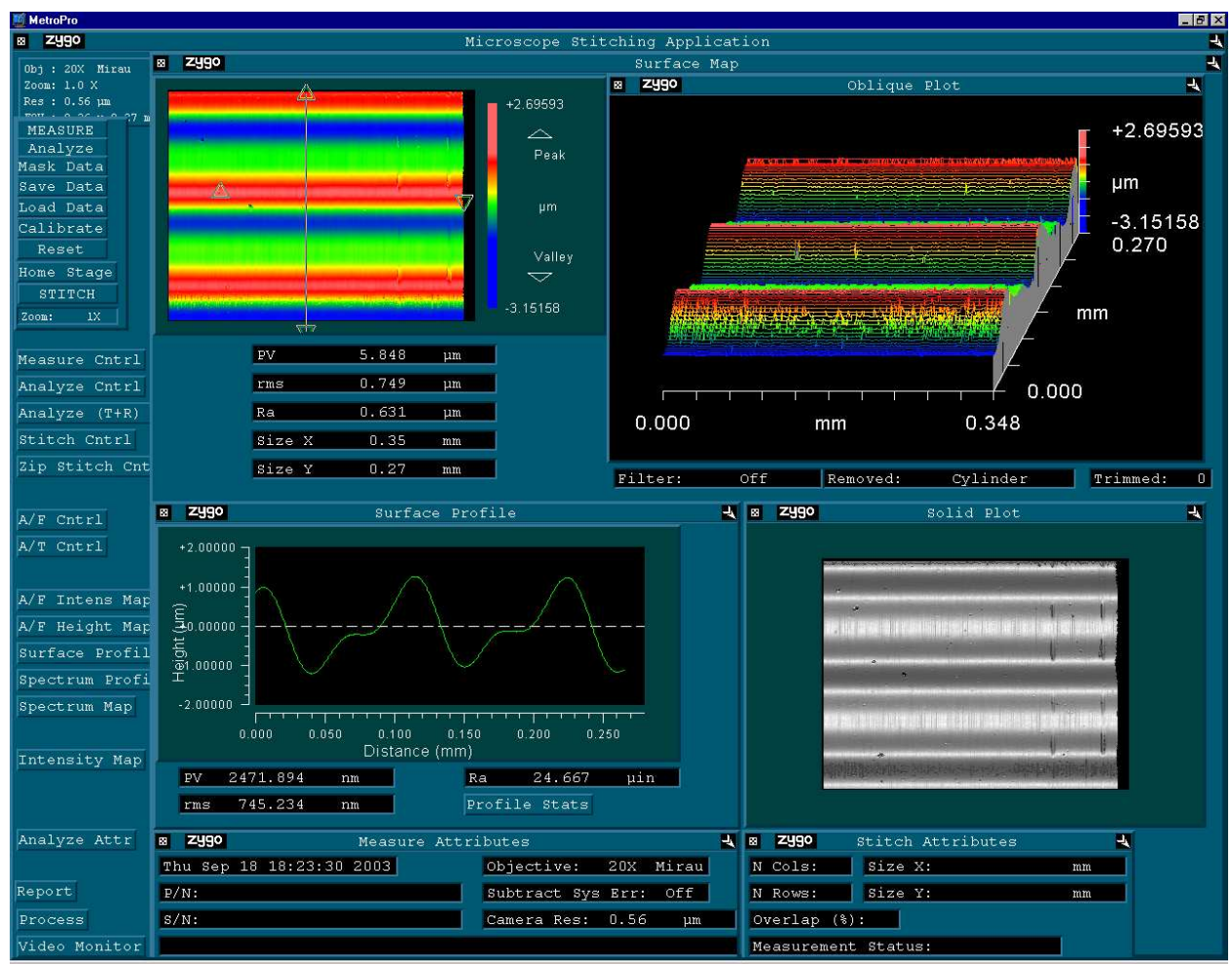

Figure C-13: Surface measurement of the outer diameter of a workpiece machined with the $2 \mathrm{kHZ}$ FTS when using an excessively aggressive first depth-of-cut with the sinusoidal tool tip trajectory.

workpiece side of the tool/workpiece interface, and is due to the lower radial-direction stiffness of the supported workpiece compared to its axial-direction stiffness.

Figure C-13 should be compared with Figure 3-63, which shows the surface of the outside diameter of a workpiece machined with the $2 \mathrm{kHz}$ FTS using a preferred sequence of machining passes that has a less aggressive series of depths-of-cut associated with the sinusoidal tool path. The surface shown in Figure 3-63 does not have a noticeable second harmonic component in it.

Referring to the preferred sequence of machining passes shown in Figure C-12, notice that the last machining pass keeps the tool constantly engaged in the workpiece. In practice, that machining pass would be followed by additional passes to improve the figure and roughness of the finished surface. 


\section{C.6 Preferred Sequences of Machining Passes used to Produce the Wavy Surfaces}

This section presents the two preferred cutting sequences that I used to machine the sinusoidal surfaces on the face and outside diameter of 6061 T6 aluminum workpieces with the $2 \mathrm{kHz}$ FTS. The first sequence uses the fast tool servo to produce a smooth surface on the workpiece and establish a known relationship between the FTS and the workpiece. The second sequence uses the fast tool servo to produce the wavy surface on the workpiece. Neither of the sequences are optimized from the standpoint of workpiece throughput, but they did allow me to produce the desired wavy surfaces with a $1012 \mathrm{~nm}$ rms surface roughness. I used food-grade mineral oil for the cutting fluid. A clean drop was applied to the tool tip while gently wiping it with a saturated cotton swab after each machining pass.

The finished surfaces have 250 grooves per revolution with a sinusoidal profile of $2.2 \mu \mathrm{m}$ PP. The workpiece was rotating at 480 RPM (2000 grooves per second). The diamond tool used in the FTS has a tool nose radius of 0.001 inch. The theoretical surface produced by a round-nose tool is a series of circular cusps. The peak-to-valley height deviation $h_{p v}$ of the theoretical surface is related to the tool nose radius $R_{t n}$ and the feed $C_{f d}$ (the distance that the tool travels across the workpiece during one revolution of the workpiece spindle) by: $h_{p v} \approx\left(C_{f d}\right)^{2} /\left(8 R_{t n}\right)$. The RMS deviation of the theoretical surface from its average height is approximately equal to $\left(h_{p v} / 3.35\right) .{ }^{1}$

It is worth mentioning that the first sequence was preceded by a "roughing" cut using a cubic boron nitride $(\mathrm{CBN})$ tool in a fixed tool holder. The roughing cut trues the surfaces of the workpiece to the spindle axis of rotation, and typically involves heavier material removal than the subsequent machining sequences. A CBN tool with a nose radius of 0.040 inch was used for faster material removal and to limit the wear on the diamond tool used in the fast tool servo. ${ }^{2}$

\footnotetext{
${ }^{1}$ The relationship between the peak-to-valley height and the root-mean-square deviation from the average height for the theoretical surface is derived by approximating the circular cusps as a series of parabolic curves. The parabolic approximation simplifies the math, and is a sufficiently accurate model because of the low aspect ratio between the height and width of the circular cusps.

${ }^{2}$ Although 6061 T6 is readily diamond turned, it can contain hard inclusions that will destroy the
} 
Table C.1: Preferred cutting sequence used to produce the initial smooth (non-wavy) surface on a workpiece using the $2 \mathrm{kHz}$ FTS. The fast tool servo was commanded to hold a constant tool tip position. Tool nose radius $=0.001$ inch, spindle speed $=480$ RPM, depth-of-cut = "DOC". Each machining pass has a total feed distance of 0.200 inch, which is what the "time" is based on. This machining sequence was preceded by a sequence that used a CBN tool in a fixed tool holder to true the part to the spindle axis of rotation.

\begin{tabular}{|c|c|c|c|c|c|c|}
\hline \multirow{2}{*}{$\begin{array}{c}\text { DOC } \\
(\mu \mathrm{in})\end{array}$} & Feedrate & Time & \multirow{2}{*}{$\begin{array}{c}\text { Feed } \\
\text { inin })\end{array}$} & $(\mathrm{min})$ & $(\mu \mathrm{in} / \mathrm{rev})$ & \multicolumn{3}{|c|}{ Theoretical surface finish } \\
\hline 500 & 0.25 & 0.8 & 520 & 34 & 850 & 250 \\
\hline 200 & 0.25 & 0.8 & 520 & 34 & 850 & 250 \\
\hline 100 & 0.025 & 8 & 52 & 0.34 & 8.5 & 2.5 \\
\hline 50 & 0.025 & 8 & 52 & 0.34 & 8.5 & 2.5 \\
\hline 50 & 0.025 & 8 & 52 & 0.34 & 8.5 & 2.5 \\
\hline
\end{tabular}

Tables C.1 and C.2 are the preferred cutting sequences that I used to produce the initial smooth surfaces and wavy surfaces on a workpiece, respectively. Referring to Table C.2, note that the amplitude $A_{s w}$ of the commanded sine wave starts off at a small value for the first two machining passes: the first pass is an interrupted cut, and the second pass is almost a continuous cut. The commanded amplitude is then incrementally increased to the desired final value during subsequent passes, which are all nominally continuous cuts. The minimum depth-of-cut of is $25-50 \mu$ in because the diamond turning machine was not in a temperature-controlled room; it was likely that a finer depth-of-cut would result in the tool and workpiece thermally drifting out of engagement during a machining pass.

cutting edge of a diamond tool. A better choice of material for the workpiece is C10100 oxygen-free electronic copper, which is what a second set of workpieces are made from. However, I produced adequate results with the aluminum workpieces so did not continue beyond them. 
Table C.2: Preferred cutting sequence used to produce the wavy surface on a workpiece using the $2 \mathrm{kHz}$ FTS. The fast tool servo was commanded to produce a sinusoidal tool tip trajectory of 250 cycles/rev. In this case, the depth-of-cut is the displacement of the average tool tip position from the previous machining pass. $A_{s w}$ is the amplitude of the commanded sine wave. Tool nose radius $=0.001 \mathrm{inch}$, spindle speed $=480$ RPM, depth-of-cut = "DOC". Each machining pass has a total feed distance of 0.200 inch, which is what the "time" is based on. This machining sequence was preceded by the sequence shown in Table C.1.

\begin{tabular}{|c|c|c|c|c|c|c|c|}
\hline DOC & $A_{s w}$ & Feedrate & Time & Feed & \multicolumn{3}{|c|}{ Theoretical surface finish } \\
\cline { 6 - 8 }$(\mu \mathrm{in})$ & $(\mu \mathrm{in})$ & $(\mathrm{in} / \mathrm{min})$ & $(\mathrm{min})$ & $(\mu \mathrm{in} / \mathrm{rev})$ & $\mathrm{pv}(\mu \mathrm{in})$ & $\mathrm{pv}(\mathrm{nm})$ & $\mathrm{rms}(\mathrm{nm})$ \\
\hline 0 & 25 & 0.025 & 8 & 52 & 0.34 & 8.5 & 2.5 \\
\hline 25 & 25 & 0.025 & 8 & 52 & 0.34 & 8.5 & 2.5 \\
\hline 25 & 25 & 0.025 & 8 & 52 & 0.34 & 8.5 & 2.5 \\
\hline 35 & 37 & 0.025 & 8 & 52 & 0.34 & 8.5 & 2.5 \\
\hline 50 & 50 & 0.025 & 8 & 52 & 0.34 & 8.5 & 2.5 \\
\hline
\end{tabular}

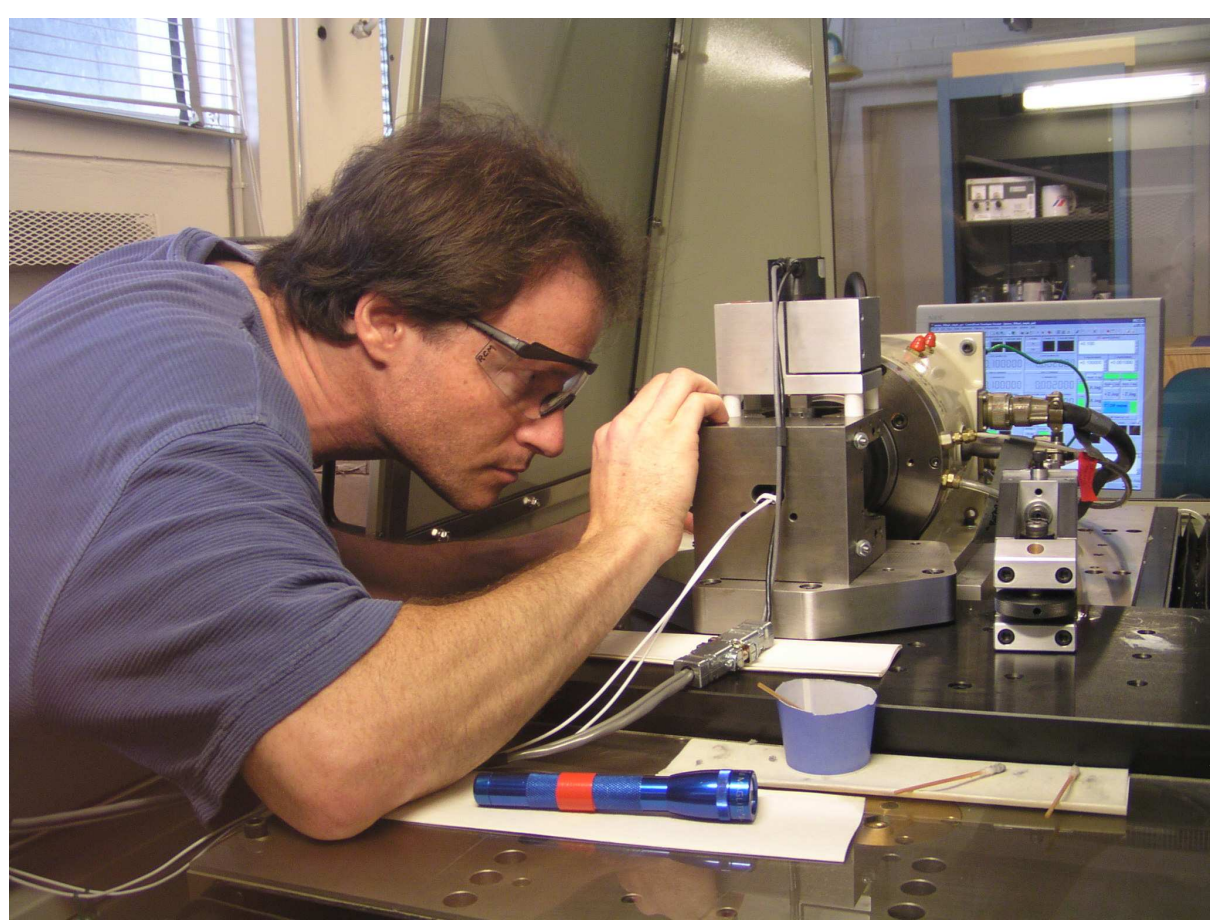

Figure C-14: The author carefully jogging the Z-axis slide of the DTM while establishing initial contact between the diamond tool carried by the $2 \mathrm{kHz}$ FTS and a rotating workpiece. My right hand is holding a magnifying glass that aids observing the first appearance of machining chips on the tool, which indicates contact between the tool and workpiece. My left hand is holding the computer mouse used to select functions in the graphical user interface for the machine, which is visible on the rightside of the figure. Note that the disturbance rejection for the Z-axis slide is sufficient enough for my arm to be resting on the slide while executing a commanded move. 


\section{Bibliography}

[1] Chardon Tool. PO Box 291, 11993 Ravenna Road, Chardon, OH 44024. http://www.chardontool.com/.

[2] The Department of Energy's Budget Request for FY 2000, Hearing of the U.S. Senate Committee on Armed Services, Subcommittee on Strategic Forces.

February 26, 1999, http://www.llnl.gov/director/testimony/cbt7_testimony/accomplish2.html.

[3] Gravure Association of America, Inc. 1200-A Scottsville Road, Rochester, NY 14624. http://www.gaa.org.

[4] HELL Gravure Systems GmbH. Philipp-Reis-Weg 5, 24148 Kiel / Germany. http://www.hell-gravure-systems.com.

[5] Max Daetwyler Corporation. 13420 Reese Boulevard West, Huntersville, NC 28078 USA. http://www.daetwyler.com.

[6] Photoengraving. Encyclopædia Britannica. 2004. Encyclopædia Britannica Online. 26 Jan. 2004 http://www.search.eb.com/eb/article?eu=117319.

[7] Precision Motion Control Laboratory at the Massachusetts Institute of Techology. 77 Massachusetts Avenue, Room 35-030, Cambridge, MA 02139. http://web.mit.edu/pmc/www/.

[8] 3M Industrial Adhesives and Tapes Division, 3M Center, Building 21-1W-10, 900 Bush Avenue, St. Paul, MN 55144. http://www.3m.com/us/mfg_industrial/adhesives/. 
[9] ADE Technologies, 77 Rowe Street, Newton, MA 02166, 617-831-8000.

[10] P.D. Agarwal. Eddy-current losses in solid and laminated iron. Trans. AIEE 78, page 169, 1959.

[11] Woronko A. Altintas, Y. A piezo tool actuator for precision turning of hardened shafts. Annals of the CIRP, 51(1):303-306, 2002.

[12] Apex Microtechnology Corporation, 5980 N. Shannon Road, Tucson, AZ 85741, 520-690-8600. http://eportal.apexmicrotech.com/mainsite/index.asp.

[13] Shunsuke Arakawa. Hitachi Metals America, Ltd. Personal communication. March 10, 2004.

[14] The Arnold Engineering Company, 770 Linden Avenue, Rochester, NY 146252764, http://www.arnoldmagnetics.com. Soft Magnetics Application Guide "February 2003 Rev. B".

[15] Walter Baar, Herbert Koehler, and Walter Muecke. Engraving device for a printing form engraving machine. U.S. Patent 3,964,382, June 1976.

[16] John R. Barnes. Electronic System Design: Interference and Noise Control Techniques. Prentice-Hall, 1987.

[17] P. Baudouin and Y. Houbaert. The study of a uniaxial deformation effect on the magnetic properties of a non-oriented electical steel using acoustic emission characterization. Journal of Magnetism and Magnetic Materials, 246(1-2):247$253,2002$.

[18] C.F. Beards. Engineering Vibration Analysis with Application to Control Systems. Halsted Press, 1996.

[19] H. Wayne Beaty and James L. Kirtley, Jr. Electric Motor Handbook. McGrawHill, 1998.

[20] Tony D. Beckett, Kenneth William Jackson, and David R. Seitz. Intaglio engraving method and apparatus. U.S. Patent 5,675,420, October 1997. 
[21] Tony D. Beckett, Kenneth William Jackson, and David R. Seitz. Engraving system and method for engraving inatglio and non-intaglio patterns. U.S. Patent $6,525,839$ B1, February 2003. Continuation of application No. 08/886,488, filed on Jul. 1, 1997, now Pat. No. 5,892,589, which is a continuation of application No. 08/376,858, filed on Jan. 23, 1995, now Pat. No. 5,675,420.

[22] James T. Berling. Magnetically powered linear displacement apparatus. U.S. Patent 5,719,543, February 1998.

[23] William H. Beyer. CRC Standard Mathematical Tables. CRC Press, 26th edition, 1981.

[24] Richard Boll. Soft Magnetic Materials: Fundamentals, Alloys, Properties, Products, Applications; The Vacuumschmelze Handbook. Heyden \& Son Ltd., 1978. Originally published as: Weichmagnetische Werkstoffe, Von Richard Boll, Siemens Aktiengesellschaft, 1977.

[25] Kenneth F. Bornhorst, Jr., David R. Seitz, and James E. Klinger. Engraving head with cartridge mounted components. U.S. Patent 5,475,914, December 1995.

[26] Branson Ultrasonics Corporation, 41 Eagle Road, Danbury, CT. 06813, 203796-2298. http://www.bransonic.com/chemistry.asp.

[27] Lester W. Buechler. Engraving apparatus and method. U.S. Patent 4,357,633, November 1982.

[28] Lester W. Buechler. Engraving apparatus and method. U.S. Patent 4,450,486, May 1984. Division of Ser. No. 056,623, Jul. 11, 1979, Pat. No. 4,357,633.

[29] Lester W. Buechler. Engraving apparatus and method. U.S. Patent 4,438,460, March 1984.

[30] Lester W. Buechler. Method and apparatus for engraving using a magnetostrictive actuator. U.S. Patent 5,491,559, February 1996. 
[31] Lester W. Buechler. Method and apparatus for engraving using a magnetostrictive actuator. U.S. Patent 5,671,064, September 1997. Continuation of Ser. No. 334,740, Nov. 4, 1994, Pat. No. 5,491,559.

[32] Lester W. Buechler. Method and apparatus for engraving using a magnetostrictive actuator. U.S. Patent 5,731,881, March 1998. Continuation-in-part of Ser. No. 433,083, May 3, 1995, Pat. No. 5,671,064, which is a continuation of Ser. No. 334,740, Nov. 4, 1994, Pat. No. 5,491,559.

[33] Marten Byl. Design and Control of a Long Stroke Fast Tool Servo. PhD thesis, Massachusetts Institute of Technology, June 2005.

[34] Cambridge Technology Incorporated, 109 Smith Place, Cambridge, MA 02138, 617-441-0600. Model 6880 Galvanometer Optical Scanner Instruction Manual, Revision 0, February 10, 1999, page 3.

[35] Carpenter Technology Corporation, 2 Meridian Blvd., Wyomissing, PA 196101339 , http://www.cartech.com. Alloy Data: Carpenter High Permeability “49” Alloy.

[36] Carr Lane Manufacturing Co., 4200 Carr Lane Ct., P.O. Box 191970, St. Louis, MS, 63119. Trigonometry Tables and Handy References for Engineers, 2001. http://www.carrlane.com.

[37] Joseph Harry Cattell. Adaptive feedforward cancellation viewed from an oscillator amplitude control perspective. Master's thesis, Massachusetts Institute of Technology, May 2003.

[38] Chih-Wen Chen. Magnetism and Metallurgy of Soft Magnetic Materials. Dover Publications, Inc., 1986. Originally published by the North-Holland Publishing Company, Amsterdam, 1977.

[39] Cooner Wire, Inc., 9265 Owensmouth Ave, Chatsworth, CA, 91311, 818-8828311. http://www.coonerwire.com. 
[40] Stephen H. Crandall and Norman C. Dahl. An Introduction to the Mechanics of Solids. McGraw-Hill, second edition, 1972.

[41] Albert Crewe, George B. Thiel, Douglas R. Adler, and Riyazhassan M. Asaria. Method and appartauts for high resolution sensing of engraving stylus movement. U.S. Patent 5,818,605, October 1998.

[42] Crystal Engineering Co., Inc., 2 Stanley Tucker Drive, Newburyport, MA 01950, 978-465-7007. http://www.crystalengineering.com.

[43] J.F. Cuttino et al. Performance optimization of a fast tool servo for single-point diamond. IEEE/ASME Transactions on Mechatronics, 4(2), June 1999.

[44] Isaac M. Daniel and Ori Ishai. Engineering Mechanics of Composite Materials. Oxford University Press, 1994.

[45] Robert A. DeBoalt. Method for tuning the resonant frequency of crossed-flexure pivot galvanometers. U.S. Patent 6,265,794, July 2001.

[46] Nicholas DeCristofaro. Amorphous Metals in Electric-Power Distribution Appications. Re-published electronically 2002, Metglas Solutions, http://www.metglas.com. Originally published by: Materials Research Society, MRS Bulletin, Volume 23, Number 5 (1998), p. 50 - 56, http://www.mrs.org/publications/bulletin.

[47] Lan Donner. Magnetic Metals Corporation, Engineer. Personal communications. February 23 and March 10, 2004.

[48] S.S. Douglass. A machining system for turning non-axis-symmetric surfaces. $\mathrm{PhD}$ thesis, University of Tennessee, Knoxville, 1983.

[49] T.A. Dow, M.H. Miller, and P.J. Falter. Application of a fast tool servo for diamond turning of nonrotationally symmetric surfaces. Precision Engineering, $13(4), 1991$. 
[50] Norman E. Dowling. Mechanical Behavior of Materials: Engineering Methods for Deformation, Fracture, and Fatigue. Prentice Hall, 1993.

[51] dSPACE Inc., 28700 Cabot Drive, Suite 1100, Novi, MI 48377, 248-567-1300. http://www.dspaceinc.com/.

[52] DuPont Performance Lubricants, Barley Mill Plaza, Bldg. 23, P.O. Box 80023 Wilmington, DE 19880, 800-424-7502. Data sheet W-400105, (9/02), http://www.krytox.com/.

[53] M. Emura et al. The influence of cutting technique on the magnetic properties of electrical steels. Journal of Magnetism and Magnetic Materials, (254).

[54] Equipment Express, 4744 E. Oak Knoll Rd., Rolling Prairie, IN 46371, 1-219778-8080. http://www.equipmentexpress24.com/.

[55] K.J. Falter and D.H. Youden. The characterization and testing of a long stroke fast tool servo. In International Progress in Precision Engineering: Proceedings of 8th International Precision Engineering Seminar, pages 225-228, 1995.

[56] A.E. Fitzgerald, Charles Kingsley, Jr., and Stephen D. Umans. Electric Machinery. McGraw-Hill, sixth edition, 2003.

[57] Charles J. Flynn. Methods for controlling the path of magnetic flux from a permanent magnet and devices incorporating the same. U.S. Patent 6,342,746, January 2002.

[58] The American Society for Metals. Metals Handbook, Volume 1, Properties and Selection: Irons, Steels, and High-Performance Alloys. ASM International, 1990.

[59] Kim R. Fowler. Electronic Instrument Design: Architecting for the Life Cycle. Oxford University Press, 1996.

[60] Gene F. Franklin, J. David Powell, and Abbas Emami-Naeini. Feedback Control of Dynamic Systems. Prentice Hall, fourth edition, 2002. 
[61] John W. Fraser. Personal communication. February 9, 2004.

[62] John W. Fraser. Engraving apparatus with oscillatory movement of tool support shaft monitored and controlled to reduce drift and vibration. U.S. Patent 5,029,011, July 1991.

[63] John W. Fraser and Curtis Woods. Apparatus and method for driving a leadscrew. U.S. Patent 5,329,215, July 1994.

[64] Thomas J. Glover. Pocket Reference. Sequoia Publishing. Third Edition, 2003.

[65] W. Greene and Shinstock D. Design of a linear voice coil actuator for fast tool servo applications. In Proceedings of the American Society for Precision Engineering $199^{` 7}$ Annual Meeting, 1997.

[66] Kohji Hada and Tadashi Syudou. Control circuit for controlling stylus overshoot in an engraving machine used for engraving gravure cylinders and method for same. U.S. Patent 5,663,801, September 1997.

[67] P. Hammond. Electromagnetism for Engineers: An Introductory Course. Oxford University Press, fourth edition, 1997.

[68] Bernard J. Hamrock, Steven R. Schmid, and O. Jackobson, Bo. Fundamentals of Machine Elements. McGraw-Hill, second edition.

[69] Francis G. Hanejko. Hoeganaes Corporation. Personal communication. March 3,2004 .

[70] Francis G. Hanejko, George W. Ellis, and Timothy J. Hale. Application of High Performance Material Processing - Electromagnetic Products. Hoeganaes Corporation, Cinnaminson, NJ, 08077, http://www.hoeganaes.com. Presented at PM2TEC'98 International Confernece on Powder Metallurgy \& Particulate Materials, Las Vegas, NV, May 31 - June 4, 1998.

[71] Francis G. Hanejko ${ }^{1}$, Hung G. Phan ${ }^{1}$, Howard G. Rutz ${ }^{1}$, and Tom L. Stuart ${ }^{2}$. Powder Metallurgy Materials for AC Magnetic Applications. (1) Hoeganaes 
Corporation, Riverton, NJ, 08077, http://www.hoeganaes.com. (2) Delphi Energy \& Engine Management Tech. Center, Delphi Automotive Systems, Division GMC, Indianapolis, IN 46256. Presented at PM²TEC '96 World Congress, Washington D.C., June 16-21, 1996.

[72] Hitachi Metals America, Ltd., Chicago Office, 2101 S.Arlington Heights Road, Suite 116, Arlington Heights, IL 60005-4142, http://www.hitachimetals.co.jp/e/prod/prod02/pdf/p02_28.pdf. Finemet EMC Components Catalog.

[73] Hitachi Metals America, Ltd., Chicago Office, 2101 S.Arlington Heights Road, Suite 116, Arlington Heights, IL 60005-4142, http://www.hitachimetals.co.jp/e/prod/prod02/pdf/hl-fm10.pdf. Finemet Nanocrystalline Soft Magnetic Material Catalog.

[74] HM Wire International, PO BOX 9181, Canton, OH, 44711, 330-244-8501. http://www.litz-wire.com/.

[75] Hoeganaes Corporation, 1001 Taylors Lane, Cinnaminson, NJ 08077-2017, 856829-2220. http://www.hoeganaes.com/.

[76] Paul L. Holowko, John W. Fraser, Eric J. Serenius, and Curtis Woods. Apparatus and method for engraving a gravure printing cylinder. U.S. Patent 5,424,845, June 1995.

[77] Paul L. Holowko, David R. Seitz, and Curtis Woods. Error detection apparatus and method for use with engravers. U.S. Patent 5,438,422, August 1995. Continuation-in-part of Ser. No. 22,127, Feb. 25, 1993.

[78] Paul Horowitz and Winfield Hill. The Art of Electronics. Cambridge University Press, second edition, 1989.

[79] Charles I. Hubert. Electric Machines: Theory, Operation, Applications, Adjustment, and Control. Prentice Hall, second edition, 2002. 
[80] ASTM International. D 2651-01, Standard Guide for Preparation of Metal Surfaces for Adhesive Bonding. 100 Barr Harbor Drive, PO Box C700, West Conshohocken, PA 19428, 2001.

[81] ASTM International. D 3933-98, Standard Guide for Preparation of Aluminum Surfaces for Strutural Adhesives Bonding (Phosphoric Acid Anodizing). 100 Barr Harbor Drive, PO Box C700, West Conshohocken, PA 19428, 2004.

[82] SAE International. ARP1575A, Surface Preparation and Priming of Aluminum Alloy Parts for High Durability Structural Adhesive Bonding: Hand Applied Phosphoric Acid Anodizing. 400 Commonwealth Drive, Warrendale, PA 15096, 1990.

[83] Tomoo Ito. Engraving head. U.S. Patent 6,099,177, August 2000.

[84] B. Jared et al. Fabrication of surface perturbation on inertial confinement fusion targets. In Proceedings of the American Society for Precision Engineering 1996 Annual Meeting, 1996.

[85] David Jiles. Magnetism and Magnetic Materials. Chapman \& Hall/CRC), second edition, 1998.

[86] Robert M. Jones. Mechanics of Composite Materials. Taylor and Francis, second edition, 1999.

[87] S. Kaji et al. Ultraprecision grinding of large silicon wafer using a rotary fast tool servo. In European Society for Precision Engineering and Nanotechnology: Proceedings of 2nd International Conference, Turin, Italy. May 27-31, 2001.

[88] Kaman Aerospace Corporation, Kaman Instrumentation Operations, 3450 North Nevada Avenue, Colorado Springs, CO 80907, 719-635-6979.

[89] J.L. Kirtley, Jr. Introduction to power systems, class notes chapter 2, ac power flow in linear networks. 
[90] E. Kuono. A fast response piezoelectric actuator for servo correction of systematic errors in precision machining. Annals of the CIRP, 33(1):369-372, 1984.

[91] Lawrence Livermore National Laboratory, 7000 East Avenue, Livermore, CA 94551. http://www.llnl.gov/.

[92] Lawrence Livermore National Laboratory. Inertial Confinement Fusion 1999 Annual Report. UCRL-LR-105820-99, October 1998-September 1999, http://www.llnl.gov/nif/icf/icfpubs/annuals/99_Annual.pdf.

[93] Lawrence Livermore National Laboratory. The Role of NIF in Developing Inertial Fusion Energy. UCRL-52000-94-12, December 1994, http://www.llnl.gov/etr/pdfs/12_94.pdf.

[94] Loctite Aerospace, Loctite Corporation, 2850 Willow Pass Road, P.O. Box 312, Bay Point, CA 94565, 925-458-8000. http://www.loctite.com.

[95] George E. Losier. Electric machine. U.S. Patent 2,092,765, September 1937.

[96] X. Lu and D.L. Trumper. High bandwidth fast tool servo control. In Proceedings of the 2004 American Control Conference, Boston, MA. June 30-July 2, 2004.

[97] X. Lu and D.L. Trumper. Electromagnetically driven fast tool servo. In Proceedings of the American Society for Precision Engineering 2003 Annual Meeting, volume 30, pages 103-106, 2003.

[98] Xiaodong Lu. Electromagnetically-Driven Ultra-Fast Tool Servos for Diamond Turning. PhD thesis, Massachusetts Institute of Technology, September 2005.

[99] Stephen Joseph Ludwick, Jr. A Rotary Fast Tool Servo for Diamond Turning of Asymmetric Optics. PhD thesis, Massachusetts Institute of Technology, June 1999.

[100] Magnetic Metals, 2475 LaPalma Avenue, Anaheim, CA 92801, 714-828-4625. http://www.magmet.com. 
[101] Magnetic Metals Corporation, 2475 LaPalma Ave., Anaheim, CA 92801, http://www.magmet.com. Wound Magnetics "Specifications02A_2001".

[102] A. Makino, T. Bitoh, A. Kojima, A. Inoue, and T. Masumoto. Low core losses of nanocrystalline Fe-Zr-Nb-B soft magnetic alloys with high magnetic flux density. Materials Science \& Engineering A, 304-306:1083-1086, 2001.

[103] Michel Mardiguian. EMI Troubleshooting Techniques. McGraw-Hill, 2000.

[104] The MathWorks, Inc., 3 Apple Hill Drive, Natick, MA 01760, 508-647-7000. http://www.mathworks.com/.

[105] Colonel William T. McLyman. Transformer and Inductor Design Handbook. Marcel Dekker, second edition, 1988.

[106] Metglas Inc., 440 Allied Drive, Conway, SC 29526, http://www.metglas.com. Magnetic Alloy 2605SA1 (Iron-based) Technical Bulletin.

[107] R.C. Montesanti and S.L. Thompson. Unpublished work, circa 1997.

[108] R.C. Montesanti and S.L. Thompson. A procedure for diamond turning KDP crystals. Lawrence Livermore National Laboratory Technical Report, UCRLID-121651, July 7, 1995.

[109] R.C. Montesanti, D.L. Trumper, and J.L. Klingmann. Extremely highbandwidth, diamond-tool axis for weapons-physics target fabrication. FY02 Engineering Technology Reports Volume 2: Laboratory Directed Research and Development. UCRL-53969-02, March 2003.

[110] Moore Nanotechnolgy Systems, LLC, 426A Winchester St., PO Box 605, Keene, NH 03431, 603-352-3030. http://www.nanotechsys.com/.

[111] MWS Wire Industries, 31200 Cedar Valley Drive, Westlake Village, CA 91362, 818-991-8553. http://www.mwswire.com.

[112] Samir Nayfeh. Some notes on the theory of elasticity. 
[113] New England Wire Technologies, 130 North Main Street, Lisbon, NH 03585, 603-838-6624. http://www.newenglandwire.com.

[114] Norman S. Nise. Control Systems Engineering. John Wiley \& Sons, third edition, 2000.

[115] Erik Oberg, Franklin D. Jones, and Holbrook L. Horton. Machinery's Handbook. Industrial Press Inc. Twenty-First Edition, Third Printing, 1980.

[116] Erik Oberg, Franklin D. Jones, Holbrook L. Horton, Henry H. Ryffel, Riccardo M. Heald, and Muhammed Igbal Hussain. Machinery's Handbook. Industrial Press Inc. Twenty-Seventh Edition, 2004.

[117] Y. Okazaki. Fast tool servo system and its application to three dimensional fine surface figures. In Proceedings of the American Society for Precision Engineering 1998 Annual Meeting, 1998.

[118] Henry W. Ott. Noise Reduction Techniques in Electronic Systems. John Wiley \& Sons, second edition, 1988.

[119] K.J. Overshott and G.E. Foot. The effect of tensile stress on the power loss of 3\% grain-oriented silicon-iron. IEEE Transactions on Magnetics, (6):1496, 1982.

[120] Parametric Technology Corporation, 140 Kendrick Street, Needham, MA 02494, 781-370-5000. http://www.ptc.com/.

[121] S.R. Patterson and E.B. Magrab. The design and testing of a fast tool servo for diamond turning. Precision Engineering, 7(3):123, 1985.

[122] Edward M. Petrie. Handbook of Adhesives and Sealants. McGraw-Hill, 2000.

[123] Egor P. Popov. Engineering Mechanics of Solids. Prentice Hall, second edition, 1999.

[124] Precitech Inc., 44 Blackbrook Road, Keene, NH 03431, 603-357-2511. http://www.precitech.com/. 
[125] Professional Instruments Company, 7800 Powell Road, Hopkins, MN 55343, 952-933-1222. http://www.airbearings.com/.

[126] Albert Oskar Raible. Machine for producing a facsimile reproduction. U.S. Patent 2,925,464, February 1960.

[127] Singiresu S. Rao. Mechanical Vibrations. Addison-Wesley, third edition, 1995.

[128] J.D. Rasmussen et al. Dynamic variable depth of cut machining using piezoelectric actuators. International Journal of Machine Tools and Manufacture, 34(3):379-392, 1994.

[129] J.D. Rasmussen et al. A piezoelectric tool servo system for variable depth of cut machining. Precision Machining: Technology and Machine Development and Improvement, PED-58:119-130, ASME 1992.

[130] E.I. Rivin. Properties and prospective applications of ultra thin layered rubbermetal laminates for limited travel bearings. Tribology International, 18(1):444, 1983.

[131] Raymond J. Roark and Warren C. Young. Formulas for Stress and Strain. McGraw-Hill. Fifth Edition, 1975.

[132] James K. Roberge. Operational Amplifiers: Theory and Practice. John Wiley \& Sons, 1975.

[133] James K. Roberge. Electronic feedback systems, 1986.

[134] H.G. Rutz, F.G. Hanejko, and G.W. Ellis. The Manufacture of Electromagnetic Components by the Powder Metallurgy Process. Hoeganaes Corporation, Riverton, NJ, 08077, http://www.hoeganaes.com. Presented at $\mathrm{PM}^{2} \mathrm{TEC}$ '97, International Conference on Poweder Metallurgy \& Particulate Materials, Chicago, IL, June 29 - July 2, 1997.

[135] Graham M. Seed. Strength of Materials: An Undergraduate Text. Saxe-Coburg Publications, 2000. 
[136] Gerald Johannes Sermund. Method and device to control an engraving device. U.S. Patent 6,421,576 B1, July 2002.

[137] Joseph Edward Shigley. Mechanical Engineering Design. McGraw-Hill. Third Edition, 1977.

[138] Stuart T. Smith. Flexures: Elements of Elastic Mechanisms. Gordon and Breach Science Publishers, 2000.

[139] Philip A. Studer. Radial and torsionally controlled magnetic bearing. U.S. Patent 4,634,191, January 1987.

[140] Charles R. Sullivan. Optimal choice for number of strands in a litz-wire transformer winding. IEEE Transactions on Power Electronics, 14(2):283, 1999.

[141] Tektronix, Sales Office for Massachusetts, 610 Professional Drive, Suite 210, Gaithersburg, MD 20879, 800-833-9200. http://www.tek.com.

[142] Texas Instruments Inc., Product Information Center, 13532 N. Central Expressway, M/S 3807, Dallas, TX, 75243, 972-644-5580. http://focus.ti.com/analog/docs/analoghomepage.jsp.

[143] M.W. Todd and J.F. Cuttino. Development of a long range, traction drive fast tool servo for diamond turning applications. In Proceedings of the American Society for Precision Engineering 1997 Annual Meeting, 1997.

[144] Stephen W. Tsai. Theory of Composites Designs. Think Composites, 1992.

[145] T. Van Doren. Grounding and shielding electroinc systems. Notes from video seminar on the NTU Satellite Network, November 11 and 12., 1991.

[146] Masanobu Wada, Yujiro Koike, and Yoshihisa Okuma. Electronic engraving system. U.S. Patent 4,052,739, October 1977.

[147] M.H. Weck et al. A new hybrid concept for a long stroke fast tool servo system. In Proceedings of the American Society for Precision Engineering 1995 Annual Meeting, 1995. 
[148] Raymond F. Wegman. Surface Preparation Techniques for Adhesive Bonding. Noyes Publications, reprint edition, 1989.

[149] Frank M. White. Fluid Mechanics. McGraw-Hill, 1979.

[150] Theodore Wildi. Electric Machines, Drives, and Power Systems. Prentice Hall, fifth edition, 2002.

[151] M.E. Williams and D.L. Trumper. Materials for effiecient high-flux magnetic bearing actuators. In NASA Conference Publication \#3247, Part 1, pages 135145, 1993. Presented at the Second International Symposium on Magnetic Suspension Technology, Seattle, WA, August 11-13, 1993.

[152] Y. Yoshizawa, S. Oguma, and K. Yamauchi. New Fe-based soft magnetic alloys composed of ultrafine grain structure. Journal of Applied Physics, 64(10):60446046, 1988.

[153] Jun Martin B. Altintas Yusuf Zhu, Wen-Hong. A fast tool servo design for precision turning of shafts on conventional cnc lathes. International Journal of Machine Tools and Manufacture, 41:953-965, 2001.

[154] Zygo Corporation, Laurel Brook Road, Middlefield, CT 06455, 860-347-8506. http://www.zygo.com/. 